\title{
Guidelines for the Prevention and Treatment of Opportunistic Infections in HIV-Exposed and HIV-Infected Children:
}

\author{
Recommendations from the National Institutes of Health, Centers for Disease Control and \\ Prevention, the HIV Medicine Association of the Infectious Diseases Society of America, \\ the Pediatric Infectious Diseases Society, and the American Academy of Pediatrics
}

George K. Siberry, MD, MPH ${ }^{1}$ [Executive Secretary], Mark J. Abzug, MD² [Co-Chair], Sharon Nachman, MD ${ }^{3}$ [Co-Chair], Michael T. Brady, MD ${ }^{4}$, Kenneth L. Dominguez, MD, MPH $^{5}$, Edward Handelsman, MD ${ }^{1, \S}$, Lynne M. Mofenson, MD ${ }^{1}$, Steve Nesheim, MD $^{5}$, and the Panel on Opportunistic Infections in HIV-Exposed and HIV-Infected Children*

\begin{abstract}
${ }^{1}$ National Institutes of Health, Bethesda, Maryland ${ }^{2}$ University of Colorado School of Medicine and Children's Hospital Colorado, Aurora, Colorado ${ }^{3}$ State University of New York at Stony Brook, Stony Brook, New York ${ }^{4}$ Nationwide Children's Hospital, Columbus, Ohio ${ }^{5}$ Centers for Disease Control and Prevention, Atlanta, Georgia
\end{abstract}

\section{Summary (Last updated November 6, 2013; last reviewed November 6, 2013)}

\begin{abstract}
This report updates the last version of the Guidelines for the Prevention and Treatment of Opportunistic Infections (OIs) in HIV-Exposed and HIV-Infected Children, published in 2009. These guidelines are intended for use by clinicians and other health-care workers providing medical care for HIV-exposed and HIV-infected children in the United States. The guidelines discuss opportunistic pathogens that occur in the United States and ones that might be acquired during international travel, such as malaria. Topic areas covered for each OI include a brief description of the epidemiology, clinical presentation, and diagnosis of the OI in children; prevention of exposure; prevention of first episode of disease; discontinuation of primary prophylaxis after immune reconstitution; treatment of disease; monitoring for adverse effects during treatment, including immune reconstitution
\end{abstract}

\footnotetext{
*Panel member authors (in alphabetical order): Debika Bhattacharya, MD; Beverly Bohannon, MS, RN; Diana Clarke, Pharm. D.; Kathryn M. Edwards, MD; Jennifer C. Esbenshade, MD, MPH; Patricia Flynn, MD; Aditya Gaur, MD; Francis Gigliotti, MD; Gail Harrison, MD; Charlotte Victoria Hobbs, MD; David Kimberlin, MD; Martin B. Kleiman, MD; Emilia H. Koumans, MD, MPH; Andrew Kroger, MD, MPH; Myron J. Levin. MD; Cara L. Mack, MD; Ben J. Marais, MD; Gabriela Maron, MD; James McAuley, MD, MPH; Heather J. Menzies, MD, MPH; Anna-Barbara Moscicki, MD; Michael R. Narkewicz, MD; Richard Rutstein, MD; Jane Seward, MBBS, MPH; Masako Shimamura, MD; William J. Steinbach, MD; Gregory J. Wilson, MD.

$\$$ Dr. Handelsman, Branch Chief of the Maternal, Adolescent, and Pediatric Research Branch of the Division of AIDS at the National Institute of Allergy and Infectious Diseases, died unexpectedly on March 5, 2012. Dr. Handelsman was a pediatrician dedicated to the care of HIV-infected infants, children, adolescents, and pregnant women whose work and advocacy saved the lives and improved the health of thousands of children around the world.

Publisher's Disclaimer: This is a PDF file of an unedited manuscript that has been accepted for publication. As a service to our customers we are providing this early version of the manuscript. The manuscript will undergo copyediting, typesetting, and review of the resulting proof before it is published in its final citable form. Please note that during the production process errors may be discovered which could affect the content, and all legal disclaimers that apply to the journal pertain.
} 
inflammatory syndrome (IRIS); management of treatment failure; prevention of disease recurrence; and discontinuation of secondary prophylaxis after immune reconstitution. A separate document providing recommendations for prevention and treatment of OIs among HIV-infected adults and post-pubertal adolescents (Guidelines for the Prevention and Treatment of Opportunistic Infections in HIV-Infected Adults and Adolescents) was prepared by a panel of adult HIV and infectious disease specialists (see http:// aidsinfo.nih.gov/guidelines).

These guidelines were developed by a panel of specialists in pediatric HIV infection and infectious diseases (the Panel on Opportunistic Infections in HIV-Exposed and HIV-Infected Children) from the U.S. government and academic institutions. For each OI, one or more pediatric specialists with subject-matter expertise reviewed the literature for new information since the last guidelines were published and then proposed revised recommendations for review by the full Panel. After these reviews and discussions, the guidelines underwent further revision, with review and approval by the Panel, and final endorsement by the National Institutes of Health (NIH), Centers for Disease Control and Prevention (CDC), the HIV Medicine Association (HIVMA) of the Infectious Diseases Society of America (IDSA), the Pediatric Infectious Disease Society (PIDS), and the American Academy of Pediatrics (AAP). So that readers can ascertain how best to apply the recommendations in their practice environments, the recommendations are rated by a letter that indicates the strength of the recommendation, a Roman numeral that indicates the quality of the evidence supporting the recommendation, and where applicable, a $*$ notation that signifies a hybrid of higher-quality adult study evidence and consistent but lowerquality pediatric study evidence.

More detailed methodologic considerations are listed in Appendix 1 (Important Guidelines Considerations), including a description of the make-up and organizational structure of the Panel, definition of financial disclosure and management of conflict of interest, funding sources for the guidelines, methods of collecting and synthesizing evidence and formulating recommendations, public commentary, and plans for updating the guidelines. The names and financial disclosures for each of the Panel members are listed in Appendices 2 and 3, respectively.

An important mode of childhood acquisition of OIs and HIV infection is from infected mothers. HIV-infected women may be more likely to have coinfections with opportunistic pathogens (e.g., hepatitis C) and more likely than women who are not HIV-infected to transmit these infections to their infants. In addition, HIV-infected women or HIV-infected family members coinfected with certain opportunistic pathogens may be more likely to transmit these infections horizontally to their children, resulting in increased likelihood of primary acquisition of such infections in young children. Furthermore, transplacental transfer of antibodies that protect infants against serious infections may be lower in HIVinfected women than in women who are HIV-uninfected. Therefore, infections with opportunistic pathogens may affect not just HIV-infected infants but also HIV-exposed, uninfected infants. These guidelines for treating OIs in children, therefore, consider treatment of infections in all children-HIV-infected and HIV-uninfected-born to HIVinfected women. 
In addition, HIV infection increasingly is seen in adolescents with perinatal infection who are now surviving into their teens and in youth with behaviorally acquired HIV infection. Guidelines for postpubertal adolescents can be found in the adult OI guidelines, but drug pharmacokinetics (PK) and response to treatment may differ in younger prepubertal or pubertal adolescents. Therefore, these guidelines also apply to treatment of HIV-infected youth who have not yet completed pubertal development.

Major changes in the guidelines from the previous version in 2009 include:

- Greater emphasis on the importance of antiretroviral therapy (ART) for prevention and treatment of OIs, especially those OIs for which no specific therapy exists;

- Increased information about diagnosis and management of IRIS;

- Information about managing ART in children with OIs, including potential drugdrug interactions;

- Updated immunization recommendations for HIV-exposed and HIV-infected children, including pneumococcal, human papillomavirus, meningococcal, and rotavirus vaccines;

- Addition of sections on influenza, giardiasis, and isosporiasis;

- Elimination of sections on aspergillosis, bartonellosis, and HHV-6 and HHV-7 infections; and

- Updated recommendations on discontinuation of OI prophylaxis after immune reconstitution in children.

The most important recommendations are highlighted in boxed major recommendations preceding each section, and a table of dosing recommendations appears at the end of each section. The guidelines conclude with summary tables that display dosing recommendations for all of the conditions, drug toxicities and drug interactions, and 2 figures describing immunization recommendations for children aged 0 to 6 years and 7 to 18 years.

The terminology for describing use of antiretroviral (ARV) drugs for treatment of HIV infection has been standardized to ensure consistency within the sections of these guidelines and with the Guidelines for the Use of Antiretroviral Agents in Pediatric HIV Infection. Combination antiretroviral therapy (cART) indicates use of multiple (generally 3 or more) ARV drugs as part of an HIV treatment regimen that is designed to achieve virologic suppression; highly active antiretroviral therapy (HAART), synonymous with cART, is no longer used and has been replaced by cART; the term ART has been used when referring to use of ARV drugs for HIV treatment more generally, including (mostly historical) use of one- or two-agent ARV regimens that do not meet criteria for cART.

Because treatment of OIs is an evolving science, and availability of new agents or clinical data on existing agents may change therapeutic options and preferences, these recommendations will be periodically updated and will be available at http:// AIDSinfo.nih.gov. 


\section{Background (Last updated November 6, 2013; last reviewed November 6, 2013)}

\section{Opportunistic Infections in HIV-Infected Children in the Era of Combination Antiretroviral Therapy}

In the era before development of potent cART regimens, OIs were the primary cause of death in HIV-infected children. ${ }^{1}$ Current ART regimens suppress viral replication, provide significant immune reconstitution, and have resulted in a substantial and dramatic decrease in AIDS-related OIs and deaths in both adults and children. ${ }^{2-5}$

Despite this progress, prevention and treatment of OIs remain critical components of care for HIV-infected children. OIs continue to be the presenting symptom of HIV infection among children whose HIV-exposure status is unknown because of lack of maternal antenatal HIV testing. For infants and children with known HIV infection, barriers such as inadequate medical care, lack of availability of suppressive ART regimens in the face of extensive prior treatment and drug resistance, caregiver substance abuse or mental illness, and multifactorial adherence difficulties may hinder effective HIV treatment and put them at risk of OIs even in the ART era. These same barriers may then impede provision of primary or secondary OI prophylaxis to children for whom such prophylaxis is indicated. In addition, concomitant OI prophylactic drugs may only exacerbate the existing difficulties in adhering to ART. Multiple drug-drug interactions between OI, ARV, and other compounds that result in increased adverse events and decreased treatment efficacy may limit the choice and continuation of both cART and prophylactic regimens. Finally, IRIS, initially described in HIV-infected adults but also seen in HIV-infected children, can complicate treatment of OIs when cART is started or when optimization of a failing regimen is attempted in patients with acute OIs. Thus, prevention and treatment of OIs in HIV-infected children remains important even in the cART era.

\section{History of the Guidelines}

In 1995, the U.S. Public Health Service (USPHS) and IDSA developed guidelines for preventing OIs in adults, adolescents, and children infected with HIV. ${ }^{6}$ These guidelines, developed for health-care providers and their HIV-infected patients, were revised in 1997, 1999, and 2002..$^{-9}$ In 2001, NIH, IDSA, and CDC convened a working group to develop guidelines for treating HIV-associated OIs, with a goal of providing evidence-based guidelines on treatment and prophylaxis. In recognition of unique considerations for HIVinfected infants, children, and adolescents-including differences between adults and children in mode of acquisition, natural history, diagnosis, and treatment of HIV-related OIs -a separate pediatric OI guidelines writing group was established. The pediatric OI treatment guidelines were initially published in December 2004. ${ }^{10}$ In 2009, recommendations for preventing and treating OIs in HIV-exposed and HIV-infected children were updated and combined into one document; a similar document on preventing and treating OIs among HIV-infected adults, prepared by a separate group of adult HIV and infectious disease specialists, was developed at the same time. Both sets of guidelines were prepared by the Opportunistic Infections Working Group under the auspices of the Office of AIDS Research (OAR) of the NIH. For the current document, the Opportunistic Infections 
Working Group, again under the auspices of OAR, convened a new panel of pediatric specialists with expertise in specific OIs. The Panel reviewed the literature since the last publication of the prevention and treatment guidelines, conferred over several months, and produced draft guidelines. These draft guidelines were revised based on review by the full Panel and review and approval by the core writing group members. The final report was further reviewed by OAR, experts at CDC, the HIVMA of IDSA, the PIDS, and AAP before final approval and publication.

\section{Why Pediatric Prevention and Treatment Guidelines?}

Mother-to-child transmission is an important mode of acquisition of HIV infection and of OIs in children. HIV-infected women coinfected with opportunistic pathogens may be more likely than HIV-uninfected women to transmit these infections to their infants. For example, higher rates of perinatal transmission of hepatitis $\mathrm{C}$ and cytomegalovirus (CMV) have been reported from HIV-infected than from HIV-uninfected women. ${ }^{11,12}$ In addition, HIVinfected women or HIV-infected family members coinfected with certain opportunistic pathogens may be more likely to transmit these infections horizontally to their children, increasing the likelihood of primary acquisition of such infections in young children. For example, Mycobacterium tuberculosis infection in children primarily reflects acquisition from family members who have active tuberculosis (TB) disease, and increased incidence and prevalence of TB among HIV-infected individuals is well documented. HIV-exposed or HIV-infected children in the United States may have a higher risk of exposure to $M$. tuberculosis than would comparably aged children in the general U.S. population because of residence in households with HIV-infected adults. ${ }^{13}$ Furthermore, HIV-infected women may have transplacental transfer of lower levels of antibodies that protect their infants against serious bacterial infections than women who are not infected with HIV. ${ }^{14}$ Therefore, these guidelines for treatment and prevention of OIs consider both HIV-infected and HIVuninfected children born to HIV-infected women.

The natural history of OIs in children may differ from that in HIV-infected adults. Many OIs in adults are secondary to reactivation of opportunistic pathogens, which often were acquired before HIV infection when host immunity was intact. However, OIs in HIVinfected children more often reflect primary infection with the pathogen. In addition, among children with perinatal HIV infection, the primary infection with the opportunistic pathogen occurs after HIV infection is established at a time when the child's immune system already may be compromised. This can lead to different manifestations of specific OIs in children than in adults. For example, young children with TB are more likely than adults to have extrapulmonary and disseminated infection, even without concurrent HIV infection.

Multiple difficulties exist in making laboratory diagnoses of various infections in children. A child's inability to describe the symptoms of disease often makes diagnosis more difficult. For infections such as hepatitis $\mathrm{C}$ (for which diagnosis is made by laboratory detection of specific antibodies), transplacental transfer of maternal antibodies that can persist in infants for up to 18 months complicates the ability to make a diagnosis in young infants. Assays capable of directly detecting the pathogen are required to diagnose such infections definitively in infants. In addition, diagnosing the etiology of lung infections in children can 
be difficult because they usually do not produce sputum, and more invasive procedures (e.g., gastric aspirates, bronchoscopy, lung biopsy) may be needed to make a more definitive diagnosis.

Data related to the efficacy of various therapies for OIs in adults are often extrapolated to children, but issues related to drug PK, formulation, ease of administration, dosing, and toxicity require special considerations for children. Young children, in particular, metabolize drugs differently from adults and older children, and the volume of distribution differs. Unfortunately, data often are lacking on appropriate drug dosing recommendations for children aged $<2$ years.

The prevalence of opportunistic pathogens in HIV-infected children during the pre-ART era varied by child age, previous OI, immunologic status, and pathogen. ${ }^{1}$ During the pre-ART era, the most common OIs in children in the United States (event rates $>1$ per 100 childyears) were serious bacterial infections (most commonly pneumonia, often presumptively diagnosed, and bacteremia), herpes zoster, disseminated Mycobacterium avium complex (MAC), Pneumocystis jirovecii pneumonia (PCP), and candidiasis (esophageal and tracheobronchial disease). Less commonly observed OIs (event rate $<1$ per 100 child-years) included CMV disease, cryptosporidiosis, TB, systemic fungal infections, and toxoplasmosis. ${ }^{3,4}$ History of a previous AIDS-defining OI predicted development of a new infection. Although most infections occurred in substantially immunocompromised children, serious bacterial infections, herpes zoster, and TB occurred across the spectrum of immune status.

Descriptions of pediatric OIs in children receiving cART have been limited. Substantial decreases in mortality and morbidity, including OIs, have been observed among children receiving cART, as in HIV-infected adults. ${ }^{3,5}$ Although the number of OIs has substantially decreased during the cART era, HIV-associated OIs and other related infections continue to occur in HIV-infected children. ${ }^{3,15}$

In contrast to recurrent serious bacterial infections, some of the protozoan, fungal, or viral OIs complicating HIV are not curable with available treatments. Sustained, effective cART, resulting in improved immune status, has been established as the most important factor in controlling OIs in both HIV-infected adults and children. For many OIs, after treatment of the initial infectious episode, secondary prophylaxis in the form of suppressive therapy is indicated to prevent a recurrence of clinical disease as a result of re-activation or reinfection.

These guidelines are a companion to the 2013 Guidelines for Prevention and Treatment of Opportunistic Infections in HIV-Infected Adults and Adolescents. ${ }^{16}$ Treatment of OIs is an evolving science, and availability of new agents or clinical data on existing agents may change therapeutic options and preferences. As a result, these recommendations will need to be periodically updated.

Because the guidelines target HIV-exposed and HIV-infected children in the United States, the opportunistic pathogens discussed are those common to the United States and do not include certain pathogens such as Penicillium marneffei that may be seen almost exclusively outside the United States, that are common but seldom cause chronic infection (e.g., chronic 
parvovirus B19 infection), or that have the same risk, disease course, and approach to prevention and treatment in all children regardless of HIV status (e.g., streptococcal pharyngitis). The document is organized to provide information about the epidemiology, clinical presentation, diagnosis, and treatment of each pathogen. Major recommendations are accompanied by ratings that include a letter that indicates the strength of the recommendation and a Roman numeral that indicates the quality of the evidence supporting the recommendation; this rating system is similar to the rating systems used in other USPHS/IDSA guidelines. Because licensure of drugs for children often relies on efficacy data from adult trials in combination with safety data in children, recommendations sometimes may need to rely on data from clinical trials or studies in adults. Thus, the quality of evidence level is accompanied by a * notation to indicate that evidence supporting the recommendation is a hybrid of higher-quality adult study evidence and consistent but lowerquality pediatric study evidence. This modification to the rating system is the same as that used by the HHS Guidelines for the Use of Antiretroviral Agents in Pediatric HIV Infection panel.

The tables at the end of this document summarize recommendations for dosing of medications used for treatment and prevention of OIs in children (Tables 1-3), drug preparation and toxicity information for children (Table 4), and drug-drug interactions (Table 5). Vaccination recommendations for HIV-infected children and adolescents are presented in Figures 1 and 2 at the end of the document.

\section{Rating Scheme for Pediatric Opportunistic Infections Recommendations}

Recommendations are rated using the rating system noted in the Pediatric Opportunistic Infections Recommendations Rating Scheme below. The rating scheme includes explanatory text that reviews the evidence and the panel's assessment. The letters A, B, and C represent the strength of the recommendation for or against a preventive or therapeutic measure and are based on assessing the balance of benefits and risks of adhering compared to not adhering to the recommendation, and Roman numerals I, I*, II, II*, and III indicate the quality of evidence supporting the recommendation and are based on study design. Roman numerals with asterisks describe types of evidence where a higher quality of evidence exists for adults compared to children.

Strength of Recommendation Rating A-Strong-The benefit associated with adhering to the recommendation nearly always outweighs the risk of not adhering to the recommendation. The recommendation applies to most patients in most circumstances and should be adhered to by clinicians unless there exists a compelling rationale for an alternative approach.

Strength of Recommendation Rating B-Moderate-The benefit associated with adhering to the recommendation outweighs the risks of not adhering to the recommendation more often than not but not as frequently as a recommendation with an A Rating. The recommendation applies to many patients in some circumstances. 
Strength of Recommendation Rating C-Optional-It is unclear whether the benefits associated with adhering to the recommendation outweigh the risks of not adhering to the recommendation; other alternatives may be equally reasonable.

Quality of Evidence Rating I-Randomized Clinical Trial Data-In the absence of large pediatric randomized trials, adult data may be used if there are substantial pediatric data consistent with high-quality adult studies. Quality of Evidence Rating I will be used if there are data from large randomized trials in children with clinical and/or validated laboratory endpoints. Quality of Evidence Rating I* will be used if there are high-quality randomized clinical trial data in adults with clinical and/or validated laboratory endpoints and pediatric data from well designed, non-randomized trials or observational cohort studies with long-term clinical outcomes that are consistent with the adult studies. A rating of I* may be used for quality of evidence if, for example, a randomized Phase III clinical trial in adults demonstrates a drug is effective in ARV-naive patients and data from a nonrandomized pediatric trial demonstrate adequate and consistent safety and PK data in the pediatric population.

\section{Quality of Evidence Rating II-Non-Randomized Clinical Trials or} Observational Cohort Data-In the absence of large, well-designed, pediatric, nonrandomized trials or observational data, adult data may be used if there are sufficient pediatric data consistent with high-quality adult studies. Quality of Evidence Rating II will be used if there are data from well-designed, non-randomized trials or observational cohorts in children. Quality of Evidence Rating II* will be used if there are well-designed, nonrandomized trials or observational cohort studies in adults with supporting and consistent information from smaller nonrandomized trials or cohort studies with clinical outcome data in children. A rating of II* may be used for quality of evidence if, for example, a large observational study in adults demonstrates clinical benefit to initiating treatment at a certain CD4 T lymphocyte (CD4) cell count and data from smaller observational studies in children indicate that a similar CD4 count is associated with clinical benefit.

Quality of Evidence Rating III-Expert Opinion-Where neither clinical trial nor observational data exist, we rely on expert opinion.

Pediatric Opportunistic Infections Recommendations Rating Scheme

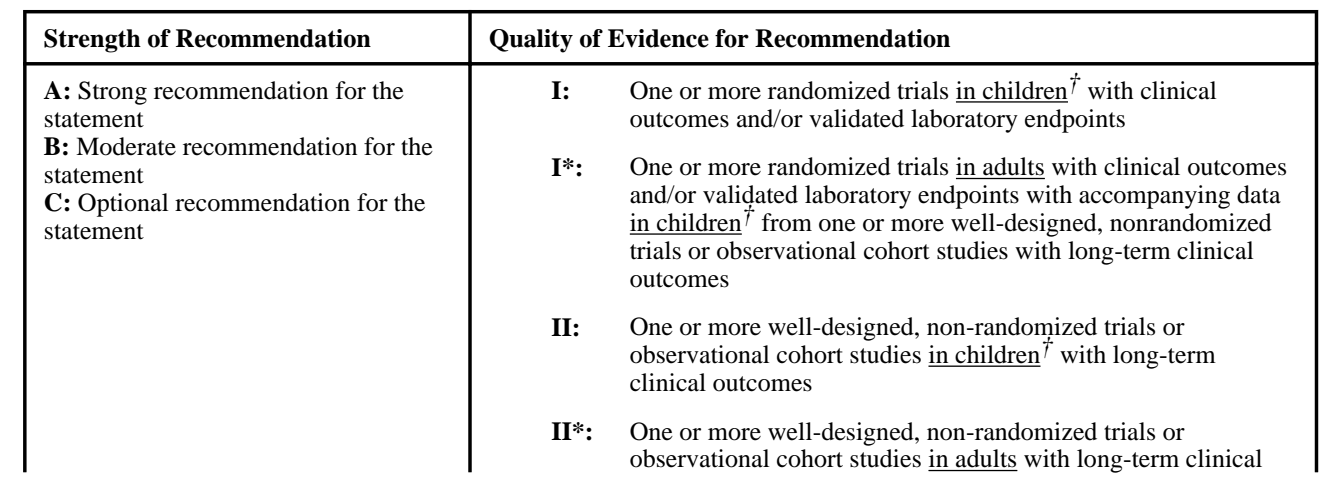




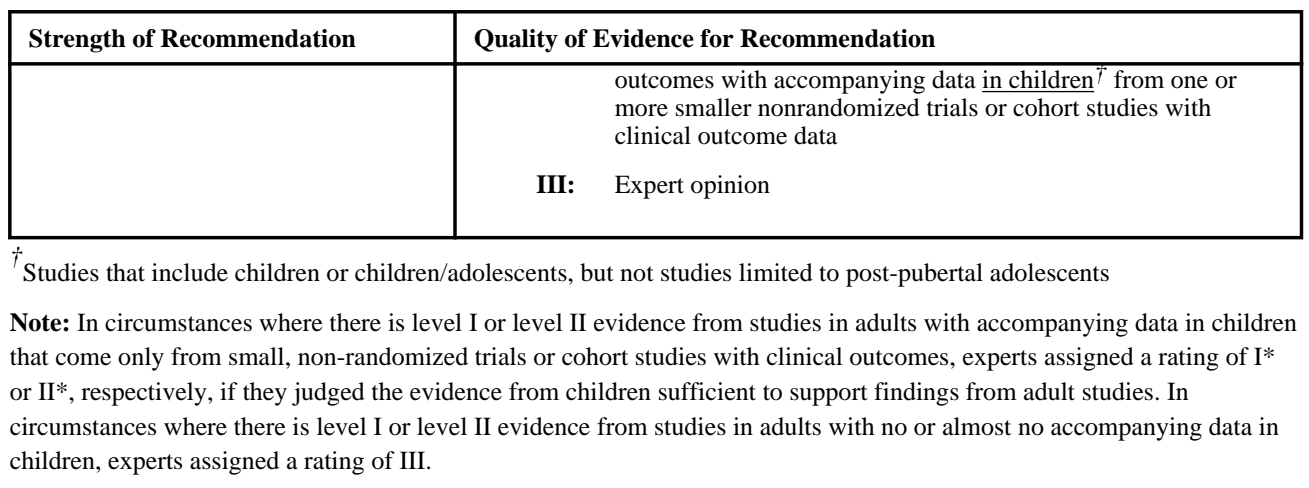

\section{Antiretroviral Therapy and Management of Opportunistic Infections}

Studies in adults and children have demonstrated that cART reduces the incidence of OIs and improves survival, independent of the use of OI antimicrobial prophylaxis.

Recommendations for cART for HIV-infected children have been developed and can be found here. cART can lead to improvement or resolution of certain OIs, such as progressive multifocal leukoencephalopathy (PML) or microsporidiosis, for which effective specific treatments are not available. However, potent cART does not replace the need for OI prophylaxis in children with severe immune suppression. In addition, initiation of cART in individuals with an acute or latent OI can lead to IRIS, an exaggerated inflammatory reaction that can present with paradoxical worsening or new appearance of an OI (see IRIS section below).

Specific data are limited to inform recommendations on when to start cART in children with an acute $\mathrm{OI}$ and how to manage cART when an acute OI occurs in a child already receiving cART. Decisions about when to start cART in children with acute or latent OIs need to be individualized and will vary by the degree of immunologic suppression in a child before he or she starts cART. The benefit of initiating cART early is improved immune function, which could result in faster resolution of the OI. However, potential problems, such as drugdrug interactions that compromise efficacy and increase toxicity, exist when cART and treatment for the OI are initiated simultaneously. The primary disadvantage of delaying cART until after initial treatment of the acute OI is risk of additional OIs or death during the delay. Similarly, in children already receiving cART who develop an OI, management will need to account for each individual's clinical, viral, and immune status on cART and the potential drug-drug interactions between cART and the required OI drug regimen. Diseasespecific information and recommendations for managing cART in context of treating an OI are included in individual sections, as appropriate.

\section{Immune Reconstitution Inflammatory Syndrome}

ART improves immune function and CD4 count in HIV-infected children as in adults; within the first few months after starting treatment, HIV viral load sharply decreases and the CD4 count rapidly increases. This results in increased capacity to mount inflammatory reactions. After initiation of cART, in some patients, reconstitution of the immune system produces a paradoxical inflammatory response to infectious or noninfectious antigens, which 
results in apparent clinical worsening of an existing OI or appearance of a new OI. IRIS primarily has been reported in adults initiating therapy, but it also has been seen in children. ${ }^{17-20}$

IRIS can occur after initiation of cART as worsening of symptoms of an existing active OI (paradoxical IRIS) or as appearance of new symptoms of a latent or occult OI (unmasking IRIS), where infectious pathogens previously not recognized by the immune system now evoke an immune response. This inflammatory response often is exaggerated in comparison with the response in patients who have normal immune systems. An example of unmaking IRIS is activation of latent or occult TB after initiation of ART in patients without TB disease at cART initiation. Clinical recrudescence or symptomatic worsening of TB disease despite microbiologic treatment success and sterile cultures is typical of paradoxical IRIS. In this case, reconstitution of antigen-specific, T-cell-mediated immunity occurs after initiation of cART, with activation of the immune system against persisting antigens, whether present as viable organisms, non-viable organisms, or organism debris.

The pathologic process of IRIS is inflammatory and not microbiologic in etiology. Thus, distinguishing IRIS from treatment failure is important. In therapeutic failure, a microbiologic culture should reveal the continued presence of an infectious organism, whereas in paradoxical IRIS, follow-up cultures most often are sterile. However, with unmasking IRIS, viable pathogens may be isolated.

IRIS is described primarily on the basis of reports of cases in adults. A proposed clinical definition is new appearance or worsening of clinical illness temporally related to starting cART accompanied by $\geq 1 \log _{10}$ decrease in plasma HIV RNA that is not explained by newly acquired infection or disease, the usual course of a previously acquired disease, or cART toxicity. ${ }^{21}$

In adults, IRIS most often has been observed after initiation of cART in patients with mycobacterial infections (including MAC and M. tuberculosis), PCP, cryptococcal infection, CMV, varicella zoster or herpes simplex virus (HSV) infections, hepatitis B and C virus infections, toxoplasmosis, and PML. The conditions most commonly associated with IRIS in children include mycobacterial infections, herpes zoster, HSV, and cryptococcal infection. In addition, reactions related to bacille Calmette-Guérin vaccine have been one of the most common IRIS manifestations in children in low-resource settings. ${ }^{17,18,20}$ In a study of 153 symptomatic children with CD4 counts $<15 \%$ at initiation of therapy in Thailand, the incidence of IRIS was 19\%, with a median time of onset of 4 weeks after start of cART; children who developed IRIS had lower baseline CD4 percentages than did children who did not develop IRIS. ${ }^{20}$

No randomized controlled trials have been published evaluating treatment of IRIS. Treatment has been based on severity of disease. For mild cases, observation alone with close clinical and laboratory monitoring may be sufficient. For moderate cases, nonsteroidal anti-inflammatory drugs have been used to ameliorate symptoms. For severe cases, corticosteroids such as dexamethasone have been used. However, the optimal dose and duration of therapy are unknown, and inflammation can take weeks to months to subside. 
During that time, cART generally is continued. Disease-specific information and recommendations for managing IRIS are included in individual sections, as appropriate.

\section{Initiation of CART for an Acute OI in Treatment-Naive Children}

The ideal time to initiate cART for an acute OI is unknown. The benefit of initiating cART is improved immune function, which could result in faster resolution of the OI. This is particularly important for OIs for which effective therapeutic options are limited or not available, such as microsporidiosis, PML, and Kaposi sarcoma (KS). However, potential problems exist when cART and treatment for the OI are initiated simultaneously. These include drug-drug interactions between the ARV and antimicrobial drugs, particularly given the more limited repertoire of ARV drugs available for children than for adults; issues related to toxicity, including potential additive toxicity of ARV and OI drugs and difficulty in distinguishing cART toxicity from OI treatment toxicity; and the potential for IRIS to complicate OI management.

The primary consideration in delaying cART until after initial treatment of the acute OI is risk of additional illness or death during the delay. Although the short-term risk of death in the United States during a 2-month cART delay may be relatively low, mortality in resource-limited countries is significant. IRIS is more likely to occur in patients with advanced HIV infection and higher OI-specific antigenic burdens, such as those who have disseminated infections or a shorter time from acute OI onset to start of cART. Most IRIS events have the potential to result in significant morbidity but do not result in death; the exception is OIs with central nervous system (CNS) involvement, the form of IRIS most commonly associated with mortality. ${ }^{22}$

Randomized trials in adults demonstrate significantly better outcomes when adults with nonCNS OIs begin cART early in the course of OI treatment, but raise concern for potential increased mortality when cART is initiated early in adults (in Africa) with cryptococcal meningitis. ${ }^{23-25}$ In the absence of trials in children, recommendations about timing of cART initiation in children undergoing OI treatment are not definitive and management should be individualized. The timing is a complex decision based on the severity of HIV disease, efficacy of standard OI-specific treatment, social support system, medical resource availability, potential drug-drug interactions, and risk of IRIS. Most experts believe that the early benefit of potent cART outweighs any increased risk to children who have OIs such as microsporidiosis, PML, or KS for which effective treatment is lacking and that they should begin it as soon as possible.

\section{Management of Acute Ols in HIV-Infected Children Receiving cART}

OIs in HIV-infected children soon after initiation of cART (within 12 weeks) may be subclinical infections unmasked by cART-related improvement in immune function (unmasking IRIS), which usually occurs in children who have more severe immune suppression at initiation of cART. This does not represent a failure of cART but rather a sign of immune reconstitution (see IRIS section). In such situations, cART should be continued and treatment for the OI begun. Assessing the potential for drug-drug interactions between 
the ARV and antimicrobial drugs and whether treatment modifications need to be made is important.

In children who develop an OI after receiving >12 weeks of cART with virologic and immunologic response to therapy, it can be difficult to distinguish between later-onset IRIS and a new OI related to persistent immunosuppression. In such situations, cART should be continued and specific OI-related therapy should be initiated, guided by results of clinical and microbiologic evaluation.

OIs that occur in HIV-infected children with poor virologic response to cART (because of poor adherence, inappropriate ARV regimens, drug resistance, or some combination of these factors) represent failure of therapy. In this situation, treatment of the OI should be initiated, viral resistance testing performed, the child's cART regimen reassessed, and adherence assessed and barriers addressed, as described in pediatric ARV guidelines.

\section{References}

1. Dankner WM, Lindsey JC, Levin MJ. Pediatric ACTGPT. Correlates of opportunistic infections in children infected with the human immunodeficiency virus managed before highly active antiretroviral therapy. Pediatr Infect Dis J. 2001 Jan; 20(1):40-48. Available at http:// www.ncbi.nlm.nih.gov/pubmed/11176565. [PubMed: 11176565]

2. Gortmaker SL, Hughes M, Cervia J, et al. Effect of combination therapy including protease inhibitors on mortality among children and adolescents infected with HIV-1. N Engl J Med. 2001 Nov 22; 345(21):1522-1528. Available at http://www.ncbi.nlm.nih.gov/pubmed/11794218. [PubMed: 11794218]

3. Gona P, Van Dyke RB, Williams PL, et al. Incidence of opportunistic and other infections in HIVinfected children in the HAART era. JAMA. 2006 Jul 19; 296(3):292-300. Available at http:// www.ncbi.nlm.nih.gov/pubmed/16849662. [PubMed: 16849662]

4. Nesheim SR, Kapogiannis BG, Soe MM, et al. Trends in opportunistic infections in the pre- and post-highly active antiretroviral therapy eras among HIV-infected children in the Perinatal AIDS Collaborative Transmission Study, 1986-2004. Pediatrics. 2007 Jul; 120(1):100-109. Available at http://www.ncbi.nlm.nih.gov/pubmed/17606567. [PubMed: 17606567]

5. Brady MT, Oleske JM, Williams PL, et al. Declines in mortality rates and changes in causes of death in HIV-1-infected children during the HAART era. J Acquir Immune Defic Syndr. 2010 Jan; 53(1):86-94. Available at http://www.ncbi.nlm.nih.gov/pubmed/20035164. [PubMed: 20035164]

6. CDC. USPHS/IDSA guidelines for the prevention of oppurtunistic infections in persons infected with human immunodeficiency virus: a summary. MMWR. 1995; 44(RR-08):1-34. Available at http://www.cdc.gov/mmwr/preview/mmwrhtml/00038328.htm.

7. CDC. Guidelines for Preventing Opportunistic Infections Among HIV-Infected Persons - 2002. Recommendations of the U.S. Public Health Service and the Infectious Diseases Society of America. MMWR. 2002; 51(RR-08):1-46. Available at http://www.cdc.gov/mmwr/preview/ mmwrhtml/rr5108a1.htm.

8. CDC. 1997 USPHS/IDSA guidelines for the prevention of opportunistic infections in persons infected with human immunodeficiency virus. USPHS/IDSA Prevention of Opportunistic Infections Working Group. MMWR Recomm Rep. 1997 Jun 27; 46(RR-12):1-46. Available at http:// www.ncbi.nlm.nih.gov/pubmed/9214702.

9. CDC. USPHS/IDSA guidelines for the prevention of oppurtunistic infections in persons infected with human immunodeficiency virus. MMWR. 1999; 48(RR-10):1-59. Available at http:// www.cdc.gov/mmwr/preview/mmwrhtml/rr4810a1.htm.

10. Mofenson LM, Oleske J, Serchuck L, Van Dyke R, Wilfert C. Treating opportunistic infections among HIV-exposed and infected children: recommendations from CDC, the National Institutes of Health, and the Infectious Diseases Society of America. Clin Infect Dis. 2005 Feb 1; 40(Suppl 1):S1-S84. Available at http://www.ncbi.nlm.nih.gov/pubmed/15655768. [PubMed: 15655768] 
11. Yeung LT, King SM, Roberts EA. Mother-to-infant transmission of hepatitis C virus. Hepatology. 2001 Aug; 34(2):223-229. Available at http://www.ncbi.nlm.nih.gov/pubmed/11481604. [PubMed: 11481604]

12. Kovacs A, Schluchter M, Easley K, et al. Cytomegalovirus infection and HIV-1 disease progression in infants born to HIV-1-infected women. Pediatric Pulmonary and Cardiovascular Complications of Vertically Transmitted HIV Infection Study Group. N Engl J Med. 1999 Jul 8; 341(2):77-84. Available at http://www.ncbi.nlm.nih.gov/pubmed/10395631. [PubMed: 10395631]

13. Gutman LT, Moye J, Zimmer B, Tian C. Tuberculosis in human immunodeficiency virus-exposed or -infected United States children. Pediatr Infect Dis J. 1994 Nov; 13(11):963-968. Available at http://www.ncbi.nlm.nih.gov/pubmed/7845749. [PubMed: 7845749]

14. Jones CE, Naidoo S, De Beer C, Esser M, Kampmann B, Hesseling AC. Maternal HIV infection and antibody responses against vaccine-preventable diseases in uninfected infants. JAMA. 2011 Feb 9; 305(6):576-584. Available at http://www.ncbi.nlm.nih.gov/pubmed/21304083. [PubMed: 21304083]

15. Kourtis AP, Bansil P, Posner SF, Johnson C, Jamieson DJ. Trends in hospitalizations of HIVinfected children and adolescents in the United States: analysis of data from the 1994-2003 Nationwide Inpatient Sample. Pediatrics. 2007 Aug; 120(2):e236-e243. Available at http:// www.ncbi.nlm.nih.gov/pubmed/17606535. [PubMed: 17606535]

16. Kaplan JE, Benson C, Holmes KH, et al. Guidelines for prevention and treatment of opportunistic infections in HIV-infected adults and adolescents: recommendations from CDC, the National Institutes of Health, and the HIV Medicine Association of the Infectious Diseases Society of America. MMWR Recomm Rep. 2009 Apr 10; 58(RR-4):1-207. Available at http:// www.ncbi.nlm.nih.gov/pubmed/19357635.

17. Wang ME, Castillo ME, Montano SM, Zunt JR. Immune reconstitution inflammatory syndrome in human immunodeficiency virus-infected children in Peru. Pediatr Infect Dis J. 2009 Oct; 28(10): 900-903. Available at http://www.ncbi.nlm.nih.gov/pubmed/19687769. [PubMed: 19687769]

18. Smith K, Kuhn L, Coovadia A, et al. Immune reconstitution inflammatory syndrome among HIVinfected South African infants initiating antiretroviral therapy. AIDS. 2009 Jun 1; 23(9):10971107. Available at http://www.ncbi.nlm.nih.gov/pubmed/19417581. [PubMed: 19417581]

19. Puthanakit T, Oberdorfer P, Ukarapol N, et al. Immune reconstitution syndrome from nontuberculous mycobacterial infection after initiation of antiretroviral therapy in children with HIV infection. Pediatr Infect Dis J. 2006 Jul; 25(7):645-648. Available at http:// www.ncbi.nlm.nih.gov/pubmed/16804438. [PubMed: 16804438]

20. Puthanakit T, Oberdorfer P, Akarathum N, Wannarit P, Sirisanthana T, Sirisanthana V. Immune reconstitution syndrome after highly active antiretroviral therapy in human immunodeficiency virus-infected thai children. Pediatr Infect Dis J. 2006 Jan; 25(1):53-58. Available at http:// www.ncbi.nlm.nih.gov/pubmed/16395104. [PubMed: 16395104]

21. Robertson J, Meier M, Wall J, Ying J, Fichtenbaum CJ. Immune reconstitution syndrome in HIV: validating a case definition and identifying clinical predictors in persons initiating antiretroviral therapy. Clin Infect Dis. 2006 Jun 1; 42(11):1639-1646. Available at http:// www.ncbi.nlm.nih.gov/pubmed/16652323. [PubMed: 16652323]

22. Torok ME, Kambugu A, Wright E. Immune reconstitution disease of the central nervous system. Curr Opin HIV AIDS. 2008 Jul; 3(4):438-445. Available at http://www.ncbi.nlm.nih.gov/pubmed/ 19373003. [PubMed: 19373003]

23. Zolopa A, Andersen J, Powderly W, et al. Early antiretroviral therapy reduces AIDS progression/ death in individuals with acute opportunistic infections: a multicenter randomized strategy trial. PLoS One. 2009; 4(5):e5575. Available at http://www.ncbi.nlm.nih.gov/pubmed/19440326. [PubMed: 19440326]

24. Abdool Karim SS, Naidoo K, Grobler A, et al. Timing of initiation of antiretroviral drugs during tuberculosis therapy. N Engl J Med. 2010 Feb 25; 362(8):697-706. Available at http:// www.ncbi.nlm.nih.gov/pubmed/20181971. [PubMed: 20181971]

25. Makadzange AT, Ndhlovu CE, Takarinda K, et al. Early versus delayed initiation of antiretroviral therapy for concurrent HIV infection and cryptococcal meningitis in sub-saharan Africa. Clin Infect Dis. 2010 Jun 1; 50(11):1532-1538. Available at http://www.ncbi.nlm.nih.gov/pubmed/ 20415574. [PubMed: 20415574] 


\section{Preventing Vaccine-Preventable Diseases in HIV-Infected Children and Adolescents (Last updated November 6, 2013; last reviewed November 6, 2013)}

Vaccines are an extremely effective primary prevention tool, and vaccines that protect against 16 diseases are recommended for routine use in children and adolescents in the United States. Vaccination schedules for children aged 0 to 18 years are published annually by the Centers for Disease Control and Prevention (see http://www.cdc.gov/vaccines/ schedules/hcp/child-adolescent.html). These schedules are compiled from approved, vaccine-specific policy recommendations and are standardized among the major vaccine policy-setting and vaccine-delivery organizations (i.e., the Advisory Committee on Immunization Practices [ACIP], American Academy of Pediatrics, and American Academy of Family Physicians).

HIV-infected children should be protected from vaccine-preventable diseases. Most vaccines recommended for routine use can be administered safely to HIV-exposed or HIVinfected children. The recommended vaccination schedules for HIV-exposed and HIVinfected children aged 0 to 18 years correspond to the ACIP-approved schedule with ACIPapproved additions specific to HIV-infected children incorporated (see Figures 1 and 2). These schedules will be updated periodically to reflect additional ACIP-approved vaccine recommendations that pertain to HIV-exposed or HIV-infected children.

All inactivated vaccines-whether killed whole organism or recombinant, subunit, toxoid, polysaccharide, or polysaccharide protein-conjugate—can be administered safely to individuals with altered immunocompetence. In addition, because of the risks of increased vaccine-preventable disease severity in HIV-infected children, specific vaccines like pneumococcal conjugate vaccine are also recommended or encouraged for children beyond the routinely recommended ages for healthy children (if not previously administered at routinely recommended ages in early childhood); additional vaccines are also recommended, such as pneumococcal polysaccharide vaccine for children aged $\geq 2$ years following receipt of pneumococcal conjugate vaccine. Similarly, before influenza vaccination was routinely recommended for children aged $\geq 6$ months, trivalent influenza vaccine (TIV) was routinely recommended for HIV-infected children because of their increased risk of severe disease. TIV continues to be recommended for HIV-infected children as part of routine prevention for influenza. ${ }^{1}$ If inactivated vaccines are indicated for individuals with altered immunocompetence, the usual doses and schedules are often recommended. However, the effectiveness of such vaccinations may be suboptimal. ${ }^{2}$

Patients with severe cell-mediated immunodeficiency should not receive live-attenuated vaccines. However, HIV-infected children are at higher risk than immunocompetent children for complications of varicella, herpes zoster, and measles-diseases for which only live vaccines are available. On the basis of limited safety, immunogenicity, and efficacy data in HIV-infected children, varicella vaccine can be considered for HIV-infected children who are not severely immunosuppressed (i.e., children with CD4 T lymphocyte (CD4) cell percentages $>15 \%$ and those aged $>5$ years with CD4 counts $\geq 200$ cells $/ \mu \mathrm{L}) .^{2-4}$ Two doses 
of measles, mumps, and rubella (MMR) vaccine are recommended for all HIV-infected individuals aged $\geq 12$ months who do not have evidence of current severe immunosuppression (i.e., individuals aged $\mathbf{5}$ years must have CD4 percentages $215 \%$ for $\geq 6$ months and those aged $>5$ years must have CD4 percentages $\geq 15 \%$ and CD4 cell counts $\geq 200$ lymphocytes $/ \mathrm{mm}^{3}$ for $\searrow 6$ months) or other current evidence of MMR immunity. ${ }^{5}$

Limited data are available from clinical trials on the safety of rotavirus vaccines in infants known to be HIV-infected; these infants were clinically asymptomatic or mildly symptomatic when vaccinated. ${ }^{6}$ The limited data available do not indicate that rotavirus vaccines have a substantially different safety profile in HIV-infected infants who are clinically asymptomatic or mildly symptomatic than in infants who are HIV-uninfected. Two other considerations support rotavirus vaccination of HIV-exposed or HIV-infected infants: first, the HIV diagnosis may not be established in infants born to HIV-infected mothers before the age of the first rotavirus vaccine dose (only about $2 \%$ of HIV-exposed infants in the United States will be determined to be HIV-infected) ${ }^{7}$ and second, vaccine strains of rotavirus are considerably attenuated. Consultation with an immunologist or infectious disease specialist is advised for infants with known or suspected altered immunocompetence, such as HIV-infected infants with low CD4 percentage or number, before rotavirus vaccine is administered.

For certain vaccines (such as Hepatitis A) the response to vaccination may be higher following combination antiretroviral therapy $(\mathrm{cART})^{8}$ or there may be variation in immunogenicity on the basis of viral load (improved immune response with lower HIV viral load), such as with yellow fever vaccine. ${ }^{9}$ For other vaccines, patients with higher CD4 cell counts have improved immune response, which also means that response (e.g., to vaccination for influenza, MMR, yellow fever) likely would be improved after cART.1,3,9,10 For children vaccinated before taking cART, there is concern about lack of protection from pre-cART vaccines and debate about need for routine re-immunization once on effective cART. ${ }^{10,11}$ On the basis of low rates of measles seroprotection in children who received MMR before cART and the safety and high rates of measles seroprotection associated with MMR re-immunization once children were receiving cART, ${ }^{12}$ the ACIP made specific recommendations for routine MMR re-immunization after cART. Individuals with perinatal HIV infection who were vaccinated prior to establishment of effective cART should receive two appropriately spaced doses of MMR vaccine once effective cART has been established (individuals aged $₫$ years must have CD4 percentages $\geq 15 \%$ for $\geq 6$ months and those aged $>5$ years must have CD4 percentages $\geq 15 \%$ and CD4 cell count $\geq 200$ lymphocytes $/ \mathrm{mm}^{3}$ for $\checkmark 6$ months) unless they have other acceptable current evidence of MMR immunity. ${ }^{5}$ For some vaccines, such as for hepatitis B, ACIP recommends performing post-vaccination serology to ensure immune response.

Consult the specific ACIP statements (available at http://www.cdc.gov/vaccines/pubs/ACIPlist.htm) for more detail regarding recommendations, precautions, and contraindications for use of specific vaccines (http://www.cdc.gov/mmwr/PDF/rr/rr4608.pdf and http:// www.cdc.gov/mmwr/pdf/rr/rr5602.pdf). $3,4,8,13-23$ 


\section{References}

1. Fiore AE, Uyeki TM, Broder K, et al. Prevention and control of influenza with vaccines: recommendations of the Advisory Committee on Immunization Practices (ACIP), 2010. MMWR Recomm Rep. 2010 Aug 6; 59(RR-8):1-62. Available at http://www.ncbi.nlm.nih.gov/pubmed/ 20689501. [PubMed: 20689501]

2. Kroger AT, Atkinson WL, Marcuse EK, Pickering LK. Advisory Committee on Immunization Practices Centers for Disease C, Prevention. General recommendations on immunization: recommendations of the Advisory Committee on Immunization Practices (ACIP). MMWR Recomm Rep. 2006 Dec 1; 55(RR-15):1-48. Available at http://www.ncbi.nlm.nih.gov/pubmed/ 17136024. [PubMed: 17136024]

3. Watson JC, Hadler SC, Dykewicz CA, Reef S, Phillips L. Measles, mumps, and rubella--vaccine use and strategies for elimination of measles, rubella, and congenital rubella syndrome and control of mumps: recommendations of the Advisory Committee on Immunization Practices (ACIP). MMWR Recomm Rep. 1998 May 22; 47(RR-8):1-57. Available at http://www.ncbi.nlm.nih.gov/ pubmed/9639369. [PubMed: 9639369]

4. Marin M, Guris D, Chaves SS, et al. Prevention of varicella: recommendations of the Advisory Committee on Immunization Practices (ACIP). MMWR Recomm Rep. 2007 Jun 22; 56(RR-4):140. Available at http://www.ncbi.nlm.nih.gov/pubmed/17585291. [PubMed: 17585291]

5. ACIP. ACIP Provisional Recommendations: Prevention of Measles, Rubella, Congenital Rubella Syndrome (CRS), and Mumps. 2012 Available at http://www.cdc.gov/vaccines/recs/provisional/ downloads/mmr-Oct-2012.pdf.

6. Steele AD, Madhi SA, Louw CE, et al. Safety, Reactogenicity, and Immunogenicity of Human Rotavirus Vaccine RIX4414 in Human Immunodeficiency Virus-positive Infants in South Africa. Pediatr Infect Dis J. 2011 Feb; 30(2):125-130. Available at http://www.ncbi.nlm.nih.gov/pubmed/ 20842070. [PubMed: 20842070]

7. Centers for Disease C, Prevention. Achievements in public health. Reduction in perinatal transmission of HIV infection-United States, 1985-2005. MMWR Morb Mortal Wkly Rep. 2006 Jun 2; 55(21):592-597. Available at http://www.ncbi.nlm.nih.gov/pubmed/16741495. [PubMed: 16741495]

8. Fiore AE, Wasley A, Bell BP. Prevention of hepatitis A through active or passive immunization: recommendations of the Advisory Committee on Immunization Practices (ACIP). MMWR Recomm Rep. 2006 May 19; 55(RR-7):1-23. Available at http://www.ncbi.nlm.nih.gov/pubmed/ 16708058.

9. Staples JE, Gershman M, Fischer M. Centers for Disease C, Prevention. Yellow fever vaccine: recommendations of the Advisory Committee on Immunization Practices (ACIP). MMWR Recomm Rep. 2010 Jul 30; 59(RR-7):1-27. Available at http://www.ncbi.nlm.nih.gov/pubmed/ 20671663.

10. Sutcliffe CG, Moss WJ. Do children infected with HIV receiving HAART need to be revaccinated? Lancet Infect Dis. 2010 Sep; 10(9):630-642. Available at http:// www.ncbi.nlm.nih.gov/pubmed/20797645. [PubMed: 20797645]

11. Melvin AJ, Mohan KM. Response to immunization with measles, tetanus, and Haemophilus influenzae type $b$ vaccines in children who have human immunodeficiency virus type 1 infection and are treated with highly active antiretroviral therapy. Pediatrics. 2003 Jun; 111(6 Pt 1):e641e644. Available at http://www.ncbi.nlm.nih.gov/pubmed/12777579. [PubMed: 12777579]

12. Abzug MJ, Qin M, Levin MJ, et al. Immunogenicity, immunologic memory, and safety following measles revaccination in HIV-infected children receiving highly active antiretroviral therapy. $\mathbf{J}$ Infect Dis. 2012 Aug 15; 206(4):512-522. Available at http://www.ncbi.nlm.nih.gov/pubmed/ 22693229. [PubMed: 22693229]

13. Cortese MM, Parashar UD. Centers for Disease C, Prevention. Prevention of rotavirus gastroenteritis among infants and children: recommendations of the Advisory Committee on Immunization Practices (ACIP). MMWR Recomm Rep. 2009 Feb 6; 58(RR-2):1-25. Available at http://www.ncbi.nlm.nih.gov/pubmed/19194371. [PubMed: 19194371]

14. MMWR. Haemophilus $b$ conjugate vaccines for prevention of Haemophilus influenzae type $b$ disease among infants and children two months of age and older. Recommendations of the 
immunization practices advisory committee (ACIP). MMWR Recomm Rep. 1991 Jan 11; 40(RR-1):1-7. Available at http://www.ncbi.nlm.nih.gov/pubmed/1899280.

15. Fiore AE, Shay DK, Broder K, et al. Prevention and control of influenza: recommendations of the Advisory Committee on Immunization Practices (ACIP), 2008. MMWR Recomm Rep. 2008 Aug 8; 57(RR-7):1-60. Available at http://www.ncbi.nlm.nih.gov/pubmed/18685555. [PubMed: 18685555]

16. Advisory Committee on Immunization P. Preventing pneumococcal disease among infants and young children. Recommendations of the Advisory Committee on Immunization Practices (ACIP). MMWR Recomm Rep. 2000 Oct 6; 49(RR-9):1-35. Available at http://www.ncbi.nlm.nih.gov/ pubmed/11055835.

17. Prevots DR, Burr RK, Sutter RW, Murphy TV. Advisory Committee on Immunization P. Poliomyelitis prevention in the United States. Updated recommendations of the Advisory Committee on Immunization Practices (ACIP). MMWR Recomm Rep. 2000 May 9; 49(RR-5):122. quiz CE21-27. Available at http://www.ncbi.nlm.nih.gov/pubmed/15580728. [PubMed: 15580728]

18. Mast EE, Margolis HS, Fiore AE, et al. A comprehensive immunization strategy to eliminate transmission of hepatitis B virus infection in the United States: recommendations of the Advisory Committee on Immunization Practices (ACIP) part 1: immunization of infants, children, and adolescents. MMWR Recomm Rep. 2005 Dec 23; 54(RR-16):1-31. Available at http:// www.ncbi.nlm.nih.gov/pubmed/16371945. [PubMed: 16371945]

19. Bilukha OO, Rosenstein N. National Center for Infectious Diseases CfDC, Prevention. Prevention and control of meningococcal disease. Recommendations of the Advisory Committee on Immunization Practices (ACIP). MMWR Recomm Rep. 2005 May 27; 54(RR-7):1-21. Available at http://www.ncbi.nlm.nih.gov/pubmed/15917737. [PubMed: 15917737]

20. CDC. Notice to readers: Recommendation from the Advisory Committee on Immunization Practices (ACIP) for use of quadrivalent meningococcal conjugate vaccine (MCV4) in children aged 2-10 years at increased risk for invasive meningococcal disease. MMWR Morb Mortal Wkly Rep. 2007; 56:1265-1266. Available at http://www.cdc.gov/mmwr/preview/mmwrhtml/ mm5648a4.htm.

21. MMWR. Pertussis vaccination: use of acellular pertussis vaccines among infants and young children. Recommendations of the Advisory Committee on Immunization Practices (ACIP). MMWR Recomm Rep. 1997 Mar 28; 46(RR-7):1-25. Available at http://www.ncbi.nlm.nih.gov/ pubmed/9091780.

22. Broder KR, Cortese MM, Iskander JK, et al. Preventing tetanus, diphtheria, and pertussis among adolescents: use of tetanus toxoid, reduced diphtheria toxoid and acellular pertussis vaccines recommendations of the Advisory Committee on Immunization Practices (ACIP). MMWR Recomm Rep. 2006 Mar 24; 55(RR-3):1-34. Available at http://www.ncbi.nlm.nih.gov/pubmed/ 16557217.

23. Centers for Disease Control and Prevention Advisory Committee on Immunization. Revised recommendations of the Advisory Committee on Immunization Practices to Vaccinate all Persons Aged 11-18 Years with Meningococcal Conjugate Vaccine. MMWR Morb Mortal Wkly Rep. 2007 Aug 10; 56(31):794-795. Available at http://www.ncbi.nlm.nih.gov/pubmed/17694617. [PubMed: 17694617]

\section{Bacterial Infections (Last updated November 6, 2013; last reviewed November 6, 2013)}

\footnotetext{
Panel's Recommendations

- Status of vaccination should be reviewed at every clinical encounter and indicated vaccinations provided, according to the established recommendations for immunization of HIV-infected children (AIII).

- Routine use of antibiotics solely for primary prevention of serious bacterial infections is not recommended (BIII).
} 
Panel's Recommendations

Discontinuation of antibiotic prophylaxis is recommended for HIV-infected children receiving antibiotics for the purpose of primary or secondary prophylaxis of serious bacterial infections once they have achieved sustained $\geq 3$ months) immune reconstitution: (CD4 T lymphocyte [CD4] cell percentage $\geq 25 \%$ if $<6$ years old; CD4 percentage $\geq 20 \%$ and CD4 count $>350$ cells $/ \mathrm{mm}^{3}$ if $\geq 6$ years old) (BII).

- Intravenous immune globulin is recommended to prevent serious bacterial infections in HIV-infected children who have hypogammaglobulinemia ( $\mathrm{IgG}<400 \mathrm{mg} / \mathrm{dL})$ (AI).

- $\quad$ HIV-infected children whose immune systems are not seriously compromised (CDC Immunologic Category I) and who are not neutropenic can be expected to respond the same as HIV-uninfected children and should be treated with the usual antimicrobial agents recommended for the most likely bacterial organisms (AIII).

- Severely immunocompromised HIV-infected children with invasive or recurrent bacterial infections require expanded empiric antimicrobial treatment covering a broad range of resistant organisms (AIII).

- Initial empiric therapy for HIV-infected children with suspected intravascular catheter sepsis should target both gram-positive and enteric gram-negative organisms, with combinations that have activity against Pseudomonas spp. and methicillin-resistant Staphylococcus aureus (MRSA) (AIII)

Rating of Recommendations: $\mathrm{A}=$ Strong; $\mathrm{B}=$ Moderate; $\mathrm{C}=$ Optional

Rating of Evidence: I = One or more randomized trials $\underline{\text { in children }}^{\dagger}$ with clinical outcomes and/or validated endpoints; $\mathrm{I}^{*}$ $=$ One or more randomized trials in adults with clinical outcomes and/or validated laboratory endpoints with accompanying data in children ${ }^{\dagger}$ from one or more well-designed, nonrandomized trials or observational cohort studies with long-term clinical outcomes; II = One or more well-designed, nonrandomized trials or observational cohort studies in children ${ }^{\dagger}$ with long-term outcomes; II* $=$ One or more well-designed, nonrandomized trials or observational studies in adults with longterm clinical outcomes with accompanying data in children ${ }^{\dagger}$ from one or more similar nonrandomized trials or cohort studies with clinical outcome data; $\mathrm{III}=$ Expert opinion

${ }^{\dagger}$ Studies that include children or children/adolescents, but not studies limited to post-pubertal adolescents

\section{Bacterial Infections, Serious and Recurrent}

Epidemiology-Before combination antiretroviral therapy (cART) was available, serious bacterial infections were the most commonly diagnosed opportunistic infections in HIVinfected children, with an event rate of 15 per 100 child-years. ${ }^{1}$ Pneumonia was the most common bacterial infection (11 per 100 child-years), followed by bacteremia ( 3 per 100 child-years), and urinary tract infection (2 per 100 child-years). Other serious bacterial infections, including osteomyelitis, meningitis, abscess, and septic arthritis, occurred at rates $<0.2$ per 100 child-years. Less serious bacterial infections such as otitis media and sinusitis were particularly common (17-85 per 100 child-years) in untreated HIV-infected children. ${ }^{2}$

Since the advent of cART, bacterial infections in HIV-infected children have decreased substantially, 3,4 and predominate in children who have not had a sustained response to cART. $^{3}$ The rate of pneumonia has decreased to 2 to 3 per 100 child-years, ${ }^{4-7}$ similar to the rate of 3 to 5 per 100 child-years in HIV-uninfected children. ${ }^{8,9}$ The rate of bacteremia/ sepsis during the cART era also has decreased dramatically to 0.35 to 0.37 per 100 childyears, ${ }^{5,6,10}$ but it remains substantially higher than that of invasive pneumococcal disease in U.S. children (0.018 and 0.0022 per 100 child years for those aged $<5$ and 5-17-year-olds, respectively). ${ }^{11}$ Rates of sinusitis and otitis in cART-treated children are substantially lower than in the pre-cART era (2.9-3.5 per 100 child-years), but remain higher than those in HIV-uninfected children. ${ }^{6}$

Pneumonia-Acute pneumonia, often presumptively diagnosed in children, was associated with increased risk of long-term mortality in HIV-infected children in one study 
during the pre-cART era. ${ }^{12}$ HIV-infected children not receiving cART who present with pneumonia are more likely to be bacteremic and to die than are HIV-uninfected children with pneumonia. ${ }^{13}$ Children with chronic lung disease, including bronchiectasis, complicating repeated episodes of infectious pneumonia or lymphocytic interstitial pneumonitis, ${ }^{14}$ are more susceptible to infectious exacerbations (similar to those in children and adults with bronchiectasis or cystic fibrosis) caused by typical respiratory bacteria (Streptococcus pneumoniae, non-typeable Haemophilus influenzae) and Pseudomonas spp.

Streptococcus pneumoniae-S. pneumoniae is the most prominent invasive bacterial pathogen in HIV-infected children both in the United States and worldwide, accounting for $>50 \%$ of bacterial bloodstream infections in HIV-infected children. ${ }^{1,10,15-19}$ HIV-infected children have a markedly higher risk of pneumococcal infection than do HIV-uninfected children. ${ }^{20,21}$ In a Philadelphia cohort, the incidence of invasive pneumococcal disease (IPD) in HIV-infected children decreased by more than $80 \%$ from 1.9 per 100 patient-years before cART to 0.3 per 100 in the cART era. ${ }^{22}$ The rate of hospitalization for IPD in HIVinfected children and youth also declined by nearly $80 \%$ since introduction of routine use of cART and pneumococcal conjugate vaccine. ${ }^{23}$ In children with invasive pneumococcal infections, study results vary on whether penicillin-resistant pneumococcal strains are more commonly isolated from HIV-infected than HIV-uninfected patients. ${ }^{17,22,24,25}$ Invasive disease caused by penicillin-nonsusceptible pneumococcus was associated with longer duration of fever and hospitalization but not with greater risk of complications or poorer outcome in a study of HIV-uninfected children; ${ }^{26}$ however, most IPD in HIV-infected children is not caused by non-susceptible pneumococci. ${ }^{22}$ In 2010 , the 7-valent pneumococcal conjugate vaccine (licensed in 2000) was replaced by a 13-valent vaccine (including coverage for serotype 19A) for routine use in all children, including HIV-infected children. ${ }^{27}$ The impact of routine use of 13 -valent conjugate vaccine on invasive pneumococcal disease in HIV-infected children is not yet known.

Haemophilus influenzae Type b-HIV-infected children are at increased risk of Haemophilus influenzae type b (Hib) infection. In a study in South African children who had not received Hib conjugate vaccine, the estimated relative annual rate of overall invasive Hib disease in children aged $<1$ year was 5.9 times greater in those who were HIV-infected than those who were uninfected, and HIV-infected children were at greater risk for bacteremic pneumonia. ${ }^{28} \mathrm{Hib}$ infection is rare in HIV-infected children in the United States because routine Hib immunization confers direct protection to immunized HIV-infected children and herd immunity confers indirect protection. ${ }^{29}$

Neisseria meningitidis (Meningococcus) - HIV infection is associated with an increased risk of meningococcal disease. ${ }^{30,31}$ In a population-based study of invasive meningococcal disease in Atlanta, Georgia, ${ }^{31}$ as expected, the annual rate of disease was higher in 18- to 24 -year-olds $(1.17$ per 100,000) than for all adults $(0.5$ per 100,000), but the estimated annual rate in HIV-infected adults was substantially higher (11.2 per 100,000). There are no studies of meningococcal disease risk in HIV-infected children in the United States. However, in a population-based surveillance study in South Africa, HIV infection significantly increased the risk of meningococcal bacteremia, which was associated with 
increased risk of death in all ages, but especially in children. Very few HIV-infected patients were receiving cART at the time of this study. ${ }^{30}$

Methicillin-Resistant Staphylococcus aureus (MRSA)-HIV infection appears to be a risk factor for MRSA infections in adults, but findings are conflicting about the relative contribution of immunosuppression vs. concomitant psychosocial risk factors to this increased risk. ${ }^{32-34}$ Limited data suggest that HIV-infected children, like their uninfected counterparts, experience predominantly non-invasive, skin, and soft tissue infections as a result of community-associated MRSA strains and that greater immunosuppression may not confer greater risk of MRSA. ${ }^{35}$

Other Pathogens-Other pathogens, including Pseudomonas aeruginosa and enteric organisms, cause infection in HIV-infected children, especially those who have indwelling vascular catheters or advanced immunosuppression or are not on cART. ${ }^{19,29,36,37}$ The most commonly isolated pathogens in catheter-associated bacteremia in HIV-infected children are similar to those in HIV-negative children with indwelling catheters, including coagulasenegative staphylococci, S. aureus, enterococci, P. aeruginosa, gram-negative enteric bacilli, Bacillus cereus, and Candida spp. ${ }^{18,37}$ In a cohort of 680 HIV-infected children in Miami, Florida, 10.6\% had 95 episodes of gram-negative bacteremia between 1980 and 1997, of which only 6 were associated with an indwelling vascular catheter. The predominant organisms were P. aeruginosa, nontyphoidal Salmonella, and Escherichia coli (15\%). ${ }^{29}$ More than $70 \%$ had advanced immunosuppression and the overall case-fatality rate was $43 \%$. In Kenyan children with bacteremia, HIV infection increased the risk of non-typhoidal Salmonella and E. coli infections. ${ }^{36}$

HIV-Exposed (but Uninfected) Children-Data are conflicting about whether infectious morbidity increases in children who have been exposed to but not infected with HIV. In studies in developing countries, HIV-exposed but uninfected (HEU) infants had higher mortality (primarily because of bacterial pneumonia and sepsis) than did those born to uninfected mothers. ${ }^{38,39}$ Advanced maternal HIV infection was associated with increased risk of infant death. ${ }^{38,39}$ In a study in Latin America and the Caribbean, $60 \%$ of $462 \mathrm{HEU}$ infants experienced infectious disease morbidity during the first 6 months of life, with the rate of neonatal infections (particularly sepsis) and respiratory infections higher than rates in comparable community-based studies. ${ }^{40}$ However, in a study from the United States, the rate of lower respiratory tract infections in HEU children was within the range reported for healthy children during the first year of life. ${ }^{41}$ There is increasing evidence for insufficient maternally derived antibody levels in HEU infants that put those infants at increased risk of pneumococcal and other vaccine-preventable infections. ${ }^{42}$

\section{Clinical Manifestations}

Clinical presentation depends on the particular type of bacterial infection (e.g., bacteremia/ sepsis, osteomyelitis/septic arthritis, pneumonia, meningitis, sinusitis/otitis media); ${ }^{43}$ HIVinfected children with invasive bacterial infections typically have a clinical presentation similar to HIV-uninfected children. ${ }^{21,44,45}$ 
The classical signs, symptoms, and laboratory test abnormalities that usually indicate invasive bacterial infection (e.g., fever, elevated white blood cell count) are usually present but may be lacking in HIV-infected children who have reduced immune competence. ${ }^{21,43}$ One-third of HIV-infected children not receiving cART who have acute pneumonia have recurrent episodes. ${ }^{12}$ Bronchiectasis and other chronic lung damage that occurs before initiation of cART can predispose to recurrent pulmonary infections, even in the presence of effective cART. ${ }^{14}$ Lower respiratory bacterial infections in children with lymphocytic interstitial pneumonitis (LIP) most often are a result of the same bacterial pathogens that cause lower respiratory infection in HIV-infected children without LIP and manifests as fever, increased sputum production, and respiratory difficulty superimposed on chronic pulmonary symptoms and radiologic abnormalities. ${ }^{46}$

In studies in Malawi and South Africa before the availability of cART, the clinical presentations of acute bacterial meningitis in HIV-infected and HIV-uninfected children were similar. ${ }^{47,48}$ However, in a study from Malawi, HIV-infected children were 6.4-fold more likely to have repeated episodes of meningitis than were HIV-uninfected children, although the study did not differentiate relapses from new infections. ${ }^{47}$ In both studies, HIVinfected children were more likely to die from meningitis than were HIV-uninfected children.

\section{Diagnosis}

Attempted isolation of a pathogenic organism from normally sterile sites (e.g., blood, cerebrospinal fluid, pleural fluid) is strongly recommended, as identification and antimicrobial resistance testing will guide effective treatment.

Because of difficulties obtaining appropriate specimens, such as sputum, from young children, bacterial pneumonia most often is a presumptive diagnosis in children with fever, pulmonary symptoms, and an abnormal chest radiograph, unless an accompanying bacteremia exists. In the absence of a laboratory isolate, differentiating viral from bacterial pneumonia using clinical criteria can be difficult. ${ }^{8}$ Mycobacterium tuberculosis (TB) and Pneumocystis jirovecii pneumonia (PCP) must always be considered in HIV-infected children with pneumonia. Presence of wheezing makes acute bacterial pneumonia less likely than other causes (e.g., viral pathogens, asthma exacerbation), atypical bacterial pathogens (e.g., Mycoplasma pneumoniae), or aspiration. Children with LIP often have episodes of bacterial respiratory infection superimposed on chronic wheezing. Sputum induction obtained by nebulization with hypertonic (5\%) saline was evaluated for diagnosis of pneumonia in 210 South African infants and children (median age: 6 months), $66 \%$ of whom were HIV-infected. ${ }^{49}$ The procedure was well-tolerated, and identified an etiology in $63 \%$ of children with pneumonia (identification of bacteria in 101, TB in 19, and PCP in 12 children). Blood and fluid from pleural effusion (if present) should be cultured.

In children with bacteremia, a source should be sought. In addition to routine chest radiographs, other diagnostic radiologic evaluations may be necessary in HIV-infected children with compromised immune systems to identify less apparent foci of infection (e.g., bronchiectasis, internal organ abscesses). ${ }^{50-52}$ In children with suspected bacteremia and central venous catheters, blood culture should be obtained through the catheter and (if 
possible) peripherally; if the catheter is removed because of suspected infection, the catheter tip should be sent for culture. ${ }^{53}$ Assays for detection of bacterial antigens or evidence by molecular biology techniques are important for diagnostic evaluation of HIV-infected children in whom unusual pathogens may be involved or difficult to identify or culture with standard techniques. For example, detection of Bordetella pertussis and Chlamydophila (formerly Chlamydia) pneumoniae with polymerase chain reaction assays of nasopharyngeal secretions may aid in the diagnosis of these infections. ${ }^{8,54,55}$

\section{Prevention Recommendations}

Preventing Exposure-Because S. pneumoniae and H. influenzae (other than type b) are common in the community, no effective way exists to eliminate exposure to these bacteria. However, routine use of conjugated pneumococcal (initially 7-valent and, more recently 13valent) and Hib vaccines in the United States has dramatically reduced vaccine-type nasopharyngeal colonization in healthy children, thus limiting the exposure of HIV-infected children to these pathogens (herd immunity).

Food: To reduce the risk of exposure to potential GI bacterial pathogens, health-care providers should advise that HIV-infected children avoid eating the following raw or undercooked foods (including other foods that contain them): eggs, poultry, meat, seafood (especially raw shellfish), and raw seed sprouts (BIII). Unpasteurized dairy products and unpasteurized fruit juices also should be avoided (BIII). Of particular concern to HIVinfected infants and children is the potential for caretakers to handle these raw foods (e.g., during meal preparation) and then unknowingly transfer bacteria from their hands to children's food, milk or formula, or directly to the children. Hands, cutting boards, counters, and knives and other utensils should be washed thoroughly after contact with uncooked foods (BIII). Produce should be washed thoroughly before being eaten (BIII). These precautions are especially important for children who are not receiving effective cART.

Pets: When obtaining a new pet, caregivers should avoid dogs or cats aged $<6$ months or stray animals (BIII). HIV-infected children and adults should avoid contact with any animals that have diarrhea and should always wash their hands after handling pets, especially before eating, and avoid contact with pets' feces (BIII). HIV-infected children should avoid contact with reptiles (e.g., snakes, lizards, iguanas, turtles) and with chicks and ducklings (as well as their uncooked eggs) because of the risk of salmonellosis (BIII). These precautions are especially important for children who are not receiving effective cART.

Travel: The risk of foodborne and waterborne infections in immunosuppressed, HIVinfected persons is magnified during travel to resource-limited settings. All children who travel to such settings should avoid foods and beverages that might be contaminated, including raw fruits and vegetables, raw or undercooked seafood or meat, tap water, ice made with tap water, unpasteurized milk and dairy products, and items sold by street vendors (AIII). Foods and beverages that are usually safe include steaming hot foods, fruits that are peeled by the traveler, bottled (including carbonated) beverages, and water brought to a rolling boil for 1 minute. Treatment of water with iodine or chlorine may not be as effective as boiling and will not eliminate Cryptosporidia but can be used when boiling is 
not practical. These precautions are especially important for children who are not receiving effective cART.

\section{Preventing Disease}

Immunization: In addition to cART, one of the most important interventions to prevent bacterial infections in HIV-infected children is to ensure that they are immunized according to the HIV-specific recommended schedule (Figures 1 and 2) (AII). Vaccines that protect against bacterial pathogens directly (e.g., pneumococcal, Hib, meningococcal, pertussis) and indirectly (e.g., influenza) have been demonstrated safe and immunogenic in HIV-infected children. ${ }^{56-60}$ HIV-infected children are at increased risk of under-immunization. ${ }^{61}$ Status of vaccination against Hib, pneumococcus, meningococcus, pertussis, influenza, and all recommended vaccines should be reviewed at every clinical encounter and indicated vaccinations provided, according to the established recommendations for immunization of HIV-infected children (AIII). Effective cART instituted before immunization offers the best means to optimize response to immunization. ${ }^{62}$ Lack of effective cART may reduce the magnitude, quality or duration of immunologic response and likely impairs memory response. Greater number or strength of vaccine doses are recommended in some circumstances to overcome suboptimal response. Evidence is mounting that protective immunity to vaccine-preventable disease is lacking in a high proportion of perinatally HIVinfected children who received many of their immunizations before the availability of effective cART. ${ }^{63}$ These data suggest that HIV-infected children may benefit from assessment of seroprotection and/or re-immunization for certain vaccines.

Hib Vaccine: HEU and HIV-infected infants and children aged $\$ 5$ years should receive Hib vaccine on the same schedule as that recommended for healthy infants, including for catchup immunization (AII). (Figure 1). Hib vaccine is recommended for routine administration to infants aged 2, 4, and 6 months (6-month dose not needed if PRP-OMP Hib conjugate vaccine used for 2- and 4-month doses), and 12 to 15 months; 1 to 3 doses are recommended for previously unvaccinated infants and children aged 7 to 23 months depending on age at first vaccination. Health-care providers should consider use of Hib vaccine for HIV-infected children aged $\geq 5$ years who have not previously received Hib vaccine (AIII). For these older children, a single dose of any Hib conjugate vaccine is recommended. ${ }^{64}$

Pneumococcal Vaccines: HEU and HIV-infected infants and children aged 2 to 59 months should receive the 13-valent pneumococcal vaccine (PCV13) on the same schedule as that recommended for healthy infants and children, including series completion for those who initiated immunization with PCV7 (AII). ${ }^{23,65,66} \mathrm{~A}$ 4-dose series of PCV13 is recommended for routine administration to infants aged 2, 4, 6, and 12 to 15 months; 2 or 3 doses are recommended for previously unvaccinated infants and children aged 7 to 23 months depending on age at first vaccination. ${ }^{64}$ Incompletely vaccinated children aged 24 to 71 months should receive 1 dose of PCV13 if 3 doses of PCV (7 or 13) were received previously, or 2 doses of PCV13 $\geq 8$ weeks apart if $<3$ doses of PCV (7 or 13) were received previously. Children who have received a complete series of $\mathrm{PCV} 7$ should receive a supplemental dose of PCV13 if they are aged 14 through 71 months. In addition, HIVinfected children aged $\geq 2$ years should receive 23 -valent pneumococcal polysaccharide 
vaccine (PPSV) ( $\geq 2$ months after their last PCV dose), with a single revaccination with PPSV 5 years later (AII). ${ }^{57,64}$ Data are limited regarding efficacy of PCV7 or PCV13 for children aged $\succ 6$ years who are at high risk of pneumococcal infection. However, the U.S. Food and Drug Administration recently approved expanded use of PCV13 for children aged 6 to 17 years. ${ }^{67}$ In addition, the Centers for Disease Control and Prevention (CDC) Advisory Committee on Immunization Practices (ACIP) recently recommended that a single dose of PCV13 be routinely administered to children aged 6 years through 18 years with immunocompromising conditions who have not previously received PCV13. ${ }^{68}$ Therefore, a single dose of PCV13 should be routinely administered to HIV-infected children aged 6 through 18 years who did not receive PCV13 before age 6 years ${ }^{64}$ (Figures 1 and 2). A multicenter study of pneumococcal vaccination in a group of HIV-infected children not administered PCV during infancy demonstrated the safety and immunogenicity of 2 doses of PCV7 followed by one dose of PPSV for cART-treated HIV-infected children aged 2 to 19 years (including some who had previously received pneumococcal polysaccharide vaccination [PPSV] $).{ }^{57}$ Based on this study, some experts recommend giving 2 doses of PCV13 to HIV-infected children aged $\nsucceq 6$ years who never received PCV7 or PCV13 (BII). PPSV may be offered $\geq 8$ weeks after PCV13 in children aged 6 to 18 years who received a PCV13 dose after having received PPSV (CII). ${ }^{57}$ The incidence of invasive pneumococcal disease was substantially lower in HIV-infected vaccine recipients in a placebo-controlled trial of a nine-valent PCV in South African children (most whom were not receiving antiretroviral therapy), but vaccine efficacy was somewhat lower in HIV-infected (65\%) than HIV-uninfected children $(85 \%) .{ }^{66}$

Meningococcal Vaccine: Like healthy children, HIV-infected children should routinely receive meningococcal conjugate vaccine (MCV) at age 11 to 12 years and again at age 16 (AII). In contrast to the 1-dose primary series for healthy children, the primary series of MCV for all HIV-infected children aged $\geq 9$ months is $2 \mathrm{MCV}$ doses at least 2 months apart for children aged 2 to 10 years, and 2 to 3 months apart for children aged 9 to 23 months in order to improve rates of seroprotection (AII). ${ }^{64,69-71}$ HIV-infected children aged 9 months to 10 years who have evidence of splenic dysfunction or complement deficiency or who plan to travel to high-incidence areas should receive the primary MCV series (AIII). While ACIP does not list HIV infection as a specific indication for MCV, some experts give MCV to all HIV-infected children aged 9 months to 10 years because of the potentially increased risk of meningococcal disease (CIII). HIV-infected children who receive their primary MCV series at ages 9 months to 10 years and who are at ongoing increased risk of meningococcal exposure should receive another MCV dose 3 years later (if primary MCV immunization was at ages 9 months to 6 years) or 5 years later (if primary MCV immunization was at $\geq 7$ years) (AIII). ${ }^{64} \mathrm{MCV}$ should be repeated every 5 years in children with splenic dysfunction or complement deficiency for as long as their splenic dysfunction persists (AIII).

Influenza Vaccine: Because influenza increases the risk of secondary bacterial respiratory infections, ${ }^{72}$ annual influenza vaccination for influenza prevention can be expected to reduce the risk of serious bacterial infections in HIV-infected children (BIII) (Figures 1 and 2). ${ }^{73} \mathrm{HIV}$-infected children should receive annual influenza vaccination according to the HIV-specific recommended immunization schedule (AII). ${ }^{60,64}$ 
Chemoprophylaxis: Trimethoprim-sulfamethoxazole (TMP-SMX) administered daily for PCP prophylaxis may decrease the rate of serious bacterial infections (predominantly respiratory) in HIV-infected children unable to take cART (BII). ${ }^{16,74}$ Atovaquone combined with azithromycin, which provides prophylaxis for Mycobacterium avium complex (MAC) as well as PCP, is well tolerated and as effective as TMP-SMX in preventing serious bacterial infections in HIV-infected children. ${ }^{75}$ However, routine use of antibiotics solely for primary prevention of serious bacterial infections (i.e., when not indicated for PCP or MAC prophylaxis or other specific reasons) promotes development of drug-resistant organisms and is not routinely recommended (BIII). Intravenous immune globulin (IVIG) is recommended to prevent serious bacterial infections in HIV-infected children who have hypogammaglobulinemia (immunoglobulin $\mathrm{G}<400 \mathrm{mg} / \mathrm{dL}$ ) (AI). ${ }^{15}$

Discontinuation of Primary Prophylaxis-The Pediatric AIDS Clinical Trials Group (PACTG) 1008 demonstrated that discontinuation of MAC and/or PCP antibiotic prophylaxis in HIV-infected children who achieved sustained ( $\geq 16$ weeks) immune reconstitution (CD4 T lymphocyte [CD4] cell percentage $>20 \%$ to $25 \%$ ) while receiving ART did not result in excessive rates of serious bacterial infections. ${ }^{6} \mathrm{HIV}$-infected children who are receiving an antibiotic for the purpose of primary prevention of serious bacterial infections should discontinue antibiotic prophylaxis once they have achieved sustained (i.e., $\geq 3$ months) immune reconstitution (CD4 percentage $\geq 25 \%$ aged $<6$ years; CD4 percentage $\geq 20 \%$ or CD4 count $>350$ cells $/ \mathrm{mm}^{3}$ if aged $\nsucceq 6$ years) $($ BII).

\section{Treatment Recommendations}

Treating Disease-The principles for treating serious bacterial infections are the same in HIV-infected and HIV-uninfected children. Specimens for microbiologic studies should be collected before initiation of antibiotic treatment. However, in patients with suspected serious bacterial infections, therapy should be administered empirically and promptly without waiting for results of such studies; therapy can be adjusted once results become available. The local prevalence of antibiotic-resistant bacteria (e.g., penicillin-resistant $S$. pneumoniae, MRSA) and the recent use of prophylactic or therapeutic antibiotics should be considered when initiating empiric therapy. When the organism is identified, antibiotic susceptibility testing should be performed, and subsequent therapy based on the results of susceptibility testing (AIII).

HIV-infected children whose immune systems are not seriously compromised (CDC Immunologic Category I ${ }^{76}$ and who are not neutropenic can be expected to respond similarly to HIV-uninfected children and should be treated for the most likely bacterial organisms (AIII). Based only on expert opinion, mild to moderate community-acquired pneumonia in HIV-infected children with only mild or no immunosuppression who are fully immunized (especially against $S$. pneumoniae and $\mathrm{Hib}$ ) and who are receiving effective cART can be treated with oral antibiotics (usually oral amoxicillin), according to the same guidelines as for healthy children (BIII). ${ }^{76}$ However, many experts have a lower threshold for hospitalizing these children to initiate treatment. In addition, broader-spectrum antimicrobial agents for initial empiric therapy are sometimes chosen because of the potentially higher risk of non-susceptible pneumococcal infections in HIV-infected 
children. ${ }^{17,22,24,25}$ Thus, options for empiric therapy for HIV-infected children outside of the neonatal period who are hospitalized for suspected community-acquired bacteremia or bacterial pneumonia include ampicillin or an extended-spectrum cephalosporin (e.g, ceftriaxone, cefotaxime) (AIII). ${ }^{8,77,78}$ The addition of vancomycin or other antibiotic for suspected bacterial meningitis should follow the same guidelines as for HIV-uninfected children. ${ }^{79}$ The addition of azithromycin or other macrolide can be considered for hospitalized patients with pneumonia to treat other common community-acquired pneumonia pathogens (M. pneumoniae, C. pneumoniae). If MRSA is suspected or the prevalence of MRSA is high (i.e., >10\%) in the community, clindamycin (for non-CNS infections), doxycycline (non-CNS, for childen aged $>8$ years) or vancomycin can be added (choice based on local susceptibility patterns) ${ }^{80-82}$ Neutropenic children also should be treated with an appropriate antipseudomonal drug with consideration for adding an aminoglycoside if infection with Pseudomonas spp. is likely. Severely immunocompromised HIV-infected children with invasive or recurrent bacterial infections require expanded empiric antimicrobial treatment covering a broad range of resistant organisms similar to that chosen for suspected catheter sepsis pending results of diagnostic evaluations and cultures (AIII).

Initial empiric therapy for HIV-infected children with suspected intravascular catheter sepsis should target both gram-positive and enteric gram-negative organisms, with combinations that include agents with anti-Pseudomonas activity and vancomycin, which is active against MRSA (AIII). Factors such as response to therapy, clinical status, identification of pathogen, and need for ongoing vascular access will determine the need for and timing of catheter removal.

Monitoring and Adverse Events (Including IRIS)—The response to appropriate antibiotic therapy should be similar in HIV-infected and HIV-uninfected children, with a clinical response usually observed within 2 to 3 days after initiation of appropriate antibiotics, recognizing that radiologic improvement in patients with pneumonia may lag behind clinical response. Whereas HIV-infected adults experience high rates of adverse and even treatment-limiting reactions to TMP-SMX, in HIV-infected children, serious adverse reactions to TMP-SMX appear to be much less of a problem. ${ }^{84}$

Immune reconstitution inflammatory syndrome (IRIS) has not clearly been described in association with treatment of bacterial infections in children. Reports of pneumonia, abscess and other bacterial infection in children during the first several weeks of effective cART have been attributed to IRIS ${ }^{85,86}$ but are more likely related to persistent immune suppression. Suspicion of IRIS in a child being treated for a bacterial infection should raise concern for the presence of a different or additional infection or for inadequately treated infection mimicking IRIS.

Preventing Recurrence-Status of vaccination against Hib, pneumococcus, meningococcus, and influenza should be reviewed and updated, according to the recommendations outlined in the section Preventing First Episode of Disease and depicted in the immunization recommendation schedules (Figures 1 and 2) (AIII). 
TMP-SMX (administered daily for PCP prophylaxis) and azithromycin or atovaquoneazithromycin (administered for MAC prophylaxis) also may reduce the incidence of serious bacterial infections in children with recurrent serious bacterial infections. Administration of antibiotic chemoprophylaxis to HIV-infected children who have frequent recurrences of serious bacterial infections despite cART (e.g., >2 serious bacterial infections in a 1-year period despite cART) can be considered (CIII); however, caution is required when using antibiotics solely to prevent recurrence of serious bacterial infections because of the potential for development of drug-resistant microorganisms and drug toxicity. In rare situations in which cART and antibiotic prophylaxis are not effective in preventing frequent recurrent serious bacterial infections, IVIG prophylaxis can be considered for secondary prophylaxis $(\mathbf{C I}) .{ }^{15}$

Discontinuing Secondary Prophylaxis-PACTG 1008 demonstrated that discontinuing MAC and/or PCP antibiotic prophylaxis in HIV-infected children who achieved sustained (i.e., $\geq 16$ weeks) immune reconstitution (CD4 percentage $>20 \%$ to $25 \%$ ) while receiving cART did not result in excessive rates of serious bacterial infections. ${ }^{6}$ Antibiotics for secondary prophylaxis of serious bacterial infections should be discontinued in HIV-infected children who have achieved sustained (i.e., 33 months) immune reconstitution (CD4 percentage $\geq 25 \%$ if $\$ 6$ years old; CD4 percentage $\geq 20 \%$ or $>350$ cells $/ \mathrm{mm}^{3}$ if $>6$ years old) (BII).

\section{References}

1. Dankner WM, Lindsey JC, Levin MJ. Pediatric ACTGPT. Correlates of opportunistic infections in children infected with the human immunodeficiency virus managed before highly active antiretroviral therapy. Pediatr Infect Dis J. 2001 Jan; 20(1):40-48. Available at http:// www.ncbi.nlm.nih.gov/pubmed/11176565. [PubMed: 11176565]

2. Mofenson LM, Korelitz J, Pelton S, Moye J Jr, Nugent R, Bethel J. Sinusitis in children infected with human immunodeficiency virus: clinical characteristics, risk factors, and prophylaxis. National Institute of Child Health and Human Development Intravenous Immunoglobulin Clinical Trial Study Group. Clin Infect Dis. 1995 Nov; 21(5):1175-1181. Available at http:// www.ncbi.nlm.nih.gov/pubmed/8589139. [PubMed: 8589139]

3. Ylitalo N, Brogly S, Hughes MD, et al. Risk factors for opportunistic illnesses in children with human immunodeficiency virus in the era of highly active antiretroviral therapy. Arch Pediatr Adolesc Med. 2006 Aug; 160(8):778-787. Available at http://www.ncbi.nlm.nih.gov/pubmed/ 16894075. [PubMed: 16894075]

4. Chiappini E, Galli L, Tovo PA, et al. Changing patterns of clinical events in perinatally HIV-1infected children during the era of HAART. AIDS. 2007 Jul 31; 21(12):1607-1615. Available at http://www.ncbi.nlm.nih.gov/pubmed/17630556. [PubMed: 17630556]

5. Gona P, Van Dyke RB, Williams PL, et al. Incidence of opportunistic and other infections in HIVinfected children in the HAART era. JAMA. 2006 Jul 19; 296(3):292-300. Available at http:// www.ncbi.nlm.nih.gov/pubmed/16849662. [PubMed: 16849662]

6. Nachman S, Gona P, Dankner W, et al. The rate of serious bacterial infections among HIV-infected children with immune reconstitution who have discontinued opportunistic infection prophylaxis. Pediatrics. 2005 Apr; 115(4):e488-e494. Available at http://www.ncbi.nlm.nih.gov/pubmed/ 15772172. [PubMed: 15772172]

7. Steenhoff AP, Josephs JS, Rutstein RM, et al. Incidence of and risk factors for community acquired pneumonia in US HIV-infected children, 2000-2005. AIDS. 2011 Mar 13; 25(5):717-720. Available at http://www.ncbi.nlm.nih.gov/pubmed/21252630. [PubMed: 21252630]

8. McIntosh K. Community-acquired pneumonia in children. N Engl J Med. 2002 Feb 7; 346(6):429437. Available at http://www.ncbi.nlm.nih.gov/pubmed/11832532. [PubMed: 11832532] 
9. Black SB, Shinefield HR, Ling S, et al. Effectiveness of heptavalent pneumococcal conjugate vaccine in children younger than five years of age for prevention of pneumonia. Pediatr Infect Dis J. 2002 Sep; 21(9):810-815. Available at http://www.ncbi.nlm.nih.gov/pubmed/12352800. [PubMed: 12352800]

10. Nesheim SR, Kapogiannis BG, Soe MM, et al. Trends in opportunistic infections in the pre- and post-highly active antiretroviral therapy eras among HIV-infected children in the Perinatal AIDS Collaborative Transmission Study, 1986-2004. Pediatrics. 2007 Jul; 120(1):100-109. Available at http://www.ncbi.nlm.nih.gov/pubmed/17606567. [PubMed: 17606567]

11. Centers for Disease Control and Prevention. Active Bacterial Core Surveillance Report, Emerging Infections Program Network, Streptococcus pneumonia. 2010 Available via the Internet: http:// www.cdc.gov/abcs/reportsfindings/survreports/spneu10.pdf. 2011.

12. Mofenson LM, Yogev R, Korelitz J, et al. Characteristics of acute pneumonia in human immunodeficiency virusinfected children and association with long term mortality risk. National Institute of Child Health and Human Development Intravenous Immunoglobulin Clinical Trial Study Group. Pediatr Infect Dis J. 1998 Oct; 17(10):872-880. Available at http:// www.ncbi.nlm.nih.gov/pubmed/9802627. [PubMed: 9802627]

13. Madhi SA, Petersen K, Madhi A, Khoosal M, Klugman KP. Increased disease burden and antibiotic resistance of bacteria causing severe community-acquired lower respiratory tract infections in human immunodeficiency virus type 1-infected children. Clin Infect Dis. $2000 \mathrm{Jul}$; 31(1):170-176. Available at http://www.ncbi.nlm.nih.gov/pubmed/10913417. [PubMed: 10913417]

14. Zar HJ. Chronic lung disease in human immunodeficiency virus (HIV) infected children. Pediatr Pulmonol. 2008 Jan; 43(1):1-10. Available at http://www.ncbi.nlm.nih.gov/pubmed/18041077. [PubMed: 18041077]

15. The National Institute of Child Health and Human Developments Intravenous Immunoglobulin Study Group. Intravenous immune globulin for the prevention of bacterial infections in children with symptomatic human immunodeficiency virus infection. N Engl J Med. 1991 Jul 11; 325(2): 73-80. Available at http://www.ncbi.nlm.nih.gov/pubmed/1675763. [PubMed: 1675763]

16. Spector SA, Gelber RD, McGrath N, et al. with Pediatric AIDS Clinical Trials Group. A controlled trial of intravenous immune globulin for the prevention of serious bacterial infections in children receiving zidovudine for advanced human immunodeficiency virus infection. N Engl J Med. 1994 Nov 3; 331(18):1181-1187. Available at http://www.ncbi.nlm.nih.gov/pubmed/7935655. [PubMed: 7935655]

17. Madhi SA, Petersen K, Madhi A, Wasas A, Klugman KP. Impact of human immunodeficiency virus type 1 on the disease spectrum of Streptococcus pneumoniae in South African children. Pediatr Infect Dis J. 2000 Dec; 19(12):1141-1147. Available at http://www.ncbi.nlm.nih.gov/ pubmed/11144373. [PubMed: 11144373]

18. Lichenstein R, King JC Jr, Farley JJ, Su P, Nair P, Vink PE. Bacteremia in febrile human immunodeficiency virusinfected children presenting to ambulatory care settings. Pediatr Infect Dis J. 1998 May; 17(5):381-385. Available at http://www.ncbi.nlm.nih.gov/pubmed/9613650. [PubMed: 9613650]

19. Kapogiannis BG, Soe MM, Nesheim SR, et al. Trends in bacteremia in the pre- and post-highly active antiretroviral therapy era among HIV-infected children in the US Perinatal AIDS Collaborative Transmission Study (1986-2004). Pediatrics. 2008 May; 121(5):e1229-e1239. Available at http://www.ncbi.nlm.nih.gov/pubmed/18450865. [PubMed: 18450865]

20. Farley JJ, King JC Jr, Nair P, Hines SE, Tressler RL, Vink PE. Invasive pneumococcal disease among infected and uninfected children of mothers with human immunodeficiency virus infection. J Pediatr. 1994 Jun; 124(6):853-858. Available at http://www.ncbi.nlm.nih.gov/pubmed/8201466. [PubMed: 8201466]

21. Andiman WA, Simpson J, Holtkamp C, Pearson HA. Invasive pneumococcal infections in children infected with HIV are not associated with splenic dysfunction. AIDS Patient Care STDS. 1996 Dec; 10(6):336-341. Available at http://www.ncbi.nlm.nih.gov/pubmed/11361548. [PubMed: 11361548]

22. Steenhoff AP, Wood SM, Rutstein RM, Wahl A, McGowan KL, Shah SS. Invasive pneumococcal disease among human immunodeficiency virus-infected children, 1989-2006. Pediatr Infect Dis J. 
2008 Oct; 27(10):886-891. Available at http://www.ncbi.nlm.nih.gov/pubmed/18776825. [PubMed: 18776825]

23. Kourtis AP, Ellington S, Bansil P, Jamieson DJ, Posner SF. Hospitalizations for invasive pneumococcal disease among HIV-1-infected adolescents and adults in the United States in the era of highly active antiretroviral therapy and the conjugate pneumococcal vaccine. J Acquir Immune Defic Syndr. 2010 Sep; 55(1):128-131. Available at http://www.ncbi.nlm.nih.gov/pubmed/ 20622675. [PubMed: 20622675]

24. Crewe-Brown HH, Karstaedt AS, Saunders GL, et al. Streptococcus pneumoniae blood culture isolates from patients with and without human immunodeficiency virus infection: alterations in penicillin susceptibilities and in serogroups or serotypes. Clin Infect Dis. 1997 Nov; 25(5):11651172. Available at http://www.ncbi.nlm.nih.gov/pubmed/9402377. [PubMed: 9402377]

25. Frankel RE, Virata M, Hardalo C, Altice FL, Friedland G. Invasive pneumococcal disease: clinical features, serotypes, and antimicrobial resistance patterns in cases involving patients with and without human immunodeficiency virus infection. Clin Infect Dis. 1996 Sep; 23(3):577-584. Available at http://www.ncbi.nlm.nih.gov/pubmed/8879783. [PubMed: 8879783]

26. Rowland KE, Turnidge JD. The impact of penicillin resistance on the outcome of invasive Streptococcus pneumoniae infection in children. Aust N Z J Med. 2000 Aug; 30(4):441-449. Available at http://www.ncbi.nlm.nih.gov/pubmed/10985508. [PubMed: 10985508]

27. Nuorti JP, Whitney CG. Centers for Disease C, Prevention. Prevention of pneumococcal disease among infants and children - use of 13-valent pneumococcal conjugate vaccine and 23-valent pneumococcal polysaccharide vaccine- recommendations of the Advisory Committee on Immunization Practices (ACIP). MMWR Recomm Rep. 2010 Dec 10; 59(RR-11):1-18. Available at http://www.ncbi.nlm.nih.gov/pubmed/21150868. [PubMed: 21150868]

28. Madhi SA, Petersen K, Khoosal M, et al. Reduced effectiveness of Haemophilus influenzae type b conjugate vaccine in children with a high prevalence of human immunodeficiency virus type 1 infection. Pediatr Infect Dis J. 2002 Apr; 21(4):315-321. Available at http:// www.ncbi.nlm.nih.gov/pubmed/12075763. [PubMed: 12075763]

29. Rongkavilit C, Rodriguez ZM, Gomez-Marin O, et al. Gram-negative bacillary bacteremia in human immunodeficiency virus type 1-infected children. Pediatr Infect Dis J. 2000 Feb; 19(2): 122-128. Available at http://www.ncbi.nlm.nih.gov/pubmed/10693998. [PubMed: 10693998]

30. Cohen C, Singh E, Wu HM, et al. Increased incidence of meningococcal disease in HIV-infected individuals associated with higher case-fatality ratios in South Africa. AIDS. 2010 Jun 1; 24(9): 1351-1360. Available at http://www.ncbi.nlm.nih.gov/pubmed/20559040. [PubMed: 20559040]

31. Stephens DS, Hajjeh RA, Baughman WS, Harvey RC, Wenger JD, Farley MM. Sporadic meningococcal disease in adults: results of a 5-year population-based study. Ann Intern Med. 1995 Dec 15; 123(12):937-940. Available at http://www.ncbi.nlm.nih.gov/pubmed/7486489. [PubMed: 7486489]

32. Crum-Cianflone NF, Burgi AA, Hale BR. Increasing rates of community-acquired methicillinresistant Staphylococcus aureus infections among HIV-infected persons. Int J STD AIDS. 2007 Aug; 18(8):521-526. Available at http://www.ncbi.nlm.nih.gov/pubmed/17686212. [PubMed: 17686212]

33. Diep BA, Chambers HF, Graber CJ, et al. Emergence of multidrug-resistant, communityassociated, methicillinresistant Staphylococcus aureus clone USA300 in men who have sex with men. Ann Intern Med. 2008 Feb 19; 148(4):249-257. Available at http://www.ncbi.nlm.nih.gov/ pubmed/18283202. [PubMed: 18283202]

34. Lee NE, Taylor MM, Bancroft E, et al. Risk factors for community-associated methicillin-resistant Staphylococcus aureus skin infections among HIV-positive men who have sex with men. Clin Infect Dis. 2005 May 15; 40(10):1529-1534. Available at http://www.ncbi.nlm.nih.gov/pubmed/ 15844078. [PubMed: 15844078]

35. Srinivasan A, Seifried S, Zhu L, et al. Short communication: methicillin-resistant Staphylococcus aureus infections in children and young adults infected with HIV. AIDS Res Hum Retroviruses. 2009 Dec; 25(12):1219-1224. Available at http://www.ncbi.nlm.nih.gov/pubmed/20001313. [PubMed: 20001313] 
36. Berkley JA, Lowe BS, Mwangi I, et al. Bacteremia among children admitted to a rural hospital in Kenya. N Engl J Med. 2005 Jan 6; 352(1):39-47. Available at http://www.ncbi.nlm.nih.gov/ pubmed/15635111. [PubMed: 15635111]

37. Roilides E, Marshall D, Venzon D, Butler K, Husson R, Pizzo PA. Bacterial infections in human immunodeficiency virus type 1-infected children: the impact of central venous catheters and antiretroviral agents. Pediatr Infect Dis J. 1991 Nov; 10(11):813-819. Available at http:// www.ncbi.nlm.nih.gov/pubmed/1661003. [PubMed: 1661003]

38. Kuhn L, Kasonde P, Sinkala M, et al. Does severity of HIV disease in HIV-infected mothers affect mortality and morbidity among their uninfected infants? Clin Infect Dis. 2005 Dec 1; 41(11): 1654-1661. Available at http://www.ncbi.nlm.nih.gov/pubmed/16267740. [PubMed: 16267740]

39. Brahmbhatt H, Kigozi G, Wabwire-Mangen F, et al. Mortality in HIV-infected and uninfected children of HIV-infected and uninfected mothers in rural Uganda. J Acquir Immune Defic Syndr. 2006 Apr 1; 41(4):504-508. Available at http://www.ncbi.nlm.nih.gov/pubmed/16652060. [PubMed: 16652060]

40. Mussi-Pinhata MM, Freimanis L, Yamamoto AY, et al. Infectious disease morbidity among young HIV-1-exposed but uninfected infants in Latin American and Caribbean countries: the National Institute of Child Health and Human Development International Site Development Initiative Perinatal Study. Pediatrics. 2007 Mar; 119(3):e694-e704. Available at http:// www.ncbi.nlm.nih.gov/pubmed/17296782. [PubMed: 17296782]

41. Kattan M, Platzker A, Mellins RB, et al. Respiratory diseases in the first year of life in children born to HIV-1-infected women. Pediatr Pulmonol. 2001 Apr; 31(4):267-276. Available at http:// www.ncbi.nlm.nih.gov/pubmed/11288208. [PubMed: 11288208]

42. Jones CE, Naidoo S, De Beer C, Esser M, Kampmann B, Hesseling AC. Maternal HIV infection and antibody responses against vaccine-preventable diseases in uninfected infants. JAMA. 2011 Feb 9; 305(6):576-584. Available at http://www.ncbi.nlm.nih.gov/pubmed/21304083. [PubMed: 21304083]

43. Abrams EJ. Opportunistic infections and other clinical manifestations of HIV disease in children. Pediatr Clin North Am. 2000 Feb; 47(1):79-108. Available at http://www.ncbi.nlm.nih.gov/ pubmed/10697643. [PubMed: 10697643]

44. Mao C, Harper M, McIntosh K, et al. Invasive pneumococcal infections in human immunodeficiency virus-infected children. J Infect Dis. 1996 Apr; 173(4):870-876. Available at http://www.ncbi.nlm.nih.gov/pubmed/8603965. [PubMed: 8603965]

45. Gesner M, Desiderio D, Kim M, et al. Streptococcus pneumoniae in human immunodeficiency virus type 1-infected children. Pediatr Infect Dis J. 1994 Aug; 13(8):697-703. Available at http:// www.ncbi.nlm.nih.gov/pubmed/7970969. [PubMed: 7970969]

46. Sharland M, Gibb DM, Holland F. Respiratory morbidity from lymphocytic interstitial pneumonitis (LIP) in vertically acquired HIV infection. Arch Dis Child. 1997 Apr; 76(4):334-336. Available at http://www.ncbi.nlm.nih.gov/pubmed/9166026. [PubMed: 9166026]

47. Molyneux EM, Tembo M, Kayira K, et al. The effect of HIV infection on paediatric bacterial meningitis in Blantyre, Malawi. Arch Dis Child. 2003 Dec; 88(12):1112-1118. Available at http:// www.ncbi.nlm.nih.gov/pubmed/14670782. [PubMed: 14670782]

48. Madhi SA, Madhi A, Petersen K, Khoosal M, Klugman KP. Impact of human immunodeficiency virus type 1 infection on the epidemiology and outcome of bacterial meningitis in South African children. Int J Infect Dis. 2001; 5(3):119-125. Available at http://www.ncbi.nlm.nih.gov/pubmed/ 11724667. [PubMed: 11724667]

49. Zar HJ, Tannenbaum E, Hanslo D, Hussey G. Sputum induction as a diagnostic tool for community-acquired pneumonia in infants and young children from a high HIV prevalence area. Pediatr Pulmonol. 2003 Jul; 36(1):58-62. Available at http://www.ncbi.nlm.nih.gov/pubmed/ 12772225. [PubMed: 12772225]

50. Selwyn PA, Pumerantz AS, Durante A, et al. Clinical predictors of Pneumocystis carinii pneumonia, bacterial pneumonia and tuberculosis in HIV-infected patients. AIDS. 1998 May 28; 12(8):885-893. Available at http://www.ncbi.nlm.nih.gov/pubmed/9631142. [PubMed: 9631142]

51. Sheikh S, Madiraju K, Steiner P, Rao M. Bronchiectasis in pediatric AIDS. Chest. 1997 Nov 5; 112(5):1202-1207. Available at http://www.ncbi.nlm.nih.gov/pubmed/9367458. [PubMed: 9367458] 
52. Midulla F, Strappini P, Sandstrom T, et al. Cellular and noncellular components of bronchoalveolar lavage fluid in HIV-1-infected children with radiological evidence of interstitial lung damage. Pediatr Pulmonol. 2001 Mar; 31(3):205-213. Available at http:// www.ncbi.nlm.nih.gov/pubmed/11276133. [PubMed: 11276133]

53. Mermel LA, Allon M, Bouza E, et al. Clinical practice guidelines for the diagnosis and management of intravascular catheter-related infection, 2009 Update by the Infectious Diseases Society of America. Clin Infect Dis. 2009 Jul 1; 49(1):1-45. Available at http:// www.ncbi.nlm.nih.gov/pubmed/19489710. [PubMed: 19489710]

54. Wood N, McIntyre P. Pertussis: review of epidemiology, diagnosis, management and prevention. Paediatr Respir Rev. 2008 Sep; 9(3):201-211. quiz 211-202. Available at http:// www.ncbi.nlm.nih.gov/pubmed/18694712. [PubMed: 18694712]

55. She RC, Thurber A, Hymas WC, et al. Limited utility of culture for Mycoplasma pneumoniae and Chlamydophila pneumoniae for diagnosis of respiratory tract infections. J Clin Microbiol. 2010 Sep; 48(9):3380-3382. Available at http://www.ncbi.nlm.nih.gov/pubmed/20610673. [PubMed: 20610673]

56. Abzug MJ, Song LY, Fenton T, et al. Pertussis booster vaccination in HIV-infected children receiving highly active antiretroviral therapy. Pediatrics. 2007 Nov; 120(5):1190-1202. Available at http://www.ncbi.nlm.nih.gov/pubmed/17938165.

57. Abzug MJ, Pelton SI, Song LY, et al. Immunogenicity, safety, and predictors of response after a pneumococcal conjugate and pneumococcal polysaccharide vaccine series in human immunodeficiency virus-infected children receiving highly active antiretroviral therapy. Pediatr Infect Dis J. 2006 Oct; 25(10):920-929. Available at http://www.ncbi.nlm.nih.gov/pubmed/ 17006288. [PubMed: 17006288]

58. Mangtani P, Mulholland K, Madhi SA, Edmond K, O'Loughlin R, Hajjeh R. Haemophilus influenzae type $\mathrm{b}$ disease in HIV-infected children: a review of the disease epidemiology and effectiveness of Hib conjugate vaccines. Vaccine. 2010 Feb 17; 28(7):1677-1683. Available at http://www.ncbi.nlm.nih.gov/pubmed/20034606. [PubMed: 20034606]

59. Siberry GK, Williams PL, Lujan-Zilbermann J, et al. Phase I/II, open-label trial of safety and immunogenicity of meningococcal (groups A, C, Y, and W-135) polysaccharide diphtheria toxoid conjugate vaccine in human immunodeficiency virus-infected adolescents. Pediatr Infect Dis J. 2010 May; 29(5):391-396. Available at http://www.ncbi.nlm.nih.gov/pubmed/20431379. [PubMed: 20431379]

60. Levin MJ, Song LY, Fenton T, et al. Shedding of live vaccine virus, comparative safety, and influenza-specific antibody responses after administration of live attenuated and inactivated trivalent influenza vaccines to HIV-infected children. Vaccine. 2008 Aug 5; 26(33):4210-4217. Available at http://www.ncbi.nlm.nih.gov/pubmed/18597900. [PubMed: 18597900]

61. Myers C, Posfay-Barbe KM, Aebi C, et al. Determinants of vaccine immunity in the cohort of human immunodeficiency virus-infected children living in Switzerland. Pediatr Infect Dis J. 2009 Nov; 28(11):996-1001. Available at http://www.ncbi.nlm.nih.gov/pubmed/19820427. [PubMed: 19820427]

62. Pensieroso S, Cagigi A, Palma P, et al. Timing of HAART defines the integrity of memory B cells and the longevity of humoral responses in HIV-1 vertically-infected children. Proc Natl Acad Sci U S A. 2009 May 12; 106(19):7939-7944. Available at http://www.ncbi.nlm.nih.gov/pubmed/ 19416836. [PubMed: 19416836]

63. Sutcliffe CG, Moss WJ. Do children infected with HIV receiving HAART need to be revaccinated? Lancet Infect Dis. 2010 Sep; 10(9):630-642. Available at http:// www.ncbi.nlm.nih.gov/pubmed/20797645. [PubMed: 20797645]

64. Centers for Disease Control and Prevention. Recommended Immunization Schedules for Persons Aged 0 Through 18 Years-United States 2011. MMWR Morb Mortal Wkly Rep. 2011 Available at http://www.cdc.gov/mmWr/preview/mmwrhtml/mm6005a6.htm.

65. Nachman S, Kim S, King J, et al. Safety and immunogenicity of a heptavalent pneumococcal conjugate vaccine in infants with human immunodeficiency virus type 1 infection. Pediatrics. 2003 Jul; 112(1 Pt 1):66-73. Available at http://www.ncbi.nlm.nih.gov/pubmed/12837869. [PubMed: 12837869] 
66. Klugman KP, Madhi SA, Huebner RE, et al. A trial of a 9-valent pneumococcal conjugate vaccine in children with and those without HIV infection. N Engl J Med. 2003 Oct 2; 349(14):1341-1348. Available at http://www.ncbi.nlm.nih.gov/pubmed/14523142. [PubMed: 14523142]

67. FDA. Summary Basis for Regulatory Action: Prevnar 13/Pneumococcal 13-valent Conjugate Vaccine (Diphtheria CRM197 Protein). 2013 Available at http://www.fda.gov/downloads/ BiologicsBloodVaccines/Vaccines/ApprovedProducts/UCM337458.pdf.

68. CDC. Immunization Works February 2013 Issue. 2013 Available at http://www.cdc.gov/vaccines/ news/newsltrs/imwrks/2013/201302.htm.

69. Lujan-Zilbermann, J.; Williams, P.; Siberry, G., et al. 28- and 72-week immunogenicity of one dose vs. two doses of Quadrivalent Meningococcal Conjugate Vaccine in HIV-infected Youth. Paper Presented at 17th Conference on Retroviruses and Opportunistic Infections; February 16-19, 2010; San Francisco, CA.

70. Warshaw, M.; Siberry, G.; Williams, P., et al. (S-103) Long-Term (72 Weeks) Immunogenicity and Increased Response Rates After a Second Dose of Quadrivalent Meningococcal Conjugate Vaccine in HIV-Infected Children and Youth. Paper presented at: 18th Conference on Retroviruses and Opportunistic Infections; February 27-March 2, 2011; Boston, MA.

71. Advisory Committee on Immunization Practices. Vaccine for Children (VFC) Resolution No. 6/11-1. Adopted and Effective: June 22, 2011. Available at: www.cdc.gov/vaccines/programs/vfc/ downloads/resolutions/06-11mening-mcv.pdf. 2011.

72. Madhi SA, Ramasamy N, Bessellar TG, Saloojee H, Klugman KP. Lower respiratory tract infections associated with influenza A and B viruses in an area with a high prevalence of pediatric human immunodeficiency type 1 infection. Pediatr Infect Dis J. 2002 Apr; 21(4):291-297. Available at http://www.ncbi.nlm.nih.gov/pubmed/12075759. [PubMed: 12075759]

73. Fiore AE, Shay DK, Broder K, et al. Prevention and control of influenza: recommendations of the Advisory Committee on Immunization Practices (ACIP), 2008. MMWR Recomm Rep. 2008 Aug 8; 57(RR-7):1-60. Available at http://www.ncbi.nlm.nih.gov/pubmed/18685555. [PubMed: 18685555]

74. Mulenga V, Ford D, Walker AS, et al. Effect of cotrimoxazole on causes of death, hospital admissions and antibiotic use in HIV-infected children. AIDS. 2007 Jan 2; 21(1):77-84. Available at http://www.ncbi.nlm.nih.gov/pubmed/17148971. [PubMed: 17148971]

75. Hughes WT, Dankner WM, Yogev R, et al. Comparison of atovaquone and azithromycin with trimethoprimsulfamethoxazole for the prevention of serious bacterial infections in children with HIV infection. Clin Infect Dis. 2005 Jan 1; 40(1):136-145. Available at http:// www.ncbi.nlm.nih.gov/pubmed/15614703. [PubMed: 15614703]

76. CDC. Revised classification system for human immunodeficiency virus infection in children less than 13 years of age. Official authorized addenda: human immunodeficiency virus infection codes and official guidelines for coding and reporting ICD-9-CM. MMWR Morb Mortal Wkly Rep. 1994; 43:1-19. Available at http://www.cdc.gov/mmwr/PDF/rr/rr4312.pdf.

77. Saez-Llorens X, McCracken GH Jr. Bacterial meningitis in children. Lancet. 2003 Jun 21; 361(9375):2139-2148. Available at http://www.ncbi.nlm.nih.gov/pubmed/12826449. [PubMed: 12826449]

78. Bradley JS, Byington CL, Shah SS, et al. The management of community-acquired pneumonia in infants and children older than 3 months of age: clinical practice guidelines by the Pediatric Infectious Diseases Society and the Infectious Diseases Society of America. Clin Infect Dis. 2011 Oct; 53(7):e25-e76. Available at http://www.ncbi.nlm.nih.gov/pubmed/21880587. [PubMed: 21880587]

79. Tunkel AR, Hartman BJ, Kaplan SL, et al. Practice guidelines for the management of bacterial meningitis. Clin Infect Dis. 2004 Nov 1; 39(9):1267-1284. Available at http:// www.ncbi.nlm.nih.gov/pubmed/15494903. [PubMed: 15494903]

80. Martinez-Aguilar G, Hammerman WA, Mason EO Jr, Kaplan SL. Clindamycin treatment of invasive infections caused by community-acquired, methicillin-resistant and methicillinsusceptible Staphylococcus aureus in children. Pediatr Infect Dis J. 2003 Jul; 22(7):593-598. Available at http://www.ncbi.nlm.nih.gov/pubmed/12867833. [PubMed: 12867833] 
81. Kaplan SL, Hulten KG, Gonzalez BE, et al. Three-year surveillance of community-acquired Staphylococcus aureus infections in children. Clin Infect Dis. 2005 Jun 15; 40(12):1785-1791. Available at http://www.ncbi.nlm.nih.gov/pubmed/15909267. [PubMed: 15909267]

82. Liu C, Bayer A, Cosgrove SE, et al. Clinical practice guidelines by the infectious diseases society of america for the treatment of methicillin-resistant Staphylococcus aureus infections in adults and children. Clin Infect Dis. 2011 Feb 1; 52(3):e18-e55. Available at http://www.ncbi.nlm.nih.gov/ pubmed/21208910. [PubMed: 21208910]

83. Moallem HJ, Garratty G, Wakeham M, et al. Ceftriaxone-related fatal hemolysis in an adolescent with perinatally acquired human immunodeficiency virus infection. J Pediatr. 1998 Aug; 133(2): 279-281. Available at http://www.ncbi.nlm.nih.gov/pubmed/9709722. [PubMed: 9709722]

84. Chintu C, et al. Co-trimoxazole as prophylaxis against opportunistic infections in HIV-infected Zambian children (CHAP): a double-blind randomised placebo-controlled trial. Lancet. 2004; 364(9448):1865-1871. Available at http://www.ncbi.nlm.nih.gov/pubmed/15555666. [PubMed: 15555666]

85. Smith K, Kuhn L, Coovadia A, et al. Immune reconstitution inflammatory syndrome among HIVinfected South African infants initiating antiretroviral therapy. AIDS. 2009 Jun 1; 23(9):10971107. Available at http://www.ncbi.nlm.nih.gov/pubmed/19417581. [PubMed: 19417581]

86. Orikiiriza J, Bakeera-Kitaka S, Musiime V, Mworozi EA, Mugyenyi P, Boulware DR. The clinical pattern, prevalence, and factors associated with immune reconstitution inflammatory syndrome in Ugandan children. AIDS. 2010 Aug 24; 24(13):2009-2017. Available at http:// www.ncbi.nlm.nih.gov/pubmed/20616700. [PubMed: 20616700]

\section{Dosing Recommendations for Prevention and Treatment of Invasive Bacterial Infections}

\begin{tabular}{|c|c|c|c|}
\hline Indication & First Choice & Alternative & Comments/Special Issues \\
\hline $\begin{array}{l}\text { Primary } \\
\text { Prophylaxis } \\
\text { S. } \\
\text { pneumoniae } \\
\text { and other } \\
\text { invasive } \\
\text { bacteria }\end{array}$ & $\begin{array}{l}\text { Pneumococcal, } \\
\text { meningococcal, } \\
\text { and Hib } \\
\text { vaccines } \\
\text { IVIG } 400 \mathrm{mg} / \mathrm{kg} \\
\text { body weight } \\
\text { every } 2-4 \text { weeks }\end{array}$ & $\begin{array}{l}\text { TMP-SMX } \\
75 / 375 \\
\mathrm{mg} / \mathrm{m}^{2} \text { body } \\
\text { surface area } \\
\text { per dose by } \\
\text { mouth twice } \\
\text { daily }\end{array}$ & $\begin{array}{l}\text { See Figures } 1 \text { and } 2 \text { for detailed vaccines } \\
\text { recommendations. } \\
\text { Vaccines Routinely Recommended for } \\
\begin{array}{l}\text { Primary } \\
\text { Prophylaxis. Additional Primary }\end{array} \\
\begin{array}{l}\text { Prophylaxis } \\
\text { Indicated For: }\end{array} \\
\begin{array}{l}\text { Hypogammaglobulinemia } \\
\text { (that is, IgG }<400 \mathrm{mg} / \mathrm{dL} \text { ) }\end{array} \\
\text { Criteria for Discontinuing Primary } \\
\begin{array}{l}\text { Prophylaxis: } \\
\text { - Resolution of } \\
\text { hypogammaglobulinemia }\end{array} \\
\begin{array}{l}\text { Criteria for Restarting Primary } \\
\text { Prophylaxis: } \\
\text { - Relapse of } \\
\quad \text { hypogammaglobulinemia }\end{array}\end{array}$ \\
\hline $\begin{array}{l}\text { Secondary } \\
\text { Prophylaxis } \\
S . \\
\text { pneumoniae } \\
\text { and other } \\
\text { invasive } \\
\text { bacteria }\end{array}$ & $\begin{array}{l}\text { TMP-SMX } \\
75 / 375 \\
\mathrm{mg} / \mathrm{m}^{2} \text { body } \\
\text { surface area per } \\
\text { dose by mouth } \\
\text { twice daily }\end{array}$ & $\begin{array}{l}\text { - } \quad \text { IVIG } 400 \\
\text { mg/kg body } \\
\text { weight every } \\
2-4 \text { weeks }\end{array}$ & $\begin{array}{l}\text { Secondary } \text { Prophylaxis Indicated: } \\
\quad \quad>2 \text { serious bacterial } \\
\text { infections in a 1-year period } \\
\text { in children who are unable to } \\
\text { take cART }\end{array}$ \\
\hline
\end{tabular}




\begin{tabular}{|c|c|c|c|}
\hline Indication & First Choice & Alternative & Comments/Special Issues \\
\hline & & & $\begin{array}{ll}- & >2 \text { serious bacterial } \\
\text { infections in a 1-year period } \\
\text { despite cART }\end{array}$ \\
\hline $\begin{array}{l}\text { Treatment } \\
\text { Bacterial } \\
\text { pneumonia; } \\
S . \\
\text { pneumoniae; } \\
\text { occasionally } \\
S . \\
\text { aureus, } H . \\
\text { influenza, } P \text {. } \\
\text { aeruginosa }\end{array}$ & $\begin{array}{l}\text { Ceftriaxone } 50- \\
100 \mathrm{mg} / \mathrm{kg} \text { body } \\
\text { weight per dose } \\
\text { once daily, or } \\
25-50 \mathrm{mg} / \mathrm{kg} \\
\text { body weight per } \\
\text { dose twice daily } \\
\text { IV or IM (max } 4 \\
\text { g/day), or } \\
\text { Cefotaxime } 40- \\
50 \mathrm{mg} / \mathrm{kg} \text { body } \\
\text { weight per dose } \\
4 \text { times daily, or } \\
50-65 \mathrm{mg} / \mathrm{kg} \\
\text { body weight } 3 \\
\text { times daily (max } \\
8-10 \mathrm{~g} / \text { day) IV }\end{array}$ & $\begin{array}{l}\text { Cefuroxime, } \\
35-50 \\
\text { mg/kg body } \\
\text { weight per } \\
\text { dose } 3 \text { times } \\
\text { daily (max } \\
4-6 \mathrm{~g} / \text { day) } \\
\text { IV }\end{array}$ & $\begin{array}{l}\text { For children who are receiving effective } \\
\text { cART, have } \\
\text { mild or no immunosuppression, and have } \\
\text { mild to } \\
\text { moderate community-acquired } \\
\text { pneumonia, oral } \\
\text { therapy option would be amoxicillin } 45 \\
\text { mg/kg } \\
\text { body weight per dose twice daily } \\
\text { (maximum dose: } \\
4 \text { g per day). } \\
\text { Add azithromycin for hospitalized } \\
\text { patients to treat } \\
\text { other common community-acquired } \\
\text { pneumonia } \\
\text { pathogens ( } M \text {. pneumoniae, } C \text {. } \\
\text { pneumoniae). } \\
\text { Add clindamycin or vancomycin if } \\
\text { methicillin- } \\
\text { resistant } S . \text { aureus is suspected (base the } \\
\text { choice } \\
\text { on local susceptibility patterns). } \\
\text { For patients with neutropenia, chronic } \\
\text { lung disease } \\
\text { other than asthma (e.g., LIP, } \\
\text { bronchiectasis) or } \\
\text { indwelling venous catheter, consider } \\
\text { regimen that } \\
\text { includes activity against } P . \text { aeruginosa } \\
\text { (such as } \\
\text { ceftazidime or cefepime instead of } \\
\text { ceftriaxone). } \\
\text { Consider PCP in patients with severe } \\
\text { pneumonia } \\
\text { or more advanced HIV disease. } \\
\text { Evaluate for tuberculosis, } \\
\text { cryptococcosis, and } \\
\text { endemic fungi as epidemiology suggests. }\end{array}$ \\
\hline
\end{tabular}

Key to Acronyms: cART = combination antiretroviral therapy; CD4 = CD4 T lymphocyte; IgG = immunoglobulin G; IM = intramuscular; IV = intravenous; IVIG = intravenous immune globulin; LIP = lymphocytic interstitial pneumonia; $\mathrm{PCP}=$ Pneumocystis jirovecii pneumonia; TMP-SMX = trimethoprim-sulfamethoxazole

\section{Candida Infections (Last updated April 2, 2014; last reviewed November 6,} 2013)

Panel's Recommendations

- Uncomplicated oropharyngeal candidiasis (OPC) infection can be effectively treated with topical therapy using clotrimazole troches or nystatin suspension (AII).

- Oral fluconazole is recommended for moderate or severe OPC disease (AI*).

- For fluconazole-refractory OPC, itraconazole oral solution is recommended, although it is less well tolerated than fluconazole (AI).

- If OPC initially is treated topically, failure or relapse should be treated with oral fluconazole or itraconazole oral solution $\left(\mathbf{A} \mathbf{I}^{*}\right)$.

- $\quad$ Systemic therapy is essential for esophageal disease (AI*).

- Oral or intravenous fluconazole, amphotericin B, or an echinocandin (caspofungin, micafungin, anidulafungin), administered for 14 to 21 days, is highly effective for treatment of Candida esophagitis (AI*). 


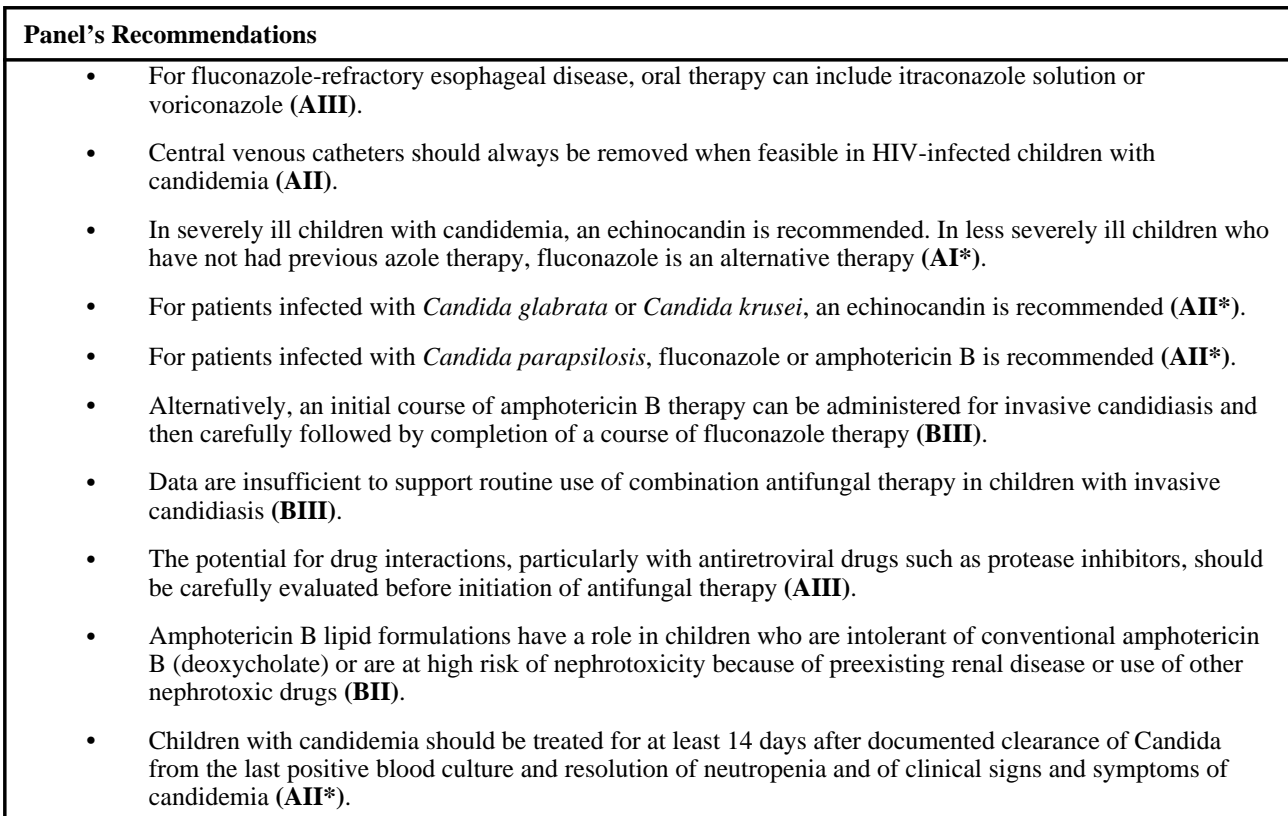

Rating of Recommendations: A = Strong; $\mathrm{B}=$ Moderate; $\mathrm{C}=$ Optional

Rating of Evidence: $\mathrm{I}=$ One or more randomized trials $\underline{\text { in children }}^{\dagger}$ with clinical outcomes and/or validated endpoints; I* $=$ One or more randomized trials in adults with clinical outcomes and/or validated laboratory endpoints with accompanying data in children ${ }^{\dagger}$ from one or more well-designed, nonrandomized trials or observational cohort studies with long-term clinical outcomes; II = One or more well-designed, nonrandomized trials or observational cohort studies in children ${ }^{\dagger}$ with long-term outcomes; II* = One or more well-designed, nonrandomized trials or observational studies in adults with longterm clinical outcomes with accompanying data in children ${ }^{\dagger}$ from one or more similar nonrandomized trials or cohort studies with clinical outcome data; III = Expert opinion

${ }^{\dagger}$ Studies that include children or children/adolescents, but not studies limited to post-pubertal adolescents.

\section{Epidemiology}

The most common fungal infections in HIV-infected children are caused by Candida spp. Localized disease caused by Candida is characterized by limited tissue invasion to the skin or mucosa. Examples of localized candidiasis include oropharyngeal and esophageal disease, vulvovaginitis, and diaper dermatitis. Once the organism penetrates the mucosal surface and widespread hematogenous dissemination occurs, invasive candidiasis ensues. This can result in candidemia, meningitis, endocarditis, renal disease, endophthalmitis, and hepatosplenic disease.

Oral thrush and diaper dermatitis occur in $50 \%$ to $85 \%$ of HIV-infected children. Oropharyngeal candidiasis (OPC) continues to be one of the most frequent opportunistic infections in HIV-infected children during the combination antiretroviral therapy (cART) era (28\% of children), with an incidence rate of 0.93 per 100 child-years. ${ }^{1}$ The incidence of esophageal or tracheobronchial candidiasis has decreased from 1.2 per 100 child-years before the pre-cART era to 0.08 per 100 child-years during the cART era (2001-2004). ${ }^{2}$ However, Candida esophagitis continues to be seen in children who are not responding to antiretroviral therapy. ${ }^{3,4}$ Children who develop esophageal candidiasis despite cART may be less likely to have typical symptoms (e.g., odynophagia, retrosternal pain) or have 
concomitant $\mathrm{OPC}^{5}$; during the pre-cART era, concomitant OPC occurred in $94 \%$ of children with Candida esophagitis. ${ }^{3}$ Risk factors for esophageal candidiasis include low CD4 T lymphocyte (CD4) cell count $\left(<100\right.$ cells $\left./ \mathrm{mm}^{3}\right)$, high viral load, and neutropenia $(<500$ cells $\left./ \mathrm{mm}^{3}\right) .{ }^{1-4}$

Disseminated candidiasis is infrequent in HIV-infected children, but Candida can disseminate from the esophagus particularly when coinfection with herpes simplex virus (HSV) or cytomegalovirus (CMV) is present. ${ }^{3,6}$ Candidemia occurs in up to $12 \%$ of HIVinfected children with chronically indwelling central venous catheters for total parental nutrition or intravenous (IV) antibiotics. ${ }^{4,7}$ Candida albicans is the most common cause of mucosal, esophageal, and invasive candidiasis, but approximately $50 \%$ of reported cases of Candida bloodstream infections in HIV-infected children are caused by non-albicans Candida spp., including Candida tropicalis, Candida pseudotropicalis, Candida parapsilosis, Candida glabrata, Candida krusei, and Candida dubliniensis. The nonalbicans Candida species are important to recognize because several are resistant to fluconazole and other antifungals. In one study of Cambodian HIV-infected children on cART who had candidiasis, seven (75\%) of nine isolated C. glabrata were resistant to fluconazole, and three (40\%) of seven C. parapsilosis isolated were resistant to more than three azole agents. ${ }^{8}$ Species-specific epidemiology also varies widely by geographic location and hospital. Many children who develop candidemia have received systemically absorbed oral antifungal azole compounds (e.g., ketoconazole, fluconazole) for control of oral and esophageal candidiasis, which may predispose to resistant isolates. ${ }^{4}$

\section{Clinical Manifestations}

Clinical manifestations of OPC vary and include pseudomembranous (thrush) and erythematous (atrophic), hyperplastic (hypertrophic), and angular cheilitis. Thrush appears as creamy white, curd-like patches with inflamed underlying mucosa that is exposed after removal of the exudate. It can be found on the oropharyngeal mucosa, palate, and tonsils. Erythematous OPC is characterized by flat erythematous lesions on the mucosal surface. Hyperplastic candidiasis comprises raised white plaques on the lower surface of the tongue, palate, and buccal mucosa and cannot be removed. Angular cheilitis occurs as red fissured lesions in the corners of the mouth.

Esophageal candidiasis often presents with odynophagia, dysphagia, or retrosternal pain, and unlike adults, many children experience nausea and vomiting. Therefore, children with esophageal candidiasis may present with dehydration and weight loss. Evidence of OPC can be absent in children with esophageal candidiasis, particularly those receiving cART.

New-onset fever in an HIV-infected child with advanced disease and a central venous catheter is the most common clinical manifestation of candidemia. Renal candidiasis presents with candiduria and ultrasonographically demonstrated renal parenchymal lesions, often without symptoms related to renal disease. ${ }^{4}$ 


\section{Diagnosis}

Oral candidiasis can be diagnosed with a potassium hydroxide preparation and culture with microscopic demonstration of budding yeast cells in wet mounts or biopsy specimens. Esophageal candidiasis has a classic cobblestoning appearance on barium swallow. Findings on endoscopy may range from few, small, white, raised plaques to elevated confluent plaques with hyperemia and extensive ulceration. Endoscopy is also helpful for ruling out other causes of refractory esophagitis, such as HSV, CMV, and Mycobacterium avium complex.

Candidemia is best diagnosed with blood cultures using lysis-centrifugation techniques ${ }^{4}$ or automated broth-based systems. ${ }^{9}$ When candidemia is present, retinal examination for endophthalmitis, cardiac echocardiogram for endocarditis, abdominal computed tomography or ultrasound for hepatic or renal involvement, and bone scans for osteomyelitis (if suspected by symptoms) should be considered.

New diagnostic techniques such as the urine $\mathrm{D}$-arabinitol/L-arabinitol ratio, ${ }^{10,11}$ serum Darabinitol/creatinine ratio, ${ }^{12,13}$ Candida antigen mannan, ${ }^{14,15}$ (1,3)-beta-D-gulcan assay, ${ }^{16,17}$ and real-time polymerase chain reaction ${ }^{18,19}$ are promising diagnostic alternatives under development for early diagnosis of invasive candidiasis. Although several of these assays are helpful in diagnosing adult patients, none of them have been validated for use in children.

\section{Prevention Recommendations}

Preventing Exposure-Candida organisms are common commensals on mucosal surfaces in healthy individuals, and no measures are available to reduce exposure to these fungi except reducing exposure to unneeded antibiotic exposure that may predispose to Candida colonization.

Preventing First Episode of Disease-Routine primary prophylaxis of candidiasis in HIV-infected infants and children is not indicated, given the low prevalence of serious Candida infections (e.g., esophageal, tracheobronchial, disseminated) during the cART era and the availability of effective treatment. Concerns exist about the potential for resistant Candida strains, drug interactions between antifungal and antiretroviral (ARV) agents, and lack of randomized controlled trials in children. ${ }^{20}$

Discontinuing Primary Prophylaxis-Not applicable.

\section{Treatment Recommendations \\ Treating Disease}

Oropharyngeal Candidiasis: Early, uncomplicated infection can be effectively treated with topical therapy using clotrimazole troches or oral nystatin suspension for 7 to 14 days (BII). ${ }^{21-24}$ Debridement can be considered as adjunctive therapy in OPC. Resistance to clotrimazole can develop as a consequence of previous exposure to clotrimazole itself or to other azole drugs; resistance correlates with refractory mucosal candidiasis. ${ }^{25}$ 
Systemic therapy with 1 of the oral azoles (e.g., fluconazole, itraconazole) for 7 to 14 days is recommended for moderate to severe OPC. ${ }^{21-23}$ Oral fluconazole is more effective than nystatin suspension for initial treatment of OPC in infants, easier to administer to children than the topical therapies, and the recommended treatment if systemic therapy is used $(\mathbf{A I} *)^{22,26}$

For fluconazole-refractory OPC, itraconazole oral solution should be used. Itraconazole solution has efficacy comparable to fluconazole and can be used to treat OPC, although it is less well tolerated than fluconazole (AI). ${ }^{27}$ Gastric acid enhances absorption of itraconazole solution; itraconazole solution should be taken without food when possible. Itraconazole capsules and oral solution should not be used interchangeably because, at the same dose, drug exposure is greater with the oral solution than with capsules and absorption of the capsule formulation varies. Ketoconazole absorption also varies, and therefore neither itraconazole capsules nor ketoconazole are recommended for treating OPC if fluconazole or itraconazole solutions are available (BII*). Additional choices for fluconazole-refractory OPC include voriconazole or posaconazole or IV treatment with amphotericin B or an echinocandin (caspofungin, micafungin, anidulafungin) if required. Chronic suppressive therapy is usually unnecessary for HIV-infected patients (AI*).

Esophageal Disease: Systemic therapy is essential for esophageal disease (AI*) and should be initiated empirically in HIV-infected children who have OPC and esophageal symptoms. In most patients, symptoms should resolve within days after the start of effective therapy. Oral fluconazole for 14 to 21 days is recommended highly effective for treatment of Candida esophagitis (AI*). ${ }^{21,28}$ IV fluconazole, amphotericin B, or an echinocandin should be used for patients who cannot tolerate oral therapy. For fluconazole-refractory disease, itraconazole solution, voriconazole, amphotericin B, or an echinocandin are alternatives. Suppressive therapy with fluconazole three times weekly is recommended for recurrent infections.

Invasive Disease: Central venous catheters should always be removed when feasible in HIV-infected children with candidemia (AI). ${ }^{4,29}$ Among children with persistent candidemia despite appropriate therapy, investigation for a deep tissue focus of infection, such as with echocardiogram, renal or abdominal ultrasound, should be conducted. The treatment of choice for invasive disease in HIV-infected children depends on severity of disease, previous azole exposure, and Candida isolate obtained (if known). An echinocandin is recommended for most severely ill children with candidiasis because of the fungicidal nature of these agents as well as the lack of adverse events (AI*). Fluconazole is a reasonable alternative for patients who are less critically ill and who have no recent fluconazole exposure. Voriconazole can be used in situations in which mold coverage is also warranted. For infections with $C$. glabrata, an echinocandin is recommended because of the increasing resistance seen against fluconazole for this species (AII). However, for patients already receiving fluconazole or voriconazole who are clinically improving despite $C$. glabrata infection, continuing use of the azole is reasonable. In addition, infection with $C$. krusei should be treated with an echinocandin because of the inherent resistance to fluconazole. Amphotericin B is an effective but less attractive alternative (BII). 
Amphotericin B lipid formulations have a role in children who are intolerant of conventional amphotericin B (deoxycholate), have disseminated candidal infection that is refractory to conventional amphotericin $\mathrm{B}$, or are at high risk of nephrotoxicity because of preexisting renal disease or use of other nephrotoxic drugs (BII). For infection with C. parapsilosis, fluconazole or amphotericin B is recommended (AII) because of data showing a decreased response from this species to the echinocandins. ${ }^{30}$ However, if a patient is receiving empiric therapy with an echinocandin and showing clinical improvement when culture of $C$. parapsilosis returns, continuing with this therapy is reasonable. Recommended duration of therapy for candidemia is 14 days after documented clearance from the blood and resolution of neutropenia and of clinical signs and symptoms of candidemia.

Conventional amphotericin B (sodium deoxycholate complex) pharmacokinetics (PK) in children are very similar to adults. In children who have azotemia or hyperkalemia or who are receiving high doses ( $\geq 1 \mathrm{mg} / \mathrm{kg}$ ), a longer infusion time of 3 to 6 hours is recommended (BIII) ${ }^{31}$ In children with life-threatening disease, the target daily dose of amphotericin B should be administered from the beginning of therapy (BIII). Flucytosine has been used in combination with amphotericin B in some children with severe invasive candidiasis, particularly in those with central nervous system disease (CIII), but it has a narrow therapeutic index and should no longer be used for this purpose.

Fluconazole PK vary significantly with age, and fluconazole is rapidly cleared in children. Daily fluconazole dose needs to be 6 to $12 \mathrm{mg} / \mathrm{kg}$ daily for children, and treatment of invasive candidiasis requires higher doses of fluconazole than are used for mucocutaneous disease. Alternatively, an initial course of amphotericin B can be administered and then carefully followed by completion of a course of fluconazole therapy (BIII). Species identification is necessary when using fluconazole because of intrinsic drug resistance among certain Candida spp. (e.g., C. krusei, C. glabrata). Because of more rapid clearance in children, fluconazole administered to children at $12 \mathrm{mg} / \mathrm{kg} /$ day provides exposure similar to standard 400-mg daily dosing in adults. Dosing of fluconazole for invasive candidiasis in children and adolescents should generally not exceed $600 \mathrm{mg} / \mathrm{day} .{ }^{32}$

Itraconazole oral solution provides levels lower than those seen in adults; therefore, dosing should be $2.5 \mathrm{mg} / \mathrm{kg}$ per dose twice daily. (BII*).

Experience with voriconazole in children is growing, both in dosing and efficacy, including esophageal candidiasis or candidemia. ${ }^{3,21,33,34}$ Usually children are started on voriconazole IV and then switched to oral administration to complete therapy after stabilization. The optimal pediatric dose of voriconazole is higher than used in adults due to differing PK. Voriconazole has been shown to be tolerated to a similar degree regardless of dosage and age; a dosage approaching $8 \mathrm{mg} / \mathrm{kg}$ in children aged 2 to 11 years was needed to attain voriconazole plasma levels achieved in adults with a 4-mg/kg IV dosage. Also, the oral bioavailability of voriconazole in children compared to adults is lower, therefore there is a need for higher weight-adjusted oral dosages than dosages used for IV therapy. ${ }^{33,34}$ The recommended voriconazole dosage for children is $9 \mathrm{mg} / \mathrm{kg}$ every 12 hours IV loading on day 1 , followed by $8 \mathrm{mg} / \mathrm{kg}$ IV every 12 hours. Conversion to oral voriconazole should be at $9 \mathrm{mg} / \mathrm{kg}$ orally every 12 hours (BII). ${ }^{35}$ In addition, therapeutic voriconazole drug levels 
should be monitored because of significant interpatient variation in PK of voriconazole in children with invasive fungal infection. ${ }^{36}$ For example, voriconazole clearance depends on allelic polymporphisms of CYP2C19, resulting in poor and extensive metabolizers of voriconazole. ${ }^{37,38}$ For example, it is estimated that $15 \%$ to $20 \%$ of Asian compared with $3 \%$ to 5\% of Caucasian and African populations are poor metabolizers, further underscoring the importance of monitoring voriconazole levels to ensure proper dosing. ${ }^{37}$

Data from studies using echinocandins including caspofungin, micafungin, and anidulafungin are now sufficient to recommend these agents as alternatives to fluconazole for esophageal candidiasis and as firstline therapy for disseminated candidiasis (AII). ${ }^{39-53}$ Caspofungin was effective in treating candidemia, renal candidiasis, and endocardial infection in 10 infants, including 1 term and 9 premature infant(s) who were unresponsive to or intolerant of deoxycholate amphotericin B. ${ }^{40} \mathrm{~A} \mathrm{PK}$ study of caspofungin in immunocompromised HIV-uninfected children aged 2 to 17 years demonstrated that 50 $\mathrm{mg} / \mathrm{m}^{2}$ body surface area/day (70 $\mathrm{mg} /$ day maximum) provides comparable exposure to that obtained in adults receiving a standard 50-mg daily regimen. ${ }^{41} \mathrm{~A}$ retrospective report in which caspofungin was administered to 20 children aged $\leq 6$ years who had invasive fungal infections (seven had invasive candidiasis) but not HIV infection, found the drug to be efficacious and well tolerated. ${ }^{42}$ In a prospective open-label study of children aged 6 months to 17 years, primary or salvage treatment with caspofungin was well tolerated and successful in $81 \%$ of Candida infections, including 30 of 37 patients with invasive candidiasis, and 1 of 1 patient with esophageal candidiasis. ${ }^{43}$ The first pediatric double-blind, randomized controlled trial of empiric antifungal therapy comparing liposomal amphotericin B with caspofungin in children aged 2 to 12 years with cancer, neutropenia, and persistent fever, found comparable efficacy between the 2 treatment arms. ${ }^{44}$

Micafungin has been studied in children treated with 2 to $4 \mathrm{mg} / \mathrm{kg}$ daily, but neonates require doses as high as 10 to $12 \mathrm{mg} / \mathrm{kg}$ daily (AII). ${ }^{45-49}$ Micafungin demonstrates doseproportional PK and an inverse relationship between age and clearance, suggesting a need for increased dosage in young children. ${ }^{50}$ Clearance of the drug in neonates was more than double that in older children and adults. ${ }^{51}$ Dosages of 10 to $15 \mathrm{mg} / \mathrm{kg} /$ day have been studied in premature neonates, resulting in area-under-the-curve values consistent with an adult dosage of 100 to $150 \mathrm{mg} /$ day. In a clinical trial comparing micafungin versus liposomal amphotericin $\mathrm{B}(\mathrm{L}-\mathrm{AmB})$ using an intent-to-treat analysis of children and adults with candidiasis/candidemia and with or without cancer, 42 children with or without neutropenia (13 with malignancies $/ 29$ without malignancies) received $2 \mathrm{mg} / \mathrm{kg} /$ day of micafungin and 3 $\mathrm{mg} / \mathrm{kg} /$ day of L-AmB. Micafungin was as effective as L-AmB in treating candidiasis/ candidemia and well tolerated in these patients. ${ }^{49}$

One PK study of anidulafungin in $25 \mathrm{HIV}$-uninfected neutropenic children aged 2 to 17 years, including 12 aged 2 to 11 years and 13 aged 12 to 17 years, showed drug concentrations at $0.75 \mathrm{mg} / \mathrm{kg}$ per dose and $1.5 \mathrm{mg} / \mathrm{kg}$ per dose were similar to drug concentrations in adults with $50 \mathrm{mg}$ per dose and $100 \mathrm{mg}$ per dose, respectively. ${ }^{52} \mathrm{In}$ a case report of a term 11-day infant with peritoneal candidiasis and failure of L-AmB therapy, an IV dose of $1.5 \mathrm{mg} / \mathrm{kg} / \mathrm{day}$ of anidulafungin was successful in treating the infection. ${ }^{53}$ 
Data in adults are limited on use of combination antifungal therapy for invasive candidal infections; combination amphotericin B and fluconazole resulted in more rapid clearance of Candida from the bloodstream but no difference in mortality. ${ }^{21}$ Data are insufficient to support routine use of combination therapy in children with invasive candidiasis (BIII). ${ }^{54}$

Monitoring and Adverse Events, Including IRIS-No adverse effects have been reported with use of oral nystatin for treatment of oral candidiasis, but bitter taste may contribute to poor adherence.

The azole drugs have relatively low rates of toxicity, but because of their ability to inhibit the cytochrome P450 (CYP450)-dependent hepatic enzymes (ketoconazole has the strongest inhibitory effect) and their metabolism by these enzymes, they can interact substantially with other drugs undergoing hepatic metabolism. These interactions can result in decreased plasma concentration of the azole because of increased metabolism induced by the coadministered drug or development of unexpected toxicity from the co-administered drug because of increased plasma concentrations secondary to azole-induced alterations in hepatic metabolism. The potential for drug interactions, particularly with ARV drugs such as protease inhibitors, should be carefully evaluated before initiation of therapy (AIII).

The most frequent adverse effects of the azole drugs are gastrointestinal, including nausea and vomiting (10\%-40\% of patients). Skin rash and pruritus can occur with all azoles; rare cases of Stevens-Johnson syndrome and alopecia have been reported with fluconazole therapy. All drugs are associated with asymptomatic increases in transaminases (1\%-13\% of patients). Hematologic abnormalities have been reported with itraconazole, including thrombocytopenia and leukopenia. Of the azoles, ketoconazole is associated with the highest frequency of side effects. Its use has been associated with endocrinologic abnormalities related to steroid metabolism, including adrenal insufficiency and gynecomastia, hemolytic anemia, and transaminitis. Dose-related, reversible visual changes such as photophobia and blurry vision have been reported in approximately $30 \%$ of patients receiving voriconazole. ${ }^{55}$ Cardiac arrhythmias and renal abnormalities including nephritis and acute tubular necrosis also have been reported with voriconazole use.

Amphotericin B deoxycholate undergoes renal excretion as inactive drug. Adverse effects of amphotericin B are primarily nephrotoxicity, defined by substantial azotemia from glomerular damage, and can be accompanied by hypokalemia from tubular damage. Nephrotoxicity is exacerbated by use of concomitant nephrotoxic drugs. Permanent nephrotoxicity is related to cumulative dose. Nephrotoxicity can be ameliorated by hydration before amphotericin B infusion. Infusion-related fevers, chills, nausea, and vomiting occur less frequently in children than in adults. Onset occurs usually within 1 to 3 hours after the infusion is started, typical duration is $<1$ hour, and the febrile reactions tend to decrease in frequency over time. Pre-treatment with acetaminophen or diphenhydramine may alleviate febrile reactions. Idiosyncratic reactions, such as hypotension, arrhythmias, and allergic reactions, including anaphylaxis, occur less frequently. Hepatic toxicity, thrombophlebitis, anemia, and rarely neurotoxicity (manifested as confusion or delirium, hearing loss, blurred vision, or seizures) also can occur. 
Lipid formulations of amphotericin B have less acute and chronic toxicity than amphotericin B deoxycholate. In approximately $20 \%$ of children, lipid formulations of amphotericin B can cause acute, infusion-related reactions, including chest pain; dyspnea; hypoxia; severe pain in the abdomen, flank, or leg; or flushing and urticaria. Compared with infusion reactions with conventional amphotericin B, most (85\%) of the reactions to the lipid formulations occur within the first 5 minutes after infusion and rapidly resolve with temporary interruption of the amphotericin B infusion and administration of IV diphenhydramine. Premedication with diphenhydramine can reduce the incidence of these reactions.

The echinocandins have an excellent safety profile. In a retrospective evaluation of 25 immunocompromised children who received caspofungin, the drug was well tolerated, although 3 patients had adverse events potentially related to the drug (hypokalemia in all 3 children, elevated bilirubin in 2, and decreased hemoglobin and elevated alanine aminotransferase in 1) ${ }^{41}$ In this study, children weighing $<50 \mathrm{~kg}$ received 0.8 to $1.6 \mathrm{mg} / \mathrm{kg}$ body weight daily, and those weighing $>50 \mathrm{~kg}$ received the adult dosage. In the PK study of 39 children who received caspofungin at $50 \mathrm{mg} / \mathrm{m}^{2}$ body surface area/day, $5(13 \%)$ patients experienced one or more drug-related clinical adverse events, including 1 patient each with fever, diarrhea, phlebitis, proteinuria, and transient extremity rash. Two patients reported one or more drug-related laboratory adverse events, including one patient each with hypokalemia and increased serum aspartate transaminase. None of the drug-related adverse events in this study were considered serious or led to discontinuation of caspofungin. ${ }^{41}$ In a prospective multicenter trial for primary or salvage treatment of Candida and Aspergillus infections in 48 children aged 6 months to 17 years, a caspofungin dose of $50 \mathrm{mg} / \mathrm{m}^{2}$ per day (maximum: $70 \mathrm{mg} /$ day; after $70 \mathrm{mg} / \mathrm{m}^{2}$ on day 1) was generally well tolerated with drugrelated clinical and laboratory adverse events occurring in $26.5 \%$ and $34.7 \%$ of patients, respectively, similar to rates seen in adults. Drug-related clinical adverse events were typically mild and did not lead to therapy discontinuation. An increased level of hepatic transaminase, often occurring in the context of other medical conditions or concomitant therapies that may have contributed to elevations in hepatic enzymes, represented the most common drug-related laboratory adverse event. None of the drug-related laboratory adverse events led to therapy interruption or discontinuation. ${ }^{43}$

In a double-blind randomized trial comparing micafungin with liposomal amphotericin B (L-amB) in 48 children aged $<16$ years with clinical signs of systemic Candida infection or culture confirmation of Candida infection, a micafungin daily dose of $2 \mathrm{mg} / \mathrm{kg}$ of body weight for patients who weighed $40 \mathrm{~kg}$ and $100 \mathrm{mg}$ for patients who weighed $>40 \mathrm{~kg}$ was well tolerated. Adverse events were similar for both treatment arms and reflected those experienced by patients with comorbid conditions. These included sepsis, fever, vomiting, diarrhea, anemia, thrombocytopenia, and hypokalemia. Patients in the micafungin group experienced significantly fewer adverse events leading to treatment discontinuation than those in the amphotericin B group (2/25 [3.8\%] versus 9/54 [16.7\%], respectively), suggesting a safety advantage for micafungin in this population. Two patients receiving micafungin experienced serious adverse events, including a worsening of renal failure, a preexisting condition, and a moderate increase in serum creatine resulting in discontinuation of therapy. Patients rarely experienced clinically meaningful changes in creatinine, aspartate 
transaminase, alanine transaminase, or bilirubin during treatment. Children aged $\geq 2$ years in the micafungin treatment arm experienced a smaller mean peak decrease in the estimated glomerular filtration rate than those in the L-amB arm. ${ }^{46}$

A multicenter, ascending-dosage study of anidulafungin in $25 \mathrm{HIV}$-uninfected neutropenic children aged 2 to 17 years showed anidulafungin to be well tolerated and observed no drugrelated serious adverse events. Fever was observed in one patient with a National Cancer Institute toxicity grade of 3 and facial erythema was observed in another patient, which resolved after slowing the infusion rate. ${ }^{52}$

Immune reconstitution inflammatory response syndrome associated with Candida infection has not been described in HIV-infected children. However, evidence suggests that candidiasis (other than Candida esophagitis) occurs with increased frequency in adults during the first 2 months after initiation of cART. ${ }^{56}$

\section{Managing Treatment Failure}

Oropharyngeal and Esophageal Candidiasis: If OPC initially is treated topically, failure or relapse should be treated with oral fluconazole or itraconazole oral solution (AI*). ${ }^{27,57}$

Approximately $50 \%$ to $60 \%$ of patients with fluconazole-refractory OPC and $80 \%$ of patients with fluconazole-refractory esophageal candidiasis will respond to itraconazole solution (AII*). ${ }^{58,59}$ Posaconazole is a second-generation orally bioavailable triazole that has been effective in HIV-infected adults with azole-refractory OPC or esophageal candidiasis. ${ }^{60}$ However, experience in children is limited, and an appropriate pediatric dosage has not been defined; thus data in children are insufficient to recommend its use in HIV-infected children (CIII). ${ }^{61,62}$

Amphotericin B (oral suspension at $1 \mathrm{~mL}$ four times daily of a $100-\mathrm{mg} / \mathrm{mL}$ suspension) sometimes has been effective in patients with OPC who do not respond to itraconazole solution; however, this product is not available in the United States (CIII) ${ }^{59}$ Low-dose IV amphotericin B (0.3-0.5 mg/kg/day) has been effective in children with refractory OPC or esophageal candidiasis (BII). ${ }^{21,59,63,64}$

Experience is limited with use of echinocandins in treatment of azole-refractory OPC or esophageal candidiasis in children (HIV-infected or -uninfected); however, given their excellent safety profile, the echinocandins ${ }^{61}$ could be considered for treatment of azolerefractory esophageal candidiasis (BIII).

Invasive Disease: Although lipid formulations appear to be at least as effective as conventional amphotericin B for treating serious fungal infections, ${ }^{65,66}$ the drugs are considerably more expensive than conventional amphotericin B. However, the lipid formulations have less acute and chronic toxicity. Two lipid formulations are used: amphotericin B lipid complex and liposomal amphotericin B lipid complex. Experience with these preparations in children is limited. ${ }^{67-69}$

For invasive candidiasis, amphotericin B lipid complex is administered as $5 \mathrm{mg} / \mathrm{kg}$ body weight IV once daily over 2 hours. ${ }^{67,68,70}$ Liposomal amphotericin B is administered IV as 3 
to $5 \mathrm{mg} / \mathrm{kg}$ body weight once daily over 1 to 2 hours. The role of the echinocandins in invasive candidiasis has not been well studied in HIV-infected children. However, invasive candidiasis associated with neutropenia in patients undergoing bone marrow transplantation has been treated successfully with this class of antifungals. These agents should be considered in treatment of invasive candidiasis but reserved as alternative, second-line therapy to currently available treatment modalities (CIII).

Preventing Recurrence-Secondary prophylaxis of recurrent OPC usually is not recommended because treatment of recurrence is typically effective, potential exists for development of resistance and drug interactions, and additional rounds of prophylaxis are costly (BIII). Immune reconstitution with cART in immunocompromised children should be a priority (AIII). However, if recurrences are severe, data from studies of HIV-infected adults with advanced disease on cART suggest that suppressive therapy with systemic azoles, either with oral fluconazole (BII*) or voriconazole or itraconazole solution (BII), can be considered. ${ }^{27,71-73}$ Potential azole resistance should be considered when long-term prophylaxis with azoles is used.

Experience with HIV-infected adults suggests that, in children with fluconazole-refractory OPC or esophageal candidiasis who responded to voriconazole or posaconazole therapy or to echinocandins, continuing the effective drug as secondary prophylaxis can be considered because of the high relapse rate until cART produces immune reconstitution (BIII).

Discontinuing Secondary Prophylaxis-In situations when secondary prophylaxis is instituted, no data exist on which to base a recommendation regarding discontinuation. On the basis of experience with HIV-infected adults with other opportunistic infections, discontinuation of secondary prophylaxis can be considered when a patient's CD4 count or percentage has risen to CDC Immunologic Category 2 or 1 (CIII) ${ }^{74}$

\section{References}

1. Gona P, Van Dyke RB, Williams PL, et al. Incidence of opportunistic and other infections in HIVinfected children in the HAART era. JAMA. 2006 Jul 19; 296(3):292-300. Available at http:// www.ncbi.nlm.nih.gov/pubmed/16849662. [PubMed: 16849662]

2. Dankner WM, Lindsey JC, Levin MJ. Pediatric ACTGPT. Correlates of opportunistic infections in children infected with the human immunodeficiency virus managed before highly active antiretroviral therapy. Pediatr Infect Dis J. 2001 Jan; 20(1):40-48. Available at http:// www.ncbi.nlm.nih.gov/pubmed/11176565. [PubMed: 11176565]

3. Chiou CC, Groll AH, Gonzalez CE, et al. Esophageal candidiasis in pediatric acquired immunodeficiency syndrome: clinical manifestations and risk factors. Pediatr Infect Dis J. 2000 Aug; 19(8):729-734. Available at http://www.ncbi.nlm.nih.gov/pubmed/10959741. [PubMed: 10959741]

4. Walsh TJ, Gonzalez C, Roilides E, et al. Fungemia in children infected with the human immunodeficiency virus: new epidemiologic patterns, emerging pathogens, and improved outcome with antifungal therapy. Clin Infect Dis. 1995 Apr; 20(4):900-906. Available at http:// www.ncbi.nlm.nih.gov/pubmed/7795092. [PubMed: 7795092]

5. Chiou CC, Groll AH, Mavrogiorgos N, Wood LV, Walsh TJ. Esophageal candidiasis in human immunodeficiency virusinfected pediatric patients after the introduction of highly active antiretroviral therapy. Pediatr Infect Dis J. 2002 May; 21(5):388-392. Available at http:// www.ncbi.nlm.nih.gov/pubmed/12150174. [PubMed: 12150174] 
6. Leibovitz E, Rigaud M, Chandwani S, et al. Disseminated fungal infections in children infected with human immunodeficiency virus. Pediatr Infect Dis J. 1991 Dec; 10(12):888-894. Available at http://www.ncbi.nlm.nih.gov/pubmed/1766703. [PubMed: 1766703]

7. Gonzalez CE, Venzon D, Lee S, Mueller BU, Pizzo PA, Walsh TJ. Risk factors for fungemia in children infected with human immunodeficiency virus: a case-control study. Clin Infect Dis. 1996 Sep; 23(3):515-521. Available at http://www.ncbi.nlm.nih.gov/pubmed/8991477. [PubMed: 8991477]

8. Krcmery V, Augustinova A, Babelova O, Doczeova A, Liskova A. Fungal resistance in Cambodian children with acquired immunodeficiency syndrome. Pediatr Infect Dis J. 2006 May.25(5):470. Available at http://www.ncbi.nlm.nih.gov/pubmed/16645523. [PubMed: 16645523]

9. Stevens DA. Diagnosis of fungal infections: current status. J Antimicrob Chemother. 2002 Feb; 49(Suppl 1):11-19. Available at http://www.ncbi.nlm.nih.gov/pubmed/11801576. [PubMed: 11801576]

10. Sigmundsdottir G, Larsson L, Wiebe T, Bjorklund LJ, Christensson B. Clinical experience of urine D-arabinitol/Larabinitol ratio in the early diagnosis of invasive candidiasis in paediatric high risk populations. Scand J Infect Dis. 2007; 39(2):146-151. Available at http://www.ncbi.nlm.nih.gov/ pubmed/17366032. [PubMed: 17366032]

11. Stradomska TJ, Sobielarska D, Mielniczuk Z, Jagiellowicz D, Syczewska M, Dzierzanowska D. Determination of urinary D-/L-arabinitol ratios as a biomarker for invasive candidiasis in children with cardiac diseases. J Med Microbiol. 2010 Dec; 59(Pt 12):1490-1496. Available at http:// www.ncbi.nlm.nih.gov/pubmed/20724507. [PubMed: 20724507]

12. Ostrosky-Zeichner L. Invasive mycoses: diagnostic challenges. Am J Med. 2012 Jan; 125(1 Suppl):S14-S24. Available at http://www.ncbi.nlm.nih.gov/pubmed/22196205. [PubMed: 22196205]

13. Yeo SF, Huie S, Sofair AN, Campbell S, Durante A, Wong B. Measurement of serum D-arabinitol/ creatinine ratios for initial diagnosis and for predicting outcome in an unselected, population-based sample of patients with Candida fungemia. J Clin Microbiol. 2006 Nov; 44(11):3894-3899. Available at http://www.ncbi.nlm.nih.gov/pubmed/16957030. [PubMed: 16957030]

14. Verduyn Lunel FM, Voss A, Kuijper EJ, et al. Detection of the Candida antigen mannan in cerebrospinal fluid specimens from patients suspected of having Candida meningitis. J Clin Microbiol. 2004 Feb; 42(2):867-870. Available at http://www.ncbi.nlm.nih.gov/pubmed/ 14766875. [PubMed: 14766875]

15. Mikulska M, Calandra T, Sanguinetti M, Poulain D, Viscoli C. Third European Conference on Infections in Leukemia G. The use of mannan antigen and anti-mannan antibodies in the diagnosis of invasive candidiasis: recommendations from the Third European Conference on Infections in Leukemia. Critical care. 2010; 14(6):R222. Available at http://www.ncbi.nlm.nih.gov/pubmed/ 21143834. [PubMed: 21143834]

16. Ostrosky-Zeichner L, Alexander BD, Kett DH, et al. Multicenter clinical evaluation of the (1-->3) beta-D-glucan assay as an aid to diagnosis of fungal infections in humans. Clin Infect Dis. 2005 Sep 1; 41(5):654-659. Available at http://www.ncbi.nlm.nih.gov/pubmed/16080087. [PubMed: 16080087]

17. Del Bono V, Delfino E, Furfaro E, et al. Clinical performance of the (1,3)-beta-D-glucan assay in early diagnosis of nosocomial Candida bloodstream infections. Clinical and vaccine immunology: CVI. 2011 Dec; 18(12):2113-2117. Available at http://www.ncbi.nlm.nih.gov/pubmed/21994353. [PubMed: 21994353]

18. Klingspor L, Jalal S. Molecular detection and identification of Candida and Aspergillus spp. from clinical samples using real-time PCR. Clin Microbiol Infect. 2006 Aug; 12(8):745-753. Available at http://www.ncbi.nlm.nih.gov/pubmed/16842569. [PubMed: 16842569]

19. Avni T, Leibovici L, Paul M. PCR diagnosis of invasive candidiasis: systematic review and metaanalysis. J Clin Microbiol. 2011 Feb; 49(2):665-670. Available at http://www.ncbi.nlm.nih.gov/ pubmed/21106797. [PubMed: 21106797]

20. Pienaar ED, Young T, Holmes H. Interventions for the prevention and management of oropharyngeal candidiasis associated with HIV infection in adults and children. Cochrane Database Syst Rev. 2006; 3(3):CD003940. Available at http://www.ncbi.nlm.nih.gov/pubmed/ 16856025. [PubMed: 16856025] 
21. Pappas PG, Kauffman CA, Andes D, et al. Clinical practice guidelines for the management of candidiasis: 2009 update by the Infectious Diseases Society of America. Clin Infect Dis. 2009 Mar 1; 48(5):503-535. Available at http://www.ncbi.nlm.nih.gov/pubmed/19191635. [PubMed: 19191635]

22. Pons V, Greenspan D, Debruin M. Therapy for oropharyngeal candidiasis in HIV-infected patients: a randomized, prospective multicenter study of oral fluconazole versus clotrimazole troches. The Multicenter Study Group. J Acquir Immune Defic Syndr. 1993 Dec; 6(12):1311-1316. Available at http://www.ncbi.nlm.nih.gov/pubmed/8254467. [PubMed: 8254467]

23. Pons V, Greenspan D, Lozada-Nur F, et al. Oropharyngeal candidiasis in patients with AIDS: randomized comparison of fluconazole versus nystatin oral suspensions. Clin Infect Dis. 1997 Jun; 24(6):1204-1207. Available at http://www.ncbi.nlm.nih.gov/pubmed/9195083. [PubMed: 9195083]

24. Lumbreras C, Cuervas-Mons V, Jara P, et al. Randomized trial of fluconazole versus nystatin for the prophylaxis of Candida infection following liver transplantation. J Infect Dis. 1996 Sep; 174(3):583-588. Available at http://www.ncbi.nlm.nih.gov/pubmed/8769617. [PubMed: 8769617]

25. Pelletier R, Peter J, Antin C, Gonzalez C, Wood L, Walsh TJ. Emergence of resistance of Candida albicans to clotrimazole in human immunodeficiency virus-infected children: in vitro and clinical correlations. J Clin Microbiol. 2000 Apr; 38(4):1563-1568. Available at http:// www.ncbi.nlm.nih.gov/pubmed/10747144. [PubMed: 10747144]

26. Goins RA, Ascher D, Waecker N, Arnold J, Moorefield E. Comparison of fluconazole and nystatin oral suspensions for treatment of oral candidiasis in infants. Pediatr Infect Dis J. 2002 Dec; 21(12): 1165-1167. Available at http://www.ncbi.nlm.nih.gov/pubmed/12506950. [PubMed: 12506950]

27. Phillips P, De Beule K, Frechette G, et al. A double-blind comparison of itraconazole oral solution and fluconazole capsules for the treatment of oropharyngeal candidiasis in patients with AIDS. Clin Infect Dis. 1998 Jun; 26(6):1368-1373. Available at http://www.ncbi.nlm.nih.gov/pubmed/ 9636865. [PubMed: 9636865]

28. Wilcox CM, Darouiche RO, Laine L, Moskovitz BL, Mallegol I, Wu J. A randomized, doubleblind comparison of itraconazole oral solution and fluconazole tablets in the treatment of esophageal candidiasis. J Infect Dis. 1997 Jul; 176(1):227-232. Available at http:// www.ncbi.nlm.nih.gov/pubmed/9207371. [PubMed: 9207371]

29. Muller FM, Groll AH, Walsh TJ. Current approaches to diagnosis and treatment of fungal infections in children infected with human immuno deficiency virus. Eur J Pediatr. 1999 Mar; 158(3):187-199. Available at http://www.ncbi.nlm.nih.gov/pubmed/10094436. [PubMed: 10094436]

30. Reboli AC, Rotstein C, Pappas PG, et al. Anidulafungin versus fluconazole for invasive candidiasis. N Engl J Med. 2007 Jun 14; 356(24):2472-2482. Available at http:// www.ncbi.nlm.nih.gov/pubmed/17568028. [PubMed: 17568028]

31. Dismukes WE. Introduction to antifungal drugs. Clin Infect Dis. 2000 Apr; 30(4):653-657. Available at http://www.ncbi.nlm.nih.gov/pubmed/10770726. [PubMed: 10770726]

32. Brammer KW, Coates PE. Pharmacokinetics of fluconazole in pediatric patients. Eur J Clin Microbiol Infect Dis. 1994 Apr; 13(4):325-329. Available at http://www.ncbi.nlm.nih.gov/ pubmed/8070441. [PubMed: 8070441]

33. Walsh TJ, Lutsar I, Driscoll T, et al. Voriconazole in the treatment of aspergillosis, scedosporiosis and other invasive fungal infections in children. Pediatr Infect Dis J. 2002 Mar; 21(3):240-248. Available at http://www.ncbi.nlm.nih.gov/pubmed/12005089. [PubMed: 12005089]

34. Walsh TJ, Karlsson MO, Driscoll T, et al. Pharmacokinetics and safety of intravenous voriconazole in children after single- or multiple-dose administration. Antimicrob Agents Chemother. 2004 Jun; 48(6):2166-2172. Available at http://www.ncbi.nlm.nih.gov/pubmed/ 15155217. [PubMed: 15155217]

35. Walsh TJ, Driscoll T, Milligan PA, et al. Pharmacokinetics, safety, and tolerability of voriconazole in immunocompromised children. Antimicrob Agents Chemother. 2010 Oct; 54(10):4116-4123. Available at http://www.ncbi.nlm.nih.gov/pubmed/20660687. [PubMed: 20660687]

36. Andes D, Pascual A, Marchetti O. Antifungal therapeutic drug monitoring: established and emerging indications. Antimicrob Agents Chemother. 2009 Jan; 53(1):24-34. Available at http:// www.ncbi.nlm.nih.gov/pubmed/18955533. [PubMed: 18955533] 
37. Goldstein JA, de Morais SM. Biochemistry and molecular biology of the human CYP2C subfamily. Pharmacogenetics. 1994 Dec; 4(6):285-299. Available at http://www.ncbi.nlm.nih.gov/ pubmed/7704034. [PubMed: 7704034]

38. Hyland R, Jones BC, Smith DA. Identification of the cytochrome P450 enzymes involved in the Noxidation of voriconazole. Drug metabolism and disposition: the biological fate of chemicals. 2003 May; 31(5):540-547. Available at http://www.ncbi.nlm.nih.gov/pubmed/12695341. [PubMed: 12695341]

39. Hoffman JA, Walsh TJ. Echinocandins in children. Pediatr Infect Dis J. 2011 Jun; 30(6):508-509. Available at http://www.ncbi.nlm.nih.gov/pubmed/21587028. [PubMed: 21587028]

40. Odio CM, Araya R, Pinto LE, et al. Caspofungin therapy of neonates with invasive candidiasis. Pediatr Infect Dis J. 2004 Dec; 23(12):1093-1097. Available at http://www.ncbi.nlm.nih.gov/ pubmed/15626944. [PubMed: 15626944]

41. Walsh TJ, Adamson PC, Seibel NL, et al. Pharmacokinetics, safety, and tolerability of caspofungin in children and adolescents. Antimicrob Agents Chemother. 2005 Nov; 49(11):4536-4545. Available at http://www.ncbi.nlm.nih.gov/pubmed/16251293. [PubMed: 16251293]

42. Merlin E, Galambrun C, Ribaud P, et al. Efficacy and safety of caspofungin therapy in children with invasive fungal infections. Pediatr Infect Dis J. 2006 Dec; 25(12):1186-1188. Available at http://www.ncbi.nlm.nih.gov/pubmed/17133169. [PubMed: 17133169]

43. Zaoutis TE, Jafri HS, Huang LM, et al. A prospective, multicenter study of caspofungin for the treatment of documented Candida or Aspergillus infections in pediatric patients. Pediatrics. 2009 Mar; 123(3):877-884. Available at http://www.ncbi.nlm.nih.gov/pubmed/19255017. [PubMed: 19255017]

44. Maertens JA, Madero L, Reilly AF, et al. A randomized, double-blind, multicenter study of caspofungin versus liposomal amphotericin B for empiric antifungal therapy in pediatric patients with persistent fever and neutropenia. Pediatr Infect Dis J. 2010 May; 29(5):415-420. Available at http://www.ncbi.nlm.nih.gov/pubmed/20431381. [PubMed: 20431381]

45. Lehrnbecher T, Groll AH. Micafungin: a brief review of pharmacology, safety, and antifungal efficacy in pediatric patients. Pediatr Blood Cancer. 2010 Aug; 55(2):229-232. Available at http:// www.ncbi.nlm.nih.gov/pubmed/20583216. [PubMed: 20583216]

46. Queiroz-Telles F, Berezin E, Leverger G, et al. Micafungin versus liposomal amphotericin B for pediatric patients with invasive candidiasis: substudy of a randomized double-blind trial. Pediatr Infect Dis J. 2008 Sep; 27(9):820-826. Available at http://www.ncbi.nlm.nih.gov/pubmed/ 18679151. [PubMed: 18679151]

47. Smith PB, Walsh TJ, Hope W, et al. Pharmacokinetics of an elevated dosage of micafungin in premature neonates. Pediatr Infect Dis J. 2009 May; 28(5):412-415. Available at http:// www.ncbi.nlm.nih.gov/pubmed/19319022. [PubMed: 19319022]

48. Benjamin DK Jr, Smith PB, Arrieta A, et al. Safety and pharmacokinetics of repeat-dose micafungin in young infants. Clin Pharmacol Ther. 2010 Jan; 87(1):93-99. Available at http:// www.ncbi.nlm.nih.gov/pubmed/19890251. [PubMed: 19890251]

49. Cornely OA, Marty FM, Stucker F, Pappas PG, Ullmann AJ. Efficacy and safety of micafungin for treatment of serious Candida infections in patients with or without malignant disease. Mycoses. 2011 Nov; 54(6):e838-e847. Available at http://www.ncbi.nlm.nih.gov/pubmed/21668522. [PubMed: 21668522]

50. Hope WW, Smith PB, Arrieta A, et al. Population pharmacokinetics of micafungin in neonates and young infants. Antimicrob Agents Chemother. 2010 Jun; 54(6):2633-2637. Available at http:// www.ncbi.nlm.nih.gov/pubmed/20308367. [PubMed: 20308367]

51. Heresi GP, Gerstmann DR, Reed MD, et al. The pharmacokinetics and safety of micafungin, a novel echinocandin, in premature infants. Pediatr Infect Dis J. 2006 Dec; 25(12):1110-1115. Available at http://www.ncbi.nlm.nih.gov/pubmed/17133155. [PubMed: 17133155]

52. Benjamin DK Jr, Driscoll T, Seibel NL, et al. Safety and pharmacokinetics of intravenous anidulafungin in children with neutropenia at high risk for invasive fungal infections. Antimicrob Agents Chemother. 2006 Feb; 50(2):632-638. Available at http://www.ncbi.nlm.nih.gov/pubmed/ 16436720. [PubMed: 16436720] 
53. Varisco BM, Benner KW, Prabhakaran P. Neonatal peritoneal candidiasis successfully treated with anidulafungin addon therapy. The Annals of pharmacotherapy. 2009 Nov; 43(11):1907-1910. Available at http://www.ncbi.nlm.nih.gov/pubmed/19826094. [PubMed: 19826094]

54. Blyth CC, Palasanthiran P, O'Brien TA. Antifungal therapy in children with invasive fungal infections: a systematic review. Pediatrics. 2007 Apr; 119(4):772-784. Available at http:// www.ncbi.nlm.nih.gov/pubmed/17403849. [PubMed: 17403849]

55. Johnson LB, Kauffman CA. Voriconazole: a new triazole antifungal agent. Clin Infect Dis. 2003 Mar 1; 36(5):630-637. Available at http://www.ncbi.nlm.nih.gov/pubmed/12594645. [PubMed: 12594645]

56. Nacher M, Vantilcke V, Huber F, et al. Increased incidence of mucosal candidiasis after HAART initiation: a benign form of immune reconstitution disease? AIDS. 2007 Nov 30; 21(18):2534 2536. Available at http://www.ncbi.nlm.nih.gov/pubmed/18025892. [PubMed: 18025892]

57. Groll AH, Wood L, Roden M, et al. Safety, pharmacokinetics, and pharmacodynamics of cyclodextrin itraconazole in pediatric patients with oropharyngeal candidiasis. Antimicrob Agents Chemother. 2002 Aug; 46(8):2554-2563. Available at http://www.ncbi.nlm.nih.gov/pubmed/ 12121932. [PubMed: 12121932]

58. Phillips P, Zemcov J, Mahmood W, Montaner JS, Craib K, Clarke AM. Itraconazole cyclodextrin solution for fluconazole-refractory oropharyngeal candidiasis in AIDS: correlation of clinical response with in vitro susceptibility. AIDS. 1996 Oct; 10(12):1369-1376. Available at http:// www.ncbi.nlm.nih.gov/pubmed/8902066. [PubMed: 8902066]

59. Fichtenbaum CJ, Powderly WG. Refractory mucosal candidiasis in patients with human immunodeficiency virus infection. Clin Infect Dis. 1998 Mar; 26(3):556-565. Available at http:// www.ncbi.nlm.nih.gov/pubmed/9524822. [PubMed: 9524822]

60. Skiest DJ, Vazquez JA, Anstead GM, et al. Posaconazole for the treatment of azole-refractory oropharyngeal and esophageal candidiasis in subjects with HIV infection. Clin Infect Dis. 2007 Feb 15; 44(4):607-614. Available at http://www.ncbi.nlm.nih.gov/pubmed/17243069. [PubMed: 17243069]

61. Zaoutis TE, Benjamin DK, Steinbach WJ. Antifungal treatment in pediatric patients. Drug Resist Updat. 2005 Aug; 8(4):235-245. Available at http://www.ncbi.nlm.nih.gov/pubmed/16054422. [PubMed: 16054422]

62. Krishna G, Sansone-Parsons A, Martinho M, Kantesaria B, Pedicone L. Posaconazole plasma concentrations in juvenile patients with invasive fungal infection. Antimicrob Agents Chemother. 2007 Mar; 51(3):812-818. Available at http://www.ncbi.nlm.nih.gov/pubmed/17210771. [PubMed: 17210771]

63. Lake DE, Kunzweiler J, Beer M, Buell DN, Islam MZ. Fluconazole versus amphotericin B in the treatment of esophageal candidiasis in cancer patients. Chemotherapy. 1996 Jul-Aug;42(4):308314. Available at http://www.ncbi.nlm.nih.gov/pubmed/8804799. [PubMed: 8804799]

64. Rex JH, Rinaldi MG, Pfaller MA. Resistance of Candida species to fluconazole. Antimicrob Agents Chemother. 1995 Jan; 39(1):1-8. Available at http://www.ncbi.nlm.nih.gov/pubmed/ 7695288. [PubMed: 7695288]

65. Walsh TJ, Whitcomb P, Piscitelli S, et al. Safety, tolerance, and pharmacokinetics of amphotericin B lipid complex in children with hepatosplenic candidiasis. Antimicrob Agents Chemother. 1997 Sep; 41(9):1944-1948. Available at http://www.ncbi.nlm.nih.gov/pubmed/9303390. [PubMed: 9303390]

66. Wiley JM, Seibel NL, Walsh TJ. Efficacy and safety of amphotericin B lipid complex in 548 children and adolescents with invasive fungal infections. Pediatr Infect Dis J. 2005 Feb; 24(2): 167-174. Available at http://www.ncbi.nlm.nih.gov/pubmed/15702047. [PubMed: 15702047]

67. Walsh TJ, Seibel NL, Arndt C, et al. Amphotericin B lipid complex in pediatric patients with invasive fungal infections. Pediatr Infect Dis J. 1999 Aug; 18(8):702-708. Available at http:// www.ncbi.nlm.nih.gov/pubmed/10462340. [PubMed: 10462340]

68. Walsh TJ, Finberg RW, Arndt C, et al. Liposomal amphotericin B for empirical therapy in patients with persistent fever and neutropenia. National Institute of Allergy and Infectious Diseases Mycoses Study Group. N Engl J Med. 1999 Mar 11; 340(10):764-771. Available at http:// www.ncbi.nlm.nih.gov/pubmed/10072411. [PubMed: 10072411] 
69. Linden P, Lee L, Walsh TJ. Retrospective analysis of the dosage of amphotericin B lipid complex for the treatment of invasive fungal infections. Pharmacotherapy. 1999 Nov; 19(11):1261-1268. Available at http://www.ncbi.nlm.nih.gov/pubmed/10555932. [PubMed: 10555932]

70. Tollemar J, Klingspor L, Ringden O. Liposomal amphotericin B (AmBisome) for fungal infections in immunocompromised adults and children. Clin Microbiol Infect. 2001; 7(Suppl 2):68-79. Available at http://www.ncbi.nlm.nih.gov/pubmed/11525221. [PubMed: 11525221]

71. Ally R, Schurmann D, Kreisel W, et al. A randomized, double-blind, double-dummy, multicenter trial of voriconazole and fluconazole in the treatment of esophageal candidiasis in immunocompromised patients. Clin Infect Dis. 2001 Nov 1; 33(9):1447-1454. Available at http:// www.ncbi.nlm.nih.gov/pubmed/11577374. [PubMed: 11577374]

72. Vazquez JA. Optimal management of oropharyngeal and esophageal candidiasis in patients living with HIV infection. Hiv/Aids. 2010; 2:89-101. Available at http://www.ncbi.nlm.nih.gov/pubmed/ 22096388. [PubMed: 22096388]

73. Goldman M, Cloud GA, Wade KD, et al. with AIDS Clinical Trials Group Study 323/Mycoses Study Group Study 40. A randomized study of the use of fluconazole in continuous versus episodic therapy in patients with advanced HIV infection and a history of oropharyngeal candidiasis. Clin Infect Dis. 2005; 41(10):1473-1480. Available at http://www.ncbi.nlm.nih.gov/ entrez/query.fcgi? $\mathrm{db}=$ pubmed \&cmd=Retrieve \&dopt=AbstractPlus\&list_uids=16231260\&query_hl=171\&itool=pub med_docsum. [PubMed: 16231260]

74. CDC C. Revised classification system for human immunodeficiency virus infection in children less than 13 years of age. Official authorized addenda: human immunodeficiency virus infection codes and official guidelines for coding and reporting ICD-9-CM. MMWR Morb Mortal Wkly Rep. 1994; 43:1-19. Available at http://www.cdc.gov/mmwr/PDF/rr/rr4312.pdf.

\section{Dosing Recommendations for Prevention and Treatment of Candidiasis}

\begin{tabular}{|c|c|c|c|}
\hline & \multicolumn{3}{|c|}{ Preventive Regimen } \\
\hline Indication & First Choice & Alternative & Comments/Special Issues \\
\hline Primary Prophylaxis & Not routinely recommended & N/A & N/A \\
\hline Secondary Prophylaxis & 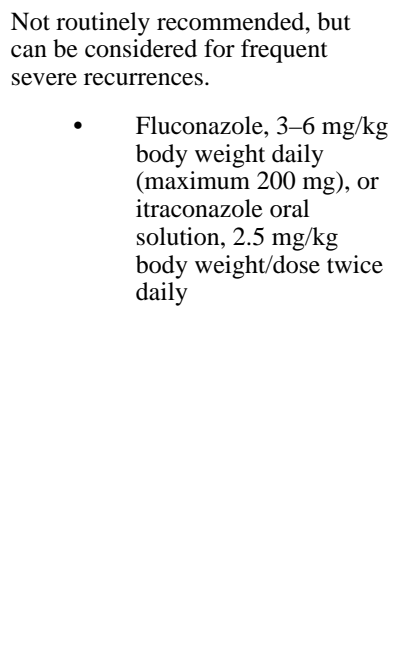 & N/A & 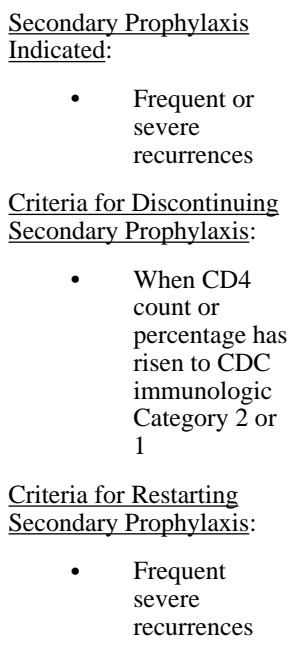 \\
\hline Treatment & 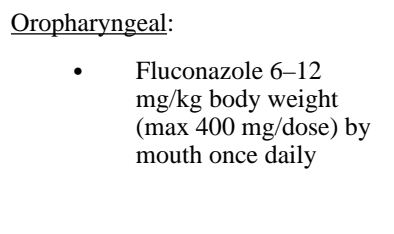 & 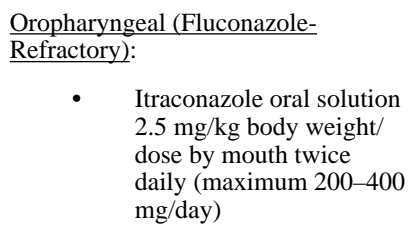 & $\begin{array}{l}\text { Itraconazole oral solution } \\
\text { should not be used } \\
\text { interchangeably with } \\
\text { itraconazole capsules. } \\
\text { Itraconazole capsules are } \\
\text { generally ineffective for } \\
\text { treatment of esophageal } \\
\text { disease. }\end{array}$ \\
\hline
\end{tabular}




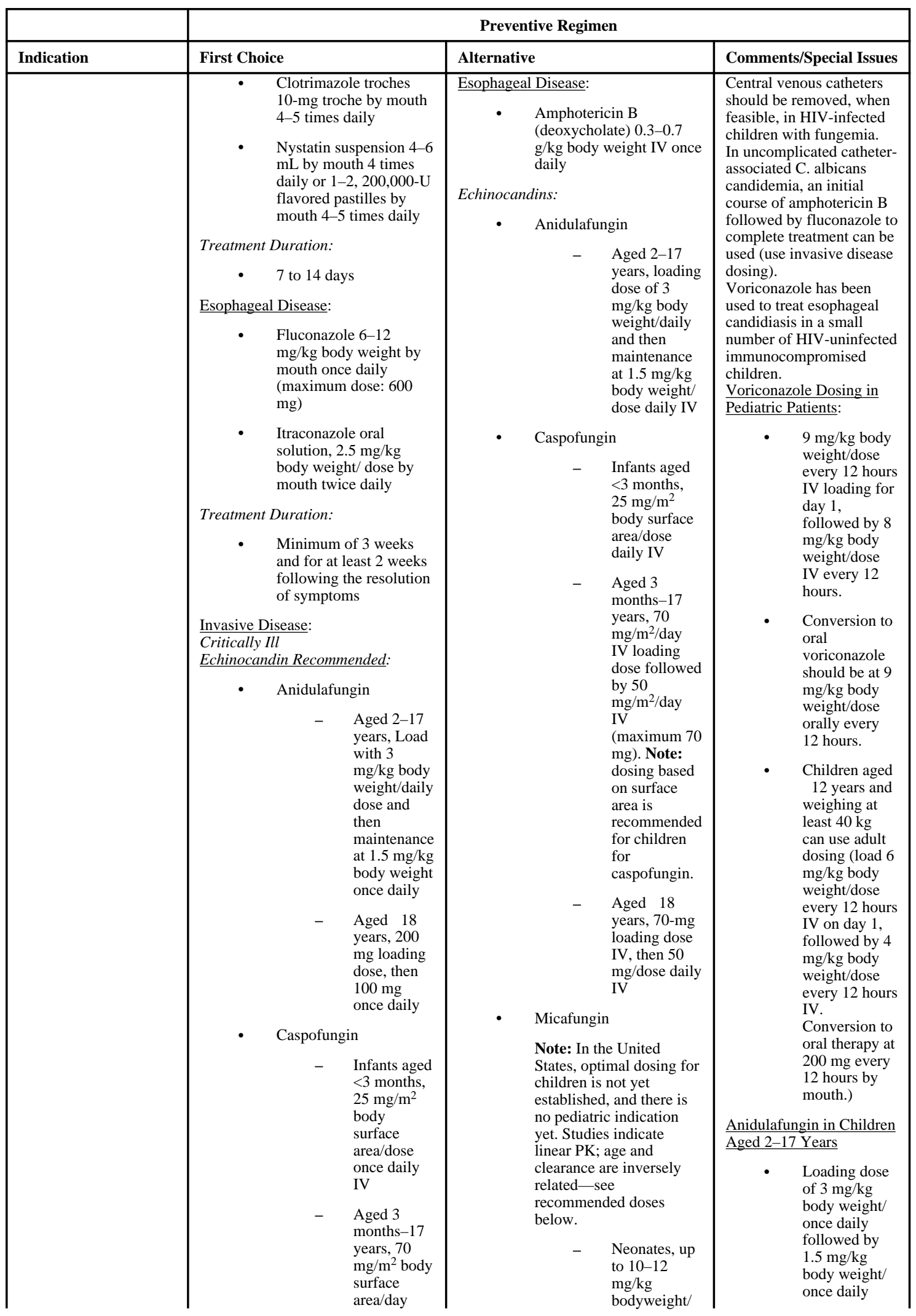




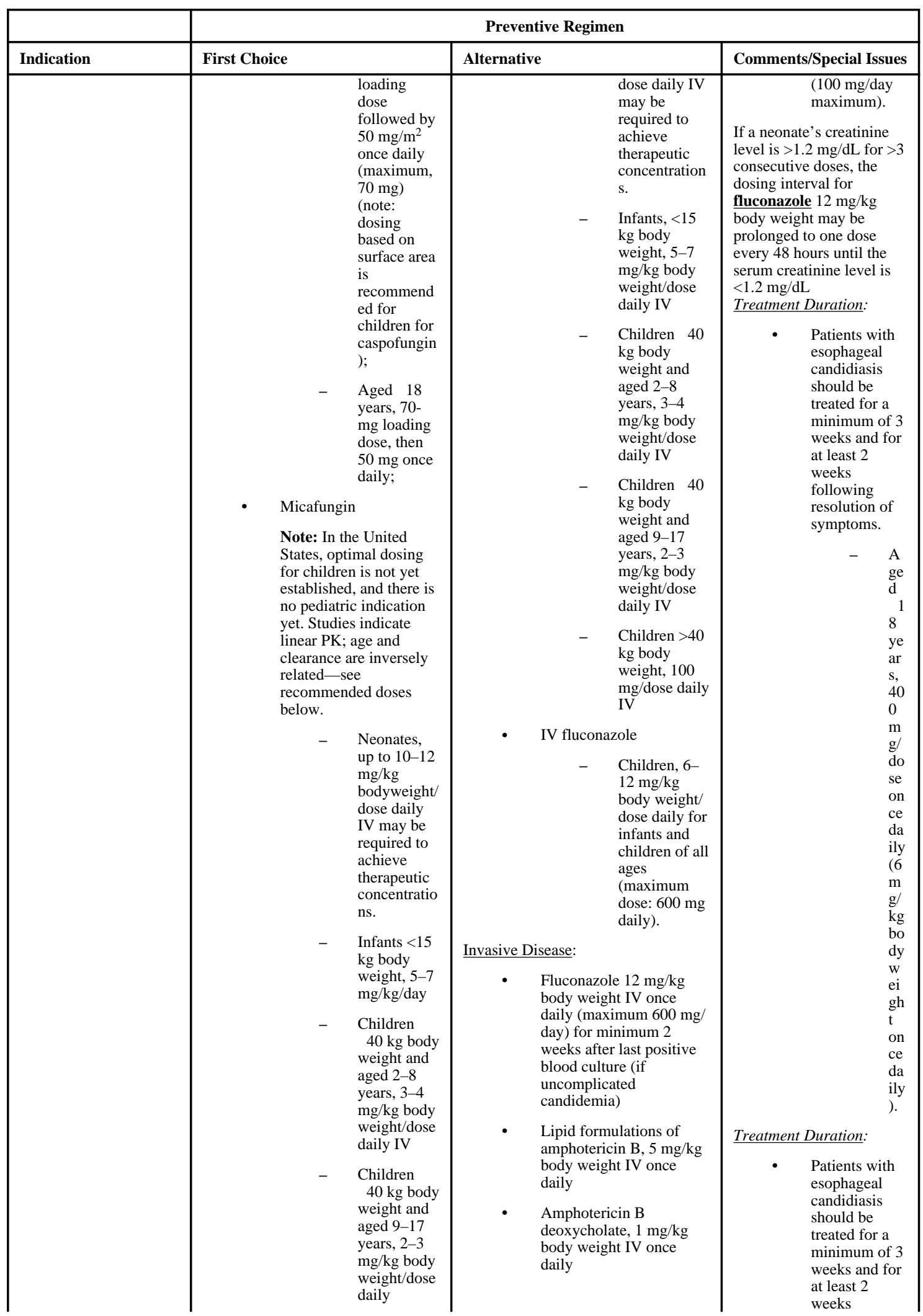




\begin{tabular}{|c|c|c|c|}
\hline & \multicolumn{3}{|c|}{ Preventive Regimen } \\
\hline Indication & First Choice & Alternative & Comments/Special Issues \\
\hline & 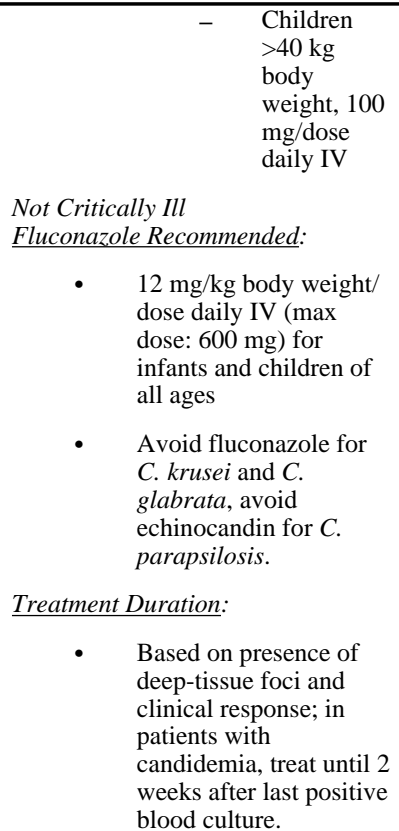 & & $\begin{array}{l}\text { following } \\
\text { resolution of } \\
\text { symptoms. }\end{array}$ \\
\hline
\end{tabular}

Key to Abbreviations: CD4 = CD4 T lymphocyte; CDC = Centers for Disease Control and Prevention; IV = intravenous; PK = pharmacokinetic

\section{Coccidioidomycosis (Last updated November 6, 2013; last reviewed November 6, 2013)}

Panel's Recommendations

- Routine use of antifungal medications for primary prophylaxis of coccidioidal infections in children is not recommended (BIII).

- Diffuse pulmonary or disseminated infection (not involving the central nervous system) should be treated initially with amphotericin B (AII*). After completion of amphotericin B, treatment with fluconazole or itraconazole should begin (BIII). Alternatively, some experts initiate therapy with amphotericin B combined with a triazole, such as fluconazole, in patients with disseminated disease and continue the triazole after amphotericin B is stopped (BIII).

- There is no evidence that lipid preparations of amphotericin are more effective than amphotericin B deoxycholate for the treatment of coccidioidomycosis. Lipid preparations are often preferred because they are better tolerated and associated with less nephrotoxicity than amphotericin B deoxycholate (AII*).

- $\quad$ For patients with mild disease (e.g., focal pneumonia), monotherapy with fluconazole or itraconazole is appropriate (BII*)

- Itraconazole is preferred for treatment of skeletal infections (AII*).

- Because absorption of itraconazole varies from patient to patient, serum concentrations should be measured to ensure effective, non-toxic levels of drug, monitor drug levels following changes in dosage, and assess compliance (BIII).

- Amphotericin B preparations are not the drugs of choice for treating coccidioidal meningitis; fluconazole is the preferred drug for treating coccidioidal meningitis (AII*).

- Lifelong antifungal suppression (secondary prophylaxis) with either fluconazole or itraconazole is recommended for treating HIV-infected children after disseminated, diffuse pulmonary, and/or meningeal coccidioidomycosis (AII*), even if immune reconstitution is achieved with combination antiretroviral therapy (cART). Lifelong secondary prophylaxis should be considered for children with mild disease and 
Panel's Recommendations

CD4 T lymphocyte cell count $<250$ cells $/ \mathrm{mm}^{3}$ or $<15 \%$, even if immune reconstitution is achieved with cART (BIII)

Rating of Recommendations: $\mathrm{A}=$ Strong; $\mathrm{B}=$ Moderate $\mathrm{C}=$ Optional

Rating of Evidence: I = One or more randomized trials in children ${ }^{\dagger}{ }^{\text {ith }}$ clinical outcomes and/or validated endpoints; $\mathrm{I}^{*}$ $=$ One or more randomized trials in adults with clinical outcomes and/or validated laboratory endpoints with accompanying data in children ${ }^{\dagger}$ from one or more well-designed, nonrandomized trials or observational cohort studies with long-term clinical outcomes; II = One or more well-designed, nonrandomized trials or observational cohort studies in children ${ }^{\dagger}$ with long-term outcomes; II $^{*}=$ One or more well-designed, nonrandomized trials or observational studies in adults with longterm clinical outcomes with accompanying data $\underline{\text { in children }}^{\dagger}$ from one or more similar nonrandomized trials or cohort studies with clinical outcome data; III = Expert opinion

${ }^{\dagger}$ Studies that include children or children/adolescents, but not studies limited to post-pubertal adolescents.

\section{Epidemiology}

Coccidioidomycosis is caused by the endemic, ${ }^{1,2}$ soil-dwelling dimorphic fungus,

Coccidioides spp. Two species, Coccidioides posadasii and C. immitis, have been identified using molecular and biogeographic characteristics. C. immitis appears to be confined mainly to California; $C$. posadasii is more widely distributed through the southwestern United States, northern Mexico, and Central and South America. Clinical illnesses caused by each are indistinguishable. Infection usually results from inhalation of spores (arthroconidia) produced by the mycelial form which grows in arid, windy environments with hot summers preceded by rainy seasons. ${ }^{3,4,5,6}$ Infection that occurs in non-endemic regions usually results from either re-activation of a previous infection or from acquisition during travel to an endemic region. ${ }^{7}$ Contaminated fomites, such as dusty clothing or agricultural products, ${ }^{8}$ also have been implicated as infrequent sources of infection. ${ }^{9}$

Most illnesses are primary infections with rates governed by both environmental conditions that are conducive to fungal growth and to activities/conditions that predispose to inhalation of spores. Increased infection rates have been attributed to population shifts to endemic regions, climatic conditions, and better recognition. ${ }^{10-12}$ A review of hospitalizations for coccidioidomycosis at children's hospitals from 2002 to 2006 found an increased incidence in 2005 to 2006 , especially among patients with comorbid conditions. ${ }^{13}$

Impairment of cellular immunity is a major risk factor for severe primary coccidioidomycosis or relapse of past infection. In HIV-infected adults, both localized pneumonia and disseminated infection usually are observed in individuals with CD4 T lymphocyte (CD4) cell counts $<250$ cells $/ \mathrm{mm}^{3}{ }^{3}{ }^{14,15}$ The threshold for increased risk in HIVinfected children has not been established; systemic fungal infection has occurred when CD4 counts were $₫ 00$ cells $/ \mathrm{mm}^{3}$ and with CD4 percentages $<15 \%$, both indicative of severe immunosuppression. ${ }^{16,17}$ Although no cases of coccidioidomycosis were reported in HIV-infected children enrolled in the Perinatal AIDS Collaborative Transmission Study, the study sites under-represented geographic regions in which coccidioidomycosis is endemic. ${ }^{18}$ Women who acquire coccidioidomycosis late in pregnancy are at risk of dissemination, but infection in their infants is infrequent. ${ }^{19}$ Infections in infants usually result from inhalation of spores in the environment. In adults, combination antiretroviral 
therapy (cART) appears to be responsible for the declining incidence and severity of coccidioidomycosis. ${ }^{20,21}$ Data are limited in children.

\section{Clinical Manifestations}

Coccidioidal infection can range from a mild, self-limited, flu-like illness to more severe, focal or disseminated illness, including pneumonia, bone and joint infection and meningitis. Immunocompromised individuals and previously healthy blacks, Hispanics, and Filipinos with coccidioidomycosis are at increased risk of dissemination, as are pregnant women who acquire coccidioidal infection during the second or third trimester ${ }^{22}$ or the postpartum period. $^{23,24}$ The severity of clinical manifestations in HIV-infected adults varies in direct proportion to the degree of immunocompromise. Diffuse pulmonary infection and extrathoracic dissemination have been associated with decreased CD4 counts, increased HIV RNA levels, and lower likelihood of having received potent antiretroviral therapy (ART) ${ }^{21}$ Focal pneumonitis can occur in mild to moderately immunocompromised patients. ${ }^{15,24}$ Pleural inflammation may result in effusion, empyema, and/or pneumothorax. ${ }^{25}$ If untreated, a coccidioidal antibody-seropositive, HIV-infected individual is at risk of serious disease, with the degree of severity inversely proportional to absolute CD4 counts $<250 / \mathrm{mm}^{3}$. Bone and joint involvement is rare in HIV-infected patients. ${ }^{20,26}$

Children with primary pulmonary infection may present with fever, malaise, and chest pain. The presence of cough varies, and hemoptysis is rare. Persistent fever may be a symptom of extrathoracic dissemination. Children with meningitis may present with headaches, altered sensorium, vomiting, and/or focal neurologic deficits. ${ }^{27-29}$ Fever is sometimes absent, and meningismus occurs in only $50 \%$ of patients. Hydrocephalus complicating basilar inflammation ${ }^{27,30}$ occurs in most $(83 \%-100 \%)$ children with coccidioidal meningitis. ${ }^{27,31}$ Generalized lymphadenopathy, skin nodules, plaques or ulcers, ${ }^{24,32}$ peritonitis, and liver abnormalities also may accompany disseminated disease.

\section{Diagnosis}

Because signs and symptoms are non-specific, the diagnosis of coccidioidomycosis should be among those considered in patients who reside in or have visited endemic areas. ${ }^{25,33}$ Culture, microscopy, and serology have been the methods used for diagnosis, but newer tests, including coccidioidal galactomannan antigen detection in urine, 1,34 are especially useful for diagnosis in immunocompromised hosts. Polymerase chain reaction (PCR) assays that target specific coccidioidal genes have been developed but are not yet commercially available. ${ }^{35,36}$

In patients with meningitis, cerebrospinal fluid (CSF) shows moderate hypoglycorrhachia, elevated protein concentration, and pleocytosis with a predominance of mononuclear cells. CSF eosinophilia may also be present. The observation of distinctive spherules containing endospores in histopathologic tissue ${ }^{37}$ or other clinical specimens is diagnostic. Stains of CSF in patients with meningitis usually are negative. Pyogranulomatous inflammation with endosporulating spherules is seen in affected tissue specimens with haematoxylin and eosin. Spherules can also be observed using Papanicolaou, Gomori methenamine silver nitrate, and periodic acid-Schiff stains. Cytologic stains are less reliable for diagnosing pulmonary 
coccidioidomycosis, and a negative cytologic stain on a clinical respiratory specimen may not exclude active pulmonary coccidioidomycosis. ${ }^{26}$ Potassium hydroxide stains are less sensitive and should not be used. ${ }^{26}$

Growth of Coccidioides spp. is supported by many conventional laboratory media used for fungal isolation; growth may occur within 5 days at $30^{\circ} \mathrm{C}$ to $37^{\circ} \mathrm{C} .{ }^{26}$ Blood cultures are positive in $<15 \%$ of cases; CSF cultures are positive in $<50 \%$ of children with meningitis. ${ }^{24,26,38}$ Cultures of respiratory specimens are often positive in adults with pulmonary coccidioidomycosis. The laboratory should be alerted to clinical suspicion of coccidioidal infection so that specimens can be handled in secure and contained fashion to minimize hazards to laboratory personnel.

Serologic assays, performed by enzyme-linked immunoassay (EIA), immunodiffusion, or classical tube precipitin or complement fixation methodology that measure coccidioidal Immunoglobulin $\mathrm{M}(\operatorname{IgM})$ and Immunoglobulin $\mathrm{G}(\mathrm{IgG})$ antibody are valuable aids in diagnosis ${ }^{39}$ but may be falsely negative in immunocompromised hosts. Presence of IgMspecific coccidioidal antibody suggests active or recent infection although, in instances in which IgG-specific antibody is absent, data are conflicting about potential false positives. ${ }^{40,41}$ IgG-specific antibody appears later and persists for 6 to 8 months. A commercial EIA appears more sensitive than the older tube precipitin and complement fixation tests and the immunodiffusion assays, although concern remains about specificity. ${ }^{42}$ The EIA, however, is not quantitative. ${ }^{24}$ Assays for coccidioidal antibody in serum or body fluids such as CSF provide diagnostic and prognostic information. Cross-reactivity can occur with other endemic mycoses. IgG-specific antibody titers often become undetectable in several months if the infection resolves. The diagnosis of meningitis is established with either a positive CSF culture or detection of IgG-specific antibody in CSF. Serial testing 36 following at least a 2-week interval may be needed to demonstrate this. Antibody titers decline during effective therapy. A Coccidiodes EIA has been developed that detects and quantifies coccidioidal galactomannan concentrations in urine samples ${ }^{34,43,44}$ and is especially useful in serious infections and/or instances in which antibody is undetectable. Dissociation of immune complexes has increased the sensitivity of detection of coccidioidal antigen in serum. ${ }^{44}$ Meningitis has been diagnosed using real-time PCR analysis of CSF. ${ }^{36}$

\section{Prevention Recommendations}

Preventing Exposure-HIV-infected patients who reside in or visit regions in which coccidioidomycosis is endemic cannot completely avoid exposure to Coccidioides spp., but risk can be reduced by avoiding activities and/or exposure to sites that may predispose to inhalation of spores. These include disturbing contaminated soil, archaeological excavation, and being outdoors during dust storms. If such activities are unavoidable, use of highefficiency respiratory filtration devices should be considered. ${ }^{36}$

Preventing First Episode of Disease-No prospective studies have been published that examine the role of prophylaxis to prevent development of active coccidioidomycosis in patients without previous (recognized) episodes of coccidioidomycosis. Although some experts would provide prophylaxis with an azole (fluconazole) to coccidioidal 
antibodypositive HIV-infected patients living in regions with endemic coccidioidomycosis, others would not. ${ }^{26}$ Chemoprophylaxis is used for coccidioidal antibody-positive HIVinfected adults living in endemic areas and with CD4 counts $<250$ cells $/ \mathrm{mm}^{3} .{ }^{45,46}$ However, given the low incidence of coccidioidomycosis in HIV-infected children, the potential for drug interactions, potential for development of antifungal drug resistance, and the cost, the routine use of antifungal medications for primary prophylaxis of coccidioidal infections in children is not recommended (BIII).

Discontinuing Primary Prophylaxis—Not applicable.

\section{Treatment Recommendations}

Treating Disease-In patients with HIV infection, effective cART, if not being administered at the time of diagnosis of coccidioidomycosis, should be started in concert with initiation of antifungal agents. Treatment protocols that are recommended for HIVinfected children are based on experience in nonrandomized, open-label studies in adults. Physicians who infrequently treat children with coccidioidomycosis should consider consulting with experts.

Antifungal therapy had been a recommendation for all HIV-infected adults with clinically active, mild coccidioidomycosis. ${ }^{47}$ More recently, treatment protocols appropriate for patients who are HIV-uninfected have been suggested ${ }^{47}$ for HIV-infected adults reliably receiving potent ART and who have CD4 counts $>250$ cells $/ \mathrm{mm}^{3} .46$ That would include patients with mild infections that are not accompanied by signs suggestive of dissemination, diffuse pulmonary infiltrates, or meningitis. In this setting, patients should be closely monitored to ensure compliance with ART, effective HIV suppression, and maintenance of CD4 counts $>250$ cells $/ \mathrm{mm}^{3}$. Management should also include education directed at reducing the probability of re-exposure to coccidioidal spores. In children, absent comparable published experience in this setting, expert consultation should be sought and, if treatment is elected, recommendations should be based upon assurance of continued compliance with ART, confirmation of continued HIV suppression, CD4 counts $>250 / \mathrm{mm}^{3}$, education directed at decreasing the likelihood of exposure to coccidioidal spores, and close medical follow up.

For patients with mild, non-meningitic disease (e.g., focal pneumonitis), monotherapy with fluconazole or itraconazole is appropriate given their effectiveness, safety, convenient oral dosing, and pharmacodynamic parameters (BII*). Fluconazole $(6-12 \mathrm{mg} / \mathrm{kg} / \mathrm{day})$ and itraconazole $(5-10 \mathrm{mg} / \mathrm{kg} /$ dose twice daily for the first 3 days, followed thereafter by $2-5$ $\mathrm{mg} / \mathrm{kg}$ per dose twice daily) are alternatives to amphotericin B for children who have mild, non-meningitic disease (BIII). In a randomized, double-blind trial in adults, fluconazole and itraconazole were equivalent for treating non-meningeal coccidioidomycosis. Itraconazole (5 $\mathrm{mg} / \mathrm{kg}$ body weight dose twice daily) appeared to be more effective than fluconazole for treating skeletal infections (AII*). ${ }^{48}$

Severely ill patients with diffuse pneumonia and/or other signs of probable disseminated infection (not involving the CNS) are initially treated with an amphotericin B preparation because these appear to evoke a faster therapeutic response than do the azoles. ${ }^{49,50}$ Although 
there is no evidence that the lipid preparations are more effective than amphotericin B deoxycholate, lipid formulations often are used because they are better tolerated (AIII). The length of amphotericin B therapy is governed by both the severity of initial symptoms and the pace of the clinical improvement. Thereafter, amphotericin B is stopped and treatment with fluconazole or itraconazole begun (BIII). Some experts initiate therapy with both amphotericin B and a triazole, such as fluconazole, in patients with severe disseminated disease and continue the triazole after amphotericin B is stopped (BIII). ${ }^{26,48}$ The total duration of therapy should be $\geq 1$ year. $^{26}$

Meningitis is a life-threatening manifestation of coccidioidomycosis and consultation with experts should be considered (BIII). Successful treatment requires an antifungal agent that achieves effective concentrations in CSF. Intravenous amphotericin B achieves poor CSF concentrations and is therefore not recommended for treating coccidioidal meningitis (AIII). The relative safety and comparatively superior ability of fluconazole to penetrate the bloodbrain barrier have made it the treatment of choice for coccidioidal meningitis (AII*). An effective dose of fluconazole in adults is $400 \mathrm{mg} /$ day, but some experts begin therapy with 800 to $1000 \mathrm{mg} / \mathrm{day} .{ }^{47}$ Children usually receive $12 \mathrm{mg} / \mathrm{kg} / \mathrm{dose}$ once daily $(800 \mathrm{mg} / \mathrm{day}$ maximum) (AII*). ${ }^{51,52} \mathrm{The} 12 \mathrm{mg} / \mathrm{kg}$ dosage may be required to attain serum concentrations equivalent to those in adults receiving $400 \mathrm{mg} /$ day. ${ }^{53}$ Some experts would begin at a dose of 15 to $23 \mathrm{mg} / \mathrm{kg} /$ day. ${ }^{24}$ Successful therapy with posaconazole ${ }^{54}$ and voriconazole has been described in adults but there is no published experience in children. ${ }^{55}$ Some experts use amphotericin B administered intrathecally ${ }^{50,56}$ in addition to an azole. Intrathecal amphotericin administration adds additional toxicity and is not used as part of initial therapy (CIII). Despite the benefits afforded by the azoles for treating meningitis, a retrospective analysis of outcomes in adults treated for coccidioidal meningitis in the preazole (earlier than 1980) compared with outcomes in the azole era found that a similar percentage developed serious complications, including stroke and hydrocephalus; risk factors for acquiring coccidioidal meningitis in the azole era included immunocompromised state, with one-third of patients in this group having HIV/AIDS. ${ }^{28}$

Monitoring and Adverse Events (Including IRIS)—In addition to monitoring patients for clinical improvement, some experts ${ }^{26}$ have recommended monitoring coccidioidal IgG antibody titers to assess response to therapy. Titers should be obtained every 12 weeks (AIII). If therapy is succeeding, titers should decrease progressively; a rise in titers suggests recurrence of clinical disease. However, if serologic tests initially were negative, titers during effective therapy may increase briefly and then decrease. ${ }^{26}$ This lag in response during the first 2 months of therapy should not necessarily be construed as treatment failure.

Adverse effects of amphotericin B are primarily those associated with nephrotoxicity. Infusion-related fevers, chills, nausea, and vomiting also can occur, although they are less frequent in children than in adults. Lipid formulations of amphotericin B have lower rates of nephrotoxicity. Hepatic toxicity, thrombophlebitis, anemia, and rarely neurotoxicity (manifested as confusion or delirium, hearing loss, blurred vision, or seizures) also can occur (see discussion on monitoring and adverse events in Candida infection). Intrathecal injection of amphotericin B may result in arachnoiditis. ${ }^{57,58}$ 
Triazoles can interact with other drugs metabolized by CYP450-dependent hepatic enzymes, ${ }^{59,60}$ and the potential for drug interactions should be assessed before initiation of therapy (AIII). Use of fluconazole or itraconazole appears to be safe in combination with ART. Voriconazole should be avoided in patients receiving protease inhibitors (BIII) ${ }^{61}$ or non-nucleoside reverse transcriptase inhibitors. ${ }^{15}$ The most frequent adverse effects of fluconazole are nausea and vomiting. Skin rash and pruritus may be observed, and cases of Stevens-Johnson syndrome have been reported. Asymptomatic increases in transaminases occur in $1 \%$ to $13 \%$ of patients receiving azole drugs. In HIV-infected patients, fluconazole at high doses can cause adrenal insufficiency. ${ }^{62}$

Because absorption of itraconazole varies from patient to patient, serum concentrations should be measured to ensure effective, non-toxic levels of drug, monitor changes in dosage, and assess compliance (BIII).

Coccidioidomycosis-associated immune reconstitution inflammatory syndrome following the initiation of ART has not been reported in children and is rarely reported in adults. ${ }^{63}$

Managing Treatment Failure-The treatment of coccidioidomycosis unresponsive to standard therapy has been reviewed; the majority of experience has been in adults. ${ }^{55}$

Posaconazole was effective in 6 adults with disease refractory to treatment with other azoles and to amphotericin $\mathrm{B}^{64}$ and has been used successfully in $73 \%$ of 15 adults whose infections were refractory to previous therapy. ${ }^{65}$ Posaconazole has also been effective for chronic refractory meningitis unresponsive to fluconazole. ${ }^{54}$ Voriconazole was effective in treating coccidioidal meningitis and non-meningeal disseminated disease in adults who did not respond to fluconazole or were intolerant of amphotericin B. ${ }^{66,67,68}$ Monotherapy with caspofungin successfully treated disseminated coccidioidomycosis in a renal transplant patient intolerant of fluconazole and other adults in whom conventional therapy failed. ${ }^{69,70}$ Others have used caspofungin in combination with fluconazole. ${ }^{71}$

Adjunctive interferon-gamma (IFN- $-\gamma)^{72}$ was successfully used in a critically ill adult with respiratory failure who did not respond to amphotericin B preparations and fluconazole. ${ }^{73}$ However, no controlled clinical studies or data exist for children; thus, adjunctive IFN- $\gamma$ is not recommended for use in HIV-infected children (BIII).

In instances in which patients with coccidioidal meningitis fail to respond to treatment with azoles, both systemic amphotericin B and direct instillation of amphotericin B into the intrathecal, ventricular, or intracisternal spaces, with or without concomitant azole treatment, have been used successfully. These regimens are recommended in such instances (AIII). ${ }^{48,52}$ The basilar inflammation that characteristically accompanies coccidioidal meningitis often results in obstructive hydrocephalus requiring placement of a CSF shunt. Thus, development of hydrocephalus in coccidioidal meningitis does not necessarily indicate treatment failure. Response rates with the azoles can be excellent, but cures are infrequent. Relapse after cessation of therapy is common, occurring in as many as $80 \%$ of patients. ${ }^{74}$ Thus, indefinite continuation of fluconazole therapy is recommended for patients who have coccidioidal meningitis (AII*). 
Preventing Recurrence-Lifelong suppression (secondary prophylaxis) is recommended for patients following successful treatment of meningitis. Relapse after successful treatment of disseminated coccidioidomycosis can occur and lifelong antifungal suppression with either fluconazole or itraconazole should be used (AII*). Secondary prophylaxis should be considered for children with mild disease and ongoing CD4 counts $<250$ cells $/ \mathrm{mm}^{3}$ or CD4 percentages $<15 \%$ (BIII). ${ }^{26,47,49,75,76}$

Discontinuing Secondary Prophylaxis-In disseminated infection, continued suppressive therapy (secondary prophylaxis) with fluconazole or itraconazole is recommended after completion of initial therapy. Patients with diffuse pulmonary disease, disseminated disease, or meningeal infection should remain on lifelong prophylaxis-even if immune reconstitution is achieved with $\mathrm{ART}^{26}$ - because of high risk of relapse (AII*). In HIV-infected adults with focal coccidioidal pneumonia who have clinically responded to antifungal therapy and have sustained CD4 counts $>250$ cells $/ \mathrm{mm}^{3}$ on ART, some experts would discontinue secondary prophylaxis after 12 months of antifungal therapy with careful monitoring for recurrence with chest radiographs and coccidioidal serology. However, only a small number of patients have been evaluated, and the safety of discontinuing secondary prophylaxis after immune reconstitution with ART in children has not been studied. Therefore, in HIV-infected children, once secondary prophylaxis is initiated for an acute episode of milder, non-meningeal coccidioidomycosis, lifelong suppressive therapy should be considered, regardless of ART and immune reconstitution (BIII).

\section{References}

1. Hage CA, Knox KS, Wheat LJ. Endemic mycoses: Overlooked causes of community acquired pneumonia. Respir Med. 2012 Mar 2. Available at http://www.ncbi.nlm.nih.gov/pubmed/22386326.

2. Thompson GR 3rd. Pulmonary coccidioidomycosis. Semin Respir Crit Care Med. 2011 Dec; 32(6): 754-763. Available at http://www.ncbi.nlm.nih.gov/pubmed/22167403. [PubMed: 22167403]

3. DiCaudo DJ. Coccidioidomycosis: a review and update. J Am Acad Dermatol. 2006 Dec; 55(6): 929-942. quiz 943-925 Available at http://www.ncbi.nlm.nih.gov/pubmed/17110216. [PubMed: 17110216]

4. Crum NF, Lederman ER, Stafford CM, Parrish JS, Wallace MR. Coccidioidomycosis: a descriptive survey of a reemerging disease. Clinical characteristics and current controversies. Medicine (Baltimore). 2004 May; 83(3):149-175. Available at http://www.ncbi.nlm.nih.gov/pubmed/ 15118543. [PubMed: 15118543]

5. Ampel NM. Coccidioidomycosis: a review of recent advances. Clin Chest Med. 2009 Jun; 30(2): 241-251. v. Available at http://www.ncbi.nlm.nih.gov/entrez/query.fcgi? $\mathrm{cmd}=$ Retrieve $\& \mathrm{db}=$ PubMed\&dopt=Citation\&list_uids=19375631. [PubMed: 19375631]

6. Tamerius JD, Comrie AC. Coccidioidomycosis incidence in Arizona predicted by seasonal precipitation. PLoS One. 2011; 6(6):e21009. Available at http://www.ncbi.nlm.nih.gov/pubmed/ 21701590. [PubMed: 21701590]

7. Desai NR, McGoey R, Troxclair D, Simeone F, Palomino J. Coccidioidomycosis in nonendemic area: case series and review of literature. J La State Med Soc. 2010 Mar-Apr;162(2):97-103. Available at http://www.ncbi.nlm.nih.gov/pubmed/20521740. [PubMed: 20521740]

8. Tang TH, Tsang OT. Images in clinical medicine. Fungal infection from sweeping in the wrong place. N Engl J Med. 2011 Jan 13.364(2):e3. Available at http://www.ncbi.nlm.nih.gov/entrez/ query.fcgi?cmd=Retrieve $\& d b=P u b M e d \& d o p t=C i t a t i o n \& l i s t \_u i d s=21226571$. [PubMed: 21226571]

9. Stagliano D, Epstein J, Hickey P. Fomite-transmitted coccidioidomycosis in an immunocompromised child. Pediatr Infect Dis J. 2007 May; 26(5):454-456. Available at http:// www.ncbi.nlm.nih.gov/pubmed/17468663. [PubMed: 17468663] 
10. Centers for Disease C, Prevention. Increase in Coccidioidomycosis - California, 2000-2007. MMWR Morb Mortal Wkly Rep. 2009 Feb 13; 58(5):105-109. Available at http:// www.ncbi.nlm.nih.gov/pubmed/19214158. [PubMed: 19214158]

11. Valdivia L, Nix D, Wright M, et al. Coccidioidomycosis as a common cause of communityacquired pneumonia. Emerg Infect Dis. 2006 Jun; 12(6):958-962. Available at http:// www.ncbi.nlm.nih.gov/pubmed/16707052. [PubMed: 16707052]

12. Ampel NM. What's Behind the Increasing Rates of Coccidioidomycosis in Arizona and California? Curr Infect Dis Rep. 2010 May; 12(3):211-216. Available at http://www.ncbi.nlm.nih.gov/ pubmed/21308532. [PubMed: 21308532]

13. Fisher BT, Chiller TM, Prasad PA, Beveridge M, Walsh TJ, Zaoutis TE. Hospitalizations for coccidioidomycosis at forty-one children's hospitals in the United States. Pediatr Infect Dis J. 2010 Mar; 29(3):243-247. Available at http://www.ncbi.nlm.nih.gov/entrez/query.fcgi? $\mathrm{cmd}=$ Retrieve $\& \mathrm{db}=$ PubMed\&dopt=Citation\&list_uids=19934792. [PubMed: 19934792]

14. Ampel NM, Dols CL, Galgiani JN. Coccidioidomycosis during human immunodeficiency virus infection: results of a prospective study in a coccidioidal endemic area. Am J Med. 1993 Mar; 94(3):235-240. Available at http://www.ncbi.nlm.nih.gov/pubmed/8095771. [PubMed: 8095771]

15. Ampel NM. Coccidioidomycosis in persons infected with HIV-1. Ann N Y Acad Sci. 2007 Sep. 1111:336-342. Available at http://www.ncbi.nlm.nih.gov/pubmed/17363429. [PubMed: 17363429]

16. Dankner WM, Lindsey JC, Levin MJ. Pediatric ACTGPT. Correlates of opportunistic infections in children infected with the human immunodeficiency virus managed before highly active antiretroviral therapy. Pediatr Infect Dis J. 2001 Jan; 20(1):40-48. Available at http:// www.ncbi.nlm.nih.gov/pubmed/11176565. [PubMed: 11176565]

17. Kaplan JE, Masur H, Holmes KK. Usphs, Infectious Disease Society of A. Guidelines for preventing opportunistic infections among HIV-infected persons-2002. Recommendations of the U.S. Public Health Service and the Infectious Diseases Society of America. MMWR Recomm Rep. 2002 Jun 14; 51(RR-8):1-52. Available at http://www.ncbi.nlm.nih.gov/pubmed/12081007.

18. Nesheim SR, Kapogiannis BG, Soe MM, et al. Trends in opportunistic infections in the pre- and post-highly active antiretroviral therapy eras among HIV-infected children in the Perinatal AIDS Collaborative Transmission Study, 1986-2004. Pediatrics. 2007 Jul; 120(1):100-109. Available at http://www.ncbi.nlm.nih.gov/pubmed/17606567. [PubMed: 17606567]

19. Hyatt HW Sr. Coccidioidomycosis in a 3-week-old infant. Am J Dis Child. 1963 Jan.105:93-98. Available at http://www.ncbi.nlm.nih.gov/pubmed/13955987. [PubMed: 13955987]

20. Ampel NM. Coccidioidomycosis among persons with human immunodeficiency virus infection in the era of highly active antiretroviral therapy (HAART). Semin Respir Infect. 2001 Dec; 16(4): 257-262. Available at http://www.ncbi.nlm.nih.gov/pubmed/11740827. [PubMed: 11740827]

21. Masannat FY, Ampel NM. Coccidioidomycosis in patients with HIV-1 infection in the era of potent antiretroviral therapy. Clin Infect Dis. 2010 Jan 1; 50(1):1-7. Available at http:// www.ncbi.nlm.nih.gov/entrez/query.fcgi?

cmd=Retrieve \&db=PubMed\&dopt=Citation\&list_uids=19995218. [PubMed: 19995218]

22. Wack EE, Ampel NM, Galgiani JN, Bronnimann DA. Coccidioidomycosis during pregnancy. An analysis of ten cases among 47,120 pregnancies. Chest. 1988 Aug; 94(2):376-379. Available at http://www.ncbi.nlm.nih.gov/pubmed/3396418. [PubMed: 3396418]

23. Adam RD, Elliott SP, Taljanovic MS. The spectrum and presentation of disseminated coccidioidomycosis. Am J Med. 2009 Aug; 122(8):770-777. Available at http:// www.ncbi.nlm.nih.gov/entrez/query.fcgi? $\mathrm{cmd}=$ Retrieve $\& \mathrm{db}=$ PubMed\&dopt=Citation\&list_uids=19635278. [PubMed: 19635278]

24. Shehab ZM. Coccidioidomycosis. Adv Pediatr. 2010; 57(1):269-286. Available at http:// www.ncbi.nlm.nih.gov/pubmed/21056742. [PubMed: 21056742]

25. Tiu CT, Cook J, Pineros DF, et al. Pneumothorax in a young man in Brooklyn, New York. Clin Infect Dis. 2011 Dec; 53(12):1255, 1296-1257. Available at http://www.ncbi.nlm.nih.gov/ pubmed/22080120. [PubMed: 22080120] 
26. Ampel NM. Coccidioidomycosis in persons infected with HIV type 1. Clin Infect Dis. 2005 Oct 15; 41(8):1174-1178. Available at http://www.ncbi.nlm.nih.gov/pubmed/16163637. [PubMed: 16163637]

27. Drake KW, Adam RD. Coccidioidal meningitis and brain abscesses: analysis of 71 cases at a referral center. Neurology. 2009 Nov 24; 73(21):1780-1786. Available at http:// www.ncbi.nlm.nih.gov/entrez/query.fcgi? cmd=Retrieve \&db=PubMed\&dopt=Citation\&list_uids=19933980. [PubMed: 19933980]

28. Mathisen G, Shelub A, Truong J, Wigen C. Coccidioidal meningitis: clinical presentation and management in the fluconazole era. Medicine (Baltimore). 2010 Sep; 89(5):251-284. Available at http://www.ncbi.nlm.nih.gov/entrez/query.fcgi? cmd=Retrieve $\& d b=$ PubMed\&dopt=Citation\&list_uids=20827104. [PubMed: 20827104]

29. Blair JE. Coccidioidal meningitis: update on epidemiology, clinical features, diagnosis, and management. Curr Infect Dis Rep. 2009 Jul; 11(4):289-295. Available at http:// www.ncbi.nlm.nih.gov/entrez/query.fcgi? cmd=Retrieve \&db=PubMed\&dopt=Citation\&list_uids=19545498. [PubMed: 19545498]

30. Winston DJ, Kurtz TO, Fleischmann J, Morgan D, Batzdorf U, Stern WE. Successful treatment of spinal arachnoiditis due to coccidioidomycosis. Case report. J Neurosurg. 1983 Aug; 59(2):328331. Available at http://www.ncbi.nlm.nih.gov/pubmed/6306182. [PubMed: 6306182]

31. Shehab ZM, Britton H, Dunn JH. Imidazole therapy of coccidioidal meningitis in children. Pediatr Infect Dis J. 1988 Jan; 7(1):40-44. Available at http://www.ncbi.nlm.nih.gov/pubmed/3340457. [PubMed: 3340457]

32. Deus Filho A, Deus AC, Meneses Ade O, Soares AS, Lira AL. Skin and mucous membrane manifestations of coccidioidomycosis: a study of thirty cases in the Brazilian states of Piaui and Maranhao. An Bras Dermatol. 2010 Feb; 85(1):45-51. Available at http://www.ncbi.nlm.nih.gov/ pubmed/20464086. [PubMed: 20464086]

33. Wheat LJ. Approach to the diagnosis of the endemic mycoses. Clin Chest Med. 2009 Jun; 30(2): 379-389. viii. Available at http://www.ncbi.nlm.nih.gov/pubmed/19375642. [PubMed: 19375642]

34. Durkin M, Connolly P, Kuberski T, et al. Diagnosis of coccidioidomycosis with use of the Coccidioides antigen enzyme immunoassay. Clin Infect Dis. 2008 Oct 15; 47(8):e69-e73. Available at http://www.ncbi.nlm.nih.gov/pubmed/18781884. [PubMed: 18781884]

35. Ampel NM. The diagnosis of coccidioidomycosis. F1000 Med Rep. 2010; 2 Available at http:// www.ncbi.nlm.nih.gov/entrez/query.fcgi? cmd=Retrieve \&db=PubMed\&dopt=Citation\&list_uids=20948866.

36. Binnicker MJ, Popa AS, Catania J, et al. Meningeal coccidioidomycosis diagnosed by real-time polymerase chain reaction analysis of cerebrospinal fluid. Mycopathologia. 2011 Apr; 171(4):285289. Available at http://www.ncbi.nlm.nih.gov/pubmed/20924686. [PubMed: 20924686]

37. Berg N, Ryscavage P, Kulesza P. The utility of fine needle aspiration for diagnosis of extrapulmonary coccidioidomycosis: a case report and discussion. Clin Med Res. 2011 Nov; 9(34):130-133. Available at http://www.ncbi.nlm.nih.gov/pubmed/21562136. [PubMed: 21562136]

38. Keckich DW, Blair JE, Vikram HR. Coccidioides fungemia in six patients, with a review of the literature. Mycopathologia. 2010 Aug; 170(2):107-115. Available at http://www.ncbi.nlm.nih.gov/ entrez/query.fcgi? $\mathrm{cmd}=$ Retrieve $\& \mathrm{db}=$ PubMed\&dopt=Citation\&list_uids=20336378. [PubMed: 20336378]

39. Pappagianis D, Zimmer BL. Serology of coccidioidomycosis. Clin Microbiol Rev. 1990 Jul; 3(3): 247-268. Available at http://www.ncbi.nlm.nih.gov/entrez/query.fcgi? $\mathrm{cmd}=$ Retrieve $\& \mathrm{db}=$ PubMed\&dopt=Citation\&list_uids=2200605. [PubMed: 2200605]

40. Kuberski T, Herrig J, Pappagianis D. False-positive IgM serology in coccidioidomycosis. J Clin Microbiol. 2010 Jun; 48(6):2047-2049. Available at http://www.ncbi.nlm.nih.gov/entrez/ query.fcgi? $\mathrm{cmd}=$ Retrieve $\& \mathrm{db}=\mathrm{PubMed} \& \mathrm{dopt}=$ Citation\&list_uids=20357210. [PubMed: 20357210]

41. Blair JE, Currier JT. Significance of isolated positive IgM serologic results by enzyme immunoassay for coccidioidomycosis. Mycopathologia. 2008 Aug; 166(2):77-82. Available at http://www.ncbi.nlm.nih.gov/pubmed/18523863. [PubMed: 18523863] 
42. Ampel NM. New perspectives on coccidioidomycosis. Proc Am Thorac Soc. 2010 May; 7(3):181185. Available at http://www.ncbi.nlm.nih.gov/entrez/query.fcgi? cmd=Retrieve \&db=PubMed\&dopt=Citation\&list_uids=20463246. [PubMed: 20463246]

43. Kuberski T, Myers R, Wheat LJ, et al. Diagnosis of coccidioidomycosis by antigen detection using cross-reaction with a Histoplasma antigen. Clin Infect Dis. 2007 Mar 1; 44(5):e50-e54. Available at http://www.ncbi.nlm.nih.gov/pubmed/17278049. [PubMed: 17278049]

44. Durkin M, Estok L, Hospenthal D, et al. Detection of Coccidioides antigenemia following dissociation of immune complexes. Clin Vaccine Immunol. 2009 Oct; 16(10):1453-1456. Available at http://www.ncbi.nlm.nih.gov/pubmed/19675225. [PubMed: 19675225]

45. Woods CW, McRill C, Plikaytis BD, et al. Coccidioidomycosis in human immunodeficiency virusinfected persons in Arizona, 1994-1997: incidence, risk factors, and prevention. J Infect Dis. 2000 Apr; 181(4):1428-1434. Available at http://www.ncbi.nlm.nih.gov/pubmed/10753734. [PubMed: 10753734]

46. Kaplan JE, Benson C, Holmes KH, et al. Guidelines for prevention and treatment of opportunistic infections in HIV-infected adults and adolescents: recommendations from CDC, the National Institutes of Health, and the HIV Medicine Association of the Infectious Diseases Society of America. MMWR Recomm Rep. 2009 Apr 10; 58(RR-4):1-207. quiz CE201-204 Available at http://www.ncbi.nlm.nih.gov/pubmed/19357635.

47. Galgiani JN, Ampel NM, Catanzaro A, Johnson RH, Stevens DA, Williams PL. Practice guideline for the treatment of coccidioidomycosis. Infectious Diseases Society of America. Clin Infect Dis. 2000 Apr; 30(4):658-661. Available at http://www.ncbi.nlm.nih.gov/pubmed/10770727. [PubMed: 10770727]

48. Galgiani JN, Catanzaro A, Cloud GA, et al. Comparison of oral fluconazole and itraconazole for progressive, nonmeningeal coccidioidomycosis. A randomized, double-blind trial. Mycoses Study Group. Ann Intern Med. 2000 Nov 7; 133(9):676-686. Available at http://www.ncbi.nlm.nih.gov/ pubmed/11074900. [PubMed: 11074900]

49. Galgiani JN, Ampel NM, Blair JE, et al. Coccidioidomycosis. Clin Infect Dis. 2005 Nov 1; 41(9): 1217-1223. Available at http://www.ncbi.nlm.nih.gov/pubmed/16206093. [PubMed: 16206093]

50. Johnson RH, Einstein HE. Amphotericin B and coccidioidomycosis. Ann N Y Acad Sci. 2007 Sep. 1111:434-441. Available at http://www.ncbi.nlm.nih.gov/pubmed/17513463. [PubMed: 17513463]

51. Mofenson LM, Oleske J, Serchuck L, Van Dyke R, Wilfert C. Treating opportunistic infections among HIV-exposed and infected children: recommendations from CDC, the National Institutes of Health, and the Infectious Diseases Society of America. Clin Infect Dis. 2005 Feb 1; 40(Suppl 1):S1-S84. Available at http://www.ncbi.nlm.nih.gov/pubmed/15655768. [PubMed: 15655768]

52. Saitoh A, Homans J, Kovacs A. Fluconazole treatment of coccidioidal meningitis in children: two case reports and a review of the literature. Pediatr Infect Dis J. 2000 Dec; 19(12):1204-1208. Available at http://www.ncbi.nlm.nih.gov/pubmed/11144385. [PubMed: 11144385]

53. Blyth CC, Palasanthiran P, O'Brien TA. Antifungal therapy in children with invasive fungal infections: a systematic review. Pediatrics. 2007 Apr; 119(4):772-784. Available at http:// www.ncbi.nlm.nih.gov/pubmed/17403849. [PubMed: 17403849]

54. Schein R, Homans J, Larsen RA, Neely M. Posaconazole for chronic refractory coccidioidal meningitis. Clin Infect Dis. 2011 Dec; 53(12):1252-1254. Available at http:// www.ncbi.nlm.nih.gov/pubmed/21987729. [PubMed: 21987729]

55. Kim MM, Vikram HR, Kusne S, Seville MT, Blair JE. Treatment of refractory coccidioidomycosis with voriconazole or posaconazole. Clin Infect Dis. 2011 Dec; 53(11):1060-1066. Available at http://www.ncbi.nlm.nih.gov/pubmed/22045955. [PubMed: 22045955]

56. Stevens DA, Shatsky SA. Intrathecal amphotericin in the management of coccidioidal meningitis. Semin Respir Infect. 2001 Dec; 16(4):263-269. Available at http://www.ncbi.nlm.nih.gov/ pubmed/11740828. [PubMed: 11740828]

57. Harrison HR, Galgiani JN, Reynolds AF Jr, Sprunger LW, Friedman AD. Amphotericin B and imidazole therapy for coccidioidal meningitis in children. Pediatr Infect Dis. 1983 May-Jun;2(3): 216-221. Available at http://www.ncbi.nlm.nih.gov/pubmed/6306607. [PubMed: 6306607] 
58. Carnevale NT, Galgiani JN, Stevens DA, Herrick MK, Langston JW. Amphotericin B-induced myelopathy. Arch Intern Med. 1980 Sep; 140(9):1189-1192. Available at http:// www.ncbi.nlm.nih.gov/pubmed/6893266. [PubMed: 6893266]

59. Hughes CA, Foisy M, Tseng A. Interactions between antifungal and antiretroviral agents. Expert Opin Drug Saf. 2010 Sep; 9(5):723-742. Available at http://www.ncbi.nlm.nih.gov/pubmed/ 20345324. [PubMed: 20345324]

60. Santhana Krishnan SG, Cobbs RK. Reversible acute adrenal insufficiency caused by fluconazole in a critically ill patient. Postgrad Med J. 2006 Sep.82(971):e23. Available at http:// www.ncbi.nlm.nih.gov/pubmed/16954446. [PubMed: 16954446]

61. Winston A, Boffito M. The management of HIV-1 protease inhibitor pharmacokinetic interactions. J Antimicrob Chemother. 2005 Jul; 56(1):1-5. Available at http://www.ncbi.nlm.nih.gov/pubmed/ 15941777. [PubMed: 15941777]

62. Huang YW, Chang CC, Sun HY, Chen MY, Hung CC, Chang SC. Primary adrenal insufficiency in patients with acquired immunodeficiency syndrome: report of four cases. J Microbiol Immunol Infect. 2004 Aug; 37(4):250-253. Available at http://www.ncbi.nlm.nih.gov/pubmed/15340655. [PubMed: 15340655]

63. Mortimer RB, Libke R, Eghbalieh B, Bilello JF. Immune reconstitution inflammatory syndrome presenting as superior vena cava syndrome secondary to Coccidioides lymphadenopathy in an HIV-infected patient. J Int Assoc Physicians AIDS Care (Chic). 2008 Nov-Dec;7(6):283-285. Available at http://www.ncbi.nlm.nih.gov/pubmed/18948432. [PubMed: 18948432]

64. Anstead GM, Corcoran G, Lewis J, Berg D, Graybill JR. Refractory coccidioidomycosis treated with posaconazole. Clin Infect Dis. 2005 Jun 15; 40(12):1770-1776. Available at http:// www.ncbi.nlm.nih.gov/pubmed/15909265. [PubMed: 15909265]

65. Stevens DA, Rendon A, Gaona-Flores V, et al. Posaconazole therapy for chronic refractory coccidioidomycosis. Chest. 2007 Sep; 132(3):952-958. Available at http://www.ncbi.nlm.nih.gov/ entrez/query.fcgi? $\mathrm{cmd}=$ Retrieve $\& \mathrm{db}=$ PubMed\&dopt=Citation\&list_uids=17573510. [PubMed: 17573510]

66. Proia LA, Tenorio AR. Successful use of voriconazole for treatment of Coccidioides meningitis. Antimicrob Agents Chemother. 2004; 48(6):2341. Available at http://www.ncbi.nlm.nih.gov/ entrez/query.fcgi?cmd=Retrieve $\& \mathrm{db}=\mathrm{PubMed} \& d o p t=$ Citation\&list_uids=15155250. [PubMed: 15155250]

67. Prabhu RM, Bonnell M, Currier BL, Orenstein R. Successful treatment of disseminated nonmeningeal coccidioidomycosis with voriconazole. Clin Infect Dis. 2004 Oct 1; 39(7):e74-e77. Available at http://www.ncbi.nlm.nih.gov/pubmed/15472837. [PubMed: 15472837]

68. Freifeld A, Proia L, Andes D, et al. Voriconazole use for endemic fungal infections. Antimicrob Agents Chemother. 2009 Apr; 53(4):1648-1651. Available at http://www.ncbi.nlm.nih.gov/ pubmed/19139290. [PubMed: 19139290]

69. Hsue G, Napier JT, Prince RA, Chi J, Hospenthal DR. Treatment of meningeal coccidioidomycosis with caspofungin. J Antimicrob Chemother. 2004 Jul; 54(1):292-294. Available at http:// www.ncbi.nlm.nih.gov/pubmed/15190025. [PubMed: 15190025]

70. Antony S. Use of the echinocandins (caspofungin) in the treatment of disseminated coccidioidomycosis in a renal transplant recipient. Clin Infect Dis. 2004 Sep 15; 39(6):879-880. Available at http://www.ncbi.nlm.nih.gov/pubmed/15472833. [PubMed: 15472833]

71. Park DW, Sohn JW, Cheong HJ, et al. Combination therapy of disseminated coccidioidomycosis with caspofungin and fluconazole. BMC Infect Dis. 2006; 6:26. Available at http:// www.ncbi.nlm.nih.gov/pubmed/16480497. [PubMed: 16480497]

72. Vinh DC, Masannat F, Dzioba RB, Galgiani JN, Holland SM. Refractory disseminated coccidioidomycosis and mycobacteriosis in interferon-gamma receptor 1 deficiency. Clin Infect Dis. 2009 Sep 15; 49(6):e62-e65. Available at http://www.ncbi.nlm.nih.gov/pubmed/19681704. [PubMed: 19681704]

73. Kuberski TT, Servi RJ, Rubin PJ. Successful treatment of a critically ill patient with disseminated coccidioidomycosis, using adjunctive interferon-gamma. Clin Infect Dis. 2004; 38(6):910-912. Available at http://www.ncbi.nlm.nih.gov/entrez/query.fcgi? cmd=Retrieve \&db=PubMed\&dopt=Citation\&list_uids=14999639. [PubMed: 14999639] 
74. Dewsnup DH, Galgiani JN, Graybill JR, et al. Is it ever safe to stop azole therapy for Coccidioides immitis meningitis? Ann Intern Med. 1996; 124(3):305-310. Available at http://

www.ncbi.nlm.nih.gov/entrez/query.fcgi?

$\mathrm{cmd}=$ Retrieve $\& \mathrm{db}=$ PubMed\&dopt=Citation\&list_uids=8554225. [PubMed: 8554225]

75. Carmichael JK. Coccidioidomycosis in HIV-infected persons. Clin Infect Dis. 2006 Apr 1.42(7): 1059. author reply 1059-1060. Available at http://www.ncbi.nlm.nih.gov/pubmed/16511783. [PubMed: 16511783]

76. Mathew G, Smedema M, Wheat LJ, Goldman M. Relapse of coccidioidomycosis despite immune reconstitution after fluconazole secondary prophylaxis in a patient with AIDS. Mycoses. 2003 Feb; 46(1-2):42-44. Available at http://www.ncbi.nlm.nih.gov/pubmed/12588482. [PubMed: 12588482]

\section{Dosing Recommendations for Prevention and Treatment of Coccidioidomycosis}

\begin{tabular}{|c|c|c|c|}
\hline Indication & First Choice & Alternative & Comments/Special Issues \\
\hline Primary Prophylaxis & N/A & N/A & $\begin{array}{l}\text { Primary prophylaxis not } \\
\text { routinely indicated in } \\
\text { children. }\end{array}$ \\
\hline Secondary Prophylaxis & $\begin{array}{l}\text { Fluconazole } 6 \mathrm{mg} / \mathrm{kg} \text { body } \\
\text { weight (maximum } 400 \\
\mathrm{mg} \text { ) by mouth once daily }\end{array}$ & $\begin{array}{l}\text { Itraconazole } 2-5 \mathrm{mg} / \mathrm{kg} \\
\text { body weight (maximum } \\
200 \mathrm{mg} \text { ) by mouth per dose } \\
\text { twice daily }\end{array}$ & $\begin{array}{l}\text { Lifelong secondary } \\
\text { prophylaxis with fluconazole } \\
\text { for patients with meningitis } \\
\text { or disseminated disease in } \\
\text { the immunocompromised } \\
\text { patient is recommended. } \\
\text { Secondary prophylaxis } \\
\text { should be considered after } \\
\text { treatment of milder disease if } \\
\text { CD4 count remains }<250 \\
\text { cells } / \mathrm{mm}^{3} \text { or CD4 percentage } \\
<15 \% \text {. }\end{array}$ \\
\hline Treatment & $\begin{array}{l}\text { Severe Illness with } \\
\text { Respiratory Compromise } \\
\text { due to Diffuse Pulmonary } \\
\text { or Disseminated Non- } \\
\text { Meningitic Disease: } \\
\\
\text { B Amphotericin } \\
\text { B deoxycholate } \\
\text { 0.5-1.0 mg/kg } \\
\text { body weight } \\
\text { IV once daily, } \\
\text { until clinical } \\
\text { improvement. } \\
\text { A lipid } \\
\text { amphotericin } \\
\text { B preparation } \\
\text { can be } \\
\text { substituted at a } \\
\text { dose of 5 } \\
\text { mg/kg body } \\
\text { weight IV } \\
\text { once daily } \\
\text { (dosage of the } \\
\text { lipid } \\
\text { preparation } \\
\text { can be } \\
\text { increased to as } \\
\text { much as } 10 \\
\text { mg/kg body } \\
\text { weight IV } \\
\text { once daily for } \\
\text { life- } \\
\text { threatening } \\
\text { infection). } \\
\text { After the } \\
\text { patient is }\end{array}$ & \begin{tabular}{l} 
Severe Illness with \\
Respiratory Compromise \\
Due to Diffuse Pulmonary \\
or Disseminated Non- \\
Meningitic Disease (If \\
Unable to Use \\
Amphotericin): \\
\multicolumn{1}{c}{ Fluconazole } \\
\\
12mg/kg body \\
weight \\
(maximum 800 \\
mg) per dose \\
IV or by mouth \\
once daily \\
Treatment is \\
continued for \\
total of 1 year, \\
followed by \\
secondary \\
prophylaxis.
\end{tabular} & $\begin{array}{l}\text { Surgical debridement of } \\
\text { bone, joint, and/or excision } \\
\text { of cavitary lung lesions may } \\
\text { be helpful. } \\
\text { Itraconazole is the preferred } \\
\text { azole for treatment of bone } \\
\text { infections. } \\
\text { Some experts initiate an } \\
\text { azole during amphotericin B } \\
\text { therapy; others defer } \\
\text { initiation of the azole until } \\
\text { after amphotericin B is } \\
\text { stopped. } \\
\text { For treatment failure, can } \\
\text { consider voriconazole, } \\
\text { caspofungin, or posaconazole } \\
\text { (or combinations). However, } \\
\text { experience is limited and } \\
\text { definitive pediatric dosages } \\
\text { have not been determined. } \\
\text { Options should be discussed } \\
\text { with an expert in the } \\
\text { treatment of } \\
\text { coccidioidomycosis. } \\
\text { Chronic suppressive therapy } \\
\text { (secondary prophylaxis) with } \\
\text { fluconazole or itraconazole is } \\
\text { routinely recommended } \\
\text { following initial induction } \\
\text { therapy for disseminated } \\
\text { disease and is continued } \\
\text { lifelong for meningeal } \\
\text { disease. } \\
\text { Therapy with amphotericin } \\
\text { results In a more rapid } \\
\text { clinical response in severe, } \\
\text { non-meningeal disease. }\end{array}$ \\
\hline
\end{tabular}




\begin{tabular}{|c|c|c|c|}
\hline Indication & First Choice & Alternative & Comments/Special Issues \\
\hline \multirow[t]{3}{*}{ 更 } & $\begin{array}{l}\text { stabilized, } \\
\text { therapy with } \\
\text { an azole } \\
\text { (fluconazole } \\
\text { or } \\
\text { itraconazole) } \\
\text { can be } \\
\text { substituted and } \\
\text { continued to } \\
\text { complete a 1- } \\
\text { year course of } \\
\text { antifungal } \\
\text { therapy. }\end{array}$ & & \\
\hline & 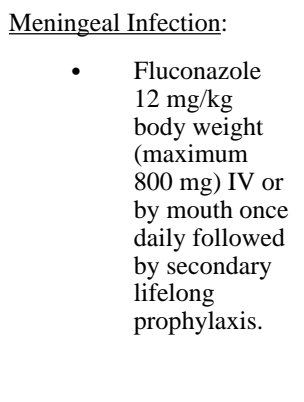 & \begin{tabular}{l}
$\frac{\text { Meningeal Infection }}{\text { (Unresponsive to }}$ \\
Fluconazole): \\
\multicolumn{1}{c}{ IV } \\
amphotericin B \\
plus intrathecal \\
amphotericin B \\
followed by \\
secondary \\
prophylaxis. \\
Note: Expert \\
consultation \\
recommended.
\end{tabular} & \\
\hline & 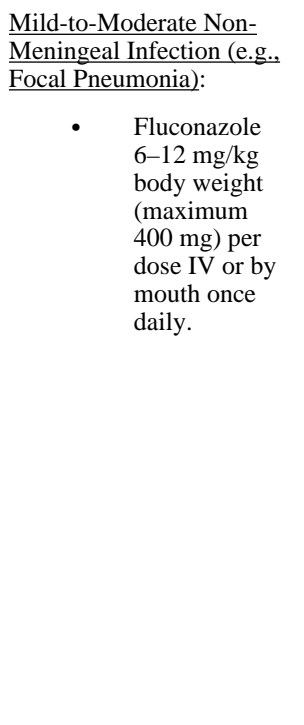 & 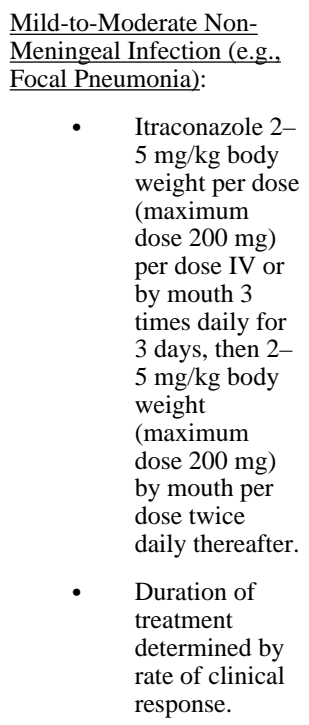 & \\
\hline
\end{tabular}

Key to Abbreviations: $\mathrm{CD} 4=\mathrm{CD} 4 \mathrm{~T}$ lymphocyte; $\mathrm{IV}=$ intravenous

\section{Cryptococcosis (Last updated November 6, 2013; last reviewed November}

\section{$6,2013)$}

Panel's Recommendations

- $\quad$ Routine use of antifungal medications is not recommended for primary prophylaxis of cryptococcal infections in children (BIII).

- Combination therapy with amphotericin B deoxycholate (or liposomal amphotericin B) and flucytosine for 2 weeks (induction therapy) followed by fluconazole for a minimum of 8 weeks (consolidation therapy) is 
Panel's Recommendations

recommended for central nervous system disease ( $\left.\mathbf{A I}^{*}\right)$. Amphotericin B lipid complex is another alternative to amphotericin B deoxycholate (BII*).

- Liposomal amphotericin B is preferred over amphotericin B deoxycholate for patients with or at risk of renal insufficiency (AI*); amphotericin B lipid complex is an alternative (BII*).

- In patients who cannot tolerate flucytosine or if flucytosine is unavailable, amphotericin B deoxycholate (or liposomal amphotericin B or amphotericin B lipid complex) with or without high-dose fluconazole can be used for initial therapy (BI*). Fluconazole plus flucytosine is superior to fluconazole alone and an option in patients who cannot tolerate any form of amphotericin (BII*).

- Echinocandins are not active against cryptococcal infections and should not be used (AIII).

- After a minimum of 2 weeks of induction therapy, if there is clinical improvement and a negative cerebrospinal fluid culture after repeat lumbar puncture, amphotericin B and flucytosine can be discontinued and consolidation therapy with fluconazole administered for a minimum of 8 weeks $\left(\mathbf{A I}^{*}\right)$; itraconazole is a less preferable alternative to fluconazole $\left(\mathbf{B I}^{*}\right)$.

- $\quad$ Secondary prophylaxis with fluconazole (AI*) or itraconazole (less preferable) $\left(\mathbf{B I}^{*}\right)$ is recommended for a minimum of 1 year.

- Discontinuing secondary prophylaxis (after receiving secondary prophylaxis for $\geq 1$ year) can be considered for asymptomatic children aged $\Varangle 6$ years with CD4 counts $\$ 100$ cells $/ \mathrm{mm}^{3}$ and an undetectable viral load on 33 months of combination antiretroviral therapy (CIII). Secondary prophylaxis should be reinitiated if the CD4 count decreases to $<100$ cells $/ \mathrm{mm}^{3}$ (AIII). Most experts would not discontinue secondary prophylaxis for patients younger than age 6 years (CIII).

- $\quad$ Patients with severe pulmonary disease or disseminated cryptococcosis should be treated with amphotericin $\mathrm{B}$ with or without the addition of flucytosine, as for CNS disease (AIII). Those with mild-to-moderate pulmonary illness or other localized disease can be managed with fluconazole monotherapy (AIII).

- In antiretroviral-naive patients newly diagnosed with cryptococcal meningitis or disseminated disease, delay in initiation of potent antiretroviral therapy may be prudent until the end of the first 2 weeks of induction therapy (CIII).

Rating of Recommendations: $\mathrm{A}=$ Strong; $\mathrm{B}=$ Moderate $\mathrm{C}=$ Optional

Rating of Evidence: I = One or more randomized trials $\underline{\text { in children }}^{\dagger}$ with clinical outcomes and/or validated endpoints; I $^{*}$ $=$ One or more randomized trials in adults with clinical outcomes and/or validated laboratory endpoints with accompanying data in children ${ }^{\dagger}$ from one or more well-designed, nonrandomized trials or observational cohort studies with long-term clinical outcomes; II = One or more well-designed, nonrandomized trials or observational cohort studies in children ${ }^{\dagger}$ with long-term outcomes; $\mathrm{II}^{*}=$ One or more well-designed, nonrandomized trials or observational studies in adults with longterm clinical outcomes with accompanying data in children ${ }^{\dagger}$ from one or more similar nonrandomized trials or cohort studies with clinical outcome data; III = Expert opinion ${ }^{\dagger}$

${ }^{\dagger}$ Studies that include children or children/adolescents, but not studies limited to post-pubertal adolescents

Given the low incidence of cryptococcosis in HIV-infected children, even during the era before combination antiretroviral therapy (cART), management of this disease in this age group has not been prospectively studied. Treatment recommendations largely reflect information extrapolated from many well-designed studies involving HIV-infected adults with cryptococcal meningitis. ${ }^{1}$

\section{Epidemiology}

Most cases of cryptococcosis in HIV-infected patients are caused by Cryptococcus neoformans; Cryptococcus gattii (formerly Cryptococcus neoformans variety gattii) infection occurs primarily in tropical and subtropical areas. Cryptococcal infections occur much less frequently in HIV-infected children than in adults. ${ }^{2-5}$ During the pre-cART era, most cases of cryptococcosis in HIV-infected children (overall incidence, 1\%) occurred in those aged 6 through 12 years and in those with CD4 T lymphocyte (CD4) cell counts indicating severe immunosuppression. ${ }^{4}$ Access to cART has further decreased the overall incidence of cryptococcal infection ${ }^{6,7}$ in HIV-infected children. Data from Pediatric AIDS 
Clinical Trials Group studies before and after the advent of cART indicate that the rate of invasive fungal infection, including cryptococcosis, has remained $<0.1$ per 100 childyears. ${ }^{8,9}$

\section{Clinical Manifestations}

Cryptococcosis often presents with subtle and non-specific findings, such as fever and headache. Early diagnosis requires consideration of this infection in symptomatic patients whose CD4 counts indicate severe immunosuppression. In both HIV-infected adults and children, meningoencephalitis is the most common initial manifestation of cryptococcosis. The disease typically evolves over days to weeks with fever and headache. Less frequent findings include nuchal rigidity, photophobia, and focal neurologic signs, as were seen among $30 \mathrm{HIV}$-infected children with cryptococcosis reported from the United States. ${ }^{4}$ In contrast to this indolent presentation, children in Zimbabwe presented with an acute form of neurologic cryptococcosis (69\% with nuchal rigidity, 38\% with seizure activity, and $23 \%$ with focal neurologic signs). ${ }^{10} \mathrm{C}$. gattii infections occur mostly in people who are not HIVinfected (or do not have other immunocompromsing conditions), and neurologic disease due to $C$. gattii in such apparently normal hosts responds more slowly to treatment and results in high risk of neurologic complications. ${ }^{11}$ C. gattii infections in HIV-infected patients, however, are uncommon and are similar in presentation to $C$. neoformans infections in HIVinfected hosts. ${ }^{12}$

Disseminated cryptococcosis can be associated with cutaneous lesions, including small, translucent, umbilicated papules (indistinguishable from molluscum contagiosum), nodules, ulcers, and infiltrated plaques resembling cellulitis. Pulmonary cryptococcosis without dissemination is unusual in children. Presenting findings include unexplained recurrent fever, cough with scant sputum, intrathoracic lymphadenopathy, and focal or diffuse pulmonary infiltrates. The infection also can be asymptomatic, with pulmonary nodules revealed on routine chest radiograph. ${ }^{3}$

\section{Diagnosis}

Detection of cryptococcal antigen in serum, cerebrospinal fluid (CSF) or other body fluids is highly effective for rapid and accurate diagnosis of cryptococcal infection.

A lumbar puncture should be done in any patient with suspected cryptococcal meningitis. CSF cell count, glucose, and protein can be virtually normal with central nervous system (CNS) cryptococcosis, but the opening pressure usually is elevated. Microscopic examination of CSF on India ink-stained wet mounts can be performed to diagnose suspected CNS disease but is largely replaced with the use of the cryptococcal antigen test. In more than $90 \%$ of patients with cryptococcal meningitis, cryptococcal antigen can be detected in CSF or serum by latex agglutination test (available from several manufacturers).

Fungal cultures from CSF, sputum, and blood can identify the organism. In some cases (meaning refractory or relapsed disease), susceptibility testing of the $C$. neoformans isolate can be beneficial. Overall, in vitro resistance to antifungal agents remains uncommon. ${ }^{13}$ 
Diffuse pulmonary disease can be diagnosed through bronchoalveolar lavage and direct examination of India ink-stained specimens, culture, and antigen detection. Focal pulmonary and skin lesions may require biopsy with culture and staining.

\section{Prevention Recommendations}

Preventing Exposure-No strategies have been proven to prevent exposure. $C$. neoformans infection is believed to be acquired through inhalation of aerosolized particles from the environment. Serologic studies of immunocompetent children in an urban setting indicate that most children have been infected by $C$. neoformans by the third year of life. ${ }^{14}$

Preventing the First Episode of Disease-Because the incidence of cryptococcal disease is so low in HIV-infected children, ${ }^{2-4,15}$ routine testing of asymptomatic children for serum cryptococcal antigen is not recommended (CIII).

A review of randomized controlled trials using antifungal interventions for the primary prevention of cryptococcal diseases indicates that fluconazole and itraconazole can reduce cryptococcal disease in adults who have advanced HIV disease and severe immunosuppression (CD4 count $<50$ cells $/ \mathrm{mm}^{3}$ ). ${ }^{16}$ However, neither of these interventions clearly affected mortality.

In addition, routine use of antifungal medications is not recommended for primary prophylaxis of cryptococcal infections in children because of the low incidence of cryptococcosis in HIV-infected children, lack of survival benefits in primary prevention studies of adults, ${ }^{16}$ possibility of drug interaction, potential resistance to antifungal drugs, and cost (BIII). Early diagnosis of HIV infection and treatment with cART (following current HIV treatment guidelines) to prevent or reverse immune suppression should further reduce risk of cryptococcal disease in HIV-infected children.

Discontinuing Primary Prophylaxis-Not applicable.

\section{Treatment Recommendations}

Treating Disease-Note: These recommendations are largely based on high-quality evidence from studies in adults.

CNS Disease: The most common and well-studied presentation of cryptococcal infection in HIV-infected patients is CNS disease. In light of studies in adults, ${ }^{17-19}$ combination therapy with amphotericin B deoxycholate (or liposomal amphotericin B) and flucytosine for 2 weeks (induction therapy) followed by fluconazole for a minimum of 8 weeks (consolidation therapy) is recommended for children (AI*). Amphotericin B lipid complex is an alternative to amphotericin B deoxycholate (BII*). ${ }^{20} \mathrm{CSF}$ was sterilized significantly more rapidly in adults with CNS cryptococcal disease who received initial therapy with amphotericin B deoxycholate $(0.7 \mathrm{mg} / \mathrm{kg} / \mathrm{day})$ and flucytosine $(100 \mathrm{mg} / \mathrm{kg} /$ day $)$ than in those who received amphotericin B deoxycholate alone, amphotericin B deoxycholate plus fluconazole, or triple-antifungal therapy. ${ }^{21,22}$ In one study of adults, liposomal amphotericin B (AmBisome ${ }^{\circledR}$ ) dosed at $4 \mathrm{mg} / \mathrm{kg} / \mathrm{day}$ resulted in significantly earlier CSF culture 
conversion than did amphotericin B deoxycholate at $0.7 \mathrm{mg} / \mathrm{kg} /$ day. ${ }^{23}$ However, a randomized, double-blind clinical trial before the routine availability of cART that compared amphotericin B $(0.7 \mathrm{mg} / \mathrm{kg} /$ day $)$, liposomal amphotericin B $(3 \mathrm{mg} / \mathrm{kg} / \mathrm{day})$, and liposomal amphotericin B $(6 \mathrm{mg} / \mathrm{kg} /$ day $)$ showed no difference in efficacy among the three arms, but significantly fewer adverse events with liposomal amphotericin B $(3 \mathrm{mg} / \mathrm{kg}$ body weight/day). ${ }^{24}$ Cost considerations aside (liposomal amphotericin is significantly more expensive than amphotericin B deoxycholate), based on the reported experience in adults, liposomal amphotericin B would be preferable to amphotericin B deoxycholate in patients with cryptococcal meningitis who have or are at risk of renal failure (AI*). Amphotericin B lipid complex is another option (BII*). ${ }^{20}$ Monitoring for and managing increased intracranial pressure (ICP) is crucial to optimal management of CNS cryptococcosis (see below).

In patients who cannot tolerate flucytosine (or if flucytosine is not available), amphotericin B deoxycholate (or its liposomal preparation) with or without fluconazole can be used for initial therapy (BI*). In a randomized Phase II trial in HIV-infected adolescents and adults, amphotericin B deoxycholate plus high-dose fluconazole ( $800 \mathrm{mg}$ daily) was found to be well tolerated and with a trend toward better outcome at days 42 and 70, compared with amphotericin B deoxycholate alone. ${ }^{25}$ Studies are needed to further validate the use of this combination. In another study 80 HIV-seropositive, antiretroviral (ARV)-naive adults presenting with cryptococcal meningitis were randomized to 4 treatment arms of 2-week duration: group 1, amphotericin B (0.7-1 mg/kg) and flucytosine ( $25 \mathrm{mg} / \mathrm{kg} 4$ times daily); group 2, amphotericin B $(0.7-1 \mathrm{mg} / \mathrm{kg}$ ) and fluconazole (800 mg daily); group 3, amphotericin B $(0.7-1 \mathrm{mg} / \mathrm{kg}$ ) and fluconazole (600 $\mathrm{mg}$ twice daily); and group 4, amphotericin B (0.7-1 mg/kg) and voriconazole (300 mg twice daily). The primary end point was the rate of clearance of infection from CSF or early fungicidal activity, as determined by results of serial, quantitative CSF cryptococcal cultures. There were no statistically significant differences in the rate of clearance of cryptococcal colony-forming units (CFU) in CSF samples among the 4 treatment groups. ${ }^{26}$ Fluconazole plus flucytosine is superior to fluconazole alone ${ }^{27,28}$ and provides an alternative to amphotericin $\mathrm{B}$ deoxycholate for acute therapy of invasive disease (BII*) that should be used only if amphotericin B-based therapy is not tolerated. Although fluconazole monotherapy was an effective alternative to amphotericin B in adults with AIDS-associated cryptococcal meningitis, ${ }^{29}$ concerns in this study about differences in early death, delayed CSF sterilization, and drug resistance 30,31 make fluconazole monotherapy less favorable for initial therapy of CNS disease. Because of rapidly developing resistance, flucytosine alone should never be used to treat cryptococcosis. Echinocandins are not active against cryptococcal infections and should not be used (AIII).

After a minimum of 2 weeks of induction therapy with evidence of clinical improvement and a negative CSF culture after repeat lumbar puncture, amphotericin B deoxycholate (or its liposomal preparation) and flucytosine can be discontinued and consolidation therapy for a minimum of 8 weeks initiated with fluconazole $\left(\mathbf{A I}^{*}\right) .{ }^{32}$ Itraconazole is a less preferable alternative to fluconazole for the consolidation phase of CNS therapy (BI*). Fluconazole is preferred because studies comparing the two agents demonstrate higher rates of CSF 
sterilization during consolidation therapy ${ }^{18}$ and less frequent relapse ${ }^{32}$ during maintenance therapy in fluconazole recipients. After completion of consolidation therapy, secondary prophylaxis (maintenance therapy or suppressive therapy) should be initiated (see below).

\section{Pulmonary and Extra Pulmonary Cryptococcosis (CNS Disease Ruled Out): No} controlled clinical studies describe the outcome of non-CNS cryptococcosis in HIV-infected patients. CNS disease should be ruled out in all patients, after which the choice of antifungal medication and length of initial therapy can be decided in light of the clinical severity of illness. Patients with severe pulmonary disease or disseminated cryptococcosis should be treated with a form of amphotericin B with or without the addition of flucytosine, as for CNS disease (AIII). Usually combination therapy should be provided until symptoms resolve. Those with mild-to-moderate pulmonary illness or other localized disease can be managed with fluconazole monotherapy (AIII). Regardless of the antifungal agent selected for initial therapy, secondary prophylaxis with fluconazole or itraconazole should be provided as for CNS disease (AIII) (see notes below on secondary prophylaxis).

\section{Monitoring and Adverse Events (Including IRIS)}

Monitoring for Raised Intracranial Pressure: At the time of diagnosis and on subsequent lumbar punctures, all patients with cryptococcal meningitis should have their lumbar opening pressure measured. Studies in adults clearly show the role of increased ICP in deaths associated with CNS cryptococcosis. ${ }^{18,33}$ Patients with severe headache, confusion, blurred vision, papilledema, or other neurologic signs or symptoms of increased ICP should be managed using measures to decrease ICP. One approach recommended for adults is to measure pressure continually or repeatedly during the lumbar puncture procedure and to remove CSF until the pressure is approximately half the opening pressure but still no lower than normal. ${ }^{34}$ This may be repeated as often as every day until symptoms and signs consistently improve. Similar data describing experience with therapeutic lumbar punctures in children with cryptococcal meningitis are not available. Not specific to cryptococcal meningitis, a cutoff opening pressure of $28 \mathrm{~cm}$ of water has been proposed in children, above which the pressure should be considered elevated. ${ }^{35} \mathrm{CSF}$ shunting through a lumbar drain or ventriculostomy can be considered for patients who continue to have symptomatic increased ICP despite multiple lumbar taps (BIII). Corticosteroids and mannitol have been shown to be ineffective in managing ICP in adults with cryptococcal meningitis and most experts would not recommend their use in children (CIII). Acetazolamide is hazardous as therapy for increased ICP management in adults without signs of immune reconstitution inflammatory syndrome (IRIS) and has not been evaluated in children with cryptococcal meningitis; acetazolamide is not recommended for adults and most experts would similarly not use it in children (BIII).

Monitoring Treatment Response: In addition to monitoring clinical response, mycological response in patients with CNS cryptococcosis typically is assessed by a repeat lumbar puncture and CSF examination at 2 weeks of treatment, with continuation of induction therapy until CSF culture is negative. 
Monitoring serial serum cryptococcal antigen titers is not useful for following treatment efficacy because changes in serum cryptococcal antigen titers do not correlate well with outcome during treatment for acute meningitis or during suppressive therapy. ${ }^{36,37}$ Serial measurement of CSF cryptococcal antigen is more useful; in one study, an unchanged or increased titer of antigen in CSF correlated with clinical and microbiologic treatment failure, and a rise in CSF antigen titer during suppressive therapy was associated with relapse of cryptococcal meningitis. ${ }^{36}$ However, monitoring of CSF cryptococcal antigen levels requires repeated lumbar punctures and is not routinely recommended for monitoring response.

Monitoring for Adverse Events: Adverse effects of amphotericin B (Table 5) are primarily nephrotoxicity; permanent nephrotoxicity is related to cumulative dose. Infusion-related fevers, chills, nausea, and vomiting can occur, but they are less frequent in children than in adults. Close monitoring for drug toxicities is needed especially when amphotericin B is used with flucytosine.

Flucytosine has the potential for marked toxicity, especially affecting the bone marrow (meaning anemia, leukopenia, and thrombocytopenia), liver, gastrointestinal (GI) tract, kidney, and skin. In patients receiving flucytosine, flucytosine blood levels should be monitored to prevent bone marrow suppression and GI toxicity; after 3-5 days of therapy, the target 2-hour post-dose serum level of flucytosine is $40-60 \mu \mathrm{g} / \mathrm{mL}$. Flucytosine should be avoided in children with severe renal impairment.

Fluconazole and the other azoles have relatively low rates of toxicity, but their potential drug interactions can limit their use. Because of their ability to inhibit the CYP450dependent hepatic enzymes, the potential for drug interactions, particularly with ARV drugs, should be carefully evaluated before initiation of therapy. Liver function tests should be monitored during treatment.

Immune Reconstitution Inflammatory Response Syndrome (IRIS): While cases of IRIS in HIV-infected children have been described, ${ }^{38}$ most of the available information comes from adult literature.

IRIS related to cryptococcosis can present within weeks (such as meningitis) or months (such as lymphadenitis) after start of cART. Symptoms of meningitis are similar to those described for meningitis presenting as the initial manifestation of cryptococcosis. In one study, about $30 \%$ of all HIV-infected adults hospitalized for infection with $C$. neoformans who received cART were re-admitted with symptoms attributed to an inflammatory response. ${ }^{39}$ Of the 18 patients with C. neoformans-related IRIS in the cited study, 17 had culture-negative meningitis, and most cases occurred during the first 30 days after initiation of cART. The most common presentation of late cryptococcal IRIS is lymphadenitis, particularly mediastinal lymphadenitis..$^{40,41}$

IRIS is a clinical diagnosis. While there are no specific laboratory tests to diagnose IRIS, presence of negative cultures in a patient with clinical signs suggestive of tissue inflammation in the face of rapidly improving cellular immunity would be suggestive of 
IRIS over treatment failure. The optimal management of cryptococcal IRIS has not been defined. Antifungal therapy should be initiated in patients not already receiving it, raised intracranial pressure managed if present and antiretroviral therapy (ART) should be continued. Although many cases resolve spontaneously, some experts also have used antiinflammatory therapy (e.g., short-course corticosteroids) in patients with severely symptomatic IRIS (CIII). ${ }^{40,42}$

Adult HIV-infected treatment-naive patients with cryptococcal meningitis who go on to develop IRIS after starting cART are more likely to have higher HIV RNA levels at baseline $^{43}$ and exhibit less initial CSF inflammation at the time of cryptococcal meningitis diagnosis, compared with those who do not develop IRIS. ${ }^{44}$ In patients with advanced immunosuppression and non-tuberculous opportunistic infections (OIs), the presence of a fungal infection, lower CD4 counts and higher HIV RNA levels at baseline, and higher CD4 counts and lower HIV RNA levels on treatment were found associated with IRIS. ${ }^{43}$ For patients not on cART at the time of diagnosis of cryptococcal meningitis, the timing of cART in relation to antifungal treatment remains controversial. One randomized trial of adult HIV-infected patients with OIs (excluding tuberculosis) primarily from the United States that included 35 patients with cryptococcal meningitis suggested that early cART treatment (within the first 14 days of diagnosis) was safe and resulted in less AIDS progression/death compared to deferred cART. ${ }^{45}$ However a randomized clinical trial in Zimbabwe was reported to show higher mortality in patients receiving cART starting within 72 hours of diagnosis compared to those waiting at least 10 weeks to initiate ART. ${ }^{46}$ Patients in this study were treated with high dose fluconazole. Differences in management of cryptococcal meningitis, raised ICP, and cART treatment options may account for some of the differences between these two studies. In ARV-naive patients newly diagnosed with cryptococcal meningitis or disseminated disease, delay in potent ART may be prudent until the end of the first 2 weeks of induction therapy (CIII); further delays in initiating cART, especially in resource-poor settings, should be individualized.

Managing Treatment Failure-Treatment failure is defined as worsening or lack of improvement in signs and symptoms after 2 weeks of appropriate therapy, including management of ICP; or relapse after an initial clinical response. Differentiating IRIS from treatment failure is important because treatment approaches and outcomes differ; persistent positive cultures indicate treatment failure. Optimal management of patients with treatment failure is unknown. If cultures remain positive, evaluation of antifungal susceptibilities can be considered, although C. neoformans resistance to fluconazole is rare in the United States. Patients in whom initial azole-based therapy fails should be switched to amphotericin Bbased therapy, ${ }^{30}$ ideally in combination with flucytosine; the possibility of drug interactions resulting in sub-therapeutic azole levels (meaning concurrent rifampin use or other drugs metabolized by the liver) should be explored. ${ }^{30}$ Use of liposomal amphotericin B should be considered, because one study suggests improved efficacy in CSF sterilization with liposomal preparations than with standard amphotericin B. ${ }^{23}$ Some data from HIV-infected adults indicate higher dosages (meaning 400-800 mg/day) of fluconazole in combination with flucytosine also can be considered for salvage therapy. ${ }^{19,47}$ Clinical experience with new antifungal agents in managing cryptococcosis is limited. A few patients with 
cryptococcal infections refractory or intolerant to standard antifungal therapy have been treated with posaconazole or voriconazole with variable success. ${ }^{48,49}$

Preventing Recurrence (Secondary Prophylaxis)—Patients who have completed initial therapy for cryptococcosis should receive secondary prophylaxis (maintenance therapy or suppressive therapy) (AI*). Fluconazole (AI*) is superior and preferable to itraconazole (BI*) for preventing relapse of cryptococcal disease. ${ }^{32,50,51}$

Discontinuing Secondary Prophylaxis (Maintenance or Suppressive Therapy)

-Until recently, lifelong secondary prophylaxis typically was recommended. The safety of discontinuing secondary prophylaxis for cryptococcosis after immune reconstitution with cART has not been studied in children, and decisions in that regard should be made on a case-by-case basis. Adults who have successfully completed a course of initial therapy (including $\geq 12$ months of secondary prophylaxis), remain asymptomatic with regard to signs and symptoms of cryptococcosis, and have a sustained ( $\ 6$ months) increase in their CD4 counts to $\geq 100$ cells $/ \mathrm{mm}^{3}$ with an undetectable viral load on ART for $>3$ months after cART are at apparent low risk of recurrence of cryptococcosis. ${ }^{52-54}$ In light of these observations and inference from data regarding discontinuing secondary prophylaxis for other OIs in adults with advanced HIV infection, discontinuing secondary prophylaxis for cryptococcosis (after receiving secondary prophylaxis for at least 1 year) can be considered for asymptomatic children aged $\geq 6$ years, with increase in their CD4 counts to $\geq 100$ cells $/ \mathrm{mm}^{3}$ and an undetectable viral load on cART for $\geq 3$ months (CIII). Secondary prophylaxis should be re-initiated if the CD4 count decreases to $<100$ cells $/ \mathrm{mm}^{3}$ (AIII). Most experts would not discontinue secondary prophylaxis for patients younger than age 6 years (CIII).

\section{References}

1. Perfect JR, Dismukes WE, Dromer F, et al. Clinical practice guidelines for the management of cryptococcal disease: 2010 update by the infectious diseases society of america. Clin Infect Dis. 2010 Feb 1; 50(3):291-322. Available at http://www.ncbi.nlm.nih.gov/pubmed/20047480. [PubMed: 20047480]

2. Leggiadro RJ, Kline MW, Hughes WT. Extrapulmonary cryptococcosis in children with acquired immunodeficiency syndrome. Pediatr Infect Dis J. 1991 Sep; 10(9):658-662. Available at http:// www.ncbi.nlm.nih.gov/pubmed/1923678. [PubMed: 1923678]

3. Gonzalez CE, Shetty D, Lewis LL, Mueller BU, Pizzo PA, Walsh TJ. Cryptococcosis in human immunodeficiency virus-infected children. Pediatr Infect Dis J. 1996 Sep; 15(9):796-800. Available at http://www.ncbi.nlm.nih.gov/pubmed/8878224. [PubMed: 8878224]

4. Abadi J, Nachman S, Kressel AB, Pirofski L. Cryptococcosis in children with AIDS. Clin Infect Dis. 1999 Feb; 28(2):309-313. Available at http://www.ncbi.nlm.nih.gov/pubmed/10064249. [PubMed: 10064249]

5. Likasitwattanakul S, Poneprasert B, Sirisanthana V. Cryptococcosis in HIV-infected children. Southeast Asian J Trop Med Public Health. 2004 Dec; 35(4):935-939. Available at http:// www.ncbi.nlm.nih.gov/pubmed/15916094. [PubMed: 15916094]

6. Mirza SA, Phelan M, Rimland D, et al. The changing epidemiology of cryptococcosis: an update from population-based active surveillance in 2 large metropolitan areas, 1992-2000. Clin Infect Dis. 2003 Mar 15; 36(6):789-794. Available at http://www.ncbi.nlm.nih.gov/pubmed/12627365. [PubMed: 12627365]

7. Dromer F, Mathoulin-Pelissier S, Fontanet A, et al. Epidemiology of HIV-associated cryptococcosis in France, (1985-2001): comparison of the pre- and post-HAART eras. AIDS. 2004 Feb 20; 18(3): 555-562. Available at http://www.ncbi.nlm.nih.gov/pubmed/15090810. [PubMed: 15090810] 
8. Dankner WM, Lindsey JC, Levin MJ. Pediatric ACTGPT. Correlates of opportunistic infections in children infected with the human immunodeficiency virus managed before highly active antiretroviral therapy. Pediatr Infect Dis J. 2001 Jan; 20(1):40-48. Available at http:// www.ncbi.nlm.nih.gov/pubmed/11176565. [PubMed: 11176565]

9. Gona P, Van Dyke RB, Williams PL, et al. Incidence of opportunistic and other infections in HIVinfected children in the HAART era. JAMA. 2006 Jul 19; 296(3):292-300. Available at http:// www.ncbi.nlm.nih.gov/pubmed/16849662. [PubMed: 16849662]

10. Gumbo T, Kadzirange G, Mielke J, Gangaidzo IT, Hakim JG. Cryptococcus neoformans meningoencephalitis in African children with acquired immunodeficiency syndrome. Pediatr Infect Dis J. 2002 Jan; 21(1):54-56. Available at http://www.ncbi.nlm.nih.gov/pubmed/11791100. [PubMed: 11791100]

11. Speed B, Dunt D. Clinical and host differences between infections with the two varieties of Cryptococcus neoformans. Clin Infect Dis. 1995 Jul; 21(1):28-34. discussion 35-26. Available at http://www.ncbi.nlm.nih.gov/pubmed/7578756. [PubMed: 7578756]

12. Morgan J, McCarthy KM, Gould S, et al. Cryptococcus gattii infection: characteristics and epidemiology of cases identified in a South African province with high HIV seroprevalence, 2002-2004. Clin Infect Dis. 2006 Oct 15; 43(8):1077-1080. Available at http:// www.ncbi.nlm.nih.gov/pubmed/16983624. [PubMed: 16983624]

13. Pfaller MA, Messer SA, Boyken L, et al. Global trends in the antifungal susceptibility of Cryptococcus neoformans (1990 to 2004). J Clin Microbiol. 2005 May; 43(5):2163-2167. Available at http://www.ncbi.nlm.nih.gov/pubmed/15872236. [PubMed: 15872236]

14. Goldman DL, Khine H, Abadi J, et al. Serologic evidence for Cryptococcus neoformans infection in early childhood. Pediatrics. 2001 May.107(5):E66. Available at http://www.ncbi.nlm.nih.gov/ pubmed/11331716. [PubMed: 11331716]

15. Nesheim SR, Kapogiannis BG, Soe MM, et al. Trends in opportunistic infections in the pre- and post-highly active antiretroviral therapy eras among HIV-infected children in the Perinatal AIDS Collaborative Transmission Study, 1986-2004. Pediatrics. 2007 Jul; 120(1):100-109. Available at http://www.ncbi.nlm.nih.gov/pubmed/17606567. [PubMed: 17606567]

16. Chang LW, Phipps WT, Kennedy GE, Rutherford GW. Antifungal interventions for the primary prevention of cryptococcal disease in adults with HIV. Cochrane Database Syst Rev. 2005(3):CD004773. Available at http://www.ncbi.nlm.nih.gov/pubmed/16034947. [PubMed: 16034947]

17. Bennett JE, Dismukes WE, Duma RJ, et al. A comparison of amphotericin B alone and combined with flucytosine in the treatment of cryptoccal meningitis. N Engl J Med. 1979 Jul 19; 301(3): 126-131. Available at http://www.ncbi.nlm.nih.gov/pubmed/449951. [PubMed: 449951]

18. van der Horst CM, Saag MS, Cloud GA, et al. Treatment of cryptococcal meningitis associated with the acquired immunodeficiency syndrome. National Institute of Allergy and Infectious Diseases Mycoses Study Group and AIDS Clinical Trials Group. N Engl J Med. 1997 Jul 3; 337(1):15-21. Available at http://www.ncbi.nlm.nih.gov/pubmed/9203426. [PubMed: 9203426]

19. Saag MS, Graybill RJ, Larsen RA, et al. Practice guidelines for the management of cryptococcal disease. Infectious Diseases Society of America. Clin Infect Dis. 2000 Apr; 30(4):710-718. Available at http://www.ncbi.nlm.nih.gov/pubmed/10770733. [PubMed: 10770733]

20. Baddour LM, Perfect JR, Ostrosky-Zeichner L. Successful use of amphotericin B lipid complex in the treatment of cryptococcosis. Clin Infect Dis. 2005; 40(Suppl 6):S409-S413. Available at http:// www.ncbi.nlm.nih.gov/entrez/query.fcgi? $\mathrm{cmd}=$ Retrieve $\& \mathrm{db}=$ PubMed\&dopt=Citation\&list_uids=15809927. [PubMed: 15809927]

21. Brouwer AE, Rajanuwong A, Chierakul W, et al. Combination antifungal therapies for HIVassociated cryptococcal meningitis: a randomised trial. Lancet. 2004 May 29; 363(9423):17641767. Available at http://www.ncbi.nlm.nih.gov/pubmed/15172774. [PubMed: 15172774]

22. Dromer F, Mathoulin-Pelissier S, Launay O, Lortholary O. French Cryptococcosis Study G. Determinants of disease presentation and outcome during cryptococcosis: the CryptoA/D study. PLoS Med. 2007 Feb.4(2):e21. Available at http://www.ncbi.nlm.nih.gov/pubmed/17284154. [PubMed: 17284154]

23. Leenders AC, Reiss P, Portegies P, et al. Liposomal amphotericin B (AmBisome) compared with amphotericin $\mathrm{B}$ both followed by oral fluconazole in the treatment of AIDS-associated 
cryptococcal meningitis. AIDS. 1997 Oct; 11(12):1463-1471. Available at http:// www.ncbi.nlm.nih.gov/pubmed/9342068. [PubMed: 9342068]

24. Hamill RJ, Sobel JD, El-Sadr W, et al. Comparison of 2 doses of liposomal amphotericin B and conventional amphotericin B deoxycholate for treatment of AIDS-associated acute cryptococcal meningitis: a randomized, doubleblind clinical trial of efficacy and safety. Clin Infect Dis. 2010 Jul 15; 51(2):225-232. Available at http://www.ncbi.nlm.nih.gov/pubmed/20536366. [PubMed: 20536366]

25. Pappas PG, Chetchotisakd P, Larsen RA, et al. A phase II randomized trial of amphotericin B alone or combined with fluconazole in the treatment of HIV-associated cryptococcal meningitis. Clin Infect Dis. 2009 Jun 15; 48(12):1775-1783. Available at http://www.ncbi.nlm.nih.gov/ pubmed/19441980. [PubMed: 19441980]

26. Loyse A, Wilson D, Meintjes G, et al. Comparison of the early fungicidal activity of high-dose fluconazole, voriconazole, and flucytosine as second-line drugs given in combination with amphotericin B for the treatment of HIV-associated cryptococcal meningitis. Clin Infect Dis. 2012 Jan 1; 54(1):121-128. Available at http://www.ncbi.nlm.nih.gov/pubmed/22052885. [PubMed: 22052885]

27. Larsen RA, Bozzette SA, Jones BE, et al. Fluconazole combined with flucytosine for treatment of cryptococcal meningitis in patients with AIDS. Clin Infect Dis. 1994 Oct; 19(4):741-745. Available at http://www.ncbi.nlm.nih.gov/pubmed/7803641. [PubMed: 7803641]

28. Mayanja-Kizza H, Oishi K, Mitarai S, et al. Combination therapy with fluconazole and flucytosine for cryptococcal meningitis in Ugandan patients with AIDS. Clin Infect Dis. 1998 Jun; 26(6): 1362-1366. Available at http://www.ncbi.nlm.nih.gov/pubmed/9636863. [PubMed: 9636863]

29. Saag MS, Powderly WG, Cloud GA, et al. Comparison of amphotericin B with fluconazole in the treatment of acute AIDS-associated cryptococcal meningitis. The NIAID Mycoses Study Group and the AIDS Clinical Trials Group. N Engl J Med. 1992 Jan 9; 326(2):83-89. Available at http:// www.ncbi.nlm.nih.gov/pubmed/1727236. [PubMed: 1727236]

30. Bicanic T, Harrison T, Niepieklo A, Dyakopu N, Meintjes G. Symptomatic relapse of HIVassociated cryptococcal meningitis after initial fluconazole monotherapy: the role of fluconazole resistance and immune reconstitution. Clin Infect Dis. 2006 Oct 15; 43(8):1069-1073. Available at http://www.ncbi.nlm.nih.gov/pubmed/16983622. [PubMed: 16983622]

31. Bicanic T, Meintjes G, Wood R, et al. Fungal burden, early fungicidal activity, and outcome in cryptococcal meningitis in antiretroviral-naive or antiretroviral-experienced patients treated with amphotericin B or fluconazole. Clin Infect Dis. 2007 Jul 1; 45(1):76-80. Available at http:// www.ncbi.nlm.nih.gov/pubmed/17554704. [PubMed: 17554704]

32. Saag MS, Cloud GA, Graybill JR, et al. A comparison of itraconazole versus fluconazole as maintenance therapy for AIDS-associated cryptococcal meningitis. National Institute of Allergy and Infectious Diseases Mycoses Study Group. Clin Infect Dis. 1999 Feb; 28(2):291-296. Available at http://www.ncbi.nlm.nih.gov/pubmed/10064246. [PubMed: 10064246]

33. Graybill JR, Sobel J, Saag M, et al. Diagnosis and management of increased intracranial pressure in patients with AIDS and cryptococcal meningitis. The NIAID Mycoses Study Group and AIDS Cooperative Treatment Groups. Clin Infect Dis. 2000 Jan; 30(1):47-54. Available at http:// www.ncbi.nlm.nih.gov/pubmed/10619732. [PubMed: 10619732]

34. Fessler RD, Sobel J, Guyot L, et al. Management of elevated intracranial pressure in patients with Cryptococcal meningitis. J Acquir Immune Defic Syndr Hum Retrovirol. 1998 Feb 1; 17(2):137142. Available at http://www.ncbi.nlm.nih.gov/pubmed/9473014. [PubMed: 9473014]

35. Avery RA, Shah SS, Licht DJ, et al. Reference range for cerebrospinal fluid opening pressure in children. N Engl J Med. 2010 Aug 26; 363(9):891-893. Available at http://www.ncbi.nlm.nih.gov/ pubmed/20818852. [PubMed: 20818852]

36. Powderly WG, Cloud GA, Dismukes WE, Saag MS. Measurement of cryptococcal antigen in serum and cerebrospinal fluid: value in the management of AIDS-associated cryptococcal meningitis. Clin Infect Dis. 1994 May; 18(5):789-792. Available at http://www.ncbi.nlm.nih.gov/ pubmed/8075272. [PubMed: 8075272]

37. Aberg JA, Watson J, Segal M, Chang LW. Clinical utility of monitoring serum cryptococcal antigen (sCRAG) titers in patients with AIDS-related cryptococcal disease. HIV Clin Trials. 2000 
Jul-Aug;1(1):1-6. Available at http://www.ncbi.nlm.nih.gov/pubmed/11590483. [PubMed: $11590483]$

38. Puthanakit T, Oberdorfer P, Akarathum N, Wannarit P, Sirisanthana T, Sirisanthana V. Immune reconstitution syndrome after highly active antiretroviral therapy in human immunodeficiency virus-infected thai children. Pediatr Infect Dis J. 2006 Jan; 25(1):53-58. Available at http:// www.ncbi.nlm.nih.gov/pubmed/16395104. [PubMed: 16395104]

39. Shelburne SA, Darcourt J, White AC, et al. The role of immune reconstitution inflammatory syndrome in AIDS-related Cryptococcus neoformans disease in the era of highly active antiretroviral therapy. Clin Infect Dis. 2005; 40(7):1049-1052. Available at http:// www.ncbi.nlm.nih.gov/entrez/query.fcgi? $\mathrm{cmd}=$ Retrieve $\& \mathrm{db}=$ PubMed\&dopt=Citation\&list_uids=15825000. [PubMed: 15825000$]$

40. Skiest DJ, Hester LJ, Hardy RD. Cryptococcal immune reconstitution inflammatory syndrome: report of four cases in three patients and review of the literature. J Infect. 2005 Dec; 51(5):e289_ e297. Available at http://www.ncbi.nlm.nih.gov/pubmed/16321643. [PubMed: 16321643]

41. Natukunda E, Musiime V, Ssali F, Kizito H, Kityo C, Mugyenyi P. A Case of Cryptococcal Lymphadenitis in an HIV-Infected Child. AIDS Res Hum Retroviruses. 2011 Apr; 27(4):373-376. Available at http://www.ncbi.nlm.nih.gov/pubmed/21087142. [PubMed: 21087142]

42. Lesho E. Evidence base for using corticosteroids to treat HIV-associated immune reconstitution syndrome. Expert Rev Anti Infect Ther. 2006 Jun; 4(3):469-478. Available at http:// www.ncbi.nlm.nih.gov/pubmed/16771623. [PubMed: 16771623]

43. Grant PM, Komarow L, Andersen J, et al. Risk factor analyses for immune reconstitution inflammatory syndrome in a randomized study of early vs. deferred ART during an opportunistic infection. PLoS One. 2010; 5(7):e11416. Available at http://www.ncbi.nlm.nih.gov/pubmed/ 20617176. [PubMed: 20617176]

44. Boulware DR, Bonham SC, Meya DB, et al. Paucity of initial cerebrospinal fluid inflammation in cryptococcal meningitis is associated with subsequent immune reconstitution inflammatory syndrome. J Infect Dis. 2010 Sep 15; 202(6):962-970. Available at http://www.ncbi.nlm.nih.gov/ pubmed/20677939. [PubMed: 20677939]

45. Zolopa A, Andersen J, Powderly W, et al. Early antiretroviral therapy reduces AIDS progression/ death in individuals with acute opportunistic infections: a multicenter randomized strategy trial. PLoS One. 2009; 4(5):e5575. Available at http://www.ncbi.nlm.nih.gov/pubmed/19440326. [PubMed: 19440326]

46. Makadzange AT, Ndhlovu CE, Takarinda K, et al. Early versus delayed initiation of antiretroviral therapy for concurrent HIV infection and cryptococcal meningitis in sub-saharan Africa. Clin Infect Dis. 2010 Jun 1; 50(11):1532-1538. Available at http://www.ncbi.nlm.nih.gov/pubmed/ 20415574. [PubMed: 20415574]

47. Lortholary O. Management of cryptococcal meningitis in AIDS: the need for specific studies in developing countries. Clin Infect Dis. 2007 Jul 1; 45(1):81-83. Available at http:// www.ncbi.nlm.nih.gov/pubmed/17554705. [PubMed: 17554705]

48. Perfect JR, Marr KA, Walsh TJ, et al. Voriconazole treatment for less-common, emerging, or refractory fungal infections. Clin Infect Dis. 2003 May 1; 36(9):1122-1131. Available at http:// www.ncbi.nlm.nih.gov/pubmed/12715306. [PubMed: 12715306]

49. Pitisuttithum P, Negroni R, Graybill JR, et al. Activity of posaconazole in the treatment of central nervous system fungal infections. J Antimicrob Chemother. 2005 Oct; 56(4):745-755. Available at http://www.ncbi.nlm.nih.gov/pubmed/16135526. [PubMed: 16135526]

50. Bozzette SA, Larsen RA, Chiu J, et al. A placebo-controlled trial of maintenance therapy with fluconazole after treatment of cryptococcal meningitis in the acquired immunodeficiency syndrome. California Collaborative Treatment Group. N Engl J Med. 1991 Feb 28; 324(9):580584. Available at http://www.ncbi.nlm.nih.gov/pubmed/1992319. [PubMed: 1992319]

51. Powderly WG, Saag MS, Cloud GA, et al. A controlled trial of fluconazole or amphotericin B to prevent relapse of cryptococcal meningitis in patients with the acquired immunodeficiency syndrome. The NIAID AIDS Clinical Trials Group and Mycoses Study Group. N Engl J Med. 1992 Mar 19; 326(12):793-798. Available at http://www.ncbi.nlm.nih.gov/pubmed/1538722. [PubMed: 1538722] 
52. Kirk O, Reiss P, Uberti-Foppa C, et al. Safe interruption of maintenance therapy against previous infection with four common HIV-associated opportunistic pathogens during potent antiretroviral therapy. Ann Intern Med. 2002 Aug 20; 137(4):239-250. Available at http:// www.ncbi.nlm.nih.gov/pubmed/12186514. [PubMed: 12186514]

53. Vibhagool A, Sungkanuparph S, Mootsikapun P, et al. Discontinuation of secondary prophylaxis for cryptococcal meningitis in human immunodeficiency virus-infected patients treated with highly active antiretroviral therapy: a prospective, multicenter, randomized study. Clin Infect Dis. 2003 May 15; 36(10):1329-1331. Available at http://www.ncbi.nlm.nih.gov/pubmed/12746781. [PubMed: 12746781]

54. Mussini C, Pezzotti P, Miro JM, et al. Discontinuation of maintenance therapy for cryptococcal meningitis in patients with AIDS treated with highly active antiretroviral therapy: an international observational study. Clin Infect Dis. 2004 Feb 15; 38(4):565-571. Available at http:// www.ncbi.nlm.nih.gov/pubmed/14765351. [PubMed: 14765351]

\section{Dosing Recommendations for Prevention and Treatment of Cryptococcosis}

\begin{tabular}{|c|c|c|c|}
\hline Indication & First Choice & Alternative & Comments/Special Issues \\
\hline Primary Prophylaxis & Not recommended & Not recommended & N/A \\
\hline Secondary Prophylaxis ${ }^{a}$ & $\begin{array}{l}\text { Fluconazole } 6 \mathrm{mg} / \mathrm{kg} \text { body } \\
\text { weight (maximum } 200 \\
\mathrm{mg} \text { ) by mouth once daily }\end{array}$ & $\begin{array}{l}\text { Itraconazole oral solution } \\
5 \mathrm{mg} / \mathrm{kg} \text { body weight } \\
\text { (maximum } 200 \mathrm{mg} \text { ) by } \\
\text { mouth once daily }\end{array}$ & 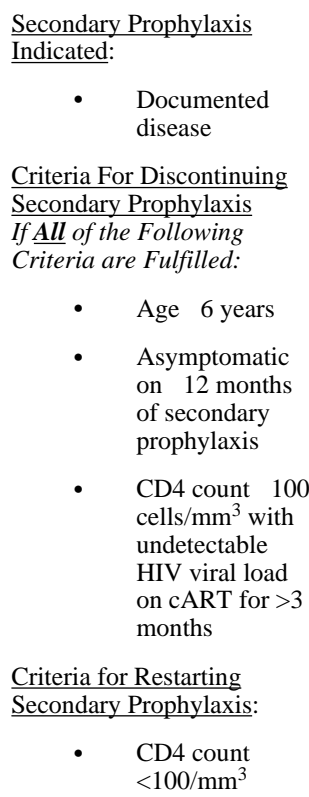 \\
\hline Treatment & 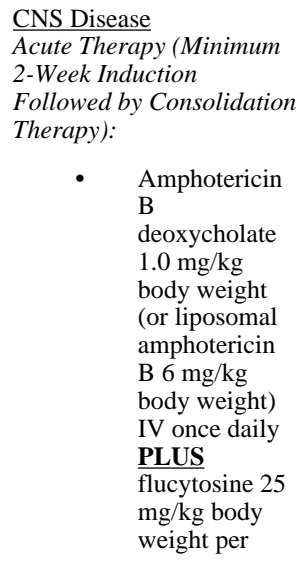 & $\begin{array}{l}\text { CNS Disease } \\
\text { Acute Therapy (Minimum } \\
\text { 2-Week Induction } \\
\text { Followed by Consolidation } \\
\text { Therapy) } \\
\text { If Flucytosine Not } \\
\text { Tolerated or Unavailable: } \\
\qquad \begin{array}{l}\text { A. Liposomal } \\
\text { amphotericin } \\
\text { B, } 6 \mathrm{mg} / \mathrm{kg} \\
\text { body weight } \\
\text { IV once daily, } \\
\text { or } \\
\text { Amphotericin } \\
\text { B Lipid } \\
\text { Complex, } 5 \\
\text { mg/kg body } \\
\text { weight IV } \\
\text { once daily, or }\end{array}\end{array}$ & $\begin{array}{l}\text { In patients with meningitis, } \\
\text { CSF culture should be } \\
\text { negative prior to initiating } \\
\text { consolidation therapy. } \\
\text { Overall, in vitro resistance } \\
\text { to antifungal agents used to } \\
\text { treat cryptococcosis remains } \\
\text { uncommon. Newer azoles } \\
\text { (voriconazole, } \\
\text { posaconazole, } \\
\text { ravuconazole) are all very } \\
\text { active in vitro against } C \text {. } \\
\text { neoformans, but published } \\
\text { clinical experience on their } \\
\text { use for cryptococcosis is } \\
\text { limited. } \\
\text { Liposomal amphotericin } \\
\text { and amphotericin B lipid } \\
\text { complex are especially } \\
\text { useful for children with }\end{array}$ \\
\hline
\end{tabular}




\begin{tabular}{|c|c|c|c|}
\hline Indication & First Choice & Alternative & Comments/Special Issues \\
\hline & 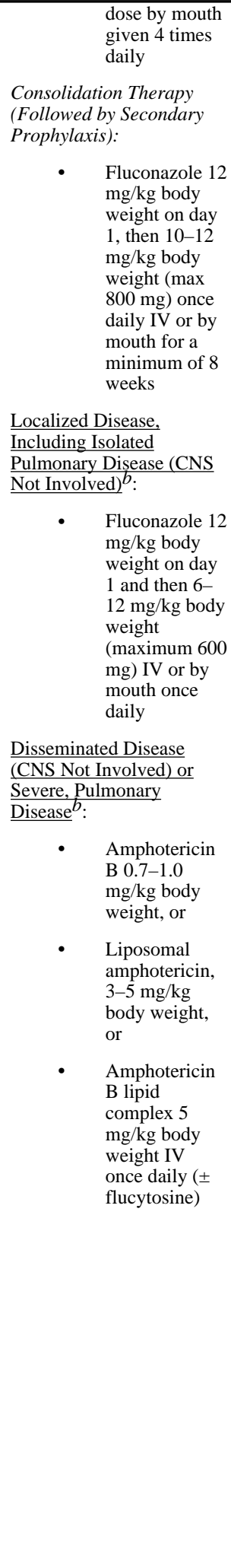 & 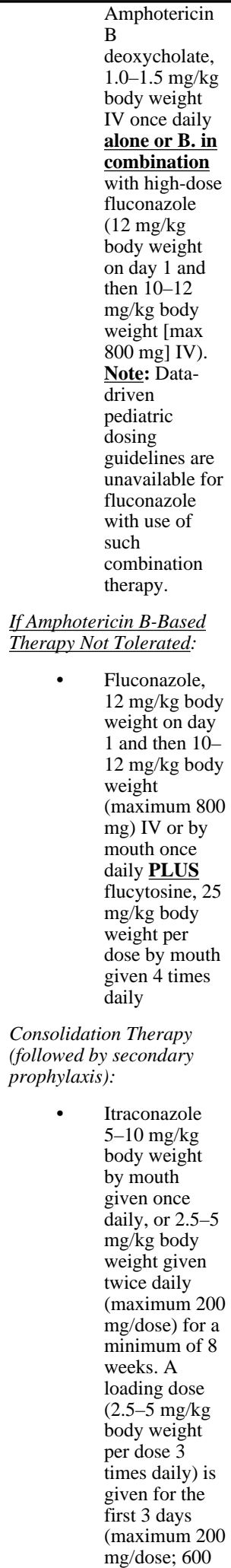 & $\begin{array}{l}\text { renal insufficiency or } \\
\text { infusion-related toxicity to } \\
\text { amphotericin B } \\
\text { deoxycholate. } \\
\text { Liposomal amphotericin } \\
\text { and amphotericin B lipid } \\
\text { complex are significantly } \\
\text { more expensive than } \\
\text { amphotericin B } \\
\text { deoxycholate. } \\
\text { Liquid preparation of } \\
\text { itraconazole (if tolerated) is } \\
\text { preferable to tablet } \\
\text { formulation because of } \\
\text { better bioavailability, but it } \\
\text { is more expensive. } \\
\text { Bioavailability of the } \\
\text { solution is better than the } \\
\text { capsule, but there were no } \\
\text { upfront differences in } \\
\text { dosing range based on } \\
\text { preparation used. Ultimate } \\
\text { dosing adjustments should } \\
\text { be guided by itraconazole } \\
\text { levels. } \\
\text { Serum itraconazole } \\
\text { concentrations should be } \\
\text { monitored to optimize drug } \\
\text { dosing. } \\
\text { Amphotericin B may } \\
\text { increase toxicity of } \\
\text { flucytosine by increasing } \\
\text { cellular uptake, or impair its } \\
\text { renal excretion, or both. } \\
\text { Flucytosine dose should be } \\
\text { adjusted to keep 2-hour } \\
\text { post-dose drug levels at } 40- \\
60 \text { ug/mL } \\
\text { Oral acetazolamide should } \\
\text { not be used for reduction of } \\
\text { ICP in cryptococcal } \\
\text { meningitis. } \\
\text { Corticosteroids and } \\
\text { mannitol have been shown } \\
\text { to be ineffective in } \\
\text { managing ICP in adults with } \\
\text { cryptococcal meningitis. } \\
\text { Secondary prophylaxis is } \\
\text { recommended following } \\
\text { completion of initial therapy } \\
\text { (induction plus } \\
\text { consolidation)-drugs and } \\
\text { dosing listed above. }\end{array}$ \\
\hline
\end{tabular}




\begin{tabular}{|c|c|c|c|}
\hline Indication & First Choice & Alternative & Comments/Special Issues \\
\hline & & 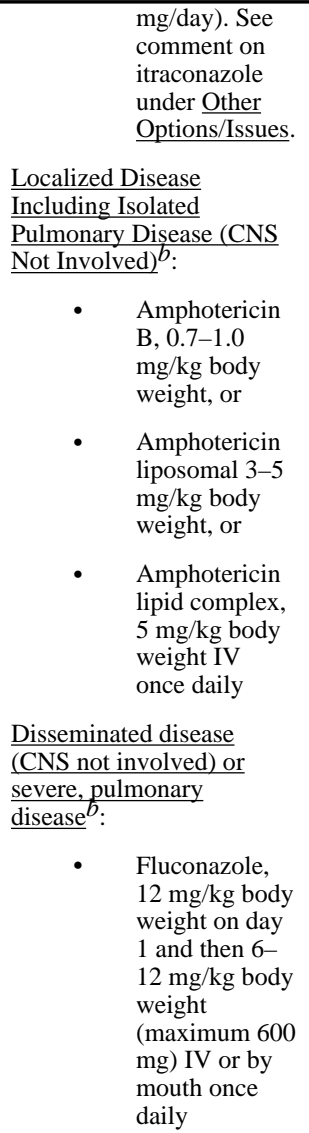 & \\
\hline
\end{tabular}

${ }^{a}$ Secondary prophylaxis is also referred to as maintenance therapy or suppressive therapy.

${ }^{b}$ Duration of therapy for non-CNS disease depends on site and severity of infection and clinical response

Key to Acronyms: $\mathrm{cART}=$ combination antiretroviral therapy; $\mathrm{CNS}=$ central nervous system; $\mathrm{CSF}=$ cerebrospinal fluid; ICP = intracranial pressure; IV = intravenous 


\title{
Cryptosporidiosis (Last updated November 6, 2013; last reviewed November 6, 2013)
}

\author{
Panel's Recommendations \\ - $\quad$ Reduce risk of Cryptosporidium infection by avoiding drinking water from public swimming pools and \\ other bodies of recreational water (AIII), touching farm animals (BIII), and having contact with known \\ Cryptosporidium-infected individuals (AIII). \\ - Combination antiretroviral therapy (cART) to prevent or reverse severe immune deficiency is the primary \\ modality for preventing chronic Cryptosporidium infection in HIV-infected children (AII*). \\ - Effective cART is the primary initial treatment for Cryptosporidium infections in HIV-infected children and \\ adults (AII*). \\ - $\quad$ Nitazoxanide can be considered in immunocompromised HIV-infected children in conjunction with cART \\ for treatment of Cryptosporidium infection (BII*). \\ - Supportive care with hydration, correction of electrolyte abnormalities, and nutritional supplementation \\ should be provided (AIII).
}

Rating of Recommendations: $\mathrm{A}=$ Strong; $\mathrm{B}=$ Moderate; $\mathrm{C}=$ Optional

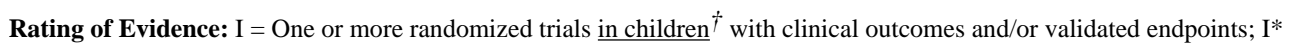
$=$ One or more randomized trials in adults with clinical outcomes and/or validated laboratory endpoints with accompanying data in children ${ }^{\dagger}$ from one or more well-designed, nonrandomized trials or observational cohort studies with long-term clinical outcomes; II = One or more well-designed, nonrandomized trials or observational cohort studies in children ${ }^{\dagger}$ with long-term outcomes; $\mathrm{II}^{*}=$ One or more well-designed, nonrandomized trials or observational studies in adults with longterm clinical outcomes with accompanying data $\underline{\text { children }}^{\dagger}$ from one or more similar nonrandomized trials or cohort studies with clinical outcome data; III = Expert opinion

${ }^{\dagger}$ Studies that include children or children/adolescents, but not studies limited to post-pubertal adolescents.

\section{Epidemiology}

Cryptosporidium spp. are protozoan parasites that primarily cause enteric illness (i.e., diarrhea) in humans and animals. They have worldwide distribution and lack host specificity. The two species that infect humans most frequently are Cryptosporidium hominis and Cryptosporidium parvum. In addition, infections caused by Cryptosporidium meleagridis, Cryptosporidium felis, and Cryptosporidium canis have been reported in HIVinfected patients. Among HIV-infected adults, risk of morbidity associated with Cryptosporidium infection is greatest in those with advanced immunosuppression, typically CD4 T-lymphocyte cell (CD4) counts $<100 / \mathrm{mm}^{3} \cdot{ }^{1-3}$ Cryptosporidium primarily infects the small intestine, but in immunocompromised hosts, extra-intestinal involvement has been documented.

Infection occurs after ingestion of infectious oocysts that were excreted in the feces of infected animals and humans. The parasite is highly infectious, with an $\mathrm{ID}_{50}$ (median dose that will infect $50 \%$ of those exposed to the parasite) ranging from 9 to 1042 oocysts, depending on the $C$. parvum isolate, ${ }^{4}$ and 10 to 83 oocysts for $C$. hominis. ${ }^{5}$ Infection occurs when the ingested oocyst releases sporozoites, which attach to and invade the intestinal epithelial cells. The parasite preferentially infects the jejunum and ileum.

Contact with infected individuals (particularly diapered children or in the child care setting) or infected animals (particularly pre-weaned calves) is an important cryptosporidiosis risk factor. ${ }^{6,7}$ Cryptosporidium oocysts can contaminate recreational water sources (such as 
swimming pools and lakes) and drinking water supplies and cause infection when contaminated water is ingested. Oocysts are environmentally hardy and extremely chlorine tolerant. They can persist for days in swimming pools despite standard chlorination, and typical pool filtration systems are only partially effective in removing oocysts. Multi-step treatment processes are often used to remove (i.e., filter) and inactivate (i.e., ultraviolet treatment) oocysts to protect public drinking water supplies. Foodborne transmission, particularly involving unpasteurized apple cider and ill food handlers, has been documented and individuals traveling internationally also may be at risk if they drink water in countries where water processing is not as strict as in the United States.

In a serosurvey of multiple U.S. cities, $21.3 \%$ of children aged $<10$ years and $21.5 \%$ of those aged 11 to 20 years had detectable response to Cryptosporidium antigen. ${ }^{8}$ Among immunocompetent pediatric patients with diarrhea, $38 \%$ of those aged 5 to 13 years and $58 \%$ of those aged 14 to 21 years were seropositive for Cryptosporidium antibodies, compared with $>80 \%$ of children aged 6 months to 13 years who resided near the U.S.Mexican border and were seeking well-child care. ${ }^{9,10}$ The incidence of reported cryptosporidiosis in the United States has dramatically increased since 2004, peaking at 4 cases per 100,000 people in 2007. ${ }^{11}$ Cases are most frequently reported in children aged 1 to 4 years, followed by those aged 5 to 9 years. However, cryptosporidiosis is a highly underdiagnosed and underreported diarrheal illness. Infected patients can be asymptomatic, those with symptoms may not seek healthcare, healthcare providers may not request laboratory diagnostics when evaluating non-bloody diarrhea, requested ova and parasite testing may not include Cryptosporidium testing, and positive laboratory results are not always reported to public health officials. ${ }^{12}$

Before effective antiretroviral therapy became available, most HIV-infected patients diagnosed with cryptosporidiosis had advanced disease or AIDS. The incidence of cryptosporidiosis in HIV-infected patients has declined dramatically since the introduction of combination antiretroviral therapy (cART) ${ }^{13-15}$ During the pre-cART era, the rate of cryptosporidiosis was 0.6 cases per 100 patient-years in children with a median age of 5.9 years and median CD4 count of $51 / \mathrm{mm}^{3}$ who were followed on 13 Pediatric AIDS Clinical Trial Group (PACTG) protocols. ${ }^{16}$ Data from the Perinatal AIDS Collaborative Transmission Study indicate that the rate of chronic intestinal cryptosporidiosis decreased from 0.2 cases per 100 person-years in the precART era to 0.0 cases per 100 person-years in the post-cART era. ${ }^{17}$ The PACTG estimates that the mortality rate in HIV-infected children significantly decreased from 7.2 to 0.8 per 100 person-years between 1994 and 2000 and subsequently stabilized through $2006 .{ }^{18}$ The proportion of deaths due to all opportunistic infections decreased between 1994 and 2006, with declines most notable in deaths caused by Cryptosporidium and Mycobacterium avium complex (MAC).

\section{Clinical Manifestations}

Symptoms of cryptosporidiosis develop after an incubation period of approximately 1 week (range, 2-14 days). Diarrhea—which can be profuse, usually non-bloody, and watery-and weight loss, abdominal pain, anorexia, fatigue, joint pain, headache, fever, and vomiting have been reported in immunocompetent children and adults infected with 
Cryptosporidium.${ }^{19}$ In immunocompetent hosts, illness is self-limiting, and symptoms most often completely resolve within 2 to 3 weeks. Recurrence of symptoms after seeming resolution often has been reported. Clinical presentation of cryptosporidiosis in HIVinfected patients varies with level of immunosuppression, ranging from no symptoms or transient disease to relapsing/chronic diarrhea or cholera-like diarrhea, which can lead to life-threatening wasting and malabsorption. ${ }^{20}$ In immunocompromised children, chronic severe diarrhea can result in malnutrition, failure to thrive, and substantial intestinal fluid losses, resulting in severe dehydration and even death.

Different Cryptosporidium spp. and genotypes are associated with different clinical manifestations in children and HIV-infected adults; vomiting is associated with $C$. hominis infection in children and C. parvum infection in adults. ${ }^{21,22}$ Neither clinical history nor physical examination allows differentiation of cryptosporidial disease from that caused by other pathogens.

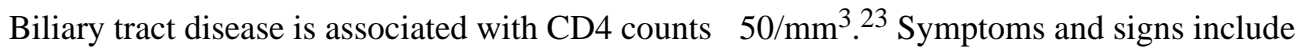
fever, right upper abdominal pain, nausea, vomiting, and elevated alkaline phosphatase. Diagnostic studies show dilatation of the common bile duct, thickening of the gall bladder wall, and pericholecystic fluid collection. Pancreatitis is rare. Although infection usually is limited to the gastrointestinal (GI) tract, respiratory cryptosporidiosis has been reported with no pathogen other than Cryptosporidium being detected in sputum. ${ }^{24,25}$

\section{Diagnosis}

Healthcare providers should specifically request Cryptosporidium testing, because standard ova and parasite testing is unlikely to include Cryptosporidium spp. Performance of diagnostic tests has not been extensively evaluated in HIV-infected children but is expected to be similar to that in HIV-uninfected children. Monoclonal antibody-based direct fluorescent antibody assay is the current test of choice for diagnosis of cryptosporidiosis because of enhanced sensitivity and specificity. ${ }^{26,27}$ Antigen-detection assays that have good sensitivity and specificity are available commercially (such as enzyme-linked immunosorbent assay [EIA] and immunochromatography). ${ }^{28,29}$

Oocyst excretion can be intermittent; therefore, the parasite may not be detected in every stool, and stool specimens collected on 3 consecutive days should be examined before considering test results to be negative. ${ }^{30}$ With EIA and rapid test methods, false-positive and false-negative results can occur, and confirmation by microscopy should be considered. If oocysts are not detected in stool specimens and if suspicion is high for cryptosporidiosis or limited oocyst excretion, polymerase chain reaction (PCR)-based detection is recommended because of its increased sensitivity. ${ }^{31}$ PCR for Cryptosporidium is not commercially available; healthcare providers should contact the state health department or Centers for Disease Control and Prevention if PCR-based detection is needed. Genotyping and subtyping tools are being increasingly used to differentiate Cryptosporidium species in outbreak investigations and infection/contamination source tracking. Cryptosporidium isolates cannot be reliably genotyped/subtyped if stool is preserved in formalin. 


\section{Prevention Recommendations}

Preventing Exposure-Caregivers and HIV-infected children should be educated and counseled about the different ways Cryptosporidium can be transmitted (AIII). Modes of transmission include having direct contact with fecal material from infected individuals (particularly children who wear diapers and infected animals), ingesting contaminated water during recreational activities, drinking contaminated water; and eating contaminated food.

Hand washing is probably the most important step to reduce the risk of Cryptosporidium infection (AIII). HIV-infected children should always wash their hands before preparing or eating food; after contact with children in diapers; after contact with clothing, bedding, toilets, or diapers soiled by someone who has diarrhea; after touching pets or other animals; and after touching anything that may have had contact with even the smallest amounts of human or animal feces (such as sand in a sandbox).

HIV-infected children should avoid contact with pre-weaned calves, ill animals, young animals (particularly dogs and cats aged $<6$ months and lambs), stray animals and stool from any animals or surfaces known to be contaminated with human or animal feces (AIII). HIVinfected children should avoid petting zoos and animal areas at farms and camps (BIII). After visiting an area with animals, an immunocompetent caregiver should clean the children's shoes and other surfaces that can become contaminated (such as clothes and stroller wheels).

HIV-infected children should avoid drinking water directly from ponds, streams, springs, lakes, or rivers, or swallowing water they swim or play in regardless of whether it is chlorinated (AIII). Caregivers and HIV-infected children should be aware that recreational water, including lakes, rivers, salt-water beaches, swimming pools, water parks, hot tubs, and interactive and ornamental water fountains may be contaminated with human or animal feces that contain Cryptosporidium. Note that children aged $<6$ years should not use a hot tub.

Some outbreaks of cryptosporidiosis have been linked to ingestion of water from contaminated municipal water supplies; the incidence of these outbreaks has dramatically decreased since the mid-1990s because of improved water treatment targeting the inactivation and removal of Cryptosporidium. To eliminate risk of cryptosporidiosis during outbreaks or in other situations in which a community advisory to boil water is issued, heat water used for preparing infant formula, drinking, and making ice at a rolling boil for 1 minute (AIII). After the boiled water cools, put it in a clean bottle or pitcher with a lid and store it in the refrigerator. Water bottles and ice trays should be cleaned with soap and water before each use. Do not touch the inside of these containers after cleaning.

Nationally distributed brands of bottled or canned carbonated soft drinks are safe to drink. Commercially packaged, non-carbonated soft drinks and fruit juices that do not require refrigeration until after they are opened (i.e., those which can be stored unrefrigerated on grocery shelves) also are safe. Nationally distributed brands of frozen fruit juice concentrate are safe if they are reconstituted by the user with water from a safe water source. Fruit juices that must be kept refrigerated from the time they are processed to the time of consumption 
may be either fresh (i.e., unpasteurized) or heat-treated (i.e., pasteurized); only juices labeled as pasteurized should be considered free of risk from Cryptosporidium. Other pasteurized beverages, such as milk, also are considered safe to drink (BIII).

Cryptosporidium-infected patients should not work as food handlers, especially if the handled food is intended to be eaten without cooking (AIII).

When traveling internationally, particularly in low-resource settings, HIV-infected patients should be warned to avoid drinking tap water and not to use it to brush teeth. Ingesting ice that may be made from tap water and raw fruits and vegetables should also be avoided (BIII). Steaming-hot foods, self-peeled fruits, bottled and canned processed drinks, and hot coffee or hot tea are probably safe.

In a hospital, standard precautions (such as the use of gloves and hand-washing after removal of gloves) should be sufficient to prevent transmission of cryptosporidiosis from an infected patient to a susceptible HIV-infected individual (AIII). However, because of the potential for fomite transmission, some experts recommend that severely immunocompromised HIV-infected patients should not share a room with a patient with cryptosporidiosis (CIII). A recent report suggests that there may be potential for respiratory transmission of Cryptosporidium. ${ }^{25}$ However, no specific modifications of current prevention efforts have been suggested.

HIV-infected adolescents who are sexually active should be counseled about avoiding sexual practices that could result in oral exposure to feces (such as oral-anal contact). To reduce the risk of exposure to feces, adolescents should use dental dams or similar barrier methods for oral-anal and oral-genital contact, wear latex gloves during digital-anal contact, and change condoms after anal intercourse. Frequent washing of hands and genitals with warm, soapy water during and after activities that could bring these body parts in contact with feces may further reduce the risk of Cryptosporidium infection.

Preventing Disease-Because chronic Cryptosporidium infection occurs most often in HIV-infected patients with advanced immunodeficiency, cART for HIV-infected children to prevent or reverse severe immune deficiency is a primary modality for prevention (AII).

Observational studies from the pre-cART era suggested that rifabutin or clarithromycin prophylaxis for MAC might be associated with decreased rates or risk of cryptosporidiosis. ${ }^{32-34}$ However, data are conflicting and insufficient to recommend using these drugs solely for prophylaxis of cryptosporidiosis.

Discontinuing Primary Prophylaxis-Not applicable.

\section{Treatment Recommendations}

Treating Disease-Immune reconstitution resulting from cART often results in clearance of Cryptosporidium infection. Effective cART is the primary initial treatment for these infections in HIV-infected children and adults (AII*). ${ }^{14,35}$ In vitro and observational studies, some of which are case series, suggest that cART containing a protease inhibitor 
(PI) may be preferable because of a direct effect of the PI on the parasite. ${ }^{35-44}$ PIs increase production of interferon-gamma, which in turn inhibits Cryptosporidium infection. Supportive care with hydration, correction of electrolyte abnormalities, and nutritional supplementation should be provided (AIII).

Antimotility agents to combat malabsorption of nutrients and drugs should be used with caution (CIII).

No consistently effective therapy is available for cryptosporidiosis, and duration of treatment in HIV-infected patients is uncertain. ${ }^{45,46}$ Multiple agents have been investigated in small randomized controlled clinical trials of HIV-infected adults, including nitazoxanide, paromomycin, spiramycin, bovine hyperimmune colostrum, and bovine dialyzable leukocyte extract. Azithromycin and roxithromycin have also been investigated in small open-label studies. ${ }^{47}$ No pharmacologic or immunologic therapy directed specifically against $C$. parvum has yet been shown consistently effective and durable when used alone without concomitant cART. ${ }^{45,46}$

A review of clinical trials of treatment for Cryptosporidia in immunocompromised patients, including those with HIV infection, found that no agent has proven efficacy for treating cryptosporidiosis in immunocompromised patients; however, in immunocompetent individuals, nitazoxanide reduces the load of parasites. Given the seriousness of this infection in immunocompromised individuals, use of nitazoxanide can be considered in immunocompromised HIV-infected children in conjunction with cART for immune restoration (BII*). ${ }^{45,46}$ Given that cART may directly inhibit the parasite, it is possible that the combination of cART and parasitic therapy may be synergistic.

Nitazoxanide is approved in the United States to treat diarrhea caused by Cryptosporidium and Giardia lamblia in children and is available in liquid and tablet formulations (BI for HIV-uninfected children and BII* for HIV-infected children). An Egyptian clinical trial in 100 HIV-uninfected adults and children randomized patients to a 3-day course of nitazoxanide or placebo. ${ }^{48}$ Nitazoxanide therapy reduced the duration of both diarrhea and oocyst shedding; in children, clinical response was $88 \%$ with nitazoxanide and $38 \%$ with placebo. No severe adverse events were reported, and adverse events that were reported were similar in the treatment and placebo groups in this study. A study in Zambia in 100 malnourished children (half of whom were HIV-infected) aged 12 to 35 months reported a clinical response in 56\% of HIV-uninfected children treated with nitazoxanide, compared with $23 \%$ receiving placebo. ${ }^{49}$ However, in the HIV-infected children, no benefit was observed from nitazoxanide (clinical response in $8 \%$ treated with nitazoxanide, compared with $25 \%$ receiving placebo). In a subsequent study of $60 \mathrm{HIV}$-infected children with cryptosporidiosis, the same investigators reported no significant benefit using twice the recommended dose administered for 28 days. ${ }^{50}$ It should be noted that the children in the Zambian studies were not receiving cART. In a study in HIV-infected adults not receiving cART who had CD4 counts $>50$ cells $/ \mathrm{mm}^{3}, 14$ days of nitazoxanide resulted in $71 \%$ (10 of 14) response using $500 \mathrm{mg}$ twice daily and $90 \%$ (9 of 10) using $1000 \mathrm{mg}$ twice daily, compared with $25 \%$ with placebo. ${ }^{51}$ The recommended dose for children is $100 \mathrm{mg}$ orally twice daily for children aged 1 to 3 years and $200 \mathrm{mg}$ twice daily for children aged 4 to 11 
years. A tablet preparation (500 mg twice daily) is available for children aged $\geq 12$ years. All medications should be administered with food.

Paromomycin, a non-absorbable aminoglycoside indicated for the treatment of intestinal amoebiasis, is not approved for treatment of cryptosporidiosis. Two small, randomized trials evaluating the efficacy of paromomycin for treatment of HIV-infected patients found clinical improvement or reduced oocyst excretion in those treated with paromomycin. ${ }^{52,53} \mathrm{~A}$ review of reports of paromomycin treatment in HIV-infected patients found repeated failure to cure. ${ }^{54}$ Therefore, data do not support a recommendation for use of paromomycin for cryptosporidiosis (BII*). Clinical or parasitological cure has been documented with use of paromomycin and azithromycin in combination in case series of HIV-infected patients with cryptosporidial diarrhea and case reports of HIV-infected patients with pulmonary cryptosporidiosis. ${ }^{55-57}$

Monitoring and Adverse Events, Including IRIS-Patients should be closely monitored for signs and symptoms of volume depletion, electrolyte imbalance, malnutrition, and weight loss. In severely ill patients, total parenteral nutrition may be indicated (CIII). One case report describes immune reconstitution inflammatory syndrome, specifically terminal ileitis, in association with treatment of cryptosporidiosis. ${ }^{58}$

In general, nitazoxanide is well tolerated and side effects are mild, transient, and limited to the GI tract.

Managing Treatment Failure-The most important steps for managing treatment failure are optimizing cART to increase CD4 counts and providing supportive treatment (AIII).

Preventing Recurrence-No pharmacologic interventions are known to be effective in preventing recurrence of cryptosporidiosis.

Discontinuing Secondary Prophylaxis—Not applicable.

\section{References}

1. Flanigan T, Whalen C, Turner J, et al. Cryptosporidium infection and CD4 counts. Ann Intern Med. 1992 May 15; 116(10):840-842. Available at http://www.ncbi.nlm.nih.gov/pubmed/1348918. [PubMed: 1348918]

2. Sorvillo F, Beall G, Turner PA, et al. Seasonality and factors associated with cryptosporidiosis among individuals with HIV infection. Epidemiol Infect. 1998 Aug; 121(1):197-204. Available at http://www.ncbi.nlm.nih.gov/pubmed/9747773. [PubMed: 9747773]

3. Inungu JN, Morse AA, Gordon C. Risk factors, seasonality, and trends of cryptosporidiosis among patients infected with human immunodeficiency virus. Am J Trop Med Hyg. 2000 Mar; 62(3):384387. Available at http://www.ncbi.nlm.nih.gov/pubmed/11037782. [PubMed: 11037782]

4. Okhuysen PC, Chappell CL, Crabb JH, Sterling CR, DuPont HL. Virulence of three distinct Cryptosporidium parvum isolates for healthy adults. J Infect Dis. 1999 Oct; 180(4):1275-1281. Available at http://www.ncbi.nlm.nih.gov/pubmed/10479158. [PubMed: 10479158]

5. Chappell CL, Okhuysen PC, Langer-Curry R, et al. Cryptosporidium hominis: experimental challenge of healthy adults. Am J Trop Med Hyg. 2006 Nov; 75(5):851-857. Available at http:// www.ncbi.nlm.nih.gov/pubmed/17123976. [PubMed: 17123976] 
6. Heijbel H, Slaine K, Seigel B, et al. Outbreak of diarrhea in a day care center with spread to household members: the role of Cryptosporidium. Pediatr Infect Dis J. 1987 Jun; 6(6):532-535. Available at http://www.ncbi.nlm.nih.gov/pubmed/3615068. [PubMed: 3615068]

7. O'Connor RM, Shaffie R, Kang G, Ward HD. Cryptosporidiosis in patients with HIV/AIDS. AIDS. 2011 Mar 13; 25(5):549-560. Available at http://www.ncbi.nlm.nih.gov/pubmed/21160413. [PubMed: 21160413]

8. Frost FJ, Muller TB, Calderon RL, Craun GF. Analysis of serological responses to Cryptosporidium antigen among NHANES III participants. Annals of epidemiology. 2004 Aug; 14(7):473-478. Available at http://www.ncbi.nlm.nih.gov/pubmed/15310525. [PubMed: 15310525]

9. Kuhls TL, Mosier DA, Crawford DL, Griffis J. Seroprevalence of cryptosporidial antibodies during infancy, childhood, and adolescence. Clin Infect Dis. 1994 May; 18(5):731-735. Available at http:// www.ncbi.nlm.nih.gov/pubmed/8075261. [PubMed: 8075261]

10. Leach CT, Koo FC, Kuhls TL, Hilsenbeck SG, Jenson HB. Prevalence of Cryptosporidium parvum infection in children along the Texas-Mexico border and associated risk factors. Am J Trop Med Hyg. 2000 May; 62(5):656-661. Available at http://www.ncbi.nlm.nih.gov/pubmed/11289680. [PubMed: 11289680]

11. Yoder JS, Harral C, Beach MJ. Centers for Disease C, Prevention. Giardiasis surveillance-United States: 2006-2008. MMWR Surveill Summ. 2010 Jun 11; 59(6):15-25. Available at http:// www.ncbi.nlm.nih.gov/pubmed/20535095. [PubMed: 20535095]

12. Yoder JS, Beach MJ. Cryptosporidium surveillance and risk factors in the United States. Exp Parasitol. 2010 Jan; 124(1):31-39. Available at http://www.ncbi.nlm.nih.gov/pubmed/19786022. [PubMed: 19786022]

13. Huang DB, White AC. An updated review on Cryptosporidium and Giardia. Gastroenterol Clin North Am. 2006 Jun; 35(2):291-314. viii. Available at http://www.ncbi.nlm.nih.gov/pubmed/ 16880067. [PubMed: 16880067]

14. Chen XM, Keithly JS, Paya CV, LaRusso NF. Cryptosporidiosis. N Engl J Med. 2002 May 30; 346(22):1723-1731. Available at http://www.ncbi.nlm.nih.gov/pubmed/12037153. [PubMed: 12037153]

15. Buchacz K, Baker RK, Palella FJ Jr, et al. AIDS-defining opportunistic illnesses in US patients, 1994-2007: a cohort study. AIDS. 2010 Jun 19; 24(10):1549-1559. Available at http:// www.ncbi.nlm.nih.gov/pubmed/20502317. [PubMed: 20502317]

16. Dankner WM, Lindsey JC, Levin MJ. Pediatric ACTGPT. Correlates of opportunistic infections in children infected with the human immunodeficiency virus managed before highly active antiretroviral therapy. Pediatr Infect Dis J. 2001 Jan; 20(1):40-48. Available at http:// www.ncbi.nlm.nih.gov/pubmed/11176565. [PubMed: 11176565]

17. Nesheim SR, Kapogiannis BG, Soe MM, et al. Trends in opportunistic infections in the pre- and post-highly active antiretroviral therapy eras among HIV-infected children in the Perinatal AIDS Collaborative Transmission Study, 1986-2004. Pediatrics. 2007 Jul; 120(1):100-109. Available at http://www.ncbi.nlm.nih.gov/pubmed/17606567. [PubMed: 17606567]

18. Brady MT, Oleske JM, Williams PL, et al. Declines in mortality rates and changes in causes of death in HIV-1-infected children during the HAART era. J Acquir Immune Defic Syndr. 2010 Jan; 53(1):86-94. Available at http://www.ncbi.nlm.nih.gov/pubmed/20035164. [PubMed: 20035164]

19. Hunter PR, Hughes S, Woodhouse S, et al. Health sequelae of human cryptosporidiosis in immunocompetent patients. Clin Infect Dis. 2004 Aug 15; 39(4):504-510. Available at http:// www.ncbi.nlm.nih.gov/pubmed/15356813. [PubMed: 15356813]

20. Hunter PR, Nichols G. Epidemiology and clinical features of Cryptosporidium infection in immunocompromised patients. Clin Microbiol Rev. 2002 Jan; 15(1):145-154. Available at http:// www.ncbi.nlm.nih.gov/pubmed/11781272. [PubMed: 11781272]

21. Cama VA, Ross JM, Crawford S, et al. Differences in clinical manifestations among Cryptosporidium species and subtypes in HIV-infected persons. J Infect Dis. 2007 Sep 1; 196(5): 684-691. Available at http://www.ncbi.nlm.nih.gov/pubmed/17674309. [PubMed: 17674309]

22. Cama VA, Bern C, Roberts J, et al. Cryptosporidium species and subtypes and clinical manifestations in children, Peru. Emerg Infect Dis. 2008 Oct; 14(10):1567-1574. Available at http://www.ncbi.nlm.nih.gov/pubmed/18826821. [PubMed: 18826821] 
23. Vakil NB, Schwartz SM, Buggy BP, et al. Biliary cryptosporidiosis in HIV-infected people after the waterborne outbreak of cryptosporidiosis in Milwaukee. N Engl J Med. 1996 Jan 4; 334(1):1923. Available at http://www.ncbi.nlm.nih.gov/pubmed/7494565. [PubMed: 7494565]

24. Clavel A, Arnal AC, Sanchez EC, et al. Respiratory cryptosporidiosis: case series and review of the literature. Infection. 1996 Sep-Oct;24(5):341-346. Available at http://www.ncbi.nlm.nih.gov/ pubmed/8923043. [PubMed: 8923043]

25. Mor SM, Tumwine JK, Ndeezi G, et al. Respiratory cryptosporidiosis in HIV-seronegative children in Uganda: potential for respiratory transmission. Clin Infect Dis. 2010 May 15; 50(10): 1366-1372. Available at http://www.ncbi.nlm.nih.gov/pubmed/20377408. [PubMed: 20377408]

26. Weber R, Bryan RT, Bishop HS, Wahlquist SP, Sullivan JJ, Juranek DD. Threshold of detection of Cryptosporidium oocysts in human stool specimens: evidence for low sensitivity of current diagnostic methods. J Clin Microbiol. 1991 Jul; 29(7):1323-1327. Available at http:// www.ncbi.nlm.nih.gov/pubmed/1715881. [PubMed: 1715881]

27. Arrowood MJ, Sterling CR. Comparison of conventional staining methods and monoclonal antibody-based methods for Cryptosporidium oocyst detection. J Clin Microbiol. 1989 Jul; 27(7): 1490-1495. Available at http://www.ncbi.nlm.nih.gov/pubmed/2475523. [PubMed: 2475523]

28. Garcia LS, Shimizu RY. Evaluation of nine immunoassay kits (enzyme immunoassay and direct fluorescence) for detection of Giardia lamblia and Cryptosporidium parvum in human fecal specimens. J Clin Microbiol. 1997 Jun; 35(6):1526-1529. Available at http:// www.ncbi.nlm.nih.gov/pubmed/9163474. [PubMed: 9163474]

29. Garcia LS, Shimizu RY, Novak S, Carroll M, Chan F. Commercial assay for detection of Giardia lamblia and Cryptosporidium parvum antigens in human fecal specimens by rapid solid-phase qualitative immunochromatography. J Clin Microbiol. 2003 Jan; 41(1):209-212. Available at http://www.ncbi.nlm.nih.gov/pubmed/12517850. [PubMed: 12517850]

30. van Gool T, Weijts R, Lommerse E, Mank TG. Triple Faeces Test: an effective tool for detection of intestinal parasites in routine clinical practice. Eur J Clin Microbiol Infect Dis. 2003 May; 22(5):284-290. Available at http://www.ncbi.nlm.nih.gov/pubmed/12736794. [PubMed: 12736794]

31. McLauchlin J, Amar CF, Pedraza-Diaz S, Mieli-Vergani G, Hadzic N, Davies EG. Polymerase chain reaction-based diagnosis of infection with Cryptosporidium in children with primary immunodeficiencies. Pediatr Infect Dis J. 2003 Apr; 22(4):329-335. Available at http:// www.ncbi.nlm.nih.gov/pubmed/12690272. [PubMed: 12690272]

32. Holmberg SD, Moorman AC, Von Bargen JC, et al. Possible effectiveness of clarithromycin and rifabutin for cryptosporidiosis chemoprophylaxis in HIV disease. HIV Outpatient Study (HOPS) Investigators. JAMA. 1998 Feb 4; 279(5):384-386. Available at http://www.ncbi.nlm.nih.gov/ pubmed/9459473. [PubMed: 9459473]

33. Fichtenbaum CJ, Zackin R, Feinberg J, Benson C, Griffiths JK. Team ACTGNWCS. Rifabutin but not clarithromycin prevents cryptosporidiosis in persons with advanced HIV infection. AIDS. 2000 Dec 22; 14(18):2889-2893. Available at http://www.ncbi.nlm.nih.gov/pubmed/11153670. [PubMed: 11153670]

34. Jordan WC. Clarithromycin prophylaxis against Cryptosporidium enteritis in patients with AIDS. Journal of the National Medical Association. 1996 Jul; 88(7):425-427. Available at http:// www.ncbi.nlm.nih.gov/pubmed/8764523. [PubMed: 8764523]

35. Miao YM, Awad-El-Kariem FM, Franzen C, et al. Eradication of cryptosporidia and microsporidia following successful antiretroviral therapy. J Acquir Immune Defic Syndr. 2000 Oct 1; 25(2):124129. Available at http://www.ncbi.nlm.nih.gov/pubmed/11103042. [PubMed: 11103042]

36. Hommer V, Eichholz J, Petry F. Effect of antiretroviral protease inhibitors alone, and in combination with paromomycin, on the excystation, invasion and in vitro development of Cryptosporidium parvum. J Antimicrob Chemother. 2003 Sep; 52(3):359-364. Available at http:// www.ncbi.nlm.nih.gov/pubmed/12888587. [PubMed: 12888587]

37. Mele R, Gomez Morales MA, Tosini F, Pozio E. Indinavir reduces Cryptosporidium parvum infection in both in vitro and in vivo models. Int J Parasitol. $2003 \mathrm{Jul}$; 33(7):757-764. Available at http://www.ncbi.nlm.nih.gov/pubmed/12814654. [PubMed: 12814654]

38. Maggi P, Larocca AM, Quarto M, et al. Effect of antiretroviral therapy on cryptosporidiosis and microsporidiosis in patients infected with human immunodeficiency virus type 1. Eur J Clin 
Microbiol Infect Dis. 2000 Mar; 19(3):213-217. Available at http://www.ncbi.nlm.nih.gov/ pubmed/10795595. [PubMed: 10795595]

39. Cabada MM, White AC Jr. Treatment of cryptosporidiosis: do we know what we think we know? Curr Opin Infect Dis. 2010 Oct; 23(5):494-499. Available at http://www.ncbi.nlm.nih.gov/ pubmed/20689422. [PubMed: 20689422]

40. Maggi P, Larocca AM, Ladisa N, et al. Opportunistic parasitic infections of the intestinal tract in the era of highly active antiretroviral therapy: is the CD4(+) count so important? Clin Infect Dis. 2001 Nov 1; 33(9):1609-1611. Available at http://www.ncbi.nlm.nih.gov/pubmed/11588705. [PubMed: 11588705]

41. Miao YM, Awad-El-Kariem FM, Gibbons CL, Gazzard BG. Cryptosporidiosis: eradication or suppression with combination antiretroviral therapy? AIDS. 1999 Apr 16; 13(6):734-735. Available at http://www.ncbi.nlm.nih.gov/pubmed/10397573. [PubMed: 10397573]

42. Bobin S, Bouhour D, Durupt S, Boibieux A, Girault V, Peyramond D. [Importance of antiproteases in the treatment of microsporidia and/or cryptosporidia infections in HIV-seropositive patients]. Pathologie-biologie. 1998 Jun; 46(6):418-419. Available at http://www.ncbi.nlm.nih.gov/pubmed/ 9769873. [PubMed: 9769873]

43. Foudraine NA, Weverling GJ, van Gool T, et al. Improvement of chronic diarrhoea in patients with advanced HIV-1 infection during potent antiretroviral therapy. AIDS. 1998 Jan 1; 12(1):35-41. Available at http://www.ncbi.nlm.nih.gov/pubmed/9456253. [PubMed: 9456253]

44. Carr A, Marriott D, Field A, Vasak E, Cooper DA. Treatment of HIV-1-associated microsporidiosis and cryptosporidiosis with combination antiretroviral therapy. Lancet. 1998 Jan 24; 351(9098):256-261. Available at http://www.ncbi.nlm.nih.gov/pubmed/9457096. [PubMed: 9457096]

45. Abubakar I, Aliyu SH, Arumugam C, Hunter PR, Usman NK. Prevention and treatment of cryptosporidiosis in immunocompromised patients. Cochrane Database Syst Rev. 2007(1):CD004932. Available at http://www.ncbi.nlm.nih.gov/pubmed/17253532. [PubMed: 17253532]

46. Abubakar I, Aliyu SH, Arumugam C, Usman NK, Hunter PR. Treatment of cryptosporidiosis in immunocompromised individuals: systematic review and meta-analysis. Br J Clin Pharmacol. 2007 Apr; 63(4):387-393. Available at http://www.ncbi.nlm.nih.gov/pubmed/17335543. [PubMed: 17335543]

47. Rossignol JF. Cryptosporidium and Giardia: treatment options and prospects for new drugs. Exp Parasitol. 2010 Jan; 124(1):45-53. Available at http://www.ncbi.nlm.nih.gov/pubmed/19632225. [PubMed: 19632225]

48. Rossignol JF, Ayoub A, Ayers MS. Treatment of diarrhea caused by Cryptosporidium parvum: a prospective randomized, double-blind, placebo-controlled study of Nitazoxanide. J Infect Dis. 2001 Jul 1; 184(1):103-106. Available at http://www.ncbi.nlm.nih.gov/pubmed/11398117. [PubMed: 11398117]

49. Amadi B, Mwiya M, Musuku J, et al. Effect of nitazoxanide on morbidity and mortality in Zambian children with cryptosporidiosis: a randomised controlled trial. Lancet. 2002 Nov 2; 360(9343):1375-1380. Available at http://www.ncbi.nlm.nih.gov/pubmed/12423984. [PubMed: 12423984]

50. Amadi B, Mwiya M, Sianongo S, et al. High dose prolonged treatment with nitazoxanide is not effective for cryptosporidiosis in HIV positive Zambian children: a randomised controlled trial. BMC Infect Dis. 2009; 9:195. Available at http://www.ncbi.nlm.nih.gov/pubmed/19954529. [PubMed: 19954529]

51. Rossignol JF, Hidalgo H, Feregrino M, et al. A double-'blind' placebo-controlled study of nitazoxanide in the treatment of cryptosporidial diarrhoea in AIDS patients in Mexico. Trans $\mathrm{R}$ Soc Trop Med Hyg. 1998 Nov-Dec;92(6):663-666. Available at http://www.ncbi.nlm.nih.gov/ pubmed/10326116. [PubMed: 10326116]

52. White AC Jr, Chappell CL, Hayat CS, Kimball KT, Flanigan TP, Goodgame RW. Paromomycin for cryptosporidiosis in AIDS: a prospective, double-blind trial. J Infect Dis. 1994 Aug; 170(2): 419-424. Available at http://www.ncbi.nlm.nih.gov/pubmed/8035029. [PubMed: 8035029]

53. Hewitt RG, Yiannoutsos CT, Higgs ES, et al. Paromomycin: no more effective than placebo for treatment of cryptosporidiosis in patients with advanced human immunodeficiency virus infection. 
AIDS Clinical Trial Group. Clin Infect Dis. 2000 Oct; 31(4):1084-1092. Available at http:// www.ncbi.nlm.nih.gov/pubmed/11049793. [PubMed: 11049793]

54. Hashmey R, Smith NH, Cron S, Graviss EA, Chappell CL, White AC Jr. Cryptosporidiosis in Houston, Texas. A report of 95 cases. Medicine (Baltimore). 1997 Mar; 76(2):118-139. Available at http://www.ncbi.nlm.nih.gov/pubmed/9100739. [PubMed: 9100739]

55. Smith NH, Cron S, Valdez LM, Chappell CL, White AC Jr. Combination drug therapy for cryptosporidiosis in AIDS. J Infect Dis. 1998 Sep; 178(3):900-903. Available at http:// www.ncbi.nlm.nih.gov/pubmed/9728569. [PubMed: 9728569]

56. Meamar AR, Rezaian M, Rezaie S, et al. Cryptosporidium parvum bovine genotype oocysts in the respiratory samples of an AIDS patient: efficacy of treatment with a combination of azithromycin and paromomycin. Parasitology research. 2006 May; 98(6):593-595. Available at http:// www.ncbi.nlm.nih.gov/pubmed/16416289. [PubMed: 16416289]

57. Palmieri F, Cicalini S, Froio N, et al. Pulmonary cryptosporidiosis in an AIDS patient: successful treatment with paromomycin plus azithromycin. Int J STD AIDS. 2005 Jul; 16(7):515-517. Available at http://www.ncbi.nlm.nih.gov/pubmed/16004637. [PubMed: 16004637]

58. Plasencia LD, Socas Mdel M, Valls RA, Fernandez EM, Higuera AC, Gutierrez AB. Terminal ileitis as a manifestation of immune reconstitution syndrome following HAART. AIDS. 2006 Sep 11; 20(14):1903-1905. Available at http://www.ncbi.nlm.nih.gov/pubmed/16954736. [PubMed: $16954736]$

\section{Dosing Recommendations for Prevention and Treatment of Cryptosporidiosis}

\begin{tabular}{|c|c|c|c|}
\hline \multicolumn{4}{|c|}{ Preventive Regimen } \\
\hline Indication & First Choice & Alternative & Comments/Special Issues \\
\hline Primary Prophylaxis & $\begin{array}{l}\text { ARV therapy to avoid } \\
\text { advanced immune } \\
\text { deficiency }\end{array}$ & N/A & N/A \\
\hline Secondary Prophylaxis & N/A & N/A & N/A \\
\hline Treatment & $\begin{array}{ll}\text { Effective cART: } \\
& \text { Immune } \\
& \text { reconstitution } \\
& \text { may lead to } \\
& \text { microbiologic } \\
& \text { and clinical } \\
& \text { response }\end{array}$ & 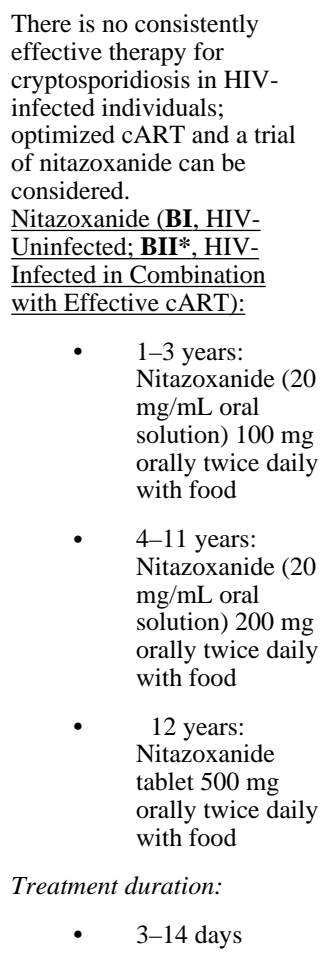 & $\begin{array}{l}\text { Supportive Care: } \\
\begin{array}{l}\text { Hydration, } \\
\text { correct } \\
\text { electrolyte } \\
\text { abnormalities, } \\
\text { nutritional } \\
\text { support }\end{array} \\
\text { Antimotility agents (such } \\
\text { as loperamide) should be } \\
\text { used with caution in young } \\
\text { children. }\end{array}$ \\
\hline
\end{tabular}


Key to Acronyms: ARV = antiretroviral; $\mathrm{cART}=$ combination antiretroviral therapy

\section{Cytomegalovirus (Last updated November 6, 2013; last reviewed November}

\section{6, 2013)}

Panel's Recommendations

- Cytomegalovirus (CMV) antibody testing is recommended at age 1 year and then annually for CMVseronegative, HIV-infected infants and children who are immunosuppressed (i.e., CD4 T-lymphocyte (CD4) cell count $<100$ cells $/ \mathrm{mm}^{3}$ or CD4 percentage $<10 \%$ ) (BII).

- $\quad$ HIV-infected children aged $<5$ years who are CMV-infected and severely immunosuppressed (i.e., CD4 cell count $<50$ cells $/ \mathrm{mm}^{3}$ or $\mathrm{CD} 4$ percentage $<5 \%$ ) should have a dilated retinal examination performed by an ophthalmologist every 6 months (AIII).

- $\quad$ CMV end-organ disease is best prevented by antiretroviral therapy (ART) to maintain the CD4 cell count $>100$ cells $/ \mathrm{mm}^{3}$ in children aged $\succ 6$ years, or CD4 percentage $>10 \%$ in children $<6$ years (BIII). Prophylaxis with valganciclovir can be considered for HIV-infected children aged $\searrow 6$ years who are CMVseropositive and have CD4 cell counts $<50$ cells $/ \mathrm{mm}^{3}$ and for HIV-infected children aged $<6$ years who are CMV-seropositive and have a CD4 percentage $<5 \%$ (CIII). Cessation of primary prophylaxis can be considered when the CD4 cell count is $>100$ cells $/ \mathrm{mm}^{3}$ for children $\succeq 6$ years of age, or $>10 \%$ in children $<6$ years (CIII).

- Intravenous (IV) ganciclovir therapy (6 mg/kg/dose administered every 12 hours) for 6 weeks can be considered for HIV-exposed or HIV-infected infants who have symptomatic congenital CMV disease involving the central nervous system (CNS) (BI).

- For HIV-infected infants and children, IV ganciclovir is the drug of choice for initial treatment for acquired CMV disease, including retinitis and other end-organ disseminated CMV disease (e.g., colitis, esophagitis, CNS disease) (AI*). Oral valganciclovir has not been evaluated in HIV-infected children with CMV retinitis, but is an option primarily for older children who weigh enough to receive the adult dose and formulation of valganciclovir (CIII). Transition from IV ganciclovir to valganciclovir oral solution can be considered for younger patients who improve on IV therapy (CIII)

- Foscarnet is an alternative drug for treating CMV disease or for use in ganciclovir-resistant CMV infections in HIV-infected children (AI*).

- Combination therapy with ganciclovir and foscarnet delays progression of retinitis in certain patients in whom monotherapy fails and can be used as initial therapy in children with sight-threatening disease (BIII).

- Combination treatment with IV ganciclovir and foscarnet may be preferable as initial therapy to stabilize CMV neurologic disease and maximize response (BII*).

- Many experts would initially treat early first relapse of retinitis with reinduction using the same drug, followed by reinstitution of maintenance therapy (AII*). If drug resistance is suspected, change to an alternative drug is reasonable (AIII). Combination IV ganciclovir and foscarnet can be considered.

- $\quad$ After induction therapy, secondary prophylaxis (chronic maintenance therapy) is given for most forms of CMV disease until immune reconstitution or, in absence of immune reconstitution, for the remainder of a patient's life (AI*). Regimens recommended for chronic suppression include IV ganciclovir, oral valganciclovir, IV foscarnet, combined IV ganciclovir and foscarnet, and parenteral cidofovir (AI*).

- Chronic maintenance therapy is not routinely recommended for gastrointestinal disease but should be considered if relapses occur (BII*). A role for maintenance therapy for CMV pneumonitis has not been established (CIII).

- Discontinuing secondary prophylaxis may be considered for children who are receiving ART and have a sustained (such as $>6$ months) increase in CD4 cell count, defined as an increase in CD4 percentage to $>15 \%$ for children aged $<6$ years, or an increase in CD4 cell count to $>100$ cells $/ \mathrm{mm}^{3}$ for children aged $\Varangle 6$ years (CIII).

- $\quad$ All patients with CMV ophthalmic disease in whom anti-CMV maintenance therapy has been discontinued should continue to undergo regular ophthalmologic monitoring at 3- to 6-month intervals for early detection of CMV relapse and for immune reconstitution uveitis (AII*).

- Secondary prophylaxis should be reinstituted in HIV-infected children in whom it was discontinued because of immune reconstitution when the CD4 percentage decreases to $<15 \%$ in those aged $<6$ years and when the CD4 cell count decreases to $<100$ cells $/ \mathrm{mm}^{3}$ in those aged $\geq 6$ years (BIII).

\section{Rating of Recommendations: $\mathrm{A}=$ Strong; $\mathrm{B}=$ Moderate $\mathrm{C}=$ Optional}

Rating of Evidence: $\mathrm{I}=$ One or more randomized trials $\underline{\text { in children }}^{\dagger}$ with clinical outcomes and/or validated endpoints; I* $=$ One or more randomized trials in adults with clinical outcomes and/or validated laboratory endpoints with accompanying data in children $^{\dagger}$ from one or more well-designed, nonrandomized trials or observational cohort studies with long-term clinical outcomes; II = One or more well-designed, nonrandomized trials or observational cohort studies in children ${ }^{\dagger}$ with 
long-term outcomes; $\mathrm{II}^{*}=$ One or more well-designed, nonrandomized trials or observational studies in adults with longterm clinical outcomes with accompanying data in children ${ }^{\dagger}$ from one or more similar nonrandomized trials or cohort studies with clinical outcome data; III = Expert opinion

${ }^{\dagger}$ Studies that include children or children/adolescents, but not studies limited to post-pubertal adolescents

\section{Epidemiology}

Infection with human cytomegalovirus (CMV) is common and usually not apparent; CMV can be acquired in utero, or during infancy, early childhood, or adolescence. Transmission can occur vertically from an infected woman to her offspring; horizontally by contact with virus-containing breast milk, saliva, urine, or sexual fluid; through transfusion of infected blood; or transplantation of infected organs. During infancy and early childhood, infection usually occurs secondary to ingestion of virions in breast milk of CMV-infected mothers or from exposure to virus shed in saliva or urine. Infection occurs at younger ages in locations where sanitation is less than optimal. Among adolescents, sexual transmission is the major mode of CMV acquisition.

Age-related prevalence of infection varies widely depending on living circumstances and social customs. Breastfeeding, child-rearing practices, crowding, sanitation, and sexual behavior most likely influence age-related variations in CMV prevalence. Where rates of maternal seropositivity are high and breastfeeding is common, more than half of infants acquire CMV during the first year of life. ${ }^{1}$ Group care of children facilitates spread of CMV, especially in toddlers, and leads to higher prevalence of infection in children who attend child care centers and in their caregivers. ${ }^{2,3}$ In Africa, Asia, and Latin America, most children are infected with CMV before adolescence. In the United States and western Europe, the prevalence of antibody to CMV in adults from middle and upper socioeconomic strata is $40 \%$ to $60 \%$, whereas the prevalence in low-income adults is $280 \% .{ }^{4}$ Overall, among U.S. women of childbearing age, the prevalence of CMV infection is $50 \%$ to $80 \%$, with the highest prevalence in women in lower socioeconomic strata. ${ }^{5,6}$ The prevalence of CMV infection among HIV-infected pregnant women is higher than in the general population, with approximately $90 \%$ of HIV-infected pregnant women coinfected with CMV. ${ }^{7,8}$

CMV is the most common congenitally transmitted infection, with incidence estimates in live-born infants in the United States ranging from $0.5 \%$ to $1.2 \% .{ }^{9}$ Congenital (in utero) CMV infection occurs most commonly among infants born to women who have primary CMV infection during pregnancy. Following primary infection during pregnancy, the rate of transmission to the fetus is approximately $30 \%$ to $40 \%{ }^{5,10}$ In comparison, the rate of congenital infection after non-primary maternal CMV infection is believed to be significantly lower (range: $0.15 \%-1.0 \%$ ). ${ }^{11-13}$ More recent studies demonstrate that in utero transmission of non-primary maternal infection can occur because of reactivation of infection in women infected before pregnancy or reinfection with a different CMV strain in CMV-seropositive women. ${ }^{14,15}$

CMV also can be transmitted from mother to infant during the intrapartum or postpartum periods. Up to 57\% of infants whose mothers shed CMV at or around delivery become infected with CMV, and up to $53 \%$ of children who are breastfed milk containing infectious 
virus can become CMV-infected. Symptomatic CMV disease in the infant is much less common when CMV is acquired intrapartum or through breastfeeding than when acquired antenatally and occurs primarily in premature neonates. Long-term sequelae are rare in premature infants who acquire CMV perinatally or postnatally. ${ }^{16-20}$

HIV-infected women with CMV infection have a higher rate of CMV shedding from the cervix than do women who are HIV-uninfected (52\%-59\% and $14 \%-35 \%$, respectively). ${ }^{21}$ The risk for mother-to-infant transmission of CMV may be higher among infants born to women dually infected with CMV and HIV. In one study of 440 infants born to HIVinfected U.S. women, the overall rate of in utero infection was $4.5 \%,{ }^{22}$ higher than the $<2 \%$ rate of in utero infection in the general U.S. population. In a more recent study of 367 U.S. infants born to HIV-infected mothers, a 3\% prevalence of congenital CMV infection was reported among HIV-uninfected infants born to HIV-infected mothers, suggesting that the rate of congenital CMV infection is similar to or slightly higher than the prevalence of congenital CMV infection among HIV-uninfected mothers. ${ }^{23}$ In a study in France, the prevalence of congenital CMV infection among HIV-infected infants was 10.3\%, compared with $2.2 \%$ in HIV-uninfected infants born to HIV-positive mothers, and the rate of in utero HIV transmission was higher among infants with congenital CMV infection compared with infants without congenital CMV infection. ${ }^{24}$

HIV-infected children appear to be at higher risk of CMV infection during early childhood than are HIV-uninfected children. ${ }^{22}$ The rate of CMV acquisition in HIV-infected children appears to be particularly high during the first 12 months of life but remains higher among HIV-infected than HIV-uninfected children through age 4 years.

CMV disease occurs less frequently among HIV-infected children than HIV-infected adults, but still contributed substantially to morbidity and mortality in the era before combination antiretroviral therapy (cART). In the pre-antiretroviral era, CMV caused $8 \%$ to $10 \%$ of pediatric AIDS-defining illnesses. ${ }^{25}$ Data in HIV-infected adults have shown a $75 \%$ to $80 \%$ decrease in the incidence of new cases of CMV end-organ disease with the advent of cART, with an incidence now estimated to be $<6$ cases per 100 person-years. ${ }^{26}$ In a study of opportunistic infections in approximately 3,000 children followed in Pediatric AIDS Clinical Trials Group studies during the pre-cART era, the frequency of CMV retinitis was 0.5 cases per 100 child-years and, of other CMV disease, 0.2 cases per 100 child-years. ${ }^{27}$ The rate varied significantly by CD4 Tlymphocyte (CD4) cell percentage; the incidence of CMV retinitis was 1.1 cases per 100 child-years in children with CD4 percentage $<15 \%$, compared with 0.1 case per 100 child-years in children with CD4 percentage $>25 \%$. In the same cohort during the cART era, the overall rate of CMV retinitis was $<0.5$ per 100 child-years. ${ }^{28}$ In the Perinatal AIDS Collaborative Transmission Study, the incidence of nonocular CMV before and after January 1997 (pre- and post-cART eras) was 1.4 per 100 child-years and 0.1 per 100 child-years, respectively. ${ }^{29}$ Similarly, CMV retinitis declined from 0.7 to 0.0 per 100 child-years.

Symptomatic HIV-infected children coinfected with CMV have a higher rate of CMV viruria than do asymptomatic HIV-infected or HIV-exposed children. Overall, up to $60 \%$ of children with AIDS shed CMV. This compares with one third of all HIV-infected children; 
$15 \%$ to $20 \%$ of $\mathrm{CMV}$-infected, HIV-exposed but uninfected children; and $<15 \%$ of CMVinfected infants not exposed to HIV. ${ }^{30}$

\section{Clinical Manifestations}

Approximately $10 \%$ of infants with in utero CMV infection are symptomatic at birth with congenital CMV syndrome (i.e., CMV inclusion disease). The rate of symptomatic CMV infection among infants infected with CMV in utero is higher in HIV-infected infants (23.1\%) than in HIV-uninfected children (6.7\%) even in the cART era. ${ }^{24}$ In studies of cohorts of neonates with symptomatic congenital CMV disease, conditions commonly observed included size that was small for gestational age, petechiae, jaundice, hepatosplenomegaly, chorioretinitis, microcephaly, intracranial calcifications, and hearing impairment. ${ }^{31,32}$ Mortality of children with symptomatic disease is as high as $30 \%$.

Approximately $40 \%$ to $58 \%$ (and in specific cohorts, as many as 90\%) of infants with symptomatic disease at birth who survive have late complications, including substantial hearing loss, mental retardation, chorioretinitis, optic atrophy, seizures, or learning disabilities. ${ }^{5,9}$ Although most children with in utero CMV infection do not have symptoms at birth, $10 \%$ to $15 \%$ are at risk of later developmental abnormalities, sensorineural hearing loss, chorioretinitis, or neurologic defects. Premature neonates who acquire CMV postnatally can be asymptomatic or can have evidence of disease such as hepatitis, thrombocytopenia, or pneumonitis.

HIV disease seems to progress more quickly in HIV-infected children coinfected with CMV than in those without CMV infection. ${ }^{22,25,33}$ In one study from the pre-cART era, 53\% of infants coinfected with HIV and CMV had progression to AIDS or had died by age 18 months, compared with $22 \%$ of HIV-infected children without CMV infection; those with HIV/CMV coinfection also were more likely to have central nervous system (CNS) manifestations (36\% versus 9\%). The relative risk of HIV disease progression in children coinfected with CMV compared with children without CMV was 2.6 (95\% CI: 1.1-6.0). ${ }^{22}$ CMV retinitis is the most frequent severe manifestation of CMV disease among HIVinfected children, accounting for approximately $25 \%$ of CMV AIDS-defining illnesses. CMV retinitis among young HIV-infected children is frequently asymptomatic and discovered on routine examination. Older children with CMV retinitis present similarly to adults, with floaters, loss of peripheral vision, or reduction in central vision. Diagnosis of CMV retinitis is based on clinical appearance with white and yellow retinal infiltrates and associated retinal hemorrhages. A more indolent, granular retinitis also can occur. HIVinfected children with CD4 cell counts $<100$ cells $/ \mathrm{mm}^{3}$ are more likely than those with higher CD4 cell counts to develop CMV retinitis; however, CD4 cell count is less predictive of risk of CMV disease in young infants, and systemic and localized CMV disease can occur in HIV-infected infants with higher, age-adjusted CD4 cell counts. ${ }^{30,34}$

End-organ CMV disease has been reported in the lung, liver, gastrointestinal (GI) tract, pancreas, kidney, sinuses, and CNS of HIV-infected children but is rare in the era of cART. ${ }^{34-37}$ In children with extraocular CMV disease, predominantly nonspecific symptoms (e.g., fever, poor weight gain, loss of developmental milestones with laboratory abnormalities of anemia, thrombocytopenia, and elevated lactic dehydrogenase) are initially

Pediatr Infect Dis J. Author manuscript; available in PMC 2014 November 01. 
observed, although the extent to which CMV or HIV infection themselves contribute to these findings is unclear. ${ }^{30} \mathrm{GI}$ manifestations among HIV-infected children include CMV colitis (the most common GI manifestation), oral and esophageal ulcers, hepatitis, ascending cholangiopathy, or gastritis. Odynophagia is a common presentation of CMV esophagitis, whereas abdominal pain and hematochezia frequently occur with CMV colitis.

Sigmoidoscopy in CMV colitis is nonspecific, demonstrating diffuse erythema, submucosal hemorrhage, and diffuse mucosal ulcerations. Esophageal or colonic ulcerations may cause perforation or hemorrhage.

The role of CMV in pulmonary disease among HIV-infected children is difficult to assess because it often is isolated with other organisms (e.g., Pneumocystis jirovecii). Histologic evidence of CMV disease is needed to determine whether active disease is present. CMV pneumonia is an interstitial process with gradual onset of shortness of breath and dry, nonproductive cough; auscultatory findings may be minimal.

CNS manifestations of CMV include subacute encephalopathy, myelitis, and polyradiculopathy (primarily observed in adults but rarely reported in children). The subacute or chronic encephalopathy of CMV can be difficult to differentiate clinically from HIV dementia, with symptoms of confusion and disorientation attributable to cortical involvement. Focal signs can be attributed to lesions in the brainstem. Cerebrospinal fluid (CSF) findings are nonspecific and may include leukocytosis with polymorphonuclear predominance ( $>50 \%$ of patients), elevated protein (75\%), and low glucose $(30 \%)$. However, up to $20 \%$ of children with CMV CNS involvement have completely normal CSF indices. CMV also can cause a rapidly progressive, often fatal, CNS disease with cranial nerve deficits, nystagmus, and increasing ventricular size. ${ }^{38}$

\section{Diagnosis}

It can be difficult to distinguish CMV infection from CMV disease in HIV-infected children. Because of transplacental transfer of antibody, a positive CMV immunoglobulin G (IgG) antibody assay in an infant aged $<12$ months can indicate infection in the mother but not necessarily in the infant. In an infant aged $>12$ months, a positive CMV IgG antibody assay indicates CMV infection of the child but not necessarily active disease. In children of any age, a positive CMV culture or polymerase chain reaction (PCR) assay indicates infection but not necessarily disease.

CMV can be isolated in cell culture from peripheral blood leukocytes, body fluids (e.g., urine, saliva), or tissues. Using centrifugation-assisted shell vial culture amplification techniques, CMV can be detected within 16 to 40 hours of culture inoculation. A positive blood buffy-coat culture establishes CMV infection and increases the likelihood that disease or symptoms were caused by CMV because children with positive blood cultures are at higher risk of end-organ disease. Recovery of virus from tissues (e.g., with endoscopically guided biopsies of GI or pulmonary tissue) provides evidence of disease causation in symptomatic patients. The limitation of this method is that detection of visible cytopathic effects in cell culture takes 1 to 6 weeks. Staining of shell vial culture with CMV monoclonal antibodies or tissue immunostaining for CMV antigens can allow earlier diagnosis of infection. Histopathology demonstrates characteristic "owl's eye" intranuclear 
and smaller intracytoplasmic inclusion bodies in biopsy specimens. Staining with monoclonal antibodies for CMV antigens also can be done on cells obtained from bronchoalveolar lavage.

Different methods have been used to detect viral antigen or DNA directly and to identify patients at risk of CMV disease; these include detection of pp65 antigenemia, qualitative and quantitative PCR, and DNA hybridization. The DNA assays are more sensitive than buffy coat or urine cultures for detecting CMV and can be used to identify patients at higher risk of clinically recognizable disease. CMV DNA detection in CSF by DNA PCR is highly sensitive for CMV CNS disease. Quantitative DNA PCR can be used as a marker for risk of disease and to monitor response to therapy. ${ }^{39}$ Anticipated international standardization of PCR assays for CMV DNA may allow for the establishment of quantitative PCR breakpoints that correlate with CMV disease and facilitate monitoring response to therapy. The National Institute of Standards and Technology and the World Health Organization Expert Committee on Biological Standardization recently developed reference standards for assays for CMV DNA. ${ }^{40,41}$

To diagnose congenital CMV infection, the gold standard remains a positive viral culture from saliva or urine within the first 21 days of life. Beyond this age, positive cultures can be due to postnatally acquired CMV infection. Other methodologies to diagnose congenital CMV infection (blood or saliva PCR) have been investigated but do not yet replace culture as a recommended diagnostic standard. ${ }^{42,43}$ To diagnose acquired CMV disease, culture, antigenemia, and PCR can be used to provide supportive laboratory evidence for clinically suspected CMV disease. However, these tests may be positive in the absence of clinical disease, due to asymptomatic reactivations, and therefore do not themselves constitute a diagnosis of CMV disease in the absence of clinical findings. Alternatively, localized CMV disease (e.g., GI disease) may not manifest with positive blood tests and laboratory diagnosis may require direct sampling of the involved organ for CMV testing.

\section{Prevention Recommendations}

Preventing Exposure-HIV-exposed infants and HIV-infected children, adolescents, and adults who are seronegative for CMV and require blood transfusion should be administered only CMV antibody-negative or leukocyte-reduced cellular blood products in nonemergency situations (BIII).

HIV-infected adults and adolescents who are child care providers or parents of children in child care facilities should be informed that they are at increased risk of CMV infection (BII*). Risk of CMV infection can be diminished by optimal hygienic practices (e.g., handwashing) (AIII). Sexually active adolescents are at risk of CMV acquisition through oraloral contact (kissing) and genital-genital contact; the latter risk may be decreased with condom use.

Preventing First Episode of Disease-The primary methods of preventing severe CMV disease are prevention of severe immunosuppression by treating with cART and recognition of the early manifestations of disease. CMV antibody testing is recommended at age 1 year and then annually thereafter for CMV-seronegative HIV-infected infants and 
children who are immunosuppressed (e.g., CD4 cell count $<100$ cells $/ \mathrm{mm}^{3}$ or CD4 percentage $<10 \%$ ) (BII). HIV-infected children aged $<5$ years who are CMV-infected and severely immunosuppressed (e.g., CD4 cell count $<50$ cells $/ \mathrm{mm}^{3}$ or CD4 percentage $<5 \%$ ) should have a dilated retinal examination performed by an ophthalmologist every 6 months (AIII). Older children should be counseled to report floaters in the eye and visual changes, similar to the recommendation for adults (BIII). Since the advent of cART, CMV end-organ disease has diminished to such an extent that primary prophylaxis with antiviral agents in CMV- and HIV-coinfected people usually is not recommended (BIII). CMV end-organ disease is best prevented by ART to maintain the CD4 cell count $>100$ cells $/ \mathrm{mm}^{3}$ (CD4 percentage $>10 \%$ in children $<6$ years). If this is not possible, prophylaxis with valganciclovir can be considered for HIV-infected children aged $\searrow 6$ years and adolescents who are CMV-seropositive and have CD4 cell counts of $<50$ cells $/ \mathrm{mm}^{3}$, and for young HIVinfected children aged $<6$ years who are CMV-seropositive and have a CD4 percentage $<5 \%$ (CIII). Data supporting the efficacy of antiviral prophylaxis against CMV in pediatric HIVinfected patients are lacking, however, and CMV disease has been observed in children with higher CD4 cell counts than those suggested for primary prophylaxis. ${ }^{27} \mathrm{~A}$ randomized study of ganciclovir prophylaxis in adult patients with AIDS and low CD4 counts did not show efficacy, and ganciclovir is associated with hematologic toxicity. ${ }^{44}$ Therefore, ART remains the preferred approach to prevent CMV disease in HIV-infected children.

Valganciclovir dosing in neonates and young infants has been defined in non-HIV-infected patients with symptomatic congenital CMV disease, with a $16 \mathrm{mg} / \mathrm{kg}$ body weight dose of oral valganciclovir producing similar systemic exposure to a $6 \mathrm{mg} / \mathrm{kg}$ body weight dose of intravenous (IV) ganciclovir. ${ }^{45}$ In children aged 4 months to 16 years, the dose should be based upon body surface area (BSA) and creatinine clearance $(\mathrm{CrCl})$, with the dose in milligrams $=7 \times \mathrm{BSA} \times \mathrm{CrCl}$ (calculated using a modified Schwartz formula); if the calculated Schwartz $\mathrm{CrCl}$ exceeds $150 \mathrm{~mL} / \mathrm{min} / 1.73 \mathrm{~m}^{2}$, then a maximum value of 150 $\mathrm{mL} / \mathrm{min} / 1.73 \mathrm{~m}^{2}$ should be used in the equation. ${ }^{46}$ All calculated doses should be rounded to the nearest 25-mg increment for the actual deliverable dose. If the calculated dose exceeds $900 \mathrm{mg}$, a maximum dose of $900 \mathrm{mg}$ should be administered. Valganciclovir oral solution is the preferred formulation for children aged 4 months to 16 years because it provides the ability to administer a dose calculated according to the formula above; however, valganciclovir tablets can be used if the calculated doses are within $10 \%$ of available tablet strength (450 mg).

Asymptomatic congenital CMV infection is associated with late-onset hearing loss in HIVuninfected children. ${ }^{32}$ Therefore, infants of mothers who were infected with CMV during pregnancy or those in whom in utero HIV transmission has been documented should be evaluated for the presence of congenital, asymptomatic CMV infection by urine shell vial testing (CIII). Some experts recommend testing all infants born to HIV-infected mothers for congenital CMV infection, because HIV transmission to infants may not be clearly defined within the 21-day window for congenital CMV testing. Infants with congenital CMV infection (symptomatic and asymptomatic) should be evaluated for hearing loss at 6-month intervals for at least the first 3 years of life (AII) ${ }^{47}$ 
Discontinuing Primary Prophylaxis-Because primary prophylaxis with antiviral agents in individuals coinfected with CMV and HIV usually is not recommended (as discussed above), consideration of discontinuing primary prophylaxis usually is unnecessary. When valganciclovir primary prophylaxis is provided, cessation of prophylactic treatment can be considered when the CD4 cell count is $>100$ cells $/ \mathrm{mm}^{3}$ for children aged $\ 6$ years, or CD4 percentage $>10 \%$ in children aged $<6$ years (CIII).

\section{Treatment Recommendations}

Treating Disease-Treatment of newborns who have symptomatic congenital CMV disease involving the CNS with IV ganciclovir for 6 weeks has been evaluated in a series of clinical trials conducted by the National Institute of Allergy and Infectious Diseases Collaborative Antiviral Study Group ${ }^{48,49}$ all infants in these studies were HIV-uninfected. Infants receiving therapy cleared their urine of CMV by culture by the end of the 6-week treatment period, but they all experienced a rebound in their viruria after the drug was discontinued. ${ }^{48}$ In a Phase III, randomized, controlled trial, infants with CNS disease who received IV ganciclovir for 6 weeks were less likely to have hearing deterioration over the first 2 years of life than were infants receiving no antiviral therapy. ${ }^{49}$ Treated infants also had more rapid resolution of liver enzyme abnormalities and a greater degree of growth during the course of therapy. They also experienced fewer neurodevelopmental delays at 1 year of life than did untreated infants. ${ }^{50}$ However, approximately two-thirds of the infants developed substantial neutropenia during therapy. ${ }^{49}$ Among patients developing neutropenia, $48 \%$ required dose modification, but most were able to complete the 6 weeks of therapy.

On the basis of these results, IV ganciclovir therapy $(6 \mathrm{mg} / \mathrm{kg}$ body weight $/ \mathrm{dose}$ administered every 12 hours) for 6 weeks can be considered for HIV-exposed or HIVinfected infants who have symptomatic congenital CMV disease involving the CNS (BI). If during the 6 weeks of therapy an infant is confirmed as HIV infected, some experts might recommend treatment for a longer period ( $>6$ weeks), but the benefit of extended therapy is unproven (CIII). A controlled trial conducted by the Collaborative Antiviral Study Group of 6 weeks versus 6 months of oral valganciclovir in HIV-uninfected infants with symptomatic congenital CMV disease is nearing completion. Neonates with symptomatic congenital CMV disease can be referred to a pediatric infectious diseases specialist for consideration of ganciclovir or valganciclovir therapy and long-term monitoring for sequelae (AI). ${ }^{45,49}$

CMV retinitis should be managed in collaboration with an experienced ophthalmologist and CMV treatment should be instituted in addition to cART. IV ganciclovir, oral valganciclovir, IV foscarnet, and IV cidofovir, and the ganciclovir intraocular implant coupled with valganciclovir are all effective treatments for CMV retinitis in HIV-infected adults (AI*). ${ }^{51-55}$ Ganciclovir intraocular implant, however, is no longer available from the manufacturer for treatment with CMV retinitis. For HIV-infected infants and children, IV ganciclovir is the drug of choice for initial treatment (induction therapy) for acquired CMV disease, including CMV retinitis and other end-organ disseminated CMV disease (e.g., colitis, esophagitis, CNS disease) (AI*). Oral valganciclovir, a prodrug of ganciclovir, is one of the first-line treatments for HIV-infected adults with CMV retinitis $\left(\mathbf{A I}^{*}\right)^{53}$ and is an 
option in older children who weigh enough to receive the adult dose and tablet formulation of valganciclovir (CIII). The drug is well absorbed from the GI tract and rapidly metabolized to ganciclovir in the intestine and liver. Valganciclovir oral solution has not been studied in pediatric patients for treatment of CMV retinitis, but consideration can be given to its use for transitioning from IV ganciclovir to oral valganciclovir to complete treatment and/or for secondary prophylaxis once improvement in retinitis is noted (CIII).

An alternative drug for treating CMV disease or for use in ganciclovir-resistant CMV infections in HIV-infected children is foscarnet (AI*). Foscarnet used as suppressive therapy has been associated with increased length of survival relative to ganciclovir in HIV-infected adults. Doses should be modified in patients with renal insufficiency. Cidofovir is effective in treating CMV retinitis in adults who are intolerant of other therapies. Cidofovir has not been studied in children with CMV disease, but can be considered when other options cannot be used (CIII).

Combination therapy with ganciclovir and foscarnet delays progression of retinitis in certain patients in whom monotherapy fails ${ }^{34,53,56,57}$ and can be used as initial therapy in children with sight-threatening disease (BIII). Combination therapy also has been used for adults with retinitis that has relapsed on single-agent therapy. However, substantial rates of adverse effects are associated with combination therapy.

Intravitreous injections of ganciclovir, foscarnet, or cidofovir have been used to control retinitis, but biweekly intraocular injections are required. Data are limited in children, and biweekly injection is impractical for use in most children (BIII). Implantation of an intravitreous ganciclovir medication-release device in the posterior chamber of the eye also has been used in HIV-infected adults and adolescents. In adults, the combination of oral valganciclovir with a ganciclovir sustained-release intraocular implant, replaced every 6 to 9 months, was superior to daily IV ganciclovir in preventing relapse of retinitis, and intraocular ganciclovir implant plus IV ganciclovir or oral valganciclovir was preferred by some adult HIV specialists for initial treatment of patients who have sight-threatening CMV lesions adjacent to the optic nerve or fovea (AI) ${ }^{51-55}$ Use of systemic therapy in addition to the ocular implant may reduce development of retinitis in the contralateral eye. Because the ganciclovir implant is no longer available from the manufacturer, this route of administration is currently not available for treatment and chronic suppression of CMV retinitis in older children large enough to receive the intraocular implant and oral valganciclovir.

Small peripheral lesions can be treated with systemic therapy without local treatment (BII*). Intraocular implants have not been studied in patients younger than age 9 years and were not recommended in children aged $<3$ years because of the small size of their eyes (AIII).

Intraocular cidofovir is not recommended in children because of lack of data and the risk of hypotony in adults (AIII).

For acquired CMV neurologic disease, prompt initiation of therapy is critical for an optimal clinical response, as well as ART to enable immune reconstitution. Levels of ganciclovir in the CSF are $24 \%$ to $70 \%$ of plasma levels, and levels in the brain are approximately $38 \%$ of 
plasma levels. ${ }^{58}$ Foscarnet concentrations in the CSF are about two-thirds of those in serum. ${ }^{59}$ Hence, combination treatment with ganciclovir and foscarnet may be preferable as initial therapy to stabilize disease and maximize response (BII*). ${ }^{60}$ However, this approach is associated with substantial rates of adverse effects, and optimal treatment for neurologic disease in children receiving optimized cART is unknown.

Patients with AIDS and recipients of solid organ transplants who have GI disease attributed to CMV appear to benefit from ganciclovir therapy (AI*). ${ }^{61,62}$ Limited and uncontrolled data suggest that ganciclovir therapy is useful in patients with AIDS and CMV pneumonia (BII*). ${ }^{63}$ As with other CMV disease, antiviral management for CMV disease should also include cART.

\section{Monitoring Response to Therapy and Adverse Events (Including IRIS)—CMV}

retinitis should be managed in concert with an experienced ophthalmologist.

Recommendations for HIV-infected adults include indirect ophthalmoscopy through a dilated pupil performed at diagnosis of CMV retinitis, after completion of induction therapy, 1 month after initiation of therapy, and monthly thereafter while patients are on anti-CMV treatment; recommendations should be similar for HIV-infected children with CMV retinitis (AIII). Monthly fundus photographs using a standardized photographic technique that documents the appearance of the retina provide the optimum method for following patients and detecting early relapse (AIII). For patients who have experienced immune recovery, the frequency of ophthalmologic follow-up can be decreased to every 3 months. However, because relapse of retinitis can occur in patients with immune recovery, regular ophthalmologic follow-up still is needed.

The major side effects of ganciclovir and valganciclovir are myelosuppression (i.e., anemia, neutropenia, and thrombocytopenia) and renal toxicity. Dose reduction or interruption because of hematologic toxicity may be necessary in up to $40 \%$ of patients receiving IV ganciclovir; granulocyte colony-stimulating factor can be used to ameliorate neutropenia. The main toxicities of foscarnet are decreased renal function and metabolic derangements. Renal toxicity and foscarnet binding to divalent metal ions, such as calcium, lead to metabolic abnormalities in approximately one-third of patients, and serious electrolyte imbalances (including abnormalities in calcium, phosphorus, magnesium, and potassium levels) and secondary seizures, cardiac dysrhythmias, abnormal liver transaminases, and CNS symptoms can occur. Metabolic disturbances can be minimized if foscarnet is administered by slow infusion, with rates not exceeding $1 \mathrm{mg} / \mathrm{kg} /$ minute. Concomitant use of other nephrotoxic drugs increases the likelihood of renal dysfunction associated with foscarnet therapy. For patients receiving ganciclovir, valganciclovir, or foscarnet, complete blood counts and serum electrolytes and renal function should be monitored twice weekly during induction therapy and once weekly thereafter (AIII).

The major side effect of cidofovir is potentially irreversible nephrotoxicity; the drug produces proximal tubular dysfunction including proteinuria, glycosuria, Fanconi syndrome, and acute renal failure. To minimize nephrotoxicity, probenecid should be administered before each infusion, and IV hydration with normal saline should be administered before and after each cidofovir infusion. For patients receiving IV cidofovir, blood urea nitrogen, 
creatinine, and urinalysis should be performed before each infusion; administration of the drug is contraindicated if renal dysfunction or proteinuria is detected. Other reported adverse events include anterior uveitis and ocular hypotony; serial ophthalmologic monitoring for anterior segment inflammation and intraocular pressure is needed while receiving the drug systemically. Cidofovir should not be administered concomitantly with other nephrotoxic agents. Cidofovir therapy must be discontinued if serum creatinine increases $\searrow 0.5 \mathrm{mg} / \mathrm{dL}$ above baseline.

Immune recovery uveitis after initiation of effective cART is an immunologic reaction to CMV associated with inflammation in the anterior chamber and/or the vitreous and therefore is a form of immune reconstitution inflammatory syndrome (IRIS) ${ }^{64}$ Ocular complications of uveitis include macular edema and development of epiretinal membranes, which can cause loss of vision. Patients with low CD4 cell counts who are starting cART are at risk of IRIS. Frequent surveillance ophthalmologic examination is warranted during the period of immune reconstitution in children who are unable to report symptoms, and ophthalmologic examination is indicated for children able to report vision changes who develop symptoms. Immune recovery uveitis may respond to periocular corticosteroids or a short course of systemic steroids. Oral valganciclovir was beneficial in one small uncontrolled study. ${ }^{65}$

Managing Treatment Failure-Resistant strains of CMV should be suspected when progressive disease and continued recovery of virus occurs despite ganciclovir therapy. Foscarnet is the drug of choice when ganciclovir resistance is suspected (AI*).

In patients with CMV retinitis, although drug resistance occurs in patients receiving longterm therapy, early relapse may be caused by the limited intraocular penetration of systemically administered drugs. In HIV-infected adults whose retinitis has relapsed during systemic treatment, placement of a ganciclovir implant was recommended because it achieved higher drug levels in the eye and often would control the retinitis for 6 to 8 months until the implant required replacement; however, the ganciclovir implant is no longer available from the manufacturer. Early first relapse of retinitis should be treated with reinduction with the same drug, followed by reinstitution of maintenance therapy (AII*). However, if drug resistance is suspected or if side effects or toxicities interfere with optimal courses of the initial agent, change to an alternative drug is reasonable (AIII). Combination ganciclovir and foscarnet can be considered but is accompanied by greater toxicity.

Preventing Recurrence-Courses of antiviral agents (e.g., ganciclovir, valganciclovir, foscarnet, cidofovir) do not cure CMV infection. After induction therapy, secondary prophylaxis (chronic maintenance therapy) is given for most forms of CMV disease until immune reconstitution, or in the absence of immune reconstitution, for the remainder of patients' lives (AI*).

Regimens that can be considered for chronic suppression in adults and adolescents include IV ganciclovir, oral valganciclovir, IV foscarnet, combined IV ganciclovir and foscarnet, and parenteral cidofovir; these regimens also are recommended for children (AI*). ${ }^{66-73}$ Repetitive intravitreous injections of ganciclovir, foscarnet, and cidofovir reportedly are effective for secondary prophylaxis of CMV retinitis, ${ }^{74,75}$ although intraocular therapy alone 
does not protect the contralateral eye or other organ systems and therefore typically is combined with systemic treatment. ${ }^{66}$ Frequent intravitreous injections also are impractical for use in most children (AIII).

A chronic maintenance regimen for patients treated for CMV disease should be chosen in consultation with a specialist. Chronic maintenance therapy is not routinely recommended for GI disease but should be considered if relapses occur $\left(\mathbf{B I I}^{*}\right)$. A role for maintenance therapy for CMV pneumonitis has not been established (CIII). For patients with retinitis, decisions should be made in consultation with an ophthalmologist, taking into consideration the anatomic location of the retinal lesion, vision in the contralateral eye, and patients' immunologic and virologic status (BIII).

Discontinuing Secondary Prophylaxis-Multiple case series have reported that maintenance therapy can be discontinued safely in adults and adolescents with CMV retinitis whose CD4 cell counts have increased substantially in response to cART. ${ }^{76-81}$ These patients have remained disease free for $>30$ and up to 95 weeks of follow up, whereas during the precART era, retinitis typically reactivated in $<6$ to 8 weeks after stopping CMV therapy. Plasma HIV RNA levels varied among these patients, supporting the hypothesis that the CD4 cell count is the primary determinant of immune recovery to CMV. However, CMV retinitis can occur in cART-treated adults with high CD4 cell counts, ${ }^{82}$ suggesting that CMV-specific cellular immunity may be important in controlling CMV in immunereconstituted HIV-infected adults ${ }^{83,84}$ and reinforcing the importance of ongoing monitoring. In HIV-infected adults with CMV retinitis, discontinuation of secondary prophylaxis can be considered for patients with a sustained increase in CD4 cell count to $>100$ cells $/ \mathrm{mm}^{3}$ in response to ART.

The safety of discontinuing secondary prophylaxis after immune reconstitution with ART in HIV-infected children has not been as well studied. Low or undetectable HIV replication in children is the strongest correlate with CMV immune reconstitution and a higher frequency of CMV-specific CD4 cells. ${ }^{85}$ Early institution of cART may help control CMV infection by maintaining normal CD4 cell count and cytotoxic T-lymphocyte responses in HIV-infected children. ${ }^{86}$ In deciding whether to discontinue secondary prophylaxis, consideration must be given to the significant toxicities associated with antiviral drugs active against CMV, including those in in vitro and animal models.

Recognizing the limitations of the data in children but drawing on the growing experience in adults, discontinuing prophylaxis can be considered in children who are receiving ART and have a sustained (i.e., $>6$ months) increase in CD4 percentage to $>15 \%$ in children aged $<6$ years, or for children aged $\Varangle 6$ years (as for adults), an increase in CD4 cell count to $>100$ cells $/ \mathrm{mm}^{3}$ (CIII). When the manifestation of CMV disease is ocular, such decisions should be made in close consultation with an ophthalmologist and should account for factors such as magnitude and duration of CD4 cell count increase, anatomic location of the retinal lesion, vision in the contralateral eye, and the feasibility of regular ophthalmologic monitoring (CIII). 
All patients with CMV ophthalmic disease in whom anti-CMV maintenance therapy has been discontinued should continue to undergo regular ophthalmologic monitoring at 3- to 6month intervals for early detection of CMV relapse and for immune reconstitution uveitis (AII*). For patients with any CMV disease, CMV viral load or other markers of CMV infection (such as antigenemia or viral DNA tests) are not well standardized; their role in predicting relapse remains to be defined, and they are not recommended for routine monitoring (BIII)..$^{87,88}$

Reinitiating Secondary Prophylaxis-Relapse of CMV retinitis occurs in adults whose anti-CMV maintenance therapies have been discontinued and whose CD4 cell counts have decreased to $<50$ cells $/ \mathrm{mm}^{3} .{ }^{74}$ Reinstitution of secondary prophylaxis is recommended for HIV-infected adults when their CD4 cell counts fall to $<100$ cells $/ \mathrm{mm}^{3}$. For HIV-infected children in whom secondary prophylaxis has been discontinued because of immune reconstitution, secondary prophylaxis should be reinstituted in those aged $<6$ years when the CD4 percentage decreases to $<15 \%$, and in those aged $\ 6$ years when the CD4 cell count decreases to $<100$ cells $/ \mathrm{mm}^{3}$ (BIII).

\section{References}

1. Stagno S, Reynolds DW, Pass RF, Alford CA. Breast milk and the risk of cytomegalovirus infection. N Engl J Med. 1980 May 8; 302(19):1073-1076. Available at http:// www.ncbi.nlm.nih.gov/pubmed/6245360. [PubMed: 6245360]

2. Pass RF, Hutto SC, Reynolds DW, Polhill RB. Increased frequency of cytomegalovirus infection in children in group day care. Pediatrics. 1984 Jul; 74(1):121-126. Available at http:// www.ncbi.nlm.nih.gov/pubmed/6330661. [PubMed: 6330661]

3. Adler SP. Cytomegalovirus and child day care. Evidence for an increased infection rate among daycare workers. N Engl J Med. 1989 Nov 9; 321(19):1290-1296. Available at http:// www.ncbi.nlm.nih.gov/pubmed/2552316. [PubMed: 2552316]

4. Pass RF. Epidemiology and transmission of cytomegalovirus. J Infect Dis. 1985 Aug; 152(2):243248. Available at http://www.ncbi.nlm.nih.gov/pubmed/2993429. [PubMed: 2993429]

5. Stagno S, Pass RF, Cloud G, et al. Primary cytomegalovirus infection in pregnancy. Incidence, transmission to fetus, and clinical outcome. JAMA. 1986 Oct 10; 256(14):1904-1908. Available at http://www.ncbi.nlm.nih.gov/pubmed/3020264. [PubMed: 3020264]

6. Yow MD, Williamson DW, Leeds LJ, et al. Epidemiologic characteristics of cytomegalovirus infection in mothers and their infants. Am J Obstet Gynecol. 1998; 158(5):1189-1195. Available at http://www.ncbi.nlm.nih.gov/entrez/query.fcgi? $\mathrm{cmd}=$ Retrieve $\& \mathrm{db}=$ PubMed\&dopt=Citation\&list_uids=2835906. [PubMed: 2835906]

7. Mussi-Pinhata MM, Yamamoto AY, Figueiredo LT, Cervi MC, Duarte G. Congenital and perinatal cytomegalovirus infection in infants born to mothers infected with human immunodeficiency virus. J Pediatr. 1998 Feb; 132(2):285-290. Available at http://www.ncbi.nlm.nih.gov/pubmed/9506642. [PubMed: 9506642]

8. Quinn TC, Piot P, McCormick JB, et al. Serologic and immunologic studies in patients with AIDS in North America and Africa. The potential role of infectious agents as cofactors in human immunodeficiency virus infection. JAMA. 1987 May 15; 257(19):2617-2621. Available at http:// www.ncbi.nlm.nih.gov/pubmed/3494857. [PubMed: 3494857]

9. Dollard SC, Grosse SD, Ross DS. New estimates of the prevalence of neurological and sensory sequelae and mortality associated with congenital cytomegalovirus infection. Rev Med Virol. 2007 Sep-Oct;17(5):355-363. Available at http://www.ncbi.nlm.nih.gov/pubmed/17542052. [PubMed: 17542052]

10. Revello MG, Zavattoni M, Furione M, Lilleri D, Gorini G, Gerna G. Diagnosis and outcome of preconceptional and periconceptional primary human cytomegalovirus infections. J Infect Dis. 
2002 Aug 15; 186(4):553-557. Available at http://www.ncbi.nlm.nih.gov/pubmed/12195384. [PubMed: 12195384]

11. Prober CG, Enright AM. Congenital cytomegalovirus (CMV) infections: hats off to Alabama. J Pediatr. 2003 Jul; 143(1):4-6. Available at http://www.ncbi.nlm.nih.gov/pubmed/12915814. [PubMed: 12915814]

12. Fowler KB, Stagno S, Pass RF. Maternal immunity and prevention of congenital cytomegalovirus infection. JAMA. 2003 Feb 26; 289(8):1008-1011. Available at http://www.ncbi.nlm.nih.gov/ pubmed/12597753. [PubMed: 12597753]

13. Azam AZ, Vial Y, Fawer CL, Zufferey J, Hohlfeld P. Prenatal diagnosis of congenital cytomegalovirus infection. Obstet Gynecol. 2001 Mar; 97(3):443-448. Available at http:// www.ncbi.nlm.nih.gov/pubmed/11239654. [PubMed: 11239654]

14. Boppana SB, Fowler KB, Britt WJ, Stagno S, Pass RF. Symptomatic congenital cytomegalovirus infection in infants born to mothers with preexisting immunity to cytomegalovirus. Pediatrics. 1999 Jul; 104(1 Pt 1):55-60. Available at http://www.ncbi.nlm.nih.gov/pubmed/10390260. [PubMed: 10390260]

15. Boppana SB, Rivera LB, Fowler KB, Mach M, Britt WJ. Intrauterine transmission of cytomegalovirus to infants of women with preconceptional immunity. N Engl J Med. 2001 May 3; 344(18):1366-1371. Available at http://www.ncbi.nlm.nih.gov/pubmed/11333993. [PubMed: 11333993]

16. Neuberger P, Hamprecht K, Vochem M, et al. Case-control study of symptoms and neonatal outcome of human milk-transmitted cytomegalovirus infection in premature infants. J Pediatr. 2006 Mar; 148(3):326-331. Available at http://www.ncbi.nlm.nih.gov/pubmed/16615961. [PubMed: 16615961]

17. Kothari A, Ramachandran VG, Gupta P. Cytomegalovirus infection in neonates following exchange transfusion. Indian J Pediatr. 2006 Jun; 73(6):519-521. Available at http:// www.ncbi.nlm.nih.gov/pubmed/16816515. [PubMed: 16816515]

18. Mussi-Pinhata MM, Yamamoto AY, do Carmo Rego MA, Pinto PC, da Motta MS, Calixto C. Perinatal or earlypostnatal cytomegalovirus infection in preterm infants under 34 weeks gestation born to CMV-seropositive mothers within a high-seroprevalence population. J Pediatr. 2004 Nov; 145(5):685-688. Available at http://www.ncbi.nlm.nih.gov/pubmed/15520780. [PubMed: 15520780]

19. Yasuda A, Kimura H, Hayakawa M, et al. Evaluation of cytomegalovirus infections transmitted via breast milk in preterm infants with a real-time polymerase chain reaction assay. Pediatrics. 2003 Jun; 111(6 Pt 1):1333-1336. Available at http://www.ncbi.nlm.nih.gov/pubmed/127775491. [PubMed: 12777549]

20. Vollmer B, Seibold-Weiger K, Schmitz-Salue C, et al. Postnatally acquired cytomegalovirus infection via breast milk: effects on hearing and development in preterm infants. Pediatr Infect Dis J. 2004 Apr; 23(4):322-327. Available at http://www.ncbi.nlm.nih.gov/pubmed/15071286. [PubMed: 15071286]

21. Mostad SB, Kreiss JK, Ryncarz A, et al. Cervical shedding of herpes simplex virus and cytomegalovirus throughout the menstrual cycle in women infected with human immunodeficiency virus type 1. Am J Obstet Gynecol. 2000 Oct; 183(4):948-955. Available at http://www.ncbi.nlm.nih.gov/pubmed/11035345. [PubMed: 11035345]

22. Kovacs A, Schluchter M, Easley K, et al. Cytomegalovirus infection and HIV-1 disease progression in infants born to HIV-1-infected women. Pediatric Pulmonary and Cardiovascular Complications of Vertically Transmitted HIV Infection Study Group. N Engl J Med. 1999 Jul 8; 341(2):77-84. Available at http://www.ncbi.nlm.nih.gov/pubmed/10395631. [PubMed: 10395631]

23. Duryea EL, Sanchez PJ, Sheffield JS, et al. Maternal human immunodeficiency virus infection and congenital transmission of cytomegalovirus. Pediatr Infect Dis J. 2010 Oct; 29(10):915-918. Available at http://www.ncbi.nlm.nih.gov/pubmed/20431424. [PubMed: 20431424]

24. Guibert G, Warszawski J, Le Chenadec J, et al. Decreased risk of congenital cytomegalovirus infection in children born to HIV-1-infected mothers in the era of highly active antiretroviral therapy. Clin Infect Dis. 2009 Jun 1; 48(11):1516-1525. Available at http:// www.ncbi.nlm.nih.gov/pubmed/19388872. [PubMed: 19388872] 
25. Kitchen BJ, Engler HD, Gill VJ, et al. Cytomegalovirus infection in children with human immunodeficiency virus infection. Pediatr Infect Dis J. 1997 Apr; 16(4):358-363. Available at http://www.ncbi.nlm.nih.gov/pubmed/9109136. [PubMed: 9109136]

26. Jabs DA, Van Natta ML, Holbrook JTKJ, Meinert CL, Davis MD. Longitudinal Study of the Ocular Complications of AIDS 1. Ocular Diagnoses at Enrollment. Ophthalmology 2007. 2007 Jan 24.

27. Dankner WM, Lindsey JC, Levin MJ. Pediatric ACTGPT. Correlates of opportunistic infections in children infected with the human immunodeficiency virus managed before highly active antiretroviral therapy. Pediatr Infect Dis J. 2001 Jan; 20(1):40-48. Available at http:// www.ncbi.nlm.nih.gov/pubmed/11176565. [PubMed: 11176565]

28. Gona P, Van Dyke RB, Williams PL, et al. Incidence of opportunistic and other infections in HIVinfected children in the HAART era. JAMA. 2006 Jul 19; 296(3):292-300. Available at http:// www.ncbi.nlm.nih.gov/pubmed/16849662. [PubMed: 16849662]

29. Nesheim SR, Kapogiannis BG, Soe MM, et al. Trends in opportunistic infections in the pre- and post-highly active antiretroviral therapy eras among HIV-infected children in the Perinatal AIDS Collaborative Transmission Study 1986-2004. Pediatrics. 2007 Jul; 120(1):100-109. Available at http://www.ncbi.nlm.nih.gov/pubmed/17606567. [PubMed: 17606567]

30. Chandwani S, Kaul A, Bebenroth D, et al. Cytomegalovirus infection in human immunodeficiency virus type 1-infected children. Pediatr Infect Dis J. 1996 Apr; 15(4):310-314. Available at http:// www.ncbi.nlm.nih.gov/pubmed/8866799. [PubMed: 8866799]

31. Boppana SB, Pass RF, Britt WJ, Stagno S, Alford CA. Symptomatic congenital cytomegalovirus infection: neonatal morbidity and mortality. Pediatr Infect Dis J. 1992 Feb; 11(2):93-99. Available at http://www.ncbi.nlm.nih.gov/pubmed/1311066. [PubMed: 1311066]

32. Fowler KB, Boppana SB. Congenital cytomegalovirus (CMV) infection and hearing deficit. J Clin Virol. 2006 Feb; 35(2):226-231. Available at http://www.ncbi.nlm.nih.gov/pubmed/16386462. [PubMed: 16386462]

33. Doyle M, Atkins JT, Rivera-Matos IR. Congenital cytomegalovirus infection in infants infected with human immunodeficiency virus type 1. Pediatr Infect Dis J. 1996 Dec; 15(12):1102-1106. Available at http://www.ncbi.nlm.nih.gov/pubmed/8970220. [PubMed: 8970220]

34. Zaknun D, Zangerle R, Kapelari K, Fischer H, Sailer M, McIntosh K. Concurrent ganciclovir and foscarnet treatment for cytomegalovirus encephalitis and retinitis in an infant with acquired immunodeficiency syndrome: case report and review. Pediatr Infect Dis J. 1997 Aug; 16(8):807811. Available at http://www.ncbi.nlm.nih.gov/pubmed/9271045. [PubMed: 9271045]

35. Mueller BU, MacKay K, Cheshire LB, et al. Cytomegalovirus ureteritis as a cause of renal failure in a child infected with the human immunodeficiency virus. Clin Infect Dis. 1995 Apr; 20(4): 1040-1043. Available at http://www.ncbi.nlm.nih.gov/pubmed/7795047. [PubMed: 7795047]

36. Olivero MT, Nelson RP Jr, Andrews T, Washington K, Good RA. Cytomegalovirus sinus disease in a human immunodeficiency virus-infected child. Pediatr Infect Dis J. 1995 Jul; 14(7):629-631. Available at http://www.ncbi.nlm.nih.gov/pubmed/7567298. [PubMed: 7567298]

37. Marriage SC, Booy R, Hermione Lyall EG, et al. Cytomegalovirus myelitis in a child infected with human immunodeficiency virus type 1. Pediatr Infect Dis J. 1996 Jun; 15(6):549-551. Available at http://www.ncbi.nlm.nih.gov/pubmed/8783359. [PubMed: 8783359]

38. Kalayjian RC, Cohen ML, Bonomo RA, Flanigan TP. Cytomegalovirus ventriculoencephalitis in AIDS. A syndrome with distinct clinical and pathologic features. Medicine (Baltimore). 1993 Mar; 72(2):67-77. Available at http://www.ncbi.nlm.nih.gov/pubmed/8386795. [PubMed: 8386795]

39. Nigro G, Krzysztofiak A, Gattinara GC, et al. Rapid progression of HIV disease in children with cytomegalovirus DNAemia. AIDS. 1996 Sep; 10(10):1127-1133. Available at http:// www.ncbi.nlm.nih.gov/pubmed/8874630. [PubMed: 8874630]

40. Haynes RJ, Kline MC, Toman B, et al. Standard reference material 2366 for measurement of human cytomegalovirus DNA. The Journal of molecular diagnostics: JMD. 2013 Mar; 15(2):177185. Available at http://www.ncbi.nlm.nih.gov/pubmed/23321018. [PubMed: 23321018]

41. Fryer, JF.; Heath, AB.; Anderson, R.; Minor, PD. Collaborative Study to Evaluate the Proposed 1st WHO International Standard for Human Cytomegalovirus (HCMV) for Nucleic Acid 
Amplification (NAT)-Based Assays. 2010. Available at http://www.nibsc.ac.uk/PDF/ HCMV_IS.pdf.

42. Boppana SB, Ross SA, Novak Z, et al. Dried blood spot real-time polymerase chain reaction assays to screen newborns for congenital cytomegalovirus infection. JAMA. 2010 Apr 14; 303(14):1375-1382. Available at http://www.ncbi.nlm.nih.gov/pubmed/20388893. [PubMed: 20388893]

43. Boppana SB, Ross SA, Shimamura M, et al. Saliva polymerase-chain-reaction assay for cytomegalovirus screening in newborns. N Engl J Med. 2011 Jun 2; 364(22):2111-2118. Available at http://www.ncbi.nlm.nih.gov/pubmed/21631323. [PubMed: 21631323]

44. Brosgart CL, Louis TA, Hillman DW, et al. A randomized, placebo-controlled trial of the safety and efficacy of oral ganciclovir for prophylaxis of cytomegalovirus disease in HIV-infected individuals. Terry Beirn Community Programs for Clinical Research on AIDS. AIDS. 1998 Feb 12; 12(3):269-277. Available at http://www.ncbi.nlm.nih.gov/pubmed/9517989. [PubMed: 9517989]

45. Kimberlin DW, Acosta EP, Sanchez PJ, et al. Pharmacokinetic and pharmacodynamic assessment of oral valganciclovir in the treatment of symptomatic congenital cytomegalovirus disease. J Infect Dis. 2008 Mar 15; 197(6):836-845. Available at http://www.ncbi.nlm.nih.gov/pubmed/18279073. [PubMed: 18279073]

46. Genetech. Valganciclovir Package Insert. 2010 , http://www.gene.com/gene/products/information/ valcyte/pdf/pi.pdf. Available at http://www.gene.com/gene/products/information/valcyte/pdf/ pi.pdf.

47. Kadambari S, Williams EJ, Luck S, Griffiths PD, Sharland M. Evidence based management guidelines for the detection and treatment of congenital CMV. Early human development. 2011 Nov; 87(11):723-728. Available at http://www.ncbi.nlm.nih.gov/pubmed/21962770. [PubMed: 21962770]

48. Whitley RJ, Cloud G, Gruber W, et al. Ganciclovir treatment of symptomatic congenital cytomegalovirus infection: results of a phase II study. National Institute of Allergy and Infectious Diseases Collaborative Antiviral Study Group. J Infect Dis. 1997 May; 175(5):1080-1086. Available at http://www.ncbi.nlm.nih.gov/pubmed/9129069. [PubMed: 9129069]

49. Kimberlin DW, Lin CY, Sanchez PJ, et al. Effect of ganciclovir therapy on hearing in symptomatic congenital cytomegalovirus disease involving the central nervous system: a randomized, controlled trial. J Pediatr. 2003 Jul; 143(1):16-25. Available at http://www.ncbi.nlm.nih.gov/ pubmed/12915819. [PubMed: 12915819]

50. Oliver SE, Cloud GA, Sanchez PJ, et al. Neurodevelopmental outcomes following ganciclovir therapy in symptomatic congenital cytomegalovirus infections involving the central nervous system. J Clin Virol. 2009 Dec; 46(Suppl 4):S22-S26. Available at http://www.ncbi.nlm.nih.gov/ pubmed/19766534. [PubMed: 19766534]

51. Studies of Ocular Complications of AIDS Research, Group in collaboration with the AIDS Clinical Trials Group. Foscarnet-Ganciclovir Cytomegalovirus Retinitis Trial. 4. Visual outcomes. Ophthalmology. 1994; 101(7):1250-1261. Available at http://www.ncbi.nlm.nih.gov/sites/entrez? $\mathrm{Db}=$ pubmed $\& \mathrm{Cmd}=$ ShowDetailView $\&$ TermToSearch $=8035989 \&$ ordinalpos=9\&itool=EntrezSyst em2.PEntrez.Pubmed.Pubmed_ResultsPanel.Pubmed_RVDocSum. [PubMed: 8035989]

52. Musch DC, Martin DF, Gordon JF, Davis MD, Kuppermann BD. Treatment of cytomegalovirus retinitis with a sustained-release ganciclovir implant. The Ganciclovir Implant Study Group. N Engl J Med. 1997 Jul 10; 337(2):83-90. Available at http://www.ncbi.nlm.nih.gov/pubmed/ 9211677. [PubMed: 9211677]

53. Martin DF, Sierra-Madero J, Walmsley S, et al. A controlled trial of valganciclovir as induction therapy for cytomegalovirus retinitis. N Engl J Med. 2002 Apr 11; 346(15):1119-1126. Available at http://www.ncbi.nlm.nih.gov/pubmed/11948271. [PubMed: 11948271]

54. Kempen JH, Jabs DA, Wilson LA, Dunn JP, West SK, Tonascia JA. Risk of vision loss in patients with cytomegalovirus retinitis and the acquired immunodeficiency syndrome. Arch Ophthalmol. 2003 Apr; 121(4):466-476. Available at http://www.ncbi.nlm.nih.gov/pubmed/12695243. [PubMed: 12695243]

55. Studies of Ocular Complications of AIDS Research Group. The AIDS Clinical Trials Group. The ganciclovir implant plus oral ganciclovir versus parenteral cidofovir for the treatment of 
cytomegalovirus retinitis in patients with acquired immunodeficiency syndrome. Am J Ophthalmol. 2001; 131:475-467.

56. Walton RC, Whitcup SM, Mueller BU, Lewis LL, Pizzo PA, Nussenblatt RB. Combined intravenous ganciclovir and foscarnet for children with recurrent cytomegalovirus retinitis. Ophthalmology. 1995 Dec; 102(12):1865-1870. Available at http://www.ncbi.nlm.nih.gov/ pubmed/9098289. [PubMed: 9098289]

57. Butler KM, De Smet MD, Husson RN, et al. Treatment of aggressive cytomegalovirus retinitis with ganciclovir in combination with foscarnet in a child infected with human immunodeficiency virus. J Pediatr. 1992 Mar; 120(3):483-486. Available at http://www.ncbi.nlm.nih.gov/pubmed/ 1311378. [PubMed: 1311378]

58. Fletcher C, Sawchuk R, Chinnock B, de Miranda P, Balfour HH Jr. Human pharmacokinetics of the antiviral drug DHPG. Clin Pharmacol Ther. 1986 Sep; 40(3):281-286. Available at http:// www.ncbi.nlm.nih.gov/pubmed/3017630. [PubMed: 3017630]

59. Hengge UR, Brockmeyer NH, Malessa R, Ravens U, Goos M. Foscarnet penetrates the blood-brain barrier: rationale for therapy of cytomegalovirus encephalitis. Antimicrob Agents Chemother. 1993 May; 37(5):1010-1014. Available at http://www.ncbi.nlm.nih.gov/pubmed/8390807. [PubMed: 8390807]

60. Anduze-Faris BM, Fillet AM, Gozlan J, et al. Induction and maintenance therapy of cytomegalovirus central nervous system infection in HIV-infected patients. AIDS. 2000 Mar 31; 14(5):517-524. Available at http://www.ncbi.nlm.nih.gov/pubmed/10780714. [PubMed: $10780714]$

61. Dieterich DT, Kotler DP, Busch DF, et al. Ganciclovir treatment of cytomegalovirus colitis in AIDS: a randomized, double-blind, placebo-controlled multicenter study. J Infect Dis. 1993 Feb; 167(2):278-282. Available at http://www.ncbi.nlm.nih.gov/pubmed/8380610. [PubMed: 8380610]

62. Gerna G, Sarasini A, Baldanti F, Percivalle E, Zella D, Revello MG. Quantitative systemic and local evaluation of the antiviral effect of ganciclovir and foscarnet induction treatment on human cytomegalovirus gastrointestinal disease of patients with AIDS. Italian Foscarnet GID Study Group. Antiviral Res. 1997 Mar; 34(1):39-50. Available at http://www.ncbi.nlm.nih.gov/pubmed/ 9107384. [PubMed: 9107384]

63. Markham A, Faulds D. Ganciclovir. An update of its therapeutic use in cytomegalovirus infection. Drugs. 1994 Sep; 48(3):455-484. Available at http://www.ncbi.nlm.nih.gov/pubmed/7527763. [PubMed: 7527763]

64. Nguyen QD, Kempen JH, Bolton SG, Dunn JP, Jabs DA. Immune recovery uveitis in patients with AIDS and cytomegalovirus retinitis after highly active antiretroviral therapy. Am J Ophthalmol. 2000 May; 129(5):634-639. Available at http://www.ncbi.nlm.nih.gov/pubmed/10844056. [PubMed: 10844056]

65. Kosobucki BR, Goldberg DE, Bessho KKH, Rodanant N, Labree L. Valganciclovir therapy for immune recovery uveitis complicated by macular edema. Am J Ophthalmol. 2004 Apr; 137(4): 636-638. 2004 Available at http://www.ncbi.nlm.nih.gov/pubmed/15059701. [PubMed: 15059701]

66. Martin DF, Kuppermann BD, Wolitz RA, Palestine AG, Li H, Robinson CA. Oral ganciclovir for patients with cytomegalovirus retinitis treated with a ganciclovir implant. Roche Ganciclovir Study Group. N Engl J Med. 1999 Apr 8; 340(14):1063-1070. Available at http:// www.ncbi.nlm.nih.gov/pubmed/10194235. [PubMed: 10194235]

67. Drew WL, Ives D, Lalezari JP, et al. Oral ganciclovir as maintenance treatment for cytomegalovirus retinitis in patients with AIDS. Syntex Cooperative Oral Ganciclovir Study Group. N Engl J Med. 1995 Sep 7; 333(10):615-620. Available at http://www.ncbi.nlm.nih.gov/ pubmed/7637721. [PubMed: 7637721]

68. Studies of Ocular complications of AIDS Research Group in Collaboration with the AIDS Clinical Trials Group. Parenteral cidofovir for cytomegalovirus retinitis in patients with AIDS: the HPMPC peripheral cytomegalovirus retinitis trial. A randomized, controlled trial. Studies of Ocular complications of AIDS Research Group in Collaboration with the AIDS Clinical Trials Group. Ann Intern Med. 1997 Feb 15; 126(4):264-274. Available at http://www.ncbi.nlm.nih.gov/ pubmed/9036798. [PubMed: 9036798] 
69. Palestine AG, Polis MA, De Smet MD, et al. A randomized, controlled trial of foscarnet in the treatment of cytomegalovirus retinitis in patients with AIDS. Ann Intern Med. 1991 Nov 1; 115(9):665-673. Available at http://www.ncbi.nlm.nih.gov/pubmed/1656826. [PubMed: 1656826]

70. Spector SA, Weingeist T, Pollard RB, et al. A randomized, controlled study of intravenous ganciclovir therapy for cytomegalovirus peripheral retinitis in patients with AIDS. AIDS Clinical Trials Group and Cytomegalovirus Cooperative Study Group. J Infect Dis. 1993 Sep; 168(3):557563. Available at http://www.ncbi.nlm.nih.gov/pubmed/8394858. [PubMed: 8394858]

71. The Studies of the Ocular Complications of AIDS Research Group icwtACTG. Combination foscarnet and ganciclovir therapy vs monotherapy for the treatment of relapsed cytomegalovirus retinitis in patients with AIDS. The cytomegalovirus retreatment trial. Arch Ophthalmol. 1996; 114(1):23-33. Available at http://www.ncbi.nlm.nih.gov/sites/entrez? $\mathrm{Db}=$ pubmed $\& \mathrm{Cmd}=$ ShowDetailView $\&$ TermToSearch=8540847\&ordinalpos=6\&itool=EntrezSyst em2.PEntrez.Pubmed.Pubmed_ResultsPanel.Pubmed_RVDocSum. [PubMed: 8540847]

72. Diaz-Llopis M, Espana E, Munoz G, et al. High dose intravitreal foscarnet in the treatment of cytomegalovirus retinitis in AIDS. The British journal of ophthalmology. $1994 \mathrm{Feb}$; 78(2):120124. Available at http://www.ncbi.nlm.nih.gov/pubmed/8123619. [PubMed: 8123619]

73. de Smet MD, Meenken CJ, van den Horn GJ. Fomivirsen - a phosphorothioate oligonucleotide for the treatment of CMV retinitis. Ocular immunology and inflammation. 1999 Dec; 7(3-4):189-198. Available at http://www.ncbi.nlm.nih.gov/pubmed/10611727. [PubMed: 10611727]

74. Kirsch LS, Arevalo JF, Chavez de la Paz E, Munguia D, de Clercq E, Freeman WR. Intravitreal cidofovir (HPMPC) treatment of cytomegalovirus retinitis in patients with acquired immune deficiency syndrome. Ophthalmology. 1995 Apr; 102(4):533-542. discussion 542-533. Available at http://www.ncbi.nlm.nih.gov/pubmed/7724170. [PubMed: 7724170]

75. Young S, Morlet N, Besen G, et al. High-dose (2000-microgram) intravitreous ganciclovir in the treatment of cytomegalovirus retinitis. Ophthalmology. 1998 Aug; 105(8):1404-1410. Available at http://www.ncbi.nlm.nih.gov/pubmed/9709750. [PubMed: 9709750]

76. Tural C, Romeu J, Sirera G, et al. Long-lasting remission of cytomegalovirus retinitis without maintenance therapy in human immunodeficiency virus-infected patients. J Infect Dis. 1998 Apr; 177(4):1080-1083. Available at http://www.ncbi.nlm.nih.gov/pubmed/9534987. [PubMed: 9534987]

77. Vrabec TR, Baldassano VF, Whitcup SM. Discontinuation of maintenance therapy in patients with quiescent cytomegalovirus retinitis and elevated CD4+ counts. Ophthalmology. 1998 Jul; 105(7): 1259-1264. Available at http://www.ncbi.nlm.nih.gov/pubmed/9663231. [PubMed: 9663231]

78. Macdonald JC, Torriani FJ, Morse LS, Karavellas MP, Reed JB, Freeman WR. Lack of reactivation of cytomegalovirus (CMV) retinitis after stopping CMV maintenance therapy in AIDS patients with sustained elevations in CD4 $\mathrm{T}$ cells in response to highly active antiretroviral therapy. J Infect Dis. 1998 May; 177(5):1182-1187. Available at http://www.ncbi.nlm.nih.gov/ pubmed/9593001. [PubMed: 9593001]

79. Whitcup SM, Fortin E, Lindblad AS, et al. Discontinuation of anticytomegalovirus therapy in patients with HIV infection and cytomegalovirus retinitis. JAMA. 1999 Nov 3; 282(17):16331637. Available at http://www.ncbi.nlm.nih.gov/pubmed/10553789. [PubMed: 10553789]

80. Jabs DA, Bolton SG, Dunn JP, Palestine AG. Discontinuing anticytomegalovirus therapy in patients with immune reconstitution after combination antiretroviral therapy. Am J Ophthalmol. 1998 Dec; 126(6):817-822. Available at http://www.ncbi.nlm.nih.gov/pubmed/9860006. [PubMed: 9860006]

81. Jouan M, Saves M, Tubiana R, et al. Discontinuation of maintenance therapy for cytomegalovirus retinitis in HIV-infected patients receiving highly active antiretroviral therapy. AIDS. 2001 Jan 5; 15(1):23-31. Available at http://www.ncbi.nlm.nih.gov/pubmed/11192865. [PubMed: 11192865]

82. Torriani FJ, Freeman WR, Macdonald JC, et al. CMV retinitis recurs after stopping treatment in virological and immunological failures of potent antiretroviral therapy. AIDS. 2000 Jan 28; 14(2): 173-180. Available at http://www.ncbi.nlm.nih.gov/pubmed/10708288. [PubMed: 10708288]

83. Lilleri D, Piccinini G, Genini E, et al. Monitoring of human cytomegalovirus (HCMV)-specific CD4+ T cell frequency by cytokine flow cytometry as a possible indicator for discontinuation of HCMV secondary prophylaxis in HAART-treated AIDS patients. J Clin Virol. 2004 Apr; 29(4): 297-307. Available at http://www.ncbi.nlm.nih.gov/pubmed/15018859. [PubMed: 15018859] 
84. Tamarit A, Alberola J, Mira JV, Tornero C, Galindo MJ, Navarro D. Assessment of human cytomegalovirus specific $\mathrm{T}$ cell immunity in human immunodeficiency virus infected patients in different disease stages following HAART and in long-term non-progressors. J Med Virol. 2004 Nov; 74(3):382-389. Available at http://www.ncbi.nlm.nih.gov/pubmed/15368523. [PubMed: 15368523]

85. Weinberg A, Wiznia AA, Lafleur BJ, Shah S, Levin MJ. Cytomegalovirus-specific cell-mediated immunity in HIV-infected children on HAART. AIDS Res Hum Retroviruses. 2006 Mar; 22(3): 283-288. Available at http://www.ncbi.nlm.nih.gov/pubmed/16545015. [PubMed: 16545015]

86. Saitoh A, Viani RM, Schrier RD, Spector SA. Treatment of infants coinfected with HIV-1 and cytomegalovirus with combination antiretrovirals and ganciclovir. J Allergy Clin Immunol. 2004 Oct; 114(4):983-985. Available at http://www.ncbi.nlm.nih.gov/pubmed/15480350. [PubMed: 15480350]

87. Spector SA, Wong R, Hsia K, Pilcher M, Stempien MJ. Plasma cytomegalovirus (CMV) DNA load predicts CMV disease and survival in AIDS patients. J Clin Invest. 1998 Jan 15; 101(2):497502. Available at http://www.ncbi.nlm.nih.gov/pubmed/9435323. [PubMed: 9435323]

88. Salmon-Ceron D, Mazeron MC, Chaput S, et al. Plasma cytomegalovirus DNA, pp65 antigenaemia and a low CD4 cell count remain risk factors for cytomegalovirus disease in patients receiving highly active antiretroviral therapy. AIDS. 2000 May 26; 14(8):1041-1049. Available at http://www.ncbi.nlm.nih.gov/pubmed/10853987. [PubMed: 10853987]

\section{Dosing Recommendations for Preventing and Treating CMV}

\begin{tabular}{|c|c|c|c|}
\hline Indication & First Choice & Alternative & Comments/Special Issues \\
\hline Primary Prophylaxis & $\begin{array}{l}\text { For older } \\
\text { children who } \\
\text { can receive } \\
\text { adult dose } \\
\text { (based on } \\
\text { their BSA), } \\
\text { valganciclovir } \\
\text { tablets } 900 \\
\text { mg orally } \\
\text { once daily } \\
\text { with food } \\
\text { For children } \\
\text { aged } 4 \\
\text { months }-16 \\
\text { years, } \\
\text { valganciclovir } \\
\text { oral solution } \\
50 \mathrm{mg} / \mathrm{mL} \text { at } \\
\text { dose in } \\
\text { milligrams }= \\
7 \times \mathrm{BSA} \times \\
\text { CrCl (up to } \\
\text { maximum } \\
\text { CrCl of } 150 \\
\mathrm{~mL} / \mathrm{min} / 1.73 \\
\mathrm{~m}^{2} \text { ) orally } \\
\text { once daily } \\
\text { with food } \\
\text { (maximum } \\
\text { dose } 900 \mathrm{mg} / \\
\text { day) }\end{array}$ & N/A & 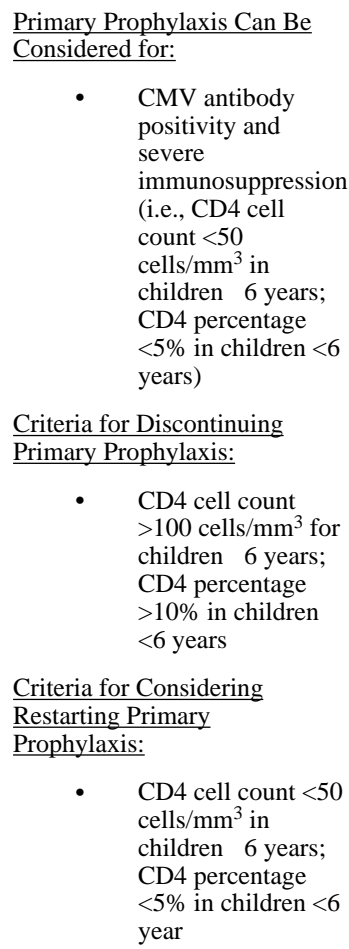 \\
\hline Secondary Prophylaxis & $\begin{array}{l}\text { - } \quad \begin{array}{l}\text { Ganciclovir } 5 \\
\mathrm{mg} / \mathrm{kg} \text { body } \\
\text { weight IV } \\
\text { once daily, or }\end{array} \\
\text { - } \begin{array}{l}\text { For older } \\
\text { children who } \\
\text { can receive }\end{array}\end{array}$ & $\begin{array}{l}\text { Cidofovir } 5 \\
\mathrm{mg} / \mathrm{kg} \text { body } \\
\text { weight per } \\
\text { dose IV every } \\
\text { other week. } \\
\text { Must be given } \\
\text { with } \\
\text { probenecid }\end{array}$ & $\begin{array}{l}\frac{\text { Secondary Prophylaxis }}{\text { Indicated For: }} \\
\qquad \begin{array}{l}\text { Prior disseminated } \\
\text { disease, retinitis, } \\
\text { neurologic disease, } \\
\text { or GI disease with } \\
\text { relapse }\end{array}\end{array}$ \\
\hline
\end{tabular}




\begin{tabular}{|c|c|c|c|}
\hline Indication & First Choice & Alternative & Comments/Special Issues \\
\hline & $\begin{array}{l}\text { adult dose } \\
\text { (based on } \\
\text { their BSA), } \\
\text { valganciclovir } \\
\text { tablets } 900 \\
\text { mg orally } \\
\text { once daily } \\
\text { with food, or } \\
\text { For children } \\
\text { age } 4 \\
\text { months-16 } \\
\text { years, } \\
\text { valganciclovir } \\
\text { oral solution } \\
50 \mathrm{mg} / \mathrm{mL} \text { (at } \\
\text { dose in } \\
\text { milligrams }= \\
7 \times \mathrm{BSA} \times \\
\mathrm{CrCl} \text { up to } \\
\text { maximum } \\
\mathrm{CrCl} \text { of } 150 \\
\mathrm{~mL} / \mathrm{min} / 1.73 \\
\mathrm{~m}^{2} \text { ) orally } \\
\text { once daily } \\
\text { with food, or } \\
\text { Foscarnet } 90- \\
120 \mathrm{mg} / \mathrm{kg} \\
\text { body weight } \\
\mathrm{IV} \text { once daily }\end{array}$ & $\begin{array}{l}\text { and IV } \\
\text { hydration. }\end{array}$ & 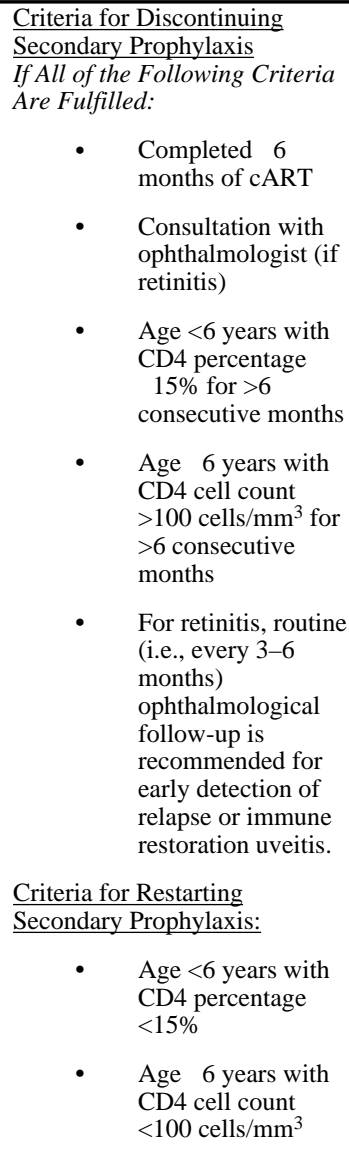 \\
\hline Treatment & 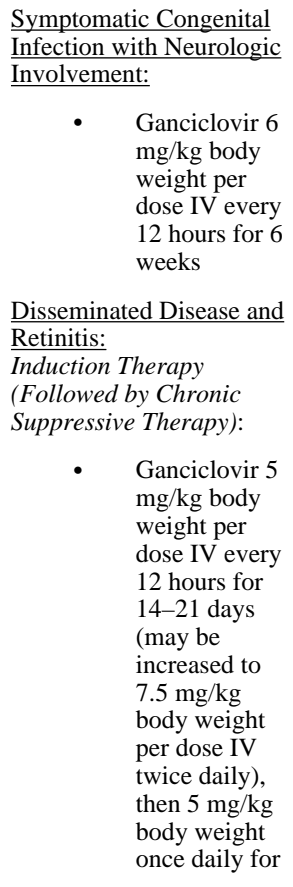 & $\begin{array}{l}\text { Disseminated Disease and } \\
\text { Retinitis: } \\
\text { Induction Therapy } \\
\text { (Followed by Chronic } \\
\text { Suppressive Therapy): } \\
\text { - } \begin{array}{l}\text { Foscarnet, } 60 \\
\text { mg/kg body } \\
\text { weight per } \\
\text { dose IV every } \\
8 \text { hours or } 90 \\
\text { mg/kg body } \\
\text { weight per } \\
\text { dose IV every } \\
\text { 12 hours } \times 14 \\
\text { to } 21 \text { days, } \\
\text { then } 90-120 \\
\text { mg/kg body } \\
\text { weight IV } \\
\text { once daily for } \\
\text { chronic } \\
\text { suppression }\end{array} \\
\begin{array}{l}\text { Alternatives for Retinitis } \\
\text { (Followed by Chronic }\end{array} \\
\text { Suppressive Therapy; See } \\
\text { Secondary Prophylaxis): } \\
\text { - } \begin{array}{l}\text { Valganciclovir } \\
\text { tablets } 900 \text { mg } \\
\text { per dose orally } \\
\text { twice daily for }\end{array}\end{array}$ & $\begin{array}{l}\text { Data on } \\
\text { valganciclovir } \\
\text { dosing in young } \\
\text { children for } \\
\text { treatment of } \\
\text { retinitis are } \\
\text { unavailable, but } \\
\text { consideration can } \\
\text { be given to } \\
\text { transitioning from } \\
\text { IV ganciclovir to } \\
\text { oral valganciclovir } \\
\text { after improvement } \\
\text { of retinitis is noted. } \\
\text { Intravitreal } \\
\text { injections of } \\
\text { ganciclovir, } \\
\text { foscarnet, or } \\
\text { cidofovir are used } \\
\text { in adults for } \\
\text { retinitis but are not } \\
\text { practical for most } \\
\text { children. } \\
\text { Combination } \\
\text { ganciclovir and } \\
\text { foscarnet is } \\
\text { associated with } \\
\text { substantial rates of } \\
\text { adverse effects, and } \\
\text { optimal treatment }\end{array}$ \\
\hline
\end{tabular}




\begin{tabular}{|c|c|c|c|}
\hline Indication & First Choice & Alternative & Comments/Special Issues \\
\hline & $\begin{array}{l}\begin{array}{l}\text { 5-7 days per } \\
\text { week for } \\
\text { chronic } \\
\text { suppression }\end{array} \\
\begin{array}{l}\text { Central Nervous System } \\
\text { Disease (Followed by }\end{array} \\
\text { Chronic Suppressive } \\
\text { Therapy; See Secondary } \\
\text { Prophylaxis): } \\
\text { Ganciclovir } 5 \\
\text { mg/kg body } \\
\text { weight per } \\
\text { dose IV every } \\
12 \text { hours } \\
\text { PLUS } \\
\text { foscarnet } 60 \\
\text { mg/kg body } \\
\text { weight per } \\
\text { dose IV every } \\
8 \text { hours (or } 90 \\
\text { mg/kg body } \\
\text { weight per } \\
\text { dose IV every } \\
12 \text { hours) } \\
\text { continued } \\
\text { until } \\
\text { symptomatic } \\
\text { improvement, } \\
\text { followed by } \\
\text { chronic } \\
\text { suppression }\end{array}$ & $\begin{array}{l}\text { 14-21 days, } \\
\text { followed by } \\
\text { chronic } \\
\text { suppressive } \\
\text { therapy (see } \\
\text { above). Note: } \\
\text { This is an } \\
\text { option in older } \\
\text { children who } \\
\text { can receive } \\
\text { the adult dose } \\
\text { (based on their } \\
\text { BSA). } \\
\text { IV ganciclovir } \\
\text { plus IV } \\
\text { foscarnet (at } \\
\text { above } \\
\text { induction } \\
\text { doses) may be } \\
\text { considered as } \\
\text { initial } \\
\text { induction } \\
\text { therapy in } \\
\text { children with } \\
\text { sight- } \\
\text { threatening } \\
\text { disease or for } \\
\text { treatment } \\
\text { following } \\
\text { failure/relapse } \\
\text { on } \\
\text { monotherapy. } \\
\text { Cidofovir is } \\
\text { also used to } \\
\text { treat CMV } \\
\text { retinitis in } \\
\text { adults } \\
\text { intolerant to } \\
\text { other } \\
\text { therapies. } \\
\text { Induction } \\
\text { dosing in } \\
\text { adults is 5 } \\
\text { mg/kg body } \\
\text { weight IV } \\
\text { once weekly } \\
\text { for } 2 \text { weeks, } \\
\text { followed by } \\
\text { chronic } \\
\text { suppressive } \\
\text { therapy (see } \\
\text { secondary } \\
\text { prophylaxis); } \\
\text { however, data } \\
\text { on dosing in } \\
\text { children are } \\
\text { unavailable. } \\
\text { Must be given } \\
\text { with } \\
\text { probenecid } \\
\text { and IV } \\
\text { hydration }\end{array}$ & $\begin{array}{l} \\
\\
\\
\\
\\
\\
\text { for neurologic } \\
\text { disease in children } \\
\text { is unknown, } \\
\text { particularly if } \\
\text { receiving optimized } \\
\text { cART. } \\
\text { Chronic } \\
\text { suppressive therapy } \\
\text { (secondary } \\
\text { prophylaxis) is } \\
\text { recommended in } \\
\text { adults and children } \\
\text { following initial } \\
\text { therapy of } \\
\text { disseminated } \\
\text { disease, retinitis, } \\
\text { neurologic disease, } \\
\text { or GI disease with } \\
\text { relapse. }\end{array}$ \\
\hline
\end{tabular}

Key to Acronyms: BSA = body surface area; $\mathrm{cART}=$ combined antiretroviral therapy; CD4 = CD4 T lymphocyte; CMV $=$ cytomegalovirus $; \mathrm{CrCl}=$ creatinine clearance $; \mathrm{GI}=$ gastrointestinal $; \mathrm{IV}=$ intravenous 


\section{Giardiasis (Last updated November 6, 2013; last reviewed November 6, 2013)}

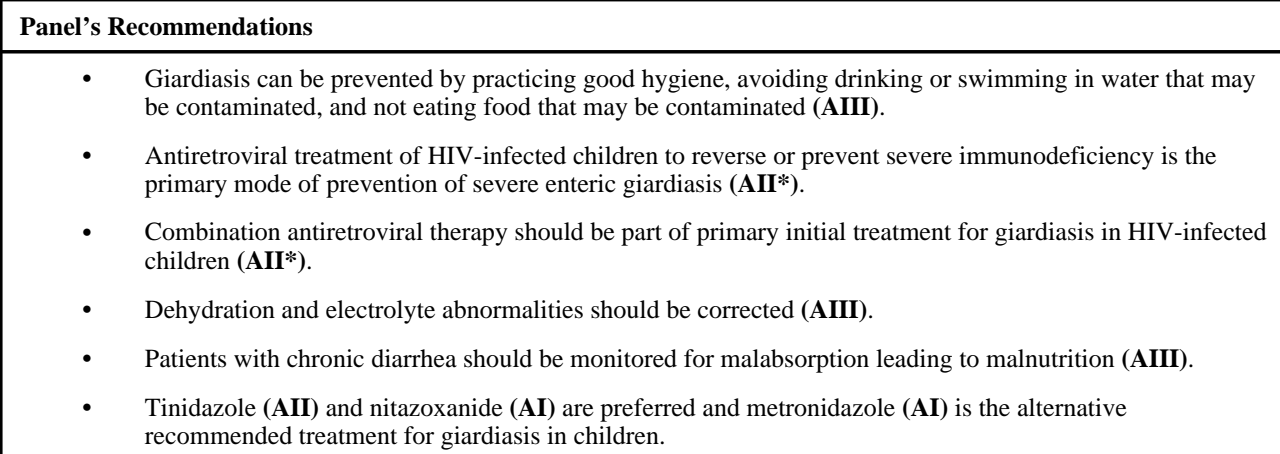

- $\quad$ Giardiasis can be prevented by practicing good hygiene, avoiding drinking or swimming in water that may be contaminated, and not eating food that may be contaminated (AIII).

- Antiretroviral treatment of HIV-infected children to reverse or prevent severe immunodeficiency is the primary mode of prevention of severe enteric giardiasis (AII*).

- Combination antiretroviral therapy should be part of primary initial treatment for giardiasis in HIV-infected children (AII*).

- Dehydration and electrolyte abnormalities should be corrected (AIII)

- $\quad$ Patients with chronic diarrhea should be monitored for malabsorption leading to malnutrition (AIII).

- Tinidazole (AII) and nitazoxanide (AI) are preferred and metronidazole (AI) is the alternative recommended treatment for giardiasis in children.

Rating of Recommendations: $\mathrm{A}=$ Strong; $\mathrm{B}=$ Moderate $\mathrm{C}=$ Optional

Rating of Evidence: I = One or more randomized trials $\underline{\text { in children }}{ }^{\dagger}$ with clinical outcomes and/or validated endpoints; I* $=$ One or more randomized trials in adults with clinical outcomes and/or validated laboratory endpoints with accompanying data in children ${ }^{\dagger}$ from one or more well-designed, nonrandomized trials or observational cohort studies with long-term clinical outcomes; II = One or more well-designed, nonrandomized trials or observational cohort studies in children ${ }^{\dagger}$ with long-term outcomes; II* $=$ One or more well-designed, nonrandomized trials or observational studies in adults with longterm clinical outcomes with accompanying data in children ${ }^{\dagger}$ from one or more similar nonrandomized trials or cohort studies with clinical outcome data; III = Expert opinion

${ }^{\dagger}$ Studies that include children or children/adolescents, but not studies limited to post-pubertal adolescents.

\section{Epidemiology}

Giardia intestinalis has a worldwide distribution and, among nationally reportable intestinal parasites, is the most commonly identified in public health laboratories in the United States. ${ }^{1}$ Surveillance data show a bimodal age distribution, with the greatest number of reported cases occurring in children aged 1 to 9 years and adults aged 35 to 44 years. In the United States, most cases are reported between early summer and early fall and are associated with recreational water activities and camping. ${ }^{1}$

Humans are the principal reservoir of G. intestinalis (also known as Giardia lamblia or Giardia duodenalis) infection. The parasite is found in many animals species, although the role of zoonotic transmission is still being unraveled. ${ }^{2}$ It is a flagellated protozoan with two forms: trophozoites and cysts. The infectious and environmentally resistant form is the cyst. After ingestion, each Giardia cyst produces two trophozoites in the proximal portion of the small intestine. Detached trophozoites pass through the intestinal tract, and form smooth, oval-shaped, thin-walled infectious cysts that are passed in feces. Duration of cyst excretion is usually selflimited but can vary and excretion may last for months. Studies in adults have shown that ingestion of as few as 10 to 100 fecally derived cysts is sufficient to initiate infection. ${ }^{3}$ Giardia cysts are infectious immediately upon being excreted in feces and remain viable for at least 3 months in water at $4^{\circ} \mathrm{C} .{ }^{4}$ Freezing does not eliminate infectivity completely, whereas heating, drying, or submersing in seawater are likely to do so. ${ }^{4,5}$

G. intestinalis is more common in certain high-risk groups, including children, employees of childcare centers, patients and staff of institutions for people with developmental disabilities, 
men who have sex with men, people who ingest contaminated drinking water or recreational water, travelers to disease-endemic areas of the world, close contacts of infected people, and people exposed to infected domestic and wild animals (i.e., dogs, cats, cattle, deer, and beavers). ${ }^{6}$ There is a paucity of information on giardiasis in HIV-infected children, although Giardia has been associated with diarrhea in children with AIDS..$^{7,8}$

Infection with Giardia can occur directly by the fecal-oral route or indirectly via ingestion of contaminated water or food, but water contaminated with cysts appears to be the major reservoir and vehicle for spread of the parasite. ${ }^{1}$ Most waterborne outbreaks have been related to ingestion of surface water treated by inadequate purification systems. ${ }^{9}$ Drinking untreated mountain stream water is a risk for hikers. Person-to-person spread occurs frequently in childcare centers and in families of children with diarrhea. ${ }^{10,6}$ Antigiardial host defenses are B-cell dependent, with secretory immunoglobulin A playing a major role in immunity. Humoral immunodeficiencies, such as X-linked agammaglobulinemia and hypogammaglobulinemia, predispose to chronic symptomatic disease..$^{11}$

Symptoms of giardiasis in HIV-infected individuals appear to be no more severe than those in HIV-negative individuals, and giardiasis is not typically considered a major cause of enteritis in HIV-infected patients. ${ }^{12}$ However, with progressive immunosuppression and reduced CD4 T lymphocyte (CD4) cell counts, the risk of symptomatic Giardia infections increases. Studies in adults have demonstrated that enteritis due to G. intestinalis is a frequent event among AIDS patients, especially in the most advanced stage of disease. ${ }^{13}$ Research in HIV-infected adults from countries where giardiasis is endemic demonstrate that risk of Giardia infections and severity of disease increased with increasing immunosuppression and lower CD4 cell counts. ${ }^{14,15}$ In a study of 75 HIV-infected adults in India, G. intestinalis was the most commonly isolated parasite, and patients with lower CD4 cell counts presented with significantly more enteric disease and chronic diarrhea. ${ }^{16} \mathrm{In}$ another study of 43 adults naive to combination antiretroviral therapy (cART), $G$. intestinalis was detected in one-third of patients and was significantly associated with lower CD4 cell counts $\left(\mathrm{OR}=3.0\right.$ for $\mathrm{CD} 4$ counts $\leq 100$ cells $\left./ \mathrm{mm}^{3}\right) .{ }^{17}$ A case-control study comparing giardiasis in HIV-infected adults in Brazil before and after the era of cART demonstrates that the incidence of enteric diseases caused by Giardia decreased after initiation of such treatment. ${ }^{14}$ Given the evidence, it is reasonable to recommend initiation of cART and immune reconstitution as a primary mode of prevention (AII*).

\section{Clinical Manifestations}

The incubation period usually lasts 1 to 2 weeks and averages 7 days. ${ }^{6}$ Symptomatic infection with $G$. intestinalis can cause a broad spectrum of clinical manifestations. Children usually present with short-lasting, acute watery diarrhea with or without low-grade fever, nausea, anorexia, and abdominal pain. Others have a more protracted intermittent course, characterized by foul-smelling stools associated with flatulence, abdominal distension, and anorexia. Malabsorption combined with anorexia can lead to significant weight loss, failure to thrive, and anemia in children. Stools can be profuse and watery initially and later become greasy and foul smelling. Blood, mucus, and fecal leukocytes are absent. Varying degrees of malabsorption can occur, and abnormal stool patterns can alternate with periods of 
constipation and normal bowel movements. Post-Giardia infection lactose intolerance can occur in $20 \%$ to $40 \%$ of patients. ${ }^{18}$ This syndrome may take several weeks to resolve and can contribute to malnutrition in children.

Asymptomatic infection is common. ${ }^{19}$ Extraintestinal invasion is unusual, but trophozoites occasionally migrate into bile or pancreatic ducts. Reactive arthritis has been associated with giardiasis. ${ }^{20}$

\section{Diagnosis}

Although performance of diagnostic tests has not been evaluated in HIV-infected children, it is expected to be similar to other populations. A definitive diagnosis is established by detection of Giardia trophozoites or cysts in stool specimens, duodenal fluid or small-bowel tissue by microscopic examination using staining methods such as trichrome; direct fluorescent antibody (DFA) assays; by detecting soluble stool antigens using enzyme immunoassays (EIA); or, by using molecular techniques including polymerase chain reaction. ${ }^{21,22}$ Identification of both trophozoites and cysts can be made on direct smears of concentrated specimens of stool. Appropriately conducted direct examination of stool establishes the diagnosis in up to $70 \%$ of patients with a single examination and in $85 \%$ with a second examination. Identification of Giardia can be difficult because of intermittent excretion of cysts. Stool specimens should be examined within 1 hour after being passed. Trophozoites are more likely to be present in unformed stools as a result of rapid bowel transit time. Cysts, but not trophozoites, are stable outside the gastrointestinal (GI) tract.

When giardiasis is suspected and stool specimens are negative, aspiration, biopsy, or both, of the duodenum or upper part of the jejunum should be performed. In a fresh specimen, trophozoites usually can be visualized on direct wet mount. The commercially available Entero-Test is an alternative method for obtaining duodenal fluid directly. ${ }^{23}$ Duodenal biopsy is the optimal method for diagnosis in patients with clinical characteristics but negative stool and duodenal fluid samples.

Use of polyclonal antisera or monoclonal antibodies against Giardia-specific antigens has improved diagnostic testing. Studies comparing EIA kits for detecting Giardia antigen in stool showed a sensitivity of $87 \%$ to $100 \%$ and specificity of $100 \%$. All fluorescent antibody tests had $100 \%$ sensitivity and specificity. ${ }^{24}$ These rapid diagnostic tests can be positive before and after detection of organisms by microscopic examination. DFA and EIA were equally sensitive, and both were more sensitive than microscopy of permanently stained smears after concentration in formalin ethyl acetate. ${ }^{25}$ Most experts recommend use of DFA testing and microscopy instead of microscopy alone (AIII). Specific antibodies to Giardia have been detected and quantified by immunodiffusion, hemagglutination, immunofluorescence, and EIA, but a serologic test is not available commercially.

\section{Prevention Recommendations}

Preventing Exposure-Because Giardia organisms are most likely transferred from contaminated water, food, or contact with an infected person or animal, avoidance of untreated water sources is recommended (AIII). This recommendation is especially 
important in individuals with severe immunosuppression. Hand washing with soap and water after exposure to potentially fecally contaminated material or contact with an infected person or animal is also recommended (AIII). Alcohol-based gels are ineffective against the cysts of Giardia and should not be substituted for hand washing when exposure to Giardia is a concern.

In a hospital, standard precautions (i.e., use of gloves and hand washing after removal of gloves) should be sufficient to prevent transmission from an infected patient to a susceptible HIV-infected person.

When traveling where water may be contaminated or where the safety of drinking water is in doubt, travelers, hikers, and campers should be advised of methods to make water safe for drinking. These measures include using bottled water, disinfecting water by heating it to a rolling boil for 1 minute, or using a filter that has been tested and rated by National Safety Foundation Standard 53 or Standard 58 for cyst and oocyst reduction. Waterborne outbreaks can be prevented with a combination of adequate filtration of water sources, chlorination, and maintenance of water distribution systems. ${ }^{1,9}$ Travelers should also be advised of the potential for transmission of giardiasis during use of contaminated recreational water (e.g., lakes, rivers, inadequately treated swimming pools).

Preventing First Episode of Disease-No chemoprophylactic regimens are known to be effective in preventing giardiasis. However, because the risk of acquisition of giardiasis and the severity of infection increase with the severity of immunosuppression, cART is a primary modality for prevention in HIV-infected children to prevent or reverse severe immunodeficiency (AII*).

Discontinuing Primary Prophylaxis—Not applicable.

\section{Treatment Recommendations}

Treating Disease-Supportive care with hydration, correction of electrolyte abnormalities, and nutritional supplementation should be provided (AIII). Effective cART and anti-parasitic therapy are the primary initial treatments for these infections in HIVinfected children and adults (AII*). ${ }^{14}$ Antimotility agents should be used with caution in young children (CIII).

Tinidazole (AII). The therapeutic efficacy against Giardia of metronidazole led to development of other nitroimidazole derivatives, such as tinidazole and secnidazole. These agents have the advantage of longer halflives, making them suitable for single-daily-dose therapies. A single, 2-g dose (or the equivalent pediatric dosing of $50 \mathrm{mg} / \mathrm{kg}$ in a single dose) of tinidazole has demonstrated cure rates ranging from $80 \%$ to $100 \%$, and is also associated with improved compliance. ${ }^{26-28}$ Tinidazole is approved for use in children aged 3 years and older. The drug is available in tablets, which can be crushed in flavored syrup for patients unable to swallow tablets.

Nitazoxanide (AI) is approved in the United States for treatment of infections due to $G$. intestinalis in patients aged 1 year or older. Two randomized, controlled clinical trials in 
HIV-uninfected children demonstrated nitazoxanide's efficacy against placebo and its comparability with metronidazole and mebendazole in treating giardiasis in children, with eradication rates for $G$. intestinalis of $71 \%$ to $94 \%$ with nitazoxanide treatment. ${ }^{29}$

Metronidazole (AI) was determined to be therapeutic against giardiasis in 1962. Since then, metronidazole and other nitroimidazoles have been used by clinicians as the mainstay of therapy of giardiasis. Metronidazole is the drug most often used for treatment worldwide. Children have been included in many of the clinical trials, with outcomes similar to those in adults (median efficacy, 94\%) for the 5- to 10-day regimens. ${ }^{30}$ Metronidazole is not available in a standard liquid form, but a suspension can be prepared by thoroughly crushing metronidazole tablets, using glycerin as a lubricant, and suspending the mixture in cherry syrup. ${ }^{31}$ In spite of its widespread and accepted use against Giardia, the U.S. Food and Drug Administration has never approved it for this indication.

Quinacrine is usually used in combination therapy for cases in which treatment failure is suspected. ${ }^{32}$ The severity of side effects has prevented clinicians from using it as an initial therapeutic choice or first-line alternative, particularly in children. A bitter taste and vomiting have led to lower efficacy in children, probably due to low compliance. Yellow/ orange discoloration of the skin, sclerae, and urine affects $4 \%$ to 5\% of those taking quinacrine, beginning about 1 week after starting treatment, and can last up to 4 months after discontinuation of therapy. Other common side effects include nausea, vomiting, headache, and dizziness. Quinacrine can precipitate hemolysis in glucose-6-phosphate dehydrogenase (G6PDH)-deficient individuals. ${ }^{33}$ Quinacrine is no longer available in the United States and has been discontinued by the manufacturer. ${ }^{34}$

Monitoring and Adverse Events (Including IRIS)—Patients with chronic diarrhea should be closely monitored for signs and symptoms of volume depletion, electrolyte and weight loss, and malnutrition. In severely ill patients, total parenteral nutrition may be indicated (BIII).

Adverse effects reported with tinidazole are not as common as with metronidazole but do include bitter taste, vertigo, and GI upset. ${ }^{30}$

Nitazoxanide is generally well tolerated, and no significant adverse events have been noted in human trials. Adverse events have been mild and transient and principally related to the GI tract, such as abdominal pain, diarrhea, and nausea. Nitazoxanide has been well tolerated up to the maximum dose of $4 \mathrm{~g}$ when taken with or without food, but the frequency of GI side effects increases significantly with the dose level. ${ }^{29}$

The most common side effects of metronidazole treatment include headache, vertigo, nausea, and a metallic taste in the mouth. Nausea occurs in 5\% to $15 \%$ of patients given standard multiday courses. In addition, pancreatitis, central nervous system toxicity at high doses, and transient, reversible neutropenia have been attributed to metronidazole. ${ }^{30}$

Immune reconstitution inflammatory syndrome has not been associated with giardiasis or its treatment. 
Managing Treatment Failure-The most important steps for management of treatment failure are supportive treatment, optimization of cART to achieve full virologic suppression, and modification of antiparasitic therapy (AII*). Treatment failures have been reported with all of the common anti-Giardia agents. It is important for clinicians to differentiate between resistance to treatment and reinfection, which is common in endemic regions and situations of poor fecal-oral hygiene. Resistance to most anti-Giardia agents has been documented but there is no consistent correlation between in vitro resistance and clinical failure. ${ }^{30}$ Clinically resistant strains have been treated with longer repeated courses or higher doses of the original agent or a drug from a different class to avoid potential cross-resistance.

Combination regimens using metronidazole-albendazole, metronidazole-quinacrine, or other active drugs or giving a nitroimidazole plus quinacrine for at least 2 weeks have proven successful against refractory infection. In AIDS patients with severe giardiasis, prolonged or combination therapy may be necessary (BII*). ${ }^{32,35}$

Preventing Recurrence-No pharmacologic interventions are known to be effective in preventing recurrence of giardiasis (CIII). Reinfection is frequent in endemic areas, in situations of poor hygiene, or inadequate treatment of contaminated water (e.g., private wells). This can be prevented by practicing good hand hygiene everywhere, but particularly after toilet use and handling of soiled diapers. Hand hygiene should also be practiced before food preparation and ingestion. To reduce risk of disease transmission, children with diarrhea should be excluded from child care settings until the diarrhea has stopped. Children with giardiasis should not use recreational water venues for 2 weeks after symptoms resolve. Additional information about recreational water illnesses and how to stop them from spreading is available at http://www.cdc.gov/healthywater/swimming.

Discontinuing Secondary Prophylaxis-Not applicable.

\section{References}

1. Yoder JS, Harral C, Beach MJ. Centers for Disease C, Prevention. Giardiasis surveillance - United States, 2006-2008. MMWR Surveill Summ. 2010 Jun 11; 59(6):15-25. Available at http:// www.ncbi.nlm.nih.gov/pubmed/20535095. [PubMed: 20535095]

2. Xiao L, Fayer R. Molecular characterisation of species and genotypes of Cryptosporidium and Giardia and assessment of zoonotic transmission. Int J Parasitol. 2008 Sep; 38(11):1239-1255. Available at http://www.ncbi.nlm.nih.gov/pubmed/18479685. [PubMed: 18479685]

3. Rendtorff RC. The experimental transmission of human intestinal protozoan parasites. II. Giardia lamblia cysts given in capsules. Am J Hyg. 1954 Mar; 59(2):209-220. Available at http:// www.ncbi.nlm.nih.gov/pubmed/13138586. [PubMed: 13138586]

4. Erickson MC, Ortega YR. Inactivation of protozoan parasites in food, water, and environmental systems. J Food Prot. 2006 Nov; 69(11):2786-2808. Available at http://www.ncbi.nlm.nih.gov/ pubmed/17133829. [PubMed: 17133829]

5. Bingham AK, Jarroll EL Jr, Meyer EA, Radulescu S. Giardia sp.: physical factors of excystation in vitro, and excystation vs eosin exclusion as determinants of viability. Exp Parasitol. 1979 Apr; 47(2):284-291. Available at http://www.ncbi.nlm.nih.gov/pubmed/35362. [PubMed: 35362]

6. Huang DB, White AC. An updated review on Cryptosporidium and Giardia. Gastroenterol Clin North Am. 2006 Jun; 35(2):291-314. viii. Available at http://www.ncbi.nlm.nih.gov/pubmed/ 16880067. [PubMed: 16880067]

7. Barrett DM, Steel-Duncan J, Christie CD, Eldemire-Shearer D, Lindo JF. Absence of opportunistic parasitic infestations in children living with HIV/AIDS in children's homes in Jamaica: pilot 
investigations. West Indian Med J. 2008 Jun; 57(3):253-256. Available at http:// www.ncbi.nlm.nih.gov/pubmed/19583124. [PubMed: 19583124]

8. Haller JO, Cohen HL. Gastrointestinal manifestations of AIDS in children. AJR Am J Roentgenol. 1994 Feb; 162(2):387-393. Available at http://www.ncbi.nlm.nih.gov/pubmed/8310932. [PubMed: 8310932]

9. Craun GF, Brunkard JM, Yoder JS, et al. Causes of outbreaks associated with drinking water in the United States from 1971 to 2006. Clin Microbiol Rev. 2010 Jul; 23(3):507-528. Available at http:// www.ncbi.nlm.nih.gov/pubmed/20610821. [PubMed: 20610821]

10. Pickering LK, Woodward WE. Diarrhea in day care centers. Pediatr Infect Dis. 1982 Jan-Feb;1(1): 47-52. Available at http://www.ncbi.nlm.nih.gov/pubmed/7177896. [PubMed: 7177896]

11. Webster AD. Giardiasis and immunodeficiency diseases. Trans R Soc Trop Med Hyg. 1980; 74(4): 440-443. Available at http://www.ncbi.nlm.nih.gov/pubmed/7445039. [PubMed: 7445039]

12. Stark D, Barratt JL, van Hal S, Marriott D, Harkness J, Ellis JT. Clinical significance of enteric protozoa in the immunosuppressed human population. Clin Microbiol Rev. 2009 Oct; 22(4):634650. Available at http://www.ncbi.nlm.nih.gov/pubmed/19822892. [PubMed: 19822892]

13. Angarano G, Maggi P, Di Bari MA, et al. Giardiasis in HIV: a possible role in patients with severe immune deficiency. Eur J Epidemiol. 1997 Jun; 13(4):485-487. Available at http:// www.ncbi.nlm.nih.gov/pubmed/9258558. [PubMed: 9258558]

14. Bachur TP, Vale JM, Coelho IC, Queiroz TR, Chaves Cde S. Enteric parasitic infections in HIV/ AIDS patients before and after the highly active antiretroviral therapy. Braz J Infect Dis. 2008 Apr; 12(2):115-122. Available at http://www.ncbi.nlm.nih.gov/pubmed/18641847. [PubMed: 18641847]

15. Daryani A, Sharif M, Meigouni M, et al. Prevalence of intestinal parasites and profile of CD4+ counts in HIV+/AIDS people in north of Iran, 2007-2008. Pak J Biol Sci. 2009 Sep 15; 12(18): 1277-1281. Available at http://www.ncbi.nlm.nih.gov/pubmed/20384282. [PubMed: 20384282]

16. Dwivedi KK, Prasad G, Saini S, Mahajan S, Lal S, Baveja UK. Enteric opportunistic parasites among HIV infected individuals: associated risk factors and immune status. Jpn J Infect Dis. 2007 May; 60(2-3):76-81. Available at http://www.ncbi.nlm.nih.gov/pubmed/17515636. [PubMed: 17515636]

17. Gautam H, Bhalla P, Saini S, et al. Epidemiology of opportunistic infections and its correlation with CD4 T-lymphocyte counts and plasma viral load among HIV-positive patients at a tertiary care hospital in India. J Int Assoc Physicians AIDS Care (Chic). 2009 Nov-Dec;8(6):333-337. Available at http://www.ncbi.nlm.nih.gov/pubmed/19755619. [PubMed: 19755619]

18. Duncombe VM, Bolin TD, Davis AE, Cummins AG, Crouch RL. Histopathology in giardiasis: a correlation with diarrhoea. Aust N Z J Med. 1978 Aug; 8(4):392-396. Available at http:// www.ncbi.nlm.nih.gov/pubmed/104699. [PubMed: 104699]

19. Hellard ME, Sinclair MI, Hogg GG, Fairley CK. Prevalence of enteric pathogens among community based asymptomatic individuals. J Gastroenterol Hepatol. 2000 Mar; 15(3):290-293. Available at http://www.ncbi.nlm.nih.gov/pubmed/10764030. [PubMed: 10764030]

20. Cantey PT, Roy S, Lee B, et al. Study of nonoutbreak giardiasis: novel findings and implications for research. Am J Med. 2011 Dec; 124(12):1175, e1171-e1178. Available at http:// www.ncbi.nlm.nih.gov/pubmed/22014792. [PubMed: 22014792]

21. Guy RA, Xiao C, Horgen PA. Real-time PCR assay for detection and genotype differentiation of Giardia lamblia in stool specimens. J Clin Microbiol. 2004 Jul; 42(7):3317-3320. Available at http://www.ncbi.nlm.nih.gov/pubmed/15243104. [PubMed: 15243104]

22. Fedorko DP, Williams EC, Nelson NA, Calhoun LB, Yan SS. Performance of three enzyme immunoassays and two direct fluorescence assays for detection of Giardia lamblia in stool specimens preserved in ECOFIX. J Clin Microbiol. $2000 \mathrm{Jul}$; 38(7):2781-2783. Available at http://www.ncbi.nlm.nih.gov/pubmed/10878088. [PubMed: 10878088]

23. Rosenthal P, Liebman WM. Comparative study of stool examinations, duodenal aspiration, and pediatric Entero-Test for giardiasis in children. J Pediatr. $1980 \mathrm{Feb}$; 96(2):278-279. Available at http://www.ncbi.nlm.nih.gov/pubmed/7351595. [PubMed: 7351595]

24. Garcia LS, Shimizu RY. Evaluation of nine immunoassay kits (enzyme immunoassay and direct fluorescence) for detection of Giardia lamblia and Cryptosporidium parvum in human fecal 
specimens. J Clin Microbiol. 1997 Jun; 35(6):1526-1529. Available at http:// www.ncbi.nlm.nih.gov/pubmed/9163474. [PubMed: 9163474]

25. Johnston SP, Ballard MM, Beach MJ, Causer L, Wilkins PP. Evaluation of three commercial assays for detection of Giardia and Cryptosporidium organisms in fecal specimens. J Clin Microbiol. 2003 Feb; 41(2):623-626. Available at http://www.ncbi.nlm.nih.gov/pubmed/ 12574257. [PubMed: 12574257]

26. Escobedo AA, Alvarez G, Gonzalez ME, et al. The treatment of giardiasis in children: single-dose tinidazole compared with 3 days of nitazoxanide. Ann Trop Med Parasitol. 2008 Apr; 102(3):199_ 207. Available at http://www.ncbi.nlm.nih.gov/pubmed/18348774. [PubMed: 18348774]

27. Canete R, Escobedo AA, Gonzalez ME, Almirall P, Cantelar N. A randomized, controlled, openlabel trial of a single day of mebendazole versus a single dose of tinidazole in the treatment of giardiasis in children. Curr Med Res Opin. 2006 Nov; 22(11):2131-2136. Available at http:// www.ncbi.nlm.nih.gov/pubmed/17076973. [PubMed: 17076973]

28. Escobedo AA, Nunez FA, Moreira I, Vega E, Pareja A, Almirall P. Comparison of chloroquine, albendazole and tinidazole in the treatment of children with giardiasis. Ann Trop Med Parasitol. 2003 Jun; 97(4):367-371. Available at http://www.ncbi.nlm.nih.gov/pubmed/12831522. [PubMed: 12831522]

29. Fox LM, Saravolatz LD. Nitazoxanide: a new thiazolide antiparasitic agent. Clin Infect Dis. 2005 Apr 15; 40(8):1173-1180. Available at http://www.ncbi.nlm.nih.gov/pubmed/15791519. [PubMed: 15791519]

30. Gardner TB, Hill DR. Treatment of giardiasis. Clin Microbiol Rev. 2001 Jan; 14(1):114-128. Available at http://www.ncbi.nlm.nih.gov/pubmed/11148005. [PubMed: 11148005]

31. Lerman SJ, Walker RA. Treatment of giardiasis: literature review and recommendations. Clin Pediatr (Phila). 1982 Jul; 21(7):409-414. Available at http://www.ncbi.nlm.nih.gov/pubmed/ 7044642. [PubMed: 7044642]

32. Nash TE, Ohl CA, Thomas E, Subramanian G, Keiser P, Moore TA. Treatment of patients with refractory giardiasis. Clin Infect Dis. 2001 Jul 1; 33(1):22-28. Available at http:// www.ncbi.nlm.nih.gov/pubmed/11389490. [PubMed: 11389490]

33. Wolfe MS. Giardiasis. Clin Microbiol Rev. 1992 Jan; 5(1):93-100. Available at http:// www.ncbi.nlm.nih.gov/pubmed/1735095. [PubMed: 1735095]

34. Thomas, Reuters. MicroMedex 2.0. http://www.micromedex.com/2/home.html.

35. Escobedo AA, Cimerman S. Giardiasis: a pharmacotherapy review. Expert Opin Pharmacother. 2007 Aug; 8(12):1885-1902. Available at http://www.ncbi.nlm.nih.gov/pubmed/17696791. [PubMed: 17696791]

\section{Dosing Recommendations for Prevention and Treatment of Giardiasis}

\begin{tabular}{|c|c|c|c|}
\hline Indication & First Choice & Alternative & Comments/Special Issues \\
\hline Primary Prophylaxis & $\begin{array}{l}\text { cART to avoid advanced } \\
\text { immunodeficiency }\end{array}$ & N/A & N/A \\
\hline Secondary Prophylaxis & N/A & N/A & N/A \\
\hline Treatment & $\begin{array}{l}\text { - Tinidazole, } 50 \mathrm{mg} / \mathrm{kg} \\
\text { by mouth, administered } \\
\text { as } 1 \text { dose given with } \\
\text { food (maximum } 2 \mathrm{~g} \text { ). } \\
\text { Note: Based on data } \\
\text { from HIV-uninfected } \\
\text { children } \\
\text { - } \quad \begin{array}{l}\text { Nitazoxanide. Note: } \\
\text { Based on data from } \\
\text { HIV-uninfected } \\
\text { children } \\
-\quad 1-3 \text { years: } \\
\quad 100 \mathrm{mg} \text { by } \\
\text { mouth } \\
\text { every } 12\end{array}\end{array}$ & $\begin{array}{l}\text { Metronidazole } \\
5 \mathrm{mg} / \mathrm{kg} \text { by } \\
\text { mouth every } 8 \\
\text { hours for 5-7 } \\
\text { days. } \\
\text { Note: Based on } \\
\text { data from HIV- } \\
\text { uninfected } \\
\text { children }\end{array}$ & \begin{tabular}{l} 
Tinidazole is approved in the \\
United States for children aged \\
$\geq 3$ years. It is available in \\
tablets that can be crushed. \\
Metronidazole has high \\
frequency of gastrointestinal \\
side effects. A pediatric \\
suspension of metronidazole is \\
not commercially available but \\
can be compounded from \\
tablets. It is not FDA-approved \\
for the treatment of giardiasis. \\
Supportive Care: \\
\multicolumn{1}{c}{ Hydration }
\end{tabular} \\
\hline
\end{tabular}




\begin{tabular}{|c|c|c|c|}
\hline Indication & First Choice & Alternative & Comments/Special Issues \\
\hline & $\begin{array}{ll}\text { hours with } \\
\text { food for } 3 \\
\text { days } \\
-\quad 4-11 \text { years: } \\
200 \mathrm{mg} \text { by } \\
\text { mouth } \\
\text { every } 12 \\
\text { hours with } \\
\text { food for } 3 \\
\text { days } \\
-\quad \geq 12 \text { years: } \\
500 \mathrm{mg} \text { by } \\
\text { mouth } \\
\text { every } 12 \\
\text { hours with } \\
\text { food for } 3 \\
\text { days }\end{array}$ & & 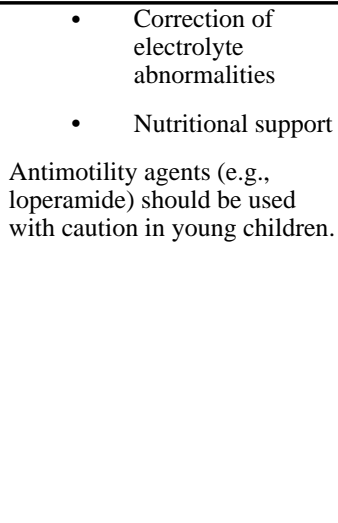 \\
\hline
\end{tabular}

Key to Abbreviations: $\mathrm{cART}=$ combination antiretroviral therapy; FDA $=$ U.S. Food and Drug Administration

\section{Hepatitis B Virus (Last updated November 6, 2013; last reviewed November}

\section{6, 2013)}

Panel's Recommendations

- All pregnant women should be tested for hepatitis B surface antigen (HBsAg) during an early prenatal visit (AI). Testing should be repeated in late pregnancy for HBsAg-negative women at high risk of hepatitis B virus (HBV) infection (e.g., injection-drug users, women with intercurrent sexually transmitted diseases, women with multiple sex partners) (BIII).

- $\quad$ All infants born to HBsAg-positive women, including HIV-co-infected women, should receive hepatitis B vaccine and hepatitis B immune globulin within 12 hours after birth, a second dose of hepatitis B vaccine at age 1 to 2 months, and a third dose at age 6 months (AI).

- HIV-infected infants, children, and adolescents should be tested for HBsAg as soon as possible after HIV diagnosis (AII)

- HIV-infected infants, children, and adolescents should be tested for quantitative anti-HBs and HBsAg 1 to 2 months after completing the vaccination series. If anti-HBs levels are $<10 \mathrm{mIU} / \mathrm{mL}$ and the $\mathrm{HBsAg}$ result is negative, they should be revaccinated with a second, 3-dose series of HBV vaccine followed in 1 to 2 months by repeat testing for anti-HBs (AIII).

- Antiviral therapy is not warranted in children without necroinflammatory liver disease (BIII). Treatment is not recommended for children with immunotolerant chronic $\mathrm{HBV}$ infection (i.e, $\mathrm{HBeAg}$ positive, normal serum transaminase levels despite detectable HBV DNA) or inactive carriers (i.e. HBeAg negative, normal serum transaminase levels despite detectable HBV DNA) (BII).

- Indications for treatment of chronic HBV infection in HIV-co-infected children are the same as in HBVinfected and HIV-uninfected children:

- $\quad$ Evidence of ongoing HBV viral replication, as indicated by serum HBV DNA $(>10,000$ 100,000 international units $/ \mathrm{ml}$ for $>6$ months) and persistent elevation of serum transaminase levels (at least twice the upper limit of normal for >6 months), or.

- $\quad$ Evidence of chronic hepatitis on liver biopsy (BII).

- Standard interferon-alfa (IFN-a), IFN-2a or IFN-2b, is recommended for treating chronic HBV infection with compensated liver disease in HIV-uninfected children aged $\geq 2$ years to $<12$ years who warrant treatment (AI). IFN-a therapy or oral antiviral therapy with adefovir or tenofovir is recommended for treating chronic HBV infection with compensated liver disease in HIV-uninfected children aged $\geq 12$ years (AI). IFN-a therapy in combination with oral antiviral therapy cannot be recommended for pediatric HBV infection in HIV-uninfected children until more data are available (BII).

- In HIV/HBV coinfected children who do not require combination antiretroviral therapy (cART) for their HIV infection, IFN-a therapy is the preferred agent to treat chronic hepatitis B (BIII), whereas adefovir can be considered in children age 12 years or older (BIII).

- Treatment options for HIV/HBV co-infected children who meet criteria for HBV therapy and who are already receiving lamivudine-or emtricitabine-containing, HIV-suppressive cART include standard IFN- $a$ 


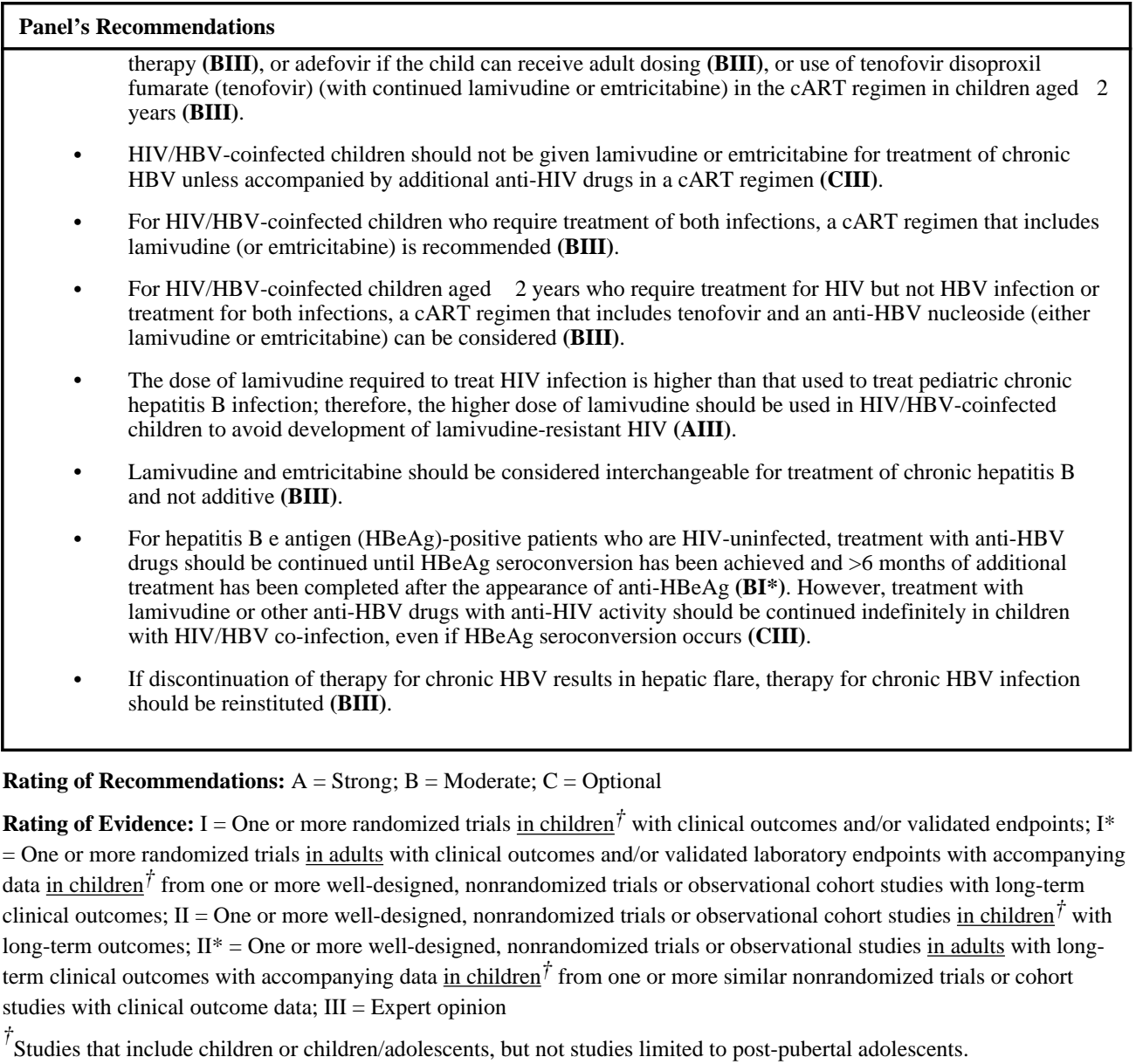

\section{Epidemiology}

Chronic hepatitis B virus (HBV) infection is defined as persistence of serum hepatitis B surface antigen (HBsAg) for $>6$ months. The risk of developing chronic HBV infection after acute infection correlates inversely with age and immune competence at $\mathrm{HBV}$ infection. In HBV-infected patients, chronic HBV infection develops in about $90 \%$ of infants, $25 \%$ to $50 \%$ of children aged 1 to 5 years, and $6 \%$ to $10 \%$ of older children and adolescents; individuals with immunocompromising conditions (e.g., renal failure) are also at increased risk of developing chronic HBV infection. ${ }^{1-4}$

Infant and childhood HBV infection can be acquired perinatally, parenterally, or postnatally through household contact. It can also be acquired parentally or through sexual transmission. HIV/HBV-coinfected pregnant women can transmit HIV, HBV, or both to their infants; it is not known if maternal HIV coinfection modifies the risk of HBV perinatal transmission. Horizontal transmission of HBV can occur through interpersonal contact with non-intact skin or mucous membranes with blood or body fluids that contain HBV (e.g., injuries, wounds) or from sharing household objects (e.g., toothbrushes, razors). Universal hepatitis $B$ vaccination of newborns has dramatically lowered chronic HBV infection in children and reduced the rates of HBV-related morbidity and mortality in the United States. The risk from 
blood transfusions in countries with blood bank screening is estimated to be very low (1.37 per million donations). ${ }^{5}$ Maternal HBV infection is not a contraindication to breastfeeding.

Adolescents are at risk of HBV infection through sexual activity or injection-drug use. In a study of HIV-infected adolescents infected through sexual activity or injection-drug use at 43 Pediatric AIDS Clinical Trial Group centers, 19\% had evidence of current or resolved HBV infection; the rate of current or resolved HBV infection in HIV-infected adolescent girls was twice the U.S. population-based rates for HIV-uninfected adolescent girls and, for adolescent boys, nearly seven times higher. ${ }^{6}$ Substance abuse and sexual activity increase the risk of HIV/HBV coinfection in adolescents, particularly in men who have sex with men $(\mathrm{MSM}){ }^{7}$

Most children who acquire HBV perinatally are initially immunotolerant to HBV and may remain immunotolerant for a decade or more. Although these children have high HBV DNA levels, serum transaminase levels are usually normal, and necroinflammatory liver disease is minimal. Childhood-acquired HBV infection, in contrast, is characterized by lower HBV DNA levels, greater serum transaminase elevation, and higher necroinflammatory liver disease than in perinatally acquired $\mathrm{HBV}$ infection. ${ }^{8}$

Data from the National Health and Nutrition Examination Survey, 1999-2004, indicate that $0.51 \%$ (95\% CI: $0.3 \%-0.9 \%$ ) of children aged 6 to 19 years had ever been infected with HBV. ${ }^{9}$ Only 1 small case series exists on the prevalence of chronic HBV infection in HIVinfected children at an inner city hospital in the United States, finding $2.6 \%$ prevalence in 228 HIV-infected children. ${ }^{10}$

\section{Clinical Manifestations}

Most acute HBV infections in children are asymptomatic. ${ }^{11}$ Prodromal symptoms of lethargy, malaise, fatigue, nausea, and anorexia can occur. Jaundice and right-upperquadrant pain can follow and, less commonly, hepatomegaly and splenomegaly. GianottiCrosti syndrome (papular acrodermatitis), urticaria, macular rash, or purpuric lesions may be seen in acute HBV infection. Extrahepatic manifestations associated with circulating immune complexes that have been reported in HBV-infected children include arthralgias, arthritis, polyarteritis nodosa, thrombocytopenia, and glomerulonephritis. However, rare cases of acute hepatic failure have occurred during perinatal and childhood HBV infection. ${ }^{12,13}$

Most children with chronic HBV infection are asymptomatic. One quarter of infants and children with chronic HBV eventually will develop cirrhosis or hepatocellular carcinoma (HCC). ${ }^{14,15}$ However, these sequelae usually develop over 2 to 3 decades and rarely occur during childhood. ${ }^{16,17}$ Development of HCC correlates with HBV DNA levels and duration of HBV infection, with the highest risk in people infected in early life. ${ }^{18} \mathrm{HIV} / \mathrm{HBV}$ coinfected adults are at increased risk of cirrhosis, end-stage liver disease, and liverrelated mortality. ${ }^{19}$ 


\section{Diagnosis}

Testing for HBV infection should be performed in any child whose mother is known to be infected with HBV as well as children from groups at high risk of HBV infection, including those who are HIV-infected and who are foreign-born in regions of high and intermediate HBV endemicity (HBsAg-positive prevalence $\geq 2 \%$ ). Adolescents and young adults with HIV infection, histories of injection-drug use, high-risk sexual contact, or MSM, should also undergo testing for HBV infection. Based on high prevalence of HBV infection in HIVinfected children and adolescents, HIV-infected children and adolescents and HIVuninfected infants born to HBsAg-positive women should be tested for HBsAg as soon as possible after HIV diagnosis (AII). ${ }^{6,7,20}$

HBsAg is the first marker detectable in serum, appearing 30 days after infection; it precedes the elevation of serum aminotransferase levels and the onset of symptoms.

Necroinflammatory liver disease then can occur, during which serum transaminase levels increase, along with high HBV DNA levels and $\mathrm{HBeAg}$ positivity. $\mathrm{HBeAg}$ correlates with viral replication, DNA polymerase activity, infectivity, and increased severity of liver disease. Antibody to hepatitis B core antigen (anti-hepatitis B core antigen [HBc] immunoglobulin $\mathrm{M}[\mathrm{IgM}]$ ) appears 2 weeks after $\mathrm{HBsAg}$ and the anti-HBc immunoglobulin $\mathrm{G}(\mathrm{IgG})$ persists for life, but should not be confused with passively transferred maternal anti$\mathrm{HBc}$ IgG that can be detectable in the infant up to ages 12 to 18 months or later. In selflimited infections, $\mathrm{HBsAg}$ is usually eliminated in 1 to 2 months, and hepatitis B surface antibody (anti-HBs) develops during convalescence. Anti-HBs indicates immunity from HBV infection. Despite immunity, HBV is incorporated into the human genome, where it can reactivate years later if a person becomes immunocompromised. ${ }^{21}$ After recovery from natural infection, both anti-HBs and anti-HBc usually are present. In patients who become chronically infected (i.e., persistently positive for HBsAg beyond 6 months), anti-HBs is undetectable. Patients who have been vaccinated may have detectable anti-HBs but not anti$\mathrm{HBc}$ or HBsAg. Patients who may have been inadvertently vaccinated after recovery from HBV infection should have detectable anti-HBs and anti-HBc upon post-vaccination testing (see Table 1, located at http://www.cdc.gov/mmwr/preview/mmwrhtml/rr5416a1.htm\#tab1, for review of interpretation of serologic test results for HBV infection).

$\mathrm{HBeAg}$ seroconversion, defined as loss of $\mathrm{HBeAg}$, followed by the production of antibodies to $\mathrm{HBeAg}$ (e.g., anti-HBe), usually heralds transition of the HBV-infected person to the inactive carrier state (HBsAg remains positive); however, some patients may develop $\mathrm{HBeAg-negative} \mathrm{chronic} \mathrm{hepatitis.} \mathrm{Variable} \mathrm{rates} \mathrm{of} \mathrm{HBeAg}$ seroconversion have been reported in children infected perinatally with $\mathrm{HBV}$ ranging from $10 \%$ to $75 \%$ in the first 2 to 4 decades but it is very infrequent in children aged $<3$ years. ${ }^{22,23}$ In contrast, higher rates of HBeAg seroconversion occur in childhood-acquired HBV infection, with $70 \%$ to $80 \%$ of children acquiring anti-HBe by the second decade of life. ${ }^{16} \mathrm{HBeAg}$ seroconversion usually is followed by reduction in serum HBV DNA levels, an initial increase and then subsequent normalization of serum transaminase levels, followed by resolution of necroinflammatory liver disease. ${ }^{16}$ Development of cirrhosis and HCC is more common in patients with delayed $\mathrm{HBeAg}$ seroconversion. ${ }^{24} \mathrm{HBeAg}$-negative infection (pre-core mutant) is uncommon in children. ${ }^{3}$ 
HBV DNA is a marker for HBV replication. In the active phase of chronic hepatitis B, high HBV DNA levels have been associated with necroinflammatory liver disease. Children infected perinatally, however, may remain in an immunotolerant phase with high levels of HBV DNA without evidence of liver damage and normal serum aminotransferase levels. Quantitative DNA assays may help determine the need for treatment and for evaluating treatment response. Although not necessary for diagnostic purposes, liver biopsy may be useful to assess the degree of liver damage and determine the need for treatment.

\section{Prevention Recommendations}

Preventing Exposure-All pregnant women should be tested for HBsAg during the first prenatal visit (AI). Testing should be repeated in late pregnancy for HBsAg-negative women at high risk of HBV infection (e.g., injection-drug users, women with intercurrent sexually transmitted diseases, women with multiple sex partners) (BIII).

Pregnancy is not a contraindication or precaution to hepatitis B vaccination for women who have not previously been vaccinated; current hepatitis B vaccines contain noninfectious HBsAg and should cause no risk to the fetus. Pregnant women who are identified as being at risk of $\mathrm{HBV}$ infection during pregnancy should be vaccinated. ${ }^{25}$

Preventing Disease-All infants born to HBV-infected women, including HIV coinfected women, should receive hepatitis B vaccine and hepatitis B immune globulin (HBIG) within 12 hours after birth, a second dose of hepatitis B vaccine at age 1 to 2 months, and a third dose at age 6 months, but not before age 24 weeks (AI) (Figures 1 and 2). ${ }^{26}$ For preterm infants weighing $<2000 \mathrm{~g}$, the initial vaccine dose (birth dose) should not be counted as part of the vaccine series because of the potentially reduced immunogenicity of hepatitis B vaccine in these infants; 3 additional doses of vaccine (for a total of 4 doses) should be administered beginning when the infant reaches 1 month of age (AI). ${ }^{26}$ In addition, term and preterm (birth weight $<2000 \mathrm{~g}$ ) infants born to women whose $\mathrm{HBsAg}$ status is unknown at delivery should receive the first dose of hepatitis B vaccine within 12 hours of birth. Infants weighing <2000 g should also receive HBIG within 12 hours of birth. Women with unknown HBsAg status should be tested as soon as possible. HBIG should be administered to term infants born to women whose HBsAg-test is found to be positive, or within 7 days of life when a mother's test results remain unknown. ${ }^{26}$

A 3-dose hepatitis B vaccine regimen is 70\% to $95 \%$ effective in preventing HBV infection in $\mathrm{HBV}$-exposed infants and combined with $\mathrm{HBIG}$, is $85 \%$ to $95 \%$ effective.

Postvaccination testing for anti-HBs and HBsAg should be performed at age 9 to 18 months in infants born to HBsAg-positive women (BIII). The level of anti-HBs that is considered protective is $\geq 10 \mathrm{mIU} / \mathrm{mL}$. Infants who are HBsAg-negative and have anti-HBs levels $<10$ $\mathrm{mIU} / \mathrm{mL}$ should be revaccinated with a second 3-dose series of hepatitis B vaccine and retested 1 to 2 months after the final vaccine dose (BIII). ${ }^{26}$

The 3-dose series of hepatitis B vaccine also is recommended for all children and adolescents aged $<19$ years who were not previously vaccinated. However, antibody responses to hepatitis B vaccination may be diminished in HIV-infected children, especially in older children or those with CD4 T lymphocyte (CD4 cell) counts $<200$ cells $/ \mathrm{mm}^{3} .{ }^{27,28}$ 
For this reason, HIV-infected infants, children, and adolescents should be tested for quantitative anti-HBs 1 to 2 months after completing the vaccination series and, if anti-HBs levels are $<10 \mathrm{mIU} / \mathrm{mL}$, revaccinated with a second 3-dose series of hepatitis B vaccine (AIII).

Limited data suggest modified hepatitis B vaccine dosing regimens, including a doubling of the standard antigen dose and use of combined hepatitis A and B (HAV/HBV) vaccine, can increase response rates in HIV-uninfected non-responders ${ }^{29}$ and in HIV-infected adults and adolescents. ${ }^{30-32}$ Therefore, use of double-dose HBV vaccine or combination HAV/HBV vaccine may be considered for HBV vaccination in HIV-infected adolescents (BI).

Waning of HBsAb levels below $10 \mathrm{mIU} / \mathrm{mL}$ after HBV re-immunization in HIV-infected children is common, but the need for booster doses of hepatitis B vaccine in HIV-infected individuals has not been determined. ${ }^{33}$ The American Academy of Pediatrics Committee on Infectious Disease recommends annual anti-HBs testing and booster doses when the antiHBs levels decline to $<10 \mathrm{mIU} / \mathrm{mL}$ for hemodialysis patients and other immunocompromised people at continued risk of hepatitis B infection (CIII) ${ }^{34} \mathrm{HBV}$ infected children should be advised not to share toothbrushes or other personal-care articles that might be contaminated with blood (e.g., razors, tweezers, nail clippers) and to cover open or draining wounds. Although efficiency of sexual transmission of HBV is relatively low, safe-sex practices should be encouraged for all sexually active HIV-infected adolescents and young adults; barrier precautions (e.g., latex condoms) are recommended to reduce the risk of exposure to sexually transmitted pathogens, including HBV.

\section{Treatment Recommendations Treating Disease}

General Issues: All children should receive HAV vaccination at age 12 to 23 months with the 2 doses in the series administered at least 6 months apart. ${ }^{35}$ Children who are not fully vaccinated by age 2 years can be vaccinated at subsequent visits. The hepatitis A vaccine is also recommended for children aged $\geq 24$ months who were not previously vaccinated and who have chronic liver disease (including chronic HBV infection) and other chronic diseases (Figures 1 and 2).

Treatment of pediatric HBV infection should be based on multiple factors, including a child's age, age at acquisition of infection, HBV DNA levels, and serum transaminase levels. Antiviral therapy regimens for chronic HBV are approved only for children aged $>2$ years who have compensated liver disease.

HIV-infected children who are not receiving anti-HBV therapy should be closely monitored with determination of serum aminotransferase levels every 6 months. If serum transaminase levels are persistently elevated (more than twofold the upper limit of normal for $\geq 6$ months), $\mathrm{HBeAg}$, anti-HBe, and HBV DNA levels should be obtained before the initiation of antiHBV therapy. Assessment of serum transaminases and HBV DNA levels over time can identify patients who may be in the process of spontaneous $\mathrm{HBeAg}$ seroconversion and who would thus not require treatment. Liver biopsy is not required before treatment but may help 
to determine the severity of hepatic inflammation and fibrosis and to exclude other causes of liver disease. ${ }^{36,37}$

No clear recommendations exist for treating chronic childhood HBV infection. HBVinfected children often have milder disease than adults and may show spontaneous HBeAg seroconversion. Few large randomized controlled trials exist of antiviral therapies for chronic HBV infection in childhood. Moreover, the long-term safety of many of the agents used to treat chronic HBV infection in adults is unknown in children. However, pediatric liver experts at a 2010 consensus meeting recommended that anti-HBV treatment be considered in children aged $>2$ years with chronic HBV infection and a duration of necroinflammatory liver disease $>6$ months. ${ }^{36}$

Indications for treatment of chronic HBV infection in HIV-coinfected children are the same as in HBV-infected, HIV-uninfected children:

- Evidence of ongoing HBV viral replication, as indicated by serum HBV DNA (>10,000-100,000 IU/mL), irrespective of HBeAg positivity, for $>6$ months and persistent elevation of serum transaminase levels (at least twice the upper limit of normal for $>6$ months), or

- Evidence of chronic hepatitis on liver biopsy (BII). . $^{3,38}$

Children without necroinflammatory liver disease do not warrant anti-HBV therapy (BIII). Anti-HBV treatment is not recommended for children with immunotolerant chronic HBV infection (i.e., $\mathrm{HBeAg}$ positive, normal serum transaminase levels despite detectable HBV DNA) or inactive carriers (i.e. HBeAg negative, normal serum transaminase levels despite detectable HBV DNA) (BII).

The goals of treatment for children with chronic HBV infection are identical to those for adults: suppression of HBV replication, normalization of serum transaminase levels, acceleration of $\mathrm{HBeAg}$ seroconversion (in those who are $\mathrm{HBeAg}$ positive), preservation of liver architecture, and prevention of long-term sequelae, such as cirrhosis and HCC.

Treatment of chronic HBV infection is evolving; consultation with providers with expertise in treating chronic HBV infection in children is recommended.

\section{Treating Chronic Hepatitis B Infection in Adults and Adolescents-Seven} medications have been approved to treat chronic HBV infection in adults: interferons (both standard and pegylated), nucleoside analogues (i.e., lamivudine, telbivudine, and entecavir), and the nucleotide analogues, adefovir and tenofovir disoproxil fumarate (tenofovir). The FDA-approved HIV antiretroviral (ARV) medication emtricitabine also has significant activity against $\mathrm{HBV}$, although it is not approved for this indication. Preferred initial therapies for adults who have chronic HBV without HIV infection include pegylated interferon-alfa (PEG-IFN-a), entecavir, or adefovir monotherapy. In HIV-infected adults who have chronic HBV infection, treatment for hepatitis B should be considered for those who are HBeAg-positive with HBV DNA $\geq 20,000 \mathrm{IU} / \mathrm{mL}$ ( $>10^{5}$ copies $\left./ \mathrm{mL}\right), \mathrm{HBeAg}$ negative with HBV DNA $\geq 2000 \mathrm{IU} / \mathrm{mL}$ ( $>10^{4}$ copies $/ \mathrm{mL}$ ), patients who have persistent serum transaminase elevation, and those with evidence of cirrhosis or fibrosis. ${ }^{19}$ Treatment 
of HBV infection is now recommended for all adults with concomitant HIV infection (Adult Opportunistic Infection and Antiretroviral Guidelines). This has not been recommended for children, however, and given the lack of data on this issue, a similar recommendation cannot be made at this point.

Treatment options for HBV in HIV-infected patients must account for the goals of therapy and the impact treatment may have on both HIV and HBV replication. In coinfected patients who require treatment for chronic HBV, HIV, or both, many experts would initiate a fully suppressive combined antiretroviral therapy (cART) regimen that includes two drugs active against HBV (tenofovir and either lamivudine or emtricitabine). This approach may reduce the risk of immune reconstitution inflammatory syndrome (IRIS), particularly in patients with advanced immunodeficiency. The combination of tenofovir with lamivudine was demonstrated to be more effective in suppressing HBV in coinfected adults than either drug alone and prevents development of lamivudine resistance. ${ }^{39}$ In instances in which HIV treatment cannot be given but treatment of HBV infection is needed, PEG-IFN- $a$ can be used alone because it does not lead to development of drug-resistant HIV or HBV mutants. Anti-HBV drugs with anti-HIV activity should not be given in the absence of a fully suppressive ARV regimen, because anti-HBV drugs such as tenofovir, entecavir, emtricitabine, lamivudine, and likely telbivudine given without additional ARV drugs in an HIV-suppressive regimen likely would produce resistant HIV in the recipient (see Guidelines for Prevention and Treatment of Opportunistic Infection in Adolescents and Adults with HIV Infection).

Treating Chronic Hepatitis B Infection in HIV-Uninfected Children-Only two drugs (IFN-a [standard] monotherapy or lamivudine monotherapy) are FDA-approved to treat chronic HBV in young children (1-11 years old) (AI) ${ }^{40,41}$ Four other drugs are approved for treatment of chronic HBV in older children: adefovir and tenofovir (children aged $\geq 12$ years) and entecavir and telbivudine (children aged $\geq 16$ years) (AI). ${ }^{42-45}$ While tenofovir is approved for treatment of HIV infection in children aged $\geq 2$ years, it is not approved for treatment of HBV in children under 12 years old.

The limited pediatric trials of these agents show that although they are well-tolerated by children, response rates are similar to adults ( $25 \%$ HBeAg seroconversion), and treatment generally does not eliminate HBV infection. ${ }^{46,47}$ There is some evidence for enhanced loss of HBsAg in children treated with IFN in comparison to those treated with lamivudine. ${ }^{40,48}$ In HIV-uninfected children, $\mathrm{HBeAg}$ seroconversion rates after 1 year of treatment are similar. ${ }^{3}$ IFN-a treatment is administered for only 6 months but requires subcutaneous administration and has more frequent side effects, including growth impairment. Although lamivudine is administered orally and has a lower rate of side effects, it requires a longer duration of therapy and has a high rate of resistance if taken for an extended time. ${ }^{3}$

Although various combination regimens involving sequential or concurrent lamivudine and standard or PEGIFN-a have been studied in children or adults with chronic HBV, superior treatment response with combination therapy over monotherapy with standard or PEG-IFN$a$ or lamivudine has not been demonstrated; however, lamivudine resistance rates may be lower with combination therapy. ${ }^{49-58}$ A recent study of children with immunotolerant HBV 
infection suggested possible benefit from sequential lamivudine and IFN-a therapy, with $78 \%$ of patients clearing HBV DNA by the end of treatment. ${ }^{57}$

However, IFN-a (standard or pegylated) therapy in combination with oral antiviral therapy cannot be recommended for HBV infection in HIV-uninfected children until more data are available (BII).

Treating HBV/HIV-Coinfected Children-None of the clinical studies of treatment of chronic HBV infection have specifically studied children with HIV/HBV coinfection. Choice of antiviral therapy for the HIV/HBV coinfected child involves consideration of whether HBV treatment, HIV treatment or treatment for both infections is warranted. Further study is needed to inform recommendations for antiviral therapy of children and adolecents with HIV/HBV coinfection.

If treatment of chronic HBV but not HIV infection is indicated, standard IFN-a is the preferred agent (BIII). Adefovir also can be considered in children aged 12 years or older (BIII). Antiviral drugs with activity against HIV (e.g., lamivudine, emtricitabine, tenofovir, entecavir, and likely telbivudine) should be avoided in the absence of a fully suppressive cART regimen to prevent development of drug-resistant HIV mutations. Despite in vitro evidence of anti-HIV activity of adefovir, there is no clinical evidence that adefovir monotherapy induces HIV drug resistance. ${ }^{59}$

If treatment of HIV infection but not chronic HBV is indicated, avoiding use of a cART regimen that contains only one ARV drug with activity against HBV (e.g., lamivudine, emtricitabine, or tenofovir) can prevent development of HBV drug resistance. Thus, in coinfected children who can receive tenofovir, use of a cART regimen that contains two drugs effective against HBV (tenofovir plus lamivudine or emtricitabine) can be considered (BIII). However, for coinfected children aged $<2$ years who need HIV but not HBV treatment, many experts would use a standard cART regimen that includes lamivudine (or emtricitabine). The optimal treatment approach needs further study.

If treatment for both HIV and chronic HBV is indicated and the child is lamivudine-naive, a cART regimen that includes lamivudine (or emtricitabine) is recommended (BIII). A regimen containing tenofovir and lamivudine (or emtricitabine) should be considered for use in HIV-infected children aged $\geq 2$ years, based on extrapolation from evidence in adults with HIV/HBV coinfection and adolescents with HBV monoinfection ${ }^{42}$ but limited by absence of data evaluating use of tenofovir for treatment of $\mathrm{HBV}$ infection in $\mathrm{HBV}$-monoinfected or HIV/HBVcoinfected children or HIV/HBV-coinfected adolescents (BIII).

If treatment for HIV and chronic HBV is indicated, a child is already receiving HIVsuppressive cART including lamivudine (or emtricitabine), and plasma HBV DNA is detectable, HBV lamivudine resistance can be assumed. However, because HBV drugresistant isolates may have lower replicative capacity, some experts recommend no change in therapy, although this recommendation is controversial (CIII). Treatment options for such children who require HBV therapy include adding standard IFN-a (BIII), or adefovir in 
children who can receive adult dosing (BIII), or use of tenofovir (with continued lamivudine or emtricitabine) in the cART regimen in children aged $\geq 2$ years (BIII).

Data are insufficient on other anti-HBV drugs in children to make recommendations.

Interferons: Standard IFN- $a-2 a$ or $-2 b$ has received the most study in children who have chronic HBV infection (without HIV infection) and is recommended for treating chronic $\mathrm{HBV}$ infection with compensated liver disease in HIV-uninfected children aged $\geq 2$ years who warrant treatment (AI).

In a review of 6 randomized clinical trials in $240 \mathrm{HBV}$-infected children aged $>1.5$ years, IFN- $a$ therapy resulted in HBV DNA clearance in $35 \%$ of treated children, $\mathrm{HBeAg}$ clearance in $10 \%$, and normalization of serum transaminase levels in $39 \%$ at treatment completion. ${ }^{60}$ Six to 18 months after therapy discontinuation, $29 \%$ of children had persistent clearance of HBV DNA, and 23\% demonstrated HBeAg clearance. Children most likely to respond to IFN treatment are younger and have higher baseline serum transaminase levels and lower baseline HBV DNA levels. ${ }^{46,61-63}$ Response is less likely (10\%) in those with normal serum transaminase levels, high HBV DNA levels, HBV genotypes $\mathrm{C}$ or D, or HBeAg-negative chronic HBV infection.

IFN-a therapy is the preferred agent to treat chronic hepatitis B in HIV-coinfected children who do not require cART for their HIV infection (BIII).

The standard course of IFN-a therapy for HIV-uninfected children is 24 weeks. PEG-IFN-a, which results in more sustained plasma interferon concentrations and can be administered by injection once weekly for 48 weeks, has proven superior to standard IFN-a in treating HBVinfected adults. ${ }^{50,64}$ However, the limited data on use of pegylated IFN-a in children come from treatment of hepatitis $\mathrm{C}$ infection, and appropriate dosing information is not available for use of pegylated IFN- $a$ to treat chronic HBV infection in children. ${ }^{65-67}$

Lamivudine: Lamivudine (3TC) is an oral nucleoside analogue that inhibits HBV replication. It is approved for use in children aged 2 to 17 years who have compensated liver disease from chronic HBV infection. In a placebo-controlled trial in HIV-uninfected children with chronic HBV infection, lamivudine was well tolerated, with virologic response (clearance of HBV DNA and $\mathrm{HBeAg}$ ) in $23 \%$ of children receiving 52 weeks of lamivudine therapy, compared with $13 \%$ in placebo recipients. ${ }^{41}$ Response rates were higher $(35 \%)$ for children with baseline serum transaminases more than two times normal. ${ }^{41}$ In a 2-year, open-label extension of this study, 213 children who remained HBeAg-positive after 1 year of therapy were continued on lamivudine treatment; virologic response was seen in $21 \%$ of the original lamivudine recipients, compared with $30 \%$ of prior placebo recipients, indicating that additional clinical response could occur over time with prolonged treatment. ${ }^{68}$ However, longer duration of lamivudine therapy also was associated with progressive development of lamivudine-resistant HBV, with base pair substitutions at the tyrosine-methionine-aspartateaspartate (YMDD) locus of HBV DNA polymerase.

Lamivudine should not be used as a single agent for treatment of chronic HBV infection in HIV-infected children who are not receiving cART because of the risk of HIV resistance to 
lamivudine (CIII); as discussed above, lamivudine should be used only in HIV/HBVcoinfected children in combination with other ARV drugs in a cART regimen (BIII). The dose of lamivudine required to treat HIV infection is higher than that for treating pediatric chronic HBV infection alone; therefore, the higher dose of lamivudine should be used in HIV/HBV-coinfected children to avoid development of lamivudine-resistant HIV (AIII).

Lamivudine resistance should be suspected if HBV DNA levels increase by 1 to 2 log during antiviral therapy. Such increases may precede increases in serum transaminase levels (hepatic flare) and liver decompensation. ${ }^{63}$

Emtricitabine: Emtricitabine is structurally similar to lamivudine and is active against HBV and HIV, although not approved for treatment of chronic HBV infection. Like lamivudine, emtricitabine also is associated with relatively rapid onset of HBV and HIV drug resistance, and patients with suspected lamivudine resistance should be assumed to have crossresistance to emtricitabine.

Lamivudine and emtricitabine should be considered interchangeable for treatment of chronic HBV infection and not additive (AIII). As with lamivudine, emtricitabine should not be used to treat chronic HBV infection in coinfected children who are not being treated with cART for their HIV infection because of the risk of HIV-associated resistance mutations (CIII).

Adefovir: Adefovir dipivoxil is an oral nucleotide analogue active against HBV. Although active against HBV, adefovir has minimal anti-HIV activity, and HIV resistance has not been observed in patients receiving a 10-mg daily dose of adefovir for 48 weeks. ${ }^{59} \mathrm{HBV}$ resistance is much lower to adefovir than to lamivudine, reportedly $2 \%$ after 2 years, $4 \%$ after 3 years, and $18 \%$ after 4 years of therapy in adults. ${ }^{69}$ These adefovir-associated mutations in HBV Pol gene result in only a modest (threefold to eightfold) increase in the $50 \%$ inhibitory concentration and are partially cross-resistant with tenofovir. Adefovir is now FDA-approved for adults who require treatment for chronic HBV infection but do not yet require treatment for HIV. Adefovir has been studied in HIV/HBV-coinfected adults with lamivudine-resistant HBV infection, and HBV suppression was demonstrated. ${ }^{59}$ Safety and effectiveness of adefovir for treating chronic HBV infection in children has been reported. ${ }^{43}$ In a randomized, placebo-controlled trial, adefovir was more effective than placebo in children age $\geq 12$ years at suppressing viral replication and normalizing transaminases.

Tenofovir Disoproxil Fumarate (Tenofovir): Tenofovir is a nucleotide analog structurally similar to adefovir that reduces HBV DNA levels in adults with lamivudine-resistant and wild-type HBV infection. A study in HIV/HBV-coinfected adults receiving stable cART comparing treatment with tenofovir or adefovir found similar efficacy in suppression of HBV DNA with no difference in toxicity. ${ }^{70}$ Another study of HIV/HBV-coinfected adults receiving tenofovir in addition to lamivudine as part of their ARV regimen found that HBV DNA became undetectable in $30 \%$ of HBeAgpositive and $82 \%$ of HBeAg-negative patients, most of whom had lamivudine-resistant HBV infection. ${ }^{59}$ As noted earlier, tenofovir is not 
approved for treatment of HBV infection in children aged $<12$ years, but tenofovir is approved as part of cART for HIV beginning at age 2 years.

However, for HIV/HBV-coinfected children aged $\geq 2$ years who require treatment of both infections, tenofovir in combination with an anti-HBV nucleoside (either lamivudine or emtricitabine) can be considered (BIII); a combined formulation of emtricitabine and tenofovir (Truvada) is available for adults. As with lamivudine and emtricitabine, tenofovir should not be used to treat chronic HBV in HIV-coinfected patients who are not receiving cART for HIV because of the risk of HIV-associated resistance mutations (CIII).

Entecavir: Entecavir is an oral nucleoside analogue that inhibits HBV DNA polymerase. When compared to lamivudine, entecavir therapy results in greater HBV viral suppression, increased normalization of serum transaminase levels, improved liver histology, and lower HBV resistance rates. ${ }^{71} \mathrm{HBV}$ viral suppression also has been demonstrated in HIV/HBVcoinfected adults. Entecavir treatment is approved for treatment of chronic HBV in adults and is preferred for lamivudine-resistant HBV infections. However, it recently was demonstrated to have suppressive activity against HIV. ${ }^{72}$ Entecavir should not be used in HIV/HBV-coinfected patients who are not receiving cART for HIV. Entecavir is approved for use in children aged $\geq 16$ years; no data are available on safety and efficacy of entecavir in younger children.

Telbivudine: Telbivudine is a thymidine nucleoside analogue that was approved to treat chronic HBV in adults. It is well tolerated, but like lamivudine, resistance emerges over time, and telbivudine is not active against lamivudine-resistant HBV. No data are available on telbivudine in HIV/HBV-coinfected adults. Telbivudine is approved for use in children aged $\geq 16$ years; no data are available on safety and efficacy of entecavir in younger children.

Duration of Therapy-The optimal duration of therapy in HIV/HBV-coinfected children is not known. The duration of IFN-a treatment in HIV-uninfected children with chronic HBV infection is 6 months. At least 1 year of lamivudine therapy is recommended for HIVuninfected children who have chronic HBV infection, with continuation of medication for $\checkmark 6$ months after documented HBeAg seroconversion. ${ }^{46}$ The duration of IFN therapy in HIVinfected children with HBV infection in whom treatment is indicated should be at least 6 months (CIII). Among HBeAg-positive children who are HIV-uninfected, treatment of chronic HBV infection with antivirals should be continued until HBeAg seroconversion has been achieved and $\succ 6$ months of additional treatment has been completed after the appearance of anti-HBe (BI*).

However, because lamivudine (or emtricitabine) and tenofovir would be administered only to HIV/HBVcoinfected children who need HIV treatment and as part of a suppressive ARV regimen, treatment with lamivudine (or other anti-HBV drugs with anti-HIV activity) should be continued indefinitely in children with HIV/HBV coinfection, even if $\mathrm{HBe} A g$ seroconversion occurs (CIII). 
Monitoring and Adverse Events (Including IRIS)—The parameters for successful therapy for chronic HBV infection are not well defined, but markers of improvement include decreased hepatic necroinflammatory disease, normalization of serum transaminase levels, reduction of $\mathrm{HBV}$ DNA levels, and $\mathrm{HBeAg}$ seroconversion. In children starting treatment for chronic HBV infection, serum transaminase levels should be measured frequently at the start of therapy and then every 3 to 6 months. In children who are also beginning cART, some experts would monitor transaminase levels more frequently during the first few months of therapy (e.g, monthly for 3 months) because of the risk of IRIS (see below). Monitoring of response to treatment for chronic HBV infection is based on testing for HBV DNA and $\mathrm{HBeAg}$ and anti-HBe antibody on the same schedule as transaminase evaluations (every 3-6 months).

Close monitoring for relapse is needed after withdrawal of therapy. In patients who are HBeAg-negative, treatment should be continued until HBsAg clearance has been achieved (BII).

In HIV/HBV-coinfected patients starting cART, serum transaminase elevations (flares) can occur as part of IRIS or secondary to cART-associated hepatotoxicity. HBV-associated liver injury is thought to be immunemediated, and restoration of immunocompetence with ARV treatment may reactivate liver inflammation and damage. Initiation of cART without antiHBV therapy can lead to re-activation of HBV. This does not represent a failure of cART but rather a sign of immune reconstitution. IRIS manifests by an increase in serum transaminase levels as the CD4 cell count increases during the first 6 to 12 weeks of cART. Thus, serum transaminase levels should be monitored closely after introduction of cART. In such situations, cART should be continued and treatment for HBV infection initiated. The prognosis for most IRIS cases is favorable because a robust inflammatory response may predict an excellent response to cART in terms of immune reconstitution, and perhaps, improved survival. In patients experiencing hepatic flare, differentiating between IRIS and druginduced liver toxicity may be difficult, and no reliable clinical or laboratory predictor exists to distinguish between the two. Close collaboration of the HIV specialist with a specialist in hepatic disease is recommended for such patients; a hepatologist should be consulted promptly if elevated aminotransferases levels are associated with clinical jaundice or other evidence of liver dysfunction (e.g., serum albumin).

Clinical and laboratory exacerbations of hepatitis and hepatic flare also can occur in coinfected children receiving cART if agents with anti-HBV activity are discontinued. Generally, once ARV drugs with anti-HBV activity are begun in coinfected children, they should be continued indefinitely unless contraindicated (CIII). If discontinuation of therapy for chronic HBV infection results in hepatic flare, therapy for chronic HBV should be reinstituted (BIII).

Some clinicians recommend monitoring HBV-infected children or adolescents for HCC with baseline screening and then annual or twice yearly determinations of serum alphafetoprotein (AFP) levels and abdominal ultrasonography; however, no data support the benefit of such surveillance. ${ }^{3,38,46,47}$ Current recommendations in HBV-infected, HIV- 
uninfected adults support abdominal ultrasonography in men aged $>40$ years and women aged $>50$ years. The use of AFP monitoring is controversial.

Adverse effects of IFN-a use in children, although frequent, usually are not severe or permanent; however, approximately $5 \%$ of children require treatment discontinuation. The most common side effects include an influenza-like syndrome, cytopenias, and neuropsychiatric effects. Influenza-like symptoms comprising fever, chills, headache, myalgia, arthralgia, abdominal pain, nausea, and vomiting are seen in $80 \%$ of patients during the first month of treatment. These side effects decrease substantially during the first 4 months of therapy; premedication with acetaminophen or ibuprofen may reduce side effects. Subtle personality changes, which resolve when therapy is discontinued, have been reported in $42 \%$ of children. ${ }^{40}$ Depression and suicidal ideation also have been reported in clinical trials of children treated with IFN-a. ${ }^{73}$ Ophthalmologic complications have been reported in clinical trials of children with pegylated IFN. ${ }^{74}$ Neutropenia, which resolves after discontinuation of therapy, is the most common laboratory abnormality; anemia and thrombocytopenia are less common. Abnormalities in thyroid function (hypothyroidism or hyperthyroidism) have been reported with IFN-a therapy. ${ }^{75}$ Loss of appetite with transient weight loss and impaired height growth can occur but usually resolves after completion of therapy. ${ }^{76}$ Less commonly observed side effects of IFN-a include epistaxis and transient mild alopecia. Antinuclear auto-antibodies have been detected in some children treated with IFN-a.

IFN- $a$ therapy is contraindicated in children with decompensated liver disease; severe cytopenia; severe renal, cardiac, or neuropsychiatric disorders; and autoimmune disease (CIII). ${ }^{77}$

Elevation of serum transaminase levels has been reported during IFN-a therapy in children and adults but usually is not an indication to stop therapy; these flares may herald impending HBeAg seroconversion. ${ }^{46}$ Children receiving IFN- $a$ therapy should be monitored with frequent complete blood count and liver function tests, and serum level of thyroidstimulating hormone should be determined at baseline and periodically (e.g., at least every 3 months) for the duration of treatment.

Lamivudine usually is well-tolerated in children; rare cases of lactic acidosis and pancreatitis have been reported in HIV/HBV-coinfected adults. tenofovir and adefovir can cause renal tubular disease. Patients receiving either drug should have baseline urinalysis and periodic urinalysis, serum creatinine and phosphate monitoring. Administration of other nephrotoxic agents increases the risk of renal toxicity. Tenofovir can lead to reduced bone density.

Managing Treatment Failure-Treatment failure is defined as ongoing HBV replication, persistent serum transaminase elevations, and the failure of $\mathrm{HBeAg}$ seroconversion in $\mathrm{HBeAg}$-positive patients at the completion of therapy (for IFN) and after an adequate trial of oral anti-HBV antivirals (generally at least 6-12 months). In individuals with $\mathrm{HBeAg-negative} \mathrm{hepatitis,} \mathrm{treatment} \mathrm{failure} \mathrm{is} \mathrm{defined} \mathrm{as} \mathrm{ongoing} \mathrm{HBV}$ replication (>10,000 IU) and persistent serum transaminase elevations. Flares of liver disease with 
increasing HBV DNA levels can be seen with the development of resistance to lamivudine or emtricitabine.

In some children who have received initial treatment for chronic HBV infection with standard-dose IFN-a monotherapy, use of higher-dose IFN-a for retreatment improves response. ${ }^{58,78,79}$

Lamivudine also has been used as secondary therapy for young ( $<12$ years old) HIVuninfected children who have not responded to standard IFN-a therapy (BI) ${ }^{80-82}$ in HIVinfected children, initiation of a lamivudinecontaining or emtricitabine-containing cART regimen (that also contains tenofovir, if aged $\geq 2$ years) can be considered (CIII).

For HIV/HBV coinfected children who develop lamivudine resistance during therapy, treatment options are more limited because of lack of data on use of adefovir, entecavir, and tenofovir for treatment of HBV infection in young children. Because these HBV drugresistant isolates may have lower replicative capacity than wild-type HBV, some experts recommend continuing lamivudine or emtricitabine therapy in such cases (CIII).

Alternatively, adding IFN-a can be considered or, in children old enough to receive adult doses of adefovir, adding that drug to the regimen can be considered (CIII).

Preventing Recurrence-Not applicable.

Discontinuing Secondary Prophylaxis—Not applicable.

\section{References}

1. Chu CM, Karayiannis P, Fowler MJ, Monjardino J, Liaw YF, Thomas HC. Natural history of chronic hepatitis B virus infection in Taiwan: studies of hepatitis B virus DNA in serum. Hepatology. 1985 May-Jun;5(3):431-434. Available at http://www.ncbi.nlm.nih.gov/pubmed/ 3997072. [PubMed: 3997072]

2. Chang MH, Sung JL, Lee CY, et al. Factors affecting clearance of hepatitis B e antigen in hepatitis B surface antigen carrier children. J Pediatr. 1989 Sep; 115(3):385-390. Available at http:// www.ncbi.nlm.nih.gov/pubmed/2769497. [PubMed: 2769497]

3. Elisofon SA, Jonas MM. Hepatitis B and C in children: current treatment and future strategies. Clin Liver Dis. 2006 Feb; 10(1):133-148. vii. Available at http://www.ncbi.nlm.nih.gov/pubmed/ 16376798. [PubMed: 16376798]

4. Hyams KC. Risks of chronicity following acute hepatitis B virus infection: a review. Clin Infect Dis. 1995 Apr; 20(4):992-1000. Available at http://www.ncbi.nlm.nih.gov/pubmed/7795104. [PubMed: 7795104]

5. Brant LJ, Reynolds C, Byrne L, Davison KL. Hepatitis B and residual risk of infection in English and Welsh blood donors, 1996 through 2008. Transfusion. $2011 \mathrm{Jul}$; 51(7):1493-1502. Available at http://www.ncbi.nlm.nih.gov/pubmed/21470235. [PubMed: 21470235]

6. Rogers AS, Lindsey JC, Futterman DC, Zimmer B, Abdalian SE, D'Angelo LJ. Serologic examination of hepatitis B infection and immunization in HIV-positive youth and associated risks. The Pediatric AIDS Clinical Trials Group Protocol 220 Team. AIDS Patient Care STDS. 2000 Dec; 14(12):651-657. Available at http://www.ncbi.nlm.nih.gov/pubmed/11119432. [PubMed: 11119432]

7. Wang EE, King S, Goldberg E, Bock B, Milner R, Read S. Hepatitis B and human immunodeficiency virus infection in street youths in Toronto, Canada. Pediatr Infect Dis J. 1991 
Feb; 10(2):130-133. Available at http://www.ncbi.nlm.nih.gov/pubmed/2062604. [PubMed: 2062604]

8. Mieli-Vergani G, Vergani D. Treatment of hepatitis B virus in children: why, whom, how? Indian J Gastroenterol. 2006 May-Jun;25(3):121-124. Available at http://www.ncbi.nlm.nih.gov/pubmed/ 16877822. [PubMed: 16877822]

9. Wasley, A.; Kruszon-Moran, D.; Kuhnert, W., et al. Hepatitis B prevalence in the U.S. in the era of vaccination [abstract 723]. Infectious Diseases Society of America 45th annual meeting; October 47, 2007; San Diego CA, Arlington VA. 2007.

10. Toussi SS, Abadi J, Rosenberg M, Levanon D. Prevalence of hepatitis B and C virus infections in children infected with HIV. Clin Infect Dis. 2007 Sep 15; 45(6):795-798. Available at http:// www.ncbi.nlm.nih.gov/pubmed/17712766. [PubMed: 17712766]

11. McMahon BJ, Alward WL, Hall DB, et al. Acute hepatitis B virus infection: relation of age to the clinical expression of disease and subsequent development of the carrier state. J Infect Dis. 1985 Apr; 151(4):599-603. Available at http://www.ncbi.nlm.nih.gov/pubmed/3973412. [PubMed: 3973412]

12. Tovo PA, Lazier L, Versace A. Hepatitis B virus and hepatitis C virus infections in children. Curr Opin Infect Dis. 2005 Jun; 18(3):261-266. Available at http://www.ncbi.nlm.nih.gov/pubmed/ 15864105. [PubMed: 15864105]

13. Delaplane D, Yogev R, Crussi F, Shulman ST. Fatal hepatitis B in early infancy: the importance of identifying HBsAg-positive pregnant women and providing immunoprophylaxis to their newborns. Pediatrics. 1983 Aug; 72(2):176-180. Available at http://www.ncbi.nlm.nih.gov/ pubmed/6683400. [PubMed: 6683400]

14. Bortolotti F, Calzia R, Cadrobbi P, et al. Liver cirrhosis associated with chronic hepatitis B virus infection in childhood. J Pediatr. 1986 Feb; 108(2):224-227. Available at http:// www.ncbi.nlm.nih.gov/pubmed/3944707. [PubMed: 3944707]

15. Chen CH, Chen YY, Chen GH, et al. Hepatitis B virus transmission and hepatocarcinogenesis: a 9 year retrospective cohort of 13676 relatives with hepatocellular carcinoma. J Hepatol. 2004 Apr; 40(4):653-659. Available at http://www.ncbi.nlm.nih.gov/pubmed/15030982. [PubMed: 15030982]

16. Bortolotti F, Guido M, Bartolacci S, et al. Chronic hepatitis B in children after e antigen seroclearance: final report of a 29-year longitudinal study. Hepatology. 2006 Mar; 43(3):556-562. Available at http://www.ncbi.nlm.nih.gov/pubmed/16496323. [PubMed: 16496323]

17. Chang MH, You SL, Chen CJ, et al. Decreased incidence of hepatocellular carcinoma in hepatitis B vaccinees: a 20-year follow-up study. J Natl Cancer Inst. 2009 Oct 7; 101(19):1348-1355. Available at http://www.ncbi.nlm.nih.gov/pubmed/19759364. [PubMed: 19759364]

18. Chen CJ, Yang HI, Su J, et al. Risk of hepatocellular carcinoma across a biological gradient of serum hepatitis B virus DNA level. JAMA. 2006 Jan 4; 295(1):65-73. Available at http:// www.ncbi.nlm.nih.gov/pubmed/16391218. [PubMed: 16391218]

19. Koziel MJ, Peters MG. Viral hepatitis in HIV infection. N Engl J Med. 2007 Apr 5; 356(14):14451454. Available at http://www.ncbi.nlm.nih.gov/pubmed/17409326. [PubMed: 17409326]

20. Weinbaum CM, Williams I, Mast EE, et al. Recommendations for identification and public health management of persons with chronic hepatitis B virus infection. MMWR Recomm Rep. 2008 Sep 19; 57(RR-8):1-20. Available at http://www.ncbi.nlm.nih.gov/pubmed/18802412. [PubMed: 18802412]

21. Lok AS, Ward JW, Perrillo RP, McMahon BJ, Liang TJ. Reactivation of hepatitis B during immunosuppressive therapy: potentially fatal yet preventable. Ann Intern Med. 2012 May 15; 156(10):743-745. Available at http://www.ncbi.nlm.nih.gov/pubmed/22586011. [PubMed: 22586011]

22. Fattovich G, Bortolotti F, Donato F. Natural history of chronic hepatitis B: special emphasis on disease progression and prognostic factors. J Hepatol. 2008 Feb; 48(2):335-352. Available at http://www.ncbi.nlm.nih.gov/pubmed/18096267. [PubMed: 18096267]

23. Chang MH, Hsu HY, Hsu HC, Ni YH, Chen JS, Chen DS. The significance of spontaneous hepatitis $\mathrm{B}$ e antigen seroconversion in childhood: with special emphasis on the clearance of 
hepatitis B e antigen before 3 years of age. Hepatology. 1995 Nov; 22(5):1387-1392. Available at http://www.ncbi.nlm.nih.gov/pubmed/7590652. [PubMed: 7590652]

24. Chu CM, Hung SJ, Lin J, Tai DI, Liaw YF. Natural history of hepatitis B e antigen to antibody seroconversion in patients with normal serum aminotransferase levels. Am J Med. 2004 Jun 15; 116(12):829-834. Available at http://www.ncbi.nlm.nih.gov/pubmed/15178498. [PubMed: 15178498]

25. Centers for Disease Control and Prevention. Guidelines for Vaccinating Pregnant Women: Abstracted recommendations from ACIP. 2013 Available at http://www.cdc.gov/vaccines/pubs/ downloads/b_preg_guide.pdf.

26. Mast EE, Margolis HS, Fiore AE, et al. A comprehensive immunization strategy to eliminate transmission of hepatitis B virus infection in the United States: recommendations of the Advisory Committee on Immunization Practices (ACIP) part 1: immunization of infants, children, and adolescents. MMWR Recomm Rep. 2005 Dec 23; 54(RR-16):1-31. Available at http:// www.ncbi.nlm.nih.gov/pubmed/16371945. [PubMed: 16371945]

27. Rutstein RM, Rudy B, Codispoti C, Watson B. Response to hepatitis B immunization by infants exposed to HIV. AIDS. 1994 Sep; 8(9):1281-1284. Available at http://www.ncbi.nlm.nih.gov/ pubmed/7802981. [PubMed: 7802981]

28. Siriaksorn S, Puthanakit T, Sirisanthana T, Sirisanthana V. Prevalence of protective antibody against hepatitis B virus in HIV-infected children with immune recovery after highly active antiretroviral therapy. Vaccine. 2006 Apr 12; 24(16):3095-3099. Available at http:// www.ncbi.nlm.nih.gov/pubmed/16488516. [PubMed: 16488516]

29. Cardell K, Akerlind B, Sallberg M, Fryden A. Excellent response rate to a double dose of the combined hepatitis A and B vaccine in previous nonresponders to hepatitis B vaccine. J Infect Dis. 2008 Aug 1; 198(3):299-304. Available at http://www.ncbi.nlm.nih.gov/pubmed/18544037. [PubMed: 18544037]

30. Flynn PM, Cunningham CK, Rudy B, et al. Hepatitis B vaccination in HIV-infected youth: a randomized trial of three regimens. J Acquir Immune Defic Syndr. 2011 Apr; 56(4):325-332. Available at http://www.ncbi.nlm.nih.gov/pubmed/21350366. [PubMed: 21350366]

31. Potsch DV, Oliveira ML, Ginuino C, et al. High rates of serological response to a modified hepatitis B vaccination schedule in HIV-infected adults subjects. Vaccine. 2010 Feb 10; 28(6): 1447-1450. Available at http://www.ncbi.nlm.nih.gov/pubmed/19995540. [PubMed: 19995540]

32. Pettit NN, DePestel DD, Malani PN, Riddell Jt. Factors associated with seroconversion after standard dose hepatitis B vaccination and high-dose revaccination among HIV-infected patients. HIV Clin Trials. 2010 Nov-Dec;11(6):332-339. Available at http://www.ncbi.nlm.nih.gov/ pubmed/21239361. [PubMed: 21239361]

33. Lao-Araya M, Puthanakit T, Aurpibul L, Taecharoenkul S, Sirisanthana T, Sirisanthana V. Prevalence of protective level of hepatitis B antibody 3 years after revaccination in HIV-infected children on antiretroviral therapy. Vaccine. 2011 May 23; 29(23):3977-3981. Available at http:// www.ncbi.nlm.nih.gov/pubmed/21473954. [PubMed: 21473954]

34. American Academy of Pediatrics. Red Book: 2012 Report of the Committee on Infectious Diseases. Elk Grove Village, IL: 2012.

35. Fiore AE, Wasley A, Bell BP. Prevention of hepatitis A through active or passive immunization: recommendations of the Advisory Committee on Immunization Practices (ACIP). MMWR Recomm Rep. 2006 May 19; 55(RR-7):1-23. Available at http://www.ncbi.nlm.nih.gov/pubmed/ 16708058.

36. Jonas MM, Block JM, Haber BA, et al. Treatment of children with chronic hepatitis B virus infection in the United States: patient selection and therapeutic options. Hepatology. 2010 Dec; 52(6):2192-2205. Available at http://www.ncbi.nlm.nih.gov/pubmed/20890947. [PubMed: 20890947]

37. Haber BA, Block JM, Jonas MM, et al. Recommendations for screening, monitoring, and referral of pediatric chronic hepatitis B. Pediatrics. 2009 Nov; 124(5):e1007-e1013. Available at http:// www.ncbi.nlm.nih.gov/pubmed/19805457. [PubMed: 19805457]

38. Shneider BL, Gonzalez-Peralta R, Roberts EA. Controversies in the management of pediatric liver disease: Hepatitis B, C and NAFLD: Summary of a single topic conference. Hepatology. 2006 
Nov; 44(5):1344-1354. Available at http://www.ncbi.nlm.nih.gov/pubmed/17058223. [PubMed: 17058223]

39. Jain MK, Comanor L, White C, et al. Treatment of hepatitis B with lamivudine and tenofovir in HIV/HBV-coinfected patients: factors associated with response. J Viral Hepat. 2007 Mar; 14(3): 176-182. Available at http://www.ncbi.nlm.nih.gov/pubmed/17305883. [PubMed: 17305883]

40. Sokal EM, Conjeevaram HS, Roberts EA, et al. Interferon alfa therapy for chronic hepatitis B in children: a multinational randomized controlled trial. Gastroenterology. 1998 May; 114(5):988995. Available at http://www.ncbi.nlm.nih.gov/pubmed/9558288. [PubMed: 9558288]

41. Jonas MM, Mizerski J, Badia IB, et al. Clinical trial of lamivudine in children with chronic hepatitis B. N Engl J Med. 2002 May 30; 346(22):1706-1713. Available at http:// www.ncbi.nlm.nih.gov/pubmed/12037150. [PubMed: 12037150]

42. Murray KF, Szenborn L, Wysocki J, et al. Randomized, placebo-controlled trial of tenofovir disoproxil fumarate in adolescents with chronic hepatitis B. Hepatology. 2012 Dec; 56(6):20182026. Available at http://www.ncbi.nlm.nih.gov/pubmed/22544804. [PubMed: 22544804]

43. Jonas MM, Kelly D, Pollack H, et al. Safety, efficacy, and pharmacokinetics of adefovir dipivoxil in children and adolescents (age 2 to < 18 years) with chronic hepatitis B. Hepatology. 2008 Jun; 47(6):1863-1871. Available at http://www.ncbi.nlm.nih.gov/pubmed/18433023. [PubMed: 18433023]

44. Chang TT, Gish RG, de Man R, et al. A comparison of entecavir and lamivudine for HBeAgpositive chronic hepatitis B. N Engl J Med. 2006 Mar 9; 354(10):1001-1010. Available at http:// www.ncbi.nlm.nih.gov/pubmed/16525137. [PubMed: 16525137]

45. Liaw YF, Gane E, Leung N, et al. 2-Year GLOBE trial results: telbivudine Is superior to lamivudine in patients with chronic hepatitis B. Gastroenterology. 2009 Feb; 136(2):486-495. Available at http://www.ncbi.nlm.nih.gov/pubmed/19027013. [PubMed: 19027013]

46. Jonas MM. Treatment of chronic hepatitis B in children. J Pediatr Gastroenterol Nutr. 2006 Jul; 43(Suppl 1):S56-S60. Available at http://www.ncbi.nlm.nih.gov/pubmed/16819403. [PubMed: 16819403]

47. Heller S, Valencia-Mayoral P. Treatment of viral hepatitis in children. Arch Med Res. 2007 Aug; 38(6):702-710. Available at http://www.ncbi.nlm.nih.gov/pubmed/17613361. [PubMed: 17613361]

48. Kobak GE, MacKenzie T, Sokol RJ, Narkewicz MR. Interferon treatment for chronic hepatitis B: enhanced response in children 5 years old or younger. J Pediatr. 2004 Sep; 145(3):340-345. Available at http://www.ncbi.nlm.nih.gov/pubmed/15343187. [PubMed: 15343187]

49. Ozgenc F, Dikici B, Targan S, et al. Comparison of antiviral effect of lamivudine with interferonalpha2a versus - alpha2b in children with chronic hepatitis B infection. Antivir Ther. 2004 Feb; 9(1):23-26. Available at http://www.ncbi.nlm.nih.gov/pubmed/15040533. [PubMed: 15040533]

50. Marcellin P, Lau GK, Bonino F, et al. Peginterferon alfa-2a alone, lamivudine alone, and the two in combination in patients with $\mathrm{HBeAg}$-negative chronic hepatitis B. N Engl J Med. 2004 Sep 16; 351(12):1206-1217. Available at http://www.ncbi.nlm.nih.gov/pubmed/15371578. [PubMed: 15371578]

51. Dikici B, Bosnak M, Kara IH, et al. Lamivudine and interferon-alpha combination treatment of childhood patients with chronic hepatitis B infection. Pediatr Infect Dis J. 2001 Oct; 20(10):988992. Available at http://www.ncbi.nlm.nih.gov/pubmed/11642634. [PubMed: 11642634]

52. Dikici B, Ozgenc F, Kalayci AG, et al. Current therapeutic approaches in childhood chronic hepatitis B infection: a multicenter study. J Gastroenterol Hepatol. 2004 Feb; 19(2):127-133. Available at http://www.ncbi.nlm.nih.gov/pubmed/14731120. [PubMed: 14731120]

53. Kuloglu Z, Krsacloglu CT, Kansu A, Erden E, Girgin N. Liver histology of children with chronic hepatitis treated with interferon-alpha alone or in combination with lamivudine. J Pediatr Gastroenterol Nutr. 2007 Nov; 45(5):564-568. Available at http://www.ncbi.nlm.nih.gov/pubmed/ 18030234. [PubMed: 18030234]

54. Sokucu S, Gokce S, Suoglu OD, Emiroglu H, Cevikbas U. Comparison of interferon monotherapy with interferonlamivudine combination treatment in children with chronic hepatitis B. Indian $\mathrm{J}$ Gastroenterol. 2006 May-Jun;25(3):136-139. Available at http://www.ncbi.nlm.nih.gov/pubmed/ 16877826. [PubMed: 16877826] 
55. Kansu A, Doganci T, Akman SA, et al. Comparison of two different regimens of combined interferon-alpha2a and lamivudine therapy in children with chronic hepatitis B infection. Antivir Ther. 2006; 11(2):255-261. Available at http://www.ncbi.nlm.nih.gov/pubmed/16640106. [PubMed: 16640106]

56. Yilmaz A, Akcam M, Gelen T, Artan R. Lamivudine and high-dose interferon alpha 2a combination treatment in naive $\mathrm{HBeAg}$-positive immunoactive chronic hepatitis B in children: an East Mediterranean center's experience. Eur J Pediatr. 2007 Mar; 166(3):195-199. Available at http://www.ncbi.nlm.nih.gov/pubmed/16944240. [PubMed: 16944240]

57. D'Antiga L, Aw M, Atkins M, Moorat A, Vergani D, Mieli-Vergani G. Combined lamivudine/ interferon-alpha treatment in"immunotolerant" children perinatally infected with hepatitis B: a pilot study. J Pediatr. 2006 Feb; 148(2):228-233. Available at http://www.ncbi.nlm.nih.gov/ pubmed/16492434. [PubMed: 16492434]

58. Saltik-Temizel IN, Kocak N, Demir H. Lamivudine and high-dose interferon-alpha combination therapy for naive children with chronic hepatitis B infection. J Clin Gastroenterol. 2005 Jan; 39(1): 68-70. Available at http://www.ncbi.nlm.nih.gov/pubmed/15599215. [PubMed: 15599215]

59. Benhamou Y, Thibault V, Vig P, et al. Safety and efficacy of adefovir dipivoxil in patients infected with lamivudineresistant hepatitis B and HIV-1. J Hepatol. 2006 Jan; 44(1):62-67. Available at http://www.ncbi.nlm.nih.gov/pubmed/16274835. [PubMed: 16274835]

60. Torre D, Tambini R. Interferon-alpha therapy for chronic hepatitis B in children: a meta-analysis. Clin Infect Dis. 1996 Jul; 23(1):131-137. Available at http://www.ncbi.nlm.nih.gov/pubmed/ 8816142. [PubMed: 8816142]

61. Gurakan F, Kocak N, Ozen H, Yuce A. Comparison of standard and high dosage recombinant interferon alpha $2 \mathrm{~b}$ for treatment of children with chronic hepatitis B infection. Pediatr Infect Dis J. 2000 Jan; 19(1):52-56. Available at http://www.ncbi.nlm.nih.gov/pubmed/10643851. [PubMed: $10643851]$

62. Yuce A, Kocak N, Ozen H, Gurakan F. Prolonged interferon alpha treatment in children with chronic hepatitis B. Ann Trop Paediatr. 2001 Mar; 21(1):77-80. Available at http:// www.ncbi.nlm.nih.gov/pubmed/11284252. [PubMed: 11284252]

63. Choe BH, Lee JH, Jang YC, et al. Long-term therapeutic efficacy of lamivudine compared with interferon-alpha in children with chronic hepatitis B: the younger the better. J Pediatr Gastroenterol Nutr. 2007 Jan; 44(1):92-98. Available at http://www.ncbi.nlm.nih.gov/pubmed/ 17204960. [PubMed: 17204960]

64. Lau GK, Piratvisuth T, Luo KX, et al. Peginterferon Alfa-2a, lamivudine, and the combination for HBeAg-positive chronic hepatitis B. N Engl J Med. 2005 Jun 30; 352(26):2682-2695. Available at http://www.ncbi.nlm.nih.gov/pubmed/15987917. [PubMed: 15987917]

65. Schwarz KB, Mohan P, Narkewicz MR, et al. Safety, efficacy and pharmacokinetics of peginterferon alpha2a (40 kd) in children with chronic hepatitis C. J Pediatr Gastroenterol Nutr. 2006 Oct; 43(4):499-505. Available at http://www.ncbi.nlm.nih.gov/pubmed/17033526. [PubMed: 17033526]

66. Wirth S, Pieper-Boustani H, Lang T, et al. Peginterferon alfa-2b plus ribavirin treatment in children and adolescents with chronic hepatitis C. Hepatology. 2005 May; 41(5):1013-1018. Available at http://www.ncbi.nlm.nih.gov/pubmed/15793840. [PubMed: 15793840]

67. Baker RD, Dee D, Baker SS. Response to pegylated interferon alpha- $2 b$ and ribavirin in children with chronic hepatitis C. J Clin Gastroenterol. 2007 Jan; 41(1):111-114. Available at http:// www.ncbi.nlm.nih.gov/pubmed/17198073. [PubMed: 17198073]

68. Sokal EM, Kelly DA, Mizerski J, et al. Long-term lamivudine therapy for children with $\mathrm{HBeAg-}$ positive chronic hepatitis B. Hepatology. $2006 \mathrm{Feb}$; 43(2):225-232. Available at http:// www.ncbi.nlm.nih.gov/pubmed/16440364. [PubMed: 16440364]

69. Locarnini S. Molecular virology and the development of resistant mutants: implications for therapy. Semin Liver Dis. 2005; 25(Suppl 1):9-19. Available at http://www.ncbi.nlm.nih.gov/ pubmed/16103977. [PubMed: 16103977]

70. Peters MG, Andersen J, Lynch P, et al. Randomized controlled study of tenofovir and adefovir in chronic hepatitis B virus and HIV infection: ACTG A5127. Hepatology. 2006 Nov; 44(5):11101116. Available at http://www.ncbi.nlm.nih.gov/pubmed/17058225. [PubMed: 17058225] 
71. Lai CL, Shouval D, Lok AS, et al. Entecavir versus lamivudine for patients with HBeAg-negative chronic hepatitis B. N Engl J Med. 2006 Mar 9; 354(10):1011-1020. Available at http:// www.ncbi.nlm.nih.gov/pubmed/16525138. [PubMed: 16525138]

72. McMahon MA, Jilek BL, Brennan TP, et al. The HBV drug entecavir - effects on HIV-1 replication and resistance. N Engl J Med. 2007 Jun 21; 356(25):2614-2621. Available at http:// www.ncbi.nlm.nih.gov/pubmed/17582071. [PubMed: 17582071]

73. Gonzalez-Peralta RP, Kelly DA, Haber B, et al. Interferon alfa-2b in combination with ribavirin for the treatment of chronic hepatitis $\mathrm{C}$ in children: efficacy, safety, and pharmacokinetics. Hepatology. 2005 Nov; 42(5):1010-1018. Available at http://www.ncbi.nlm.nih.gov/pubmed/ 16250032. [PubMed: 16250032]

74. Narkewicz MR, Rosenthal P, Schwarz KB, et al. Ophthalmologic complications in children with chronic hepatitis $C$ treated with pegylated interferon. J Pediatr Gastroenterol Nutr. 2010 Aug; 51(2):183-186. Available at http://www.ncbi.nlm.nih.gov/pubmed/20512062. [PubMed: 20512062]

75. Kuloglu Z, Kansu A, Berberoglu M, Adiyaman P, Ocal G, Girgin N. The incidence and evolution of thyroid dysfunction during interferon-alpha therapy in children with chronic hepatitis B infection. J Pediatr Endocrinol Metab. 2007 Feb; 20(2):237-245. Available at http:// www.ncbi.nlm.nih.gov/pubmed/17396441. [PubMed: 17396441]

76. Comanor L, Minor J, Conjeevaram HS, et al. Impact of chronic hepatitis B and interferon-alpha therapy on growth of children. J Viral Hepat. 2001 Mar; 8(2):139-147. Available at http:// www.ncbi.nlm.nih.gov/pubmed/11264734. [PubMed: 11264734]

77. Jara P, Bortolotti F. Interferon-alpha treatment of chronic hepatitis B in childhood: a consensus advice based on experience in European children. J Pediatr Gastroenterol Nutr. 1999 Aug; 29(2): 163-170. Available at http://www.ncbi.nlm.nih.gov/pubmed/10435653. [PubMed: 10435653]

78. Vajro P, Migliaro F, Fontanella A, Orso G. Interferon: a meta-analysis of published studies in pediatric chronic hepatitis B. Acta Gastroenterol Belg. 1998 Apr-Jun;61(2):219-223. Available at http://www.ncbi.nlm.nih.gov/pubmed/9658614. [PubMed: 9658614]

79. Ozen H, Kocak N, Yuce A, Gurakan F. Retreatment with higher dose interferon alpha in children with chronic hepatitis B infection. Pediatr Infect Dis J. 1999 Aug; 18(8):694-697. Available at http://www.ncbi.nlm.nih.gov/pubmed/10462338. [PubMed: 10462338]

80. Kocak N, Saltik IN, Ozen H, Yuce, Gurakan F. Lamivudine treatment for children with interferon refractory chronic hepatitis B. Hepatology. 2000 Feb.31(2):545. Available at http:// www.ncbi.nlm.nih.gov/pubmed/10691379. [PubMed: 10691379]

81. Sokal EM, Roberts EA, Mieli-Vergani G, et al. A dose ranging study of the pharmacokinetics, safety, and preliminary efficacy of lamivudine in children and adolescents with chronic hepatitis B. Antimicrob Agents Chemother. 2000 Mar; 44(3):590-597. Available at http:// www.ncbi.nlm.nih.gov/pubmed/10681323. [PubMed: 10681323]

82. Hartman C, Berkowitz D, Eshach-Adiv O, et al. Long-term lamivudine therapy for chronic hepatitis B infection in children unresponsive to interferon. J Pediatr Gastroenterol Nutr. 2006 Oct; 43(4):494-498. Available at http://www.ncbi.nlm.nih.gov/pubmed/17033525. [PubMed: 17033525]

\section{Dosing Recommendations for Prevention and Treatment of HBV in HIV/HBV Coinfected Children}

\begin{tabular}{|c|c|c|c|}
\hline \multicolumn{4}{|c|}{ Preventive Regimen } \\
\hline Indication & First Choice & Alternative & Comments/Special Issues \\
\hline Primary Prophylaxis & $\begin{array}{ll}\text { - } & \text { Hepatitis B } \\
\text { vaccine } \\
\text { - } \\
\text { Combination of } \\
\text { hepatitis B } \\
\text { immunoglobulin } \\
\text { and hepatitis B } \\
\text { vaccine for infants }\end{array}$ & $\begin{array}{l}\text { Hepatitis B } \\
\text { immunoglobulin } \\
\text { following exposure }\end{array}$ & $\begin{array}{l}\text { See Figures } 1 \text { and } 2 \text { for } \\
\text { detailed vaccine } \\
\text { recommendations. } \\
\text { Primary Prophylaxis } \\
\underline{\text { Indicated for: }}\end{array}$ \\
\hline
\end{tabular}




\begin{tabular}{|c|c|c|c|c|}
\hline \multicolumn{5}{|c|}{ Preventive Regimen } \\
\hline Indication & First Choice & Alternat & & Comments/Special Issues \\
\hline & $\begin{array}{l}\text { born to mothers } \\
\text { with hepatitis B } \\
\text { infection }\end{array}$ & & & $\begin{array}{l}\begin{array}{l}\text { All individuals } \\
\text { who are not } \\
\text { HBV infected }\end{array} \\
\text { Criteria for Discontinuing } \\
\text { Primary Prophylaxis: } \\
\qquad \quad \text { N/A } \\
\text { Criteria for Restarting } \\
\text { Primary Prophylaxis: } \\
\text { N/A }\end{array}$ \\
\hline Secondary Prophylaxis & Hepatitis A Vaccine & N/A & & $\begin{array}{l}\frac{\text { Secondary Prophylaxis }}{\text { Indicated for: }} \\
\qquad \quad \begin{array}{l}\text { Chronically } \\
\text { HBV-infected } \\
\text { individuals to } \\
\text { prevent further } \\
\text { liver injury }\end{array} \\
\begin{array}{c}\text { Criteria for Discontinuing } \\
\text { Secondary Prophylaxis: }\end{array} \\
\text { N/A } \\
\begin{array}{c}\text { Criteria for Restarting } \\
\text { Secondary Prophylaxis: }\end{array} \\
\text { N/A }\end{array}$ \\
\hline Treatment & 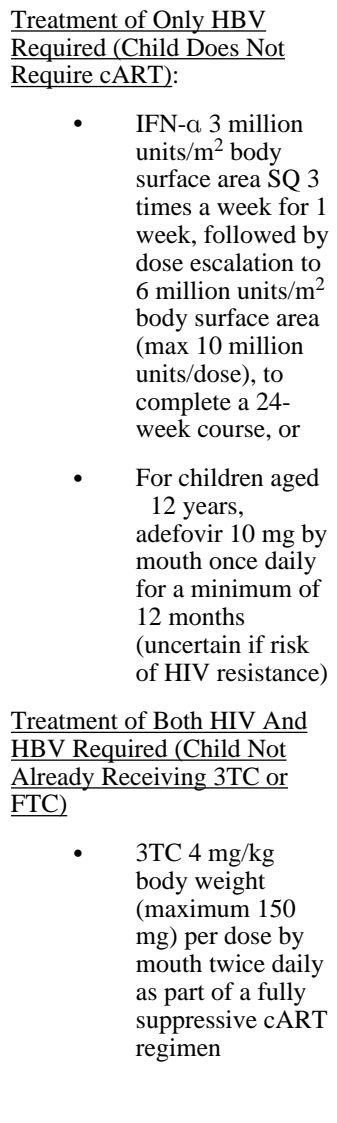 & • & $\begin{array}{l}\text { IFN-a } 10 \\
\text { million } \\
\text { units/m² } \\
\text { body } \\
\text { surface area } \\
\text { SQ } 3 \text { times } \\
\text { a week for } \\
6 \text { months } \\
\text { (sometimes } \\
\text { used for } \\
\text { retreatment } \\
\text { of failed } \\
\text { lower-dose } \\
\text { interferon } \\
\text { therapy) } \\
\text { Alternative } \\
\text { for } \\
\text { 3TC:FTC 6 } \\
\text { mg/kg body } \\
\text { weight } \\
\text { (maximum } \\
\text { 200 mg) } \\
\text { once daily }\end{array}$ & 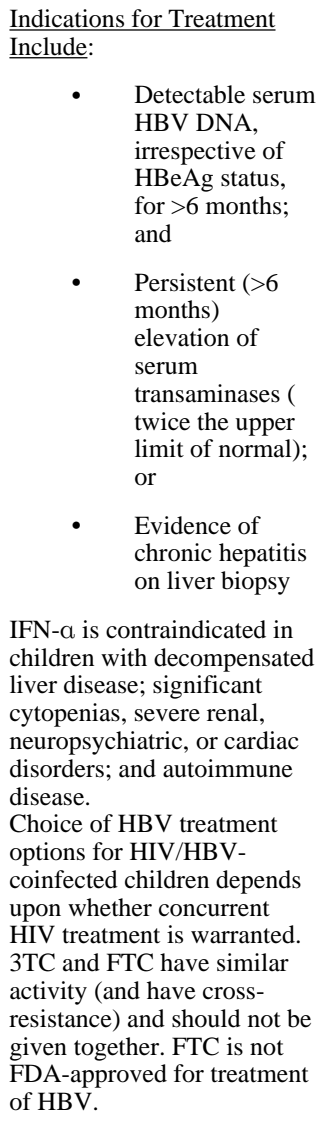 \\
\hline
\end{tabular}




\begin{tabular}{|c|c|c|c|}
\hline \multicolumn{4}{|c|}{ Preventive Regimen } \\
\hline Indication & First Choice & Alternative & Comments/Special Issues \\
\hline & 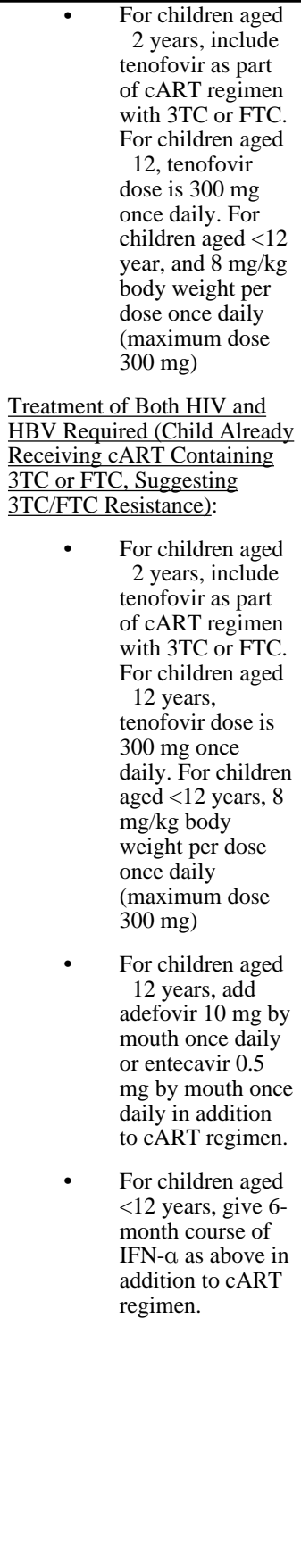 & & $\begin{array}{l}\text { Tenofovir is approved for } \\
\text { use in treatment of HIV } \\
\text { infection in children aged } 22 \\
\text { years but it is not approved } \\
\text { for treatment of HBV } \\
\text { infection in children aged } \\
<12 \text { years. It should only be } \\
\text { used for HBV in HIV/HBV- } \\
\text { infected children as part of a } \\
\text { cART regimen. } \\
\text { Adefovir is approved for use } \\
\text { in children aged } \geq 12 \text { years. } \\
\text { ETV is not approved for use } \\
\text { in children younger than age } \\
16 \text { years, but is under study } \\
\text { in HIV-uninfected children } \\
\text { for treatment of chronic } \\
\text { hepatitis B. Can be } \\
\text { considered for older HIV- } \\
\text { infected children who can } \\
\text { receive adult dosage. It } \\
\text { should only be used for HBV } \\
\text { in HIV/HBV-infected } \\
\text { children who also receive an } \\
\text { HIV-suppressive cART } \\
\text { regimen. } \\
\text { IRIS may be manifested by } \\
\text { dramatic increase in } \\
\text { transaminases as CD4 cell } \\
\text { counts rise within the first } 6 \\
\text { to } 12 \text { weeks of cART. It may } \\
\text { be difficult to distinguish } \\
\text { between drug-induced } \\
\text { hepatotoxicity and other } \\
\text { causes of hepatitis and IRIS. } \\
\text { In children receiving } \\
\text { tenofovir and } 3 T C \text { or FTC, } \\
\text { clinical and laboratory } \\
\text { exacerbations of hepatitis } \\
\text { (flare) may occur if the drug } \\
\text { is discontinued; thus, once } \\
\text { anti-HIV/HBV therapy has } \\
\text { begun, it should be continued } \\
\text { unless contraindicated or } \\
\text { until the child has been } \\
\text { treated for }>6 \text { months after } \\
\text { HBeAdg seroconversion and } \\
\text { can be closely monitored on } \\
\text { discontinuation. } \\
\text { If anti-HBV therapy is } \\
\text { discontinued and a flare } \\
\text { occurs, reinstitution of } \\
\text { therapy is recommended } \\
\text { because a flare can be life } \\
\text { threatening. } \\
\text { Telbivudine has been } \\
\text { approved for use in people } \\
\text { aged } \geq 16 \text { years with HBV; } \\
\text { there are no data on safety or } \\
\text { efficacy in children aged }<16 \\
\text { years; a pharmacokinetic } \\
\text { study is under way in HIV- } \\
\text { uninfected children. }\end{array}$ \\
\hline
\end{tabular}

Key to Acronyms: $3 \mathrm{TC}=$ lamivudine; $\mathrm{cART}=$ combined antiretroviral therapy; $\mathrm{CD} 4=\mathrm{CD} 4 \mathrm{~T}$ lymphocyte; $\mathrm{FTC}=$ emtricitabine; $\mathrm{HBeAg}=$ hepatitis $\mathrm{B}$ antigen; $\mathrm{HBV}=$ hepatitis $\mathrm{B}$ virus; $\mathrm{IFN}-\mathrm{a}=$ interferon alfa; IRIS = immune reconstitution inflammatory syndrome; $\mathrm{SQ}=$ subcutaneous; tenofovir = tenofovir disoproxil fumarate 


\section{Hepatitis C Virus (Last updated November 6, 2013; last reviewed November 6, 2013)}

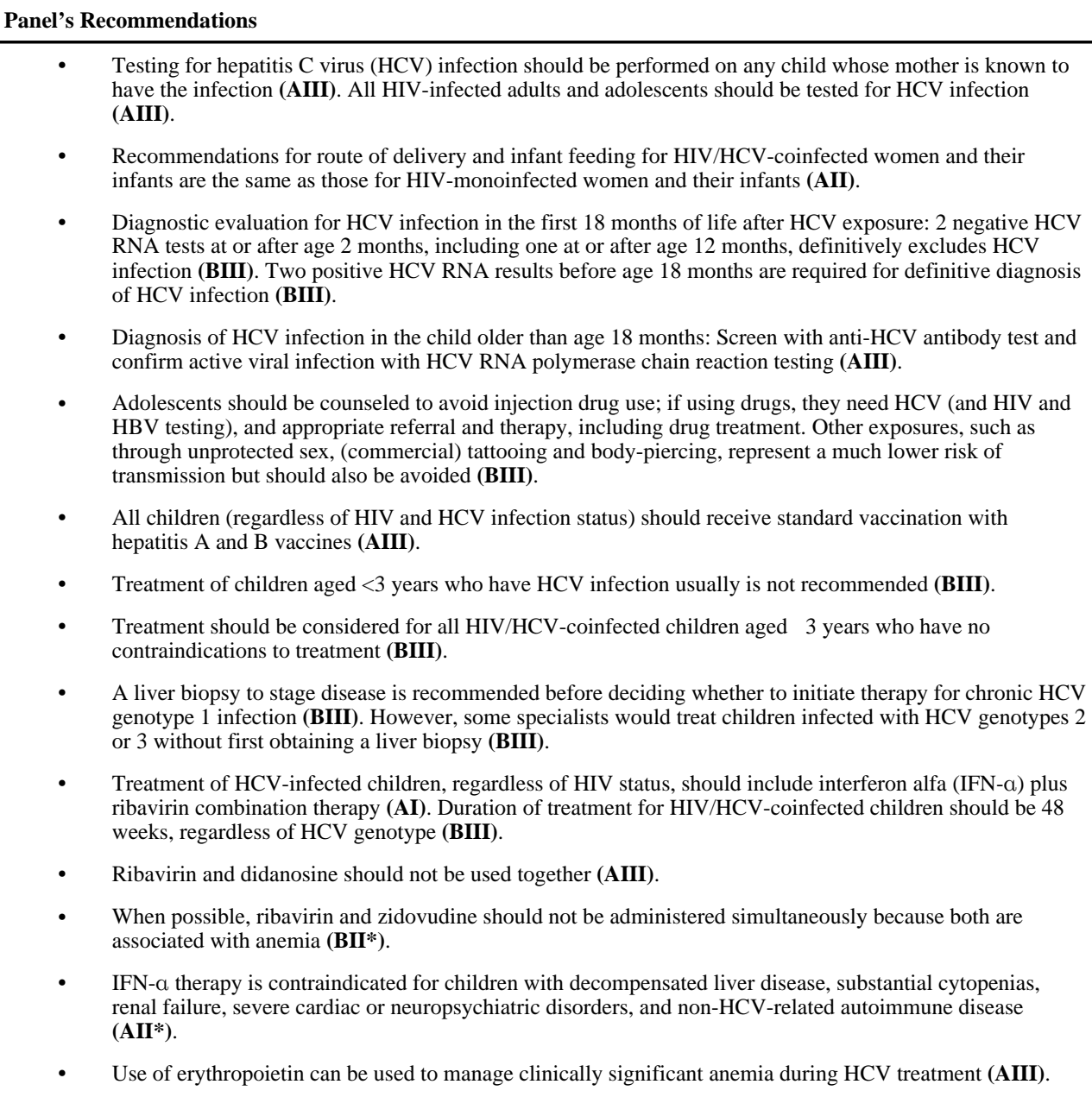

- Testing for hepatitis $\mathrm{C}$ virus (HCV) infection should be performed on any child whose mother is known to have the infection (AIII). All HIV-infected adults and adolescents should be tested for HCV infection (AIII).

- Recommendations for route of delivery and infant feeding for HIV/HCV-coinfected women and their infants are the same as those for HIV-monoinfected women and their infants (AII).

- Diagnostic evaluation for $\mathrm{HCV}$ infection in the first 18 months of life after $\mathrm{HCV}$ exposure: 2 negative $\mathrm{HCV}$ RNA tests at or after age 2 months, including one at or after age 12 months, definitively excludes HCV infection (BIII). Two positive HCV RNA results before age 18 months are required for definitive diagnosis of HCV infection (BIII).

- Diagnosis of HCV infection in the child older than age 18 months: Screen with anti-HCV antibody test and confirm active viral infection with HCV RNA polymerase chain reaction testing (AIII).

- Adolescents should be counseled to avoid injection drug use; if using drugs, they need HCV (and HIV and HBV testing), and appropriate referral and therapy, including drug treatment. Other exposures, such as through unprotected sex, (commercial) tattooing and body-piercing, represent a much lower risk of transmission but should also be avoided (BIII).

- All children (regardless of HIV and HCV infection status) should receive standard vaccination with hepatitis A and B vaccines (AIII).

- Treatment of children aged <3 years who have HCV infection usually is not recommended (BIII).

- Treatment should be considered for all HIV/HCV-coinfected children aged $\geq 3$ years who have no contraindications to treatment (BIII).

- A liver biopsy to stage disease is recommended before deciding whether to initiate therapy for chronic HCV genotype 1 infection (BIII). However, some specialists would treat children infected with HCV genotypes 2 or 3 without first obtaining a liver biopsy (BIII).

- Treatment of HCV-infected children, regardless of HIV status, should include interferon alfa (IFN-a) plus ribavirin combination therapy (AI). Duration of treatment for HIV/HCV-coinfected children should be 48 weeks, regardless of $\mathrm{HCV}$ genotype (BIII).

- Ribavirin and didanosine should not be used together (AIII).

- When possible, ribavirin and zidovudine should not be administered simultaneously because both are associated with anemia (BII*).

- IFN-a therapy is contraindicated for children with decompensated liver disease, substantial cytopenias, renal failure, severe cardiac or neuropsychiatric disorders, and non-HCV-related autoimmune disease (AII*).

- Use of erythropoietin can be used to manage clinically significant anemia during HCV treatment (AIII).

Rating of Recommendations: $\mathrm{A}=$ Strong; $\mathrm{B}=$ Moderate; $\mathrm{C}=$ Optional

Rating of Evidence: I = One or more randomized trials in children ${ }^{\dagger}$ with clinical outcomes and/or validated endpoints; I $^{*}$ $=$ One or more randomized trials in adults with clinical outcomes and/or validated laboratory endpoints with accompanying data in children ${ }^{\dagger}$ from one or more well-designed, nonrandomized trials or observational cohort studies with long-term clinical outcomes; II = One or more well-designed, nonrandomized trials or observational cohort studies in children $\underline{\text { with }}^{\dagger}$ long-term outcomes; $\mathrm{II}^{*}=$ One or more well-designed, nonrandomized trials or observational studies in adults with longterm clinical outcomes with accompanying data in children ${ }^{\dagger}$ from one or more similar nonrandomized trials or cohort studies with clinical outcome data; III = Expert opinion

${ }^{\dagger}$ Studies that include children or children/adolescents, but not studies limited to post-pubertal adolescents.

\section{Epidemiology}

In the United States, the prevalence of hepatitis $\mathrm{C}$ virus $(\mathrm{HCV})$ infection is $0.2 \%$ among children aged 1 to 11 years and $0.4 \%$ among adolescents aged 12 to 19 years. ${ }^{1,2}$ Modeling based on a recent U.S. census predicts that $\sim 7,200$ new cases of pediatric HCV infection occur annually. ${ }^{3}$ At least six HCV genotypes are known (genotypes 1-6), with genotype 1 
occurring most commonly in the United States. ${ }^{4}$ The prevalence of HCV infection among HIV-infected children may be higher. In a serostudy of $535 \mathrm{HIV}$-infected children followed in pediatric HIV clinical trials, the prevalence of $\mathrm{HCV}$ infection by HCV antibody and RNA testing was $1.5 \% .^{5}$ In a more recent study of 228 HIV-infected children at an inner-city hospital in the Bronx, seven HIV-infected children had chronic HCV infection (3.1\% [95\% $\mathrm{CI}, 1.4 \%-6.5 \%])$, defined as a reactive $\mathrm{HCV}$ antibody and positive $\mathrm{HCV}$ real-time polymerase chain reaction (PCR) ${ }^{6}$ The mean age of HIV/HCV-coinfected children was 16 years, and 57\% had mild elevation (up to twofold above upper limit of normal) in serum transaminase levels.

Mother-to-child transmission (MTCT) is the predominant mode of HCV acquisition in children. ${ }^{7,8}$ Other potential sources of $\mathrm{HCV}$ infection in older children, as for adults, include injection-drug use and, to a lesser extent, non-commercial body piercing or tattoos, unintentional needle stick injury, household contact, and sexual exposure. ${ }^{9,10}$ Before 1992, blood transfusion was a source of $\mathrm{HCV}$ infection in children. A recent retrospective study found that $3 \%$ of infants who had received blood transfusions in a neonatal intensive-care unit between 1975 and1992 were anti-HCV-antibody positive. ${ }^{11}$ However, the incidence of HCV infection from transfusion has dramatically declined since 1992, when secondgeneration HCV enzyme-linked immunosorbent assay (EIA) screening was implemented. With the current additional use of nucleic acid amplification testing, the risk of $\mathrm{HCV}$ infection through transfusion is approximately 1 in 2 million. ${ }^{12}$

The overall risk for MTCT of HCV from a woman infected with HCV alone ranges from $4 \%$ to $10 \%{ }^{7,13-21}$ The primary risk factor for perinatal $\mathrm{HCV}$ transmission is maternal $\mathrm{HCV}$ viremia at delivery, although an absolute threshold for $\mathrm{HCV}$ transmission has not been identified. ${ }^{14,22-27}$ Data do not indicate that HCV genotype is related to risk of perinatal HCV transmission. ${ }^{14,20}$ Although a few studies have suggested that vaginal delivery increases risk of $\mathrm{HCV}$ transmission ${ }^{13,15,17,22}$ and that $\mathrm{HCV}$ can be transmitted during the intrapartum period, ${ }^{28}$ most studies have found that mode of delivery does not appear to influence perinatal HCV transmission. $8,15,16,18,29-33$ In addition, even though HCV RNA can be detected in breast milk, studies of infants born to HCV-infected women have not demonstrated a higher risk of HCV transmission in breastfed infants than in those who are formula-fed. ${ }^{8,13-16,18,25,28,29,34}$

Maternal HIV coinfection increases the risk of perinatal transmission, with perinatal HCV transmission rates of $6 \%$ to $23 \%$ reported for infants born to women who are HIV/HCVcoinfected $^{7,13-15,19,26,30-32,35-41}$ Furthermore, a few studies suggest that children who are infected with HIV during the perinatal period may be more likely than HIV-uninfected children to acquire HCV infection from mothers who are HIV/HCV-coinfected. 30,31,38,40 Dual virus transmission has been reported in $4 \%$ to $10 \%$ of children born to HIV/HCVcoinfected mothers. ${ }^{13,30,36,38,39}$ HCV RNA levels are hypothesized to be higher among women coinfected with HIV than in those infected with HCV alone, which could account, in part, for the increased risk of MTCT of HCV from HIV/HCV-coinfected women; however, not all studies have found higher levels of HCV viremia among HIV-infected mothers. ${ }^{24,31,35}$ One European study suggested that perinatal transmission of HCV may be reduced in HIV-infected women receiving combination antiretroviral therapy (cART). ${ }^{32}$ 
Acute $\mathrm{HCV}$ infection appears to spontaneously resolve in $15 \%$ to $25 \%$ of adults. ${ }^{4}$ Findings from a limited number of longitudinal studies suggest that $\mathrm{HCV}$ infection resolves spontaneously in $17 \%$ to $59 \%$ of children with perinatal HCV infection. ${ }^{42-47}$ Spontaneous viral clearance in perinatal $\mathrm{HCV}$ infection was more common with $\mathrm{HCV}$ genotype 3 and usually occurred by age 3 years. ${ }^{46,48}$ Spontaneous viral clearance also has been associated with the presence of CC interleukin-28 (IL28B) host genotype in perinatally HCV-infected infants. ${ }^{49}$

Chronic HCV infection is defined as the presence of HCV RNA for $>6$ months. A study from Italy reported on long-term outcome in more than 350 children with chronic, untreated $\mathrm{HCV}$ infection (mean follow up 5.9 \pm 3.8 years), encompassing both perinatal and parenteral modes of transmission. The overall proportion of children who had spontaneous viral clearance was $7.5 \%$. The rate of spontaneous viral clearance in the vertically acquired cases was 11.5\%: half of these cases were genotype 3 and clearance occurred within the first 3 years of life. Evidence of chronic liver disease and cirrhosis was present in $1.8 \%$ of $\mathrm{HCV}$ infected children. The average time from diagnosis of HCV infection to development of cirrhosis was $9.87 \pm 5.9$ years. ${ }^{50}$ In a study comparing children with perinatal HIV/HCV coinfection with those with perinatal HCV infection alone, spontaneous clearance of HCV infection occurred in $10(17.5 \%)$ of 57 with HCV monoinfection but none of the 13 children with $\mathrm{HIV} / \mathrm{HCV}$ coinfection. ${ }^{51}$

\section{Clinical Manifestations}

Children with perinatal HCV infection appear to have a more benign clinical course than do adults with newly acquired HCV infection. ${ }^{9,52,53}$ Most HCV-infected children are asymptomatic, with minor abnormalities such as hepatomegaly, or mild nonspecific symptoms such as fatigue, myalgias, and poor weight gain; $9,53,54$ however, intermittent asymptomatic elevations in transaminase levels are common during the first 2 years of life. ${ }^{45,54-56}$ In a large European cohort of HCV-infected children, about $20 \%$ of children had apparent clearance of $\mathrm{HCV}$ viremia; $50 \%$ had chronic asymptomatic infection, characterized by intermittent viremia, rare hepatomegaly, and usually normal liver transaminase levels; and 30\% had chronic active infection with persistent viremia and abnormal transaminase levels. 46

Histopathologic inflammatory changes of chronic hepatitis may be present in patients with chronic HCV infection despite lack of symptoms, normal serum transaminase levels, and low HCV RNA levels. ${ }^{54}$ Analysis of liver histology in 121 treatment-naive pediatric patients showed some degree of inflammation in all samples, mild fibrosis (Ishak stage 1-2) in 80\% and cirrhosis in only $2 \%$ of patients. ${ }^{57}$ Most children with chronic HCV infection who have undergone liver biopsy and are included in published studies typically have mild-tomoderate liver disease as determined by signs of structural alterations, inflammatory activity, and necrosis. ${ }^{9,24,53,55}$ Similar proportions of vertically and parenterally HCVinfected children have signs of chronic hepatitis on liver biopsy. ${ }^{56} \mathrm{~A}$ small subset of children may develop severe liver disease. In a study of 60 children with perinatally acquired or transfusion-acquired $\mathrm{HCV}$ infection who were infected for a mean duration of 13 years, $12 \%$ had significant fibrosis on liver biopsy. ${ }^{53}$ Older age at time of infection and 
elevated serum gamma-glutamyltranspeptidase correlated with fibrosis; serum transaminase levels correlated with inflammation. ${ }^{53}$

In HIV/ HCV-coinfected adults, the natural history of HCV infection appears to be accelerated, with more rapid progression to cirrhosis, decompensated liver disease, hepatocellular carcinoma (HCC), and death. ${ }^{58,59}$ In HIV/HCV-coinfected adults, there are conflicting reports about the effect of cART and immune reconstitution on liver-related mortality, with some studies showing decreases and others little difference in liver-related mortality. ${ }^{60,61}$ Data are minimal on the effect of HIV/HCV-coinfection on the natural history of $\mathrm{HCV}$ infection in children and insufficient to draw conclusions about $\mathrm{HCV}$ disease progression in coinfected children. ${ }^{7}$

Data are conflicting on the impact of HCV infection on HIV disease progression in adults; some studies suggest higher rates of HIV progression and others do not. ${ }^{7}$ The effect of pediatric coinfection on HIV disease progression also is unclear because the number of coinfected children is small, and few studies have evaluated this. Two studies of children with perinatal HIV/HCV coinfection found no increase in HIV progression. On the other hand, in a study from Spain comparing children with perinatal HIV/HCV coinfection with those with perinatal $\mathrm{HCV}$ infection alone, $\mathrm{HCV}$ viremia and maximum transaminase levels were higher in the coinfected children than in those with $\mathrm{HCV}$ infection alone. ${ }^{51}$ In a study of older children with thalassemia who were infected through transfusion, disease progression was more rapid and mortality higher in those with HIV/HCV-coinfection than in those with HIV monoinfection. ${ }^{30,39,62}$

\section{Making the Diagnosis}

Testing for HCV infection should be performed on any child whose mother is known to have HCV infection (AIII). All HIV-infected adults and adolescents should be tested for HCV infection (AIII).

Serologic and nucleic acid tests are used to diagnose HCV infection. HCV RNA first becomes detectable 1 to 3 weeks after $\mathrm{HCV}$ infection and precedes serologic response to HCV. ${ }^{4}$ A third-generation EIA is available for detecting antibody to HCV (anti-HCV). Passively transferred maternal anti-HCV can be detected for up to 18 months in infants born to HCV-infected mothers. In a large cohort of HCV-exposed but -uninfected children, anti$\mathrm{HCV}$ was present in $15 \%$ of children at 12 months, $5 \%$ at 15 months, and $2 \%$ at 18 months. ${ }^{24}$ Therefore, only the presence of persistent HCV viremia can be used to reliably verify $\mathrm{HCV}$ infection in atrisk children aged $<18$ months. ${ }^{63} \mathrm{HCV}$ infection can be diagnosed in such children using a nucleic acid test to detect HCV RNA after age 1 month; the sensitivity of the HCV RNA testing is low at birth (22\%), but increases to $85 \%$ at 6 months. ${ }^{64}$ Most children with perinatal HCV infection will have a positive HCV RNA test by age 12 months. However, because of intermittent viremia, a single negative HCV RNA test is not conclusive evidence of lack of infection. Thus, two negative HCV RNA results obtained at or after age 2 months, including at least one test at or after age 12 months, definitively excludes HCV infection in an HCV-exposed infant (BIII). Two positive HCV RNA results before age 18 months are required for definitive diagnosis of $\mathrm{HCV}$ infection (BIII). ${ }^{64}$ 
A positive anti-HCV antibody test in a child aged $>18$ months indicates prior $\mathrm{HCV}$ infection. Supplemental testing with a more specific assay, such as HCV RNA testing, is recommended to clarify whether the positive antibody test indicates a chronic active or a resolved infection (AIII). A positive HCV RNA test confirms current $\mathrm{HCV}$ infection, and if positive for $>6$ months, indicates chronic infection. HCV RNA can be measured qualitatively or quantitatively. Qualitative nucleic acid tests include qualitative PCR and transcription-mediated amplification. Quantitative tests include branched-chain DNA amplification, quantitative PCR, and real-time PCR and are most useful for monitoring response to anti-HCV therapy. Quantitative HCV RNA level (i.e., HCV viral load) does not correlate with degree of liver damage and does not serve as a surrogate for measuring disease severity, but it does provide important information about response to antiviral therapy. Assays vary substantially, and if serial values are required to monitor treatment, continued use of the same quantitative assay for all assessments is strongly recommended.

Liver biopsy is the most accurate test to assess the severity of hepatic disease and measure the amount of hepatic fibrosis present. The degree of liver injury found on biopsy can be used to determine the need for treatment. A liver biopsy is recommended before initiating therapy for chronic HCV genotype 1 infection, but is often used for other genotype infections (2, 3 or 4$)$ as well. ${ }^{65,66}$ Virus eradication from anti-HCV therapy is much more likely in HCV genotypes 2 and $3(\sim 80 \%)$, compared with genotype $1(<50 \%)$. Thus, the need for liver biopsy before treatment of HCV genotypes 2 or 3 is debatable. ${ }^{67}$

\section{Prevention Recommendations}

Preventing Exposure-All HIV-infected patients should be screened for HCV. No reliable strategy exists to prevent perinatal $\mathrm{HCV}$ transmission. Cesarean delivery is not associated with reduced perinatal transmission of $\mathrm{HCV}$ infection and is not recommended for this purpose for women with chronic HCV infection (AII). The presence of maternal $\mathrm{HCV}$ coinfection does not alter the current recommendation for scheduled cesarean delivery for HIV-infected women who have HIV RNA levels $>1,000$ copies $/ \mathrm{mL}$ near delivery to prevent perinatal HIV transmission. Limited data suggest that breastfeeding does not transmit HCV; maternal HCV infection is not a reason to avoid breastfeeding. The presence of maternal HCV coinfection does not alter the current recommendation that HIV-infected women in the United States should not breastfeed their infants (see Recommendations for Use of Antiretroviral Drugs in Pregnant HIV-1-Infected Women for Maternal Health and Interventions to Reduce Perinatal HIV Transmission in the United States).

No vaccines are available to prevent $\mathrm{HCV}$ infection. Adolescents considering tattooing or body-piercing should be informed about potential risks of acquiring $\mathrm{HCV}$, which could be transmitted if equipment is not sterile or if proper infection-control procedures are not followed, and to avoid injection-drug use and unprotected sex (BIII). ${ }^{68} \mathrm{HCV}$-infected persons should be advised not to share toothbrushes, razors, tweezers, nail clippers and other personal-care articles that might be contaminated with blood to prevent transmission of HCV. 
Preventing First Episode of Disease-Patients with chronic liver disease can develop fulminant hepatitis from hepatitis A (HAV) or B (HAB) infection; all children (regardless of $\mathrm{HIV}$ and HCV infection status) should receive standard vaccination with HAV and HAB vaccines (AIII) ${ }^{68-70}$ Patients with advanced HCV-related liver disease and/or HIV infection may not mount an appropriate immune response to vaccines. ${ }^{71}$ Therefore, measurement of HBV antibody titers 3 months after completion of the vaccination series is recommended. ${ }^{72}$

\section{Treatment Recommendations}

Treating Disease-The standard of care for treatment of chronic HCV infection in children, in the absence of HIV infection, is combination therapy with pegylated interferonalfa (Peg-IFN- $a$ ) administered as a subcutaneous (SQ) injection once a week and twicedaily oral ribavirin. ${ }^{73}$ For HIV-uninfected individuals, the length of therapy is 48 weeks for treating HCV genotype 1, and 24 weeks for genotypes 2 or 3 . Recent studies demonstrate improved response rates in adults with the addition of protease inhibitors (PIs) (telaprevir or boceprevir) to pegylated-IFN- $\alpha$ and ribavirin in adults with $\mathrm{HCV}$ genotype 1 infection. ${ }^{74,75}$ A recently completed randomized, double-blind, placebo-controlled trial of peg-IFN-a with and without ribavirin in $\mathrm{HCV}$-infected children has shown superior efficacy with combination therapy. ${ }^{76}$ Improved viral eradication was previously noted with combination therapy in a non-randomized European study as well. ${ }^{67}$ There is a paucity of studies on the treatment of $\mathrm{HIV} / \mathrm{HCV}$-coinfected children. Consultation with experts in treating chronic $\mathrm{HCV}$ infection in children is recommended.

The PIs telaprevir and boceprevir have been approved for use in adults for treatment of HCV genotype 1 , in concert with peg-IFN-a and ribavirin therapy. ${ }^{77}$ This "triple therapy" was associated with markedly improved viral clearance, with sustained virologic responses demonstrated in up to $68 \%$ of treated patients. ${ }^{75}$

HIV/HCV-coinfected Adults and Adolescents-Regardless of HIV coinfection status, treatment should be considered in all non-pregnant, $\mathrm{HCV}$-infected adults or adolescents who have abnormal serum transaminase levels and liver biopsies that show chronic hepatitis with inflammation, fibrosis, and compensated liver disease. ${ }^{78}$ Because of the high rate of $\mathrm{HCV}$ eradication with treatment for $\mathrm{HCV}$ genotypes 2 or 3, a liver biopsy is optional before initiating therapy. Treatment should be considered for HIV/HCV-coinfected adults and adolescents for whom potential benefits of treatment are judged to outweigh potential risks, including those infected with HCV genotypes 2 or 3, those with stable HIV infection not requiring cART, and those with $\mathrm{HCV}$-related cryoglobulinemic vasculitis or glomerulonephritis. ${ }^{65,79}$ Baseline serum HCV RNA level and HCV genotype are the primary predictors of response to treatment. Younger age, higher CD4 T lymphocyte (CD4 cell) count, elevated transaminase levels, lack of liver fibrosis, low body mass index, lack of insulin resistance, and white race are other variables associated with better treatment response. ${ }^{79}$ The recommended treatment for HCV genotypes 2 and 3 is combined peg-IFN$\mathrm{a} 2 \mathrm{a}$ (or $2 \mathrm{~b}$ ) plus ribavirin for 48 weeks, while telaprevir is added to that regimen for the first 12 weeks in most adults with HCV genotype 1 infection (see Adult OI Guidelines). In HIV/ HCV-coinfected adults, rates of sustained virologic response to treatment with peg-IFN-a 
plus ribavirin range from $44 \%$ to $73 \%$ for treatment of $\mathrm{HCV}$ genotypes 2 and 3 infection and from $14 \%$ to $29 \%$ for $\mathrm{HCV}$ genotype 1 infection. ${ }^{73,80,81}$ Response to anti-HCV treatment improves in HIV/HCV-coinfected adults with CD4 cell counts $>200$ cells $/ \mathrm{mm}^{3}$; therefore, cART should be considered before anti-HCV therapy is initiated in HIV/HCV-coinfected patients with CD4 cell counts $<200$ cells $/ \mathrm{mm}^{3}$. Anti-HCV treatment is not recommended during pregnancy for $\mathrm{HCV}$-infected women because ribavirin is teratogenic.

HCV-Infected, HIV-Uninfected Children-Treatment usually is not recommended for HIV-uninfected children aged $<3$ years who have $\mathrm{HCV}$ infection because spontaneous $\mathrm{HCV}$ clearance can occur in this age group (BIII). All decisions about treatment of HCV infection in children should be individualized because HCV usually causes mild disease in this population and few data exist to identify risk factors differentiating those at greater risk for progression of liver disease. ${ }^{80,82}$

HCV-infected, HIV-uninfected children $\geq 3$ years old who are chosen for treatment should receive combination therapy with peg-IFN- $\alpha$ and ribavirin for 48 weeks for genotype 1 and 24 weeks for genotypes 2 or 3 (AI). This recommendation is based on the results of a recently completed pediatric trial in the United States on the efficacy of peg-IFN-a with or without ribavirin. ${ }^{76}$ In this trial, children aged 5 to 17 years were defined as having chronic $\mathrm{HCV}$ infection based on at least 2 positive $\mathrm{HCV}$ RNA blood tests for $>6$ months duration and liver histology consistent with $\mathrm{HCV}$ infection. The primary outcome measured was a sustained virologic response (SVR) defined as non-detectable HCV RNA in plasma at 24 weeks after treatment completion. The overall SVR was $53 \%$ with combination therapy and $21 \%$ with peg-IFN-a monotherapy. Combination therapy resulted in SVR in $47 \%$ of patients with genotype $1 \mathrm{HCV}$ and $80 \%$ of patients with genotypes $2-6 \mathrm{HCV}$. A non-randomized trial using peg-IFN- $a$ and ribavirin for pediatric $\mathrm{HCV}$ infection in Europe found similar efficacy for combination therapy. SVR was achieved in $48 \%$ of patients with genotype 1 and $100 \%$ of patients with genotypes 2 or $3 .{ }^{67}$

Previous studies on the use of combination therapy with standard IFN-a (SQ injections 3 times weekly) and ribavirin reported overall rates of SVR ranging from $46 \%$ to $65 \%{ }^{66,83-87}$ In these studies, children infected with genotype 1 were less likely to have a SVR (36\%) than those infected with genotypes 2 or 3 (SVR 84\%). ${ }^{83}$ Other factors associated with favorable response to anti-HCV treatment in children include lower pretreatment HCV RNA levels, white race, and possibly younger age. ${ }^{66}$

HIV/HCV-coinfected Children-No specific studies have been done of treatment of children with HIV/HCV-coinfection, and recommendations are based primarily on data from adults. Because therapy for $\mathrm{HCV}$ infection is more likely to be effective in younger patients and in those without advanced disease or immunodeficiency, treatment should be considered for all $\mathrm{HIV} / \mathrm{HCV}$-coinfected children aged $\geq 3$ years who have no contraindications to treatment (BIII) (see Dosing Table for contraindications to anti-HCV drugs). Treatment of $\mathrm{HIV} / \mathrm{HCV}$-coinfected children aged $<3$ years usually is not recommended (BIII), even though spontaneous HCV clearance in HIV/HCV-coinfected children may occur at lower rates than in HIV-uninfected children. ${ }^{51}$ 
In HIV/HCV-coinfected adults, the recommended duration of combination treatment is 48 weeks for infections with all HCV genotypes, including 2 and 3, because coinfected adults may not respond as well as those who are HIV-uninfected and they may have higher rates of relapse. Moreover, the efficacy of shorter treatment has not been adequately evaluated in HIV-infected individuals. ${ }^{79}$ By extrapolation, 48 weeks of therapy also are recommended for HIV/HCV-coinfected children, regardless of genotype (BIII). Potential drug interactions complicate the concomitant use of cART and anti-HCV therapy. Ribavirin enhances phosphorylation of didanosine, which could increase the risk of toxicity; therefore, these drugs should not be used together (AIII). Ribavirin and zidovudine both are associated with anemia and should not be administered together (BII*). ${ }^{79}$

The PIs telaprevir and boceprevir are approved only for use in adults with genotype $1 \mathrm{HCV}$ infection. These agents may be tested and approved for use in children in the near future. No recommendations for use of these agents in children can be made at this time. See Adult OI Guidelines for important warnings about drug interactions between HCV PIs and HIV PIs and other antiretroviral drugs.

\section{Monitoring and Adverse Events (Including IRIS)}

Monitoring in Children Not Receiving Anti-HCV Therapy: Although no evidence-based long-term monitoring guidelines exist for children with perinatally acquired HCV, many experts monitor HCV RNA levels and serum transaminase levels every 6 to 12 months and complete blood counts $(\mathrm{CBC})$ and serum alpha fetoprotein levels annually. ${ }^{82}$ Serum transaminase levels can fluctuate and do not necessarily correlate with histologic liver damage because significant liver disease can be present in patients with normal serum transaminase levels. In HCV-infected persons who are HIV-uninfected, HCC rarely is seen in the absence of cirrhosis. The benefits of serum alpha-fetoprotein (AFP) and abdominal sonography as screening tools for HCC have not been studied in children. Some experts perform periodic sonographic screening at defined intervals (every 2-5 years) in children with chronic $\mathrm{HCV}$ infection; others do these tests only in those with advanced liver disease and/or rising serum AFP concentrations. ${ }^{82}$ The risk of HCC in HCV-infected children, with or without HIV infection, is unknown.

As with HIV/HBV-coinfection, use of cART in HIV/HCV-coinfected patients can worsen hepatitis, with increases in serum transaminase levels and clinical signs of liver disease, including hepatomegaly and jaundice (also called "hepatic flare"). This does not represent a failure of ART, but rather, is a sign of immune reconstitution. Immune reconstitution inflammatory syndrome (IRIS) manifests by an increase in serum transaminase levels as the CD4 cell count increases during the first 6 to 12 weeks of cART. Thus, serum transaminase levels should be monitored closely after introduction of cART in HIV/HCV-coinfected children. The prognosis for most patients with IRIS is favorable. Consultation with a hepatologist should be sought if elevated aminotransferases are associated with clinical jaundice or other evidence of liver dysfunction, in other words, low serum albumin.

Monitoring During Combination Therapy (Interferon and Ribavirin): HCV RNA quantitation is used to monitor response to antiviral therapy. HCV RNA levels should be 
performed at baseline; after 5, 12, and 24 weeks of antiviral therapy; at treatment completion (48 weeks); and 6 months after treatment cessation. Some experts continue to perform serial HCV RNA testing at 6- to 12-month intervals for an additional 1 to 5 years to exclude late virologic relapse.

The following are outcomes measured during the treatment of HCV:

- Rapid Virological Response (RVR): Non-detectable plasma HCV RNA after 4 weeks of therapy;

- Early Virologic Response (EVR): Decrease in HCV RNA $\geq 2 \log _{10} \mathrm{IU} / \mathrm{mL}$ below baseline after 12 weeks of therapy;

- End Of Treatment Virologic Response: Non-detectable HCV RNA at time of treatment completion;

- $\quad$ Sustained Virologic Response (SVR): Non-detectable HCV RNA at 24 weeks after treatment completion;

- Virologic Relapse: Achievement of end of treatment response followed by return of HCV RNA positivity after treatment completion;

- Nonresponse: Failure to suppress HCV RNA below detection at any time during treatment; and

- Breakthrough Response: Reemergence of detectable HCV RNA from nondetectable status despite the continuation of therapy. ${ }^{4}$

In the absence of specific data for HIV/HCV-coinfected children, the criteria for determining response to therapy in HCV-monoinfected children and HIV/HCV-coinfected adults are used. Failure to achieve EVR with treatment with peg-IFN-a and ribavirin correlates with a low chance ( $<3 \%)$ of achieving SVR (based on adult data) and treatment can be discontinued after 12 weeks. Treatment should be discontinued in patients who achieve an EVR but still have detectable HCV RNA at 24 weeks of therapy. For all other $\mathrm{HIV} / \mathrm{HCV}$-coinfected children, treatment should be given for 48 weeks, regardless of genotype (BIII). In addition to HCV RNA quantification, patients receiving antiviral therapy for $\mathrm{HCV}$ infection should be closely monitored for medication side effects with $\mathrm{CBC}$, measurement of serum transaminase levels, thyroid function tests, ophthalmologic exams, and assessment of mental status/mood disorders. Some experts would monitor transaminase levels more frequently during the first few months of therapy, such as monthly for 3 months, in HIV/HCV-coinfected children who are also starting cART because of the risk of IRIS.

Side effects of IFN-a in children are common but usually not severe; approximately 5\% of children need to discontinue treatment because of side effects. The most common side effects include influenza-like symptoms (e.g., fever, chills, headache, myalgias, arthralgias, abdominal pain, nausea, vomiting) in $80 \%$ of patients during the first month of treatment. However, these symptoms usually resolve over time and usually are not treatment-limiting; pre-medication with acetaminophen or ibuprofen may reduce the incidence of side effects. In $42 \%$ of children subtle personality changes that resolve when therapy is discontinued 
have been reported ${ }^{88}$ Depression and suicidal ideation also have been reported in clinical trials of children treated with IFN-a. ${ }^{83}$ Neutropenia, which usually improves with dosereduction, is the most common laboratory abnormality; anemia and thrombocytopenia are less common. Abnormalities in thyroid function (hypothyroidism or hyperthyroidism) have been reported with IFN-a therapy. ${ }^{89}$ Loss of appetite, with transient weight loss and impaired height growth, can occur but usually resolves after completion of therapy. ${ }^{90}$

Less commonly observed side effects of IFN-a include epistaxis and transient mild alopecia. Some children develop antinuclear autoantibodies. The incidence of interferon-associated ophthalmologic complications in HCV-infected children on combination therapy was recently reported. ${ }^{91}$ Three of 114 patients developed significant eye disease, including ischemic retinopathy with cotton wool spots, uveitis, and transient monocular blindness. Despite the low incidence of disease, the severity of the ophthalmologic findings warrants follow-up with eye exams at 24 and 48 weeks of therapy. IFN-a therapy is contraindicated in children with decompensated liver disease, substantial cytopenias, renal failure, severe cardiac or neuropsychiatric disorders, and non-HCV-related autoimmune disease (AII*). ${ }^{92}$

Side effects of ribavirin include hemolytic anemia and lymphopenia. Ribavirin-induced hemolytic anemia is dose-dependent and usually presents with a substantial decrease in hemoglobin within 1 to 2 weeks after ribavirin initiation, but the hemoglobin usually stabilizes. Significant anemia (hemoglobin $<10 \mathrm{~g} / \mathrm{dL}$ ) occurs in about $10 \%$ of ribavirintreated children. ${ }^{82}$ Erythropoietin can be used to manage clinically significant anemia during HCV treatment (BIII). Coadministration of didanosine is contraindicated in children receiving ribavirin because this combination can increase the risk of mitochondrial toxicity and hepatic decompensation (AIII). Children receiving concomitant zidovudine may be more likely to experience bone marrow suppression; if possible, zidovudine should be avoided in children receiving ribavirin (BII*). Children who are receiving zidovudine and ribavirin together should be monitored closely for neutropenia and anemia. Ribavirin is teratogenic and should not be used by pregnant women. Sexually active adolescent girls or those likely to become sexually active who are receiving ribavirin should be counseled about the risks and need for consistent contraceptive use during and for 6 months after completion of ribavirin therapy.

In patients on $\mathrm{HCV}$ therapy who start cART and experience hepatic flares, differentiating between IRIS and drug-induced liver toxicity may be difficult, and no reliable clinical or laboratory predictors exist to distinguish between the two. Close interaction of the HIV specialist with a specialist in hepatic disease - usually a hepatologist—is recommended for such patients; prompt consultation with a hepatologist should be sought if elevated aminotransferases are associated with clinical jaundice or other evidence of liver dysfunction (such as low serum albumin).

Managing Treatment Failure-No data exist on which to base recommendations for treatment of HIV/HCV-coinfected children in whom initial HCV treatment fails. In HIV/ HCV-coinfected adults, a second course of treatment has a limited chance of resulting in sustained virologic response in nonresponders (those who do not achieve early virologic response by week 12 or undetectable HCV load at week 24) or patients whose HCV 
relapses. Therapeutic interventions for such adults need to be individualized according to prior response, tolerance, and adherence to therapy; severity of liver disease; viral genotype; and other underlying factors that might influence response. Some experts might extend the duration of treatment (e.g., to 72 weeks) in adults who experience a virologic response followed by relapse after adequate HCV therapy or in patients with advanced fibrosis. In the setting of treatment failure, the addition of PIs (telaprevir or boceprevir) to peg-IFN-a and ribavirin may increase rates of eradication. ${ }^{74,75}$ In a clinical trial, the addition of boceprevir to peg-IFN-ribavirin resulted in significantly higher rates of sustained virologic response (up to $66 \%$ ) in previously treated adults with chronic HCV genotype 1 infection, as compared with peg-interferon-ribavirin alone. ${ }^{93} \mathrm{HIV} / \mathrm{HCV}$-coinfected adults with prior suboptimal treatment of $\mathrm{HCV}$ genotypes 2 or 3 infection may benefit from optimized retreatment; coinfected adults with treatment failure for HCV genotype 1 infection may benefit from retreatment with a combination regimen that includes boceprevir or telaprevir (see Adult OI Guidelines). See Adult OI Guidelines for important warnings about drug interactions between HCV PIs and HIV PIs and other antiretroviral drugs. No data exist on which to base a recommendation for management of $\mathrm{HCV}$ treatment failure in HIV/HCV-coinfected children, and pediatric trials of triple therapy are warranted.

Preventing Recurrence-Not applicable.

Discontinuing Secondary Prophylaxis—Not applicable.

\section{References}

1. Alter MJ, Kruszon-Moran D, Nainan OV, et al. The prevalence of hepatitis C virus infection in the United States, 1988 through 1994. N Engl J Med. 1999 Aug 19; 341(8):556-562. Available at http://www.ncbi.nlm.nih.gov/pubmed/10451460. [PubMed: 10451460]

2. El-Kamary SS, Serwint JR, Joffe A, Santosham M, Duggan AK. Prevalence of hepatitis C virus infection in urban children. J Pediatr. 2003 Jul; 143(1):54-59. Available at http:// www.ncbi.nlm.nih.gov/pubmed/12915824. [PubMed: 12915824]

3. Jhaveri R, Grant W, Kauf TL, McHutchison J. The burden of hepatitis C virus infection in children: estimated direct medical costs over a 10-year period. J Pediatr. 2006 Mar; 148(3):353-358. Available at http://www.ncbi.nlm.nih.gov/pubmed/16615966. [PubMed: 16615966]

4. Scott JD, Gretch DR. Molecular diagnostics of hepatitis C virus infection: a systematic review. JAMA. 2007 Feb 21; 297(7):724-732. Available at http://www.ncbi.nlm.nih.gov/pubmed/ 17312292. [PubMed: 17312292]

5. Schuval S, Van Dyke RB, Lindsey JC, et al. Hepatitis C prevalence in children with perinatal human immunodeficiency virus infection enrolled in a long-term follow-up protocol. Arch Pediatr Adolesc Med. 2004 Oct; 158(10):1007-1013. Available at http://www.ncbi.nlm.nih.gov/pubmed/15466691. [PubMed: 15466691]

6. Toussi SS, Abadi J, Rosenberg M, Levanon D. Prevalence of hepatitis B and C virus infections in children infected with HIV. Clin Infect Dis. 2007 Sep 15; 45(6):795-798. Available at http:// www.ncbi.nlm.nih.gov/pubmed/17712766. [PubMed: 17712766]

7. England K, Thorne C, Newell ML. Vertically acquired paediatric coinfection with HIV and hepatitis C virus. Lancet Infect Dis. 2006 Feb; 6(2):83-90. Available at http://www.ncbi.nlm.nih.gov/ pubmed/16439328. [PubMed: 16439328]

8. Tajiri H, Miyoshi Y, Funada S, et al. Prospective study of mother-to-infant transmission of hepatitis C virus. Pediatr Infect Dis J. 2001 Jan; 20(1):10-14. Available at http://www.ncbi.nlm.nih.gov/ pubmed/11176560. [PubMed: 11176560] 
9. Jara P, Resti M, Hierro L, et al. Chronic hepatitis $\mathrm{C}$ virus infection in childhood: clinical patterns and evolution in 224 white children. Clin Infect Dis. 2003 Feb 1; 36(3):275-280. Available at http:// www.ncbi.nlm.nih.gov/pubmed/12539067. [PubMed: 12539067]

10. Murray KF, Richardson LP, Morishima C, Owens JW, Gretch DR. Prevalence of hepatitis C virus infection and risk factors in an incarcerated juvenile population: a pilot study. Pediatrics. $2003 \mathrm{Jan}$; 111(1):153-157. Available at http://www.ncbi.nlm.nih.gov/pubmed/12509569. [PubMed: 12509569]

11. Cagle HH, Jacob J, Homan CE, Williams JL, Christensen CJ, McMahon BJ. Results of a general hepatitis $\mathrm{C}$ lookback program for persons who received blood transfusions in a neonatal intensive care unit between January 1975 and July 1992. Arch Pediatr Adolesc Med. 2007 Feb; 161(2):125130. Available at http://www.ncbi.nlm.nih.gov/pubmed/17283296. [PubMed: 17283296]

12. Stramer SL. Current risks of transfusion-transmitted agents: a review. Arch Pathol Lab Med. 2007 May; 131(5):702-707. Available at http://www.ncbi.nlm.nih.gov/pubmed/17488155. [PubMed: 17488155]

13. Tovo PA, Palomba E, Ferraris G, et al. Increased risk of maternal-infant hepatitis $\mathrm{C}$ virus transmission for women coinfected with human immunodeficiency virus type 1. Italian Study Group for HCV Infection in Children. Clin Infect Dis. 1997 Nov; 25(5):1121-1124. Available at http://www.ncbi.nlm.nih.gov/pubmed/9402369. [PubMed: 9402369]

14. Zanetti AR, Tanzi E, Romano L, et al. A prospective study on mother-to-infant transmission of hepatitis C virus. Intervirology. 1998; 41(4-5):208-212. Available at http:// www.ncbi.nlm.nih.gov/pubmed/10213898. [PubMed: 10213898]

15. Gibb DM, Goodall RL, Dunn DT, et al. Mother-to-child transmission of hepatitis C virus: evidence for preventable peripartum transmission. Lancet. 2000 Sep 9; 356(9233):904-907. Available at http://www.ncbi.nlm.nih.gov/pubmed/11036896. [PubMed: 11036896]

16. European Paediatric Hepatitis CVN. Effects of mode of delivery and infant feeding on the risk of mother-to-child transmission of hepatitis $\mathrm{C}$ virus. European Paediatric Hepatitis C Virus Network. BJOG: an international journal of obstetrics and gynaecology. 2001 Apr; 108(4):371-377. Available at http://www.ncbi.nlm.nih.gov/pubmed/11305543.

17. Granovsky MO, Minkoff HL, Tess BH, et al. Hepatitis C virus infection in the mothers and infants cohort study. Pediatrics. 1998 Aug; 102(2 Pt 1):355-359. Available at http:// www.ncbi.nlm.nih.gov/pubmed/9685438. [PubMed: 9685438]

18. Resti M, Azzari C, Mannelli F, et al. Mother to child transmission of hepatitis C virus: prospective study of risk factors and timing of infection in children born to women seronegative for HIV-1. Tuscany Study Group on Hepatitis C Virus Infection. BMJ. 1998 Aug 15; 317(7156):437-441. Available at http://www.ncbi.nlm.nih.gov/pubmed/9703524. [PubMed: 9703524]

19. Thomas SL, Newell ML, Peckham CS, Ades AE, Hall AJ. A review of hepatitis C virus (HCV) vertical transmission: risks of transmission to infants born to mothers with and without $\mathrm{HCV}$ viraemia or human immunodeficiency virus infection. Int J Epidemiol. 1998 Feb; 27(1):108-117. Available at http://www.ncbi.nlm.nih.gov/pubmed/9563703. [PubMed: 9563703]

20. Mazza C, Ravaggi A, Rodella A, et al. Prospective study of mother-to-infant transmission of hepatitis C virus (HCV) infection. Study Group for Vertical Transmission. J Med Virol. 1998 Jan; 54(1):12-19. Available at http://www.ncbi.nlm.nih.gov/pubmed/9443104. [PubMed: 9443104]

21. McMenamin MB, Jackson AD, Lambert J, et al. Obstetric management of hepatitis C-positive mothers: analysis of vertical transmission in 559 mother-infant pairs. Am J Obstet Gynecol. 2008 Sep; 199(3):315, e311-e315. Available at http://www.ncbi.nlm.nih.gov/pubmed/18771997. [PubMed: 18771997]

22. Okamoto M, Nagata I, Murakami J, et al. Prospective reevaluation of risk factors in mother-tochild transmission of hepatitis $\mathrm{C}$ virus: high virus load, vaginal delivery, and negative anti-NS4 antibody. J Infect Dis. 2000 Nov; 182(5):1511-1514. Available at http://www.ncbi.nlm.nih.gov/ pubmed/11023474. [PubMed: 11023474]

23. Dal Molin G, D'Agaro P, Ansaldi F, et al. Mother-to-infant transmission of hepatitis C virus: rate of infection and assessment of viral load and IgM anti-HCV as risk factors. J Med Virol. 2002 Jun; 67(2):137-142. Available at http://www.ncbi.nlm.nih.gov/pubmed/11992574. [PubMed: 11992574] 
24. Mast EE, Hwang LY, Seto DS, Nolte FS NO, Wurtzel H, et al. Risk factors for perinatal transmission of hepatitis $\mathrm{C}$ virus $(\mathrm{HCV})$ and the natural history of $\mathrm{HCV}$ infection acquired in infancy. J Infect Dis. 2005; 192(11):1880-1889. Available at http://www.ncbi.nlm.nih.gov/ pubmed/16267758. [PubMed: 16267758]

25. Ruiz-Extremera A, Salmeron J, Torres C, et al. Follow-up of transmission of hepatitis C to babies of human immunodeficiency virus-negative women: the role of breast-feeding in transmission. Pediatr Infect Dis J. 2000 Jun; 19(6):511-516. Available at http://www.ncbi.nlm.nih.gov/pubmed/ 10877164. [PubMed: 10877164]

26. Polis CB, Shah SN, Johnson KE, Gupta A. Impact of maternal HIV coinfection on the vertical transmission of hepatitis C virus: a meta-analysis. Clin Infect Dis. 2007; 44(8):1123-1131. Available at http://www.ncbi.nlm.nih.gov/pubmed/17366462. [PubMed: 17366462]

27. Shebl FM, El-Kamary SS, Saleh DA, et al. Prospective cohort study of mother-to-infant infection and clearance of hepatitis C in rural Egyptian villages. J Med Virol. 2009 Jun; 81(6):1024-1031. Available at http://www.ncbi.nlm.nih.gov/pubmed/19382251. [PubMed: 19382251]

28. Mok J, Pembrey L, Tovo PA, Newell ML. European Paediatric Hepatitis CVN. When does mother to child transmission of hepatitis C virus occur? Arch Dis Child Fetal Neonatal Ed. 2005 Mar; 90(2):F156-F160. Available at http://www.ncbi.nlm.nih.gov/pubmed/15724041. [PubMed: 15724041]

29. Conte D, Fraquelli M, Prati D, Colucci A, Minola E. Prevalence and clinical course of chronic hepatitis $\mathrm{C}$ virus (HCV) infection and rate of $\mathrm{HCV}$ vertical transmission in a cohort of 15,250 pregnant women. Hepatology. 2000 Mar; 31(3):751-755. Available at http:// www.ncbi.nlm.nih.gov/pubmed/10706568. [PubMed: 10706568]

30. Papaevangelou V, Pollack H, Rochford G, et al. Increased transmission of vertical hepatitis C virus (HCV) infection to human immunodeficiency virus (HIV)-infected infants of HIV- and HCVcoinfected women. J Infect Dis. 1998 Oct; 178(4):1047-1052. Available at http:// www.ncbi.nlm.nih.gov/pubmed/9806033. [PubMed: 9806033]

31. Marine-Barjoan E, Berrebi A, Giordanengo V, et al. HCV/HIV co-infection, HCV viral load and mode of delivery: risk factors for mother-to-child transmission of hepatitis C virus? AIDS. 2007 Aug 20; 21(13):1811-1815. Available at http://www.ncbi.nlm.nih.gov/pubmed/17690581. [PubMed: 17690581]

32. European Paefiatric Hepatiis C Virus Network. A significant sex-but not elective cesarean section-effect on motherto-child transmission of hepatitis C virus infection. J Infect Dis. 2005; 192(11):1872-1879. Available at http://www.ncbi.nlm.nih.gov/pubmed/16267757. [PubMed: 16267757]

33. Ghamar Chehreh ME, Tabatabaei SV, Khazanehdari S, Alavian SM. Effect of cesarean section on the risk of perinatal transmission of hepatitis C virus from HCV-RNA+/HIV-mothers: a metaanalysis. Arch Gynecol Obstet. 2011 Feb; 283(2):255-260. Available at http:// www.ncbi.nlm.nih.gov/pubmed/20652289. [PubMed: 20652289]

34. Lin HH, Kao JH, Hsu HY, et al. Absence of infection in breast-fed infants born to hepatitis C virus-infected mothers. J Pediatr. 1995 Apr; 126(4):589-591. Available at http:// www.ncbi.nlm.nih.gov/pubmed/7535353. [PubMed: 7535353]

35. Pappalardo BL. Influence of maternal human immunodeficiency virus (HIV) co-infection on vertical transmission of hepatitis C virus (HCV): a meta-analysis. Int J Epidemiol. 2003 Oct; 32(5):727-734. Available at http://www.ncbi.nlm.nih.gov/pubmed/14559740. [PubMed: 14559740]

36. Thomas DL, Villano SA, Riester KA, et al. Perinatal transmission of hepatitis C virus from human immunodeficiency virus type 1-infected mothers. Women and Infants Transmission Study. J Infect Dis. 1998 Jun; 177(6):1480-1488. Available at http://www.ncbi.nlm.nih.gov/pubmed/9607823. [PubMed: 9607823]

37. Paccagnini S, Principi N, Massironi E, et al. Perinatal transmission and manifestation of hepatitis C virus infection in a high risk population. Pediatr Infect Dis J. 1995 Mar; 14(3):195-199. Available at http://www.ncbi.nlm.nih.gov/pubmed/7761184. [PubMed: 7761184]

38. Hershow RC, Riester KA, Lew J, et al. Increased vertical transmission of human immunodeficiency virus from hepatitis $\mathrm{C}$ virus-coinfected mothers. Women and Infants 
Transmission Study. J Infect Dis. 1997 Aug; 176(2):414-420. Available at http:// www.ncbi.nlm.nih.gov/pubmed/9237706. [PubMed: 9237706]

39. Nigro G, D'Orio F, Catania $S$, et al. Mother to infant transmission of coinfection by human immunodeficiency virus and hepatitis $\mathrm{C}$ virus: prevalence and clinical manifestations. Arch Virol. 1997; 142(3):453-457. Available at http://www.ncbi.nlm.nih.gov/pubmed/9349291. [PubMed: 9349291]

40. Giovannini M, Tagger A, Ribero ML, et al. Maternal-infant transmission of hepatitis C virus and HIV infections: a possible interaction. Lancet. 1990 May 12.335(8698):1166. Available at http:// www.ncbi.nlm.nih.gov/pubmed/1971901. [PubMed: 1971901]

41. Ngo-Giang-Huong N, Jourdain G, Sirirungsi W, et al. Human immunodeficiency virus-hepatitis C virus co-infection in pregnant women and perinatal transmission to infants in Thailand. Int $\mathrm{J}$ Infect Dis. 2010 Jul; 14(7):e602-e607. Available at http://www.ncbi.nlm.nih.gov/pubmed/20047847. [PubMed: 20047847]

42. El-Sherbini A, Hassan W, Abdel-Hamid M, Naeim A. Natural history of hepatitis C virus among apparently normal schoolchildren: follow-up after 7 years. J Trop Pediatr. 2003 Dec; 49(6):384385. Available at http://www.ncbi.nlm.nih.gov/pubmed/14725420. [PubMed: 14725420]

43. Rerksuppaphol S, Hardikar W, Dore GJ. Long-term outcome of vertically acquired and posttransfusion hepatitis C infection in children. J Gastroenterol Hepatol. 2004 Dec; 19(12):13571362. Available at http://www.ncbi.nlm.nih.gov/pubmed/15610308. [PubMed: 15610308]

44. England K, Pembrey L, Tovo PA, Newell ML. European Paediatric Hcv N. Growth in the first 5 years of life is unaffected in children with perinatally-acquired hepatitis $\mathrm{C}$ infection. J Pediatr. 2005 Aug; 147(2):227-232. Available at http://www.ncbi.nlm.nih.gov/pubmed/16126055. [PubMed: 16126055]

45. Resti M, Jara P, Hierro L, et al. Clinical features and progression of perinatally acquired hepatitis C virus infection. J Med Virol. 2003 Jul; 70(3):373-377. Available at http://www.ncbi.nlm.nih.gov/ pubmed/12766999. [PubMed: 12766999]

46. European Paediatric Hepatitis CVN. Three broad modalities in the natural history of vertically acquired hepatitis C virus infection. Clin Infect Dis. 2005 Jul 1; 41(1):45-51. Available at http:// www.ncbi.nlm.nih.gov/pubmed/15937762. [PubMed: 15937762]

47. Bortolotti F, Resti M, Marcellini M, et al. Hepatitis C virus (HCV) genotypes in 373 Italian children with $\mathrm{HCV}$ infection: changing distribution and correlation with clinical features and outcome. Gut. 2005 Jun; 54(6):852-857. Available at http://www.ncbi.nlm.nih.gov/pubmed/ 15888796. [PubMed: 15888796]

48. Guido M, Rugge M, Jara P, et al. Chronic hepatitis C in children: the pathological and clinical spectrum. Gastroenterology. 1998 Dec; 115(6):1525-1529. Available at http:// www.ncbi.nlm.nih.gov/pubmed/9834281. [PubMed: 9834281]

49. Ruiz-Extremera A, Munoz-Gamez JA, Salmeron-Ruiz MA, et al. Genetic variation in interleukin $28 \mathrm{~B}$ with respect to vertical transmission of hepatitis $\mathrm{C}$ virus and spontaneous clearance in $\mathrm{HCV}$ infected children. Hepatology. 2011 Jun; 53(6):1830-1838. Available at http:// www.ncbi.nlm.nih.gov/pubmed/21413051. [PubMed: 21413051]

50. Bortolotti F, Verucchi G, Camma C, et al. Long-term course of chronic hepatitis C in children: from viral clearance to end-stage liver disease. Gastroenterology. 2008 Jun; 134(7):1900-1907. Available at http://www.ncbi.nlm.nih.gov/pubmed/18439604. [PubMed: 18439604]

51. Claret-Teruel G, Noguera-Julian A, Esteva C, et al. Impact of human immunodeficiency virus coinfection on the progression of mother-to-child transmitted hepatitis $\mathrm{C}$ virus infection. Pediatr Infect Dis J. 2011 Sep; 30(9):801-804. Available at http://www.ncbi.nlm.nih.gov/pubmed/ 21772231. [PubMed: 21772231]

52. Aach RD, Yomtovian RA, Hack M. Neonatal and pediatric posttransfusion hepatitis C: a look back and a look forward. Pediatrics. 2000 Apr; 105(4 Pt 1):836-842. Available at http:// www.ncbi.nlm.nih.gov/pubmed/10742329. [PubMed: 10742329]

53. Mohan P, Colvin C, Glymph C, et al. Clinical spectrum and histopathologic features of chronic hepatitis C infection in children. J Pediatr. 2007 Feb; 150(2):168-174. 174 e161. Available at http://www.ncbi.nlm.nih.gov/pubmed/17236895. [PubMed: 17236895] 
54. Davison SM, Mieli-Vergani G, Sira J, Kelly DA. Perinatal hepatitis C virus infection: diagnosis and management. Arch Dis Child. 2006 Sep; 91(9):781-785. Available at http:// www.ncbi.nlm.nih.gov/pubmed/16923861. [PubMed: 16923861]

55. Tovo PA, Pembrey LJ, Newell ML. Persistence rate and progression of vertically acquired hepatitis C infection. European Paediatric Hepatitis C Virus Infection. J Infect Dis. 2000 Feb; 181(2):419-424. Available at http://www.ncbi.nlm.nih.gov/pubmed/10669321. [PubMed: 10669321]

56. England K, Thorne C, Harris H, Ramsay M, Newell ML. The impact of mode of acquisition on biological markers of paediatric hepatitis C virus infection. J Viral Hepat. 2011 Aug; 18(8):533541. Available at http://www.ncbi.nlm.nih.gov/pubmed/21762285. [PubMed: 21762285]

57. Goodman ZD, Makhlouf HR, Liu L, et al. Pathology of chronic hepatitis C in children: liver biopsy findings in the Peds-C Trial. Hepatology. 2008 Mar; 47(3):836-843. Available at http:// www.ncbi.nlm.nih.gov/pubmed/18167062. [PubMed: 18167062]

58. Graham CS, Baden LR, Yu E, et al. Influence of human immunodeficiency virus infection on the course of hepatitis C virus infection: a meta-analysis. Clin Infect Dis. 2001 Aug 15; 33(4):562569. Available at http://www.ncbi.nlm.nih.gov/pubmed/11462196. [PubMed: 11462196]

59. Merchante N, Giron-Gonzalez JA, Gonzalez-Serrano M, et al. Survival and prognostic factors of HIV-infected patients with HCV-related end-stage liver disease. AIDS. 2006 Jan 2; 20(1):49-57. Available at http://www.ncbi.nlm.nih.gov/pubmed/16327319. [PubMed: 16327319]

60. Mehta SH, Thomas DL, Torbenson M, Brinkley S, Mirel L, et al. The effect of antiretroviral therapy on liver disease among adults with HIV and hepatitis C coinfection. Hepatology. 2005; 41(1):123-131. 2005 Available at http://www.ncbi.nlm.nih.gov/pubmed/15619237. [PubMed: 15619237]

61. Qurishi N, Kreuzberg CL, GEWKBST, Rockstroh JK SU. Effect of antiretroviral therapy on liverrelated mortality in patients with HIV and hepatitis C coinfection. Lancet. 2004; 362(9397):17081713. Available at http://www.ncbi.nlm.nih.gov/pubmed/14643119. [PubMed: 14643119]

62. Shivraj SO, Chattopadhya D, Grover G, Kumar A, Baveja UK. Role of HCV coinfection towards disease progression and survival in HIV-1 infected children: a follow-up study of 10 years. J Trop Pediatr. 2006 Jun; 52(3):206-211. Available at http://www.ncbi.nlm.nih.gov/pubmed/16339160. [PubMed: 16339160]

63. Dunn DT, Gibb DM, Healy M, et al. Timing and interpretation of tests for diagnosing perinatally acquired hepatitis C virus infection. Pediatr Infect Dis J. 2001 Jul; 20(7):715-716. Available at http://www.ncbi.nlm.nih.gov/pubmed/11465848. [PubMed: 11465848]

64. Polywka S, Pembrey L, Tovo PA, Newell ML. Accuracy of HCV-RNA PCR tests for diagnosis or exclusion of vertically acquired HCV infection. J Med Virol. 2006 Feb; 78(2):305-310. Available at http://www.ncbi.nlm.nih.gov/pubmed/16372293. [PubMed: 16372293]

65. Koziel MJ, Peters MG. Viral hepatitis in HIV infection. N Engl J Med. 2007 Apr 5; 356(14):14451454. Available at http://www.ncbi.nlm.nih.gov/pubmed/17409326. [PubMed: 17409326]

66. Shneider BL, Gonzalez-Peralta R, Roberts EA. Controversies in the management of pediatric liver disease: Hepatitis B, C and NAFLD: Summary of a single topic conference. Hepatology. 2006 Nov; 44(5):1344-1354. Available at http://www.ncbi.nlm.nih.gov/pubmed/17058223. [PubMed: 17058223]

67. Wirth S, Pieper-Boustani H, Lang T, et al. Peginterferon alfa-2b plus ribavirin treatment in children and adolescents with chronic hepatitis C. Hepatology. 2005 May; 41(5):1013-1018. Available at http://www.ncbi.nlm.nih.gov/pubmed/15793840. [PubMed: 15793840]

68. CDC. Recommendations for prevention and control of hepatitis C virus (HCV) infection and HCVrelated chronic disease. Centers for Disease Control and Prevention. MMWR Recomm Rep. 1998 Oct 16; 47(RR-19):1-39. Available at http://www.ncbi.nlm.nih.gov/pubmed/9790221.

69. Fiore AE, Wasley A, Bell BP. Prevention of hepatitis A through active or passive immunization: recommendations of the Advisory Committee on Immunization Practices (ACIP). MMWR Recomm Rep. 2006 May 19; 55(RR-7):1-23. Available at http://www.ncbi.nlm.nih.gov/pubmed/ 16708058.

70. Bilukha OO, Rosenstein N. National Center for Infectious Diseases CfDC, Prevention. Prevention and control of meningococcal disease. Recommendations of the Advisory Committee on 
Immunization Practices (ACIP). MMWR Recomm Rep. 2005 May 27; 54(RR-7):1-21. Available at http://www.ncbi.nlm.nih.gov/pubmed/15917737. [PubMed: 15917737]

71. Kramer ES, Hofmann C, Smith PG, Shiffman ML, Sterling RK. Response to hepatitis A and B vaccine alone or in combination in patients with chronic hepatitis $\mathrm{C}$ virus and advanced fibrosis. Dig Dis Sci. 2009 Sep; 54(9):2016-2025. Available at http://www.ncbi.nlm.nih.gov/pubmed/ 19517231. [PubMed: 19517231]

72. Mast EE, Weinbaum CM, Fiore AE, et al. A comprehensive immunization strategy to eliminate transmission of hepatitis B virus infection in the United States: recommendations of the Advisory Committee on Immunization Practices (ACIP) Part II: immunization of adults. MMWR Recomm Rep. 2006 Dec 8; 55(RR-16):1-33. quiz CE31-34 Available at http://www.ncbi.nlm.nih.gov/ pubmed/17159833. [PubMed: 17159833]

73. Fried MW, Shiffman ML, Reddy KR, et al. Peginterferon alfa-2a plus ribavirin for chronic hepatitis C virus infection. N Engl J Med. 2002 Sep 26; 347(13):975-982. Available at http:// www.ncbi.nlm.nih.gov/pubmed/12324553. [PubMed: 12324553]

74. Marcellin P, Forns X, Goeser T, et al. Telaprevir is effective given every 8 or 12 hours with ribavirin and peginterferon alfa-2a or- $2 b$ to patients with chronic hepatitis $\mathrm{C}$. Gastroenterology. $2011 \mathrm{Feb} ; 140(2): 459-468$. e451. quiz e414. Available at http://www.ncbi.nlm.nih.gov/pubmed/ 21034744. [PubMed: 21034744]

75. Poordad F, McCone J Jr, Bacon BR, et al. Boceprevir for untreated chronic HCV genotype 1 infection. N Engl J Med. 2011 Mar 31; 364(13):1195-1206. Available at http:// www.ncbi.nlm.nih.gov/pubmed/21449783. [PubMed: 21449783]

76. Schwarz KB, Gonzalez-Peralta RP, Murray KF, et al. The combination of ribavirin and peginterferon is superior to peginterferon and placebo for children and adolescents with chronic hepatitis C. Gastroenterology. 2011 Feb; 140(2):450-458. e451. Available at http:// www.ncbi.nlm.nih.gov/pubmed/21036173. [PubMed: 21036173]

77. Nelson DR. The role of triple therapy with protease inhibitors in hepatitis $C$ virus genotype 1 naive patients. Liver Int. 2011 Jan; 31(Suppl 1):53-57. Available at http://www.ncbi.nlm.nih.gov/ pubmed/21205138. [PubMed: 21205138]

78. Strader DB, Wright T, Thomas DL, Seeff LB. American Association for the Study of Liver D. Diagnosis, management, and treatment of hepatitis C. Hepatology. 2004 Apr; 39(4):1147-1171. Available at http://www.ncbi.nlm.nih.gov/pubmed/15057920. [PubMed: 15057920]

79. Soriano V, Puoti M, Sulkowski M, et al. Care of patients coinfected with HIV and hepatitis C virus: 2007 updated recommendations from the HCV-HIV International Panel. AIDS. 2007 May 31; 21(9):1073-1089. Available at http://www.ncbi.nlm.nih.gov/pubmed/17502718. [PubMed: 17502718]

80. Elisofon SA, Jonas MM. Hepatitis B and C in children: current treatment and future strategies. Clin Liver Dis. 2006 Feb; 10(1):133-148. vii. Available at http://www.ncbi.nlm.nih.gov/pubmed/ 16376798. [PubMed: 16376798]

81. Chung RT, Andersen J, Volberding P, et al. Peginterferon Alfa-2a plus ribavirin versus interferon alfa-2a plus ribavirin for chronic hepatitis C in HIV-coinfected persons. N Engl J Med. 2004 Jul 29; 351(5):451-459. Available at http://www.ncbi.nlm.nih.gov/pubmed/15282352. [PubMed: 15282352]

82. Narkewicz MR, Cabrera R, Gonzalez-Peralta RP. The "C" of viral hepatitis in children. Semin Liver Dis. 2007 Aug; 27(3):295-311. Available at http://www.ncbi.nlm.nih.gov/pubmed/ 17682976. [PubMed: 17682976]

83. Gonzalez-Peralta RP, Kelly DA, Haber B, et al. Interferon alfa-2b in combination with ribavirin for the treatment of chronic hepatitis $\mathrm{C}$ in children: efficacy, safety, and pharmacokinetics. Hepatology. 2005 Nov; 42(5):1010-1018. Available at http://www.ncbi.nlm.nih.gov/pubmed/ 16250032. [PubMed: 16250032]

84. Christensson B, Wiebe T, Akesson A, Widell A. Interferon-alpha and ribavirin treatment of hepatitis C in children with malignancy in remission. Clin Infect Dis. 2000 Mar; 30(3):585-586. Available at http://www.ncbi.nlm.nih.gov/pubmed/10722449. [PubMed: 10722449]

85. Lackner H, Moser A, Deutsch J, et al. Interferon-alpha and ribavirin in treating children and young adults with chronic hepatitis C after malignancy. Pediatrics. 2000 Oct.106(4):E53. Available at http://www.ncbi.nlm.nih.gov/pubmed/11015548. [PubMed: 11015548] 
86. Kowala-Piaskowska A, Sluzewski W, Figlerowicz M, Mozer-Lisewska I. Early virological response in children with chronic hepatitis $\mathrm{C}$ treated with pegylated interferon and ribavirin. Infection. 2007 Jun; 35(3):175-179. Available at http://www.ncbi.nlm.nih.gov/pubmed/17565459. [PubMed: 17565459]

87. Wirth S, Lang T, Gehring S, Gerner P. Recombinant alfa-interferon plus ribavirin therapy in children and adolescents with chronic hepatitis C. Hepatology. 2002 Nov; 36(5):1280-1284. Available at http://www.ncbi.nlm.nih.gov/pubmed/12395341. [PubMed: 12395341]

88. Sokal EM, Conjeevaram HS, Roberts EA, et al. Interferon alfa therapy for chronic hepatitis B in children: a multinational randomized controlled trial. Gastroenterology. 1998 May; 114(5):988995. Available at http://www.ncbi.nlm.nih.gov/pubmed/9558288. [PubMed: 9558288]

89. Kuloglu Z, Kansu A, Berberoglu M, Adiyaman P, Ocal G, Girgin N. The incidence and evolution of thyroid dysfunction during interferon-alpha therapy in children with chronic hepatitis B infection. J Pediatr Endocrinol Metab. 2007 Feb; 20(2):237-245. Available at http:// www.ncbi.nlm.nih.gov/pubmed/17396441. [PubMed: 17396441]

90. Comanor L, Minor J, Conjeevaram HS, et al. Impact of chronic hepatitis B and interferon-alpha therapy on growth of children. J Viral Hepat. 2001 Mar; 8(2):139-147. Available at http:// www.ncbi.nlm.nih.gov/pubmed/11264734. [PubMed: 11264734]

91. Narkewicz MR, Rosenthal P, Schwarz KB, et al. Ophthalmologic complications in children with chronic hepatitis $\mathrm{C}$ treated with pegylated interferon. J Pediatr Gastroenterol Nutr. 2010 Aug; 51(2):183-186. Available at http://www.ncbi.nlm.nih.gov/pubmed/20512062. [PubMed: 20512062]

92. Jara P, Bortolotti F. Interferon-alpha treatment of chronic hepatitis B in childhood: a consensus advice based on experience in European children. J Pediatr Gastroenterol Nutr. 1999 Aug; 29(2): 163-170. Available at http://www.ncbi.nlm.nih.gov/pubmed/10435653. [PubMed: 10435653]

93. Bacon BR, Gordon SC, Lawitz E, et al. Boceprevir for previously treated chronic HCV genotype 1 infection. N Engl J Med. 2011 Mar 31; 364(13):1207-1217. Available at http:// www.ncbi.nlm.nih.gov/pubmed/21449784. [PubMed: 21449784]

\section{Dosing Recommendations for Prevention and Treatment of Hepatitis C Virus (HCV)}

\begin{tabular}{|c|c|c|c|c|}
\hline \multicolumn{5}{|c|}{ Preventive Regimen } \\
\hline Indication & First $\mathrm{Cl}$ & & Alternative & Comments/Special Issues \\
\hline $\begin{array}{l}\text { Primary } \\
\text { Prophylaxis }\end{array}$ & None & & N/A & N/A \\
\hline $\begin{array}{l}\text { Secondary } \\
\text { Prophylaxis }\end{array}$ & None & & N/A & N/A \\
\hline Treatment & $\begin{array}{c}\frac{\text { IFN-a P P }}{\text { Therapy }} \\
\text { PLUS } \\
\text { • }\end{array}$ & 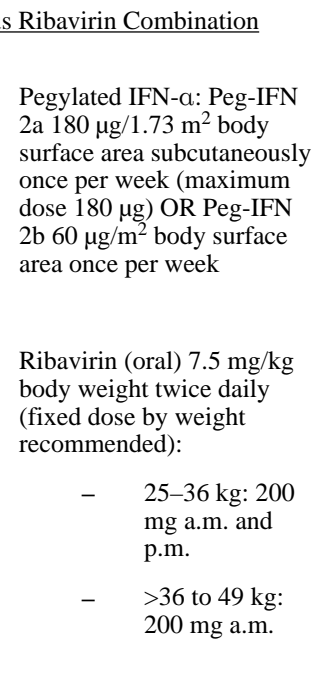 & None & $\begin{array}{l}\text { Optimal duration of treatment for HIV/ } \\
\text { HCV-coinfected children is unknown and } \\
\text { based on recommendations for HIV/HCV- } \\
\text { coinfected adults } \\
\text { Treatment of HCV in children <3 years } \\
\text { generally is not recommended. } \\
\text { Indications for treatment are based on } \\
\text { recommendations in HIV/HCV-coinfected } \\
\text { adults; because HCV therapy is more likely } \\
\text { to be effective in younger patients and in } \\
\text { those without advanced disease or } \\
\text { immunodeficiency, treatment should be } \\
\text { considered for all HIV/HCV-coinfected } \\
\text { children aged >3 years in whom there are } \\
\text { no contraindications to treatment } \\
\text { For recommendations related to use of } \\
\text { telaprevir or boceprevir in adults, including } \\
\text { warnings about drug interactions between } \\
\text { HCV protease inhibitors and HIV protease } \\
\text { inhibitors and other antiretroviral drugs, see } \\
\text { Adult OI guidelines. } \\
\text { IRIS may be manifested by dramatic } \\
\text { increase in transaminases as CD4 cell } \\
\text { counts rise within the first 6-12 weeks of }\end{array}$ \\
\hline
\end{tabular}




\begin{tabular}{|c|c|c|c|c|}
\hline \multicolumn{5}{|c|}{ Preventive Regimen } \\
\hline Indication & First Choice & & Alternative & Comments/Special Issues \\
\hline & $\begin{array}{r}- \\
- \\
- \\
\text { Treatment Duration } \\
48 \text { week } \\
\text { HCV ge }\end{array}$ & $\begin{array}{l}\text { and } 400 \mathrm{mg} \\
\text { p.m. } \\
>49 \text { to } 61 \mathrm{~kg} \text { : } \\
400 \mathrm{mg} \text { a.m. } \\
\text { and p.m. } \\
>61 \text { to } 75 \mathrm{~kg} \text { : } \\
400 \mathrm{mg} \text { a.m. } \\
\text { and } 600 \mathrm{mg} \\
\text { p.m. } \\
>75 \mathrm{~kg}: 600 \mathrm{mg} \\
\text { a.m. and p.m. } \\
\text { regardless of } \\
\text { type }\end{array}$ & & $\begin{array}{l}\text { cART. It may be difficult to distinguish } \\
\text { between IRIS and drug-induced } \\
\text { hepatotoxicity or other causes of hepatitis. } \\
\text { IFN-a is contraindicated in children with } \\
\text { decompensated liver disease, significant } \\
\text { cytopenias, renal failure, severe cardiac } \\
\text { disorders and non-HCV-related } \\
\text { autoimmune disease. } \\
\text { Ribavirin is contraindicated in children with } \\
\text { unstable cardiopulmonary disease, severe } \\
\text { pre-existing anemia or hemoglobinopathy. } \\
\text { Didanosine combined with ribavirin may } \\
\text { lead to increased mitochondrial toxicities; } \\
\text { concomitant use is contraindicated. } \\
\text { Ribavirin and zidovudine both are } \\
\text { associated with anemia, and when possible, } \\
\text { should not be administered together }\end{array}$ \\
\hline
\end{tabular}

Key to Acronyms: $\mathrm{cART}=$ combined antiretroviral therapy; $\mathrm{HCV}=$ hepatitis $\mathrm{C}$ virus; IFN = interferon; IRIS = immune reconstitution inflammatory syndrome; Peg-IFN = pegylated interferon; $\mathrm{SQ}=$ subcutaneous

\section{Herpes Simplex Virus Infections (Last updated November 6, 2013; last reviewed November 6, 2013)}

Panel's Recommendations

- $\quad$ HIV-infected patients should use male latex condoms consistently and correctly during sexual intercourse to reduce the risk of exposure to herpes simplex virus (HSV) and other sexually transmitted pathogens (AI*). They should specifically avoid sexual contact when herpetic lesions (genital or orolabial) are evident (AIII).

- Although use of acyclovir or valacyclovir, beginning at 36 weeks of pregnancy to reduce the need for cesarean delivery, is recommended in HIV-uninfected women with recurrent genital herpes, data are insufficient to make a specific recommendation for HIV and HSV coinfected women (BIII).

- For pregnant women who have active genital HSV lesions at the onset of labor, delivery by elective cesarean delivery, preferably before rupture of membranes, is recommended (BII*).

- Acyclovir is the drug of choice for treatment of local and disseminated HSV in infants and children, regardless of HIV-infection status (AI).

- $\quad$ Neonatal HSV disease should be treated with high-dose intravenous (IV) acyclovir ( $20 \mathrm{mg} / \mathrm{kg}$ body weight), administered for 21 days for central nervous system (CNS) and disseminated disease and for 14 days for disease of the skin, eyes, and mouth (AI). IV acyclovir therapy should not be discontinued in neonates with CNS disease unless a repeat cerebrospinal fluid HSV DNA polymerase chain reaction assay is negative near the end of treatment (BIII). Oral acyclovir prophylaxis for 6 months after treatment of neonatal disease involving the CNS or skin, eyes, and mouth can prevent cutaneous recurrences and may be associated with superior neurodevelopmental outcome in those with CNS disease (AI).

- $\quad$ Beyond the neonatal period, HSV encephalitis should be treated with IV acyclovir for 21 days (AIII).

- $\quad$ First-episode orolabial or genital lesions in HIV-infected children or adolescents can be treated with oral acyclovir for 7 to 10 days (AI). Children or adolescents with severe immunosuppression and moderate-tosevere mucocutaneous HSV lesions should be treated initially with IV acyclovir and may require longer therapy $(\mathbf{A I} *)$.

- $\quad$ Recurrent mucocutaneous lesions, if treated, are generally treated with oral acyclovir for 5 days (AI*).

- $\quad$ Alternatives to oral acyclovir in adolescents and adults include valacyclovir and famciclovir (AI*)

- $\quad$ Patients with acute retinal necrosis should receive combination antiretroviral therapy and high-dose IV acyclovir for 10 to 14 days, followed by prolonged (i.e., 4-6 weeks) oral therapy, such as valacyclovir or acyclovir (AIII).

- HSV keratoconjunctivitis is usually treated with topical trifluridine or oral acyclovir alone, although many experts recommend the combination (AII*).

- $\quad$ The treatment of choice for acyclovir-resistant HSV is IV foscarnet (AI*). 
Panel's Recommendations

- Children or adolescents who have frequent or severe orolabial or genital recurrences can be given daily suppressive therapy with oral acyclovir (AI*). Valacyclovir and famciclovir also are options for adolescents (AI*).

Rating of Recommendations: $\mathrm{A}=$ Strong; $\mathrm{B}=$ Moderate; $\mathrm{C}=$ Optional

Rating of Evidence: I = One or more randomized trials $\underline{\text { in children }}^{\dagger}$ with clinical outcomes and/or validated endpoints; $\mathrm{I}^{*}$ $=$ One or more randomized trials in adults with clinical outcomes and/or validated laboratory endpoints with accompanying data in children $^{\dagger}$ from one or more well-designed, nonrandomized trials or observational cohort studies with long-term clinical outcomes; II = One or more well-designed, nonrandomized trials or observational cohort studies in children $\underline{\text { with }}^{\dagger}$ long-term outcomes; II* = One or more well-designed, nonrandomized trials or observational studies in adults with longterm clinical outcomes with accompanying data in children ${ }^{\dagger}$ from one or more similar nonrandomized trials or cohort studies with clinical outcome data; III = Expert opinion

${ }^{\dagger}$ Studies that include children or children/adolescents, but not studies limited to post-pubertal adolescents

\section{Epidemiology}

Herpes simplex virus type 1 (HSV-1) and HSV-2 can cause disease at any age. HSV-1 is transmitted primarily through contact with infected oral secretions; HSV-2 is acquired primarily through contact with infected genital secretions. In the United States, HSV-1 seroprevalence in children increases from about 30\% at ages 6 to 13 years to 39\% in adolescence and is higher among children who live below the poverty level compared to those who live at or above poverty level. ${ }^{1,2}$ Seroprevalence in children is higher in nonHispanic blacks and in those born in Mexico. The seroprevalence of HSV-1 approaches 60\% in older adults. ${ }^{2} \mathrm{HSV}-2$ seroprevalence prior to reported sexual debut is low (2.6\%) and rises to $22 \%$ to $26 \%$ in 30- to 49 -year-olds and is higher in non-Hispanic blacks, individuals with large numbers of sex partners, females, and in those living below the poverty level. ${ }^{2}$ HSV-2 seroprevalence is higher among individuals who were age 17 years or younger compared with 18 years or older at time of sexual debut. ${ }^{2}$ Among young adolescent girls, a longer period of sexual activity and having had another sexually transmitted disease in the past 6 months was associated with HSV-2 seropositivity. ${ }^{3}$ Among some populations of older adolescents and young adults, HSV-1 is the cause of a large proportion of first episodes of genital HSV infection. ${ }^{4-6}$ These epidemiologic data indicate that children are at significant risk for primary infection or reactivation with HSV throughout childhood and adolescence. The age-specific seroprevalence of both HSV types is higher in many developing countries.

Young children generally acquire HSV-1 from oral secretions of caretakers or playmates. Rarely is this the result of contact with active herpetic lesions; infection most often results from exposure to HSV shed asymptomatically in the saliva of the contact. Salivary shedding of HSV detected by polymerase chain reaction (PCR) in HSV-1-seropositive adults is frequent (9\% of days). ${ }^{7,8}$ While older individuals may acquire HSV-1 in this manner, HSV-1 also can be acquired via sexual activity in adults who were not infected earlier during childhood or adolescence. HSV-2 is more likely to be acquired during adulthood or adolescence, rather than childhood, as it is typically sexually transmitted. Genital shedding of HSV-2 by HSV-infected women who are not HIV-infected, as detected by PCR, is very frequent (19\% of days). ${ }^{8}$ Either virus type can be spread by oral-oral, oral-genital, and genital-genital contact. In general, shedding of oral HSV persists longer in young children. Oral and genital HSV shedding are more common both in close proximity to the first 
episode of infection and also in HIV-infected patients. HSV infection can be acquired as a neonatal infection, primarily through exposure to HSV-infected maternal fluids during vaginal delivery; less commonly, infection may occur in utero. ${ }^{9}$ Newborns also infrequently are infected from oral secretions of an adult caretaker. The risk of transmitting HSV during delivery is approximately $1 \%$ in pregnant women with remote primary HSV infection, whereas the risk is much higher for infants born to women with recent HSV infection (range: 30\%-50\%). ${ }^{9}$ Maternal HSV antibody status before delivery likely influences the probability of transmission to infants and the severity of neonatal infection. ${ }^{10,11}$ Genital shedding of HSV at delivery increases the risk of transmission, as does prolonged rupture of membranes ( $>6$ hours), probably because of ascending HSV infection from the cervix. Importantly, mothers of neonates with HSV often do not provide a history of either past genital HSV infection or incident genital lesions. ${ }^{12,13}$

Dual HSV and HIV infection of pregnant women is likely to be common, because both viral infections share risk factors (race, socioeconomic status, and number of sexual partners). Genital HSV was detected by PCR in $31 \%$ of HSV-seropositive, HIV-infected women at the time of delivery, compared with $9.5 \%$ of HSV-seropositive, HIV-uninfected pregnant women. ${ }^{14}$ Shedding is greatest when the CD4 T-lymphocyte count is low. ${ }^{15}$ In spite of the potential risk factors for the infant, there is no evidence that in utero HSV infection occurs more frequently in HIV-infected pregnant woman coinfected with HSV-2 or that infants born to these women are at increased risk of perinatal (intrapartum) HSV infection. In the general population, the neonatal HSV infection rate is 1 case per 2,000 to 10,000 deliveries, ${ }^{9,16}$ indicating that neonatal HSV will rarely be observed at clinics caring for dually-infected pregnant women.

Conversely, numerous studies have shown that coinfection with genital HSV in adults is associated with higher titers of HIV RNA in plasma and genital secretions; HSVseropositivity increases the risk of HIV transmission to sexual partners, even in the absence of genital ulcer disease. ${ }^{17,18}$ Three studies suggest that maternal HSV coinfection increases the risk of intrapartum HIV transmission. ${ }^{19-21}$

\section{Clinical Manifestations}

In most immunologically competent children, HSV infection causes minimal signs and symptoms and is usually not recognized as a distinct illness. Up to one third of children may develop a characteristic orolabial syndrome (primary gingivostomatitis), usually associated with HSV-1 infection, which consists of fever, irritability, tender submandibular lymphadenopathy, and superficial, painful ulcers on the gingival and oral mucosa and perioral area. ${ }^{22,23} \mathrm{HSV}$ viremia occurs in approximately one-third of patients with primary herpetic gingivostomatitis. ${ }^{24} \mathrm{HSV}$ is a common cause of severe posterior pharyngitis in older children and adolescents. ${ }^{25}$ Children with advanced HIV infection may have primary infection with multiple lesions that are atypical in appearance and delayed in healing. Very rarely, disseminated HSV occurs with visceral involvement (including liver, adrenals, lung, and brain) and generalized skin lesions. Small crops of recurrent perioral vesicles ("cold sores") that heal quickly can occur throughout life in both healthy and HIV-infected children, but those with AIDS are at risk of frequent recurrences, which can be associated 
with severe ulcerative disease and symptoms similar to primary infection. ${ }^{26} \mathrm{HIV}$-infected children also may have prolonged shedding of HSV after both primary and reactivation infection. HSV esophagitis, which occurs in severely immunocompromised children, can result from failure to limit replication of HSV present in saliva, although a study of adults found that evidence of oral HSV infection often is not present simultaneously. ${ }^{27}$ Prolonged cutaneous HSV infection and organ involvement are AIDS-indicator conditions. These illnesses are uncommon in the era of combination antiretroviral therapy (cART), with a documented incidence rate of systemic HSV of 0.14 per 100 child-years. $^{28}$

Genital infection is the most common manifestation of HSV-2 infection in sexually active adolescents. Most primary infections are asymptomatic or subclinical; however, when symptoms do occur, they are characterized by painful, ulcerative lesions on the perineum, penis, and vaginal and urethral mucosae. Mucosal disease often is accompanied by dysuria and/or vaginal or urethral discharge. Inguinal lymphadenopathy, particularly in primary infection, is common with perineal disease. ${ }^{29}$ Frequent recurrences and delayed healing are more likely in severely immunosuppressed patients. Severe proctitis and perianal infection occur in patients who practice receptive anal intercourse. ${ }^{7,30}$

In HIV-infected patients, HSV keratitis and herpetic whitlow are similar in presentation to diseases in HIV-uninfected individuals, but may be more severe. Acute retinal necrosis is a rare sight-threatening complication that occurs more frequently in immunocompromised individuals. HSV encephalitis occurs in HIV-infected patients, but is not more frequent or severe than in HIV-uninfected individuals and has similar signs and symptoms (encephalopathy, neurologic abnormalities/seizures, and mononuclear pleocytosis in cerebrospinal fluid $[\mathrm{CSF}])$. Focal deficits and temporal lobe abnormalities on neuroimaging are typical. ${ }^{31,32}$

Neonatal infection in infants born to dually-infected mothers is similar in presentation to that seen in HIV-uninfected infants. Neonatal HSV can appear as disseminated multiorgan disease; localized disease of the central nervous system (CNS); or disease localized to the skin, eyes, and mouth. ${ }^{33}$ Vesicular rash occurs in only approximately $60 \%$ of infants with CNS or disseminated disease..$^{33,34}$

\section{Diagnosis}

Clinical diagnosis is based on the typical location and appearance of vesicles and ulcers. The virus is readily isolated in tissue culture within 1 to 3 days, especially when samples are from first episode infections or obtained soon after the appearance of recurrent lesions (especially when vesicles are present). ${ }^{35,36}$ Speed and accuracy are maximized with the shell vial method, which combines centrifugation and staining with fluorescein-conjugated monoclonal antibodies to detect synthesis of early HSV proteins, thereby providing an etiologic diagnosis after 24 hours. Detection of HSV DNA by PCR, which is very sensitive and specific, is the gold standard method for diagnosis of HSV infection. DNA PCR may be especially useful when assessing skin lesions that are recurrent or that are being evaluated long after their appearance. In these cases, the HSV DNA remains in the healing lesions, even though HSV can no longer be cultured. Direct immunofluorescence for HSV antigen can be performed on cells scraped from skin, conjunctiva, or mucosal lesions. ${ }^{37}$ The 
sensitivity of this method may not exceed $75 \%$, often because it is difficult to obtain evaluable specimens.

Detection of HSV DNA in the CSF is the preferred diagnostic test for evaluation of children with suspected HSV encephalitis, because cultures of CSF are usually negative. Sensitivity of HSV PCR is generally $\geq 95 \%$ for CSF, especially if obtained more than 3 days after onset of herpes encephalitis. ${ }^{32,38}$ During therapy for HSV-Iproven encephalitis, the CSF HSV PCR remains positive for a mean of 10 days after neurologic onset. ${ }^{39}$ In neonatal CNS HSV disease, CSF PCR has a sensitivity of $75 \%$ to $100 \%$ and a specificity of $71 \%$ to $100 \%{ }^{34}$

Specimens from newborns with suspected neonatal HSV should be obtained from blood, skin vesicles, mouth or nasopharynx, conjunctiva, and stool or rectum. Positive cultures obtained from any of these sites more than 48 hours after birth indicate viral replication rather than contamination after intrapartum exposure.

Definitive diagnosis of HSV esophagitis requires endoscopy with biopsy. Histologic evidence of multinucleated giant cells with intranuclear viral inclusions and positive staining with monoclonal antibodies supplement culture or PCR results.

The rapid onset of poor vision, red eye, or eye pain should result in an immediate referral to an ophthalmologist, because these may be caused by herpesviruses or other pathogens that require specialized diagnostic (including fluorescein staining to detect characteristic dendritic corneal ulceration and fundoscopic exam) and treatment approaches.

Typing of HSV isolates (or genotyping of amplicons) can provide important prognostic information, since recurrence frequency after genital HSV-1 infection in HIV-uninfected patients is significantly less than after HSV-2 infection. ${ }^{40,41}$

\section{Prevention Recommendations}

Preventing Exposure-Exposure to HSV-1 is an inevitable part of childhood. Although avoiding direct contact with secretions from adult caretakers, siblings, or other close contacts with active herpes labialis is intuitive, it is likely that most infections occur as a result of unrecognized exposure to the frequent asymptomatic shedding of HSV by individuals with prior infection.

When used consistently and correctly, male latex condoms reduce the risk of genital herpes when the infected site is covered, although data for this effect are limited (see http:// www.cdc.gov/condomeffectiveness/latex.htm). ${ }^{42}$ Data pooled from 6 prospective studies estimated the odds of HSV-2 acquisition with every sexual act as increased by $3.6 \%, 2.7 \%$, and $0 \%$ when condoms were never used, sometimes used, or always used, respectively. ${ }^{43} \mathrm{In}$ another pooled analysis, individuals who always used condoms had a 30\% lower risk of HSV-2 acquisition compared with those who never used condoms, and risk of HSV-2 acquisition increased steadily with each unprotected sex act. ${ }^{42}$ Some data suggest that condom usage decreases the acquisition of genital HSV-2 infection by women, but may not be protective for heterosexual men or against HSV-1 infection; ${ }^{44}$ however, neither of the aforementioned pooled analyses detected such a difference between men and women. ${ }^{42,43}$ 
HIV-infected patients should use latex condoms consistently and correctly during sexual intercourse to reduce the risk of HSV and other sexually transmitted pathogens (AI*). They should specifically avoid sexual contact when herpetic lesions (genital or orolabial) are evident (AIII); however, most genital herpes infections are transmitted by individuals unaware that they are infected. Chronic suppressive therapy with valacyclovir in individuals with genital herpes reduces HSV-2 transmission to susceptible heterosexual partners by $50 \% .{ }^{45}$ Use of suppressive antiviral drugs against HSV in HIV-infected adults receiving cART resulted in fewer symptomatic lesions than in HIV-infected patients receiving such prophylaxis without cART, but subclinical mucosal HSV-2 shedding was similar regardless of cART. ${ }^{46}$

The rate of HSV transmission to fetuses and neonates of HIV-infected pregnant women coinfected with HSV is unknown. Effective cART regimens may decrease, but not prevent, maternal genital HSV shedding and recurrence of genital lesions. ${ }^{47}$

Use of acyclovir or valacyclovir near term suppresses genital HSV outbreaks and shedding in late pregnancy in HIV-uninfected women with recurrent genital herpes and reduces the need for cesarean delivery for recurrent HSV. ${ }^{48}$ Although the sample size was insufficient to determine the effect of prophylaxis on neonatal infection, it is recommended that HIVuninfected pregnant women with recurrent genital herpes be offered suppressive antiviral therapy at or beyond 36 weeks' gestation. ${ }^{49}$ The safety and efficacy of this strategy have not been evaluated in HIV/HSV coinfected women, who may have less HSV-2-specific antibody and/or T-cell function and are more likely to have both symptomatic and asymptomatic reactivation of genital HSV. Although suppressive antiviral therapy in late gestation is likely to also have efficacy in HIV-seropositive women, data are insufficient to make a specific recommendation (BIII). ${ }^{11}$ Elective cesarean delivery, preferably before rupture of membranes, is recommended for HIV-infected and HIV-uninfected women who have active genital HSV lesions at the onset of labor (BII*). ${ }^{50-53}$

Preventing Disease-Antiviral prophylaxis before or after exposure to HSV has been used successfully, but has not been studied in HIV-infected patients and is not recommended.

\section{Treatment Recommendations}

Treating Disease-Acyclovir is the drug of choice for treatment of local and disseminated HSV in infants and children, regardless of HIV-infection status (AI). Neonatal HSV disease should be treated with high-dose intravenous (IV) acyclovir $(20 \mathrm{mg} / \mathrm{kg}$ body weight three times a day) administered for 21 days for CNS and disseminated disease and for 14 days for disease of the skin, eyes, and mouth (AI). ${ }^{54}$ IV acyclovir therapy should not be discontinued in neonates with CNS disease unless a repeat CSF HSV DNA PCR assay is negative near the end of treatment (BIII).

IV acyclovir is the drug of choice for disseminated HSV and HSV encephalitis beyond the neonatal period. Beyond the neonatal period, HSV encephalitis should be treated (10-20 $\mathrm{mg} / \mathrm{kg}$ body weight three times a day) for 21 days (AIII). 
First-episode orolabial or genital lesions in HIV-infected children or adolescents can be treated with oral acyclovir for 7 to 10 days as indicated by the response to therapy (AI). ${ }^{22,23}$ Children or adolescents with severe immunosuppression and moderate-to-severe mucocutaneous HSV lesions should be treated initially with IV acyclovir and may need longer therapy, adjusted to the rate and character of healing (AI*). Patients can be switched to oral therapy after their lesions have begun to regress, and therapy continued until lesions have completely healed.

Recurrent mucocutaneous lesions, if treated, are generally treated with oral acyclovir for 5 days (AI*). Patients in whom frequent or severe recurrences are an unacceptable burden may benefit from daily suppressive therapy with acyclovir (AI*).

Alternatives to oral acyclovir in older adolescents and adults include valacyclovir and famciclovir (AI*). Valacyclovir is a prodrug of acyclovir with improved bioavailability that is rapidly converted to acyclovir after absorption. Sufficient information exists to support the use of valacyclovir in children, especially given its two- to threefold improved bioavailability compared with acyclovir, at a dose of 20 to $25 \mathrm{mg} / \mathrm{kg}$ body weight administered 2 to 3 times a day. ${ }^{55,56}$ No pediatric formulation is available and valacyclovir can generally only be used for children old enough to swallow the large valacyclovir tablets, although crushed valacyclovir tablets can be used to make a suspension with good bioavailability. ${ }^{57}$ The database on the pharmacokinetics and dosing of famciclovir in children is insufficient to make recommendations, and no pediatric preparation is available. ${ }^{58}$ Because of their improved bioavailability, valacyclovir and famciclovir administration at higher doses for only 1 to 3 days often is sufficient to manage recurrent genital HSV infection in HIV-uninfected adults and oral infections in HIV-infected adults. ${ }^{59}$

Treatment for acute retinal disease caused by HSV should be guided by an ophthalmologist. Patients with acute retinal necrosis should be on cART and receive high-dose IV acyclovir (10-15 mg/kg body weight IV every 8 hours for 10-14 days), followed by prolonged (i.e., 4-6 weeks) oral therapy, such as with valacyclovir or acyclovir (AIII). ${ }^{60} \mathrm{HSV}$ keratoconjunctivitis is usually treated with topical trifluridine or acyclovir, although many experts recommend combination therapy (AII*). ${ }^{61}$ Because of potential corneal toxicity of topical therapy, close follow-up by an ophthalmologist is recommended and the duration of therapy should be individualized.

Monitoring and Adverse Events (Including IRIS)—Primary toxicities of acyclovir are phlebitis (when administered IV), renal toxicity, nausea, vomiting, and rash. Toxicities are similar for valacyclovir. In infants receiving high-dose acyclovir for neonatal disease, the major side effect was neutropenia (defined as absolute neutrophil count $<1,000 / \mathrm{mm}^{3}$ ). ${ }^{54}$ Significant nephrotoxicity was observed in $6 \%$ of patients. For infants and children receiving high-dose IV acyclovir, monitoring of complete blood counts (CBCs) and renal function is recommended at initiation of treatment and once or twice weekly for the duration of treatment, particularly in those with underlying renal dysfunction and who are receiving prolonged therapy. If possible, avoid other nephrotoxic drugs. IV acyclovir must be adequately diluted and administered slowly over 1 to 2 hours. Acyclovir is excreted 
primarily by the kidney; as a result, dose adjustment based on creatinine clearance is needed in patients with renal insufficiency or renal failure.

Anogenital HSV has been included by some investigators as a potential manifestation of immune reconstitution inflammatory syndrome, but this has not been validated by comparing the anogenital HSV incidence after cART with the incidence during a similar period prior to cART.

Managing Treatment Failure-Resistance of HSV to acyclovir occurs in 5\% to 10\% of immunocompromised patients. ${ }^{62}$ This reflects the fact that acyclovir is a virostatic drug and patients with inadequate HSV-specific cell-mediated immunity fail to rapidly clear the HSV infection. Resistance to antiviral drugs should be suspected if systemic involvement and skin lesions do not begin to resolve within 5 to 7 days after initiation of therapy, skin lesions are atypical in appearance, or satellite lesions appear after 3 to 4 days of therapy. If possible, a lesion culture should be obtained and, if virus is isolated, susceptibility testing performed to confirm resistance. This may be difficult to arrange and will involve significant delay. Thus, the decision to change therapy is often based on clinical observations. All acyclovir-resistant HSV strains are resistant to valacyclovir, and it is very rare that they are sensitive to famciclovir. The therapeutic choice for acyclovir-resistant herpes is foscarnet (AI*). ${ }^{63,64}$ Foscarnet has significant nephrotoxic potential; up to $30 \%$ of patients experience increases in serum creatinine levels. It also causes serious electrolyte imbalances (including abnormalities in calcium, phosphorus, magnesium, and potassium levels) in many patients, and secondary seizures or cardiac dysrhythmias can occur. Abnormal liver transaminases and CNS symptoms can also occur. For patients receiving foscarnet, $\mathrm{CBC}$, serum electrolytes, and renal function should be monitored twice weekly during induction therapy and once weekly thereafter. Infusing foscarnet after saline fluid loading can minimize renal toxicity. Doses should be modified in patients with renal insufficiency (see package insert).

IV cidofovir is used to treat patients with HSV resistant to acyclovir and foscarnet. ${ }^{65}$ For disease limited to a small number of indolent, non-healing lesions, topical formulations of trifluridine, foscarnet, and cidofovir have been used successfully, although this will require local preparation, and prolonged application for 21 to 28 days or longer may be required. ${ }^{66}$

Preventing Recurrence-Administration of oral acyclovir prophylaxis (suppressive therapy) for 6 months can prevent cutaneous recurrences of HSV after neonatal disease of the CNS or skin, eyes, and mouth and may be associated with superior neurodevelopmental outcome in those with CNS disease (AI) ${ }^{67}$

Beyond the neonatal period, because recurrent episodes of mucocutaneous HSV disease can be treated successfully, chronic prophylaxis with acyclovir or other available antivirals against HSV is not required after lesions resolve in most patients. Effective cART may decrease recurrences. Children who have frequent or severe recurrences (i.e., 4 to 6 severe episodes a year) can be given daily prophylaxis with oral acyclovir (AI*). Valacyclovir or famciclovir also are options for prophylaxis in adolescents (AI*). Because corneal clouding can occur as a result of the stromal reaction of recurrent keratoconjunctivitis, some ophthalmologists use acyclovir prophylaxis to reduce the frequency of recurrences. 
However, resistance to acyclovir has been reported in this circumstance in HIV-uninfected patients.

Discontinuing Secondary Prophylaxis-Patients receiving prophylactic therapy should be evaluated annually for the need to continue prophylaxis. Cessation of secondary prophylaxis will be determined by the level of immune reconstitution, frequency and severity of subsequent recurrences, and each individual's tolerance for recurrent episodes.

\section{References}

1. Xu F, Lee FK, Morrow RA, et al. Seroprevalence of herpes simplex virus type 1 in children in the United States. J Pediatr. 2007 Oct; 151(4):374-377. Available at http://www.ncbi.nlm.nih.gov/ pubmed/17889072. [PubMed: 17889072]

2. Xu F, Sternberg MR, Kottiri BJ, et al. Trends in herpes simplex virus type 1 and type 2 seroprevalence in the United States. JAMA. 2006 Aug 23; 296(8):964-973. Available at http:// www.ncbi.nlm.nih.gov/pubmed/16926356. [PubMed: 16926356]

3. Stanberry LR, Rosenthal SL, Mills L, et al. Longitudinal risk of herpes simplex virus (HSV) type 1, HSV type 2, and cytomegalovirus infections among young adolescent girls. Clin Infect Dis. 2004 Nov 15; 39(10):1433-1438. Available at http://www.ncbi.nlm.nih.gov/pubmed/15546077. [PubMed: 15546077]

4. Roberts CM, Pfister JR, Spear SJ. Increasing proportion of herpes simplex virus type 1 as a cause of genital herpes infection in college students. Sex Transm Dis. 2003 Oct; 30(10):797-800. Available at http://www.ncbi.nlm.nih.gov/pubmed/14520181. [PubMed: 14520181]

5. Samra Z, Scherf E, Dan M. Herpes simplex virus type 1 is the prevailing cause of genital herpes in the Tel Aviv area, Israel. Sex Transm Dis. 2003 Oct; 30(10):794-796. Available at http:// www.ncbi.nlm.nih.gov/pubmed/14520180. [PubMed: 14520180]

6. Ryder N, Jin F, McNulty AM, Grulich AE, Donovan B. Increasing role of herpes simplex virus type 1 in first-episode anogenital herpes in heterosexual women and younger men who have sex with men, 1992-2006. Sex Transm Infect. 2009 Oct; 85(6):416-419. Available at http:// www.ncbi.nlm.nih.gov/pubmed/19273479. [PubMed: 19273479]

7. Krone MR, Wald A, Tabet SR, Paradise M, Corey L, Celum CL. Herpes simplex virus type 2 shedding in human immunodeficiency virus-negative men who have sex with men: frequency, patterns, and risk factors. Clin Infect Dis. 2000 Feb; 30(2):261-267. Available at http:// www.ncbi.nlm.nih.gov/pubmed/10671325. [PubMed: 10671325]

8. Mark KE, Wald A, Magaret AS, et al. Rapidly cleared episodes of herpes simplex virus reactivation in immunocompetent adults. J Infect Dis. 2008 Oct 15; 198(8):1141-1149. Available at http:// www.ncbi.nlm.nih.gov/pubmed/18783315. [PubMed: 18783315]

9. Corey L, Wald A. Maternal and neonatal herpes simplex virus infections. N Engl J Med. 2009 Oct 1; 361(14):1376-1385. Available at http://www.ncbi.nlm.nih.gov/pubmed/19797284. [PubMed: 19797284]

10. Ashley RL, Dalessio J, Burchett S, et al. Herpes simplex virus-2 (HSV-2) type-specific antibody correlates of protection in infants exposed to HSV-2 at birth. J Clin Invest. 1992 Aug; 90(2):511514. Available at http://www.ncbi.nlm.nih.gov/pubmed/1322941. [PubMed: 1322941]

11. Kimberlin DW. Herpes simplex virus infections in neonates and early childhood. Semin Pediatr Infect Dis. 2005 Oct; 16(4):271-281. Available at http://www.ncbi.nlm.nih.gov/pubmed/ 16210107. [PubMed: 16210107]

12. Tookey P, Peckham CS. Neonatal herpes simplex virus infection in the British Isles. Paediatr Perinat Epidemiol. 1996 Oct; 10(4):432-442. Available at http://www.ncbi.nlm.nih.gov/pubmed/ 8931058. [PubMed: 8931058]

13. Whitley R, Davis EA, Suppapanya N. Incidence of neonatal herpes simplex virus infections in a managed-care population. Sex Transm Dis. 2007 Sep; 34(9):704-708. Available at http:// www.ncbi.nlm.nih.gov/pubmed/17413535. [PubMed: 17413535] 
14. Patterson J, Hitti J, Selke S, et al. Genital HSV detection among HIV-1-infected pregnant women in labor. Infect Dis Obstet Gynecol. 2011; 2011:157680. Available at http:// www.ncbi.nlm.nih.gov/pubmed/21527986. [PubMed: 21527986]

15. Mostad SB, Kreiss JK, Ryncarz A, et al. Cervical shedding of herpes simplex virus and cytomegalovirus throughout the menstrual cycle in women infected with human immunodeficiency virus type 1. Am J Obstet Gynecol. 2000 Oct; 183(4):948-955. Available at http://www.ncbi.nlm.nih.gov/pubmed/11035345. [PubMed: 11035345]

16. Flagg EW, Weinstock H. Incidence of neonatal herpes simplex virus infections in the United States, 2006. Pediatrics. 2011 Jan; 127(1):e1-e8. Available at http://www.ncbi.nlm.nih.gov/ pubmed/21149432. [PubMed: 21149432]

17. Wald A, Link K. Risk of human immunodeficiency virus infection in herpes simplex virus type 2seropositive persons: a meta-analysis. J Infect Dis. 2002 Jan 1; 185(1):45-52. Available at http:// www.ncbi.nlm.nih.gov/pubmed/11756980. [PubMed: 11756980]

18. Freeman EE, Weiss HA, Glynn JR, Cross PL, Whitworth JA, Hayes RJ. Herpes simplex virus 2 infection increases HIV acquisition in men and women: systematic review and meta-analysis of longitudinal studies. AIDS. 2006 Jan 2; 20(1):73-83. Available at http://www.ncbi.nlm.nih.gov/ pubmed/16327322. [PubMed: 16327322]

19. Chen KT, Segu M LL, Kuhn L, Carter RJ, Bulterys M, et al. Genital herpes simplex virus infection and perinatal transmission of human immunodeficiency virus. Obstet Gynecol. 2005; 106(6): 1341-1348. 2005. Available at http://www.ncbi.nlm.nih.gov/pubmed/16319261. [PubMed: 16319261]

20. Cowan FM, Humphrey JH, Ntozini R, Mutasa K, Morrow R, Iliff P. Maternal Herpes simplex virus type 2 infection, syphilis and risk of intra-partum transmission of HIV-1: results of a case control study. AIDS. 2008 Jan 11; 22(2):193-201. Available at http://www.ncbi.nlm.nih.gov/ pubmed/18097221. [PubMed: 18097221]

21. Drake AL, John-Stewart GC, Wald A, et al. Herpes simplex virus type 2 and risk of intrapartum human immunodeficiency virus transmission. Obstet Gynecol. 2007 Feb; 109(2 Pt 1):403-409. Available at http://www.ncbi.nlm.nih.gov/pubmed/17267842. [PubMed: 17267842]

22. Amir J, Harel L, Smetana Z, Varsano I. The natural history of primary herpes simplex type 1 gingivostomatitis in children. Pediatr Dermatol. 1999 Jul-Aug;16(4):259-263. Available at http:// www.ncbi.nlm.nih.gov/pubmed/10469407. [PubMed: 10469407]

23. Arvin, AM. Chapter 163: Herpes simplex 1 \& 2. In: Feigin, RD.; Cherry, JD.; Demmler, GJ.; Kaplan, SL., editors. Textbook of Pediatric Infectious Disease. 5th Edition. Philadelphia: Saunders; 2004. p. 1884-1912.

24. Harel L, Smetana Z, Prais D, et al. Presence of viremia in patients with primary herpetic gingivostomatitis. Clin Infect Dis. 2004 Sep 1; 39(5):636-640. Available at http:// www.ncbi.nlm.nih.gov/pubmed/15356775. [PubMed: 15356775]

25. Glezen WP, Fernald GW, Lohr JA. Acute respiratory disease of university students with special reference to the etiologic role of Herpesvirus hominis. Am J Epidemiol. 1975 Feb; 101(2):111121. Available at http://www.ncbi.nlm.nih.gov/pubmed/164768. [PubMed: 164768]

26. Salvini F, Carminati G, Pinzani R, Carrera C, Rancilio L, Plebani A. Chronic ulcerative herpes simplex virus infection in HIV-infected children. AIDS Patient Care STDS. 1997 Dec; 11(6):421428. Available at http://www.ncbi.nlm.nih.gov/pubmed/11361863. [PubMed: 11361863]

27. Genereau T, Lortholary O, Bouchaud O, et al. Herpes simplex esophagitis in patients with AIDS: report of 34 cases. The Cooperative Study Group on Herpetic Esophagitis in HIV Infection. Clin Infect Dis. 1996 Jun; 22(6):926-931. Available at http://www.ncbi.nlm.nih.gov/pubmed/8783688. [PubMed: 8783688]

28. Gona P, Van Dyke RB, Williams PL, et al. Incidence of opportunistic and other infections in HIVinfected children in the HAART era. JAMA. 2006 Jul 19; 296(3):292-300. Available at http:// www.ncbi.nlm.nih.gov/pubmed/16849662. [PubMed: 16849662]

29. Corey L, Adams HG, Brown ZA, Holmes KK. Genital herpes simplex virus infections: clinical manifestations, course, and complications. Ann Intern Med. 1983 Jun; 98(6):958-972. Available at http://www.ncbi.nlm.nih.gov/pubmed/6344712. [PubMed: 6344712] 
30. Siegal FP, Lopez C, Hammer GS, et al. Severe acquired immunodeficiency in male homosexuals, manifested by chronic perianal ulcerative herpes simplex lesions. N Engl J Med. 1981 Dec 10; 305(24):1439-1444. Available at http://www.ncbi.nlm.nih.gov/pubmed/6272110. [PubMed: 6272110]

31. Whitley RJ, Kimberlin DW. Herpes simplex encephalitis: children and adolescents. Semin Pediatr Infect Dis. 2005 Jan; 16(1):17-23. Available at http://www.ncbi.nlm.nih.gov/pubmed/15685145. [PubMed: 15685145]

32. De Tiege X, Rozenberg F, Heron B. The spectrum of herpes simplex encephalitis in children. Eur J Paediatr Neurol. 2008 Mar; 12(2):72-81. Available at http://www.ncbi.nlm.nih.gov/pubmed/ 17870623. [PubMed: 17870623]

33. Kimberlin DW, Lin CY, Jacobs RF, et al. Natural history of neonatal herpes simplex virus infections in the acyclovir era. Pediatrics. 2001 Aug; 108(2):223-229. Available at http:// www.ncbi.nlm.nih.gov/pubmed/11483781. [PubMed: 11483781]

34. Kimberlin DW. Herpes simplex virus infections of the newborn. Semin Perinatol. 2007 Feb; 31(1): 19-25. Available at http://www.ncbi.nlm.nih.gov/pubmed/17317423. [PubMed: 17317423]

35. Cone RW, Hobson AC, Palmer J, Remington M, Corey L. Extended duration of herpes simplex virus DNA in genital lesions detected by the polymerase chain reaction. J Infect Dis. 1991 Oct; 164(4):757-760. Available at http://www.ncbi.nlm.nih.gov/pubmed/1654360. [PubMed: 1654360]

36. Kimberlin DW. Diagnosis of herpes simplex virus in the era of polymerase chain reaction. Pediatr Infect Dis J. 2006 Sep; 25(9):841-842. Available at http://www.ncbi.nlm.nih.gov/pubmed/ 16940845. [PubMed: 16940845]

37. Slomka MJ, Emery L, Munday PE, Moulsdale M, Brown DW. A comparison of PCR with virus isolation and direct antigen detection for diagnosis and typing of genital herpes. J Med Virol. 1998 Jun; 55(2):177-183. Available at http://www.ncbi.nlm.nih.gov/pubmed/9598940. [PubMed: 9598940]

38. Weil AA, Glaser CA, Amad Z, Forghani B. Patients with suspected herpes simplex encephalitis: rethinking an initial negative polymerase chain reaction result. Clin Infect Dis. 2002 Apr 15; 34(8):1154-1157. Available at http://www.ncbi.nlm.nih.gov/pubmed/11915008. [PubMed: 11915008]

39. Kimura H, Aso K, Kuzushima K, Hanada N, Shibata M, Morishima T. Relapse of herpes simplex encephalitis in children. Pediatrics. 1992 May; 89(5 Pt 1):891-894. Available at http:// www.ncbi.nlm.nih.gov/pubmed/1315949. [PubMed: 1315949]

40. Lafferty WE, Coombs RW, Benedetti J, Critchlow C, Corey L. Recurrences after oral and genital herpes simplex virus infection. Influence of site of infection and viral type. N Engl J Med. 1987 Jun 4; 316(23):1444-1449. Available at http://www.ncbi.nlm.nih.gov/pubmed/3033506. [PubMed: 3033506]

41. Engelberg R, Carrell D, Krantz E, Corey L, Wald A. Natural history of genital herpes simplex virus type 1 infection. Sex Transm Dis. 2003 Feb; 30(2):174-177. Available at http:// www.ncbi.nlm.nih.gov/pubmed/12567178. [PubMed: 12567178]

42. Martin ET, Krantz E, Gottlieb SL, et al. A pooled analysis of the effect of condoms in preventing HSV-2 acquisition. Arch Intern Med. 2009 Jul 13; 169(13):1233-1240. Available at http:// www.ncbi.nlm.nih.gov/pubmed/19597073. [PubMed: 19597073]

43. Stanaway JD, Wald A, Martin ET, Gottlieb SL, Magaret AS. Case-crossover analysis of condom use and herpes simplex virus type 2 acquisition. Sex Transm Dis. 2012 May; 39(5):388-393. Available at http://www.ncbi.nlm.nih.gov/pubmed/22504606. [PubMed: 22504606]

44. Wald A, Langenberg AG, Krantz E, et al. The relationship between condom use and herpes simplex virus acquisition. Ann Intern Med. 2005; 143(10):707-713. Available at http:// www.ncbi.nlm.nih.gov/entrez/query.fcgi? $\mathrm{cmd}=$ Retrieve $\& \mathrm{db}=$ PubMed\&dopt=Citation\&list_uids=16287791. [PubMed: 16287791]

45. Corey L, Wald A, Patel R, et al. Once-daily valacyclovir to reduce the risk of transmission of genital herpes. N Engl J Med. 2004 Jan 1; 350(1):11-20. Available at http:// www.ncbi.nlm.nih.gov/pubmed/14702423. [PubMed: 14702423] 
46. Strick LB, Wald A, Celum C. Management of herpes simplex virus type 2 infection in HIV type 1infected persons. Clin Infect Dis. 2006 Aug 1; 43(3):347-356. Available at http:// www.ncbi.nlm.nih.gov/pubmed/16804851. [PubMed: 16804851]

47. Posavad CM, Wald A, Kuntz S, et al. Frequent reactivation of herpes simplex virus among HIV-1infected patients treated with highly active antiretroviral therapy. J Infect Dis. 2004 Aug 15; 190(4):693-696. Available at http://www.ncbi.nlm.nih.gov/pubmed/15272395. [PubMed: 15272395]

48. Hollier LM, Wendel GD. Third trimester antiviral prophylaxis for preventing maternal genital herpes simplex virus (HSV) recurrences and neonatal infection. Cochrane Database Syst Rev. 2008(1):CD004946. Available at http://www.ncbi.nlm.nih.gov/pubmed/18254066. [PubMed: 18254066]

49. Bulletins ACoP. ACOG Practice Bulletin. Clinical management guidelines for obstetriciangynecologists. No. 82 June 2007. Management of herpes in pregnancy. Obstet Gynecol. 2007 Jun; 109(6):1489-1498. Available at http://www.ncbi.nlm.nih.gov/pubmed/17569194. [PubMed: 17569194]

50. American College of Obstetricians and Gynecologists. Management of herpes in pregnancy, ACLG Practice Bulletin 8. Washington, DC: 1999.

51. Whitley RJ, Kimberlin DW, Roizman B. Herpes simplex viruses. Clin Infect Dis. 1998 Mar; 26(3): 541-553. quiz 554-545. Available at http://www.ncbi.nlm.nih.gov/pubmed/9524821. [PubMed: 9524821]

52. Prober CG, Corey L, Brown ZA, et al. The management of pregnancies complicated by genital infections with herpes simplex virus. Clin Infect Dis. 1992 Dec; 15(6):1031-1038. Available at http://www.ncbi.nlm.nih.gov/pubmed/1457634. [PubMed: 1457634]

53. Brown ZA, Wald A, Morrow RA, Selke S, Zeh J, Corey L. Effect of serologic status and cesarean delivery on transmission rates of herpes simplex virus from mother to infant. JAMA. 2003 Jan 8; 289(2):203-209. Available at http://www.ncbi.nlm.nih.gov/pubmed/12517231. [PubMed: 12517231]

54. Kimberlin DW, Lin CY, Jacobs RF, et al. Safety and efficacy of high-dose intravenous acyclovir in the management of neonatal herpes simplex virus infections. Pediatrics. 2001 Aug; 108(2):230238. Available at http://www.ncbi.nlm.nih.gov/pubmed/11483782. [PubMed: 11483782]

55. Eksborg S, Pal N, Kalin M, Palm C, Soderhall S. Pharmacokinetics of acyclovir in immunocompromized children with leukopenia and mucositis after chemotherapy: can intravenous acyclovir be substituted by oral valacyclovir? Med Pediatr Oncol. 2002 Apr; 38(4):240-246. Available at http://www.ncbi.nlm.nih.gov/pubmed/11920787. [PubMed: 11920787]

56. GlaxoSmithKline. Valtrex. 2010. Available at http://us.gsk.com/products/assets/us_valtrex.pdf

57. PDR Network. Physicians Desk Reference. 65th Edition. Montvale, NJ: PDR Network, LLC; 2011.

58. Novartis Pharmaceuticals Corporation. Famvir. 2009 Available at http://pharma.us.novartis.com/ product/pi/pdf/Famvir.pdf.

59. Workowski KA, Berman SM. CDC. Sexually transmitted diseases treatment guidelines, 2006. MMWR Recomm Rep. 2006; 55(RR-11):1-94. Available at http://www.ncbi.nlm.nih.gov/sites/ entrez? $\mathrm{Db}=$ pubmed $\& \mathrm{Cmd}=$ ShowDetailView $\&$ TermToSearch=16888612\&ordinalpos=6\&itool=EntrezSy stem2.PEntrez.Pubmed.Pubmed_ResultsPanel.Pubmed_RVDocSum. [PubMed: 16888612]

60. Dworkin RH, Johnson RW, Breuer J, et al. Recommendations for the management of herpes zoster. Clin Infect Dis. 2007 Jan 1; 44(Suppl 1):S1-S26. Available at http:// www.ncbi.nlm.nih.gov/pubmed/17143845. [PubMed: 17143845]

61. Wilhelmus KR. Antiviral treatment and other therapeutic interventions for herpes simplex virus epithelial keratitis. Cochrane Database Syst Rev. 2010(12):CD002898. Available at http:// www.ncbi.nlm.nih.gov/pubmed/21154352. [PubMed: 21154352]

62. Bacon TH, Levin MJ, Leary JJ, Sarisky RT, Sutton D. Herpes simplex virus resistance to acyclovir and penciclovir after two decades of antiviral therapy. Clin Microbiol Rev. 2003 Jan; 16(1):114128. Available at http://www.ncbi.nlm.nih.gov/pubmed/12525428. [PubMed: 12525428] 
63. Balfour HH Jr. Antiviral drugs. N Engl J Med. 1999 Apr 22; 340(16):1255-1268. Available at http://www.ncbi.nlm.nih.gov/pubmed/10210711. [PubMed: 10210711]

64. Balfour HH Jr, Benson C, Braun J, et al. Management of acyclovir-resistant herpes simplex and varicella-zoster virus infections. J Acquir Immune Defic Syndr. 1994 Mar; 7(3):254-260. Available at http://www.ncbi.nlm.nih.gov/pubmed/8106965. [PubMed: 8106965]

65. Chen Y, Scieux C, Garrait V, et al. Resistant herpes simplex virus type 1 infection: an emerging concern after allogeneic stem cell transplantation. Clin Infect Dis. 2000 Oct; 31(4):927-935. Available at http://www.ncbi.nlm.nih.gov/pubmed/11049772. [PubMed: 11049772]

66. Lateef F, Don PC, Kaufmann M, White SM, Weinberg JM. Treatment of acyclovir-resistant, foscarnet-unresponsive HSV infection with topical cidofovir in a child with AIDS. Arch Dermatol. 1998 Sep; 134(9):1169-1170. Available at http://www.ncbi.nlm.nih.gov/pubmed/9762047. [PubMed: 9762047]

67. Kimberlin DW, Whitley RJ, Wan W, et al. Oral acyclovir suppression and neurodevelopment after neonatal herpes. N Engl J Med. 2011 Oct 6; 365(14):1284-1292. Available at http:// www.ncbi.nlm.nih.gov/pubmed/21991950. [PubMed: 21991950]

\section{Dosing Recommendations for Prevention and Treatment of Herpes Simplex Virus (HSV) Infections}

\begin{tabular}{|c|c|c|c|}
\hline Indication & First Choice & Alternative & $\begin{array}{l}\text { Comments/Special } \\
\text { Issues }\end{array}$ \\
\hline $\begin{array}{l}\text { Primary } \\
\text { Prophylaxis }\end{array}$ & None. & None. & $\begin{array}{l}\text { Primary prophylaxis is not } \\
\text { indicated. }\end{array}$ \\
\hline $\begin{array}{l}\text { Secondary } \\
\text { Prophylaxis }\end{array}$ & 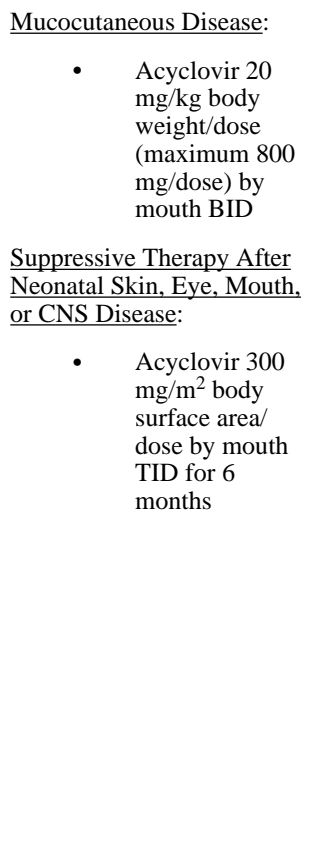 & $\begin{array}{ll}\frac{\text { Mucocutaneous Disease, For }}{\text { Adolescents Old Enough to }} \\
\text { Receive Adult Dosing: } \\
\text { - } \quad \begin{array}{l}\text { Valacyclovir } 500 \mathrm{mg} \\
\text { by mouth BID, or }\end{array} \\
\text { - } \quad \begin{array}{l}\text { Famciclovir } 500 \mathrm{mg} \text { by } \\
\text { mouth BID }\end{array}\end{array}$ & $\begin{array}{ll}\text { Secondary } & \text { Prophylaxis } \\
\underline{\text { Indicated: }} & \\
& \begin{array}{l}\text { Suppressive } \\
\text { secondary } \\
\text { prophylaxis can be } \\
\text { considered for } \\
\text { children with severe } \\
\text { and recurrent } \\
\text { mucocutaneous (oral } \\
\text { or genital) disease }\end{array} \\
\text { Criteria for Discontinuing } \\
\text { Secondary Prophylaxis: } \\
\text { - } \\
\text { After a prolonged } \\
\text { period (e.g., 1 year) } \\
\text { of prophylaxis, } \\
\text { consider suspending } \\
\text { prophylaxis and } \\
\text { determine with the } \\
\text { patient whether } \\
\text { additional } \\
\text { prophylaxis is } \\
\text { necessary. Although } \\
\text { level of immune } \\
\text { reconstitution is a } \\
\text { consideration, no } \\
\text { specific CD4 } \\
\text { threshold has been } \\
\text { established. }\end{array}$ \\
\hline Treatment & $\begin{array}{l}\text { Neonatal CNS or } \\
\text { Disseminated Disease: } \\
\begin{array}{l}\text { Acyclovir } 20 \\
\text { mg/kg body } \\
\text { weight IV/dose } \\
\text { TID for } \geq 21 \\
\text { days }\end{array} \\
\text { Neonatal Skin, Eye, or } \\
\text { Mouth Disease: }\end{array}$ & $\begin{array}{l}\text { Valacyclovir is } \\
\text { approved for } \\
\text { immunocompetent } \\
\text { adults and adolescents } \\
\text { with first-episode } \\
\text { mucocutaneous HSV at } \\
\text { a dose of } 1 \mathrm{~g} / \text { dose by } \\
\text { mouth BID for } 7-10 \\
\text { days; also approved for } \\
\text { recurrent herpes }\end{array}$ & $\begin{array}{l}\text { For Neonatal CNS Disease: } \\
\text { Repeat CSF HSV } \\
\text { DNA PCR should } \\
\text { be performed on } \\
\text { days } 19 \text { to } 21 \text { of } \\
\text { therapy; do not stop } \\
\text { acyclovir until } \\
\text { repeat CSF HSV } \\
\text { DNA PCR is } \\
\text { negative. }\end{array}$ \\
\hline
\end{tabular}




\begin{tabular}{|c|c|c|c|}
\hline Indication & First Choice & Alternative & $\begin{array}{l}\text { Comments/Special } \\
\text { Issues }\end{array}$ \\
\hline & 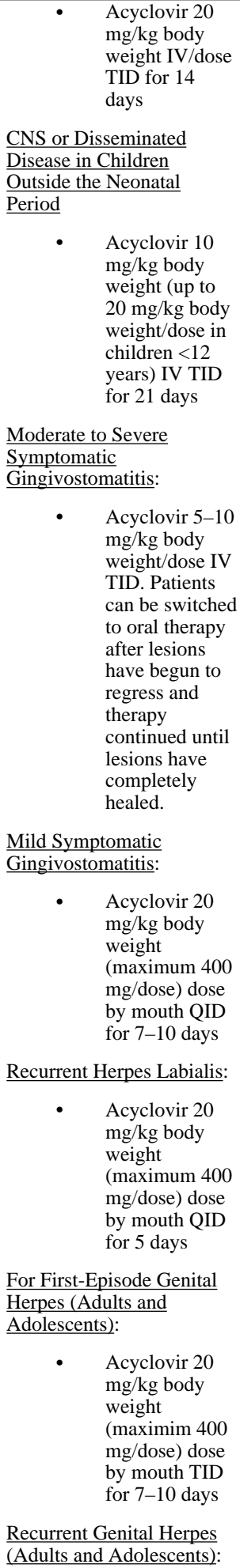 & $\begin{array}{l}\text { labialis in children } \geq 12 \\
\text { years using two, } 2 \mathrm{~g} \\
\text { doses by mouth } \\
\text { separated by } 12 \text { hours } \\
\text { as single-day therapy. } \\
\text { - Recurrent genital HSV } \\
\text { can be treated with } \\
\text { valacyclovir } 500 \mathrm{mg} \\
\text { BID for } 3 \text { days or } 1 \mathrm{~g} \\
\text { by mouth daily for } 5 \\
\text { days. } \\
\text { Immunocompetent } \\
\text { adults with recurrent } \\
\text { herpes labialis can be } \\
\text { treated with } \\
\text { famciclovir, } 1 \mathrm{~g} / \mathrm{dose} \\
\text { by mouth BID for } 1 \\
\text { day. } \\
\text { Famciclovir is } \\
\text { approved to treat } \\
\text { primary genital HSV in } \\
\text { immunocompetent } \\
\text { adults at a dose of } 250 \\
\text { mg/dose by mouth TID } \\
\text { for } 7-10 \text { days. } \\
\text { Recurrent genital HSV } \\
\text { is treated with } \\
\text { famciclovir } 1 \mathrm{~g} / \text { dose } \\
\text { by mouth BID at a } 12- \\
\text { hour interval for } 2 \\
\text { doses } \\
\text { Famciclovir is } \\
\text { approved for use in } \\
\text { HIV- infected adults } \\
\text { and adolescents with } \\
\text { recurrent } \\
\text { mucocutaneous HSV } \\
\text { infection at a dose of } \\
500 \text { mg/dose by mouth } \\
\text { BID for } 7 \text { days. } \\
\text { Infection: } \\
\text { - } \text { - } \\
\text { Fesistant HSV } \\
\text { Foscarnet } 40 \text { mg/kg } \\
\text { body weight/dose } \\
\text { given IV TID (or } 60 \\
\text { mg/kg body weight/ } \\
\text { dose BID) should be } \\
\text { administered slowly } \\
\text { over the course of } 2 \\
\text { hours (i.e., no faster } \\
\text { than } 1 \text { mg/kg/minute). }\end{array}$ & $\begin{array}{l}\text { There is no pediatric } \\
\text { preparation of } \\
\text { valacyclovir } \\
\text { (although crushed } \\
\text { capsules can be used } \\
\text { to make a } \\
\text { suspension) and data } \\
\text { on dosing in } \\
\text { children are limited; } \\
\text { can be used by } \\
\text { adolescents able to } \\
\text { receive adult dosing. } \\
\text { - There is no pediatric } \\
\text { preparation of } \\
\text { famciclovir and data } \\
\text { on dosing in } \\
\text { children are } \\
\text { unavailable; can be } \\
\text { used by adolescents } \\
\text { able to receive adult } \\
\text { dosing. }\end{array}$ \\
\hline
\end{tabular}




\begin{tabular}{|c|c|c|c|}
\hline Indication & First Choice & Alternative & $\begin{array}{l}\text { Comments/Special } \\
\text { Issues }\end{array}$ \\
\hline & 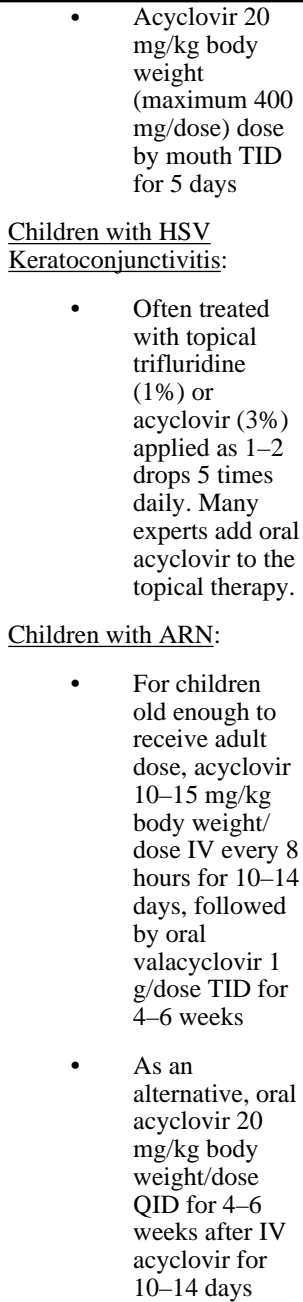 & & \\
\hline
\end{tabular}

Key to Acronyms: $\mathrm{ARN}=$ acute retinal necrosis; $\mathrm{BID}=$ twice daily; $\mathrm{CD} 4=\mathrm{CD} 4 \mathrm{~T}$ lymphocyte; $\mathrm{CNS}=$ central nervous system; $\mathrm{CSF}$ = cerebrospinal fluid; HSV = herpes simplex virus; IV = intravenous; $\mathrm{PCR}=$ polymerase chain reaction; $\mathrm{QID}$

$=$ four times daily; TID $=$ three times daily

\section{Histoplasmosis (Last updated November 6, 2013; last reviewed November}

\section{6, 2013)}

Panel's Recommendations

- Routine use of antifungal medications for primary prophylaxis of histoplasmosis in children is not recommended (BIII).

- Amphotericin B is preferred for initial treatment of moderately severe to severe infections (AI*).

- Itraconazole is the azole preferred for treatment of histoplasmosis (AIII).

- In manifestations of histoplasmosis in which antigenuria is demonstrated, antigen levels should be monitored during therapy and for 1 year thereafter to identify relapse (AIII). 
Panel's Recommendations

- $\quad$ For severe or moderately severe acute primary pulmonary histoplasmosis, amphotericin B should be administered for at least 1 to 2 weeks (and clinical improvement) (AIII). After treatment with amphotericin, patients with intact immunity should receive itraconazole for at least 12 weeks (AIII). Adults with CD4 T lymphocyte (CD4) cell counts $<150$ cells $/ \mathrm{mm}^{3}$ and HIV-infected children with severe immunosuppression should receive itraconazole consolidation therapy for at least 12 months (AIII).

- The preferred treatment for severe or moderately severe progressive disseminated histoplasmosis is initial (induction) therapy with amphotericin B for $\geq 2$ weeks (and favorable clinical response), followed by consolidation therapy with itraconazole for at least 12 months (AI*).

- Itraconazole monotherapy for 12 months is recommended for HIV-infected children with mild to moderate progressive disseminated histoplasmosis (AII*).

- Liposomal amphotericin B for 4 to 6 weeks is the preferred initial treatment in the presence of focal brain lesions (BIII*). Thereafter, children should receive itraconazole consolidation therapy for at least 12 months and until cerebrospinal fluid abnormalities, including histoplasma antigen, have resolved (AII*).

- In the event of immune reconstitution inflammatory syndrome, antiretroviral therapy should be continued along with antifungal therapy (AIII).

- $\quad$ Longer-term suppressive therapy (secondary prophylaxis) with itraconazole may be required in HIVinfected children who are severely immunosuppressed (meaning CD4 percentage $<15 \%$ at any age or CD4 count $<150$ cells $/ \mathrm{mm}^{3}$ in children aged $\ 6$ years) and patients who experience relapse despite receipt of appropriate therapy (AIII).

Rating of Recommendations: $\mathrm{A}=$ Strong; $\mathrm{B}=$ Moderate; $\mathrm{C}=$ Optional

Rating of Evidence: $\mathrm{I}=$ One or more randomized trials $\underline{\text { in children }}^{\dagger}$ with clinical outcomes and/or validated endpoints; $\mathrm{I}^{*}$ $=$ One or more randomized trials in adults with clinical outcomes and/or validated laboratory endpoints with accompanying data in children ${ }^{\dagger}$ from one or more well-designed, nonrandomized trials or observational cohort studies with long-term clinical outcomes; II = One or more well-designed, nonrandomized trials or observational cohort studies in children ${ }^{\dagger}$ with long-term outcomes; II* $=$ One or more well-designed, nonrandomized trials or observational studies in adults with longterm clinical outcomes with accompanying data in children ${ }^{\dagger}$ from one or more similar nonrandomized trials or cohort studies with clinical outcome data; III = Expert opinion

${ }^{\dagger}$ Studies that include children or children/adolescents, but not studies limited to post-pubertal adolescents

\section{Epidemiology}

Histoplasmosis is caused by inhalation of microconidia produced by the mycelial form of Histoplasma capsulatum, an endemic dimorphic fungus, and cases have been reported from all continents except Antarctica. In the United States, it is most highly endemic in the Ohio and Mississippi river valleys. Infections in regions in which histoplasmosis is not endemic often result from travel to endemic regions within and outside the United States (e.g., Mexico, Central and South America). Risk factors predisposing to infection are exposure to activities that disturb contaminated sites and are accompanied by aerosolization of spores and (in HIV-infected adults) a CD4 T lymphocyte (CD4) cell count $<150$ cells $/ \mathrm{mm}^{3}$. Because yeast forms of the fungus may remain viable within granulomas formed after successful treatment or spontaneous resolution of infection, late relapse can occur if cellular immune function wanes, although the magnitude of this risk appears very low. ${ }^{1}$ Infection can occur during pregnancy, and transplacental infection has rarely been reported. ${ }^{2}$

During the era before combination antiretroviral therapy (cART), histoplasmosis was reported in 2\% to 5\% of HIV-infected adults living in regions with endemic disease; rates of $25 \%$ have been reported in some cities. ${ }^{3}$ In a highly endemic region, histoplasmosis was the AIDS-defining illness in $25 \%$ of adults and $8 \%$ of children. ${ }^{4}$ Progressive disseminated histoplasmosis (PDH) occurred in 5\% of HIV-infected children in another highly endemic region (M. Kleiman, unpublished data). The overall incidence of histoplasmosis in children 
has not been examined systematically but appeared to be low, even during the pre-cART era. ${ }^{5}$ An HIV-positive infant with probable congenital histoplasmosis has been reported in a non-endemic area. ${ }^{6}$

Few epidemiologic data have been reported on disseminated histoplasmosis in HIV-infected children and adolescents treated with cART. In several combined Pediatric AIDS Clinical Trial Group cohorts, the incidence rate of all non-Candida invasive fungal infection was 0.10 infections per 100 child-years (95\% CI $0.05-0.20$ ) during the pre-cART era, and 0.08 infections per 100 child-years (95\% CI 0.03-0.17) since the advent of cART. ${ }^{5,7}$ These data were contributed from centers that underrepresented the geographic regions of maximal histoplasmosis prevalence, so the statistical power to detect decreases in incidence rates associated with cART may have been limited. However, none of the rates of domestic endemic fungal infections (e.g., histoplasmosis, coccidioidomycosis, and blastomycosis) are likely to exceed these estimates in HIV-infected children and adolescents.

\section{Clinical Manifestations}

In HIV-uninfected children, acute pulmonary manifestations are common; chronic pulmonary infection has not been described. Because of greater airway pliability in children, airway obstruction from mediastinal lymphadenopathy is more common in children. ${ }^{8}$ Meningitis often accompanies progressive disseminated infection in infancy; subacute meningitis and parenchymal lesions characteristic of central nervous system (CNS) disease in adults are unusual in children. ${ }^{9}$ Isolated pulmonary granulomas resulting from past infections are common incidental findings in chest radiographs of asymptomatic persons who have resided in histoplasmosis-endemic regions.

The most frequent clinical manifestation of histoplasmosis in HIV-infected children with AIDS is PDH, which is fatal if untreated. Prolonged fever and failure to thrive are uniform presenting complaints. Few reports have been published of presenting signs and symptoms in children with PDH complicating AIDS. ${ }^{40-12}$ However, most are similar to those seen in PDH in otherwise normal infants and in infections in patients with other primary or acquired cellular immunodeficiencies. These include splenomegaly, cough, respiratory distress, hepatomegaly, septic appearance, generalized lymphadenopathy, interstitial pneumonitis, cytopenia(s), coagulopathy, oropharyngeal/gastrointestinal (GI) ulcerations, and erythematous nodular/ulcerative cutaneous lesions. ${ }^{13-15}$

\section{Diagnosis}

Culture and histopathologic, serologic, antigen-detection, and molecular diagnostic techniques have been developed to aid in diagnosing histoplasmosis. ${ }^{16,17}$ Understanding their uses and limitations is essential to interpreting results.

Histoplasmin skin tests are no longer available and were not useful in diagnosing disseminated disease. ${ }^{14,15}$ Although isolation of the fungus using culture is diagnostic, it often requires invasive procedures, is insensitive, and may take 10 to 30 days for growth to occur. Lysis-centrifugation methodology facilitates growth of $H$. capsulatum, and a DNA probe permits prompt identification of isolates. ${ }^{18}$ Histopathologic demonstration of typical 
yeast forms in tissue specimens, bone marrow, or peripheral blood can be performed rapidly and, when positive, is highly suggestive of active infection. However, results are positive in only $12 \%$ to $43 \%$ of adults with PDH. ${ }^{16}$ Polymerase chain reaction and DNA probes have been developed to detect $H$. capsulatum DNA in tissues ${ }^{19}$ and body fluids ${ }^{20}$ but neither is sufficiently sensitive and DNA probes may lack adequate specificity. ${ }^{16,17}$

Interpretation of serologic testing using complement fixation $(\mathrm{CF})$ and immunodiffusion methods is problematic in immunocompromised hosts with PDH. CF titers of $\geq 1: 32$ to the yeast and/or mycelial antigens or detection of $\mathrm{H}$ and/or $\mathrm{M}$ bands with the immunodiffusion test are considered strongly suggestive of active or recent infection. However, only $41 \%$ to $69 \%$ of HIV-infected adults are seropositive, compared with $82 \%$ of adults with PDH and no underlying immunodeficiency. ${ }^{21,22}$ Thus, seronegativity cannot be used to exclude active infection, especially PDH. Although a fourfold increase in CF antibody is diagnostic of active infection, 2 to 4 weeks is needed to determine this. CF antibody titers of cerebrospinal fluid (CSF) may be useful for diagnosing meningitis. In these instances, the assay should begin with undiluted specimens. Concurrent serum titers should be evaluated to exclude false positivity caused by blood contamination of the CSF. ${ }^{9}$

An enzyme-linked immunoassay (EIA) that rapidly identifies and quantifies histoplasma antigen in body fluids fills most of the gaps left by other diagnostic methods. ${ }^{22}$ EIA is especially suited for evaluating patients with large fungal burdens, a feature of infection in immunocompromised hosts. EIA can detect antigen in serum, bronchoalveolar lavage, and CSF specimens. The reported sensitivity of antigen detection is $91 \%$ to $92 \%$ in adults with PDH, and 95\% in adults with AIDS; ${ }^{16,17}$ sensitivity in children with underlying cellular immunodeficiency, including those who are HIV-infected, and in otherwise normal infants approaches $100 \% .^{14,23}$

The third-generation EIA is standardized by extrapolating antigen concentrations from a calibration curve that is linear to a value of $39 \mathrm{ng} / \mathrm{mL}$. However, urine antigen concentrations in serious infections frequently exceed this value. In these instances, serum specimens should be followed because maximum serum concentrations are lower than those in urine and thus more likely to be in a range in which differences can be accurately measured. After resolution of the antigenemia, urine concentrations can be followed to monitor the effectiveness of treatment and, thereafter, to identify relapse. Antigenuria is identified in $90 \%$ of patients whose histoplasmosis relapses. ${ }^{8}$ Interpretation is complicated by cross-reactions with blastomycosis, paracoccidioidomycosis, and Penicillium marneffei infections. ${ }^{16,17}$ Distinctive clinical and geographic features of these endemic fungal infections permit accurate differentiation. Urine antigen is detectable in $75 \%$ to $81 \%$ of immunocompetent hosts with acute, primary pulmonary infection. This occurs early in infection, reflecting the primary fungemia that is aborted by an effective cellular immune response. Thus, antigenuria in a patient with HIV who retains normal cellular immunity may not necessarily presage development of disseminated infection. Based on adult data, testing both serum and urine following high inoculum exposure may improve sensitivity of detecting antigen in acute primary pulmonary infection, especially in patients with less severe CD4 depletion and milder illness, in whom sensitivity in urine may be lower. ${ }^{24}$ 
Diagnosis of CNS infection is difficult, particularly in patients who have isolated meningitis without disseminated disease. ${ }^{9}$ Highest sensitivity is achieved by testing CSF for histoplasma antigen, antibody, and large-volume culture. In adults, CSF culture is positive in $20 \%$ to $60 \%$ of patients, CSF antigen is positive in $40 \%$ to $70 \%$, and CSF antibody is positive in $70 \%$ to $90 \% .{ }^{16,17}$ Meningitis frequently accompanies PDH of infancy, ${ }^{13}$ an entity that has not been associated with a recognized immunodeficiency disorder.

\section{Prevention Recommendations}

Preventing Exposure-Most infections occur without a recognized history of exposure to a high-risk site or activity. Therefore, complete avoidance of exposure in histoplasmosisendemic regions is not possible. Sites and conditions sometimes implicated in high-risk exposure and point-source outbreaks include disturbances of contaminated areas resulting in aerosolization of spores. These include soil contaminated with bird or bat droppings, older urban and rural structures, decaying vegetation or trees, and caves. Dry and windy conditions, excavation, demolition, renovation, gardening, and agricultural activities often predispose to aerosolization of spores. Education should be directed toward avoidance of these activities. If not feasible, reducing the release of spores by wetting soil, renovation sites, and other potentially contaminated areas, and use of protective respiratory devices, ${ }^{25}$ should be recommended.

Preventing First Episode of Disease-Prophylaxis with itraconazole is recommended for HIV-infected adults with CD4 counts $<150$ cells $/ \mathrm{mm}^{3}$ and who reside in areas where histoplasmosis is highly endemic (that is, incidence $>10$ cases per 100 patient-years) and in instances in which risk of occupational exposure is high. Prophylaxis has no effect on survival. ${ }^{8}$ Given the low incidence of histoplasmosis in HIV-infected children, possibility for drug interaction, development of antifungal drug resistance, and cost, routine use of antifungal medications for primary prophylaxis of histoplasma infections in children is not recommended (BIII).

Discontinuing Primary Prophylaxis-Not applicable.

\section{Treatment Recommendations}

Treating Disease-PDH is fatal without treatment. The clinical response to amphotericin $\mathrm{B}$ is faster than that of itraconazole and it is preferred for initial treatment of severe infections (AI*). Following amphotericin B induction, itraconazole, the azole preferred for treatment of histoplasmosis $\left(\mathbf{A I} \mathbf{I}^{*}\right){ }^{8}$ is used to complete the course of therapy. A trial in adults ${ }^{26}$ demonstrated that induction with liposomal amphotericin B was associated with less toxicity and improved survival, compared with induction using amphotericin B deoxycholate. Recommendations for HIV-infected children are derived from trials in adults and from anecdotal experience in children. ${ }^{8}$ Because of important differences in managing PDH in children, consultation with experts should be considered.

Itraconazole is usually well tolerated in children. Itraconazole has a long half-life and reaches steady-state levels at 2 weeks. The interval needed to achieve desired serum concentrations can be shortened if the recommended dose is administered 3 times daily for 
the first 3 days of therapy (i.e., loading dose); the recommended dose, administered twice daily, should be started thereafter. Itraconazole solution is preferred to the capsule formulation because it is better absorbed and serum concentrations are $30 \%$ higher than those achieved with the capsules. The solution should be taken on an empty stomach or with a carbonated beverage. If capsules are used, they should be taken with meals. Because absorption of itraconazole varies considerably from patient to patient, serum concentrations should be measured to ensure effective levels of drug, monitor changes in dosage, and assess compliance (BIII). The minimal inhibitory concentration of $H$. capsulatum is $0.01 \mu \mathrm{g} / \mathrm{mL}$, and although minimally effective serum concentrations have not been determined, a serum concentration of $1.0 \mu \mathrm{g} / \mathrm{mL}$ is recommended; dosage should be reduced if concentrations exceed $10 \mu \mathrm{g} / \mathrm{mL}^{8}$

Fluconazole is an alternative for patients with mild histoplasmosis and who are intolerant of itraconazole or in whom desired serum levels of itraconazole cannot be attained.

Fluconazole is less effective than itraconazole and has been associated with development of drug resistance. ${ }^{27}$

Acute Primary Pulmonary Histoplasmosis: Patients with acute primary pulmonary histoplasmosis can present with a wide spectrum of symptoms, ranging from dyspnea with high fever to only mild respiratory symptoms, and variable fever. Chest radiographs may show mediastinal adenopathy with or without focal pulmonary infiltrate and/or a diffuse miliary-like pattern in high-inoculum exposure; radiographic findings may mimic those of tuberculosis. For severe or moderately severe symptoms, liposomal amphotericin B should be administered for 1 to 2 weeks (AI*). ${ }^{8}$ After clinical improvement, adults with CD4 counts $>300$ cells $/ \mathrm{mm}^{3}$ and, by extrapolation, HIV-infected children with CD4 percentage $>20 \%$ or, if $\geq 6$ years, CD4 count $>300$ cells $/ \mathrm{mm}^{3}$, should receive itraconazole, beginning with a loading dose (see above) for the first 3 days, followed by the recommended doses administered twice daily for at least 12 weeks (AIII). All other HIV-infected children should receive itraconazole for 12 months (AIII). Urine antigen usually is elevated in these situations and should be monitored to gauge clinical response and, after treatment, identify relapse (AIII).

HIV-infected children, particularly those with CD4 percentage $>20 \%$ (or, if $\geq 6$ years, CD4 counts $>300$ cells $/ \mathrm{mm}^{3}$ ) compatible with functional cellular immunity, occasionally present with fever, mild primary pulmonary infection, and histoplasma antigenuria. Although an effective cellular immune response may limit such illnesses, it may be prudent to treat with itraconazole for 12 weeks and monitor histoplasma urine antigen concentrations to ensure that concentrations decrease (BIII).

Moderately Severe to Severe PDH: Data derived from experience in HIV-infected adults suggest that HIV-infected children with moderately severe to severe disseminated histoplasmosis should be treated with an IV amphotericin B formulation for $\geq 2$ weeks (and until they clinically improve), followed by itraconazole for 12 months (AI*). HIV-infected adults with moderately severe to severe PDH have a higher response rate to treatment with liposomal amphotericin B than with the deoxycholate formulation (88\% vs. 64\%) and a lower death rate ( $2 \%$ vs. $13 \%$ ); therefore liposomal preparations are preferred in adults and, 
by extrapolation, in children $\left(\mathbf{A I}^{*}\right) .{ }^{8} \mathrm{~A}$ loading dose (see above) of itraconazole should be used for the initial 3 days. If itraconazole is not well tolerated, a 4- to 6-week course of amphotericin B can be used (AIII). Progressive decline in histoplasma urine and serum antigen levels is expected with effective treatment, and monitoring levels for lack of such decline can detect relapse.

Although therapeutic trials of amphotericin B deoxycholate used to treat PDH in HIVinfected children have not been performed, this formulation is effective for treating severe PDH in infants, ${ }^{13,28}$ including those with CNS infection, ${ }^{13}$ and in children with other primary or acquired immunodeficiency states. Amphotericin B deoxycholate is better tolerated by children than by adults, and it is less costly than other formulations. It can be used if cost or availability of lipid formulations precludes their use (AIII).

Mild to Moderate PDH: In $80 \%$ to $100 \%$ of patients without signs of CNS infection, mild to moderate PDH responds favorably to itraconazole monotherapy for 12 months (AII*). ${ }^{8,29}$ This regimen also is recommended for HIV-infected children with mild to moderate PDH (AII*). A loading dose of itraconazole (see above) should be administered at the onset of treatment and serum concentrations monitored. Urine antigen concentrations should also be monitored.

CNS Infection: CNS infection that accompanies PDH is expected to respond to the regimen recommended for moderately severe to severe PDH. Isolated CNS infection is unusual in children. In adults, frequent failure and relapse are common, and aggressive therapy is recommended. Penetration into the CSF is poor with all amphotericin B formulations. Liposomal amphotericin B is preferred for CNS disease in children and adults because it achieves higher concentrations in the brain (AII*); the deoxycholate formulation is an alternative. Another lipid formulation can be used at the same dosage if cost is a concern or in patients who cannot tolerate liposomal amphotericin B (AIII). Amphotericin should be administered for 4 to 6 weeks. Thereafter, a child should receive a loading dose of itraconazole and continuation of itraconazole for 12 months and until CSF abnormalities, including histoplasma antigen, have resolved (AII*).

Itraconazole levels should be followed and the dose adjusted to ensure optimal serum concentrations (AIII).

Asymptomatic Histoplasma Granuloma: In asymptomatic HIV-infected children who have intact cellular immunity (meaning CD4 $>15 \%$ for all ages and CD4 cell count $>150$ cells $/ \mathrm{mm}^{3}$ for ages $\ 6$ years) and have resided in an area with endemic histoplasmosis, the presence of a typical granuloma in a chest radiograph should prompt evaluation of histoplasma urine antigen and both CF and immunodiffusion antibody. If any of these tests are positive, treatment with itraconazole for 12 weeks is prudent (BIII). If these tests are negative, therapy need not be used, and close clinical follow-up is recommended. In either instance, histoplasma urine antigen testing should be considered if unexplained fever, weight loss, or other systemic symptoms occur. 
Monitoring and Adverse Events (Including IRIS)_-In manifestations of histoplasmosis in which antigenuria is demonstrated, antigen levels should be monitored during therapy and for a year thereafter to identify relapse (AIII). ${ }^{8}$ After a recommended course of therapy and in the absence of symptoms, low-level, stable antigenuria may not constitute a basis for prolonging the recommended course of therapy. Serum levels of itraconazole should be monitored in patients receiving treatment (AIII).

Adverse effects of amphotericin B are primarily nephrotoxicity; permanent nephrotoxicity is related to cumulative dose. Infusion-related fevers, chills, nausea, and vomiting can occur, especially early in treatment, although they are less frequent in children than in adults. Renal dysfunction and electrolyte imbalances are the primary toxicities; these parameters should be monitored during therapy.

Itraconazole, like other azoles, has relatively low rates of toxicity. GI upset is seen occasionally and its principal toxicity is hepatic. Because the azole drugs inhibit CYP450dependent hepatic enzymes, drug interactions-particularly with antiretroviral drugsshould be carefully evaluated before initiation of therapy.

Immune reconstitution inflammatory syndrome (IRIS) caused by an inflammatory response to histoplasmosis unmasked by cART-induced improvement in cellular immunity is unusual, and symptoms are often mild. ${ }^{30}$ In the event of IRIS, cART should be continued along with antifungal therapy (AIII). IRIS related to histoplasmosis has not been reported in children.

Managing Treatment Failure-Both voriconazole and posaconazole have been used successfully in a small number of refractory cases in adults. ${ }^{8}$ Because little experience has been reported using the newer azoles and data are limited on use of these agents in children, expert consultation is recommended for cases refractory to first-line agents.

Preventing Recurrence-Following initial amphotericin B treatment (induction) and subsequent oral itraconazole consolidation therapy for at least 1 year, longer-term suppressive therapy with itraconazole may be required in HIV-infected children who remain immunosuppressed (i.e., CD4 percentage $<15 \%$ at any age or $<150$ cells $/ \mathrm{mm}^{3}$ in children aged $\ 6$ years) and in those who experience relapse despite receipt of appropriate therapy (AII*). ${ }^{8,31}$ Fluconazole is less effective than itraconazole (CII*), and experience with voriconazole is limited in children. Adherence to both antifungal treatment and cART should be monitored carefully, as nonadherence can increase the risk of relapse.

Discontinuing Secondary Prophylaxis-Discontinuation of secondary prophylaxis (suppressive therapy) has not been examined in children. Based on data from a clinical trial, adults with immune restoration on cART can discontinue itraconazole if itraconazole has been received for $\geq 1$ year, blood cultures are negative, histoplasma serum antigen is $<2$ $\mathrm{ng} / \mathrm{mL}, \mathrm{CD} 4$ counts are $>150$ cells $/ \mathrm{mm}^{3}$, and there is good adherence to cART. ${ }^{31}$ Extrapolating these recommendations to HIV-infected children on cART with immune restoration (meaning CD4 percentage $\geq 15 \%$ at any age; $\mathrm{CD} 4$ count $>150$ cells $/ \mathrm{mm}^{3}$ in children aged $\searrow 6$ years) seems reasonable (CIII). Secondary prophylaxis should resume if 
these parameters are not met. Chronic suppressive therapy is recommended for relapse that occurs despite appropriate treatment (BIII).

\section{References}

1. Hage CA, Davis TE, Fuller D, et al. Diagnosis of histoplasmosis by antigen detection in BAL fluid. Chest. 2010 Mar; 137(3):623-628. Available at http://www.ncbi.nlm.nih.gov/pubmed/19837826. [PubMed: 19837826]

2. Whitt SP, Koch GA, Fender B, Ratnasamy N, Everett ED. Histoplasmosis in pregnancy: case series and report of transplacental transmission. Arch Intern Med. 2004 Feb 23; 164(4):454-458. Available at http://www.ncbi.nlm.nih.gov/pubmed/14980998. [PubMed: 14980998]

3. Wheat LJ, Chetchotisakd P, Williams B, Connolly P, Shutt K, Hajjeh R. Factors associated with severe manifestations of histoplasmosis in AIDS. Clin Infect Dis. 2000 Jun; 30(6):877-881. Available at http://www.ncbi.nlm.nih.gov/pubmed/10854363. [PubMed: 10854363]

4. Schutze GE, Tucker NC, Jacobs RF. Histoplasmosis and perinatal human immunodeficiency virus. Pediatr Infect Dis J. 1992 Jun; 11(6):501-502. Available at http://www.ncbi.nlm.nih.gov/pubmed/ 1608693. [PubMed: 1608693]

5. Dankner WM, Lindsey JC, Levin MJ. Pediatric ACTGPT. Correlates of opportunistic infections in children infected with the human immunodeficiency virus managed before highly active antiretroviral therapy. Pediatr Infect Dis J. 2001 Jan; 20(1):40-48. Available at http:// www.ncbi.nlm.nih.gov/pubmed/11176565. [PubMed: 11176565]

6. Alverson B, Alexander N, LeGolvan MP, Dunlap W, Levy C. A human immunodeficiency viruspositive infant with probable congenital histoplasmosis in a nonendemic area. Pediatr Infect Dis J. 2010 Nov; 29(11):1055-1057. Available at http://www.ncbi.nlm.nih.gov/pubmed/20526228. [PubMed: 20526228]

7. Gona P, Van Dyke RB, Williams PL, et al. Incidence of opportunistic and other infections in HIVinfected children in the HAART era. JAMA. 2006 Jul 19; 296(3):292-300. Available at http:// www.ncbi.nlm.nih.gov/pubmed/16849662. [PubMed: 16849662]

8. Wheat LJ, Freifeld AG, Kleiman MB, et al. Clinical practice guidelines for the management of patients with histoplasmosis: 2007 update by the Infectious Diseases Society of America. Clin Infect Dis. 2007 Oct 1; 45(7):807-825. Available at http://www.ncbi.nlm.nih.gov/pubmed/ 17806045. [PubMed: 17806045]

9. Wheat LJ, Musial CE, Jenny-Avital E. Diagnosis and management of central nervous system histoplasmosis. Clin Infect Dis. 2005 Mar 15; 40(6):844-852. Available at http:// www.ncbi.nlm.nih.gov/pubmed/15736018. [PubMed: 15736018]

10. Saidinejad M, Burns MM, Harper MB. Disseminated histoplasmosis in a nonendemic area. Pediatr Infect Dis J. 2004 Aug; 23(8):781-782. Available at http://www.ncbi.nlm.nih.gov/pubmed/ 15295232. [PubMed: 15295232]

11. Byers M, Feldman S, Edwards J. Disseminated histoplasmosis as the acquired immunodeficiency syndrome-defining illness in an infant. Pediatr Infect Dis J. 1992 Feb; 11(2):127-128. Available at http://www.ncbi.nlm.nih.gov/pubmed/1741185. [PubMed: 1741185]

12. Pillay T, Pillay DG, Bramdev A. Disseminated histoplasmosis in a human immunodeficiency virus-infected African child. Pediatr Infect Dis J. 1997 Apr; 16(4):417-418. Available at http:// www.ncbi.nlm.nih.gov/pubmed/9109150. [PubMed: 9109150]

13. Odio CM, Navarrete M, Carrillo JM, Mora L, Carranza A. Disseminated histoplasmosis in infants. Pediatr Infect Dis J. 1999 Dec; 18(12):1065-1068. Available at http://www.ncbi.nlm.nih.gov/ pubmed/10608625. [PubMed: 10608625]

14. Leggiadro RJ, Barrett FF, Hughes WT. Disseminated histoplasmosis of infancy. Pediatr Infect Dis J. 1988 Nov; 7(11):799-805. Available at http://www.ncbi.nlm.nih.gov/pubmed/3068620. [PubMed: 3068620]

15. Hughes WT. Hematogenous histoplasmosis in the immunocompromised child. J Pediatr. 1984 Oct; 105(4):569-575. Available at http://www.ncbi.nlm.nih.gov/pubmed/6090628. [PubMed: 6090628] 
16. Wheat LJ. Antigen detection, serology, and molecular diagnosis of invasive mycoses in the immunocompromised host. Transpl Infect Dis. 2006 Sep; 8(3):128-139. Available at http:// www.ncbi.nlm.nih.gov/pubmed/16913971. [PubMed: 16913971]

17. Wheat LJ. Improvements in diagnosis of histoplasmosis. Expert Opin Biol Ther. 2006 Nov; 6(11): 1207-1221. Available at http://www.ncbi.nlm.nih.gov/pubmed/17049017. [PubMed: 17049017]

18. Brandt, ME.; Warnock, DW. Histoplasma, Blastomyces, Coccidioides, and other dimorphic fungi causing systemic mycoses. In: Murray, P., editor. Manual of clinical microbiology. 9th ed. Vol. 9. 2007. p. 1857-1865.

19. Babady NE, Miranda E, Gilhuley KA. Evaluation of Luminex xTAG fungal analyte-specific reagents for rapid identification of clinically relevant fungi. J Clin Microbiol. 2011 Nov; 49(11): 3777-3782. Available at http://www.ncbi.nlm.nih.gov/pubmed/21880976. [PubMed: 21880976]

20. Tang YW, Li H, Durkin MM, et al. Urine polymerase chain reaction is not as sensitive as urine antigen for the diagnosis of disseminated histoplasmosis. Diagn Microbiol Infect Dis. 2006 Apr; 54(4):283-287. Available at http://www.ncbi.nlm.nih.gov/pubmed/16466889. [PubMed: 16466889]

21. Tobon AM, Agudelo CA, Rosero DS, et al. Disseminated histoplasmosis: a comparative study between patients with acquired immunodeficiency syndrome and non-human immunodeficiency virus-infected individuals. Am J Trop Med Hyg. 2005; 73(3):576-582. Available at http:// www.ncbi.nlm.nih.gov/entrez/query.fcgi? $\mathrm{cmd}=$ Retrieve $\& \mathrm{db}=$ PubMed\&dopt=Citation\&list_uids=16172484. [PubMed: 16172484]

22. Hage CA, Ribes JA, Wengenack NL, et al. A multicenter evaluation of tests for diagnosis of histoplasmosis. Clin Infect Dis. 2011 Sep; 53(5):448-454. Available at http:// www.ncbi.nlm.nih.gov/pubmed/21810734. [PubMed: 21810734]

23. Fojtasek MF, Kleiman MB, Connolly-Stringfield P, Blair R, Wheat LJ. The Histoplasma capsulatum antigen assay in disseminated histoplasmosis in children. Pediatr Infect Dis J. 1994 Sep; 13(9):801-805. Available at http://www.ncbi.nlm.nih.gov/pubmed/7808850. [PubMed: 7808850]

24. Swartzentruber S, LeMonte A, Witt J, et al. Improved detection of Histoplasma antigenemia following dissociation of immune complexes. Clinical and vaccine immunology: CVI. 2009 Mar; 16(3):320-322. Available at http://www.ncbi.nlm.nih.gov/pubmed/19144790. [PubMed: 19144790]

25. Lenhart, SW.; Schafer, MP.; Singal, M., et al. Histoplasmosis-protecting workers at risk. Atlanta, GA: U.S. Department of Health and Human Services, CDC; 2004. DHHS (NIOSH) Publication No. 2005-109. Available at http://www.cdc.gov/niosh/docs/2005-109/\#a

26. Johnson PC, Wheat LJ, Cloud GA, et al. Safety and efficacy of liposomal amphotericin B compared with conventional amphotericin B for induction therapy of histoplasmosis in patients with AIDS. Ann Intern Med. 2002 Jul 16; 137(2):105-109. Available at http:// www.ncbi.nlm.nih.gov/pubmed/12118965. [PubMed: 12118965]

27. Wheat LJ, Connolly P, Smedema M, et al. Emergence of resistance to fluconazole as a cause of failure during treatment of histoplasmosis in patients with acquired immunodeficiency disease syndrome. Clin Infect Dis. 2001 Dec 1; 33(11):1910-1913. Available at http:// www.ncbi.nlm.nih.gov/pubmed/11692303. [PubMed: 11692303]

28. Adderson EE. Histoplasmosis in a pediatric oncology center. J Pediatr. 2004 Jan; 144(1):100-106. Available at http://www.ncbi.nlm.nih.gov/pubmed/14722526. [PubMed: 14722526]

29. Dismukes WE, Bradsher RW Jr, Cloud GC, et al. Itraconazole therapy for blastomycosis and histoplasmosis. NIAID Mycoses Study Group. Am J Med. 1992 Nov; 93(5):489-497. Available at http://www.ncbi.nlm.nih.gov/pubmed/1332471. [PubMed: 1332471]

30. Nacher M, Sarazin F, El Guedj M, et al. Increased incidence of disseminated histoplasmosis following highly active antiretroviral therapy initiation. J Acquir Immune Defic Syndr. 2006; 41(4):468-470. Available at http://www.ncbi.nlm.nih.gov/entrez/query.fcgi? $\mathrm{cmd}=$ Retrieve $\& \mathrm{db}=$ PubMed\&dopt=Citation\&list_uids=16652055. [PubMed: 16652055$]$

31. Goldman M, Zackin R, Fichtenbaum CJ, et al. Safety of discontinuation of maintenance therapy for disseminated histoplasmosis after immunologic response to antiretroviral therapy. Clin Infect Dis. 2004 May 15; 38(10):1485-1489. Available at http://www.ncbi.nlm.nih.gov/pubmed/ 15156489. [PubMed: 15156489] 


\section{Dosing Recommendations for Preventing and Treating Histoplasmosis}

\begin{tabular}{|c|c|c|c|}
\hline Indication & First Choice & Alternative & Comments/Special Issues \\
\hline $\begin{array}{l}\text { Primary } \\
\text { Prophylaxis }\end{array}$ & N/A & N/A & $\begin{array}{l}\text { Primary Prophylaxis indicated for } \\
\text { selected HIV-infected adults but } \\
\text { not children. } \\
\text { Criteria for Discontinuing } \\
\text { Primary Prophylaxis: } \\
\qquad \quad \text { N/A } \\
\text { Criteria for Restarting Primary } \\
\text { Prophylaxis: } \\
\quad \text { N/A }\end{array}$ \\
\hline $\begin{array}{l}\text { Secondary } \\
\text { Prophylaxis } \\
\text { (Suppressive } \\
\text { Therapy) }\end{array}$ & $\begin{array}{l}\text { Itraconazole oral solution } 5-10 \\
\mathrm{mg} / \mathrm{kg} \text { body weight (maximum } \\
200 \mathrm{mg} \text { ) per dose by mouth } \\
\text { daily }\end{array}$ & $\begin{array}{l}\text { Fluconazole } 3-6 \mathrm{mg} / \mathrm{kg} \text { body } \\
\text { weight (maximum } 200 \mathrm{mg} \text { ) } \\
\text { by mouth once daily }\end{array}$ & 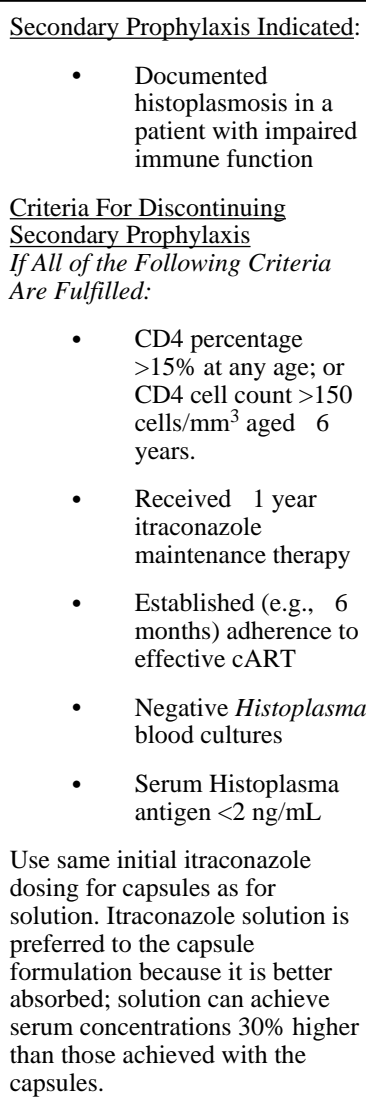 \\
\hline Treatment & $\begin{array}{ll}\text { Acute Primary Pulmonary } \\
\text { Histoplasmosis: } \\
\\
\text { Itraconazole oral } \\
\text { solution loading } \\
\text { dose of } 2-5 \mathrm{mg} / \mathrm{kg} \\
\text { body weight } \\
\text { (maximum } 200 \\
\text { mg) per dose by } \\
\text { mouth } 3 \text { times } \\
\text { daily for first } 3 \\
\text { days of therapy, } \\
\text { followed by } 2-5 \\
\text { mg/kg body weight } \\
\text { (max } 200 \text { mg) per } \\
\text { dose by mouth } \\
\text { twice daily for } 12 \\
\text { months. Duration } \\
\text { of } 12 \text { weeks is }\end{array}$ & 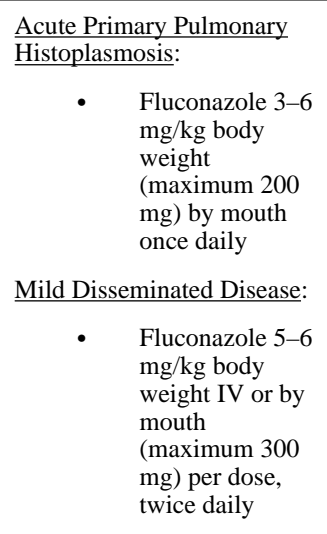 & $\begin{array}{l}\text { Use same initial itraconazole } \\
\text { dosing for capsules as for } \\
\text { solution. Itraconazole solution is } \\
\text { preferred to the capsule } \\
\text { formulation because it is better } \\
\text { absorbed; solution can achieve } \\
\text { serum concentrations } 30 \% \text { higher } \\
\text { than those achieved with the } \\
\text { capsules. } \\
\text { Urine antigen concentration } \\
\text { should be assessed at diagnosis. If } \\
>39 \text { ng/mL, serum concentrations } \\
\text { should be followed. When serum } \\
\text { levels become undetectable, urine } \\
\text { concentrations should be } \\
\text { monitored monthly during } \\
\text { treatment and followed thereafter } \\
\text { to identify relapse. }\end{array}$ \\
\hline
\end{tabular}




\begin{tabular}{|c|c|c|c|}
\hline Indication & First Choice & Alternative & Comments/Special Issues \\
\hline & 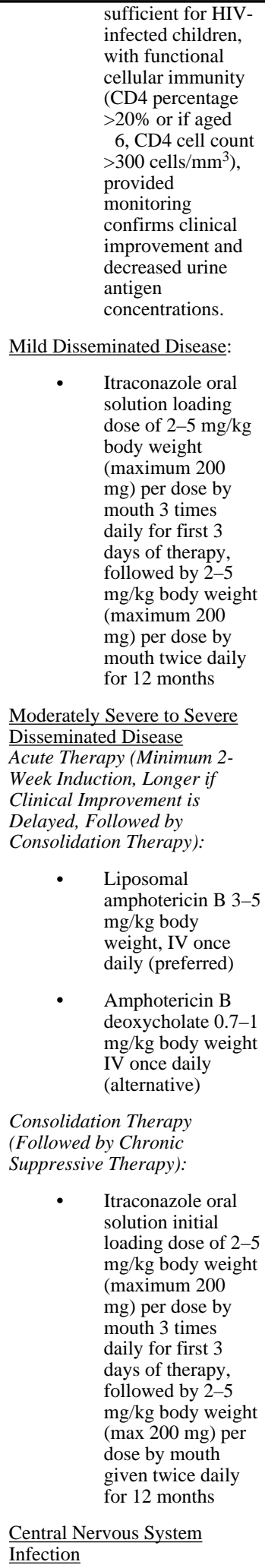 & $\begin{array}{ll} & \begin{array}{l}\text { (maximum 600 } \\
\text { mg/day) for 12 } \\
\text { months }\end{array} \\
\text { Moderately Severe to Severe } \\
\text { Disseminated Disease: } \\
\text { If itraconazole } \\
\text { not tolerated, } \\
\text { amphotericin } \\
\text { alone for 4-6 } \\
\text { weeks can be } \\
\text { used with } \\
\text { monitoring that } \\
\text { confirms decline } \\
\text { in histoplasma } \\
\text { urine and serum } \\
\text { antigen levels. } \\
\text { Liposomal } \\
\text { amphotericin B } \\
\text { 3-5 mg/kg body } \\
\text { weight IV once } \\
\text { daily (preferred) } \\
\text { for 4-6 weeks } \\
\text { Amphotericin B } \\
\text { deoxycholate } \\
0.7-1 \text { mg/kg } \\
\text { body weight IV } \\
\text { once daily } \\
\text { (alternative) for } \\
\text { 4-6 weeks }\end{array}$ & $\begin{array}{l}\text { Serum concentrations of } \\
\text { itraconazole should be monitored } \\
\text { and achieve a level of } 1 \mu \mathrm{g} / \mathrm{mL} \text { at } \\
\text { steady-state. Levels exceeding } 10 \\
\mu \mathrm{g} / \mathrm{mL} \text { should be followed by } \\
\text { dose reduction. } \\
\text { High relapse rate with CNS } \\
\text { infection occurs in adults and } \\
\text { longer therapy may be required; } \\
\text { treatment in children is anecdotal } \\
\text { and expert consultation should be } \\
\text { considered. } \\
\text { Chronic suppressive therapy } \\
\text { (secondary prophylaxis) with } \\
\text { itraconazole is recommended in } \\
\text { adults and children following } \\
\text { initial therapy. } \\
\text { Amphotericin B deoxycholate is } \\
\text { better tolerated in children than in } \\
\text { adults. Liposomal amphotericin B } \\
\text { is preferred for treatment of } \\
\text { parenchymal cerebral lesions. }\end{array}$ \\
\hline
\end{tabular}




\begin{tabular}{|c|c|c|c|}
\hline Indication & First Choice & Alternative & Comments/Special Issues \\
\hline & $\begin{array}{ll}\text { Acute Therapy (4-6 Weeks, } \\
\text { Followed by Consolidation } \\
\text { Therapy): } \\
\text { - } \\
\text { Liposomal } \\
\text { amphotericin B, } 5 \\
\text { mg/kg body weight } \\
\text { IV once daily (AII) } \\
\text { Consolidation Therapy } \\
\text { (Followed by Chronic } \\
\text { Suppressive Therapy): } \\
\text { - } \quad \text { Itraconazole oral } \\
\text { solution initial } \\
\text { loading dose of 2-5 } \\
\text { mg/kg body weight } \\
\text { (maximum } 200 \\
\text { mg) per dose by } \\
\text { mouth } 3 \text { times } \\
\text { daily for first } 3 \\
\text { days of therapy, } \\
\text { followed by } 2-5 \\
\text { mg/kg body weight } \\
\text { (max } 200 \text { mg) per } \\
\text { dose by mouth } \\
\text { given twice daily } \\
\text { for } \geq 12 \text { months and } \\
\text { until histoplasma } \\
\text { antigen is no longer } \\
\text { detected in } \\
\text { cerebrospinal fluid }\end{array}$ & & \\
\hline
\end{tabular}

Key to Acronyms: $\mathrm{cART}=$ combination antiretroviral therapy; $\mathrm{CD} 4=\mathrm{CD} 4 \mathrm{~T}$ lymphocyte; $\mathrm{CNS}=$ central nervous system; IV = intravenous

\section{Human Herpesvirus 8 Disease (Last updated November 6, 2013; last reviewed November 6, 2013)}

Panel's Recommendations

- $\quad$ Effective suppression of HIV replication with combination antiretroviral therapy (cART) is recommended to reduce the risk of human herpesvirus 8- (HHV-8) —associated Kaposi sarcoma (AIII).

- $\quad$ Routine testing to identify HHV-8 - seropositive, HIV-infected patients is not recommended (BIII).

- Effective suppression of HIV replication with cART is recommended for all patients with evidence of active KS and other human herpesvirus 8-associated malignant lymphoproliferative disorders (AIII).

- The use of intravenous ganciclovir or oral valganciclovir is recommended for treatment of HHV-8 associated multicentric Castleman disease (BIII) and may be a useful adjunct for treating HHV-8associated primary effusion lymphoma (BIII).

- $\quad$ Appropriate chemotherapy, in combination with potent cART, should be considered for patients with visceral KS or primary effusion lymphoma (BII*)

Rating of Recommendations: $\mathrm{A}=$ Strong; $\mathrm{B}=$ Moderate; $\mathrm{C}=$ Optional

Rating of Evidence: $\mathrm{I}=$ One or more randomized trials $\underline{\text { in children }}^{\dagger}$ with clinical outcomes and/or validated endpoints; I* $=$ One or more randomized trials in adults with clinical outcomes and/or validated laboratory endpoints with accompanying data in children ${ }^{\dagger}$ from one or more well-designed, nonrandomized trials or observational cohort studies with long-term clinical outcomes; II = One or more well-designed, nonrandomized trials or observational cohort studies in children ${ }^{\dagger}$ with long-term outcomes; II* = One or more well-designed, nonrandomized trials or observational studies in adults with longterm clinical outcomes with accompanying data in children ${ }^{\dagger}$ from one or more similar nonrandomized trials or cohort studies with clinical outcome data; III = Expert opinion

${ }^{\dagger}$ Studies that include children or children/adolescents, but not studies limited to post-pubertal adolescents. 


\section{Epidemiology}

Human herpesvirus 8 (HHV-8) is a transmissible DNA virus, with similarities in DNA structure to Epstein-Barr virus. HHV-8 has been causally linked to all forms of Kaposi sarcoma (KS) (HIV-related and endemic) and with two rare neoplastic conditions usually associated with HIV infection: body cavity-based lymphoma, also known as primary effusion lymphoma (a B-cell lymphoma that typically arises in body cavities such as the pleural space) and multicentric Castleman disease (non-cancerous tumors that may develop in lymph nodes in a single site or in multiple sites throughout the body). The exact mechanism by which HHV-8 infection leads to neoplastic disease has not been fully elucidated, but seroconversion to HHV-8 antibody positivity virtually always precedes development of the tumors. ${ }^{1}$ Higher plasma HHV-8 DNA titers are associated with increased risk of $\mathrm{KS}^{2}$

The prevalence of antibodies to HHV-8 varies widely with age and geography. In the United States and Europe, $1 \%$ to $3 \%$ of the general adult population is seropositive, with higher rates ( $8 \%$ ) among men who have sex with men (MSM). ${ }^{3}$ Among other adult men in the general population, HHV-8-seropositivity was marginally associated with duration of heterosexual activity and positively associated with the number of lifetime sex partners and co-infection with hepatitis B virus and herpes simplex virus type 2 viruses; none of these were significantly associated with risk for women. In contrast, the adult seropositivity rate in Mediterranean countries ranges from 10 to $25 \%$. In areas where HHV-8 is endemic, such as eastern and central Sub-Saharan Africa, HHV-8 seropositivity rates as high as $80 \%$ have been reported in adults. ${ }^{4-8}$

HHV-8 is transmitted through oral and genital secretions. Immunocompetent HHV-8infected adults frequently shed HHV-8 in their oropharyngeal secretions, with viral DNA detected in saliva on $22 \%$ of test days. ${ }^{9}$ In areas where HHV- 8 infection is endemic, the seroprevalence increases quickly during the first 5 years of life (especially when other family members are HHV-8-positive), then plateaus until adolescence and young adult years. In studies from rural Tanzania and Uganda, the rate of positivity for HHV-8 was 3.7\% to $16 \%$ among infants, $58 \%$ among children aged 4 to 5 years, and $49 \%$ to $89 \%$ among adults aged $>45$ years. ${ }^{10,11}$ The seroprevalence among infants and children increased with the number of HHV-8-positive parents and siblings in the home, indicating non-sexual transmission for pre-pubertal children, with a limited role for perinatal transmission. ${ }^{10-17}$

In the United States, among a cohort of HIV-infected and at-risk HIV-negative adolescents with a median age of 19 years, $11.2 \%$ were HHV-8 seropositive. ${ }^{18}$ The highest rates were in adolescent HIV-infected males reporting sex with males (23\%). Seropositivity was associated with HIV infection, MSM, a history of syphilis, and injection-drug use. ${ }^{18,19}$

HHV-8 can be transmitted through exposure to infected blood. Adult injection-drug users have an increased rate of HHV-8 positivity. ${ }^{18,19}$ In addition, recent evidence suggests that HHV-8 may be transmitted through blood product transfusions. In one study in an area of Uganda with a high incidence of HHV-8-seropositivity, the excess risk of acquiring HHV-8 through transfusion was nearly 3\% when recipients of HHV-8 antibody-positive blood were compared with those receiving HHV-8 antibody-negative blood. ${ }^{20}$ 
A small study suggested that maternal HHV-8 infection might increase risk for perinatal transmission of HIV, although no evidence of mother-to-child transmission of HHV-8 infection was identified among HIV-infected infants. ${ }^{15}$ Women coinfected with HHV-8 and HIV had increases in both HHV-8 and HIV viral load in serum and/or cervical fluid during pregnancy, when compared to their pre-pregnancy levels and to HHV-8 and HIV coinfected, non-pregnant women. ${ }^{21}$

For HIV-infected individuals, coinfection with HHV-8 places them at risk of KS. The risk is highest in adults (compared to children). Before the advent of combination antiretroviral therapy (cART), the overall incidence of KS in HIV-infected adults was as high as $20 \%$. However, the rate among children was low. In the United States and England, KS represented $<1 \%$ of pediatric AIDS-defining illnesses. The risk of KS is also highest in individuals with severe immunodeficiency. KS, primary effusion lymphoma, and multicentric Castleman disease can occur at any CD4 T lymphocyte (CD4) count level, but they are described most often in HIV-infected patients with more advanced immunosuppression (CD4 cell count $<200$ cells $/ \mathrm{mm}^{3}$ in adults).

The incidence of KS appeared to decline even before the widespread use of cART. The reason is unclear but may have been related to the use of other antiviral agents, such as those used to treat cytomegalovirus (CMV) (i.e., foscarnet, ganciclovir, and cidofovir), which may inhibit HHV-8. ${ }^{22-28}$ With the advent of earlier and more aggressive cART, the incidence of KS in adults has continued to decrease. In a well-characterized, HIV-infected adult cohort, the incidence fell from 33/1,000 person-years before 1996, to 5.1/1,000 personyears in 1996 to 1998 and 1.4/1,000 since 1999. Of note, the risk of KS decreased sharply after the first few months on cART and remained low for 7 to 10 years after the initiation of antiretroviral therapy. ${ }^{29,30}$

Although KS occurs primarily in adults, the incidence in children has increased substantially as a result of the HIV pandemic, particularly in Africa and because of frequent use of immunosuppressive drugs. One series reported a 40 -fold increase in incidence of childhood KS in Uganda in the era of AIDS; ${ }^{31}$ a 30 -fold increase was also noted recently in South Africa, ${ }^{32}$ where KS represented $5 \%$ of pediatric cancers. In a cohort of 6,530 HIV-infected children in Uganda, $1.7 \%$ were found to have a malignancy, $91 \%$ of which were $\mathrm{KS}^{33}$

\section{Clinical Manifestations}

A febrile illness with mild respiratory symptoms and a maculopapular rash and a mononucleosis-like illness have been associated with primary infection in young, immunocompetent children. ${ }^{34,35}$ A similar self-limited illness has been described in adults with primary infection. Evidence suggests more significant symptomatology in immunodeficient adults with primary infection, including reports of fever, arthralgia, splenomegaly, and bone marrow suppression. ${ }^{36,37}$

KS presentation varies widely, but most patients have non-tender, purplish, indurated skin lesions. Intraoral lesions can be seen and visceral dissemination can occur, occasionally without skin lesions. Multicentric Castleman disease presents with generalized adenopathy and fever and may progress to multiorgan failure. Primary effusion lymphoma presents with 
symptoms related to fluid accumulation in the pleural or pericardial space or with abdominal distention. In South Africa ${ }^{32}$ the average age at the time of diagnosis of KS in HIV-infected children was 72 months; median CD4 percentage and count at the time of diagnosis were $12 \%$ and 440 cells $/ \mu \mathrm{L}$, respectively. Just over half presented with skin lesions; $30 \%$ had adenopathy and 25\% had oral lesions. In a study in Mozambique, biopsy-proven KS was observed in $32 \mathrm{HIV}$-infected children $(0.8 \%$ of all HIV-infected children seen between 2003-2008); mean age was 8.3 years and median CD4 percentage was $16 \% .{ }^{38} \mathrm{KS}$ affected the lymph glands in 5 children, the skin in 10 children, and was mixed in 13 children (both cutaneous and nodal in 12 children and cutaneous and lung in 1 child). ${ }^{38}$

\section{Making the Diagnosis}

Laboratory diagnosis of HHV-8 infection is most commonly based on serologic assays, such as immunofluorescence, enzyme-linked immunosorbent assay, and Western blot. However, without a standard for diagnosing HHV-8 infection, these tests range in sensitivity from $80 \%$ to $\geq 90 \%$ and interassay agreement is poor. ${ }^{39}$ Combination assays containing both lytic and late-phase antigens may improve detection rates. Nucleic acid-based tests, such as in situ DNA hybridization and polymerase chain reaction (PCR), are important for tissue diagnosis. Although these tests have high levels of sensitivity, specificity and reproducibility are highly variable. Only $40 \%$ to $60 \%$ of patients with proven KS will have detectable HHV-8 DNA in their blood by PCR.

Laboratory diagnosis of KS is based on histologic examination of affected tissues.

\section{Prevention Recommendations}

Preventing Exposure-Routine testing of children and adults for HHV-8 is not recommended; therefore, the serostatus of HIV-infected patients usually is unknown. Although the efficacy of condoms in preventing HHV-8 exposure has not been established, HIV-infected patients should use male latex condoms correctly and consistently during sexual intercourse to reduce exposure to sexually transmitted pathogens. HIV-infected injection-drug users should be counseled not to share drug-injection equipment, even if both users are already HIV-infected, because of the risk of becoming infected with HHV-8 or other blood-borne pathogens. Adolescents who are diagnosed with KS should be counseled about the possibility of transmitting HHV-8 to their sexual contacts through intercourse and, possibly, kissing.

In the future, HHV-8 testing of donated blood products before use in immunodeficient patients may be considered. In addition, routine use of leukocyte reduction for red cell transfusions may lower the transmission risk.

Infants can acquire HHV-8 perinatally or through contact with infected family members and playmates. No effective intervention is known to prevent childhood acquisition of HHV-8, although avoidance of salivary exposure (e.g, via pre-mastication of food) may theoretically prevent transmission.

Preventing First Episode of Disease-The use of cART with suppression of HIV replication has markedly decreased the incidence of KS in HIV-infected adults (AIII). 
Routine testing to identify HHV-8-seropositive, HIV-infected individuals is not recommended (BIII). Although several antiviral agents (namely, ganciclovir, foscarnet, and cidofovir) inhibit HHV-8 replication in vitro, no data exist on their use to prevent KS in patients who are HIV/HHV-8 coinfected.

\section{Treatment Recommendations}

Treating Disease-Specific treatment recommendations are not included in this report because the HIV-related clinical entities associated with HHV-8, such as KS and Castleman disease, are oncologic and traditionally have been treated with cytotoxic chemotherapy. However, in HIV-infected patients with KS, effective suppression of HIV replication with cART may prevent KS progression or occurrence of new lesions. Therefore, cART is recommended for all HIV-infected patients with evidence of active KS and other HHV-8associated malignant lymphoproliferative disorders (AIII). Appropriate chemotherapy, in combination with potent cART, should be considered for patients with visceral KS or primary effusion lymphoma (BII*). A retrospective analysis of $28 \mathrm{HIV}$-infected children in Mozambique treated with cART and 6 cycles of monthly paclitaxel showed that treatment was well-tolerated and resulted in complete and sustained remission of KS in 19 children. ${ }^{38}$

In HIV-infected adults with KS, HHV-8 cellular viremia and higher viral load have been associated with disease progression. ${ }^{40}$ Treatment with specific antiviral agents that have in vitro activity against the lytic but not latent phase of HHV-8 (e.g., ganciclovir, foscarnet, cidofovir), has not been widely studied. No therapeutic effect was noted when oral valganciclovir was given to five HIV-negative adults with $\mathrm{KS} .{ }^{41}$ In addition, the vast majority of infected cells are not undergoing lytic replication, and anti-herpesvirus medications have had little or no effect on established KS or HHV-8 cellular viremia. Studies are under way of methods that induce lytic replication or attack the episomal (latent) HHV-8 genome. ${ }^{42,43}$

In contrast to KS, in Castleman disease, many of the cells support lytic replication of HHV-8, and treatment with anti-herpesvirus drugs has led to substantial clinical improvement in some studies. ${ }^{43}$ IV ganciclovir or oral valganciclovir is recommended for treating multicentric Castleman disease (BIII) ${ }^{44}$ and may be a useful adjunct for treating primary effusion lymphoma (BIII). ${ }^{45,46}$

Monitoring and Adverse Events (Including IRIS)—Rapid progression of KS after initiation of cART and after a change from a failing regimen to a more potent one has been reported, representing immune reconstitution inflammatory syndrome (IRIS) associated with immunologic improvement. IRIS-related progression of KS usually appeared within 8 weeks after start of a potent cART regimen. Most patients experienced rapid progression of cutaneous lesions; however, sudden worsening of pulmonary KS, with resultant deaths, was reported in at least 4 patients. All reported fatalities were linked to pulmonary KS. In most cases, cART was continued with stabilization and then regression of lesions. In more severe cases, especially those involving visceral lesions, chemotherapy was instituted, and in combination with cART, led to regression of the KS. ${ }^{47,48}$ 
For patients with disease manifestations of HHV-8 infection who are treated with ganciclovir or valganciclovir, refer to the chapter on CMV infections (Monitoring and Adverse Events) for information on treatment-associated adverse events.

Managing Treatment Failure-No recommendations exist for management of treatment failure.

Preventing Recurrence-Effective suppression of HIV replication with cART in HIVinfected patients with KS may prevent KS progression or occurrence of new lesions and is recommended for all individuals with evidence of active KS and other HHV-8-associated malignant lymphoproliferative disorders (AIII).

Discontinuing Secondary Prophylaxis—Not applicable.

\section{References}

1. Gao SJ, Kingsley L, Hoover DR, et al. Seroconversion to antibodies against Kaposi's sarcomaassociated herpesvirus-related latent nuclear antigens before the development of Kaposi's sarcoma. N Engl J Med. 1996 Jul 25; 335(4):233-241. Available at http://www.ncbi.nlm.nih.gov/pubmed/ 8657239. [PubMed: 8657239]

2. Lennette ET, Blackbourn DJ, Levy JA. Antibodies to human herpesvirus type 8 in the general population and in Kaposi's sarcoma patients. Lancet. 1996 Sep 28; 348(9031):858-861. Available at http://www.ncbi.nlm.nih.gov/pubmed/8826812. [PubMed: 8826812]

3. Engels EA, Atkinson JO, Graubard BI, et al. Risk factors for human herpesvirus 8 infection among adults in the United States and evidence for sexual transmission. J Infect Dis. 2007 Jul 15; 196(2): 199-207. Available at http://www.ncbi.nlm.nih.gov/pubmed/17570106. [PubMed: 17570106]

4. Whitby D, Smith NA, Matthews S, et al. Human herpesvirus 8: seroepidemiology among women and detection in the genital tract of seropositive women. J Infect Dis. 1999 Jan; 179(1):234-236. Available at http://www.ncbi.nlm.nih.gov/pubmed/9841845. [PubMed: 9841845]

5. Goedert JJ, Kedes DH, Ganem D. Antibodies to human herpesvirus 8 in women and infants born in Haiti and the USA. Lancet. 1997 May 10.349(9062):1368. Available at http:// www.ncbi.nlm.nih.gov/pubmed/9149705. [PubMed: 9149705]

6. Huang LM, Huang SY, Chen MY, et al. Geographical differences in human herpesvirus 8 seroepidemiology: a survey of 1,201 individuals in Asia. J Med Virol. 2000 Mar; 60(3):290-293. Available at http://www.ncbi.nlm.nih.gov/pubmed/10630961. [PubMed: 10630961]

7. Serraino D, Locatelli M, Songini M, et al. Human herpes virus-8 infection among pregnant women and their children: results from the Sardinia-IDDM Study 2. Int J Cancer. 2001 Mar 1; 91(5):740741. Available at http://www.ncbi.nlm.nih.gov/pubmed/11267990. [PubMed: 11267990]

8. Martin JN. The epidemiology of KSHV and its association with malignant disease. 2007 Available at http://www.ncbi.nlm.nih.gov/entrez/query.fcgi? $\mathrm{cmd}=$ Retrieve $\& \mathrm{db}=$ PubMed\&dopt=Citation\&list_uids $=21348075$.

9. Casper C, Krantz E, Selke S, et al. Frequent and asymptomatic oropharyngeal shedding of human herpesvirus 8 among immunocompetent men. J Infect Dis. 2007 Jan 1; 195(1):30-36. Available at http://www.ncbi.nlm.nih.gov/pubmed/17152006. [PubMed: 17152006]

10. Butler LM, Were WA, Balinandi S, et al. Human herpesvirus 8 infection in children and adults in a population-based study in rural Uganda. J Infect Dis. 2011 Mar 1; 203(5):625-634. Available at http://www.ncbi.nlm.nih.gov/pubmed/21273188. [PubMed: 21273188]

11. Mbulaiteye SM, Pfeiffer RM, Whitby D, Brubaker GR, Shao J, Biggar RJ. Human herpesvirus 8 infection within families in rural Tanzania. J Infect Dis. 2003 Jun 1; 187(11):1780-1785. Available at http://www.ncbi.nlm.nih.gov/pubmed/12751036. [PubMed: 12751036]

12. He J, Bhat G, Kankasa C, et al. Seroprevalence of human herpesvirus 8 among Zambian women of childbearing age without Kaposi's sarcoma (KS) and mother-child pairs with KS. J Infect Dis. 
1998 Dec; 178(6):1787-1790. Available at http://www.ncbi.nlm.nih.gov/pubmed/9815235. [PubMed: 9815235]

13. Gessain A, Mauclere $P$, van Beveren M, et al. Human herpesvirus 8 primary infection occurs during childhood in Cameroon, Central Africa. Int J Cancer. 1999 Apr 12; 81(2):189-192. Available at http://www.ncbi.nlm.nih.gov/pubmed/10188717. [PubMed: 10188717]

14. Sitas F, Newton R, Boshoff C. Increasing probability of mother-to-child transmission of HHV-8 with increasing maternal antibody titer for HHV-8. N Engl J Med. 1999 Jun 17.340(24):1923. Available at http://www.ncbi.nlm.nih.gov/pubmed/10375309. [PubMed: 10375309]

15. Calabro ML, Gasperini P, Barbierato M, et al. A search for human herpesvirus 8 (HHV-8) in HIV-1 infected mothers and their infants does not suggest vertical transmission of HHV-8. Int J Cancer. 2000 Jan 15; 85(2):296-297. Available at http://www.ncbi.nlm.nih.gov/pubmed/ 10629092. [PubMed: 10629092]

16. Plancoulaine S, Abel L, van Beveren M, et al. Human herpesvirus 8 transmission from mother to child and between siblings in an endemic population. Lancet. 2000 Sep 23; 356(9235):1062-1065. Available at http://www.ncbi.nlm.nih.gov/pubmed/11009141. [PubMed: 11009141]

17. Malope BI, Pfeiffer RM, Mbisa G, et al. Transmission of Kaposi sarcoma-associated herpesvirus between mothers and children in a South African population. J Acquir Immune Defic Syndr. 2007 Mar 1; 44(3):351-355. Available at http://www.ncbi.nlm.nih.gov/pubmed/17195763. [PubMed: 17195763]

18. Casper C, Meier AS, Wald A, Morrow RA, Corey L, Moscicki AB. Human herpesvirus 8 infection among adolescents in the REACH cohort. Arch Pediatr Adolesc Med. 2006 Sep; 160(9):937-942. Available at http://www.ncbi.nlm.nih.gov/pubmed/16953017. [PubMed: 16953017]

19. Cannon MJ, Dollard SC, Smith DK, et al. Blood-borne and sexual transmission of human herpesvirus 8 in women with or at risk for human immunodeficiency virus infection. $\mathrm{N}$ Engl J Med. 2001 Mar 1; 344(9):637-643. Available at http://www.ncbi.nlm.nih.gov/pubmed/11228278. [PubMed: 11228278]

20. Hladik W, Dollard SC, Mermin J, et al. Transmission of human herpesvirus 8 by blood transfusion. N Engl J Med. 2006 Sep 28; 355(13):1331-1338. Available at http://www.ncbi.nlm.nih.gov/ pubmed/17005950. [PubMed: 17005950]

21. Lisco A, Barbierato M, Fiore JR, et al. Pregnancy and human herpesvirus 8 reactivation in human immunodeficiency virus type 1-infected women. J Clin Microbiol. 2006 Nov; 44(11):3863-3871. Available at http://www.ncbi.nlm.nih.gov/pubmed/16943357. [PubMed: 16943357]

22. Glesby MJ, Hoover DR, Weng S, et al. Use of antiherpes drugs and the risk of Kaposi's sarcoma: data from the Multicenter AIDS Cohort Study. J Infect Dis. 1996 Jun; 173(6):1477-1480. Available at http://www.ncbi.nlm.nih.gov/pubmed/8648224. [PubMed: 8648224]

23. Mocroft A, Youle M, Gazzard B, Morcinek J, Halai R, Phillips AN. Anti-herpesvirus treatment and risk of Kaposi's sarcoma in HIV infection. Royal Free/Chelsea and Westminster Hospitals Collaborative Group. AIDS. 1996 Sep; 10(10):1101-1105. Available at http:// www.ncbi.nlm.nih.gov/pubmed/8874626. [PubMed: 8874626]

24. Cannon JS, Hamzeh F, Moore S, Nicholas J, Ambinder RF. Human herpesvirus 8-encoded thymidine kinase and phosphotransferase homologues confer sensitivity to ganciclovir. J Virol. 1999 Jun; 73(6):4786-4793. Available at http://www.ncbi.nlm.nih.gov/pubmed/10233939. [PubMed: 10233939]

25. Neyts J, De Clercq E. Antiviral drug susceptibility of human herpesvirus 8. Antimicrob Agents Chemother. 1997 Dec; 41(12):2754-2756. Available at http://www.ncbi.nlm.nih.gov/pubmed/ 9420052. [PubMed: 9420052]

26. Kedes DH, Ganem D. Sensitivity of Kaposi's sarcoma-associated herpesvirus replication to antiviral drugs. Implications for potential therapy. J Clin Invest. 1997 May 1; 99(9):2082-2086. Available at http://www.ncbi.nlm.nih.gov/pubmed/9151779. [PubMed: 9151779]

27. Robles R, Lugo D, Gee L, Jacobson MA. Effect of antiviral drugs used to treat cytomegalovirus end-organ disease on subsequent course of previously diagnosed Kaposi's sarcoma in patients with AIDS. J Acquir Immune Defic Syndr Hum Retrovirol. 1999 Jan 1; 20(1):34-38. Available at http://www.ncbi.nlm.nih.gov/pubmed/9928727. [PubMed: 9928727] 
28. Cannon MJ, Laney AS, Pellett PE. Human herpesvirus 8: current issues. Clin Infect Dis. 2003 Jul 1; 37(1):82-87. Available at http://www.ncbi.nlm.nih.gov/pubmed/12830412. [PubMed: 12830412]

29. Franceschi S, Maso LD, Rickenbach M, et al. Kaposi sarcoma incidence in the Swiss HIV Cohort Study before and after highly active antiretroviral therapy. Br J Cancer. 2008 Sep 2; 99(5):800804. Available at http://www.ncbi.nlm.nih.gov/pubmed/18665172. [PubMed: 18665172]

30. Ledergerber B, Egger M, Erard V, et al. AIDS-related opportunistic illnesses occurring after initiation of potent antiretroviral therapy: the Swiss HIV Cohort Study. JAMA. 1999 Dec 15; 282(23):2220-2226. Available at http://www.ncbi.nlm.nih.gov/pubmed/10605973. [PubMed: 10605973]

31. Ziegler JL, Katongole-Mbidde E. Kaposi's sarcoma in childhood: an analysis of 100 cases from Uganda and relationship to HIV infection. Int J Cancer. 1996 Jan 17; 65(2):200-203. Available at http://www.ncbi.nlm.nih.gov/pubmed/8567117. [PubMed: 8567117]

32. Stefan DC, Stones DK, Wainwright L, Newton R. Kaposi sarcoma in South African children. Pediatr Blood Cancer. 2011 Mar; 56(3):392-396. Available at http://www.ncbi.nlm.nih.gov/ pubmed/21225916. [PubMed: 21225916]

33. Tukei VJ, Kekitiinwa A, Beasley RP. Prevalence and outcome of HIV-associated malignancies among children. AIDS. 2011 Sep 10; 25(14):1789-1793. Available at http:// www.ncbi.nlm.nih.gov/pubmed/21673560. [PubMed: 21673560]

34. Chen RL, Lin JC, Wang PJ, Lee CP, Hsu YH. Human herpesvirus 8-related childhood mononucleosis: a series of three cases. Pediatr Infect Dis J. 2004 Jul; 23(7):671-674. Available at http://www.ncbi.nlm.nih.gov/pubmed/15247609. [PubMed: 15247609]

35. Andreoni M, Sarmati L, Nicastri E, et al. Primary human herpesvirus 8 infection in immunocompetent children. JAMA. 2002 Mar 13; 287(10):1295-1300. Available at http:// www.ncbi.nlm.nih.gov/pubmed/11886321. [PubMed: 11886321]

36. Luppi M, Barozzi P, Schulz TF, et al. Bone marrow failure associated with human herpesvirus 8 infection after transplantation. N Engl J Med. 2000 Nov 9; 343(19):1378-1385. Available at http:// www.ncbi.nlm.nih.gov/pubmed/11070102. [PubMed: 11070102]

37. Luppi M, Barozzi P, Rasini V, et al. Severe pancytopenia and hemophagocytosis after HHV-8 primary infection in a renal transplant patient successfully treated with foscarnet. Transplantation. 2002 Jul 15; 74(1):131-132. Available at http://www.ncbi.nlm.nih.gov/pubmed/12134112. [PubMed: 12134112]

38. Vaz P, Macassa E, Jani I, et al. Treatment of Kaposi sarcoma in human immunodeficiency virus-1infected Mozambican children with antiretroviral drugs and chemotherapy. Pediatr Infect Dis J. 2011 Oct; 30(10):891-893. Available at http://www.ncbi.nlm.nih.gov/pubmed/21730886. [PubMed: 21730886]

39. Bhaduri-McIntosh S. Human herpesvirus-8: clinical features of an emerging viral pathogen. Pediatr Infect Dis J. 2005 Jan; 24(1):81-82. Available at http://www.ncbi.nlm.nih.gov/pubmed/ 15665715. [PubMed: 15665715]

40. Laney AS, Cannon MJ, Jaffe HW, et al. Human herpesvirus 8 presence and viral load are associated with the progression of AIDS-associated Kaposi's sarcoma. AIDS. 2007 Jul 31; 21(12): 1541-1545. Available at http://www.ncbi.nlm.nih.gov/pubmed/17630548. [PubMed: 17630548]

41. Krown SE, Dittmer DP, Cesarman E. Pilot study of oral valganciclovir therapy in patients with classic Kaposi sarcoma. J Infect Dis. 2011 Apr 15; 203(8):1082-1086. Available at http:// www.ncbi.nlm.nih.gov/pubmed/21450998. [PubMed: 21450998]

42. Anderson LA, Goedert JJ. Tumor markers and treatments for Kaposi sarcoma. AIDS. 2007 Jul 31; 21(12):1637-1639. Available at http://www.ncbi.nlm.nih.gov/pubmed/17630560. [PubMed: 17630560]

43. Klass CM, Offermann MK. Targeting human herpesvirus-8 for treatment of Kaposi's sarcoma and primary effusion lymphoma. Curr Opin Oncol. 2005 Sep; 17(5):447-455. Available at http:// www.ncbi.nlm.nih.gov/pubmed/16093794. [PubMed: 16093794]

44. Casper C, Nichols WG, Huang ML, Corey L, Wald A. Remission of HHV-8 and HIV-associated multicentric Castleman disease with ganciclovir treatment. Blood. 2004 Mar 1; 103(5):1632-1634. Available at http://www.ncbi.nlm.nih.gov/pubmed/14615380. [PubMed: 14615380] 
45. Aboulafia DM. Interleukin-2, ganciclovir, and high-dose zidovudine for the treatment of AIDSassociated primary central nervous system lymphoma. Clin Infect Dis. 2002 Jun 15; 34(12):1660 1662. Available at http://www.ncbi.nlm.nih.gov/pubmed/12032910. [PubMed: 12032910]

46. Crum-Cianflone NF, Wallace MR, Looney D. Successful secondary prophylaxis for primary effusion lymphoma with human herpesvirus 8 therapy. AIDS. 2006 Jul 13; 20(11):1567-1569. Available at http://www.ncbi.nlm.nih.gov/pubmed/16847420. [PubMed: 16847420]

47. Leidner RS, Aboulafia DM. Recrudescent Kaposi's sarcoma after initiation of HAART: a manifestation of immune reconstitution syndrome. AIDS Patient Care STDS. 2005 Oct; 19(10): 635-644. Available at http://www.ncbi.nlm.nih.gov/pubmed/16232048. [PubMed: 16232048]

48. Bower M, Nelson M, Young AM, et al. Immune reconstitution inflammatory syndrome associated with Kaposi's sarcoma. J Clin Oncol. 2005 Aug 1; 23(22):5224-5228. Available at http:// www.ncbi.nlm.nih.gov/pubmed/16051964. [PubMed: 16051964]

\section{Human Papillomavirus (HPV) (Last updated November 6, 2013; last reviewed November 6, 2013)}

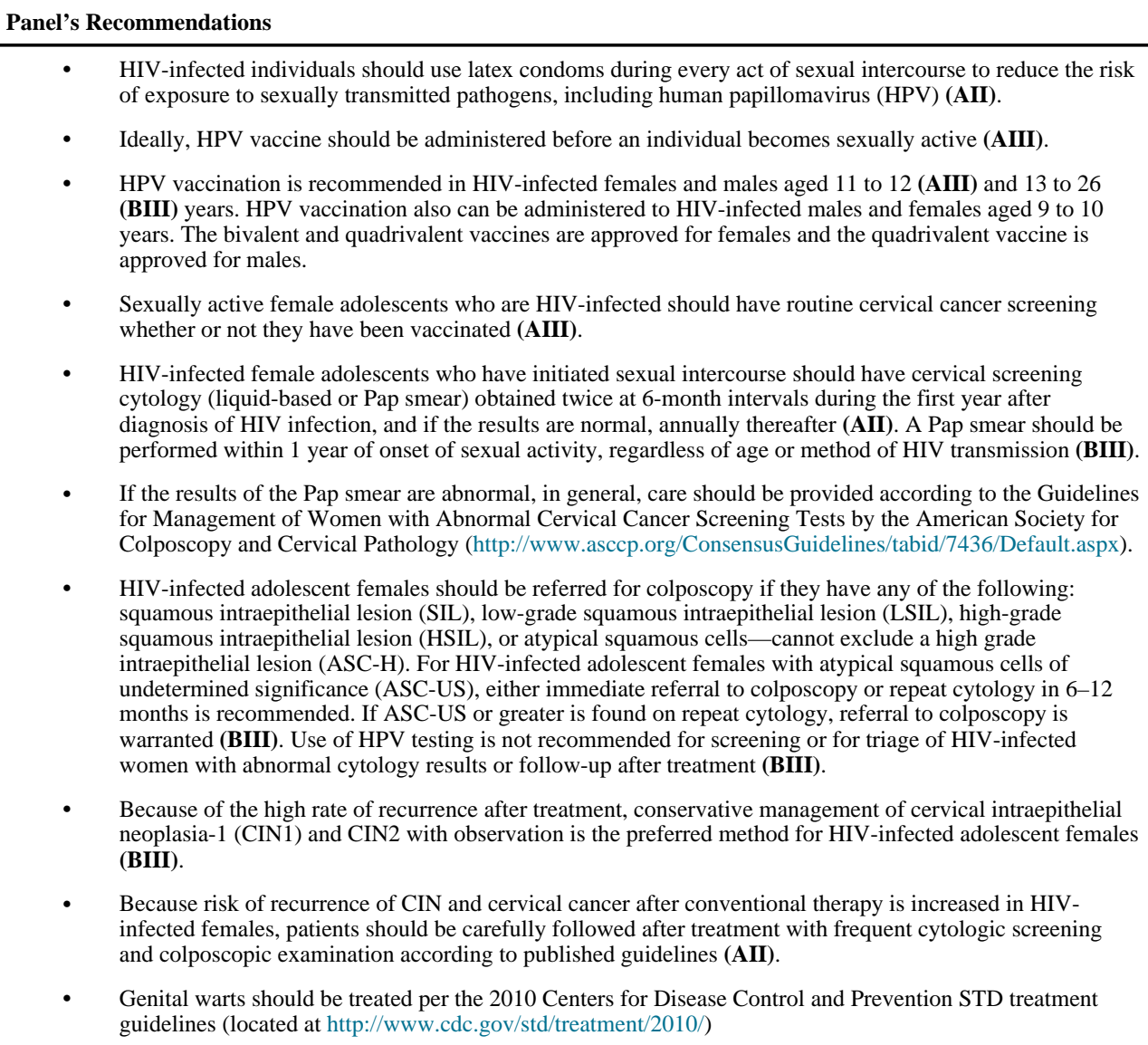

- $\quad$ HIV-infected individuals should use latex condoms during every act of sexual intercourse to reduce the risk of exposure to sexually transmitted pathogens, including human papillomavirus (HPV) (AII).

- Ideally, HPV vaccine should be administered before an individual becomes sexually active (AIII).

- HPV vaccination is recommended in HIV-infected females and males aged 11 to 12 (AIII) and 13 to 26 (BIII) years. HPV vaccination also can be administered to HIV-infected males and females aged 9 to 10 years. The bivalent and quadrivalent vaccines are approved for females and the quadrivalent vaccine is approved for males.

- Sexually active female adolescents who are HIV-infected should have routine cervical cancer screening whether or not they have been vaccinated (AIII).

- HIV-infected female adolescents who have initiated sexual intercourse should have cervical screening cytology (liquid-based or Pap smear) obtained twice at 6-month intervals during the first year after diagnosis of HIV infection, and if the results are normal, annually thereafter (AII). A Pap smear should be performed within 1 year of onset of sexual activity, regardless of age or method of HIV transmission (BIII).

- If the results of the Pap smear are abnormal, in general, care should be provided according to the Guidelines for Management of Women with Abnormal Cervical Cancer Screening Tests by the American Society for Colposcopy and Cervical Pathology (http://www.asccp.org/ConsensusGuidelines/tabid/7436/Default.aspx).

- $\quad$ HIV-infected adolescent females should be referred for colposcopy if they have any of the following: squamous intraepithelial lesion (SIL), low-grade squamous intraepithelial lesion (LSIL), high-grade squamous intraepithelial lesion (HSIL), or atypical squamous cells-cannot exclude a high grade intraepithelial lesion (ASC-H). For HIV-infected adolescent females with atypical squamous cells of undetermined significance (ASC-US), either immediate referral to colposcopy or repeat cytology in 6-12 months is recommended. If ASC-US or greater is found on repeat cytology, referral to colposcopy is warranted (BIII). Use of HPV testing is not recommended for screening or for triage of HIV-infected women with abnormal cytology results or follow-up after treatment (BIII).

- Because of the high rate of recurrence after treatment, conservative management of cervical intraepithelial neoplasia-1 (CIN1) and CIN2 with observation is the preferred method for HIV-infected adolescent females (BIII).

- Because risk of recurrence of CIN and cervical cancer after conventional therapy is increased in HIVinfected females, patients should be carefully followed after treatment with frequent cytologic screening and colposcopic examination according to published guidelines (AII).

- Genital warts should be treated per the 2010 Centers for Disease Control and Prevention STD treatment guidelines (located at http://www.cdc.gov/std/treatment/2010/)

Rating of Recommendations: $\mathrm{A}=$ Strong; $\mathrm{B}=$ Moderate $\mathrm{C}=$ Optional

Rating of Evidence: $\mathrm{I}=$ One or more randomized trials $\underline{\text { in children }}^{\dagger}$ with clinical outcomes and/or validated endpoints; $\mathrm{I}^{*}$ $=$ One or more randomized trials in adults with clinical outcomes and/or validated laboratory endpoints with accompanying data in children ${ }^{\dagger}$ from one or more well-designed, nonrandomized trials or observational cohort studies with long-term clinical outcomes; II = One or more well-designed, nonrandomized trials or observational cohort studies in children ${ }^{\dagger}$ with long-term outcomes; II* $=$ One or more well-designed, nonrandomized trials or observational studies in adults with longterm clinical outcomes with accompanying data in children ${ }^{\dagger}$ from one or more similar nonrandomized trials or cohort studies with clinical outcome data; III = Expert opinion 
${ }^{\dagger}$ Studies that include children or children/adolescents, but not studies limited to post-pubertal adolescents

\section{Epidemiology}

The majority of human papillomaviruses (HPV) fall predominantly into the alpha HPV genus. Alpha HPV infects cutaneous and mucosal squamous epithelium. More than 100 distinct types of alpha HPV exist. ${ }^{1}$ HPV can be detected on normal healthy mucosal and cutaneous surfaces but also is associated with warts and anogenital pre-cancers and cancers and oropharyngeal cancers in adults, and in rare cases, in adolescents and children. Certain types are found predominantly in cutaneous warts (such as HPV2) whereas other distinct mucosal types are associated with anogenital and oropharyngeal cancers. The mucosal HPV types found in cancers are referred to as high-risk and those not associated with cancers are referred to as low-risk types. Of the approximately 40-plus genital (i.e., mucosal) HPV types, 12 types have been established as high-risk $(16,18,31,33,35,39,45,51,52,56,58$, 59), and 6 as probable high-risk $(26,53,66,68,73,82) .{ }^{1} \mathrm{HPV} 16$ alone accounts for $50 \%$ of all squamous cell (SC) cervical cancers and $80 \%$ to $90 \%$ of all SC anal cancers. Of the HPV-associated vulvar, vaginal, penile, and oropharyngeal cancers, HPV16 is attributed to $50 \%$ to $80 \%$ as well. $^{2-4}$

Skin warts associated with HPV are common in children, ${ }^{5-7}$ whereas mucosal warts, including anogenital ${ }^{8,9}$ and oral warts, are less common. ${ }^{10}$

HPV-associated cutaneous warts are transmitted by close person-to-person contact that is facilitated by minor trauma to the skin. Skin warts are most commonly associated with cutaneous HPV types 1, 2, 3, 4, 27, and 57, and are associated with distinct wart histology. The estimated prevalence of skin warts in immunocompetent children varies by population from approximately $5 \%$ to $50 \%{ }^{5-7}$ In comparison, children with compromised cellular immunity often have intense and widespread appearance of both cutaneous and mucosal warts. Unfortunately, no data are available on prevalence or incidence of skin warts in HIVinfected children.

HPV-associated anogenital warts are known to be transmitted by sexual contact, thereby raising the concern of sexual abuse when diagnosed in pre-pubertal children. ${ }^{9,11}$ The prevalence of HPV-associated anogenital warts varies by population and risk factors, For example, varying prevalences of HPV-associated anogenital warts have been reported in children; $0 \%$ in non-abused pre-pubertal children, ${ }^{8} 1.7 / 1000$ in children referred to a tertiary care hospital ${ }^{9}$ and $1.8 \%$ in children with suspected sexual abuse. ${ }^{12}$ Several studies have shown that anogenital warts can be found in children with no evidence of sexual abuse, suggesting that transmission may occur through other means such as perinatally ${ }^{13}$ or through other non-sexual means (e.g., autoinoculation or transmission from the hands or mouth of a caretaker). ${ }^{14-16}$ HPV6 and 11 are the most common types detected in anogenital warts in children. ${ }^{17}$ In one study of children with anogenital warts, $24 \%$ of children had an adult family member with anogenital warts, $63 \%$ had a mother with cervical intraepithelial neoplasia (CIN), and $48 \%$ had a family member with extra-genital warts, ${ }^{18}$ suggesting nonsexual transmission as the route of infection. ${ }^{19}$ Rarely, cutaneous HPV types also have been associated with anogenital warts in children. ${ }^{20}$ Oral papillomas also have been described in children as well as sexually active adolescents and are commonly associated with HPV6 and 
11. Juvenile Onset Recurrent Respiratory Papillomatosis (JORRP), which is also associated with HPV6 and 11, can be life-threatening due to the ability of the lesions to cause airway obstruction. Incidence of JORRP in the United States is around 1.7 to 4.3 per 100,000.

Detection of HPV DNA in normal tissue of infants has been documented, suggesting that perinatal transmission also can occur. Rates of HPV DNA detected in newborns vary significantly (0\%-70\%), and when found in the infant, concordance between the mother and infant also is quite variable $(<1 \%-100 \%) .{ }^{21-23}$ Studies completed before 2000 tended to have higher rates of detection, whereas more recent studies find low rates of HPV DNA detected in infants ( $>5 \%)$. A systematic quantitative review of maternal-neonatal transmission concluded that pooled mother-to-child HPV transmission was around $6.5 \%{ }^{21}$ Several authors have suggested that the rate of HPV detection in infants depends on the rate found in pregnant mothers. ${ }^{22,24}$ Risks of DNA detection in newborns include mother's HPV status at delivery and presence of anogenital lesions (i.e., condyloma or squamous intraepithelial lesion [SIL]) in the mother. ${ }^{22,23}$ Recent studies have concluded that pregnancy itself, even in HIV-infected women, is not associated with increased vulnerability to HPV. ${ }^{25}$

In a recent study, $19.7 \%$ of infants born to HPV-infected mothers and $16.9 \%$ of infants born to mothers who were HPV-negative at delivery were found to be HPV-positive in their orogenital area at some time during a 14-month follow-up period, suggesting that vertical transmission is not the sole source of oral or genital HPV infection in infants. ${ }^{22}$ Although maternal history of condyloma at time of delivery has been a well-described risk factor for appearance of genital condyloma in infants months later, the risk remains quite low, with estimates of 7 per 1,000 births with a maternal history of genital warts. ${ }^{26}$ In a parent-child study in Finland, ${ }^{27}$ the cumulative detection rates for high-risk HPV from the child's genital and oral samples were $36 \%$ and $42 \%$, respectively. ${ }^{28}$ However, persistence of HPV was less common, with persistent oral HPV in 10\% of infants and persistent genital HPV in $1.5 \%$ of infants. Together, these data show that while oral and genital perinatal transmission can occur, persistence is unusual when infection is acquired (whether through vertical or horizontal transmission).

Genital HPV is most commonly a result of sexual transmission. Young age at first sexual intercourse and a higher number of recent sex partners are strong risk factors for HPV in both women and men. ${ }^{29-34}$ Prevalence of HPV is common in sexually active adolescent girls, with prevalence of $12 \%$ to $64 \%$, compared with $2 \%$ to $7 \%$ in women aged $>35$ years. ${ }^{32,35-37}$ Cervical HPV is acquired shortly after onset of sexual activity, with $50 \%$ cumulative exposure within 3 years, ${ }^{29,30}$ even among young women with one sex partner. ${ }^{38}$ Recent data on young men suggest similarly high rates of genital HPV acquisition associated with number of sexual partners. ${ }^{39}$ Rates of HPV are higher in HIV-infected adolescents and adult women than in HIV-uninfected women. ${ }^{40-42}$ As with HPV, CIN and condyloma also are more common in HIV-infected women than uninfected women. ${ }^{43-47}$

Although the incidence of anogenital HPV infection in sexually active youth is high, longitudinal studies have demonstrated that $80 \%$ to $90 \%$ of infections in HIV-uninfected youth are transient, and spontaneously regress. ${ }^{48,49}$ Repeated infections with new types are 
common ${ }^{49}$ but whether repeat detection of same-HPV-type infections result from new exposures or from reactivation of latent infection is unknown. ${ }^{50}$ Rates of clearance of genital HPV infection are even higher in men. ${ }^{39}$ Overall prevalence of HPV remains above $50 \%$ in men across all age groups, suggesting that repeated infections are even more common in men than in women. ${ }^{51} \mathrm{~A}$ risk for HPV in the anus in women is associated with anal intercourse. ${ }^{52,53}$ One study also showed that anal HPV acquisition was associated with cervical HPV infection and was quite common even without reported anal intercourse, suggesting that other sexual and non-sexual routes of anal acquisition are possible. ${ }^{53}$

The higher prevalence of HPV infections in HIV-infected populations may result partly from increased HPV persistence in these patients. In one study of adolescents with HIV, only $50 \%$ cleared their HPV infections. ${ }^{54}$ Detection of anal HPV also is higher in HIVinfected youth. ${ }^{55}$ Receptive anal sex is a risk factor for anal HPV in HIV-infected and HIVuninfected men; ${ }^{56}$ the association between anal HPV infection and anal sex is not as clear for women. ${ }^{55,57}$ In studies of HIV-infected and -uninfected women, anal HPV infection is equal to if not more prevalent than cervical infection. ${ }^{53,58}$

Persistent infection with high-risk HPV types is associated with increased risk of CIN and cervical and vulvovaginal carcinoma in women and of anal intraepithelial neoplasia (AIN) and anal carcinoma in both women and men. Rates of HPV-associated cancers including cervical, vulvar, vaginal, penile, anal (men and women), and oropharyngeal are higher in HIV-infected individuals ${ }^{59-61}$ and believed to result predominantly from the increased risk of persistent infection in this group. The rates are highest in HIV-infected young people. ${ }^{59}$ Adolescent girls, whether HIV-infected or -uninfected, differ biologically from adult women (e.g., increased areas of cervical squamous metaplasia in adolescents, resulting in an increased susceptibility to either persistent infection or disease). ${ }^{40,62}$

Even though combination antiretroviral therapy (cART) has dramatically altered HIV's natural history, its impact on HPV and HPV-associated neoplasia is less clear. Several studies have shown that HPV prevalence and rates of CIN and AIN have not been reduced with cART, ${ }^{54,63,64}$ in contrast to rates of Kaposi sarcoma, which have fallen dramatically since the advent of cART. Current data suggest that cervical cancer rates have decreased in most racial/ethnic groups, while anal cancer rates have increased in HIV-infected individuals. ${ }^{65}$

Other risks associated with increasing rates of cervical cancer include lack of cervical cancer screening, prolonged use of hormonal contraception, parity, smoking, and immunocompromising conditions (other than HIV) ${ }^{31} \mathrm{~A}$ recent study of perinatally infected adolescents showed that $30 \%$ of HIV-infected girls had an abnormal (atypical squamous cells of undetermined significance [ASC-US] or greater) Pap smear. ${ }^{66}$ The mean age at the time of the first Pap smear was 16.7 years (range 13-23 years). The observational study also noted that 23 cases of condyloma were reported in those younger than age 13. In a small study of Brazilian infants, HIV in the mother was noted to be a risk factor for neonatal transmission. ${ }^{24}$ These data suggest that perinatally infected children may be more vulnerable to maternal transmission of HPV, because of higher rates of HPV in this group, and higher rates of HPV persistence in the neonatal and infant period due to immunosuppression. 


\section{Clinical Manifestations}

Genital, Anal, Oral and Skin Warts-Genital HPV types cause hyperplastic, papillomatous, and verrucous squamous epithelial lesions (warts) on skin and mucus membranes, including anal, genital, oral, nasal, conjunctiva, gastrointestinal, bladder, and respiratory tract mucosa. Lesions in the genital area are often referred to as condyloma accuminata. Warts can be single or present with multiple lesions and often appear as papules, flat, smooth or pedunculated lesions. Common sites for skin warts are the hand, elbows, knees, and feet. JORRP can present with hoarseness and difficulty breathing.

Precancerous and Cancerous Lesions-Genital lesions associated with HPV include high grade CIN; vulvar intraepithelial neoplasia (VIN), vaginal intraepithelial neoplasia (VaIN), and AIN. Most intraepithelial neoplasias are asymptomatic. Cancers associated with high-risk HPV types include cervical, vulvar, vaginal, penile, anal, and oropharyngeal, specifically at the base of the tongue and tonsils. Cancers are often asymptomatic but also can be associated with bleeding, pain or a palpable mass.

\section{Diagnosis}

Genital, Anal, Oral and Skin Warts-Most cutaneous and anogenital warts can be diagnosed by visual inspection. A speculum examination may be required for cervical and vaginal lesions and anoscopy for intra-anal lesions. If the lesions do not respond to standard therapy or the warts are pigmented, indurated, fixed, or ulcerated, biopsy may be needed.

Patients in whom cancer or JORRP is suspected should be referred to an expert for diagnosis and management.

Intraepithelial and Squamous Cell Cancers-The same cytology and colposcopic techniques used to detect CIN in HIV-uninfected patients should be used in HIV-infected patients. Cytology is a screening test for cervical cancer (see Prevention section). However, histology remains the gold standard for confirming CIN and invasive cancers. In sexually active individuals, the entire genitalia and anal canal should be inspected carefully for visual signs of warts, intraepithelial neoplasia or invasive cancers. Vaginal, vulvar, and anal cancers often can be palpated by digital examination of the vaginal, vulvar, and intra-anal regions. Diagnosis is by histology; CIN, AIN, VaIN, VIN, and oral cancer are recognized through visual inspection, which includes colposcopy and high-resolution anoscopy (HRA), and biopsy to confirm diagnosis.

\section{Role of HPV Testing}

HPV DNA can be detected using several platforms. ${ }^{67} \mathrm{HPV}$ tests available can detect from 2 to 13 to 14 oncogenic HPV types in clinical specimens. Currently, data are insufficient for use of HPV testing in triage of HIV-infected women with abnormal cytology results or for follow-up after treatment (BIII), and it is not recommended for primary screening for any women younger than age 30. HPV testing also is not helpful in diagnosing or managing visible genital, skin or oral warts. HPV testing is not recommended in any circumstance for adolescent girls (aged <20 years), ${ }^{68}$ regardless of whether they are HIV-infected or HIVuninfected, because of the high rates of HPV infection. 


\section{Prevention Recommendations}

Preventing Exposure-HIV-infected individuals should use latex condoms during every act of sexual intercourse to reduce the risk of exposure to (or transmission of) sexually transmitted pathogens (AII). Condom use has been shown to reduce HPV genital acquisition, reduce risk of genital warts, and enhance clearance of CIN. ${ }^{33,69,70}$ This is true in both HIV-infected men and women. ${ }^{71}$ In all circumstances where a male condom cannot be used properly, the use of a female condom may be protective for vaginal intercourse (AII), but may not be protective for anal intercourse involving either women (BIII) or men who have sex with men (BIII). ${ }^{72,73}$

HPV Vaccine-The quadrivalent and bivalent vaccines have been shown to prevent HPV16 and 18 infections and associated precancers in females and the quadrivalent has been shown to prevent HPV16 and 18 infections and precancers in males. The quadrivalent vaccine also protects against HPV6 and 11 infections and associated genital warts in females and males. ${ }^{74-77}$ Because the HPV vaccine prevents infection and is not therapeutic, it ideally should be administered before potential exposure to HPV through sexual contact (AIII). Data from clinical trials ${ }^{75}$ of both vaccines showed that if previous exposure to the vaccine HPV types was documented, no efficacy was noted for that type, underscoring the fact that the vaccine is not therapeutic.

A randomized clinical trial of the quadrivalent HPV vaccine in the United States found the vaccine to be safe and immunogenic in HIV-infected children aged 8 to 11 years. ${ }^{78}$ Serum antibodies to HPV6 and 18 were $30 \%$ to $50 \%$ lower than in historic age-matched immunocompetent controls. In addition, at 18 months after the third dose of vaccine, $94 \%$ to 99\% had antibody to HPV6, 11, and 16, however, only $76 \%$ had antibody to HPV18. This group was also given a fourth dose which demonstrated an excellent amnestic response for all the vaccine associated HPV types. ${ }^{79}$ The clinical significance of this observation is unknown. Ongoing studies will continue to evaluate the efficacy and duration of immune response in HIV-infected boys and girls. Although no studies in HIV-infected adolescents and adult women have yet been published, a study in HIV-infected men found the vaccine to be safe and immunogenic. 80

Data on prior exposure to vaccine types in HIV-positive individuals aged 13 to 26 years are insufficient to determine the proportion that would benefit from vaccination.

HPV vaccination in HIV-infected youth is recommended (AIII). Either bivalent or quadrivalent HPV vaccine offers protection against the two most common types that are associated with HPV-associated genital cancers. Quadrivalent vaccine also offers protection against the two most common types that cause genital warts. Either the bivalent or quadrivalent HPV vaccine is recommended for routine vaccination of HIV-infected females aged 11 to 12 years; quadrivalent HPV vaccine is recommended for routine vaccination of HIV-infected males aged 11 to 12 years.

The first dose of the HPV vaccine series should be administered to males and females aged 11 to 12 years, but can be administered as early as age 9 years. The second dose should be administered 1 to 2 months after the first dose, and the third dose should be administered 6 
months after the first dose. HIV-infected adolescents aged 13 to 26 years who have not been previously vaccinated or have not completed the vaccine series should be vaccinated (see http://www.cdc.gov/mmwr/preview/mmwrhtml/mm6050a3.htm) (AIII).

\section{Preventing Disease}

Circumcision: There is evidence that circumcision reduces the rates of oncogenic HPV infection of the penis, ${ }^{81-85}$ and is associated with lower risk of penile cancer ${ }^{86,87}$ and cervical cancer in sexual partners. ${ }^{88}$ Because other studies suggest no benefit, ${ }^{89}$ evidence is insufficient to recommend adult male circumcision solely for the purpose of reducing the risk of oncogenic HPV infection in HIV-infected men or their sex partners in the United States, or infant male circumcision solely for the purpose of reducing the future risk of oncogenic HPV infection before or after they initiate sex.

Preventing Cervical Cancer-HIV-infected adolescents and women who have initiated sexual intercourse should have cervical screening cytology (liquid-based or Pap smear) obtained twice at 6-month intervals during the first year after diagnosis of HIV infection, and if the results are normal, annually thereafter (AII). Because of the reportedly high rate of progression of abnormal cytology in HIV-infected adolescents ${ }^{46}$ and young women who were infected through sexual intercourse, providers should consider screening within 1 year of onset of sexual activity, regardless of age or method of HIV acquisition (BIII). Although no similar prospective data are available for perinatally infected adolescents, Brogly et al ${ }^{66}$ reported that $30 \%$ of perinatally infected adolescents had an abnormality (ASCUS or greater ) on their first Pap smear. HIV-infected adolescents and women who have become sexually active, whether vaccinated or not, should continue screening annually throughout their lives (BIII). Evidence is insufficient to recommend cervical cancer screening in HIVinfected girls who are not sexually active.

If Pap smear results are abnormal, care should be provided according to the Guidelines for Management of Women with Abnormal Cervical Cancer Screening Tests by American Society for Colposcopy and Cervical Pathology. ${ }^{68}$ Exceptions include the role of HPV testing in women age 21 and older (see section HPV Testing above). It is recommended that triage be done in HIV-infected adolescents similar to that in adult women, in that any SIL, low-grade squamous intraepithelial lesion (LSIL), high-grade squamous intraepithelial lesion (HSIL), or atypical squamous cells cannot exclude a high-grade lesion (ASC-H) should be referred for colposcopy (BIII). For ASC-US, either immediate referral to colposcopy or repeat cytology in 6 to 12 months is recommended. Some clinicians may opt for colposcopy in HIV-infected adolescents/women. If ASC-US or greater is found on repeat cytology, referral to colposcopy is warranted.

Preventing Vaginal and Vulvar Cancer-No routine screening for vaginal or vulvar cancer is recommended for HIV-infected children and adolescents. Women with a history of high-grade CIN or invasive cervical cancer are at increased risk of vulvar and vaginal cancer and should be referred to a specialist (AIII). 
Preventing Anal Cancer-At this time, no national recommendations exist for routine screening for anal cancer; some specialists recommend anal cytologic screening for HIVseropositive men and women (CIII). ${ }^{69}$ An annual digital anal examination may be useful to detect on palpation masses that could be anal cancer (BIII). ${ }^{90}$ If anal cytology is performed and indicates ASC-US, ASC-H, LSIL, or HSIL, then it should be followed by HRA (BIII). Visible lesions should be biopsied to determine the level of histologic changes and to rule out invasive cancer (BIII) (see section on treatment for details of treatment of AIN).

\section{Treatment Recommendations Treating Disease}

Genital Warts: Multiple treatments for HPV-associated skin and external genital lesions exist, but no one treatment is ideal for all patients or all lesions (CIII). ${ }^{91}$ Treatment can induce wart-free periods, but the underlying viral infection can persist, resulting in recurrence. Treatment modalities for external genital warts are the same for HIV-infected and -uninfected populations. Guidelines for the treatment of warts found in the Centers for Disease Control and Prevention (CDC) Sexually Transmitted Diseases Treatment Guidelines, 2010, should be followed. ${ }^{92}$ Individuals who are immunosuppressed because of HIV may have larger or more numerous warts, and may not respond as well as immunocompetent individuals to therapy for genital warts. Recurrences after therapy also are an issue for these patients. ${ }^{92-95}$ Topical treatments may be ineffective in patients with large or extensive lesions. Self-applied therapies include podofilox $(0.5 \%)$ solution or gel, imiquimod (5\%) cream, and sinecatechin ointment. Provider-applied agents include trichloroacetic or bichloroacetic acid (TCA; BCA) (80\%-90\% aqueous solution).

Other treatments include intralesional interferon-alfa (IFN-a) or 5-fluorouracil [5-FU]/ epinephrine gel implant, and cidofovir topical gel (1\%). Cidofovir gel (1\%) is a topical preparation that has been evaluated in a limited number of adults for treatment of anogenital HPV infection (CIII). Topical cidofovir can be absorbed systemically and associated with renal toxicity. ${ }^{96}$ Injectable therapy (such as with IFN-a or 5-FU/epinephrine gel implant) should be offered in only severe recalcitrant cases because of inconvenient routes of administration, frequent office visits, and a high frequency of systemic adverse effects.

Lesions can be removed by cryotherapy or surgery (BIII). Cryotherapy (application of liquid nitrogen or dry ice) must be applied until each lesion is thoroughly frozen. Treatment can be repeated every 1 to 2 weeks up to 4 times. The major toxicity is local pain. Adequate local pain management is essential for all caustic treatments. Topical anesthetics are favored. Lesions can be removed surgically by tangential scissor, tangential shave excision, curettage, or electrosurgery.

Limited data are available on treatment of oral warts in HIV-infected patients. Limited lesions can be treated with provider-applied therapies such as TCA or BCA or surgical excision. Extensive lesions should be referred to an expert. ${ }^{97}$

Treatment of Histologically Confirmed CIN: HIV-infected female adolescents should be evaluated by a clinician with experience in colposcopy and treatment of cervical cancer 
precursors, and managed according to The American Society for Colposcopy and Cervical Pathology (ASCCP) guidelines. ${ }^{68}$ Not only is progression of lesions more common in HIVinfected women, recurrence is also more common, thus close observation as outlined in the CDC Sexually Transmitted Diseases Treatment Guidelines, 2010, should be considered for management of CIN1 and 2. Follow-up with annual cytologic assessment is recommended for adolescents with CIN1 (AII). ${ }^{68}$ At the 12-month follow-up, only adolescents with HSIL or greater on repeat cytology should be referred back to colposcopy. At the 24-month follow-up, those with an ASCUS or greater result should be referred back to colposcopy (AII).

For adolescent girls and young women with a histologic diagnosis of CIN2 or 3 not otherwise specified or cytologic diagnosis of HSIL, either treatment or observation for up to 24 months using both colposcopy and cytology at 6-month intervals is acceptable, provided colposcopy is satisfactory (BIII). ${ }^{68}$ When a histologic diagnosis of CIN2 is specified, observation is preferred, but treatment is acceptable. If compliance with follow-up is a concern, then treatment may be preferable for CIN2. When CIN3 is histologically diagnosed or when colposcopy is unsatisfactory, treatment is recommended (BIII).

If the colposcopic appearance of the lesion worsens or if HSIL cytology or a high-grade colposcopic lesion persists for 1 year, repeat biopsy is recommended (BIII). After 2 consecutive Negative for Intraepithelial Lesion or Malignancy results, adolescents and young women with normal colposcopy can return to routine cytologic screening (BII). Treatment is recommended if CIN3 is subsequently identified or if CIN2 or 3 persists for 24 months (BII).

Persistent CIN1, 2, and 3 lesions in HIV-infected women should be treated as in HIVuninfected women. ${ }^{68}$ Conventional therapies used to treat CIN2 or 3 include cryotherapy, laser therapy, cone biopsy, and a loop electrosurgical excision procedure (LEEP). Excisional methods are recommended for women with abnormal colposcopy and for women with recurrent disease (AII). Recurrence rates of $40 \%$ to $60 \%$ after treatment have been reported in HIV-infected women undergoing these procedures. ${ }^{98-100}$ Management of invasive cervical cancer should follow the National Comprehensive Cancer Network (NCCN) guidelines (http://www.nccn.org).

Treatment of VIN and Vulvar Cancer and of VaIN and Vaginal Cancer: Treatment of VIN/VaIN should be made in consultation with a specialist. Low-grade VIN/VaIN (VIN 1/ VAIN 1) can be observed or managed as per recommendations for vulvovaginal warts. Various treatment modalities for VIN are available, including TCA, local excision, laser vaporization or ablation, and imiquimod therapy. Treatment options for VaIN include topical 5-FU, laser vaporization with a $\mathrm{CO} 2$ laser, and excisional procedures with electrosurgical loops or a scalpel excision. Fluorouracil cream and ointments should not be used in pregnant women. Management of invasive vulvar or vaginal cancer should follow the NCCN guidelines (http://www.nccn.org).

Treatment of AIN: There are no adequate randomized, controlled, therapeutic trials reported for the treatment of AIN. Treatment decisions are based on size, location, and 
severity of histology. Several different treatments have been described in small open-label studies, including topical 5-FU or imiquimod, infrared coagulation, laser therapy, and surgical excision. ${ }^{101-104}$ These data do not indicate that treatment for HIV-infected women with AIN should be modified for patients receiving cART nor is there evidence indicating that cART should be instituted or modified for the purpose of treating AIN.

Treatment of HPV-associated disease at other sites, including oral and penile lesions, does not differ in HIV-infected versus uninfected men and women.

Role of Antiretroviral Therapy: Severe immunosuppression is associated with greater HPV-associated morbidity and mortality. However, studies show conflicting findings in reducing risk of HPV-related cervical and anal HPV disease, therefore, intraepithelial neoplasia by itself is not an indication for initiating cART.

Monitoring of Adverse Events (Including IRIS)—Monitoring for toxicity and recurrences is required during and after treatment of genital warts. The major toxicity of podofilox, imiquimod, and sinecatechin ointment is inflammation at the application site. The major toxicity of cryotherapy is local pain. The major toxicities of surgical treatment for genital warts are local pain, bleeding, and secondary infection. The major toxicities associated with acid cauterization are local pain and irritation or ulceration of adjacent normal skin. Intralesional IFN- $a$ can be associated with systemic toxicities of IFN-a, including fever, fatigue, myalgia, malaise, depression, and other influenza-like symptoms. Infrared coagulation may lead to bleeding and abscess formation. Scarring can occur with any of the above treatment modalities. Topical cidofovir may result in systemic absorption and be associated with renal toxicity. ${ }^{96}$

Secondary infections are not uncommon if ulcerations occur, and close monitoring posttreatment for treatment-related toxicity is warranted. Treatment of CIN with ablative and excisional modalities can be associated with several adverse events such as pain and discomfort, intraoperative hemorrhage, postoperative hemorrhage, infection, and cervical stenosis. Treatment of AIN is associated with adverse events, including ulcerations, abscesses, fissures, and fistulas.

An immune reconstitution-like syndrome related to HPV-associated oral warts in HIVinfected adults has been observed in which occurrence of oral warts was associated with decreased HIV RNA levels with cART. ${ }^{105}$ Immune reconstitution in response to viral load reduction may result in a return of marked inflammatory responses against latent oral HPV infection. Some studies, ${ }^{105,106}$ but not others, ${ }^{107}$ have reported an increase in oral warts following cART initiation.

Preventing Recurrence-Monitoring after therapy for cervical disease should follow the ASCCP guidelines. ${ }^{108}$ No recommendations exist for preventing recurrence of external genital warts. Patients should be monitored with cytologic screening according to published guidelines and, when indicated, colposcopic examination for recurrent lesions (AI).$^{90,109}$ 
Managing Treatment Failure-Treatment failure is defined as the persistence or recurrence of lesions after appropriate therapy. For persistent or recurrent genital warts, retreatment with any of the modalities previously described should be considered, preferably with an alternative modality to the one that previously failed (AIII). Genital warts often require more than one course of treatment. Recalcitrant warts should be managed by experienced clinicians and referred for excisional therapy. Recurrence of CIN may require additional treatments (e.g., LEEP, laser). Excisional therapy is recommended for recurrent lesions. Recurrent cytologic and histologic abnormalities after therapy for CIN should be managed according to the ASCCP guidelines. ${ }^{68}$ There is no consensus on the treatment of biopsy-proven recurrent VIN, VaIN or AIN. Risk of recurrence of CIN and cervical cancer after conventional therapy is increased in HIV-infected women, and patients should be carefully followed after treatment with frequent cytologic screening and colposcopic examination according to published guidelines (AII). ${ }^{99,110}$

\section{Discontinuing Secondary Prophylaxis-Not applicable.}

\section{References}

1. Munoz N, Castellsague X, de Gonzalez AB, Gissmann L. Chapter 1: HPV in the etiology of human cancer. Vaccine. 2006 Aug 31; 24(Suppl 3):S3/1-S3/10. Available at http://www.ncbi.nlm.nih.gov/ pubmed/16949995.

2. D'Souza G, Kreimer AR, Viscidi R, et al. Case-control study of human papillomavirus and oropharyngeal cancer. N Engl J Med. 2007 May 10; 356(19):1944-1956. Available at http:// www.ncbi.nlm.nih.gov/pubmed/17494927. [PubMed: 17494927]

3. Miralles-Guri C, Bruni L, Cubilla AL, Castellsague X, Bosch FX, de Sanjose S. Human papillomavirus prevalence and type distribution in penile carcinoma. J Clin Pathol. 2009 Oct; 62(10):870-878. Available at http://www.ncbi.nlm.nih.gov/pubmed/19706632. [PubMed: 19706632]

4. Smith JS, Backes DM, Hoots BE, Kurman RJ, Pimenta JM. Human papillomavirus type-distribution in vulvar and vaginal cancers and their associated precursors. Obstet Gynecol. 2009 Apr; 113(4): 917-924. Available at http://www.ncbi.nlm.nih.gov/pubmed/19305339. [PubMed: 19305339]

5. American Academy of Pediatrics. Red Book: 2012 Report of the Committee on Infectious Diseases. Elk Grove Village, IL: 2012.

6. Williams HC, Pottier A, Strachan D. The descriptive epidemiology of warts in British schoolchildren. Br J Dermatol. 1993 May; 128(5):504-511. Available at http:// www.ncbi.nlm.nih.gov/pubmed/8504040. [PubMed: 8504040]

7. van Haalen FM, Bruggink SC, Gussekloo J, Assendelft WJ, Eekhof JA. Warts in primary schoolchildren: prevalence and relation with environmental factors. Br J Dermatol. 2009 Jul; 161(1):148-152. Available at http://www.ncbi.nlm.nih.gov/pubmed/19438464. [PubMed: 19438464]

8. Gutman LT, Herman-Giddens ME, Phelps WC. Transmission of human genital papillomavirus disease: comparison of data from adults and children. Pediatrics. 1993 Jan; 91(1):31-38. Available at http://www.ncbi.nlm.nih.gov/pubmed/8416503. [PubMed: 8416503]

9. Marcoux D, Nadeau K, McCuaig C, Powell J, Oligny LL. Pediatric anogenital warts: a 7-year review of children referred to a tertiary-care hospital in Montreal, Canada. Pediatr Dermatol. 2006 May-Jun;23(3):199-207. Available at http://www.ncbi.nlm.nih.gov/pubmed/16780463. [PubMed: 16780463]

10. Pinheiro RS, de Franca TR, Rocha B, et al. Human papillomavirus coinfection in the oral cavity of HIV-infected children. J Clin Pathol. 2011 Dec; 64(12):1083-1087. Available at http:// www.ncbi.nlm.nih.gov/pubmed/21965827. [PubMed: 21965827] 
11. Sinclair KA, Woods CR, Kirse DJ, Sinal SH. Anogenital and respiratory tract human papillomavirus infections among children: age, gender, and potential transmission through sexual abuse. Pediatrics. 2005 Oct; 116(4):815-825. Available at http://www.ncbi.nlm.nih.gov/pubmed/ 16199688. [PubMed: 16199688]

12. Ingram DL, Everett VD, Lyna PR, White ST, Rockwell LA. Epidemiology of adult sexually transmitted disease agents in children being evaluated for sexual abuse. Pediatr Infect Dis J. 1992 Nov; 11(11):945-950. Available at http://www.ncbi.nlm.nih.gov/pubmed/1454437. [PubMed: 1454437]

13. Jones V, Smith SJ, Omar HA. Nonsexual transmission of anogenital warts in children: a retrospective analysis. The Scientific World Journal. 2007; 7:1896-1899. Available at http:// www.ncbi.nlm.nih.gov/pubmed/18060328.

14. Davis AJ, et al. HPV autoinoculation: A case report. J Ped Adol Gyn. 1989; 2(3):165-166. Available at http://www.journals.elsevierhealth.com/periodicals/jpgy/article/ S0932-8610(89)80009-X/abstract.

15. Fairley CK, Gay NJ, Forbes A, Abramson M, Garland SM. Hand-genital transmission of genital warts? An analysis of prevalence data. Epidemiol Infect. 1995 Aug; 115(1):169-176. Available at http://www.ncbi.nlm.nih.gov/pubmed/7641831. [PubMed: 7641831]

16. Rintala MA, Grenman SE, Puranen MH, et al. Transmission of high-risk human papillomavirus (HPV) between parents and infant: a prospective study of HPV in families in Finland. J Clin Microbiol. 2005 Jan; 43(1):376-381. Available at http://www.ncbi.nlm.nih.gov/pubmed/ 15634997. [PubMed: 15634997]

17. Gibson PE, Gardner SD, Best SJ. Human papillomavirus types in anogenital warts of children. J Med Virol. 1990 Feb; 30(2):142-145. Available at http://www.ncbi.nlm.nih.gov/pubmed/2156007. [PubMed: 2156007]

18. Handley J, Dinsmore W, Maw R, et al. Anogenital warts in prepubertal children; sexual abuse or not? Int J STD AIDS. 1993 Sep-Oct;4(5):271-279. Available at http://www.ncbi.nlm.nih.gov/ pubmed/8218514. [PubMed: 8218514]

19. Hernandez BY, Wilkens LR, Zhu X, et al. Transmission of human papillomavirus in heterosexual couples. Emerg Infect Dis. 2008 Jun; 14(6):888-894. Available at http://www.ncbi.nlm.nih.gov/ pubmed/18507898. [PubMed: 18507898]

20. Syrjanen S. Current concepts on human papillomavirus infections in children. APMIS. 2010 Jun; 118(6-7):494-509. Available at http://www.ncbi.nlm.nih.gov/pubmed/20553530. [PubMed: 20553530]

21. Medeiros LR, Ethur AB, Hilgert JB, et al. Vertical transmission of the human papillomavirus: a systematic quantitative review. Cad Saude Publica. 2005 Jul-Aug;21(4):1006-1015. Available at http://www.ncbi.nlm.nih.gov/pubmed/16021238. [PubMed: 16021238]

22. Castellsague X, Drudis T, Canadas MP, et al. Human Papillomavirus (HPV) infection in pregnant women and mother-to-child transmission of genital HPV genotypes: a prospective study in Spain. BMC Infect Dis. 2009; 9:74. Available at http://www.ncbi.nlm.nih.gov/pubmed/19473489. [PubMed: 19473489]

23. Smith EM, Parker MA, Rubenstein LM, Haugen TH, Hamsikova E, Turek LP. Evidence for vertical transmission of HPV from mothers to infants. Infect Dis Obstet Gynecol. 2010; 2010:326369. Available at http://www.ncbi.nlm.nih.gov/pubmed/20300545. [PubMed: 20300545]

24. Rombaldi RL, Serafini EP, Mandelli J, Zimmermann E, Losquiavo KP. Perinatal transmission of human papilomavirus DNA. Virol J. 2009; 6:83. Available at http://www.ncbi.nlm.nih.gov/ pubmed/19545396. [PubMed: 19545396]

25. Minkoff H, Shen X, Watts DH, et al. Relationship of pregnancy to human papillomavirus among human immunodeficiency virus-infected women. Obstet Gynecol. 2006 Oct; 108(4):953-960. Available at http://www.ncbi.nlm.nih.gov/pubmed/17012459. [PubMed: 17012459]

26. Silverberg M, Thorsen P, Lindeberg H, Grant LA, Shah KV. Condyloma in pregnancy is strongly predictive of juvenileonset recurrent respiratory papillomatosis. Obstet Gynecol. 2003 Apr; 101(4):645-652. 2003. Available at http://www.ncbi.nlm.nih.gov/pubmed/12681865. [PubMed: 12681865] 
27. Rintala M, Grenman S, Puranen M, Syrjanen S. Natural history of oral papillomavirus infections in spouses: a prospective Finnish HPV Family Study. J Clin Virol. 2006 Jan; 35(1):89-94. Available at http://www.ncbi.nlm.nih.gov/pubmed/16112613. [PubMed: 16112613]

28. Rintala MA, Grenman SE, Jarvenkyla ME, Syrjanen KJ, Syrjanen SM. High-risk types of human papillomavirus (HPV) DNA in oral and genital mucosa of infants during their first 3 years of life: experience from the Finnish HPV Family Study. Clin Infect Dis. 2005 Dec 15; 41(12):1728-1733. Available at http://www.ncbi.nlm.nih.gov/pubmed/16288396. [PubMed: 16288396]

29. Moscicki AB, Hills N, Shiboski S, et al. Risks for incident human papillomavirus infection and low-grade squamous intraepithelial lesion development in young females. JAMA. 2001 Jun 20; 285(23):2995-3002. Available at http://www.ncbi.nlm.nih.gov/pubmed/11410098. [PubMed: $11410098]$

30. Winer RL, Lee SK, Hughes JP, Adam DE, Kiviat NB, Koutsky LA. Genital human papillomavirus infection: incidence and risk factors in a cohort of female university students. Am J Epidemiol. 2003 Feb 1; 157(3):218-226. Available at http://www.ncbi.nlm.nih.gov/pubmed/12543621. [PubMed: 12543621]

31. Munoz N, Mendez F, Posso H, et al. Incidence, duration, and determinants of cervical human papillomavirus infection in a cohort of Colombian women with normal cytological results. J Infect Dis. 2004 Dec 15; 190(12):2077-2087. Available at http://www.ncbi.nlm.nih.gov/pubmed/ 15551205. [PubMed: 15551205]

32. Burchell AN, Winer RL, de Sanjose S, Franco EL. Chapter 6: Epidemiology and transmission dynamics of genital HPV infection. Vaccine. 2006 Aug 31; 24(Suppl 3):S3/52-S3/61. Available at http://www.ncbi.nlm.nih.gov/pubmed/16950018. [PubMed: 16950018]

33. Winer RL, Hughes JP, Feng Q, et al. Condom use and the risk of genital human papillomavirus infection in young women. N Engl J Med. 2006; 354(25):2645-2654. Available at http:// www.ncbi.nlm.nih.gov/sites/entrez?

Db=pubmed \&Cmd=ShowDetailView\&TermToSearch=16790697\&ordinalpos=1\&itool=EntrezSy stem2.PEntrez.Pubmed.Pubmed_ResultsPanel.Pubmed_RVDocSum. [PubMed: 16790697]

34. Forhan SE, Gottlieb SL, Sternberg MR, et al. Prevalence of sexually transmitted infections among female adolescents aged 14 to 19 in the United States. Pediatrics. 2009 Dec; 124(6):1505-1512. Available at http://www.ncbi.nlm.nih.gov/pubmed/19933728. [PubMed: 19933728]

35. Moscicki AB, Shiboski S, Broering J, et al. The natural history of human papillomavirus infection as measured by repeated DNA testing in adolescent and young women. J Pediatr. $1998 \mathrm{Feb}$; 132(2):277-284. Available at http://www.ncbi.nlm.nih.gov/pubmed/9506641. [PubMed: 9506641]

36. Tarkowski TA, Koumans EH, Sawyer M, et al. Epidemiology of human papillomavirus infection and abnormal cytologic test results in an urban adolescent population. J Infect Dis. 2004 Jan 1; 189(1):46-50. Available at http://www.ncbi.nlm.nih.gov/pubmed/14702152. [PubMed: 14702152]

37. Brown DR, Shew ML, Qadadri B, et al. A longitudinal study of genital human papillomavirus infection in a cohort of closely followed adolescent women. J Infect Dis. 2005 Jan 15; 191(2):182192. Available at http://www.ncbi.nlm.nih.gov/pubmed/15609227. [PubMed: 15609227]

38. Winer RL, Feng Q, Hughes JP, O'Reilly S, Kiviat NB, Koutsky LA. Risk of female human papillomavirus acquisition associated with first male sex partner. J Infect Dis. 2008 Jan 15; 197(2): 279-282. Available at http://www.ncbi.nlm.nih.gov/pubmed/18179386. [PubMed: 18179386]

39. Lu B, Wu Y, Nielson CM, et al. Factors associated with acquisition and clearance of human papillomavirus infection in a cohort of US men: a prospective study. J Infect Dis. 2009 Feb 1; 199(3):362-371. Available at http://www.ncbi.nlm.nih.gov/pubmed/19133808. [PubMed: 19133808]

40. Moscicki AB, Ellenberg JH, Vermund SH, et al. Prevalence of and risks for cervical human papillomavirus infection and squamous intraepithelial lesions in adolescent girls: impact of infection with human immunodeficiency virus. Arch Pediatr Adolesc Med. 2000 Feb; 154(2):127134. Available at http://www.ncbi.nlm.nih.gov/pubmed/10665598. [PubMed: 10665598]

41. Hagensee ME, Cameron JE, Leigh JE, Clark RA. Human papillomavirus infection and disease in HIV-infected individuals. Am J Med Sci. 2004 Jul; 328(1):57-63. Available at http:// www.ncbi.nlm.nih.gov/pubmed/15254442. [PubMed: 15254442]

42. Bollen LJ, Chuachoowong R, Kilmarx PH, et al. Human papillomavirus (HPV) detection among human immunodeficiency virus-infected pregnant Thai women: implications for future HPV 
immunization. Sex Transm Dis. 2006 Apr; 33(4):259-264. Available at http:// www.ncbi.nlm.nih.gov/pubmed/16452834. [PubMed: 16452834]

43. Delmas MC, Larsen C vBB, Hamers FF, Bergeron C, Poveda JD, et al. Cervical squamous intraepithelial lesions in HIV-infected women: prevalence, incidence and regression. European Study Group on Natural History of HIV Infection in Women. 14(12):1775-84. AIDS. 2000 Available at http://www.ncbi.nlm.nih.gov/pubmed/10985315.

44. Ahdieh L, Klein RS, Burk R, et al. Prevalence, incidence, and type-specific persistence of human papillomavirus in human immunodeficiency virus (HIV)-positive and HIV-negative women. J Infect Dis. 2001 Sep 15; 184(6):682-690. Available at http://www.ncbi.nlm.nih.gov/pubmed/ 11517428. [PubMed: 11517428]

45. Schuman P, Ohmit SE, Klein RS, Duerr A, Cu-Uvin S, Jamieson DJ, et al. Longitudinal study of cervical squamous intraepithelial lesions in human immunodeficiency virus (HIV)-seropositive and at-risk HIV-seronegative women. J Infect Dis. 2003 Jul 1; 188(1):128-136. 2003. Available at http://www.ncbi.nlm.nih.gov/pubmed/12825181. [PubMed: 12825181]

46. Moscicki AB, Ellenberg JH, Farhat S, Xu J. Persistence of human papillomavirus infection in HIVinfected and - uninfected adolescent girls: risk factors and differences, by phylogenetic type. J Infect Dis. 2004 Jul 1; 190(1):37-45. Available at http://www.ncbi.nlm.nih.gov/pubmed/ 15195241. [PubMed: 15195241]

47. Dolev JC, Maurer T, Springer G, et al. Incidence and risk factors for verrucae in women. AIDS. 2008 Jun 19; 22(10):1213-1219. Available at http://www.ncbi.nlm.nih.gov/pubmed/18525267. [PubMed: 18525267]

48. Ho GY, Bierman R, Beardsley L, Chang CJ, Burk RD. Natural history of cervicovaginal papillomavirus infection in young women. N Engl J Med. 1998 Feb 12; 338(7):423-428. Available at http://www.ncbi.nlm.nih.gov/pubmed/9459645. [PubMed: 9459645]

49. Moscicki AB, Ma Y, Jonte J, et al. The role of sexual behavior and human papillomavirus persistence in predicting repeated infections with new human papillomavirus types. Cancer Epidemiol Biomarkers Prev. 2010 Aug; 19(8):2055-2065. Available at http:// www.ncbi.nlm.nih.gov/pubmed/20696663. [PubMed: 20696663]

50. Strickler HD, Burk RD, Fazzari M, et al. Natural history and possible reactivation of human papillomavirus in human immunodeficiency virus-positive women. J Natl Cancer Inst. 2005 Apr 20; 97(8):577-586. Available at http://www.ncbi.nlm.nih.gov/pubmed/15840880. [PubMed: 15840880]

51. Giuliano AR, Lazcano-Ponce E, Villa LL, et al. The human papillomavirus infection in men study: human papillomavirus prevalence and type distribution among men residing in Brazil, Mexico, and the United States. Cancer Epidemiol Biomarkers Prev. 2008 Aug; 17(8):2036-2043. Available at http://www.ncbi.nlm.nih.gov/pubmed/18708396. [PubMed: 18708396]

52. Moscicki AB, Hills NK, Shiboski S, et al. Risk factors for abnormal anal cytology in young heterosexual women. Cancer Epidemiol Biomarkers Prev. 1999 Feb; 8(2):173-178. Available at http://www.ncbi.nlm.nih.gov/pubmed/10067816. [PubMed: 10067816]

53. Goodman MT, Shvetsov YB, McDuffie K, et al. Acquisition of anal human papillomavirus (HPV) infection in women: the Hawaii HPV Cohort study. J Infect Dis. 2008 Apr 1; 197(7):957-966. Available at http://www.ncbi.nlm.nih.gov/pubmed/18429348. [PubMed: 18429348]

54. Moscicki AB, Ellenberg JH, Crowley-Nowick P, Darragh TM, Xu J, Fahrat S. Risk of high-grade squamous intraepithelial lesion in HIV-infected adolescents. J Infect Dis. 2004 Oct 15; 190(8): 1413-1421. Available at http://www.ncbi.nlm.nih.gov/pubmed/15378433. [PubMed: 15378433]

55. Moscicki AB, Durako SJ, Houser J, et al. Human papillomavirus infection and abnormal cytology of the anus in HIV-infected and uninfected adolescents. AIDS. 2003 Feb 14; 17(3):311-320. Available at http://www.ncbi.nlm.nih.gov/pubmed/12556684. [PubMed: 12556684]

56. Nyitray AG, Smith D, Villa L, et al. Prevalence of and risk factors for anal human papillomavirus infection in men who have sex with women: a cross-national study. J Infect Dis. 2010 May 15; 201(10):1498-1508. Available at http://www.ncbi.nlm.nih.gov/pubmed/20367457. [PubMed: 20367457]

57. Palefsky J. HPV infection and HPV-associated neoplasia in immunocompromised women. Int J Gynaecol Obstet. 2006; 94(Suppl 1):S56-S64. Available at http://screening.iarc.fr/doc/HPV $\% 20$ supplement $\% 20-\% 20$ chapter\%2005.pdf. 
58. Palefsky JM, Holly EA, Ralston ML, Da Costa M, Greenblatt RM. Prevalence and risk factors for anal human papillomavirus infection in human immunodeficiency virus (HIV)-positive and highrisk HIV-negative women. J Infect Dis. 2001 Feb 1; 183(3):383-391. Available at http:// www.ncbi.nlm.nih.gov/pubmed/11133369. [PubMed: 11133369]

59. Frisch M, Biggar RJ, Goedert JJ. Human papillomavirus-associated cancers in patients with human immunodeficiency virus infection and acquired immunodeficiency syndrome. J Natl Cancer Inst. 2000 Sep 20; 92(18):1500-1510. Available at http://www.ncbi.nlm.nih.gov/pubmed/10995805. [PubMed: 10995805]

60. Patel P, Hanson DL, Sullivan PS, et al. Incidence of types of cancer among HIV-infected persons compared with the general population in the United States, 1992-2003. Ann Intern Med. May 20; 2008 148(10):728-736. Available at http://www.ncbi.nlm.nih.gov/pubmed/18490686. [PubMed: 18490686]

61. Chaturvedi AK, Madeleine MM, Biggar RJ, Engels EA. Risk of human papillomavirus-associated cancers among persons with AIDS. J Natl Cancer Inst. 2009 Aug 19; 101(16):1120-1130. Available at http://www.ncbi.nlm.nih.gov/pubmed/19648510. [PubMed: 19648510]

62. Moscicki AB, Burt VG, Kanowitz S, Darragh T, Shiboski S. The significance of squamous metaplasia in the development of low grade squamous intraepithelial lesions in young women. Cancer. 1999 Mar 1; 85(5):1139-1144. Available at http://www.ncbi.nlm.nih.gov/pubmed/ 10091799. [PubMed: 10091799]

63. Palefsky J, Holly EA, Efirdc JT, Da Costa M, Jay N, Berry JM, et al. Anal intraepithelial neoplasia in the highly active antiretroviral therapy era among HIV-positive men who have sex with men. 19(13):1407-14. AIDS. 2005 Available at http://www.ncbi.nlm.nih.gov/pubmed/16103772.

64. Shrestha S, Sudenga SL, Smith JS, Bachmann LH, Wilson CM, Kempf MC. The impact of highly active antiretroviral therapy on prevalence and incidence of cervical human papillomavirus infections in HIV-positive adolescents. BMC Infect Dis. 2010; 10:295. Available at http:// www.ncbi.nlm.nih.gov/pubmed/20946655. [PubMed: 20946655]

65. Jemal A, Simard EP, Dorell C, et al. Annual Report to the Nation on the Status of Cancer, 19752009, featuring the burden and trends in human papillomavirus(HPV)-associated cancers and HPV vaccination coverage levels. J Natl Cancer Inst. 2013 Feb 6; 105(3):175-201. Available at http:// www.ncbi.nlm.nih.gov/pubmed/23297039. [PubMed: 23297039]

66. Brogly SB, Watts DH, Ylitalo N, et al. Reproductive health of adolescent girls perinatally infected with HIV. Am J Public Health. 2007 Jun; 97(6):1047-1052. Available at http:// www.ncbi.nlm.nih.gov/pubmed/17463385. [PubMed: 17463385]

67. Arbyn M, Sasieni P, Meijer CJ, Clavel C, Koliopoulos G, Dillner J. Chapter 9: Clinical applications of HPV testing: a summary of meta-analyses. Vaccine. 2006 Aug 31; 24(Suppl 3):S3/78-S3/89. Available at http://www.ncbi.nlm.nih.gov/pubmed/16950021. [PubMed: 16950021]

68. Wright TC Jr, Massad LS, Dunton CJ, et al. 2006 consensus guidelines for the management of women with cervical intraepithelial neoplasia or adenocarcinoma in situ. Am J Obstet Gynecol. 2007 Oct; 197(4):340-345. Available at http://www.ncbi.nlm.nih.gov/pubmed/17904956. [PubMed: 17904956]

69. Hogewoning CJ, Bleeker MC, van den Brule AJ, et al. Condom use promotes regression of cervical intraepithelial neoplasia and clearance of human papillomavirus: a randomized clinical trial. Int J Cancer. 2003 Dec 10; 107(5):811-816. Available at http://www.ncbi.nlm.nih.gov/ pubmed/14566832. [PubMed: 14566832]

70. Nielson CM, Harris RB, Nyitray AG, Dunne EF, Stone KM, Giuliano AR. Consistent condom use is associated with lower prevalence of human papillomavirus infection in men. J Infect Dis. 2010 Aug 15; 202(3):445-451. Available at http://www.ncbi.nlm.nih.gov/pubmed/20569156. [PubMed: 20569156]

71. Fukuchi E, Sawaya GF, Chirenje M, et al. Cervical human papillomavirus incidence and persistence in a cohort of HIV-negative women in Zimbabwe. Sex Transm Dis. 2009 May; 36(5): 305-311. Available at http://www.ncbi.nlm.nih.gov/pubmed/19295468. [PubMed: 19295468]

72. French PP, Latka M, Gollub EL, Rogers C, Hoover DR, Stein ZA. Use-effectiveness of the female versus male condom in preventing sexually transmitted disease in women. Sex Transm Dis. 2003 
May; 30(5):433-439. Available at http://www.ncbi.nlm.nih.gov/pubmed/12916135. [PubMed: 12916135]

73. Kelvin EA, Smith RA, Mantell JE, Stein ZA. Adding the female condom to the public health agenda on prevention of HIV and other sexually transmitted infections among men and women during anal intercourse. Am J Public Health. 2009 Jun; 99(6):985-987. Available at http:// www.ncbi.nlm.nih.gov/pubmed/19372513. [PubMed: 19372513]

74. Garland SM, Hernandez-Avila M, Wheeler CM, et al. Quadrivalent vaccine against human papillomavirus to prevent anogenital diseases. N Engl J Med. 2007 May 10; 356(19):1928-1943. Available at http://www.ncbi.nlm.nih.gov/pubmed/17494926. [PubMed: 17494926]

75. Group FIS. Quadrivalent vaccine against human papillomavirus to prevent high-grade cervical lesions. N Engl J Med. 2007 May 10; 356(19):1915-1927. Available at http:// www.ncbi.nlm.nih.gov/pubmed/17494925. [PubMed: 17494925]

76. Paavonen J, Naud P, Salmeron J, et al. Efficacy of human papillomavirus (HPV)-16/18 AS04adjuvanted vaccine against cervical infection and precancer caused by oncogenic HPV types (PATRICIA): final analysis of a double-blind, randomised study in young women. Lancet. 2009 Jul 25; 374(9686):301-314. Available at http://www.ncbi.nlm.nih.gov/pubmed/19586656. [PubMed: 19586656]

77. Giuliano AR, Palefsky JM, Goldstone S, et al. Efficacy of quadrivalent HPV vaccine against HPV Infection and disease in males. N Engl J Med. 2011 Feb 3; 364(5):401-411. Available at http:// www.ncbi.nlm.nih.gov/pubmed/21288094. [PubMed: 21288094]

78. Levin MJ, Moscicki AB, Song LY, et al. Safety and immunogenicity of a quadrivalent human papillomavirus (types $6,11,16$, and 18) vaccine in HIV-infected children 7 to 12 years old. J Acquir Immune Defic Syndr. 2010 Oct; 55(2):197-204. Available at http://www.ncbi.nlm.nih.gov/ pubmed/20574412. [PubMed: 20574412]

79. Weinberg A, Song LY, Saah A, et al. Humoral, mucosal and cell-mediated immunity against vaccine and non-vaccine genotypes after administration of quadrivalent human papillomavirus vaccine to HIV-infected children. J Infect Dis. 2012 Aug 2. Available at http:// www.ncbi.nlm.nih.gov/pubmed/22859825.

80. Wilkin T, Lee JY, Lensing SY, et al. Safety and immunogenicity of the quadrivalent human papillomavirus vaccine in HIV-1-infected men. J Infect Dis. 2010 Oct 15; 202(8):1246-1253. Available at http://www.ncbi.nlm.nih.gov/pubmed/20812850. [PubMed: 20812850]

81. Auvert B, Sobngwi-Tambekou J, Cutler E, et al. Effect of male circumcision on the prevalence of high-risk human papillomavirus in young men: results of a randomized controlled trial conducted in Orange Farm, South Africa. J Infect Dis. 2009 Jan 1; 199(1):14-19. Available at http:// www.ncbi.nlm.nih.gov/pubmed/19086814. [PubMed: 19086814]

82. Giuliano AR, Lazcano E, Villa LL, et al. Circumcision and sexual behavior: factors independently associated with human papillomavirus detection among men in the HIM study. Int J Cancer. 2009 Mar 15; 124(6):1251-1257. Available at http://www.ncbi.nlm.nih.gov/pubmed/19089913. [PubMed: 19089913]

83. Tobian AA, Serwadda D, Quinn TC, et al. Male circumcision for the prevention of HSV-2 and HPV infections and syphilis. N Engl J Med. 2009 Mar 26; 360(13):1298-1309. Available at http:// www.ncbi.nlm.nih.gov/pubmed/19321868. [PubMed: 19321868]

84. Gray RH, Serwadda D, Kong X, et al. Male circumcision decreases acquisition and increases clearance of high-risk human papillomavirus in HIV-negative men: a randomized trial in Rakai, Uganda. J Infect Dis. 2010 May 15; 201(10):1455-1462. Available at http:// www.ncbi.nlm.nih.gov/pubmed/20370483. [PubMed: 20370483]

85. Serwadda D, Wawer MJ, Makumbi F, et al. Circumcision of HIV-infected men: effects on highrisk human papillomavirus infections in a randomized trial in Rakai, Uganda. J Infect Dis. 2010 May 15; 201(10):1463-1469. Available at http://www.ncbi.nlm.nih.gov/pubmed/20370481. [PubMed: 20370481]

86. Schoen EJ, Oehrli M, Colby C, Machin G. The highly protective effect of newborn circumcision against invasive penile cancer. Pediatrics. 2000 Mar.105(3):E36. Available at http:// www.ncbi.nlm.nih.gov/pubmed/10699138. [PubMed: 10699138] 
87. Daling JR, Madeleine MM, Johnson LG, et al. Penile cancer: importance of circumcision, human papillomavirus and smoking in in situ and invasive disease. Int J Cancer. 2005 Sep 10; 116(4): 606-616. Available at http://www.ncbi.nlm.nih.gov/pubmed/15825185. [PubMed: 15825185]

88. Castellsague X, Bosch FX, Munoz N, et al. Male circumcision, penile human papillomavirus infection, and cervical cancer in female partners. N Engl J Med. 2002 Apr 11; 346(15):1105-1112. Available at http://www.ncbi.nlm.nih.gov/pubmed/11948269. [PubMed: 11948269]

89. Dickson NP, Ryding J, van Roode T, et al. Male circumcision and serologically determined human papillomavirus infection in a birth cohort. Cancer Epidemiol Biomarkers Prev. 2009 Jan; 18(1): 177-183. Available at http://www.ncbi.nlm.nih.gov/pubmed/19124496. [PubMed: 19124496]

90. Goldie SJ, Kuntz KM, Weinstein MC, Freedberg KA, Welton ML, Palefsky JM. The clinical effectiveness and costeffectiveness of screening for anal squamous intraepithelial lesions in homosexual and bisexual HIV-positive men. JAMA. 1999 May 19; 281(19):1822-1829. Available at http://www.ncbi.nlm.nih.gov/pubmed/10340370. [PubMed: 10340370]

91. Beutner KR, Reitano MV, Richwald GA, Wiley DJ. External genital warts: report of the American Medical Association Consensus Conference. AMA Expert Panel on External Genital Warts. Clin Infect Dis. 1998 Oct; 27(4):796-806. Available at http://www.ncbi.nlm.nih.gov/pubmed/9798036. [PubMed: 9798036]

92. Workowski KA, Berman S. Centers for Disease C, Prevention. Sexually transmitted diseases treatment guidelines, 2010. MMWR Recomm Rep. 2010 Dec 17; 59(RR-12):1-110. Available at http://www.ncbi.nlm.nih.gov/pubmed/21160459. [PubMed: 21160459]

93. De Panfilis G, Melzani G, Mori G, Ghidini A, Graifemberghi S. Relapses after treatment of external genital warts are more frequent in HIV-positive patients than in HIV-negative controls. Sex Transm Dis. 2002 Mar; 29(3):121-125. Available at http://www.ncbi.nlm.nih.gov/pubmed/ 11875372. [PubMed: 11875372]

94. Silverberg MJ, Ahdieh L, Munoz A, et al. The impact of HIV infection and immunodeficiency on human papillomavirus type 6 or 11 infection and on genital warts. Sex Transm Dis. 2002 Aug; 29(8):427-435. Available at http://www.ncbi.nlm.nih.gov/pubmed/12172526. [PubMed: 12172526]

95. Conley LJ, Ellerbrock TV, Bush TJ, Chiasson MA, Sawo D, Wright TC. HIV-1 infection and risk of vulvovaginal and perianal condylomata acuminata and intraepithelial neoplasia: a prospective cohort study. Lancet. 2002 Jan 12; 359(9301):108-113. Available at http://www.ncbi.nlm.nih.gov/ pubmed/11809252. [PubMed: 11809252]

96. Bienvenu B, Martinez F, Devergie A, et al. Topical use of cidofovir induced acute renal failure. Transplantation. 2002 Feb 27; 73(4):661-662. Available at http://www.ncbi.nlm.nih.gov/pubmed/ 11889450. [PubMed: 11889450]

97. Baccaglini L, Atkinson JC, Patton LL, Glick M, Ficarra G, Peterson DE. Management of oral lesions in HIV-positive patients. Oral Surg Oral Med Oral Pathol Oral Radiol Endod. 2007 Mar; 103(Suppl):S50, e51-e23. Available at http://www.ncbi.nlm.nih.gov/pubmed/17379155. [PubMed: 17379155]

98. Reimers LL, Sotardi S, Daniel D, et al. Outcomes after an excisional procedure for cervical intraepithelial neoplasia in HIV-infected women. Gynecol Oncol. 2010 Oct; 119(1):92-97. Available at http://www.ncbi.nlm.nih.gov/pubmed/20605046. [PubMed: 20605046]

99. Wright TC Jr, Ellerbrock TV, Chiasson MA, Van Devanter N, Sun XW. Cervical intraepithelial neoplasia in women infected with human immunodeficiency virus: prevalence, risk factors, and validity of Papanicolaou smears. New York Cervical Disease Study. Obstet Gynecol. 1994 Oct; 84(4):591-597. Available at http://www.ncbi.nlm.nih.gov/pubmed/8090399. [PubMed: 8090399]

100. Ramchandani SM, Houck KL, Hernandez E, Gaughan JP. Predicting persistent/recurrent disease in the cervix after excisional biopsy. MedGenMed: Medscape general medicine. 2007; 9(2):24. Available at http://www.ncbi.nlm.nih.gov/pubmed/17955080. [PubMed: 17955080]

101. Scholefield JH. Treatment of grade III anal intraepithelial neoplasia with photodynamic therapy: report of a case. Dis Colon Rectum, 2003; 46(11):1555-1559. Tech Coloproctol. 2004 Nov.8(3): 200. Available at http://www.ncbi.nlm.nih.gov/pubmed/15654532. [PubMed: 15654532]

102. Webber J, Fromm D. Photodynamic therapy for carcinoma in situ of the anus. Arch Surg. 2004 Mar; 139(3):259-261. Available at http://www.ncbi.nlm.nih.gov/pubmed/15006881. [PubMed: 15006881] 
103. Goldstone SE, Kawalek AZ, Huyett JW. Infrared coagulator: a useful tool for treating anal squamous intraepithelial lesions. Dis Colon Rectum. 2005 May; 48(5):1042-1054. Available at http://www.ncbi.nlm.nih.gov/pubmed/15868241. [PubMed: 15868241]

104. Graham BD, Jetmore AB, Foote JE, Arnold LK. Topical 5-fluorouracil in the management of extensive anal Bowen's disease: a preferred approach. Dis Colon Rectum. 2005 Mar; 48(3):444450. Available at http://www.ncbi.nlm.nih.gov/pubmed/15747068. [PubMed: 15747068]

105. King MD, Reznik DA, O'Daniels CM, Larsen NM, Osterholt D, Blumberg HM. Human papillomavirus-associated oral warts among human immunodeficiency virus-seropositive patients in the era of highly active antiretroviral therapy: an emerging infection. Clin Infect Dis. 2002 Mar 1; 34(5):641-648. Available at http://www.ncbi.nlm.nih.gov/pubmed/11803508. [PubMed: 11803508]

106. Greenspan D, Canchola AJ, MacPhail LA, Cheikh B, Greenspan JS. Effect of highly active antiretroviral therapy on frequency of oral warts. Lancet. 2001 May 5; 357(9266):1411-1412. Available at http://www.ncbi.nlm.nih.gov/pubmed/11356441. [PubMed: 11356441]

107. Hamza OJ, Matee MI, Simon EN, et al. Oral manifestations of HIV infection in children and adults receiving highly active anti-retroviral therapy [HAART] in Dar es Salaam, Tanzania. BMC Oral Health. 2006; 6:12. Available at http://www.ncbi.nlm.nih.gov/pubmed/16916469. [PubMed: 16916469]

108. Massad LS, Einstein MH, Huh WK, et al. 2012 updated consensus guidelines for the management of abnormal cervical cancer screening tests and cancer precursors. Journal of lower genital tract disease. 2013 Apr; 17(5 Suppl 1):S1-S27. Available at http://www.ncbi.nlm.nih.gov/pubmed/ 23519301. [PubMed: 23519301]

109. Kurman RJ, Henson DE, Herbst AL, Noller KL, Schiffman MH. Interim guidelines for management of abnormal cervical cytology. The 1992 National Cancer Institute Workshop. JAMA. 1994 Jun 15; 271(23):1866-1869. Available at http://www.ncbi.nlm.nih.gov/pubmed/ 8196145. [PubMed: 8196145]

110. Fruchter RG, Maiman M, Sedlis A, Bartley L, Camilien L, Arrastia CD. Multiple recurrences of cervical intraepithelial neoplasia in women with the human immunodeficiency virus. Obstet Gynecol. 1996 Mar; 87(3):338-344. Available at http://www.ncbi.nlm.nih.gov/pubmed/8598951. [PubMed: 8598951]

\section{Dosing Recommendations for Prevention and Treatment of Human Papillomavirus (HPV)}

\begin{tabular}{|c|c|c|c|}
\hline Indication & First Choice & Alternative & Comments/Special Issues \\
\hline Primary Prophylaxis & HPV vaccine & N/A & $\begin{array}{l}\text { See Figure } 2 \text { for detailed } \\
\text { vaccine recommendations. }\end{array}$ \\
\hline Secondary Prophylaxis & $\mathrm{N} / \mathrm{A}$ & N/A & N/A \\
\hline Treatment & $\begin{array}{l}\text { Podofilox } \\
\text { solution/gel } \\
(0.5 \%) \text { applied } \\
\text { topically BID } \\
\text { for } 3 \\
\text { consecutive } \\
\text { days a week up } \\
\text { to } 4 \text { weeks } \\
\text { (patient } \\
\text { applied). } \\
\text { Withhold } \\
\text { treatment for } 4 \\
\text { days and repeat } \\
\text { the cycle } \\
\text { weekly up to } 4 \\
\text { times (BIII) } \\
\text { Imiquimod } \\
\text { cream (5\%) } \\
\text { applied } \\
\text { topically at } \\
\text { night and } \\
\text { washed off in }\end{array}$ & $\begin{array}{l}\text { - Intralesional } \\
\text { IFN-a is } \\
\text { generally not } \\
\text { recommended } \\
\text { because of high } \\
\text { cost, difficult } \\
\text { administration, } \\
\text { and potential } \\
\text { for systemic } \\
\text { side effects } \\
\text { (CIII) } \\
\text { Cidofovir } \\
\text { topical gel } \\
\text { (1\%) is an } \\
\text { experimental } \\
\text { therapy studied } \\
\text { in HIV-infected } \\
\text { adults that is } \\
\text { commercially } \\
\text { available } \\
\text { through } \\
\text { compounding }\end{array}$ & $\begin{array}{l}\text { Adequate topical anesthetics } \\
\text { to the genital area should be } \\
\text { given before caustic } \\
\text { modalities are applied. } \\
\text { Sexual contact should be } \\
\text { limited while solutions or } \\
\text { creams are on the skin. } \\
\text { Although sinecatechins } \\
\text { (15\% ointment) applied TID } \\
\text { up to } 16 \text { weeks is } \\
\text { recommended in } \\
\text { immunocompetent } \\
\text { individuals, data are } \\
\text { insufficient on safety and } \\
\text { efficacy in HIV-infected } \\
\text { individuals. } \\
\text { cART has not been } \\
\text { consistently associated with } \\
\text { reduced risk of HPV-related } \\
\text { cervical abnormalities in } \\
\text { HIV-infected women. } \\
\text { Laryngeal papillomatosis } \\
\text { generally requires referral to }\end{array}$ \\
\hline
\end{tabular}




\begin{tabular}{|c|c|c|c|}
\hline Indication & First Choice & Alternative & Comments/Special Issues \\
\hline & $\begin{array}{l}\text { the morning for } \\
3 \text { non- } \\
\text { consecutive } \\
\text { nights a week } \\
\text { for up to } 16 \\
\text { weeks (patient } \\
\text { applied) (BII) } \\
\text { TCA or BCA } \\
\text { (80\%-90\%) } \\
\text { applied } \\
\text { topically } \\
\text { weekly for up } \\
\text { to } 3 \text { to } 6 \text { weeks } \\
\text { (provider } \\
\text { applied) (BIII) } \\
\text { Podophyllin } \\
\text { resin (10\%- } \\
25 \% \\
\text { suspension in } \\
\text { tincture of } \\
\text { benzoin) } \\
\text { applied } \\
\text { topically and } \\
\text { washed off } \\
\text { several hours } \\
\text { later, repeated } \\
\text { weekly for } 3 \text { to } \\
6 \text { weeks } \\
\text { (provider } \\
\text { applied) (CIII) } \\
\text { Cryotherapy } \\
\text { with liquid } \\
\text { nitrogen or } \\
\text { cryoprobe } \\
\text { applied every } \\
\text { 1-2 weeks } \\
\text { (BIII) } \\
\text { Surgical } \\
\text { removal either } \\
\text { by tangential } \\
\text { excision, } \\
\text { tangential } \\
\text { shave excision, } \\
\text { curettage, or } \\
\text { electrosurgery }\end{array}$ & $\begin{array}{l}\text { pharmacies and } \\
\text { has very } \\
\text { limited use in } \\
\text { children; } \\
\text { systemic } \\
\text { absorption can } \\
\text { occur (CIII). } \\
\text { 5-FU/ } \\
\text { epinephrine gel } \\
\text { implant should } \\
\text { be offered in } \\
\text { only severe } \\
\text { recalcitrant } \\
\text { cases because } \\
\text { of inconvenient } \\
\text { routes of } \\
\text { administration, } \\
\text { frequent office } \\
\text { visits, and a } \\
\text { high frequency } \\
\text { of systemic } \\
\text { adverse effects. }\end{array}$ & $\begin{array}{l}\text { a pediatric otolaryngologist. } \\
\text { Treatment is directed at } \\
\text { maintaining the airway, } \\
\text { rather than removing all } \\
\text { disease. } \\
\text { For women who have } \\
\text { exophytic cervical warts, a } \\
\text { biopsy to exclude HSIL } \\
\text { must be performed before } \\
\text { treatment. } \\
\text { Liquid nitrogen or } \\
\text { TCA/BCA is recommended } \\
\text { for vaginal warts. Use of a } \\
\text { cryoprobe in the vagina is } \\
\text { not recommended. } \\
\text { Cryotherapy with liquid } \\
\text { nitrogen or podophyllin } \\
\text { resin (10\%-25\%) is } \\
\text { recommended for urethral } \\
\text { meatal warts. } \\
\text { Cryotherapy with liquid } \\
\text { nitrogen or TCA/BCA or } \\
\text { surgical removal is } \\
\text { recommended for anal } \\
\text { warts. } \\
\text { Abnormal Pap smear } \\
\text { cytology should be referred } \\
\text { to colposcopy for diagnosis } \\
\text { and management. }\end{array}$ \\
\hline
\end{tabular}

Key to Acronyms: 5-FU = 5-fluorouracil; $\mathrm{BCA}=$ bichloroacetic acid; $\mathrm{BID}=$ twice daily; $\mathrm{cART}=$ combination antiretroviral therapy; HPV = human papillomavirus; HSIL = high-grade squamous intraepithelial lesion; IFN- $\mathrm{a}=$ interferon alfa; $\mathrm{TCA}=$ trichloroacetic acid; $\mathrm{TID}=$ three times daily

Influenza (Last updated November 6, 2013; last reviewed November 6, 2013)

Panel's Recommendations

- The approach to evaluation and treatment of HIV-infected children on stable combination antiretroviral therapy with suspected or confirmed influenza should be similar to that of HIV-uninfected children (AIII). HIV-infected children with evidence of moderate-to-severe immunosuppression by CD4-defined or clinical disease-defined categories may be at increased risk of influenza-related complications and should be monitored closely until illness resolution (BII).

- $\quad$ Prevention of influenza in HIV-infected children aged 6 months and older should include annual administration of trivalent inactivated influenza vaccine, according to Advisory Committee on Immunization Practices recommendations (see annual updated recommendations at http://www.cdc.gov/ vaccines/pubs/acip-list.htm) (AII).

- Influenza-specific antiviral chemoprophylaxis should be considered for HIV-infected children based on level of immunosuppression and other preexisting co-morbidities, influenza vaccination status, and degree 
Panel's Recommendations

of exposure to suspected or confirmed influenza, according to CDC guidelines (http://www.cdc.gov/mmwr/ preview/mmwrhtml/rr6001a1.htm) (BII).

- $\quad$ HIV-infected children with confirmed influenza should be considered for prompt antiviral therapy, according to CDC guidelines (see http://www.cdc.gov/flu/antivirals/ and http://www.cdc.gov/mmwr/pdf/rr/ rr6001.pdf) (AII).

Rating of Recommendations: $\mathrm{A}=$ Strong; $\mathrm{B}=$ Moderate; $\mathrm{C}=$ Optional

Rating of Evidence: $\mathrm{I}=$ One or more randomized trials in children ${ }^{\dagger}$ with clinical outcomes and/or validated endpoints; I* $=$ One or more randomized trials in adults with clinical outcomes and/or validated laboratory endpoints with accompanying data in children ${ }^{\dagger}$ from one or more well-designed, nonrandomized trials or observational cohort studies with long-term clinical outcomes; II = One or more well-designed, nonrandomized trials or observational cohort studies in children ${ }^{\dagger}$ with long-term outcomes; II* $=$ One or more well-designed, nonrandomized trials or observational studies in adults with longterm clinical outcomes with accompanying data in children ${ }^{\dagger}$ from one or more similar nonrandomized trials or cohort studies with clinical outcome data; III = Expert opinion

$\dagger$

\section{Epidemiology}

Influenza viruses are spread directly from person to person across distances less than 6 feet via large or small droplets generated by coughing or sneezing or indirectly from contaminated surfaces to hands to mucosal membranes. ${ }^{1}$ Influenza has an incubation period of 1 to 4 days (mean: 2 days), ${ }^{2}$ and can be shed by adults from 1 day before to 5 to 10 days after onset of symptoms and by children from several days before to $\geq 10$ days after illness onset. ${ }^{3}$ Viral shedding can occur over longer periods of time in those with chronic diseases, including patients with immunologic suppression or those receiving systemic corticosteroid therapy. ${ }^{4-7}$

Seasonal influenza viruses can be divided into three types: A, B, and C. Influenza A viruses are further subdivided based on surface glycoproteins: hemagglutinin $(\mathrm{H})$ and neuraminidase (N). Influenza A viruses circulate primarily among aquatic birds, but also among humans and other animals, including pigs, horses, and seals. Influenza A subtypes H1N1 and H3N2 currently circulate among humans. Influenza B circulates primarily among humans. ${ }^{8}$ Influenza $\mathrm{C}$ circulates primarily among animals such as swine and dogs and rarely in humans. ${ }^{9,10}$ Influenza A and B cause seasonal outbreaks and impose a higher disease burden than influenza $\mathrm{C}$, which is associated with milder illness, sporadic cases, and rarely, localized outbreaks. Two influenza A subtypes and one influenza B strain are included in current seasonal influenza vaccines. Influenza viruses cause annual outbreaks in the United States lasting from winter through early spring.

Certain groups have been identified by the Centers for Disease Control and Prevention (CDC) to be at risk of complications from influenza, including individuals with immunosuppression caused by HIV infection. ${ }^{11}$ The burden of influenza virus in HIVinfected children has been characterized in limited case reports and case series, but assessment of its impact has been confounded by the stage of HIV infection, type of antiretroviral therapy (ART), and other comorbidities. ${ }^{12}$ In the era before combination antiretroviral therapy (cART), multiple large epidemiological studies suggested high hospitalization and mortality rates with influenza in HIV-infected individuals. ${ }^{13,14}$ However, 
observations reported during the cART era suggest that better control of HIV infection is associated with a milder course of influenza. In an outbreak of 2009 H1N1 influenza virus infection in Germany involving 15 HIV-infected schoolchildren receiving cART, the clinical course in HIV-infected children was similar to that in HIV-uninfected children. ${ }^{15}$ A case series of 13 HIV-infected children with 2009 H1N1 in Barcelona also reported outcomes similar to those in HIV-uninfected groups. ${ }^{16}$ In both reports, half of the children were aged $<13$ years, had CD4 counts $>500$ cells $/ \mathrm{mm}^{3}$, and very low or undetectable HIV viral loads. Existing data suggest that HIV-infected children receiving cART who have natural influenza infection develop influenza-specific antibody levels similar to those seen in HIV-uninfected children. ${ }^{15}$ Larger observational studies of HIV-infected children are needed to further substantiate these findings.

\section{Clinical Manifestations}

Signs and symptoms related to influenza are similar between HIV-infected and HIVuninfected children and include fever, cough, and rhinorrhea in the majority of patients. ${ }^{15-17}$ Loss of appetite was more common in HIV-infected patients than in HIV-uninfected patients in one study. ${ }^{18}$ In a prospective cohort study conducted in South Africa from 1997 to 1999, prior to cART availability, hospitalized HIV-infected children with laboratory-confirmed influenza had more radiographic evidence of alveolar consolidation when compared with HIV-uninfected children. However, other types of bacterial complications did not vary by HIV status. Clinical outcomes including duration of hospitalization and in-hospital mortality were similar for both HIV-infected and -uninfected groups. ${ }^{18,19}$ The differential diagnosis for pneumonia in an HIV-infected child during the influenza season includes primary influenza pneumonia or influenza complicated by secondary bacterial pneumonia with Streptococcus pneumoniae, Staphylococcus aureus, Streptococcus pyogenes, or Haemophilus influenzae, the most commonly identified bacterial co-pathogens. Consideration also must be given to other potential pathogens including Pneumocystis jirovecii, Mycobacterium tuberculosis, atypical mycobacteria, endemic mycoses, and other respiratory viruses, according to the child's level of immunosuppression, potential exposures, and local epidemiology.

\section{Diagnosis}

The laboratory approach to diagnosis of influenza in HIV-infected and -uninfected children is identical. This includes rapid influenza diagnostic tests (RIDTs), immunofluorescence assays, reverse transcription-polymerase chain reaction (RT-PCR) assays, and viral culture. RT-PCR and viral culture are considered the gold standard influenza tests. Viral culture results are not immediately available and culture is not as sensitive as RT-PCR. RIDTs offer point-of-care diagnosis, but sensitivity that is substantially lower than for viral culture or RT-PCR and false-positivity-particularly when prevalence is low-limit their reliability for patient management. ${ }^{20}$ Regardless of the method of laboratory diagnosis, clinical diagnosis with laboratory confirmation of influenza is important, especially in hospitalized patients and outpatients at higher risk of influenza complications. 


\section{Prevention Recommendations}

Preventing Exposure-Basic personal hygiene, including hand hygiene and proper cough etiquette, are mainstays of influenza prevention. Individuals should avoid touching their eyes, nose, and mouth and avoid contact with sick individuals. Hands should be washed often with soap and water or with alcohol-based hand rub containing at least $60 \%$ alcohol, if soap and water are unavailable. Proper hand washing technique involves wetting hands with clean running water, applying soap, and rubbing and scrubbing all hand surfaces and under the fingernails for at least 20 seconds. Hands should be dried with a clean towel or air dried. When using alcohol-based hand rub, the hand rub should be applied to one hand, and the hands (including all hand surfaces and fingers) should be rubbed together until dry.

Cough etiquette directs the individual to cough or sneeze into a tissue (preferred) or, when a tissue is unavailable, into the upper sleeve or elbow rather than into the hands. A soiled tissue should be disposed of in a waste basket. Other prevention methods include reducing crowding, maintaining a few feet of distance from others, avoiding shaking hands or hugging at gatherings, and avoiding gatherings altogether (see http://www.cdc.gov/flu/ protect/habits.htm, http://www.cdc.gov/handwashing, and http://www.cdc.gov/h1n1flu/ faithbased/factsheet2.htm).

Prolonged influenza viral shedding in a hospitalized immunocompromised patient has implications for preventing spread of influenza in the health care setting. Strategies to prevent the spread of influenza in health care facilities include use of droplet precautions by health care workers according to Healthcare Infection Control Practices Advisory Committee guidelines (see http://www.cdc.gov/hicpac). ${ }^{21}$

Preventing First Episode of Disease-Annual influenza vaccination is a cornerstone of influenza prevention, at the individual and community level. ${ }^{22}$ Past concerns about an increase in HIV viral load following influenza vaccination have not been substantiated, particularly in the presence of cART. ${ }^{11,23-27}$ Currently in the United States, trivalent inactivated influenza vaccine (TIV) is recommended for HIV-infected patients according to the CDC Advisory Committee on Immunization Practices (ACIP) recommendations. Multiple studies examining the immune response of HIV-infected children and adolescents on ART to TIV have shown immune responses comparable to those seen in HIV-uninfected individuals when matched for age and gender. ${ }^{26,28}$

ART to maintain adequate CD4 lymphocytes is the cornerstone of adequate immune responses to vaccine. ${ }^{29,30} \mathrm{In}$ a multivariate analysis of the immune response to TIV in HIVinfected children during the 1994-1995 influenza season, a higher pre-immunization CD4:CD8 ratio was associated with a greater antibody response. ${ }^{25}$ Multiple studies have shown that HIV-infected individuals with depleted T lymphocytes have a suboptimal immune response to vaccines. ${ }^{31-33} \mathrm{~A}$ randomized controlled trial of the immune response of HIV-infected children on cART to either TIV or live-attenuated influenza vaccine (LAIV) showed protective antibody titers after both vaccines (96\%-98\% for influenza A; 81\%-88\% for influenza B), with low baseline HIV viral loads correlating with improved antibody responses to both vaccines. ${ }^{34}$ Although LAIV is not licensed for use in HIV-infected 
children, many experts would consider using it on the basis of demonstrated safety and immunogenicity in HIV-infected children on cART without CD4-defined immunosuppression. ${ }^{35}$ Quadrivalent LAIV is Food and Drug Administration (FDA)approved and is anticipated to be available in upcoming influenza seasons. ACIP recommends TIV over LAIV for HIV-infected children. Household contacts (aged 6 months and older) of HIV-infected children should receive influenza vaccine annually as a means to help protect against influenza illness.

Contraindications to use of inactivated influenza vaccine are the same for HIV-infected and HIV-uninfected individuals. A physician should be consulted before flu vaccine is administered to children who have a severe allergy to chicken eggs, have had a severe reaction to influenza vaccine in the past, are less than 6 months of age (influenza vaccine is not approved for this age group), or who have a moderate-to-severe illness with a fever (in which case the child should be vaccinated after recovery).

Antiviral chemoprophylaxis according to current $\mathrm{CDC}$ guidelines ${ }^{36}$ is recommended for unvaccinated HIV-infected children who are close contacts of a person suspected of having or confirmed to have influenza. Selection of an antiviral drug for chemoprophylaxis should be based on current ACIP and CDC influenza antiviral recommendations and consider the antiviral susceptibility testing data for circulating influenza virus strains that can be obtained from the CDC (see http://www.cdc.gov/flu/weekly or http://gis.cdc.gov/grasp/fluview/ fluportaldashboard.html). Ideally, antiviral chemoprophylaxis should be started within 48 hours of exposure to a known influenza contact. Either oseltamivir or zanamivir, part of the antiviral class of medications called neuraminidase inhibitors, are approved and recommended for chemoprophylaxis against influenza A and B viruses. Oseltamivir prophylaxis is not FDA-approved for children aged $<1$ year, but the American Academy of Pediatrics and CDC have issued recommendations for prophylaxis of children 3 months of age and older; zanamivir prophylaxis is not recommended for children aged $<5$ years (see Table). Although oseltamivir resistance has been documented previously among circulating seasonal influenza A (H1N1) virus strains during the 2008-2009 influenza season, since September 2009, most (99\%) circulating influenza A and B viruses have been susceptible to oseltamivir. ${ }^{36}$ Amantadine and rimantadine, adamantane derivatives, are approved but not currently recommended for chemoprophylaxis of influenza A viruses because of resistance of current influenza A (H3N2 and $2009 \mathrm{H} 1 \mathrm{~N} 1)$ virus strains to adamantanes. ${ }^{36,37}$ Amantadine (generic) and rimantadine (Flumadine ${ }^{\circledR}$, generic) are approved to prevent only influenza A virus infection in people aged $>1$ year. Because chemoprophylaxis does not totally eliminate risk of influenza illness, children who develop fever and respiratory symptoms should be evaluated by a health care provider. ${ }^{36}$

Discontinuing Primary Prophylaxis-Duration of chemoprophylaxis is typically 10 days.

\section{Treatment Recommendations}

Treating Disease-Treatment of influenza in HIV-infected patients is recommended according to the ACIP and CDC guidelines. The recommended duration of treatment is 5 
days, but may need to be extended in severely ill hospitalized or immunocompromised patients. ${ }^{38}$ As with primary prophylaxis, selection of an antiviral drug for treatment should be based on current ACIP and CDC influenza antiviral recommendations and consider the antiviral susceptibility testing data for circulating influenza virus strains that can be obtained from the CDC (see http://www.cdc.gov/flu/weekly or http://gis.cdc.gov/grasp/fluview/ fluportaldashboard.html). Currently recommended influenza antiviral medications are the neuraminidase inhibitor drugs, oseltamivir (orally administered) and zanamivir (inhaled); both are effective for treatment against influenza A and B viruses. Oseltamivir is approved for treatment of influenza in children aged $\geq 2$ weeks. Although oseltamivir resistance was documented in circulating seasonal influenza A (H1N1) virus strains during the 2008-2009 influenza season, since September 2009, most (99\%) of circulating influenza A and B viruses have been susceptible to oseltamavir. ${ }^{36}$ Zanamivir is approved for treatment of influenza in children aged $\geq 7$ years (see Table). Alternative dosing of influenza-specific antiviral drugs and duration of therapy are under investigation. Amantadine and rimantadine, adamantane derivatives, are both effective treatments for influenza A viruses, but not influenza B viruses. Of the adamantanes, amantadine (generic) is approved to treat only influenza A viruses in people aged $>1$ year and rimantadine (Flumadine $®$; generic) is approved to treat only influenza A virus infections in people aged $\geq 13$ years. However, some pediatric influenza specialists may consider rimantadine appropriate for treatment of children aged $>1$ year. Adamantanes are not currently recommended for treatment of influenza A because of resistance of current influenza A (H3N2 and 2009 H1N1) strains. 36,37

A fundamental feature of influenza in the immunocompromised host is prolonged influenza viral shedding from the respiratory tract, which may persist weeks to months in an individual with poorly controlled HIV infection. ${ }^{39,40}$ However, patients with well-controlled HIV on cART will have diminished influenza viral shedding, with further attenuation with antiviral treatment. During the influenza pandemic of 2009, 2009 H1N1 viral RNA was detectable by RT-PCR for a median of 4 days (range 4-5) in 5 HIV-infected, oseltamivirtreated children, compared with a median of 8 days (range 8-13) in 10 HIV-infected, oseltamivir-untreated children. ${ }^{15}$

Monitoring of Adverse Events including IRIS—Clinicians should take into account patients' age, weight, renal function, history of seizures, level of immunosuppression, presence of other medical conditions, and potential drug interactions when considering administration of influenza antiviral medications. ${ }^{36}$

Zanamivir: Because of cases of respiratory deterioration manifested as decreased forced expiratory volume or bronchospasm in patients with asthma or chronic obstructive pulmonary disease, zanamivir is not recommended for treatment of patients with underling pulmonary disease. In clinical treatment studies involving patients with uncomplicated influenza, common adverse events were similar in those treated with inhaled zanamivir and those treated with inhaled placebo. ${ }^{36,38}$

Oseltamivir: For patients with creatinine clearance $10-30 \mathrm{~mL} / \mathrm{min}$, a reduction in chemoprophylaxis dosage to $75 \mathrm{mg}$ every other day and in treatment dosage to $75 \mathrm{mg}$ once 
daily is recommended. Pharmacokinetic (PK) data are limited for dosing recommendations for patients with severe renal insufficiency on dialysis. In studies of adults and children, mild nausea and vomiting has been the most common side effect of treatment with oseltamivir; ${ }^{41,42}$ however, that can be somewhat alleviated if the medication is taken with food. ${ }^{43}$ Despite earlier post-market reports of transient neuropsychiatric events manifested as self-injury or delirium in Japan, ${ }^{44}$ more recently, oseltamivir has not been associated with increased risk of neuropsychiatric events. ${ }^{45}$ The FDA recommends close monitoring for abnormal behavior in patients treated with oseltamivir. ${ }^{43}$ The FDA and CDC also recommend that clinicians and pharmacists pay careful attention to the possibility of dosing errors in young children. ${ }^{46}$

Amantadine: For patients with creatinine clearance $<50 \mathrm{~mL} / \mathrm{min}$, the following dosage frequency reductions are recommended: 30 to $50 \mathrm{~mL} / \mathrm{min}$ - once daily; 15 to $29 \mathrm{~mL} / \mathrm{min}$ once every 48 hours; $<15 \mathrm{~mL} / \mathrm{min}$ - once weekly. These patients should be observed carefully for adverse reactions that would necessitate a further decrease in dose. ${ }^{36,47}$ Because an increased incidence of seizures has been noted in patients with a history of seizure disorders exposed to amantadine, such patients should be observed for increased seizure activity when given the drug. ${ }^{36,48}$

Rimantadine: For patients with creatinine clearance $<10 \mathrm{~mL} / \mathrm{min}$, a reduction of dosage to $100 \mathrm{mg} /$ day is recommended. Patients with any degree of renal insufficiency, including older individuals, should be carefully observed for adverse reactions necessitating a further decrease in dose. Also, in patients with severe hepatic dysfunction, a reduction in dosage to $100 \mathrm{mg} /$ day is recommended. ${ }^{36,49}$ Seizure or seizure-like activity was seen in patients taking rimantadine who had a history of seizure disorders and were not taking anticonvulsants. ${ }^{50}$

Patients aged $\leq 8$ years with suspected influenza should not be given aspirin or aspirincontaining products such as bismuth subsalicylate (Pepto Bismol) because of the risk of Reye syndrome. In such cases, it is recommended that fever be treated with other antipyretics such as acetaminophen or non-steroidal antiinflammatory medications. ${ }^{51}$

Drug Interactions: Clinical data are limited with respect to drug interactions between zanamivir or oseltamivir and antiretroviral drugs, and no clinical trials to date have evaluated the safety or efficacy of using combinations of different classes of influenza antiviral drugs. ${ }^{36}$ However, information derived from pharmacology and PK studies of oseltamivir suggests that clinically significant drug interactions are unlikely, and zanamivir is not a substrate nor does it affect cytochrome P450 (CYP450) isoenzymes; no clinically significant drug interactions are predicted based on in vitro studies.

Managing Treatment Failure-Clinicians developing management plans in response to treatment failure or severe illness associated with influenza viral infections can consider changes in dosing or route of administration, increasing duration of therapy, or tailoring therapy based on viral resistance. Patients who are severely ill and hospitalized or who are immunosuppressed may require longer treatment. ${ }^{38}$ In treating severely ill patients with avian influenza A (H5N1), doubling of the dose of oseltamivir (e.g., $150 \mathrm{mg}$ twice daily in adults) was well tolerated in one case report ${ }^{52}$ and may be more effective. ${ }^{53}$ For oseltamivir- 
resistant influenza virus infection, treatment under compassionate use and Emergency Act Authorization using nebulized ${ }^{54}$ and intravenous (IV) zanamivir and IV peramivir have been described. Although the Emergency Act Authorization for use of these drugs through these routes of administration has expired, clinical trials of IV zanamivir, peramivir, and oseltamivir are under way through the National Institutes of Health. Clinicians interested in learning more about these trials of IV antiviral products should go to the http:// www.clinicaltrials.gov website.

Zanamivir: IV zanamivir has occasionally been used as therapy for patients with proven resistance because of the $\mathrm{H} 275 \mathrm{Y}$ mutation in neuraminidase. The $\mathrm{H} 275 \mathrm{Y}$ mutation is associated with no change in susceptibility to zanamivir, but high-level oseltamivir resistance and intermediate-level resistance to peramivir, an investigational neuraminidase inhibitor. ${ }^{55}$ IV zanamivir was previously used to treat cases of oseltamivir-resistant 2009 H1N1 infection, although it is not approved for this use. In one case report, an 18-month-old child with relapsed hematologic malignancy was admitted for a stem cell transplant and was started on oseltamivir early in the hospital course after developing cough and fever and being diagnosed with influenza A virus infection. After the child was diagnosed with 2009 $\mathrm{H} 1 \mathrm{~N} 1$ virus and experienced clinical deterioration, IV zanamivir was administered on a compassionate use basis (GlaxoSmithKline), $320 \mathrm{mg}$ ( $20 \mathrm{mg} / \mathrm{kg}$ per dose) every 12 hours. Sequence analysis of the neuraminidase gene from virus detected in endotracheal aspirates demonstrated absence of $\mathrm{H} 275 \mathrm{Y}$ mutation on day 2, but presence of the mutation beginning on day 13. Although this patient died on hospital day 57, administration of IV zanamivir was associated with a logarithmic drop in $2009 \mathrm{H} 1 \mathrm{~N} 1$ viral RNA levels. ${ }^{56}$ In another case report, a 10-year-old girl with acute lymphoblastic leukemia and PCR-documented 2009 pandemic influenza A (H1N1) infection failed oseltamivir therapy and had progressive respiratory clinical deterioration leading to intubation. The $\mathrm{H} 275 \mathrm{Y}$ neuraminidase mutation was detected and the patient was changed to IV zanamivir for 15 days (600 mg every 12 hours) under an emergency investigational new drug application. Treatment with IV zanamivir was associated with a substantial decrease in influenza A viral loads to undetectable levels by PCR testing and the patient was weaned off the ventilator approximately 3 weeks after zanamivir initiation. The patient tolerated zanamivir well with no adverse effects. ${ }^{57}$

Peramivir: Both adults and children with severe, progressively worsening 2009 H1N1 influenza viral pneumonia and respiratory failure experienced recovery associated with administration of IV peramivir, an investigational neuraminidase inhibitor, despite most having received oseltamivir therapy. Administration of the drug was made possible through the Emergency Investigational New Drug (eIND) regulations. From April through October 2009 , in 20 adults and 11 children aged $<18$ years who received oseltamivir for a median of 10 days (range 1-14 days), the 14-, 28-, and 56-day survival rates were $76.7 \%, 66.7 \%$, and $59.0 \%$, respectively. The adult dosage was $600 \mathrm{mg}$ IV once daily with adjustments for renal impairment, and the pediatric dose ranged from $6 \mathrm{mg} / \mathrm{kg}$ to $12 \mathrm{mg} / \mathrm{kg}$, not to exceed $600 \mathrm{mg}$ IV per day. Seventeen of 31 patients continued oseltamivir administration after initiating peramivir. Survival was associated with earlier administration of peramivir, on hospital days 2 to 8 , compared with hospital days 10 to 16 in those who died. Delay in administration of the drug was mainly secondary to a delay in requesting the drug after hospitalization. The 
investigational drug was generally well tolerated with no reports of associated serious adverse events. Peramivir has been approved in Japan and South Korea and is undergoing U.S. Phase III trials in hospitalized patients with influenza. ${ }^{58}$ It is important to reiterate that for patients with the $\mathrm{H} 275 \mathrm{Y}$ mutation, peramivir is not recommended because of associated intermediate-level resistance.

Preventing Recurrence-See sections Preventing Exposure and Preventing First Episode of Disease.

Discontinuing Secondary Prophylaxis-Not applicable.

\section{References}

1. Brankston G, Gitterman L, Hirji Z, Lemieux C, Gardam M. Transmission of influenza A in human beings. Lancet Infect Dis. 2007 Apr; 7(4):257-265. Available at http://www.ncbi.nlm.nih.gov/ pubmed/17376383. [PubMed: 17376383]

2. Cox NJ, Subbarao K. Influenza. Lancet. 1999 Oct 9; 354(9186):1277-1282. Available at http:// www.ncbi.nlm.nih.gov/pubmed/10520648. [PubMed: 10520648]

3. Hall CB, Douglas RG Jr. Nosocomial influenza infection as a cause of intercurrent fevers in infants. Pediatrics. 1975 May; 55(5):673-677. Available at http://www.ncbi.nlm.nih.gov/pubmed/1168894. [PubMed: 1168894]

4. Giannella M, Alonso M, Garcia de Viedma D, et al. Prolonged viral shedding in pandemic influenza A(H1N1): clinical significance and viral load analysis in hospitalized patients. Clin Microbiol Infect. 2011 Aug; 17(8):1160-1165. Available at http://www.ncbi.nlm.nih.gov/pubmed/20946412. [PubMed: 20946412]

5. Lee N, Chan PK, Hui DS, et al. Viral loads and duration of viral shedding in adult patients hospitalized with influenza. J Infect Dis. 2009 Aug 15; 200(4):492-500. Available at http:// www.ncbi.nlm.nih.gov/pubmed/19591575. [PubMed: 19591575]

6. Klimov AI, Rocha E, Hayden FG, Shult PA, Roumillat LF, Cox NJ. Prolonged shedding of amantadine-resistant influenzae A viruses by immunodeficient patients: detection by polymerase chain reaction-restriction analysis. J Infect Dis. 1995 Nov; 172(5):1352-1355. Available at http:// www.ncbi.nlm.nih.gov/pubmed/7594676. [PubMed: 7594676]

7. Frank AL, Taber LH, Wells CR, Wells JM, Glezen WP, Paredes A. Patterns of shedding of myxoviruses and paramyxoviruses in children. J Infect Dis. 1981 Nov; 144(5):433-441. Available at http://www.ncbi.nlm.nih.gov/pubmed/6273473. [PubMed: 6273473]

8. Bridges, CB.; Fry, A., et al. Influenza. In: DL, H., editor. Control of Comunicable Diseases Manual. 19th edition. Washington, DC: 2008. p. 315-331.

9. Youzbashi E, Marschall M, Chaloupka I, Meier-Ewert H. [Distribution of influenza C virus infection in dogs and pigs in Bavaria]. Tierarztliche Praxis. 1996 Aug; 24(4):337-342. Available at http://www.ncbi.nlm.nih.gov/pubmed/9012016. [PubMed: 9012016]

10. Matsuzaki Y, Sugawara K, Mizuta K, et al. Antigenic and genetic characterization of influenza C viruses which caused two outbreaks in Yamagata City, Japan, in 1996 and 1998. J Clin Microbiol. 2002 Feb; 40(2):422-429. Available at http://www.ncbi.nlm.nih.gov/pubmed/11825952. [PubMed: 11825952]

11. Fiore AE, Uyeki TM, Broder K, et al. Prevention and control of influenza with vaccines: recommendations of the Advisory Committee on Immunization Practices (ACIP), 2010. MMWR Recomm Rep. 2010 Aug 6; 59(RR-8):1-62. Available at http://www.ncbi.nlm.nih.gov/pubmed/ 20689501. [PubMed: 20689501]

12. Dolin R. Editorial commentary: Perspectives on the role of immunization against influenza in HIVinfected patients. Clin Infect Dis. 2011 Jan 1; 52(1):147-149. Available at http:// www.ncbi.nlm.nih.gov/pubmed/21148533. [PubMed: 21148533]

13. Neuzil KM, Coffey CS, Mitchel EF Jr, Griffin MR. Cardiopulmonary hospitalizations during influenza season in adults and adolescents with advanced HIV infection. J Acquir Immune Defic 
Syndr. 2003 Nov 1; 34(3):304-307. Available at http://www.ncbi.nlm.nih.gov/pubmed/14600576. [PubMed: 14600576]

14. Lin JC, Nichol KL. Excess mortality due to pneumonia or influenza during influenza seasons among persons with acquired immunodeficiency syndrome. Arch Intern Med. 2001 Feb 12; 161(3):441-446. Available at http://www.ncbi.nlm.nih.gov/pubmed/11176770. [PubMed: 11176770]

15. Feiterna-Sperling C, Edelmann A, Nickel R, et al. Pandemic influenza A (H1N1) outbreak among 15 school-aged HIV-1-infected children. Clin Infect Dis. 2010 Dec 1; 51(11):e90-e94. Available at http://www.ncbi.nlm.nih.gov/pubmed/21039216. [PubMed: 21039216]

16. Noguera-Julian A, Provens AC, Soler-Palacin P, et al. Pandemic influenza a (2009 H1N1) in human immunodeficiency virus-infected catalan children. Pediatr Infect Dis J. 2011 Feb; 30(2): 173-175. Available at http://www.ncbi.nlm.nih.gov/pubmed/20802374. [PubMed: 20802374]

17. Poehling KA, Edwards KM, Weinberg GA, et al. The underrecognized burden of influenza in young children. N Engl J Med. 2006 Jul 6; 355(1):31-40. Available at http:// www.ncbi.nlm.nih.gov/pubmed/16822994. [PubMed: 16822994]

18. Madhi SA, Ramasamy N, Bessellar TG, Saloojee H, Klugman KP. Lower respiratory tract infections associated with influenza A and B viruses in an area with a high prevalence of pediatric human immunodeficiency type 1 infection. Pediatr Infect Dis J. 2002 Apr; 21(4):291-297. Available at http://www.ncbi.nlm.nih.gov/pubmed/12075759. [PubMed: 12075759]

19. Janoff EN, Breiman RF, Daley CL, Hopewell PC. Pneumococcal disease during HIV infection. Epidemiologic, clinical, and immunologic perspectives. Ann Intern Med. 1992 Aug 15; 117(4): 314-324. Available at http://www.ncbi.nlm.nih.gov/pubmed/1637028. [PubMed: 1637028]

20. Centers for Disease C, Prevention. Evaluation of rapid influenza diagnostic tests for detection of novel influenza A (H1N1) Virus - United States, 2009. MMWR Morb Mortal Wkly Rep. 2009 Aug 7; 58(30):826-829. Available at http://www.ncbi.nlm.nih.gov/pubmed/19661856. [PubMed: 19661856]

21. Siegel JD, Rhinehart E, Jackson M, Chiarello L. Health Care Infection Control Practices Advisory C. 2007 Guideline for Isolation Precautions: Preventing Transmission of Infectious Agents in Health Care Settings. Am J Infect Control. 2007 Dec; 35(10 Suppl 2):S65-S164. Available at http://www.ncbi.nlm.nih.gov/pubmed/18068815. [PubMed: 18068815]

22. Hurwitz ES, Haber M, Chang A, et al. Effectiveness of influenza vaccination of day care children in reducing influenza-related morbidity among household contacts. JAMA. 2000 Oct 4; 284(13): 1677-1682. Available at http://www.ncbi.nlm.nih.gov/pubmed/11015798. [PubMed: 11015798]

23. Sullivan PS, Hanson DL, Dworkin MS, et al. Effect of influenza vaccination on disease progression among HIV-infected persons. AIDS. 2000 Dec 1; 14(17):2781-2785. Available at http://www.ncbi.nlm.nih.gov/pubmed/11125897. [PubMed: 11125897]

24. Zanetti AR, Amendola A, Besana S, Boschini A, Tanzi E. Safety and immunogenicity of influenza vaccination in individuals infected with HIV. Vaccine. 2002 Dec 20; 20(Suppl 5):B29-B32. Available at http://www.ncbi.nlm.nih.gov/pubmed/12477415. [PubMed: 12477415]

25. Jackson CR, Vavro CL, Valentine ME, et al. Effect of influenza immunization on immunologic and virologic characteristics of pediatric patients infected with human immunodeficiency virus. Pediatr Infect Dis J. 1997 Feb; 16(2):200-204. Available at http://www.ncbi.nlm.nih.gov/pubmed/ 9041601. [PubMed: 9041601]

26. Esposito S, Tagliaferri L, Daleno C, et al. Pandemic influenza A/H1N1 vaccine administered sequentially or simultaneously with seasonal influenza vaccine to HIV-infected children and adolescents. Vaccine. 2011 Feb 11; 29(8):1677-1682. Available at http://www.ncbi.nlm.nih.gov/ pubmed/21199699. [PubMed: 21199699]

27. Keller M, Deveikis A, Cutillar-Garcia M, et al. Pneumococcal and influenza immunization and human immunodeficiency virus load in children. Pediatr Infect Dis J. 2000 Jul; 19(7):613-618. Available at http://www.ncbi.nlm.nih.gov/pubmed/10917218. [PubMed: 10917218]

28. Levin MJ, Song LY, Fenton T, et al. Shedding of live vaccine virus, comparative safety, and influenza-specific antibody responses after administration of live attenuated and inactivated trivalent influenza vaccines to HIV-infected children. Vaccine. 2008 Aug 5; 26(33):4210-4217. Available at http://www.ncbi.nlm.nih.gov/pubmed/18597900. [PubMed: 18597900] 
29. Montoya CJ, Toro MF, Aguirre C, et al. Abnormal humoral immune response to influenza vaccination in pediatric type-1 human immunodeficiency virus infected patients receiving highly active antiretroviral therapy. Mem Inst Oswaldo Cruz. 2007 Jun; 102(4):501-508. Available at http://www.ncbi.nlm.nih.gov/pubmed/17612772. [PubMed: 17612772]

30. Amendola A, Pariani E, Vigano A, et al. Influenza surveillance in a cohort of HIV-infected children and adolescents immunized against seasonal influenza. Vaccine. 2010 Mar 24; 28(15): 2700-2704. Available at http://www.ncbi.nlm.nih.gov/pubmed/20105427. [PubMed: 20105427]

31. Cagigi A, Nilsson A, Pensieroso S, Chiodi F. Dysfunctional B-cell responses during HIV-1 infection: implication for influenza vaccination and highly active antiretroviral therapy. Lancet Infect Dis. 2010 Jul; 10(7):499-503. Available at http://www.ncbi.nlm.nih.gov/pubmed/20610332. [PubMed: 20610332]

32. Chadwick EG, Chang G, Decker MD, Yogev R, Dimichele D, Edwards KM. Serologic response to standard inactivated influenza vaccine in human immunodeficiency virus-infected children. Pediatr Infect Dis J. 1994 Mar; 13(3):206-211. Available at http://www.ncbi.nlm.nih.gov/pubmed/ 8177629. [PubMed: 8177629]

33. Crum-Cianflone NF, Eberly LE, Duplessis C, et al. Immunogenicity of a monovalent 2009 influenza $\mathrm{A}(\mathrm{H} 1 \mathrm{~N} 1)$ vaccine in an immunocompromised population: a prospective study comparing HIV-infected adults with HIV-uninfected adults. Clin Infect Dis. 2011 Jan 1; 52(1): 138-146. Available at http://www.ncbi.nlm.nih.gov/pubmed/21148532. [PubMed: 21148532]

34. Weinberg A, Song LY, Walker R, et al. Anti-influenza serum and mucosal antibody responses after administration of live attenuated or inactivated influenza vaccines to HIV-infected children. J Acquir Immune Defic Syndr. 2010 Oct; 55(2):189-196. Available at http://www.ncbi.nlm.nih.gov/ pubmed/20581690. [PubMed: 20581690]

35. King JC Jr, Fast PE, Zangwill KM, et al. Safety, vaccine virus shedding and immunogenicity of trivalent, cold-adapted, live attenuated influenza vaccine administered to human immunodeficiency virus-infected and noninfected children. Pediatr Infect Dis J. 2001 Dec; 20(12): 1124-1131. Available at http://www.ncbi.nlm.nih.gov/pubmed/11740317. [PubMed: 11740317]

36. Fiore AE, Fry A, Shay D, et al. Antiviral agents for the treatment and chemoprophylaxis of influenza - recommendations of the Advisory Committee on Immunization Practices (ACIP). MMWR Recomm Rep. 2011 Jan 21; 60(1):1-24. Available at http://www.ncbi.nlm.nih.gov/ pubmed/21248682. [PubMed: 21248682]

37. Centers for Disease Control and Prevention. FluView: week ending. Atlanta, GA: US Department of Health and Human Services, CDC; 2010 May 20. 2010. http://www.cdc.gov/flu/weekly. [Accessed December 16, 2010]

38. Glaxo Wellcome Inc. Relenza (zanimivir for inhalation) [Package insert]. 2011 Available at http:// www.accessdata.fda.gov/drugsatfda_docs/label/2011/021036s027lbl.pdf.

39. Evans KD, Kline MW. Prolonged influenza A infection responsive to rimantadine therapy in a human immunodeficiency virus-infected child. Pediatr Infect Dis J. 1995 Apr; 14(4):332-334. Available at http://www.ncbi.nlm.nih.gov/pubmed/7603824. [PubMed: 7603824]

40. King JC Jr. Community respiratory viruses in individuals with human immunodeficiency virus infection. Am J Med. 1997 Mar 17; 102(3A):19-24. discussion 25-16. Available at http:// www.ncbi.nlm.nih.gov/pubmed/10868138. [PubMed: 10868138]

41. Nicholson KG, Aoki FY, Osterhaus AD, et al. Efficacy and safety of oseltamivir in treatment of acute influenza: a randomised controlled trial. Neuraminidase Inhibitor Flu Treatment Investigator Group. Lancet. 2000 May 27; 355(9218):1845-1850. Available at http://www.ncbi.nlm.nih.gov/ pubmed/10866439. [PubMed: 10866439]

42. Whitley RJ, Hayden FG, Reisinger KS, et al. Oral oseltamivir treatment of influenza in children. Pediatr Infect Dis J. 2001 Feb; 20(2):127-133. Available at http://www.ncbi.nlm.nih.gov/pubmed/ 11224828. [PubMed: 11224828]

43. Roche Laboratories Inc. Tamiflu (oseltamivir phosphate) capsules and oral suspension [package insert]. 2011. Available at http://www.accessdata.fda.gov/drugsatfda_docs/label/ 2011/021087s057lbl.pdf

44. New concerns about oseltamivir. Lancet. 2007 Mar 31.369(9567):1056. Available at http:// www.ncbi.nlm.nih.gov/pubmed/17398285. 
45. Toovey S, Rayner C, Prinssen E, et al. Assessment of neuropsychiatric adverse events in influenza patients treated with oseltamivir: a comprehensive review. Drug safety: an international journal of medical toxicology and drug experience. 2008; 31(12):1097-1114. Available at http:// www.ncbi.nlm.nih.gov/pubmed/19026027. [PubMed: 19026027]

46. Budnitz DS, Lewis LL, Shehab N, Birnkrant D. CDC and FDA response to risk of confusion in dosing Tamiflu oral suspension. N Engl J Med. 2009 Nov 5; 361(19):1913-1914. Available at http://www.ncbi.nlm.nih.gov/pubmed/19797275. [PubMed: 19797275]

47. Soung LS, Ing TS, Daugirdas JT, et al. Amantadine hydrochloride pharmacokinetics in hemodialysis patients. Ann Intern Med. 1980 Jul; 93(1):46-49. Available at http:// www.ncbi.nlm.nih.gov/pubmed/7396313. [PubMed: 7396313]

48. Atkinson WL, Arden NH, Patriarca PA, Leslie N, Lui KJ, Gohd R. Amantadine prophylaxis during an institutional outbreak of type A (H1N1) influenza. Arch Intern Med. 1986 Sep; 146(9):17511756. Available at http://www.ncbi.nlm.nih.gov/pubmed/3753115. [PubMed: 3753115]

49. Forest Pharmaceuticals. Flumadine syrup (rimantadine hydrochloride syrup) [Package insert]. 2006 Available at http://www.accessdata.fda.gov/drugsatfda_docs/label/ 2007/019649s010,019650s007lbl.pdf.

50. Soo W. Adverse effects of rimantadine: summary from clinical trials. J Respir Dis. 1980; 10(Suppl):S26-S31.

51. Food and Drug Administration. [Accessed July 10, 2012] FDA statement following CHPA's announcement on nonprescription over-the-counter cough and cold medicines in children. 2010. http:/www.fda.gov/NewsEvents/Newsroom/PressAnnouncements/2008/ucm116964.htm.

52. He G, Massarella J, Ward P. Clinical pharmacokinetics of the prodrug oseltamivir and its active metabolite Ro 64-0802. Clinical pharmacokinetics. 1999 Dec; 37(6):471-484. Available at http:// www.ncbi.nlm.nih.gov/pubmed/10628898. [PubMed: 10628898]

53. Abdel-Ghafar AN, Chotpitayasunondh T, et al. Writing Committee of the Second World Health Organization Consultation on Clinical Aspects of Human Infection with Avian Influenza AV. Update on avian influenza A (H5N1) virus infection in humans. N Engl J Med. 2008 Jan 17; 358(3):261-273. Available at http://www.ncbi.nlm.nih.gov/pubmed/18199865. [PubMed: 18199865]

54. Da Dalt L, Calistri A, Chillemi C, et al. Oseltamivir-resistant pandemic (H1N1) 2009 treated with nebulized zanamivir. Emerg Infect Dis. 2010 Nov; 16(11):1813-1815. Available at http:// www.ncbi.nlm.nih.gov/pubmed/21029559. [PubMed: 21029559]

55. Baz M, Abed Y, Boivin G. Characterization of drug-resistant recombinant influenza A/H1N1 viruses selected in vitro with peramivir and zanamivir. Antiviral Res. 2007 May; 74(2):159-162. Available at http://www.ncbi.nlm.nih.gov/pubmed/17137644. [PubMed: 17137644]

56. Dulek DE, Williams JV, Creech CB, et al. Use of intravenous zanamivir after development of oseltamivir resistance in a critically Ill immunosuppressed child infected with 2009 pandemic influenza A (H1N1) virus. Clin Infect Dis. 2010 Jun 1; 50(11):1493-1496. Available at http:// www.ncbi.nlm.nih.gov/pubmed/20415572. [PubMed: 20415572]

57. Gaur AH, Bagga B, Barman S, et al. Intravenous zanamivir for oseltamivir-resistant 2009 H1N1 influenza. N Engl J Med. 2010 Jan 7; 362(1):88-89. Available at http://www.ncbi.nlm.nih.gov/ pubmed/20032317. [PubMed: 20032317]

58. Hernandez JE, Adiga R, Armstrong R, et al. Clinical experience in adults and children treated with intravenous peramivir for 2009 influenza A (H1N1) under an Emergency IND program in the United States. Clin Infect Dis. 2011 Mar 15; 52(6):695-706. Available at http:// www.ncbi.nlm.nih.gov/pubmed/21367722. [PubMed: 21367722]

\section{Dosing Recommendations for Chemoprophylaxis and Treatment of Influenza}

\begin{tabular}{|l|l|l|l|}
\hline Indication & First Choice & Alternative & Comments/Special Issues \\
\hline Primary Prophylaxis & $\underline{\text { Influenza vaccine }}$ & None & $\begin{array}{l}\text { Note: See Figures 1 and 2 } \\
\text { for detailed vaccines } \\
\text { recommendations. }\end{array}$ \\
\hline
\end{tabular}




\begin{tabular}{|c|c|c|c|}
\hline Indication & First Choice & Alternative & Comments/Special Issues \\
\hline $\begin{array}{l}\text { Primary Chemoprophylaxis } \\
\text { Influenza A and B }\end{array}$ & 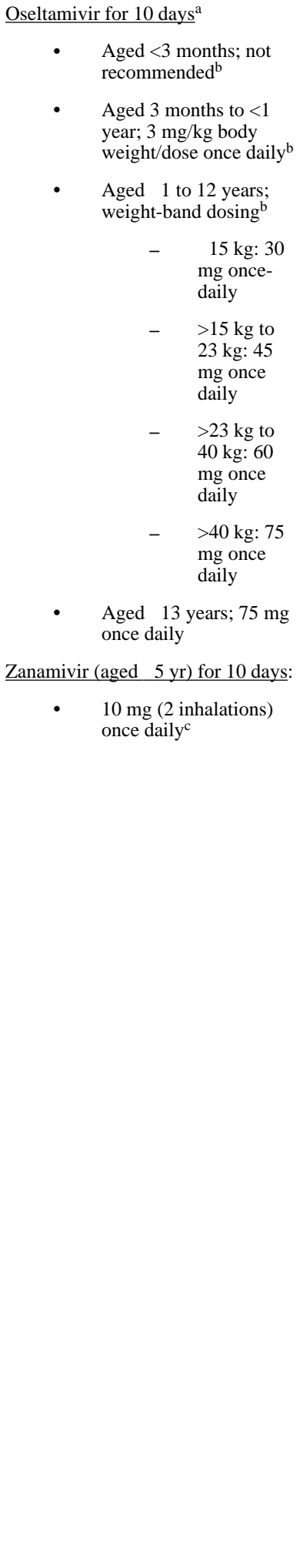 & None & $\begin{array}{l}\text { Primary chemoprophylaxis } \\
\text { is indicated for unvaccinated } \\
\text { HIV-infected children with } \\
\text { moderate-to-severe } \\
\text { immunosuppression (as } \\
\text { assessed by immunologic } \\
\text { and/or clinical diagnostic } \\
\text { categories) who are } \\
\text { household contacts or close } \\
\text { contacts of individuals with } \\
\text { confirmed or suspected } \\
\text { influenza. } \\
\text { Chemoprophylaxis of } \\
\text { vaccinated HIV-infected } \\
\text { children with severe } \\
\text { immunosuppression also } \\
\text { may be indicated based on } \\
\text { health-care provider } \\
\text { assessment of the exposure } \\
\text { situation. Post-exposure } \\
\text { antiviral chemoprophylaxis } \\
\text { should be initiated as soon } \\
\text { as possible after exposure. } \\
\text { a Oseltamivir } \\
\text { chemoprophylaxis } \\
\text { duration: Recommended } \\
\text { duration is } 10 \text { days when } \\
\text { administered after a } \\
\text { household exposure and } 7 \\
\text { days after the most recent } \\
\text { known exposure in other } \\
\text { situations. For control of } \\
\text { outbreaks in long-term care } \\
\text { facilities and hospitals, CDC } \\
\text { recommends antiviral } \\
\text { chemoprophylaxis for a } \\
\text { minimum of } 2 \text { weeks and up } \\
\text { to } 1 \text { week after the most } \\
\text { recent known case was } \\
\text { identified (see http:// } \\
\text { www.cdc.gov/mmwr/ } \\
\text { preview/mmwrhtml/ } \\
\text { rr6001a1.htm). } \\
\text { b Oseltamivir is approved by } \\
\text { the FDA for treatment of } \\
\text { influenza in children aged } \\
\geq 2 \text { weeks. It is not approved } \\
\text { for prophylaxis in children } \\
\text { aged <1 year. However, the } \\
\text { CDC recommends that } \\
\text { health-care providers who } \\
\text { treat children ages } 33 \\
\text { months to < } 1 \text { year } \\
\text { administer a } \\
\text { chemoprophylaxis dose of } 3 \\
\text { mg/kg body weight/dose } \\
\text { once daily. } \\
\text { Chemoprophylaxis for } \\
\text { infants aged <3 months is } \\
\text { not recommended unless the } \\
\text { exposure situation is judged } \\
\text { to be critical. } \\
\text { Premature infants: Current } \\
\text { weight-based dosing } \\
\text { recommendations for } \\
\text { oseltamivir are not } \\
\text { appropriate for premature } \\
\text { infants (i.e., gestational age } \\
\text { at delivery < } 38 \text { weeks). See } \\
\text { J Infect Dis } 202 \text { [4]:563- } \\
566,2010 \text { for dosing } \\
\text { recommendations in } \\
\text { premature infants. }\end{array}$ \\
\hline
\end{tabular}




\begin{tabular}{|c|c|c|c|}
\hline Indication & First Choice & Alternative & Comments/Special Issues \\
\hline & & & $\begin{array}{l}\text { Renal insufficiency: A } \\
\text { reduction in dose of } \\
\text { oseltamivir is recommended } \\
\text { for patients with creatinine } \\
\text { clearance }<30 \mathrm{~mL} / \mathrm{min} \text {. } \\
{ }^{\mathrm{c}} \text { Zanamivir: Zanamivir is } \\
\text { not recommended for } \\
\text { chemoprophylaxis in } \\
\text { children aged }<5 \text { years old. }\end{array}$ \\
\hline $\begin{array}{l}\text { Primary Chemoprophylaxis } \\
\text { Influenza A (ONLY) } \\
\text { Oseltamivir-resistant, } \\
\text { adamantane-sensitive strains } \\
\text { Based on CDC influenza } \\
\text { surveillance; http:// } \\
\text { www.cdc.gov/flu/weekly/ } \\
\text { fluactivitysurv.htm }\end{array}$ & 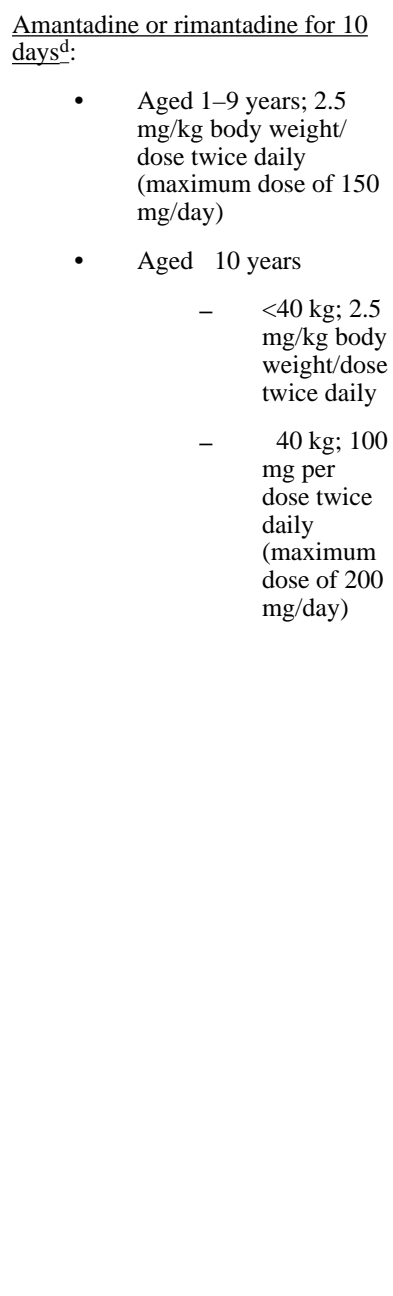 & & $\begin{array}{l}\text { d } \text { Adamantanes: Because of } \\
\text { resistance in currently } \\
\text { circulating influenza A virus } \\
\text { strains, amantadine and } \\
\text { rimantadine are not } \\
\text { currently recommended for } \\
\text { chemoprophylaxis or } \\
\text { treatment (adamantanes are } \\
\text { not active against influenza } \\
\text { B virus). However, potential } \\
\text { exists for emergence of } \\
\text { oseltamivir-resistant, } \\
\text { adamantane-sensitive } \\
\text { circulating influenza A } \\
\text { strains. Therefore, } \\
\text { verification of antiviral } \\
\text { sensitivity of circulating } \\
\text { influenza A strains should } \\
\text { be done using the CDC } \\
\text { influenza surveillance } \\
\text { website: http:// } \\
\text { www.cdc. gov/flu/weekly/ } \\
\text { fluactivitysurv.htm } \\
\text { If administered based on } \\
\text { CDC antiviral sensitivity } \\
\text { surveillance data, both } \\
\text { amantadine and rimantadine } \\
\text { are recommended for } \\
\text { chemoprophylaxis of } \\
\text { influenza A in children aged } \\
\geq 1 \text { yr. For treatment, } \\
\text { rimantadine is only } \\
\text { approved for use in } \\
\text { adolescents aged } \geq 13 \text { years. } \\
\text { Rimantadine is preferred } \\
\text { over amantadine because of } \\
\text { less frequent adverse events. } \\
\text { Some pediatric influenza } \\
\text { specialists may consider it } \\
\text { appropriate for treatment of } \\
\text { children aged > } 1 \text { year. } \\
\text { Renal insufficiency: A } \\
\text { reduction in dose of } \\
\text { amantadine is recommended } \\
\text { for patients with creatinine } \\
\text { clearance <30 mL/min. }\end{array}$ \\
\hline Secondary Chemoprophylaxis & N/A & N/A & $\begin{array}{l}\text { No role for secondary } \\
\text { chemoprophylaxis }\end{array}$ \\
\hline $\begin{array}{l}\text { Treatment } \\
\text { Influenza A and B }\end{array}$ & 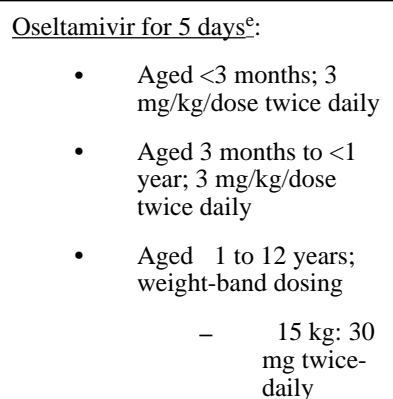 & None & $\begin{array}{l}\text { e Oseltamivir is FDA- } \\
\text { approved for treatment of } \\
\text { influenza in children aged } \\
\geq 2 \text { weeks. The CDC } \\
\text { recommends that clinicians } \\
\text { who treat children ages } \geq 3 \\
\text { months to < } 1 \text { year } \\
\text { administer a dose of } 3 \\
\mathrm{mg} / \mathrm{kg} \text { twice daily. A dose } \\
\text { of } 3 \mathrm{mg} / \mathrm{kg} / \text { dose twice daily } \\
\text { also is recommended for } \\
\text { infants aged <3 months. } \\
\text { Premature Infants: } \\
\text { Current weight-based }\end{array}$ \\
\hline
\end{tabular}




\begin{tabular}{|c|c|c|c|}
\hline Indication & First Choice & Alternative & Comments/Special Issues \\
\hline & 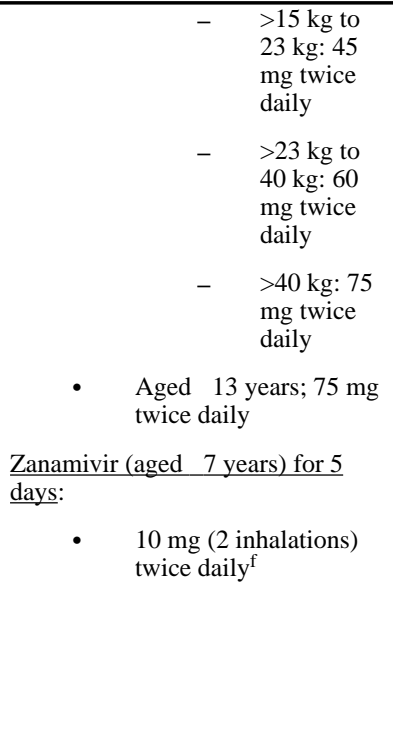 & & $\begin{array}{l}\text { dosing recommendations for } \\
\text { oseltamivir are not } \\
\text { appropriate for premature } \\
\text { infants: gestational age at } \\
\text { delivery <38 weeks. See } J \\
\text { Infect Dis } 202 \text { [4]:563-566, } \\
2010 \text { for dosing } \\
\text { recommendations in } \\
\text { premature infants. } \\
\text { Oseltamivir treatment } \\
\text { duration: Recommended } \\
\text { duration for antiviral } \\
\text { treatment is 5 days; longer } \\
\text { treatment courses can be } \\
\text { considered for patients who } \\
\text { remain severely ill after 5 } \\
\text { days of treatment. } \\
\text { Renal insufficiency: A } \\
\text { reduction in dose of } \\
\text { oseltamivir is recommended } \\
\text { for patients with creatinine } \\
\text { clearance <30 mL/min. } \\
\text { f Zanamivir: Zanamivir is } \\
\text { not recommended for } \\
\text { treatment in children aged } \\
<7 \text { years. }\end{array}$ \\
\hline $\begin{array}{l}\text { Treatment } \\
\text { Influenza A (ONLY) } \\
\text { Oseltamivir-resistant, } \\
\text { adamantane-sensitive strains } \\
\text { Based on CDC influenza } \\
\text { surveillance; http:// } \\
\text { www.cdc.gov/flu/weekly/ } \\
\text { fluactivitysurv.htm }\end{array}$ & 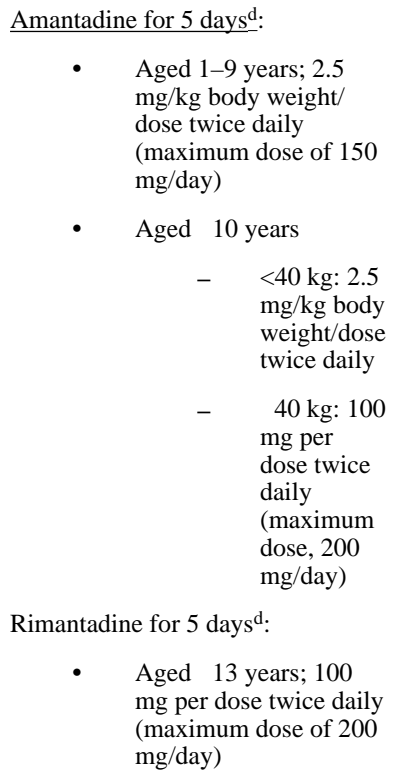 & & $\begin{array}{l}\text { Please see comment }{ }^{\mathrm{d}} \text {, } \\
\text { above, about adamantane } \\
\text { use and resistance. }\end{array}$ \\
\hline
\end{tabular}

Key to Acronyms: $\mathrm{CDC}=$ Centers for Disease Control and Prevention; FDA $=$ Food and Drug Administration

Isosporiasis (Cystoisosporiasis) (Last updated November 6, 2013; last reviewed November 6, 2013)

Panel's Recommendations

- Antiretroviral treatment of HIV-infected children to reverse or prevent severe immunodeficiency may reduce the incidence or prevent recurrence of isosporiasis (CIII).

- Careful hand washing and thorough washing of fruits and vegetables are recommended to prevent exposure (AIII). 
Panel's Recommendations

- $\quad$ Travelers to endemic areas should avoid untreated water for drinking, brushing teeth, and in ice, as well as unpeeled fruits and vegetables, all of which can be contaminated (BIII).

- Trimethoprim-sulfamethoxazole (TMP-SMX) is recommended for treatment of isosporiasis in HIVinfected children $\left(\mathbf{A I}^{*}\right)$.

- In those with severe immunosuppression, treatment should be followed by secondary prophylaxis with TMP-SMX until severe immunosuppression resolves (AII*).

- As with all causes of diarrhea, supportive care, including replenishment of fluids and electrolytes, is essential (AIII).

Rating of Recommendations: $\mathrm{A}=$ Strong; $\mathrm{B}=$ Moderate; $\mathrm{C}=$ Optional

Rating of Evidence: $\mathrm{I}=$ One or more randomized trials $\underline{\mathrm{in} \mathrm{children}}^{\dagger}$ with clinical outcomes and/or validated endpoints; $\mathrm{I}^{*}$ $=$ One or more randomized trials in adults with clinical outcomes and/or validated laboratory endpoints with accompanying data in children ${ }^{\dagger}$ from one or more well-designed, nonrandomized trials or observational cohort studies with long-term clinical outcomes; II = One or more well-designed, nonrandomized trials or observational cohort studies in children ${ }^{\dagger}$ with long-term outcomes; II* $=$ One or more well-designed, nonrandomized trials or observational studies in adults with longterm clinical outcomes with accompanying data in children ${ }^{\dagger}$ from one or more similar nonrandomized trials or cohort studies with clinical outcome data; III = Expert opinion ${ }^{\dagger}$ Stu

Studies that include children or children/adolescents, but not studies limited to post-pubertal adolescents.

\section{Epidemiology}

Isospora belli (Cystoisospora belli) is an intestinal coccidian parasite in the phylum Apicomplexa. It was first linked with human disease in 1915 and is believed to infect only humans. ${ }^{1}$ Isosporiasis, also known as cystoisosporiasis, occurs worldwide but is more prevalent in tropical and subtropical regions; it has been reported as an etiologic agent of traveler's diarrhea. ${ }^{2-4}$ Prior to the availability of combination antiretroviral therapy (cART), the prevalence of isosporiasis among adults with AIDS was reported to be $15 \%$ in Haiti but $<0.2 \%$ in the United States. ${ }^{1,5}$

Infected individuals pass non-infective, unsporulated (immature) oocysts in their stools. The oocysts must sporulate (mature) outside the host, in favorable environmental conditions, to become infective. ${ }^{1,4}$ Therefore, direct person-to-person transmission is unlikely. Infection results from ingestion of sporulated oocysts, such as in contaminated food or water. In the proximal small intestine, the ingested oocysts release sporozoites that invade the intestinal epithelial cells. They then enter an asexual reproduction stage that infects neighboring epithelial cells. Sexual gametocytes are also produced; their fertilization results in unsporulated oocysts, which are shed in stool. ${ }^{1,6}$

\section{Clinical Manifestations}

On the basis of limited data, the incubation period averages approximately 1 week but may range from several days to 2 or more weeks; symptom onset may be acute or insidious. ${ }^{1,2,4,5}$ The most common symptom is watery (non-bloody) diarrhea, which can be profuse and result in dehydration, weight loss, and malabsorption. Affected people also can have crampy abdominal pain, flatulence, nausea, vomiting, anorexia, and low-grade fever. Biliary disease (cholecystitis/cholangiopathy) and reactive arthritis also have been reported. ${ }^{7,8}$ Whereas immunocompetent hosts typically have self-limited infection, chronic, debilitating diarrhea is common in untreated HIV-infected patients. 


\section{Diagnosis}

Isosporiasis is diagnosed by identifying I. belli oocysts in stool (or duodenal aspirates using the Entero-Test) or developmental stages of the parasite in biopsy specimens (such as of the small intestine). The oocysts are relatively large (23-33 $\mu \mathrm{m}$ long by $10-19 \mu \mathrm{m}$ wide) but may be difficult to find. Oocysts may be shed in low numbers even by individuals who have severe diarrhea, which underscores the utility of repeated stool examinations, using methods that concentrate and highlight the parasite. Although staining is frequently variable, the organism can be identified with use of a modified acid-fast stain, staining bright red on a green background. ${ }^{5,6}$ The organism also autofluoresces when viewed by ultraviolet fluorescence microscopy. ${ }^{1}$ Blunting and clubbing of villi and hypertrophied crypts can be seen on small bowel biopsy. There also may be an increase in lymphocytes, plasma cells, and eosinophils in the lamina propria. ${ }^{6}$ Serologic tests for diagnosing I. belli infection are not available. Peripheral eosinophilia occurs in up to half of patients. Polymerase chain reaction is a promising diagnostic tool but is not yet commercially available. ${ }^{9}$

\section{Prevention Recommendations}

Preventing Exposure-Careful hand washing and thorough washing of fruits and vegetables are recommended (AIII). As always, travelers to endemic areas should avoid untreated water for drinking, brushing teeth, and in ice, as well as unpeeled fruits and vegetables (BIII).

Preventing Disease-There are no U.S. recommendations for primary prophylaxis of isosporiasis. Prophylaxis with trimethoprimsulfamethoxazole (TMP-SMX, $160 \mathrm{mg}$ and 800 $\mathrm{mg}$, respectively) was effective in preventing isosporiasis in adults with World Health Organization stage 2 or 3 HIV infection in Cote d'Ivoire. ${ }^{10}$ In addition, in an observational study, the incidence of isosporiasis decreased after widespread availability of cART, except among persons with CD4 counts less than 50 cells $/ \mu \mathrm{L} .{ }^{11}$ Although there have been no studies in children, the relationship between severe immunosuppression and disease in adults suggests that initiation of cART in HIV-infected children before development of severe immunodeficiency may reduce the incidence or prevent recurrence of isosporiasis (CIII).

\section{Treatment Recommendations}

Treating Disease-TMP-SMX is the recommended treatment for isosporiasis. Three randomized trials performed in HIV-infected adults in Haiti not receiving antiretroviral therapy have demonstrated the effectiveness of various regimens. ${ }^{5,12,13}$ In the first study, TMP-SMX (160 mg and $800 \mathrm{mg}$, respectively) was administered 4 times daily for 10 days and then twice daily for 3 weeks. Improvement in diarrheal symptoms occurred within a few days, but 7 of 15 patients (47\%) had recurrent symptoms within a mean of $8+/-5.8$ weeks following completion of therapy. ${ }^{5}$ In the second study, TMP-SMX (160 mg and $800 \mathrm{mg}$, respectively) was administered 4 times daily for 10 days; subjects were then randomized to 1 of 3 secondary prophylaxis arms. At the completion of the initial 10 days of TMP-SMX, all 32 participants had resolution of diarrhea and abdominal pain as well as stool samples that tested negative for I. belli. ${ }^{12}$ In the third study, subjects were randomized to receive 
either TMPSMX (160 mg and $800 \mathrm{mg}$, respectively) or ciprofloxacin $(500 \mathrm{mg}$ ) twice daily for 7 days. TMP-SMX treatment resulted in cessation of diarrhea in all 10 patients and negative results on stool examination at day 7 in 9 of the 10, while ciprofloxacin resulted in resolution of diarrhea in 10 of 12 patients and 9 of 12 with negative stool examinations. ${ }^{13}$ On the basis of these studies in adults, the recommended treatment for HIV-infected children is TMP-SMX, $5 \mathrm{mg} / \mathrm{kg}$ per dose of the trimethoprim component, given twice daily, for 10 days (AI*). If symptoms worsen or persist, the TMP-SMX dose may be increased to $5 \mathrm{mg} / \mathrm{kg} / \mathrm{dose}$ of the trimethoprim component, 3 to 4 times daily, for 10 days or the duration of treatment lengthened (up to 3-4 weeks) (CIII). ${ }^{5,14}$ Intravenous administration of TMPSMX should be considered for patients with potential or documented malabsorption.

Daily pyrimethamine (50-75 $\mathrm{mg}$ in adults), with folinic acid (10-25 $\mathrm{mg} /$ day) to prevent myelosuppression, may be an effective therapy and is typically the alternative for patients who are intolerant of TMP-SMX (BIII).${ }^{15}$ Other agents to consider in a TMP-SMXintolerant patient include ciprofloxacin (CI*) or nitazoxanide (CIII). Based on the study previously cited, ${ }^{13}$ ciprofloxacin is less effective than TMP-SMX, and nitazoxanide has only been studied in small numbers of HIV-uninfected children and adults. ${ }^{16}$ As reviewed above, the relationship between the use of cART and recovery from isosporiasis remains unknown. However, because the incidence of isosporiasis has been reported to be higher in those with more severe immune suppression, it seems reasonable to initiate cART in children with isosporiasis not already receiving cART to prevent recurrence (CIII).

As with all causes of diarrhea, supportive care, including replenishment of fluids and electrolytes, is essential (AIII).

Monitoring and Adverse Events (Including IRIS)—Immune reconstitution inflammatory syndrome has not been reported in association with treatment of isosporiasis. In general, recommended treatment regimens are well tolerated.

Managing Treatment Failure-Reports of treatment failure are relatively uncommon. Mixed data regarding treatment outcomes are available for albendazole, ${ }^{17-19}$ doxycycline, ${ }^{20}$ roxithromycin, ${ }^{21}$ and spiramycin. ${ }^{22}$

Preventing Recurrence-Following treatment of an acute episode, secondary prophylaxis should be continued in those with severe immunosuppression (Centers for Disease Control and Prevention [CDC] immunologic category 3) for an indefinite period until sustained immunologic recovery is observed (AII*). Pape et al., randomized HIVinfected adults completing therapy for acute infection to one of three regimens: TMP-SMX (160 mg and $800 \mathrm{mg}$, respectively) three times per week, sulfadoxine (500 mg) plus pyrimethamine $\left(25 \mathrm{mg}\right.$ ) once weekly, or placebo. ${ }^{12}$ The two active treatment arms were equally effective in preventing relapse. However, the combination of sulfadoxine and pyrimethamine is not recommended in the United States because of increased risk of severe cutaneous reactions. In another study, adult patients with a clinical response following treatment of acute infection with TMP-SMX or ciprofloxacin received secondary prophylaxis for 10 weeks with the same agent as treatment, but at reduced doses: TMP-SMX (160 mg and $800 \mathrm{mg}$, respectively) or ciprofloxacin $(500 \mathrm{mg}$ ) three times per week. The two 
agents were equally effective in preventing recurrence during the monitoring period. ${ }^{13}$

Based on these findings in adults, acceptable regimens in HIV-infected children include TMP-SMX, $2.5 \mathrm{mg} / \mathrm{kg}$ body weight twice daily of the trimethoprim component, administered 3 days per week. The 3 days per week can be three consecutive days or an alternating-day schedule (e.g., Monday-Wednesday-Friday) (AII*). Patients intolerant of TMP-SMX may receive pyrimethamine (plus folinic acid) as secondary prophylaxis ${ }^{15}$ (BIIII). Ciprofloxacin three times weekly can be considered as a second-line alternative (CI*). ${ }^{22}$

Discontinuing Secondary Prophylaxis-There are no data to provide guidance regarding the duration of secondary prophylaxis. All patients should be monitored for recurrence (BIII) and those with severe immunosuppression may require secondary prophylaxis indefinitely (CIII). Secondary prophylaxis can probably be discontinued in patients who demonstrate sustained recovery from severe immunosuppression. In adults, a CD4 count $>200$ cells $/ \mu \mathrm{L}$ for at least 6 months is recommended to discontinue secondary prophylaxis. In children, a reasonable time to discontinue secondary prophylaxis would be after sustained improvement in CD4 count or CD4 percentage from CDC immunologic category 3 to 1 or 2 .

\section{References}

1. Lindsay DS, Dubey JP, Blagburn BL. Biology of Isospora spp. from humans, nonhuman primates, and domestic animals. Clin Microbiol Rev. 1997 Jan; 10(1):19-34. Available at http:// www.ncbi.nlm.nih.gov/pubmed/8993857. [PubMed: 8993857]

2. Shaffer N, Moore L. Chronic travelers' diarrhea in a normal host due to Isospora belli. J Infect Dis. 1989 Mar; 159(3):596-597. Available at http://www.ncbi.nlm.nih.gov/pubmed/2915177. [PubMed: 2915177]

3. Godiwala T, Yaeger R. Isospora and traveler's diarrhea. Ann Intern Med. 1987 Jun; 106(6):908909. Available at http://www.ncbi.nlm.nih.gov/pubmed/3579077. [PubMed: 3579077]

4. Wittner M, Tanowitz HB, Weiss LM. Parasitic infections in AIDS patients. Cryptosporidiosis, isosporiasis, microsporidiosis, cyclosporiasis. Infect Dis Clin North Am. 1993 Sep; 7(3):569-586. Available at http://www.ncbi.nlm.nih.gov/pubmed/8254160. [PubMed: 8254160]

5. DeHovitz JA, Pape JW, Boncy M, Johnson WD Jr. Clinical manifestations and therapy of Isospora belli infection in patients with the acquired immunodeficiency syndrome. N Engl J Med. $1986 \mathrm{Jul}$ 10; 315(2):87-90. Available at http://www.ncbi.nlm.nih.gov/pubmed/3487730. [PubMed: 3487730]

6. Pape JW, Johnson WD Jr. Isospora belli infections. Prog Clin Parasitol. 1991; 2:119-127. Available at http://www.ncbi.nlm.nih.gov/pubmed/1893117. [PubMed: 1893117]

7. Bialek R, Overkamp D, Rettig I, Knobloch J. Case report: Nitazoxanide treatment failure in chronic isosporiasis. Am J Trop Med Hyg. 2001 Aug; 65(2):94-95. Available at http:// www.ncbi.nlm.nih.gov/pubmed/11508398. [PubMed: 11508398]

8. Gonzalez-Dominguez J, Roldan R, Villanueva JL, Kindelan JM JR, Torre-Cisneros J. Isospora belli reactive arthritis in a patient with AIDS [Letter]. Ann Rheum Di. 1994; 53:618-619. Available at http://www.ncbi.nlm.nih.gov/pmc/articles/PMC1005417/.

9. ten Hove RJ, van Lieshout L, Brienen EA, Perez MA, Verweij JJ. Real-time polymerase chain reaction for detection of Isospora belli in stool samples. Diagn Microbiol Infect Dis. $2008 \mathrm{Jul}$; 61(3):280-283. Available at http://www.ncbi.nlm.nih.gov/pubmed/18424043. [PubMed: 18424043]

10. Anglaret X, Chene G, Attia A, et al. Early chemoprophylaxis with trimethoprimsulphamethoxazole for HIV-1-infected adults in Abidjan, Cote d'Ivoire: a randomised trial. Cotrimo-CI Study Group. Lancet. 1999 May 1; 353(9163):1463-1468. Available at http:// www.ncbi.nlm.nih.gov/pubmed/10232311. [PubMed: 10232311] 
11. Guiguet M, Furco A, Tattevin P, Costgagliola D MJ-M. HIV-associated Isospora belli infection: incidence and risk factors in the French Hospital Database on HIV. HIV Medicine. 2007; 8:124 130. 2007. Available at http://www.ncbi.nlm.nih.gov/pubmed/17352769. [PubMed: 17352769]

12. Pape JW, Verdier RI, Johnson WD Jr. Treatment and prophylaxis of Isospora belli infection in patients with the acquired immunodeficiency syndrome. N Engl J Med. 1989 Apr 20; 320(16): 1044-1047. Available at http://www.ncbi.nlm.nih.gov/pubmed/2927483. [PubMed: 2927483]

13. Verdier RI, Fitzgerald DW, Johnson WD Jr, Pape JW. Trimethoprim-sulfamethoxazole compared with ciprofloxacin for treatment and prophylaxis of Isospora belli and Cyclospora cayetanensis infection in HIV-infected patients. A randomized, controlled trial. Ann Intern Med. 2000 Jun 6; 132(11):885-888. Available at http://www.ncbi.nlm.nih.gov/pubmed/10836915. [PubMed: 10836915]

14. Whiteside ME, Barkin JS, May RG, et al. Enteric coccidiosis among patients with the acquired immunodeficiency syndrome. Am J Trop Med Hyg. 1984; 33(6):1065-1072. Available at http:// www.ncbi.nlm.nih.gov/sites/entrez?

$\mathrm{Db}=$ pubmed\&Cmd=ShowDetailView\&TermToSearch=6334448\&ordinalpos=16\&itool=EntrezSy stem2.PEntrez.Pubmed.Pubmed_ResultsPanel.Pubmed_RVDocSum. [PubMed: 6334448]

15. Weiss LM, Perlman DC, Sherman J, Tanowitz H, Wittner M. Isospora belli infection: treatment with pyrimethamine. Ann Intern Med. 1988 Sep 15; 109(6):474-475. Available at http:// www.ncbi.nlm.nih.gov/pubmed/3261956. [PubMed: 3261956]

16. Romero Cabello R, Guerrero LR, Munoz Garcia MR, Geyne Cruz A. Nitazoxanide for the treatment of intestinal protozoan and helminthic infections in Mexico. Trans R Soc Trop Med Hyg. 1997 Nov-Dec;91(6):701-703. Available at http://www.ncbi.nlm.nih.gov/pubmed/9580117. [PubMed: 9580117]

17. Jongwutiwes S, Sampatanukul P, Putaporntip C. Recurrent isosporiasis over a decade in an immunocompetent host successfully treated with pyrimethamine. Scand J Infect Dis. 2002; 34:859-862. Available at http://www.ncbi.nlm.nih.gov/pubmed/12578164. [PubMed: 12578164]

18. Dionisio D, Sterrantino G, Meli M, Leoncini F, Orsi A, Nicoletti P. Treatment of isosporiasis with combined albendazole and ornidazole in patients with AIDS. AIDS. 1996 Sep; 10(11):1301-1302. Available at http://www.ncbi.nlm.nih.gov/pubmed/8883600. [PubMed: 8883600]

19. Zulu I, Veitch A, Sianongo S, et al. Albendazole chemotherapy for AIDS-related diarrhoea in Zambia--clinical, parasitological and mucosal responses. Aliment Pharmacol Ther. 2002; 16(3): 595-601. Available at http://www.ncbi.nlm.nih.gov/entrez/query.fcgi? $\mathrm{cmd}=$ Retrieve $\& \mathrm{db}=$ PubMed\&dopt=Citation\&list_uids=11876715. [PubMed: 11876715]

20. Meyohas MC, Capella F, Poirot JL LI, Binet D, Eliaszewicz M, Frottier J. Treatment with doxycycline and nifuroxazide of Isospora belli infection in AIDS. Pathol Biol (Paris). 1990; 38:589-591. 1990. Available at http://www.ncbi.nlm.nih.gov/pubmed/2385457. [PubMed: 2385457]

21. Musey KL, Chidiac C, Beaucaire G, Houriez S, Fourrier A. Effectiveness of roxithromycin for treating Isospora belli infection. J Infect Dis. 1988 Sep.158(3):646. Available at http:// www.ncbi.nlm.nih.gov/pubmed/3411149. [PubMed: 3411149]

22. Gaska JA, Tietze KJ, Cosgrove EM. Unsuccessful treatment of enteritis due to Isospora belli with spiramycin: a case report. J Infect Dis. 1985 Dec; 152(6):1336-1338. Available at http:// www.ncbi.nlm.nih.gov/pubmed/4067332. [PubMed: 4067332]

\section{Dosing Recommendations for Prevention and Treatment of Isosporiasis (Cystoisosporiasis)}

\begin{tabular}{|l|l|l|l|}
\hline Indication & First Choice & Alternative & Comments/Special Issues \\
\hline Primary Prophylaxis & $\begin{array}{l}\text { There are no U.S. } \\
\text { recommendations for } \\
\text { primary prophylaxis of } \\
\text { isosporiasis. }\end{array}$ & N/A & $\begin{array}{l}\text { Initiation of cART to } \\
\text { avoid advanced } \\
\text { immunodeficiency may } \\
\text { reduce incidence; TMP- } \\
\text { SMX prophylaxis may } \\
\text { reduce incidence. }\end{array}$ \\
\hline
\end{tabular}




\begin{tabular}{|c|c|c|c|}
\hline Indication & First Choice & Alternative & Comments/Special Issues \\
\hline Secondary Prophylaxis & $\begin{array}{ll}\underline{\text { If Severe }} & \\
\underline{\text { Immunosuppression: }} \\
& \text { Administer } \\
& \text { TMP- } \\
& \text { SMX 2.5 } \\
\text { mg/kg } & \\
\text { body } & \\
\text { weight of } \\
\text { TMP } \\
\text { component } \\
\text { twice daily } \\
\text { by mouth } \\
\text { 3 times per } \\
\text { week }\end{array}$ & $\begin{array}{l}\text { Pyrimethamine } 1 \mathrm{mg} / \mathrm{kg} \text { body } \\
\text { weight (maximum } 25 \mathrm{mg} \text { ) plus } \\
\text { folinic acid, } 10-25 \mathrm{mg} \text { by mouth } \\
\text { once daily. } \\
\text { Second-Line Alternative: } \\
\qquad \begin{array}{l}\text { Ciprofloxacin, } 10-20 \\
\mathrm{mg} / \mathrm{kg} \text { body weight } \\
\text { given twice daily by } \\
\text { mouth } 3 \text { times per week }\end{array}\end{array}$ & $\begin{array}{l}\text { Consider discontinuing } \\
\text { secondary prophylaxis in a } \\
\text { patient receiving cART } \\
\text { after sustained } \\
\text { improvement from severe } \\
\text { immunosuppression (from } \\
\text { CDC immunologic } \\
\text { category } 3 \text { to CD4 values } \\
\text { that fall within category } 1 \\
\text { or } 2 \text { ) for longer than } 6 \\
\text { months. } \\
\text { In adults, the dose of } \\
\text { pyrimethamine for } \\
\text { secondary prophylaxis ( } 25 \\
\text { mg daily) is lower than the } \\
\text { dose for treatment (50-75 } \\
\text { mg daily), but no similar } \\
\text { data exist for children. } \\
\text { Thus, the recommended } \\
\text { dosing for secondary } \\
\text { prophylaxis in children is } \\
1 \text { mg/kg per dose } \\
\text { (maximum } 25 \text { mg) once } \\
\text { daily. } \\
\text { Ciprofloxacin is generally } \\
\text { not a drug of first choice } \\
\text { in children due to } \\
\text { increased incidence of } \\
\text { adverse events, including } \\
\text { events related to joints } \\
\text { and/or surrounding } \\
\text { tissues. }\end{array}$ \\
\hline Treatment & $\begin{array}{l}\text { TMP-SMX } 5 \mathrm{mg} / \mathrm{kg} \\
\text { body weight of TMP } \\
\text { component given } \\
\text { twice daily by mouth } \\
\text { for } 10 \text { days }\end{array}$ & $\begin{array}{l}\text { Pyrimethamine } 1 \mathrm{mg} / \mathrm{kg} \text { body } \\
\text { weight plus folinic acid 10-25 mg } \\
\text { by mouth once daily for } 14 \text { days } \\
\text { Second-Line Alternatives: } \\
\text { - } \quad \begin{array}{l}\text { Ciprofloxacin } 10-20 \\
\text { mg/kg body weight/day } \\
\text { twice daily by mouth } \\
\text { for } 7 \text { days }\end{array} \\
\text { Nitazoxanide (see doses } \\
\text { below) for } 3 \\
\text { consecutive days } \\
-\quad \text { Children } 1- \\
3 \text { years: } 100 \\
\text { mg by } \\
\text { mouth } \\
\text { every } 12 \\
\text { hours } \\
-\quad \text { Children } 4- \\
11 \text { years: } \\
200 \mathrm{mg} \text { by } \\
\text { mouth } \\
\text { every } 12 \\
\text { hours } \\
-\quad \text { Adolescents } \\
\geq 12 \text { years } \\
\text { and adults: } \\
500 \mathrm{mg} \text { by } \\
\text { mouth } \\
\text { every } 12 \\
\text { hours }\end{array}$ & $\begin{array}{l}\text { If symptoms worsen or } \\
\text { persist, the TMP-SMX } \\
\text { dose may be increased to } \\
5 \mathrm{mg} / \mathrm{kg} / \text { day given } 3-4 \\
\text { times daily by mouth for } \\
10 \text { days or the duration of } \\
\text { treatment may be } \\
\text { lengthened. Duration of } \\
\text { treatment with } \\
\text { pyrimethamine has not } \\
\text { been well established. } \\
\text { Ciprofloxacin is generally } \\
\text { not a drug of first choice } \\
\text { in children due to } \\
\text { increased incidence of } \\
\text { adverse events, including } \\
\text { events related to joints } \\
\text { and/or surrounding } \\
\text { tissues. }\end{array}$ \\
\hline
\end{tabular}

Key to Acronyms: $\mathrm{CD} 4=\mathrm{CD} 4 \mathrm{~T}$ lymphocyte; $\mathrm{CDC}=$ Centers for Disease Control and Prevention; $\mathrm{cART}=$ combination antiretroviral therapy; TMP-SMX = trimethoprim-sulfamethoxazole 
Malaria (Last updated November 6, 2013; last reviewed November 6, 2013)

Panel's Recommendations

- $\quad$ Families traveling to malaria-endemic countries should receive pre-travel counseling, including information on insecticide-treated bed nets, N,N-Diethyl-meta-toluamide, and country-specific antimalarial prophylaxis (AII).

- Trimethoprim-sulfamethoxazole is not recommended for antimalarial prophylaxis (AIII).

- Treatment of malaria is based on disease severity, patient age, parasite species, pregnancy status, and local resistance patterns where the malaria infection was acquired (AI).

- The choice of malaria therapy is not affected by HIV status but can be modified based on potential interactions between antiretroviral and antimalarial drugs (AIII). Quinidine is not recommended for patients who are taking ritonavir (AIII) (ritonavir may be replaced if quinidine is needed for severe malaria) and should be administered with caution with atazanavir, darunavir and fosamprenavir (AIII).

- The treatment options for uncomplicated chloroquine-susceptible Plasmodium falciparum malaria include chloroquine phosphate, atovaquone-proguanil, artemether-lumefantrine, and quinine sulfate plus either doxycycline, tetracycline (in children aged $\geq 8$ years), or clindamycin. Mefloquine is considered an alternative regimen (AIII).

- Chloroquine should not be used to treat malaria infections acquired in areas with chloroquine resistance (AIII).

- Treatment of uncomplicated chloroquine-resistant malaria may include atovaquone-proguanil, quinine sulfate plus either doxycycline or tetracycline (specifically in children aged $\geq 8$ years) or clindamycin or artemether-lumefantrine (AIII).

- $\quad$ Treat for presumptive chloroquine-resistant P. falciparum malaria in symptomatic patients who have traveled to a region with chloroquine-resistant $P$. falciparum and for whom reliable identification of the malaria species is not possible or who are severely ill (AIII).

- $\quad$ After initial treatment for Plasmodium vivax and Plasmodium ovale (same as for uncomplicated $P$. falciparum), primaquine is recommended for treatment of the dormant liver stage (hypnozoites) (AIII).

- Glucose-6-phosphate dehydrogenase deficiency must be excluded before use of primaquine because of risk of severe hemolytic anemia (AIII).

- Treatment of severe malaria includes both IV quinidine gluconate plus either doxycycline $\underline{\mathbf{O R}}$ clindamycin OR tetracycline. Alternatives include artesunate IV (under Investigational New Drug protocol: Contact the Centers for Disease Control and Prevention Malaria Hotline at (770) 488-7788) followed by either doxycycline $\underline{\mathbf{O R}}$ atovaquone-proguanil $\underline{\mathbf{O R}}$ mefloquine $\underline{\mathbf{O R}}$ clindamycin (AIII).

Rating of Recommendations: $\mathrm{A}=$ Strong; $\mathrm{B}=$ Moderate; $\mathrm{C}=$ Optional

Rating of Evidence: $\mathrm{I}=$ One or more randomized trials $\underline{\text { in children }}^{\dagger}$ with clinical outcomes and/or validated endpoints; $\mathrm{I}^{*}$ $=$ One or more randomized trials in adults with clinical outcomes and/or validated laboratory endpoints with accompanying data in children ${ }^{\dagger}$ from one or more well-designed, nonrandomized trials or observational cohort studies with long-term clinical outcomes; II = One or more well-designed, nonrandomized trials or observational cohort studies in children ${ }^{\dagger}$ with long-term outcomes; II* $=$ One or more well-designed, nonrandomized trials or observational studies in adults with longterm clinical outcomes with accompanying data in children ${ }^{\dagger}$ from one or more similar nonrandomized trials or cohort studies with clinical outcome data; III = Expert opinion ts

\section{Epidemiology}

Malaria is caused by the obligate, intracellular protozoa of the genus Plasmodium, and is transmitted by the bite of an infective female Anopheles mosquito. Worldwide, malaria is a leading killer of children and pregnant women. In the United States, most malaria cases occur in patients who have returned from travels to areas of endemic malaria transmission. Rarely, cases occur as a result of exposure to infected blood products, local mosquito-borne transmission (i.e., autochthonous transmission), or mother-to-child transmission (MTCT) (congenital malaria). Prompt recognition and treatment are essential, and failure to act quickly and appropriately can have grave consequences. 
In 2009, 1484 cases of malaria were reported in the United States, of which 4 were fatal. ${ }^{1}$ In the majority of cases in which species were identified, Plasmodium falciparum was the pathogen involved; however, in $38 \%$ of cases, the species was either not reported or unidentified. Lack of adherence to prophylaxis is the key identified risk factor for acquisition of malaria in those for whom data are available.

\section{High-Risk Groups}

United States-born children visiting family in malaria-endemic regions are at highest risk of malaria infection. Children of foreign citizenship, children of unknown resident status, and adopted children who come from countries of endemic malaria transmission are also at high risk. Education regarding the misconception that prior exposure to malaria confers protection against re-infection is important; families should be prepared (with malaria chemoprophylaxis) and educated with travel advice (e.g., such as recommending use of insecticidetreated nets and insect repellants) before returning to endemic areas (AII). Although some parents may assume that their children are protected from disease because of their ethnic background (from high malaria endemic countries), ${ }^{2,3,4}$ the converse is true, with patients in this group at high risk because of factors such as visiting private residences, sleeping in homes that lack screens or air conditioning, and having longer visits, all of which contribute to a higher risk of contracting malaria (http://www.cdc.gov/malaria/travelers/ vfr.html). Adults living in the United States but born in malaria-endemic areas often believe they are not susceptible to malaria because of naturally acquired immunity. Such acquired immunity develops after age 5 years in people who reside in areas of stable malaria transmission, but it is partial (providing relative protection against disease, not infection), wanes quickly once people are no longer living in malaria-endemic areas, and may not be present in HIV-infected populations with advanced immunodeficiency. Therefore, both adults and children living in the United States who were born in malaria-endemic areas should be prescribed the same prophylaxis as any other patients traveling to malariaendemic areas.

\section{Prevention Recommendations}

Recommendations for preventing exposure and for primary chemoprophylaxis are identical for HIV-infected and HIV-uninfected individuals (see http://www.cdc.gov/malaria/travelers/ index.html). All travelers to malaria-endemic regions should receive pre-travel counseling on appropriate chemoprophylaxis and avoidance of mosquitos (AII). ${ }^{4,5}$ Families should be counseled regarding signs and symptoms of malaria and the need for early medical intervention if these signs and symptoms are present. An early appropriate medical evaluation should be completed on all patients returning from a malaria-endemic area who have unexplained fever or other signs or symptoms of malaria.

Preventing Exposure-All travelers should use personal protective measures to prevent mosquito bites when traveling to malariaendemic areas (AII), ${ }^{6}$ including sleeping under an insecticide-treated bed net and wearing clothing impregnated with permethrin (effective for weeks and through several washings, but not dry cleaning). Discussions regarding the routine use of bed nets should be individualized as per specific sleeping arrangements (airconditioned hotel vs. open windows). Long-acting N,N-Diethyl-meta-toluamide (DEET) 
mosquito repellents are safe, practical, and effective, and the duration of protection increases with increasing DEET concentrations, plateauing between $30 \%$ and 50\%. DEET should be applied (by patients or their caregivers when appropriate) to skin, but not to wounds, cuts, irritated areas, the mouth, or hands of young children (AIII). Additional information about other recommended mosquito repellants can be found at http://www.cdc.gov/ncidod/dvbid/ westnile/qa/insect_repellent.htm.

Depending on the level of risk, it may be appropriate to recommend to travelers no specific interventions, mosquito-avoidance measures only, or mosquito-avoidance measures plus chemoprophylaxis (Centers for Disease Control and Prevention [CDC] Yellow book; http:// wwwnc.cdc.gov/travel/yellowbook/2012/chapter-3-infectious-diseases-related-to-travel/ malaria.htm). Pregnant women should discuss travel to endemic areas with a travel medicine expert.

Primary Chemoprophylaxis-Primary chemoprophylaxis should be prescribed to all individuals traveling to malaria-endemic areas, regardless of ethnicity or prior exposure to or illness with malaria. Antimalarial medications may need special preparation, and some are not easily delivered to children. Therefore, families planning to travel to malaria-endemic areas are advised to visit a travel medicine specialist with training and experience in pediatrics at least 2 weeks before departure (AII). If that is not possible, families can still see a travel medicine specialist up to the day of departure, because some antimalarial prophylaxis regimens can still be prescribed and effectively used even at that late date.

For patients traveling to areas with chloroquine-sensitive malaria, chloroquine phosphate (5 $\mathrm{mg} / \mathrm{kg}$ body weight base, up to 300-mg base) given once weekly is acceptable. Other acceptable choices include primaquine, atovaquone/proguanil, doxycycline, and mefloquine. For travelers to areas with mainly Plasmodium vivax, primaquine is a very good option. Travellers who will be given primaquine should have glucose-6-phosphate dehydrogenase (G6PD) testing before this medication is started. Travelers to areas with chloroquineresistant malaria should take atovaquone/proguanil daily (dosed on a sliding scale by weight bands), or daily doxycycline ( $2.2 \mathrm{mg} / \mathrm{kg}$ body weight for children aged $\geq 8$ years) or weekly mefloquine, dosed based on weight. Medications for prophylaxis should be started before leaving and continued after returning from travel, as per their specific schedule. Trimethoprim-sulfamethoxazole (TMP-SMX) is not a surrogate for antimalarial prophylaxis, and is not recommended as effective prophylaxis for malaria (AIII). Although TMP-SMX prophylaxis appears to reduce episodes of clinical malaria to varying degrees, with the already almost universal resistance to sulfadoxine pyrimethamine, it is extremely unlikely that TMP-SMX would be useful alone as primary prophylaxis. ${ }^{7}$

Discontinuing Primary Prophylaxis-Travel-related chemoprophylaxis with chloroquine, mefloquine, or doxycycline usually should be continued for 4 weeks after departure from a malaria-endemic area because these drugs are not effective against malarial parasites developing in the liver and kill the parasite only once it has emerged to infect the red blood cells. Atovaquone-proguanil and primaquine may be discontinued 1 week after departure from malaria-endemic areas. 
Clinical and Laboratory Manifestations-HIV increases the frequency and severity of clinical malaria episodes in more severely immunosuppressed adults, pregnant women, and older children, possibly reflecting HIV-mediated interference with acquisition of malaria immunity, but not related to failure of initial antimalarial therapy. ${ }^{7,8}$ In young children, there is no clear evidence that HIV infection is associated with more severe malaria disease, although one case-control study in Uganda found an association between HIV infection and cerebral malaria in children. ${ }^{9}$

In a case series of returning travelers, symptoms most commonly reported include fever $(100 \%)$, headache $(100 \%)$, weakness $(94 \%)$, profuse night sweats $(91 \%)$, insomnia (69\%), arthralgias (59\%), myalgias (56\%), diarrhea (13\%), and abdominal cramps (8\%). ${ }^{10}$ Patients may also have pallor, hepatosplenomegaly, or jaundice. Altered consciousness or seizures may indicate progression to severe malaria. Splenic rupture can be a rare presentation of malaria, requiring urgent medical and surgical management. Rash, lymphadenopathy, and signs of pulmonary consolidation are not characteristic of malaria. Laboratory values may include anemia; high, normal, or low neutrophil counts; normal or low platelets; low sodium (usually because of syndrome of inappropriate antidiuretic hormone secretion and/or dehydration); lactic acidosis; renal insufficiency, increased creatinine, proteinuria, and hemoglobinuria; and elevated lactate dehydrogenase. ${ }^{11,12}$ Severe malaria may present before severe anemia (hemoglobin $<7 \mathrm{~g} / \mathrm{dL}$ ) is documented.

Although fever is often the most common clinical presentation of malaria in people coming from areas of endemic malaria transmission, it is not uniformly present in children. Nonspecific clinical findings often predominate in children and clinical diagnosis in them can be difficult. Malaria fever patterns in children also often do not follow the classically described tertian or quartan patterns described in adults. ${ }^{13,14}$ Children more often present with hepatomegaly, jaundice, or splenomegaly than do adults. They are also more likely to have fever $>40^{\circ} \mathrm{C}$ and may present with febrile convulsions. Laboratory findings may include low serum glucose (seen with falciparum malaria), whereas serum glucose measurements in adults may be normal. Children who have severe malaria also may have concomitant bacteremia/sepsis. ${ }^{2,11,12}$ In returning travelers, when children are diagnosed with malaria, their siblings might present with malaria at the same time. ${ }^{2}$

Splenomegaly, fever, and thrombocytopenia are highly specific for malaria in immigrant children and need appropriate evaluation. ${ }^{13,15}$ Congenital malaria is rare but should be considered in febrile neonates whose mothers migrated from areas where malaria is endemic; however, empiric therapy should not be administered without a confirmed diagnosis. ${ }^{13} \mathrm{HIV} /$ malaria coinfection during pregnancy has been shown to have additional detrimental effects on maternal and infant survival and to confer increased risk of MTCT of both HIV and malaria. ${ }^{16}$

\section{Diagnosis}

For early and prompt recognition of malaria, physicians must obtain a complete travel history from every febrile patient and maintain a high index of suspicion for malaria in travelers returning from areas of endemic malaria, remembering that signs and symptoms also can vary depending on chemoprophylaxis and prior partial treatment for malaria (see 
Table 7 from ${ }^{17}$ for list of resources or http://wwwnc.cdc.gov/travel/destinations/list.htm). Children who have recently migrated from regions where malaria is endemic should be evaluated for malarial infection upon arrival and/or if they become ill after arriving in the United States. A Giemsa-stained thick blood smear is the most sensitive smear technique for detecting infection, whereas a thin blood smear is used for determination of parasite species and burden (for an example of malaria parasites on smear, please visit http:// www.dpd.cdc.gov/dpdx/HTML/Image_Library.htm). Smear accuracy depends upon proper preparation and interpretation of thick and thin smears by experienced laboratory personnel. ${ }^{17}$ Because symptoms can develop before parasitemia is detectable in a nonimmune person, the initial blood-smear examination may be misleadingly negative. Blood smears should be obtained every 12 to 24 hours for a total of 3 sets to fully evaluate for malaria; if all 3 sets are negative, the probability of malaria is extremely low. In all patients in whom malaria is suspected, smears should be read immediately. A qualified person who can perform and read smears should always be available, even at off-hours. Every effort should be made to establish a diagnosis before therapy is initiated. However, if severe malaria is strongly suspected and diagnostic interpretation is not readily available, empiric intravenous therapy for presumed $P$. falciparum infection should be initiated, with a blood smear preserved for reading as soon as possible. Consultation and aid in the initial diagnosis, speciation, and treatment plan is available via the CDC Malaria Hotline at (770) 488-7788 (Monday-Friday, 9 a.m.-5 p.m., eastern time. For emergency consultation after hours, call (770) 488-7100, and ask to speak with a CDC Malaria Branch clinician).

Performance of rapid diagnostic tests (RDTs) varies greatly, and only one test (Binax) currently is Food and Drug Administration (FDA)-approved. Such tests may have limited usefulness early in infection because their sensitivity is decreased with lower parasite density (see http://www.wpro.who.int/sites/rdt/who_rdt_evaluation/). However, if microscopy is not immediately available, these tests can be used to aid in establishing a diagnosis of malaria. Microscopy must still be performed on all suspected cases of malaria, despite positive and negative RDTs, for confirmation.

Malaria in the United States is a reportable disease. Directions on case definitions and reporting can be found at http://www.cdc.gov/malaria/report.html.

\section{Treating Disease}

Chemoprophylaxis is not completely effective, and malaria should be included in the differential diagnosis of fever or other signs or symptoms consistent with malaria in anyone who traveled to malaria-endemic areas during the previous 12 months (see http:// www.cdc.gov/immigrantrefugeehealth/guidelines/overseas/malaria-guidelinesoverseas.html\#sect2). Malaria medications purchased in sub-Saharan Africa or Southeast Asia may be counterfeit; therefore, the index of suspicion must remain high when evaluating children with fever coming from endemic areas, regardless of prior history of antimalarial therapy.

CDC recommends presumptive treatment for malaria for all refugees and adoptees resettling to the United States from sub-Saharan Africa, including those who were treated for malaria before departing from Africa but who did not receive primaquine for treatment of dormant 
liver stage forms (hypnozoites) of Plasmodium ovale and P. vivax infection. These patients remain at risk of developing malaria after arrival in the United States and should be evaluated with a high index of suspicion for malaria. Children with past or current $P$. vivax or $P$. ovale infection should receive treatment with primaquine to eradicate the dormant liver stage, if the drug was not previously administered (see CDC Guidance located at http:// www.cdc.gov/malaria/resources/pdf/treatmenttable.pdf).

Treatment of malaria is based on the disease severity, patient age at onset, parasite species, pregnancy status, and known resistance patterns in the area where the malaria infection was acquired (AI). Drug dosing for pediatric patients must be adjusted for weight, and dosing should never exceed the recommended adult dose. Recommendations for treatmentincluding drug dosing in HIV-infected children and adolescents with malaria-by species are described below and summarized in Table 1, and can also be found at http:// www.cdc.gov/malaria/diagnosis_treatment/treatment.html. Additional information can be found at http://www.malaria.org/ABOUT\%20MALARIA/Treatment\%20of\%20MalariaGuidelines\%20for\%20clinicians\%20WHO.pdf for further clinical guidance.

HIV infection status does not affect choice or dosing of antimalarial therapy. However, choice of antimalarial therapy may be affected by interactions between antiretroviral (ARV) and antimalarial drugs; clinicians are urged to evaluate for drug interactions before initiating antimalarial therapy (please see Drug Interactions section below).

Unknown Species-Clinicians should always treat patients who traveled to a region in which chloroquine-resistant $P$. falciparum malaria is present for chloroquine-resistant $P$. falciparum malaria if reliable identification of the malaria species is not possible or the patient is severely ill (AIII).

Uncomplicated Malaria-Uncomplicated malaria is defined by the World Health Organization as "symptomatic infection with malaria parasitemia without signs of severity and/or evidence of vital organ dysfunction." 18 The preferred treatment options for uncomplicated malaria include chloroquine phosphate (if chloroquine-susceptible), atovaquoneproguanil, artemether-lumefantrine, or quinine sulfate plus a second medicine (either tetracycline, doxycycline [in children aged $\geq 8$ years] or clindamycin) (see Dosing Table for details) (AI). Mefloquine also can be used for treatment, but has a higher rate of side effects (AIII). Primaquine also must be administered for radical cure of $P$. vivax and $P$. ovale infection. G6PD deficiency must be excluded before first use of primaquine because of the risk of severe hemolytic anemia. Primaquine should not be used in pregnant women because the presence of G6PD deficiency cannot be determined in the unborn child (AIII).

Severe Malaria-Severe malaria is defined as acute malaria "with signs of severity and/or evidence of vital organ dysfunction" 18 and is most often caused by $P$. falciparum, but can also be caused by $P$. vivax. Mixed infections can also occur. These signs, symptoms, and laboratory parameters include diminished consciousness or seizures, respiratory distress (acute respiratory distress syndrome [ARDS], Kussmaul's respiration), prostration, hyperparasitemia ( $>5 \%$ ), severe anemia (hemoglobin $<7 \mathrm{~g} / \mathrm{dL}$ ), hypoglycemia, jaundice/ icterus, renal insufficiency, hemoglobinuria, shock, cessation of eating and drinking, 
repetitive vomiting, or hyperpyrexia. Cerebral malaria is usually defined by presence of coma (Glasgow coma scale $<11$, Blantyre coma scale $<3$ ). Severe malaria can present long before hemoglobin goes below the $7 \mathrm{mg} / \mathrm{dL}$ threshold because of the hemo-concentrating effects of dehydration.

Patients diagnosed with severe malaria should be treated aggressively with intravenous (IV) antimalarial therapy. The only FDA-approved regimen includes quinidine gluconate plus one of the following: doxycycline, tetracycline, or clindamycin. A promising ${ }^{19}$ alternative parenteral therapy is IV artesunate (available under Investigational New Drug protocol from $\mathrm{CDC}$ for certain patients meeting criteria). Additional alternative therapies include atovaquone-proguanil, clindamycin, mefloquine, or (for children aged $\geq 8$ years) doxycycline. Treatment with IV quinidine or artesunate should be initiated as soon as possible after the diagnosis has been made. Patients with severe malaria treated with quinidine should be given an IV loading dose unless they have received more than $40 \mathrm{mg} / \mathrm{kg}$ body weight of quinine in the preceding 48 hours or if they have received mefloquine within the preceding 12 hours. Consultation with a cardiologist and a physician with experience treating malaria is advised when treating malaria patients with quinidine because of the known complications of quinidine, including widening of the QRS complex and/or lengthening of the QTc interval. Cardiac complications, if severe, may warrant temporary discontinuation of the drug or slowing of the IV infusion. IV quinidine administration should not be delayed for an exchange transfusion and can be given concurrently throughout it.

Exchange transfusion should be considered (BII) only for treatment of very severe malaria when children have a parasite density of more than $10 \%$ and if complications such as cerebral malaria, ARDS or renal complications exist. The risks of exchange transfusion include fluid overload, febrile and allergic reactions, metabolic disturbances (e.g., hypocalcaemia), red blood cell alloantibody sensitization, blood-borne transmissible infection, and line sepsis. ${ }^{20-22}$ The parasite density should be monitored every 12 hours until it falls below 1\%, which usually requires the exchange of 8 to 10 units of blood in adults.

Malaria Despite Chemoprophylaxis-Medication used for chemoprophylaxis should not be used as a part of a new treatment regimen in individuals who develop malaria despite taking chemoprophylaxis; rather, treatment with one of the other options is recommended.

\section{Drug Interactions}

There are multiple potential interactions between ARV and antimalarial drugs, but data from HIV-infected children and adults remain limited. ${ }^{7,23-25}$ Many antimalarials are metabolized by cytochrome p450 enzymes, while certain non-nucleoside reverse transcriptase inhibitors (NNRTIs) and protease inhibitors (PIs) either inhibit or induce cytochrome p450 enzymes. ${ }^{26-28}$ Tetracyclines have no clinically significant interactions expected with PIs or NNRTIs. Atovaquone is not expected to have any significant interaction with common nucleoside reverse transcriptase inhibitors, although no data are available for proguanil. Ritonavir inhibits quinidine metabolism; therefore, concomitant administration of ritonavir (including co-formulated products like lopinavir/ritonavir that contain ritonavir) and 
quinidine is not recommended. Replacement of ritonavir in ritonavir-containing cART should be considered. The inhibitory action of ritonavir will still be present for several days after dosing is interrupted; thus, in patients with severe malaria already on ritonavir, artesunate should be considered. Caution is also advised before co-administering quinidine with other PIs (including atazanavir, darunavir, and fosamprenavir).

Other drug-drug interactions exist but have not been studied. The CDC Malaria Hotline is an excellent resource for additional assistance with drug-drug interactions, as are the World Health Organization's Guidelines for the Treatment of Malaria (http://whqlibdoc.who.int/ publications/2010/9789241547925_eng.pdf). An interactive web-based resource for checking on drug interactions involving ARV drugs is found at the University of Liverpool website http://www.hiv-druginteractions.org.

Potential Clinically Relevant Interactions between Antimalarial and Antiretroviral Drugs*

\begin{tabular}{|c|c|c|c|}
\hline Antimalarial Drug & Protease Inhibitors & NRTI & NNRTI \\
\hline Quinine & $\begin{array}{l}\text { PIs: increase } \\
\text { quinine levels }\end{array}$ & No available data & $\begin{array}{l}\text { Efavirenz, } \\
\text { Nevirapine: } \\
\text { reduces } \\
\text { quinine levels }\end{array}$ \\
\hline Atovaquone/Proguanil & $\begin{array}{l}\text { Lopinavir/ } \\
\text { Ritonavir:, } \\
\text { Atazanavir/ } \\
\text { Ritonavir reduces } \\
\text { atovaquone and } \\
\text { proguanil levels }\end{array}$ & & $\begin{array}{l}\text { Efavirenz } \\
\text { reduces } \\
\text { atovaquone } \\
\text { and proguanil } \\
\text { levels }\end{array}$ \\
\hline Mefloquine & $\begin{array}{l}\text { Ritonavir reduces } \\
\text { ritonavir levels }\end{array}$ & & $\begin{array}{l}\text { Efavirenz, } \\
\text { Nevirapine: } \\
\text { reduces } \\
\text { mefloquine } \\
\text { levels }\end{array}$ \\
\hline Lumefantrine, Halofantrine & $\begin{array}{l}\text { PIs: increase } \\
\text { lumefantrine or } \\
\text { halofantrine levels, } \\
\text { which can prolong } \\
\text { QT interval }\end{array}$ & & $\begin{array}{l}\text { Efavirenz, } \\
\text { Nevirapine: } \\
\text { increases } \\
\text { lumefantrine } \\
\text { or halofantrine } \\
\text { levels, which } \\
\text { can prolong } \\
\text { QT interval }\end{array}$ \\
\hline Amodiaquine plus Artesunate & & & $\begin{array}{l}\text { Efavirenz: } \\
\text { increases } \\
\text { amodiaquine } \\
\text { concentration } \\
\text { which can } \\
\text { increase } \\
\text { hepatic } \\
\text { toxicity; do } \\
\text { not co- } \\
\text { administer }\end{array}$ \\
\hline Chloroquine, Pyrimethamine, Sulfadoxine-Pyrimethamine & $\begin{array}{l}\text { Ritonavir: alters } \\
\text { anti-malarial drug } \\
\text { metabolism, may } \\
\text { increase } \\
\text { chloroquine levels }\end{array}$ & & \\
\hline Sulfadoxine-Pyrimethamine & & $\begin{array}{l}\text { Zidovudine: } \\
\text { possibly } \\
\text { increases risk of } \\
\text { anemia }\end{array}$ & $\begin{array}{l}\text { Nevirapine: } \\
\text { possibly } \\
\text { increases } \\
\text { adverse skin or } \\
\text { liver adverse } \\
\text { reactions; do }\end{array}$ \\
\hline
\end{tabular}




\begin{tabular}{|l|l|l|l|}
\hline Antimalarial Drug & Protease Inhibitors & NRTI & NNRTI \\
\hline Artemisinin & & & $\begin{array}{l}\text { not start both } \\
\text { drugs } \\
\text { simultaneously }\end{array}$ \\
\hline Dapsone & $\begin{array}{l}\text { PIs: alter } \\
\text { artemisinin } \\
\text { metabolism }\end{array}$ & & $\begin{array}{l}\text { Nevirapine: } \\
\text { may decrease } \\
\text { artemisinin } \\
\text { levels }\end{array}$ \\
\hline
\end{tabular}

Key to Acronyms: NRTI=nucleoside reverse transcriptase inhibitor; NNRTI=non-nucleoside reverse transcriptase inhibitor; $\mathrm{PI}=$ protease inhibitor *

Modified from: Flateau, C., G. Le Loup, et al. Consequences of HIV infection on malaria and therapeutic implications: a systematic review. Lancet Infect Dis. 2011. 11(7);541-556.

Special Populations-Because primaquine is not routinely prescribed for immigrants as part of a post-treatment/pre-departure regimen, patients who may have had $P$. vivax or $P$. ovale infection in the past would be at continued risk of developing malaria months to years after arrival in the United States. Presumptive treatment on arrival (preferable) or laboratory screening to detect Plasmodium infection is recommended for refugees originating in subSaharan Africa who have not received pre-departure therapy with a recommended regimen (see http://www.cdc.gov/immigrantrefugeehealth/guidelines/domestic/malaria-guidelinesdomestic.html).

Monitoring and Adverse Events (Including IRIS)—Severe malaria commonly induces hypoglycemia in children, especially when treated with IV quinine/quinidine because of inhibition of gluconeogenesis and induction of endogenous insulin production. Therefore, monitoring glucose levels and use of a glucose-containing crystalloid solution for fluid maintenance is prudent until IV quinine/quinidine therapy has been completed. Monitoring glucose is especially important for children with altered mental status. Cardiac and intensive-care monitoring is also recommended because IV quinine/quinidine can cause hypotension and widening of the QRS interval. Quinine toxicity, a cluster of symptoms that includes tinnitus, dizziness, disorientation, nausea, visual changes, and auditory deficits, can occur. Many of the adverse events associated with quinine are dose-related, and because of age-related differences in the rate at which quinine is eliminated from the body, the frequency and severity of adverse effects associated with quinine drug products may be lower in children. Tinnitus alone, a common (50\%-75\%) adverse reaction to both oral and IV quinine, usually resolves after treatment. Use of mefloquine at treatment doses may be associated with neuropsychiatric symptoms. Following antimalarial therapy, HIV-infected children should be monitored closely for hematologic complications (especially anemia and neutropenia), which are more frequent because of both the direct hematologic effects of HIV infection and of HIV treatment with other bone-marrow-suppressive drugs such as TMPSMX and zidovudine. Immune reconstitution inflammatory syndrome caused by malaria has not been reported.

Managing Treatment Failure-Failure of treatment for $P$. falciparum is uncommon in children who receive a full course of appropriate antimalarial therapy. Patients should be 
monitored for clinical and laboratory response (thick and thin smear) and for signs of recrudescence after therapy completion. Relapse of $P$. vivax and $P$. ovale can occur from the dormant (hypnozoite) liver form but is less common following primaquine treatment. When treatment failure occurs, malaria speciation should be confirmed, as should the geography of where the malaria was acquired. Retreatment with an appropriate first-line regimen (but not the same regimen as initially used) should be given. Discussion with a Pediatric Infectious Disease specialist or consultation through the CDC malaria hotline is appropriate when complex situations arise.

Preventing Recurrence-Except for re-activation of $P$. vivax and $P$. ovale hypnozoites, malaria once successfully treated does not recur, unless re-exposure and re-infection occur. One or even several episodes of malaria infection does not imply protective immunity, and continued exposure to malaria parasites can result in repeated infection, which should be treated as aggressively as the initial event.

\section{References}

1. Mali S, Tan KR, Arguin PM, et al. Malaria surveillance-United States, 2009. MMWR Surveill Summ. 2011 Apr 22; 60(3):1-15. Available at http://www.ncbi.nlm.nih.gov/pubmed/21508921. [PubMed: 21508921]

2. Ladhani S, Aibara RJ, Riordan FA, Shingadia D. Imported malaria in children: a review of clinical studies. Lancet Infect Dis. 2007 May; 7(5):349-357. Available at http://www.ncbi.nlm.nih.gov/ pubmed/17448938. [PubMed: 17448938]

3. Bradley D, Warhurst D, Blaze M, Smith V. Malaria imported into the United Kingdom in 1992 and 1993. Commun Dis Rep CDR Rev. 1994 Dec 9; 4(13):R169-R172. Available at http:// www.ncbi.nlm.nih.gov/pubmed/7531566. [PubMed: 7531566]

4. Hill DR, Ericsson CD, Pearson RD, et al. The practice of travel medicine: guidelines by the Infectious Diseases Society of America. Clin Infect Dis. 2006 Dec 15; 43(12):1499-1539. Available at http://www.ncbi.nlm.nih.gov/pubmed/17109284. [PubMed: 17109284]

5. Stauffer WM, Kamat D, Magill AJ. Traveling with infants and children. Part IV: insect avoidance and malaria prevention. J Travel Med. 2003 Jul-Aug;10(4):225-240. Available at http:// www.ncbi.nlm.nih.gov/pubmed/12946301. [PubMed: 12946301]

6. Bacaner N, Stauffer B, Boulware DR, Walker PF, Keystone JS. Travel medicine considerations for North American immigrants visiting friends and relatives. JAMA. 2004; 291:2856-2864. 2004. Available at http://www.ncbi.nlm.nih.gov/pubmed/15199037. [PubMed: 15199037]

7. Flateau C, Le Loup G, Pialoux G. Consequences of HIV infection on malaria and therapeutic implications: a systematic review. Lancet Infect Dis. 2011 Jul; 11(7):541-556. Available at http:// www.ncbi.nlm.nih.gov/pubmed/21700241. [PubMed: 21700241]

8. Achan J, Gasasira AF, Aweeka F, Havlir D, Rosenthal PJ, Kamya AR. Prophylaxis and treatment of malaria in HIV-infected populations. Future HIV Ther. 2008; 2(5):453-464. Available at http:// www.futuremedicine.com/doi/abs/10.2217/17469600.2.5.453.

9. Imani PD, Musoke P, Byarugaba J, Tumwine JK. Human immunodeficiency virus infection and cerebral malaria in children in Uganda: a case-control study. BMC Pediatr. 2011; 11:5. Available at http://www.ncbi.nlm.nih.gov/pubmed/21235797. [PubMed: 21235797]

10. Jelinek T, Nothdurft HD, Loscher T. Malaria in Nonimmune Travelers: A Synopsis of History, Symptoms, and Treatment in 160 Patients. J Travel Med. 1994 Dec 1; 1(4):199-202. Available at http://www.ncbi.nlm.nih.gov/pubmed/9815339. [PubMed: 9815339]

11. Mandell GL, Bennett JE, Dolin R. Elsevier. Malaria Chapter. Principles and Practices of Infectious Diseases (7th edition). 2011

12. Taylor SM, Molyneux ME, Simel DL, Meshnick SR, Juliano JJ. Does this patient have malaria? JAMA. 2010 Nov 10; 304(18):2048-2056. Available at http://www.ncbi.nlm.nih.gov/pubmed/ 21057136. [PubMed: 21057136] 
13. Skarbinski J, James EM, Causer LM, et al. Malaria surveillance-United States, 2004. MMWR Surveill Summ. 2006 May 26; 55(4):23-37. Available at http://www.ncbi.nlm.nih.gov/pubmed/ 16723971. [PubMed: 16723971]

14. Shingadia D, Shulman ST. Recognition and management of imported malaria in children. Seminars in Pediatr Infect Dis. 2000; 11(3):172-177.

15. Maroushek SR, Aguilar EF, Stauffer W, Abd-Alla MD. Malaria among refugee children at arrival in the United States. Pediatr Infect Dis J. 2005 May; 24(5):450-452. Available at http:// www.ncbi.nlm.nih.gov/pubmed/15876946. [PubMed: 15876946]

16. Ticconi C, Mapfumo M, Dorrucci M, et al. Effect of maternal HIV and malaria infection on pregnancy and perinatal outcome in Zimbabwe. J Acquir Immune Defic Syndr. 2003 Nov 1; 34(3): 289-294. Available at http://www.ncbi.nlm.nih.gov/pubmed/14600573. [PubMed: 14600573]

17. Mali S, Steele S, Slutsker L, Arguin PM. Centers for Disease C, Prevention. Malaria surveillance United States, 2008. MMWR Surveill Summ. 2010 Jun 25; 59(7):1-15. Available at http:// www.ncbi.nlm.nih.gov/pubmed/20577158. [PubMed: 20577158]

18. World Health Organization. Guidelines for the Treatment of Malaria. Second Edition2010. Available at http://whqlibdoc.who.int/publications/2010/9789241547925_eng.pdf

19. Dondorp A, Nosten F, Stepniewska K, Day N, White N. South East Asian Quinine Artesunate Malaria Trial g. Artesunate versus quinine for treatment of severe falciparum malaria: a randomised trial. Lancet. 2005; 366(9487):717-725. Aug 27-Sep-2 Available at http:// www.ncbi.nlm.nih.gov/pubmed/16125588. [PubMed: 16125588]

20. van Genderen PJ, Hesselink DA, Bezemer JM, Wismans PJ, Overbosch D. Efficacy and safety of exchange transfusion as an adjunct therapy for severe Plasmodium falciparum malaria in nonimmune travelers: a 10-year single-center experience with a standardized treatment protocol. Transfusion. 2010 Apr; 50(4):787-794. Available at http://www.ncbi.nlm.nih.gov/pubmed/ 19951317. [PubMed: 19951317]

21. Gulprasutdilog S, Chongkolwatana V, Buranakitjaroen P, Jaroonvesama N. Exchange transfusion in severe falciparum malaria. J Med Assoc Thai. 1999 Jan; 82(1):1-8. Available at http:// www.ncbi.nlm.nih.gov/pubmed/10087731. [PubMed: 10087731]

22. Shanbag P, Juvekar M, More V, Vaidya M. Exchange transfusion in children with severe falciparum malaria and heavy parasitaemia. Ann Trop Paediatr. 2006 Sep; 26(3):199-204. Available at http://www.ncbi.nlm.nih.gov/pubmed/16925956. [PubMed: 16925956]

23. Fehintola FA, Akinyinka OO, Adewole IF, Maponga CC, Ma Q, Morse GD. Drug interactions in the treatment and chemoprophylaxis of malaria in HIV infected individuals in sub Saharan Africa. Curr Drug Metab. 2011 Jan; 12(1):51-56. Available at http://www.ncbi.nlm.nih.gov/pubmed/ 21222586. [PubMed: 21222586]

24. Tseng A, Foisy M. Important Drug-Drug Interactions in HIV-Infected Persons on Antiretroviral Therapy: An Update on New Interactions Between HIV and Non-HIV Drugs. Curr Infect Dis Rep. 2012 Feb; 14(1):67-82. Available at http://www.ncbi.nlm.nih.gov/pubmed/22125049. [PubMed: 22125049]

25. Kredo T, Mauff K, Van der Walt JS, et al. Interaction between artemether-lumefantrine and nevirapine-based antiretroviral therapy in HIV-1-infected patients. Antimicrob Agents Chemother. 2011 Dec; 55(12):5616-5623. Available at http://www.ncbi.nlm.nih.gov/pubmed/21947399. [PubMed: 21947399]

26. Asimus S, Elsherbiny D, Hai TN, et al. Artemisinin antimalarials moderately affect cytochrome P450 enzyme activity in healthy subjects. Fundam Clin Pharmacol. 2007 Jun; 21(3):307-316. Available at http://www.ncbi.nlm.nih.gov/pubmed/17521300. [PubMed: 17521300]

27. Dooley KE, Flexner C, Andrade AS. Drug interactions involving combination antiretroviral therapy and other antiinfective agents: repercussions for resource-limited countries. J Infect Dis. 2008 Oct 1; 198(7):948-961. Available at http://www.ncbi.nlm.nih.gov/pubmed/18713054. [PubMed: 18713054]

28. Khoo S, Back D, Winstanley P. The potential for interactions between antimalarial and antiretroviral drugs. AIDS. 2005 Jul 1; 19(10):995-1005. Available at http:// www.ncbi.nlm.nih.gov/pubmed/15958830. [PubMed: 15958830] 


\section{Dosing Recommendations for Prevention and Treatment of Malaria}

\begin{tabular}{|c|c|c|}
\hline Indication & First Choice & Comments/Special Issues \\
\hline Primary Prophylaxis & 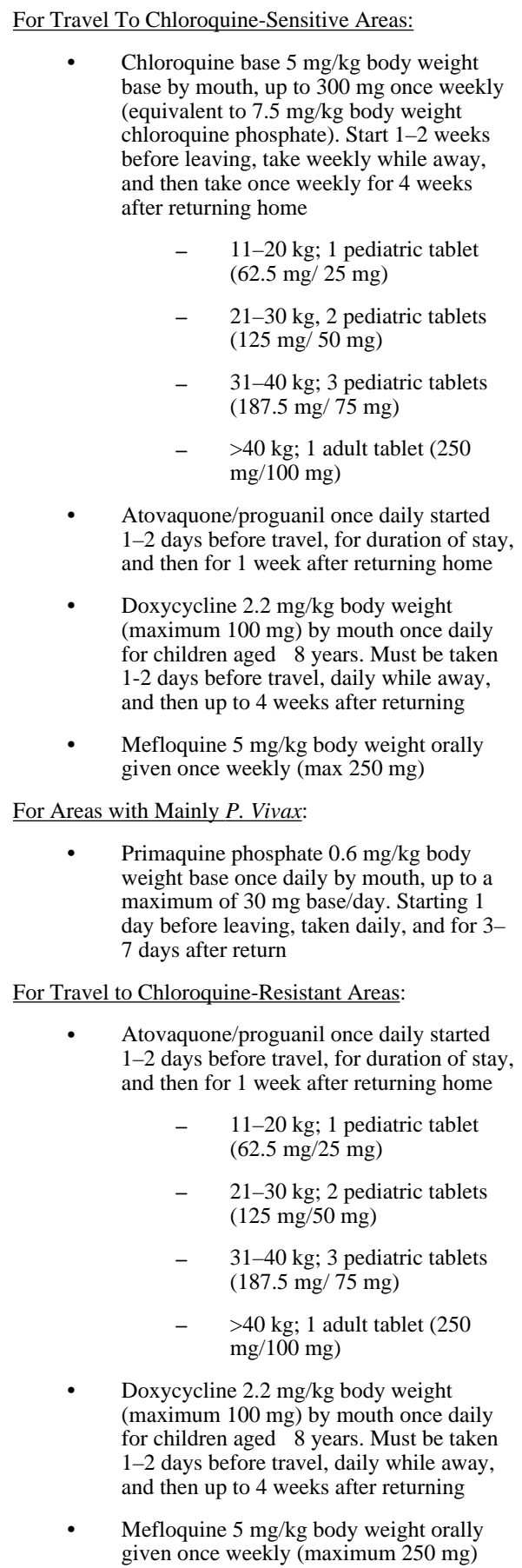 & $\begin{array}{l}\text { Recommendations are the same } \\
\text { for HIV-infected and HIV- } \\
\text { uninfected children. Please refer } \\
\text { to the following website for the } \\
\text { most recent recommendations } \\
\text { based on region and drug } \\
\text { susceptibility: http:// } \\
\text { www.cdc.gov/malaria/ } \\
\text { For travel to chloroquine- } \\
\text { sensitive areas. Equally } \\
\text { recommended options include } \\
\text { chloroquine, atovaquone/ } \\
\text { proguanil, doxycycline (for } \\
\text { children aged } \ 8 \text { years), and } \\
\text { mefloquine; primaquine is } \\
\text { recommended for areas with } \\
\text { mainly } P \text {. vivax. } \\
\text { G6PD screening must be } \\
\text { performed prior to primaquine } \\
\text { use. } \\
\text { Chloroquine phosphate is the } \\
\text { only formulation of chloroquine } \\
\text { available in the United States; } \\
10 \mathrm{mg} \text { of chloroquine phosphate } \\
=6 \mathrm{mg} \text { of chloroquine base. } \\
\text { For travel to chloroquine- } \\
\text { resistant areas, preferred drugs } \\
\text { are atovaquone/proguanil, } \\
\text { doxycycline (for children aged } \\
\geq 8 \text { years) or mefloquine. }\end{array}$ \\
\hline Secondary Prophylaxis & $\begin{array}{l}\text { For } P \text {. vivax or } P \text {. ovale: } \\
\begin{array}{l}\text { Primaquine } 0.5 \mathrm{mg} / \mathrm{kg} \text { base }(0.8 \mathrm{mg} / \mathrm{kg} \\
\text { salt) up to adult dose orally, daily for } 14\end{array}\end{array}$ & $\begin{array}{l}\text { This regimen, known as PART, } \\
\text { is recommended only for } \\
\text { individuals who have resided in } \\
\text { a malaria-endemic area for an } \\
\text { extended period of time. Adult }\end{array}$ \\
\hline
\end{tabular}




\begin{tabular}{|c|c|c|}
\hline Indication & First Choice & Comments/Special Issues \\
\hline & $\begin{array}{l}\text { days after departure from the malarious } \\
\text { area }\end{array}$ & $\begin{array}{l}\text { dose: } 30 \mathrm{mg} \text { base }(52.6 \mathrm{mg} \text { salt }) \\
\text { orally, daily for } 14 \text { days after } \\
\text { departure from the malarious } \\
\text { area. } \\
\text { http://wwwnc.cdc.gov/travel/ } \\
\text { yellowbook/2012/chapter-3- } \\
\text { infectious-diseases-related-to- } \\
\text { travel/malaria.htm\#1939 }\end{array}$ \\
\hline Treatment & 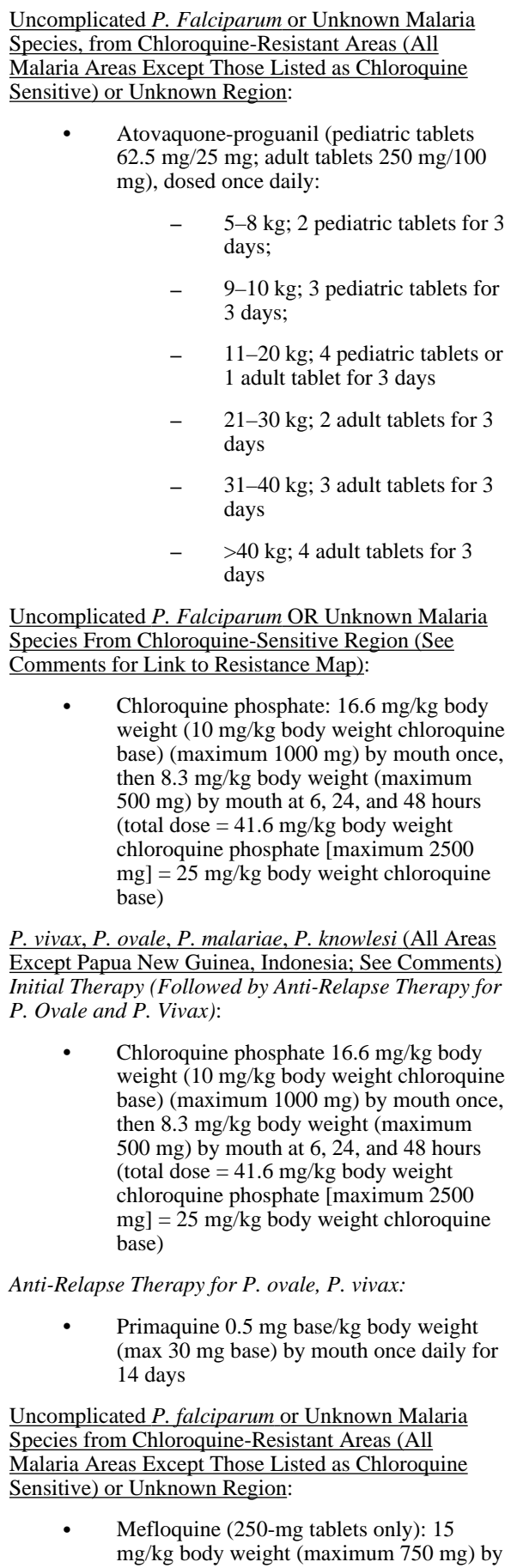 & 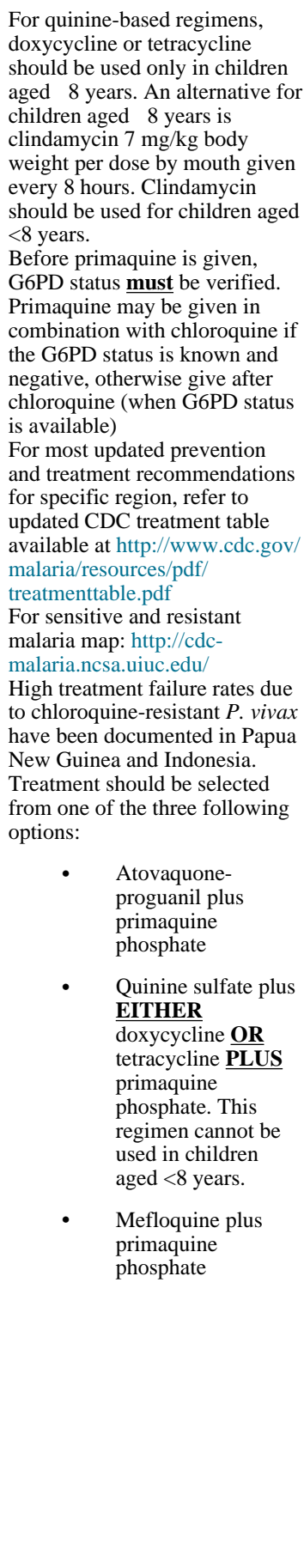 \\
\hline
\end{tabular}




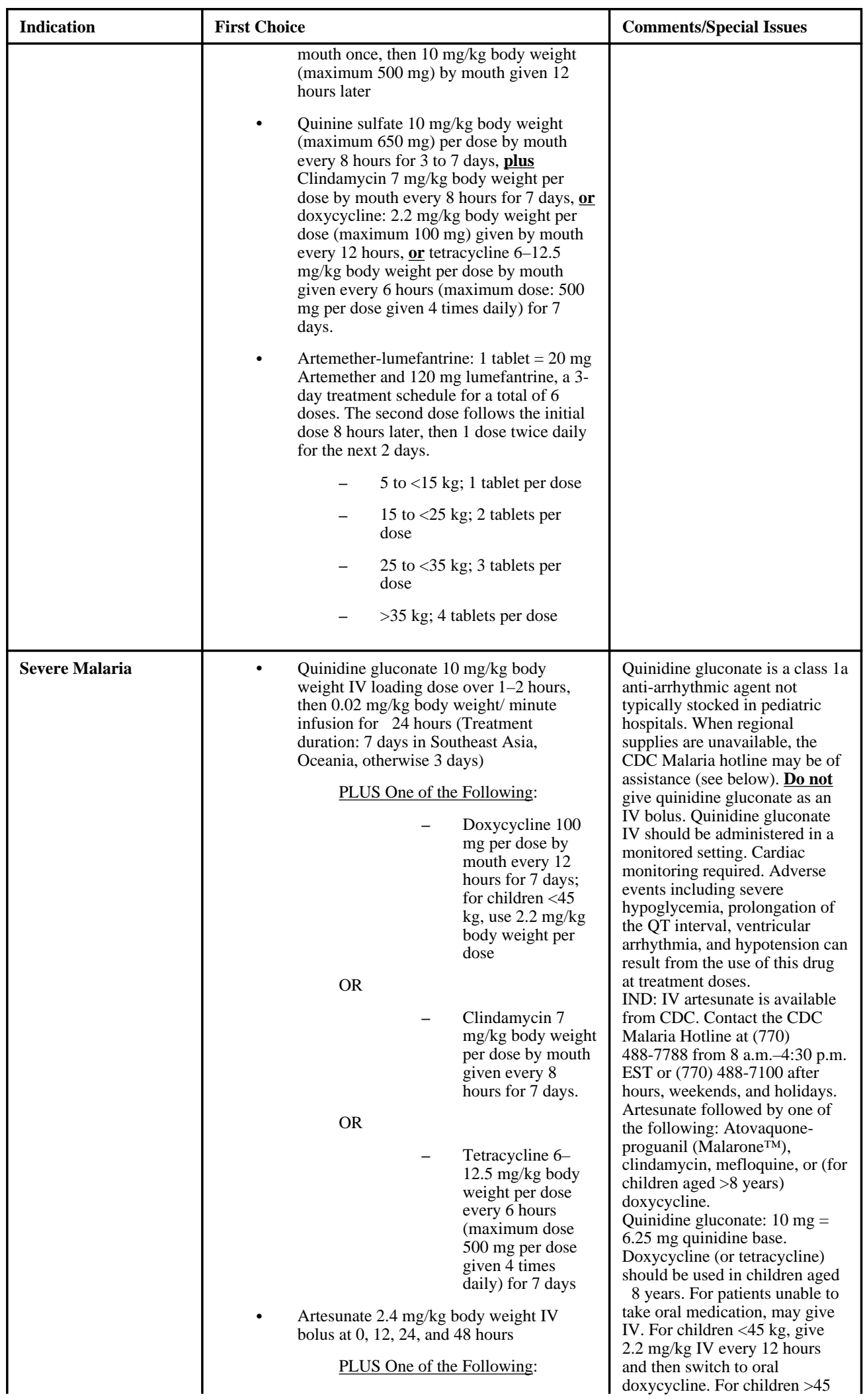




\begin{tabular}{|c|c|c|c|c|}
\hline Indication & First Choice & & & Comments/Special Issues \\
\hline & & $\begin{array}{l}- \\
- \\
- \\
-\end{array}$ & 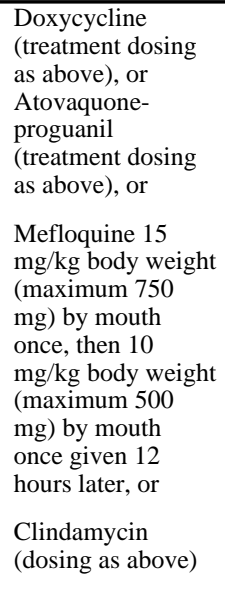 & \begin{tabular}{l} 
kg, use the same dosing as per \\
adults. For IV use, avoid rapid \\
administration. \\
For patients unable to take oral \\
clindamycin, give $10 \mathrm{mg}$ \\
base/kg loading dose IV, \\
followed by $5 \mathrm{mg}$ base/kg IV \\
every 8 hours. Switch to oral \\
clindamycin (oral dose as \\
above) as soon as a patient can \\
take oral medication. For IV \\
use, avoid rapid administration. \\
Drug Interactions: \\
\multicolumn{1}{c}{ Avoid co- } \\
administration of \\
quinidine with \\
ritonavir \\
Use quinidine with \\
caution with other \\
protease inhibitors.
\end{tabular} \\
\hline
\end{tabular}

Key to Acronyms: $\mathrm{CDC}=$ Centers for Disease Control and Prevention; G6PD = glucose-6-phosphate dehydrogenase; IND $=$ investigational new drug; IV = intravenous; PART = presumptive anti-relapse therapy

\section{Microsporidiosis (Last updated November 6, 2013; last reviewed November 6, 2013)}

Panel's Recommendations

- $\quad$ Avoiding untreated water sources, washing fresh fruit and vegetables, and washing the hands after possible exposure to an infected person or animal (AIII) are recommended.

- Effective combination antiretroviral therapy is the primary initial treatment for microsporidiosis in HIVinfected children (AII*).

- Supportive care with hydration, correction of electrolyte abnormalities, and nutritional supplementation should be provided (AIII).

- Albendazole is recommended for initial therapy of microsporidiosis caused by microsporidia other than Enterocytozoon bieneusi and Vittaforma corneae (AII*).

- $\quad$ Topical therapy with fumagillin eye drops can be considered in HIV-infected children with keratoconjunctivitis caused by microsporidia (BII*). Oral albendazole is recommended in addition to topical therapy for keratoconjunctivitis (BIII).

- Treatment for microsporidiosis should be continued until improvement in severe immunosuppression is sustained (more than 6 months at CDC immunologic category 1 or 2) and clinical signs and symptoms of infection are resolved (BIII).

Rating of Recommendations: $\mathrm{A}=$ Strong; $\mathrm{B}=$ Moderate; $\mathrm{C}=$ Optional

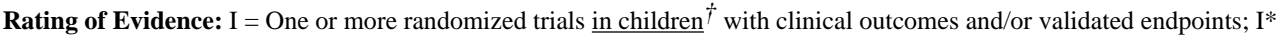
$=$ One or more randomized trials in adults with clinical outcomes and/or validated laboratory endpoints with accompanying data in children ${ }^{\dagger}$ from one or more well-designed, nonrandomized trials or observational cohort studies with long-term clinical outcomes; II = One or more well-designed, nonrandomized trials or observational cohort studies in children ${ }^{\dagger}$ with long-term outcomes; II* = One or more well-designed, nonrandomized trials or observational studies in adults with longterm clinical outcomes with accompanying data in children ${ }^{\dagger}$ from one or more similar nonrandomized trials or cohort studies with clinical outcome data; III = Expert opinion

${ }^{\dagger}$ Studies that include children or children/adolescents, but not studies limited to post-pubertal adolescents 


\section{Epidemiology}

Microsporidia are obligate, intracellular, spore-forming organisms that primarily cause moderate to severe diarrhea in children. They are ubiquitous and infect most animal species. They are classified as fungi and defined by their unique single polar tube that coils around the interior of the spore. ${ }^{1}$ Many microsporidia were reported as pathogens in humans, but Enterocytozoon bieneusi and Encephalitozoon intestinalis are the most common microsporidia that cause infection in HIV-infected patients. Other microsporidia, such as Encephalitozoon cuniculi, Encephalitozoon hellem, Trachipleistophora hominis, Trachipleistophora anthropophthera, Pleistophora spp., Pleistophora ronneeafiei, Vittaforma (Nosema) corneae, Mycobacterium africanum, Mycobacterium ceylonensis, Nosema ocularum, Anncaliia (syns Brachiola/Nosema) connori, Anncaliia (syn Brachiola) vesicularum, and Anncaliia (syns Brachiola/Nosema) algerae also have been implicated in human infections. The organisms develop in enterocytes, and are excreted in feces and transmitted by the fecal-oral route, including through ingestion of contaminated food or water, and possibly through contact with infected animals. ${ }^{2,3}$ Vertical transmission of infection from an infected mother to her child has not been demonstrated in humans but it does occur in animals. ${ }^{3}$

Prior to the era of combination antiretroviral therapy (cART), prevalence rates for microsporidiosis were reported to be as high as $70 \%$ in HIV-infected adults with diarrhea. ${ }^{1,4-6}$ The role of microsporidiosis in chronic diarrhea was questioned early in the HIV epidemic but is now believed to be causal. ${ }^{7,8}$ The incidence of microsporidiosis has declined with the widespread use of effective cART, but it is still being detected in HIVinfected individuals who are not receiving effective cART. ${ }^{9}$ Among HIV-uninfected individuals, microsporidiosis is increasingly recognized in children, travelers, organ transplant recipients, contact lens wearers, and the elderly.

\section{Clinical Manifestations}

The most common manifestation of microsporidiosis is gastrointestinal (GI) tract infection. Microsporidiaassociated diarrhea is intermittent, copious, watery, and non-bloody. It may be accompanied by crampy abdominal pain; fever is uncommon. Chronic severe diarrhea can result in dehydration, malnutrition, and failure to thrive. Microsporidia species have been described as causing disease in multiple other organs besides the GI tract, as well as disseminated disease. ${ }^{4,10}$ Different infecting species may result in different clinical manifestations. E. bieneusi is associated with malabsorption, diarrhea, pulmonary disease, and cholangitis. E. cuniculi is associated with hepatitis, encephalitis, peritonitis, keratoconjunctivitis, sinusitis, osteomyelitis, pulmonary disease, and disseminated disease. Encephalitozoon (syn Septata) intestinalis is associated with diarrhea, cholangitis, dermatitis, disseminated infection, and superficial keratoconjunctivitis. E. hellem is associated with superficial keratoconjunctivitis, sinusitis, respiratory disease, prostatic abscesses, nephritis, urethritis, cystitis, and disseminated infection. Nosema, Vittaforma, and Microsporidium spp. are associated with stromal keratitis following trauma in immunocompetent hosts. Pleistophora, Anncaliia, and Trachipleistophora spp. are associated with myositis. Trachipleistophora spp. is associated with encephalitis, cardiac disease, and disseminated disease. 


\section{Diagnosis}

To diagnose microsporidia GI infection, thin smears of un-concentrated stool-formalin suspension or duodenal aspirates can be stained with modified trichrome stain. Chemofluorescence agents such as chromotrope $2 \mathrm{R}$, calcofluor white (a fluorescent brightener), or Uvitex $2 \mathrm{~B}$ are useful as selective stains for microsporidia in stool and other body fluids. Microsporidia spores are small (1-5 $\mu \mathrm{m}$ diameter), ovoid, stain pink to red with modified trichrome stain, and contain a distinctive equatorial belt-like stripe. They can also be visualized with hematoxylin-eosin, Giemsa, and acid-fast staining but are often overlooked because of their small size.

Urine sediment examination by light microscopy can be used to identify microsporidia spores causing disseminated disease (such as Encephalitozoonidae or Trachipleistophora). Transmission electron microscopy, staining with species-specific antibodies, or polymerase chain reaction (using specific primers) is needed for speciation.

Endoscopic biopsy should be considered for all patients with chronic diarrhea of longer than 2 months' duration and negative stool examinations (CIII). Touch preparations are useful for rapid diagnosis (i.e., within 24 hours). The organisms can be visualized with Giemsa, tissue Gram stain, calcofluor white or Uvitex 2B, Warthin-Starry silver staining, or Chromotrope 2A. ${ }^{11}$ Sensitive assays using PCR amplification of DNA sequences extracted from stool or biopsy specimens have been developed for E. bieneusi ${ }^{12,13}$ but are research tools and not commercially available.

\section{Prevention Recommendations}

Preventing Exposure-Because microsporidia are most likely transferred from contaminated water, food, or contact with an infected individual or animal, direct contact should be avoided. Untreated water sources (drinking water that has not been chemically treated, filtered, or boiled to eliminate infectious agents) should also be avoided (AIII). Fresh fruit and vegetables should be thoroughly washed or peeled prior to eating. This recommendation is especially important for individuals with severe immunosuppression. Hand-washing after exposure to potentially contaminated material or contact with infected individuals or animals also is recommended (AIII).

In a hospital, standard precautions (such as use of gloves and hand-washing after removal of gloves) should be sufficient to prevent transmission from an infected patient to a susceptible HIV-infected individual. However, contact precautions should be used in the case of a diapered or incontinent child.

Preventing Disease-No chemoprophylactic regimens are known to be effective in preventing microsporidiosis. Whether initiating cART will help prevent microsporidiosis is unknown.

Discontinuing Primary Prophylaxis-Not applicable. 


\section{Treatment Recommendations}

Treating Disease-Immune reconstitution resulting from cART often results in clearance of microsporidia infections. Effective cART is the primary initial treatment for these infections in HIV-infected children and adults (AII*). ${ }^{14,15}$ Supportive care with hydration, correction of electrolyte abnormalities, and nutritional supplementation should be provided (AIII). Albendazole has activity against many species of microsporidia, but it is not effective against Enterocytozoon infections or V. corneae. ${ }^{16,17}$ This agent decreased diarrhea and sometimes eliminated the organism. ${ }^{17,18}$ Albendazole is recommended for initial therapy of microsporidiosis caused by microsporidia other than E. bieneusi and V. corneae (AII*).

Although two drugs - fumagillin and nitazoxanide-have been studied in small numbers of patients for treatment of E. bieneusi infection, neither has definitive evidence for efficacy in adequate and controlled trials. Fumagillin (Sanofi-Synthelabo Laboratories, Gentilly, France) (a water-insoluble antibiotic made by Aspergillus fumigatus) and its synthetic analog, TNP-470, ${ }^{19}$ have both been used to treat microsporidiosis in animals and humans. In a placebo-controlled study of immunocompromised adults (10 of 12 of whom were HIVinfected adults) with $E$. bieneusi microsporidiosis, fumagillin ( $20 \mathrm{mg} /$ dose orally 3 times daily for 2 weeks) was associated with decreased diarrhea and clearance of microsporidia spores, which was not observed in placebo patients. ${ }^{20}$ Placebo patients received fumagillin at the conclusion of the trial and all 6 demonstrated clearance of microsporidia.

Thrombocytopenia occurred in 2 of the 6 patients randomized to receive fumagillin. No data are available on use of fumagillin or TNP-470 in HIV-infected children, and neither drug is available for systemic use in the United States. Data are insufficient to make recommendations on the use of these drugs in children (CIII). Consultation with an expert is recommended. One report indicated that treatment with nitazoxanide for 60 days might resolve chronic diarrhea caused by E. bieneusi in the absence of antiretroviral therapy, ${ }^{21}$ but this effect was minimal in patients with low CD4 T lymphocyte counts, and therefore, may be of limited utility (CIII).

Keratoconjunctivitis caused by microsporidia in HIV-infected adults responds to topical therapy with investigational fumagillin eye drops prepared from Fumidil-B ${ }^{\circledR}$ (fumagillin bicyclohexylammonium, a commercial product used to control a microsporidia disease of honeybees) in saline (to achieve a concentration of $70 \mu \mathrm{g} / \mathrm{mL}$ of fumagillin). ${ }^{22}$ Topical therapy with investigational fumagillin eye drops can be considered for HIV-infected children with keratoconjunctivitis caused by microsporidia (BII*). The addition of oral albendazole to topical fumagillin is recommended for keratoconjunctivitis because microsporidia may remain systemically despite clearance from the eye with topical therapy alone (BIII) ${ }^{23}$ Children with suspected keratoconjunctivitis that is non-responsive to antibacterial or antiviral therapy should be referred to a pediatric ophthalmologist for evaluation for possible microsporidiosis.

Other agents including nitazoxanide, atovaquone, metronidazole, and fluoroquinolones have been reported to reduce diarrhea associated with microsporidia infection. However, metronidazole and atovaquone are not active in vitro or in animal models and should not be 
used to treat microsporidiosis (AII*). The role of alternative agents or the use of combination regimens for initial therapy is unknown and albendazole remains the preferred therapy for GI tract and disseminated infection caused by microsporidia other than $E$. bieneusi and $V$. corneae (AII*). ${ }^{17,18}$

Monitoring and Adverse Events (Including IRIS)_Patients with diarrhea should be closely monitored for signs and symptoms of volume depletion, electrolyte and weight loss, and malnutrition. In severely ill patients, total parenteral nutrition may be indicated (AIII).

Albendazole side effects are rare, but hypersensitivity (e.g., rash, pruritus, fever), neutropenia (reversible), central nervous system effects (e.g., dizziness, headache), GI disturbances (e.g., abdominal pain, diarrhea, nausea, vomiting), hair loss (reversible), and elevated hepatic enzymes (reversible) have been reported. Dose-related bone marrow toxicity is the principal adverse effect of systemic fumagillin, with reversible thrombocytopenia and neutropenia being the most frequent adverse events; topical fumagillin has not been associated with substantial side effects.

There has been one report of immune reconstitution inflammatory syndrome (IRIS) following initiation of cART in a patient with E. bieneusi infection ${ }^{24}$ but IRIS has not been described in association with treatment for non-E. bieneusi microsporidiosis. Concern for IRIS should not delay institution of cART in the presence of microsporidia infection (AIII).

Managing Treatment Failure-The only feasible approaches to managing treatment failure are supportive treatment and optimization of cART to achieve full virologic suppression (AIII). The role of alternative and combination therapy is unknown.

Preventing Recurrence-No pharmacologic interventions are known to be effective in preventing recurrence of microsporidiosis. However, the use of cART alone in patients with microsporidiosis has resulted in clearance of infection and symptoms ${ }^{15}$ suggesting that improvements in the immune system after successful cART are critical to recovery. Continued albendazole therapy after treatment for an acute episode of GI or disseminated infection caused by microsporidia other than E. bieneusi and V. corneae should be considered in those with severe immunosuppression (Centers for Disease Control and Prevention $[\mathrm{CDC}]$ immunologic category 3 ) until immune recovery is observed (longer than 6 months at CDC immunologic category 1 or 2) (BIII).

For keratoconjunctivitis, albendazole treatment can be discontinued after resolution of infection in patients without severe immunosuppression (CDC immunologic category 3 ) but should be continued indefinitely if severe immunosuppression persists because recurrence or relapse may follow treatment discontinuation (BIII).

Discontinuing Secondary Prophylaxis-See above. 


\section{References}

1. Mathis A. Microsporidia: emerging advances in understanding the basic biology of these unique organisms. Int J Parasitol. 2000 Jun; 30(7):795-804. Available at http://www.ncbi.nlm.nih.gov/ pubmed/10899524. [PubMed: 10899524]

2. Hutin YJ, Sombardier MN, Liguory O, et al. Risk factors for intestinal microsporidiosis in patients with human immunodeficiency virus infection: a case-control study. J Infect Dis. 1998 Sep; 178(3): 904-907. Available at http://www.ncbi.nlm.nih.gov/pubmed/9728570. [PubMed: 9728570]

3. Didier ES, Stovall ME, Green LC, Brindley PJ, Sestak K, Didier PJ. Epidemiology of microsporidiosis: sources and modes of transmission. Vet Parasitol. 2004 Dec 9; 126(1-2):145-166. Available at http://www.ncbi.nlm.nih.gov/pubmed/15567583. [PubMed: 15567583]

4. Kotler DP, Orenstein JM. Clinical syndromes associated with microsporidiosis. Advances in parasitology. 1998; 40:321-349. Available at http://www.ncbi.nlm.nih.gov/pubmed/9554078. [PubMed: 9554078]

5. Wittner, M.; Weiss, L. The Microsporidia and Microsporidiosis. Washington, DC: ASM Press; 1999.

6. Deplazes P, Mathis A, Weber R. Epidemiology and zoonotic aspects of microsporidia of mammals and birds. Contributions to microbiology. 2000; 6:236-260. Available at http:// www.ncbi.nlm.nih.gov/pubmed/10943515. [PubMed: 10943515]

7. Eeftinck Schattenkerk JK, van Gool T, van Ketel RJ, et al. Clinical significance of small-intestinal microsporidiosis in HIV-1-infected individuals. Lancet. 1991 Apr 13; 337(8746):895-898. Available at http://www.ncbi.nlm.nih.gov/pubmed/1672978. [PubMed: 1672978]

8. Molina JM, Sarfati C, Beauvais B, et al. Intestinal microsporidiosis in human immunodeficiency virus-infected patients with chronic unexplained diarrhea: prevalence and clinical and biologic features. J Infect Dis. 1993 Jan; 167(1):217-221. Available at http://www.ncbi.nlm.nih.gov/ pubmed/8418171. [PubMed: 8418171]

9. Stark D, Barratt JL, van Hal S, Marriott D, Harkness J, Ellis JT. Clinical significance of enteric protozoa in the immunosuppressed human population. Clin Microbiol Rev. 2009 Oct; 22(4):634650. Available at http://www.ncbi.nlm.nih.gov/pubmed/19822892. [PubMed: 19822892]

10. Didier ES, Weiss LM. Microsporidiosis: current status. Curr Opin Infect Dis. 2006 Oct; 19(5):485492. Available at http://www.ncbi.nlm.nih.gov/pubmed/16940873. [PubMed: 16940873]

11. Weiss LM, Vossbrinck CR. Microsporidiosis: molecular and diagnostic aspects. Advances in parasitology. 1998; 40:351-395. Available at http://www.ncbi.nlm.nih.gov/pubmed/9554079. [PubMed: 9554079]

12. McLauchlin J, Amar CF, Pedraza-Diaz S, Mieli-Vergani G, Hadzic N, Davies EG. Polymerase chain reaction-based diagnosis of infection with Cryptosporidium in children with primary immunodeficiencies. Pediatr Infect Dis J. 2003 Apr; 22(4):329-335. Available at http:// www.ncbi.nlm.nih.gov/pubmed/12690272. [PubMed: 12690272]

13. Menotti J, Cassinat B, Porcher R, Sarfati C, Derouin F, Molina JM. Development of a real-time polymerase-chain-reaction assay for quantitative detection of Enterocytozoon bieneusi DNA in stool specimens from immunocompromised patients with intestinal microsporidiosis. J Infect Dis. 2003 May 1; 187(9):1469-1474. Available at http://www.ncbi.nlm.nih.gov/pubmed/12717629. [PubMed: 12717629]

14. Chen XM, Keithly JS, Paya CV, LaRusso NF. Cryptosporidiosis. N Engl J Med. 2002 May 30; 346(22):1723-1731. Available at http://www.ncbi.nlm.nih.gov/pubmed/12037153. [PubMed: 12037153]

15. Miao YM, Awad-El-Kariem FM, Franzen C, et al. Eradication of cryptosporidia and microsporidia following successful antiretroviral therapy. J Acquir Immune Defic Syndr. 2000 Oct 1; 25(2):124129. Available at http://www.ncbi.nlm.nih.gov/pubmed/11103042. [PubMed: 11103042]

16. Weber R, Sauer B, Luthy R, Nadal D. Intestinal coinfection with Enterocytozoon bieneusi and Cryptosporidium in a human immunodeficiency virus-infected child with chronic diarrhea. Clin Infect Dis. 1993 Sep; 17(3):480-483. Available at http://www.ncbi.nlm.nih.gov/pubmed/8218693. [PubMed: 8218693] 
17. Molina JM, Chastang C, Goguel J, et al. Albendazole for treatment and prophylaxis of microsporidiosis due to Encephalitozoon intestinalis in patients with AIDS: a randomized doubleblind controlled trial. J Infect Dis. 1998 May; 177(5):1373-1377. Available at http:// www.ncbi.nlm.nih.gov/pubmed/9593027. [PubMed: 9593027]

18. Hicks P, Zwiener RJ, Squires J, Savell V. Azithromycin therapy for Cryptosporidium parvum infection in four children infected with human immunodeficiency virus. J Pediatr. 1996 Aug; 129(2):297-300. Available at http://www.ncbi.nlm.nih.gov/pubmed/8765631. [PubMed: 8765631]

19. Didier PJ, Phillips JN, Kuebler DJ, et al. Antimicrosporidial activities of fumagillin, TNP-470, ovalicin, and ovalicin derivatives in vitro and in vivo. Antimicrob Agents Chemother. 2006 Jun; 50(6):2146-2155. Available at http://www.ncbi.nlm.nih.gov/pubmed/16723577. [PubMed: 16723577]

20. Molina JM, Tourneur M, Sarfati C, et al. Fumagillin treatment of intestinal microsporidiosis. N Engl J Med. 2002 Jun 20; 346(25):1963-1969. Available at http://www.ncbi.nlm.nih.gov/pubmed/ 12075057. [PubMed: 12075057]

21. Bicart-See A, Massip P, Linas MD, Datry A. Successful treatment with nitazoxanide of Enterocytozoon bieneusi microsporidiosis in a patient with AIDS. Antimicrob Agents Chemother. 2000 Jan; 44(1):167-168. Available at http://www.ncbi.nlm.nih.gov/pubmed/10602740. [PubMed: 10602740]

22. Diesenhouse MC, Wilson LA, Corrent GF, Visvesvara GS, Grossniklaus HE, Bryan RT. Treatment of microsporidial keratoconjunctivitis with topical fumagillin. Am J Ophthalmol. 1993 Mar 15; 115(3):293-298. Available at http://www.ncbi.nlm.nih.gov/pubmed/8117342. [PubMed: 8117342]

23. Didier ES. Effects of albendazole, fumagillin, and TNP-470 on microsporidial replication in vitro. Antimicrob Agents Chemother. 1997 Jul; 41(7):1541-1546. Available at http:// www.ncbi.nlm.nih.gov/pubmed/9210681. [PubMed: 9210681]

24. Sriaroon C, Mayer CA, Chen L, Accurso C, Greene JN, Vincent AL. Diffuse intra-abdominal granulomatous seeding as a manifestation of immune reconstitution inflammatory syndrome associated with microsporidiosis in a patient with HIV. AIDS Patient Care STDS. 2008 Aug; 22(8):611-612. Available at http://www.ncbi.nlm.nih.gov/pubmed/18627278. [PubMed: $18627278]$

\section{Dosing Recommendations for Preventing and Treating Microsporidiosis}

\begin{tabular}{|c|c|c|c|}
\hline \multicolumn{4}{|l|}{ Preventive Regimen } \\
\hline Indication & First Choice & Alternative & Comments/Special Issues \\
\hline Primary Prophylaxis & N/A & N/A & Not recommended \\
\hline Secondary Prophylaxis & $\begin{array}{l}\text { Disseminated, Non-Ocular Infection or GI } \\
\text { Infection Caused by Microsporidia Other } \\
\text { Than E. Bieneusi or V. Corneae: } \\
\begin{array}{l}\text { Albendazole } 7.5 \mathrm{mg} / \mathrm{kg} \text { body } \\
\text { weight (maximum } 400 \mathrm{mg} / \\
\text { dose) by mouth twice daily }\end{array} \\
\text { Ocular Infection: } \\
\text { Topical fumagillin } \\
\text { bicyclohexylammonium } \\
\text { (Fumidil B) } 3 \mathrm{mg} / \mathrm{mL} \text { in saline } \\
\text { (fumagillin } 70 \mu \mathrm{gg} / \mathrm{mL} \text { ) eye } \\
\text { drops: } 2 \text { drops every } 2 \text { hours } \\
\text { for } 4 \text { days, then } 2 \text { drops QID } \\
\text { (investigational use only in } \\
\text { United States) plus albendazole } \\
7.5 \mathrm{mg} / \mathrm{kg} \text { body weight } \\
\text { (maximum } 400 \mathrm{mg} / \mathrm{dose} \text { ) by } \\
\text { mouth twice daily for } \\
\text { management of systemic } \\
\text { infection }\end{array}$ & N/A & $\begin{array}{ll}\text { Criteria For Discontinuing } \\
\text { Secondary Prophylaxis: } \\
& \text { Continue until } \\
& \text { sustained } \\
\text { immune } & \text { reconstitution } \\
\text { (more than 6 } & \text { months at CDC } \\
\text { immunologic } & \\
\text { category } 1 \text { or 2), } & \text { or } \\
\text { or } & \text { After initiation } \\
& \text { of cART and } \\
\text { resolution of } & \text { signs and } \\
\text { symptoms }\end{array}$ \\
\hline
\end{tabular}




\begin{tabular}{|c|c|c|c|}
\hline \multicolumn{4}{|c|}{ Preventive Regimen } \\
\hline Indication & First Choice & Alternative & Comments/Special Issues \\
\hline Treatment & 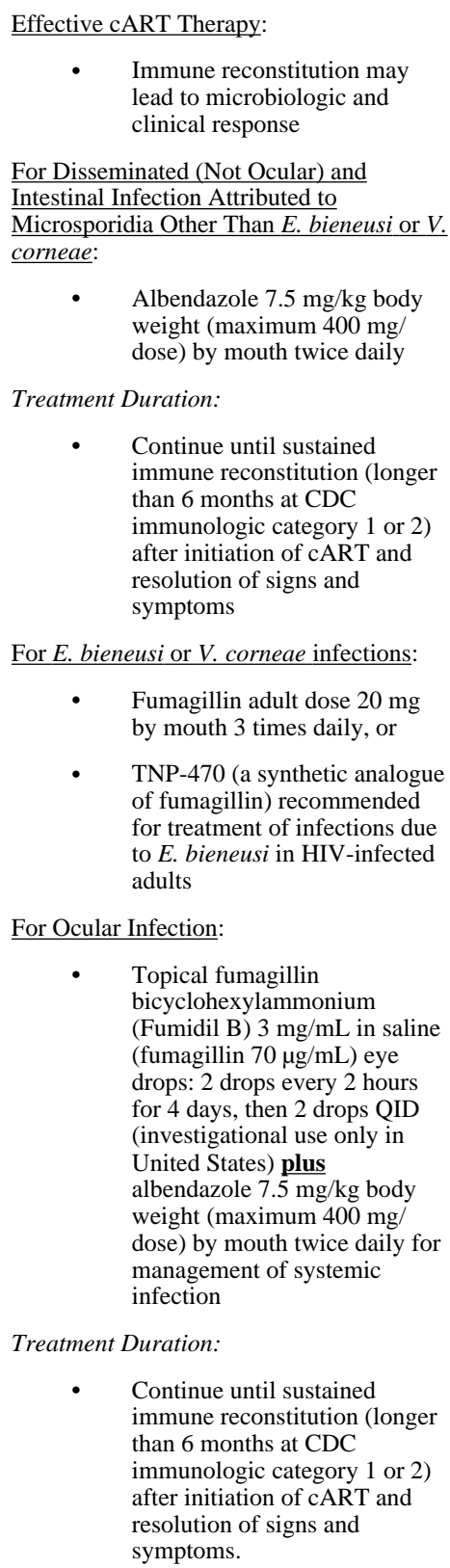 & N/A & $\begin{array}{l}\text { - Supportive care: } \\
\text { Hydration, } \\
\text { correct } \\
\text { electrolyte } \\
\text { abnormalities, } \\
\text { nutritional } \\
\text { support } \\
\text { - } \\
\text { Fumagillin for } \\
\text { systemic use is } \\
\text { unavailable in } \\
\text { the United } \\
\text { States and data } \\
\text { on dosing in } \\
\text { children are } \\
\text { unavailable. } \\
\text { Consultation } \\
\text { with an expert is } \\
\text { recommended. }\end{array}$ \\
\hline
\end{tabular}

Key to Acronyms: $\mathrm{cART}=$ combination antiretroviral therapy; $\mathrm{CDC}=$ Centers for Disease Control and Prevention; $\mathrm{GI}=$ gastrointestinal; $\mathrm{QID}=$ four times a day 


\section{Mycobacterium avium Complex Disease (Last updated November 6, 2013; last reviewed November 6, 2013)}

Panel's Recommendations

- $\quad$ Routine screening of respiratory or gastrointestinal specimens for Mycobacterium avium complex (MAC) microorganisms is not recommended (BIII), but a blood culture for MAC should be obtained to rule out disseminated disease before initiating prophylaxis (AIII).

- Prophylaxis with either clarithromycin or azithromycin should be offered to HIV-infected children who have advanced immunosuppression (AII).

- Children aged $<1$ year: $<750$ cells $/ \mathrm{mm}^{3}$.

- Children aged 1 to $<2$ years: $<500$ cells $/ \mathrm{mm}^{3}$.

- $\quad$ Children aged 2 to $<6$ years: $<75$ cells $/ \mathrm{mm}^{3}$.

- Children aged $\ 6$ years: $<50$ cells $/ \mathrm{mm}^{3}$

Discontinuing Primary Prophylaxis:

- $\quad$ Primary prophylaxis can be discontinued in HIV-infected children aged $\geq 2$ years receiving stable combination antiretroviral therapy (cART) for $\ 6$ months and experiencing sustained ( $>3$ months) CD4 T lymphocyte (CD4) cell count recovery well above the age-specific target for initiation of prophylaxis (i.e., as in adults, $>100$ cells $/ \mathrm{mm}^{3}$ for children aged $\Varangle 6$ years (AI); and $>200$ cells $/ \mathrm{mm}^{3}$ for children aged 2 to $<6$ years) (BII*)

Treating Disease:

- Testing of MAC isolates for susceptibility to clarithromycin or azithromycin is recommended (BIII) Combination therapy with a minimum of two drugs (e.g., clarithromycin or azithromycin plus ethambutol) is recommended to prevent or delay the emergence of resistance (AI*). Some experts use clarithromycin as the preferred first agent $\left(\mathbf{A} \mathbf{I}^{*}\right)$, reserving azithromycin for patients with substantial intolerance to clarithromycin or when drug interactions with clarithromycin are a concern (AII*).

- Use of rifabutin as a third drug added to the macrolide/ethambutol regimen is controversial. Some experts would add rifabutin as a third drug to the clarithromycin/ethambutol regimen, particularly in the absence of cART and in the presence of high mycobacterial counts (CIII); however, drug interactions should be checked carefully, and more intensive toxicity monitoring may be warranted with such combination therapy (AIII). Other experts recommend against using this third agent in children because of rifabutin's increased cytochrome P450 activity, which leads to increased clearance of other drugs such as protease inhibitors and non-nucleoside reverse transcriptase inhibitors, and the potential for increased toxicity associated with concomitant administration of drugs (CIII).

- Treatment failure is defined as the absence of clinical response and the persistence of mycobacteremia afte 8 to 12 weeks of treatment. Repeat susceptibility testing of MAC isolates is recommended in this situation, and a new multidrug regimen of two or more drugs not previously used and to which the isolate is susceptible should be administered (AIII). Drugs that should be considered for this scenario include rifabutin, amikacin, and a quinolone.

Secondary Prophylaxis:

- $\quad$ Children with a history of disseminated MAC and continued immunosuppression should receive lifelong prophylaxis to prevent recurrence (AII*). Secondary prophylaxis typically consists of continued multidrug therapy used in treatment of disease.

- Some experts recommend discontinuation of therapy in HIV-infected children who meet all of the following criteria:

- $\quad$ Aged $\geq 2$ years and have completed $\geq 12$ months of treatment for MAC;

- $\quad$ Remain asymptomatic for MAC;

- $\quad$ Receiving stable cART (i.e., cART not requiring change for virologic or immunologic failure);

- Have sustained ( $\ 6$ months) CD4 count recovery well above the age-specific target for initiation of primary prophylaxis (i.e., as in adults, $>100$ cells $/ \mathrm{mm}^{3}$ for children aged $\geq 6$ years (AII*) and $>200$ cells $/ \mathrm{mm}^{3}$ for children aged 2 to $<6$ years) (CIII).

Rating of Recommendations: $\mathrm{A}=$ Strong; $\mathrm{B}=$ Moderate $\mathrm{C}=$ Optional

Rating of Evidence: $\mathrm{I}=$ One or more randomized trials $\underline{\underline{\text { in children }}}{ }^{\dagger}$ with clinical outcomes and/or validated endpoints; $\mathrm{I}^{*}$ = One or more randomized trials in adults with clinical outcomes and/or validated laboratory endpoints with accompanying 
data in children $^{\dagger}$ from one or more well-designed, nonrandomized trials or observational cohort studies with long-term clinical outcomes; II = One or more well-designed, nonrandomized trials or observational cohort studies in children ${ }^{\dagger}$ with long-term outcomes; II* = One or more well-designed, nonrandomized trials or observational studies in adults with longterm clinical outcomes with accompanying data in children ${ }^{\dagger}$ from one or more similar nonrandomized trials or cohort studies with clinical outcome data; III = Expert opinion

${ }^{\dagger}$ Studies that include children or children/adolescents, but not studies limited to post-pubertal adolescents

\section{Epidemiology}

Mycobacterium avium complex (MAC) refers to multiple related species of nontuberculous mycobacteria (NTM) (e.g., Mycobacterium avium, Mycobacterium intracellulare, and Mycobacterium paratuberculosis) that are widely distributed in the environment. Recent surveillance data have shown an increasing rate of MAC infection in some regions within the United States. ${ }^{1}$ Comprehensive guidelines on the diagnosis, prevention, and treatment of nontuberculous mycobacterial diseases were published in $2007 .^{2}$ These guidelines highlight the tremendous advances in laboratory methods in mycobacteriology that have expanded the number of known NTM species from 50 in 1997 to 125 in 2006. In the United States, NTM infections outnumber Mycobacterium tuberculosis infections and have become an important cause of pulmonary morbidity in adults. ${ }^{3}$

MAC was the second most common opportunistic infection (OI) in HIV-infected children in the United States after Pneumocystis jirovecii pneumonia during the era before combination antiretroviral therapy (cART), but its incidence has greatly decreased from 1.3 to 1.8 episodes per 100 person-years during that time to 0.14 to 0.2 episodes per 100 person-years during the cART era. ${ }^{4,5} \mathrm{MAC}$ is ubiquitous in the environment and presumably is acquired by routine exposures through inhalation, ingestion, or inoculation. ${ }^{6}$ A recent populationbased study in Florida of adults and children associated soil exposure, along with black race and birth outside the United States, with MAC infection. ${ }^{7}$ Respiratory and gastrointestinal (GI) colonization can act as portals from which infection can disseminate. ${ }^{8}$

MAC can appear as isolated lymphadenitis in both HIV-infected and HIV-uninfected children. Disseminated infection with MAC in pediatric HIV infection rarely occurs during the first year of life; its frequency increases with age and declining CD4 T lymphocyte (CD4) cell count, but can occur at higher CD4 counts in younger HIV-infected children than in older children or adults. It is a recognized complication of advanced immunologic deterioration among HIV-infected children. ${ }^{6,9,10}$

\section{Clinical Manifestations}

Respiratory symptoms are uncommon in HIV-infected children who have disseminated $\mathrm{MAC}$, and isolated pulmonary disease is rare. Early symptoms can be minimal and may precede mycobacteremia by several weeks. Symptoms commonly associated with disseminated MAC infection in children include persistent or recurrent fever, weight loss or failure to gain weight, sweats, fatigue, persistent diarrhea, and persistent or recurrent abdominal pain. Mesenteric adenitis may mimic acute appendicitis. GI symptoms can occur alone or in combination with systemic findings. Lymphadenopathy, hepatomegaly, and splenomegaly may occur. Laboratory abnormalities include anemia, leukopenia, and thrombocytopenia. Although serum chemistries are usually normal, some children may have elevated alkaline phosphatase or lactate dehydrogenase levels. These signs and symptoms 
also are relatively common in the absence of disseminated MAC in HIV-infected children with advanced immunosuppression.

\section{Diagnosis}

Procedures used to diagnose MAC in children are the same as those used for HIV-infected adults. ${ }^{11}$ MAC is definitively diagnosed by isolation of the organism from blood or from biopsy specimens from normally sterile sites (e.g., bone marrow, lymph node). Multiple mycobacterial blood cultures over time may be required to yield a positive result. The volume of blood sent for culture also influences yield, with increased volume leading to increased yield. Use of a radiometric broth medium or lysis-centrifugation culture technique can enhance recovery of organisms from blood.

Histology demonstrating macrophage-containing acid-fast bacilli is strongly indicative of MAC infection in a patient with typical signs and symptoms, but culture is essential to differentiate nontuberculous mycobacteria from M. tuberculosis, to determine which nontuberculous mycobacterium is causing infection, and to perform drug-susceptibility testing. Testing of MAC isolates for susceptibility to clarithromycin or azithromycin is recommended (BIII). The BACTEC ${ }^{\mathrm{TM}}$ method for radiometric susceptibility testing can be used. Resistance for clarithromycin is defined as a minimal inhibitory concentration $\geq 32$ $\mu \mathrm{g} / \mathrm{mL}$ and a minimal inhibitory concentration of $2256 \mu \mathrm{g} / \mathrm{mL}$ for azithromycin. ${ }^{12}$ As with tuberculosis testing, multiplex polymerase chain reaction systems have been developed for rapid identification and drug susceptibility testing, but these are currently only available in research laboratories. ${ }^{13,14}$

\section{Prevention Recommendations}

Preventing Exposure-MAC is ubiquitous in the environment. Available information does not support specific recommendations regarding exposure avoidance. ${ }^{1}$ Person-toperson transmission is not believed to be common.

Preventing First Episode of Disease-The most effective way to prevent disseminated MAC among HIV-infected children is to preserve immune function through use of effective cART. HIV-infected children who have advanced immunosuppression should be offered prophylaxis against disseminated MAC disease according to the following CD4 count thresholds (AII): ${ }^{15,16}$

- Children aged $<1$ year: $<750$ cells $/ \mathrm{mm}^{3}$

- Children aged 1 to $<2$ years: $<500$ cells $/ \mathrm{mm}^{3}$

- Children aged 2 to $<6$ years: $<75$ cells $/ \mathrm{mm}^{3}$

- Children aged $\succ 6$ years: $<50$ cells $/ \mathrm{mm}^{3}$

For the same reasons that clarithromycin and azithromycin are the preferred prophylactic agents for adults, either one is recommended for prophylaxis in children (AI*); oral suspensions of both agents are commercially available in the United States. Before prophylaxis is initiated, at-risk children should be evaluated for disseminated MAC disease, including obtaining a blood culture for MAC (AIII). For children who cannot tolerate 
azithromycin or clarithromycin, rifabutin is an alternative prophylactic agent for MAC, although drug interactions and a lack of efficacy data in children limit its use (CIII). Combination therapy for prophylaxis has not been shown to be cost effective and increases rates of adverse events, and therefore, generally should be avoided in children (AIII).

Although detection of MAC in stool or the respiratory tract may precede disseminated disease, no data demonstrate a correlation between initiation of prophylaxis in patients with detectable organisms at these sites and reduced risk of developing disseminated MAC. Therefore, routine screening of respiratory or GI specimens for MAC is not recommended (BIII).

Discontinuing Primary Prophylaxis-On the basis of both randomized controlled trials and observational data, primary prophylaxis for MAC can be safely discontinued in HIVinfected adults who respond to cART with an increase in CD4 count. ${ }^{17,18}$ In a study of discontinuing OI prophylaxis among HIV-infected children whose CD4 percentages were $\geq 20 \%$ for those aged $>6$ years and $\geq 25 \%$ for those aged 2 to 6 years, 63 HIV-infected children discontinued MAC prophylaxis, and no MAC events were observed during $\geq 2$ years of follow up. ${ }^{19}$ On the basis of both these findings and data from studies in adults, primary prophylaxis can be discontinued in HIV-infected children aged $\geq 2$ years receiving stable cART for $\ 6$ months who experience sustained (>3 months) CD4 cell recovery well above the age-specific target for initiation of prophylaxis (i.e., as in adults, $>100$ cells $/ \mathrm{mm}^{3}$ for children aged $\succ 6$ years and $>200$ cells $/ \mathrm{mm}^{3}$ for children aged 2 to $<6$ years) (BII*). No specific recommendations exist for discontinuing MAC prophylaxis in HIV-infected children aged $<2$ years.

\section{Treatment Recommendations}

Treating Disease-Disseminated MAC infection should be treated in consultation with a pediatric infectious disease specialist who has expertise in pediatric HIV infection (AIII). Combination therapy of MAC with a minimum of 2 drugs is recommended to prevent or delay the emergence of resistance $\left(\mathbf{A} \mathbf{I}^{*}\right) .{ }^{20-23}$ Monotherapy with a macrolide results in emergence of high-level drug resistance within weeks. ${ }^{24}$

Improved immunologic status is important for controlling disseminated MAC disease; cART should be initiated in children with MAC disease who are antiretroviral (ARV) naive. However, the optimal time to start cART in this situation is unknown; many experts treat MAC with antimycobacterial therapy for 2 weeks before starting cART to try to minimize immune reconstitution inflammatory syndrome (IRIS), although whether this makes a difference is unknown (CIII). For children already receiving cART, it should be continued and optimized with careful attention to potential drug interactions between the ARV and antimycobacterial drugs.

Initial MAC empiric therapy should include 2 or more drugs (AI*): clarithromycin or azithromycin plus ethambutol. ${ }^{25}$ Some experts use clarithromycin as the preferred first agent $\left(\mathbf{A I}^{*}\right)$, reserving azithromycin for patients with substantial intolerance to clarithromycin or when drug interactions with clarithromycin are a concern (AII*). ${ }^{26}$ Clarithromycin levels can be increased by protease inhibitors (PI) and decreased by efavirenz, but no data are 
available to recommend dose adjustments for children. Azithromycin is not metabolized by the cytochrome P450 (CYP450) system; therefore, it can be used without concern for significant drug interactions with PIs and non-nucleoside reverse transcriptase inhibitors (NNRTIs).

Because a study in adults demonstrated a survival benefit with the addition of rifabutin to clarithromycin plus ethambutol, some experts would add rifabutin as a third drug to the clarithromycin/ethambutol regimen (CIII); ${ }^{23}$ however, drug interactions should be checked carefully, and more intensive toxicity monitoring may be warranted if such drugs are administered concomitantly (AIII). ${ }^{27}$ Because rifabutin increases CYP450 activity that leads to increased clearance of other drugs (e.g., PIs, NNRTIs), and toxicity might increase with concomitant administration of drugs, other experts recommend against using this third agent in children (CIII). Guidelines and recommendations exist for dose adjustments necessary in adults treated with rifabutin and PIs, but the absence of data in children precludes extrapolating these to HIV-infected children undergoing treatment for disseminated MAC. No pediatric formulation of rifabutin exists, but the drug can be administered mixed with foods such as applesauce. It can also be compounded in a liquid formulation by a pharmacist. Limited safety data are available from 22 HIV-infected children (median age: 9 years) who received rifabutin in combination with 2 or more other antimycobacterial drugs for treatment of MAC for 1 to 183 weeks; doses ranged from $4 \mathrm{mg} / \mathrm{kg}$ to $18.5 \mathrm{mg} / \mathrm{kg}$, and reported adverse effects were similar to those reported in adults. ${ }^{28}$ The most commonly reported dose in children has been $5 \mathrm{mg} / \mathrm{kg}$.

Therapy is typically prolonged and depends upon response and immune reconstitution as discussed under cessation of secondary prophylaxis.

Monitoring and Adverse Events, Including IRIS-Clinically, most patients improve substantially during the first 4 to 6 weeks of therapy. A repeat blood culture for MAC should be obtained 4 to 8 weeks after initiation of antimycobacterial therapy in patients who fail to respond clinically to their initial treatment regimen. Some experts would consider a repeat blood culture for all patients with an initial positive culture, regardless of clinical response to therapy. Improvement in fever can be expected within 2 to 4 weeks after initiation of appropriate therapy. However, for those with more extensive disease or advanced immunosuppression, clinical response may be delayed, and elimination of the organism from the blood may require up to 12 weeks of effective therapy.

IRIS in patients receiving MAC therapy during cART has been reported in HIV-infected adults and children. ${ }^{29-32}$ New onset of systemic symptoms, especially fever or abdominal pain, leukocytosis, and focal lymphadenitis (cervical, thoracic, or abdominal) associated with preexisting but relatively asymptomatic MAC infection has occurred after the start of cART. In addition, paradoxical worsening of systemic or local symptoms of MAC may occur as the immune system is reconstituted.

In children with very low CD4 counts, the decision to begin immediate cART must take into consideration not only the urgent need for rapid immunologic improvement, but also the possibility of IRIS due to MAC. If symptoms suggestive of MAC infection are present at the 
time of cART initiation, the clinician should evaluate for MAC and can consider treating for MAC presumptively. cART generally should be withheld until after the first 2 weeks of antimycobacterial therapy have been completed in patients with disseminated MAC disease who have not been treated previously with or are not receiving effective cART to reduce the risk of drug interactions and complications associated with IRIS and to lower the pill burden (CIII). However, ART should be started as soon as possible after the first 2 weeks of antimycobacterial therapy in order to reduce the risk of developing additional AIDSdefining OIs, and to facilitate immune reconstitution and further improve the response to antimycobacterial therapy (CIII). Children with moderate symptoms of IRIS can be treated symptomatically with nonsteroidal anti-inflammatory drugs (NSAIDs) or, if unresponsive to NSAIDS, a short course (such as 4 weeks) of systemic corticosteroid therapy while continuing to receive cART (CIII).

Adverse effects from clarithromycin and azithromycin include nausea, vomiting, abdominal pain, abnormal taste, and elevations in liver transaminase levels or hypersensitivity reactions. The major toxicity associated with ethambutol is optic neuritis, with symptoms of blurry vision, central scotomata, and red-green color blindness, which usually is reversible and rare at doses of $15-25 \mathrm{mg} / \mathrm{kg}$ in children with normal renal function. Assessments of renal function, ophthalmoscopy, and (if possible) visual acuity and color vision should be performed before starting ethambutol and monitored regularly during treatment with the agent (AIII). Use of ethambutol in very young children whose visual acuity cannot be monitored requires careful consideration of risks and benefits. ${ }^{33,34}$

Patients receiving clarithromycin plus rifabutin should be observed for the rifabutin-related development of leukopenia, uveitis, polyarthralgias, and pseudojaundice. Tiny, almost transparent, asymptomatic peripheral and central corneal deposits that do not impair vision have been observed in some HIV-infected children receiving rifabutin as part of a multidrug regimen for MAC. ${ }^{28}$

Managing Treatment Failure-Treatment failure is defined as the absence of clinical response and the persistence of mycobacteremia after 8 to 12 weeks of treatment. Repeat susceptibility testing of MAC isolates is recommended in this situation, and a new multidrug regimen of 2 or more drugs not previously used and to which the isolate is susceptible should be administered (AIII). Drugs that should be considered for this scenario include rifabutin, amikacin, and a quinolone. Data from treating MAC in HIV-uninfected patients indicate that an injectable agent such as amikacin or streptomycin should be considered (CIII) ${ }^{2,3}$ Because dosing of these agents in children can be problematic, drug-resistant disseminated MAC should be treated with input from an expert in this disease (AIII). Optimization of cART is an especially important adjunct to treatment of patients in whom initial MAC therapy has failed.

Preventing Recurrence-Children with a history of disseminated MAC should be given prophylaxis to prevent recurrence (AII*) until their immune systems are reconstituted. ${ }^{35}$ Prophylaxis in this setting means continuation of multidrug therapy, because use of a single agent (clarithromycin or azithromycin) for secondary prophylaxis carries a high risk of inducing drug-resistant MAC infection. 
Discontinuing Secondary Prophylaxis-On the basis of immune reconstitution data in adults ${ }^{34,36}$ and data in children discontinuing primary prophylaxis, some experts recommend discontinuation of secondary prophylaxis in HIV-infected children aged $\geq 2$ years who have completed $\geq 12$ months of treatment for MAC, remain asymptomatic for $\mathrm{MAC}$, and are receiving stable cART (i.e., cART not requiring change for viral or immune failure) and who have sustained ( $\ 6$ months) CD4 count recovery well above the agespecific target for initiation of primary prophylaxis (as in adults, $>100$ cells $/ \mathrm{mm}^{3}$ for children aged $\succ 6$ years (AII*) and $>200$ cells $/ \mathrm{mm}^{3}$ for children aged 2 to $<6$ years) (CIII). Multidrug secondary prophylaxis should be reintroduced if the CD4 count falls below the age-related threshold.

\section{References}

1. Cassidy PM, Hedberg K, Saulson A, McNelly E, Winthrop KL. Nontuberculous mycobacterial disease prevalence and risk factors: a changing epidemiology. Clin Infect Dis. 2009 Dec 15; 49(12):e124-e129. Available at http://www.ncbi.nlm.nih.gov/pubmed/19911942. [PubMed: 19911942]

2. Griffith DE, Aksamit T, Brown-Elliott BA, et al. An official ATS/IDSA statement: diagnosis, treatment, and prevention of nontuberculous mycobacterial diseases. Am J Respir Crit Care Med. 2007 Feb 15; 175(4):367-416. Available at http://www.ncbi.nlm.nih.gov/pubmed/17277290. [PubMed: 17277290]

3. Kasperbauer SH, Daley CL. Diagnosis and treatment of infections due to Mycobacterium avium complex. Semin Respir Crit Care Med. 2008 Oct; 29(5):569-576. Available at http:// www.ncbi.nlm.nih.gov/pubmed/18810690. [PubMed: 18810690]

4. Gona P, Van Dyke RB, Williams PL, et al. Incidence of opportunistic and other infections in HIVinfected children in the HAART era. JAMA. 2006 Jul 19; 296(3):292-300. Available at http:// www.ncbi.nlm.nih.gov/pubmed/16849662. [PubMed: 16849662]

5. Nesheim SR, Kapogiannis BG, Soe MM, et al. Trends in opportunistic infections in the pre- and post-highly active antiretroviral therapy eras among HIV-infected children in the Perinatal AIDS Collaborative Transmission Study, 1986-2004. Pediatrics. 2007 Jul; 120(1):100-109. Available at http://www.ncbi.nlm.nih.gov/pubmed/17606567. [PubMed: 17606567]

6. Perez Mato S, Van Dyke RB. Pulmonary infections in children with HIV infection. Semin Respir Infect. 2002 Mar; 17(1):33-46. Available at http://www.ncbi.nlm.nih.gov/pubmed/11891517. [PubMed: 11891517]

7. Reed C, von Reyn CF, Chamblee S, et al. Environmental risk factors for infection with Mycobacterium avium complex. Am J Epidemiol. 2006 Jul 1; 164(1):32-40. Available at http:// www.ncbi.nlm.nih.gov/pubmed/16675537. [PubMed: 16675537]

8. Peacock KH, Lewis L, Lavoie S. Erosive mediastinal lymphadenitis associated with Mycobacterium avium infection in a pediatric acquired immunodeficiency syndrome patient. Pediatr Infect Dis J. 2000 Jun; 19(6):576-578. Available at http://www.ncbi.nlm.nih.gov/pubmed/10877180. [PubMed: 10877180]

9. Hartmann P, Plum G. Immunological defense mechanisms in tuberculosis and MAC-infection. Diagn Microbiol Infect Dis. 1999 Jun; 34(2):147-152. Available at http://www.ncbi.nlm.nih.gov/ pubmed/10354865. [PubMed: 10354865]

10. Keller C, Kirkpatrick S, Lee K, Paul M, Hanson IC, Gilger M. Disseminated Mycobacterium avium complex presenting as hematochezia in an infant with rapidly progressive acquired immunodeficiency syndrome. Pediatr Infect Dis J. 1996 Aug; 15(8):713-715. Available at http:// www.ncbi.nlm.nih.gov/pubmed/8858681. [PubMed: 8858681]

11. Kaplan JE, Benson C, Holmes KH, et al. Guidelines for prevention and treatment of opportunistic infections in HIV-infected adults and adolescents: recommendations from CDC, the National Institutes of Health, and the HIV Medicine Association of the Infectious Diseases Society of America. MMWR Recomm Rep. 2009 Apr 10; 58(RR-4):1-207. quiz CE201-204. Available at http://www.ncbi.nlm.nih.gov/pubmed/19357635. 
12. Wong DA, Yip PC, Cheung DT, Kam KM. Simple and rational approach to the identification of Mycobacterium tuberculosis, Mycobacterium avium complex species, and other commonly isolated mycobacteria. J Clin Microbiol. 2001 Oct; 39(10):3768-3771. Available at http:// www.ncbi.nlm.nih.gov/pubmed/11574614. [PubMed: 11574614]

13. Shin SJ, Lee BS, Koh WJ, et al. Efficient differentiation of Mycobacterium avium complex species and subspecies by use of five-target multiplex PCR. J Clin Microbiol. 2010 Nov; 48(11):40574062. Available at http://www.ncbi.nlm.nih.gov/pubmed/20810779. [PubMed: 20810779]

14. Iamsawat S, Surawut S, Prammananan T, Leelaporn A, Jearanaisilavong J. Multiplex PCR for detection of clarithromycin resistance and simultaneous species identification of Mycobacterium avium complex. Southeast Asian J Trop Med Public Health. 2010 May; 41(3):590-601. Available at http://www.ncbi.nlm.nih.gov/pubmed/20578547. [PubMed: 20578547]

15. Lewis LL, Butler KM, Husson RN, et al. Defining the population of human immunodeficiency virus-infected children at risk for Mycobacterium avium-intracellulare infection. J Pediatr. 1992 Nov; 121(5 Pt 1):677-683. Available at http://www.ncbi.nlm.nih.gov/pubmed/1432413. [PubMed: 1432413]

16. Rutstein RM, Cobb P, McGowan KL, Pinto-Martin J, Starr SE. Mycobacterium avium intracellulare complex infection in HIV-infected children. AIDS. 1993 Apr; 7(4):507-512. Available at http://www.ncbi.nlm.nih.gov/pubmed/8099487. [PubMed: 8099487]

17. Currier JS, Williams PL, Koletar SL, et al. with the AIDS Clinical Trials Group 362 Study Team. Discontinuation of Mycobacterium avium complex prophylaxis in patients with antiretroviral therapy-induced increases in CD4+ cell count. A randomized, double-blind, placebo-controlled trial. Ann Intern Med. 2000 Oct 3; 133(7):493-503. Available at http://www.ncbi.nlm.nih.gov/ pubmed/11015162. [PubMed: 11015162]

18. Brooks JT, Song R, Hanson DL, et al. Discontinuation of primary prophylaxis against Mycobacterium avium complex infection in HIV-infected persons receiving antiretroviral therapy: observations from a large national cohort in the United States, 1992-2002. Clin Infect Dis. 2005 Aug 15; 41(4):549-553. Available at http://www.ncbi.nlm.nih.gov/pubmed/16028167. [PubMed: 16028167]

19. Nachman S, Gona P, Dankner W, et al. The rate of serious bacterial infections among HIVinfected children with immune reconstitution who have discontinued opportunistic infection prophylaxis. Pediatrics. 2005 Apr; 115(4):e488-e494. Available at http://www.ncbi.nlm.nih.gov/ pubmed/15772172. [PubMed: 15772172]

20. Cohn DL, Fisher EJ, Peng GT, et al. A prospective randomized trial of four three-drug regimens in the treatment of disseminated Mycobacterium avium complex disease in AIDS patients: excess mortality associated with high-dose clarithromycin. Terry Beirn Community Programs for Clinical Research on AIDS. Clin Infect Dis. 1999 Jul; 29(1):125-133. Available at http:// www.ncbi.nlm.nih.gov/pubmed/10433575. [PubMed: 10433575]

21. Dunne M, Fessel J, Kumar P, et al. A randomized, double-blind trial comparing azithromycin and clarithromycin in the treatment of disseminated Mycobacterium avium infection in patients with human immunodeficiency virus. Clin Infect Dis. 2000 Nov; 31(5):1245-1252. Available at http:// www.ncbi.nlm.nih.gov/pubmed/11073759. [PubMed: 11073759]

22. Benson CA, Williams PL, Cohn DL, et al. Clarithromycin or rifabutin alone or in combination for primary prophylaxis of Mycobacterium avium complex disease in patients with AIDS: A randomized, double-blind, placebo-controlled trial. The AIDS Clinical Trials Group 196/Terry Beirn Community Programs for Clinical Research on AIDS 009 Protocol Team. J Infect Dis. 2000 Apr; 181(4):1289-1297. Available at http://www.ncbi.nlm.nih.gov/pubmed/10762562. [PubMed: 10762562]

23. Benson CA, Williams PL, Currier JS, et al. A prospective, randomized trial examining the efficacy and safety of clarithromycin in combination with ethambutol, rifabutin, or both for the treatment of disseminated Mycobacterium avium complex disease in persons with acquired immunodeficiency syndrome. Clin Infect Dis. 2003 Nov 1; 37(9):1234-1243. Available at http:// www.ncbi.nlm.nih.gov/pubmed/14557969. [PubMed: 14557969]

24. Grosset J, Ji B. Prevention of the selection of clarithromycin-resistant Mycobacterium aviumintracellulare complex. Drugs. 1997; 54(Suppl 2):23-27. discussion 28-29. Available at http:// www.ncbi.nlm.nih.gov/pubmed/9358197. [PubMed: 9358197] 
25. Corti M, Palmero D. Mycobacterium avium complex infection in HIV/AIDS patients. Expert Rev Anti Infect Ther. 2008 Jun; 6(3):351-363. Available at http://www.ncbi.nlm.nih.gov/pubmed/ 18588499. [PubMed: 18588499]

26. Ward TT, Rimland D, Kauffman C, Huycke M, Evans TG, Heifets L. Randomized, open-label trial of azithromycin plus ethambutol vs. clarithromycin plus ethambutol as therapy for Mycobacterium avium complex bacteremia in patients with human immunodeficiency virus infection. Veterans Affairs HIV Research Consortium. Clin Infect Dis. 1998 Nov; 27(5):1278-1285. Available at http://www.ncbi.nlm.nih.gov/pubmed/9827282. [PubMed: 9827282]

27. Powderly WG. Treatment of infection due to Mycobacterium avium complex. Pediatr Infect Dis J. 1999 May; 18(5):468-469. Available at http://www.ncbi.nlm.nih.gov/pubmed/10353523. [PubMed: 10353523]

28. Smith JA, Mueller BU, Nussenblatt RB, Whitcup SM. Corneal endothelial deposits in children positive for human immunodeficiency virus receiving rifabutin prophylaxis for Mycobacterium avium complex bacteremia. Am J Ophthalmol. 1999 Feb; 127(2):164-169. Available at http:// www.ncbi.nlm.nih.gov/pubmed/10030558. [PubMed: 10030558]

29. Race EM, Adelson-Mitty J, Kriegel GR, et al. Focal mycobacterial lymphadenitis following initiation of proteaseinhibitor therapy in patients with advanced HIV-1 disease. Lancet. 1998 Jan 24; 351(9098):252-255. Available at http://www.ncbi.nlm.nih.gov/pubmed/9457095. [PubMed: 9457095]

30. Phillips P, Chan K, Hogg R, et al. Azithromycin prophylaxis for Mycobacterium avium complex during the era of highly active antiretroviral therapy: evaluation of a provincial program. Clin Infect Dis. 2002 Feb 1; 34(3):371-378. Available at http://www.ncbi.nlm.nih.gov/pubmed/ 11774085. [PubMed: 11774085]

31. Steenhoff AP, Wood SM, Shah SS, Rutstein RM. Cutaneous Mycobacterium avium complex infection as a manifestation of the immune reconstitution syndrome in a human immunodeficiency virus-infected child. Pediatr Infect Dis J. 2007 Aug; 26(8):755-757. Available at http:// www.ncbi.nlm.nih.gov/pubmed/17848894. [PubMed: 17848894]

32. Babiker ZO, Beeston C, Purcell J, Desai N, Ustianowski A. Mycobacterium avium complex suppurative parotitis in a patient with human immunodeficiency virus infection presenting with immune reconstitution inflammatory syndrome. J Med Microbiol. 2010 Nov; 59(Pt 11):13651367. Available at http://www.ncbi.nlm.nih.gov/pubmed/20634331. [PubMed: 20634331]

33. American Academy of Pediatrics. Red Book: 2009 Report of the Committee on Infectious Diseases. 28th ed. 28th ed. Elk Grove Village, IL: 2009.

34. Lange CG, Woolley IJ, Brodt RH. Disseminated mycobacterium avium-intracellulare complex (MAC) infection in the era of effective antiretroviral therapy: is prophylaxis still indicated? Drugs. 2004; 64(7):679-692. Available at http://www.ncbi.nlm.nih.gov/pubmed/15025543. [PubMed: 15025543]

35. Aberg J, Powderly W. HIV: primary and secondary prophylaxis for opportunistic infections. Clin Evid (Online). 2010; 2010 Available at http://www.ncbi.nlm.nih.gov/pubmed/21418688.

36. Powderly WG. Prophylaxis for opportunistic infections in an era of effective antiretroviral therapy. Clin Infect Dis. 2000 Aug; 31(2):597-601. Available at http://www.ncbi.nlm.nih.gov/pubmed/ 10987727. [PubMed: 10987727]

\section{Dosing Recommendations for Prevention and Treatment of Mycobacterium avium Complex (MAC)}

\begin{tabular}{|c|c|c|c|}
\hline \multicolumn{4}{|c|}{ Preventive Regimen } \\
\hline Indication & First Choice & Alternative & Comments/Special Issues \\
\hline Primary Prophylaxis & $\begin{array}{l}\text { - Clarithromycin } \\
7.5 \mathrm{mg} / \mathrm{kg} \\
\text { body weight } \\
\text { (maximum } \\
500 \mathrm{mg} \text { ) by }\end{array}$ & $\begin{array}{l}\text { - } \quad \text { Azithromycin } \\
5 \mathrm{mg} / \mathrm{kg} \\
\text { body weight } \\
\text { (maximum } \\
250 \mathrm{mg} \text { ) }\end{array}$ & $\begin{array}{cl}\text { Primary Prophylaxis Indicated for } \\
\text { Children: } \\
1 & \text { Aged }<1 \text { year with } \\
& \text { CD4 count }<750 \\
& \text { cells } / \mathrm{mm}^{3} ;\end{array}$ \\
\hline
\end{tabular}




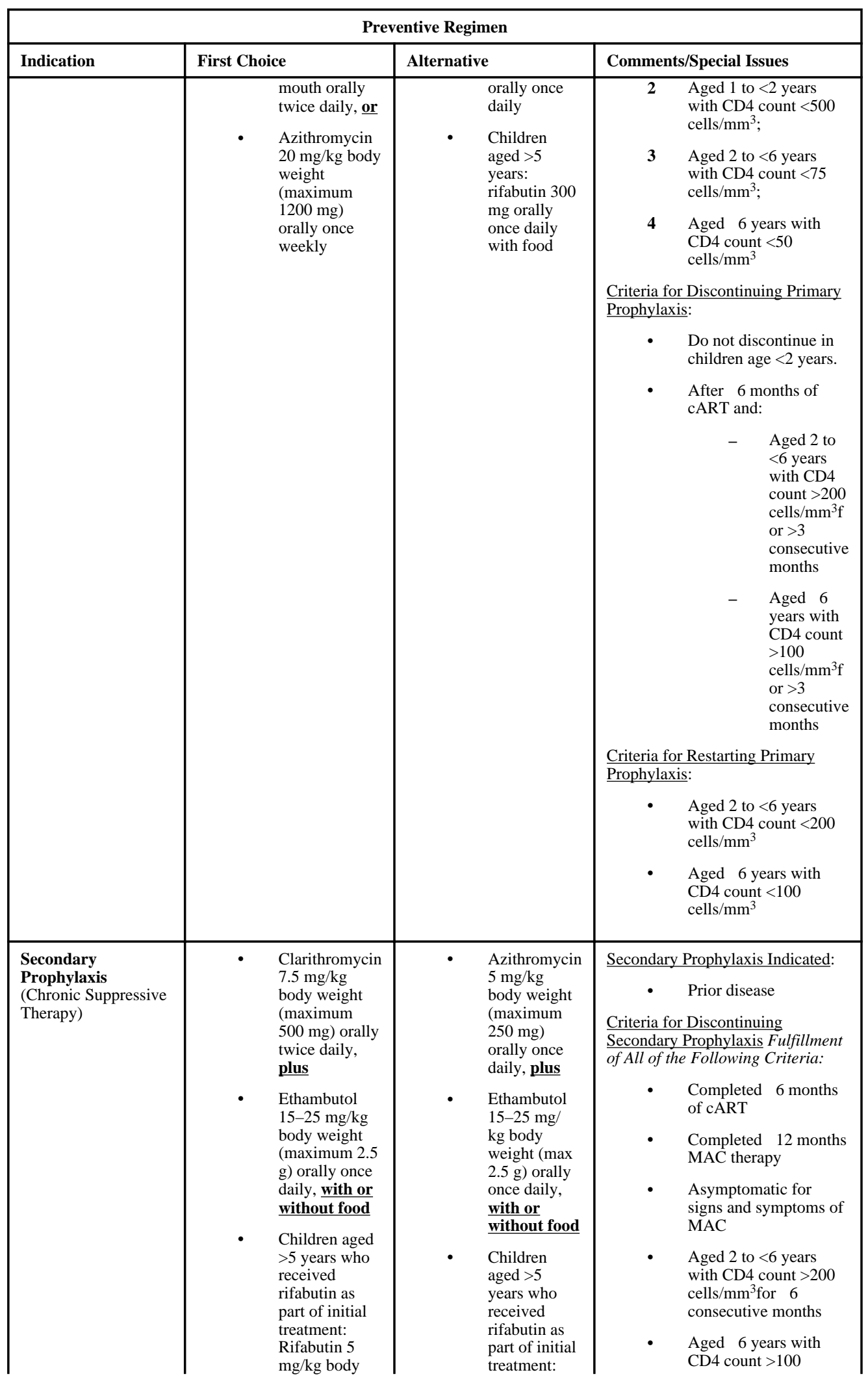




\begin{tabular}{|c|c|c|c|}
\hline \multicolumn{4}{|c|}{ Preventive Regimen } \\
\hline Indication & First Choice & Alternative & Comments/Special Issues \\
\hline & $\begin{array}{l}\text { weight } \\
\text { (maximum } \\
300 \mathrm{mg} \text { ) orally } \\
\text { once daily } \\
\text { with food }\end{array}$ & $\begin{array}{l}\text { Rifabutin } 5 \\
\text { mg/kg body } \\
\text { weight } \\
\text { (maximum } \\
300 \mathrm{mg} \text { ) } \\
\text { orally once } \\
\text { daily with } \\
\text { food. }\end{array}$ & 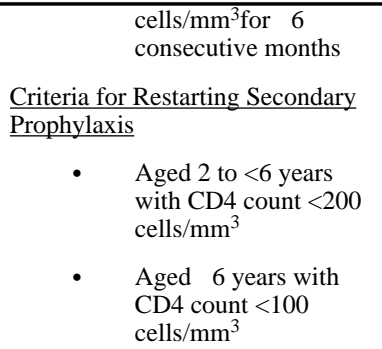 \\
\hline Treatment & 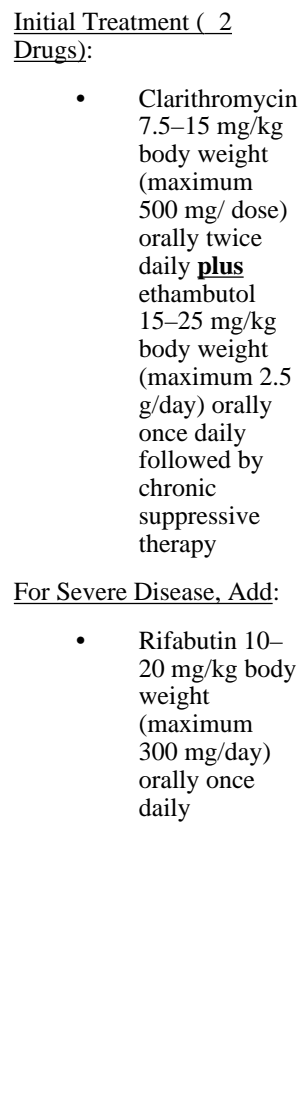 & 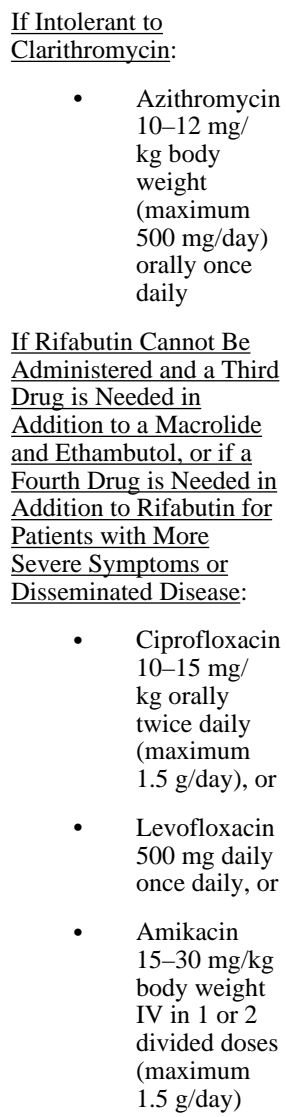 & $\begin{array}{l}\text { Combination therapy with a } \\
\text { minimum of } 2 \text { drugs is } \\
\text { recommended for at least } 12 \\
\text { months. } \\
\text { Clofazimine is associated with } \\
\text { increased mortality in HIV-infected } \\
\text { adults and should not be used. } \\
\text { Children receiving ethambutol who } \\
\text { are old enough to undergo routine } \\
\text { eye testing should have monthly } \\
\text { monitoring of visual acuity and } \\
\text { color discrimination. } \\
\text { Fluoroquinolones (e.g., } \\
\text { ciprofloxacin and levofloxacin) are } \\
\text { not labeled for use in children aged } \\
\text { <18 years because of concerns } \\
\text { regarding potential effects on } \\
\text { cartilage; use in younger } \\
\text { individuals requires an assessment } \\
\text { of potential risks and benefits } \\
\text { Chronic suppressive therapy } \\
\text { (secondary prophylaxis) is } \\
\text { recommended in children and } \\
\text { adults following initial therapy. }\end{array}$ \\
\hline
\end{tabular}

Key to Acronyms: $\mathrm{cART}=$ combination antiretroviral therapy; $\mathrm{CD} 4=\mathrm{CD} 4 \mathrm{~T}$ lymphocyte; $\mathrm{MAC}=$ Mycobacterium avium Complex; IV = intravenous

\section{Mycobacterium tuberculosis (Last updated November 6, 2013; last reviewed November 6, 2013)}

\begin{tabular}{|l|}
\hline Panel's Recommendations \\
\hline Detection of Latent TB Infection \\
$\begin{array}{l}\text { Diagnostic methods for latent tuberculosis (TB) infection (LTBI) include the tuberculin skin test (TST), } \\
\text { administered by the Mantoux method with an Food and Drug Administration (FDA)-approved purified }\end{array}$
\end{tabular}


Panel's Recommendations

protein derivative, or FDA-approved interferon gamma release assays (IGRA) (QuantiFERON ${ }^{\circledR}$-TB Gold

In-Tube, and T SPOT $\left.{ }^{\circledR} . \mathrm{TB}\right)$; TST is preferred over IGRA in children aged $<5$ years (BII).

- $\quad$ TST and IGRA should NOT be used to rule out disease and cannot replace regular screening for TB exposure (AII). In high-TB-burden settings, screening for TB exposure and for signs or symptoms suggestive of TB disease is universally applicable and should occur at every health care visit (AII)

\section{Treatment for LTBI}

- $\quad$ HIV-infected children should receive preventive therapy if they have a positive TST or IGRA result or if they are exposed to an individual with infectious TB (regardless of previous treatment for TB or the TST or IGRA result), after TB disease has been excluded (AII).

- The preferred preventive therapy regimen is isoniazid daily for 9 months (AII). If adherence with daily isoniazid cannot be ensured, then consider twice-weekly isoniazid by directly observed therapy (DOT) by a trained worker, not a family member (BII).

- With exposure to an isoniazid mono-resistant source case, preventive therapy consisting of daily rifampin for 6 months is recommended, with adjustment of combination antiretroviral therapy (cART) as required (BII).

- A 12-dose combination regimen of once-weekly isoniazid and rifapentine by DOT is as safe and effective as other regimens in preventing TB disease, and the completion rate is greater than for longer regimens. However, pediatric experience with this regimen is limited, and drug-drug interactions between rifapentine and other antiretroviral drugs have not been determined. This regimen is not recommended for children aged $<2$ years, nor for HIV-infected adults or children who are receiving cART or individuals who have LTBI with presumed isoniazid or rifampin resistance; the preferred regimen for children aged 2 to 11 years remains daily isoniazid for 9 months.

\section{Treatment of TB Disease}

- In children diagnosed with TB, DOT must be started immediately (AII) and all cases of suspected and confirmed TB disease must be reported to the relevant health authorities.

- $\quad$ All children diagnosed with TB should be tested for HIV infection (AIII)

- In HIV-infected children, the recommended treatment for fully-drug-susceptible TB is a 4-drug regimen consisting of isoniazid, rifampin, pyrazinamide, and ethambutol given daily during the 2-month intensive phase, followed by a 7-month continuation phase using only isoniazid and rifampin (AII), with adjustment of cART as required. With good adherence and treatment response, thrice- weekly treatment under DOT during the continuation phase can be considered (CII).

- $\quad$ For children with extrapulmonary disease caused by drug susceptible TB involving the bones or joints, central nervous system (CNS), or disseminated/miliary disease, the recommended duration of treatment is 12 months (AIII)

- $\quad$ For TB meningitis (TBM), pending drug-susceptibility testing results, ethionamide can replace ethambutol (or an injectable aminoglycoside) as the fourth drug because of its superior cerebrospinal fluid penetration (CII).

- $\quad$ Children with suspected and confirmed multidrug resistant (MDR) TB (i.e., resistance to both isoniazid and rifampin) should be managed in consultation with an expert. In the United States, treatment of MDR-TB should be individualized based on drug susceptibility test (DST) results (in cases where DST results for the child are not available, then DST results for the source case should be used to guide initial choice of regimen) (AII).

- $\quad$ Treatment for TB must commence as soon as the diagnosis is established in HIV-infected children, both those who are already on cART and those not yet receiving cART; those not yet on cART should be evaluated for early cART initiation, preferably within 2 to 8 weeks of starting TB therapy (AII).

- Depending on age and previous cART exposure, an efavirenz-based regimen usually is preferable because such regimens are associated with better treatment outcomes (AII). Nevirapine with potential dose adjustment with concomitant rifampin administration can also be considered (CIII).

- If a protease inhibitor-based regimen is used, superboosting with ritonavir (using a ritonavir dose equal to the lopinavir dose) for the full duration of rifampin treatment (and 2 weeks after termination) is required (AII).

- $\quad$ Pyridoxine supplementation (1-2 $\mathrm{mg} / \mathrm{kg}$ body weight/day, max $50 \mathrm{mg} / \mathrm{day}$ ) is recommended for all HIVinfected children who are taking isoniazid (AII) or cycloserine (AIII)

- $\quad$ Adjunctive corticosteroids treatment (with ongoing treatment for TB) is indicated for children with TBM or pericardial effusion (AII). It can also be considered with severe immune reconstitution inflammatory syndrome, airway compression, or pleural effusion (BII). 
Panel's Recommendations

- $\quad$ Liver chemistry tests should be performed before initiation and after 2, 4, and 8 weeks of treatment for TB (the same for cART initiation while receiving treatment for TB) (BIII). Beyond 2 months, routine testing every 2 to 3 months is advisable for all children receiving cART, or more frequently if clinically indicated (BIII).

Rating of Recommendations: $\mathrm{A}=$ Strong; $\mathrm{B}=$ Moderate; $\mathrm{C}=$ Optional

Rating of Evidence: $\mathrm{I}=$ One or more randomized trials in children ${ }^{\dagger}$ with clinical outcomes and/or validated endpoints; $\mathrm{I}^{*}$ $=$ One or more randomized trials in adults with clinical outcomes and/or validated laboratory endpoints with accompanying data in children $^{\dagger}$ from one or more well-designed, nonrandomized trials or observational cohort studies with long-term clinical outcomes; II = One or more well-designed, nonrandomized trials or observational cohort studies in children $\underline{\text { with }}^{\dagger}$ long-term outcomes; II $^{*}=$ One or more well-designed, nonrandomized trials or observational studies in adults with longterm clinical outcomes with accompanying data in children ${ }^{\dagger}$ from one or more similar nonrandomized trials or cohort studies with clinical outcome data; III = Expert opinion

${ }^{\dagger}$ Studies that include children or children/adolescents, but not studies limited to post-pubertal adolescents

\section{Epidemiology}

Of the 11,182 cases of tuberculosis (TB) reported in the United States in 2010, $637(6 \%)$ occurred in children aged $<15 .{ }^{1}$ Information on the epidemiology of TB in the United States can be found at http://www.cdc.gov/tb/statistics/default.htm. Among TB cases with known HIV tests results reported in the United States between 1994 and 2007, HIV coinfection was reported in $20 \%$ of adults and $3 \%$ of children and adolescents ( $<18$ years) overall. ${ }^{2}$ The actual rate of HIV coinfection in U.S. children and adolescents with TB is unknown because of the very low rate of HIV testing in this population-more than $70 \%$ did not have an HIV result reported to the National TB Surveillance System $;^{2}$ however, routine HIV testing is indicated in all individuals with confirmed or suspected TB.

Numerous studies have documented the increased risk of TB in HIV-infected adults. Domestic and international studies have documented a similar increased risk of TB in HIVinfected children. ${ }^{3-5}$ Unlike other AIDS-related opportunistic infections, a decreasing or low CD4 T lymphocyte (CD4) cell count is not necessary for increased risk of TB in HIVinfected children. Congenital TB is rare, but has been reported with possible increased frequency in children born to HIV-infected mothers with TB. ${ }^{6,7}$

Children with TB usually have been infected by an adult in their immediate environment, and their disease represents progression of primary infection rather than reactivation disease. ${ }^{8}$ Discovery and treatment of the source case and evaluation of all exposed members of the household are particularly important to terminate ongoing transmission (from primary and secondary cases) and to find and diagnose high-risk individuals with latent Mycobacterium tuberculosis infection who may benefit from preventive therapy. ${ }^{9}$ All confirmed and suspected cases of TB disease must be reported to state and local health departments, which will assist in contact evaluation.

Disease caused by Mycobacterium bovis is less common than disease caused by $M$. tuberculosis in the United States, but pediatric cases have been reported. ${ }^{10,11}$ Among 11,860 TB cases reported in the United States between 1995 and 2005 for which genotyping information was available, $165(1.4 \%)$ were caused by M. bovis; of these, $12(7.3 \%)$ of the patients were aged 0 to 4 years and $19(11.5 \%)$ were aged 5 to 14 years. Risk factors for $M$. 
bovis disease in the United States include Hispanic ethnicity, age $<15$ years, HIV infection, and extrapulmonary TB (EPTB). ${ }^{12}$ Several reports demonstrate that $M$. bovis is primarily transmitted via ingestion of unpasteurized dairy products, ${ }^{10,12}$ which may have been consumed outside the United States or imported casually. Although ingestion is the usual route of entry, human-to-human airborne transmission has been observed and its likelihood may be increased by HIV coinfection. Distinction between M. tuberculosis and M. bovis is important, because nearly all $M$. bovis isolates are resistant to pyrazinamide and the public health interventions are different.

The emergence and effective transmission of drug-resistant TB is a major obstacle to global TB control. ${ }^{13-15}$ In the United States, comprehensive public health measures successfully reduced the rates of drug-resistant TB; the proportion of primary multidrug-resistant TB (MDR-TB) cases declined from 2.5\% in 1993 to approximately $1.1 \%$ in 1997 and has remained at about $1 \%$ since. ${ }^{16}$ Between 1994 and 2007, M. tuberculosis resistance to any first-line TB drug was found in $17 \%$ of children and adolescents ( $<18$ years) who had culture-confirmed TB and drug-susceptibility testing results reported to the Centers for Disease Control and Prevention (CDC), with higher rates in foreign-born (20\%) than in U.S.-born children (15\%). ${ }^{2}$ The fraction of culture-confirmed TB that was MDR-TB (resistant to at least isoniazid and rifampin) was $2 \%$ in foreignborn and $1 \%$ in U.S.-born children. ${ }^{2}$ However, the fraction of foreign-born TB patients in the United States continues to rise, ${ }^{16}$ many originating from countries with high rates of drug-resistant TB. Parents, guardians, or visiting relatives may expose children to drug-resistant infection.

Extensively drug-resistant TB (XDR-TB), defined as resistance to isoniazid and rifampin (MDR-TB) with additional resistance to any fluoroquinolone and at least one of three second-line injectable drugs (capreomycin, kanamycin, and amikacin), emerged globally as an important new threat, particularly in HIV-infected individuals. ${ }^{13,14,17}$ Of the 49 cases of XDR-TB reported in the United States from 1993 to 2006, one (2\%) was in a child aged <15 years. ${ }^{18}$ However, this number possibly underestimates the burden in children, because most TB cases in children are not culture-positive; thus, a definitive diagnosis of drug-resistant TB is not achieved.

\section{Clinical Manifestations}

Once infected with TB, young (aged $<5$ years) and/or immunocompromised children such as those who are HIV-infected are highly susceptible to developing TB disease, with the first 12 months after primary infection representing the period of greatest risk for progression to TB disease. ${ }^{5,19}$ Generally, the clinical features of TB in HIV-infected and HIV-uninfected children are similar, with non-localizing signs such as failure to thrive, cough, and intermittent fever present, although disease progression may be more rapid and the development of complicated or disseminated disease more likely in HIV-infected children. ${ }^{8,20,21}$ Both HIV-infected and HIV-uninfected children may present with characteristic pulmonary involvement such as hilar and/or mediastinal adenopathy, which may cause airway compression. Immunocompromised children, including those who are HIV-infected, may also have atypical findings, such as multi-lobar infiltrates and diffuse interstitial disease. ${ }^{4}$ Rapidly progressive disease, including meningitis or mycobacterial 
sepsis, is more likely in the very young and/or immunocompromised, including HIVinfected children. Descriptions of the disease's natural history provide the following general patterns that characterize childhood $\mathrm{TB}$, although exceptions to the rule are common and HIV-infected children of all ages are more likely to have disease manifestations similar to those seen in very young (immune immature) children: ${ }^{8}$

- Aged <1 year: Greatest risk of disease progression and disease manifestations reflecting poor containment such as disseminated (miliary) $\mathrm{TB}$, tuberculous meningitis (TBM), extensive pneumonic infiltration.

- Aged 1-4 years: Persistent but declining risk of disseminated forms of disease. Children $<5$ years are at greatest risk of complications resulting from airway compression, because of their small, pliable airways and exuberant lymph node responses. Extra-thoracic manifestations are not uncommon (see below).

- Aged 5-9 years: Period of lowest risk for immunocompetent children, but they may contribute significantly to the total case load, depending on the average age at which primary infection occurs in the epidemiological setting. In this age group, a wide range of disease manifestations is seen, including disease patterns seen in young children and adult-type disease. Adult-type pulmonary disease, with upper lobe infiltration, cavitation, and sputum production, is more common starting at age 8 years, and in high-TB-burden settings, and is seen more frequently in adolescent girls than in boys.

- Aged >10 years: Adult-type pulmonary disease is more common. Children in this age group are more likely to have positive results from acid-fast bacteria (AFB) sputum-smear microscopy and should be regarded as a potential infectious source. $^{22}$

Approximately $25 \%$ of children with TB have extra-thoracic involvement, with disseminated forms more common in HIV-infected children. ${ }^{20,23-25}$ Extra-thoracic disease manifestations include:

- Peripheral lymphadenitis (usually cervical). Features include a matted mass of lymph nodes $>2 \times 2 \mathrm{~cm} .{ }^{26}$ Axillary adenitis ipsilateral to bacille calmette Guerin (BCG) vaccination site is suggestive of BCG adenitis (also see immune reconstitution inflammatory syndrome [IRIS] discussion).

- $\quad$ TBM is most common in children aged $<3$ years, but especially with HIV coinfection, can occur at any age. Disease manifestations are often similar, but the list of differential diagnoses is greatly expanded in immunocompromised individuals, including HIV-infected children. ${ }^{27,28}$

- Osteo-articular disease can involve any bone or joint, but vertebral involvement with typical TB gibbus formation with/without para-vertebral abscess formation is most common.

- Cold abscesses can occur at any site, but often develop in association with bone involvement or in deep muscle groups, such as psoas muscle. 
- A great variety of disease manifestations are possible, including hypersensitivity reactions such as erythema nodosum and phlyctenular keratoconjunctivitis. ${ }^{29}$

\section{Diagnosis}

TB Infection-Latent TB infection (LTBI), which by definition is a symptomless condition, can be diagnosed using the tuberculin skin test (TST), administered by the Mantoux method, or by interferon-gamma release assays (IGRAs). Both categories of testing methods are indirect ways of detecting M. tuberculosis infection and require T-cell immune activity; thus, HIV infection and the degree of immune alteration diminish the utility of these tests and change interpretation of results. A negative result with any of these tests cannot be regarded as exclusionary for M. tuberculosis infection (AII), whether latent or active, especially in the context of HIV infection, and the interpretation of any result with any of these tests must take into account an individual patient's epidemiological and medical factors and the circumstances of testing. The QuantiFERON-TB Gold In-Tube (QFT) (Cellestis Limited, Valencia, California) and the T SPOT ${ }^{\circledR}$.TB assay (Oxford Immunotec, Marlborough, Massachusetts) are U.S. Food and Drug Administration (FDA)-approved. An IGRA is preferred for testing BCG-vaccinated patients and for use in settings when the return rate for TST reading is poor; however, studies of IGRA performance in HIV-infected children and in very young children are limited, and results from these studies have shown inconsistent results, with data on sensitivity and specificity in this age group not available. ${ }^{30}$ TST is preferred over IGRAs for children younger than age 5 years (AII). ${ }^{31}$ When increased sensitivity for diagnosing $M$. tuberculosis infection is sought, TST and an IGRA can be done simultaneously, with a positive result from either being diagnostic. Younger age, HIV infection, and reduced numbers of CD4 cells increase the rate of indeterminate IGRA results. ${ }^{32}$ A recent systematic review and meta-analysis also found reduced QFT sensitivity in young children with greatly reduced diagnostic utility in TB-endemic areas. ${ }^{33}$

Because HIV-infected children are at high risk of TB, annual LTBI testing is recommended beginning at ages 3 to 12 months and annually thereafter for those who tested negative in the past (AIII), ${ }^{34}$ depending on the local epidemiology, region of birth, and travel history. In HIV-infected patients, a TST induration $\geq 5 \mathrm{~mm}$ is considered positive, but even with this reduced cut-off, sensitivity remains poor; in U.S. recommendations, cut-off points for IGRAs are not adjusted for HIV infection. It is important that skin tests be administered and read correctly (http://www.cdc.gov/tb/education/Mantoux/default.htm). ${ }^{34}$ The use of control skin antigens to assess cutaneous anergy is of uncertain value and not recommended (AII). Sensitivity to tuberculin is reduced by severe malnutrition and some viral infections, including measles; the additive effect of HIV infection in these circumstances has not been determined. As a precaution, skin testing scheduled around the time of live-virus vaccination should be done at the same time as, or delayed until 4 weeks after vaccination to avoid potentially suppressed sensitivity (AIII). Test characteristics for IGRAs in these situations have not been determined, but the same scheduling adjustments as for TST are advisable. ${ }^{31}$ Two-step skin testing may boost sensitivity in adults, but its utility has not been assessed in children nor in the presence of HIV infection and its use is not recommended. Patients who test positive should undergo chest radiography and clinical evaluation to exclude TB disease. 
TB Disease-The most rigorous diagnosis of TB requires culture confirmation. However, in clinical practice, a diagnosis of TB in children frequently depends on a combination of TB exposure or infection together with symptoms and clinical signs suggestive of TB and chest imaging studies with findings suggestive of active disease; where EPTB is suspected, histopathology and other laboratory results (such as evidence of granuloma formation on histological examination of biopsy specimens) also may aid diagnosis. Chest radiography should include both posteroanterior (or anteroposterior) and lateral views for optimal assessment of hilar adenopathy; in cases of uncertainty, ongoing symptom review and repeat radiography in 1 to 2 weeks may be highly informative. All children diagnosed with TB should be tested for HIV infection (AIII).

Direct methods for detection of M. tuberculosis include AFB microscopy, nucleic-acid amplification tests (NAATs), and isolation in culture. Sputum smears are positive on AFB microscopy in $50 \%$ to $70 \%$ of adults with pulmonary TB; however, young children and children infected with HIV often have paucibacillary disease (low bacterial load), resulting in lower yield from sputum smear microscopy and culture, and specimens may be difficult to obtain because young children are unable to expectorate. ${ }^{35}$ A positive smear result is suggestive of TB, but it does not differentiate M. tuberculosis from other mycobacterial species. Mycobacterial culture improves both sensitivity and specificity beyond that of AFB microscopy and permits species identification, drug-susceptibility testing, and genotyping. Confirming the presence of $M$. tuberculosis is most helpful in HIV-infected children because of the expansive differential diagnosis. ${ }^{36}$ Obtaining a total of 3 sputum specimens ${ }^{37}$ for microscopic evaluation and mycobacterial culture is advisable. ${ }^{38-40}$ Performing NAAT on at least one respiratory specimen is advisable in adults and also has added value in children. ${ }^{41,42}$ For children who are unable to produce sputum spontaneously, specimens should be collected via early-morning gastric aspirates or sputum induction; the first gastric aspirate collected gives the very highest yield and should be undertaken carefully. ${ }^{43}$ The sensitivity and specificity of AFB microscopy of gastric aspirate specimens is poor. Bronchoscopy can be considered for patients unable to produce sputum. ${ }^{37}$ When extrapulmonary involvement is suspected, relevant specimens should be obtained as clinically indicated and sent for histology and culture carefully. ${ }^{43}$ Overall yield is increased by collecting multiple specimens.

A single FDA-approved commercial NAAT for direct detection of M. tuberculosis in sputum samples with positive or negative smear-microscopy results is available in the U.S. market: Amplified M. tuberculosis Direct Test (Gen-Probe). Newer direct tests that also can detect genetic markers of drug resistance, such as GenXpert (Cepheid), have been developed for point-of-care applications; these tests have been adopted at some sites in the United States after local validation but are not yet FDA-approved. GeneXpert testing of non sputum samples is not recommended. Data on the use of urine lipoarabinomannan (LAM) in children is unavailable. For children who can produce sputum, consideration should be given to performing NAAT on at least one respiratory specimen if a diagnosis of TB is being considered and if a positive test result would alter case management; however, further research is needed before specific recommendations can be made on the use of NAAT in the diagnosis of TB in children who cannot produce sputum and in the diagnosis of EPTB. 
Individual case reports have shown the utility of such testing without determining the overall test characteristics for this off-label usage. ${ }^{41}$ Use of NAATs on gastric aspirate and cerebrospinal fluid specimens proved disappointing in the past; ${ }^{44-46}$ they may be useful for increasing specificity of diagnosis (confirming disease) but sensitivity is inadequate to exclude disease. ${ }^{47}$

Because of the challenges of specimen collection and poor bacteriologic yield in children including those who are HIV-infected, the epidemiologic risk factors and a TB exposure history are critical determinants for making the diagnosis. In clinical practice, diagnosis often rests on indirect tests for TB infection (positive result from TST or IGRA) together with symptoms and chest radiograph findings suggestive of active disease. ${ }^{19} \mathrm{~A}$ high index of suspicion is important, together with awareness that the stage of HIV infection affects the frequency of symptoms and radiologic signs and the characteristic performance of the indirect tests for infection such as TST, as well as the likelihood of alternative diagnoses (such as chronic lymphoid interstitial pneumonitis or recurrent bacterial infections). ${ }^{4}$

Drug-resistant TB should be suspected in the following situations: ${ }^{43}$

- Exposure to a person with drug-resistant TB,

- Residence in or travel to a region with high rates of drug-resistant TB,

- Residence in or work in an institution or setting in which drug-resistant TB is documented,

- Treatment of pulmonary problems with a prolonged course of multiple medicines or an injectable agent for more than a few weeks in a foreign country (i.e., the patient may not realize that he or she was treated for TB),

- Treatment of a pulmonary illness with a fluoroquinolone, and

- Treatment for LTBI when TB disease was not recognized.

Careful inquiry about the drug susceptibility pattern and treatment history of the likely source case (this should be routinely available for all newly diagnosed adult TB cases) ${ }^{48}$ is essential to guide clinical management and choice of treatment regimen in children. TB drug-susceptibility testing (genotypic and phenotypic) should be performed in all cases where $M$. tuberculosis is isolated from a child; obtaining specimen(s) for mycobacterial culture and TB-drug susceptibility testing is particularly important for those who meet any of the risk criteria for drug resistance or if treatment failure occurs. A service for the molecular (i.e., genotypic) detection of drug resistance, provided by CDC through public health microbiology laboratories, provides rapid assessment of drug resistance, but phenotypic testing, using well standardized techniques, remains the reference standard. ${ }^{41}$

\section{Prevention Recommendations}

The most effective way to reduce TB-related morbidity and mortality is to prevent TB disease, which can be achieved by preventing TB exposure, minimizing HIV-related immunocompromise with early initiation of combination antiretroviral therapy (cART), ${ }^{49,50}$ and preventing progression to disease by diagnosing infection or high-risk exposure early 
and treating it. ${ }^{36} \mathrm{~TB}$ infection control has proven to be critical in healthcare and high-risk congregate settings.

Preventing Exposure-Most childhood infections with M. tuberculosis come from exposure in the immediate environment, often the household. Risk factors for TB disease (such as homelessness, incarceration, exposure to institutional settings, birth or residence in a high TB burden region) in close contacts of HIV-infected children also should be considered. The peripartum period seems to be a particularly vulnerable period for HIVinfected mothers; they should be evaluated for TB if they develop any symptoms suggestive of disease. ${ }^{51}$

Preventing Disease-BCG vaccine, which is not routinely administered in the United States, should not be administered to HIV-infected infants and children (AII).

In the United States, where TB exposure is uncommon and BCG is not routinely administered at birth, HIV-infected children should have a TST (IGRA has uncertain value) during infancy (3-12 months of age) and annually thereafter (AIII). ${ }^{34}$ However, the value of this strategy will depend on the local TB epidemiology, region of birth, and travel history.

After TB disease has been excluded, all HIV-infected children who have had close contact with an infectious TB case (regardless of their TST or IGRA result or previous history of TB diagnosis) or who test positive for the first time (AI) should receive preventive therapy (AII). The preferred regimen is isoniazid (10-15 $\mathrm{mg} / \mathrm{kg}$ body weight/day for 9 months) (AII); if adherence with daily treatment supervised by the parent or other family member cannot be ensured, then isoniazid (20-30 $\mathrm{mg} / \mathrm{kg}$ body weight twice weekly as directlyobserved therapy [DOT] by a trained worker, not a family member) can be considered (BII). For HIV-infected children, liver chemistry tests (serum alanine aminotransferase [ALT] concentration at a minimum) should be performed before initiating isoniazid (AII) and monthly thereafter or if any symptoms or signs suggestive of possible hepatotoxicity develop; medical providers should emphasize to patients that isoniazid treatment should be stopped immediately upon the earliest onset of toxicity (such as excess fatigue, nausea, vomiting, abdominal pain, or jaundice), even before a clinical evaluation has been conducted, and that initial symptoms can be subtle and may not include jaundice. ${ }^{52}$ If isoniazid mono-resistance is known or suspected in the source case, daily rifampin for 6 months is recommended (BII). A 2-month regimen of rifampin and pyrazinamide has been associated with severe and fatal hepatotoxicity in adults and was never recommended for children (AII). Children exposed to other drug-resistant TB should receive individualized medical management in consultation with an expert, taking into account the susceptibility pattern and treatment history of the likely source-case. ${ }^{53,54}$

As noted above, in the United States, treatment for LTBI should be given to all HIV-infected patients following exposure to an infectious TB case or who test positive for the first time (i.e., positive on TST or IGRA) after TB disease has been excluded.

Ongoing prophylaxis after treatment for TB is completed (secondary or post-treatment prophylaxis) is not recommended. TB exposure screening should be ongoing and post- 
exposure prophylaxis provided following documented close contact with an infectious TB case, irrespective of previous exposure or treatment.

A 12-dose combination regimen of once-weekly isoniazid and rifapentine by DOT is safe and as effective as other regimens in preventing TB disease, and the completion rate is greater than for longer regimens. ${ }^{1,55-57}$ However, pediatric experience with this regimen is limited, and the drug-drug interactions between rifapentine and antiretroviral drugs have not been determined. This regimen is not recommended for children aged $<2$ years, for HIVinfected adults or children who are receiving cART, or for individuals who have LTBI with presumed isoniazid or rifampin resistance; the preferred regimen for children aged 2 to 11 years remains daily isoniazid for 9 months. ${ }^{1}$

\section{Treatment Recommendations}

Treating Disease-Empiric therapy for TB should be started in HIV-infected infants and children in whom the diagnosis is strongly suspected and continued until the diagnosis is definitively excluded. The use of DOT (by a trained worker, not a family member) is recommended to maximize adherence (AII). Principles for treatment of TB are similar in HIV-infected and HIV-uninfected children. However, treating TB in an HIV-infected child is complicated by cART interactions and overlapping toxicities. Once TB is diagnosed, treatment must be started immediately (AII). The recommended total treatment duration is a minimum of 9 months for HIV-infected children (AIII). ${ }^{34,58}$ An overview of dosing recommendations for the prevention and treatment of TB in HIV-infected children is provided in the Dosing Recommendations Table.

In HIV-infected children, treatment of drug-susceptible TB consists of a 4-drug regimen: isoniazid, rifampin, pyrazinamide, and ethambutol given daily during the 2-month intensivetherapy phase, followed by a 7-month continuation phase using only isoniazid and rifampin (AII). ${ }^{34}$ Therapy for HIV-infected children should be given as daily DOT. With good adherence and treatment response, thrice-weekly treatment during the continuation phase can be considered (CIII); once- or twice-weekly dosing has been associated with an increased rate of relapse or treatment failure with rifamycin resistance in HIV-infected adults with low CD4 counts and, therefore, is not recommended. ${ }^{59,60}$ For children without significant immune compromise and with minimal disease with fully drug-susceptible TB, some experts would consider a standard 3-drug regimen (isoniazid, rifampin, pyrazinamide) during the 2-month intensive phase and a continuation phase (using isoniazid and rifampin) of 4 months (BII).

Ethionamide can be used as an alternative to ethambutol (or an injectable aminoglycoside) in TBM cases (CII), because of its superior cerebrospinal fluid penetration. ${ }^{61-64}$ For children with extrapulmonary disease involving the bones or joints, central nervous system (CNS), or miliary disease, the minimum recommended total duration of treatment is 12 months (2-month intensive phase followed by 10-month continuation phase) (AIII); ${ }^{34,62,65}$ see the Dosing Recommendations Table. These recommendations assume that the organism is believed to be fully susceptible, that adherence is ensured by DOT, and that a child responds well clinically (and, if laboratory confirmed, microbiologically) to therapy. 
Co-Treatment of TB and HIV-Concomitant treatment of TB and HIV is complicated by unfavorable pharmacokinetic (PK) interactions and overlapping toxicities and should be managed by a specialist with expertise in treating both conditions. Issues to consider when treating both conditions include:

- The critical role of rifampin because of its bactericidal and sterilizing properties, but also its potent induction of the CYP3A enzyme system and p-glycoproteinmediated efflux that lowers cART drug levels, especially those of the protease inhibitors (PIs);

- Overlapping toxicities; and

- The challenges of adhering to a medication regimen that may include seven or more drugs. See the Summary of Recommendations Table.

Standard anti-TB treatment must start as soon as TB is diagnosed (AII). For children already receiving cART, the cART regimen should be reviewed to minimize potential toxicities and drug-drug interactions. For children not yet receiving cART, early cART initiation should be planned, preferably within 2 to 8 weeks of starting treatment for TB (AII). Results from treating TB/HIV coinfection in adults suggest that early initiation of cART after the start of treatment for TB (within 2-8 weeks) may increase the risk of IRIS, but it is associated with a significant reduction in mortality. ${ }^{66}$ Results from treating TB/HIV coinfection in children also support early cART initiation. ${ }^{49}$ For severely ill children, immediate cART initiation may be advisable (CIII). The timing of cART initiation with CNS TB remains more controversial because of the potentially devastating effects of CNS IRIS. ${ }^{67,68}$

The choice of cART regimen in an HIV-infected child receiving a rifampin-based TB treatment regimen should be carefully considered. Rifampin is a potent inducer of the CYP3A enzyme system, with resultant severe reductions in PI levels (except ritonavir, which partially reverses this effect) and moderate reductions in nevirapine levels; nucleoside reverse transcriptase inhibitor (NRTIs) and efavirenz drug levels are least affected.

Rifabutin, a rifamycin-class semi-synthetic antibiotic related to rifampin, exhibits minimal CYP3A induction and has been used in this context. However, drug dose adjustments are still required and data on its use in children remain limited; use only with expert guidance. NRTI drug levels are least affected by rifampin; therefore, a classic double NRTI backbone is maintained. However, because a triple NRTI strategy is associated with inferior virologic outcomes $^{69}$ (unless the viral load is sufficiently suppressed), the third drug of choice is usually a non-nucleoside reverse transcriptase inhibitor (NNRTI); efavirenz is the preferred NNRTI, but alternative options need to be considered in children in whom efavirenz is contraindicated or intolerable. Efavirenz is the preferred NNRTI in children and evidence suggests that no dosage adjustment is necessary (AII). ${ }^{70}$ Efavirenz was FDA approved in 2013 for children aged 3 months (and at least $3.5 \mathrm{~kg}$ ) to 3 years old, but experience in this age group remains very limited. Nevirapine can be considered, but serum drug levels are reduced by more than $30 \%$ to $40 \%$ during rifampin co-treatment. ${ }^{71}$ Adult data suggest that no dosage adjustment is necessary, apart from omitting the lead-in dose, ${ }^{32}$ but many pediatric experts still recommend a $\approx 30 \%$ increase in the nevirapine dose in children, given the low risk of hepatic toxicity (a particular concern in healthy in young women) and the 
need to ensure optimal drug levels in young children with high viral loads (CIII) (See the Summary of Recommendations Table).

If a PI-based regimen is used, then a super-boosted PI regimen is advised, such as lopinavir/ ritonavir with additional ritonavir to equal the lopinavir dose. ${ }^{73}$ The super-boosted PI regimen should be continued for the full duration of rifampin treatment and 2 weeks after termination of TB therapies (AIII). For children already receiving cART, the issues are similar. cART must continue and concurrent treatment of TB must be started immediately (AII). The cART regimen should be reviewed to ensure optimal treatment of both TB and HIV and to minimize potential toxicities and drug-drug interactions. Combined use of integrase inhibitors and other cART classes with rifampin-based treatment has not been evaluated in children. Ongoing studies in adults suggest that dosage adjustment also is required with integrase inhibitors (See the Summary of Recommendations Table).

When available, therapeutic drug monitoring can be used to help guide drug dose adjustments during HIV/TB co-treatment.

Treatment of Drug-Resistant TB-For treatment of drug-resistant TB, a minimum of 4 drugs to which the isolate is susceptible should be administered, including two or more bactericidal drugs (AII). Therapeutic regimens are individualized on the basis of the resistance pattern of the $M$. tuberculosis isolate and treatment history of the patient and the likely source case, considering the relative activities of each drug, the extent of disease, and any comorbid conditions. Children with suspected or confirmed drug-resistant TB should be managed in consultation with an expert.

Mono-Drug Resistance-If the strain is resistant only to isoniazid, isoniazid should be discontinued and the patient treated with 9 to 12 months of a rifampin-containing regimen (e.g., rifampin, pyrazinamide, ethambutol) (BII). Rifampin mono-resistance is rare, and rifampin resistance usually is a marker of MDR-TB. Therefore, if rifampin mono-resistance is detected with a rapid test, it should be regarded as MDR-TB until the susceptibility or resistance to both isoniazid and rifampin is confirmed by phenotypic testing, because the rapid molecular (genotypic) methods for detecting resistance are not as sensitive to isoniazid as they are to rifampin.

MDR-TB-Children with suspected and confirmed MDR-TB (resistance to both isoniazid and rifampin) should be managed in consultation with an expert. In the United States, treatment of MDR-TB should be individualized based on drug susceptibility test (DST) results. In cases where DST results for a child are unavailable, DST results for the source case should be used to guide initial choice of regimen. For treatment of drug-resistant TB, a minimum of 3 to 4 drugs to which the isolate is susceptible should be administered, including two or more bactericidal drugs (AII). Children with extensive or disseminated disease should be treated with at least 5 active drugs, because early aggressive treatment provides the best chance for cure. ${ }^{14,53,54}$ All treatment for MDR-TB in HIV-infected children should be given daily with DOT. ${ }^{34,74}$ 
XDR-TB-Children with suspected or confirmed XDR-TB should be managed in consultation with an expert. XDR-TB is a form of MDR-TB for which the principles of management are similar, albeit with even greater challenges. ${ }^{53}$

Adjunctive Treatment-Adjunctive treatment with corticosteroids is indicated for children with TBM, since it reduces mortality and long-term neurologic impairment (AII).

Adjunctive corticosteroid use reduces long-term constrictive complications in TB pericarditis (AII) and is associated with more rapid symptom resolution in TB pleural effusion (relative indication). It also can be considered with severe airway obstruction related to endobronchial TB and highly symptomatic TB IRIS (BIII). Prednisone (1-2 $\mathrm{mg} / \mathrm{kg}$ body weight/day) for 4 to 6 weeks is advisable, tapered over 2 weeks. Pyridoxine (1$2 \mathrm{mg} / \mathrm{kg}$ body weight/day, $\max 50 \mathrm{mg} /$ day) is recommended for all $\mathrm{HIV}$-infected children treated with isoniazid, because of persistent low pyridoxine levels and possible increased risk of peripheral neuropathy (AII) ${ }^{75}$

Monitoring of Adverse Events (Including IRIS)—Regular monitoring of clinical and bacteriologic response to therapy is important (AII). For children with pulmonary TB, chest radiographs should be obtained 2 months after the start of treatment to evaluate acute response to therapy and then serially as needed, judging by clinical response. ${ }^{34}$ Hilar adenopathy may persist or even worsen despite successful treatment, and normalization of the chest radiograph is not a criterion for shortening or discontinuing therapy. The most important indicators of treatment response are bacteriologic conversion, symptom resolution, and weight gain; all children with culture-confirmed disease should be monitored regularly for bacteriologic response; ${ }^{37}$ this is critical in all children with extensive lung disease or culture-confirmed drug-resistant TB (CIII).

Gastric upset can occur during the initial weeks of isoniazid treatment, but it usually can be avoided by having some food in the stomach when the drug is administered. Hepatotoxicity is the most common serious adverse effect. It includes subclinical hepatic enzyme elevation, which usually resolves spontaneously during continuation of treatment, and clinical hepatitis that usually resolves when the drug is discontinued. It rarely progresses to hepatic failure, but the likelihood increases when isoniazid is continued despite hepatitis symptoms (jaundice; tender, enlarged liver). Hepatotoxicity is less frequent in children than in adults, but no age group is risk-free. Transient asymptomatic serum transaminase elevations have been noted in $3 \%$ to $10 \%$ and clinical hepatitis in $<1 \%$ of children receiving isoniazid; $<1 \%$ required treatment discontinuation. ${ }^{65,76}$ The rate of hepatotoxicity may be higher in children who take multiple hepatotoxic medications.

Although the risk in HIV-infected children has not been quantified, excessive hepatotoxicity has not been documented. Liver chemistry tests (serum ALT at a minimum; AST and bilirubin also should be considered) should be performed before initiation and after 2, 4, and 8 weeks of treatment for TB (the same for cART initiation while receiving treatment for TB) (BIII). Beyond 2 months, routine testing every 2 to 3 months is advisable for all children receiving ART, and more frequently if clinically indicated (BIII). Patients and their families should be educated about the signs and symptoms of hepatotoxicity; for children who develop them, treatment should be stopped and evaluation done on an urgent basis and liver 
enzymes measured (AIII). Mild elevations in serum transaminase concentration (i.e., less than 3 times the upper limit of normal [ULN]) do not require drug discontinuation in children who are asymptomatic and in whom other findings (including bilirubin) are normal (AII). If transaminase levels exceed five times the ULN or three times the ULN in the presence of any symptoms or signs indicative of hepatotoxicity (e.g., anorexia, jaundice, raised bilirubin), then all hepatotoxic drugs should be immediately discontinued. Discussion with an expert on further management using non-hepatotoxic drugs, and future careful rechallenge with first-line TB drugs should be considered. With transaminase levels three to five times the ULN in the absence of any symptoms or signs indicative of hepatotoxicity, treatment can cautiously continue with regular (at least weekly) liver chemistry tests and ongoing expert consultation. $.22,77$

Rifampin is also associated with hepatotoxicity. If transaminase levels exceed 5 times ULN or 3 times the ULN in the presence of any symptoms or signs indicative of hepatotoxicity (e.g., anorexia, jaundice, raised bilirubin), then all hepatotoxic drugs should be immediately discontinued. Discussion with an expert on further management using non-hepatotoxic drugs, and future careful re-challenge with first-line TB drugs should be considered. Rifampin may lead to color changes in secretions including urine and saliva, and may lead to discoloration of contact lenses. Ethambutol can cause optic neuritis, with symptoms of blurry vision, central scotomata, and redgreen color blindness, but it is rare at the recommended daily dose of 20 to $25 \mathrm{mg} / \mathrm{kg}$ body weight ${ }^{34,37,62}$ and is usually reversible ${ }^{78,79}$ (see http://whqlibdoc.who.int/hq/2006/WHO_HTM_TB_2006.365_eng.pdf). Because ethambutol should be given daily as part of a 4-drug regimen for TB treatment, intermittent dosing (i.e., two or three times weekly) in children is not recommended. The maximum recommended dose of ethambutol given as daily dosing is $1.6 \mathrm{~g}$. Visual acuity should be evaluated before starting ethambutol and monitored regularly during treatment (AIII). Use of ethambutol in very young children whose visual acuity cannot be monitored requires careful consideration of risks and benefits. ${ }^{34}$

Hypothyroidism has been associated with ethionamide and 4 (para)-aminosalicylic acid use; ${ }^{80}$ periodic (i.e., every 3 months) monitoring of thyroid function is recommended (AIII). Major adverse effects of aminoglycoside drugs are ototoxicity and nephrotoxicity; periodic (i.e., every 3 months) audiometry and blood urea and creatinine measurements are recommended (AIII). Audiometry should be continued until 6 months after treatment completion, because ototoxicity can progress after termination of prolonged aminoglycoside use. Co-administration of pyridoxine (1-2 $\mathrm{mg} / \mathrm{kg}$ body weight/day) with cycloserine is recommended to reduce CNS side-effects (AIII).

Immune Reconstitution Inflammatory Syndrome (IRIS)-TB IRIS after initiation of cART was first reported in HIV-infected adults. ${ }^{81-83}$ It may present with new onset of systemic symptoms, especially high fever; expanding CNS lesions; and worsening adenopathy, pulmonary infiltrates, or pleural effusions. Similar cases in children have been reported. ${ }^{65,84,85}$ IRIS should be suspected in children with advanced immunosuppression who initiate cART and develop new symptoms shortly thereafter (within 3-6 months), despite evidence of good HIV control (increased weight and CD4 count, reduced viral load). It represents a temporary exacerbation of symptoms and occurs in two clinical scenarios. In 
patients who have occult TB before cART initiation, TB may be unmasked by subsequent immune recovery. ${ }^{86}$ This unmasking or incident TB-IRIS usually occurs within 3 months of cART initiation and the pathogen typically is detectable. ${ }^{87}$ IRIS also can result in paradoxical worsening of TB disease in HIV/TB-coinfected patients after CART initiation; treatment failure because of microbial resistance or poor adherence also must be excluded in these cases. In prospective observational studies, IRIS occurred in nearly $20 \%$ of children, usually within 4 weeks of cART initiation, resulting mostly from atypical mycobacteria, BCG (in young vaccinated infants) and TB (more prevalent in older children). ${ }^{88,89}$ Mild-tomoderate symptoms of IRIS can be treated symptomatically with nonsteroidal antiinflammatory agents, while short-term use of systemic corticosteroids can be considered in more severe cases (BIII); ${ }^{81-83,90}$ treatment for TB and ART should not be discontinued.

Managing Treatment Failure-Most children with TB, including those who are HIVinfected, respond well to standard treatment. If clinical response is poor, then adherence to therapy, drug absorption, and the possibility of drug resistance should be addressed. Mycobacterial culture, drug-susceptibility testing, and serum concentrations of TB drugs should be done whenever possible. Drug resistance should be suspected in any child whose smear or culture fails to convert after 2 months of DOT or in any of the situations previously emphasized. Also consider possible alternative diagnoses or dual pathology.

Preventing Recurrence-TB recurrence can represent relapse or re-infection disease. The relapse rate is low in children with drugsusceptible TB who receive DOT and cART. Recurrence within 6 to 12 months of treatment completion should be regarded as relapse and managed the same as treatment failure. Recurrence more than 6 to 12 months after treatment completion is probably re-infection disease, especially after new TB exposure or a visit to a TB endemic setting. Re-infection disease should be managed the same as first-time TB. Secondary (post-treatment) prophylaxis is not recommended. However, regular TB exposure screening should continue after completion of treatment, and preventive therapy should be considered whenever repeat exposure occurs.

International Guidelines-These guidelines were developed for the United States. Guidelines for resource-limited countries may be different and are available from the World Health Organization and International Union Against Tuberculosis and Lung Disease. ${ }^{91}$

Additional Resources-

- CDC Division of TB Elimination

- http://www.cdc.gov/tb/

- $\quad$ 800-CDC-INFO

(800-232-4636)

TTY: (888) 232-6348

24 Hours/Every Day

- cdcinfo@cdc.gov 
- U.S. Regional Training and Medical Consultation Centers

- http://www.cdc.gov/tb/education/rtmc/default.htm

- Drug-Resistant Tuberculosis: A Survival Guide for Clinicians

- http://www.currytbcenter.ucsf.edu/drtb/

- World Health Organization Childhood TB website

- http://www.who.int/tb/challenges/children/en/index.html

- International Union Against TB and Lung Disease Childhood TB website

- http://www.theunion.org/index.php/en/what-we-do/child-lung-health-/ childhood-tb

\section{Table}

Summary of Recommendations for Concurrent Use of Antiretroviral Therapy and TB Treatment

\begin{tabular}{|c|c|}
\hline Age/Weight & Combination Antiretroviral Therapy (cART) ${ }^{a}$ \\
\hline Aged $<3$ years or weight $<10 \mathrm{~kg}$ & 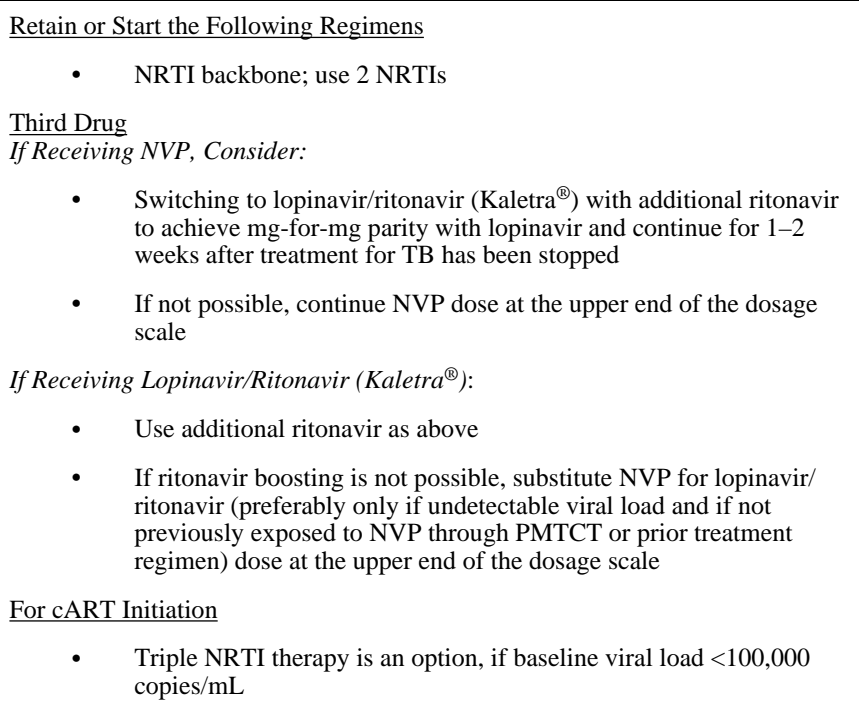 \\
\hline $\begin{array}{l}\text { Aged } \geq 3 \text { years and weight } \geq 10 \\
\text { kg }\end{array}$ & $\begin{array}{l}\text { Retain or Start the Following Regimens } \\
\text { - } \quad 2 \text { NRTIs as backbone } \\
\text { Third drug } \\
\text { If Receiving EFV: } \\
\text { - } \quad \text { Retain efavirenz (no dosage adjustment necessary) } \\
\text { If Receiving NVP: } \\
\text { - Substitute efavirenz for nevirapine } \\
\text { - If efavirenz not available, continue nevirapine; dose at the upper end } \\
\text { of the dosage scale } \\
\text { If Receiving Lopinavir/Ritonavir (Kaletra }{ }^{\circledR} \text { ): } \\
\text { - Consider substituting efavirenz for lopinavir/ritonavir, preferably } \\
\text { only if viral load is undetectable } b \text { and no prior NNRTI exposure } \\
\text { - Alternatively use additional ritonavir as above }\end{array}$ \\
\hline
\end{tabular}

Pediatr Infect Dis J. Author manuscript; available in PMC 2014 November 01. 


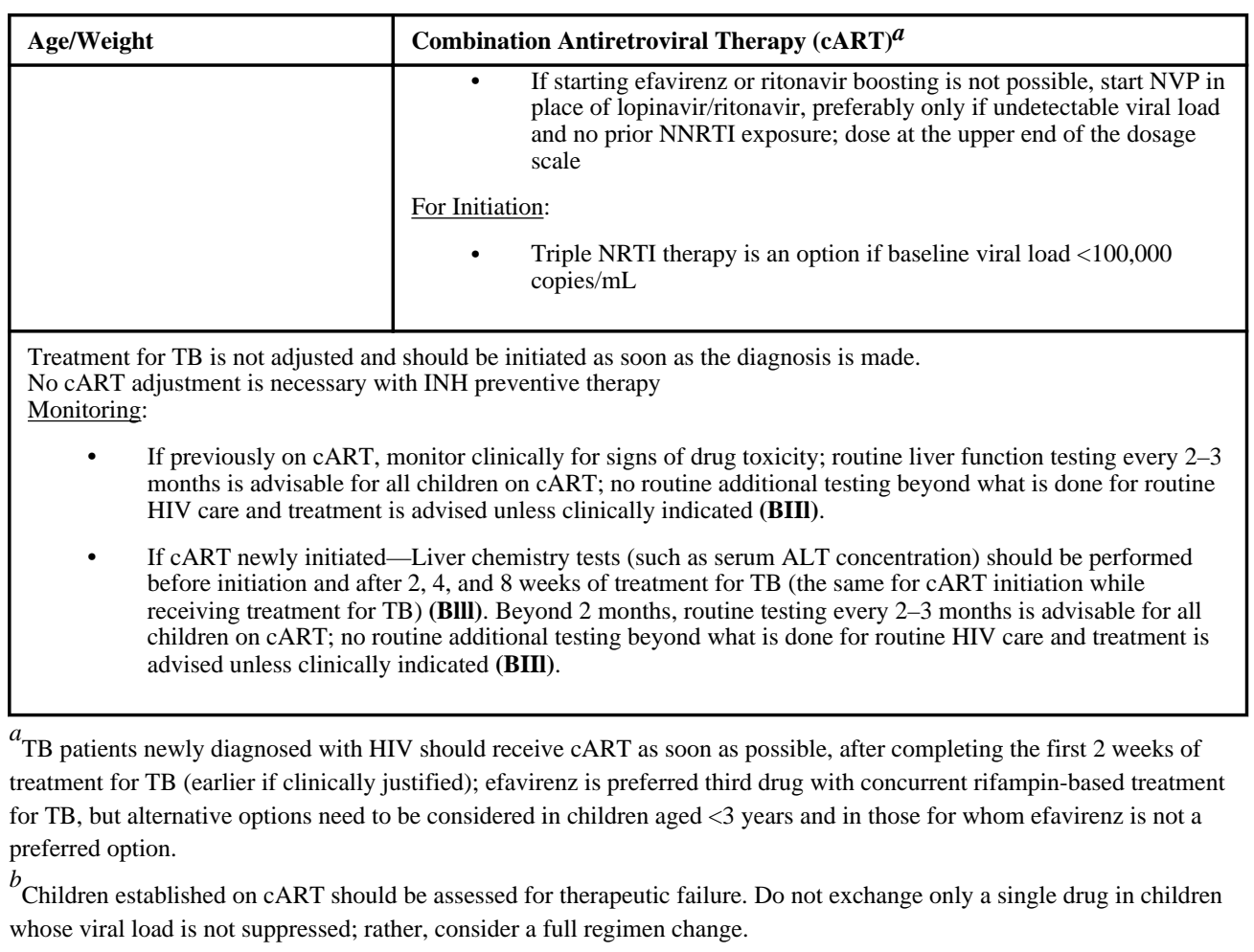

Adapted from Marais, Rabie, Cotton (2011)

Key to Acronyms: $\mathrm{cART}=$ combined antiretroviral therapy; $\mathrm{EFV}=$ efavirenz; NRTI = nucleoside reverse transcriptase inhibitor; NVP = nevirapine; $\mathrm{TB}=$ tuberculosis

\section{References}

1. CDC. Prevent TB Study. 2011 May. Available at http://www.cdc.gov/nchhstp/newsroom/docs/ PREVENTTB-Factsheet.pdf.

2. Menzies HJ, Winston CA, Holtz TH, Cain KP, Mac Kenzie WR. Epidemiology of tuberculosis among US- and foreignborn children and adolescents in the United States, 1994-2007. Am J Public Health. 2010 Sep; 100(9):1724-1729. Available at http://www.ncbi.nlm.nih.gov/pubmed/20634457. [PubMed: 20634457]

3. Shah SR, Tullu MS, Kamat JR. Clinical profile of pediatric HIV infection from India. Arch Med Res. 2005 Jan-Feb;36(1):24-31. Available at http://www.ncbi.nlm.nih.gov/pubmed/15777991. [PubMed: 15777991]

4. Marais BJ, Graham SM, Cotton MF, Beyers N. Diagnostic and management challenges for childhood tuberculosis in the era of HIV. J Infect Dis. 2007 Aug 15; 196(Suppl 1):S76-S85. Available at http://www.ncbi.nlm.nih.gov/pubmed/17624829. [PubMed: 17624829]

5. Hesseling AC, Cotton MF, Jennings T, et al. High incidence of tuberculosis among HIV-infected infants: evidence from a South African population-based study highlights the need for improved tuberculosis control strategies. Clin Infect Dis. 2009 Jan 1; 48(1):108-114. Available at http:// www.ncbi.nlm.nih.gov/pubmed/19049436. [PubMed: 19049436]

6. Adhikari M, Pillay T, Pillay DG. Tuberculosis in the newborn: an emerging disease. Pediatr Infect Dis J. 1997 Dec; 16(12):1108-1112. Available at http://www.ncbi.nlm.nih.gov/pubmed/9427454. [PubMed: 9427454]

7. Pillay T, Sturm AW, Khan M, et al. Vertical transmission of Mycobacterium tuberculosis in KwaZulu Natal: impact of HIV-1 co-infection. Int J Tuberc Lung Dis. 2004 Jan; 8(1):59-69. Available at http://www.ncbi.nlm.nih.gov/pubmed/14974747. [PubMed: 14974747] 
8. Marais BJ, Gie RP, Schaaf HS, et al. The natural history of childhood intra-thoracic tuberculosis: a critical review of literature from the pre-chemotherapy era. Int J Tuberc Lung Dis. 2004 Apr; 8(4): 392-402. Available at http://www.ncbi.nlm.nih.gov/pubmed/15141729. [PubMed: 15141729]

9. Marais BJ, Ayles H, Graham SM, Godfrey-Faussett P. Screening and preventive therapy for tuberculosis. Clin Chest Med. 2009 Dec; 30(4):827-846. x. Available at http:// www.ncbi.nlm.nih.gov/pubmed/19925970. [PubMed: 19925970]

10. Centers for Disease Contro and Prevention. Human tuberculosis caused by Mycobacterium bovis —New York City, 2001-2004. MMWR Morb Mortal Wkly Rep. 2005 Jun 24; 54(24):605-608. Available at http://www.ncbi.nlm.nih.gov/pubmed/15973241. [PubMed: 15973241]

11. LoBue PA, LeClair JJ, Moser KS. Contact investigation for cases of pulmonary Mycobacterium bovis. Int J Tuberc Lung Dis. 2004 Jul; 8(7):868-872. Available at http://www.ncbi.nlm.nih.gov/ pubmed/15260279. [PubMed: 15260279]

12. Hlavsa MC, Moonan PK, Cowan LS, et al. Human tuberculosis due to Mycobacterium bovis in the United States, 1995-2005. Clin Infect Dis. 2008 Jul 15; 47(2):168-175. Available at http:// www.ncbi.nlm.nih.gov/pubmed/18532886. [PubMed: 18532886]

13. Gandhi NR, Nunn P, Dheda K, et al. Multidrug-resistant and extensively drug-resistant tuberculosis: a threat to global control of tuberculosis. Lancet. 2010 May 22; 375(9728):1830 1843. Available at http://www.ncbi.nlm.nih.gov/pubmed/20488523. [PubMed: 20488523]

14. World Health Organization. Multidrug and extensively drug-resistant TB (M/XDR-TB): 2010 global report on surveillance and response. Geneva, Switzerland: 2010. (WHO/HTM/TB/2010.3) Available at http://www.who.int/tb/publications/2010/978924599191/en/

15. World Health Organization. Global tuberculosis control: WHO report 2010. Geneva, Switzerland: 2010. (WHO/HTM/TB/2010.7) Available at http://www.who.int/tb/publications/global_report/ 2010/en/

16. Centers for Disease C, Prevention. Decrease in reported tuberculosis cases - United States, 2009. MMWR Morb Mortal Wkly Rep. 2010 Mar 19; 59(10):289-294. Available at http:// www.ncbi.nlm.nih.gov/pubmed/20300055. [PubMed: 20300055]

17. Raviglione MC, Smith IM. XDR tuberculosis--implications for global public health. N Engl J Med. 2007 Feb 15; 356(7):656-659. Available at http://www.ncbi.nlm.nih.gov/pubmed/17301295. [PubMed: 17301295]

18. CDC. Extensively Drug Resistant Tuberculosis - United States 1993-2006. MMWR. 2007; 56(11):250-253. Available at http://www.cdc.gov/mmwr/preview/mmwrhtml/mm5611a3.htm. [PubMed: 17380107]

19. Marais BJ, Gie RP, Schaaf HS, Hesseling AC, Enarson DA, Beyers N. The spectrum of disease in children treated for tuberculosis in a highly endemic area. Int J Tuberc Lung Dis. 2006 Jul; 10(7): 732-738. Available at http://www.ncbi.nlm.nih.gov/pubmed/16848333. [PubMed: 16848333]

20. Mukadi YD, Wiktor SZ, Coulibaly IM, et al. Impact of HIV infection on the development, clinical presentation, and outcome of tuberculosis among children in Abidjan, Cote d'Ivoire. AIDS. 1997 Jul 15; 11(9):1151-1158. Available at http://www.ncbi.nlm.nih.gov/pubmed/9233463. [PubMed: 9233463]

21. Chintu C, Bhat G, Luo C, et al. Seroprevalence of human immunodeficiency virus type 1 infection in Zambian children with tuberculosis. Pediatr Infect Dis J. 1993 Jun; 12(6):499-504. Available at http://www.ncbi.nlm.nih.gov/pubmed/7688450. [PubMed: 7688450]

22. Hoffman ND, Kelly C, Futterman D. Tuberculosis infection in human immunodeficiency viruspositive adolescents and young adults: a New York City cohort. Pediatrics. 1996 Feb; 97(2):198203. Available at http://www.ncbi.nlm.nih.gov/pubmed/8584377. [PubMed: 8584377]

23. Schaaf HS, Geldenduys A, Gie RP, Cotton MF. Culture-positive tuberculosis in human immunodeficiency virus type 1-infected children. Pediatr Infect Dis J. 1998 Jul; 17(7):599-604. Available at http://www.ncbi.nlm.nih.gov/pubmed/9686725. [PubMed: 9686725]

24. Khouri YF, Mastrucci MT, Hutto C, Mitchell CD, Scott GB. Mycobacterium tuberculosis in children with human immunodeficiency virus type 1 infection. Pediatr Infect Dis J. 1992 Nov; 11(11):950-955. Available at http://www.ncbi.nlm.nih.gov/pubmed/1454438. [PubMed: 1454438] 
25. Chan SP, Birnbaum J, Rao M, Steiner P. Clinical manifestation and outcome of tuberculosis in children with acquired immunodeficiency syndrome. Pediatr Infect Dis J. 1996 May; 15(5):443447. Available at http://www.ncbi.nlm.nih.gov/pubmed/8724068. [PubMed: 8724068]

26. Marais BJ, Wright CA, Schaaf HS, et al. Tuberculous lymphadenitis as a cause of persistent cervical lymphadenopathy in children from a tuberculosis-endemic area. Pediatr Infect Dis J. 2006 Feb; 25(2):142-146. Available at http://www.ncbi.nlm.nih.gov/pubmed/16462291. [PubMed: 16462291]

27. Marais S, Pepper DJ, Marais BJ, Torok ME. HIV-associated tuberculous meningitis-diagnostic and therapeutic challenges. Tuberculosis (Edinb). 2010 Nov; 90(6):367-374. Available at http:// www.ncbi.nlm.nih.gov/pubmed/20880749. [PubMed: 20880749]

28. Marais S, Thwaites G, Schoeman JF, et al. Tuberculous meningitis: a uniform case definition for use in clinical research. Lancet Infect Dis. 2010 Nov; 10(11):803-812. Available at http:// www.ncbi.nlm.nih.gov/pubmed/20822958. [PubMed: 20822958]

29. Schaaf, HS.; Zumla, A., editors. Tuberculosis - a comprehensive clinical reference. UK: 2009.

30. Lewinsohn DA, Lobato MN, Jereb JA. Interferon-gamma release assays: new diagnostic tests for Mycobacterium tuberculosis infection, and their use in children. Curr Opin Pediatr. 2010 Feb; 22(1):71-76. Available at http://www.ncbi.nlm.nih.gov/pubmed/19952926. [PubMed: 19952926]

31. Mazurek GH, Jereb J, Vernon A, et al. Updated guidelines for using Interferon Gamma Release Assays to detect Mycobacterium tuberculosis infection - United States, 2010. MMWR Recomm Rep. 2010 Jun 25; 59(RR-5):1-25. Available at http://www.ncbi.nlm.nih.gov/pubmed/20577159. [PubMed: 20577159]

32. Ling DI, Zwerling AA, Steingart KR, Pai M. Immune-based diagnostics for TB in children: what is the evidence? Paediatr Respir Rev. 2011 Mar; 12(1):9-15. Available at http:// www.ncbi.nlm.nih.gov/pubmed/21172669. [PubMed: 21172669]

33. Machingaidze S, Wiysonge CS, Gonzalez-Angulo Y, et al. The utility of an interferon gamma release assay for diagnosis of latent tuberculosis infection and disease in children: a systematic review and meta-analysis. Pediatr Infect Dis J. 2011 Aug; 30(8):694-700. Available at http:// www.ncbi.nlm.nih.gov/pubmed/21427627. [PubMed: 21427627]

34. American Academy of Pediatrics. Red Book: 2009 Report of the Committee on Infectious Diseases. 28th ed. 28th ed. Elk Grove Village, IL: 2009.

35. Marais BJ, Pai M. New approaches and emerging technologies in the diagnosis of childhood tuberculosis. Paediatr Respir Rev. 2007 Jun; 8(2):124-133. Available at http:// www.ncbi.nlm.nih.gov/pubmed/17574156. [PubMed: 17574156]

36. Marais BJ, Rabie H, Cotton MF. TB and HIV in children - advances in prevention and management. Paediatr Respir Rev. 2011 Mar; 12(1):39-45. Available at http:// www.ncbi.nlm.nih.gov/pubmed/21172674. [PubMed: 21172674]

37. CDC. Treatment of tuberculosis. MMWR. 2003; 52(RR-11) Available at http://www.cdc.gov/ mmwr/preview/mmwrhtml/rr5211a1.htm.

38. Iriso R, Mudido PM, Karamagi C, Whalen C. The diagnosis of childhood tuberculosis in an HIVendemic setting and the use of induced sputum. Int J Tuberc Lung Dis. 2005 Jul; 9(7):716-726. Available at http://www.ncbi.nlm.nih.gov/pubmed/16013765. [PubMed: 16013765]

39. Zar HJ, Hanslo D, Apolles P, Swingler G, Hussey G. Induced sputum versus gastric lavage for microbiological confirmation of pulmonary tuberculosis in infants and young children: a prospective study. Lancet. 2005 Jan 8-14; 365(9454):130-134. Available at http:// www.ncbi.nlm.nih.gov/pubmed/15639294. [PubMed: 15639294]

40. Hatherill M, Hawkridge T, Zar HJ, et al. Induced sputum or gastric lavage for community-based diagnosis of childhood pulmonary tuberculosis? Arch Dis Child. 2009 Mar; 94(3):195-201. Available at http://www.ncbi.nlm.nih.gov/pubmed/18829621. [PubMed: 18829621]

41. Centers for Disease C, Prevention. Updated guidelines for the use of nucleic acid amplification tests in the diagnosis of tuberculosis. MMWR Morb Mortal Wkly Rep. 2009 Jan 16; 58(1):7-10. Available at http://www.ncbi.nlm.nih.gov/pubmed/19145221. [PubMed: 19145221]

42. Nicol MP, Workman L, Isaacs W, et al. Accuracy of the Xpert MTB/RIF test for the diagnosis of pulmonary tuberculosis in children admitted to hospital in Cape Town, South Africa: a descriptive 
study. Lancet Infect Dis. 2011 Nov; 11(11):819-824. Available at http://www.ncbi.nlm.nih.gov/ pubmed/21764384. [PubMed: 21764384]

43. Francis J. Curry National Tuberculosis Center. Tuberculosis Contact Investigation in Jail: A Facilitator Guide. 2008 Available at http://health.state.ga.us/pdfs/forms/tb/CI_jail.pdf.

44. Delacourt C, Poveda JD, Chureau C, et al. Use of polymerase chain reaction for improved diagnosis of tuberculosis in children. J Pediatr. 1995 May; 126(5 Pt 1):703-709. Available at http://www.ncbi.nlm.nih.gov/pubmed/7751992. [PubMed: 7751992]

45. Pierre C, Olivier C, Lecossier D, Boussougant Y, Yeni P, Hance AJ. Diagnosis of primary tuberculosis in children by amplification and detection of mycobacterial DNA. Am Rev Respir Dis. 1993 Feb; 147(2):420-424. Available at http://www.ncbi.nlm.nih.gov/pubmed/8430968. [PubMed: 8430968]

46. Smith KC, Starke JR, Eisenach K, Ong LT, Denby M. Detection of Mycobacterium tuberculosis in clinical specimens from children using a polymerase chain reaction. Pediatrics. 1996 Feb; 97(2): 155-160. Available at http://www.ncbi.nlm.nih.gov/pubmed/8584370. [PubMed: 8584370]

47. Dinnes J, Deeks J, Kunst H, et al. A systematic review of rapid diagnostic tests for the detection of tuberculosis infection. Health Technol Assess. 2007 Jan; 11(3):1-196. Available at http:// www.ncbi.nlm.nih.gov/pubmed/17266837. [PubMed: 17266837]

48. Diagnostic Standards and Classification of Tuberculosis in Adults and Children. This official statement of the American Thoracic Society and the Centers for Disease Control and Prevention was adopted by the ATS Board of Directors, July 1999. This statement was endorsed by the Council of the Infectious Disease Society of America, September 1999. Am J Respir Crit Care Med. 2000; 161(4 Pt 1):1376-1395. Available at http://www.ncbi.nlm.nih.gov/entrez/query.fcgi? $\mathrm{db}=$ pubmed\&cmd=Retrieve\&dopt=AbstractPlus\&list_uids=10764337\&itool=iconfft\&query_hl=1 16\&itool=pubmed_docsum. [PubMed: 10764337]

49. Violari A, Cotton MF, Gibb DM, et al. Early antiretroviral therapy and mortality among HIVinfected infants. N Engl J Med. 2008 Nov 20; 359(21):2233-2244. Available at http:// www.ncbi.nlm.nih.gov/pubmed/19020325. [PubMed: 19020325]

50. Walters E, Cotton MF, Rabie H, Schaaf HS, Walters LO, Marais BJ. Clinical presentation and outcome of tuberculosis in human immunodeficiency virus infected children on anti-retroviral therapy. BMC Pediatr. 2008; 8:1. Available at http://www.ncbi.nlm.nih.gov/pubmed/18186944. [PubMed: 18186944]

51. Gupta A, Nayak U, Ram M, et al. Postpartum tuberculosis incidence and mortality among HIVinfected women and their infants in Pune, India, 2002-2005. Clin Infect Dis. 2007 Jul 15; 45(2): 241-249. Available at http://www.ncbi.nlm.nih.gov/pubmed/17578786. [PubMed: 17578786]

52. Centers for Disease Control and Prevention. Severe isoniazid-associated liver injuries among persons being treated for latent tuberculosis infection - United States, 2004-2008. MMWR Morb Mortal Wkly Rep. 2010 Mar 5; 59(8):224-229. Available at http://www.ncbi.nlm.nih.gov/ pubmed/20203555. [PubMed: 20203555]

53. Schaaf HS, Marais BJ. Management of multidrug-resistant tuberculosis in children: a survival guide for paediatricians. Paediatr Respir Rev. 2011 Mar; 12(1):31-38. Available at http:// www.ncbi.nlm.nih.gov/pubmed/21172673. [PubMed: 21172673]

54. Al-Dabbagh M, Lapphra K, McGloin R, et al. Drug-resistant tuberculosis: pediatric guidelines. Pediatr Infect Dis J. 2011 Jun; 30(6):501-505. Available at http://www.ncbi.nlm.nih.gov/pubmed/ 21297522. [PubMed: 21297522]

55. Schechter M, Zajdenverg R, Falco G, et al. Weekly rifapentine/isoniazid or daily rifampin/ pyrazinamide for latent tuberculosis in household contacts. Am J Respir Crit Care Med. 2006 Apr 15; 173(8):922-926. Available at http://www.ncbi.nlm.nih.gov/pubmed/16474028. [PubMed: 16474028]

56. Martinson NA, Barnes GL, Moulton LH, et al. New regimens to prevent tuberculosis in adults with HIV infection. N Engl J Med. 2011 Jul 7; 365(1):11-20. Available at http:// www.ncbi.nlm.nih.gov/pubmed/21732833. [PubMed: 21732833]

57. Sterling TR, Villarino ME, Borisov AS, et al. Three months of rifapentine and isoniazid for latent tuberculosis infection. N Engl J Med. 2011 Dec 8; 365(23):2155-2166. Available at http:// www.ncbi.nlm.nih.gov/pubmed/22150035. [PubMed: 22150035] 
58. CDC. Acquired rifamycin resistance in persons with advanced HIV disease being treated for active tuberculosis with intermittent rifamycin-based regimens. MMWR. 2002; 51:214-215. Available at http://www.cdc.gov/mmwr/preview/mmwrhtml/mm5110a5.htm. [PubMed: 11922192]

59. Rieder HL, Arnadottir T, Trebucq A, Enarson DA. Tuberculosis treatment: dangerous regimens? Int J Tuberc Lung Dis. 2001 Jan; 5(1):1-3. Available at http://www.ncbi.nlm.nih.gov/pubmed/ 11263509. [PubMed: 11263509]

60. Vernon A, Burman W, Benator D, Khan A, Bozeman L. Acquired rifamycin monoresistance in patients with HIV-related tuberculosis treated with once-weekly rifapentine and isoniazid. Tuberculosis Trials Consortium. Lancet. 1999 May 29; 353(9167):1843-1847. Available at http:// www.ncbi.nlm.nih.gov/pubmed/10359410. [PubMed: 10359410]

61. Marais BJ, Schaaf HS, Donald PR. Pediatric TB: issues related to current and future treatment options. Future Microbiol. 2009 Aug; 4(6):661-675. Available at http://www.ncbi.nlm.nih.gov/ pubmed/19659423. [PubMed: 19659423]

62. World Health Organization. Rapid Advice: treatment of tuberculosis in children. Geneva, Switzerland: 2010. (WHO/HTM/TB/2010.13). Available at http://whqlibdoc.who.int/publications/ 2010/9789241500449_eng.pdf

63. Ellard GA, Humphries MJ, Allen BW. Cerebrospinal fluid drug concentrations and the treatment of tuberculous meningitis. Am Rev Respir Dis. 1993 Sep; 148(3):650-655. Available at http:// www.ncbi.nlm.nih.gov/pubmed/8368635. [PubMed: 8368635]

64. Donald PR, Seifart HI. Cerebrospinal fluid concentrations of ethionamide in children with tuberculous meningitis. J Pediatr. 1989 Sep; 115(3):483-486. Available at http:// www.ncbi.nlm.nih.gov/pubmed/2769511. [PubMed: 2769511]

65. Starke JR, Correa AG. Management of mycobacterial infection and disease in children. Pediatr Infect Dis J. 1995 Jun; 14(6):455-469. quiz 469-470. Available at http://www.ncbi.nlm.nih.gov/ pubmed/7667049. [PubMed: 7667049]

66. Abdool Karim SS, Naidoo K, Grobler A, et al. Timing of initiation of antiretroviral drugs during tuberculosis therapy. N Engl J Med. 2010 Feb 25; 362(8):697-706. Available at http:// www.ncbi.nlm.nih.gov/pubmed/20181971. [PubMed: 20181971]

67. Asselman V, Thienemann F, Pepper DJ, et al. Central nervous system disorders after starting antiretroviral therapy in South Africa. AIDS. 2010 Nov 27; 24(18):2871-2876. Available at http:// www.ncbi.nlm.nih.gov/pubmed/21045634. [PubMed: 21045634]

68. Lawn SD, Wood R. Poor prognosis of HIV-associated tuberculous meningitis regardless of the timing of antiretroviral therapy. Clin Infect Dis. 2011 Jun; 52(11):1384-1387. Available at http:// www.ncbi.nlm.nih.gov/pubmed/21596681. [PubMed: 21596681]

69. Gerstoft J, Kirk O, Obel N, et al. Low efficacy and high frequency of adverse events in a randomized trial of the triple nucleoside regimen abacavir, stavudine and didanosine. AIDS. 2003 Sep 26; 17(14):2045-2052. Available at http://www.ncbi.nlm.nih.gov/pubmed/14502007. [PubMed: 14502007]

70. Ren Y, Nuttall JJ, Eley BS, et al. Effect of rifampicin on efavirenz pharmacokinetics in HIVinfected children with tuberculosis. J Acquir Immune Defic Syndr. 2009 Apr 15; 50(5):439-443. Available at http://www.ncbi.nlm.nih.gov/pubmed/19223781. [PubMed: 19223781]

71. Elsherbiny D, Cohen K, Jansson B, Smith P, McIlleron H, Simonsson US. Population pharmacokinetics of nevirapine in combination with rifampicin-based short course chemotherapy in HIV- and tuberculosis-infected South African patients. Eur J Clin Pharmacol. 2009 Jan; 65(1): 71-80. Available at http://www.ncbi.nlm.nih.gov/pubmed/18751690. [PubMed: 18751690]

72. Palumbo P, Lindsey JC, Hughes MD, et al. Antiretroviral treatment for children with peripartum nevirapine exposure. N Engl J Med. 2010 Oct 14; 363(16):1510-1520. Available at http:// www.ncbi.nlm.nih.gov/pubmed/20942667. [PubMed: 20942667]

73. Ren Y, Nuttall JJ, Egbers C, et al. Effect of rifampicin on lopinavir pharmacokinetics in HIVinfected children with tuberculosis. J Acquir Immune Defic Syndr. 2008 Apr 15; 47(5):566-569. Available at http://www.ncbi.nlm.nih.gov/pubmed/18197120. [PubMed: 18197120]

74. World Health Organization. Guidance for national tuberculosis and HIV programmes on the management of tuberculosis in HIV-infected children: recommendations for a public health approach. Paris, France: IUATLD; 2010. Available at http://www.theunion.org/index.php/en/ 
resources/technical-publications/item/759-guidance-for-national-tuberculosis-and-hivprogrammes-on-the-management-of-tuberculosis-in-hiv-infected-children-recommendations-for-apublic-health-approach

75. Donald P, Cilliers K, Willemse M, et al. Pyridoxine serum concentrations in children hospitalized with tuberculosis. Int J Tuberc Lung Dis. 2007; 11(Suppl 1):S225. 2007.

76. Palusci VJ, O'Hare D, Lawrence RM. Hepatotoxicity and transaminase measurement during isoniazid chemoprophylaxis in children. Pediatr Infect Dis J. 1995 Feb; 14(2):144-148. Available at http://www.ncbi.nlm.nih.gov/pubmed/7746698. [PubMed: 7746698]

77. Saukkonen JJ, Cohn DL, Jasmer RM, et al. An official ATS statement: hepatotoxicity of antituberculosis therapy. Am J Respir Crit Care Med. 2006 Oct 15; 174(8):935-952. Available at http://www.ncbi.nlm.nih.gov/pubmed/17021358. [PubMed: 17021358]

78. Donald PR, Maher D, Maritz JS, Qazi S. Ethambutol dosage for the treatment of children: literature review and recommendations. Int J Tuberc Lung Dis. 2006 Dec; 10(12):1318-1330. Available at http://www.ncbi.nlm.nih.gov/pubmed/17167947. [PubMed: 17167947]

79. World Health Organization. Ethambutol efficacy and toxicity. 2006 Available at http:// apps.who.int/iris/bitstream/10665/69366/1/WHO_HTM_TB_2006.365_eng.pdf.

80. Thee, S.; Zollner, EW.; Willemse, M.; Hesseling, AC.; Magdorf, K.; Schaaf, HS. Hypothyroidism in children on ethionamide therapy. Abstract. Union World Conference on Lung Health; Berlin, Germany. 2010.

81. Narita M, Ashkin D, Hollender ES, Pitchenik AE. Paradoxical worsening of tuberculosis following antiretroviral therapy in patients with AIDS. Am J Respir Crit Care Med. 1998 Jul; 158(1):157161. Available at http://www.ncbi.nlm.nih.gov/pubmed/9655723. [PubMed: 9655723]

82. Wendel KA, Alwood KS, Gachuhi R, Chaisson RE, Bishai WR, Sterling TR. Paradoxical worsening of tuberculosis in HIV-infected persons. Chest. $2001 \mathrm{Jul}$; 120(1):193-197. Available at http://www.ncbi.nlm.nih.gov/pubmed/11451837. [PubMed: 11451837]

83. Chien JW, Johnson JL. Paradoxical reactions in HIV and pulmonary TB. Chest. 1998 Sep; 114(3): 933-936. Available at http://www.ncbi.nlm.nih.gov/pubmed/9743188. [PubMed: 9743188]

84. Puthanakit T, Oberdorfer P, Punjaisee S, Wannarit P, Sirisanthana T, Sirisanthana V. Immune reconstitution syndrome due to bacillus Calmette-Guerin after initiation of antiretroviral therapy in children with HIV infection. Clin Infect Dis. 2005 Oct 1; 41(7):1049-1052. Available at http:// www.ncbi.nlm.nih.gov/pubmed/16142674. [PubMed: 16142674]

85. Zampoli M, Kilborn T, Eley B. Tuberculosis during early antiretroviral-induced immune reconstitution in HIV-infected children. Int J Tuberc Lung Dis. 2007 Apr; 11(4):417-423. Available at http://www.ncbi.nlm.nih.gov/pubmed/17394688. [PubMed: 17394688]

86. Lawn SD, Wilkinson RJ, Lipman MC, Wood R. Immune reconstitution and"unmasking" of tuberculosis during antiretroviral therapy. Am J Respir Crit Care Med. 2008 Apr 1; 177(7):680685. Available at http://www.ncbi.nlm.nih.gov/pubmed/18202347. [PubMed: 18202347]

87. Meintjes G, Lawn SD, Scano F, et al. Tuberculosis-associated immune reconstitution inflammatory syndrome: case definitions for use in resource-limited settings. Lancet Infect Dis. 2008 Aug; 8(8): 516-523. Available at http://www.ncbi.nlm.nih.gov/pubmed/18652998. [PubMed: 18652998]

88. Puthanakit T, Oberdorfer P, Akarathum N, Wannarit P, Sirisanthana T, Sirisanthana V. Immune reconstitution syndrome after highly active antiretroviral therapy in human immunodeficiency virus-infected thai children. Pediatr Infect Dis J. 2006 Jan; 25(1):53-58. Available at http:// www.ncbi.nlm.nih.gov/pubmed/16395104. [PubMed: 16395104]

89. Rabie, H.; Violari, A.; Madhi, S., et al. Complications of BCG Vaccination in HIV-infected and uninfected Children: CHER Study. 15th Conference On Retroviruses and Opportunistic Infections (CROI); February 3-6, 2008; Boston, MA. 2008.

90. Meintjes G, Wilkinson RJ, Morroni C, et al. Randomized placebo-controlled trial of prednisone for paradoxical tuberculosis-associated immune reconstitution inflammatory syndrome. AIDS. 2010 Sep 24; 24(15):2381-2390. Available at http://www.ncbi.nlm.nih.gov/pubmed/20808204. [PubMed: 20808204]

91. World Health Organization. WHO/IUATLD Guidance for national TB and HIV programmes on the management of TB in HIV-infected children: Recommendations for a public health approach. 2010 . 
Table: Dosing Recommendations for Preventing and Treating TB in HIV-infected Children

\begin{tabular}{|c|c|c|c|}
\hline Indication & First Choice & Alternative & Comments/Special Issues \\
\hline $\begin{array}{l}\text { Prophylaxis } \\
\text { Post-exposure }\end{array}$ & $\begin{array}{l}\text { Source Case Drug } \\
\text { Susceptible: } \\
\qquad \begin{array}{l}\text { Isoniazid } 10-15 \\
\text { mg/kg body } \\
\text { weight (maximum } \\
\text { 300 mg/day) by } \\
\text { mouth daily for } 9 \\
\text { months }\end{array} \\
\text { Source Case Drug Resistant } \\
\quad \begin{array}{l}\text { Consult expert } \\
\text { and local public } \\
\text { health authorities. }\end{array}\end{array}$ & $\begin{array}{l}\text { - If adherence } \\
\text { with daily } \\
\text { isoniazid cannot } \\
\text { be ensured, } \\
\text { consider } \\
\text { isoniazid } 20-30 \\
\text { mg/ kg body } \\
\text { weight } \\
\text { (maximum } 900 \\
\text { mg/day) by } \\
\text { mouth } 2 \text { times a } \\
\text { week by DOT } \\
\text { for } 9 \text { months } \\
\text { Isoniazid } 10-15 \\
\text { mg/kg body } \\
\text { weight } \\
\text { (maximum } 300 \\
\text { mg/ day) and } \\
\text { rifampin } 10-20 \\
\text { mg/ kg body } \\
\text { weight } \\
\text { (maximum } 600 \\
\text { mg/day) by } \\
\text { mouth daily for } \\
3-4 \text { months } \\
\text { Rifampin } 10-20 \\
\text { mg/kg body } \\
\text { weight } \\
\text { (maximum } 600 \\
\text { mg/ day) by } \\
\text { mouth daily for } \\
4-6 \text { months }\end{array}$ & 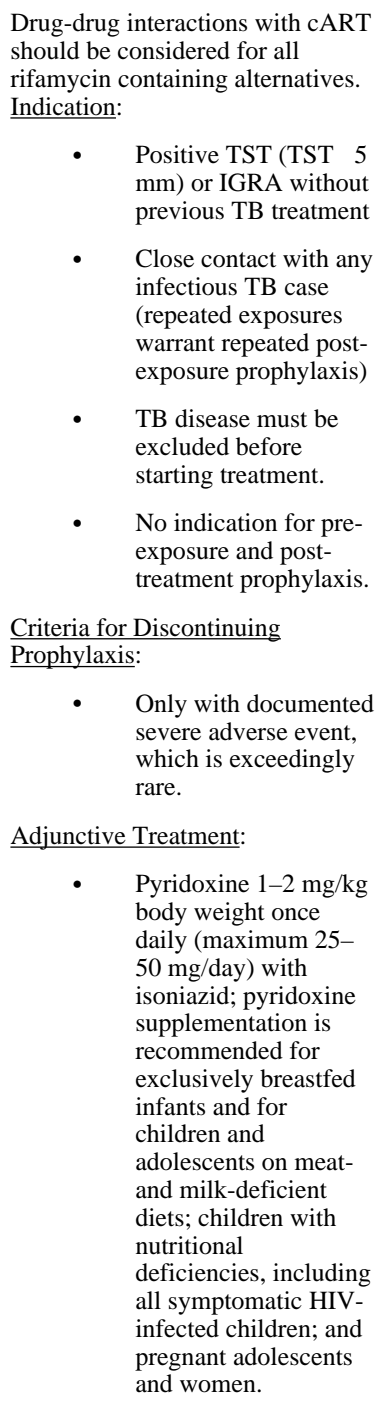 \\
\hline Treatmen & \begin{tabular}{ll}
\multicolumn{2}{l}{ Intrathoracic Disease } \\
Drug-Susceptible TB \\
Intensive Phase (2 Months): \\
- & Isoniazid, 10-15 \\
mg/kg body \\
weight (maximum \\
300 mg/day) by \\
mouth once daily, \\
plus \\
Rifampin 10-20 \\
mg/kg body \\
weight (maximum \\
$600 \mathrm{mg} /$ day) by \\
mouth once daily, \\
plus \\
Pyrazinamide 30- \\
$40 \mathrm{mg} / \mathrm{kg}$ body \\
weight (maximum
\end{tabular} & 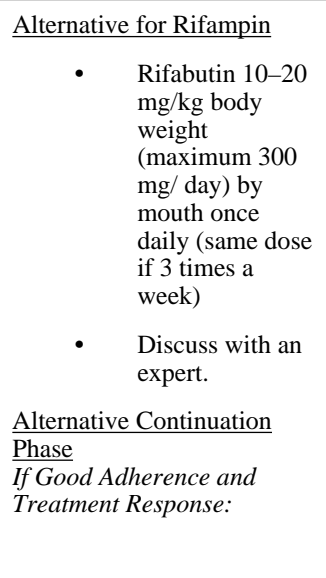 & $\begin{array}{l}\text { Only DOT. } \\
\text { If cART-naive, start TB therapy } \\
\text { immediately and initiate cART } \\
\text { within 2-8 weeks. } \\
\text { Already on cART; review to } \\
\text { minimize potential toxicities and } \\
\text { drug-drug interactions; start TB } \\
\text { treatment immediately. } \\
\text { Potential drug toxicity and } \\
\text { interactions should be reviewed at } \\
\text { every visit. } \\
\text { Adjunctive Treatment: } \\
\text { Co-trimoxazole } \\
\quad \text { prophylaxis } \\
\quad \text { Pyridoxine } 1-2 \\
\text { mg/kg/ body } \\
\text { weight/day (maximum } \\
25-50 \mathrm{mg} / \text { day) with }\end{array}$ \\
\hline
\end{tabular}




\begin{tabular}{|c|c|c|c|}
\hline Indication & First Choice & Alternative & Comments/Special Issues \\
\hline & 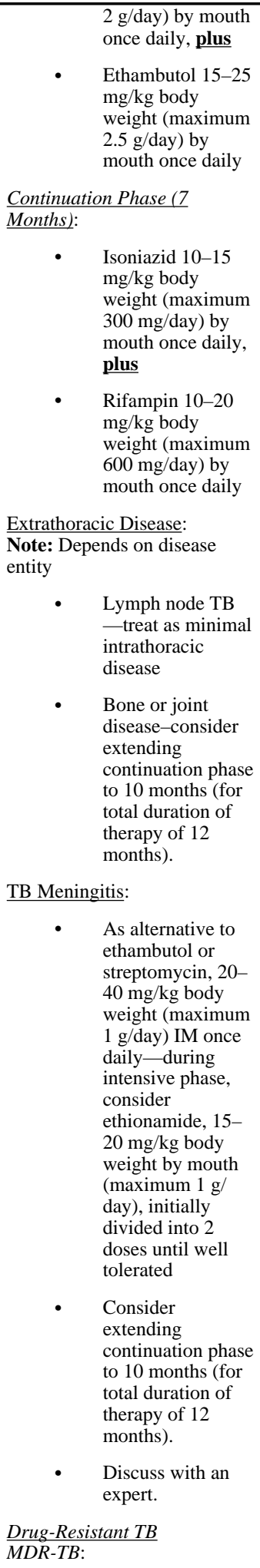 & $\begin{array}{l}\text { Isoniazid 20-30 } \\
\text { mg/kg body } \\
\text { weight } \\
\text { (maximum } 900 \\
\text { mg/ day) by } \\
\text { mouth, plus } \\
\text { Rifampin 10-20 } \\
\text { mg/kg body } \\
\text { weight } \\
\text { (maximum } 600 \\
\text { mg/day) three } \\
\text { times a week. } \\
\text { In children with } \\
\text { minimal disease } \\
\text { with fully drug- } \\
\text { susceptible TB } \\
\text { in the absence of } \\
\text { significant } \\
\text { immune } \\
\text { compromise, a } \\
\text { 3-drug intensive } \\
\text { phase regimen } \\
\text { (excluding } \\
\text { ethambutol) and } \\
\text { a continuation } \\
\text { phase of } 4 \\
\text { months can be } \\
\text { considered (total } \\
\text { duration of } \\
\text { therapy of } 6 \\
\text { months). }\end{array}$ & $\begin{array}{l}\text { isoniazid or } \\
\text { cycloserine/terizidone } \\
\text { or, if malnourished; } \\
\text { pyridoxine } \\
\text { supplementation is } \\
\text { recommended for } \\
\text { exclusively breastfed } \\
\text { infants and for } \\
\text { children and } \\
\text { adolescents on meat- } \\
\text { and milk-deficient } \\
\text { diets; children with } \\
\text { nutritional } \\
\text { deficiencies, including } \\
\text { all symptomatic HIV- } \\
\text { infected children; and } \\
\text { pregnant adolescents } \\
\text { and women. } \\
\text { Corticosteroids }(2 \\
\text { mg/kg body weight } \\
\text { per day of prednisone } \\
\text { [maximum, } 60 \text { mg/ } \\
\text { day] or its equivalent } \\
\text { for } 4-6 \text { weeks } \\
\text { followed by tapering) } \\
\text { with CNS disease or } \\
\text { pericardial effusion; } \\
\text { may be considered } \\
\text { with pleural effusions, } \\
\text { severe airway } \\
\text { compression, or severe } \\
\text { IRIS. } \\
\text { - } \\
\text { - } \\
\text { Terizidone } 10-20 \\
\text { mg/kg body weight } \\
\text { (maximum } 1 \text { g/day) by } \\
\text { mouth once daily }\end{array}$ \\
\hline
\end{tabular}




\begin{tabular}{|c|c|c|c|}
\hline Indication & First Choice & Alternative & Comments/Special Issues \\
\hline & $\begin{array}{ll}\text { - } & \begin{array}{l}\text { Therapy should be } \\
\text { based on } \\
\text { resistance pattern } \\
\text { of child (or of } \\
\text { source case where } \\
\text { child's isolate is } \\
\text { not available); } \\
\text { consult an expert. }\end{array} \\
\text { Treatment } & \text { Duration: } \\
& \begin{array}{l}\text { 18-24 months } \\
\text { after non- } \\
\text { bacteriological } \\
\text { diagnosis or after } \\
\text { culture } \\
\text { conversion; } \geq 12 \\
\text { months if minimal } \\
\text { disease }\end{array} \\
\text { Discuss with an } \\
\text { expert. }\end{array}$ & & $\begin{array}{ll}\text { Ethionamide/ } \\
\text { prothionamide, } 15-20 \\
\text { mg/ kg body weight } \\
\text { (maximum 1 g/day) by } \\
\text { mouth in 2-3 divided } \\
\text { doses } \\
\\
\text { Para-aminosalicylic } \\
\text { acid 200-300 mg/kg } \\
\text { body weight by mouth } \\
\text { divided into 3-4 doses } \\
\text { per day (maximum 10 } \\
\text { g/day). } \\
\text { Thiacetazone can } \\
\text { cause severe reactions } \\
\text { in HIV-infected } \\
\text { children including } \\
\text { rash and aplastic } \\
\text { anemia, and should } \\
\text { not be used. }\end{array}$ \\
\hline
\end{tabular}

Key to Acronyms: cART = combined antiretroviral therapy; $\mathrm{CNS}=$ central nervous system; DOT = directly observed therapy; FDA = Food and Drug Administration; IGRA = interferon-gamma release assay; IM = intramuscular; IRIS = immune reconstitution inflammatory syndrome; IV = intravenous; $\mathrm{MDR}-\mathrm{TB}=$ multi-drug-resistant tuberculosis; $\mathrm{TB}=$ tuberculosis; TST $=$ tuberculin skin test

\section{References}

1210. Pickering, LK.; Baker, CJ.; Kimberlin, DW.; Long, SS. the American Academy of Pediatrics. Red Book: 2009 Report of the Committee on Infectious Diseases. Elk Grove Village, IL: American Academy of Pediatrics; 2009. Tuberculosis; p. 680-701.

1211. Centers for Disease Control and Prevention. Tuberculin Testing and Treatment of Latent Tuberculosis Infection. MMWR. 2003; 49(RR06):1-54.

1212. Centers for Disease Control and Prevention. Treatment of Tuberculosis. MMWR. 2003; 52(RR11):1-77.

1213. Schaaf HS, Marais BJ. Management of multidrug-resistant tuberculosis in children: a survival guide for paediatricians. Paediatr Respir Rev. 2011; 12:31-38. [PubMed: 21172673]

1214. World Health Organization. Rapid Advice: treatment of tuberculosis in children. Paper presented at Geneva, Switzerland: (WHO/HTM/TB/2010.13).

1215. World Health Organization. Guidance for national tuberculosis and HIV programmes on the management of tuberculosis in HIV-infected children: recommendations for a public health approach. Paper presented at: Paris, France: IUATLD. 2010

\section{Pneumocystis jirovecii Pneumonia (Last updated November 6, 2013; last reviewed November 6, 2013)}

\footnotetext{
Panel's Recommendations

Prevention of Primary Exposure

- Some experts recommend that consideration be given to not placing a patient with Pneumocystis jirovecii pneumonia (PCP) in a hospital room with another patient and not placing an at-risk immunocompromised patient in a room with a patient who has a respiratory tract infection (BIII).

Chemoprophylaxis

- Chemoprophylaxis is highly effective in preventing PCP. Prophylaxis is recommended for all HIV-infected children aged $\nsucceq 6$ years who have CD4 T lymphocyte (CD4) cell counts $<200$ cells $/ \mathrm{mm}^{3}$ or CD4 percentage
} 
Panel's Recommendations

$<15 \%$, for children aged 1 to $<6$ years with CD 4 counts $<500$ cells $/ \mathrm{mm}^{3}$ or CD 4 percentage $<15 \%$, and for all HIV-infected infants aged $<12$ months regardless of CD4 count or percentage (AII).

- Infants with indeterminate HIV infection status should receive prophylaxis until they are determined to be HIV-uninfected or presumptively HIV-uninfected (AIII). HIV-infected infants should be administered prophylaxis until age 1 year, at which time they should be reassessed on the basis of the age-specific CD4 count or percentage thresholds mentioned above (AII).

- Trimethoprim-sulfamethoxazole (TMP-SMX; cotrimoxazole), administered either on 3 consecutive days/ week or daily, is the drug of choice for prophylaxis because of its high efficacy, relative safety, low cost, and broad antimicrobial spectrum (AI).

- $\quad$ Other effective and safe prophylaxis regimens are available for patients unable to take TMP-SMX. A second choice would be either atovaquone (AI) or dapsone (BI*).

- Aerosolized pentamidine is recommended for children who cannot take TMP-SMX, atovaquone, or dapsone and who are old enough to use nebulization with a Respirgard II ${ }^{\circledR}$ nebulizer (Marquest; Englewood, CO) (BI*).

- Intravenous (IV) pentamidine is not recommended for prophylaxis unless no other options are available (BII).

- Discontinuation of PCP prophylaxis should be considered for HIV-infected children when, after receiving combination antiretroviral therapy for $\geq 6$ months, CD4 percentage is $\geq 15 \%$ or CD4 count is $\geq 200$ cells $/ \mathrm{mm}^{3}$ for patients aged $\geq 6$ years (BII) and CD4 percentage is $\geq 15 \%$ or CD4 count is 2500 cells $/ \mathrm{mm}^{3}$ for patients aged 1 to $<6$ years (BII) for $>3$ consecutive months. Thereafter, CD4 percentage and CD4 count should be reevaluated at least every 3 months and prophylaxis reinstituted if the age-specific criteria for prophylaxis are reached (BIII).

Treatment

- $\quad$ TMP-SMX, administered IV, is the recommended treatment for PCP (AI). As the acute pneumonitis subsides, children with mild-to-moderate disease who do not have malabsorption or diarrhea can be transitioned to oral treatment with the same total daily dose of TMP-SMX administered in 3 or 4 divided doses to complete a 21-day course (AII)

- IV pentamidine isethionate once daily is recommended for patients who cannot tolerate TMP-SMX or who demonstrate clinical treatment failure after 5 to 7 days of TMP-SMX therapy (AI*).

- $\quad$ Atovaquone is an alternative for treatment of mild-to-moderately severe PCP (BI*).

- Dapsone/TMP is effective in treating mild-to-moderate PCP (BI*).

- Clindamycin/primaquine has been used to treat mild-to-moderate PCP; data in children are unavailable (BIII).

- A short course of corticosteroids is recommended in cases of moderate or severe PCP, starting within 72 hours of diagnosis (AI*).

- $\quad$ Patients who have experienced an episode of PCP should continue on PCP prophylaxis after completion of treatment until CD4 counts exceed the threshold for initiating prophylaxis (AI).

- Children who present with clinical signs and symptoms compatible with PCP after discontinuation of prophylaxis should be evaluated thoroughly despite normal or high CD4 counts or percentages (BI*).

Rating of Recommendations: $\mathrm{A}=$ Strong; $\mathrm{B}=$ Moderate; $\mathrm{C}=$ Optional

Rating of Evidence: $\mathrm{I}=$ One or more randomized trials in children ${ }^{\dagger}$ with clinical outcomes and/or validated endpoints; $\mathrm{I}^{*}$ $=$ One or more randomized trials in adults with clinical outcomes and/or validated laboratory endpoints with accompanying data in children $^{\dagger}$ from one or more well-designed, nonrandomized trials or observational cohort studies with long-term clinical outcomes; II = One or more well-designed, nonrandomized trials or observational cohort studies in children ${ }^{\dagger}$ with long-term outcomes; II* = One or more well-designed, nonrandomized trials or observational studies in adults with longterm clinical outcomes with accompanying data in children ${ }^{\dagger}$ from one or more similar nonrandomized trials or cohort studies with clinical outcome data; III = Expert opinion †

\section{Epidemiology}

Pneumocystis spp. are found worldwide in the lungs of humans and lower animals. The organisms are host specific, and cross-infection between humans and other species does not occur. Pneumocystis spp. from all sources are morphologically, tinctorially, and biologically 
similar, but surface antigens and gene sequencing have demonstrated host-specific differences. Since the original designation of Pneumocystis carinii a century ago, several changes in terminology have been suggested. The most recent proposal was to change $P$. carinii to Pneumocystis jirovecii for isolates from human lungs. Pneumocystis has been designated a fungus on the basis of DNA analysis, but it has several biologic features of protozoa. Most humans are infected with Pneumocystis early in life. By ages 2 to 4 years, more than $80 \%$ of children in most countries have acquired antibodies to Pneumocystis. ${ }^{1-3}$ Immunocompetent infants with the infection are either asymptomatic or have mild respiratory symptoms. Pneumocystis jirovecii pneumonia (PCP) occurs almost exclusively in the immunocompromised host.

PCP remains an important AIDS-indicator disease among HIV-infected children. The highest incidence of PCP in HIV-infected children is in the first year of life, with cases peaking at ages 3 to 6 months. ${ }^{4-6}$ Data from the Centers for Disease Control and Prevention Pediatric Spectrum of Disease Project (1994-2001) indicate a decline in PCP infection rates (cases per 1000 HIV-infected children) from 25 in 1994 to 18 in 1996 to 6 in 2001.7 Similarly, analyses of data from the Perinatal AIDS Collaborative Transmission Study revealed a 95\% decline in PCP (cases per 100 child-years) from 5.8 (pre-combination antiretroviral therapy [cART] era) to 0.3 (cART era) ${ }^{8}$ Finally, the incidence rate for PCP (cases per 100 child-years) was 1.3 during the pre-cART era (1981-1988) and $<0.5$ during the cART era (2001-2004). ${ }^{9}$ This decline probably resulted from implementation of interventions to prevent mother-to-child transmission of HIV, introduction of cART in HIVinfected children in 1995, and chemoprophylaxis for PCP.

PCP continues to be a major cause of death among HIV-infected infants and children in the developing world. Autopsies done in Africa revealed PCP in 16\% of children who died with HIV/AIDS during 1992 and 1993, ${ }^{10}$ in 29\% of those who died during 1997 and $2000,{ }^{11}$ and in $44 \%$ of those who died during 2000 and $2001 .^{12}$

The mode of transmission of Pneumocystis among HIV-infected infants, children, and adults is not firmly established, but airborne human-to-human transmission is likely. Animal studies show Pneumocystis is transmitted by air from infected to susceptible rats. ${ }^{13,14}$ Furthermore, Pneumocystis can infect normal mice, produce subclinical disease and be transmitted to normal or immunocompromised mice. ${ }^{15}$ Human-to-human transmission has been suggested by molecular epidemiology and global clustering of PCP cases in recent studies. ${ }^{16-18}$ Intrauterine transmission is considered rare. However, in one report, 1 of 8 infants born to women who had AIDS and PCP during pregnancy had evidence of Pneumocystis infection. ${ }^{19}$

The single most important factor in susceptibility of HIV-infected patients of all ages to PCP is the status of cell-mediated immunity of the host. Severe compromise, reflected by a marked decrease in CD4 T lymphocyte (CD4) cell count and percentage, is the hallmark of high risk for $\mathrm{PCP}$ and is discussed further in the prevention section. 


\section{Clinical Manifestations}

Prominent clinical features of PCP among HIV-infected children are fever, tachypnea, dyspnea, and cough. The severity of these signs and symptoms varies from child to child. Onset can be abrupt or insidious with nonspecific symptoms such as mild cough, dyspnea, poor feeding, diarrhea, and weight loss. Some patients may not be febrile, but almost all will have tachypnea by the time pneumonitis is evident on chest radiograph. Physical examination sometimes shows bilateral basilar rales with evidence of respiratory distress and hypoxia.

In HIV-infected children with pneumonia, four clinical variables are independently associated with PCP: aged $<6$ months, respiratory rate $>59$ breaths per minute, arterial percentage hemoglobin saturation $\$ 2 \%$, and absence of vomiting. ${ }^{20}$ A high plasma HIV RNA concentration strongly predicts PCP and other opportunistic infections (OIs). ${ }^{21}$

Extrapulmonary Pneumocystis organisms, often associated with a localized inflammatory reaction, are found in $<2.5 \%$ of HIV-infected adults and children. ${ }^{22,23}$ This can occur without concurrent PCP and can be located at multiple noncontiguous sites. Involved sites have included ear, eye, thyroid, spleen, gastrointestinal (GI) tract, peritoneum, stomach, duodenum, small intestine, transverse colon, liver, and pancreas. Less frequently involved sites include adrenal glands, muscle, bone marrow, heart, kidney, ureter, lymph nodes, meninges, and cerebral cortex.

\section{Diagnosis}

Most children with PCP have substantial hypoxia with low arterial oxygen pressure $\left(\mathrm{PaO}_{2}\right.$ typically $<70 \mathrm{~mm} \mathrm{Hg}$ ) and an A-a gradient $>30 \mathrm{mmHg}$. CD4 percentage is often $<15 \%$ and CD4 counts are usually $<200$ cells $/ \mathrm{mm}^{3}$ in children aged 6 years and older. Lactic dehydrogenase is often increased, but this is not specific for PCP. Serum albumin may be depressed. Chest radiographs most commonly reveal bilateral diffuse parenchymal infiltrates with "ground-glass" or reticulogranular appearance, but they also can be normal or have only mild parenchymal infiltrates. The earliest infiltrates are perihilar, progressing peripherally before reaching the apical portions of the lung. Rarely, lobar, cavitary, nodular, or miliary lesions; pneumothorax; or pneumomediastinum are observed.

A definitive diagnosis of PCP requires demonstration of the organism in pulmonary tissues or fluids in the presence of pneumonitis. Diagnostic procedures are the same as for adults suspected of having PCP (see Guidelines for the Prevention and Treatment of Opportunistic Infections in HIV-Infected Adults), ${ }^{24}$ but some procedures may be more difficult to perform in children.

Induced sputum analysis, during which the patient produces sputum after inhalation of nebulized 3\% hypertonic saline, may be difficult in children aged $<2$ years because of small airways and poor ability to produce sputum. Complications from the procedure include nausea, vomiting, and bronchospasm. Sensitivity of sputum analysis in adults ranges from $25 \%$ to $90 \%$. After a negative induced sputum sample, a bronchoalveolar lavage may be necessary for definitive diagnosis. 
Nasogastric aspirates, if positive, are of diagnostic value. Pneumocystis organisms were found in $48.6 \%$ of HIV-infected children with respiratory illnesses in whom gastric aspirates were obtained on three consecutive mornings. ${ }^{25}$ Other studies have shown the organism only found in gastric contents of patients with PCP. ${ }^{26}$

Bronchoscopy with bronchoalveolar lavage is the diagnostic procedure of choice for most infants and children. Sensitivity ranges from $55 \%$ to $97 \%$ and results may be positive for $\geq 72$ hours after initiation of PCP treatment; treatment should not be delayed while awaiting results. Complications include hemoptysis, pneumothorax, transient increase in hypoxemia, a transient increase in pulmonary infiltrates at the lavage site, and post-bronchoscopy fever.

Fiberoptic bronchoscopy with trans-bronchial biopsy is recommended only when bronchoalveolar lavage is negative or non-diagnostic despite a clinical picture consistent with PCP. Sensitivity is $87 \%$ to $95 \%$, and cysts can typically be identified up to 10 days after initiation of treatment. Complications include pneumothorax and hemorrhage; this procedure is contraindicated in children with thrombocytopenia.

Open-lung biopsy is the most sensitive and specific diagnostic technique, but not recommended routinely because it requires thoracotomy and often chest tube drainage. It has the advantage of revealing the type and extent of disease as well as the organism.

Histopathology shows alveoli filled with eosinophilic, acellular, proteinaceous material that contains cysts and trophozoites but few inflammatory cells. Complications include pneumothorax, pneumomediastinum, and hemorrhage.

Three types of stains can be used to identify Pneumocystis organisms in specimens. Gomori methenaminesilver method stains the cyst wall brown or black. Toluidine blue stains the cyst wall blue or lavender. Both methods stain fungal elements. Giemsa, Diff-Quick ${ }^{\circledR}$, and Wright stains depict the trophozoites and intracystic sporozoites pale blue with a punctate red nucleus, but unlike other stains, these do not stain the cyst wall. Monoclonal immunofluorescent antibodies (MERIFLUOR ${ }^{\circledR}$, Meridian Bioscience, Inc.; Cincinnati, OH) that identify the cyst wall also can be used for diagnosis and have enhanced specificity and sensitivity compared with the other staining methods. A cyst wall, trophozoite, and immunofluorescent antibody stain is recommended for each specimen studied.

Polymerase chain reaction assays to amplify the human Pneumocystis MSG/gpA gene, mitochondrial large subunit (mtLSU) RNA, the dihydropteroate synthase gene, and the internal transcribed spacer region genes have been developed for diagnostic evaluation. These tests are usually more sensitive but less specific than microscopic methods and are not standardized or available in most centers. ${ }^{27,28}$ Pneumocystis-specific DNA is found in $18 \%$ of bronchoalveolar lavage samples from patients without clinical PCP, HIV, or other infections. $^{29}$

Coinfection with other organisms such as cytomegalovirus (CMV) or pneumococcus has been reported in HIV-infected children. 6,30,31 Children with dual infections may have more severe disease. Although CMV in lung secretions of children with PCP indicates colonization, it usually does not require therapy in the absence of histopathologic evidence of invasive CMV disease. 


\section{Prevention Recommendations}

Preventing Exposure-Clinical data are unavailable upon which to make a decision regarding isolation of patients with PCP. However, animal model experiments, which generally provide an accurate demonstration of the pathophysiology seen in humans, suggest that transmission occurs easily; therefore, isolation should be strongly considered (AIII). ${ }^{32}$ Immunocompromised patients who are compliant with PCP prophylaxis, especially with trimethoprim-sulfamethoxazole (TMP-SMX), are unlikely to acquire PCP. However, some experts still suggest that such at-risk patients not be placed in a room with another patient with PCP. Caution is also advised in having an at-risk patient share a room with another patient with an undiagnosed respiratory illness that could be PCP (AIII). This is especially true of respiratory illnesses occurring during the first 2 years of life when $85 \%$ of children undergo a primary infection with Pneumocystis. ${ }^{1}$

Preventing First Episode of Disease-Chemoprophylaxis is highly effective in preventing PCP. Criteria for its use are based on a patient's age and CD4 count or percentage. ${ }^{33}$ Prophylaxis is recommended for all HIV-infected children aged $\geq 6$ years who have CD4 counts $<200$ cells $/ \mathrm{mm}^{3}$ or CD4 percentage $<15 \%$, for children aged 1 to $<6$ years with CD4 counts $<500$ cells $/ \mathrm{mm}^{3}$ or CD4 percentage $<15 \%$, and for all HIV-infected infants aged $<12$ months regardless of CD4 count or percentage (AII). ${ }^{33}$

Infants born to HIV-infected mothers should be considered for prophylaxis beginning at 4 to 6 weeks of age. HIV-infected infants should be administered prophylaxis until age 1 year, at which time they should be reassessed on the basis of the age-specific CD4 count or percentage thresholds mentioned previously (AII). ${ }^{34}$ Infants with indeterminate HIV infection status should receive prophylaxis until they are determined to be definitively HIVuninfected ${ }^{34}$ or presumptively HIV-uninfected (AIII). ${ }^{35-37}$ Prophylaxis is not recommended for infants who meet criteria for being definitively or presumptively HIV-uninfected. In nonbreastfeeding infants with no positive HIV virologic test results, presumptive exclusion of HIV infection can be based on two negative virologic test results, one obtained at $\geq 2$ weeks and one obtained at $\geq 4$ weeks of age; one negative virologic test result obtained at $\geq$ weeks of age; or one negative HIV-antibody test result obtained at $\geq 6$ months of age. Definitive exclusion of HIV infection is based on two negative virologic test results: 1 obtained at $\geq 1$ month of age and one obtained at $\geq 4$ months of age, or on 2 negative HIV-antibody test results from separate specimens obtained at $\succeq$ months of age. For both presumptive and definitive exclusion of infection, a child should have no other laboratory (e.g., no positive virologic test results) or clinical conditions (e.g., no AIDS-defining conditions that cannot be explained on the basis of other causes of immunosuppression) or evidence of HIV infection. ${ }^{35-37}$

Four drug regimens have been found effective and relatively safe for preventing PCP in high-risk HIV-infected children and adults.

TMP-SMX (cotrimoxazole) is the drug of choice for prophylaxis because of its high efficacy, relative safety, low cost, and broad antimicrobial spectrum (AI) ${ }^{38-40} \mathrm{TMP}$ alone has little, if any, anti-Pneumocystis activity, but it enhances the activity of the sulfonamide. The prophylactic dosage is $150 \mathrm{mg} / \mathrm{m}^{2}$ body surface area per day TMP and $750 \mathrm{mg} / \mathrm{m}^{2}$ body 
surface area per day SMX (approximately $5.0-10 \mathrm{mg} / \mathrm{kg}$ body weight per day TMP and 25$50 \mathrm{mg} / \mathrm{kg}$ body weight per day SMX; dosing based on TMP component) administered orally either every day (AI) ${ }^{41}(5.0-10 \mathrm{mg} / \mathrm{kg}$ body weight/dose once daily TMP and $25-50 \mathrm{mg} / \mathrm{kg}$ body weight/dose once daily SMX) or on 3 consecutive days per week $(2.5-5.0 \mathrm{mg} / \mathrm{kg}$ body weight/dose TMP and $12.5-25 \mathrm{mg} / \mathrm{kg}$ body weight/dose SMX twice per day) ${ }^{42}$ or every other day (e.g., Monday, Wednesday, Friday). The total daily dose should not exceed 320 mg TMP and $1600 \mathrm{mg}$ SMX. In patients with impaired renal function, a reduced dose may be necessary.

TMP-SMX, preferably given daily, also is effective in preventing toxoplasmosis ${ }^{43}$ and some bacterial infections (e.g., Salmonella, Haemophilus, Staphylococcus). ${ }^{41,44-46}$

Dihydropteroate synthase gene mutations in Pneumocystis from humans have been observed with TMPSMX and dapsone prophylaxis, suggestive of possible drug resistance, but studies for clinical correlates have not provided conclusive results. ${ }^{27}$ More apparent is the association of prolonged TMP-SMX prophylaxis for PCP with the emergence of TMP-SMX resistant bacterial species due to selective pressure, a point to be considered in managing bacterial infections in patients receiving prophylaxis. ${ }^{47,48}$

Other effective and safe prophylaxis regimens are available for patients unable to take TMPSMX. A second choice would be either atovaquone (AI) ${ }^{49}$ or dapsone $(\mathbf{B I} *) .{ }^{39}$ Atovaquone is effective and safe but expensive. Dapsone is effective and inexpensive but associated with more serious adverse effects than atovaquone.

Atovaquone is administered with a meal as an oral yellow suspension as a single daily dose of $30 \mathrm{mg} / \mathrm{kg}$ body weight/day for patients aged 1 to 3 months and $>24$ months to 12 years, as $45 \mathrm{mg} / \mathrm{kg}$ body weight/day for infants aged $>3$ months to 24 months, ${ }^{50}$ and as $1500 \mathrm{mg}$ (10 cc) for adolescents and adults aged $\geq 13$ years $\left(\mathbf{B I}^{*}\right) .{ }^{39,40}$ Outcomes with atovaquone equaled those of dapsone for the prevention of PCP in patients with HIV infection who cannot tolerate trimethoprim, sulfonamides, or both. ${ }^{39}$ Unlike TMP-SMX, atovaquone has no antibacterial activity but is effective against Toxoplasma gondii. Azithromycin, in a single dosage of $5.0 \mathrm{mg} / \mathrm{kg}$ body weight/day, has been used to supplement atovaquone for greater broad-spectrum prophylaxis. The randomized, double-blind, placebo-controlled study the Pediatric AIDS Clinical Trial Group (PACTG) 254 compared TMP-SMX and atovaquone plus azithromycin for 3 years (median) in $366 \mathrm{HIV}$-infected children qualifying for PCP prophylaxis. ${ }^{49}$ Results showed atovaquone-azithromycin to be as effective as TMPSMX for preventing serious bacterial infections, as well as PCP.

Dapsone can be administered on a daily or weekly schedule as $2.0 \mathrm{mg} / \mathrm{kg}$ body weight/day (maximum total dosage $100 \mathrm{mg} /$ day) or $4.0 \mathrm{mg} / \mathrm{kg}$ body weight/week (maximum total dosage $200 \mathrm{mg} /$ week) orally (AI) ${ }^{51}$ Approximately two-thirds of patients intolerant to TMP-SMX can take dapsone successfully. Studies in adults show dapsone is as effective as atovaquone or aerosolized pentamidine but slightly less effective than TMP-SMX. ${ }^{39,50}$

Aerosolized pentamidine is recommended for children who cannot take TMP-SMX, atovaquone, or dapsone and are old enough to use nebulization with a Respirgard II ${ }^{\circledR}$ nebulizer (Marquest; Englewood, CO) (BI*). ${ }^{33}$ The dosage for all ages is $300 \mathrm{mg}$ once a 
month. ${ }^{45}$ Adverse reactions in HIV-infected children include cough, sneezing, and bronchospasm. ${ }^{52}$ Atypical systemic presentations of PCP can occur in children on aerosolized pentamidine.

Pyrimethamine-sulfadoxine (Fansidar ${ }^{\circledR}$ ) also is recognized as an effective prophylactic regimen in adults (CIII) ${ }^{53}$ Although this drug was effective in preventing PCP in Iranian orphanages in the 1960s, it has not been evaluated adequately in HIV-infected children.

Intravenous (IV) pentamidine can be considered in children older than age 2 years when other options are unavailable $\left(\mathbf{B I I}{ }^{*}\right) .54$

Discontinuing Primary Prophylaxis-Studies of HIV-infected adults and children following immune reconstitution after receipt of cART demonstrate acceptably low risks for PCP after discontinuation of prophylaxis. ${ }^{55-60}$ Data from the PACTG 1008 study evaluated $235 \mathrm{HIV}$-infected children and adolescents on antiretroviral therapy who received PCP prophylaxis for $\geq 6$ months and achieved CD4 percentages $\geq 20 \%$ for patients aged $>6$ years and $25 \%$ for patients aged 2 to 6 years, after which the prophylaxis was stopped. ${ }^{55} \mathrm{At}$ median follow-up of 2.5 years (547 person-years), no cases of PCP occurred in children not receiving prophylaxis; $9.4 \%$ of patients enrolled required reinstitution of PCP prophylaxis because of low CD4 counts during the observation period. These data, along with data from studies in adults, support the expectation for very low risk for PCP after prophylaxis is discontinued in children who have achieved immune reconstitution.

Discontinuation of PCP prophylaxis should be considered for HIV-infected children when, after receiving cART for $\geq 6$ months, CD4 percentage is $\geq 15 \%$ or CD4 count is $\geq 200$ cells $/ \mathrm{mm}^{3}$ for patients aged $\ 6$ years (BII) and CD4 percentage is $\geq 15 \%$ or CD4 count is 2500 cells $/ \mathrm{mm}^{3}$ for patients aged 1 to $<6$ years (BII) for $>3$ consecutive months. ${ }^{55,61}$

Subsequently, the CD4 percentage and CD4 count should be reevaluated at least every 3 months and prophylaxis reinstituted if the original criteria for prophylaxis are reached (BIII). PCP prophylaxis should not be discontinued in HIV-infected infants aged $<1$ year. ${ }^{62}$

\section{Treatment Recommendations}

Treating Disease-TMP-SMX is the recommended treatment for PCP (AI) ${ }^{38,63,64}$ The dose for HIV-infected children aged $>2$ months is 3.75 to $5 \mathrm{mg} / \mathrm{kg}$ body weight/dose of the TMP component and 19 to $25 \mathrm{mg} / \mathrm{kg}$ body weight/dose of the SMX component administered IV every 6 hours, with each IV dose infused over 1 hour for 21 days (AI). ${ }^{62}$ As the acute pneumonitis subsides, children with mild to moderate disease who do not have malabsorption or diarrhea can be transitioned to oral treatment with the same total daily dose of TMP-SMX administered in 3 or 4 divided doses to complete a 21-day course (AII). ${ }^{62}$ Effective therapeutic serum concentrations of 5 to $10 \mu \mathrm{g} / \mathrm{mL}$ TMP can be reached with the recommended dose administered orally in HIV-infected children. 65

IV pentamidine isethionate ( $4 \mathrm{mg} / \mathrm{kg}$ body weight) once daily is recommended for patients who cannot tolerate TMP-SMX or who demonstrate clinical treatment failure after 5 to 7 days of TMP-SMX therapy (AI*). ${ }^{62,66,67}$ No evidence exists for synergistic or additive 
effects on efficacy of these agents; ${ }^{68}$ therefore, because of potential increased toxicity, their combined use is not recommended (BIII). ${ }^{62}$ In patients with clinical improvement after 7 to 10 days of IV therapy with pentamidine, an oral regimen (i.e., atovaquone [BI] or TMP/ dapsone [CIII]) can be considered to complete a 21-day course. ${ }^{62}$

Atovaquone is an alternative for treatment of mild to moderately severe PCP in adults (BI). ${ }^{39,63,69}$ The dosage for adolescents aged $\geq 13$ years is $750 \mathrm{mg} /$ dose $(5 \mathrm{~mL})$ administered orally twice daily with food. ${ }^{39,63,69-71}$ Therapeutic data are limited for children, but based on studies of prophylaxis, the primary dosage for children $<3$ months and $>24$ months to 12 years of age is 30 to $40 \mathrm{mg} / \mathrm{kg}$ body weight/dose administered orally once a day with food, and for children aged 2 to 24 months of age is a higher dose of $45 \mathrm{mg} / \mathrm{kg}$ body weight/dose once daily (BI*). ${ }^{49,50}$ Based on adult studies that use twice-daily dosing, some experts also use an alternate dosing regimen for children $<3$ months and $>24$ months to age 12 years of 15 to $20 \mathrm{mg} / \mathrm{kg}$ body weight/dose administered orally twice daily with food, and for children aged 2 to 24 months, a dose of $22.5 \mathrm{mg} / \mathrm{kg}$ body weight/dose twice daily with food (CIII).

Food increases the bioavailability of atovaquone approximately threefold compared with that achieved with the fasting state. Atovaquone concentration increases with co administration of fluconazole and prednisone and decreases with co-administration of acyclovir, opiates, cephalosporins, rifampin, and benzodiazepines. Some experts suggest desensitizing the patient to allow for use of TMP-SMX.

Dapsone/TMP is effective in treating mild-to-moderate PCP in adults (BI) $;{ }^{72}$ data on toxicity and efficacy among children are limited. The dosage of dapsone for adolescents and adults is $100 \mathrm{mg}$ (total dose)/dose orally once daily and TMP $5 \mathrm{mg} / \mathrm{kg}$ body weight/dose three times per day administered for 21 days. In children aged < 13 years, a dapsone dosage of $2 \mathrm{mg} / \mathrm{kg}$ body weight/dose once daily is required to achieve therapeutic levels (AI). ${ }^{73}$ The pediatric dose of TMP is $5 \mathrm{mg} / \mathrm{kg}$ body weight/day/dose three times per day. Dapsone is less effective than the combination. ${ }^{74}$ Clindamycin/primaquine has been found to be effective in treating mild to moderate $\mathrm{PCP}$ in adults but can be considered as an alternative therapy for PCP in children despite lack of pediatric data. (CIII). Primaquine is contraindicated in patients with glucose-6-dehydrogenase deficiency because of the possibility of inducing hemolytic anemia. Dosing information for treating PCP is available only for adults. For patients who weigh $>60 \mathrm{~kg}$, clindamycin $600 \mathrm{mg}$ IV every 6 hours for 10 days, then 300 to $450 \mathrm{mg}$ orally every 6 hours to complete 21 days of treatment, is recommended. Primaquine is administered as $30 \mathrm{mg}$ of base orally for 21 days. Dosing for children is based on use of these drugs for treating other infections; the usual pediatric dose of clindamycin for treating bacterial infection is $10 \mathrm{mg} / \mathrm{kg}$ body weight/dose every 6 hours, and the pediatric dose of primaquine equivalent to an adult dose of $20 \mathrm{mg}$ base (when used for malaria) is $0.3 \mathrm{mg} / \mathrm{kg}$ body weight/day of the base.

On the basis of studies in both adults ${ }^{75-79}$ and children, ${ }^{80}$ a short course of corticosteroids is recommended in cases of moderate or severe PCP, starting within 72 hours of diagnosis (AI*). Pediatric studies have indicated reduced acute respiratory failure, decreased need for ventilation, and decreased mortality with early use of corticosteroids in HIV-infected children who have PCP. ${ }^{80-82}$ Indications for corticosteroid treatment include a $\mathrm{PaO}_{2}$ value of $<70 \mathrm{~mm} \mathrm{Hg}$ or an alveolar-arterial gradient of $>35 \mathrm{~mm} \mathrm{Hg}$. Doses for children vary 
between studies. A commonly used scheme is prednisone $1 \mathrm{mg} / \mathrm{kg}$ of body weight $/ \mathrm{dose}$ twice daily on days 1 through $5 ; 0.5 \mathrm{mg} / \mathrm{kg} /$ dose twice daily on days 6 through 10 ; and 0.5 $\mathrm{mg} / \mathrm{kg}$ of body weight/dose once daily on days 11 through 21 . Alternative regimens include:

1. Adult dosage of prednisone: $40 \mathrm{mg} /$ dose twice daily on days 1 through $5 ; 40 \mathrm{mg} /$ dose once daily on days 6 through $10 ; 20 \mathrm{mg} /$ dose once daily on days 11 through 21 , and

2. Methylprednisolone IV $1 \mathrm{mg} / \mathrm{kg} /$ dose every 6 hours on days 1 through $7 ; 1 \mathrm{mg} / \mathrm{kg} /$ dose twice daily on days 8 through $9 ; 0.5 \mathrm{mg} / \mathrm{kg} /$ dose twice daily on days 10 and 11 ; and $1 \mathrm{mg} / \mathrm{kg} / \mathrm{dose}$ once daily on days $12-16$.

Some case reports have documented improved pulmonary function with use of surfactant in cases of severe disease such as respiratory distress syndrome with established respiratory failure requiring ventilation. ${ }^{83-85}$ Alterations in surfactant function and composition have been demonstrated in HIV-infected adults with PCP. ${ }^{86}$ Data are insufficient to recommend surfactant administration for PCP in children.

Monitoring and Adverse Events (Including IRIS)—Clinical parameters for monitoring disease status include temperature, respiratory rate, arterial oxygen saturation, and chest radiograph. ${ }^{87}$ Clinical improvement can be expected at a mean of approximately $4.5 \pm 2.5$ days and radiographic improvement at approximately $7.7 \pm 4.5$ days. ${ }^{87}$

Immune reconstitution inflammatory syndrome (IRIS) has been less frequently associated with Pneumocystis infection (2\% of 44 adults with IRIS) than with several other OIs in HIVinfected adults and children. ${ }^{88}$ Whether this low rate is related to PCP prophylaxis is unknown.

In children, adverse reactions to TMP-SMX include rash (mild maculopapular in most cases but rarely erythema multiforme and Stevens-Johnson syndrome [SJS]), hematologic abnormalities (e.g., neutropenia, thrombocytopenia, megaloblastic or aplastic anemia), GI complaints (usually mild), hepatitis, and renal disorders (e.g., interstitial nephritis). ${ }^{89,90}$ Data from a PACTG study of HIV-infected children at high risk of PCP receiving TMP-SMX for a median of 3 years showed $28 \%$ had a rash, $9.3 \%$ had neutropenia, $8.8 \%$ had thrombocytopenia, and $2.2 \%$ had anemia. ${ }^{49}$ None were fatal or irreversible reactions. Some very mild reactions will resolve while the drug is continued. With any significant adverse effect, TMP-SMX should be withheld until the reaction has subsided. Based on adult randomized clinical trials, unless the reaction has been life-threatening, TMP-SMX prophylaxis can be resumed in children, preferably by beginning with low desensitizing daily doses and gradually increasing to therapeutic dosing (CIII). ${ }^{91,92}$ In adults, $75 \%$ of patients affected tolerated re-challenge with TMP-SMX. ${ }^{92}$ The overall frequency of adverse reactions appears to be lower in HIV-infected children than in adults; approximately $15 \%$ of children have substantial adverse reactions to TMP-SMX. ${ }^{57}$ If an urticarial rash or SJS occurs, TMP-SMX should be discontinued and not readministered (AIII). ${ }^{89,90,92}$

The most common adverse drug reaction to pentamidine isethionate is renal toxicity, which usually occurs after 2 weeks of therapy and can be averted by adequate hydration and careful monitoring of renal function and electrolytes. Severe hypotension (particularly if 
infused rapidly), prolonged QT interval (torsades de pointes), and cardiac arrhythmias can occur. Hypoglycemia (usually after 5-7 days of therapy) or hyperglycemia, hypercalcemia, hyperkalemia, pancreatitis, and insulin-dependent diabetes mellitus also have been reported. Patients may report a metallic or bitter taste. Serious adverse reactions to pentamidine have been reported in approximately $17 \%$ of children receiving the drug. ${ }^{93}$ This drug should not be administered with other nephrotoxic drugs (e.g., aminoglycosides, amphotericin B, cisplatin, or vancomycin) or with agents associated with pancreatitis (e.g., didanosine).

With dapsone and TMP, the primary adverse reaction is reversible neutropenia; other reactions include skin rashes, elevated serum transaminases, methemoglobinemia, anemia, and thrombocytopenia. ${ }^{72,74}$ Dapsone is the problematic component of the combination and accounts for most of the adverse reactions. ${ }^{50}$

Skin rashes (10\%-15\%), nausea, and diarrhea can occur with atovaquone administration. Liver enzymes may increase briefly. No serious toxicity or fatality has been demonstrated from use of atovaquone in adults or children.

Adverse reactions to clindamycin/primaquine include skin rash, nausea, and diarrhea.

Managing Treatment Failure-Occasionally an inflammatory reaction, thought to be due to antibiotic-induced killing of the organism in the lungs, can result in an initial early and reversible deterioration during the first 3 to 5 days of therapy, so an adequate trial of therapy is needed before switching drugs because of lack of clinical improvement. Clinical failure is defined by lack of improvement or worsening of respiratory function documented by arterial blood gases after at least 4 to 8 days of anti-PCP treatment. Other concomitant infections need to be excluded as causes of clinical failure. With evidence of treatment failure after the use of TMP-SMX, therapy can be changed. If tolerated, pentamidine isethionate is the drug of next choice (AI*). ${ }^{94,95}$ No evidence exists for synergistic or additive therapeutic effects; therefore, because of potential increased toxicity, their combination is not recommended.

Preventing Recurrence-None of the drugs administered to treat and prevent PCP completely eliminates Pneumocystis, and prophylaxis is effective only while the selected drug is administered. Patients who have experienced an episode of PCP should remain on a prophylactic regimen after completion of treatment unless they meet criteria for discontinuing secondary prophylaxis (AIII). ${ }^{95}$

Discontinuing Secondary Prophylaxis-In most patients, secondary prophylaxis can be discontinued using the same criteria as for discontinuing primary prophylaxis. PCP prophylaxis is not to be discontinued in HIV-infected infants aged $<1$ year. Children who present with clinical signs and symptoms compatible with PCP after discontinuation of prophylaxis should be evaluated thoroughly despite normal or high CD4 counts or percentages (AIII). ${ }^{96}$ If PCP recurs at a CD4 count $\geq 200$ cells $/ \mathrm{mm}^{3}$, lifelong prophylaxis should be administered (CIII). 


\section{References}

1. Vargas SL, Hughes WT, Santolaya ME, et al. Search for primary infection by Pneumocystis carinii in a cohort of normal, healthy infants. Clin Infect Dis. 2001 Mar 15; 32(6):855-861. Available at http://www.ncbi.nlm.nih.gov/pubmed/11247708. [PubMed: 11247708]

2. Respaldiza N, Medrano FJ, Medrano AC, et al. High seroprevalence of Pneumocystis infection in Spanish children. Clin Microbiol Infect. 2004 Nov; 10(11):1029-1031. Available at http:// www.ncbi.nlm.nih.gov/pubmed/15522012. [PubMed: 15522012]

3. Pifer LL, Hughes WT, Stagno S, Woods D. Pneumocystis carinii infection: evidence for high prevalence in normal and immunosuppressed children. Pediatrics. 1978 Jan; 61(1):35-41. Available at http://www.ncbi.nlm.nih.gov/pubmed/400818. [PubMed: 400818]

4. Simonds RJ, Oxtoby MJ, Caldwell MB, Gwinn ML, Rogers MF. Pneumocystis carinii pneumonia among US children with perinatally acquired HIV infection. JAMA. 1993 Jul 28; 270(4):470-473. Available at http://www.ncbi.nlm.nih.gov/pubmed/8320786. [PubMed: 8320786]

5. Gibb DM, Davison CF, Holland FJ, Walters S, Novelli V, Mok J. Pneumocystis carinii pneumonia in vertically acquired HIV infection in the British Isles. Arch Dis Child. 1994 Mar; 70(3):241-244. Available at http://www.ncbi.nlm.nih.gov/pubmed/8135571. [PubMed: 8135571]

6. Williams AJ, Duong T, McNally LM, et al. Pneumocystis carinii pneumonia and cytomegalovirus infection in children with vertically acquired HIV infection. AIDS. 2001 Feb 16; 15(3):335-339. Available at http://www.ncbi.nlm.nih.gov/pubmed/11273213. [PubMed: 11273213]

7. Morris A, Lundgren JD, Masur H, et al. Current epidemiology of Pneumocystis pneumonia. Emerg Infect Dis. 2004 Oct; 10(10):1713-1720. Available at http://www.ncbi.nlm.nih.gov/pubmed/ 15504255. [PubMed: 15504255]

8. Nesheim SR, Kapogiannis BG, Soe MM, et al. Trends in opportunistic infections in the pre- and post-highly active antiretroviral therapy eras among HIV-infected children in the Perinatal AIDS Collaborative Transmission Study, 1986-2004. Pediatrics. 2007 Jul; 120(1):100-109. Available at http://www.ncbi.nlm.nih.gov/pubmed/17606567. [PubMed: 17606567]

9. Gona P, Van Dyke RB, Williams PL, et al. Incidence of opportunistic and other infections in HIVinfected children in the HAART era. JAMA. 2006 Jul 19; 296(3):292-300. Available at http:// www.ncbi.nlm.nih.gov/pubmed/16849662. [PubMed: 16849662]

10. Ikeogu MO, Wolf B, Mathe S. Pulmonary manifestations in HIV seropositivity and malnutrition in Zimbabwe. Arch Dis Child. 1997 Feb; 76(2):124-128. Available at http:/www.ncbi.nlm.nih.gov/ pubmed/9068301. [PubMed: 9068301]

11. Chintu C, Mudenda V, Lucas S, et al. Lung diseases at necropsy in African children dying from respiratory illnesses: a descriptive necropsy study. Lancet. 2002 Sep 28; 360(9338):985-990. Available at http://www.ncbi.nlm.nih.gov/pubmed/12383668. [PubMed: 12383668]

12. Madhi SA, Cutland C, Ismail K, O'Reilly C, Mancha A, Klugman KP. Ineffectiveness of trimethoprim-sulfamethoxazole prophylaxis and the importance of bacterial and viral coinfections in African children with Pneumocystis carinii pneumonia. Clin Infect Dis. 2002 Nov 1; 35(9): 1120-1126. Available at http://www.ncbi.nlm.nih.gov/pubmed/12384847. [PubMed: 12384847]

13. Hendley JO, Weller TH. Activation and transmission in rats of infection with Pneumocystis. Proc Soc Exp Biol Med. 1971 Sep; 137(4):1401-1404. Available at http://www.ncbi.nlm.nih.gov/ pubmed/5316452. [PubMed: 5316452]

14. Hughes WT. Natural mode of acquisition for de novo infection with Pneumocystis carinii. J Infect Dis. 1982 Jun; 145(6):842-848. Available at http://www.ncbi.nlm.nih.gov/pubmed/6979590. [PubMed: 6979590]

15. Gigliotti F, Harmsen AG, Wright TW. Characterization of transmission of Pneumocystis carinii f. sp. muris through immunocompetent BALB/c mice. Infect Immun. 2003 Jul; 71(7):3852-3856. Available at http://www.ncbi.nlm.nih.gov/pubmed/12819069. [PubMed: 12819069]

16. de Boer MG, Bruijnesteijn van Coppenraet LE, Gaasbeek A, et al. An outbreak of Pneumocystis jiroveci pneumonia with 1 predominant genotype among renal transplant recipients: interhuman transmission or a common environmental source? Clin Infect Dis. 2007 May 1; 44(9):1143-1149. Available at http://www.ncbi.nlm.nih.gov/pubmed/17407029. [PubMed: 17407029] 
17. Hocker B, Wendt C, Nahimana A, Tonshoff B, Hauser PM. Molecular evidence of Pneumocystis transmission in pediatric transplant unit. Emerg Infect Dis. 2005 Feb; 11(2):330-332. Available at http://www.ncbi.nlm.nih.gov/pubmed/15752458. [PubMed: 15752458]

18. Rabodonirina M, Vanhems P, Couray-Targe S, et al. Molecular evidence of interhuman transmission of Pneumocystis pneumonia among renal transplant recipients hospitalized with HIVinfected patients. Emerg Infect Dis. 2004 Oct; 10(10):1766-1773. Available at http:// www.ncbi.nlm.nih.gov/pubmed/15504262. [PubMed: 15504262]

19. Mortier E, Pouchot J, Bossi P, Molinie V. Maternal-fetal transmission of Pneumocystis carinii in human immunodeficiency virus infection. N Engl J Med. 1995 Mar 23.332(12):825. Available at http://www.ncbi.nlm.nih.gov/pubmed/7862196. [PubMed: 7862196]

20. Fatti GL, Zar HJ, Swingler GH. Clinical indicators of Pneumocystis jiroveci pneumonia (PCP) in South African children infected with the human immunodeficiency virus. Int J Infect Dis. 2006 Jul; 10(4):282-285. Available at http://www.ncbi.nlm.nih.gov/pubmed/16460981. [PubMed: 16460981]

21. Podlekareva D, Mocroft A, Dragsted UB, et al. Factors associated with the development of opportunistic infections in HIV-1-infected adults with high CD4+ cell counts: a EuroSIDA study. J Infect Dis. 2006 Sep 1; 194(5):633-641. Available at http://www.ncbi.nlm.nih.gov/pubmed/ 16897662. [PubMed: 16897662]

22. Ng VL, Yajko DM, Hadley WK. Extrapulmonary pneumocystosis. Clin Microbiol Rev. 1997 Jul; 10(3):401-418. Available at http://www.ncbi.nlm.nih.gov/pubmed/9227859. [PubMed: 9227859]

23. Chen A, Zaidi AK, Mueller BU, Huskins WC, Perez-Atayde AR, McIntosh K. Pneumocystis carinii presenting as a mediastinal mass in a child with acquired immunodeficiency syndrome. Pediatr Infect Dis J. 1999 Sep; 18(9):827-831. Available at http://www.ncbi.nlm.nih.gov/pubmed/ 10493348. [PubMed: 10493348]

24. Kaplan JE, Benson C, Holmes KH, et al. Guidelines for prevention and treatment of opportunistic infections in HIV-infected adults and adolescents: recommendations from CDC, the National Institutes of Health, and the HIV Medicine Association of the Infectious Diseases Society of America. MMWR Recomm Rep. 2009 Apr 10; 58(RR-4):1-207. quiz CE201-204. Available at http://www.ncbi.nlm.nih.gov/pubmed/19357635.

25. Surve TY, Rathod AD. Role of naso-gastric aspirate in HIV-positive children presenting with respiratory symptoms. J Trop Pediatr. 2006 Dec; 52(6):451-453. Available at http:// www.ncbi.nlm.nih.gov/pubmed/16870685. [PubMed: 16870685]

26. Chan H, Pifer L, Hughes WT, Feldman S, Pearson TA, Woods D. Comparison of gastric contents to pulmonary aspirates for the cytologic diagnosis of Pneuomcystis carinii pneumonia. J Pediatr. 1977 Feb; 90(2):243-244. Available at http://www.ncbi.nlm.nih.gov/pubmed/63544. [PubMed: 63544]

27. Huang L, Morris A, Limper AH, Beck JM. Participants ATSPW. An Official ATS Workshop Summary: Recent advances and future directions in pneumocystis pneumonia (PCP). Proc Am Thorac Soc. 2006 Nov; 3(8):655-664. Available at http://www.ncbi.nlm.nih.gov/pubmed/ 17065370. [PubMed: 17065370]

28. Leibovitz E, Pollack H, Rigaud M, et al. Polymerase chain reaction is more sensitive than standard cytologic stains in detecting Pneumocystis carinii in bronchoalveolar lavages from human immunodeficiency virus type 1-infected infants and children with pneumonia. Pediatr Infect Dis J. 1995 Aug; 14(8):714-716. Available at http://www.ncbi.nlm.nih.gov/pubmed/8532434. [PubMed: 8532434]

29. Maskell NA, Waine DJ, Lindley A, et al. Asymptomatic carriage of Pneumocystis jiroveci in subjects undergoing bronchoscopy: a prospective study. Thorax. 2003 Jul; 58(7):594-597. Available at http://www.ncbi.nlm.nih.gov/pubmed/12832674. [PubMed: 12832674]

30. Glatman-Freedman A, Ewig JM, Dobroszycki J, Mitsudo S, Glaser JH. Simultaneous Pneumocystis carinii and pneumococcal pneumonia in human immunodeficiency virus-infected children. J Pediatr. 1998 Jan; 132(1):169-171. Available at http://www.ncbi.nlm.nih.gov/pubmed/ 9470024. [PubMed: 9470024]

31. Jeena PM, Coovadia HM, Chrystal V. Pneumocystis carinii and cytomegalovirus infections in severely ill, HIV-infected African infants. Ann Trop Paediatr. 1996 Dec; 16(4):361-368. Available at http://www.ncbi.nlm.nih.gov/pubmed/8985536. [PubMed: 8985536] 
32. Hughes WT, Bartley DL, Smith BM. A natural source of infection due to pneumocystis carinii. J Infect Dis. Mar.1983 147(3):595. Available at http://www.ncbi.nlm.nih.gov/pubmed/6601170. [PubMed: 6601170]

33. CDC. Guidelines for prophylaxis against Pneumocystis carinii pneumonia for children infected with human immunodeficiency virus. MMWR Recomm Rep. 1991 Mar 15; 40(RR-2):1-13. Available at http://www.ncbi.nlm.nih.gov/pubmed/1672036.

34. CDC. 1995 revised guidelines for prophylaxis against Pneumocystis carinii pneumonia for children infected with or perinatally exposed to human immunodeficiency virus. National Pediatric and Family HIV Resource Center and National Center for Infectious Diseases, Centers for Disease Control and Prevention. MMWR Recomm Rep. 1995 Apr 28; 44(RR-4):1-11. Available at http:// www.ncbi.nlm.nih.gov/pubmed/7565543.

35. Panel on Antiretroviral Therapy and Medical Management of HIV-Infected Children. Guidelines for the Use of Antiretroviral Agents in Pediatric HIV Infection. 2011 Aug 11.:1-268. Available at http://aidsinfo.nih.gov/ContentFiles/PediatricGuidelines.pdf. 2011.

36. Read JS. Committee on Pediatric Aids AAoP. Diagnosis of HIV-1 infection in children younger than 18 months in the United States. Pediatrics. 2007 Dec; 120(6):e1547-e1562. Available at http://www.ncbi.nlm.nih.gov/pubmed/18055670. [PubMed: 18055670]

37. Schneider E, Whitmore S, Glynn KM, et al. Revised surveillance case definitions for HIV infection among adults, adolescents, and children aged $<18$ months and for HIV infection and AIDS among children aged 18 months to <13 years-United States 2008. MMWR Recomm Rep. 2008 Dec 5; 57(RR-10):1-12. Available at http://www.ncbi.nlm.nih.gov/pubmed/19052530. [PubMed: 19052530]

38. Thea DM, Lambert G, Weedon J, et al. Benefit of primary prophylaxis before 18 months of age in reducing the incidence of Pneumocystis carinii pneumonia and early death in a cohort of 112 human immunodeficiency virus-infected infants. New York City Perinatal HIV Transmission Collaborative Study Group. Pediatrics. 1996 Jan; 97(1):59-64. Available at http:// www.ncbi.nlm.nih.gov/pubmed/8545225. [PubMed: 8545225]

39. El-Sadr WM, Murphy RL, Yurik TM, et al. Atovaquone compared with dapsone for the prevention of Pneumocystis carinii pneumonia in patients with HIV infection who cannot tolerate trimethoprim, sulfonamides, or both. Community Program for Clinical Research on AIDS and the AIDS Clinical Trials Group. N Engl J Med. 1998 Dec 24; 339(26):1889-1895. Available at http:// www.ncbi.nlm.nih.gov/pubmed/9862944. [PubMed: 9862944]

40. Chan C, Montaner J, Lefebvre EA, et al. Atovaquone suspension compared with aerosolized pentamidine for prevention of Pneumocystis carinii pneumonia in human immunodeficiency virusinfected subjects intolerant of trimethoprim or sulfonamides. J Infect Dis. 1999 Aug; 180(2):369376. Available at http://www.ncbi.nlm.nih.gov/pubmed/10395851. [PubMed: 10395851]

41. Hughes WT, Kuhn S, Chaudhary S, et al. Successful chemoprophylaxis for Pneumocystis carinii pneumonitis. N Engl J Med. 1977 Dec 29; 297(26):1419-1426. Available at http:// www.ncbi.nlm.nih.gov/pubmed/412099. [PubMed: 412099]

42. Hughes WT, Rivera GK, Schell MJ, Thornton D, Lott L. Successful intermittent chemoprophylaxis for Pneumocystis carinii pneumonitis. N Engl J Med. 1987 Jun 25; 316(26):1627-1632. Available at http://www.ncbi.nlm.nih.gov/pubmed/3495732. [PubMed: 3495732]

43. Carr A, Tindall B, Brew BJ, et al. Low-dose trimethoprim-sulfamethoxazole prophylaxis for toxoplasmic encephalitis in patients with AIDS. Ann Intern Med. 1992 Jul 15; 117(2):106-111. Available at http://www.ncbi.nlm.nih.gov/pubmed/1351371. [PubMed: 1351371]

44. Bozzette SA, Finkelstein DM, Spector SA, et al. A randomized trial of three antipneumocystis agents in patients with advanced human immunodeficiency virus infection. NIAID AIDS Clinical Trials Group. N Engl J Med. 1995 Mar 16; 332(11):693-699. Available at http:// www.ncbi.nlm.nih.gov/pubmed/7854375. [PubMed: 7854375]

45. Hardy WD, Feinberg J, Finkelstein DM, et al. A controlled trial of trimethoprim-sulfamethoxazole or aerosolized pentamidine for secondary prophylaxis of Pneumocystis carinii pneumonia in patients with the acquired immunodeficiency syndrome. AIDS Clinical Trials Group Protocol 021. N Engl J Med. 1992 Dec 24; 327(26):1842-1848. Available at http://www.ncbi.nlm.nih.gov/ pubmed/1448121. [PubMed: 1448121] 
46. Dworkin MS, Williamson J, Jones JL, Kaplan JE. Adult, Adolescent Spectrum of HIVDP. Prophylaxis with trimethoprim-sulfamethoxazole for human immunodeficiency virus-infected patients: impact on risk for infectious diseases. Clin Infect Dis. 2001 Aug 1; 33(3):393-398. Available at http://www.ncbi.nlm.nih.gov/pubmed/11438910. [PubMed: 11438910]

47. Martin JN, Rose DA, Hadley WK, Perdreau-Remington F, Lam PK, Gerberding JL. Emergence of trimethoprim-sulfamethoxazole resistance in the AIDS era. J Infect Dis. 1999 Dec; 180(6):18091818. Available at http://www.ncbi.nlm.nih.gov/pubmed/10558935. [PubMed: 10558935]

48. Huovinen P. Resistance to trimethoprim-sulfamethoxazole. Clin Infect Dis. 2001 Jun 1; 32(11): 1608-1614. Available at http://www.ncbi.nlm.nih.gov/pubmed/11340533. [PubMed: 11340533]

49. Hughes WT, Dankner WM, Yogev R, et al. Comparison of atovaquone and azithromycin with trimethoprim-sulfamethoxazole for the prevention of serious bacterial infections in children with HIV infection. Clin Infect Dis. 2005 Jan 1; 40(1):136-145. Available at http:// www.ncbi.nlm.nih.gov/pubmed/15614703. [PubMed: 15614703]

50. Hughes WT. Use of dapsone in the prevention and treatment of Pneumocystis carinii pneumonia: a review. Clin Infect Dis. 1998 Jul; 27(1):191-204. Available at http://www.ncbi.nlm.nih.gov/ pubmed/9675476. [PubMed: 9675476]

51. McIntosh K, Cooper E, Xu J, et al. Toxicity and efficacy of daily vs. weekly dapsone for prevention of Pneumocystis carinii pneumonia in children infected with human immunodeficiency virus. ACTG 179 Study Team. AIDS Clinical Trials Group. Pediatr Infect Dis J. 1999 May; 18(5): 432-439. Available at http://www.ncbi.nlm.nih.gov/pubmed/10353516. [PubMed: 10353516]

52. Principi N, Marchisio P, Onorato J, et al. Long-term administration of aerosolized pentamidine as primary prophylaxis against Pneumocystis carinii pneumonia in infants and children with symptomatic human immunodeficiency virus infection. The Italian Pediatric Collaborative Study Group on Pentamidine. J Acquir Immune Defic Syndr Hum Retrovirol. 1996 Jun 1; 12(2):158163. Available at http://www.ncbi.nlm.nih.gov/pubmed/8680887. [PubMed: 8680887]

53. Schurmann D, Bergmann F, Albrecht $\mathrm{H}$, et al. Twice-weekly pyrimethamine-sulfadoxine effectively prevents Pneumocystis carinii pneumonia relapse and toxoplasmic encephalitis in patients with AIDS. J Infect. 2001 Jan; 42(1):8-15. Available at http://www.ncbi.nlm.nih.gov/ pubmed/11243747. [PubMed: 11243747]

54. Kim SY, Dabb AA, Glenn DJ, Snyder KM, Chuk MK, Loeb DM. Intravenous pentamidine is effective as second line Pneumocystis pneumonia prophylaxis in pediatric oncology patients. Pediatr Blood Cancer. 2008 Apr; 50(4):779-783. Available at http://www.ncbi.nlm.nih.gov/ pubmed/17635000. [PubMed: 17635000]

55. Nachman S, Gona P, Dankner W, et al. The rate of serious bacterial infections among HIVinfected children with immune reconstitution who have discontinued opportunistic infection prophylaxis. Pediatrics. 2005 Apr; 115(4):e488-e494. Available at http://www.ncbi.nlm.nih.gov/ pubmed/15772172. [PubMed: 15772172]

56. Furrer H, Egger M, Opravil M, et al. Discontinuation of primary prophylaxis against Pneumocystis carinii pneumonia in HIV-1-infected adults treated with combination antiretroviral therapy. Swiss HIV Cohort Study. N Engl J Med. 1999 Apr 29; 340(17):1301-1306. Available at http:// www.ncbi.nlm.nih.gov/pubmed/10219064. [PubMed: 10219064]

57. Schneider MM, Borleffs JC, Stolk RP, Jaspers CA, Hoepelman AI. Discontinuation of prophylaxis for Pneumocystis carinii pneumonia in HIV-1-infected patients treated with highly active antiretroviral therapy. Lancet. 1999 Jan 16; 353(9148):201-203. Available at http:// www.ncbi.nlm.nih.gov/pubmed/9923876. [PubMed: 9923876]

58. Dworkin MS, Hanson DL, Kaplan JE, Jones JL, Ward JW. Risk for preventable opportunistic infections in persons with AIDS after antiretroviral therapy increases CD4+ T lymphocyte counts above prophylaxis thresholds. J Infect Dis. 2000 Aug; 182(2):611-615. Available at http:// www.ncbi.nlm.nih.gov/pubmed/10915098. [PubMed: 10915098]

59. Ledergerber B, Mocroft A, Reiss P, et al. Discontinuation of secondary prophylaxis against Pneumocystis carinii pneumonia in patients with HIV infection who have a response to antiretroviral therapy. Eight European Study Groups. N Engl J Med. 2001 Jan 18; 344(3):168-174. Available at http://www.ncbi.nlm.nih.gov/pubmed/11188837. [PubMed: 11188837]

60. Lopez Bernaldo de Quiros JC, Miro JM, Pena JM, et al. A randomized trial of the discontinuation of primary and secondary prophylaxis against Pneumocystis carinii pneumonia after highly active 
antiretroviral therapy in patients with HIV infection. Grupo de Estudio del SIDA 04/98. N Engl J Med. 2001 Jan 18; 344(3):159-167. Available at http://www.ncbi.nlm.nih.gov/pubmed/11172138. [PubMed: 11172138]

61. Urschel S, Ramos J, Mellado M, et al. Withdrawal of Pneumocystis jirovecii prophylaxis in HIVinfected children under highly active antiretroviral therapy. AIDS. 2005 Dec 2; 19(18):2103-2108. Available at http://www.ncbi.nlm.nih.gov/pubmed/16284459. [PubMed: 16284459]

62. Panel on Opportunistic Infections in HIV-Infected Adults and Adolescents. Guidelines for the prevention and treatment of opportunistic infections in HIV-infected adults and adolescents: Recommendations from the Centers for Disease Control and Prevention, the National Institutes of Health, and the HIV Medicine Association of the Infectious Diseases Society of America. Available at http://aidsinfo.nih.gov/contentfiles/lvguidelines/adult_oi.pdf.

63. Hughes W, Leoung G, Kramer F, et al. Comparison of atovaquone (566C80) with trimethoprimsulfamethoxazole to treat Pneumocystis carinii pneumonia in patients with AIDS. N Engl J Med. 1993 May 27; 328(21):1521-1527. Available at http://www.ncbi.nlm.nih.gov/pubmed/8479489. [PubMed: 8479489]

64. Safrin S, Finkelstein DM, Feinberg J, et al. Comparison of three regimens for treatment of mild to moderate Pneumocystis carinii pneumonia in patients with AIDS. A double-blind, randomized, trial of oral trimethoprim-sulfamethoxazole, dapsone-trimethoprim, and clindamycin-primaquine. ACTG 108 Study Group. Ann Intern Med. 1996 May 1; 124(9):792-802. Available at http:// www.ncbi.nlm.nih.gov/pubmed/8610948. [PubMed: 8610948]

65. Zar HJ, Langdon G, Apolles P, Eley B, Hussey G, Smith P. Oral trimethoprim-sulphamethoxazole levels in stable HIV-infected children. S Afr Med J. 2006 Jul; 96(7):627-629. Available at http:// www.ncbi.nlm.nih.gov/pubmed/16909188. [PubMed: 16909188]

66. Ivady G, Paldy L. Treatment of Pneumocystis carinii pneumonia in infancy. Natl Cancer Inst Monogr. 1976; 43:201-209. [PubMed: 1087956]

67. Pearson RD, Hewlett EL. Pentamidine for the treatment of Pneumocystis carinii pneumonia and other protozoal diseases. Ann Intern Med. 1985 Nov; 103(5):782-786. Available at http:// www.ncbi.nlm.nih.gov/pubmed/3901852. [PubMed: 3901852]

68. Walzer, PD. Isselbacher, KJ.; Martin EB, JB.; Fauci, AS.; Wilson , JD.; Kasper, DL. Harrison's Principles of Internal Medicine. New York, NY: McGraw-Hill; 1994. Pneumocystis carinii pneumonia; p. 908-910.

69. Dohn MN, Weinberg WG, Torres RA, et al. Oral atovaquone compared with intravenous pentamidine for Pneumocystis carinii pneumonia in patients with AIDS. Atovaquone Study Group. Ann Intern Med. 1994 Aug 1; 121(3):174-180. Available at http://www.ncbi.nlm.nih.gov/ pubmed/7880228. [PubMed: 7880228]

70. Falloon J, Sargent S, Piscitelli SC, et al. Atovaquone suspension in HIV-infected volunteers: pharmacokinetics, pharmacodynamics, and TMP-SMX interaction study. Pharmacotherapy. 1999 Sep; 19(9):1050-1056. Available at http://www.ncbi.nlm.nih.gov/pubmed/10610011. [PubMed: $10610011]$

71. Rosenberg DM, McCarthy W, Slavinsky J, et al. Atovaquone suspension for treatment of Pneumocystis carinii pneumonia in HIV-infected patients. AIDS. 2001 Jan 26; 15(2):211-214. Available at http://www.ncbi.nlm.nih.gov/pubmed/11216929. [PubMed: 11216929]

72. Leoung GS, Mills J, Hopewell PC, Hughes W, Wofsy C. Dapsone-trimethoprim for Pneumocystis carinii pneumonia in the acquired immunodeficiency syndrome. Ann Intern Med. $1986 \mathrm{Jul}$; 105(1):45-48. Available at http://www.ncbi.nlm.nih.gov/pubmed/2940954. [PubMed: 2940954]

73. Mirochnick M, Cooper E, McIntosh K, et al. Pharmacokinetics of dapsone administered daily and weekly in human immunodeficiency virus-infected children. Antimicrob Agents Chemother. 1999 Nov; 43(11):2586-2591. Available at http://www.ncbi.nlm.nih.gov/pubmed/10543733. [PubMed: 10543733]

74. Mills J, Leoung G, Medina I, Hopewell PC, Hughes WT, Wofsy C. Dapsone treatment of Pneumocystis carinii pneumonia in the acquired immunodeficiency syndrome. Antimicrob Agents Chemother. 1988 Jul; 32(7):1057-1060. Available at http://www.ncbi.nlm.nih.gov/pubmed/ 3263834. [PubMed: 3263834]

75. Bozzette SA, Sattler FR, Chiu J, et al. A controlled trial of early adjunctive treatment with corticosteroids for Pneumocystis carinii pneumonia in the acquired immunodeficiency syndrome. 
California Collaborative Treatment Group. N Engl J Med. 1990 Nov 22; 323(21):1451-1457. Available at http://www.ncbi.nlm.nih.gov/pubmed/2233917. [PubMed: 2233917]

76. Montaner JS, Lawson LM, Levitt N, Belzberg A, Schechter MT, Ruedy J. Corticosteroids prevent early deterioration in patients with moderately severe Pneumocystis carinii pneumonia and the acquired immunodeficiency syndrome (AIDS). Ann Intern Med. 1990 Jul 1; 113(1):14-20. Available at http://www.ncbi.nlm.nih.gov/pubmed/2190515. [PubMed: 2190515]

77. Nielsen TL, Eeftinck Schattenkerk JK, Jensen BN, et al. Adjunctive corticosteroid therapy for Pneumocystis carinii pneumonia in AIDS: a randomized European multicenter open label study. $\mathrm{J}$ Acquir Immune Defic Syndr. 1992; 5(7):726-731. Available at http://www.ncbi.nlm.nih.gov/ pubmed/1613673. [PubMed: 1613673]

78. Gallant JE, Chaisson RE, Moore RD. The effect of adjunctive corticosteroids for the treatment of Pneumocystis carinii pneumonia on mortality and subsequent complications. Chest. 1998 Nov; 114(5):1258-1263. Available at http://www.ncbi.nlm.nih.gov/pubmed/9823998. [PubMed: 9823998]

79. Briel M, Bucher HC, Boscacci R, Furrer H. Adjunctive corticosteroids for Pneumocystis jiroveci pneumonia in patients with HIV-infection. Cochrane Database Syst Rev. 2006; 3 CD006150(3):CD006150. Available at http://www.ncbi.nlm.nih.gov/pubmed/16856118.

80. Bye MR, Cairns-Bazarian AM, Ewig JM. Markedly reduced mortality associated with corticosteroid therapy of Pneumocystis carinii pneumonia in children with acquired immunodeficiency syndrome. Arch Pediatr Adolesc Med. 1994 Jun; 148(6):638-641. Available at http://www.ncbi.nlm.nih.gov/pubmed/8193693. [PubMed: 8193693]

81. Sleasman JW, Hemenway C, Klein AS, Barrett DJ. Corticosteroids improve survival of children with AIDS and Pneumocystis carinii pneumonia. Am J Dis Child. 1993 Jan; 147(1):30-34. Available at http://www.ncbi.nlm.nih.gov/pubmed/8093422. [PubMed: 8093422]

82. McLaughlin GE, Virdee SS, Schleien CL, Holzman BH, Scott GB. Effect of corticosteroids on survival of children with acquired immunodeficiency syndrome and Pneumocystis carinii-related respiratory failure. J Pediatr. 1995 May; 126(5 Pt 1):821-824. Available at http:// www.ncbi.nlm.nih.gov/pubmed/7752016. [PubMed: 7752016]

83. Creery WD, Hashmi A, Hutchison JS, Singh RN. Surfactant therapy improves pulmonary function in infants with Pneumocystis carinii pneumonia and acquired immunodeficiency syndrome. Pediatr Pulmonol. 1997 Nov; 24(5):370-373. Available at http://www.ncbi.nlm.nih.gov/pubmed/ 9407571. [PubMed: 9407571]

84. Marriage SC, Underhill H, Nadel S. Use of natural surfactant in an HIV-infected infant with Pneumocystis carinii pneumonia. Intensive Care Med. 1996 Jun; 22(6):611-612. Available at http://www.ncbi.nlm.nih.gov/pubmed/8814483. [PubMed: 8814483]

85. Slater AJ, Nichani SH, Macrae D, Wilkinson KA, Novelli V, Tasker RC. Surfactant adjunctive therapy for Pneumocystis carinii pneumonitis in an infant with acute lymphoblastic leukaemia. Intensive Care Med. 1995 Mar; 21(3):261-263. Available at http://www.ncbi.nlm.nih.gov/ pubmed/7790617. [PubMed: 7790617]

86. Schmidt R, Markart P, Ruppert C, et al. Pulmonary surfactant in patients with Pneumocystis pneumonia and acquired immunodeficiency syndrome. Crit Care Med. 2006 Sep; 34(9):23702376. Available at http://www.ncbi.nlm.nih.gov/pubmed/16849999. [PubMed: 16849999]

87. Datta D, Ali SA, Henken EM, Kellet H, Brown S, Metersky ML. Pneumocystis carinii pneumonia: the time course of clinical and radiographic improvement. Chest. 2003 Nov; 124(5):1820-1823. Available at http://www.ncbi.nlm.nih.gov/pubmed/14605054. [PubMed: 14605054]

88. Ratnam I, Chiu C, Kandala NB, Easterbrook PJ. Incidence and risk factors for immune reconstitution inflammatory syndrome in an ethnically diverse HIV type 1-infected cohort. Clin Infect Dis. 2006 Feb 1; 42(3):418-427. Available at http://www.ncbi.nlm.nih.gov/pubmed/ 16392092. [PubMed: 16392092]

89. Gutman LT. The use of trimethoprim-sulfamethoxazole in children: a review of adverse reactions and indications. Pediatr Infect Dis. 1984 Jul-Aug;3(4):349-357. Available at http:// www.ncbi.nlm.nih.gov/pubmed/6473140. [PubMed: 6473140]

90. Rieder MJ, King SM, Read S. Adverse reactions to trimethoprim-sulfamethoxazole among children with human immunodeficiency virus infection. Pediatr Infect Dis J. 1997 Nov; 16(11): 1028-1031. Available at http://www.ncbi.nlm.nih.gov/pubmed/9384334. [PubMed: 9384334] 
91. Para MF, Finkelstein D, Becker S, Dohn M, Walawander A, Black JR. Reduced toxicity with gradual initiation of trimethoprim-sulfamethoxazole as primary prophylaxis for Pneumocystis carinii pneumonia: AIDS Clinical Trials Group 268. J Acquir Immune Defic Syndr. 2000 Aug 1; 24(4):337-343. Available at http://www.ncbi.nlm.nih.gov/pubmed/11015150. [PubMed: 11015150]

92. Leoung GS, Stanford JF, Giordano MF, et al. Trimethoprim-sulfamethoxazole (TMP-SMZ) dose escalation versus direct rechallenge for Pneumocystis Carinii pneumonia prophylaxis in human immunodeficiency virus-infected patients with previous adverse reaction to TMP-SMZ. J Infect Dis. 2001 Oct 15; 184(8):992-997. Available at http://www.ncbi.nlm.nih.gov/pubmed/11574913. [PubMed: 11574913]

93. Goodwin SD. Pneumocystis carinii pneumonia in human immunodeficiency virus-infected infants and children. Pharmacotherapy. 1993 Nov-Dec;13(6):640-646. Available at http:// www.ncbi.nlm.nih.gov/pubmed/8302691. [PubMed: 8302691]

94. Gigliotti, F.; Wright, TW. Pneumocystosis. In: Rinaldi, DRHaMG., editor. Infectious Disease: Diagnosis and Treatment of Human Mycoses. Totowa, NJ: Humana Press Inc; 2008. p. 245-254.

95. Miller RF, Le Noury J, Corbett EL, Felton JM, De Cock KM. Pneumocystis carinii infection: current treatment and prevention. J Antimicrob Chemother. 1996 May; 37(Suppl B):33-53. Available at http://www.ncbi.nlm.nih.gov/pubmed/8818828. [PubMed: 8818828]

96. Mussini C, Pezzotti P, Antinori A, et al. Discontinuation of secondary prophylaxis for Pneumocystis carinii pneumonia in human immunodeficiency virus-infected patients: a randomized trial by the CIOP Study Group. Clin Infect Dis. 2003 Mar 1; 36(5):645-651. Available at http://www.ncbi.nlm.nih.gov/pubmed/12594647. [PubMed: 12594647]

\section{Dosing Recommendations for Prevention and Treatment of Pneumocystis Pneumonia}

\begin{tabular}{|c|c|c|c|}
\hline Indication & First Choice & Alternative & Comments/Special Issues \\
\hline Primary Prophylaxis & $\begin{array}{l}\text { - TMP-SMX } \\
\text { (Cotrimoxazole): TMP } \\
\text { 2.5-5 mg/kg body } \\
\text { weight/dose with SMX } \\
\text { 12.5-25 mg/kg body } \\
\text { weight/dose twice per } \\
\text { day. Dosing based on } \\
\text { TMP component. } \\
\text { The total daily dose } \\
\text { should not exceed } 320 \\
\text { mg TMP and } 1600 \mathrm{mg} \\
\text { SMX. Several dosing } \\
\text { schemes have been } \\
\text { used successfully- } \\
\text { - } \quad \text { Given } 3 \\
\text { days per } \\
\text { week on } \\
\text { consecutive } \\
\text { days or on } \\
\text { alternate } \\
\text { days } \\
\text { - } \quad \text { Given } 2 \\
\text { days per } \\
\text { week on } \\
\text { consecutive } \\
\text { days or on } \\
\text { alternate } \\
\text { days } \\
\text { Given } \\
\text { every day } \\
\text { (total daily } \\
\text { dose of } \\
\text { TMP 5-10 } \\
\text { mg/kg } \\
\text { body } \\
\text { weight }\end{array}$ & 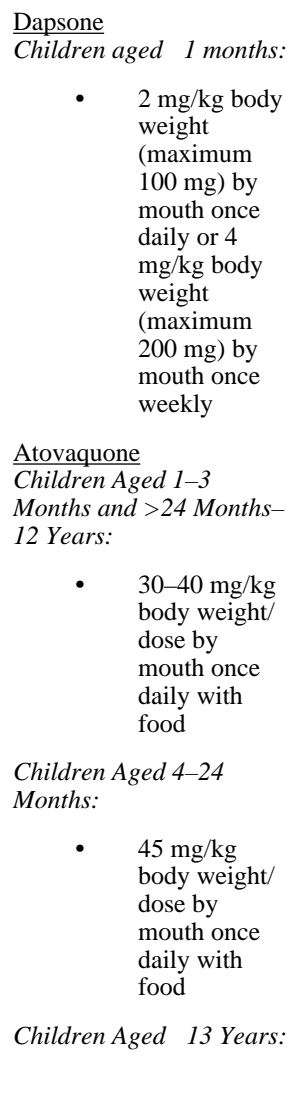 & 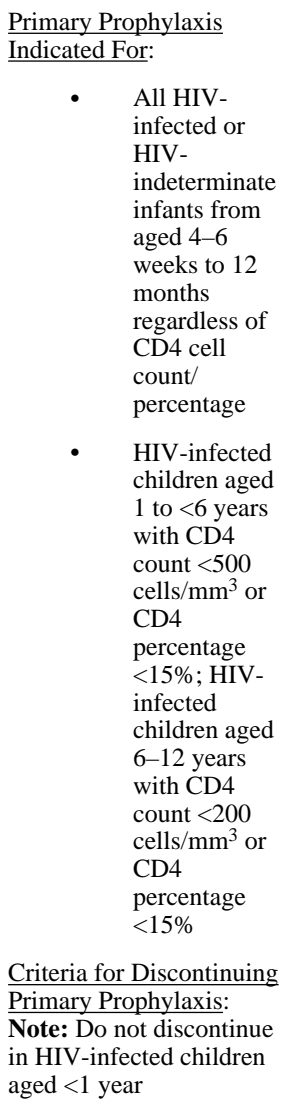 \\
\hline
\end{tabular}




\begin{tabular}{|c|c|c|c|}
\hline Indication & First Choice & Alternative & Comments/Special Issues \\
\hline & $\begin{array}{l}\text { given as a } \\
\text { single dose } \\
\text { each day) }\end{array}$ & $\begin{array}{cl}\bullet & \begin{array}{l}1500 \mathrm{mg}(10 \\
\text { cc oral yellow } \\
\text { suspension) } \\
\text { per dose by } \\
\text { mouth once } \\
\text { daily }\end{array} \\
\text { Aerosolized Pentamidine } \\
\text { Children Aged } \geq 5 \text { Years: } \\
\text { 300 mg every } \\
\text { month via } \\
\text { Respirgard } \\
\text { II'T nebulizer } \\
\text { (manufactured } \\
\text { by Marquest; } \\
\text { Englewood, } \\
\text { Colorado) }\end{array}$ & 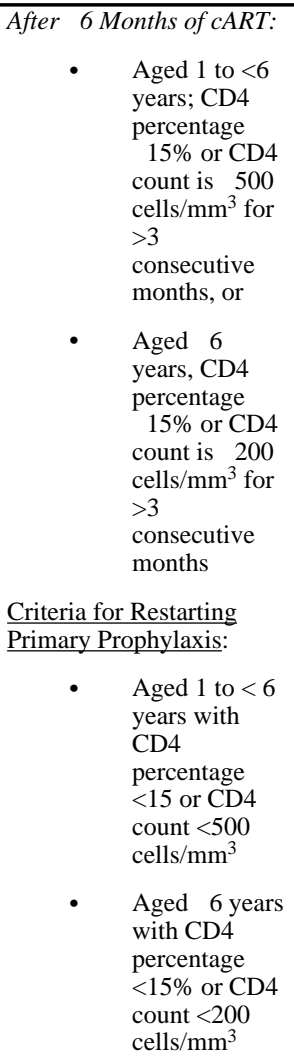 \\
\hline $\begin{array}{l}\text { Secondary Prophylaxis } \\
\text { Prior PCP }\end{array}$ & Same as for primary prophylaxis. & $\begin{array}{l}\text { Same as for primary } \\
\text { prophylaxis. }\end{array}$ & $\begin{array}{l}\frac{\text { Secondary Prophylaxis }}{\text { Indicated For: }} \\
\text { - } \begin{array}{l}\text { Children with } \\
\text { prior episode } \\
\text { of PCP }\end{array} \\
\text { Criteria for Discontinuing } \\
\text { Secondary Prophylaxis: } \\
\begin{array}{l}\text { Same as for } \\
\text { primary } \\
\text { prophylaxis }\end{array} \\
\text { Criteria for Restarting } \\
\text { Secondary Prophylaxis: } \\
\begin{array}{l}\text { Same as for } \\
\text { primary } \\
\text { prophylaxis }\end{array}\end{array}$ \\
\hline Treatment & $\begin{array}{l}\text { TMP-SMX } 3.75-5 \mathrm{mg} / \mathrm{kg} \text { body } \\
\text { weight/dose TMP (based on TMP } \\
\text { component) every } 6 \text { hours IV or } \\
\text { orally given for } 21 \text { days (followed } \\
\text { by secondary prophylaxis dosing) }\end{array}$ & $\begin{array}{l}\frac{\text { If TMP-SMX-Intolerant }}{\text { or Clinical Treatment }} \\
\frac{\text { Failure After 5-7 Days of }}{\text { TMP-SMX Therapy }} \\
\text { Pentamidine: } \\
\text { - } \quad 4 \mathrm{mg} / \mathrm{kg} \text { body } \\
\text { weight/dose } \\
\text { IV/IM once } \\
\text { daily is the } \\
\text { first choice } \\
\text { alternative } \\
\text { regimen. }\end{array}$ & $\begin{array}{l}\text { After acute pneumonitis } \\
\text { resolved in mild-moderate } \\
\text { disease, IV TMP-SMX } \\
\text { can be changed to oral. } \\
\text { For oral administration, } \\
\text { total daily dose of TMP- } \\
\text { SMX can also be } \\
\text { administered in } 3 \text { divided } \\
\text { doses (every } 8 \text { hours). } \\
\text { Dapsone } 2 \mathrm{mg} / \mathrm{kg} \text { body } \\
\text { weight by mouth once } \\
\text { daily (maximum } 100 \mathrm{mg} / \\
\text { day) plus trimethoprim } 5 \\
\text { mg/kg body weight by }\end{array}$ \\
\hline
\end{tabular}

Pediatr Infect Dis J. Author manuscript; available in PMC 2014 November 01. 


\begin{tabular}{|c|c|c|c|}
\hline Indication & First Choice & Alternative & Comments/Special Issues \\
\hline & & $\begin{array}{l}\text { Note: } \\
\text { Pentamidine } \\
\text { can be } \\
\text { changed to } \\
\text { atovaquone } \\
\text { after } 7-10 \\
\text { days IV } \\
\text { therapy. } \\
\text { Atovaquone } \\
\text { Daily Dosing: } \\
\text { - Children aged } \\
1-3 \text { months } \\
\text { and }>24 \\
\text { months-12 } \\
\text { years: } 30-40 \\
\text { mg/kg body } \\
\text { weight/dose } \\
\text { by mouth } \\
\text { once daily } \\
\text { with food } \\
\text { Children aged } \\
4-24 \text { months: } \\
45 \text { mg/kg } \\
\text { body weight/ } \\
\text { dose by } \\
\text { mouth once } \\
\text { daily with } \\
\text { food } \\
\\
\text { Twice-Daily Dosing }\end{array}$ & $\begin{array}{l}\text { mouth every } 8 \text { hours has } \\
\text { been used in adults but } \\
\text { data in children are } \\
\text { limited. } \\
\text { Primaquine base } 0.3 \\
\text { mg/kg body weight by } \\
\text { mouth once daily } \\
\text { (maximum } 30 \mathrm{mg} / \mathrm{day}) \\
\text { plus clindamycin } 10 \\
\mathrm{mg} / \mathrm{kg} \text { body weight/dose } \\
\text { IV or by mouth } \\
\text { (maximum } 600 \mathrm{mg} \text { given } \\
\text { IV and } 300-450 \mathrm{mg} \text { given } \\
\text { orally) every } 6 \text { hours has } \\
\text { been used in adults, but } \\
\text { data in children are not } \\
\text { available. } \\
\text { Indications for } \\
\text { Corticosteroids: } \\
\end{array}$ \\
\hline
\end{tabular}




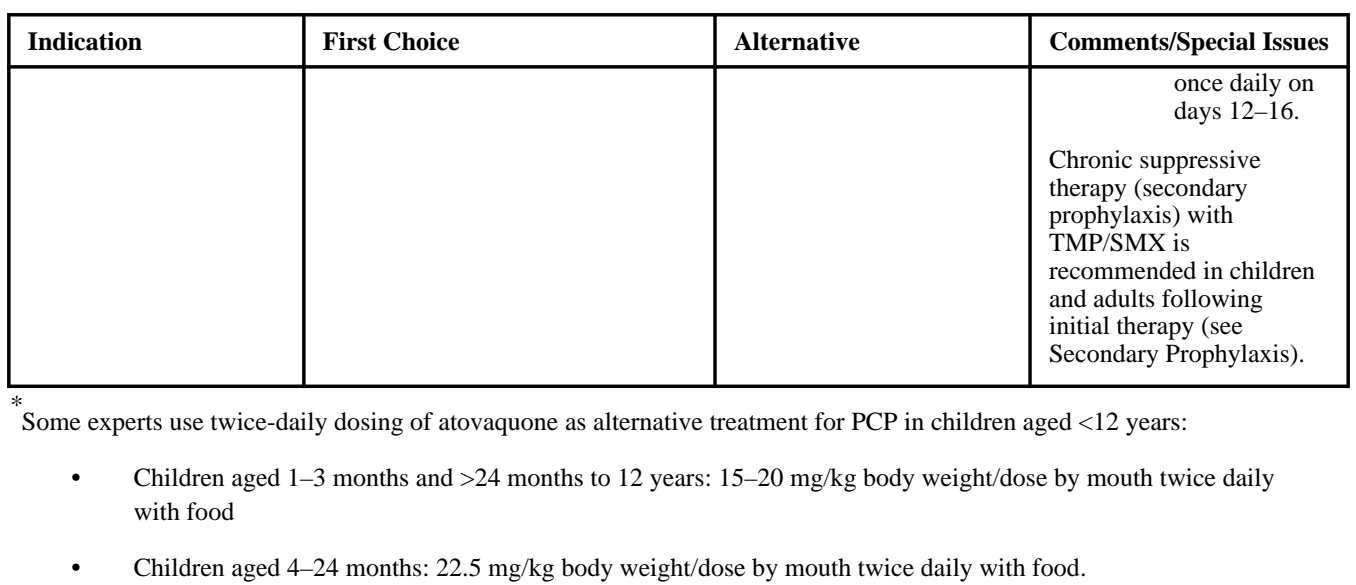

Key to Acronyms: cART = combination antiretroviral therapy; CD4 = CD4 T lymphocyte cell; IM = intramuscular; IV = intravenous; $\mathrm{PCP}=$ Pneumocystis jirovecii pneumonia; TMP-SMX = trimethoprim-sulfamethoxazole

Note: Information included in these guidelines might not represent Food and Drug Administration (FDA) approval or approved labeling for products or indications. Specifically, the terms safe and effective might not be synonymous with the FDA-defined legal standards for product approval.

\section{Progressive Multifocal Leukoencephalopathy (Last updated November 6, 2013; last reviewed November 6, 2013)}

Panel's Recommendations

- The main approach to treatment of Progressive Multifocal Leukoencephalopathy (PML) is treatment with an effective antiretroviral regimen that suppresses HIV viremia and preserves or restores CD4 Tlymphocyte (CD4) cell-defined immune function (AII).

- Intrathecal cytosine arabinoside and cidofovir are not routinely recommended for treatment of PML (BIII).

- Immunomodulatory approaches, such as interferon alfa, are not routinely recommended for treatment of PML (BIII).

Rating of Recommendations: $\mathrm{A}=$ Strong; $\mathrm{B}=$ Moderate $\mathrm{C}=$ Optional

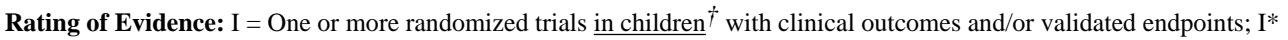
= One or more randomized trials in adults with clinical outcomes and/or validated laboratory endpoints with accompanying data in children ${ }^{\dagger}$ from one or more well-designed, nonrandomized trials or observational cohort studies with long-term clinical outcomes; II = One or more well-designed, nonrandomized trials or observational cohort studies in children ${ }^{\dagger}$ with long-term outcomes; II* $=$ One or more well-designed, nonrandomized trials or observational studies in adults with longterm clinical outcomes with accompanying data in children ${ }^{\dagger}$ from one or more similar nonrandomized trials or cohort studies with clinical outcome data; III = Expert opinion

${ }^{\dagger}$ Studies that include children or children/adolescents, but not studies limited to post-pubertal adolescents

\section{Epidemiology}

First described in association with disorders of B-cell function, such as chronic lymphocytic leukemia and Hodgkin disease, progressive multifocal leukoencephalopathy (PML) is a rare demyelinating disease of the central nervous system (CNS) that occurs in immunocompromised patients. ${ }^{1}$ In HIV-infected adults, CD4 T lymphocyte (CD4 cell) counts less than 100 cells $/ \mathrm{mm}^{3}$ are associated with development of PML, and persistence of CD4 counts less than 50 to 100 cells $/ \mathrm{mm}^{3}$ are associated with fatal PML. Not all patients with PML have severe immune dysfunction, however, and PML has been reported in HIV- 
infected patients with high CD4 counts who are receiving successful combination antiretroviral therapy (cART).

PML is caused by JC virus (JCV), a ubiquitous polyomavirus, named using the initials of the patient, John Cunningham, from whom it was first isolated. Most humans are infected with JCV early in life; in a seroepidemiology study, $50 \%$ of Swedish children were seropositive for JCV by ages 9 to 11 years, and $72 \%$ of adult women aged $\geq 25$ years in the Finnish Maternity Cohort were JCV seropositive. ${ }^{2}$ The exact mode of transmission of JCV between individuals is unknown. Because the virus is commonly detected in urine, JCV has been detected in sewage effluent. It is also detectable in peripheral blood mononuclear cells of both healthy and immunocompromised individuals. Vertical transmission from mother to newborn also has been documented. ${ }^{3,4}$ Lymphocytes, renal tubular epithelium, bone marrow, and possibly spleen and lymphoid tissue likely represent sites of viral latency, and lymphocytes also may be a vehicle for spread of the virus to other organ systems, including the CNS. ${ }^{5,6}$

The evolution of asymptomatic infection with JCV to symptomatic PML probably involves a series of events that are both virologic and immunologic. The original infecting strain of $\mathrm{JCV}$ - the strain that is commonly detected in urine and blood—-mutates and alters a regulatory gene through rearrangement of a non-coding region (at-NCCR to rr-NCCR) to become a neurotropic strain of JCV capable of replicating in neuronal glial cells. ${ }^{7}$ Failed immune surveillance allows replicating virus to persist in peripheral blood cells and serum. If the neurotropic form of JCV gains entry into the brain, it can then establish a productive infection in oligodendrocyte cells, which leads to PML in the absence of proper CNS immune surveillance. ${ }^{8}$ Serotonin receptor 5-HT(2a) appears important for JCV infection of brain glial cells. ${ }^{9}$ Recently, in HIV-uninfected adults, an increased incidence of PML has been associated with use of therapeutic monoclonal antibodies, including natalizumab (an alpha 4 beta 1 and alpha 4 beta 7 antagonist that targets activated lymphocytes), efalizumab (an anti CD-11a antibody that targets T-lymphocytes), rituximab (an anti CD-20 antibody that targets B-lymphocytes), and alemtuzumab (an anti-CD52 antibody that depletes both T and $\mathrm{B}$ cells). ${ }^{8,10-12}$

PML is an AIDS-defining illness in HIV-infected individuals. It has rarely been seen in reports from large series of HIV-infected children, ${ }^{13-15}$ but cases have been reported in children with a wide range of ages and a broad geographical distribution. ${ }^{16-22}$ The incidence of PML has decreased from 3.3 cases per 1000 person-years at risk during the era before cART, to 1.3 cases per 1000 person-years after the introduction of $\mathrm{cART} .{ }^{23}$ During the precART era, survival was extremely poor in adults and children with PML. ${ }^{15}$ Survival among adults has improved during the cART era ${ }^{24-26}$ from $10 \%$ to $50 \%$, and mean survival time from time of diagnosis of PML has increased from 0.4 years to 1.8 years. ${ }^{27}$ No comparable data exist for children.

\section{Clinical Manifestations}

No symptoms are known to be associated with acute or latent JCV infection. Asymptomatic urinary shedding is common. PML is the primary disease caused by JCV and clinical manifestations in children are similar to those in adults. The disease has an insidious onset 
and produces a neurologic syndrome that steadily progresses over weeks or months, characterized by confusion, disorientation, lack of energy, loss of balance, cognitive dysfunction, dementia, seizures, ataxia, aphasia, cranial nerve deficits, visual abnormalities (blurred or double vision or loss of vision), hemiparesis or quadriparesis, and eventually coma.

Demyelination is at first patchy, involving subcortical regions, and then spreads to deep white matter in a confluent pattern; thus, PML initially may present with focal neurologic deficits that involve different brain regions.

\section{Diagnosis}

The established criteria for clinical diagnosis are focal signs and symptoms on neurologic examination, focal white matter lesions on magnetic resonance imaging (MRI) or computerized tomography (CT) without mass effect, and exclusion of other causes of the clinical and neuroradiologic findings. ${ }^{28} \mathrm{~A}$ confirmed diagnosis of PML requires a compatible clinical syndrome and radiographic findings, coupled with brain biopsy demonstrating a characteristic triad of pathologic foci of demyelination, enlarged hyperchromatic oligodendrocytes with enlarged nuclei and basophilic-staining intranuclear material, and enlarged astrocytes with bizarre hyperchromatic nuclei. When only two of these features are present, JCV can be demonstrated by in situ hybridization or by electron microscopy for definitive diagnosis.

Brain biopsy remains the gold standard confirmatory test for diagnosis of PML, but brain imaging with MRI or CT can reveal characteristic lesions. The radiologic features of PML are typically non-inflammatory (unless associated with immune reconstitution inflammatory syndrome [IRIS] related to initiation of cART). Typical CT abnormalities include single or multiple hypodense, non-enhancing cerebral white matter lesions; cerebellum and brain stem occasionally are involved. MRI may be more sensitive for detecting changes in the brain associated with PML, and may be positive before JCV DNA is detected in the cerebrospinal fluid (CSF). MRI depicts white matter lesions of low T1 signal intensity and high proton density on T2-weighted images with absence of edema or mass effect. Post-contrast enhancement is unusual, and when present, usually is sparse, with a thin or reticulated appearance adjacent to the edge of the lesions.

PML diagnosis is now facilitated by use of a polymerase chain reaction (PCR) assay to detect JCV DNA in CSF, which may obviate the need for brain biopsy in patients with a compatible clinical syndrome and radiographic findings. Nested JCV DNA PCR on CSF is highly sensitive (90\%-100\%) and specific (92\%-100\%) for PML in adults, and in the absence of comparative data for children, similar performance characteristics are anticipated but not proven in that population..$^{29}$ False-negative tests occur, however, and PML may be present and diagnosed by brain biopsy in patients with a negative JCV DNA PCR test in the CSF. Measurement of JCV DNA levels in CSF samples can be a useful virologic marker for managing PML in patients receiving cART. ${ }^{30} \mathrm{With}$ the advent of multiple modalities to support PML diagnosis, diagnostic criteria can be stratified according to the following terminology and levels of certainty of diagnosis: 
- Biopsy-confirmed PML: JCV antigens detected by immunohistochemistry, JCV DNA detected by in situ nucleic acid hybridization, or JC virions detected by electron microscopy in brain tissue obtained by cerebral biopsy, associated with typical histology, in patients with typical clinical and radiological findings

- Laboratory-confirmed PML: JCV DNA detected by PCR of CSF in patients with typical, clinical, and radiological findings (detection of intrathecal antibody production may also support the diagnosis)

- Possible PML: Patients with typical clinical and radiological findings, without virologic or histologic confirmation in brain tissue or CSF. ${ }^{31,32}$

Presence of antibodies to JCV in the serum or presence of JCV DNA in the blood or urine of patients does not establish the diagnosis of PML because these studies can be positive in individuals without PML. Conversely, while most patients with JCV-associated PML have moderate to high anti-JCV antibodies and JCV DNA in their peripheral blood, serum, and CSF, some patients with PML diagnosed by brain biopsy will not have detectable anti-JCV antibody or JCV DNA in their blood or CSF. Most patients with JCV-associated PML, however, have moderate to high anti-JCV antibodies and JCV DNA in their peripheral blood, serum, and CSF.

\section{Prevention Recommendations \\ Preventing Exposure-There is no known way to prevent exposure to JCV.}

Preventing First Episode of Disease-Use of cART can prevent or reverse the severe immunosuppression that increases the risk of PML. Incidence of PML has decreased in the cART era. There are no means of preventing PML in severely immunosuppressed individuals.

Discontinuing Primary Prophylaxis-No means of primary prophylaxis of JCV infection or development of PML have been demonstrated.

\section{Treatment Recommendations}

Treating Disease-No effective specific therapy has been established for JCV infection or PML. Survival in HIV-infected adults with PML has substantially improved during the post-cART era, with an increase in median survival from 14 to 64 weeks. ${ }^{27,33}$ A CD4 count $>100$ cells $/ \mathrm{mm}^{3}$ at PML diagnosis is associated with improved survival, and use of cART after diagnosis of PML is strongly associated with improved survival. ${ }^{33}$ Thus, the main approach to treatment involves optimizing cART to reverse the immunosuppression that interferes with normal host response to this virus (AII).

A number of agents have been proposed or reported anecdotally as more specific treatments for PML, but none has proven effective after greater scrutiny or more extensive study. In a randomized, open-label trial of intravenous (IV) and intrathecal cytosine arabinoside ${ }^{34}$ and a non-randomized, open-label trial of IV cidofovir, ${ }^{35}$ neither drug was effective in producing clinical improvement of PML in HIV-infected adults, and neither agent is routinely recommended (BIII). Immunomodulatory approaches such as interferon-alfa (IFN-a) also 
have been described in case reports in HIV-infected adults; however, none have been studied in a controlled clinical trial and, in one analysis, these approaches did not provide any benefit beyond that with cART. ${ }^{36}$ Thus, they are also not routinely recommended (BIII). Anecdotal reports have been published about use of mirtazapine (a 5-HT(2a) receptor antagonist) plus either cidofovir or cytosine-arabinoside, with tapering of immunosuppressive therapy, to treat PML in HIV-uninfected adults who developed the disease while on immunosuppressive therapy. While the results with this adjunctive treatment are encouraging, there is insufficient evidence to recommend it at this time. ${ }^{31,37,38}$ In addition, recent in vitro studies have shown that CMX001, an investigational oral ester form of cidofovir, suppresses JCV replication in human brain cell cultures, and the compound may be evaluated in clinical trials in the near future. ${ }^{39,40}$ No therapeutic trials have been conducted in children.

Monitoring and Adverse Events, Including IRIS-Patients may develop PML before starting cART or may manifest PML as an unmasking IRIS event after immune reconstitution with antiretroviral therapy (ART). Neurologic stability or improvement and prolonged survival are associated with reduced levels of JCV DNA in CSF, appearance of JCV-specific antibody in CSF, and presence of JCV-specific cytotoxic T-cell responses in patients receiving cART. ${ }^{41}$

After cART is initiated and CD4 counts rise, some patients will experience neurologic improvement; however, reports have documented worsening neurologic manifestations after initiation of ART. ${ }^{26}$ Clinical worsening may represent the natural history of PML in these patients. However, this apparent worsening may also be a paradoxical reaction from inflammatory responses to JCV potentiated by cART-induced immune reconstitution, called IRIS, ${ }^{26,42-44}$ examples of which have occurred in children. ${ }^{45}$ The underlying mechanism of cARTassociated PML IRIS is controversial. One hypothesis is that a reduction in inhibitory cytokines (e.g., IFN-a and interleukin-12) after cART promotes JCV re-activation within the brain or increases trafficking of JCV-infected peripheral lymphocytes into the brain. ${ }^{46}$ Another possibility is that JCV infection occurring coincidental to cART initiation results in a beneficial inflammatory response, with lack of disease progression. ${ }^{46}$ This may be particularly likely in cases of perinatal HIV infection, because JCV acquisition is most common early in life. The overall prevalence of PML-associated IRIS in children is unknown. Inflammatory PML should be suspected in cART-treated children with advanced HIV who show acute neurologic deterioration and contrast-enhancing demyelinating lesions on MRI, even if immunological and virological measures show improvement in HIV status. ${ }^{22}$ Retrospective data suggest that early and prolonged treatment with steroids may be beneficial for some patients in whom immune reconstitution with ART activates an inflammatory response to JCV. No clinical trial data exist, however, to substantiate the anecdotal evidence. ${ }^{47}$

Managing Treatment Failure-PML remission with cART may take several weeks, and no criteria exist that define progression of disease. A working definition of treatment failure used for HIV-infected adults is continued clinical worsening and continued detection of CSF JCV DNA at 3 months (see Guidelines for the Prevention and Treatment of Opportunistic 
Infections in HIV-Infected Adults). ${ }^{48}$ In addition, lack of JCV antibody response or JCVspecific cytotoxic T-cell immune responses are associated with poor prognosis. In some patients, PML worsens despite cART, either because of IRIS or because of the natural history of PML. Whichever is the case, cART should be continued. If cART fails to suppress HIV RNA or to increase the CD4 count, then attention should focus on modifying and optimizing the cART (AII). In HIV-infected children responding well to cART but with continued worsening of PML, an expert in pediatric HIV infection should be consulted for consideration of investigational therapies.

Preventing Recurrence-On the basis of its role in reversing the disease, the main measure for preventing PML recurrence is an effective cART regimen that suppresses HIV viremia and preserves or restores CD4-defined immune function (AII).

Discontinuing Secondary Prophylaxis-No methods for secondary prophylaxis of JCV infection or PML have been proven effective.

\section{References}

1. Astrom KE, Mancall EL, Richardson EP Jr. Progressive multifocal leuko-encephalopathy; a hitherto unrecognized complication of chronic lymphatic leukaemia and Hodgkin's disease. Brain. 1958 Mar; 81(1):93-111. Available at http://www.ncbi.nlm.nih.gov/pubmed/13523006. [PubMed: 13523006]

2. Stolt A, Sasnauskas K, Koskela P, Lehtinen M, Dillner J. Seroepidemiology of the human polyomaviruses. J Gen Virol. 2003 Jun; 84(Pt 6):1499-1504. Available at http:// www.ncbi.nlm.nih.gov/pubmed/12771419. [PubMed: 12771419]

3. White MK, Khalili K. Pathogenesis of progressive multifocal leukoencephalopathy-revisited. J Infect Dis. 2011 Mar 1; 203(5):578-586. Available at http://www.ncbi.nlm.nih.gov/pubmed/ 21227915. [PubMed: 21227915]

4. Boldorini R, Allegrini S, Miglio U, et al. Serological evidence of vertical transmission of JC and BK polyomaviruses in humans. J Gen Virol. 2011 May; 92(Pt 5):1044-1050. Available at http:// www.ncbi.nlm.nih.gov/pubmed/21307224. [PubMed: 21307224]

5. Rodrigues C, Pinto D, Medeiros R. Molecular epidemiology characterization of the urinary excretion of polyomavirus in healthy individuals from Portugal-a Southern European population. $\mathrm{J}$ Med Virol. 2007 Aug; 79(8):1194-1198. Available at http://www.ncbi.nlm.nih.gov/pubmed/ 17596822. [PubMed: 17596822]

6. Gu ZY, Li Q, Si YL, Li X, Hao HJ, Song HJ. Prevalence of BK virus and JC virus in peripheral blood leukocytes and normal arterial walls in healthy individuals in China. J Med Virol. 2003 Aug; 70(4):600-605. Available at http://www.ncbi.nlm.nih.gov/pubmed/12794723. [PubMed: 12794723]

7. Gosert R, Kardas P, Major EO, Hirsch HH. Rearranged JC virus noncoding control regions found in progressive multifocal leukoencephalopathy patient samples increase virus early gene expression and replication rate. J Virol. 2010 Oct; 84(20):10448-10456. Available at http:// www.ncbi.nlm.nih.gov/pubmed/20686041. [PubMed: 20686041]

8. Berger JR, Houff SA, Major EO. Monoclonal antibodies and progressive multifocal leukoencephalopathy. MAbs. 2009 Nov-Dec;1(6):583-589. Available at http:// www.ncbi.nlm.nih.gov/pubmed/20073129. [PubMed: 20073129]

9. Focosi D, Kast RE, Maggi F, Ceccherini-Nelli L, Petrini M. Sialic acid moieties and 5-HT2a: two faces of the same receptor for JC virus? J Clin Virol. 2008 Sep; 43(1):132-133. Available at http:// www.ncbi.nlm.nih.gov/pubmed/18534904. [PubMed: 18534904]

10. Carson KR, Focosi D, Major EO, et al. Monoclonal antibody-associated progressive multifocal leucoencephalopathy in patients treated with rituximab, natalizumab, and efalizumab: a Review from the Research on Adverse Drug Events and Reports (RADAR) Project. Lancet Oncol. 2009 
Aug; 10(8):816-824. Available at http://www.ncbi.nlm.nih.gov/pubmed/19647202. [PubMed: 19647202]

11. Major EO. Progressive multifocal leukoencephalopathy in patients on immunomodulatory therapies. Annu Rev Med. 2010; 61:35-47. Available at http://www.ncbi.nlm.nih.gov/pubmed/ 19719397. [PubMed: 19719397]

12. Gea-Banacloche JC. Rituximab-associated infections. Semin Hematol. 2010 Apr; 47(2):187-198. Available at http://www.ncbi.nlm.nih.gov/pubmed/20350666. [PubMed: 20350666]

13. Dankner WM, Lindsey JC, Levin MJ. Pediatric ACTGPT. Correlates of opportunistic infections in children infected with the human immunodeficiency virus managed before highly active antiretroviral therapy. Pediatr Infect Dis J. 2001 Jan; 20(1):40-48. Available at http:// www.ncbi.nlm.nih.gov/pubmed/11176565. [PubMed: 11176565]

14. Nesheim SR, Kapogiannis BG, Soe MM, et al. Trends in opportunistic infections in the pre- and post-highly active antiretroviral therapy eras among HIV-infected children in the Perinatal AIDS Collaborative Transmission Study, 1986-2004. Pediatrics. 2007 Jul; 120(1):100-109. Available at http://www.ncbi.nlm.nih.gov/pubmed/17606567. [PubMed: 17606567]

15. Ciuta ST, Boros S, Napoli PA, Pezzotti P, Rezza G. Predictors of survival in children with acquired immunodeficiency syndrome in Italy, 1983 to 1995. AIDS Patient Care STDS. 1998 Aug; 12(8):629-637. Available at http://www.ncbi.nlm.nih.gov/pubmed/15468435. [PubMed: 15468435]

16. Araujo AP, Pereira HS, Oliveira RH, Frota AC, Esperanca JC, Duarte F. Progressive multifocal leukoencephalopathy in a child with acquired immunodeficiency syndrome (AIDS). Arq Neuropsiquiatr. 1997 Mar; 55(1):122-125. Available at http://www.ncbi.nlm.nih.gov/pubmed/ 9332571. [PubMed: 9332571]

17. Robinson LG, Chiriboga CA, Champion SE, Ainyette I, DiGrado M, Abrams EJ. Progressive multifocal leukoencephalopathy successfully treated with highly active antiretroviral therapy and cidofovir in an adolescent infected with perinatal human immunodeficiency virus (HIV). J Child Neurol. 2004 Jan; 19(1):35-38. Available at http://www.ncbi.nlm.nih.gov/pubmed/15032381. [PubMed: 15032381]

18. Wilmshurst JM, Burgess J, Hartley P, Eley B. Specific neurologic complications of human immunodeficiency virus type 1 (HIV-1) infection in children. J Child Neurol. 2006 Sep; 21(9): 788-794. Available at http://www.ncbi.nlm.nih.gov/pubmed/16970887. [PubMed: 16970887]

19. Shah I, Chudgar P. Progressive multifocal leukoencephalopathy (PML) presenting as intractable dystonia in an HIV-infected child. J Trop Pediatr. 2005 Dec; 51(6):380-382. Available at http:// www.ncbi.nlm.nih.gov/pubmed/15927949. [PubMed: 15927949]

20. Berger JR, Scott G, Albrecht J, Belman AL, Tornatore C, Major EO. Progressive multifocal leukoencephalopathy in HIV-1-infected children. AIDS. 1992 Aug; 6(8):837-841. Available at http://www.ncbi.nlm.nih.gov/pubmed/1418781. [PubMed: 1418781]

21. Liptai Z, Papp E, Barsi P, et al. Progressive multifocal leukoencephalopathy in an HIV-infected child. Neuropediatrics. 2007 Feb; 38(1):32-35. Available at http://www.ncbi.nlm.nih.gov/pubmed/ 17607602. [PubMed: 17607602]

22. Oberdorfer P, Washington CH, Katanyuwong K, Jittamala P. Progressive Multifocal Leukoencephalopathy in HIV-Infected Children: A Case Report and Literature Review. Int J Pediatr. 2009; 2009:348507. Available at http://www.ncbi.nlm.nih.gov/pubmed/20041004. [PubMed: 20041004]

23. Engsig FN, Hansen AB, Omland LH, et al. Incidence, clinical presentation, and outcome of progressive multifocal leukoencephalopathy in HIV-infected patients during the highly active antiretroviral therapy era: a nationwide cohort study. J Infect Dis. 2009 Jan 1; 199(1):77-83. Available at http://www.ncbi.nlm.nih.gov/pubmed/19007313. [PubMed: 19007313]

24. Antinori A, Cingolani A, Lorenzini P, et al. Clinical epidemiology and survival of progressive multifocal leukoencephalopathy in the era of highly active antiretroviral therapy: data from the Italian Registry Investigative Neuro AIDS (IRINA). J Neurovirol. 2003; 9(Suppl 1):47-53. Available at http://www.ncbi.nlm.nih.gov/pubmed/12709872. [PubMed: 12709872]

25. Koralnik IJ. New insights into progressive multifocal leukoencephalopathy. Curr Opin Neurol. 2004 Jun; 17(3):365-370. Available at http://www.ncbi.nlm.nih.gov/pubmed/15167073. [PubMed: 15167073] 
26. Berenguer J, Miralles P, Arrizabalaga J, et al. Clinical course and prognostic factors of progressive multifocal leukoencephalopathy in patients treated with highly active antiretroviral therapy. Clin Infect Dis. 2003 Apr 15; 36(8):1047-1052. Available at http://www.ncbi.nlm.nih.gov/pubmed/ 12684918. [PubMed: 12684918]

27. Lima MA, Bernal-Cano F, Clifford DB, Gandhi RT, Koralnik IJ. Clinical outcome of long-term survivors of progressive multifocal leukoencephalopathy. J Neurol Neurosurg Psychiatry. 2010 Nov; 81(11):1288-1291. Available at http://www.ncbi.nlm.nih.gov/pubmed/20710013. [PubMed: 20710013]

28. Angelini L, Pietrogrande MC, Delle Piane MR, et al. Progressive multifocal leukoencephalopathy in a child with hyperimmunoglobulin E recurrent infection syndrome and review of the literature. Neuropediatrics. 2001 Oct; 32(5):250-255. Available at http://www.ncbi.nlm.nih.gov/pubmed/ 11748496. [PubMed: 11748496]

29. Mamidi A, DeSimone JA, Pomerantz RJ. Central nervous system infections in individuals with HIV-1 infection. J Neurovirol. 2002 Jun; 8(3):158-167. Available at http://www.ncbi.nlm.nih.gov/ pubmed/12053271. [PubMed: 12053271]

30. Bossolasco S, Calori G, Moretti F, et al. Prognostic significance of JC virus DNA levels in cerebrospinal fluid of patients with HIV-associated progressive multifocal leukoencephalopathy. Clin Infect Dis. 2005 Mar 1; 40(5):738-744. Available at http://www.ncbi.nlm.nih.gov/pubmed/ 15714422. [PubMed: 15714422]

31. Focosi D, Marco T, Kast RE, Maggi F, Ceccherini-Nelli L, Petrini M. Progressive multifocal leukoencephalopathy: what's new? Neuroscientist. 2010 Jun; 16(3):308-323. Available at http:// www.ncbi.nlm.nih.gov/pubmed/20479473. [PubMed: 20479473]

32. Cinque P, Koralnik IJ, Gerevini S, Miro JM, Price RW. Progressive multifocal leukoencephalopathy in HIV-1 infection. Lancet Infect Dis. 2009 Oct; 9(10):625-636. Available at http://www.ncbi.nlm.nih.gov/pubmed/19778765. [PubMed: 19778765]

33. Drake AK, Loy CT, Brew BJ, et al. Human immunodeficiency virus-associated progressive multifocal leucoencephalopathy: epidemiology and predictive factors for prolonged survival. Eur J Neurol. Apr; 2007 14(4):418-423. Available at http://www.ncbi.nlm.nih.gov/pubmed/17388991. [PubMed: 17388991]

34. Hall CD, Dafni U, Simpson D, et al. Failure of cytarabine in progressive multifocal leukoencephalopathy associated with human immunodeficiency virus infection. AIDS Clinical Trials Group 243 Team. N Engl J Med. 1998 May 7; 338(19):1345-1351. Available at http:// www.ncbi.nlm.nih.gov/pubmed/9571254. [PubMed: 9571254]

35. Marra CM, Rajicic N, Barker DE, et al. A pilot study of cidofovir for progressive multifocal leukoencephalopathy in AIDS. AIDS. 2002 Sep 6; 16(13):1791-1797. Available at http:// www.ncbi.nlm.nih.gov/pubmed/12218391. [PubMed: 12218391]

36. Geschwind MD, Skolasky RI, Royal WS, McArthur JC. The relative contributions of HAART and alpha-interferon for therapy of progressive multifocal leukoencephalopathy in AIDS. J Neurovirol. 2001 Aug; 7(4):353-357. Available at http://www.ncbi.nlm.nih.gov/pubmed/11517416. [PubMed: 11517416]

37. Vulliemoz S, Lurati-Ruiz F, et al. Favourable outcome of progressive multifocal leucoencephalopathy in two patients with dermatomyositis. J Neurol Neurosurg Psychiatry. 2006; 77(9):1079-1082. Available at http://www.ncbi.nlm.nih.gov/pmc/articles/PMC2077730/. [PubMed: 16914758]

38. Owczarczyk K, Hilker R, Brunn A, Hallek M, Rubbert A. Progressive multifocal leucoencephalopathy in a patient with sarcoidosis - successful treatment with cidofovir and mirtazapine. Rheumatology (Oxford). 2007 May; 46(5):888-890. Available at http:// www.ncbi.nlm.nih.gov/pubmed/17389659. [PubMed: 17389659]

39. Jiang ZG, Cohen J, Marshall LJ, Major EO. Hexadecyloxypropyl-cidofovir (CMX001) suppresses JC virus replication in human fetal brain SVG cell cultures. Antimicrob Agents Chemother. 2010 Nov; 54(11):4723-4732. Available at http://www.ncbi.nlm.nih.gov/pubmed/20823288. [PubMed: 20823288]

40. Gosert R, Rinaldo CH, Wernli M, Major EO, Hirsch HH. CMX001 (1-O-hexadecyloxypropylcidofovir) inhibits polyomavirus JC replication in human brain progenitor-derived astrocytes. 
Antimicrob Agents Chemother. 2011 May; 55(5):2129-2136. Available at http:// www.ncbi.nlm.nih.gov/pubmed/21402853. [PubMed: 21402853]

41. Giudici B, Vaz B, Bossolasco S, et al. Highly active antiretroviral therapy and progressive multifocal leukoencephalopathy: effects on cerebrospinal fluid markers of JC virus replication and immune response. Clin Infect Dis. 2000 Jan; 30(1):95-99. Available at http:// www.ncbi.nlm.nih.gov/pubmed/10619739. [PubMed: 10619739]

42. Safdar A, Rubocki RJ, Horvath JA, Narayan KK, Waldron RL. Fatal immune restoration disease in human immunodeficiency virus type 1-infected patients with progressive multifocal leukoencephalopathy: impact of antiretroviral therapy-associated immune reconstitution. Clin Infect Dis. 2002 Nov 15; 35(10):1250-1257. Available at http://www.ncbi.nlm.nih.gov/pubmed/ 12410486. [PubMed: 12410486]

43. Cinque P, Koralnik IJ, Clifford DB. The evolving face of human immunodeficiency virus-related progressive multifocal leukoencephalopathy: defining a consensus terminology. J Neurovirol. 2003; 9(Suppl 1):88-92. Available at http://www.ncbi.nlm.nih.gov/pubmed/12709878. [PubMed: 12709878]

44. D'Amico R, Sarkar S, Yusuff J, Azar E, Perlman DC. Immune reconstitution after potent antiretroviral therapy in AIDS patients with progressive multifocal leukoencephalopathy. Scand. J Infect Dis. 2007; 39(4):347-350. Available at http://www.ncbi.nlm.nih.gov/pubmed/17454900.

45. Nuttall JJ, Wilmshurst JM, Ndondo AP, et al. Progressive multifocal leukoencephalopathy after initiation of highly active antiretroviral therapy in a child with advanced human immunodeficiency virus infection: a case of immune reconstitution inflammatory syndrome. Pediatr Infect Dis J. 2004 Jul; 23(7):683-685. Available at http://www.ncbi.nlm.nih.gov/pubmed/15247614. [PubMed: 15247614]

46. Du Pasquier RA, Koralnik IJ. Inflammatory reaction in progressive multifocal leukoencephalopathy: harmful or beneficial? J Neurovirol. 2003; 9(Suppl 1):25-31. Suppl 1 Available at http://www.ncbi.nlm.nih.gov/pubmed/12709868. [PubMed: 12709868]

47. Tan K, Roda R, Ostrow L, McArthur J, Nath A. PML-IRIS in patients with HIV infection: clinical manifestations and treatment with steroids. Neurology. 2009 Apr 28; 72(17):1458-1464. Available at http://www.ncbi.nlm.nih.gov/pubmed/19129505. [PubMed: 19129505]

48. Kaplan JE, Benson C, Holmes KH, et al. Guidelines for prevention and treatment of opportunistic infections in HIV-infected adults and adolescents: recommendations from CDC, the National Institutes of Health, and the HIV Medicine Association of the Infectious Diseases Society of America. MMWR Recomm Rep. 2009 Apr 10; 58(RR-4):1-207. quiz CE201-204. Available at http://www.ncbi.nlm.nih.gov/pubmed/19357635.

\section{Syphilis (Last updated November 6, 2013; last reviewed November 6, 2013)}

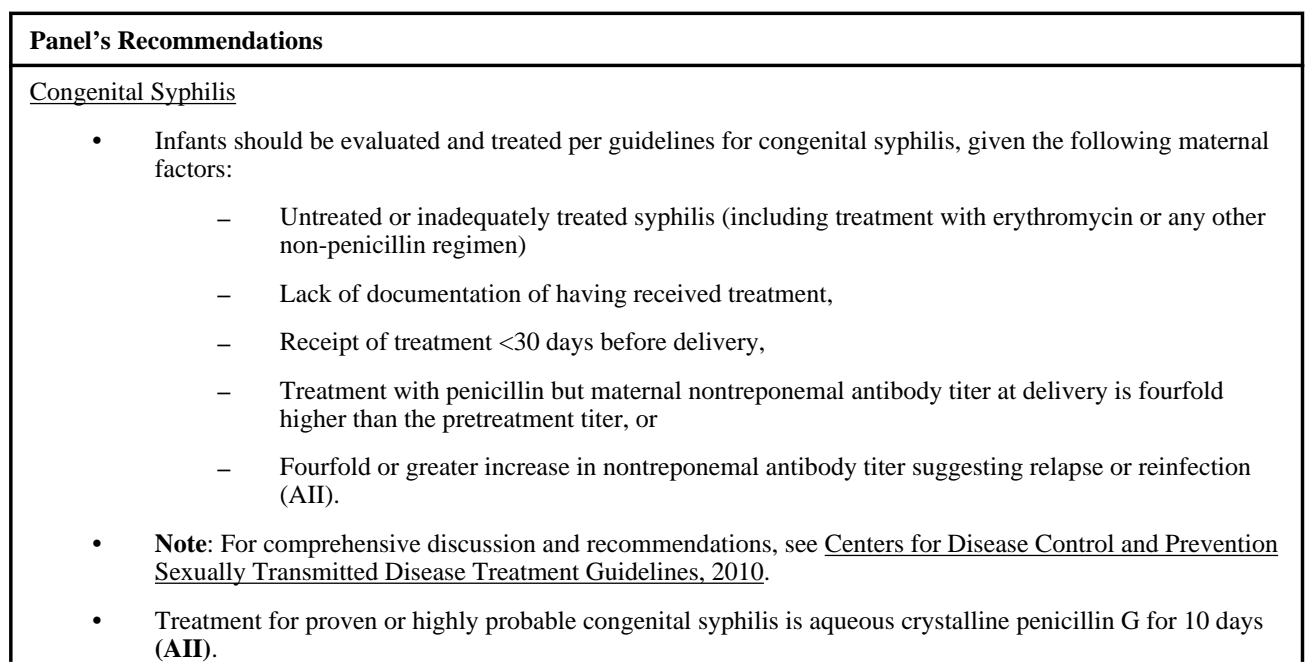


Panel's Recommendations

- If congenital syphilis is diagnosed after age 1 month, the dosage of aqueous crystalline penicillin G should be increased per treatment guidelines (AII).

- An alternative to aqueous crystalline penicillin $\mathrm{G}$ is procaine penicillin $\mathrm{G}$ for 10 days (BII).

- All seroreactive infants (or infants whose mothers were seroreactive at delivery) should receive careful follow-up examinations and serologic testing (a nontreponemal test) every 2 to 3 months until the test becomes nonreactive or the titer has decreased fourfold (AIII). Infants whose initial cerebrospinal fluid (CSF) evaluations are abnormal should undergo repeat lumbar puncture approximately every 6 months until the results are normal (AII).

- $\quad$ After treatment of congenital syphilis, children with increasing or stable nontreponemal titers at ages 6 to 12 months should be evaluated (i.e., including a CSF examination) and treated with a 10-day course of parenteral penicillin (AIII).

- Infants in whom the nontreponemal test is reactive at age 18 months should be fully evaluated or reevaluated (physical, serological, CSF, radiographic exams) and treated or re-treated for congenital syphilis (AIII).

Sexually-Acquired Syphilis

Early Syphilis

- $\quad$ Acquired syphilis in children and adolescents is treated with a single dose of benzathine penicillin $\mathrm{G}$ for early-stage disease (i.e., primary, secondary, and early latent disease) (AII).

- $\quad$ HIV-infected children and adolescents with early syphilis (i.e., primary, secondary, early latent) should receive a single dose of benzathine penicillin G. Those with primary and secondary syphilis should have clinical and serologic response monitored at 3, 6, 9, 12, and 24 months after therapy, and those with early latent syphilis should have clinical and serologic response monitored at 6,12,18, and 24 months after therapy (AIII). (For comprehensive discussion and recommendations, see the Centers for Disease Control and Prevention STD Treatment Guidelines, 2010).

- Re-treatment of patients with early-stage syphilis (i.e., primary, secondary, early latent) and evaluation for HIV infection is recommended for those who:

- $\quad$ Do not experience at least a fourfold decrease in serum nontreponemal test titers 6 to 12 months after therapy,

- Have a sustained fourfold increase in serum nontreponemal test titers after an initial reduction post-treatment, or

- Have persistent or recurring clinical signs or symptoms of disease.

- Individuals whose titers do not decline should at a minimum receive additional clinical and serologic follow-up. If such additional follow-up cannot be ensured, re-treatment is recommended. Because occult central nervous system infection may be signaled by persistently elevated serum nontreponemal test titers, evaluation of CSF can be considered in the event of such persistently elevated titers (BIII).

- If initial CSF examination demonstrates pleocytosis, repeat lumbar puncture should be conducted, and then every 6 months until the cell count is normal (AIII).

Late Latent Syphilis

- For late latent disease, 3 doses of benzathine penicillin G should be administered over 3 weeks (AIII).

- Patients with late-latent syphilis should have CSF examination if they have clinical signs or symptoms attributable to syphilis, a fourfold increase in serum nontreponemal test titer, or experience an inadequate serologic response (i.e., less than fourfold decline in nontreponemal test titer) within 12 to 24 months after therapy if initial titer was high (>1:32) (BIII). CSF examination should also be performed. Treatment for neurosyphilis should be initiated if CSF examination is positive for neurosyphilis.

- $\quad$ Benzathine penicillin G should be administered at 1-week intervals for 3 weeks to patients in whom CSF examination does not confirm the diagnosis of neurosyphilis (AIII).

Neurosyphilis

- $\quad$ Neurosyphilis should be treated with aqueous penicillin $\mathrm{G}$ for 10 to 14 days (AII).

- If a patient has signs or symptoms consistent with neurosyphilis, and repeat CSF examination is consistent with CNS involvement and cannot be attributable to other ongoing illness, re-treatment for neurosyphilis is recommended (AIII) 


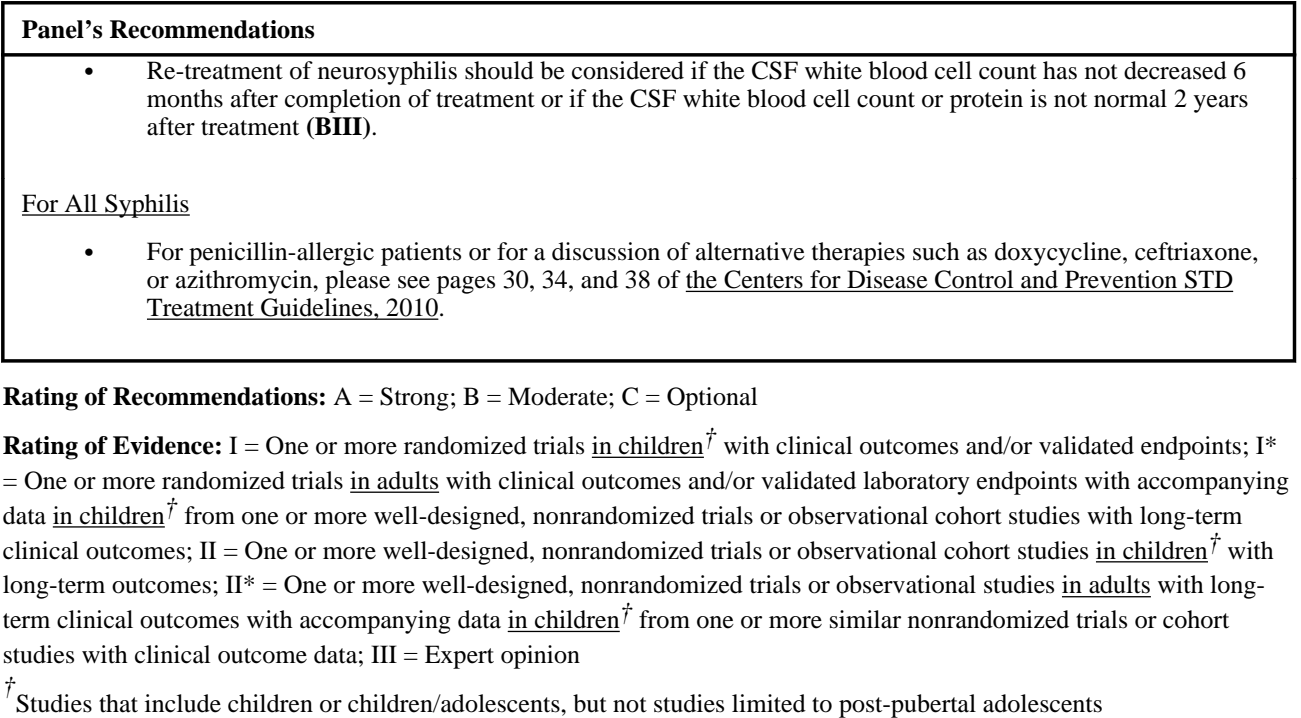

\section{Epidemiology}

Treponema pallidum can be transmitted from mother to child at any stage of pregnancy or during delivery. Among women with untreated primary, secondary, early latent (lacking clinical manifestations within first year after infection), or late latent (lacking clinical manifestations $>1$ year since infection) syphilis at delivery, approximately $30 \%, 60 \%, 40 \%$, and $7 \%$ of infants, respectively, will be infected. Treatment of the mother for syphilis $\geq 30$ days before delivery is required for effective in utero treatment.

Congenital syphilis has been reported despite adequate maternal treatment. Factors that contribute to treatment failure include maternal stage of syphilis (early stage, including primary, secondary, or early latent syphilis), advancing gestational age at treatment, higher nontreponemal titers at treatment and delivery, and short interval from treatment to delivery (<30 days). ${ }^{1,2}$ Since 1991, rates of congenital syphilis have trended downward $92 \%$ to 8.5 cases per 100,000 live births in $2011 .{ }^{3}$ The continuing decline in the rate of congenital syphilis probably reflects the substantially reduced rate of primary and secondary syphilis in women during the last decade.

Drug use during pregnancy, particularly cocaine use, has been associated with increased risk of maternal syphilis and congenital infection. ${ }^{4}$ Similarly, HIV-infected women have a higher prevalence of untreated or inadequately treated syphilis during pregnancy, which places their newborns at higher risk of congenital syphilis. ${ }^{5}$ Rates of mother-to-child HIV transmission may be higher when syphilis coinfection is present during pregnancy. ${ }^{5-7}$ Risk of HIV transmission does not appear to be higher in mothers whose syphilis is effectively treated before pregnancy. ${ }^{5}$

Although individuals aged 15 to 24 represent one-quarter of the ever-sexually-active population aged 15 to 44, approximately half of sexually transmitted diseases (STDs) diagnosed annually in the United States occur in individuals aged 15 to 24 years. ${ }^{8,9}$ 
Furthermore, individuals in this age group accounted for $28 \%$ of primary and secondary syphilis cases during 2011. ${ }^{3}$ In 2011, the rate of primary and secondary syphilis was highest among individuals aged 20 to 24 years and 25 to 29 years (13.8 and 12.1 cases per 100,000 population, respectively). Nevertheless, the prevalence and incidence of syphilis in HIVinfected youth and of HIV infection in youth with syphilis are appreciable; in a study of 320 HIV-infected and HIV-uninfected U.S. adolescents aged 12 to 19 years, the prevalence of syphilis was $9 \%$ in HIV-infected girls and $6 \%$ in HIV-infected boys. ${ }^{10}$ In a meta-analysis of 30 studies including individuals of all ages, the median HIV seroprevalence in those infected with syphilis in the United States was $15.7 \%$ (27.5\% in men and $12.4 \%$ in women with syphilis). ${ }^{11}$ In 2010, coinfection with HIV was reported in $46 \%$ of 15 - to 29 -year-old men who have sex with men with primary and secondary syphilis who knew their HIV status. ${ }^{12}$

\section{Clinical Manifestations}

Untreated early syphilis during pregnancy can lead to spontaneous abortion, stillbirth, hydrops fetalis, preterm delivery, and perinatal death in up to $40 \%$ of pregnancies. ${ }^{13}$ In children with congenital syphilis, two characteristic syndromes of clinical disease exist: early and late congenital syphilis. Early congenital syphilis refers to clinical manifestations that appear during the first 2 years of life. Late congenital syphilis refers to clinical manifestations that appear in children older than age 2 years.

At birth, infected infants may manifest signs such as hepatosplenomegaly, jaundice, mucocutaneous lesions (e.g., skin rash, nasal discharge, mucous patches, condyloma lata), lymphadenopathy, pseudoparalysis of an extremity, anemia, thrombocytopenia, pneumonia, and skeletal lesions (e.g., osteochondritis, periostitis, or osteitis). In a study of 148 infants born to mothers with untreated or inadequately treated syphilis, $47 \%$ had clinical, radiographic, or conventional laboratory findings consistent with congenital syphilis, and $44 \%$ had a positive rabbit infectivity test, polymerase chain reaction assay, or immunoglobulin M (IgM) immunoblot of serum, blood, or cerebrospinal fluid (CSF). ${ }^{14}$ Manifestations of congenital syphilis in infants of HIV-infected women are expected to be similar to those in HIV-unexposed infants. However, as many as $60 \%$ of infants with congenital syphilis do not have any clinical signs at birth. ${ }^{15}$ If untreated, these asymptomatic infants can develop clinically apparent disease in the ensuing 3 weeks to 6 months. In addition, fever, nephrotic syndrome, and hypopituitarism can occur. Clinical manifestations of late congenital syphilis are similar to late manifestations of syphilis in adults (e.g., involvement of bone and soft tissue, eyes, ears, and the central nervous system [CNS]).

The manifestations of sexually acquired syphilis in older children and adolescents are similar to those in adults (see Guidelines for the Prevention and Treatment of Opportunistic Infections in HIV-Infected Adults).${ }^{16} \mathrm{HIV}$-infected individuals with early syphilis may be at increased risk of neurologic complications and may have higher rates of serologic treatment failure. ${ }^{17}$

\section{Diagnosis}

The standard serologic tests for syphilis are based on measurement of immunoglobulin $\mathrm{G}$ (IgG) antibody. Because $\operatorname{IgG}$ antibody in an infant reflects transplacental passively 
transferred antibody from the mother, interpretation of reactive serologic tests for syphilis in infants is difficult. Therefore, the diagnosis of neonatal congenital syphilis depends on a combination of results from physical, laboratory, radiographic, and direct microscopic examinations.

All infants born to women with reactive nontreponemal and treponemal test results should be evaluated with a quantitative nontreponemal test (e.g., Venereal Disease Research Laboratory [VDRL] slide test, rapid plasma reagin [RPR], the automated reagin test) from the infant and compared with the same test done at the same laboratory on the mother's serum. Umbilical cord specimens should not be tested because of the potential for maternal blood contamination. Specific treponemal tests, such as the fluorescent treponemal antibody absorption (FTA-ABS) test and T. pallidum particle agglutination (TP-PA) test, are not necessary to evaluate congenital syphilis in the neonate. There is no commercially available IgM test recommended for diagnostic use. Note: Some laboratories use treponemal tests (e.g., enzyme immunoassay, chemiluminescence) for initial screening, and nontreponemal tests for confirmation of positive specimens. ${ }^{18}$ However, such an approach with congenital syphilis has not been published.

Congenital syphilis can be definitively diagnosed if $T$. pallidum is detected by using darkfield microscopic examination or special stains of lesions or body fluids such as umbilical cord, placenta, nasal discharge, or skin lesion material from an infant. Failure to detect $T$. pallidum does not definitively rule out infection because false-negative results are common. ${ }^{19}$ A quantitative nontreponemal serologic titer in an infant that is fourfold higher than the mother's is suggestive of infection. Infection also should be assumed in infants born to mothers who were untreated or inadequately treated for syphilis prior to delivery (e.g., non-penicillin regimen or treatment completion $<30$ days before delivery), regardless of lack of physical, radiographic, or laboratory findings in the infants suggestive of congenital syphilis.

Evaluation of suspected cases of congenital syphilis should include a careful and complete physical examination. Physical signs and symptoms of congenital syphilis include, but are not limited to, non-immune hydrops, jaundice, hepatosplenomegaly, rhinitis, skin rash, and pseudoparalysis of an extremity. Further evaluation to support a diagnosis of congenital syphilis depends on maternal treatment history for syphilis, findings on physical examination, and planned infant treatment. and may include a complete blood count and differential and platelet count, long bone radiographs, and CSF analysis for VDRL, cell count, and protein. A positive CSF VDRL test, elevated CSF protein, and/or elevated CSF white blood cell (WBC) count without other causes may be due to congenital syphilis. Other tests should be performed as clinically indicated (e.g., chest radiograph, liver-function tests, cranial ultrasound, ophthalmologic examination, auditory brainstem response). Individuals with latent syphilis who have neurologic or ophthalmologic signs or symptoms, active tertiary syphilis, or serologic treatment failure should have a CSF examination. Different scenarios indicating clinical management and follow-up recommendations for congenital syphilis are provided on page 36 through 37 of the Centers for Disease Control and Prevention STD Treatment Guidelines, 2010. 
For diagnosis of acquired syphilis, a reactive nontreponemal test must be confirmed by a specific treponemal test such as FTA-ABS or TP-PA. Treponemal tests usually remain positive for life, even with successful treatment. The prozone phenomenon (a weakly reactive or falsely negative) reaction is more common in HIV-infected patients. ${ }^{20}$ Treponemal antibody titers do not correlate with disease activity and should not be used to monitor treatment response.

\section{Prevention Recommendations \\ Preventing Exposure}

Congenital Syphilis: Effective identification and treatment of congenital syphilis depends on the identification of syphilis in pregnant women and, therefore, on routine serologic screening of pregnant women during the first prenatal visit. In communities and populations in which the risk of congenital syphilis is high, serologic testing and a sexual history also should be obtained at 28 weeks' gestation and at delivery. Moreover, as part of management of pregnant women who have syphilis, information about treatment of sex partners should be obtained to assess the risk of reinfection. Serologic testing at delivery of the mother's serum is preferred over testing of the infant's serum because the serologic tests performed on infant serum can be non-reactive if the mother's serologic test result is of low titer or the mother was infected late in pregnancy. No HIV-exposed infant should leave the hospital unless the maternal syphilis serologic status has been documented at least once during pregnancy and at delivery in communities and populations in which the risk of congenital syphilis is high. ${ }^{21,22}$ Routine screening of serum from newborns or umbilical cord blood is not recommended.

Acquired Syphilis: Primary prevention of syphilis includes routine discussion of sexual behaviors that may place individuals at risk of infection. Providers should discuss risk reduction messages that are client-centered and provide specific actions that can reduce the risk of STD acquisition and HIV transmission. ${ }^{23-25}$

Routine serologic screening for syphilis is recommended at least annually for all sexually active HIV-infected individuals, with more frequent screening (i.e., 3-6 months) depending on individual risk behaviors (e.g., as multiple partners, sex in conjunction with illicit drug use, methamphetamine use, partners who participate in such activities). ${ }^{17,26}$ Syphilis in an HIV-infected individual indicates high-risk behavior and should prompt intensified counseling messages and consideration of referral for behavioral intervention. Patients undergoing screening or treatment for syphilis also should be evaluated for other STDs. ${ }^{27}$

Discontinuing Primary Prophylaxis-Not applicable.

\section{Treatment Recommendations}

Treating Disease-Penicillin remains the treatment of choice for syphilis, congenital or acquired, regardless of HIV status (AI*).

Congenital Syphilis: Data are insufficient to determine whether infants who have congenital syphilis and whose mothers are coinfected with HIV require different evaluation, 
therapy, or follow-up for syphilis than that recommended for infants born to mothers who are not HIV-coinfected. Response to standard treatment may differ in HIV-infected mothers. For example, some studies in adults have shown a lag in serologic improvement in appropriately treated HIV-infected patients. ${ }^{28,29}$

Treatment for congenital syphilis should be administered to infants whose mothers:

- Have been untreated or inadequately treated for syphilis (including treatment with erythromycin or any other non-penicillin regimen),

- Have no documentation of receiving treatment,

- Received treatment $<30$ days before delivery, or

- Have experienced a fourfold or greater increase in nontreponemal antibody titer suggestive of relapse or reinfection (AII) (proven or highly probable disease). (Sexually Transmitted Disease Treatment Guidelines, 2010) ${ }^{27}$

Infants should be treated regardless of maternal treatment history if they have an abnormal physical examination consistent with congenital syphilis, positive darkfield or fluorescent antibody test of body fluid(s), or serum quantitative nontreponemal serologic titer that is at least fourfold greater than maternal titer (AII) (proven or highly probable disease). ${ }^{27}$

Treatment for proven or highly probable congenital syphilis is aqueous crystalline penicillin G 100,000 to 150,000 units/kg body weight/day, administered as 50,000 units $/ \mathrm{kg}$ body weight/dose intravenously (IV) every 12 hours during the first 7 days of life and every 8 hours thereafter for a total of 10 days (AII). If congenital syphilis is diagnosed after age 1 month, the dosage of aqueous penicillin G should be increased to 200,000 to 300,000 units $/ \mathrm{kg} /$ day IV, administered as 50,000 units $/ \mathrm{kg}$ body weight/dose IV every 4 to 6 hours for 10 days (AII). If 1 day of therapy is missed, the entire course should be restarted. An alternative to aqueous penicillin $\mathrm{G}$ is procaine penicillin $\mathrm{G} 50,000$ units $/ \mathrm{kg}$ body weight/dose intramuscularly (IM) in a single dose daily for 10 days (BII). However, aqueous penicillin $\mathrm{G}$ is preferred because of its higher penetration into the CSF. Insufficient data are available on the effectiveness of ampicillin or other therapies for treatment of congenital syphilis.

For infants who do not meet criteria for proven or highly probable disease, treatment options are influenced by several factors, including maternal treatment, maternal serologic results, and response to therapy, and infant physical exam, infant serologic results, and other laboratory test results. Scenarios that include variations of these factors with treatment recommendations are provided in detail in on pages 36 and 37 of the Centers for Disease Control and Prevention STD Treatment Guidelines, 2010. ${ }^{27}$ In the setting of maternal and possible infant HIV infection, the more conservative choices among scenario-specific treatment options may be preferable.

Acquired Syphilis: Acquired syphilis in children and adolescents is treated with a single dose of benzathine penicillin G 50,000 units/kg body weight IM (up to the adult dose of 2.4 million units) for early-stage disease (i.e., primary, secondary, and early latent disease) (AII). For late latent disease, three doses of benzathine penicillin G 50,000 units $/ \mathrm{kg}$ body weight (up to the adult dose of 2.4 million units) should be administered IM once weekly for 
3 doses (total 150,000 units $/ \mathrm{kg}$ body weight, up to the adult total dose of 7.2 million units) (AIII). Alternative therapies (e.g., ceftriaxone, azithromycin) should be administered to HIV-infected patients only when treatment with penicillin is not feasible, and with close clinical and serologic monitoring because data on their use are limited (BII). See the Sexually Transmitted Disease Treatment Guidelines, 2010. ${ }^{27}$ Neurosyphilis should be treated with aqueous penicillin G 200,000 to 300,000 units/kg body weight per dose IV every 4 to 6 hours (maximum dosage: 18-24 million units/day) for 10 to 14 days (AII). ${ }^{30}$ See Guidelines for the Prevention and Treatment of Opportunistic Infections in HIVInfected Adults for dosing recommendations for older HIV-infected adolescents with acquired syphilis. ${ }^{16}$

Monitoring and Adverse Events (Including IRIS)_All infants with a reactive nontreponemal test for syphilis (or infants whose mothers were seroreactive at delivery) should receive careful follow-up examinations and serologic testing (i.e., a nontreponemal test) every 2 to 3 months until the test becomes non-reactive or the titer has decreased fourfold (AIII). Nontreponemal antibody titers should decline by age 3 months and should be non-reactive by age 6 months in infants who were not infected (i.e., if the reactive test result was caused by passive transfer of maternal $\operatorname{IgG}$ antibody) or who were infected but have been adequately treated. The serologic response after therapy may be slower in infants treated after the neonatal period. Whether children with congenital syphilis who also are HIV-infected take longer to become nonreactive and require retreatment is unknown.

Treponemal tests should not be used to evaluate treatment response because in infected children, the results can remain positive despite effective therapy or be related to maternal infection. Passively transferred maternal treponemal antibodies can be present in infants until age 15 months. A reactive treponemal test after age 18 months is diagnostic of congenital syphilis. If the nontreponemal test is non-reactive at that time, no further evaluation or treatment is necessary. Infants in whom the nontreponemal test is reactive at age 18 months should be fully (re)evaluated and (re)treated for congenital syphilis (AIII).

Infants whose initial CSF evaluations are abnormal should undergo repeat lumbar puncture approximately every 6 months until the results are normal (AII). A repeat reactive CSF VDRL test or abnormal CSF indices that cannot be attributed to other ongoing illness requires retreatment for possible neurosyphilis.

HIV-infected children and adolescents with acquired primary and secondary syphilis should have clinical and serologic response monitored at 3, 6, 9, 12, and 24 months after therapy (AIII); nontreponemal test titers should decline by at least fourfold by 6 to 12 months after successful therapy, with examination of CSF and re-treatment strongly considered in the absence of such decline. For acquired syphilis of longer duration (e.g., early and late latent syphilis), follow up is indicated at $6,12,18$, and 24 months; fourfold decline should be expected by 12 to 24 months. If initial CSF examination demonstrated pleocytosis, repeat lumbar puncture should be conducted at 6 months after therapy, and then every 6 months until the cell count is normal (AIII). Follow-up CSF examinations also can be used to evaluate changes in the CSF-VDRL or CSF protein levels after therapy, but changes in these parameters occur more slowly than changes in CSF cell counts. Data from HIV-infected 
adults with neurosyphilis suggest that CSF abnormalities may persist for extended times, and close clinical follow up is warranted. ${ }^{31}$

Syphilis in HIV-infected children (congenital or acquired) manifesting as immune response inflammatory syndrome (IRIS) has not been reported, and only very rare reports of syphilisassociated IRIS in adults (primarily syphilitic ocular inflammatory disease) have been reported. ${ }^{32,33}$

Managing Treatment Failure-After treatment of congenital syphilis, children with increasing or stable nontreponemal titers at ages 6 to 12 months or children who are seropositive with any nontreponemal titer at 18 months should be evaluated (including with a CSF examination) and considered for retreatment with a 10-day course of parenteral penicillin G (AIII).

Management of failed treatment of acquired syphilis in older children and adolescents is identical to that in adults. ${ }^{17}$ Re-treatment of patients with primary or secondary syphilis should be considered for those who:

- Do not experience at least a fourfold decrease in serum nontreponemal test titers 6 to 12 months after therapy,

- Have a sustained fourfold increase in serum nontreponemal test titers after an initial reduction post-treatment, or

- Have persistent or recurring clinical signs or symptoms of disease (BIII).

Adolescents or adults in whom CSF examination does not confirm a neurosyphilis diagnosis should receive benzathine penicillin G 2.4 million units IM, at 1-week intervals for 3 weeks (BIII). If titers fail to respond appropriately after re-treatment, the value of repeat CSF evaluation or re-treatment is unclear, but not recommended.

Re-treatment is warranted for patients with early or late-latent syphilis who have new or sustained clinical signs or symptoms of syphilis, have a fourfold increase in serum nontreponemal test titer, or experience an inadequate serologic response (less than fourfold decline in nontreponemal test titer) within 12 to 24 months after therapy if initial titer was high (>1:32) (BIII). Repeat CSF examination should be performed on these patients, and if the results are consistent with CNS involvement, re-treatment should follow the neurosyphilis recommendations (AIII). Adolescents or adults whose CSF profile is not indicative of CNS disease should receive a repeat course of benzathine penicillin 2.4 million units IM weekly for 3 weeks (BIII); re-treatment of neurosyphilis should be considered in patients whose CSF WBC count has not decreased 6 months after completion of treatment or in whom CSF WBC count or protein is not normal after 2 years (BIII).

Preventing Recurrence-No recommendations have been developed for secondary prophylaxis or chronic maintenance therapy for syphilis in HIV-infected children.

Discontinuing Secondary Prophylaxis-Not applicable. 


\section{References}

1. Alexander JM, Sheffield JS, Sanchez PJ, Mayfield J, Wendel GD Jr. Efficacy of treatment for syphilis in pregnancy. Obstet Gynecol. 1999 Jan; 93(1):5-8. Available at http:// www.ncbi.nlm.nih.gov/pubmed/9916946. [PubMed: 9916946]

2. Sheffield JS, Sanchez PJ, Morris G, et al. Congenital syphilis after maternal treatment for syphilis during pregnancy. Am J Obstet Gynecol. 2002 Mar; 186(3):569-573. Available at http:// www.ncbi.nlm.nih.gov/pubmed/11904625. [PubMed: 11904625]

3. Centers for Disease Control and Prevention. Sexually Transmitted Disease Surveillance 2011. 2012. Available at http://www.cdc.gov/std/stats11/default.htm

4. Sison CG, Ostrea EM Jr, Reyes MP, Salari V. The resurgence of congenital syphilis: a cocainerelated problem. J Pediatr. 1997 Feb; 130(2):289-292. Available at http://www.ncbi.nlm.nih.gov/ pubmed/9042134. [PubMed: 9042134]

5. Schulte JM, Burkham S, Hamaker D, et al. Syphilis among HIV-infected mothers and their infants in Texas from 1988 to 1994. Sex Transm Dis. 2001 Jun; 28(6):315-320. Available at http:// www.ncbi.nlm.nih.gov/pubmed/11403187. [PubMed: 11403187]

6. Lee MJ, Hallmark RJ, Frenkel LM, Del Priore G. Maternal syphilis and vertical perinatal transmission of human immunodeficiency virus type-1 infection. Int J Gynaecol Obstet. 1998 Dec; 63(3):247-252. Available at http://www.ncbi.nlm.nih.gov/pubmed/9989893. [PubMed: 9989893]

7. Mwapasa V, Rogerson SJ, Kwiek JJ, Wilson PE, Milner D MM, Kamwendo DD, et al. Maternal syphilis infection is associated with increased risk of mother-to-child transmission of HIV in Malawi. AIDS. 2006; 20(14):1869-1877. Available at http://www.ncbi.nlm.nih.gov/pubmed/ 16954728. [PubMed: 16954728]

8. Weinstock H, Berman S, Cates W Jr. Sexually transmitted diseases among American youth: incidence and prevalence estimates, 2000. Perspectives On Sexual And Reproductive Health. 2004 Jan-Feb;36(1):6-10. Available at http://www.ncbi.nlm.nih.gov/pubmed/14982671. [PubMed: 14982671]

9. Satterwhite CL, Torrone E, Meites E, et al. Sexually transmitted infections among US women and men: prevalence and incidence estimates, 2008. Sex Transm Dis. 2013 Mar; 40(3):187-193. Available at http://www.ncbi.nlm.nih.gov/pubmed/23403598. [PubMed: 23403598]

10. Vermund SH, Wilson CM, Rogers AS, Partlow C, Moscicki AB. Sexually transmitted infections among HIV infected and HIV uninfected high-risk youth in the REACH study. Reaching for Excellence in Adolescent Care and Health. J Adolesc Health. 2001 Sep; 29(3 Suppl):49-56. Available at http://www.ncbi.nlm.nih.gov/pubmed/11530303. [PubMed: 11530303]

11. Blocker ME, Levine WC, St Louis ME. HIV prevalence in patients with syphilis, United States. Sex Transm Dis. 2000 Jan; 27(1):53-59. Available at http://www.ncbi.nlm.nih.gov/pubmed/ 10654870. [PubMed: 10654870]

12. Su, J. Paper presented at National STD Prevention Conference. Minneapolis, MN: 2012. Increases in Syphilis Among Young Men in the United States. Available at https://cdc.confex.com/cdc/ std2012/webprogram/Session12901.html

13. Singh R MJ. Syphilis in pregnancy. Venereology. 2001; 14:121-131.

14. Michelow IC, Wendel GD Jr, Norgard MV, et al. Central nervous system infection in congenital syphilis. N Engl J Med. 2002 Jun 6; 346(23):1792-1798. Available at http:// www.ncbi.nlm.nih.gov/pubmed/12050339. [PubMed: 12050339]

15. Glaser JH. Centers for Disease Control and Prevention guidelines for congenital syphilis. J Pediatr. 1996 Oct; 129(4):488-490. Available at http://www.ncbi.nlm.nih.gov/pubmed/8859252. [PubMed: 8859252]

16. Kaplan JE, Benson C, Holmes KH, et al. Guidelines for prevention and treatment of opportunistic infections in HIV-infected adults and adolescents: recommendations from CDC, the National Institutes of Health, and the HIV Medicine Association of the Infectious Diseases Society of America. MMWR Recomm Rep. 2009 Apr 10; 58(RR-4):1-207. quiz CE201-204. Available at http://www.ncbi.nlm.nih.gov/pubmed/19357635. 
17. Centers for Disease Control and Prevention. Sexually Transmitted Diseases Treatment Guidelines. MMWR. 2010; 59(12) Available at http://www.cdc.gov/std/treatment/2010/genitalulcers.htm\#syphhiv.

18. Centers for Disease Control and Prevention. Discordant results from reverse sequence syphilis screening-five laboratories, United States, 2006-2010. MMWR. 2011 Feb 11; 60(5):133-137. Available at http://www.ncbi.nlm.nih.gov/pubmed/21307823. [PubMed: 21307823]

19. Association of Public Health Laboratories. Laboratory Diagnostic Testing for Treponema pallidum. 2009 Available at http://www.aphl.org/aphlprograms/infectious/std/Documents/ LaboratoryGuidelinesTreponemapallidumMeetingReport.pdf.

20. Jurado RL, Campbell J, Martin PD. Prozone phenomenon in secondary syphilis. Has its time arrived? Arch Intern Med. 1993 Nov 8; 153(21):2496-2498. Available at http:// www.ncbi.nlm.nih.gov/pubmed/7832818. [PubMed: 7832818]

21. Centers for Disease Control and Prevention. Congenital syphilis-United States, 2002. MMWR. 2004 Aug 13; 53(31):716-719. Available at http://www.ncbi.nlm.nih.gov/pubmed/15306757. [PubMed: 15306757]

22. Beltrami J, Berman S. Congenital syphilis: a persisting sentinel public health event. Sex Transm Dis. 2006 Nov; 33(11):675-676. Available at http://www.ncbi.nlm.nih.gov/pubmed/16794558. [PubMed: 16794558]

23. Kamb ML, Fishbein M, Douglas J J, et al. Efficacy of risk-reduction counseling to prevent human immunodefiency virus and sexually transmitted diseases: a randomized controlled trial. JAMA. 1998; 280:1161-1167. 1998. Available at http://www.ncbi.nlm.nih.gov/pubmed/9777816. [PubMed: 9777816]

24. Fisher JD, Cornman DH, Osborn CY, et al. Clinician-initiated HIV risk reduction intervention for HIV-positive persons: formative research, acceptability, and fidelity of the Options Project. J Acquir Immune Defic Syndr. 2004; 37(Suppl 2):S88-S94. Available at http:// www.ncbi.nlm.nih.gov/pubmed/15385903. [PubMed: 15385904]

25. Richardson JL, Milam J SS, et al. Using patient risk indicators to plan prevention strategies in the clinical care setting. J Acquir Immune Defic Syndr. 2004; 37(suppl 2):S88-S94. Available at http://www.ncbi.nlm.nih.gov/pubmed/15385904. [PubMed: 15385904]

26. CDC, HRSA NIH, HIVMA/IDSA, and the HIV Prevention in Clinical Care Working Group. Recommendations for Incorporating Human Immunodeficiency Virus (HIV) Prevention into the Medical Care of Persons Living with HIV. Clin Infect Dis. 2004; 38:104-121. [PubMed: 14679456]

27. Workowski KA, Berman S. Centers for Disease C, Prevention. Sexually transmitted diseases treatment guidelines, 2010. MMWR Recomm Rep. 2010 Dec 17; 59(RR-12):1-110. Available at http://www.ncbi.nlm.nih.gov/pubmed/21160459. [PubMed: 21160459]

28. Yinnon AM, Coury-Doniger P, Polito R, Reichman RC. Serologic response to treatment of syphilis in patients with HIV infection. Arch Intern Med. 1996 Feb 12; 156(3):321-325. Available at http://www.ncbi.nlm.nih.gov/pubmed/8572843. [PubMed: 8572843]

29. Rolfs RT, Joesoef MR, Hendershot EF, et al. A randomized trial of enhanced therapy for early syphilis in patients with and without human immunodeficiency virus infection. The Syphilis and HIV Study Group. N Engl J Med. 1997 Jul 31; 337(5):307-314. Available at http:// www.ncbi.nlm.nih.gov/pubmed/9235493. [PubMed: 9235493]

30. American Academy of Pediatrics. Red Book: 2012 Report of the Committee on Infectious Diseases. Elk Grove Village, IL: 2012.

31. Centers for Disease Control and Prevention. Symptomatic early neurosyphilis among HIV-positive men who have sex with men-four cities, United States, January 2002-June 2004. MMWR. 2007 Jun 29; 56(25):625-628. Available at http://www.ncbi.nlm.nih.gov/pubmed/17597693. [PubMed: 17597693]

32. Moloney G, Branley M, Kotsiou G, Rhodes D. Syphilis presenting as scleritis in an HIV-positive man undergoing immune reconstitution. Clin Experiment Ophthalmol. 2004 Oct; 32(5):526-528. Available at http://www.ncbi.nlm.nih.gov/pubmed/15498066. [PubMed: 15498066] 
33. Bernal E, Munoz A, Ortiz Mdel M, Cano A. Syphilitic panuveitis in an HIV-infected patient after immune restoration. Enferm Infecc Microbiol Clin. 2009 Oct; 27(8):487-489. Available at http:// www.ncbi.nlm.nih.gov/pubmed/19406524. [PubMed: 19406524]

\section{Dosing Recommendations for Prevention and Treatment of Syphilis}

\begin{tabular}{|c|c|c|c|}
\hline \multicolumn{4}{|c|}{ Preventive Regimen } \\
\hline Indication & First Choice & Alternative & Comments/Special Issues \\
\hline Primary Prophylaxis & N/A & N/A & $\begin{array}{l}\frac{\text { Primary Prophylaxis }}{\text { Indicated for: }} \\
\qquad \quad \text { N/A } \\
\text { Criteria for Discontinuing } \\
\text { Primary Prophylaxis: } \\
\text { N/A } \\
\frac{\text { Criteria for Restarting }}{\text { Primary Prophylaxis: }} \\
\text { N/A }\end{array}$ \\
\hline Secondary Prophylaxis & N/A & N/A & $\begin{array}{l}\frac{\text { Secondary Prophylaxis }}{\text { Indicated: }} \\
\qquad \quad \text { N/A } \\
\frac{\text { Criteria For Discontinuing }}{\text { Secondary Prophylaxis: }} \\
\bullet \quad \text { N/A } \\
\frac{\text { Criteria For Restarting }}{\text { Secondary Prophylaxis: }} \\
\qquad \quad \text { N/A }\end{array}$ \\
\hline Treatment & $\begin{array}{ll}\text { Congenital } \\
\text { Proven or Highly Probable } \\
\text { Disease: }\end{array}$ & $\begin{array}{l}\text { Congenital } \\
\text { Proven or Highly Probable } \\
\text { Disease (Less Desirable if } \\
\text { CNS Involvement): } \\
\text { Procaine } \\
\text { penicillin G } \\
50,000 \text { units/kg } \\
\text { body weight IM } \\
\text { once daily for } 10 \\
\text { days } \\
\text { Possible Disease: } \\
\text { Treatment } \\
\text { options are } \\
\text { influenced by } \\
\text { several factors, } \\
\text { including } \\
\text { maternal } \\
\text { treatment, titer, } \\
\text { and response to } \\
\text { therapy; and } \\
\text { infant physical } \\
\text { exam, titer, and } \\
\text { test results. } \\
\text { Scenarios that } \\
\text { include } \\
\text { variations of } \\
\text { these factors are } \\
\text { described and } \\
\text { treatment } \\
\text { recommendations } \\
\text { are provided in } \\
\text { detail on pages } \\
\text { 36-37 of the }\end{array}$ & $\begin{array}{l}\text { For treatment of } \\
\text { congenital syphilis, repeat } \\
\text { the entire course of } \\
\text { treatment if }>1 \text { day of } \\
\text { treatment is missed. } \\
\text { Examinations and } \\
\text { serologic testing for } \\
\text { children with congenital } \\
\text { syphilis should occur } \\
\text { every } 2-3 \text { months until the } \\
\text { test becomes non-reactive } \\
\text { or there is a fourfold } \\
\text { decrease in titer. Children } \\
\text { with increasing titers or } \\
\text { persistently positive titers } \\
\text { (even if low levels) at ages } \\
6-12 \text { months should be } \\
\text { evaluated and considered } \\
\text { for retreatment. } \\
\text { In the setting of maternal } \\
\text { and possible infant HIV } \\
\text { infection, the more } \\
\text { conservative choices } \\
\text { among scenario-specific } \\
\text { treatment options may be } \\
\text { preferable. } \\
\text { Children and adolescents } \\
\text { with acquired syphilis } \\
\text { should have clinical and } \\
\text { serologic response } \\
\text { monitored at } 3,6,9,12, \\
\text { and } 24 \text { months after } \\
\text { therapy. }\end{array}$ \\
\hline
\end{tabular}




\begin{tabular}{|c|c|c|c|}
\hline \multicolumn{4}{|c|}{ Preventive Regimen } \\
\hline Indication & First Choice & Alternative & Comments/Special Issues \\
\hline & 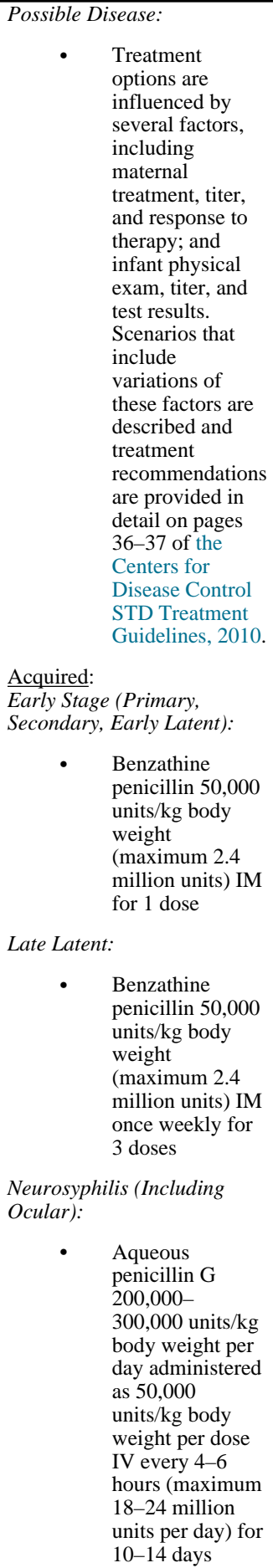 & $\begin{array}{l}\text { Centers for } \\
\text { Disease Control } \\
\text { STD Treatment } \\
\text { Guidelines, } 2010 .\end{array}$ & \\
\hline
\end{tabular}

Key to Acronyms: $\mathrm{CDC}=$ Centers for Disease Control and Prevention; $\mathrm{IM}=$ intramuscular; $\mathrm{IV}=$ intravenous; $\mathrm{STD}=$ sexually transmitted disease 


\title{
Toxoplasmosis (Last updated November 6, 2013; last reviewed November
}

\section{6, 2013)}

\author{
Panel's Recommendations \\ Preventing Exposure \\ - Ingestion of undercooked meats that could contain tissue cysts and contact with cat feces that could contain \\ sporulated oocysts should be avoided (AIII). \\ Initiating Primary Prophylaxis \\ - Toxoplasma-seropositive children aged $<6$ years with CD4 T lymphocyte (CD4) cell percentage $<15 \%$ and \\ children aged $\Varangle 6$ years with $\mathrm{CD} 4<100$ cells $/ \mathrm{mm}^{3}$ should be administered prophylaxis against Toxoplasma \\ encephalitis (TE) (AIII). The preferred agent for prophylaxis of TE is trimethoprim-sulfamethoxazole, one \\ double-strength tablet daily for adolescents and adults (or weight-equivalent dosing for children) (AII*).
}

- Primary preventive therapy can be discontinued once a child responds to combination antiretroviral therapy (cART) with a sustained rise in CD4 percentage above $15 \%$ for children $<6$ years of age, and $>200$ cells $/ \mathrm{mm}^{3}$ for children aged $\nsucceq 6$ years $(\mathbf{B I I I})$.

- Most experts recommend treating pregnant women with acute toxoplasmosis in an attempt to prevent fetal infection (BII). For more extensive information on diagnosis, prevention, and treatment of pregnant women with toxoplasmosis, please see the Guidelines for the Prevention and Treatment of Opportunistic Infections in HIV-Infected Adults and Adolescents.

- $\quad$ Empiric therapy should be strongly considered for newborns of HIV-infected mothers who had symptomatic or asymptomatic primary Toxoplasma infection during pregnancy, regardless of whether treatment was administered during pregnancy (BIII).

- The preferred treatment for congenital toxoplasmosis is pyrimethamine combined with sulfadiazine, with supplementary leucovorin (AII).

- The recommended duration of treatment of congenital toxoplasmosis in HIV-infected infants is 12 months (AIII).

- Therapy for acquired toxoplasmosis in HIV-infected children is sulfadiazine plus pyrimethamine and leucovorin $(\mathbf{A I})$.

- Corticosteroids are recommended for HIV-infected children with central nervous system toxoplasmosis when cerebrospinal fluid protein is highly elevated (i.e., $>1,000 \mathrm{mg} / \mathrm{dL}$ ) or who have focal lesions with substantial mass effect (BIII). Anticonvulsants should be administered only to children with TE who have a history of or current seizures (AIII).

- Complete blood count should be monitored weekly in patients taking daily pyrimethamine (AIII). Patients who have completed initial therapy for TE should be given suppressive therapy (i.e., secondary prophylaxis or chronic maintenance therapy) unless cART results in immune reconstitution (AI*).

- The preferred regimen for suppressive therapy for TE is sulfadiazine plus pyrimethamine and leucovorin (AI*).

Rating of Recommendations: $\mathrm{A}=$ Strong; $\mathrm{B}=$ Moderate C $=$ Optional

Rating of Evidence: I = One or more randomized trials in children ${ }^{\dagger}$ with clinical outcomes and/or validated endpoints; $\mathrm{I}^{*}$ $=$ One or more randomized trials in adults with clinical outcomes and/or validated laboratory endpoints with accompanying data in children ${ }^{\dagger}$ from one or more well-designed, nonrandomized trials or observational cohort studies with long-term clinical outcomes; II = One or more well-designed, nonrandomized trials or observational cohort studies in children ${ }^{\dagger}$ with long-term outcomes; II* $=$ One or more well-designed, nonrandomized trials or observational studies in adults with longterm clinical outcomes with accompanying data in children ${ }^{\dagger}$ from one or more similar nonrandomized trials or cohort studies with clinical outcome data; III = Expert opinion †

Studies that include children or children/adolescents, but not studies limited to post-pubertal adolescents

\section{Epidemiology}

The major mode of transmission of Toxoplasma gondii infection to infants and young children is congenital, occurring almost exclusively in neonates born to women who sustain primary Toxoplasma infection during pregnancy. The estimated incidence of congenital toxoplasmosis in the United States is one case per 1,000 to 12,000 live-born infants. ${ }^{1,2}$ The 
seroprevalence of $T$. gondii in U.S.-born individuals aged 12 to 49 years declined from 14.1\% in the National Health and Nutrition Examination Survey from 1988 to 1994 to 9.0\% from 1999 to $2004 .{ }^{3}$ Older children, adolescents, and adults typically acquire Toxoplasma infection by eating undercooked meat that contains parasitic cysts or by unintentionally ingesting sporulated oocysts from cat feces in soil or contaminated food or water. ${ }^{4}$ In the United States, eating raw shellfish including oysters, clams, and mussels was recently identified as a novel risk factor for acute infection. ${ }^{5}$ Cats are the only definitive host for $T$. gondii. However, cats excrete oocysts in their feces only transiently after initial infection, and most studies have failed to show a correlation between cat ownership and Toxoplasma infection in humans. Indeed, Toxoplasma infection in humans in the United States has declined despite increased cat ownership. ${ }^{4}$

The overall risk of maternal-fetal transmission in HIV-uninfected women who acquire primary Toxoplasma infection during pregnancy is $29 \%$ (95\% confidence interval [CI], $25 \%-33 \%$ ), with variation depending upon the trimester during which primary maternal infection occurs. ${ }^{6}$ The risk of congenital infection is low among infants born to women who become infected during the first trimester (range: $2 \%-6 \%$ ) but increases sharply thereafter, with a risk as high as $81 \%$ in women who become infected during the last few weeks of pregnancy. 6,7 Infection of the fetus in early gestation usually results in more severe disease than does infection late in gestation.

The prevalence of latent Toxoplasma infection in HIV-infected and HIV-uninfected women in the United States was assessed in a cross-sectional study of 2,525 non-pregnant women enrolled in the Women's Interagency Health Study. ${ }^{8}$ The prevalence of Toxoplasma seropositivity was $15 \%$ and did not differ by HIV infection status. The overall rate of mother-to-child transmission (MTCT) of Toxoplasma in HIV-infected pregnant women is unknown; however, a few cases of MTCT of Toxoplasma in HIV-infected women have been reported. ${ }^{9-13}$ HIV-infected women may be at increased risk of transmitting $T$. gondii to their fetuses, and serologic testing for Toxoplasma should be performed on all HIV-infected pregnant women. Prenatal transmission of $T$. gondii is rare from women without HIV infection who acquired chronic Toxoplasma infection before pregnancy. ${ }^{14}$ However, with HIV coinfection, perinatal transmission of Toxoplasma has been observed in women with chronic Toxoplasma infection (transmission rate: $<4 \%$ ), presumably because of reactivation of replication of the organism in women who are severely immunosuppressed. ${ }^{9-12}$

Central nervous system (CNS) infection with $T$. gondii was reported as an AIDS-indicator condition in $<1 \%$ of pediatric AIDS cases before the advent of combination antiretroviral therapy (cART). ${ }^{15}$ Since then, this condition is rarely encountered in HIV-infected U.S. children. CNS toxoplasmosis occurred in 5 of 2,767 (0.2\%) HIV-infected children enrolled in the long-term follow-up study Pediatric AIDS Clinical Trials Group 219c since cART has been available. ${ }^{16}$ Infection is considered to have occurred in utero in most cases of Toxoplasma encephalitis (TE) seen in HIV-infected children.

More rarely, it has been reported in older HIV-infected children, who presumably had primary acquired toxoplasmosis. ${ }^{17-19}$ As in adults, the greatest risk is among severely immunosuppressed children (i.e., CD4 T lymphocyte [CD4] cell count $<50$ cells $/ \mathrm{mm}^{3}$ ). 


\section{Clinical Manifestations}

In studies of non-immunocompromised infants with congenital toxoplasmosis, most infants (70\%-90\%) are asymptomatic at birth. However, most asymptomatic children develop late sequelae (i.e., retinitis, visual impairment, and intellectual or neurologic impairment), with onset of symptoms ranging from several months to years after birth. Symptoms in newborns take either of two presentations: generalized disease or predominantly neurologic disease. Symptoms can include maculopapular rash; generalized lymphadenopathy; hepatosplenomegaly; jaundice; hematologic abnormalities including anemia, thrombocytopenia, and neutropenia; and substantial CNS disease including hydrocephalus, intracerebral calcification, microcephaly, chorioretinitis, and seizures. ${ }^{20}$

Toxoplasmosis acquired after birth most often is initially asymptomatic. When symptoms occur, they are frequently nonspecific and can include malaise, fever, sore throat, myalgia, lymphadenopathy (cervical), and a mononucleosis-like syndrome featuring a maculopapular rash and hepatosplenomegaly. ${ }^{21}$

TE should be considered in all HIV-infected children with new neurologic findings, but especially those with severe immunosuppression. Although focal findings are typical, the initial presentation can vary and reflect diffuse CNS disease. Generalized symptoms include fever, reduced alertness, and seizures.

Isolated ocular toxoplasmosis is rare in immunocompromised children and usually occurs in association with CNS infection. As a result, a neurologic examination is indicated for children in whom Toxoplasma chorioretinitis is diagnosed. Ocular toxoplasmosis appears as white retinal lesions with little associated hemorrhage; visual loss can occur initially.

Less frequent presentations in HIV-infected children with reactivated chronic toxoplasmosis include systemic toxoplasmosis, pneumonitis, hepatitis, and cardiomyopathy/ myocarditis. ${ }^{12,22}$

\section{Diagnosis}

All infants whose mothers are both HIV-infected and seropositive for Toxoplasma should be evaluated for congenital toxoplasmosis (AIII). ${ }^{23}$ Congenital toxoplasmosis can be diagnosed by enzyme-linked immunoassay or an immunosorbent assay to detect Toxoplasma-specific immunoglobulin $\mathrm{M}(\operatorname{IgM}), \operatorname{Ig} \mathrm{A}$, or $\operatorname{IgE}$ in neonatal serum within the first 6 months of life or persistence of specific immunoglobulin $\mathrm{G}$ antibody beyond age 12 months. ${ }^{24-28}$ IgA may be more sensitive for detecting congenital infection than IgM or IgE. ${ }^{25}$ However, approximately $20 \%$ to $30 \%$ of infants with congenital toxoplasmosis will not be identified during the neonatal period with IgA or IgM assays. ${ }^{26}$

Serologic testing is the major method of diagnosis, but interpretation of assays often is confusing and difficult. When considering a diagnosis of congenital toxoplasmosis, specialized reference laboratories can perform serology, isolation of organisms and polymerase chain reaction (PCR) and can offer assistance in interpreting results. ${ }^{25,28}$ 
Additional methods that can be used to diagnose infection in the newborn include isolation of the Toxoplasma parasite by mouse inoculation or inoculation in tissue cultures of cerebrospinal fluid (CSF), urine, placental tissue, amniotic fluid, or infant blood. T. gondii DNA can be detected by PCR performed on clinical specimens (e.g., white blood cells, CSF, amniotic fluid, tissue) in a reference laboratory. ${ }^{25,26}$ The following evaluation should be undertaken for all newborns in whom a diagnosis of toxoplasmosis is suspected: ophthalmologic, auditory, and neurologic examinations; lumbar puncture; and imaging of the head (either CT or magnetic resonance imaging [MRI] scans) to determine whether hydrocephalus or calcifications are present.

CNS toxoplasmosis is presumptively diagnosed on the basis of clinical symptoms, serologic evidence of infection, and presence of a space-occupying lesion on imaging studies of the brain. ${ }^{29} \mathrm{TE}$ rarely has been reported in individuals without Toxoplasma-specific IgG antibodies; therefore, negative serology does not definitively exclude that diagnosis. Brain computer tomography (CT) that demonstrates multiple, bilateral, ring-enhancing lesions, especially in the basal ganglia and cerebral corticomedullary junction, would be typical of TE. Calcifications are more typical in congenital toxoplasmosis than in TE seen later in life. Magnetic resonance imaging (MRI) is more sensitive and will confirm basal ganglia lesions in most patients. ${ }^{30}$ F-fluoro-2-deoxyglucose-positive emission tomography reportedly is helpful in adults in distinguishing Toxoplasma abscesses from primary CNS lymphoma, but the accuracy is not high, and this test is not widely available.

Definitive diagnosis of TE requires histologic or cytologic confirmation by brain biopsy, which may demonstrate leptomeningeal inflammation, microglial nodules, gliosis, and Toxoplasma cysts. Brain biopsy is reserved by some experts for patients who do not respond to specific therapy.

\section{Prevention Recommendations}

Preventing Exposure-All HIV-infected children and adolescents and their caregivers should be counseled about sources of $T$. gondii infection. They should be advised not to eat raw or undercooked meat, including undercooked lamb, beef, pork, or venison (BIII). All meat (lamb, beef, and pork) should be cooked to an internal temperature of $145^{\circ} \mathrm{F}$ for 3 minutes. ${ }^{31}$ However, a study has found that $T$. gondii can survive at $64^{\circ} \mathrm{C}\left(147.2^{\circ} \mathrm{F}\right)$ for 3 minutes, so higher temperatures than this seem best for immunosuppressed patients. ${ }^{32}$ Ground meat and wild game meat should be cooked to $71^{\circ} \mathrm{C}\left(160^{\circ} \mathrm{F}\right)$. Poultry should be cooked to $74^{\circ} \mathrm{C}\left(165^{\circ} \mathrm{F}\right)$. Hands should be washed after contact with raw meat and after gardening or other contact with soil; in addition, fruits and vegetables should be washed well before being eaten raw (BIII). Stray cats should not be handled or adopted; a cat already in the household should be kept inside and the litter box changed daily, preferably by an HIVuninfected individual who is not pregnant (BIII). Cats should be fed only canned or dried commercial food or well-cooked table food, not raw or undercooked meats (BIII). Patients need not be advised to part with their cats or to have their cats tested for toxoplasmosis (AII). 
Preventing Disease-In the United States, routine Toxoplasma serologic screening of HIV-infected children whose mothers do not have toxoplasmosis is not recommended because of its low incidence. However, in regions with high incidence of Toxoplasma infection ( $\geq 1 \%$ per year), or for children immigrating from such regions, serologic testing can be selectively considered for HIV-infected children aged $>12$ months (CIII). HIVinfected adolescents without previous Toxoplasma infection should undergo serologic testing (CIII). Toxoplasmaseronegative adults and adolescents who are not taking Pneumocystis pneumonia (PCP) prophylaxis known to be active against TE should be retested for IgG antibody to Toxoplasma if their CD4 cell counts decline to $<100$ cells $/ \mathrm{mm}^{3}$ to determine whether they have seroconverted to Toxoplasma.

Toxoplasma-seropositive adolescents and adults who have CD4 cell counts $<100 \mathrm{cells} / \mathrm{mm}^{3}$ should be given prophylaxis against TE. ${ }^{33}$ Specific levels of immunosuppression that increase the risk of TE in children are less well defined. Toxoplasma-seropositive children with CD4 percentages $<15 \%$ should be given prophylaxis against TE (AIII). For children aged $\ 6$ years, the same absolute CD4 count level used for HIV-infected adults can be used (AIII).

In HIV-infected adolescents and adults, the double-strength-tablet daily dose of trimethoprimsulfamethoxazole (TMP-SMX) recommended as the preferred regimen for PCP prophylaxis is effective TE prophylaxis (AII). ${ }^{33}$ Data from case series in children and from trials in adults support this as the preferred regimen in children using age-based dosing (See Table: Dosing Recommendations for the Prevention and Treatment of Toxoplasma gondii) (BIII). TMP-SMX, one double-strength tablet 3 times weekly (or 3 consecutive days a week), is an alternative (BIII). If patients cannot tolerate TMP-SMX, the recommended alternative is dapsone-pyrimethamine, which also is effective against PCP (BI*). ${ }^{34,35}$ Atovaquone with or without pyrimethamine also can be considered (CIII). Single-drug prophylaxis with dapsone, pyrimethamine, azithromycin, or clarithromycin cannot be recommended (AIII). Aerosolized pentamidine does not protect against TE and is not recommended. ${ }^{33,36}$ Severely immunosuppressed children who are not receiving TMP-SMX or atovaquone who are seropositive for Toxoplasma should be given prophylaxis for both PCP and toxoplasmosis (i.e., dapsone plus pyrimethamine) (BIII).

Discontinuing Primary Prophylaxis-Multiple observational studies ${ }^{37-39}$ and two randomized trials ${ }^{40,41}$ have reported that primary prophylaxis can be discontinued with minimal risk of TE in patients who have responded to cART with an increase in CD4 cell count to $\geq 200$ cells $/ \mathrm{mm}^{3}$ for $>6$ months. Although patients with CD4 cell counts of $<100$ cells $/ \mathrm{mm}^{3}$ are at greatest risk of TE, the risk of TE when CD4 cell counts increase to 100 to 200 cells $/ \mathrm{mm}^{3}$ has not been studied as rigorously as an increase to $>200$ cells $/ \mathrm{mm}^{3}$. Thus, the recommendation for adults and adolescents specifies discontinuing prophylaxis after an increase to $>200$ cells $/ \mathrm{mm}^{3}$. Discontinuing primary TE prophylaxis when CD4 cell counts have increased to $>200$ cells $/ \mathrm{mm}^{3}$ is recommended because prophylaxis adds limited disease prevention for toxoplasmosis and because discontinuing drugs reduces pill burden, the potential for drug toxicity, drug interactions, selection of drug-resistant pathogens, and cost. Data do not exist on the safety of discontinuing primary TE prophylaxis for HIV-infected 
children whose immunologic status improves on cART. Data on adults suggest discontinuation of TMP-SMX may be safe once a child responds to cART with a sustained rise in CD4 percentage above 15\%; for children aged $\searrow 6$ years, the same CD4 cell count used for HIV-infected adults can be used (BIII). A sustained response in children has been defined as a CD4 count or percentage above the threshold level for $>3$ consecutive months after receiving cART for $>6$ months.

Prophylaxis should be reintroduced in HIV-infected adults (AIII), adolescents (AIII), and children $\Varangle 6$ years old (BIII) if the CD4 cell count decreases to $<100$ to 200 cells $/ \mathrm{mm}^{3}$ or the CD4 percentage falls below 15\% for HIV-infected children aged $<6$ years (BIII).

\section{Treatment Recommendations}

Treating Disease-Pregnant women with suspected or confirmed primary toxoplasmosis and newborns with possible or documented congenital toxoplasmosis should be managed in consultation with an appropriate infectious disease specialist. Although controversy exists about the efficacy of treating pregnant women who have acute toxoplasmosis in an attempt to prevent infection of the fetus, ${ }^{42}$ most experts would recommend such therapy (BII) ${ }^{23}$ Empiric therapy should be strongly considered for newborns of HIV-infected mothers who had symptomatic or asymptomatic primary Toxoplasma infection during pregnancy, regardless of whether treatment was administered during pregnancy (BIII).

The preferred treatment for congenital toxoplasmosis is pyrimethamine combined with sulfadiazine, with supplementary leucovorin (folinic acid) to minimize pyrimethamineassociated hematologic toxicity (AII). ${ }^{20,43}$ Although the optimal duration of therapy is undefined, the recommended duration of treatment for congenital toxoplasmosis in HIVuninfected infants is 12 months (AII). ${ }^{43}$ Older HIV-infected children with acquired CNS, ocular, or systemic toxoplasmosis should be treated with pyrimethamine and leucovorin plus sulfadiazine (AI*). Acute therapy should be continued for 6 weeks, assuming clinical and radiologic improvement (BII*). Longer courses of treatment may be required for extensive disease or poor response after 6 weeks. The primary alternative for sulfadiazine in patients who develop sulfonamide hypersensitivity is clindamycin, administered with pyrimethamine and leucovorin $\left(\mathbf{A I}^{*}\right)$. Azithromycin instead of clindamycin also has been used with pyrimethamine and leucovorin in sulfa-allergic adults, but this regimen has not been studied in children. Extrapolation of doses used in adults corresponds to a dose of $20 \mathrm{mg} / \mathrm{kg}$ given every 24 hours (maximum 1,000 mg) but this dose has not been evaluated in children.

Another alternative in adults is atovaquone plus pyrimethamine and leucovorin, or atovaquone with sulfadiazine alone, or atovaquone as a single agent in patients intolerant to both pyrimethamine and sulfadiazine; however, these regimens have not been studied in children (BII*). In adults, atovaquone is dosed at twice the total daily dose used for PCP prophylaxis and is divided into four doses per day, but such dosing for treatment of acquired toxoplasmosis in children has not been evaluated. In a small (77 subjects) randomized trial in adults, TMP-SMX was reported to be effective and better tolerated than pyrimethaminesulfadiazine. ${ }^{44}$ Others have reported similar efficacy in open-label observational studies. ${ }^{45}$ However, this has not yet been studied in children. 
For isolated ocular toxoplasmosis in immunocompetent hosts, TMP-SMX alone is as effective as pyrimethamine-sulfadiazine. ${ }^{46}$ However, these data have not been duplicated in HIV-infected patients; therefore, this regimen cannot be recommended for this group of patients.

Based upon treatment of congenital toxoplasmosis in HIV-uninfected children, corticosteroids such as dexamethasone and prednisone are recommended for all HIVinfected children with CNS disease when CSF protein is highly elevated (i.e., >1,000 $\mathrm{mg} / \mathrm{dL}$ ) or who have focal lesions with substantial mass effects (BIII). Because of the potential immunosuppressive effects of steroids, they should be discontinued as soon as possible.

Anticonvulsants should be given to children with TE who have a history of seizures (AIII) but should not be administered prophylactically to children without a history of seizures (BIII). Anticonvulsants, if administered, should be continued at least through acute therapy.

Although the initiation of cART aids in the treatment of many opportunistic infections and malignancies, it has not been definitively shown to improve the outcome of TE therapy.

Monitoring and Adverse Events, Including IRIS—Children with TE should be routinely monitored for clinical and radiologic improvement and for adverse effects of treatment; changes in antibody titers are not useful for monitoring responses to therapy.

Toxoplasmosis-associated immune reconstitution inflammatory syndrome (IRIS) has been described rarely in HIV-infected adults and has not been described in HIV-infected children, although it could presumably occur. ${ }^{47,48}$ IRIS in HIV-infected pregnant women may pose additional risk to the fetuses ${ }^{49}$ although any unique risk for pregnant women co-infected with HIV and Toxoplasma has not been defined.

Pyrimethamine can be associated with rash (including Stevens-Johnson syndrome) and nausea. The primary toxicity of pyrimethamine is reversible bone marrow suppression (i.e., neutropenia, anemia, and thrombocytopenia). A complete blood count should be performed at least weekly in children who are on daily pyrimethamine and at least monthly in those on less-than-daily dosing (AIII). Leucovorin (folinic acid) always should be administered with pyrimethamine; increased doses of leucovorin may be required in the event of marrow suppression. Because of the long half-life of pyrimethamine, leucovorin should be continued 1 week after pyrimethamine has been discontinued.

Adverse effects of sulfadiazine include rash, fever, leukopenia, hepatitis, gastrointestinal (GI) symptoms (e.g., nausea, vomiting, diarrhea), and crystalluria. Clindamycin can be associated with fever, rash, and GI symptoms (e.g., nausea; vomiting, and diarrhea, and including pseudomembranous colitis) and hepatotoxicity.

Drug interactions between anticonvulsant and antiretroviral drugs should be evaluated. Patients receiving corticosteroids should be closely monitored for development of other opportunistic infections. 
Managing Treatment Failure-Brain biopsy should be considered in the event of early clinical or radiologic neurologic deterioration despite adequate empiric treatment or in children who do not clinically respond to anti-Toxoplasma therapy after 10 to 14 days. In children who undergo brain biopsy and have confirmed histopathologic evidence of TE despite treatment, a switch to an alternative regimen as previously described should be considered (BIII).

Preventing Recurrence-Patients who have completed initial therapy for acquired TE should be given suppressive therapy (i.e., secondary prophylaxis or chronic maintenance therapy) (AI*) ${ }^{50,51}$ until immune reconstitution occurs with cART. The combination of pyrimethamine, sulfadiazine, and leucovorin is highly effective for this purpose (AI*). A commonly used regimen for patients who cannot tolerate sulfa drugs is pyrimethamine plus clindamycin with leucovorin (BI*); however, only the combination of pyrimethamine plus sulfadiazine provides protection against PCP as well. Data on adults indicate atovaquone with or without pyrimethamine also can be considered for children (CIII). Limited data support the use of TMP-SMX for secondary prophylaxis $;{ }^{52}$ this regimen should be used only for patients who do not tolerate pyrimethamine plus sulfadiazine or pyrimethamine plus clindamycin (CIII).

Discontinuing Secondary Prophylaxis-Adults and adolescents receiving secondary prophylaxis for acquired TE are at low risk of recurrence of TE when they have successfully completed their initial therapy, continue to have no signs or symptoms of TE, and have a sustained increase in CD4 cell count of $>200$ cells $/ \mathrm{mm}^{3}$ after cART (i.e., $>6$ months). ${ }^{38,39,41,53,54}$ Discontinuing chronic maintenance therapy in HIV-infected adolescents and adults who meet these criteria is a reasonable consideration. The highest risk of relapse appears to occur within the first 6 months after stopping secondary prophylaxis. Some specialists would obtain an MRI of the brain as part of their evaluation to determine whether discontinuing therapy is appropriate. The safety of discontinuing secondary prophylaxis after immune reconstitution with cART in children has not been studied extensively. However, given the data in adults, clinicians caring for HIV-infected children aged 1 to $<6$ years can consider discontinuing secondary prophylaxis against $T$. gondii after they have completed TE therapy and $\succ$ months of stable cART and are asymptomatic and once the CD4 percentage has risen to $\geq 15 \%$ for $>6$ consecutive months (BIII). For children aged $\succ 6$ years, the same CD4 cell count used in adults (CD4 count $>200$ cells $/ \mathrm{mm}^{3}$ ) also can be used (BIII). Prophylaxis should be re-instituted if these parameters are not met.

\section{References}

1. Guerina NG, Hsu HW, Meissner HC, et al. Neonatal serologic screening and early treatment for congenital Toxoplasma gondii infection. The New England Regional Toxoplasma Working Group. N Engl J Med. 1994 Jun 30; 330(26):1858-1863. Available at http://www.ncbi.nlm.nih.gov/ pubmed/7818637. [PubMed: 7818637]

2. Jara M, Hsu HW, Eaton RB, Demaria A Jr. Epidemiology of congenital toxoplasmosis identified by population-based newborn screening in Massachusetts. Pediatr Infect Dis J. 2001 Dec; 20(12): 1132-1135. Available at http://www.ncbi.nlm.nih.gov/pubmed/11740319. [PubMed: 11740319] 
3. Smith KL, Wilson M, Hightower AW, et al. Prevalence of Toxoplasma gondii antibodies in US military recruits in 1989: comparison with data published in 1965. Clin Infect Dis. 1996 Nov; 23(5): 1182-1183. Available at http://www.ncbi.nlm.nih.gov/pubmed/8922828. [PubMed: 8922828]

4. Jones JL, Kruszon-Moran D, Wilson M, McQuillan G, Navin T, McAuley JB. Toxoplasma gondii infection in the United States: seroprevalence and risk factors. Am J Epidemiol. 2001 Aug 15; 154(4):357-365. Available at http://www.ncbi.nlm.nih.gov/pubmed/11495859. [PubMed: 11495859]

5. Jones JL, Dargelas V, Roberts J, Press C, Remington JS, Montoya JG. Risk factors for Toxoplasma gondii infection in the United States. Clin Infect Dis. 2009 Sep 15; 49(6):878-884. Available at http://www.ncbi.nlm.nih.gov/pubmed/19663709. [PubMed: 19663709]

6. Dunn D, Wallon M, Peyron F, Petersen E, Peckham C, Gilbert R. Mother-to-child transmission of toxoplasmosis: risk estimates for clinical counselling. Lancet. 1999 May 29; 353(9167):1829-1833. Available at http://www.ncbi.nlm.nih.gov/pubmed/10359407. [PubMed: 10359407]

7. Montoya JG. Laboratory diagnosis of Toxoplasma gondii infection and toxoplasmosis. J Infect Dis. 2002 Feb 15; 185(Suppl 1):S73-S82. Available at http://www.ncbi.nlm.nih.gov/pubmed/11865443. [PubMed: 11865443]

8. Falusi O, French AL, Seaberg EC, et al. Prevalence and predictors of Toxoplasma seropositivity in women with and at risk for human immunodeficiency virus infection. Clin Infect Dis. 2002 Dec 1; 35(11):1414-1417. Available at http://www.ncbi.nlm.nih.gov/pubmed/12439806. [PubMed: 12439806]

9. Minkoff H, Remington JS, Holman S, Ramirez R, Goodwin S, Landesman S. Vertical transmission of toxoplasma by human immunodeficiency virus-infected women. Am J Obstet Gynecol. 1997 Mar; 176(3):555-559. Available at http://www.ncbi.nlm.nih.gov/pubmed/9077606. [PubMed: 9077606]

10. Dunn D, Newell ML, Gilbert R. Low risk of congenital toxoplasmosis in children born to women infected with human immunodeficiency virus. Pediatr Infect Dis J. 1997 Jan.16(1):84. Available at http://www.ncbi.nlm.nih.gov/pubmed/9002113. [PubMed: 9002113]

11. Dunn D, Newell ML, Gilbert R. Low incidence of congenital toxoplasmosis in children born to women infected with human immunodeficiency virus. European Collaborative Study and Research Network on Congenital Toxoplasmosis. Eur J Obstet Gynecol Reprod Biol. 1996 Sep; 68(1-2):9396. Available at http://www.ncbi.nlm.nih.gov/pubmed/8886688. [PubMed: 8886688]

12. Mitchell CD, Erlich SS, Mastrucci MT, Hutto SC, Parks WP, Scott GB. Congenital toxoplasmosis occurring in infants perinatally infected with human immunodeficiency virus 1 . Pediatr Infect Dis J. 1990 Jul; 9(7):512-518. Available at http://www.ncbi.nlm.nih.gov/pubmed/2371084. [PubMed: 2371084]

13. D'Offizi G, Topino S, Anzidei G, Frigiotti D, Narciso P. Primary Toxoplasma gondii infection in a pregnant human immunodeficiency virus-infected woman. Pediatr Infect Dis J. 2002 Oct; 21(10): 981-982. Available at http://www.ncbi.nlm.nih.gov/pubmed/12400531. [PubMed: 12400531]

14. Vogel N, Kirisits M, Michael E, et al. Congenital toxoplasmosis transmitted from an immunologically competent mother infected before conception. Clin Infect Dis. 1996 Nov; 23(5): 1055-1060. Available at http://www.ncbi.nlm.nih.gov/pubmed/8922802. [PubMed: 8922802]

15. Centers for Disease Control and Prevention (CDC). HIV/AIDS surveillance report. 1996

16. Gona P, Van Dyke RB, Williams PL, et al. Incidence of opportunistic and other infections in HIVinfected children in the HAART era. JAMA. 2006 Jul 19; 296(3):292-300. Available at http:// www.ncbi.nlm.nih.gov/pubmed/16849662. [PubMed: 16849662]

17. Sobanjo A, Ferguson DJ, Gross U. Primary acquired toxoplasmosis in a five-year-old child with perinatal human immunodeficiency virus type 1 infection. Pediatr Infect Dis J. 1999 May; 18(5): 476-478. Available at http://www.ncbi.nlm.nih.gov/pubmed/10353529. [PubMed: 10353529]

18. Wahn V, Kramer HH, Voit T, Bruster HT, Scrampical B, Scheid A. Horizontal transmission of HIV infection between two siblings. Lancet. 1986 Sep 20.2(8508):694. Available at http:// www.ncbi.nlm.nih.gov/pubmed/2876170. [PubMed: 2876170]

19. King SM, Matlow A, Al-Hajjar S, et al. Toxoplasmic encephalitis in a child with HIV infectionUnited States. Pediatr AIDS and HIV Infect. Fetus to Adolesc. 1992; 3:242-244. 
20. McAuley J, Boyer KM, Patel D, et al. Early and longitudinal evaluations of treated infants and children and untreated historical patients with congenital toxoplasmosis: the Chicago Collaborative Treatment Trial. Clin Infect Dis. 1994 Jan; 18(1):38-72. Available at http:// www.ncbi.nlm.nih.gov/pubmed/8054436. [PubMed: 8054436]

21. McAuley, JB.; Boyer, KM. Feigin and Cherry's Textbook of Pediatric Infectious Diseases. 6th Ed. Amsterdam, The Netherlands: Elsevier Press; 2009. Toxoplasmosis.

22. Medlock MD, Tilleli JT, Pearl GS. Congenital cardiac toxoplasmosis in a newborn with acquired immunodeficiency syndrome. Pediatr Infect Dis J. 1990 Feb; 9(2):129-132. Available at http:// www.ncbi.nlm.nih.gov/pubmed/2314952. [PubMed: 2314952]

23. American Academy of Pediatrics. Red Book: 2009 Report of the Committee on Infectious Diseases. 28th ed. 28th ed. Elk Grove Village, IL: 2009.

24. Pinon JM, Dumon H, Chemla C, et al. Strategy for diagnosis of congenital toxoplasmosis: evaluation of methods comparing mothers and newborns and standard methods for postnatal detection of immunoglobulin G, M, and A antibodies. J Clin Microbiol. 2001 Jun; 39(6):22672271. Available at http://www.ncbi.nlm.nih.gov/pubmed/11376068. [PubMed: 11376068]

25. Wilson, M.; Jones, JL.; McAuley, JB. Toxoplasmaeds. In: Murray, PR.; Baron, EJ.; Jorgensen, JH.; Landry, ML.; Pfaller, MA., editors. Manual of clinical microbiology. 9th ed. St. Louis, MO: ASM Press; 2007. p. 2070-2081.

26. Montoya JG, Liesenfeld O. Toxoplasmosis. Lancet. 2004 Jun 12; 363(9425):1965-1976. Available at http://www.ncbi.nlm.nih.gov/pubmed/15194258. [PubMed: 15194258]

27. Wong SY, Hajdu MP, Ramirez R, Thulliez P, McLeod R, Remington JS. Role of specific immunoglobulin $\mathrm{E}$ in diagnosis of acute toxoplasma infection and toxoplasmosis. J Clin Microbiol. 1993 Nov; 31(11):2952-2959. Available at http://www.ncbi.nlm.nih.gov/pubmed/ 8263181. [PubMed: 8263181]

28. McAuley, JB.; Jones, JL., et al. Manual of Clinical Microbiology. 10th Ed. Washington, DC: American Society of Microbiology Press; 2011. Toxoplasma.

29. Portegies P, Solod L, Cinque P, et al. Guidelines for the diagnosis and management of neurological complications of HIV infection. Eur J Neurol. 2004 May; 11(5):297-304. Available at http:// www.ncbi.nlm.nih.gov/pubmed/15142222. [PubMed: 15142222]

30. Offiah CE, Turnbull IW. The imaging appearances of intracranial CNS infections in adult HIV and AIDS patients. Clin Radiol. 2006 May; 61(5):393-401. Available at http://www.ncbi.nlm.nih.gov/ pubmed/16679111. [PubMed: 16679111]

31. US Department of Agriculture. FoodSafety.gov: gateway to government food safety information. 2002 Available at FoodSafety.gov.

32. Dubey JP, Kotula AW, Sharar A, Andrews CD, Lindsay DS. Effect of high temperature on infectivity of Toxoplasma gondii tissue cysts in pork. The Journal of parasitology. 1990 Apr; 76(2):201-204. Available at http://www.ncbi.nlm.nih.gov/pubmed/2319420. [PubMed: 2319420]

33. Carr A, Tindall B, Brew BJ, et al. Low-dose trimethoprim-sulfamethoxazole prophylaxis for toxoplasmic encephalitis in patients with AIDS. Ann Intern Med. 1992 Jul 15; 117(2):106-111. Available at http://www.ncbi.nlm.nih.gov/pubmed/1351371. [PubMed: 1351371]

34. Podzamczer D, Salazar A, Jimenez J, et al. Intermittent trimethoprim-sulfamethoxazole compared with dapsonepyrimethamine for the simultaneous primary prophylaxis of Pneumocystis pneumonia and toxoplasmosis in patients infected with HIV. Ann Intern Med. 1995 May 15; 122(10):755-761. Available at http://www.ncbi.nlm.nih.gov/pubmed/7717598. [PubMed: 7717598]

35. Opravil M, Hirschel B, Lazzarin A, et al. Once-weekly administration of dapsone/pyrimethamine vs. aerosolized pentamidine as combined prophylaxis for Pneumocystis carinii pneumonia and toxoplasmic encephalitis in human immunodeficiency virus-infected patients. Clin Infect Dis. 1995 Mar; 20(3):531-541. Available at http://www.ncbi.nlm.nih.gov/pubmed/7756472. [PubMed: 7756472]

36. Bozzette SA, Finkelstein DM, Spector SA, et al. A randomized trial of three antipneumocystis agents in patients with advanced human immunodeficiency virus infection. NIAID AIDS Clinical Trials Group. N Engl J Med. 1995 Mar 16; 332(11):693-699. Available at http:// www.ncbi.nlm.nih.gov/pubmed/7854375. [PubMed: 7854375] 
37. Dworkin MS, Hanson DL, Kaplan JE, Jones JL, Ward JW. Risk for preventable opportunistic infections in persons with AIDS after antiretroviral therapy increases CD4+ T lymphocyte counts above prophylaxis thresholds. J Infect Dis. 2000 Aug; 182(2):611-615. Available at http:// www.ncbi.nlm.nih.gov/pubmed/10915098. [PubMed: 10915098]

38. Kirk O, Lundgren JD, Pedersen C, Nielsen H, Gerstoft J. Can chemoprophylaxis against opportunistic infections be discontinued after an increase in CD4 cells induced by highly active antiretroviral therapy? AIDS. 1999 Sep 10; 13(13):1647-1651. Available at http:// www.ncbi.nlm.nih.gov/pubmed/10509565. [PubMed: 10509565]

39. Furrer H, Opravil M, Bernasconi E, Telenti A, Egger M. Stopping primary prophylaxis in HIV-1infected patients at high risk of toxoplasma encephalitis. Swiss HIV Cohort Study. Lancet. 2000 Jun 24; 355(9222):2217-2218. Available at http://www.ncbi.nlm.nih.gov/pubmed/10881897. [PubMed: 10881897]

40. Mussini C, Pezzotti P, Govoni A, et al. Discontinuation of primary prophylaxis for Pneumocystis carinii pneumonia and toxoplasmic encephalitis in human immunodeficiency virus type I-infected patients: the changes in opportunistic prophylaxis study. J Infect Dis. 2000 May; 181(5):16351642. Available at http://www.ncbi.nlm.nih.gov/pubmed/10823763. [PubMed: 10823763]

41. Miro JM, Lopez JC, Podzamczer D, et al. Discontinuation of primary and secondary Toxoplasma gondii prophylaxis is safe in HIV-infected patients after immunological restoration with highly active antiretroviral therapy: results of an open, randomized, multicenter clinical trial. Clin Infect Dis. 2006 Jul 1; 43(1):79-89. Available at http://www.ncbi.nlm.nih.gov/pubmed/16758422. [PubMed: 16758422]

42. group, Ss; Thiebaut, R.; Leproust, S.; Chene, G.; Gilbert, R. Effectiveness of prenatal treatment for congenital toxoplasmosis: a meta-analysis of individual patients' data. Lancet. 2007 Jan 13; 369(9556):115-122. Available at http://www.ncbi.nlm.nih.gov/pubmed/17223474. [PubMed: 17223474]

43. McLeod R, Boyer K, Karrison T, et al. Outcome of treatment for congenital toxoplasmosis, 19812004: the National Collaborative Chicago-Based, Congenital Toxoplasmosis Study. Clin Infect Dis. 2006 May 15; 42(10):1383-1394. Available at http://www.ncbi.nlm.nih.gov/pubmed/ 16619149. [PubMed: 16619149]

44. Torre D, Casari S, Speranza F, et al. Randomized trial of trimethoprim-sulfamethoxazole versus pyrimethamine-sulfadiazine for therapy of toxoplasmic encephalitis in patients with AIDS. Italian Collaborative Study Group. Antimicrob Agents Chemother. 1998 Jun; 42(6):1346-1349. Available at http://www.ncbi.nlm.nih.gov/pubmed/9624473. [PubMed: 9624473]

45. Beraud G, Pierre-Francois S, Theodose R, et al. Anicteric cholestasis among HIV infected patients with syphilis. Scand J Infect Dis. 2009; 41(6-7):524-527. Available at http:// www.ncbi.nlm.nih.gov/pubmed/19263273. [PubMed: 19263273]

46. Soheilian M, Sadoughi MM, Ghajarnia M, et al. Prospective randomized trial of trimethoprim/ sulfamethoxazole versus pyrimethamine and sulfadiazine in the treatment of ocular toxoplasmosis. Ophthalmology. 2005 Nov; 112(11):1876-1882. Available at http://www.ncbi.nlm.nih.gov/ pubmed/16171866. [PubMed: 16171866]

47. Lawn SD. Immune reconstitution disease associated with parasitic infections following initiation of antiretroviral therapy. Curr Opin Infect Dis. 2007 Oct; 20(5):482-488. Available at http:// www.ncbi.nlm.nih.gov/pubmed/17762781. [PubMed: 17762781]

48. Shah I. Immune Reconstitution Syndrome in HIV-1 infected children - a study from India. Indian J Pediatr. 2011 May; 78(5):540-543. Available at http://www.ncbi.nlm.nih.gov/pubmed/21203868. [PubMed: 21203868]

49. Caby F, Lemercier D, Coulomb A, et al. Fetal death as a result of placental immune reconstitution inflammatory syndrome. J Infect. $2010 \mathrm{Jul}$; 61(2):185-188. Available at http:// www.ncbi.nlm.nih.gov/pubmed/20361998. [PubMed: 20361998]

50. Katlama C, De Wit S, O'Doherty E, Van Glabeke M, Clumeck N. Pyrimethamine-clindamycin vs. pyrimethamine-sulfadiazine as acute and long-term therapy for toxoplasmic encephalitis in patients with AIDS. Clin Infect Dis. 1996 Feb; 22(2):268-275. Available at http:// www.ncbi.nlm.nih.gov/pubmed/8838183. [PubMed: 8838183]

51. Dannemann B, McCutchan JA, Israelski D, et al. Treatment of toxoplasmic encephalitis in patients with AIDS. A randomized trial comparing pyrimethamine plus clindamycin to pyrimethamine plus 
sulfadiazine. The California Collaborative Treatment Group. Ann Intern Med. 1992 Jan 1; 116(1): 33-43. Available at http://www.ncbi.nlm.nih.gov/pubmed/1727093. [PubMed: 1727093]

52. Duval X, Pajot O, Le Moing V, et al. Maintenance therapy with cotrimoxazole for toxoplasmic encephalitis in the era of highly active antiretroviral therapy. AIDS. 2004; 18:1342-1344. Available at http://www.ncbi.nlm.nih.gov/pubmed/15362670. [PubMed: 15362670]

53. Soriano V, Dona C, Rodriguez-Rosado R, Barreiro P, Gonzalez-Lahoz J. Discontinuation of secondary prophylaxis for opportunistic infections in HIV-infected patients receiving highly active antiretroviral therapy. AIDS. 2000 Mar 10; 14(4):383-386. Available at http:// www.ncbi.nlm.nih.gov/pubmed/10770540. [PubMed: 10770540]

54. Bertschy S, Opravil M, Cavassini M, et al. Discontinuation of maintenance therapy against toxoplasma encephalitis in AIDS patients with sustained response to anti-retroviral therapy. Clin Microbiol Infect. 2006 Jul; 12(7):666-671. Available at http://www.ncbi.nlm.nih.gov/pubmed/ 16774564. [PubMed: 16774564]

\section{Dosing Recommendations for the Prevention and Treatment of Toxoplasmosis}

\begin{tabular}{|c|c|c|c|}
\hline Indication & First Choice & Alternative & Comments/Special Issues \\
\hline Primary Prophylaxis & $\begin{array}{l}\text { TMP-SMX } 150 / 750 \mathrm{mg} / \mathrm{m}^{2} \\
\text { body surface area once daily } \\
\text { by mouth }\end{array}$ & 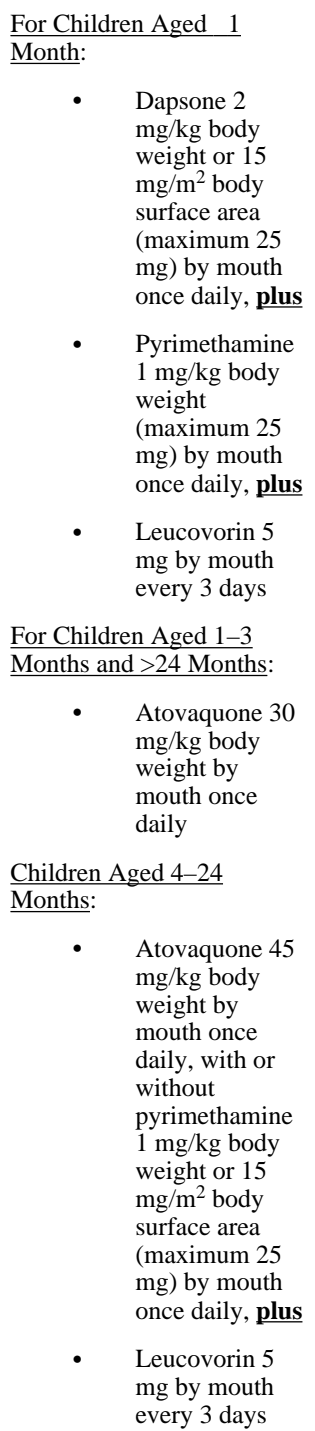 & 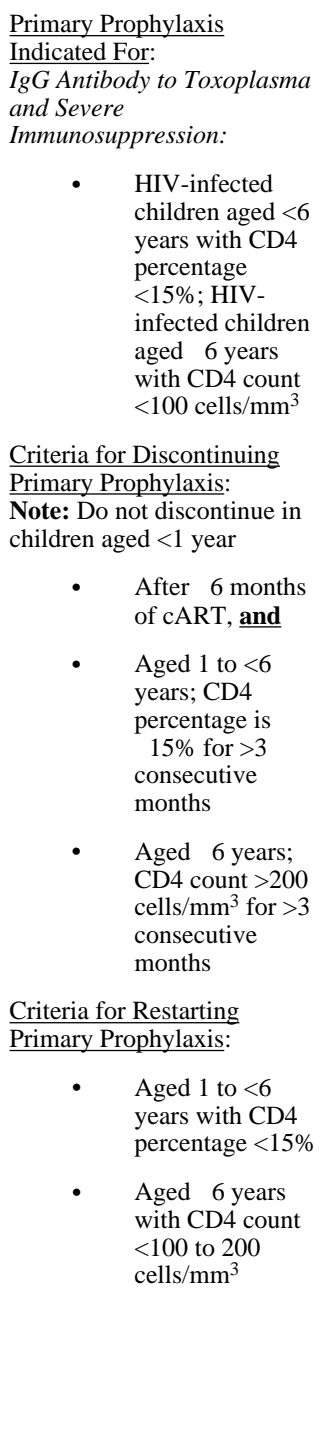 \\
\hline
\end{tabular}




\begin{tabular}{|c|c|c|c|}
\hline Indication & First Choice & Alternative & Comments/Special Issues \\
\hline & & 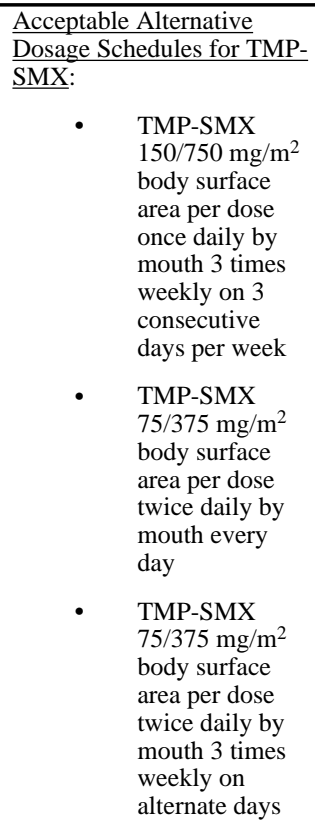 & \\
\hline $\begin{array}{l}\text { Secondary } \\
\text { Prophylaxis } \\
\text { (Suppressive } \\
\text { Therapy) }\end{array}$ & 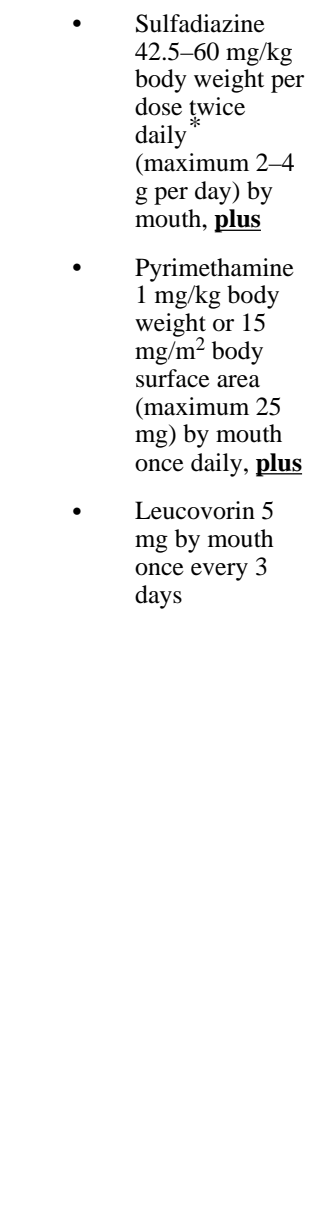 & 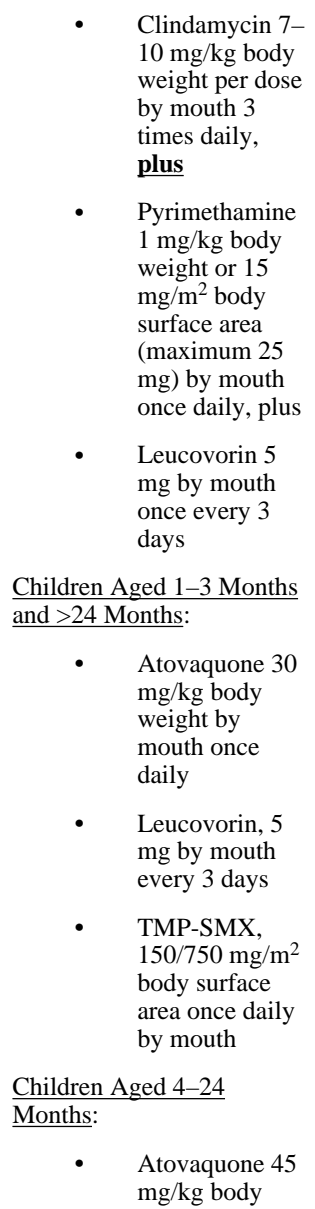 & 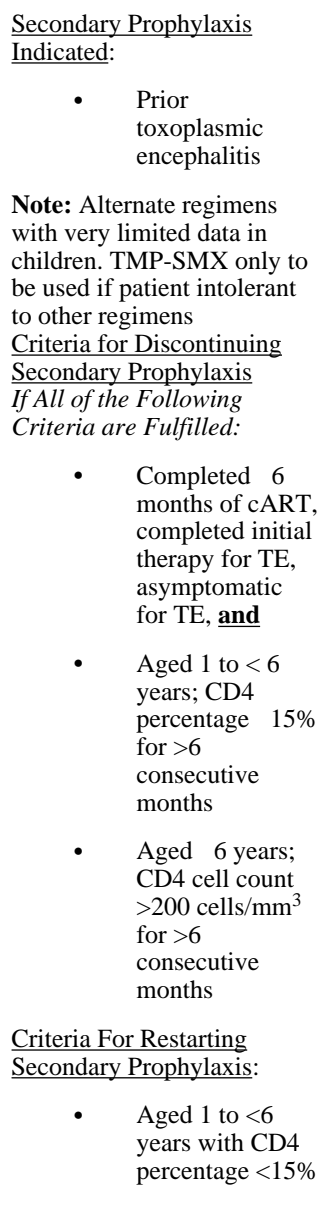 \\
\hline
\end{tabular}

Pediatr Infect Dis J. Author manuscript; available in PMC 2014 November 01. 


\begin{tabular}{|c|c|c|c|}
\hline Indication & First Choice & Alternative & Comments/Special Issues \\
\hline & & $\begin{array}{l}\text { weight by } \\
\text { mouth once } \\
\text { daily, with or } \\
\text { without } \\
\text { pyrimethamine } \\
1 \mathrm{mg} / \mathrm{kg} \text { body } \\
\text { weight or } 15 \\
\mathrm{mg} / \mathrm{m}^{2} \text { body } \\
\text { surface area } \\
\text { (maximum } 25 \\
\mathrm{mg} \text { ) by mouth } \\
\text { once daily, plus } \\
\text { Leucovorin, } 5 \\
\text { mg by mouth } \\
\text { every } 3 \text { days } \\
\text { TMP-SMX, } \\
150 / 750 \mathrm{mg} / \mathrm{m}^{2} \\
\text { body surface } \\
\text { area once daily } \\
\text { by mouth }\end{array}$ & $\begin{array}{l}\text { Aged } \ 6 \text { years } \\
\text { with CD4 cell } \\
\text { count }<200 \\
\text { cells } / \mathrm{mm}^{3}\end{array}$ \\
\hline Treatment & $\begin{array}{l}\text { Congenital Toxoplasmosis: } \\
\text { - } \\
\text { Pyrimethamine } \\
\text { loading dose-2 } \\
\text { mg/kg body } \\
\text { weight by } \\
\text { mouth once } \\
\text { daily for } 2 \text { days, } \\
\text { then } 1 \mathrm{mg} / \mathrm{kg} \\
\text { body weight by } \\
\text { mouth once } \\
\text { daily for } 2-6 \\
\text { months, then } 1 \\
\text { mg/kg body } \\
\text { weight by } \\
\text { mouth } 3 \text { times } \\
\text { weekly, plus } \\
\text { Leucovorin } \\
\text { (folinic acid) } 10 \\
\text { mg by mouth or } \\
\text { IM with each } \\
\text { dose of } \\
\text { pyrimethamine, } \\
\text { plus } \\
\text { - } \\
\text { Sulfadiazine } 50 \\
\text { mg/kg body } \\
\text { weight by } \\
\text { mouth twice } \\
\text { daily }\end{array}$ & $\begin{array}{ll}\text { For Sulfonamide-Intolerant } \\
\text { Patients: } & \\
& \text { Clindamycin 5- } \\
& 7.5 \mathrm{mg} / \mathrm{kg} \text { body } \\
& \text { weight } \\
& \text { (maximum 600 } \\
& \text { mg/dose) by } \\
& \text { mouth or IV per } \\
& \text { dose given 4 } \\
& \text { times a day can } \\
& \text { be substituted } \\
& \text { for sulfadiazine } \\
& \text { combined with } \\
& \text { pyrimethamine } \\
& \text { and leucovorin }\end{array}$ & $\begin{array}{l}\text { Congenital Toxoplasmosis: } \\
\text { For infants born } \\
\text { to mothers with } \\
\text { symptomatic } \\
\text { Toxoplasma } \\
\text { infection during } \\
\text { pregnancy, } \\
\text { empiric therapy } \\
\text { of the newborn } \\
\text { should be } \\
\text { strongly } \\
\text { considered } \\
\text { irrespective of } \\
\text { the mothers } \\
\text { treatment during } \\
\text { pregnancy. } \\
\text { Acquired Toxoplasmosis: } \\
\text { Pyrimethamine } \\
\text { Pse requires } \\
\text { CBC monitoring } \\
\text { at least weekly } \\
\text { while on daily } \\
\text { dosing and at } \\
\text { least monthly } \\
\text { while on less } \\
\text { than daily } \\
\text { dosing. } \\
\text { TMP-SMX- } \\
\text { TMP } 5 \text { mg/kg } \\
\text { body weight plus } \\
\text { SMX } 25 \text { mg/kg } \\
\text { body weight per } \\
\text { dose IV or by } \\
\text { mouth given } \\
\text { twice daily has } \\
\text { been used as an } \\
\text { alternative to } \\
\text { pyrimethamine- } \\
\text { sulfadiazine in } \\
\text { adults, but has } \\
\text { not been studied } \\
\text { in children. } \\
\text { Atovaquone (for } \\
\text { adults, } 1.5 \mathrm{~g} \text { by } \\
\text { mouth twice } \\
\text { daily-double } \\
\text { the prophylaxis }\end{array}$ \\
\hline
\end{tabular}




\begin{tabular}{|c|c|c|c|}
\hline Indication & First Choice & Alternative & Comments/Special Issues \\
\hline & 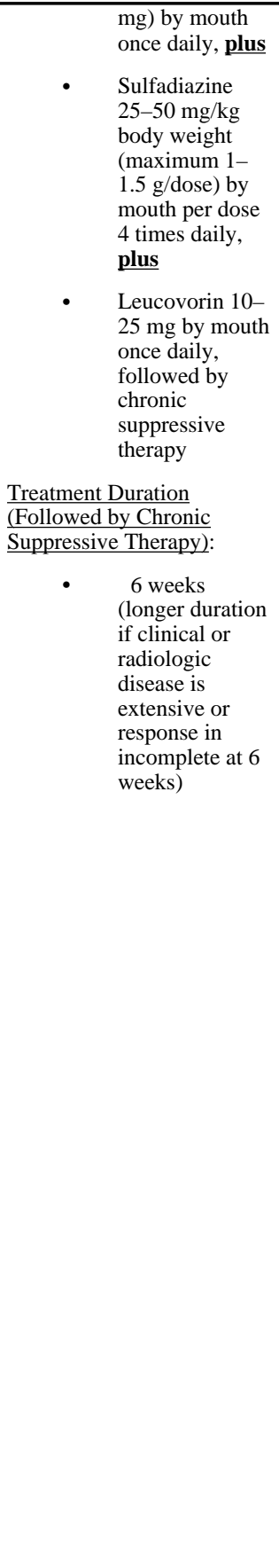 & & $\begin{array}{l}\text { dose) in } \\
\text { regimens } \\
\text { combined with } \\
\text { pyrimethamine/ } \\
\text { leucovorin, with } \\
\text { sulfadiazine } \\
\text { alone, or as a } \\
\text { single agent in } \\
\text { patients } \\
\text { intolerant to both } \\
\text { pyrimethamine } \\
\text { and sulfadiazine, } \\
\text { has been used in } \\
\text { adults, but these } \\
\text { regimens have } \\
\text { not been studied } \\
\text { in children. } \\
\text { Azithromycin } \\
\text { (for adults, } 900- \\
1,200 \text { mg } / \text { day, } \\
\text { corresponding to } \\
20 \text { mg/ kg/day in } \\
\text { children) has } \\
\text { also been used in } \\
\text { adults combined } \\
\text { with } \\
\text { pyrimethamine- } \\
\text { sulfadiazine, but } \\
\text { has not been } \\
\text { studied in } \\
\text { children. } \\
\text { Corticosteroids } \\
\text { (e.g., prednisone, } \\
\text { dexamethasone) } \\
\text { have been used } \\
\text { in children with } \\
\text { CNS disease } \\
\text { when CSF } \\
\text { protein is very } \\
\text { elevated (>1,000 } \\
\text { mg/dL) or there } \\
\text { are focal lesions } \\
\text { with significant } \\
\text { mass effects, } \\
\text { with } \\
\text { discontinuation } \\
\text { as soon as } \\
\text { clinically } \\
\text { feasible. } \\
\text { Anticonvulsants } \\
\text { should be } \\
\text { administered to } \\
\text { patients with a } \\
\text { history of } \\
\text { seizures and } \\
\text { continued } \\
\text { through the acute } \\
\text { treatment; but } \\
\text { should not be } \\
\text { used } \\
\text { prophylactically. }\end{array}$ \\
\hline
\end{tabular}

Note: Sulfadiazine may be given as $2-4$ equal doses per day as long as the total daily dose is $85-120 \mathrm{mg} / \mathrm{kg}$ body weight.

Key to Acronyms: $\mathrm{cART}=$ combination antiretroviral therapy; $\mathrm{CBC}=$ complete blood count; $\mathrm{CD} 4=\mathrm{CD} 4 \mathrm{~T}$ lymphocyte; $\mathrm{CNS}=$ central nervous system; $\mathrm{CSF}=$ cerebrospinal fluid; $\mathrm{IgG}=$ Immunoglobulin $\mathrm{G} ; \mathrm{IM}=$ intramuscular; $\mathrm{IV}=$ intravenous; $\mathrm{TE}=$ toxoplasmic encephalitis; TMP-SMX = trimethoprim-sulfamethoxazole 


\section{Varicella-Zoster Virus (Last updated November 6, 2013; last reviewed November 6, 2013)}

\begin{abstract}
Panel's Recommendations
- $\quad$ HIV-infected children and adults who have no evidence of immunity to varicella should avoid exposure to people with varicella or zoster (AIII). Household contacts of HIV-infected patients should receive varicella vaccine if they lack evidence of immunity to avoid the possibility of transmitting wild-type varicella-zoster virus (VZV) to their HIV-infected contacts (AIII).

- $\quad$ HIV-infected children aged 1 through 8 years without evidence of varicella immunity and whose CD4 T lymphocyte (CD4) cell counts are $\geq 15 \%$ should be considered for 2 doses of varicella vaccine, the first dose administered as early as age 12 to 15 months (or as soon as possible after the first birthday) and the second dose 3 months later (BII). Older children with comparable levels of immune function (i.e., CD4 cell counts $\geq 200$ cells $/ \mathrm{mm}^{3}$ ) who lack varicella immunity may be considered for 2 doses of varicella vaccine administered 3 months apart (BIII).
\end{abstract}

- $\quad$ Combination measles-mumps-rubella-varicella vaccine should not be administered to HIV-infected children (AIII).

- $\quad$ HIV-infected children with low CD4 percentages $(<15 \%)$ should not be vaccinated against varicella (AIII) Vaccination of such children can be safely undertaken after reconstitution of their immune systems (CD4 percentage $\geq 15 \%$ ) with combination antiretroviral therapy (cART) for at least 3 months (AII). Herpes zoster (HZ) vaccine should not be given to HIV-infected children (AIII).

- $\quad$ HIV-infected children and adolescents who:

1. lack evidence of immunity to varicella, and

2. have a non-transient exposure to a contact with varicella or herpes zoster

should receive VZV immunoglobulin prophylaxis as soon as possible (ideally within 96 hours but potentially beneficial up to 10 days) after the close contact (AII). Many experts limit this recommendation to varicella- or zoster-exposed HIV-infected children who are considered to be severely immunocompromised (i.e., CDC Immunologic Category 3) especially if they have high HIV viral loads and would be classified in CDC Clinical Category C (BIII). When passive immunization is impossible, some experts recommend prophylaxis with acyclovir beginning 7 to 10 days after exposure, while others consider it prudent to wait until the first appearance of rash to start acyclovir therapy in VZV-susceptible and VZVexposed, HIV-infected children (CIII).

- $\quad$ Acyclovir is the drug of choice for treating VZV infection in HIV-infected children (AI). Intravenous (IV) acyclovir is recommended for treating varicella in HIV-infected children with severe immunosuppression (i.e., CDC Immunologic Category 3) and those who have high fever, abdominal pain, respiratory symptoms, or numerous or deep, necrotic, or hemorrhagic skin lesions (AIII). Oral acyclovir should only be used to treat varicella in HIV-infected children who are in CDC Immunologic Category 1 or 2 and who have mild varicella disease (BIII).

- Acyclovir is the oral treatment of choice for zoster in HIV-infected children, given for 7 to 10 days, although longer durations of therapy should be considered if lesions are slow to resolve (AII*). Oral administration of acyclovir for $\mathrm{HZ}$ is considered safe for children with mild to moderate immune suppression (AII). Initial IV administration is recommended for HIV-infected children with severe immunosuppression (i.e., CDC Immunologic Category 3), extensive multidermatomal HZ, disseminated infection, visceral involvement, or otherwise complicated HZ (AII*). It can also be considered for trigeminal nerve or sacral dermatomal involvement. IV acyclovir should be continued until cutaneous lesions and visceral disease are clearly resolving (AIII), after which oral administration can be considered to complete the course of therapy-10 to 14 days in this situation (AIII).

- Recommended treatment for progressive outer retinal necrosis includes optimization of cART and IV antiVZV therapy that includes combinations of systemic antivirals (acyclovir or ganciclovir plus foscarnet), frequently with twice-weekly intravitreal injections of ganciclovir and/or foscarnet (AIII). Adjunctive retinal surgery is sometimes recommended, along with corticosteroids and/or low-dose aspirin (BIII). Acute retinal necrosis can be treated with IV acyclovir for 10 to 14 days, followed by prolonged (i.e., 4-6 weeks) oral treatment (AIII).

- $\quad$ Alternatives to oral acyclovir in older adolescents include valacyclovir and famciclovir (AI*).

- The treatment of choice for acyclovir-resistant VZV is IV foscarnet for 7 days (AII*) or until no new lesions for at least 48 hours (AIII).

Rating of Recommendations: $\mathrm{A}=$ Strong; $\mathrm{B}=$ Moderate $\mathrm{C}=$ Optional

Rating of Evidence: $\mathrm{I}=$ One or more randomized trials $\underline{\text { in children }}^{\dagger}$ with clinical outcomes and/or validated endpoints; I* $=$ One or more randomized trials in adults with clinical outcomes and/or validated laboratory endpoints with accompanying data in children ${ }^{\dagger}$ from one or more well-designed, nonrandomized trials or observational cohort studies with long-term clinical outcomes; II = One or more well-designed, nonrandomized trials or observational cohort studies in children ${ }^{\dagger}$ with 
long-term outcomes; $\mathrm{II}^{*}=$ One or more well-designed, nonrandomized trials or observational studies in adults with longterm clinical outcomes with accompanying data in children ${ }^{\dagger}$ from one or more similar nonrandomized trials or cohort studies with clinical outcome data; III = Expert opinion

${ }^{\dagger}$ Studies that include children or children/adolescents, but not studies limited to post-pubertal adolescents

\section{Epidemiology}

Varicella-zoster virus (VZV) infections are endemic worldwide. Prior to the universal administration of varicella vaccine, approximately 4 million cases of varicella occurred annually in the United States. The annual incidence in children aged $<10$ years was $9 \%$; by adulthood more than $95 \%$ of individuals had antibodies to VZV, indicating prior primary infection. ${ }^{1}$ In tropical and subtropical areas, varicella may be acquired later in childhood or early adulthood, but seroprevalence is high by age 30 . Because of universal vaccination in the United States, the incidence of varicella and its associated morbidity and mortality have decreased by $74 \%$ to more than $90 \% .^{2,3} \mathrm{VZV}$ is transmitted by an airborne route. ${ }^{4}$ It is highly contagious; clinical infection develops in about $80 \%$ of susceptible individuals exposed in a household. ${ }^{5}$ Second attacks of varicella are uncommon.

Vertical mother-to-child transmission (MTCT) of VZV can occur. However, because most pregnant women are immune, varicella complicating pregnancy is unusual. Congenital varicella syndrome (multiple anomalies) occurs in approximately $2 \%$ (95\% CI: $0 \%-5 \%$ ) of infants born to women who have varicella at 13 to 20 weeks of pregnancy, ${ }^{6,7}$ but is not seen in infants born to women who develop herpes zoster (HZ) during pregnancy. MTCT of VZV has not been reported in HIV-infected pregnant women who develop varicella.

VZV also can be transmitted to fetuses in late gestation, resulting in neonatal varicella. In mothers who develop varicella in the interval of 5 days before to 2 days after delivery, the attack rate for infants is approximately $20 \%$ and mortality, before the availability of antiviral therapy, was approximately $30 \% .{ }^{8}$ In comparison, if maternal varicella precedes delivery long enough to allow transfer of VZV antibodies across the placenta, infants can still develop varicella in the first 5 days of life, but it is rarely severe.

VZV causes both varicella (primary infection; chickenpox) and HZ, which represents reactivation of VZV that resides in a latent state in dorsal root and cranial sensory ganglia following varicella.

Once established, VZV latency persists for life, but reactivation to cause $\mathrm{HZ}$ occurs in $25 \%$ to $30 \%$ of people who had varicella. $\mathrm{HZ}$ is less contagious than varicella, but virus from $\mathrm{HZ}$ lesions can spread by direct contact or by an airborne route from immunocompetent and immunocompromised individuals to cause varicella in people who never had this infection. $\mathrm{HZ}$ occurs because the VZV-specific cellular immunity that is acquired with varicella and is needed to maintain latency declines with age. This same immunity is typically lost with HIV infection, explaining why $\mathrm{HZ}$ is common in HIV-infected people. ${ }^{9,10} \mathrm{HZ}$ was a very common complication in HIV-infected children in the era before combination antiretroviral therapy (cART) (approximately 10 cases/100 patient-years prior to 1996); the incidence of $\mathrm{HZ}$ remains at 2 to 3 cases per 100 patient-years in the cART era, which is 10 to 25 times higher than in the general population. ${ }^{11-13}$ Risk factors for development of $\mathrm{HZ}$ include low 
incident or nadir CD4 T lymphocyte (CD4) cell count/percentage; high HIV viral load; and acquisition of varicella when the CD4 percentage is $<15 \%$. ${ }^{13-15}$

As in adults, the frequency of $\mathrm{HZ}$ recurrences in children correlates inversely with the CD4 cell count. ${ }^{13,16}$ The incidence of $\mathrm{HZ}$ increases with age; this trend extends into adulthood, particularly in individuals younger than age 50 .

In addition to cART and immune reconstitution, another reason for the declining incidence of $\mathrm{HZ}$ in HIV-infected children in countries with varicella vaccination programs is that many received the licensed varicella vaccine. This is associated with a decrease in $\mathrm{HZ}$ in HIV-uninfected children. ${ }^{17} \mathrm{HZ}$ is also less common in vaccinated HIV-infected children compared with those who had wild-type infection. ${ }^{18}$

\section{Clinical Manifestations}

The incubation period for varicella ranges from 10 to 21 days (average: 14 days) in immunocompetent children. Varicella can be associated with a brief prodrome of malaise and fever, followed by skin lesions that are more numerous on the face and trunk than on the extremities. These evolve over 5 days through macular, papular, vesicular, and pustular stages, culminating in crusts. Combinations of these types of lesions are present simultaneously. Complications of varicella include superinfection of skin with bacterial pathogens, primarily staphylococci and streptococci. Clinically important systemic involvement can include neurologic manifestations, such as encephalitis, cerebellar ataxia, and transverse myelitis; hepatitis; pneumonia; and multiorgan failure with intravascular coagulation.

Varicella causes more morbidity in HIV-infected patients than in the general population. Initial reports of varicella in HIV-infected children suggested severe disease manifestations and chronic atypical skin lesions, ${ }^{19,20}$ but more recent studies support less complicated courses, particularly in children receiving cART or who have higher CD4 cell counts at the time of infection. ${ }^{14,21}$ However, the disease may last longer than normal, and the rate of complications is higher than in otherwise healthy children with varicella. ${ }^{15,22}$

Uncommonly, severely immunocompromised HIV-infected children can have persistent chronic infection, with continued appearance of new VZV lesions for $>1$ month after primary or recurrent infection..$^{20,23}$ The lesions are characteristically varicelliform at onset but evolve into non-healing ulcers or necrotic, crusted, and hyperkeratotic verrucous lesions. Chronic VZV was reported in 14\% of HIV-infected children with VZV in the pre-cART era, usually in children with low CD4 cell counts. ${ }^{16}$

The classical presentation of $\mathrm{HZ}$ is an often painful or pruritic, vesicular dermatomal rash. Typically pain precedes the rash by 2 to 3 days. Less typical rashes, similar to those described for chronic varicella, including rashes that extend beyond dermatomal boundaries or are bilaterally distributed or generalized, can occur during HZ in HIV-infected children. They often have multiple recurrent episodes of HZ. ${ }^{13,16}$ Disseminated zoster with multiorgan involvement can occur, with or without the typical rash of HZ. Encephalitis long after HZ, or without rash, has been reported in HIV-infected children. ${ }^{24}$ Ruling out herpes 
simplex virus infection, which can be confused with VZV skin manifestations, is important in evaluating HIV-infected children with possible $\mathrm{HZ}$ infection.

Retinitis is a complication of VZV infection in HIV-infected children and adolescents ${ }^{25,26}$ that can be confused with cytomegalovirus retinitis. ${ }^{27}$ Progressive outer retinal necrosis is a VZV-associated entity that typically occurs with CD4 cell counts $<50$ cells $/ \mathrm{mm}^{3}$ and is often associated with HZ. Acute retinal necrosis can occur in HIV-infected children at any CD4 cell count. A rapid decrease in visual acuity, or occurrence of red eye or eye pain, should result in an immediate consultation with an ophthalmologist for diagnosis and specific therapy.

\section{Diagnosis}

Typical presentations of varicella and $\mathrm{HZ}$ are readily diagnosed clinically. Laboratory methods are required for atypical presentations, prolonged course, and non-response to therapy. VZV DNA polymerase chain reaction (PCR) is the most sensitive and specific method for diagnosing a VZV infection. It can provide an etiologic diagnosis within 24 to 48 hours and some research laboratories can differentiate between wild-type and vaccine strain VZV. In addition to lesion specimens, PCR can be applied to blood, cerebrospinal fluid, and pharyngeal and conjunctival swabs. Direct immunofluorescence for VZV antigen can be performed on cells collected from skin, conjunctiva, or mucosal lesion scrapings. Optimal sensitivity requires obtaining cells from the base of a lesion after unroofing a fresh vesicle. This method requires only a 3-hour turnaround time, but is significantly less sensitive ( $<75 \%)$ than PCR. ${ }^{28,29} \mathrm{VZV}$ can be isolated in cell culture from vesicular fluid or ulcer swabs, but the virus is labile and specimens must be processed rapidly. Typical cytopathic effect is noted only after 5 to 7 days. Even the more rapid shell vial method, which combines centrifugation of samples onto tissue culture monolayers and staining with fluorescein-conjugated monoclonal antibodies to detect synthesis of early VZV proteins, requires at least 48 hours and is less sensitive than PCR. ${ }^{29}$ Culture methods are most often positive when an ulcer or vesicle is sampled, especially during the early days after illness onset and before initiation of antiviral therapy, whereas PCR often remains positive late in the illness and when scabs are used as a sample. PCR is critical for evaluating atypical presentations of HZ. Serologic tests are of little value in diagnosing active VZV infection in either HIV-infected or HIV-uninfected children.

\section{Prevention Recommendations}

Preventing Exposure-HIV-infected children who are susceptible to VZV infection (with no verified history of varicella or $\mathrm{HZ}$ and lack of evidence of appropriate vaccination or varicella immunity by a sensitive, specific antibody assay) should avoid exposure to individuals with varicella or HZ (AIII). Commercially available VZV antibody assays can have false-negative and false-positive results, however, limiting the ability to determine varicella immunity with certainty. ${ }^{30}$ Household contacts who lack evidence of immunity should receive varicella vaccine to reduce the possibility of transmitting wild-type VZV to their HIV-infected contacts (AIII). ${ }^{31}$ For the same reason, elderly household contacts should receive the $\mathrm{HZ}$ vaccine according to Advisory Committee on Immunization Practices recommendations. 


\section{Preventing Disease}

Active Immunization: HIV-infected children aged 1 to 8 years without evidence of varicella immunity and whose CD4 percentages are $\geq 15 \%$ should be considered for two doses of varicella vaccine, the first dose administered as early as age 12 to 15 months or as soon as possible after the first birthday and the second dose 3 months later (BII). Limited data from a clinical trial in HIV-infected children with these characteristics indicate that the vaccine was well-tolerated and that $>80 \%$ of subjects had detectable VZV-specific immune responses (either antibody or cell-mediated immune response or both) at 1 year after vaccination..$^{32,33}$ This has been validated by other investigators. ${ }^{34-36}$ In the absence of specific safety and immunogenicity data, the combination measles-mumps-rubella-varicella vaccine should not be administered in place of the single-antigen varicella vaccine to HIVinfected children (AIII).

Data are limited on use of varicella vaccine in older HIV-infected children and adolescents. However, the safety of varicella vaccine in HIV-infected individuals aged $>8$ years who have comparable levels of immune function (i.e., CD4 cell count $\geq 200$ cells $/ \mathrm{mm}^{3}$ ) is likely to be similar to that in children aged $<8$ years; however, based on observations with HIVuninfected individuals, immunogenicity may be lower in older HIV-infected children, adolescents, and adults. Weighing the risk of severe wild-type VZV disease against the potential benefit of vaccination, older children with CD4 percentages $\geq 15 \%$ and CD4 cell counts $\geq 200$ cells $/ \mathrm{mm}^{3}$ who lack varicella immunity can be considered for vaccination on the same schedule (BIII). The response to vaccination should be optimal in patients on cART for an extended period and in those with high CD4 cell counts and very low viral loads. This should be considered in scheduling this (and other) immunizations.

Although HIV-infected children who are not severely immunocompromised tolerate the vaccine well, they, like healthy children, infrequently develop mild rashes around 2 to 3 weeks after vaccination. They rarely require antiviral therapy, and skin lesions usually clear in 3 to 5 days without treatment. Vaccine-strain VZV is susceptible to acyclovir. Because there is still varicella in some communities, VZV rashes (especially when they are extensive) that develop shortly after vaccination require virologic investigation to distinguish vaccine-associated rashes from those caused by wild-type VZV. HZ from the vaccine (Oka strain) is described in some healthy children and some children with acute lymphocytic leukemia.

HIV-infected children with low CD4 percentages $(<15 \%)$ may rarely develop systemic disease (i.e., pneumonia and neurologic manifestations) from vaccine-strain VZV and should not be vaccinated against varicella (AIII). ${ }^{37}$ Vaccination of such children can be safely undertaken after stable reconstitution of their immune systems (CD4 percentage 15\%) with cART for 3 months (AII). ${ }^{35}$ Efficacy of the varicella vaccine in HIV-infected children is suggested by long-term follow-up studies of vaccinees at several institutions. ${ }^{12,18}$ Vaccination was $>80 \%$ effective against varicella and no cases of $\mathrm{HZ}$ were observed in vaccinees. This compares favorably with the efficacy in vaccinated healthy children (after one dose) and in children with underlying leukemia (after two doses), where an efficacy of $80 \%$ to $85 \%$ was observed for prevention of clinical infection. In vaccinated HIV-uninfected 
children, most breakthrough varicella cases are mild, with few lesions (commonly <50), less fever, and a shorter duration of illness. ${ }^{31,38}$

Because $\mathrm{HZ}$ vaccine is licensed only for use in healthy people aged $\geq 50$ years to prevent $\mathrm{HZ}$ and has not been studied in HIV-infected children, it should not be given to HIV-infected children (AIII).

\section{Passive Immunization}

HIV-infected children and adolescents who

1. lack evidence of immunity to varicella (as defined above), and

2. have a non-transient exposure to a contact with varicella or herpes zoster should receive human VZV immunoglobulin (VariZIG) prophylaxis as soon as possible after the close contact, ideally within 96 hours but potentially beneficial up to 10 days ${ }^{39,40}$ (AII). Many experts limit this recommendation to varicella- or zoster-exposed HIV-infected children who are considered to be severely immunocompromised (i.e., in the Centers for Disease Control and Prevention [CDC] Immunologic Category 3), especially if also classified as CDC Clinical Category $\mathrm{C}^{41}$ and experiencing a high HIV RNA plasma viral load (BIII). When passive immunization is not possible, some experts recommend oral acyclovir for post-exposure prophylaxis (see below) for less immunocompromised HIVinfected patients. Passive immunization is achieved with VariZIG, a lyophilized preparation which, when properly reconstituted, is a 5\% solution of hyperimmune Immunoglobulin G that can be administered intramuscularly. Previously available under an investigational new drug application expanded access protocol, VariZIG was approved by the FDA in December 2012. It is now available commercially and can be obtained 24 hours a day from the sole authorized U.S. distributor (FFF Enterprises, Temecula, California) at 1-800-843-7477 or online at http://www.fffenterprises.com. The duration of the incubation period for varicella may be prolonged up to 28 days after VariZIG administration, thus also extending the period of potential infectiousness. Subsequent active immunization, provided the vaccine is not contraindicated, should be delayed for 5 months. An alternative to VariZIG is intravenous immune globulin (IVIG), $400 \mathrm{mg} / \mathrm{kg}$ body weight, administered once as soon as possible (ideally within 96 hours after exposure). Patients who have received this dose of IVIG within the prior 3 weeks should be protected.

\section{Post-Exposure Antiviral Prophylaxis}

Several small studies suggest that post-exposure prophylaxis with acyclovir in healthy children often prevents or attenuates varicella. ${ }^{42-44}$ Note that this approach is dependent upon adequate specific immune responses developing in exposed children during the incubation period. Thus, when passive immunization is not possible, some experts recommend prophylaxis with oral acyclovir $20 \mathrm{mg} / \mathrm{kg}$ body weight (maximum dose 800 $\mathrm{mg}$ ), administered 4 times daily for 7 days, beginning 7 to 10 days after exposure. ${ }^{45}$ The use of acyclovir for prophylaxis in HIV-infected, VZV-exposed children has not been studied. For that reason, some experts consider it prudent to wait until rash appears to start acyclovir therapy in VZV-susceptible and VZV-exposed, HIV-infected children who were not given passive immunization (CIII). 


\section{Post-Exposure Prophylaxis with Varicella Vaccine}

Post-exposure prophylaxis with varicella vaccine has been successfully used in HIVuninfected children and adults. ${ }^{46}$ However, this preventive approach is predicated on a prompt and robust immune response, which is why it has not been studied in HIV-infected patients and is not recommended.

Treating Disease-On the basis of controlled trials in children with malignancies, and response to therapy in HIV-infected children severely ill with varicella, ${ }^{19}$ acyclovir is the drug of choice for treating VZV infections (AI). Acyclovir should be initiated as soon as possible after varicella lesions appear. In immune competent children, new lesions can continue to appear for 72 hours after initiation of acyclovir and crusting of all lesions may take 5 to 7 days. Intravenous (IV) acyclovir is recommended for treating varicella in HIVinfected children with severe immunosuppression (i.e., CDC Immunologic Category 3) and those who have high fever, abdominal pain, respiratory symptoms, or numerous or deep, necrotic, or hemorrhagic skin lesions (AIII). For children aged $<1$ year, the dose of acyclovir is $10 \mathrm{mg} / \mathrm{kg}$ body weight administered IV every 8 hours as a 1-hour infusion. Some health-care providers administer the same dose to older children, while others base the dose of acyclovir in older children on body surface area $\left(500 \mathrm{mg} / \mathrm{m}^{2} \mathrm{IV}\right.$ every 8 hours as a 1-hour infusion). ${ }^{45}$ Administration is for 7 to 10 days, provided that new lesions have ceased to appear for at least 48 hours. Oral administration should only be used to treat varicella in HIV-infected children who are in CDC Immunologic Category 1 or 2 and who have mild varicella disease (BIII).

Acyclovir $20 \mathrm{mg} / \mathrm{kg}$ body weight ( $800 \mathrm{mg}$ maximum dose) administered 4 times per day for 7 to 10 days is the oral treatment of choice for HZ in HIV-infected children, although longer therapy should be considered when lesions are slow to resolve (AII*). Oral administration of acyclovir for $\mathrm{HZ}$ is considered safe because the risk of disseminated, life-threatening disease is lower with $\mathrm{HZ}$ than with varicella. However, initial IV administration is recommended for HIV-infected children with severe immunosuppression (i.e., CDC Immunologic Category 3), extensive multidermatomal HZ, disseminated infection, visceral involvement, or otherwise complicated HZ (AII*). It also can be considered for trigeminal nerve or sacral dermatomal involvement. IV acyclovir should be continued until cutaneous lesions and visceral disease are clearly resolving (AIII), after which oral administration can be considered to complete the course of therapy-10 to 14 days in this situation (AIII). Doses of IV acyclovir for treating HZ are the same as those for treating varicella.

Progressive outer retinal necrosis is rapidly progressive and prognosis for visual preservation is poor despite aggressive therapy. Optimal therapy has not been defined. ${ }^{25,47,48}$ Regardless of specific VZV antiviral therapy, optimization of cART is recommended. Most experts recommend IV anti-VZV therapy that includes combinations of systemic antivirals (acyclovir or ganciclovir plus foscarnet), frequently with twice-weekly intravitreal injections of ganciclovir and/or foscarnet (AIII). ${ }^{49}$ Adjunctive retinal surgery is sometimes recommended, along with corticosteroids and/or low-dose aspirin for associated occlusive vasculopathy and optic neuropathy (BIII). In contrast, acute retinal necrosis appears more responsive to high-dose IV acyclovir (10-15 $\mathrm{mg} / \mathrm{kg}$ body weight IV every 8 
hours for 10-14 days), followed by prolonged (i.e., 4-6 weeks) oral treatment with acyclovir, or valacyclovir for older patients (AIII). ${ }^{49}$

Alternatives to oral acyclovir in older adolescents and adults include valacyclovir and famciclovir (AI*). Valacyclovir is a prodrug of acyclovir with improved bioavailability that is rapidly converted to acyclovir after absorption. Sufficient information exists to support the use of valacyclovir in children, especially given its two- to three-fold improved bioavailability compared with acyclovir, at a dose of $20 \mathrm{mg} / \mathrm{kg}$ body weight (maximum dose $1 \mathrm{~g}$ ) three times a day. ${ }^{50,51}$ However, because no pediatric formulation is available, valacyclovir can generally only be used for children old enough to swallow the large valacyclovir tablets. Alternatively, crushed valacyclovir tablets, which can be used to make an extemporaneous suspension, provide good bioavailability. ${ }^{52}$ Data on the pharmacokinetics and dosing of famciclovir in children are insufficient to make recommendations, and no pediatric preparation is available..$^{53}$

Monitoring and Adverse Events, Including IRIS-Acyclovir is excreted primarily by the kidney; as a result, dose adjustment based on creatinine clearance is needed in patients with renal insufficiency or renal failure. Primary toxicities of acyclovir are phlebitis (with IV administration), renal toxicity, neutropenia, nausea, vomiting, and rash. Toxicities are similar for valacyclovir. If possible, avoid other nephrotoxic drugs when acyclovir is administered. IV acyclovir must be adequately diluted and administered slowly over 1 to 2 hours. Adequate hydration should be assured and may require IV fluid administration for ill patients. For infants and children receiving high-dose IV acyclovir, monitoring of the complete blood count (CBC) and renal function is recommended at initiation of treatment and once or twice weekly for the duration of treatment, particularly for those with underlying renal dysfunction and those receiving prolonged therapy. Periodic monitoring of $\mathrm{CBCs}$ and renal function is also recommended for children receiving prolonged oral therapy.

HZ has been considered an immune reconstitution inflammatory syndrome event in numerous reports where the incidence of $\mathrm{HZ}$ was increased transiently after institution of cART. ${ }^{54}$ However, an analysis comparing HZ incidence rates in the 3 months prior to cART with HZ incidence in the 3 months after cART indicated no difference. ${ }^{13}$ This suggests that the high incidence occurring in the 3 months after cART represents persistence of the inability to develop a robust VZV-specific cell-mediated immune response in this early postcART period. The incidence of $\mathrm{HZ}$ falls in the subsequent follow-up period as immune reconstitution proceeds. This relationship has been demonstrated with numerous opportunistic infections ${ }^{55}$ and confirmed for HZ. ${ }^{12}$

Managing Treatment Failure-Children who continue to develop lesions, whose lesions fail to heal, or whose lesions progress after 7 days of treatment may be infected with acyclovir-resistant VZV. ${ }^{56}$ This reflects the fact that acyclovir is a virostatic drug, and that such patients have inadequate VZV-specific cell-mediated immunity to rapidly clear the VZV infection. If possible, a lesion culture should be obtained and, if virus is isolated, susceptibility testing should be performed to confirm drug resistance. This may be difficult to arrange and will involve significant delay. Thus, the decision to change therapy is often based on clinical observations. All acyclovirresistant VZV strains are resistant to 
valacyclovir, famciclovir, and ganciclovir. The therapeutic choice for acyclovir-resistant VZV is foscarnet 40 to $60 \mathrm{mg} / \mathrm{kg}$ body weight, which should be administered IV 3 times daily for 7 days (AII*) or until no new lesions have appeared for at least 48 hours (AIII) ${ }^{49,57}$ Foscarnet should be administered slowly IV over the course of 2 hours (no faster than $1 \mathrm{mg} / \mathrm{kg} / \mathrm{minute}$ ).

Foscarnet has significant nephrotoxic potential; $\geq 30 \%$ of patients experience increases in serum creatinine levels. It also causes serious electrolyte imbalances (including abnormalities in calcium, phosphorus, magnesium, and potassium levels) in many patients, and secondary seizures or cardiac dysrhythmias can occur. Abnormal liver transaminases and central nervous system symptoms can occur. Infusing foscarnet with saline fluid loading can minimize renal toxicity, and infusion through a central venous catheter can avoid thrombophlebitis. Doses should be modified in patients with renal insufficiency (see package insert). For patients receiving foscarnet, $\mathrm{CBCs}$, serum electrolytes, and renal function should be monitored at least 2 to 3 times per week during induction therapy and once weekly thereafter.

Preventing Recurrence-No preventive measures are available for $\mathrm{HZ}$ in $\mathrm{HIV}$-infected children and adolescents. However, varicella vaccination reduces the incidence (perhaps severity) of HZ compared with that in healthy or HIV-infected children who had natural infection. ${ }^{12,17,18}$ The likelihood of initial or recurrent attacks of $\mathrm{HZ}$ is reduced with effective cART. ${ }^{13}$ A vaccine to prevent $\mathrm{HZ}$ has been approved for use in immunocompetent adults aged $\geq 50$ years. Data regarding safety and efficacy of this vaccine in HIV-infected persons of any age are lacking, and use of the vaccine in HIV-infected patients is not recommended. However, prospective clinical trials are under way to evaluate the safety and immunogenicity of the $\mathrm{HZ}$ vaccine in HIV-infected adults.

Discontinuing Secondary Prophylaxis-Not applicable.

\section{References}

1. Kilgore PE, Kruszon-Moran D, Seward JF, et al. Varicella in Americans from NHANES III: implications for control through routine immunization. J Med Virol. 2003; 70(Suppl 1):S111-S118. Available at http://www.ncbi.nlm.nih.gov/pubmed/12627498. [PubMed: 12627498]

2. Guris D, Jumaan AO, Mascola L, et al. Changing varicella epidemiology in active surveillance sites —United States, 1995-2005. J Infect Dis. 2008 Mar 1; 197(Suppl 2):S71-S75. Available at http:// www.ncbi.nlm.nih.gov/pubmed/18419413. [PubMed: 18419413]

3. Marin M, Zhang JX, Seward JF. Near elimination of varicella deaths in the US after implementation of the vaccination program. Pediatrics. 2011 Aug; 128(2):214-220. Available at http:// www.ncbi.nlm.nih.gov/pubmed/21788222. [PubMed: 21788222]

4. Suzuki K, Yoshikawa T, Ihira M, Ohashi M, Suga S, Asano Y. Spread of varicella-zoster virus DNA to the environment from varicella patients who were treated with oral acyclovir. Pediatr Int. 2003 Aug; 45(4):458-460. Available at http://www.ncbi.nlm.nih.gov/pubmed/12911484. [PubMed: 12911484]

5. Ross AH. Modification of chicken pox in family contacts by administration of gamma globulin. N Engl J Med. 1962 Aug 23.267:369-376. Available at http://www.ncbi.nlm.nih.gov/pubmed/ 14494142. [PubMed: 14494142] 
6. Schulze A, Dietzsch HJ. The natural history of varicella embryopathy: a 25-year follow-up. J Pediatr. 2000 Dec; 137(6):871-874. Available at http://www.ncbi.nlm.nih.gov/pubmed/11113846. [PubMed: 11113846]

7. Enders G, Miller E, Cradock-Watson J, Bolley I, Ridehalgh M. Consequences of varicella and herpes zoster in pregnancy: prospective study of 1739 cases. Lancet. 1994 Jun 18; 343(8912):15481551. Available at http://www.ncbi.nlm.nih.gov/pubmed/7802767. [PubMed: 7802767]

8. Meyers JD. Congenital varicella in term infants: risk reconsidered. J Infect Dis. 1974 Feb; 129(2): 215-217. Available at http://www.ncbi.nlm.nih.gov/pubmed/4129828. [PubMed: 4129828]

9. Levin, MJ. Zoster Vaccine. In: Plotkin, S.; Orenstein, W.; Offitt, P., editors. Vaccines 5th Edition. 5th ed. USA: Saunders Elsevier; 2008. p. 1057-1068.

10. Oxman MN. Zoster vaccine: current status and future prospects. Clin Infect Dis. 2010 Jul 15; 51(2):197-213. Available at http://www.ncbi.nlm.nih.gov/pubmed/20550454. [PubMed: 20550454]

11. Veenstra J, Krol A, van Praag RM, et al. Herpes zoster, immunological deterioration and disease progression in HIV-1 infection. AIDS. 1995 Oct; 9(10):1153-1158. Available at http:// www.ncbi.nlm.nih.gov/pubmed/8519451. [PubMed: 8519451]

12. Wood SM, Shah SS, Steenhoff AP, Rutstein RM. Primary varicella and herpes zoster among HIVinfected children from 1989 to 2006. Pediatrics. 2008 Jan; 121(1):e150-e156. Available at http:// www.ncbi.nlm.nih.gov/pubmed/18086820. [PubMed: 18086820]

13. Levin MJ, Anderson JP, Seage GR 3rd, Williams PL. Team PIC. Short-term and long-term effects of highly active antiretroviral therapy on the incidence of herpes zoster in HIV-infected children. $\mathbf{J}$ Acquir Immune Defic Syndr. 2009 Feb 1; 50(2):182-191. Available at http:// www.ncbi.nlm.nih.gov/pubmed/19131890. [PubMed: 19131890]

14. Gershon AA, Mervish N, LaRussa P, et al. Varicella-zoster virus infection in children with underlying human immunodeficiency virus infection. J Infect Dis. 1997 Dec; 176(6):1496-1500. Available at http://www.ncbi.nlm.nih.gov/pubmed/9395360. [PubMed: 9395360]

15. Derryck A, LaRussa P, Steinberg S, Capasso M, Pitt J, Gershon AA. Varicella and zoster in children with human immunodeficiency virus infection. Pediatr Infect Dis J. 1998 Oct; 17(10): 931-933. Available at http://www.ncbi.nlm.nih.gov/pubmed/9802645. [PubMed: 9802645]

16. von Seidlein L, Gillette SG, Bryson Y, et al. Frequent recurrence and persistence of varicellazoster virus infections in children infected with human immunodeficiency virus type 1 . J Pediatr. 1996 Jan; 128(1):52-57. Available at http://www.ncbi.nlm.nih.gov/pubmed/8551421. [PubMed: 8551421]

17. Civen R, Chaves SS, Jumaan A, et al. The incidence and clinical characteristics of herpes zoster among children and adolescents after implementation of varicella vaccination. Pediatr Infect Dis J. 2009 Nov; 28(11):954-959. Available at http://www.ncbi.nlm.nih.gov/pubmed/19536039. [PubMed: 19536039]

18. Son M, Shapiro ED, LaRussa P, et al. Effectiveness of varicella vaccine in children infected with HIV. J Infect Dis. 2010 Jun 15; 201(12):1806-1810. Available at http://www.ncbi.nlm.nih.gov/ pubmed/20441519. [PubMed: 20441519]

19. Jura E, Chadwick EG, Josephs SH, et al. Varicella-zoster virus infections in children infected with human immunodeficiency virus. Pediatr Infect Dis J. 1989 Sep; 8(9):586-590. Available at http:// www.ncbi.nlm.nih.gov/pubmed/2797953. [PubMed: 2797953]

20. Srugo I, Israele V, Wittek AE, Courville T, Vimal VM, Brunell PA. Clinical manifestations of varicella-zoster virus infections in human immunodeficiency virus-infected children. Am J Dis Child. 1993 Jul; 147(7):742-745. Available at http://www.ncbi.nlm.nih.gov/pubmed/8322744. [PubMed: 8322744]

21. Kelley R, Mancao M, Lee F, Sawyer M, Nahmias A, Nesheim S. Varicella in children with perinatally acquired human immunodeficiency virus infection. J Pediatr. 1994 Feb; 124(2):271273. Available at http://www.ncbi.nlm.nih.gov/pubmed/8301436. [PubMed: 8301436]

22. Leibovitz E, Cooper D, Giurgiutiu D, et al. Varicella-zoster virus infection in Romanian children infected with the human immunodeficiency virus. Pediatrics. 1993 Dec; 92(6):838-842. Available at http://www.ncbi.nlm.nih.gov/pubmed/7901834. [PubMed: 7901834] 
23. Leibovitz E, Kaul A, Rigaud M, Bebenroth D, Krasinski K, Borkowsky W. Chronic varicella zoster in a child infected with human immunodeficiency virus: case report and review of the literature. Cutis. 1992 Jan; 49(1):27-31. Available at http://www.ncbi.nlm.nih.gov/pubmed/ 1733656. [PubMed: 1733656]

24. Silliman CC, Tedder D, Ogle JW, et al. Unsuspected varicella-zoster virus encephalitis in a child with acquired immunodeficiency syndrome. J Pediatr. 1993 Sep; 123(3):418-422. Available at http://www.ncbi.nlm.nih.gov/pubmed/8394901. [PubMed: 8394901]

25. Purdy KW, Heckenlively JR, Church JA, Keller MA. Progressive outer retinal necrosis caused by varicella-zoster virus in children with acquired immunodeficiency syndrome. Pediatr Infect Dis J. 2003 Apr; 22(4):384-386. Available at http://www.ncbi.nlm.nih.gov/pubmed/12712978. [PubMed: 12712978]

26. Friedman SM, Mames RN, Sleasman JW, Whitcup SM. Acute retinal necrosis after chickenpox in a patient with acquired immunodeficiency syndrome. Arch Ophthalmol. 1993 Dec; 111(12):16071608. Available at http://www.ncbi.nlm.nih.gov/pubmed/8155026. [PubMed: 8155026]

27. Hellinger WC, Bolling JP, Smith TF, Campbell RJ. Varicella-zoster virus retinitis in a patient with AIDS-related complex: case report and brief review of the acute retinal necrosis syndrome. Clin Infect Dis. 1993 Feb; 16(2):208-212. Available at http://www.ncbi.nlm.nih.gov/pubmed/8382963. [PubMed: 8382963]

28. Leung J, Harpaz R, Baughman AL, et al. Evaluation of laboratory methods for diagnosis of varicella. Clin Infect Dis. 2010 Jul 1; 51(1):23-32. Available at http://www.ncbi.nlm.nih.gov/ pubmed/20504232. [PubMed: 20504232]

29. Schmid DS, Jumaan AO. Impact of varicella vaccine on varicella-zoster virus dynamics. Clin Microbiol Rev. 2010 Jan; 23(1):202-217. Available at http://www.ncbi.nlm.nih.gov/pubmed/ 20065330. [PubMed: 20065330]

30. Weinberg A, Hayward AR, Masters HB, Ogu IA, Levin MJ. Comparison of two methods for detecting varicella-zoster virus antibody with varicella-zoster virus cell-mediated immunity. J Clin Microbiol. 1996 Feb; 34(2):445-446. Available at http://www.ncbi.nlm.nih.gov/pubmed/8789035. [PubMed: 8789035]

31. Marin M, Guris D, Chaves SS, et al. Prevention of varicella: recommendations of the Advisory Committee on Immunization Practices (ACIP). MMWR Recomm Rep. 2007 Jun 22; 56(RR-4):140. Available at http://www.ncbi.nlm.nih.gov/pubmed/17585291. [PubMed: 17585291]

32. Levin MJ, Gershon AA, Weinberg A, et al. Immunization of HIV-infected children with varicella vaccine. J Pediatr. 2001 Aug; 139(2):305-310. Available at http://www.ncbi.nlm.nih.gov/pubmed/ 11487761. [PubMed: 11487761]

33. Levin MJ, Gershon AA, Weinberg A, et al. Administration of live varicella vaccine to HIVinfected children with current or past significant depression of CD4(+) T cells. J Infect Dis. 2006 Jul 15; 194(2):247-255. Available at http://www.ncbi.nlm.nih.gov/pubmed/16779732. [PubMed: 16779732]

34. Armenian SH, Han JY, Dunaway TM, Church JA. Safety and immunogenicity of live varicella virus vaccine in children with human immunodeficiency virus type 1. Pediatr Infect Dis J. 2006 Apr; 25(4):368-370. Available at http://www.ncbi.nlm.nih.gov/pubmed/16567993. [PubMed: 16567993]

35. Bekker V, Westerlaken GH, Scherpbier H, et al. Varicella vaccination in HIV-1-infected children after immune reconstitution. AIDS. 2006 Nov 28; 20(18):2321-2329. Available at http:// www.ncbi.nlm.nih.gov/pubmed/17117018. [PubMed: 17117018]

36. Taweesith W, Puthanakit T, Kowitdamrong E, et al. The immunogenicity and safety of live attenuated varicella-zoster virus vaccine in human immunodeficiency virus-infected children. Pediatr Infect Dis J. 2011 Apr; 30(4):320-324. Available at http://www.ncbi.nlm.nih.gov/pubmed/ 20975615. [PubMed: 20975615]

37. Kramer JM, LaRussa P, Tsai WC, et al. Disseminated vaccine strain varicella as the acquired immunodeficiency syndrome-defining illness in a previously undiagnosed child. Pediatrics. 2001 Aug.108(2):E39. Available at http://www.ncbi.nlm.nih.gov/pubmed/11483849. [PubMed: 11483849]

38. Gershon, A.; Seward, J.; Takahashi, M. Plotkin S, OW.; Offit, PA. Vaccine. 5th ed. Philadelphia, PA: Saunders; 2008. Varicella vaccine; p. 915-958. 
39. Cangene. VARIZIG Drug Label. 2012 Available at http://www.fda.gov/downloads/ biologicsBloodVaccines/BloodBloodProducts/ApprovedProducts/Licensed\%20ProductsBLAs/ Fractionated\%20PlasmsProduct/UCM333220.pdf.

40. FDA. Varicella Zoster Immune Globulin (VZIG) Anticipated Short Supply and Alternate Product Availability Under an Investigational New Drug Application Expanded Access Protocol. 2006 Available at http://www.fda.gov/biologicsbloodvaccines/safetyavailability/ucm176029.htm.

41. CDC. Revised classification system for human immunodeficiency virus infection in children less than 13 years of age. Official authorized addenda: human immunodeficiency virus infection codes and official guidelines for coding and reporting ICD-9-CM. MMWR Morb Mortal Wkly Rep. 1994; 43:1-19. Available at http://www.cdc.gov/mmwr/PDF/rr/rr4312.pdf.

42. Asano Y, Yoshikawa T, Suga S, et al. Postexposure prophylaxis of varicella in family contact by oral acyclovir. Pediatrics. 1993 Aug; 92(2):219-222. Available at http://www.ncbi.nlm.nih.gov/ pubmed/8393173. [PubMed: 8393173]

43. Huang YC, Lin TY, Chiu CH. Acyclovir prophylaxis of varicella after household exposure. Pediatr Infect Dis J. 1995 Feb; 14(2):152-154. Available at http://www.ncbi.nlm.nih.gov/pubmed/ 7746701. [PubMed: 7746701]

44. Lin TY, Huang YC, Ning HC, Hsueh C. Oral acyclovir prophylaxis of varicella after intimate contact. Pediatr Infect Dis J. 1997 Dec; 16(12):1162-1165. Available at http:// www.ncbi.nlm.nih.gov/pubmed/9427463. [PubMed: 9427463]

45. American Academy of Pediatrics. Varicealla-zoster Infection. In: Pickering, LK.; Baker, CJ.; Kinberlin, DW.; Long, SS., editors. Red book: 2009 report of the Committee on Infectious Diseases. 29th Edition. Elk Grove Village, IL: 2009. p. 714-727.

46. Macartney K, McIntyre P. Vaccines for post-exposure prophylaxis against varicella (chickenpox) in children and adults. Cochrane Database of Systematic reviews. 2009(3):CD001833. Available at http://www.ncbi.nlm.nih.gov/pubmed/18646079.

47. Yin PD, Kurup SK, Fischer SH, et al. Progressive outer retinal necrosis in the era of highly active antiretroviral therapy: successful management with intravitreal injections and monitoring with quantitative PCR. J Clin Virol. 2007 Mar; 38(3):254-259. Available at http:// www.ncbi.nlm.nih.gov/pubmed/17280866. [PubMed: 17280866]

48. Austin RB. Progressive outer retinal necrosis syndrome: a comprehensive review of its clinical presentation, relationship to immune system status, and management. Clin Eye Vis Care. 2000 Dec; 12(3-4):119-129. Available at http://www.ncbi.nlm.nih.gov/pubmed/11137426. [PubMed: 11137426]

49. Dworkin RH, Johnson RW, Breuer J, et al. Recommendations for the management of herpes zoster. Clin Infect Dis. 2007 Jan 1; 44(Suppl 1):S1-S26. Available at http:// www.ncbi.nlm.nih.gov/pubmed/17143845. [PubMed: 17143845]

50. Eksborg S. The pharmacokinetics of antiviral therapy in paediatric patients. Herpes. 2003 Dec; 10(3):66-71. Available at http://www.ncbi.nlm.nih.gov/pubmed/14759338. [PubMed: 14759338]

51. GlaxoSmithKline. Valtrex. 2010. http://us.gsk.com/products/assets/us_valtrex.pdf.

52. PDR Network. Physicians Desk Reference. 65th Edition. Montvale, NJ: PDR Network, LLC; 2011.

53. Novartis Pharmaceuticals Corporation. 2009. Famvir - pharma.us.novartis.com/product/pi/pdf/ Famvir.pdf.

54. Tangsinmankong N, Kamchaisatian W, Lujan-Zilbermann J, Brown CL, Sleasman JW, Emmanuel PJ. Varicella zoster as a manifestation of immune restoration disease in HIV-infected children. $\mathbf{J}$ Allergy Clin Immunol. 2004 Apr; 113(4):742-746. Available at http://www.ncbi.nlm.nih.gov/ pubmed/15100682. [PubMed: 15100682]

55. Ledergerber B, Egger M, Erard V, et al. AIDS-related opportunistic illnesses occurring after initiation of potent antiretroviral therapy: the Swiss HIV Cohort Study. JAMA. 1999 Dec 15; 282(23):2220-2226. Available at http://www.ncbi.nlm.nih.gov/pubmed/10605973. [PubMed: 10605973]

56. Levin MJ, Dahl KM, Weinberg A, Giller R, Patel A, Krause PR. Development of resistance to acyclovir during chronic infection with the Oka vaccine strain of varicella-zoster virus, in an 
immunosuppressed child. J Infect Dis. 2003 Oct 1; 188(7):954-959. Available at http://

www.ncbi.nlm.nih.gov/pubmed/14513413. [PubMed: 14513413]

57. Heininger U, Seward JF. Varicella. Lancet. 2006 Oct 14; 368(9544):1365-1376. Available at http://www.ncbi.nlm.nih.gov/pubmed/17046469. [PubMed: 17046469]

\section{Dosing Recommendations for Preventing and Treating Varicella-Zoster Virus}

\begin{tabular}{|c|c|c|c|}
\hline Indication & First Choice & Alternative & Comments/Special Issues \\
\hline Pre-Exposure Prophylaxis & Varicella vaccine & N/A & $\begin{array}{l}\text { See Figures } 1 \text { and } 2 \text { for detailed } \\
\text { vaccine recommendations. }\end{array}$ \\
\hline Primary (Post-Exposure) Prophylaxis & $\begin{array}{l}\text { VariZIG } 125 \text { IU/10 } \\
\text { kg body weight IM } \\
\text { (maximum 625 IU), } \\
\text { administered ideally } \\
\text { within } 96 \text { hours } \\
\text { (potentially } \\
\text { beneficial up to } 10 \\
\text { days) after exposure }\end{array}$ & $\begin{array}{l}\text { If VariZIG } \\
\text { cannot be } \\
\text { administered } \\
\text { within } 96 \\
\text { hours (up to } \\
10 \text { days), } \\
\text { IVIG 400 } \\
\text { mg/kg body } \\
\text { weight, } \\
\text { administered } \\
\text { once should } \\
\text { be } \\
\text { considered. } \\
\text { IVIG should } \\
\text { ideally be } \\
\text { administered } \\
\text { within } 96 \\
\text { hours of } \\
\text { exposure } \\
\text { When } \\
\text { passive } \\
\text { immunization } \\
\text { is not } \\
\text { possible, } \\
\text { some experts } \\
\text { recommend } \\
\text { prophylaxis } \\
\text { with } \\
\text { acyclovir } 20 \\
\text { mg/kg body } \\
\text { weight/dose } \\
\text { (maximum } \\
\text { dose } 800 \\
\text { mg), } \\
\text { administered } \\
\text { QID for } 7 \\
\text { days, } \\
\text { beginning 7- } \\
10 \text { days after } \\
\text { exposure }\end{array}$ & $\begin{array}{l}\text { Primary Post-Exposure } \\
\text { Prophylaxis Indicated for: } \\
\text { Patients with } \\
\text { substantial exposure } \\
\text { to varicella or zoster } \\
\text { with no verified } \\
\text { history of varicella or } \\
\text { zoster or who are } \\
\text { seronegative for VZV } \\
\text { on a sensitive, } \\
\text { specific antibody } \\
\text { assay or who lack } \\
\text { evidence of } \\
\text { vaccination. Many } \\
\text { experts limit this } \\
\text { recommendation to } \\
\text { varicella or zoster- } \\
\text { exposed HIV-infected } \\
\text { children who are } \\
\text { considered to be } \\
\text { severely } \\
\text { immunocompromised, } \\
\text { (i.e., in CDC } \\
\text { Immunologic } \\
\text { Category } 3 \text { ), } \\
\text { especially if also } \\
\text { classified as CDC } \\
\text { Clinical Category Ca } \\
\text { and experiencing a } \\
\text { high HIV RNA } \\
\text { plasma viral load } \\
\text { (BIII). } \\
\text { Some experts start } \\
\text { acyclovir at first } \\
\text { appearance of rash. } \\
\text { - }\end{array}$ \\
\hline Secondary Prophylaxis & N/A & N/A & $\begin{array}{l}\text { There is no indication for } \\
\text { secondary prophylaxis }\end{array}$ \\
\hline Treatment & $\begin{array}{l}\text { Chickenpox } \\
\text { Children with No or } \\
\text { Moderate Immune }\end{array}$ & $\begin{array}{l}\text { Patients Unresponsive to } \\
\text { Acyclovir: }\end{array}$ & $\begin{array}{l}\text { In children } \geq 1 \text { year of age, some } \\
\text { experts base IV acyclovir dosing } \\
\text { on body surface area }\left(500 \mathrm{mg} / \mathrm{m}^{2}\right.\end{array}$ \\
\hline
\end{tabular}




\begin{tabular}{|c|c|c|c|}
\hline Indication & First Choice & Alternative & Comments/Special Issues \\
\hline & 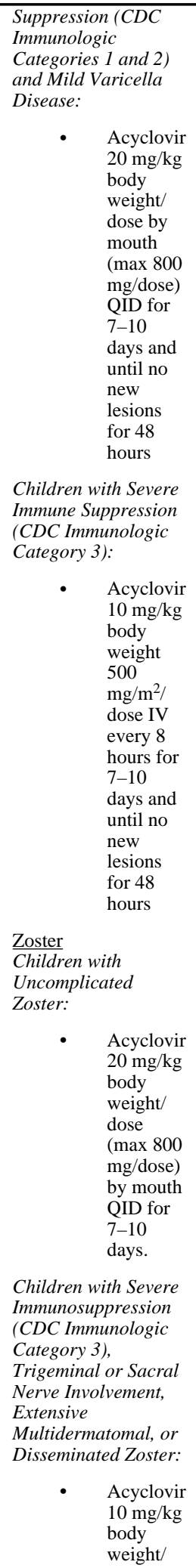 & $\begin{array}{ll}\text { Foscarnet } \\
\text { (40-60 } \\
\text { mg/kg body } \\
\text { weight/dose } \\
\text { IV every } 8 \\
\text { hours) for 7- } \\
10 \text { days or } \\
\text { until no new } \\
\text { lesions have } \\
\text { appeared for } \\
\text { 48 hours }\end{array}$ & $\begin{array}{l}\text { body surface area/dose IV every } 8 \\
\text { hours) instead of body weight. } \\
\text { Valacyclovir is approved for use } \\
\text { in adults and adolescents with } \\
\text { zoster at } 1 \mathrm{~g} / \text { dose by mouth TID } \\
\text { for } 7 \text { days; the same dose has } \\
\text { been used for varicella infections. } \\
\text { Data on dosing in children are } \\
\text { limited and there is no pediatric } \\
\text { preparation, although } 500 \mathrm{mg} \\
\text { capsules can be extemporaneously } \\
\text { compounded to make a } \\
\text { suspension to administer } 20 \\
\text { mg/kg body weight/dose } \\
\text { (maximum dose } 1 \mathrm{~g} \text { ) given TID } \\
\text { (see prescribing information). } \\
\text { Famciclovir is approved for use in } \\
\text { adults and adolescents with zoster } \\
\text { at } 500 \text { mg/dose by mouth TID for } \\
7 \text { days; the same dose has been } \\
\text { used for varicella infections. } \\
\text { There is no pediatric preparation } \\
\text { and data on dosing in children are } \\
\text { limited; can be used by } \\
\text { adolescents able to receive adult } \\
\text { dosing. } \\
\text { Involvement of an } \\
\text { ophthalmologist with experience } \\
\text { in managing herpes zoster } \\
\text { ophthalmicus and its } \\
\text { complications in children is } \\
\text { strongly recommended when } \\
\text { ocular involvement is evident. } \\
\text { Optimal management of PORN } \\
\text { has not been defined. }\end{array}$ \\
\hline
\end{tabular}




\begin{tabular}{|c|c|c|c|}
\hline Indication & First Choice & Alternative & Comments/Special Issues \\
\hline
\end{tabular}




\begin{tabular}{|l|l|l|l|}
\hline Indication & First Choice & Alternative & Comments/Special Issues \\
\hline & Oral valacyclovir 1 g/ & & \\
dose TID for 4-6 & & \\
& weeks (for children & & \\
old enough to receive & & \\
adult dose). & & \\
Alternative oral & & \\
acyclovir dose: 20 & & \\
mg/kg body weight/ & & \\
& dose QID for 4-6 & & \\
& weeks & & \\
\hline
\end{tabular}

Key to Acronyms: $\mathrm{ARN}=$ acute retinal necrosis; $\mathrm{CDC}=$ Centers for Diseases Control and Prevention; $\mathrm{IM}=$ intramuscular; IU = international units; IV = intravenous; IVIG = intravenous immunoglobulin; PORN = progressive outer retinal necrosis; $\mathrm{QID}=$ four times a day; TID = three times daily; VariZIG = varicella zoster immune globulin; VZV = varicella zoster virus

\section{Appendix 1. NIH-CDC-HIVMA/IDSA-PIDS-AAP Guidelines for the Prevention and Treatment of Opportunistic Infections in HIV-Exposed and HIV-Infected Children A Working Group of the Office of AIDS Research Advisory Council (OARAC) (Last updated November 6, 2013; last reviewed November 6, 2013)}

Important Guideline Considerations

\begin{tabular}{|c|c|}
\hline Topic & Comment \\
\hline Goal of the Guidelines & $\begin{array}{l}\text { Provide guidance to HIV care practitioners on the prevention and management of HIV- } \\
\text { related opportunistic infections for HIV-exposed and HIV-infected children in the } \\
\text { United States. }\end{array}$ \\
\hline Panel Members & $\begin{array}{l}\text { The panel is composed of the Executive Secretary and two non-governmental Co- } \\
\text { Chairs with expertise in pediatric HIV infection and infectious diseases. The panel has } \\
\text { members who represent the National Institutes of Health (NIH), the Centers for Disease } \\
\text { Control and Prevention (CDC), the HIV Medical Association of the Infectious Disease } \\
\text { Society of America (HIVMA/IDSA), the Pediatric Infectious Disease Society (PIDS), } \\
\text { the American Academy of Pediatrics (AAP), plus approximately } 30 \text { members with } \\
\text { expertise in HIV clinical care, infectious disease management, and research in children. } \\
\text { The panel members are selected from government, academia, and the healthcare } \\
\text { community by the Executive Secretary and Co-Chairs and assigned to a working group } \\
\text { for one or more of the guideline's sections based on the member's area of subject } \\
\text { matter expertise. Each working group is chaired by a panel member selected by the co- } \\
\text { chairs. Members serve on the panel for a four-year term, with an option to be } \\
\text { reappointed for additional terms. The list of the current working group members can be } \\
\text { found in Appendix } 2 \text {. }\end{array}$ \\
\hline $\begin{array}{l}\text { Financial Disclosure and } \\
\text { Management of Conflicts } \\
\text { of Interest }\end{array}$ & $\begin{array}{l}\text { All members of the panel submit a written financial disclosure annually reporting any } \\
\text { associations with manufacturers of drugs, vaccines, medical devices, or diagnostics } \\
\text { used to manage HIV-related opportunistic infections. A list of these disclosures and the } \\
\text { date of their last update are available in Appendix } 3 \text {. The panel co-editors review each } \\
\text { reported association for potential conflict of interest and determine the appropriate } \\
\text { action: disqualification from the panel, disqualification/recusal from topic review and } \\
\text { discussion, or no disqualification needed. A conflict of interest is defined as any direct } \\
\text { financial interest related to a product addressed in the section of the guideline to which } \\
\text { a panel member contributes content. Financial interests include direct receipt by the } \\
\text { panel member of payments, gratuities, consultancies, honoraria, employment, grants, } \\
\text { support for travel or accommodation, or gifts from an entity having a commercial } \\
\text { interest in that product. Financial interest also includes direct compensation for } \\
\text { membership on an advisory board, data safety monitoring board, or speakers' bureau. } \\
\text { Compensation and support that filters through a working group member's university or } \\
\text { institution (e.g., grants, research funding) is not considered a conflict of interest. }\end{array}$ \\
\hline Users of the Guidelines & Pediatric HIV treatment providers in the United States \\
\hline Developer & $\begin{array}{l}\text { Panel on Guidelines for Prevention and Treatment of Opportunistic Infections in HIV- } \\
\text { Exposed and HIV-Infected Children—a working group of the Office of AIDS Research } \\
\text { (OAR) Advisory Council }\end{array}$ \\
\hline
\end{tabular}




\begin{tabular}{|c|c|}
\hline Topic & Comment \\
\hline Funding Source & OAR, NIH \\
\hline Evidence Collection & $\begin{array}{l}\text { The recommendations in the guidelines are generally based on studies published in } \\
\text { peer-reviewed journals. On some occasions, particularly when new information may } \\
\text { affect patient safety, unpublished data presented at major conferences or information } \\
\text { prepared by the U.S. Food and Drug Administration or manufacturers (e.g., warnings to } \\
\text { the public) may be used as evidence to revise the guidelines. Panel members of each } \\
\text { working group are responsible for conducting a systematic comprehensive review of } \\
\text { the literature, for conducting updates of that review, and for bringing to their working } \\
\text { group's attention all relevant literature and documents that merit evaluation based on } \\
\text { the quality and content of data. }\end{array}$ \\
\hline $\begin{array}{l}\text { Method of Synthesizing } \\
\text { Data and Formulating } \\
\text { Recommendations }\end{array}$ & $\begin{array}{l}\text { Each section of the guidelines is assigned to a panel member with expertise in the area } \\
\text { of interest. The panel member synthesizes the available evidence. Recommendations } \\
\text { are reviewed and updated by each working group after an assessment of the quality and } \\
\text { impact of the existing and any new data. Aspects of evidence that are considered } \\
\text { include, but are not necessarily limited to, the type of study (e.g., case series, } \\
\text { prospective cohort, randomized controlled trial), the quality and appropriateness of the } \\
\text { methods, the number of subjects and effect sizes observed, and data from both adult and } \\
\text { pediatric studies. Finally, all material is reviewed by the Executive Secretary, Co- } \\
\text { Chairs, by the OAR, by subject matter experts at the CDC, the HIVMA/IDSA, the } \\
\text { PIDS, and AAP prior to final approval and publication. In general, these } \\
\text { recommendations were developed based on combining scientific evidence with expert } \\
\text { opinion. Randomized clinical trials in children remain the gold standard; however, } \\
\text { because studies focusing on HIV-infected children in relation to each pathogen are } \\
\text { uncommon, all studies including observational studies, were considered. When there } \\
\text { were no pediatric studies available, recommendations were based on adult studies. } \\
\text { Finally, when there was lack of published literature altogether, recommendations were } \\
\text { based on expert opinion. Summary tables of evidence for these guidelines were not } \\
\text { developed because of the small number of studies available for each question in } \\
\text { consideration. }\end{array}$ \\
\hline Recommendation Rating & $\begin{array}{l}\text { Recommendations are rated using a revised version of the previous rating system (see } \\
\text { Table in Introduction: Rating System for Prevention and Treatment Recommendations) } \\
\text { and accompanied, as needed, by explanatory text that reviews the evidence and the } \\
\text { working group's assessment. All proposals are discussed at teleconferences and by } \\
\text { email and then assessed by the panel's Executive Secretary and Co-Chairs and } \\
\text { reviewed by the OAR, the CDC, the HIVMA/IDSA, the PIDS, and the AAP before } \\
\text { being endorsed as official recommendations. }\end{array}$ \\
\hline Other Guidelines & $\begin{array}{l}\text { These guidelines focus on prevention and treatment of HIV-related opportunistic } \\
\text { infections for HIV-exposed and HIV-infected children in the United States. A separate } \\
\text { guideline outlines similar recommendations for adults. These guidelines are also } \\
\text { available on the AIDSinfo website (http://www.aidsinfo.nih.gov). }\end{array}$ \\
\hline Update Plan & $\begin{array}{l}\text { Each work group and the co-editors meet at least every } 6 \text { months by teleconference to } \\
\text { review data that may warrant modification of the guidelines. Updates may be prompted } \\
\text { by approvals of new drugs, vaccines, medical devices or diagnostics; by new } \\
\text { information regarding indications or dosing; by new safety or efficacy data; or by other } \\
\text { information that may affect prevention and treatment of HIV-related opportunistic } \\
\text { infections. Updates that may significantly affect patient safety or treatment and that } \\
\text { warrant rapid notification may be posted temporarily on the AIDSinfo website (http:// } \\
\text { www.aidsinfo.nih.gov) until the guideline document can be updated. }\end{array}$ \\
\hline Public Comments & $\begin{array}{l}\text { After release of an update on the AIDSinfo website, the public is given a 2-week period } \\
\text { to submit comments to the panel. These comments are reviewed, and a determination is } \\
\text { made by the appropriate work group and the co-editors as to whether revisions are } \\
\text { indicated. The public may also submit comments to the Panel at any time at } \\
\text { contactus @ aidsinfo.nih.gov. }\end{array}$ \\
\hline
\end{tabular}

\section{Appendix 2. NIH-CDC-HIVMA/IDSA-PIDS-AAP Guidelines for the Prevention and Treatment of Opportunistic Infections in HIV-Exposed and HIV-Infected}




\section{Children A Working Group of the Office of AIDS Research Advisory Council (OARAC)}

Panel Members: January 2011-October 2013

\begin{tabular}{|c|c|c|}
\hline Topic & Principal Author(s) & Co-Author(s) \\
\hline Bacterial Infections & $\begin{array}{l}\text { George K. Siberry, MD, MPH } \\
\text { National Institutes of Health } \\
\text { Rockville, MD }\end{array}$ & \\
\hline Candidal Infections & $\begin{array}{l}\text { William J. Steinbach, MD } \\
\text { Duke University Medical Center } \\
\text { Durham, NC }\end{array}$ & \\
\hline Coccidioidomycosis & $\begin{array}{l}\text { Martin B. Kleiman, MD } \\
\text { Riley Hospital for Children } \\
\text { Indianapolis, Indiana }\end{array}$ & \\
\hline Cryptococcal Infections & $\begin{array}{l}\text { Aditya Gaur, MD } \\
\text { St. Jude Children's Research Hospital } \\
\text { Memphis, TN }\end{array}$ & $\begin{array}{l}\text { Patricia Flynn, MD } \\
\text { St. Jude Children's Research } \\
\text { Hospital } \\
\text { Memphis, TN }\end{array}$ \\
\hline Cryptosporidium Infections & $\begin{array}{l}\text { Patricia Flynn, MD } \\
\text { St. Jude Children's Research Hospital } \\
\text { Memphis, TN }\end{array}$ & $\begin{array}{l}\text { Gabriela Maron, MD } \\
\text { St. Jude Children's Research } \\
\text { Hospital } \\
\text { Memphis, TN }\end{array}$ \\
\hline Cytomegalovirus Infections & $\begin{array}{l}\text { Masako Shimamura, MD } \\
\text { University of Alabama at Birmingham } \\
\text { Birmingham, AL }\end{array}$ & $\begin{array}{l}\text { David Kimberlin, MD } \\
\text { University of Alabama at } \\
\text { Birmingham } \\
\text { Birmingham, AL }\end{array}$ \\
\hline Giardiasis & $\begin{array}{l}\text { Patricia Flynn, MD } \\
\text { St. Jude Children's Research Hospital } \\
\text { Memphis, TN }\end{array}$ & $\begin{array}{l}\text { Gabriela Maron, MD } \\
\text { St. Jude Children's Research } \\
\text { Hospital } \\
\text { Memphis, TN }\end{array}$ \\
\hline Hepatitis B Virus Infections & $\begin{array}{l}\text { Michael R. Narkewicz, MD } \\
\text { Children's Hospital Colorado/University of } \\
\text { Colorado School of Medicine; Department of } \\
\text { Pediatrics; Section of Pediatric } \\
\text { Gastroenterology, Hepatology, and Nutrition } \\
\text { Aurora, CO }\end{array}$ & $\begin{array}{l}\text { Cara L. Mack, MD } \\
\text { Children's Hospital Colorado; } \\
\text { Gastroenterology, } \\
\text { Hepatology, and Nutrition } \\
\text { Aurora, CO } \\
\text { Debika Bhattacharya, MD } \\
\text { David Geffen School of } \\
\text { Medicine at UCLA } \\
\text { Los Angeles, CA }\end{array}$ \\
\hline Hepatitis C Virus Infections & $\begin{array}{l}\text { Cara L. Mack, MD } \\
\text { Children's Hospital Colorado; } \\
\text { Gastroenterology, Hepatology, and Nutrition } \\
\text { Aurora, CO }\end{array}$ & $\begin{array}{l}\text { Michael R. Narkewicz, MD } \\
\text { Children's Hospital Colorado/ } \\
\text { University of } \\
\text { Colorado School of Medicine; } \\
\text { Department of } \\
\text { Pediatrics; Section of Pediatric } \\
\text { Gastroenterology, Hepatology, } \\
\text { and Nutrition } \\
\text { Aurora, CO } \\
\text { Debika Bhattacharya, MD } \\
\text { David Geffen School of } \\
\text { Medicine at UCLA } \\
\text { Los Angeles, CA }\end{array}$ \\
\hline $\begin{array}{l}\text { Herpes Simplex Virus } \\
\text { Infections }\end{array}$ & $\begin{array}{l}\text { Myron J. Levin, MD } \\
\text { University of Colorado School of Medicine } \\
\text { Aurora, CO }\end{array}$ & \\
\hline Histoplasmosis & $\begin{array}{l}\text { Martin B. Kleiman, MD } \\
\text { Riley Hospital for Children } \\
\text { Indianapolis, Indiana }\end{array}$ & \\
\hline $\begin{array}{l}\text { Human Herpesvirus } 8 \\
\text { Infections }\end{array}$ & $\begin{array}{l}\text { Richard Rutstein, MD } \\
\text { Children's Hospital of Philadelphia } \\
\text { Philadelphia, PA }\end{array}$ & \\
\hline
\end{tabular}




\begin{tabular}{|c|c|c|}
\hline Topic & Principal Author(s) & Co-Author(s) \\
\hline $\begin{array}{l}\text { Human Papillomavirus } \\
\text { Infections }\end{array}$ & $\begin{array}{l}\text { Anna-Barbara Moscicki, MD } \\
\text { University of California San Francisco } \\
\text { San Francisco, CA } \\
\text { Sharon Nachman, MD } \\
\text { State University of New York at Stony Brook } \\
\text { Stony Brook, NY }\end{array}$ & \\
\hline $\begin{array}{l}\text { Immunizations Text and } \\
\text { Figures }\end{array}$ & $\begin{array}{l}\text { Andrew Kroger, MD, MPH } \\
\text { Centers for Disease Control and Prevention } \\
\text { Atlanta, GA }\end{array}$ & $\begin{array}{l}\text { Jane Seward, MBBS, MPH } \\
\text { Centers for Disease Control and } \\
\text { Prevention } \\
\text { Atlanta, GA }\end{array}$ \\
\hline Influenza & $\begin{array}{l}\text { Kathryn M. Edwards, MD } \\
\text { Vanderbilt University School of Medicine } \\
\text { Nashville, TN }\end{array}$ & $\begin{array}{l}\text { Jennifer C. Esbenshade, MD, } \\
\text { MPH } \\
\text { Vanderbilt University School of } \\
\text { Medicine } \\
\text { Nashville, TN } \\
\text { Gregory J. Wilson, MD } \\
\text { Vanderbilt University School of } \\
\text { Medicine } \\
\text { Nashville, TN }\end{array}$ \\
\hline Isosporiasis & $\begin{array}{l}\text { Patricia Flynn, MD } \\
\text { St. Jude Children's Research Hospital } \\
\text { Memphis, TN }\end{array}$ & $\begin{array}{l}\text { Gabriela Maron, MD } \\
\text { St. Jude Children's Research } \\
\text { Hospital } \\
\text { Memphis, TN }\end{array}$ \\
\hline Malaria & $\begin{array}{l}\text { Charlotte Victoria Hobbs, MD } \\
\text { National Institutes of Health } \\
\text { Rockville, MD } \\
\text { Sharon Nachman, MD } \\
\text { State University of New York at Stony Brook } \\
\text { Stony Brook, NY }\end{array}$ & \\
\hline Microsporidiosis & $\begin{array}{l}\text { Patricia Flynn, MD } \\
\text { St. Jude Children's Research Hospital } \\
\text { Memphis, TN }\end{array}$ & $\begin{array}{l}\text { Gabriela Maron, MD } \\
\text { St. Jude Children's Research } \\
\text { Hospital } \\
\text { Memphis, TN }\end{array}$ \\
\hline $\begin{array}{l}\text { Mycobacterium avium } \\
\text { Complex Disease }\end{array}$ & $\begin{array}{l}\text { James McAuley, MD, MPH } \\
\text { Rush University Medical Center } \\
\text { Chicago, IL }\end{array}$ & \\
\hline $\begin{array}{l}\text { Mycobacterium tuberculosis } \\
\text { Infections }\end{array}$ & $\begin{array}{l}\text { Ben J. Marais, MD } \\
\text { University of Sydney, Children's Hospital at } \\
\text { Westmead } \\
\text { Sydney, Australia }\end{array}$ & $\begin{array}{l}\text { Heather J. Menzies, MD, MPH } \\
\text { Centers for Disease Control and } \\
\text { Prevention } \\
\text { Atlanta, GA }\end{array}$ \\
\hline Pharmacology Review & $\begin{array}{l}\text { Diana Clarke, Pharm. D. } \\
\text { Boston Medical Center } \\
\text { Boston, MA }\end{array}$ & \\
\hline Pneumocystis Pneumonia & $\begin{array}{l}\text { Francis Gigliotti, MD } \\
\text { University of Rochester, School of Medicine } \\
\text { and Dentistry } \\
\text { Rochester, NY }\end{array}$ & \\
\hline $\begin{array}{l}\text { Progressive Multifocal } \\
\text { Leukoencephalopathy }\end{array}$ & $\begin{array}{l}\text { Gail Harrison, MD } \\
\text { Baylor College of Medicine } \\
\text { Texas Children's Hospital } \\
\text { Houston, TX }\end{array}$ & \\
\hline Syphilis & $\begin{array}{l}\text { Emilia H. Koumans, MD, MPH } \\
\text { Centers for Disease Control and Prevention } \\
\text { Atlanta, GA }\end{array}$ & \\
\hline Toxoplasmosis & $\begin{array}{l}\text { James McAuley, MD, MPH } \\
\text { Rush University Medical Center } \\
\text { Chicago, IL }\end{array}$ & \\
\hline $\begin{array}{l}\text { Varicella-Zoster Virus } \\
\text { Infections }\end{array}$ & $\begin{array}{l}\text { Myron J. Levin, MD } \\
\text { University of Colorado School of Medicine } \\
\text { Aurora, CO }\end{array}$ & \\
\hline
\end{tabular}

Pediatr Infect Dis J. Author manuscript; available in PMC 2014 November 01. 


\section{Appendix 3. NIH-CDC-HIVMA/IDSA-PIDS-AAP Guidelines for the Prevention and Treatment of Opportunistic Infections in HIV-Exposed and HIV-Infected Children A Working Group of the Office of AIDS Research Advisory Council (OARAC)}

Financial Disclosures: January 2009-May 2013

\begin{tabular}{|c|c|c|c|}
\hline Member & Panel Role & Company & Relationship \\
\hline $\begin{array}{l}\text { Abzug, Mark } \\
\text { Children's Hospital Colorado } \\
\text { Denver, CO }\end{array}$ & Co-Chair & None & N/A \\
\hline $\begin{array}{l}\text { Bhattacharya, Debika } \\
\text { David Geffen School of } \\
\text { Medicine at UCLA } \\
\text { Los Angeles, CA }\end{array}$ & $\begin{array}{l}\text { Co-Author: Hepatitis B Virus } \\
\text { Infections } \\
\text { Co-Author: Hepatitis C Virus } \\
\text { Infections }\end{array}$ & - Vertex & - Research Support \\
\hline $\begin{array}{l}\text { Bohannon, Beverly } \\
\text { Centers for Disease Control } \\
\text { and Prevention } \\
\text { Atlanta, GA }\end{array}$ & Core Leadership Group & None & N/A \\
\hline $\begin{array}{l}\text { Brady, Michael } \\
\text { Nationwide Children's } \\
\text { Hospital } \\
\text { Columbus, Ohio }\end{array}$ & Core Leadership Group & None & N/A \\
\hline $\begin{array}{l}\text { Clarke, Diana } \\
\text { Boston Medical Center } \\
\text { Boston, MA }\end{array}$ & Author: Pharmacology Review & None & N/A \\
\hline $\begin{array}{l}\text { Dominiguez, Kenneth } \\
\text { Centers for Disease Control } \\
\text { and Prevention } \\
\text { Atlanta, GA }\end{array}$ & Core Leadership Group & None & N/A \\
\hline \multirow{2}{*}{$\begin{array}{l}\text { Edwards, Kathryn } \\
\text { Vanderbilt University School } \\
\text { of Medicine } \\
\text { Nashville, TN }\end{array}$} & \multirow[t]{2}{*}{ Principal Author: Influenza } & $\cdot \mathrm{CDC}, \mathrm{NIH}$ & - Research Support \\
\hline & & •IDSA & - Governing Body \\
\hline $\begin{array}{l}\text { Esbenshade, Jennifer } \\
\text { Vanderbilt University School } \\
\text { of Medicine } \\
\text { Nashville, TN }\end{array}$ & Co-Author: Influenza & None & N/A \\
\hline \multirow{3}{*}{$\begin{array}{l}\text { Flynn, Patricia } \\
\text { St. Jude Children's Research } \\
\text { Hospital } \\
\text { Memphis, TN }\end{array}$} & \multirow{3}{*}{$\begin{array}{l}\text { Author: Cryptosporidium } \\
\text { Infections, Giardiasis, } \\
\text { Isosporiasis, Microsporidiosis } \\
\text { Co-Author: Cryptococcal } \\
\text { Infections }\end{array}$} & - Tibotec & - Research Support \\
\hline & & - Bristol-Myers Squibb & - Research Support \\
\hline & & • Merck & $\begin{array}{l}\text { - Safety Monitoring } \\
\text { Committee }\end{array}$ \\
\hline \multirow{2}{*}{$\begin{array}{l}\text { Gaur, Aditya } \\
\text { St. Jude Children's Research } \\
\text { Hospital } \\
\text { Memphis, TN }\end{array}$} & \multirow[t]{2}{*}{ Author: Cryptococcal Infections } & - Gilead & - Research Support \\
\hline & & • GSK & - Research Support \\
\hline $\begin{array}{l}\text { Gigliotti, Francis } \\
\text { University of Rochester, } \\
\text { School of Medicine } \\
\text { and Dentistry } \\
\text { Rochester, NY }\end{array}$ & $\begin{array}{l}\text { Author: Pneumocystis } \\
\text { Pneumonia }\end{array}$ & • GSK & - Speakers' Bureau \\
\hline $\begin{array}{l}\text { Handelsman, Edward } \\
\text { National Institutes of Health } \\
\text { Rockville, MD }\end{array}$ & Core Leadership Group & None & N/A \\
\hline \multirow{2}{*}{$\begin{array}{l}\text { Harrison, Gail } \\
\text { Baylor College of Medicine } \\
\text { Texas Children's Hospital } \\
\text { Houston, TX }\end{array}$} & \multirow{2}{*}{$\begin{array}{l}\text { Author: Progressive Multifocal } \\
\text { Leukoencephalopathy }\end{array}$} & $\cdot \mathrm{CDC}$ & - Research Support \\
\hline & & - AAP & - Advisory Board \\
\hline
\end{tabular}




\begin{tabular}{|c|c|c|c|}
\hline Member & Panel Role & Company & Relationship \\
\hline & & • Elsevier & - Royalties \\
\hline & & - UpToDate, Inc & - Royalties \\
\hline $\begin{array}{l}\text { Hobbs, Charlottle } \\
\text { National Institutes of Health } \\
\text { Rockville, MD }\end{array}$ & Author: Malaria & None & N/A \\
\hline \multirow{4}{*}{$\begin{array}{l}\text { Kimberlin, David } \\
\text { University of Alabama at } \\
\text { Birmingham } \\
\text { Birmingham, AL }\end{array}$} & \multirow{4}{*}{$\begin{array}{l}\text { Co-Author: Cytomegalovirus } \\
\text { Infections }\end{array}$} & • GSK & - Research Support \\
\hline & & - Cellex & - Research Support \\
\hline & & - Cubist & - Research Support \\
\hline & & • Gilead & - Research Support \\
\hline $\begin{array}{l}\text { Kleiman, Martin } \\
\text { Riley Hospital for Children } \\
\text { Indianapolis, Indiana }\end{array}$ & $\begin{array}{l}\text { Author: Coccidioidomycosis, } \\
\text { Histoplasmosis }\end{array}$ & None & N/A \\
\hline $\begin{array}{l}\text { Koumans, Emilia } \\
\text { Centers for Disease Control } \\
\text { and Prevention } \\
\text { Atlanta, GA }\end{array}$ & Author: Syphilis & None & N/A \\
\hline $\begin{array}{l}\text { Kroger, Andrew } \\
\text { Centers for Disease Control } \\
\text { and Prevention } \\
\text { Atlanta, GA }\end{array}$ & $\begin{array}{l}\text { Author: Immunizations Text and } \\
\text { Figures }\end{array}$ & None & N/A \\
\hline \multirow[t]{2}{*}{$\begin{array}{l}\text { Levin, Myron } \\
\text { University of Colorado School } \\
\text { of Medicine } \\
\text { Aurora, CO }\end{array}$} & \multirow[t]{2}{*}{$\begin{array}{l}\text { Author: Herpes Simplex Virus } \\
\text { Infections, Varicella-Zoster } \\
\text { Virus Infections }\end{array}$} & - Merck & $\begin{array}{l}\text { - Advisory Board } \\
\text { - Patent } \\
\text { - Adjudication } \\
\text { Committee } \\
\text { - Research Support }\end{array}$ \\
\hline & & - GlaxoSmith Kline & $\begin{array}{l}\text { - Adjudication } \\
\text { Committee } \\
\text { - Research Support }\end{array}$ \\
\hline $\begin{array}{l}\text { Mack, Cara } \\
\text { Children's Hospital Colorado; } \\
\text { Gastroenterology, Hepatology, } \\
\text { and Nutrition } \\
\text { Aurora, CO }\end{array}$ & $\begin{array}{l}\text { Author: Hepatitis C Virus } \\
\text { Infections } \\
\text { Co-Author: Hepatitis B Virus } \\
\text { Infections }\end{array}$ & None & N/A \\
\hline $\begin{array}{l}\text { Marais, Ben J } \\
\text { University of Sydney, } \\
\text { Children's Hospital at } \\
\text { Westmead } \\
\text { Sydney, Australia }\end{array}$ & $\begin{array}{l}\text { Author: Mycobacterium } \\
\text { tuberculosis Infections }\end{array}$ & None & N/A \\
\hline $\begin{array}{l}\text { Maron, Gabriela } \\
\text { St. Jude Children's Research } \\
\text { Hospital } \\
\text { Memphis, TN }\end{array}$ & $\begin{array}{l}\text { Co-Author: Cryptosporidium } \\
\text { Infections, Giardiasis, } \\
\text { Isosporiasis, Microsporidiosis }\end{array}$ & None & N/A \\
\hline $\begin{array}{l}\text { McAuley, James } \\
\text { Rush University Medical } \\
\text { Center } \\
\text { Chicago, IL }\end{array}$ & $\begin{array}{l}\text { Author: } \text { Mycobacterium avium } \\
\text { Complex Disease, } \\
\text { Toxoplasmosis }\end{array}$ & • NIH-NICHD & - Research Support \\
\hline $\begin{array}{l}\text { Menzies, Heather } \\
\text { Centers for Disease Control } \\
\text { and Prevention } \\
\text { Atlanta, GA }\end{array}$ & $\begin{array}{l}\text { Co-Author: } \text { Mycobacterium } \\
\text { tuberculosis Infections }\end{array}$ & None & N/A \\
\hline $\begin{array}{l}\text { Mofenson, Lynne } \\
\text { National Institutes of Health } \\
\text { Rockville, MD }\end{array}$ & Core Leadership Group & None & N/A \\
\hline \multirow{3}{*}{$\begin{array}{l}\text { Moscicki, Anna-Barbara } \\
\text { University of California San } \\
\text { Francisco } \\
\text { San Francisco, CA }\end{array}$} & \multirow{3}{*}{$\begin{array}{l}\text { Author: Human Papillomavirus } \\
\text { Infections }\end{array}$} & - Merck & - Advisory Board \\
\hline & & - GSK & - Honoraria \\
\hline & & - Becton-Dickenson & • Honoraria \\
\hline
\end{tabular}




\begin{tabular}{|c|c|c|c|}
\hline Member & Panel Role & Company & Relationship \\
\hline $\begin{array}{l}\text { Nachman, Sharon } \\
\text { State University of New York } \\
\text { at Stony Brook } \\
\text { Stony Brook, NY }\end{array}$ & $\begin{array}{l}\text { Co-Chair } \\
\text { Author: Human Papillomavirus } \\
\text { Infections, Malaria }\end{array}$ & None & N/A \\
\hline \multirow{4}{*}{$\begin{array}{l}\text { Narkewicz, Michael R. } \\
\text { Children's Hospital Colorado/ } \\
\text { University of } \\
\text { Colorado School of Medicine; } \\
\text { Department } \\
\text { of Pediatrics; Section of } \\
\text { Pediatric } \\
\text { Gastroenterology, Hepatology, } \\
\text { and Nutrition } \\
\text { Aurora, CO }\end{array}$} & \multirow{4}{*}{$\begin{array}{l}\text { Author: Hepatitis B Virus } \\
\text { Infections } \\
\text { Co-Author: Hepatitis C Virus } \\
\text { Infections }\end{array}$} & - Vertex & $\begin{array}{l}\text { - Research Support } \\
\text { - Consultant }\end{array}$ \\
\hline & & - Merck & $\begin{array}{l}- \text { Stock Holder } \\
\text { (spouse) }\end{array}$ \\
\hline & & - Schering Plough & - Research Support \\
\hline & & - Novartis & - Research Support \\
\hline $\begin{array}{l}\text { Nesheim, Steve } \\
\text { Centers for Disease Control } \\
\text { and Prevention } \\
\text { Atlanta, GA }\end{array}$ & Core Leadership Group & None & N/A \\
\hline $\begin{array}{l}\text { Rutstein, Richard } \\
\text { Children's Hospital of } \\
\text { Philadelphia } \\
\text { Philadelphia, PA }\end{array}$ & $\begin{array}{l}\text { Author: Human Herpesvirus } 8 \\
\text { Infections }\end{array}$ & None & N/A \\
\hline $\begin{array}{l}\text { Seward, Jane } \\
\text { Centers for Disease Control } \\
\text { and Prevention } \\
\text { Atlanta, GA }\end{array}$ & $\begin{array}{l}\text { Co-Author: Immunizations Text } \\
\text { and Figures }\end{array}$ & None & N/A \\
\hline $\begin{array}{l}\text { Shimamura, Masako } \\
\text { University of Alabama at } \\
\text { Birmingham } \\
\text { Birmingham, AL }\end{array}$ & $\begin{array}{l}\text { Author: Cytomegalovirus } \\
\text { Infections }\end{array}$ & None & N/A \\
\hline $\begin{array}{l}\text { Siberry, George } \\
\text { National Institutes of Health } \\
\text { Rockville, MD }\end{array}$ & $\begin{array}{l}\text { Executive Secretary } \\
\text { Author: Bacterial Infections }\end{array}$ & None & N/A \\
\hline \multirow{2}{*}{$\begin{array}{l}\text { Steinbach, William } \\
\text { Duke University Medical } \\
\text { Center } \\
\text { Durham, NC }\end{array}$} & \multirow[t]{2}{*}{ Author: Candidal Infections } & - Merck & - Research Support \\
\hline & & - Astellas & - Research Support \\
\hline $\begin{array}{l}\text { Wilson, Gregory } \\
\text { Vanderbilt University School } \\
\text { of Medicine } \\
\text { Nashville, TN }\end{array}$ & Co-Author: Influenza & None & N/A \\
\hline
\end{tabular}




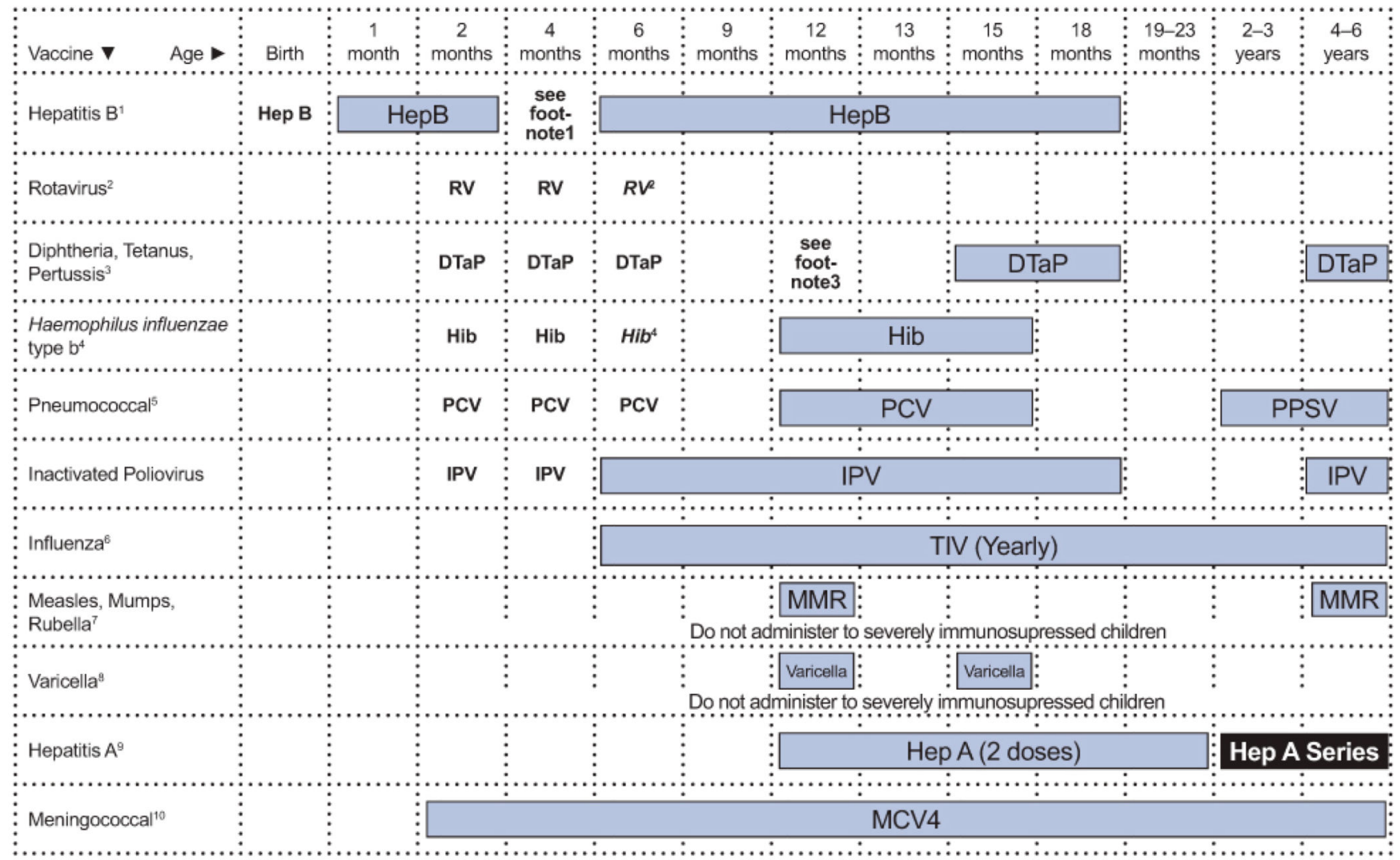

*These recommendations should also be used for perinatally HIV-exposed children who are awaiting laboratory confirmation that they are HIV-uninfected although HIV can be reasonably excluded in most infants in the U.S. by 4 weeks of age (DHHS Pediatric ARV Guidelines).

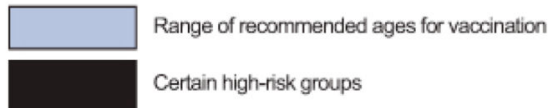

Figure 1. Recommended Immunization Schedule for HIV-Infected Children Aged 0-6 Years; United States, 2013 (Last updated November 6, 2013; last reviewed November 6, 2013)

This schedule summarizes recommendations for routine administration of vaccines for HIVinfected children 0-6 years and indicates the recommended ages for vaccine administration in this population for childhood vaccines licensed in the United States. Any dose not administered at the recommended age should be administered at a subsequent visit, when indicated and feasible. Licensed combination vaccines may be used whenever any component of the combination is indicated and other components of the vaccine are not contraindicated and if approved by the Food and Drug Administration for that dose of the series. Providers should consult the relevant Advisory Committee on Immunization Practices statement for detailed recommendations. Clinically significant adverse events that follow immunization should be reported to the Vaccine Adverse Event Reporting System (VAERS). Guidance about how to obtain and complete a VAERS form is available at http:// www.vaers.hhs.gov or telephone 800-822-7967.

Hepatitis B vaccine (HepB)

Minimum age: Birth

At Birth: 
- Administer monovalent HepB to all newborns before hospital discharge.

- If mother is hepatitis B surface antigen (HBsAg)-positive, administer HepB and 0.5 $\mathrm{mL}$ of hepatitis B immune globulin (HBIG) within 12 hours after birth.

- If mother's HBsAg status is unknown, administer HepB within 12 hours after birth. Determine mother's HBsAg status as soon as possible and if HBsAg-positive, administer HBIG as soon as possible

After the Birth Dose:

- The HepB series should be completed with either monovalent HepB or a combination vaccine containing HepB. The second dose should be administered at ages 1 through 2 months. Monovalent HepB should be used for doses administered before 6 weeks. The final dose should be administered no earlier than age 24 weeks. Infants who did not receive a birth dose should receive 3 doses of a HepBcontaining vaccine on an age-appropriate schedule

Four-Month Dose:

- It is permissible to administer 4 doses of HepB when combination vaccines are administered after the birth dose. If monovalent HepB is used for doses after the birth dose, a dose at age 4 months is not needed.

Post-Vaccination:

- Infants born to HBsAg-positive mothers should be tested for HBsAg and the antibody to HBsAg (anti-HBs) after completion of at least 3 doses of a licensed HepB series, at ages 9 through 18 months(generally at the next well-child visit).

- Testing is recommended for HIV-infected children and should be performed 1 to 2 months after administration of the last dose of the vaccine series using a method that allows determination of a protective level of anti-HBs ( $\geq 10 \mathrm{mIU} / \mathrm{mL})$.

- Children with anti-HBs $<10 \mathrm{mIU} / \mathrm{mL}$ after the primary schedule should receive a second series, followed by anti-HBs testing 1 to 2 months after the third dose, which usually is more practical than serologic testing after one or more doses of vaccine.

Booster Dose:

- In HIV-infected children, the need for booster doses has not been determined. Annual anti-HBs testing and booster doses when anti-HBs levels decline to $<10$ $\mathrm{mIU} / \mathrm{mL}$ should be considered in individuals with ongoing risk for exposure. See MMWR 2005:54(No. RR-16).

Rotavirus vaccine (RV)

Minimum age: 6 weeks

- Practitioners should consider the potential risks and benefits of administering rotavirus vaccine to infants with known or suspected altered immunocompetence. Consultation with an immunologist or infectious disease specialist is advised. Limited safety and efficacy data are available for the administration of rotavirus 
vaccines to infants who are potentially immunocompromised, including those who are HIV-infected. However, the following considerations support vaccination of HIV-exposed or HIV-infected infants:

a. In infants born to HIV-positive mothers, the HIV diagnosis may not be established before the age of the first rotavirus vaccine dose (only 1.5\%$3 \%$ of HIV-exposed infants in the United States will eventually be determined to be HIV-infected), and

b. Vaccine strains of rotavirus are considerably attenuated.

- The maximum age for the first dose in the series is 14 weeks and 6 days; for the final dose in the series, it is 8 months and 0 days. Vaccination should not be initiated for infants aged 15 weeks and 0 days or older.

- If Rotarix ${ }^{\circledR}$ is administered at ages 2 and 4 months, a dose at 6 months is not indicated.

Diphtheria and Tetanus Toxoids and Acellular Pertussis Vaccine (Dtap)

Minimum age: 6 weeks

- The fourth dose may be administered as early as age 12 months, provided that at least 6 months have elapsed since the third dose.

Haemophilus Influenzae Type B Conjugate Vaccine (Hib)

Minimum age: 6 weeks

- If PRP-OMP (PedvaxHIB ${ }^{\circledR}$ or ComVax ${ }^{\circledR}[\mathrm{Hep} \mathrm{B-Hib]}$ ) is administered at ages 2 and 4 months, a dose at age 6 months is not indicated.

- Hiberix should not be used for doses at ages 2, 4, or 6 months for the primary series but may be used as the final dose in children aged 12 months through 4 years.

- One dose of Hib vaccine should be administered to unvaccinated or partially vaccinated persons aged 5 years or older who have leukemia, malignant neoplasms, anatomic or functional asplenia (including sickle cell disease), HIV infection, or other immunocompromising conditions.

Pneumococcal Vaccine

Minimum age: 6 weeks for pneumococcal conjugate vaccine (PCV); 2 years for pneumococcal polysaccharide vaccine (PPSV)

- $\quad$ A PCV series begun with 7-valent PCV (PCV7) should be completed with 13valent PCV (PCV13). A single supplemental dose of PCV13 is recommended for children aged 14 months through 71 months who have received an age-appropriate series of PCV7. For incompletely vaccinated children aged 24 months through 71 months, administer 2 doses of PCV13 at least 8 weeks apart. Children who have previously received $3 \mathrm{PCV}$ doses need only 1 dose.

- Children aged 2 years or older also should receive PPSV after their last PCV dose.

Trivalent Inactivated Influenza Vaccine (TIV) 
- Administer annually to HIV-infected children aged 6 months through 6 years and to all their eligible close contacts (including household members). TIV is recommended for HIV-infected children.

- For healthy, non-pregnant close contacts aged 2 years through 49 years, either live, attenuated influenza vaccine (LAIV) or TIV may be used.

- Children receiving TIV should receive $0.25 \mathrm{~mL}$ if aged 6 through 35 months or 0.5 $\mathrm{mL}$ if aged 3 years or older.

- Administer 2 doses (separated by at least 4 weeks) to children aged younger than 9 years per current influenza vaccine recommendations.

Inactivated Polio Vaccine (IPV)

Minimum age: 6 weeks

- If 4 or more doses are administered prior to age 4 years, an additional dose should be administered at ages 4 through 6 years.

- The final dose in the series should be administered on or after the fourth birthday and at least 6 months after the previous dose.

Measles, Mumps, and Rubella Vaccine (MMR)

Minimum age: 12 months

- Two doses of MMR vaccine for all HIV-infected individuals aged $\geq 12$ months who do not have evidence of current severe immunosuppression (i.e., individuals aged $\leq 5$ years must have CD4 T lymphocyte [CD4] percentages $\geq 15 \%$ for $\geq 6$ months; and individuals aged $>5$ years must have CD4 percentages $\geq 15 \%$ and CD4 $\geq 200$ lymphocytes $/ \mathrm{mm}^{3}$ for $\geq 6$ months) or other current evidence of measles, rubella, and mumps immunity. In cases when only CD4 cell counts or only CD4 percentages are available for those older than age 5 years, the assessment of severe immunosuppression can be based on the CD4 values (count or percentage) that are available. In cases when CD4 percentages are not available for those aged $\mathbf{5}$ years, the assessment of severe immunosuppression can be based on age-specific CD4 counts at the time CD4 counts were measured; i.e., absence of severe immunosuppression is defined as $\Varangle 6$ months above age-specific CD4 count criteria: CD4 count $>750$ lymphocytes $/ \mathrm{mm}^{3}$ while aged $\leq 2$ months and CD4 count $\geq 500$ lymphocytes $/ \mathrm{mm}^{3}$ while aged 1 through 5 years.

- The first dose should be administered at ages 12 months through 15 months and the second dose at ages 4 years through 6 years, or as early as 28 days after the first dose.

- Individuals with perinatal HIV infection who were vaccinated prior to establishment of effective combination antiretroviral therapy (cART) should receive 2 appropriately spaced doses of MMR vaccine once effective cART has

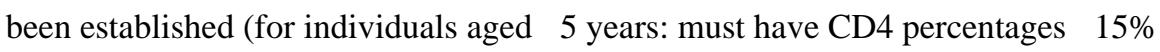
for $\geq 6$ months; and for individuals aged $>5$ years: must have CD4 percentages $\geq 15 \%$ and CD4 $\geq 200$ lymphocytes $/ \mathrm{mm}^{3}$ for $\geq 6$ months) unless they have other acceptable current evidence of measles, rubella, and mumps immunity. 
Varicella Vaccine

Minimum age: 12 months

- Limited data are available on safety and immunogenicity of varicella vaccine in HIV-infected children aged 1 year through 8 years in CDC immunologic categories 1 and 2 (CD4 T-lymphocyte percentages $15 \%$ or greater) and clinical categories $\mathrm{N}$, $\mathrm{A}$, and $\mathrm{B}$.

- Single-antigen varicella vaccine should be considered for HIV-infected children who have CD4 percentages $\geq 15 \%$. Eligible children should receive 2 doses 3 months apart, with the first dose administered as soon as possible after the first birthday.

- Varicella vaccine is not recommended for HIV-infected children who have evidence of severe immunosuppression (CD4 percentage $<15 \%$ at any age; for those older than age 5 years, CD4 count $<200$ cells $/ \mathrm{mm}^{3}$ ).

- MMRV vaccine has not been studied in HIV-infected children and should not be substituted for singleantigen varicella vaccine.

Hepatitis A Vaccine (HepA)

Minimum age: 12 months

- Administer to all children aged 12 months through 23 months. The 2 doses in the series should be administered at least 6 months apart.

- Children who are not fully vaccinated by age 2 years can be vaccinated at subsequent visits.

- HepA is also recommended for children 24 months and older who live in areas where vaccination programs target older children, who are at increased risk of infection, or for whom immunity against hepatitis A is desired. See $M M W R$ 2006;55(No. RR-7).

Meningococcal Vaccine

Minimum age: 6 weeks for combination Haemophilus influenzae type b-meningococcal conjugate vaccine serogroup CY (Hib-MenCY); 9 months for meningococcal conjugate vaccine (MCV4); 2 years for meningococcal polysaccharide vaccine (MPSV4)

- Administer MCV4 to children aged 2 years through 6 years who have functional asplenia and certain other high-risk groups. A primary series of 2 doses should be administered with a minimum interval of 8 weeks. See $M M W R$ 2007;56(48):12656.

- Administer MCV4-D (Menactra) to infants/children 9 months through 23 months who have persistent complement component deficiency, are traveling to an area endemic for meningococcal disease, or are involved in a meningococcal outbreak.

- Children who received MPSV4 $\geq 3$ years previously and remain at increased risk of meningococcal disease should be revaccinated with MCV4. 
- HIV-infected children are not considered at increased risk of meningococcal disease because of HIV infection, per se. Although the efficacy of MCV4 among HIV-infected children is unknown, providers can vaccinate HIV-infected children.

- HIV-infected children who remain at increased risk of meningococcal disease should be vaccinated every 5 years thereafter. 


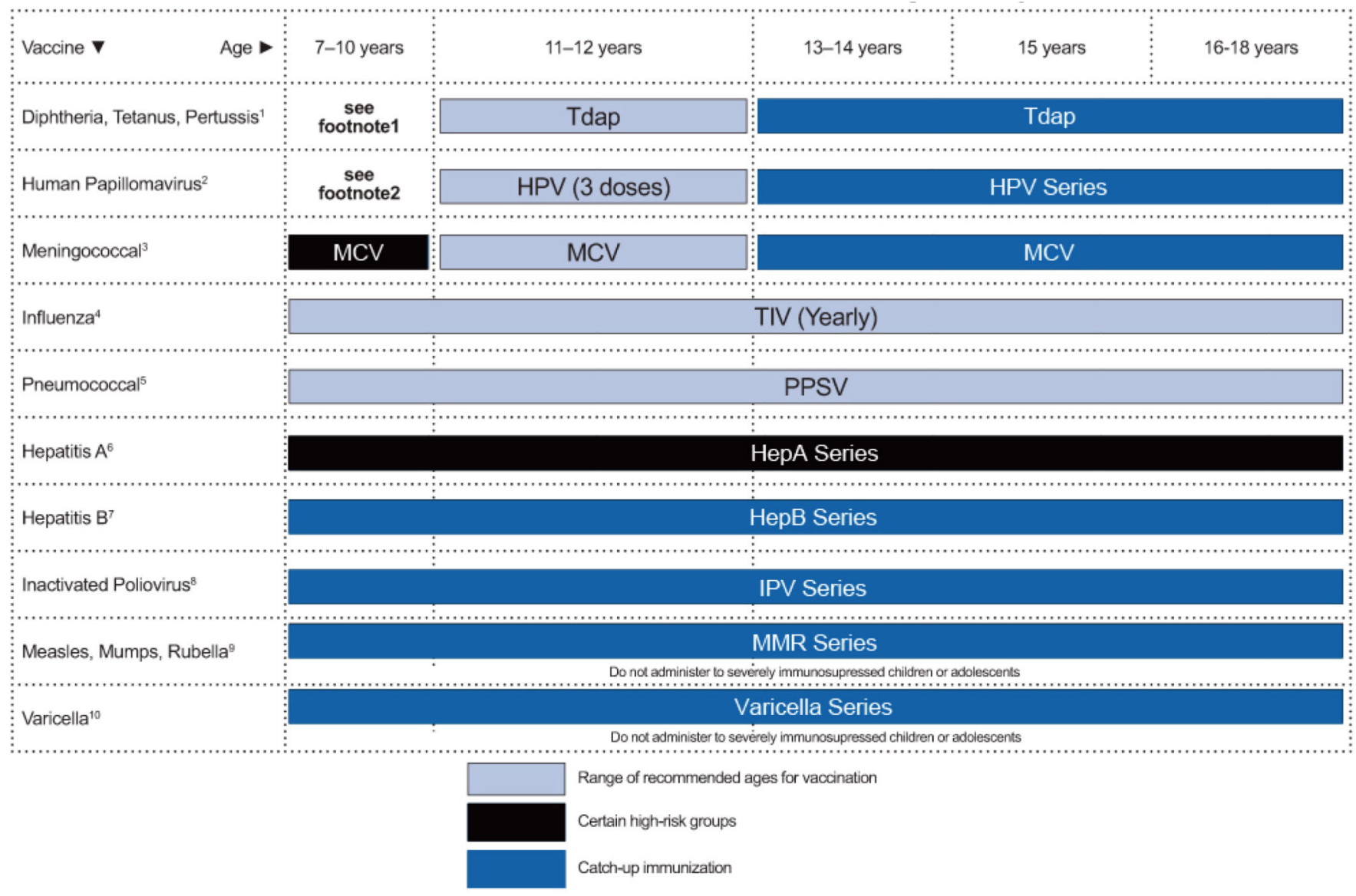

Figure 2. Recommended Immunization Schedule for HIV-Infected Children Aged 7-18 Years; United States 2013 (Last updated November 6, 2013; last reviewed November 6, 2013)

This schedule summarizes recommendations for routine administration of vaccines for HIVinfected children and adolescents aged 7 through 18 years and indicates the recommended ages for vaccine administration for vaccines licensed in the United States in 2013. Licensed combination vaccines may be used whenever a component of the combination is indicated and other components of the vaccine are not contraindicated and if approved by the Food and Drug Administration for that dose of the series, unless otherwise specified. Clinically significant adverse events that follow immunization should be reported to the Vaccine Adverse Event Reporting System (VAERS). Guidance about how to obtain and complete a VAERS form is available at http://www.vaers.hhs.gov or telephone 800-822-7967.

Tetanus And Diphtheria Toxoids And Acellular Pertussis Vaccine (Tdap) Minimum age: 7 years

- Individuals aged 11 through 18 years who have not received Tdap should receive a dose followed by Tetanus Diphtheria (Td) booster doses every 10 years thereafter.

- Individuals aged 7 through 10 years who are not fully immunized against pertussis (including those never vaccinated or with unknown pertussis status) should receive a single dose of Tdap. Refer to the catch-up schedule if additional doses of tetanus and diphtheria toxoid-containing vaccine are needed. 
- Tdap can be administered regardless of the interval since the last tetanus dose.

Human Papillomavirus Vaccine (HPV)

Minimum age: 9 years

Note: Two HPV vaccines are licensed. A quadrivalent vaccine (HPV4) is licensed for use in females and males; a bivalent vaccine (HPV2) is licensed for use in females. Because these are not live virus vaccines, they can be administered to individuals who are

immunosuppressed because of disease or medication, including those who are HIV-infected. However, the immune response and vaccine efficacy may be less than in immunocompetent individuals.

- HPV vaccines are most effective for both males and females when given before exposure to HPV through sexual contact.

- Administer the first dose at ages 11 or 12 years.

- Administer the second dose 1 to 2 months after the first dose and the third dose 6 months after the first dose (at least 24 weeks after the first dose).

- Administer the series at ages 13 through 18 years if not previously vaccinated.

- HPV4 can be administered in a 3-dose series to individuals aged 9 through 10 years.

Meningococcal Vaccine (Meningococcal Conjugate Vaccine [MCV4])

- Individuals who receive the second dose of the primary series at or before age 1112 years should receive a booster dose at age 16 years. Although the efficacy of MCV4 among HIV-infected patients is unknown, HIV-infected patients aged 7 through 10 years may elect vaccination.

Influenza Vaccine (Trivalent Inactivated Influenza Vaccine [TIV])

- Administer annually to HIV-infected children and adolescents. Only TIV should be used in HIV-infected individuals. For healthy non-pregnant close contacts aged 2 through 49 years, either live, attenuated influenza vaccine (LAIV) or TIV can be used.

- Administer 2 doses (separated by at least 4 weeks) to children aged younger than 9 years who are receiving influenza vaccine for the first time or based on previous influenza vaccine history, per current influenza vaccine recommendations.

Pneumococcal Vaccine (Pneumococcal Conjugate Vaccine [PCV]; Pneumococcal Polysaccharide Vaccine [PPSV])

- A single dose of 13-valent pneumococcal conjugate vaccine (PCV13) should be routinely administered to HIV-infected children aged 6 through 18 years who did not previously receive a dose of PCV13 before age 6 years. The dose should be administered at least 8 weeks after the previous dose of PCV.

- Administer 23-valent pneumococcal polysaccharide vaccine (PPSV23) at least 8 weeks after the last dose of PCV13 to children aged $\geq 2$ years. A single revaccination dose should be administered 5 years thereafter. 
- HIV-infected children who have already received a dose or doses of PPSV23 should receive a dose of PCV13 a minimum of 8 weeks after the dose of PPSV23. A second dose of PPSV23 is recommended 5 years later.

Hepatitis A Vaccine (HepA)

- HepA is recommended for children older than age 23 months who live in areas where vaccination programs target older children, who are at increased risk of infection, or for whom immunity against Hepatitis A is desired. See $M M W R$ 2006;55(No. RR-7).

Hepatitis B Vaccine (HepB)

- Administer the 3-dose series to those who were not previously vaccinated.

- Post-vaccination testing is recommended for HIV-infected individuals. Testing should be performed 1 to 2 months after administration of the final dose. Individuals found to have anti-HBs levels of $<10 \mathrm{mIU} / \mathrm{mL}$ after the primary series should be revaccinated. Administration of 3 doses on an appropriate schedule, followed by anti-HBs testing 1 to 2 months after the third dose, is usually more practical than serologic testing after 1 or 2 doses of vaccine. Modified dosing regimens, including doubling of the standard antigen dose, may increase response rates. However, data are limited on response to these alternative vaccination schedules.

- In HIV-infected individuals, the need for booster doses has not been determined. Annual anti-HBs testing and booster doses when anti-HBs levels decline to $<10$ $\mathrm{mIU} / \mathrm{mL}$ should be considered in individuals with ongoing risk of exposure. See MMWR 2005:54(No. RR-16).

Inactivated Poliovirus Vaccine (IPV)

- The final dose in the series should be administered on or after the fourth birthday and at least 6 months after the previous dose.

- For children who received an all-IPV or all-oral poliovirus (OPV) series, a fourth dose is not necessary if the third dose was administered at age $\geq 4$ years.

- If both OPV and IPV were administered as part of a series, a total of 4 doses should be administered, regardless of a child's current age.

Measles, Mumps, and Rubella Vaccine (MMR)

- If eligible and not previously vaccinated, administer 2 doses with the second dose at least 28 days after the first dose, or administer the second dose for those who received only 1 dose, with at least 28 days between doses.

- Two doses of MMR vaccine are recommended for all HIV-infected individuals aged $\geq 12$ months who do not have evidence of current severe immunosuppression (i.e., individuals aged $>5$ years must have CD4 T lymphocyte [CD4] percentages $\geq 15 \%$ and CD4 $\geq 200$ lymphocytes $/ \mathrm{mm}^{3}$ for $\geq 6$ months) or other current evidence of measles, rubella, and mumps immunity. In cases when only CD4 counts or only 
CD4 percentages are available for those older than age 5 years, assessment of severe immunosuppression can be based on the CD4 values (count or percentage) that are available.

- Individuals with perinatal HIV infection who were vaccinated prior to establishment of effective combination antiretroviral therapy (cART) should receive two appropriately spaced doses of MMR vaccine once effective cART has been established (individuals aged $>5$ years: must have CD4 percentages $\geq 15 \%$ and CD4 200 lymphocytes $/ \mathrm{mm}^{3}$ for $\geq 6$ months) unless they have other acceptable current evidence of measles, rubella, and mumps immunity.

Varicella Vaccine

- Limited data are available on safety and immunogenicity of varicella vaccine in HIV-infected children aged 1 through 8 years in Centers for Disease Control and Prevention immunologic categories 1 and 2 (CD4 percentages $215 \%$ ) and clinical categories N, A, and B. Varicella vaccine should be considered for HIV-infected children aged 1 through 8 years with CD4 percentages $\geq 15 \%$. Eligible children should receive 2 doses at least 3 months apart.

- Data are lacking on use of varicella vaccine in HIV-infected children older than age 8 years. However, on the basis of expert opinion, the safety of varicella vaccine in HIV-infected individuals older than age 8 years with similar levels of immune function (CD4 age-specific percentages $\geq 15 \%$ or count $\geq 200$ cells $/ \mathrm{mm}^{3}$ ) is likely to be similar to that for children aged $\$ 8$ years. Immunogenicity may be lower in HIV-infected adolescents (and adults). However, weighing the risk of severe disease from wild varicella zoster virus and the potential benefit of vaccination, vaccination ( 2 doses administered 3 months apart) can be considered for children and adolescents aged 9 through 18 years who lack evidence of immunity.

- Varicella vaccine is not recommended for HIV-infected children or adolescents who have evidence of severe immunosuppression (CD4 percentage $<15 \%$ at any age; for those older than age 5 years, CD4 count $<200$ cells $/ \mathrm{mm}^{3}$ ).

- MMRV vaccine has not been studied in HIV-infected children and should not be substituted for single-antigen varicella vaccine.

- For evidence of immunity guidance and other details, see $M M W R$ 2007;56(No.RR-4).

Note: Haemophilus Influenzae Type B Conjugate Vaccine (Hib)

- Hib conjugate vaccines are available in single- or combined-antigen preparations. Hib is recommended routinely for all children through age 59 months. One dose of Hib vaccine should be administered to unvaccinated or partially vaccinated individuals aged 5 years or older who have leukemia, malignant neoplasms, anatomic or functional asplenia (including sickle cell disease), are HIV-infected, or who have other immunocompromising conditions. 
Table 1

Primary Prophylaxis of Opportunistic Infections in HIV-Exposed and HIV-Infected Children-Summary of Recommendations (Last updated November 6, 2013; last reviewed November 6, 2013)

\begin{tabular}{|c|c|c|c|}
\hline Indication & First Choice & Alternative & Comments/Special Issues \\
\hline $\begin{array}{l}\text { Bacterial Infections } S \text {. pneumoniae and other } \\
\text { invasive bacteria }\end{array}$ & 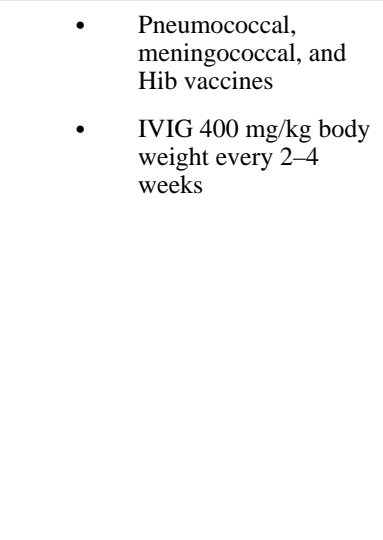 & $\begin{array}{l}\text { TMP-SMX } \\
75 / 375 \mathrm{mg} / \mathrm{m}^{2} \\
\text { body surface } \\
\text { area per dose } \\
\text { by mouth } \\
\text { twice daily }\end{array}$ & 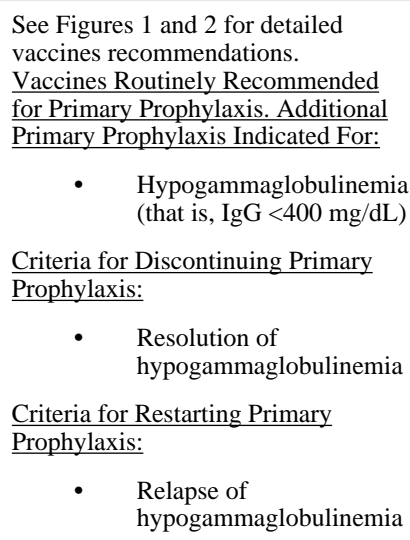 \\
\hline Candidiasis & Not routinely recommended & N/A & N/A \\
\hline Coccidioidomycosis & N/A & N/A & $\begin{array}{l}\text { Primary prophylaxis not routinely } \\
\text { indicated in children. }\end{array}$ \\
\hline Cryptococcosis & Not recommended & Not recommended & N/A \\
\hline Cryptosporidiosis & $\begin{array}{l}\text { ARV therapy to avoid advanced } \\
\text { immune deficiency }\end{array}$ & N/A & N/A \\
\hline Cytomegalovirus (CMV) & $\begin{array}{l}\text { - For older children who } \\
\text { can receive adult dose } \\
\text { (based on their BSA), } \\
\text { valganciclovir tablets } \\
900 \mathrm{mg} \text { orally once } \\
\text { daily with food } \\
\text { - } \quad \text { For children aged } 4 \\
\text { months } 16 \text { years, } \\
\text { valganciclovir oral } \\
\text { solution } 50 \mathrm{mg} / \mathrm{mL} \text { at } \\
\text { dose in milligrams }=7 \\
\times \mathrm{BSA} \times \mathrm{CrCl}(\text { up to } \\
\text { maximum } \mathrm{CrCl} \text { of } 150 \\
\left.\mathrm{~mL} / \mathrm{min} / 1.73 \mathrm{~m}^{2}\right) \\
\text { orally once daily with } \\
\text { food (maximum dose } \\
900 \mathrm{mg} / \text { day) }\end{array}$ & N/A & 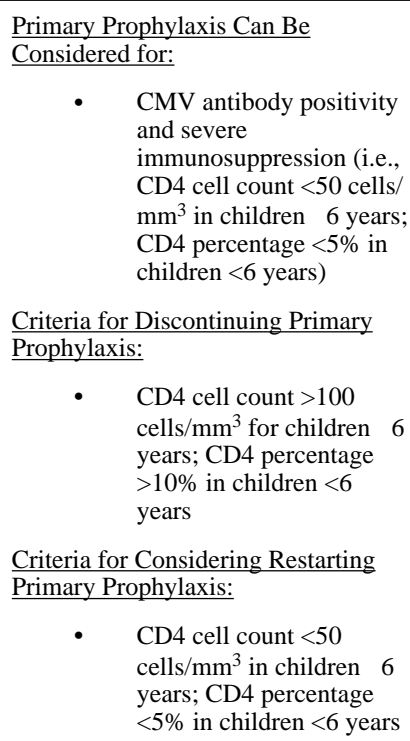 \\
\hline Giardiasis & $\begin{array}{l}\text { cART to avoid advanced } \\
\text { immunodeficiency }\end{array}$ & N/A & N/A \\
\hline Hepatitis B Virus (HBV) & $\begin{array}{ll}\text { - } & \text { Hepatitis B vaccine } \\
\text { - } & \text { Combination of } \\
\text { hepatitis B } \\
\text { immunoglobulin and } \\
\text { hepatitis B vaccine for }\end{array}$ & $\begin{array}{l}\text { Hepatitis B } \\
\text { immunoglobulin following } \\
\text { exposure }\end{array}$ & 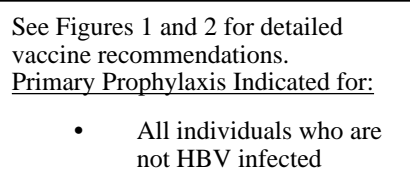 \\
\hline
\end{tabular}




\begin{tabular}{|c|c|c|c|}
\hline Indication & First Choice & Alternative & Comments/Special Issues \\
\hline & $\begin{array}{l}\text { infants born to mothers } \\
\text { with hepatitis B } \\
\text { infection }\end{array}$ & & $\begin{array}{l}\text { Criteria for Discontinuing Primary } \\
\text { Prophylaxis: } \\
\text { - N/A } \\
\text { Criteria for Restarting Primary } \\
\text { Prophylaxis: } \\
\text { - N/A }\end{array}$ \\
\hline Hepatitis C Virus (HCV) & None & N/A & N/A \\
\hline Herpes Simplex Virus Infections (HSV) & None & None & Primary prophylaxis is not indicated. \\
\hline Histoplasmosis & N/A & N/A & $\begin{array}{l}\text { Primary Prophylaxis indicated for } \\
\text { selected HIV-infected adults but not } \\
\text { children. } \\
\text { Criteria for Discontinuing Primary } \\
\begin{array}{c}\text { Prophylaxis: } \\
\text { - N/A }\end{array} \\
\text { Criteria for Restarting Primary } \\
\begin{array}{l}\text { Prophylaxis: } \\
\quad \text { N/A }\end{array}\end{array}$ \\
\hline Human Papillomavirus (HPV) & HPV vaccine & N/A & $\begin{array}{l}\text { See Figure } 2 \text { for detailed vaccine } \\
\text { recommendations. }\end{array}$ \\
\hline Influenza Primary Prophylaxis & Influenza vaccine & None & $\begin{array}{l}\text { Note: See Figures } 1 \text { and } 2 \text { for } \\
\text { detailed vaccines recommendations. }\end{array}$ \\
\hline $\begin{array}{l}\text { Primary Chemoprophylaxis Influenza A } \\
\text { and B }\end{array}$ & 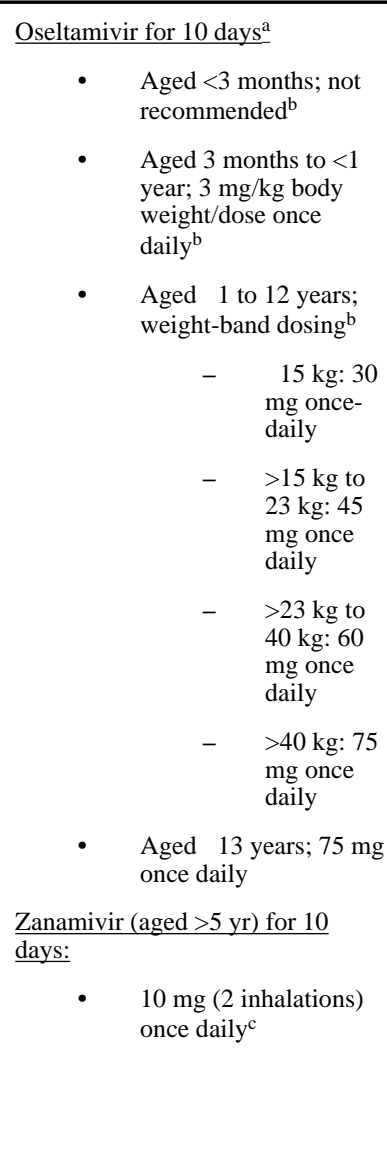 & None & $\begin{array}{l}\text { Primary chemoprophylaxis is } \\
\text { indicated for unvaccinated HIV- } \\
\text { infected children with moderate-to- } \\
\text { severe immunosuppression (as } \\
\text { assessed by immunologic and/or } \\
\text { clinical diagnostic categories) who } \\
\text { are household contacts or close } \\
\text { contacts of individuals with } \\
\text { confirmed or suspected influenza. } \\
\text { Chemoprophylaxis of vaccinated } \\
\text { HIV-infected children with severe } \\
\text { immunosuppression also may be } \\
\text { indicated based on health-care } \\
\text { provider assessment of the exposure } \\
\text { situation. Post-exposure antiviral } \\
\text { chemoprophylaxis should be initiated } \\
\text { as soon as possible after exposure. } \\
\text { a Oseltamivir chemoprophylaxis } \\
\text { duration: Recommended duration is } \\
10 \text { days when administered after a } \\
\text { household exposure and } 7 \text { days after } \\
\text { the most recent known exposure in } \\
\text { other situations. For control of } \\
\text { outbreaks in long-term care facilities } \\
\text { and hospitals, CDC recommends } \\
\text { antiviral chemoprophylaxis for a } \\
\text { minimum of } 2 \text { weeks and up to } 1 \\
\text { week after the most recent known } \\
\text { case was identified (see http:// } \\
\text { www.cdc.gov/mmwr/preview/ } \\
\text { mmwrhtml/rr6001al.htm). } \\
\text { b Oseltamivir is approved by the FDA } \\
\text { for treatment of influenza in children } \\
\text { aged } 22 \text { weeks. It is not approved for } \\
\text { prophylaxis in children aged }<1 \text { year. } \\
\text { However, the CDC recommends that } \\
\text { health-care providers who treat } \\
\text { children ages } 23 \text { months to < } 1 \text { year } \\
\text { administer a chemoprophylaxis dose } \\
\text { of } 3 \text { mg/kg body weight/dose once } \\
\text { daily. Chemoprophylaxis for infants }\end{array}$ \\
\hline
\end{tabular}




\begin{tabular}{|c|c|c|c|}
\hline Indication & First Choice & Alternative & Comments/Special Issues \\
\hline & & & $\begin{array}{l}\text { aged }<3 \text { months is not recommended } \\
\text { unless the exposure situation is } \\
\text { judged to be critical. } \\
\text { Premature infants: Current weight- } \\
\text { based dosing recommendations for } \\
\text { oseltamivir are not appropriate for } \\
\text { premature infants (i.e., gestational } \\
\text { age at delivery <38 weeks). See J } \\
\text { Infect Dis } 202 \text { [4]:563-566, } 2010 \text { for } \\
\text { dosing recommendations in } \\
\text { premature infants. } \\
\text { Renal insufficiency: A reduction in } \\
\text { dose of oseltamivir is recommended } \\
\text { for patients with creatinine clearance } \\
<30 \text { mL/min. } \\
\text { c Zanamivir: Zanamivir is not } \\
\text { recommended for chemoprophylaxis } \\
\text { in children aged <5 years old. }\end{array}$ \\
\hline $\begin{array}{l}\text { Primary Chemoprophylaxis Influenza A } \\
\text { (ONLY) Oseltamivir-resistant, adamantane- } \\
\text { sensitive strains } \\
\text { Based on CDC influenza surveillance; http:// } \\
\text { www.cdc.gov/flu/weekly/fluactivitysurv.htm }\end{array}$ & 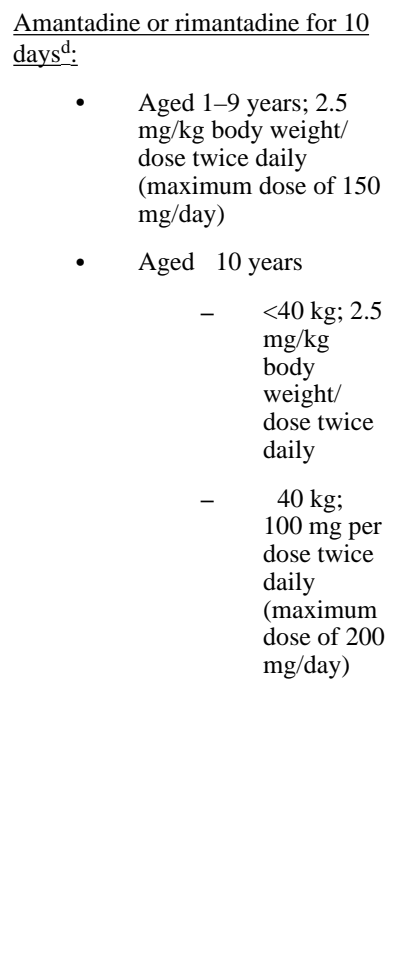 & & $\begin{array}{l}\text { dAdamantanes: Because of } \\
\text { resistance in currently circulating } \\
\text { influenza A virus strains, amantadine } \\
\text { and rimantadine are not currently } \\
\text { recommended for chemoprophylaxis } \\
\text { or treatment (adamantanes are not } \\
\text { active against influenza B virus). } \\
\text { However, potential exists for } \\
\text { emergence of oseltamivir-resistant, } \\
\text { adamantane-sensitive circulating } \\
\text { influenza A strains. Therefore, } \\
\text { verification of antiviral sensitivity of } \\
\text { circulating influenza A strains should } \\
\text { be done using the CDC influenza } \\
\text { surveillance website: http:// } \\
\text { www.cdc.gov/flu/weekly/ } \\
\text { fluactivitysurv.htm } \\
\text { If administered based on CDC } \\
\text { antiviral sensitivity surveillance data, } \\
\text { both amantadine and rimantadine are } \\
\text { recommended for chemoprophylaxis } \\
\text { of influenza A in children aged } \geq 1 \text { yr. } \\
\text { For treatment, rimantadine is only } \\
\text { approved for use in adolescents aged } \\
\geq 13 \text { years. Rimantadine is preferred } \\
\text { over amantadine because of less } \\
\text { frequent adverse events. Some } \\
\text { pediatric influenza specialists may } \\
\text { consider it appropriate for treatment } \\
\text { of children aged }>1 \text { year. } \\
\text { Renal insufficiency: A reduction in } \\
\text { dose of amantadine is recommended } \\
\text { for patients with creatinine clearance } \\
<30 \text { mL/min. }\end{array}$ \\
\hline Isosporiasis (Cystoisosporiasis) & $\begin{array}{l}\text { There are no U.S. } \\
\text { recommendations for primary } \\
\text { prophylaxis of isosporiasis. }\end{array}$ & N/A & $\begin{array}{l}\text { Initiation of cART to avoid advanced } \\
\text { immunodeficiency may reduce } \\
\text { incidence; TMP-SMX prophylaxis } \\
\text { may reduce incidence. }\end{array}$ \\
\hline Malaria & $\begin{array}{ll}\text { For Travel To Chloroquine- } \\
\text { Sensitive Areas } \\
& \text { Chloroquine base 5 } \\
\text { mg/kg body weight } \\
\text { base by mouth, up to } \\
300 \text { mg once weekly } \\
\text { (equivalent to } 7.5 \\
\text { mg/kg body weight } \\
\text { chloroquine } \\
\text { phosphate). Start } 1-2 \\
\text { weeks before leaving, } \\
\text { take weekly while } \\
\text { away, and then take } \\
\text { once weekly for 4 }\end{array}$ & N/A & $\begin{array}{l}\text { Recommendations are the same for } \\
\text { HIV-infected and HIV-uninfected } \\
\text { children. Please refer to the following } \\
\text { website for the most recent } \\
\text { recommendations based on region } \\
\text { and drug susceptibility: http:// } \\
\text { www.cdc.gov/ malaria/ } \\
\text { For travel to chloroquine-sensitive } \\
\text { areas. Equally recommended options } \\
\text { include chloroquine, atovaquone/ } \\
\text { proguanil, doxycycline (for children } \\
\text { aged } \geq 8 \text { years), and mefloquine; } \\
\text { primaquine is recommended for areas } \\
\text { with mainly } P \text {. vivax. }\end{array}$ \\
\hline
\end{tabular}




\begin{tabular}{|c|c|c|c|}
\hline Indication & First Choice & Alternative & Comments/Special Issues \\
\hline & 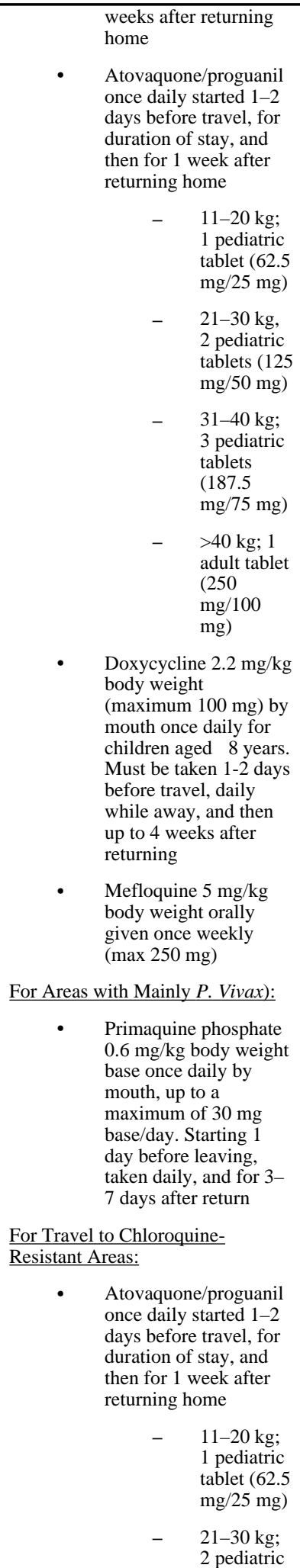 & & $\begin{array}{l}\text { G6PD screening must be performed } \\
\text { prior to primaquine use. } \\
\text { Chloroquine phosphate is the only } \\
\text { formulation of chloroquine available } \\
\text { in the United States; } 10 \mathrm{mg} \text { of } \\
\text { chloroquine phosphate }=6 \mathrm{mg} \text { of } \\
\text { chloroquine base. } \\
\text { For travel to chloroquine-resistant } \\
\text { areas, preferred drugs are } \\
\text { atovaquone/proguanil, doxycycline } \\
\text { (for children aged } \geq 8 \text { years) or } \\
\text { mefloquine. }\end{array}$ \\
\hline
\end{tabular}




\begin{tabular}{|c|c|c|c|}
\hline Indication & First Choice & Alternative & Comments/Special Issues \\
\hline & $\begin{array}{l}\text { tablets (125 } \\
\mathrm{mg} / 50 \mathrm{mg}) \\
-\quad 31-40 \mathrm{~kg} ; \\
3 \text { pediatric } \\
\text { tablets } \\
(187.5 \\
\mathrm{mg} / 75 \mathrm{mg}) \\
-\quad>40 \mathrm{~kg} ; 1 \\
\text { adult tablet } \\
(250 \\
\mathrm{mg} / 100 \\
\mathrm{mg} \text { ) }\end{array}$ & & \\
\hline Microsporidiosis & N/A & N/A & Not recommended \\
\hline Mycobacterium avium Complex (MAC) & $\begin{array}{l}\text { - Clarithromycin } 7.5 \\
\text { mg/kg body weight } \\
\text { (maximum } 500 \mathrm{mg} \text { ) by } \\
\text { mouth orally twice } \\
\text { daily, or } \\
\text { - } \quad \text { Azithromycin } 20 \\
\mathrm{mg} / \mathrm{kg} \text { body weight } \\
\text { (maximum } 1200 \mathrm{mg} \text { ) } \\
\text { orally once weekly }\end{array}$ & $\begin{array}{ll}\text { - Azithromycin } \\
5 \mathrm{mg} / \mathrm{kg} \text { body } \\
\text { weight } \\
\text { (maximum } \\
250 \mathrm{mg} \text { ) orally } \\
\text { once daily } \\
\text { - Children aged } \\
>5 \text { years: } \\
\text { rifabutin } 300 \\
\text { mg orally once } \\
\text { daily with food }\end{array}$ & 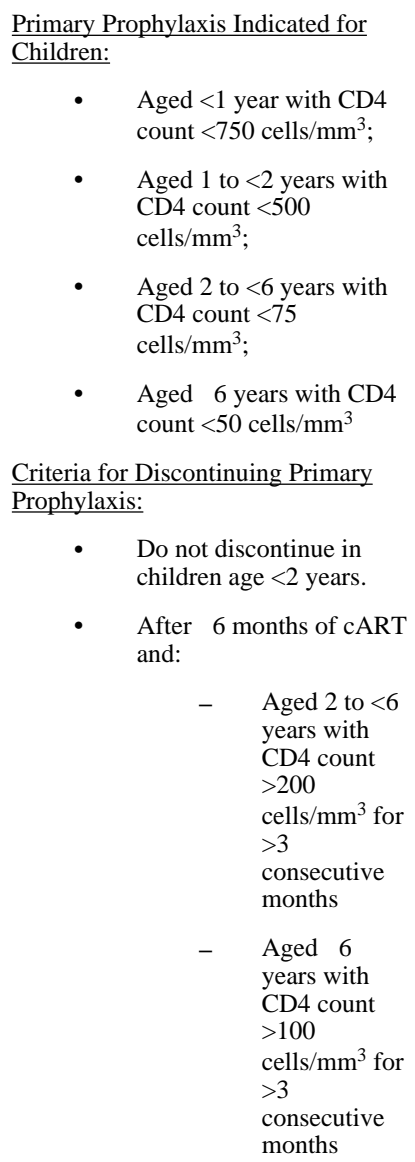 \\
\hline
\end{tabular}




\begin{tabular}{|c|c|c|c|}
\hline Indication & First Choice & Alternative & Comments/Special Issues \\
\hline & & & $\begin{array}{l}\text { Criteria for Restarting Primary } \\
\text { Prophylaxis: } \\
\\
\text { Aged } 2 \text { to }<6 \text { years with } \\
\text { CD4 count }<200 \\
\text { cells } / \mathrm{mm}^{3} \\
\text { - } \quad \begin{array}{l}\text { Aged } \succ 6 \text { years with CD4 } \\
\text { count }<100 \text { cells } / \mathrm{mm}^{3}\end{array}\end{array}$ \\
\hline Mycobacterium Tuberculosis (post-exposure) & $\begin{array}{l}\text { Source Case Drug Susceptible: } \\
\begin{array}{l}\text { Isoniazid } 10-15 \mathrm{mg} / \mathrm{kg} \\
\text { body weight } \\
\text { (maximum } 300 \mathrm{mg} / \\
\text { day) by mouth daily for } \\
9 \text { months }\end{array} \\
\text { Source Case Drug Resistant: } \\
\text { - } \quad \begin{array}{l}\text { Consult expert and } \\
\text { local public health } \\
\text { authorities. }\end{array}\end{array}$ & $\begin{array}{l}\text { - If adherence } \\
\text { with daily } \\
\text { isoniazid } \\
\text { cannot be } \\
\text { ensured, } \\
\text { consider } \\
\text { isoniazid 20- } \\
30 \mathrm{mg} / \mathrm{kg} \text { body } \\
\text { weight } \\
\text { (maximum } \\
900 \mathrm{mg} / \mathrm{day} \text { ) } \\
\text { by mouth } 2 \\
\text { times a week } \\
\text { by DOT for } 9 \\
\text { months } \\
\text { Isoniazid } 10- \\
15 \mathrm{mg} / \mathrm{kg} \\
\text { body weight } \\
\text { (maximum } \\
300 \mathrm{mg} / \text { day) } \\
\text { and rifampin } \\
10-20 \mathrm{mg} / \mathrm{kg} / \\
\text { body weight } \\
\text { (maximum } \\
600 \mathrm{mg} / \mathrm{day} \text { ) } \\
\text { by mouth daily } \\
\text { for } 3-4 \text { months } \\
\text { Rifampin } 10- \\
20 \mathrm{mg} / \mathrm{kg} \\
\text { body weight } \\
\text { (maximum } \\
600 \mathrm{mg} / \text { day) } \\
\text { by mouth daily } \\
\text { for } 4-6 \mathrm{months}\end{array}$ & 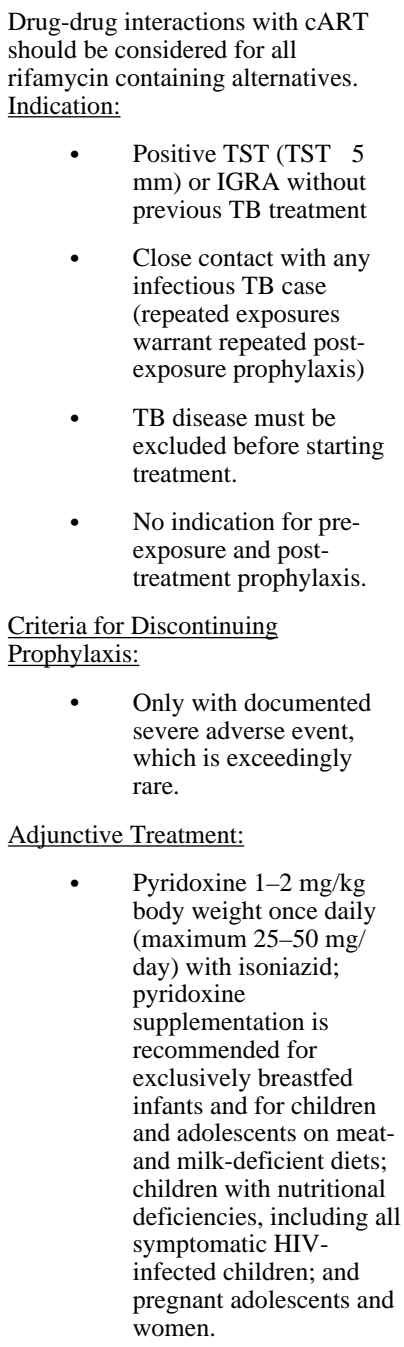 \\
\hline Pneumocystis jirovecii Pneumonia & $\begin{array}{l}\text { TMP-SMX } \\
\text { (Cotrimoxazole): TMP } \\
\text { 2.5-5 mg/kg body } \\
\text { weight/dose with SMX } \\
12.5-25 \mathrm{mg} / \mathrm{kg} \text { body } \\
\text { weight/dose twice per } \\
\text { day. Dosing based on } \\
\text { TMP component. } \\
\text { The total daily dose } \\
\text { should not exceed } 320 \\
\text { mg TMP and } 1600 \mathrm{mg} \\
\text { SMX. Several dosing }\end{array}$ & $\begin{array}{l}\text { Dapsone } \\
\text { Children aged } \geq 1 \text { months: } \\
\qquad \quad 2 \mathrm{mg} / \mathrm{kg} \text { body } \\
\text { weight } \\
\text { (maximum } \\
100 \mathrm{mg} \text { ) by } \\
\text { mouth once } \\
\text { daily or } 4 \\
\text { mg/kg body } \\
\text { weight } \\
\text { (maximum } \\
200 \mathrm{mg} \text { ) by }\end{array}$ & $\begin{array}{ll}\text { Primary Prophylaxis Indicated For: } \\
\text { All HIV-infected or HIV- } \\
\text { indeterminate infants } \\
\text { from aged 4-6 weeks to } \\
12 \text { months. regardless of } \\
\text { CD4 cell count/ } \\
\text { percentage } \\
\text { HIV-infected children } \\
\text { aged } 1 \text { to }<6 \text { years with } \\
\text { CD4 count }<500 \\
\text { cells } / \mathrm{mm}^{3} \text { or CD4 } \\
\text { percentage }<15 \% ; \text { HIV- }\end{array}$ \\
\hline
\end{tabular}




\begin{tabular}{|c|c|c|c|}
\hline Indication & First Choice & Alternative & Comments/Special Issues \\
\hline & $\begin{array}{cl}\text { schemes have been } \\
\text { used successfully- } \\
\text { - } & \text { Given } 3 \\
& \text { days per } \\
& \text { week on } \\
\text { consecutive } & \text { days or on } \\
\text { alternate } & \text { days } \\
\text { - } & \text { Given } 2 \\
& \text { days per } \\
\text { week on } \\
\text { consecutive } \\
\text { days or on } \\
\text { alternate } \\
\text { days } \\
\text { - Given } \\
\text { every day } \\
\text { (total daily } \\
\text { dose of } \\
\text { TMP 5-10 } \\
\text { mg/kg } \\
\text { body } \\
\text { weight } \\
\text { given as a } \\
\text { single dose } \\
\text { each day) }\end{array}$ & $\begin{array}{l}\text { mouth once } \\
\text { weekly } \\
\text { Atovaquone } \\
\text { Children Aged 1-3 Months } \\
\text { and >24 Months-12 } \\
\text { Years: } \\
\text { - } \quad \text { 30-40 mg/kg } \\
\text { body weight/ } \\
\text { dose by mouth } \\
\text { once daily } \\
\text { with food }\end{array}$ & 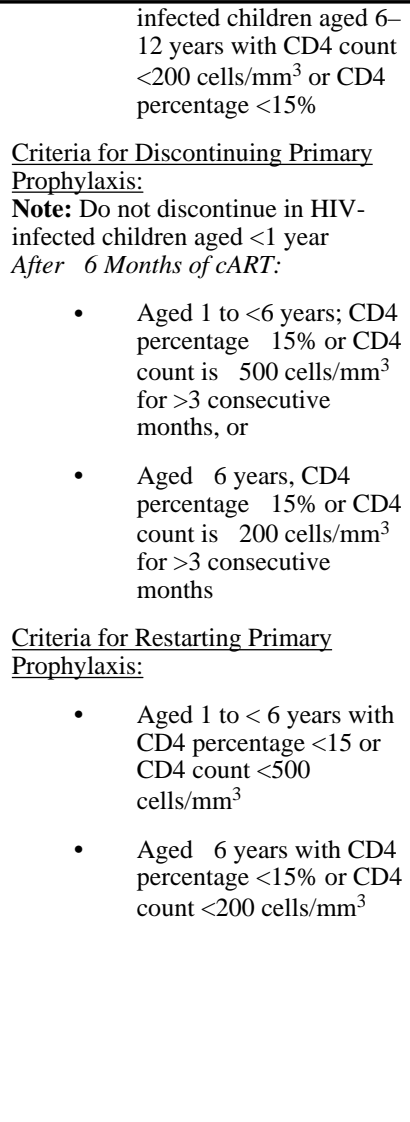 \\
\hline Syphilis & N/A & N/A & $\begin{array}{l}\text { Primary Prophylaxis Indicated for: } \\
\text { - N/A } \\
\text { Criteria for Discontinuing Primary } \\
\text { Prophylaxis: } \\
\text { - N/A } \\
\text { Criteria for Restarting Primary } \\
\text { Prophylaxis: } \\
\quad \text { N/A }\end{array}$ \\
\hline Toxoplasmosis & $\begin{array}{l}\text { TMP-SMX } 150 / 750 \mathrm{mg} / \mathrm{m}^{2} \text { body } \\
\text { surface area once daily by mouth }\end{array}$ & $\begin{array}{ll}\text { For Children Aged } \geq 1 \\
\text { Month: } & \\
\text { - } & \text { Dapsone } 2 \\
& \text { mg/kg body } \\
& \text { weight or } 15 \\
& \mathrm{mg} / \mathrm{m}^{2} \text { body } \\
\text { surface area } & \text { (maximum } 25 \\
& \mathrm{mg} \text { ) by mouth } \\
& \text { once daily, } \\
& \text { plus } \\
\text { - } & \text { Pyrimethamine } \\
& 1 \mathrm{mg} / \mathrm{kg} \text { body } \\
& \text { weight } \\
\text { (maximum } 25 \\
\text { mg) by mouth }\end{array}$ & 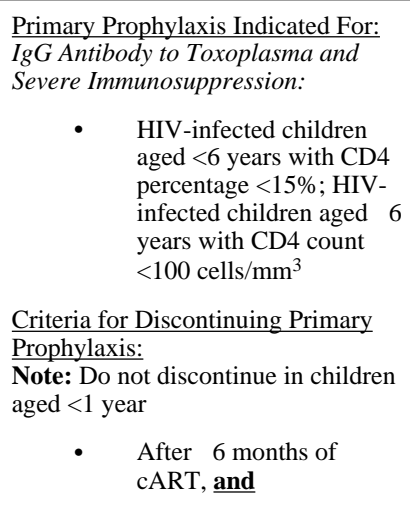 \\
\hline
\end{tabular}




\begin{tabular}{|c|c|c|c|}
\hline Indication & First Choice & Alternative & Comments/Special Issues \\
\hline & & 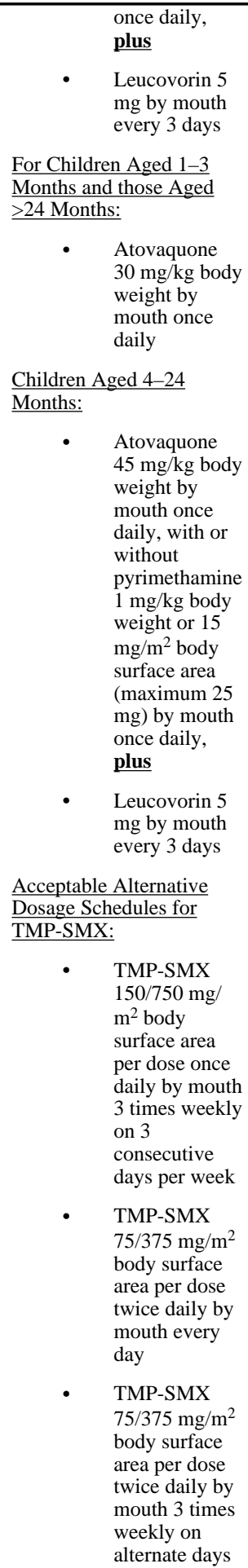 & 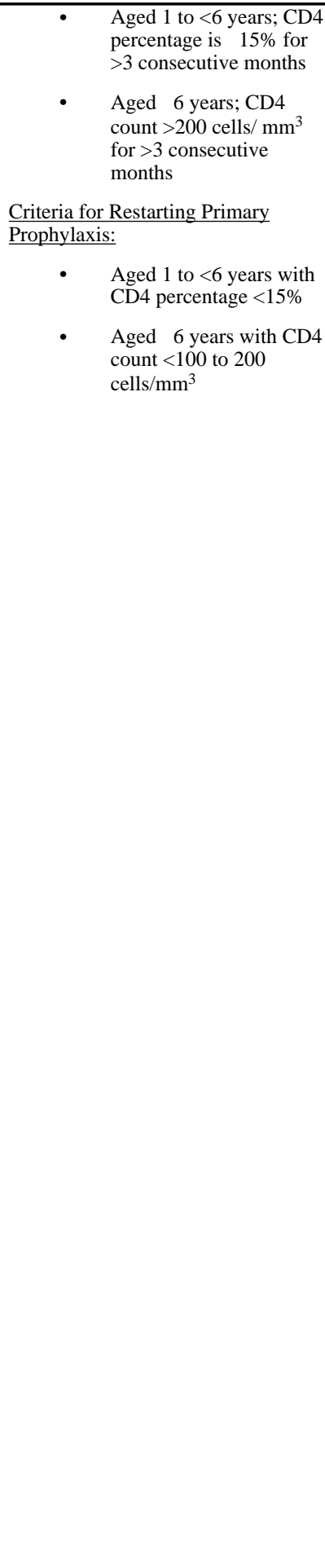 \\
\hline $\begin{array}{l}\text { Varicella-Zoster Virus (VZV) Pre-Exposure } \\
\text { Prophylaxis }\end{array}$ & Varicella vaccine & N/A & $\begin{array}{l}\text { See Figures } 1 \text { and } 2 \text { for detailed } \\
\text { vaccine recommendations. }\end{array}$ \\
\hline
\end{tabular}




\begin{tabular}{|c|c|c|c|}
\hline Indication & First Choice & Alternative & Comments/Special Issues \\
\hline $\begin{array}{l}\text { Varicella-Zoster Virus (VZV) Primary } \\
\text { (Post-Exposure) Prophylaxis }\end{array}$ & $\begin{array}{l}\text { VariZIG } 125 \mathrm{IU} / 10 \mathrm{~kg} \text { body weight } \\
\text { IM (maximum } 625 \mathrm{IU}) \\
\text { administered ideally within } 96 \\
\text { hours (potentially beneficial up to } \\
10 \text { days) after exposure }\end{array}$ & $\begin{array}{l}\text { - If VariZIG } \\
\text { cannot be } \\
\text { administered } \\
\text { within } 96 \\
\text { hours (up to } 10 \\
\text { days), IVIG } \\
400 \mathrm{mg} / \mathrm{kg} \\
\text { body weight, } \\
\text { administered } \\
\text { once should be } \\
\text { considered. } \\
\text { IVIG should } \\
\text { ideally be } \\
\text { administered } \\
\text { within } 96 \\
\text { hours of } \\
\text { exposure } \\
\text { When passive } \\
\text { immunization } \\
\text { is not possible, } \\
\text { some experts } \\
\text { recommend } \\
\text { prophylaxis } \\
\text { with acyclovir } \\
20 \mathrm{mg} / \mathrm{kg} \text { body } \\
\text { weight/dose } \\
\text { (maximum } \\
\text { dose } 800 \mathrm{mg} \text { ), } \\
\text { administered } \\
\text { QID for } 7 \\
\text { days, } \\
\text { beginning 7- } \\
10 \text { days after } \\
\text { exposure }\end{array}$ & 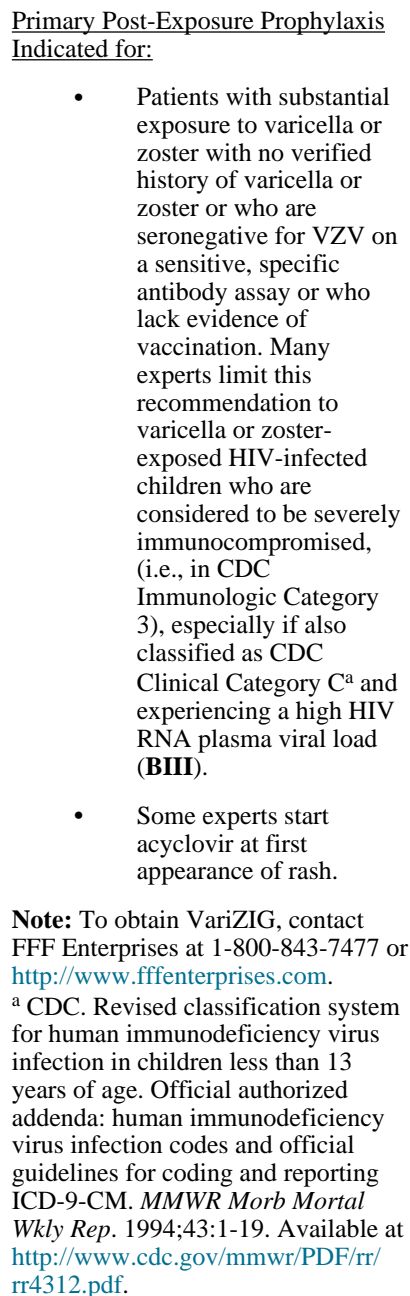 \\
\hline
\end{tabular}

Key to Acronyms: $\mathrm{ARV}=$ antiretroviral; $\mathrm{BSA}=$ body surface area; $\mathrm{cART}=$ combination antiretroviral therapy; $\mathrm{CrCl}=($ estimated $)$ creatinine clearance; DOT = directly observed therapy; HBV = hepatitis B virus; IGRA = interferon-gamma release assay; $\mathrm{QID}=$ four times daily; TB = tuberculosis; TMP-SMX = trimethoprim-sulfamethoxazole 
Table 2

Secondary Prophylaxis of Opportunistic Infections in HIV-Exposed and HIV-Infected Children-Summary of Recommendations (Last updated November 6, 2013; last reviewed November 6, 2013)

\begin{tabular}{|c|c|c|c|}
\hline Indication & First Choice & Alternative & Comments/Special Issues \\
\hline $\begin{array}{l}\text { Bacterial Infections } S \text {. pneumoniae } \\
\text { and other invasive bacteria }\end{array}$ & $\begin{array}{l}\text { TMP-SMX } 75 / 375 \\
\mathrm{mg} / \mathrm{m}^{2} \text { body surface } \\
\text { area per dose by mouth } \\
\text { twice daily }\end{array}$ & $\begin{array}{l}\text { IVIG } 400 \\
\text { mg/kg body } \\
\text { weight every } \\
\text { 2-4 weeks }\end{array}$ & 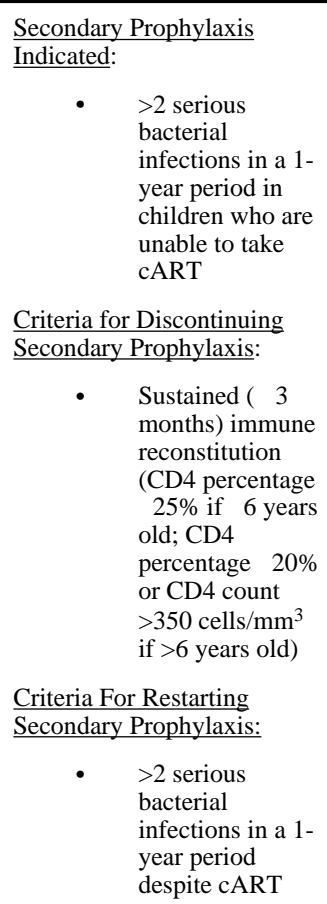 \\
\hline Candidiasis & 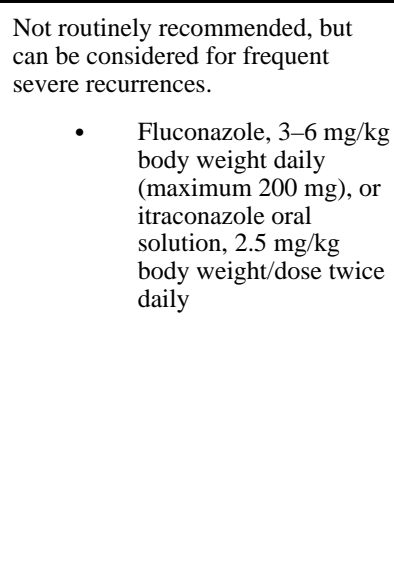 & N/A & $\begin{array}{ll}\begin{array}{l}\text { Secondary Prophylaxis } \\
\text { Indicated: }\end{array} & \begin{array}{l}\text { Frequent or } \\
\text { severe } \\
\text { recurrences }\end{array} \\
\text { Criteria for Discontinuing } \\
\text { Secondary Prophylaxis: }\end{array}$ \\
\hline Coccidioidomycosis & $\begin{array}{l}\text { Fluconazole } 6 \mathrm{mg} / \mathrm{kg} \text { body weight } \\
\text { (maximum } 400 \mathrm{mg} \text { ) by mouth once } \\
\text { daily }\end{array}$ & $\begin{array}{l}\text { Itraconazole } 2-5 \mathrm{mg} / \mathrm{kg} \\
\text { body weight (maximum } \\
200 \mathrm{mg} \text { ) by mouth per } \\
\text { dose twice daily }\end{array}$ & $\begin{array}{l}\text { Lifelong secondary } \\
\text { prophylaxis with fluconazole } \\
\text { for patients with meningitis } \\
\text { or disseminated disease in the } \\
\text { immunocompromised patient } \\
\text { is recommended. Secondary } \\
\text { prophylaxis should be } \\
\text { considered after treatment of } \\
\text { milder disease if CD4 count } \\
\text { remains }<250 \text { cells } / \mathrm{mm}^{3} \text { or } \\
\text { CD4 percentage }<15 \% \text {. }\end{array}$ \\
\hline
\end{tabular}




\begin{tabular}{|c|c|c|c|}
\hline Indication & First Choice & Alternative & Comments/Special Issues \\
\hline Cryptococcosis $^{\mathrm{a}}$ & $\begin{array}{l}\text { Fluconazole } 6 \mathrm{mg} / \mathrm{kg} \text { body weight } \\
\text { (maximum } 200 \mathrm{mg} \text { ) by mouth once } \\
\text { daily }\end{array}$ & $\begin{array}{l}\text { Itraconazole oral solution } 5 \\
\mathrm{mg} / \mathrm{kg} \text { body weight } \\
\text { (maximum } 200 \mathrm{mg} \text { ) by } \\
\text { mouth once daily }\end{array}$ & 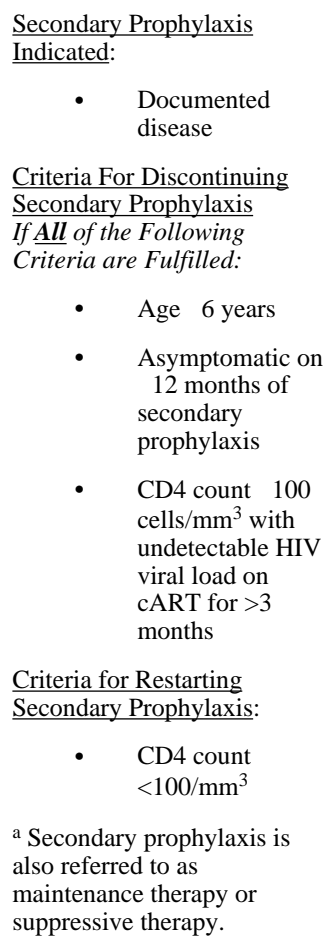 \\
\hline Cryptosporidiosis & $\mathrm{N} / \mathrm{A}$ & N/A & N/A \\
\hline Cytomegalovirus (CMV) & $\begin{array}{l}\text { Ganciclovir } 5 \mathrm{mg} / \mathrm{kg} \\
\text { body weight IV once } \\
\text { daily, or } \\
\text { For older children who } \\
\text { can receive adult dose } \\
\text { (based on their BSA), } \\
\text { valganciclovir tablets } \\
900 \mathrm{mg} \text { orally once } \\
\text { daily with food, or } \\
\text { For children age } 4 \\
\text { months }-16 \text { years, } \\
\text { valganciclovir oral } \\
\text { solution } 50 \mathrm{mg} / \mathrm{mL} \text { (at } \\
\text { dose in milligrams }=7 \times \\
\text { BSA } \times \text { CrCl up to } \\
\text { maximum CrCl of } 150 \\
\text { mL/min/1.73 } \mathrm{m}^{2} \text { ) orally } \\
\text { once daily with food, or } \\
\text { Foscarnet } 90-120 \\
\text { mg/kg body weight IV } \\
\text { once daily }\end{array}$ & $\begin{array}{l}\text { Cidofovir } 5 \\
\text { mg/kg body } \\
\text { weight per } \\
\text { dose IV every } \\
\text { other week. } \\
\text { Must be given } \\
\text { with } \\
\text { probenecid and } \\
\text { IV hydration. }\end{array}$ & 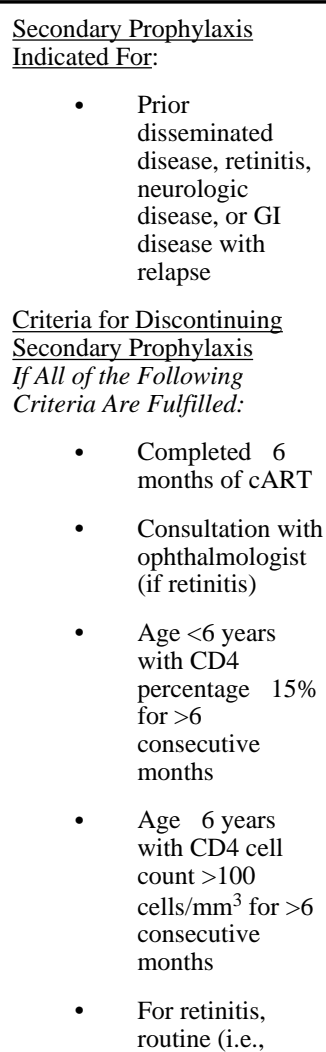 \\
\hline
\end{tabular}




\begin{tabular}{|c|c|c|c|}
\hline Indication & First Choice & Alternative & Comments/Special Issues \\
\hline & & & \begin{tabular}{ll} 
every 3-6 & \multicolumn{1}{c}{$\begin{array}{l}\text { months) } \\
\text { ophthalmological } \\
\text { follow-up is } \\
\text { recommended for } \\
\text { early detection of } \\
\text { relapse or } \\
\text { immune } \\
\text { restoration } \\
\text { uveitis. }\end{array}$} \\
Criteria for Restarting \\
Secondary Prophylaxis: \\
\end{tabular} $\begin{array}{l}\text { Age }<6 \text { years } \\
\text { with CD4 } \\
\text { percentage }<15 \% \\
\text { Age } 86 \text { years } \\
\text { with CD4 cell } \\
\text { count }<100 \\
\text { cells } / \mathrm{mm}^{3}\end{array}$ \\
\hline Giardiasis & N/A & N/A & N/A \\
\hline Hepatitis B Virus (HBV) & Hepatitis A Vaccine & N/A & $\begin{array}{l}\frac{\text { Secondary Prophylaxis }}{\text { Indicated for: }} \\
\qquad \quad \begin{array}{l}\text { Chronically } \\
\text { HBV-infected } \\
\text { individuals to } \\
\text { prevent further } \\
\text { liver injury }\end{array} \\
\text { Criteria for Discontinuing } \\
\text { Secondary Prophylaxis: } \\
\text { N/A }\end{array}$ \\
\hline Hepatitis C Virus (HCV) & None & N/A & N/A \\
\hline $\begin{array}{l}\text { Herpes Simplex Virus (HSV) } \\
\text { Infections }\end{array}$ & $\begin{array}{l}\text { Mucocutaneous Disease: } \\
\text { - } \quad \begin{array}{l}\text { Acyclovir } 20 \mathrm{mg} / \mathrm{kg} \\
\text { body weight } / \mathrm{dose} \\
\text { (maximum } 800 \mathrm{mg} / \\
\text { dose) by mouth BID }\end{array} \\
\text { Suppressive Therapy After } \\
\text { Neonatal Skin, Eye, Mouth, or CNS } \\
\text { Disease: } \\
\text { - } \begin{array}{l}\text { Acyclovir } 300 \mathrm{mg} / \mathrm{m}^{2} \\
\text { body surface area/dose } \\
\text { by mouth TID for } 6 \\
\text { months }\end{array}\end{array}$ & 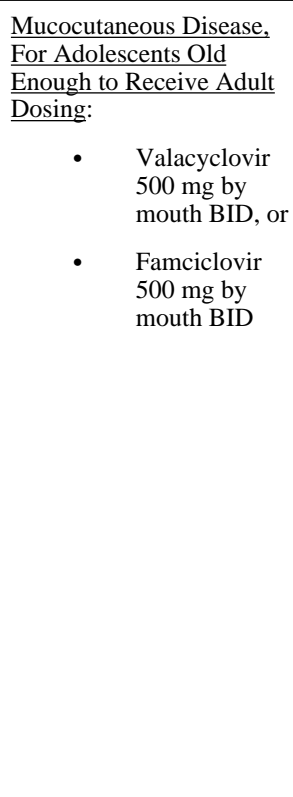 & $\begin{array}{ll}\text { Secondary } & \text { Prophylaxis } \\
\text { Indicated: } & \\
& \begin{array}{l}\text { Suppressive } \\
\text { secondary } \\
\text { prophylaxis can } \\
\text { be considered for } \\
\text { children with } \\
\text { severe and } \\
\text { recurrent } \\
\text { mucocutaneous } \\
\text { (oral or genital) } \\
\text { disease }\end{array} \\
\text { Criteria for Discontinuing } \\
\text { Secondary } & \text { Prophylaxis: } \\
\text { - } & \begin{array}{l}\text { After a prolonged } \\
\text { period (e.g., } 1\end{array} \\
\text { year) of } \\
\text { prophylaxis, } \\
\text { consider } \\
\text { suspending } \\
\text { prophylaxis and } \\
\text { determine with } \\
\text { the patient } \\
\text { whether } \\
\text { additional } \\
\text { prophylaxis is }\end{array}$ \\
\hline
\end{tabular}




\begin{tabular}{|c|c|c|c|}
\hline Indication & First Choice & Alternative & Comments/Special Issues \\
\hline & & & $\begin{array}{l}\text { necessary. } \\
\text { Although level of } \\
\text { immune } \\
\text { reconstitution is a } \\
\text { consideration, no } \\
\text { specific CD4 } \\
\text { threshold has } \\
\text { been established. }\end{array}$ \\
\hline Histoplasmosis (Suppressive Therapy) & $\begin{array}{l}\text { Itraconazole oral solution } 5-10 \\
\mathrm{mg} / \mathrm{kg} \text { body weight (maximum } 200 \\
\mathrm{mg} \text { ) per dose by mouth daily }\end{array}$ & $\begin{array}{l}\text { Fluconazole } 3-6 \mathrm{mg} / \mathrm{kg} \\
\text { body weight (maximum } \\
200 \mathrm{mg} \text { ) by mouth once } \\
\text { daily }\end{array}$ & 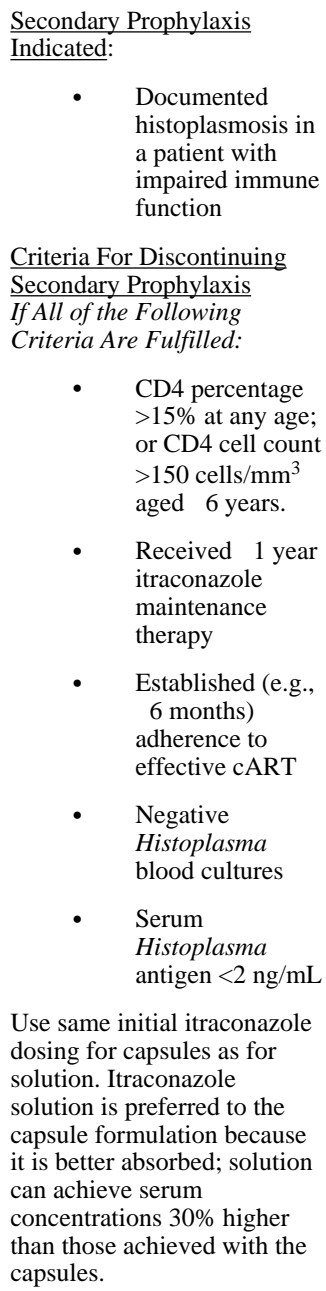 \\
\hline Human Papillomavirus (HPV) & N/A & N/A & N/A \\
\hline Influenza & N/A & N/A & $\begin{array}{l}\text { No role for secondary } \\
\text { chemoprophylaxis }\end{array}$ \\
\hline Isosporiasis (Cystoisosporiasis) & $\begin{array}{ll}\text { If Severe Immunosuppression: } \\
\text { Administer TMP-SMX } \\
2.5 \mathrm{mg} / \mathrm{kg} \text { body weight } \\
\text { of TMP component } \\
\text { twice daily by mouth } 3 \\
\text { times per week }\end{array}$ & $\begin{array}{l}\text { Pyrimethamine } 1 \mathrm{mg} / \mathrm{kg} \\
\text { body weight (maximum } 25 \\
\mathrm{mg} \text { ) plus folinic acid, } 10- \\
25 \mathrm{mg} \text { by mouth once } \\
\text { daily. } \\
\text { Second-Line Alternative: } \\
\qquad \begin{array}{l}\text { Ciprofloxacin, } \\
10-20 \mathrm{mg} / \mathrm{kg} \\
\text { body weight } \\
\text { given twice } \\
\text { daily by mouth }\end{array}\end{array}$ & $\begin{array}{l}\text { Consider discontinuing } \\
\text { secondary prophylaxis in a } \\
\text { patient receiving cART after } \\
\text { sustained improvement from } \\
\text { severe immunosuppression } \\
\text { (from CDC immunologic } \\
\text { category } 3 \text { to CD } 4 \text { values that } \\
\text { fall within category } 1 \text { or } 2) \\
\text { for longer than } 6 \text { months. } \\
\text { In adults, the dose of } \\
\text { pyrimethamine for secondary } \\
\text { prophylaxis ( } 25 \mathrm{mg} \text { daily) is }\end{array}$ \\
\hline
\end{tabular}




\begin{tabular}{|c|c|c|c|}
\hline Indication & First Choice & Alternative & Comments/Special Issues \\
\hline & & $\begin{array}{l}3 \text { times per } \\
\text { week }\end{array}$ & $\begin{array}{l}\text { lower than the dose for } \\
\text { treatment ( } 50-75 \mathrm{mg} \text { daily), } \\
\text { but no similar data exist for } \\
\text { children. Thus, the } \\
\text { recommended dosing for } \\
\text { secondary prophylaxis in } \\
\text { children is } 1 \mathrm{mg} / \mathrm{kg} \text { per dose } \\
\text { (maximum } 25 \mathrm{mg} \text { ) once } \\
\text { daily. } \\
\text { Ciprofloxacin is generally not } \\
\text { a drug of first choice in } \\
\text { children due to increased } \\
\text { incidence of adverse events, } \\
\text { including events related to } \\
\text { joints and/or surrounding } \\
\text { tissues. }\end{array}$ \\
\hline Malaria & $\begin{array}{l}\text { For P. vivax or P. ovale: } \\
\begin{array}{l}\text { Primaquine } 0.5 \mathrm{mg} / \mathrm{kg} \\
\text { base }(0.8 \mathrm{mg} / \mathrm{kg} \text { salt) up } \\
\text { to adult dose orally, } \\
\text { daily for } 14 \text { days after } \\
\text { departure from the } \\
\text { malarious area }\end{array}\end{array}$ & N/A & $\begin{array}{l}\text { This regimen, known as } \\
\text { PART, is recommended only } \\
\text { for individuals who have } \\
\text { resided in a malaria-endemic } \\
\text { area for an extended period } \\
\text { of time. Adult dose: } 30 \mathrm{mg} \\
\text { base ( } 52.6 \mathrm{mg} \text { salt) orally, } \\
\text { daily for } 14 \text { days after } \\
\text { departure from the malarious } \\
\text { area. } \\
\text { http://wwwnc.cdc.gov/travel/ } \\
\text { yellowbook/2012/chapter-3- } \\
\text { infectious-diseases-related- } \\
\text { to-travel/malaria.htm\#1939 }\end{array}$ \\
\hline Microsporidiosis & $\begin{array}{l}\text { Disseminated, Non-Ocular Infection } \\
\text { or GI Infection Caused by } \\
\text { Microsporidia Other Than } E . \\
\text { Bieneusi or V. Corneae: } \\
\begin{array}{l}\text { Albendazole } 7.5 \mathrm{mg} / \mathrm{kg} \\
\text { body weight (maximum } \\
400 \mathrm{mg} / \text { dose) by mouth } \\
\text { twice daily }\end{array} \\
\text { Ocular Infection: } \\
\text { Topical fumagillin } \\
\text { bicyclohexylammonium } \\
\text { (Fumidil B) } 3 \mathrm{mg} / \mathrm{mL} \text { in } \\
\text { saline (fumagillin } 70 \\
\mu \mathrm{g} / \mathrm{mL} \text { ) eye drops: } 2 \\
\text { drops every } 2 \text { hours for } \\
4 \text { days, then } 2 \text { drops } \\
\text { QID (investigational } \\
\text { use only in United } \\
\text { States) plus albendazole } \\
7.5 \mathrm{mg} / \mathrm{kg} \text { body weight } \\
\text { (maximum } 400 \mathrm{mg} / \\
\text { dose) by mouth twice } \\
\text { daily for management } \\
\text { of systemic infection }\end{array}$ & N/A & $\begin{array}{ll}\text { Criteria For Discontinuing } \\
\text { Secondary Prophylaxis: } \\
& \text { Continue until } \\
& \text { sustained } \\
\text { immune } \\
\text { reconstitution } \\
\text { (more than } 6 \\
\text { months at CDC } \\
\text { immunologic } \\
\text { category 1 or 2), } \\
\text { or } \\
\text { After initiation of } \\
\text { cART and } \\
\text { resolution of } \\
\text { signs and } \\
\text { symptoms }\end{array}$ \\
\hline $\begin{array}{l}\text { Mycobacterium avium Complex } \\
\text { (MAC) (Chronic Suppressive } \\
\text { Therapy) }\end{array}$ & $\begin{array}{ll}\text { - } & \begin{array}{l}\text { Clarithromycin } 7.5 \\
\text { mg/kg body weight } \\
\text { (maximum } 500 \mathrm{mg} \text { ) } \\
\text { orally twice daily, plus }\end{array} \\
\text { - } & \begin{array}{l}\text { Ethambutol } 15-25 \\
\text { mg/kg body weight } \\
\text { (maximum } 2.5 \mathrm{~g} \text { ) orally }\end{array} \\
\text { once daily, with or } \\
\text { without food } \\
\text { - Children aged }>5 \text { years } \\
\text { who received rifabutin } \\
\text { as part of initial } \\
\text { treatment: Rifabutin } 5\end{array}$ & $\begin{array}{l}\text { - Azithromycin } \\
5 \mathrm{mg} / \mathrm{kg} \text { body } \\
\text { weight } \\
\text { (maximum } 250 \\
\text { mg) orally } \\
\text { once daily, } \\
\text { plus } \\
\text { - } \\
\text { Ethambutol } \\
15-25 \mathrm{mg} / \mathrm{kg} \\
\text { body weight } \\
\text { (max } 2.5 \mathrm{~g} \text { ) } \\
\text { orally once }\end{array}$ & $\begin{array}{l}\text { Secondary Prophylaxis } \\
\text { Indicated: } \\
\bullet \quad \text { Prior disease } \\
\text { Criteria for Discontinuing } \\
\text { Secondary Prophylaxis } \\
\text { Fulfillment of All of the } \\
\text { Following Criteria: } \\
\text { - Completed } 26 \\
\text { months of cART }\end{array}$ \\
\hline
\end{tabular}




\begin{tabular}{|c|c|c|c|}
\hline Indication & First Choice & Alternative & Comments/Special Issues \\
\hline & $\begin{array}{l}\mathrm{mg} / \mathrm{kg} \text { body weight } \\
\text { (maximum } 300 \mathrm{mg} \text { ) } \\
\text { orally once daily with } \\
\text { food }\end{array}$ & $\begin{array}{l}\text { daily, with or } \\
\text { without food } \\
\text { Children aged } \\
>5 \text { years who } \\
\text { received } \\
\text { rifabutin as } \\
\text { part of initial } \\
\text { treatment: } \\
\text { Rifabutin } 5 \\
\text { mg/kg body } \\
\text { weight } \\
\text { (maximum } 300 \\
\text { mg) orally } \\
\text { once daily with } \\
\text { food. }\end{array}$ & 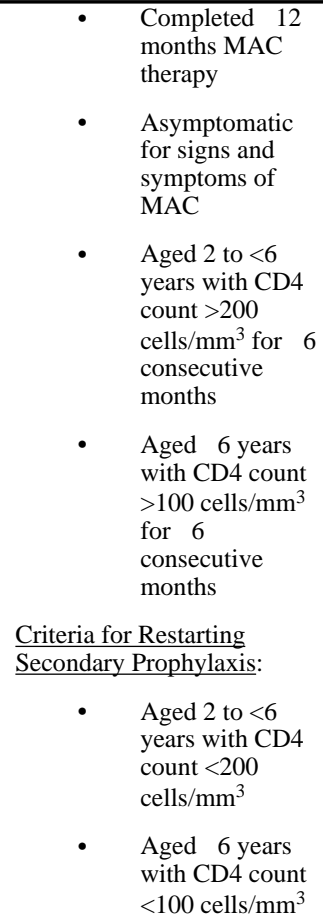 \\
\hline Mycobacterium Tuberculosis & N/A & N/A & N/A \\
\hline Pneumocystis Pneumonia & $\begin{array}{l}\text { TMP-SMX } \\
\text { (Cotrimoxazole): TMP } \\
\text { 2.5-5 mg/kg body } \\
\text { weight/dose with SMX } \\
\text { 12.5-25 mg/kg body } \\
\text { weight/dose twice per } \\
\text { day. Dosing based on } \\
\text { TMP component. } \\
\text { The total daily dose } \\
\text { should not exceed } 320 \\
\text { mg TMP and } 1600 \mathrm{mg} \\
\text { SMX. Several dosing } \\
\text { schemes have been used } \\
\text { successfully- } \\
\text { - } \quad \text { Given } 3 \\
\text { days per } \\
\text { week on } \\
\text { consecutive } \\
\text { days or on } \\
\text { alternate } \\
\text { days } \\
-\quad \text { Given } 2 \\
\text { days per } \\
\text { week on } \\
\text { consecutive } \\
\text { days or on } \\
\text { alternate } \\
\text { days } \\
\text { - } \\
\text { Given every } \\
\text { day (total } \\
\text { daily dose } \\
\text { of TMP } 5- \\
10 \mathrm{mg} / \mathrm{kg} \\
\text { body weight }\end{array}$ & 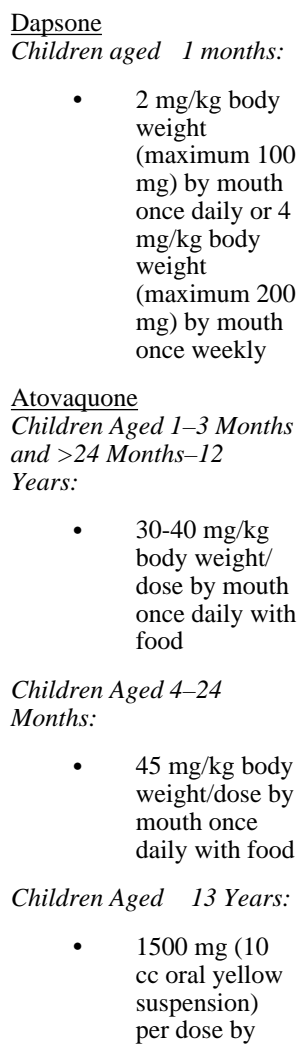 & $\begin{array}{c}\frac{\text { Secondary Prophylaxis }}{\text { Indicated For: }} \\
\qquad \quad \begin{array}{l}\text { Children with } \\
\text { prior episode of } \\
\text { PCP }\end{array} \\
\text { Criteria for Discontinuing } \\
\text { Secondary Prophylaxis: } \\
\qquad \quad \begin{array}{l}\text { Same as for } \\
\text { primary } \\
\text { prophylaxis }\end{array} \\
\text { Criteria for Restarting } \\
\text { Secondary Prophylaxis: } \\
\text { - } \quad \begin{array}{l}\text { Same as for } \\
\text { primary } \\
\text { prophylaxis }\end{array}\end{array}$ \\
\hline
\end{tabular}




\begin{tabular}{|c|c|c|c|}
\hline Indication & First Choice & Alternative & Comments/Special Issues \\
\hline & $\begin{array}{l}\text { given as a } \\
\text { single dose } \\
\text { each day) }\end{array}$ & 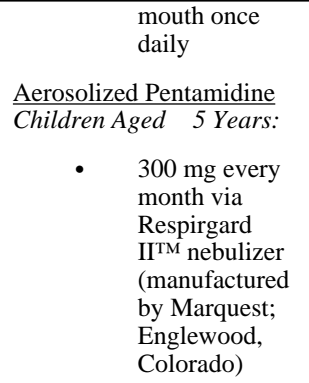 & \\
\hline Syphilis & N/A & N/A & $\begin{array}{l}\frac{\text { Secondary Prophylaxis }}{\underline{\text { Indicated: }}} \\
\bullet \quad \text { N/A } \\
\text { Criteria For Discontinuing } \\
\frac{\text { Secondary Prophylaxis: }}{\bullet \quad \text { N/A }} \\
\frac{\text { Criteria For Restarting }}{\text { Secondary Prophylaxis: }} \\
\quad \text { N/A }\end{array}$ \\
\hline Toxoplasmosis (Suppressive Therapy) & $\begin{array}{l}\text { - Sulfadiazine } 42.5-60 \\
\text { mg/kg body weight per } \\
\text { dose twice daily* } \\
\text { (maximum } 2-4 \text { g per } \\
\text { day) by mouth, plus } \\
\text { - } \quad \text { Pyrimethamine } 1 \mathrm{mg} / \mathrm{kg} \\
\text { body weight or } 15 \\
\mathrm{mg} / \mathrm{m}^{2} \text { body surface } \\
\text { area (maximum } 25 \mathrm{mg} \text { ) } \\
\text { by mouth once daily, } \\
\text { plus } \\
\text { Leucovorin } 5 \text { mg by } \\
\text { mouth once every } 3 \\
\text { days }\end{array}$ & 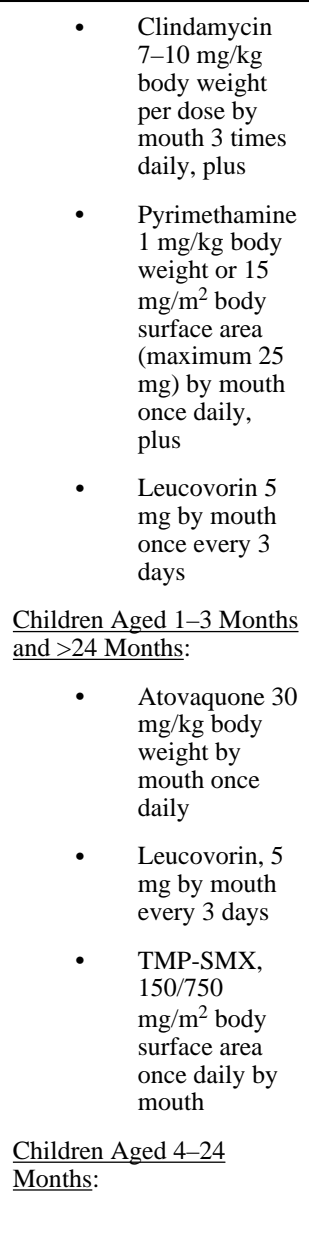 & 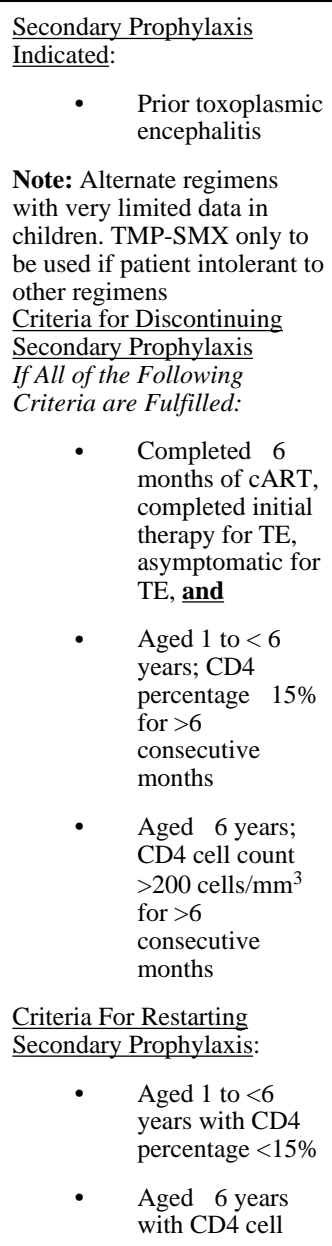 \\
\hline
\end{tabular}




\begin{tabular}{|c|c|c|c|}
\hline Indication & First Choice & Alternative & Comments/Special Issues \\
\hline & & $\begin{array}{l}\text { Atovaquone } 45 \\
\text { mg/kg body } \\
\text { weight by } \\
\text { mouth once } \\
\text { daily, with or } \\
\text { without } \\
\text { pyrimethamine } \\
1 \mathrm{mg} / \mathrm{kg} \text { body } \\
\text { weight or } 15 \\
\mathrm{mg} / \mathrm{m}^{2} \text { body } \\
\text { surface area } \\
\text { (maximum } 25 \\
\mathrm{mg} \text { ) by mouth } \\
\text { once daily, } \\
\text { plus } \\
\text { Leucovorin, } 5 \\
\text { mg by mouth } \\
\text { every } 3 \text { days } \\
\text { TMP-SMX, } \\
150 / 750 \\
\text { mg/m }{ }^{2} \text { body } \\
\text { surface area } \\
\text { once daily by } \\
\text { mouth }\end{array}$ & $\begin{array}{l}\text { count }<200 \\
\text { cells } / \mathrm{mm}^{3}\end{array}$ \\
\hline Varicella-Zoster Virus (VZV) & N/A & N/A & $\begin{array}{l}\text { There is no indication for } \\
\text { secondary prophylaxis }\end{array}$ \\
\hline
\end{tabular}

Key to Acronyms: $\mathrm{BID}=$ twice daily; $\mathrm{BSA}=$ body surface area; $\mathrm{cART}=$ combination antiretroviral therapy; $\mathrm{CNS}=$ central nervous system; $\mathrm{CrCl}$ $=($ estimated $)$ creatinine clearance, $\mathrm{CSF}=$ cerebrospinal fluid; $\mathrm{GI}=$ gastrointestinal; HBV $=$ hepatitis $\mathrm{B}$ virus; HSV $=$ herpes simplex virus; $\mathrm{IV}=$ intravenous; $\mathrm{SQ}=$ subcutaneous; $\mathrm{TE}=$ toxoplasmic encephalitis; TID = three times daily; TMP-SMX = trimethoprim-sulfamethoxazole 
Table 3

Treatment of Opportunistic Infections in HIV-Exposed and HIV-Infected Children-Summary of Recommendations (Last updated April 2, 2014; last reviewed November 6, 2013)

\begin{tabular}{|c|c|c|c|}
\hline Indication & First Choice & Alternative & Comments/Special Issues \\
\hline $\begin{array}{l}\text { Bacterial Infections } \\
\text { Bacterial pneumonia } S . \\
\text { pneumoniae; occasionally } S \text {. } \\
\text { aureus, } H \text {. influenzae, } P \text {. } \\
\text { aeruginosa }\end{array}$ & $\begin{array}{l}\text { Ceftriaxone } 50-100 \\
\mathrm{mg} / \mathrm{kg} \text { body weight per } \\
\text { dose once daily, or } 25- \\
50 \mathrm{mg} / \mathrm{kg} \text { body weight } \\
\text { per dose twice daily IV } \\
\text { or IM (max } 4 \mathrm{~g} / \text { day), or } \\
\text { Cefotaxime } 40-50 \\
\mathrm{mg} / \mathrm{kg} \text { body weight per } \\
\text { dose } 4 \text { times daily, or } \\
50-65 \mathrm{mg} / \mathrm{kg} \text { body } \\
\text { weight } 3 \text { times daily } \\
\text { (max } 8-10 \text { g/day) IV }\end{array}$ & $\begin{array}{l}\text { Cefuroxime, } 35-50 \\
\text { mg/kg body weight per } \\
\text { dose } 3 \text { times daily(max } \\
4-6 \mathrm{~g} / \text { day) IV }\end{array}$ & $\begin{array}{l}\text { For children who are } \\
\text { receiving effective cART, } \\
\text { have mild or no } \\
\text { immunosuppression, and } \\
\text { have mild to moderate } \\
\text { community-acquired } \\
\text { pneumonia, oral therapy } \\
\text { option would be amoxicillin } \\
45 \text { mg/kg body weight per } \\
\text { dose twice daily (maximum } \\
\text { dose: } 4 \text { g per day). } \\
\text { Add azithromycin for } \\
\text { hospitalized patients to treat } \\
\text { other common community- } \\
\text { acquired pneumonia } \\
\text { pathogens (M. pneumoniae, } \\
C . \text { pneumoniae). } \\
\text { Add clindamycin or } \\
\text { vancomycin if methicillin- } \\
\text { resistant } S . \text { aureus is } \\
\text { suspected (base the choice } \\
\text { on local susceptibility } \\
\text { patterns). } \\
\text { For patients with } \\
\text { neutropenia, chronic lung } \\
\text { disease other than asthma } \\
\text { (e.g., LIP, bronchiectasis) } \\
\text { or indwelling venous } \\
\text { catheter, consider regimen } \\
\text { that includes activity } \\
\text { against } P \text {. aeruginosa (such } \\
\text { as ceftazidime or cefepime } \\
\text { instead of ceftriaxone). } \\
\text { Consider PCP in patients } \\
\text { with severe pneumonia or } \\
\text { more advanced HIV } \\
\text { disease. } \\
\text { Evaluate for tuberculosis, } \\
\text { cryptococcosis, and } \\
\text { endemic fungi as } \\
\text { epidemiology suggests. }\end{array}$ \\
\hline Candidiasis & 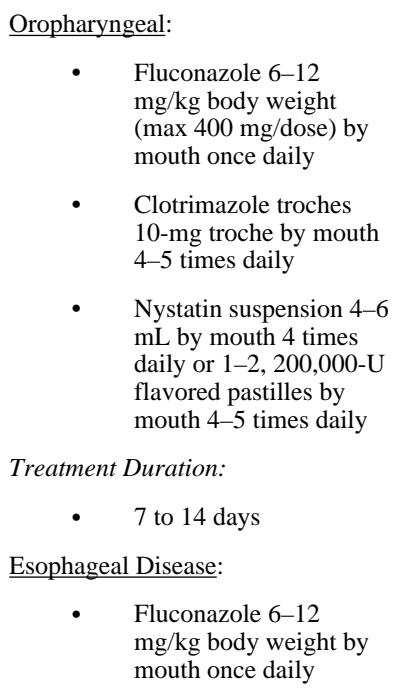 & 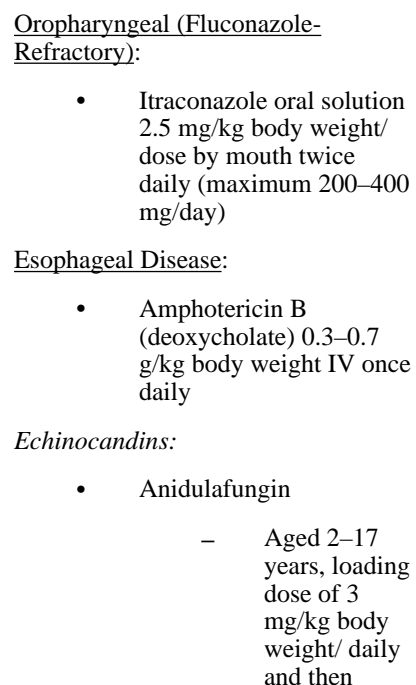 & $\begin{array}{l}\text { Itraconazole oral solution } \\
\text { should not be used } \\
\text { interchangeably with } \\
\text { itraconazole capsules. } \\
\text { Itraconazole capsules are } \\
\text { generally ineffective for } \\
\text { treatment of esophageal } \\
\text { disease. } \\
\text { Central venous catheters } \\
\text { should be removed, when } \\
\text { feasible, in HIV-infected } \\
\text { children with fungemia. } \\
\text { In uncomplicated catheter- } \\
\text { associated C. albicans } \\
\text { candidemia, an initial } \\
\text { course of amphotericin B } \\
\text { followed by fluconazole to } \\
\text { complete treatment can be } \\
\text { used (use invasive disease } \\
\text { dosing). } \\
\text { Voriconazole has been used } \\
\text { to treat esophageal } \\
\text { candidiasis in a small } \\
\text { number of HIV-uninfected }\end{array}$ \\
\hline
\end{tabular}




\begin{tabular}{|c|c|c|c|}
\hline Indication & First Choice & Alternative & Comments/Special Issues \\
\hline & 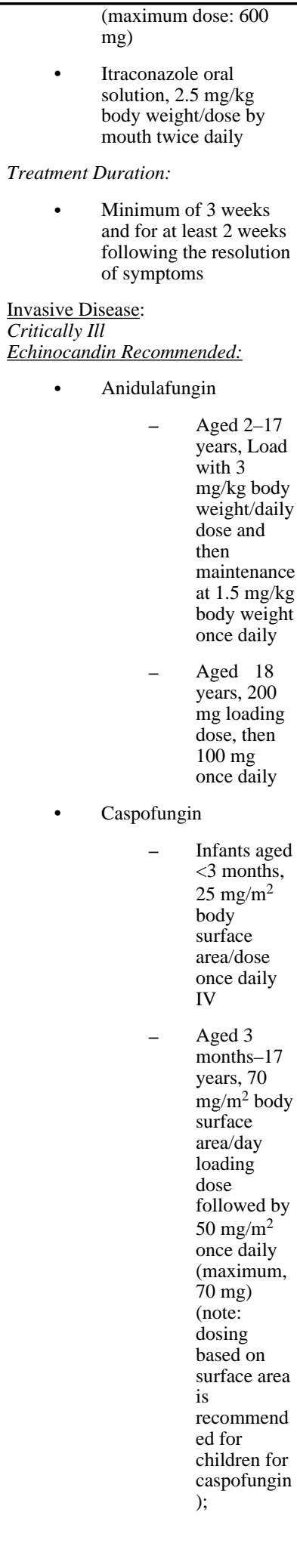 & 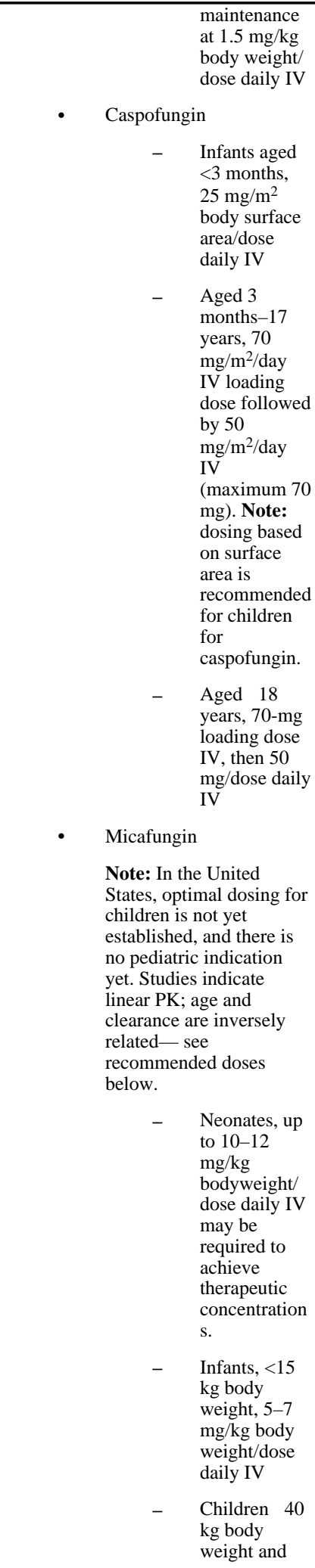 & 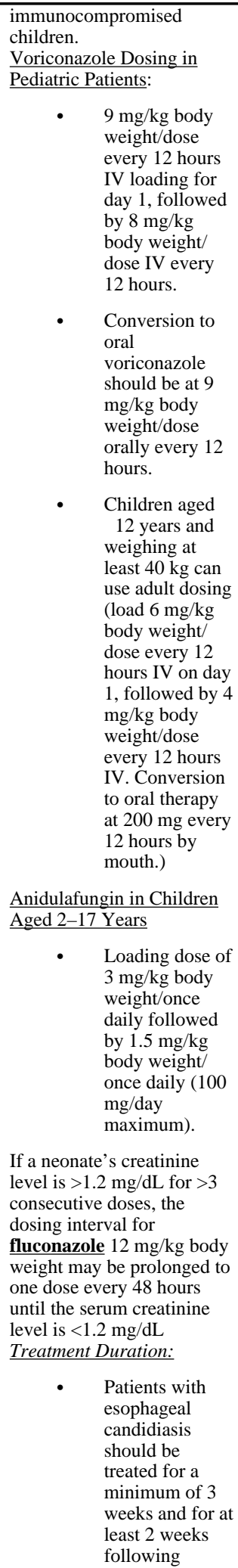 \\
\hline
\end{tabular}




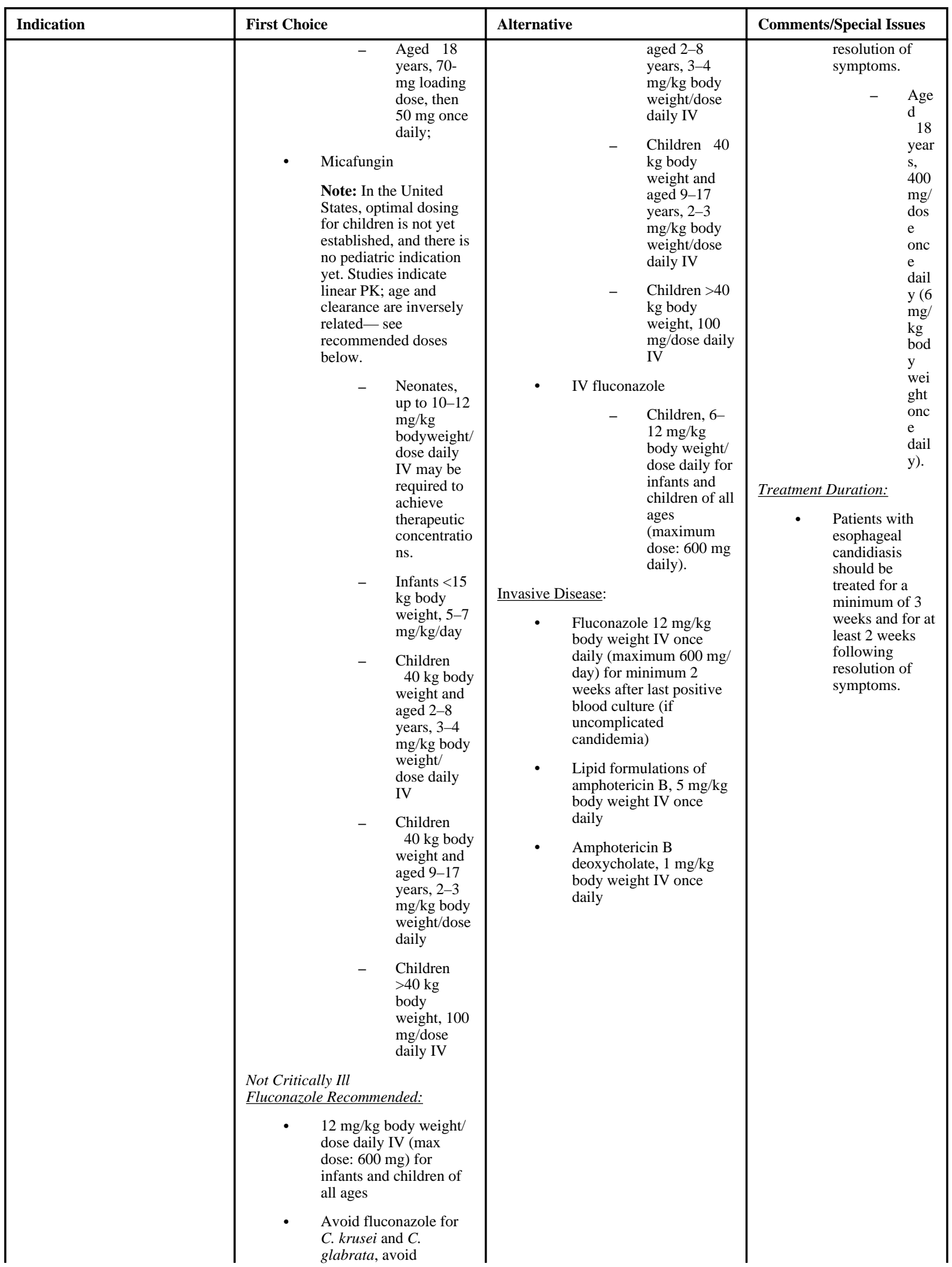




\begin{tabular}{|c|c|c|c|}
\hline Indication & First Choice & Alternative & Comments/Special Issues \\
\hline & $\begin{array}{ll} & \begin{array}{l}\text { echinocandin for } C . \\
\text { parapsilosis. }\end{array} \\
\text { Treatment } & \text { Duration: } \\
& \text { Based on presence of } \\
\text { deep-tissue foci and } \\
\text { clinical response; in } \\
\text { patients with } \\
\text { candidemia, treat until } 2 \\
\text { weeks after last positive } \\
\text { blood culture. }\end{array}$ & & \\
\hline \multirow[t]{2}{*}{ Coccidioidomycosis } & $\begin{array}{l}\text { Severe Illness with Respiratory } \\
\text { Compromise due to Diffuse } \\
\text { Pulmonary or Disseminated Non- } \\
\text { Meningitic Disease: } \\
\text { Amphotericin B } \\
\text { deoxycholate } 0.5-1.0 \\
\text { mg/kg body weight IV } \\
\text { once daily, until clinical } \\
\text { improvement. } \\
\text { A lipid amphotericin B } \\
\text { preparation can be } \\
\text { substituted at a dose of } \\
5 \text { mg/kg body weight IV } \\
\text { once daily (dosage of } \\
\text { the lipid preparation can } \\
\text { be increased to as much } \\
\text { as } 10 \text { mg/kg body } \\
\text { weight IV once daily for } \\
\text { life-threatening } \\
\text { infection). } \\
\text { After the patient is } \\
\text { stabilized, therapy with } \\
\text { an azole (fluconazole or } \\
\text { itraconazole) can be } \\
\text { substituted and } \\
\text { continued to complete a } \\
1 \text {-year course of } \\
\text { antifungal therapy. }\end{array}$ & 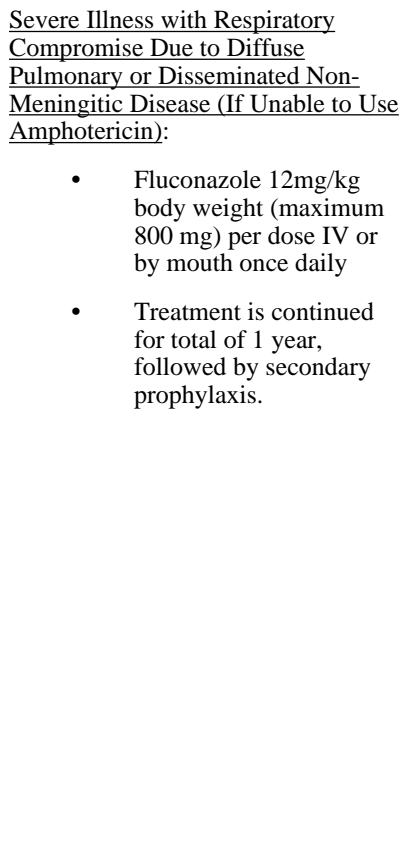 & \multirow[t]{2}{*}{$\begin{array}{l}\text { Surgical debridement of } \\
\text { bone, joint, and/or excision } \\
\text { of cavitary lung lesions may } \\
\text { be helpful. } \\
\text { Itraconazole is the preferred } \\
\text { azole for treatment of bone } \\
\text { infections. } \\
\text { Some experts initiate an } \\
\text { azole during amphotericin } \\
\text { B therapy; others defer } \\
\text { initiation of the azole until } \\
\text { after amphotericin B is } \\
\text { stopped. } \\
\text { For treatment failure, can } \\
\text { consider voriconazole, } \\
\text { caspofungin, or } \\
\text { posaconazole (or } \\
\text { combinations). However, } \\
\text { experience is limited and } \\
\text { definitive pediatric dosages } \\
\text { have not been determined. } \\
\text { Options should be discussed } \\
\text { with an expert in the } \\
\text { treatment of } \\
\text { coccidioidomycosis. } \\
\text { Chronic suppressive therapy } \\
\text { (secondary prophylaxis) } \\
\text { with fluconazole or } \\
\text { itraconazole is routinely } \\
\text { recommended following } \\
\text { initial induction therapy for } \\
\text { disseminated disease and is } \\
\text { continued lifelong for } \\
\text { meningeal disease. } \\
\text { Therapy with amphotericin } \\
\text { results In a more rapid } \\
\text { clinical response in severe, } \\
\text { non-meningeal disease. }\end{array}$} \\
\hline & 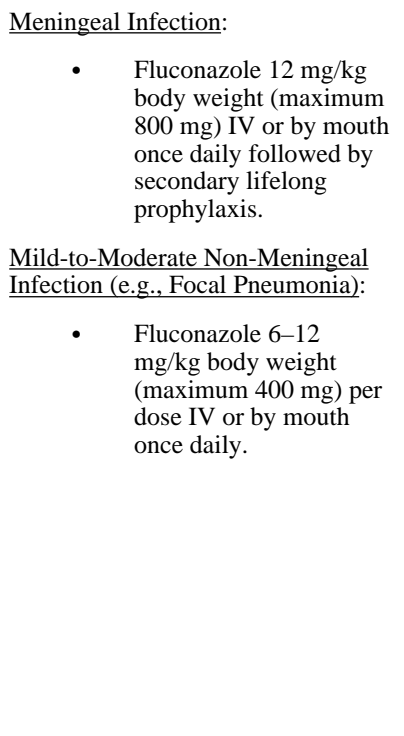 & $\begin{array}{l}\text { Meningeal Infection (Unresponsive to } \\
\text { Fluconazole): } \\
\text { IV amphotericin B plus } \\
\text { intrathecal amphotericin } \\
\text { B followed by secondary } \\
\text { prophylaxis. Note: } \\
\text { Expert consultation } \\
\text { recommended. } \\
\text { Mild-to-Moderate Non-Meningeal } \\
\text { Infection (e.g., Focal Pneumonia): } \\
\\
\text { Itraconazole } 2-5 \mathrm{mg} / \mathrm{kg} \\
\text { body weight per dose } \\
\text { (maximum dose } 200 \mathrm{mg} \text { ) } \\
\text { per dose IV or by mouth } \\
\text { 3 times daily for } 3 \text { days, } \\
\text { then } 2-5 \mathrm{mg} / \mathrm{kg} \text { body } \\
\text { weight (maximum dose } \\
\text { 200 mg) by mouth per } \\
\text { dose twice daily } \\
\text { thereafter. } \\
\text { Duration of treatment } \\
\text { determined by rate of } \\
\text { clinical response. }\end{array}$ & \\
\hline
\end{tabular}




\begin{tabular}{|c|c|c|c|}
\hline Indication & First Choice & Alternative & Comments/Special Issues \\
\hline Cryptococcosis & $\begin{array}{l}\text { CNS Disease } \\
\text { Acute Therapy (Minimum 2-Week } \\
\text { Induction Followed by } \\
\text { Consolidation Therapy): } \\
\text { • } \quad \begin{array}{l}\text { Amphotericin B } \\
\text { deoxycholate } 1.0 \mathrm{mg} / \mathrm{kg}\end{array} \\
\text { body weight (or } \\
\text { liposomal amphotericin } \\
\text { B } 6 \mathrm{mg} / \mathrm{kg} \text { body weight) } \\
\text { IV once daily PLUS } \\
\text { flucytosine } 25 \mathrm{mg} / \mathrm{kg} \\
\text { body weight per dose by } \\
\text { mouth given } 4 \text { times } \\
\text { daily }\end{array}$ & 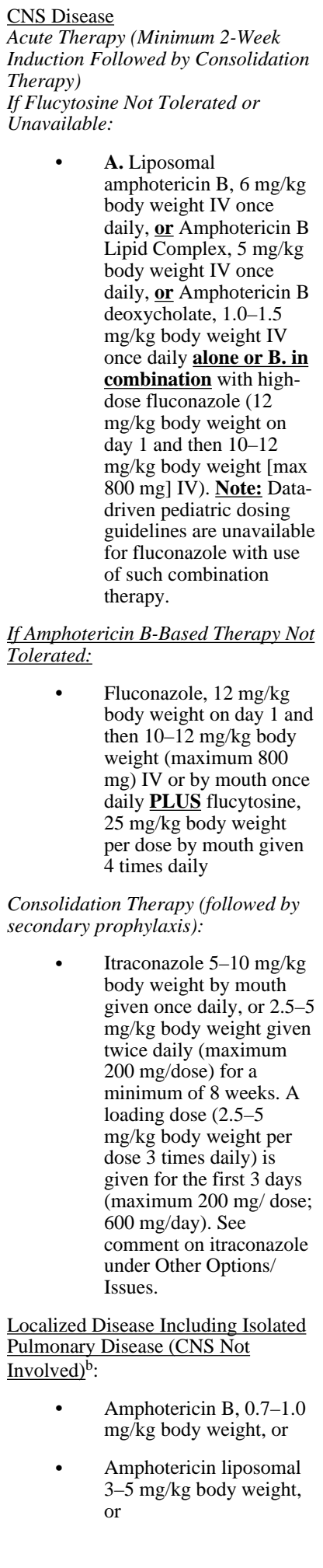 & $\begin{array}{l}\text { In patients with meningitis, } \\
\text { CSF culture should be } \\
\text { negative prior to initiating } \\
\text { consolidation therapy. } \\
\text { Overall, in vitro resistance } \\
\text { to antifungal agents used to } \\
\text { treat cryptococcosis remains } \\
\text { uncommon. Newer azoles } \\
\text { (voriconazole, } \\
\text { posaconazole, } \\
\text { ravuconazole) are all very } \\
\text { active in vitro against } C \text {. } \\
\text { neoformans, but published } \\
\text { clinical experience on their } \\
\text { use for cryptococcosis is } \\
\text { limited. } \\
\text { Liposomal amphotericin } \\
\text { and amphotericin B lipid } \\
\text { complex are especially } \\
\text { useful for children with } \\
\text { renal insufficiency or } \\
\text { infusion-related toxicity to } \\
\text { amphotericin B } \\
\text { deoxycholate. } \\
\text { Liposomal amphotericin } \\
\text { and amphotericin B lipid } \\
\text { complex are significantly } \\
\text { more expensive than } \\
\text { amphotericin B } \\
\text { deoxycholate. } \\
\text { Liquid preparation of } \\
\text { itraconazole (if tolerated) is } \\
\text { preferable to tablet } \\
\text { formulation because of } \\
\text { better bioavailability, but it } \\
\text { is more expensive. } \\
\text { Bioavailability of the } \\
\text { solution is better than the } \\
\text { capsule, but there were no } \\
\text { upfront differences in } \\
\text { dosing range based on } \\
\text { preparation used. Ultimate } \\
\text { dosing adjustments should } \\
\text { be guided by itraconazole } \\
\text { levels. } \\
\text { Serum itraconazole } \\
\text { concentrations should be } \\
\text { monitored to optimize drug } \\
\text { dosing. } \\
\text { Amphotericin B may } \\
\text { increase toxicity of } \\
\text { flucytosine by increasing } \\
\text { cellular uptake, or impair its } \\
\text { renal excretion, or both. } \\
\text { Flucytosine dose should be } \\
\text { adjusted to keep 2-hour } \\
\text { post-dose drug levels at } 40- \\
\text { 60 } 4 \text { go/mLL } \\
\text { Oral acetazolamide should } \\
\text { not be used for reduction of } \\
\text { ICP in cryptococcal } \\
\text { meningitis. } \\
\text { Corticosteroids and } \\
\text { mannitol have been shown } \\
\text { to be ineffective in } \\
\text { managing ICP in adults } \\
\text { with cryptococcal } \\
\text { meningitis. } \\
\text { Secondary prophylaxis is } \\
\text { recommended following } \\
\text { completion of initial } \\
\text { therapy (induction plus }\end{array}$ \\
\hline
\end{tabular}




\begin{tabular}{|c|c|c|c|}
\hline Indication & First Choice & Alternative & Comments/Special Issues \\
\hline & & 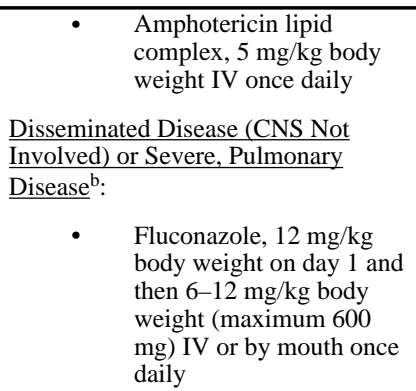 & $\begin{array}{l}\text { consolidation)—drugs and } \\
\text { dosing listed above. } \\
\text { buration of therapy for } \\
\text { non-CNS disease depends } \\
\text { on site and severity of } \\
\text { infection and clinical } \\
\text { response }\end{array}$ \\
\hline Cryptosporidiosis & $\begin{array}{ll}\text { Effective cART: } \\
\\
\begin{array}{l}\text { Immune reconstitution } \\
\text { may lead to } \\
\text { microbiologic and } \\
\text { clinical response }\end{array}\end{array}$ & $\begin{array}{l}\text { There is no consistently effective } \\
\text { therapy for cryptosporidiosis in HIV- } \\
\text { infected individuals; optimized cART } \\
\text { and a trial of nitazoxanide can be } \\
\text { considered. } \\
\text { Nitazoxanide (BI, HIV-Uninfected; } \\
\text { BII*, HIV-Infected in Combination } \\
\text { with Effective cART): } \\
\begin{array}{l}1-3 \text { years: Nitazoxanide } \\
\text { (20 mg/mL oral solution) }\end{array} \\
100 \mathrm{mg} \text { orally twice daily } \\
\text { with food } \\
\quad 4-11 \text { years: Nitazoxanide } \\
\text { (20 mg/mL oral solution) } \\
200 \mathrm{mg} \text { orally twice daily } \\
\text { with food } \\
\text { - } 12 \text { years: Nitazoxanide } \\
\text { tablet 500 mg orally } \\
\text { twice daily with food } \\
\text { Treatment Duration: } \\
\text { 3-14 days }\end{array}$ & $\begin{array}{l}\text { Supportive Care: } \\
\qquad \begin{array}{l}\text { Hydration, } \\
\text { correct } \\
\text { electrolyte } \\
\text { abnormalities, } \\
\text { nutritional } \\
\text { support }\end{array} \\
\begin{array}{l}\text { Antimotility agents (such as } \\
\text { loperamide) should be used } \\
\text { with caution in young } \\
\text { children. }\end{array}\end{array}$ \\
\hline Cytomegalovirus (CMV) & $\begin{array}{l}\text { Symptomatic Congenital Infection } \\
\text { with Neurologic Involvement: } \\
\begin{array}{l}\text { Ganciclovir } 6 \mathrm{mg} / \mathrm{kg} \\
\text { body weight per dose } \\
\text { IV every } 12 \text { hours for } 6 \\
\text { weeks }\end{array} \\
\text { Disseminated Disease and Retinitis: } \\
\text { Induction Therapy (Followed by } \\
\text { Chronic Suppressive Therapy): } \\
\text { - Ganciclovir } 5 \mathrm{mg} / \mathrm{kg} \\
\text { body weight per dose } \\
\text { IV every } 12 \text { hours for } \\
\text { 14-21 days (may be } \\
\text { increased to } 7.5 \mathrm{mg} / \mathrm{kg} \\
\text { body weight per dose } \\
\text { IV twice daily), then } 5 \\
\text { mg/kg body weight } \\
\text { once daily for } 5-7 \text { days } \\
\text { per week for chronic } \\
\text { suppression } \\
\text { Central Nervous System Disease } \\
\text { (Followed by Chronic Suppressive } \\
\text { Therapy; See Secondary } \\
\text { Prophylaxis): } \\
\text { - } \\
\text { Ganciclovir } 5 \mathrm{mg} / \mathrm{kg} \\
\text { body weight per dose } \\
\text { IV every } 12 \text { hours } \\
\text { PLUS foscarnet } 60\end{array}$ & $\begin{array}{l}\text { Disseminated Disease and Retinitis: } \\
\text { Induction Therapy (Followed by } \\
\text { Chronic Suppressive Therapy): } \\
\text { - } \quad \text { Foscarnet, } 60 \mathrm{mg} / \mathrm{kg} \text { body } \\
\text { weight per dose IV every } \\
8 \text { hours or } 90 \mathrm{mg} / \mathrm{kg} \text { body } \\
\text { weight per dose IV every } \\
12 \text { hours } 14 \text { to } 21 \text { days, } \\
\text { then } 90-120 \mathrm{mg} / \mathrm{kg} \text { body } \\
\text { weight IV once daily for } \\
\text { chronic suppression } \\
\text { Alternatives for Retinitis (Followed } \\
\text { by Chronic Suppressive Therapy; See } \\
\text { Secondary Prophylaxis): } \\
\text { Valganciclovir tablets } \\
\text { 900 mg per dose orally } \\
\text { twice daily for } 14-21 \\
\text { days, followed by chronic } \\
\text { suppressive therapy (see } \\
\text { above). Note: This is an } \\
\text { option in older children } \\
\text { who can receive the adult } \\
\text { dose (based on their } \\
\text { BSA). } \\
\text { IV ganciclovir plus IV } \\
\text { foscarnet (at above } \\
\text { induction doses) may be } \\
\text { considered as initial } \\
\text { induction therapy in }\end{array}$ & $\begin{array}{l}\text { Data on } \\
\text { valganciclovir } \\
\text { dosing in young } \\
\text { children for } \\
\text { treatment of } \\
\text { retinitis are } \\
\text { unavailable, but } \\
\text { consideration } \\
\text { can be given to } \\
\text { transitioning } \\
\text { from IV } \\
\text { ganciclovir to } \\
\text { oral } \\
\text { valganciclovir } \\
\text { after } \\
\text { improvement of } \\
\text { retinitis is } \\
\text { noted. } \\
\text { Intravitreal } \\
\text { injections of } \\
\text { ganciclovir, } \\
\text { foscarnet, or } \\
\text { cidofovir are } \\
\text { used in adults } \\
\text { for retinitis but } \\
\text { are not practical } \\
\text { for most } \\
\text { children. } \\
\text { Combination } \\
\text { ganciclovir and }\end{array}$ \\
\hline
\end{tabular}




\begin{tabular}{|c|c|c|c|}
\hline Indication & First Choice & Alternative & Comments/Special Issues \\
\hline & $\begin{array}{l}\mathrm{mg} / \mathrm{kg} \text { body weight per } \\
\text { dose IV every } 8 \text { hours } \\
\text { (or } 90 \mathrm{mg} / \mathrm{kg} \text { body } \\
\text { weight per dose IV } \\
\text { every } 12 \text { hours) } \\
\text { continued until } \\
\text { symptomatic } \\
\text { improvement, followed } \\
\text { by chronic suppression }\end{array}$ & $\begin{array}{l}\text { children with sight- } \\
\text { threatening disease or for } \\
\text { treatment following } \\
\text { failure/relapse on } \\
\text { monotherapy. } \\
\text { Cidofovir is also used to } \\
\text { treat CMV retinitis in } \\
\text { adults intolerant to other } \\
\text { therapies. Induction } \\
\text { dosing in adults is } 5 \\
\text { mg/kg body weight IV } \\
\text { once weekly for } 2 \text { weeks, } \\
\text { followed by chronic } \\
\text { suppressive therapy (see } \\
\text { secondary prophylaxis); } \\
\text { however, data on dosing } \\
\text { in children are } \\
\text { unavailable. Must be } \\
\text { given with probenecid } \\
\text { and IV hydration }\end{array}$ & $\begin{array}{l}\text { foscarnet is } \\
\text { associated with } \\
\text { substantial rates } \\
\text { of adverse } \\
\text { effects, and } \\
\text { optimal } \\
\text { treatment for } \\
\text { neurologic } \\
\text { disease in } \\
\text { children is } \\
\text { unknown, } \\
\text { particularly if } \\
\text { receiving } \\
\text { optimized } \\
\text { cART. } \\
\text { Chronic } \\
\text { suppressive } \\
\text { therapy } \\
\text { (secondary } \\
\text { prophylaxis) is } \\
\text { recommended } \\
\text { in adults and } \\
\text { children } \\
\text { following initial } \\
\text { therapy of } \\
\text { disseminated } \\
\text { disease, } \\
\text { retinitis, } \\
\text { neurologic } \\
\text { disease, or GI } \\
\text { disease with } \\
\text { relapse. }\end{array}$ \\
\hline Giardiasis & $\begin{array}{l}\text { - Tinidazole, } 50 \mathrm{mg} / \mathrm{kg} \text { by } \\
\text { mouth, administered as } \\
\text { 1 dose given with food } \\
\text { (maximum } 2 \mathrm{~g} \text { ). Note: } \\
\text { Based on data from } \\
\text { HIV-uninfected } \\
\text { children } \\
\text { - Nitazoxanide. Note: } \\
\text { Based on data from } \\
\text { HIV-uninfected } \\
\text { children } \\
-\quad 1-3 \text { years: } \\
100 \text { mg by } \\
\text { mouth every } \\
12 \text { hours } \\
\text { with food } \\
\text { for } 3 \text { days } \\
-\quad 4-11 \text { years: } \\
200 \text { mg by } \\
\text { mouth every } \\
12 \text { hours } \\
\text { with food } \\
\text { for } 3 \text { days } \\
-\quad \text { >12 years: } \\
500 \text { mg by } \\
\text { mouth every } \\
12 \text { hours } \\
\text { with food } \\
\text { for } 3 \text { days }\end{array}$ & $\begin{array}{l}\text { Metronidazole } 5 \mathrm{mg} / \mathrm{kg} \text { by mouth } \\
\text { every } 8 \text { hours for } 5-7 \text { days. } \\
\text { Note: Based on data from HIV- } \\
\text { uninfected children }\end{array}$ & $\begin{array}{l}\text { Tinidazole is approved in } \\
\text { the United States for } \\
\text { children aged } 23 \text { years. It is } \\
\text { available in tablets that can } \\
\text { be crushed. } \\
\text { Metronidazole has high } \\
\text { frequency of } \\
\text { gastrointestinal side effects. } \\
\text { A pediatric suspension of } \\
\text { metronidazole is not } \\
\text { commercially available but } \\
\text { can be compounded from } \\
\text { tablets. It is not FDA- } \\
\text { approved for the treatment } \\
\text { of giardiasis. } \\
\text { Supportive Care: } \\
\quad \text { - Hydration } \\
\quad \text { Correction of } \\
\quad \text { electrolyte } \\
\quad \text { abnormalities } \\
\quad \text { Nutritional } \\
\quad \text { support }\end{array}$ \\
\hline Hepatitis B Virus (HBV) & $\frac{\frac{\text { Treatment of Only HBV Required }}{\text { (Child Does Not Require cART): }}}{\text { - } \quad \begin{array}{l}\text { IFN-a } 3 \text { million } \\
\text { units } / \mathrm{m}^{2} \text { body surface } \\
\text { area SQ } 3 \text { times a week }\end{array}}$ & $\begin{array}{l}\text { IFN-a } 10 \text { million } \\
\text { units/m² body surface } \\
\text { area SQ } 3 \text { times a week } \\
\text { for } 6 \text { months (sometimes } \\
\text { used for retreatment of }\end{array}$ & 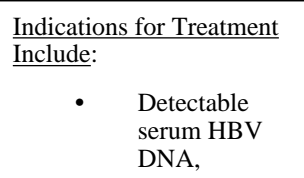 \\
\hline
\end{tabular}




\begin{tabular}{|c|c|c|c|}
\hline Indication & First Choice & Alternative & Comments/Special Issues \\
\hline & 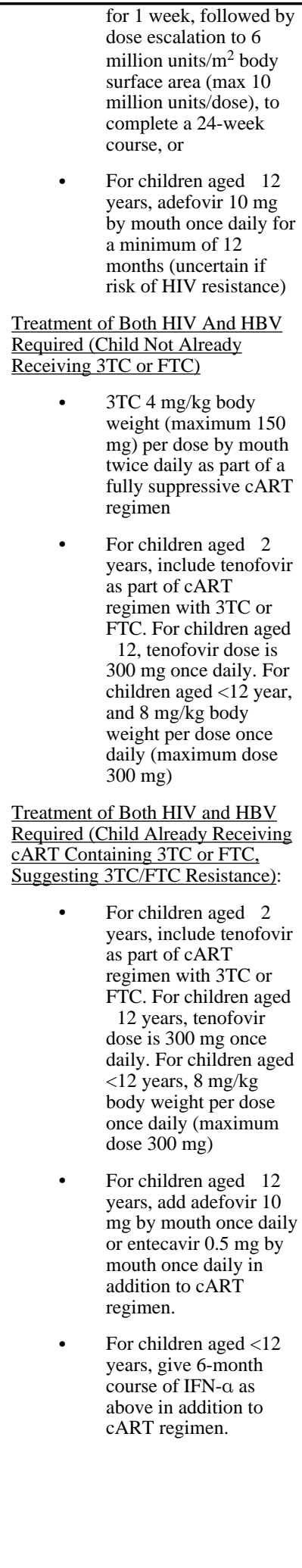 & $\begin{array}{l}\text { failed lower-dose } \\
\text { interferon therapy) } \\
\text { Alternative for 3TC: FTC } \\
6 \mathrm{mg} / \mathrm{kg} \text { body weight } \\
\text { (maximum } 200 \mathrm{mg} \text { ) once } \\
\text { daily }\end{array}$ & $\begin{array}{l}\text { irrespective of } \\
\quad \text { HBeAg status, } \\
\quad \text { for }>6 \text { months; } \\
\quad \text { and } \\
\quad \text { Persistent (>6 } \\
\quad \text { months) } \\
\quad \text { elevation of } \\
\quad \text { serum } \\
\text { transaminases } \\
\quad \text { ( } 2 \text { twice the } \\
\quad \text { upper limit of } \\
\quad \text { normal); or } \\
\quad \text { Evidence of } \\
\quad \text { chronic } \\
\quad \text { hepatitis on } \\
\quad \text { liver biopsy } \\
\text { IFN-a is contraindicated in } \\
\text { children with } \\
\text { decompensated liver } \\
\text { disease; significant } \\
\text { cytopenias, severe renal, } \\
\text { neuropsychiatric, or cardiac } \\
\text { disorders; and autoimmune } \\
\text { disease. } \\
\text { Choice of HBV treatment } \\
\text { options for HIV/HBV-co- } \\
\text { infected children depends } \\
\text { upon whether concurrent } \\
\text { HIV treatment is warranted. } \\
\text { 3TC and FTC have similar } \\
\text { activity (and have cross- } \\
\text { resistance) and should not } \\
\text { be given together. FTC is } \\
\text { not FDA-approved for } \\
\text { treatment of HBV. } \\
\text { Tenofovir is approved for } \\
\text { use in treatment of HIV } \\
\text { infection in children aged } \\
22 \text { years but it is not } \\
\text { approved for treatment of } \\
\text { HBV infection in children } \\
\text { aged <18 years. It should } \\
\text { only be used for HBV in } \\
\text { HIV/HBV-infected children } \\
\text { as part of a cART regimen. } \\
\text { Adefovir is approved for } \\
\text { use in children aged } \geq 12 \\
\text { years. } \\
\text { ETV is not approved for use } \\
\text { in children younger than } \\
\text { age 16 years, but is under } \\
\text { study in HIV-uninfected } \\
\text { children for treatment of } \\
\text { chronic hepatitis B. Can be } \\
\text { considered for older HIV- } \\
\text { infected children who can } \\
\text { receive adult dosage. It } \\
\text { should only be used for } \\
\text { HBV in HIV/HBV-infected } \\
\text { children who also receive } \\
\text { an HIV-suppressive cART } \\
\text { regimen. } \\
\text { IRIS may be manifested by } \\
\text { dramatic increase in } \\
\text { transaminases as CD4 cell } \\
\text { counts rise within the first } 6 \\
\text { to 12 weeks of cART. It } \\
\text { may be difficult to } \\
\text { distinguish between drug- } \\
\text { induced hepatotoxicity and } \\
\text { other causes of hepatitis and } \\
\text { IRIS. }\end{array}$ \\
\hline
\end{tabular}




\begin{tabular}{|c|c|c|c|}
\hline Indication & First Choice & Alternative & Comments/Special Issues \\
\hline & & & $\begin{array}{l}\text { In children receiving } \\
\text { tenofovir and 3TC or FTC, } \\
\text { clinical and laboratory } \\
\text { exacerbations of hepatitis } \\
\text { (flare) may occur if the drug } \\
\text { is discontinued; thus, once } \\
\text { anti-HIV/HBV therapy has } \\
\text { begun, it should be } \\
\text { continued unless } \\
\text { contraindicated or until the } \\
\text { child has been treated for } \\
>6 \text { months after HBeAg } \\
\text { seroconversion and can be } \\
\text { closely monitored on } \\
\text { discontinuation. } \\
\text { If anti-HBV therapy is } \\
\text { discontinued and a flare } \\
\text { occurs, reinstitution of } \\
\text { therapy is recommended } \\
\text { because a flare can be life } \\
\text { threatening. } \\
\text { Telbivudine has been } \\
\text { approved for use in people } \\
\text { aged } \geq 16 \text { years with HBV; } \\
\text { there are no data on safety } \\
\text { or efficacy in children aged } \\
<16 \text { years; a } \\
\text { pharmacokinetic study is } \\
\text { under way in HIV- } \\
\text { uninfected children. }\end{array}$ \\
\hline Hepatitis C Virus & 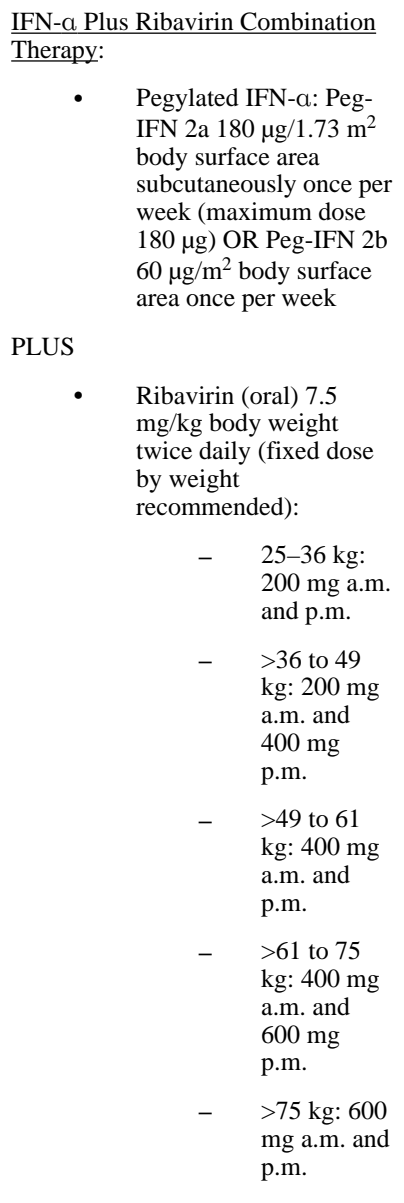 & None & $\begin{array}{l}\text { Optimal duration of } \\
\text { treatment for HIV/HCV- } \\
\text { coinfected children is } \\
\text { unknown and based on } \\
\text { recommendations for HIV/ } \\
\text { HCV-coinfected adults } \\
\text { Treatment of HCV in } \\
\text { children <3 years generally } \\
\text { is not recommended. } \\
\text { Indications for treatment are } \\
\text { based on recommendations } \\
\text { in HIV/HCV-coinfected } \\
\text { adults; because HCV } \\
\text { therapy is more likely to be } \\
\text { effective in younger } \\
\text { patients and in those } \\
\text { without advanced disease or } \\
\text { immunodeficiency, } \\
\text { treatment should be } \\
\text { considered for all HIV/ } \\
\text { HCV-coinfected children } \\
\text { aged >3 years in whom } \\
\text { there are no } \\
\text { contraindications to } \\
\text { treatment } \\
\text { For recommendations } \\
\text { related to use of telaprevir } \\
\text { or boceprevir in adults, } \\
\text { including warnings about } \\
\text { drug interactions between } \\
\text { HCV protease inhibitors } \\
\text { and HIV protease inhibitors } \\
\text { and other antiretroviral } \\
\text { drugs, see Adult OI } \\
\text { guidelines. } \\
\text { IRIS may be manifested by } \\
\text { dramatic increase in } \\
\text { transaminases as CD4 cell } \\
\text { counts rise within the first } \\
6-12 \text { weeks of cART. It } \\
\text { may be difficult to } \\
\text { distinguish between IRIS } \\
\text { and drug-induced }\end{array}$ \\
\hline
\end{tabular}




\begin{tabular}{|c|c|c|c|}
\hline Indication & First Choice & Alternative & Comments/Special Issues \\
\hline & $\begin{array}{l}\text { Treatment Duration: } \\
\text { - } 48 \text { weeks, regardless of } \\
\text { HCV genotype }\end{array}$ & & $\begin{array}{l}\text { hepatotoxicity or other } \\
\text { causes of hepatitis. } \\
\text { IFN-a is contraindicated in } \\
\text { children with } \\
\text { decompensated liver } \\
\text { disease, significant } \\
\text { cytopenias, renal failure, } \\
\text { severe cardiac disorders and } \\
\text { non-HCV-related } \\
\text { autoimmune disease. } \\
\text { Ribavirin is contraindicated } \\
\text { in children with unstable } \\
\text { cardiopulmonary disease, } \\
\text { severe pre-existing anemia } \\
\text { or hemoglobinopathy. } \\
\text { Didanosine combined with } \\
\text { ribavirin may lead to } \\
\text { increased mitochondrial } \\
\text { toxicities; concomitant use } \\
\text { is contraindicated. } \\
\text { Ribavirin and zidovudine } \\
\text { both are associated with } \\
\text { anemia, and when possible, } \\
\text { should not be administered } \\
\text { together }\end{array}$ \\
\hline $\begin{array}{l}\text { Herpes Simplex Virus } \\
\text { Infections (HSV) }\end{array}$ & 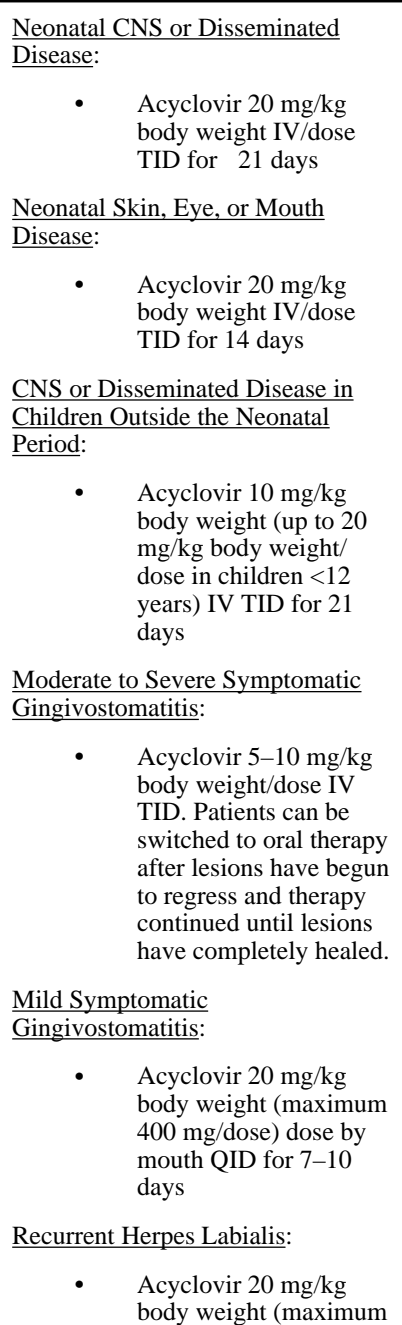 & $\begin{array}{l}\text { - Valacyclovir is approved } \\
\text { for immuno-competent } \\
\text { adults and adolescents } \\
\text { with first-episode } \\
\text { mucocutaneous HSV at a } \\
\text { dose of } 1 \mathrm{~g} / \text { dose by } \\
\text { mouth BID for } 7-10 \\
\text { days; also approved for } \\
\text { recurrent herpes labialis } \\
\text { in children } \geq 12 \text { years } \\
\text { using two, } 2 \mathrm{~g} \text { doses by } \\
\text { mouth separated by } 12 \\
\text { hours as single-day } \\
\text { therapy. } \\
\text { Recurrent genital HSV } \\
\text { can be treated with } \\
\text { valacyclovir } 500 \text { mg BID } \\
\text { for } 3 \text { days or } 1 \mathrm{~g} \text { by } \\
\text { mouth daily for } 5 \text { days. } \\
\text { Immunocompetent adults } \\
\text { with recurrent herpes } \\
\text { labialis can be treated } \\
\text { with famciclovir, } 1 \mathrm{~g} / \\
\text { dose by mouth BID for } 1 \\
\text { day. } \\
\text { Famciclovir is approved } \\
\text { to treat primary genital } \\
\text { HSV in } \\
\text { immunocompetent adults } \\
\text { at a dose of } 250 \text { mg/dose } \\
\text { by mouth TID for } 7-10 \\
\text { days. } \\
\text { Recurrent genital HSV is } \\
\text { treated with famciclovir } 1 \\
\text { g/dose by mouth BID at a } \\
12 \text {-hour interval for } 2 \\
\text { doses } \\
\text { Famciclovir is approved } \\
\text { for use in HIV-infected } \\
\text { adults and adolescents } \\
\text { with recurrent } \\
\text { mucocutaneous HSV } \\
\text { infection at a dose of } 500 \\
\text { - }\end{array}$ & $\begin{array}{ll}\text { For Neonatal CNS Disease: } \\
& \text { Repeat CSF } \\
& \text { HSV DNA PCR } \\
\text { should be } \\
\text { performed on } \\
\text { days } 19 \text { to } 21 \text { of } \\
\text { therapy; do not } \\
\text { stop acyclovir } \\
\text { until repeat CSF } \\
\text { HSV DNA PCR } \\
\text { is negative. } \\
\text { There is no } \\
\text { pediatric } \\
\text { preparation of } \\
\text { valacyclovir } \\
\text { (although } \\
\text { crushed } \\
\text { capsules can be } \\
\text { used to make a } \\
\text { suspension) and } \\
\text { data on dosing } \\
\text { in children are } \\
\text { limited; can be } \\
\text { used by } \\
\text { adolescents able } \\
\text { to receive adult } \\
\text { dosing. } \\
\text { There is no } \\
\text { pediatric } \\
\text { preparation of } \\
\text { famciclovir and } \\
\text { data on dosing } \\
\text { in children are } \\
\text { unavailable; can } \\
\text { be used by } \\
\text { adolescents able } \\
\text { to receive adult } \\
\text { dosing. }\end{array}$ \\
\hline
\end{tabular}




\begin{tabular}{|c|c|c|c|}
\hline Indication & First Choice & Alternative & Comments/Special Issues \\
\hline & 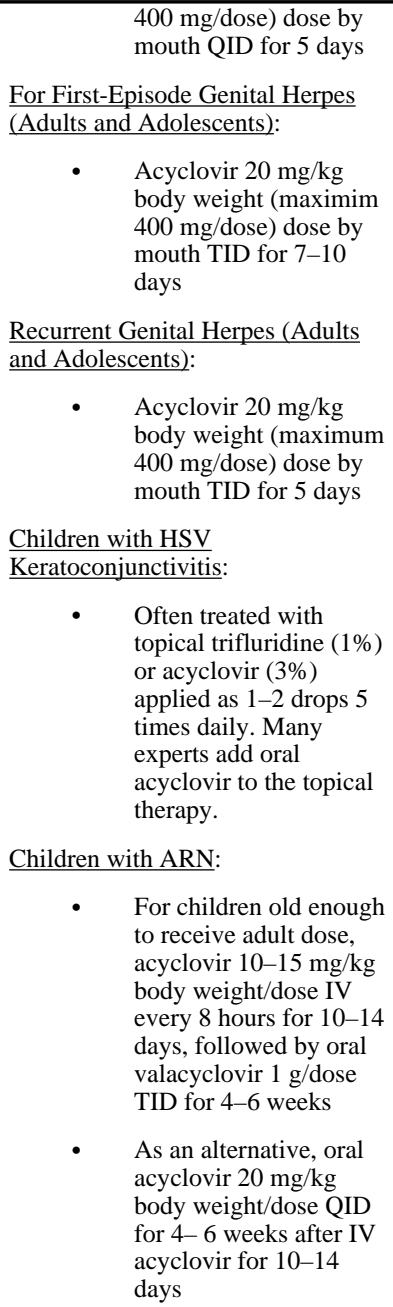 & $\begin{array}{ll}\begin{array}{l}\mathrm{mg} / \text { dose by mouth BID } \\
\text { for } 7 \text { days. }\end{array} \\
\text { Acyclovir-Resistant HSV Infection: } \\
\text { Foscarnet } 40 \mathrm{mg} / \mathrm{kg} \text { body } \\
\text { weight } / \text { dose given IV } \\
\text { TID (or } 60 \mathrm{mg} / \mathrm{kg} \text { body } \\
\text { weight } / \text { dose BID) should } \\
\text { be administered slowly } \\
\text { over the course of } 2 \text { hours } \\
\text { (i.e., no faster than } 1 \\
\mathrm{mg} / \mathrm{kg} / \text { minute). }\end{array}$ & $\begin{array}{l}\begin{array}{l}\text { Acyclovir 800 } \\
\text { mg per dose by } \\
\text { mouth BID for } \\
5 \text { days }\end{array} \\
\quad \begin{array}{l}\text { Acyclovir } 800 \\
\text { mg per dose by } \\
\text { mouth TID for } \\
2 \text { days }\end{array} \\
\text { Note: Consultation with an } \\
\text { ophthalmologist } \\
\text { experienced in managing } \\
\text { herpes simplex infection } \\
\text { involving the eye and its } \\
\text { complications in children is } \\
\text { strongly recommended } \\
\text { when ocular disease is } \\
\text { present. }\end{array}$ \\
\hline Histoplasmosis & 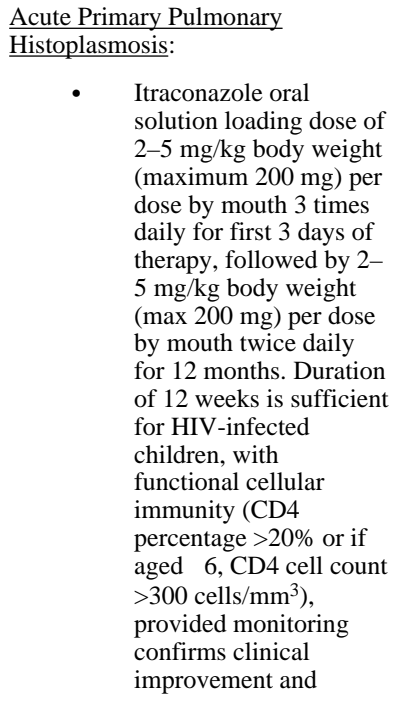 & 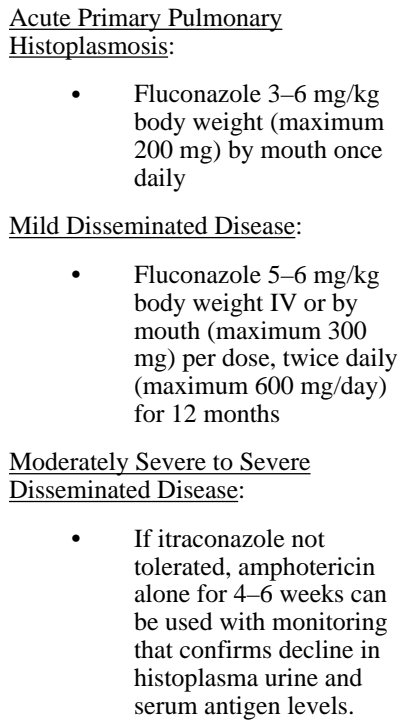 & $\begin{array}{l}\text { Use same initial } \\
\text { itraconazole dosing for } \\
\text { capsules as for solution. } \\
\text { Itraconazole solution is } \\
\text { preferred to the capsule } \\
\text { formulation because it is } \\
\text { better absorbed; solution } \\
\text { can achieve serum } \\
\text { concentrations } 30 \% \text { higher } \\
\text { than those achieved with the } \\
\text { capsules. } \\
\text { Urine antigen concentration } \\
\text { should be assessed at } \\
\text { diagnosis. If }>39 \text { ng/mL, } \\
\text { serum concentrations } \\
\text { should be followed. When } \\
\text { serum levels become } \\
\text { undetectable, urine } \\
\text { concentrations should be } \\
\text { monitored monthly during } \\
\text { treatment and followed } \\
\text { thereafter to identify } \\
\text { relapse. } \\
\text { Serum concentrations of } \\
\text { itraconazole should be }\end{array}$ \\
\hline
\end{tabular}




\begin{tabular}{|c|c|c|c|}
\hline Indication & First Choice & Alternative & Comments/Special Issues \\
\hline & 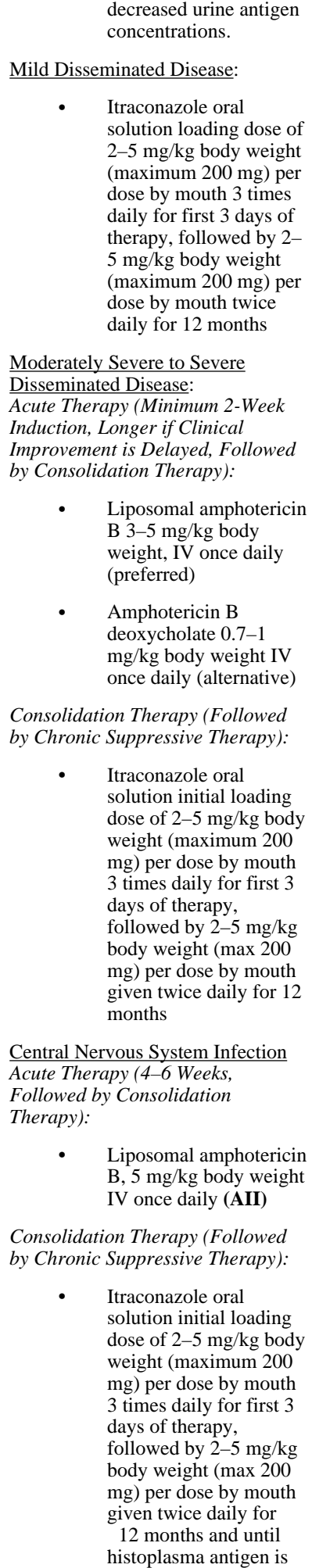 & $\begin{array}{l}\text { Liposomal amphotericin } \\
\text { B 3-5 mg/kg body weight } \\
\text { IV once daily (preferred) } \\
\text { for 4-6 weeks } \\
\text { - Amphotericin B } \\
\text { deoxycholate } 0.7-1 \\
\text { mg/kg body weight IV } \\
\text { once daily (alternative) } \\
\text { for 4-6 weeks }\end{array}$ & $\begin{array}{l}\text { monitored and achieve a } \\
\text { level of } 1 \mu \mathrm{g} / \mathrm{mL} \text { at steady- } \\
\text { state. Levels exceeding } 10 \\
\mu \mathrm{g} / \mathrm{mL} \text { should be followed } \\
\text { by dose reduction. } \\
\text { High relapse rate with CNS } \\
\text { infection occurs in adults } \\
\text { and longer therapy may be } \\
\text { required; treatment in } \\
\text { children is anecdotal and } \\
\text { expert consultation should } \\
\text { be considered. } \\
\text { Chronic suppressive therapy } \\
\text { (secondary prophylaxis) } \\
\text { with itraconazole is } \\
\text { recommended in adults and } \\
\text { children following initial } \\
\text { therapy. } \\
\text { Amphotericin B } \\
\text { deoxycholate is better } \\
\text { tolerated in children than in } \\
\text { adults. Liposomal } \\
\text { amphotericin B is preferred } \\
\text { for treatment of } \\
\text { parenchymal cerebral } \\
\text { lesions. }\end{array}$ \\
\hline
\end{tabular}




\begin{tabular}{|c|c|c|c|}
\hline Indication & First Choice & Alternative & Comments/Special Issues \\
\hline & $\begin{array}{l}\text { no longer detected in } \\
\text { cerebrospinal fluid }\end{array}$ & & \\
\hline Human Papillomavirus (HPV) & $\begin{array}{l}\text { Podofilox solution/gel } \\
\text { (0.5\%) applied topically } \\
\text { BID for } 3 \text { consecutive } \\
\text { days a week up to } 4 \\
\text { weeks (patient applied). } \\
\text { Withhold treatment for } \\
4 \text { days and repeat the } \\
\text { cycle weekly up to } 4 \\
\text { times (BIII) } \\
\text { Imiquimod cream (5\%) } \\
\text { applied topically at } \\
\text { night and washed off in } \\
\text { the morning for } 3 \text { non- } \\
\text { consecutive nights a } \\
\text { week for up to } 16 \text { weeks } \\
\text { (patient applied) (BII) } \\
\text { TCA or BCA (80\%- } \\
\text { 90\%) applied topically } \\
\text { weekly for up to } 3 \text { to } 6 \\
\text { weeks (provider } \\
\text { applied) (BIII) } \\
\text { Podophyllin resin } \\
\text { (10\%-25\% suspension } \\
\text { in tincture of benzoin) } \\
\text { applied topically and } \\
\text { washed off several } \\
\text { hours later, repeated } \\
\text { weekly for } 3 \text { to } 6 \text { weeks } \\
\text { (provider applied) } \\
\text { (CIII) } \\
\text { Cryotherapy with liquid } \\
\text { nitrogen or cryoprobe } \\
\text { applied every } 1-2 \\
\text { weeks (BIII) } \\
\text { Surgical removal either } \\
\text { by tangential excision, } \\
\text { tangential shave } \\
\text { excision, curettage, or } \\
\text { electrosurgery } \\
\text { - }\end{array}$ & $\begin{array}{l}\text { - Intralesional IFN-a is } \\
\text { generally not } \\
\text { recommended because of } \\
\text { high cost, difficult } \\
\text { administration, and } \\
\text { potential for systemic } \\
\text { side effects (CIII) } \\
\text { - Cidofovir topical gel } \\
\text { (1\%) is an experimental } \\
\text { therapy studied in HIV- } \\
\text { infected adults that is } \\
\text { commercially available } \\
\text { through compounding } \\
\text { pharmacies and has very } \\
\text { limited use in children; } \\
\text { systemic absorption can } \\
\text { occur (CIII). } \\
\text { 5-FU/epinephrine gel } \\
\text { implant should be offered } \\
\text { in only severe recalcitrant } \\
\text { cases because of } \\
\text { inconvenient routes of } \\
\text { administration, frequent } \\
\text { office visits, and a high } \\
\text { frequency of systemic } \\
\text { adverse effects. }\end{array}$ & $\begin{array}{l}\text { Adequate topical } \\
\text { anesthetics to the genital } \\
\text { area should be given before } \\
\text { caustic modalities are } \\
\text { applied. } \\
\text { Sexual contact should be } \\
\text { limited while solutions or } \\
\text { creams are on the skin. } \\
\text { Although sinecatechins } \\
\text { (15\% ointment) applied } \\
\text { TID up to } 16 \text { weeks is } \\
\text { recommended in } \\
\text { immunocompetent } \\
\text { individuals, data are } \\
\text { insufficient on safety and } \\
\text { efficacy in HIV-infected } \\
\text { individuals. } \\
\text { cART has not been } \\
\text { consistently associated with } \\
\text { reduced risk of HPV-related } \\
\text { cervical abnormalities in } \\
\text { HIV-infected women. } \\
\text { Laryngeal papillomatosis } \\
\text { generally requires referral } \\
\text { to a pediatric } \\
\text { otolaryngologist. Treatment } \\
\text { is directed at maintaining } \\
\text { the airway, rather than } \\
\text { removing all disease. } \\
\text { For women who have } \\
\text { exophytic cervical warts, a } \\
\text { biopsy to exclude HSIL } \\
\text { must be performed before } \\
\text { treatment. } \\
\text { Liquid nitrogen or } \\
\text { TCA/BCA is recommended } \\
\text { for vaginal warts. Use of a } \\
\text { cryoprobe in the vagina is } \\
\text { not recommended. } \\
\text { Cryotherapy with liquid } \\
\text { nitrogen or podophyllin } \\
\text { resin (10\%-25\%) is } \\
\text { recommended for urethral } \\
\text { meatal warts. } \\
\text { Cryotherapy with liquid } \\
\text { nitrogen or TCA/BCA or } \\
\text { surgical removal is } \\
\text { recommended for anal } \\
\text { warts. } \\
\text { Abnormal Pap smear } \\
\text { cytology should be referred } \\
\text { to colposcopy for diagnosis } \\
\text { and management. }\end{array}$ \\
\hline Influenza $A$ and $B$ & 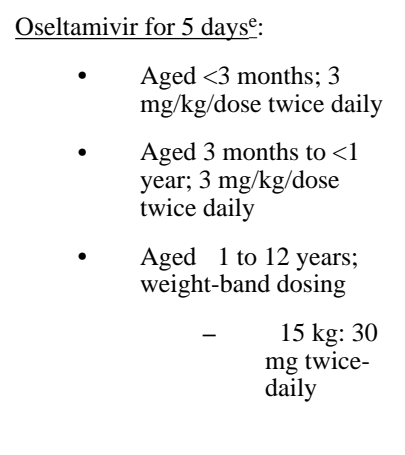 & None & $\begin{array}{l}{ }^{\mathrm{e}} \text { Oseltamivir is FDA- } \\
\text { approved for treatment of } \\
\text { influenza in children aged } \\
22 \text { weeks. The CDC } \\
\text { recommends that clinicians } \\
\text { who treat children ages } \geq 3 \\
\text { months to }<1 \text { year } \\
\text { administer a dose of } 3 \\
\mathrm{mg} / \mathrm{kg} \text { twice daily. A dose } \\
\text { of } 3 \mathrm{mg} / \mathrm{kg} / \text { dose twice daily } \\
\text { also is recommended for } \\
\text { infants aged < } 3 \text { months. } \\
\text { Premature Infants: } \\
\text { Current weight-based } \\
\text { dosing recommendations } \\
\text { for oseltamivir are not }\end{array}$ \\
\hline
\end{tabular}




\begin{tabular}{|c|c|c|c|}
\hline Indication & First Choice & Alternative & Comments/Special Issues \\
\hline & 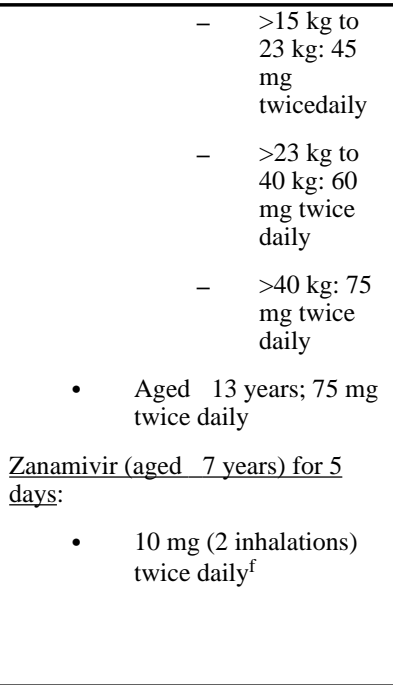 & & $\begin{array}{l}\text { appropriate for premature } \\
\text { infants: gestational age at } \\
\text { delivery <38 weeks. See } J \\
\text { Infect Dis } 202 \text { [4]:563-566, } \\
2010 \text { for dosing } \\
\text { recommendations in } \\
\text { premature infants. } \\
\text { Oseltamivir treatment } \\
\text { duration: Recommended } \\
\text { duration for antiviral } \\
\text { treatment is 5 days; longer } \\
\text { treatment courses can be } \\
\text { considered for patients who } \\
\text { remain severely ill after } 5 \\
\text { days of treatment. } \\
\text { Renal insufficiency: A } \\
\text { reduction in dose of } \\
\text { oseltamivir is recommended } \\
\text { for patients with creatinine } \\
\text { clearance <30 mL/min. } \\
\text { } \text { Zanamivir: Zanamivir is } \\
\text { not recommended for } \\
\text { treatment in children aged } \\
<7 \text { years. }\end{array}$ \\
\hline $\begin{array}{l}\text { Influenza A (ONLY) } \\
\text { Oseltamivir-resistant, } \\
\text { adamantane-sensitive strains } \\
\text { (Based on CDC influenza } \\
\text { surveillance www.cdc.gov/flu/ } \\
\text { weekly/fluactivitysurv.htm) }\end{array}$ & 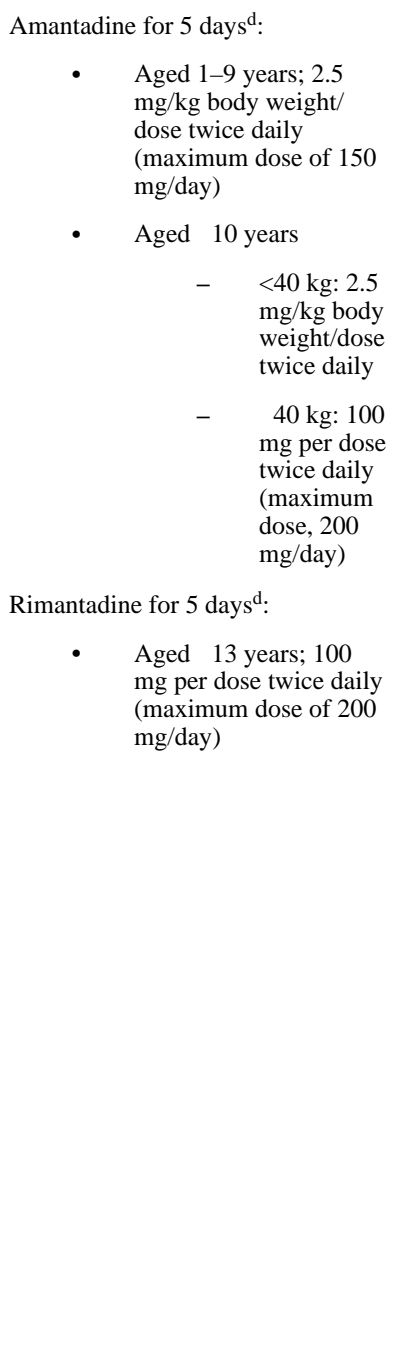 & & $\begin{array}{l}\text { d Adamantanes: Because of } \\
\text { resistance in currently } \\
\text { circulating influenza A } \\
\text { virus strains, amantadine } \\
\text { and rimantadine are not } \\
\text { currently recommended for } \\
\text { chemoprophylaxis or } \\
\text { treatment (adamantanes are } \\
\text { not active against influenza } \\
\text { B virus). However, } \\
\text { potential exists for } \\
\text { emergence of oseltamivir- } \\
\text { resistant, adamantane- } \\
\text { sensitive circulating } \\
\text { influenza A strains. } \\
\text { Therefore, verification of } \\
\text { antiviral sensitivity of } \\
\text { circulating influenza A } \\
\text { strains should be done using } \\
\text { the CDC influenza } \\
\text { surveillance website: http:// } \\
\text { www.cdc.gov/flu/weekly/ } \\
\text { fluactivitysurv.htm } \\
\text { If administered based on } \\
\text { CDC antiviral sensitivity } \\
\text { surveillance data, both } \\
\text { amantadine and rimantadine } \\
\text { are recommended for } \\
\text { chemoprophylaxis of } \\
\text { influenza A in children aged } \\
\geq 1 \text { yr. For treatment, } \\
\text { rimantadine is only } \\
\text { approved for use in } \\
\text { adolescents aged } \geq 13 \text { years. } \\
\text { Rimantadine is preferred } \\
\text { over amantadine because of } \\
\text { less frequent adverse } \\
\text { events. Some pediatric } \\
\text { influenza specialists may } \\
\text { consider it appropriate for } \\
\text { treatment of children aged } \\
>1 \text { year. } \\
\text { Renal insufficiency: A } \\
\text { reduction in dose of } \\
\text { amantadine is } \\
\text { recommended for patients } \\
\text { with creatinine clearance } \\
<30 \text { mL/min. }\end{array}$ \\
\hline
\end{tabular}




\begin{tabular}{|c|c|c|c|}
\hline Indication & First Choice & Alternative & Comments/Special Issues \\
\hline Isosporiasis (Cystoisosporiasis) & $\begin{array}{l}\text { TMP-SMX } 5 \mathrm{mg} / \mathrm{kg} \text { body weight of } \\
\text { TMP component given twice daily } \\
\text { by mouth for } 10 \text { days }\end{array}$ & $\begin{array}{l}\text { Pyrimethamine } 1 \mathrm{mg} / \mathrm{kg} \text { body weight } \\
\text { plus folinic acid } 10-25 \mathrm{mg} \text { by mouth } \\
\text { once daily for } 14 \text { days } \\
\text { Second-Line Alternatives: } \\
\\
\begin{array}{l}\text { Ciprofloxacin } 10-20 \\
\text { mg/kg body weight/day } \\
\text { twice daily by mouth for } \\
7 \text { days }\end{array} \\
\text { Nitazoxanide (see doses } \\
\text { below) for } 3 \text { consecutive } \\
\text { days }\end{array}$ & $\begin{array}{l}\text { If symptoms worsen or } \\
\text { persist, the TMP-SMX dose } \\
\text { may be increased to } 5 \\
\mathrm{mg} / \mathrm{kg} / \mathrm{day} \text { given } 3-4 \text { times } \\
\text { daily by mouth for } 10 \text { days } \\
\text { or the duration of treatment } \\
\text { may be lengthened. } \\
\text { Duration of treatment with } \\
\text { pyrimethamine has not been } \\
\text { well established. } \\
\text { Ciprofloxacin is generally } \\
\text { not a drug of first choice in } \\
\text { children due to increased } \\
\text { incidence of adverse events, } \\
\text { including events related to } \\
\text { joints and/or surrounding } \\
\text { tissues. }\end{array}$ \\
\hline Malaria & 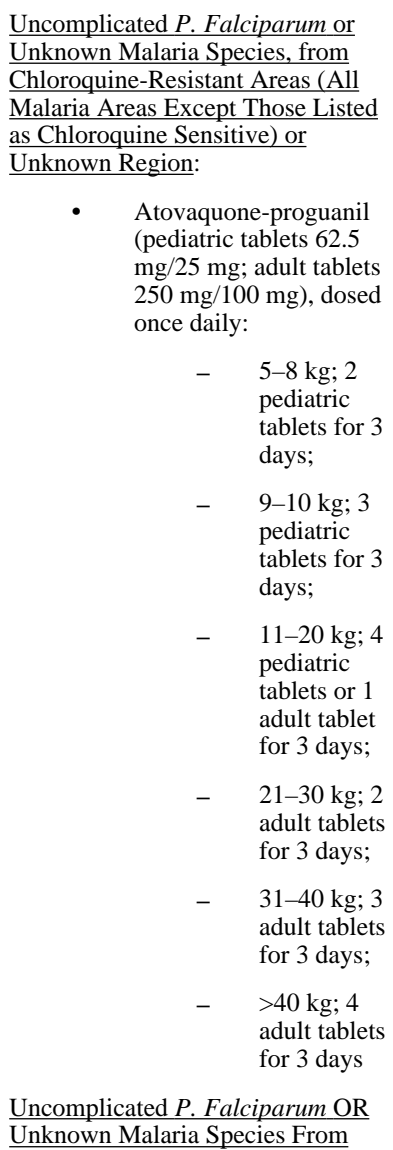 & N/A & $\begin{array}{l}\text { For quinine-based } \\
\text { regimens, doxycycline or } \\
\text { tetracycline should be used } \\
\text { only in children aged } 88 \\
\text { years. An alternative for } \\
\text { children aged } \geq 8 \text { years is } \\
\text { clindamycin } 7 \text { mg/kg body } \\
\text { weight per dose by mouth } \\
\text { given every } 8 \text { hours. } \\
\text { Clindamycin should be used } \\
\text { for children aged <8 years. } \\
\text { Before primaquine is given, } \\
\text { G6PD status must be } \\
\text { verified. Primaquine may be } \\
\text { given in combination with } \\
\text { chloroquine if the G6PD } \\
\text { status is known and } \\
\text { negative, otherwise give } \\
\text { after chloroquine (when } \\
\text { G6PD status is available) } \\
\text { For most updated } \\
\text { prevention and treatment } \\
\text { recommendations for } \\
\text { specific region, refer to } \\
\text { updated CDC treatment } \\
\text { table available at http:// } \\
\text { www.cdc.gov/malaria/ } \\
\text { resources/pdf/ } \\
\text { treatmenttable.pdf } \\
\text { For sensitive and resistant } \\
\text { malaria map: http://cdc- } \\
\text { malaria.ncsa.uiuc.edu/ } \\
\text { High treatment failure rates } \\
\text { due to chloroquine-resistant } \\
P . \text { vivax have been } \\
\text { documented in Papua New } \\
\text { Guinea and Indonesia. } \\
\text { Treatment should be } \\
\text { selected from one of the } \\
\text { three following options: }\end{array}$ \\
\hline
\end{tabular}




\begin{tabular}{|c|c|c|c|}
\hline Indication & First Choice & Alternative & Comments/Special Issues \\
\hline & 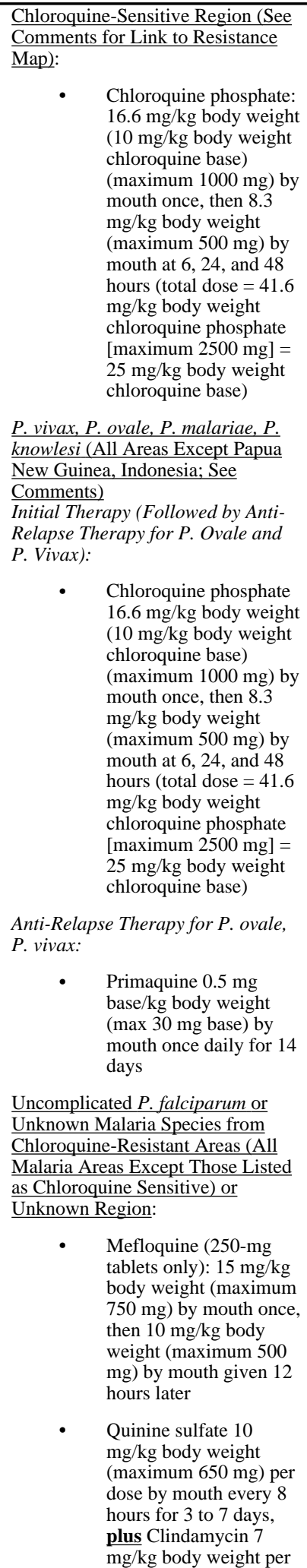 & & $\begin{array}{ll}\text { Atovaquone- } \\
\text { proguanil plus } \\
\text { primaquine } \\
\text { phosphate } \\
\text { Quinine sulfate } \\
\text { plus EITHER } \\
\text { doxycycline } \\
\text { OR tetracycline } \\
\text { PLUS } \\
\text { primaquine } \\
\text { phosphate. This } \\
\text { regimen cannot } \\
\text { be used in } \\
\text { children aged } \\
<8 \text { years. } \\
\text { Mefloquine plus } \\
\text { primaquine } \\
\text { phosphate } \\
\end{array}$ \\
\hline
\end{tabular}




\begin{tabular}{|c|c|c|c|}
\hline Indication & First Choice & Alternative & Comments/Special Issues \\
\hline & 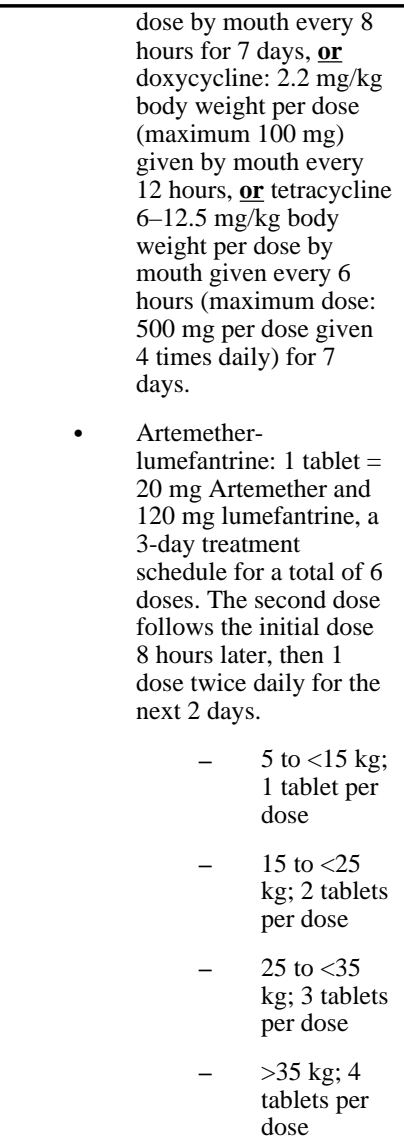 & & \\
\hline Severe Malaria & $\begin{array}{l}\text { Quinidine gluconate } 10 \\
\text { mg/kg body weight IV } \\
\text { loading dose over } 1-2 \\
\text { hours, then } 0.02 \mathrm{mg} / \mathrm{kg} \\
\text { body weight/ minute } \\
\text { infusion for } 224 \text { hours } \\
\text { (Treatment duration: } 7 \\
\text { days in Southeast Asia, } \\
\text { Oceania, otherwise } 3 \\
\text { days) } \\
\text { PLUS One of the Following: } \\
\text { Doxycycline } 100 \mathrm{mg} \\
\text { per dose by mouth } \\
\text { every } 12 \text { hours for } 7 \\
\text { days; for children }<45 \\
\text { kg, use } 2.2 \mathrm{mg} / \mathrm{kg} \text { body } \\
\text { weight per dose } \\
\text { OR } \\
\text { Clindamycin } 7 \mathrm{mg} / \mathrm{kg} \\
\text { body weight per dose by } \\
\text { mouth given every } 8 \\
\text { hours for } 7 \text { days. } \\
\text { OR } \\
\text { Tetracycline } 6-12.5 \\
\text { mg/kg body weight per } \\
\text { dose every } 6 \text { hours } \\
\text { (maximum dose } 500 \mathrm{mg}\end{array}$ & N/A & $\begin{array}{l}\text { Quinidine gluconate is a } \\
\text { class 1a anti-arrhythmic } \\
\text { agent not typically stocked } \\
\text { in pediatric hospitals. When } \\
\text { regional supplies are } \\
\text { unavailable, the CDC } \\
\text { Malaria hotline may be of } \\
\text { assistance (see below). Do } \\
\text { not give quinidine } \\
\text { gluconate as an IV bolus. } \\
\text { Quinidine gluconate IV } \\
\text { should be administered in a } \\
\text { monitored setting. Cardiac } \\
\text { monitoring required. } \\
\text { Adverse events including } \\
\text { severe hypoglycemia, } \\
\text { prolongation of the QT } \\
\text { interval, ventricular } \\
\text { arrhythmia, and } \\
\text { hypotension can result from } \\
\text { the use of this drug at } \\
\text { treatment doses. } \\
\text { IND: IV artesunate is } \\
\text { available from CDC. } \\
\text { Contact the CDC Malaria } \\
\text { Hotline at (770) } 488-7788 \\
\text { from } 8 \text { a.m.- 4:30 p.m. EST } \\
\text { or (770) 488-7100 after } \\
\text { hours, weekends, and } \\
\text { holidays. Artesunate } \\
\text { followed by one of the }\end{array}$ \\
\hline
\end{tabular}




\begin{tabular}{|c|c|c|c|}
\hline Indication & First Choice & Alternative & Comments/Special Issues \\
\hline & 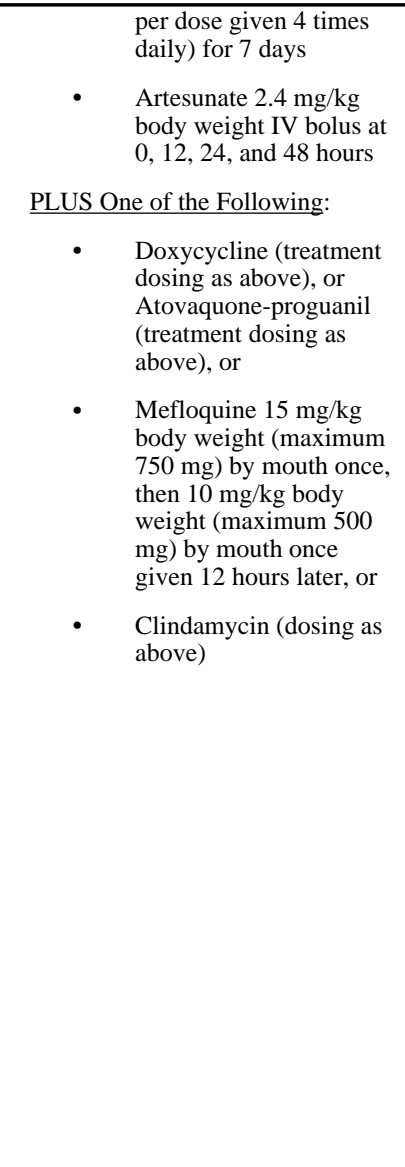 & & 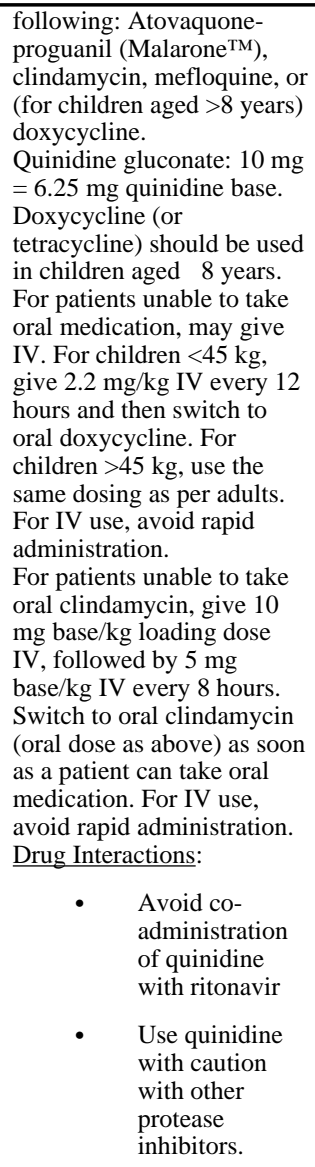 \\
\hline Microsporidiosis & 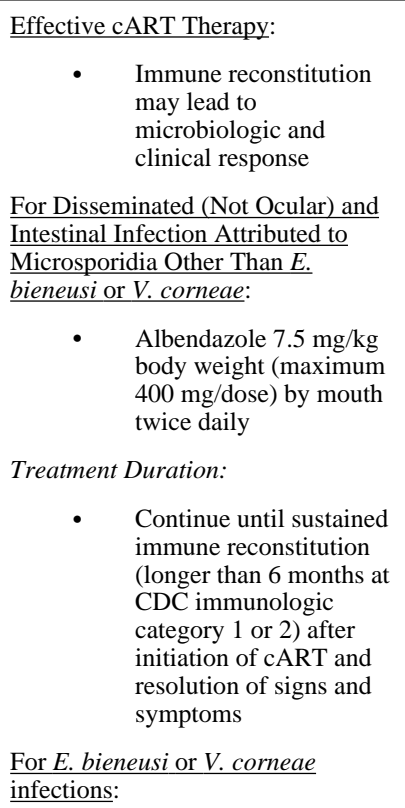 & N/A & $\begin{array}{l}\text { - Supportive care: } \\
\text { Hydration, } \\
\text { correct } \\
\text { electrolyte } \\
\text { abnormalities, } \\
\text { nutritional } \\
\text { support } \\
\text { Fumagillin for } \\
\text { systemic use is } \\
\text { unavailable in } \\
\text { the United } \\
\text { States and data } \\
\text { on dosing in } \\
\text { children are } \\
\text { unavailable. } \\
\text { Consultation } \\
\text { with an expert } \\
\text { is } \\
\text { recommended. }\end{array}$ \\
\hline
\end{tabular}




\begin{tabular}{|c|c|c|c|}
\hline Indication & First Choice & Alternative & Comments/Special Issues \\
\hline & 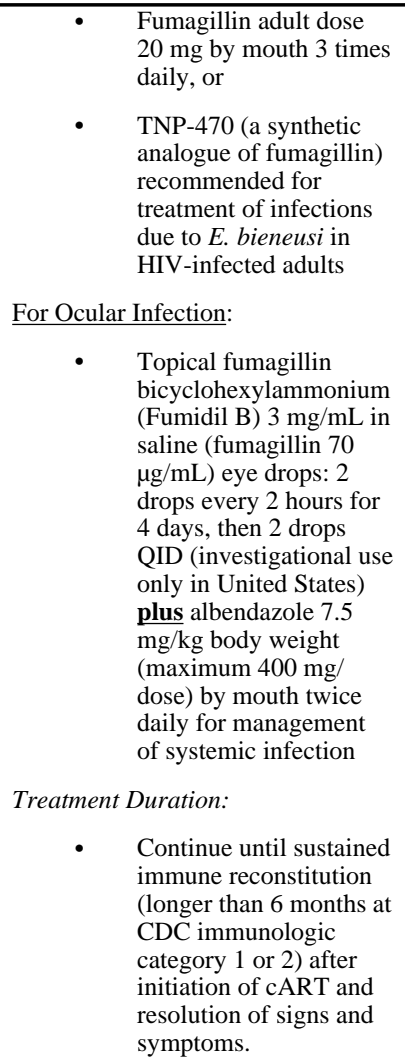 & & \\
\hline $\begin{array}{l}\text { Mycobacterium avium } \\
\text { Complex (MAC) }\end{array}$ & 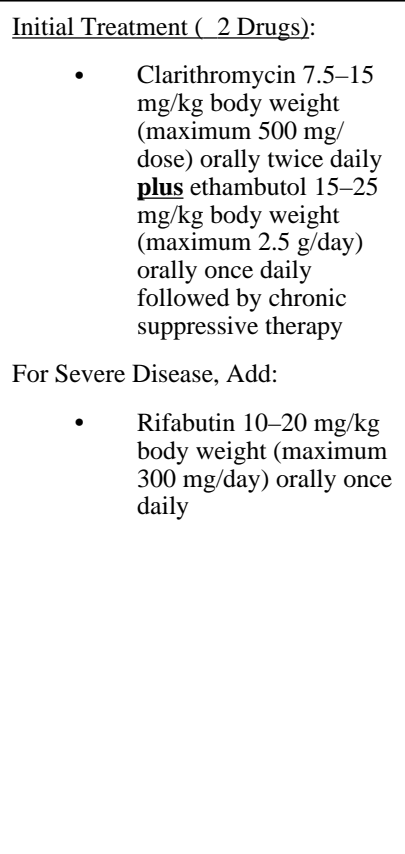 & 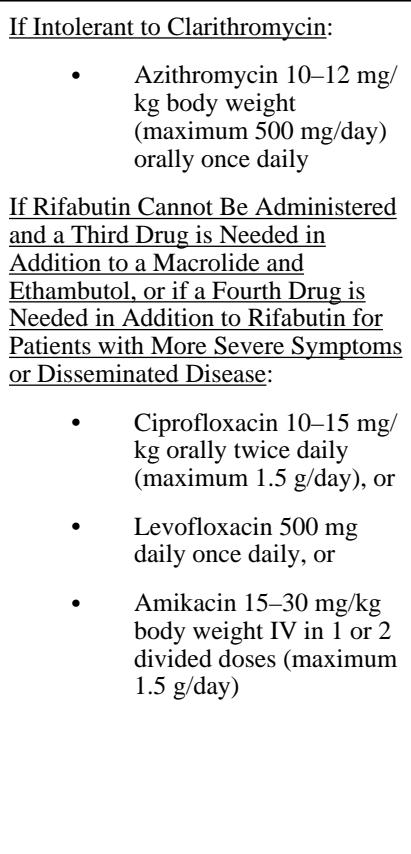 & $\begin{array}{l}\text { Combination therapy with a } \\
\text { minimum of } 2 \text { drugs is } \\
\text { recommended for at least } 12 \\
\text { months. } \\
\text { Clofazimine is associated } \\
\text { with increased mortality in } \\
\text { HIV-infected adults and } \\
\text { should not be used. } \\
\text { Children receiving } \\
\text { ethambutol who are old } \\
\text { enough to undergo routine } \\
\text { eye testing should have } \\
\text { monthly monitoring of } \\
\text { visual acuity and color } \\
\text { discrimination. } \\
\text { Fluoroquinolones (e.g., } \\
\text { ciprofloxacin and } \\
\text { levofloxacin) are not } \\
\text { labeled for use in children } \\
\text { aged <18 years because of } \\
\text { concerns regarding potential } \\
\text { effects on cartilage; use in } \\
\text { younger individuals } \\
\text { requires an assessment of } \\
\text { potential risks and benefits } \\
\text { Chronic suppressive therapy } \\
\text { (secondary prophylaxis) is } \\
\text { recommended in children } \\
\text { and adults following initial } \\
\text { therapy. }\end{array}$ \\
\hline Mycobacterium Tuberculosis & $\begin{array}{l}\text { Intrathoracic Disease } \\
\text { Drug-Susceptible TB } \\
\text { Intensive Phase (2 Months): }\end{array}$ & $\begin{array}{l}\text { Alternative for Rifampin: } \\
\begin{array}{ll}\text { Rifabutin } 10-20 \mathrm{mg} / \mathrm{kg} \\
\text { body weight (maximum }\end{array}\end{array}$ & $\begin{array}{l}\text { Only DOT. } \\
\text { If cART-naive, start TB } \\
\text { therapy immediately and }\end{array}$ \\
\hline
\end{tabular}




\begin{tabular}{|c|c|c|c|}
\hline Indication & First Choice & Alternative & Comments/Special Issues \\
\hline & 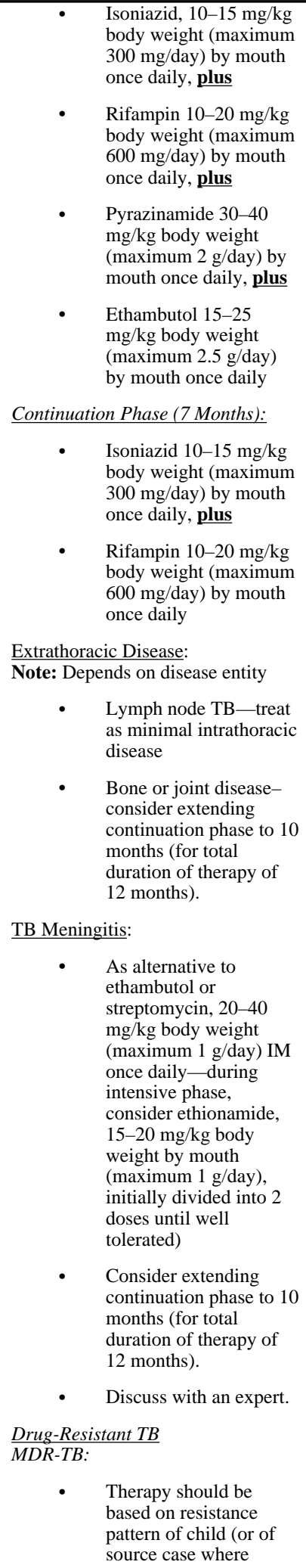 & $\begin{array}{ll} & 300 \mathrm{mg} / \mathrm{day} \text { ) by mouth } \\
& \text { once daily (same dose if } \\
& 3 \text { times a week) } \\
& \text { Discuss with an expert. } \\
\text { Alternative Continuation Phase } \\
\text { If Good Adherence and Treatment } \\
\text { Response: } \\
\text { - } \quad \text { Isoniazid } 20-30 \mathrm{mg} / \mathrm{kg} \\
\text { body weight (maximum } \\
\text { 900 mg/day) by mouth, } \\
\text { plus } \\
\text { Rifampin } 10-20 \mathrm{mg} / \mathrm{kg} \\
\text { body weight (maximum } \\
\text { 600 mg/day) three times a } \\
\text { week. } \\
\text { In children with minimal } \\
\text { disease with fully drug- } \\
\text { susceptible TB in the } \\
\text { absence of significant } \\
\text { immune compromise, a } \\
\text { 3-drug intensive phase } \\
\text { regimen (excluding } \\
\text { ethambutol) and a } \\
\text { continuation phase of } 4 \\
\text { months can be considered } \\
\text { (total duration of therapy } \\
\text { of } 6 \text { months). }\end{array}$ & 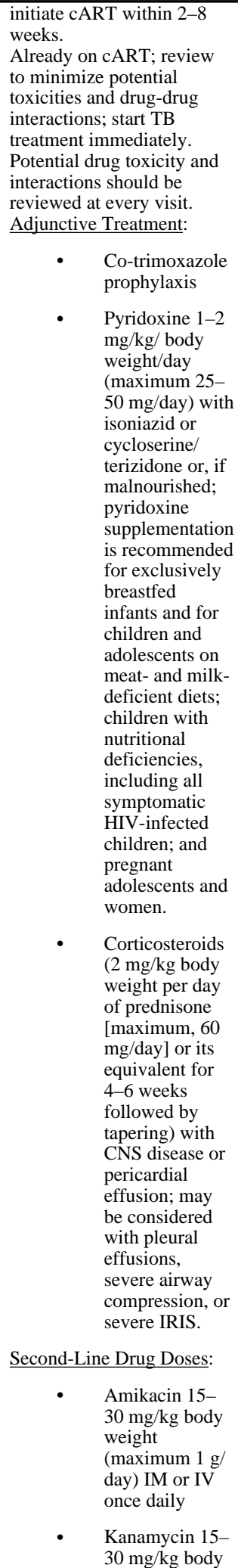 \\
\hline
\end{tabular}




\begin{tabular}{|c|c|c|c|}
\hline Indication & First Choice & Alternative & Comments/Special Issues \\
\hline & $\begin{array}{l}\text { child's isolate is not } \\
\text { available); consult an } \\
\text { expert. }\end{array}$ & & $\begin{array}{l}\text { weight } \\
\text { (maximum } 1 \mathrm{~g} / \\
\text { day) IM or IV } \\
\text { once daily } \\
\text { Capreomycin } \\
15-30 \mathrm{mg} / \mathrm{kg} \\
\text { body weight } \\
\text { (maximum } 1 \mathrm{~g} / \\
\text { day) IM once } \\
\text { daily } \\
\text { Ofloxacin } 15- \\
\text { 20 mg/kg body } \\
\text { weight } \\
\text { (maximum } 800 \\
\text { mg/day), or } \\
\text { levofloxacin } \\
7.5-10 \text { mg/kg } \\
\text { body weight } \\
\text { (maximum } 750 \\
\text { mg/day) by } \\
\text { mouth once } \\
\text { daily. Because } \\
\text { some } \\
\text { fluoroquinolone } \\
\text { s are approved } \\
\text { by the FDA for } \\
\text { use only in } \\
\text { people aged } 18 \\
\text { years and older, } \\
\text { their use in } \\
\text { younger } \\
\text { patients } \\
\text { necessitates } \\
\text { careful } \\
\text { assessment of } \\
\text { the potential } \\
\text { risks and } \\
\text { benefits. } \\
\text { Cycloserine/ } \\
\text { Terizidone } 10- \\
20 \text { mg/kg body } \\
\text { weight } \\
\text { (maximum } 1 \mathrm{~g} / \\
\text { day) by mouth } \\
\text { once daily } \\
\text { Ethionamide/ } \\
\text { prothionamide, } \\
15-20 \text { mg/kg } \\
\text { body weight } \\
\text { (maximum } 1 \mathrm{~g} / \\
\text { day) by mouth } \\
\text { in } 2-3 \text { divided } \\
\text { doses } \\
\text { Para- } \\
\text { aminosalicylic } \\
\text { acid } 200-300 \\
\text { mg/kg body } \\
\text { weight by } \\
\text { mouth divided } \\
\text { into } 3-4 \text { doses } \\
\text { per day } \\
\text { (maximum } 10 \\
\text { g/day). } \\
\text { Thiacetazone } \\
\text { can cause } \\
\text { severe reactions } \\
\text { in HIV-infected } \\
\text { children } \\
\text { including rash } \\
\text { and aplastic }\end{array}$ \\
\hline
\end{tabular}




\begin{tabular}{|c|c|c|c|}
\hline Indication & First Choice & Alternative & Comments/Special Issues \\
\hline & & & $\begin{array}{l}\text { anemia, and } \\
\text { should not be } \\
\text { used. }\end{array}$ \\
\hline Pneumocystis Pneumonia & $\begin{array}{l}\text { TMP-SMX } 3.75-5 \mathrm{mg} / \mathrm{kg} \text { body } \\
\text { weight } / \text { dose TMP (based on TMP } \\
\text { component) every } 6 \text { hours IV or } \\
\text { orally given for } 21 \text { days (followed } \\
\text { by secondary prophylaxis dosing) }\end{array}$ & $\begin{array}{l}\text { If TMP-SMX-Intolerant or Clinical } \\
\text { Treatment Failure After 5-7 Days of } \\
\text { TMP-SMX Therapy } \\
\text { Pentamidine: } \\
\text { - } \quad 4 \text { mg/kg body weight/ } \\
\text { dose IV/IM once daily is } \\
\text { the first choice alternative } \\
\text { regimen. Note: } \\
\text { Pentamidine can be } \\
\text { changed to atovaquone } \\
\text { after } 7-10 \text { days IV } \\
\text { therapy. }\end{array}$ & 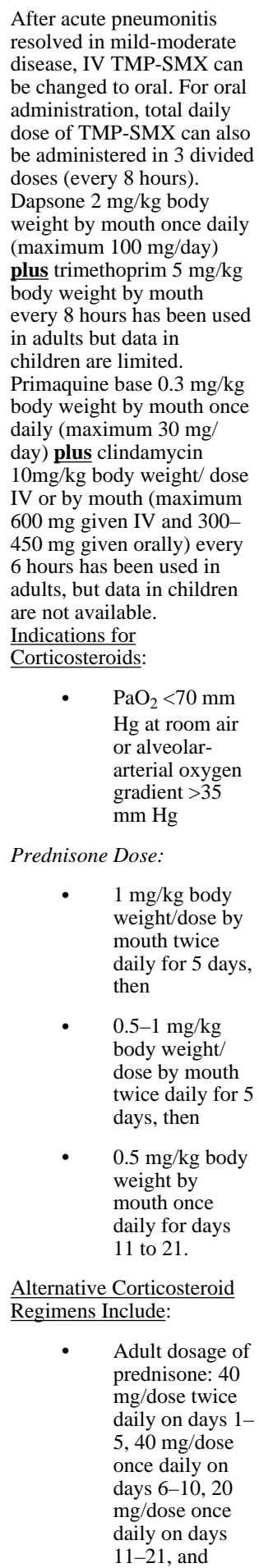 \\
\hline
\end{tabular}




\begin{tabular}{|c|c|c|c|}
\hline Indication & First Choice & Alternative & Comments/Special Issues \\
\hline & & & $\begin{array}{l}\text { Methylprednisol } \\
\text { one IV } 1 \mathrm{mg} / \mathrm{kg} / \\
\text { dose every 6 } \\
\text { hours on days } \\
1-7,1 \mathrm{mg} / \mathrm{kg} / \\
\text { dose twice daily } \\
\text { on days } 8-9,0.5 \\
\text { mg/kg/dose } \\
\text { twice daily on } \\
\text { days } 10 \text { and } 11, \\
\text { and } 1 \mathrm{mg} / \mathrm{kg} / \\
\text { dose once daily } \\
\text { on days } 12-16 .\end{array}$ \\
\hline Syphilis & 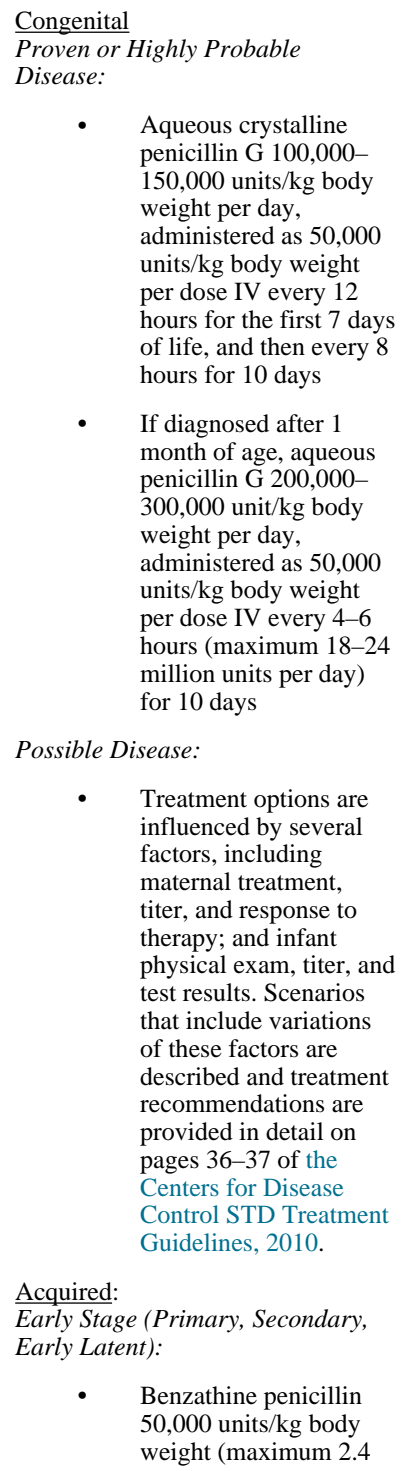 & $\begin{array}{l}\text { Congenital } \\
\text { Proven or Highly Probable Disease } \\
\text { (Less Desirable if CNS Involvement): } \\
\text { - } \quad \text { Procaine penicillin G } \\
\text { 50,000 units/kg body } \\
\text { weight IM once daily for } \\
10 \text { days } \\
\text { Possible Disease: } \\
\text { Treatment options are } \\
\text { influenced by several } \\
\text { factors, including } \\
\text { maternal treatment, titer, } \\
\text { and response to therapy; } \\
\text { and infant physical exam, } \\
\text { titer, and test results. } \\
\text { Scenarios that include } \\
\text { variations of these factors } \\
\text { are described and } \\
\text { treatment } \\
\text { recommendations are } \\
\text { provided in detail on } \\
\text { pages 36-37 of the } \\
\text { Centers for Disease } \\
\text { Control STD Treatment } \\
\text { Guidelines, 2010. }\end{array}$ & $\begin{array}{l}\text { For treatment of congenital } \\
\text { syphilis, repeat the entire } \\
\text { course of treatment if }>1 \\
\text { day of treatment is missed. } \\
\text { Examinations and serologic } \\
\text { testing for children with } \\
\text { congenital syphilis should } \\
\text { occur every } 2-3 \text { months } \\
\text { until the test becomes non- } \\
\text { reactive or there is a } \\
\text { fourfold decrease in titer. } \\
\text { Children with increasing } \\
\text { titers or persistently positive } \\
\text { titers (even if low levels) at } \\
\text { ages } 6-12 \text { months should be } \\
\text { evaluated and considered } \\
\text { for re-treatment. } \\
\text { In the setting of maternal } \\
\text { and possible infant HIV } \\
\text { infection, the more } \\
\text { conservative choices among } \\
\text { scenario-specific treatment } \\
\text { options may be preferable. } \\
\text { Children and adolescents } \\
\text { with acquired syphilis } \\
\text { should have clinical and } \\
\text { serologic response } \\
\text { monitored at } 3,6,9,12 \text {, and } \\
24 \text { months after therapy. }\end{array}$ \\
\hline
\end{tabular}




\begin{tabular}{|c|c|c|c|}
\hline Indication & First Choice & Alternative & Comments/Special Issues \\
\hline & $\begin{array}{l}\text { million units) IM for 1 } \\
\text { dose } \\
\text { Late Latent: } \\
\text { • } \quad \text { Benzathine penicillin } \\
\text { 50,000 units/kg body } \\
\text { weight (maximum 2.4 } \\
\text { million units) IM once } \\
\text { weekly for 3 doses } \\
\text { Neurosyphilis (Including Ocular): } \\
\text { • } \quad \text { Aqueous penicillin G } \\
\text { 200,000- 300,000 } \\
\text { units/kg body weight } \\
\text { per day administered as } \\
\text { 50,000 units/kg body } \\
\text { weight per dose IV } \\
\text { every 4-6 hours } \\
\text { (maximum 18-24 } \\
\text { million units per day) } \\
\text { for 10-14 days }\end{array}$ & & \\
\hline Toxoplasmosis & 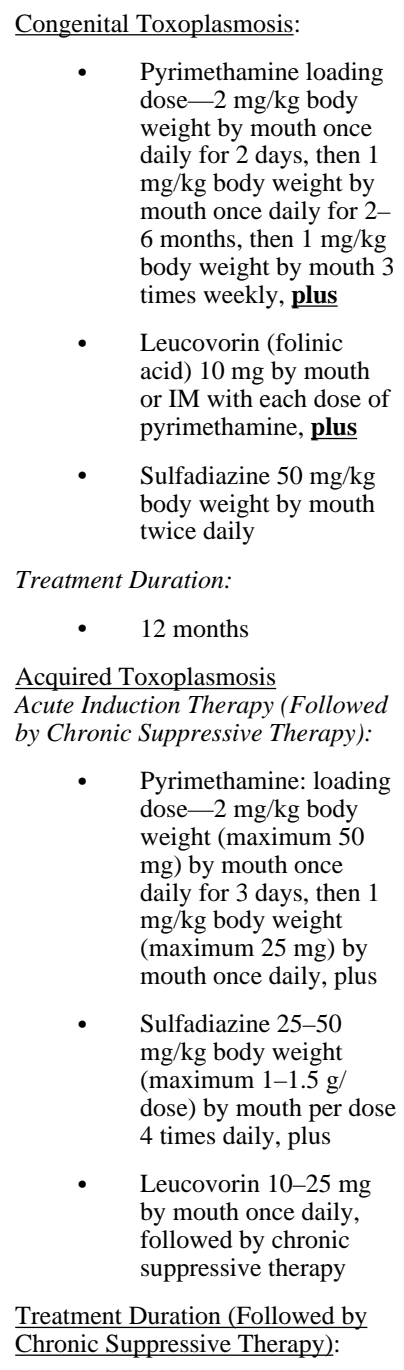 & $\begin{array}{cl}\text { For Sulfonamide-Intolerant Patients: } \\
\\
\text { Clindamycin 5-7.5 } \\
\text { mg/kg body weight } \\
\text { (maximum 600 mg/dose) } \\
\text { by mouth or IV per dose } \\
\text { given } 4 \text { times a day can } \\
\text { be substituted for } \\
\text { sulfadiazine combined } \\
\text { with pyrimethamine and } \\
\text { leucovorin }\end{array}$ & $\begin{array}{l}\text { Congenital Toxoplasmosis: } \\
\text { For infants born } \\
\text { to mothers with } \\
\text { symptomatic } \\
\text { Toxoplasma } \\
\text { infection during } \\
\text { pregnancy, } \\
\text { empiric therapy } \\
\text { of the newborn } \\
\text { should be } \\
\text { strongly } \\
\text { considered } \\
\text { irrespective of } \\
\text { the mother's } \\
\text { treatment } \\
\text { during } \\
\text { pregnancy. } \\
\text { Toxoplasmosis: } \\
\text { Pyrimethamine } \\
\text { use requires } \\
\text { CBC } \\
\text { monitoring at } \\
\text { least weekly } \\
\text { while on daily } \\
\text { dosing and at } \\
\text { least monthly } \\
\text { while on less } \\
\text { than daily } \\
\text { dosing. } \\
\text { TMP-SMX- } \\
\text { TMP } 5 \text { mg/kg } \\
\text { body weight } \\
\text { plus SMX } 25 \\
\text { mg/kg body } \\
\text { weight per dose } \\
\text { IV or by mouth } \\
\text { given twice } \\
\text { daily has been } \\
\text { used as an } \\
\text { alternative to } \\
\text { pyrimethamine- } \\
\text { sulfadiazine in } \\
\text { adults, but has } \\
\text { not been studied } \\
\text { in children. } \\
\text { - }\end{array}$ \\
\hline
\end{tabular}




\begin{tabular}{|c|c|c|c|}
\hline Indication & First Choice & Alternative & Comments/Special Issues \\
\hline & $\begin{array}{ll}6 \text { weeks (longer } \\
\text { duration if clinical or } \\
\text { radiologic disease is } \\
\text { extensive or response in } \\
\text { incomplete at } 6 \text { weeks) }\end{array}$ & & $\begin{array}{l}\text { Atovaquone } \\
\text { (for adults, } 1.5 \\
\text { g by mouth } \\
\text { twice daily- } \\
\text { double the } \\
\text { prophylaxis } \\
\text { dose) in } \\
\text { regimens } \\
\text { combined with } \\
\text { pyrimethamine/ } \\
\text { leucovorin, with } \\
\text { sulfadiazine } \\
\text { alone, or as a } \\
\text { single agent in } \\
\text { patients } \\
\text { intolerant to } \\
\text { both } \\
\text { pyrimethamine } \\
\text { and } \\
\text { sulfadiazine, } \\
\text { has been used in } \\
\text { adults, but these } \\
\text { regimens have } \\
\text { not been studied } \\
\text { in children. } \\
\text { Azithromycin } \\
\text { (for adults, } \\
\text { 900-1,200 mg/ } \\
\text { day, } \\
\text { corresponding } \\
\text { to } 20 \text { mg/kg/day } \\
\text { in children) has } \\
\text { also been used } \\
\text { in adults } \\
\text { combined with } \\
\text { pyrimethamine- } \\
\text { sulfadiazine, but } \\
\text { has not been } \\
\text { studied in } \\
\text { children. } \\
\text { Corticosteroids } \\
\text { (e.g.. } \\
\text { prednisone, } \\
\text { dexamethasone) } \\
\text { have been used } \\
\text { in children with } \\
\text { CNS disease } \\
\text { when CSF } \\
\text { protein is very } \\
\text { elevated } \\
\text { (>1,000 mg/dL) } \\
\text { or there are } \\
\text { focal lesions } \\
\text { with significant } \\
\text { mass effects, } \\
\text { with } \\
\text { discontinuation } \\
\text { as soon as } \\
\text { clinically } \\
\text { feasible. } \\
\text { Anticonvulsants } \\
\text { should be } \\
\text { administered to } \\
\text { patients with a } \\
\text { history of } \\
\text { seizures and } \\
\text { continued } \\
\text { through the } \\
\text { acute treatment; } \\
\text { but should not } \\
\text { be used } \\
\text { - }\end{array}$ \\
\hline
\end{tabular}




\begin{tabular}{|c|c|c|c|}
\hline Indication & First Choice & Alternative & Comments/Special Issues \\
\hline & & & $\begin{array}{l}\text { prophylactically } \\
\text {. }\end{array}$ \\
\hline Varicella-Zoster Virus (VZV) & 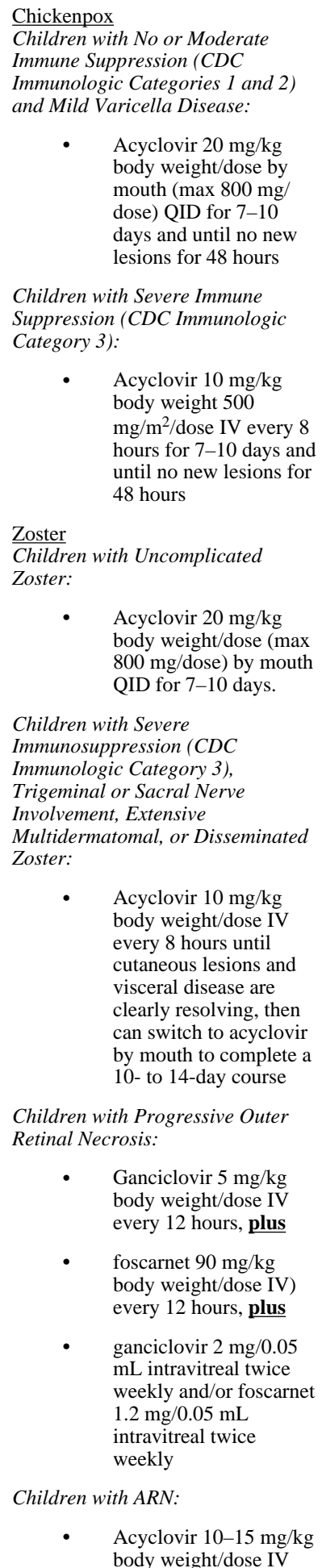 & 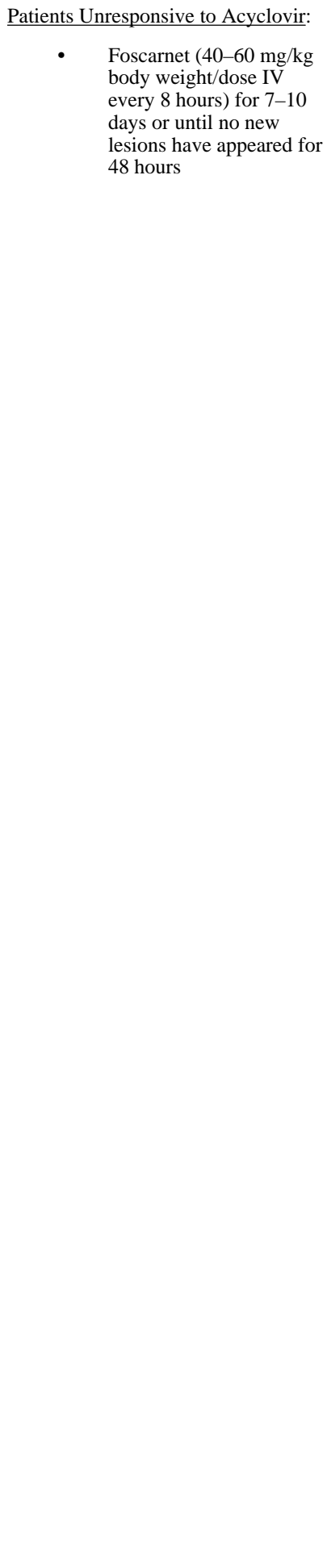 & $\begin{array}{l}\text { In children } \geq 1 \text { year of age, } \\
\text { some experts base IV } \\
\text { acyclovir dosing on body } \\
\text { surface area ( } 500 \mathrm{mg} / \mathrm{m}^{2} \\
\text { body surface area/dose IV } \\
\text { every } 8 \text { hours) instead of } \\
\text { body weight. } \\
\text { Valacyclovir is approved } \\
\text { for use in adults and } \\
\text { adolescents with zoster at } 1 \\
\text { g/dose by mouth TID for } 7 \\
\text { days; the same dose has } \\
\text { been used for varicella } \\
\text { infections. Data on dosing } \\
\text { in children are limited and } \\
\text { there is no pediatric } \\
\text { preparation, although } 500 \\
\text { mg capsules can be } \\
\text { extemporaneously } \\
\text { compounded to make a } \\
\text { suspension to administer } 20 \\
\text { mg/kg body weight/dose } \\
\text { (maximum dose } 1 \text { g) given } \\
\text { TID (see prescribing } \\
\text { information). } \\
\text { Famciclovir is approved for } \\
\text { use in adults and } \\
\text { adolescents with zoster at } \\
500 \text { mg/dose by mouth TID } \\
\text { for } 7 \text { days; the same dose } \\
\text { has been used for varicella } \\
\text { infections. There is no } \\
\text { pediatric preparation and } \\
\text { data on dosing in children } \\
\text { are limited; can be used by } \\
\text { adolescents able to receive } \\
\text { adult dosing. } \\
\text { Involvement of an } \\
\text { ophthalmologist with } \\
\text { experience in managing } \\
\text { herpes zoster ophthalmicus } \\
\text { and its complications in } \\
\text { children is strongly } \\
\text { recommended when ocular } \\
\text { involvement is evident. } \\
\text { Optimal management of } \\
\text { PORN has not been } \\
\text { defined. }\end{array}$ \\
\hline
\end{tabular}




\begin{tabular}{|c|c|c|c|}
\hline Indication & First Choice & Alternative & Comments/Special Issues \\
\hline & $\begin{array}{l}\text { every } 8 \text { hours daily for } \\
10-14 \text { days, followed } \\
\text { by } \\
\text { Oral valacyclovir } 1 \mathrm{~g} / \text { dose TID for } \\
\text { 4-6 weeks (for children old enough } \\
\text { to receive adult dose). Alternative } \\
\text { oral acyclovir dose: } 20 \mathrm{mg} / \mathrm{kg} \text { body } \\
\text { weight/dose QID for } 4-6 \text { weeks }\end{array}$ & & \\
\hline
\end{tabular}

Key to Acronyms: LIP = lymphocytic interstitial pneumonia; $\mathrm{PCP}=$ pneumocystis jirovecii pneumonia; $\mathrm{IV}=$ intravenous; $\mathrm{PK}=$ pharmacokinetic; $\mathrm{CSF}=$ cerebrospinal fluid; $\mathrm{CNS}=$ central nervous system; ICP = intracranial pressure; $\mathrm{cART}=$ combination antiretroviral therapy; $\mathrm{ART}=$ antiretroviral therapy; $\mathrm{BSA}=$ body surface area; $\mathrm{CrCl}=$ (estimated) creatinine clearance; $\mathrm{HBV}=$ hepatitis $\mathrm{B}$ virus; $\mathrm{SQ}=$ subcutaneous; $\mathrm{HCV}=$ hepatitis $\mathrm{C}$ virus; IFN- $\mathrm{a}=$ interferon-alfa; $\mathrm{BID}=$ twice daily; $\mathrm{TID}=$ three times daily; $\mathrm{QID}=$ four times daily; $\mathrm{CNS}=$ central nervous system; CSF $=$ cerebrospinal fluid; $\mathrm{HSV}=$ herpes simplex virus; $\mathrm{PCR}=$ polymerase chain reaction; $\mathrm{BCA}=$ bichloroacetic acid; $\mathrm{IFN}=$ interferon; $\mathrm{TCA}=$ trichloroacetic acid; TMP-SMX = trimethoprim-sulfamethoxazole; DOT = directly observed therapy; IGRA = interferon-gamma release assay; IM $=$ intramuscular; $\mathrm{TB}=$ tuberculosis; IRIS $=$ immune reconstitution inflammatory syndrome; $\mathrm{TE}=$ toxoplasmic encephalitis 
Table 4

Common Drugs Used for Treatment of Opportunistic Infections in HIV-Infected Children: Preparations and Major Toxicities (Last updated November 6, 2013; last reviewed November 6, 2013)

\begin{tabular}{|c|c|c|c|c|}
\hline \multirow[t]{2}{*}{ Drug } & Preparations & \multicolumn{2}{|l|}{ Major Toxicities $a$} & \multirow[t]{2}{*}{ Special Instructions } \\
\hline & & $\begin{array}{l}\text { Indicating Need for Medical } \\
\text { Attention }\end{array}$ & $\begin{array}{l}\text { Indicating Need for } \\
\text { Medical Attention if } \\
\text { Persistent or Bothersome }\end{array}$ & \\
\hline Acyclovir (Zovirax) & 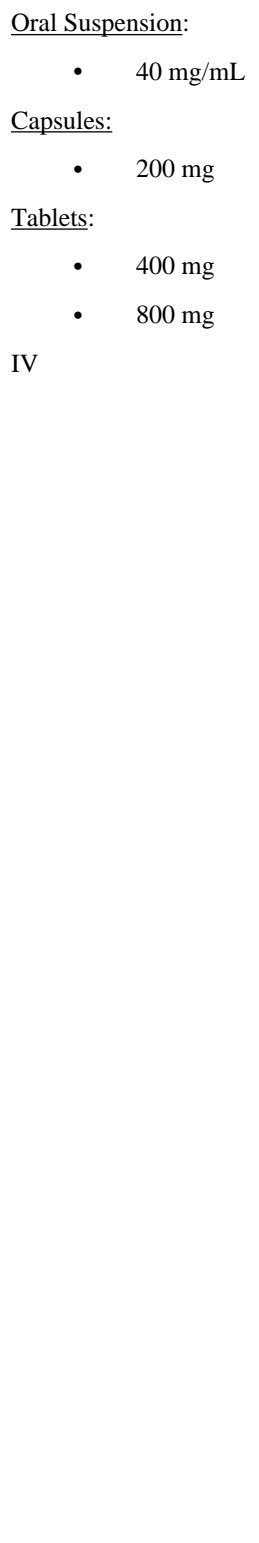 & 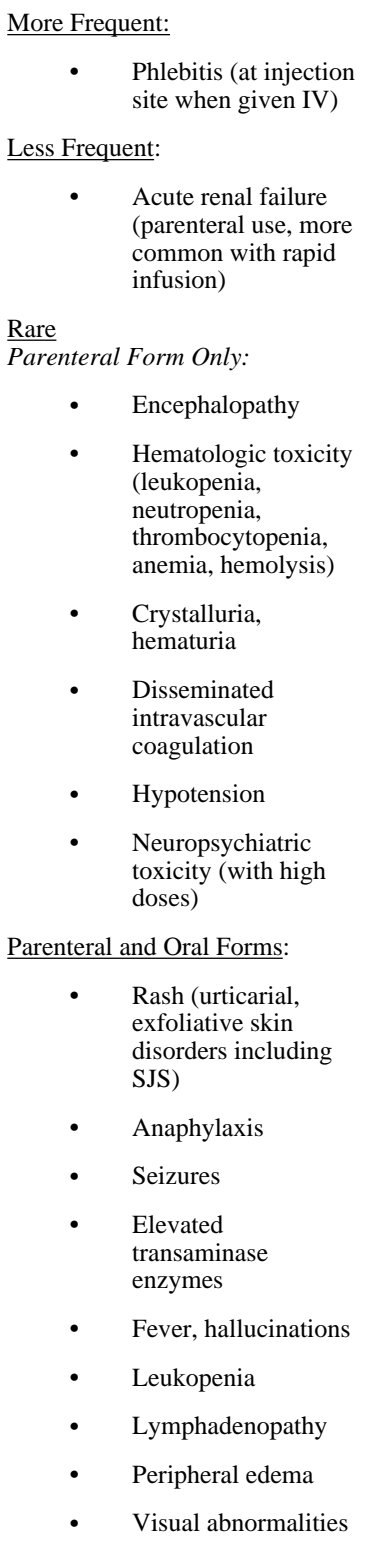 & 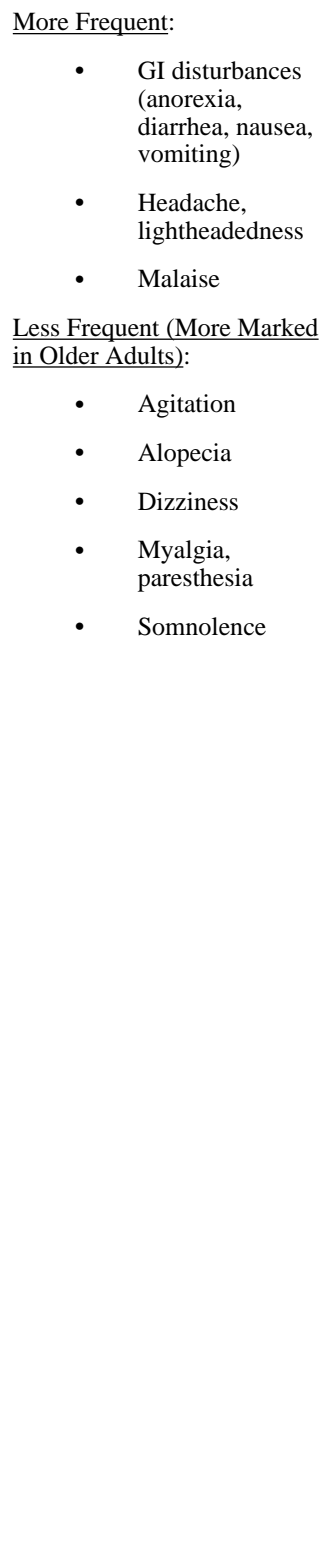 & $\begin{array}{l}\text { Requires dose adjustment } \\
\text { in patients with renal } \\
\text { impairment. } \\
\text { Avoid other nephrotoxic } \\
\text { drugs. } \\
\text { Administer IV preparation } \\
\text { by slow IV infusion over } \\
\text { at least } 1 \text { hour at a final } \\
\text { concentration not to } \\
\text { exceed } 7 \mathrm{mg} / \mathrm{mL} \text {. This is } \\
\text { to avoid renal tubular } \\
\text { damage related to } \\
\text { crystalluria; must be } \\
\text { accompanied by adequate } \\
\text { hydration. }\end{array}$ \\
\hline Albendazole (Albenza) & $\begin{array}{c}\text { Tablets: } \\
\text { • } \\
\quad 200 \mathrm{mg}\end{array}$ & $\begin{array}{l}\text { More Frequent: } \\
\quad \begin{array}{l}\text { Abnormal liver } \\
\text { function tests (LFTs) }\end{array} \\
\text { Less Frequent: }\end{array}$ & \begin{tabular}{ll}
\multicolumn{2}{l}{ Less frequent: } \\
$\qquad \begin{array}{l}\text { CNS effects } \\
\text { (dizziness, } \\
\text { headache) }\end{array}$
\end{tabular} & $\begin{array}{l}\text { Should be given with } \\
\text { food. } \\
\text { May crush or chew tablets } \\
\text { and give with water. } \\
\text { Monitor CBC and LFTs } \\
\text { prior to each cycle. }\end{array}$ \\
\hline
\end{tabular}




\begin{tabular}{|c|c|c|c|c|}
\hline \multirow[t]{2}{*}{ Drug } & \multirow[t]{2}{*}{ Preparations } & \multicolumn{2}{|l|}{ Major Toxicities ${ }^{a}$} & \multirow[t]{2}{*}{ Special Instructions } \\
\hline & & $\begin{array}{l}\text { Indicating Need for Medical } \\
\text { Attention }\end{array}$ & $\begin{array}{l}\text { Indicating Need for } \\
\text { Medical Attention if } \\
\text { Persistent or Bothersome }\end{array}$ & \\
\hline & & $\begin{array}{cl}\text { - } & \begin{array}{l}\text { Hypersensitivity } \\
\text { (rash, pruritus) }\end{array} \\
\text { - } & \begin{array}{l}\text { Neutropenia (with } \\
\text { high doses) }\end{array} \\
\text { Rare: } & \\
\text { - } & \text { Pancytopenia }\end{array}$ & $\begin{array}{ll} & \begin{array}{l}\text { GI disturbances } \\
\text { (abdominal pain, } \\
\text { diarrhea, nausea, } \\
\text { vomiting) }\end{array} \\
\text { Rare: } & \\
\text { - } & \text { Alopecia }\end{array}$ & \\
\hline Amikacin & IV & $\begin{array}{cl}\text { More Frequent: } \\
\text { - } & \text { Nephrotoxicity } \\
& \begin{array}{l}\text { Neurotoxicity } \\
\text { (including muscle } \\
\text { twitching, seizures) }\end{array} \\
\text { - } & \begin{array}{l}\text { Ototoxicity, both } \\
\text { auditory and } \\
\text { vestibular }\end{array} \\
\text { Less Frequent: } & \begin{array}{l}\text { Hypersensitivity } \\
\text { (skin rash, redness, or } \\
\text { swelling) }\end{array} \\
\text { Rare: } & \begin{array}{l}\text { Neuromuscular } \\
\text { blockade }\end{array}\end{array}$ & N/A & $\begin{array}{l}\text { Must be infused over } 30 \text { to } \\
60 \text { minutes to avoid } \\
\text { neuromuscular blockade. } \\
\text { Requires dose adjustment } \\
\text { in patients with impaired } \\
\text { renal function. } \\
\text { Should monitor renal } \\
\text { function and hearing } \\
\text { periodically (e.g., } \\
\text { monthly) in children on } \\
\text { prolonged therapy. } \\
\text { Therapeutic drug } \\
\text { monitoring (TDM). } \\
\text { indicated }\end{array}$ \\
\hline $\begin{array}{l}\text { Amphotericin B Deoxycholate } \\
\text { (Fungizone) }\end{array}$ & IV & 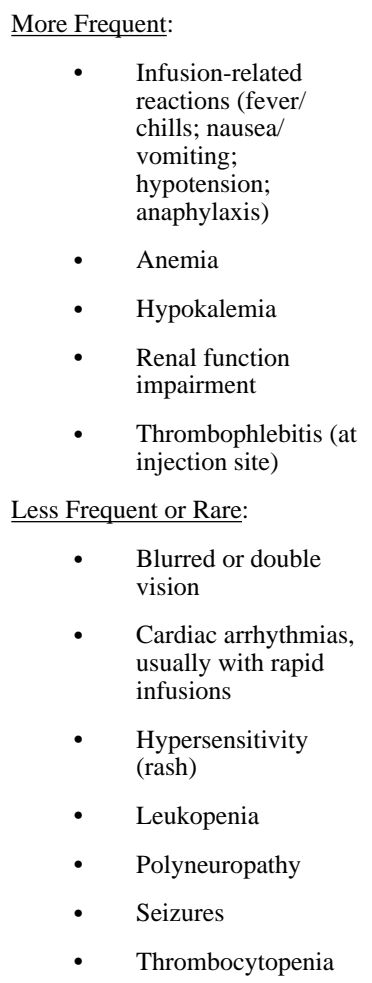 & 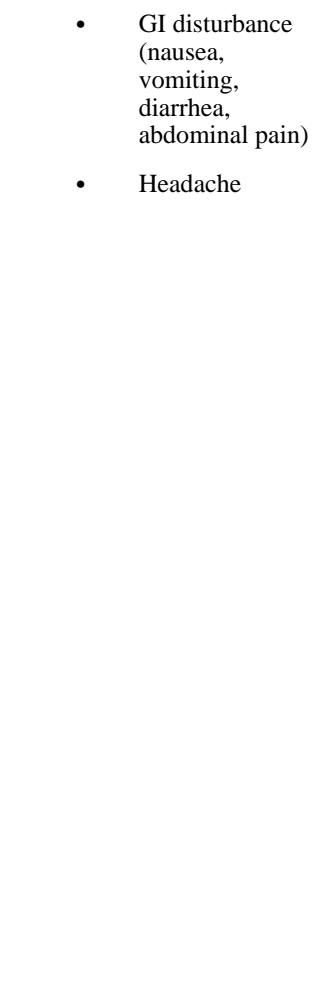 & $\begin{array}{l}\text { Monitor BUN, Cr, CBC, } \\
\text { electrolytes, LFTs. } \\
\text { Infuse over } 1 \text { to } 2 \text { hours; } \\
\text { in patients with azotemia, } \\
\text { hyperkalemia, or getting } \\
\text { doses }>1 \mathrm{mg} / \mathrm{kg} \text {, infuse } \\
\text { over } 3 \text { to } 6 \text { hours. } \\
\text { Requires dose reduction in } \\
\text { patients with impaired } \\
\text { renal function. } \\
\text { Avoid other nephrotoxic } \\
\text { drugs, when possible, } \\
\text { because nephrotoxicity is } \\
\text { exacerbated with } \\
\text { concomitant use of other } \\
\text { nephrotoxic drugs; } \\
\text { permanent nephrotoxicity } \\
\text { is related to cumulative } \\
\text { dose. } \\
\text { Nephrotoxicity may be } \\
\text { ameliorated by hydration } \\
\text { with } 0.9 \% \text { saline IV over } \\
30 \text { minutes prior to the } \\
\text { amphotericin B infusion. } \\
\text { Infusion-related reactions } \\
\text { less frequent in children } \\
\text { than adults; the onset is } \\
\text { usually } 1 \text { to } 3 \text { hours after } \\
\text { infusion, duration }<1 \text { hour; } \\
\text { frequency decreases over } \\
\text { time. } \\
\text { Pre-treatment with } \\
\text { acetaminophen and/or } \\
\text { diphenhydramine may } \\
\text { alleviate febrile reactions. }\end{array}$ \\
\hline
\end{tabular}




\begin{tabular}{|c|c|c|c|c|}
\hline \multirow[t]{2}{*}{ Drug } & Preparations & \multicolumn{2}{|l|}{ Major Toxicities ${ }^{a}$} & \multirow[t]{2}{*}{ Special Instructions } \\
\hline & & $\begin{array}{l}\text { Indicating Need for Medical } \\
\text { Attention }\end{array}$ & $\begin{array}{l}\text { Indicating Need for } \\
\text { Medical Attention if } \\
\text { Persistent or Bothersome }\end{array}$ & \\
\hline $\begin{array}{l}\text { Amphotericin B Lipid Complex } \\
\text { (Abelcet) }\end{array}$ & IV & $\begin{array}{ll}\text { More Frequent: } \\
\text { - } & \begin{array}{l}\text { Infusion-related } \\
\text { reactions (fever/ } \\
\text { chills, nausea/ } \\
\text { vomiting; headache, } \\
\text { nausea and vomiting) }\end{array} \\
\text { Less Frequent: } \\
\text { - } & \text { Anemia } \\
\text { - } & \text { Leukopenia } \\
\text { - } & \text { Respiratory distress } \\
\text { - } & \text { Thrombocytopenia } \\
\text { - } & \begin{array}{l}\text { Renal function } \\
\text { impairment }\end{array}\end{array}$ & $\begin{array}{l}\text { GI disturbance } \\
\text { (loss of appetite, } \\
\text { nausea, vomiting, } \\
\text { diarrhea, } \\
\text { abdominal pain) }\end{array}$ & $\begin{array}{l}\text { Monitor BUN, Cr, CBC, } \\
\text { electrolytes, and LFTs. } \\
\text { Infuse diluted solution at } \\
\text { rate of } 2.5 \mathrm{mg} / \mathrm{kg} / \text { hour. } \\
\text { In-line filters should not } \\
\text { be used. } \\
\text { Use with caution with } \\
\text { other drugs that are bone } \\
\text { marrow suppressants or } \\
\text { that are nephrotoxic; renal } \\
\text { toxicity is dose-dependent, } \\
\text { but less renal toxicity than } \\
\text { seen with conventional } \\
\text { amphotericin B. } \\
\text { Consider dose reduction in } \\
\text { patients with impaired } \\
\text { renal function. }\end{array}$ \\
\hline $\begin{array}{l}\text { Amphotericin B Liposome } \\
\text { (AmBisome) }\end{array}$ & IV & \begin{tabular}{ll}
\multicolumn{2}{l}{ More Frequent: } \\
• & Fever, chills \\
- & Hypokalemia \\
Less Frequent: \\
- & Back pain \\
- & Chest pain \\
- & Dark urine \\
- & Dyspnea \\
- & Infusion-related \\
& reaction (fever/chills, \\
- & headache) \\
- & Jaundice \\
& Renal function \\
impairment
\end{tabular} & 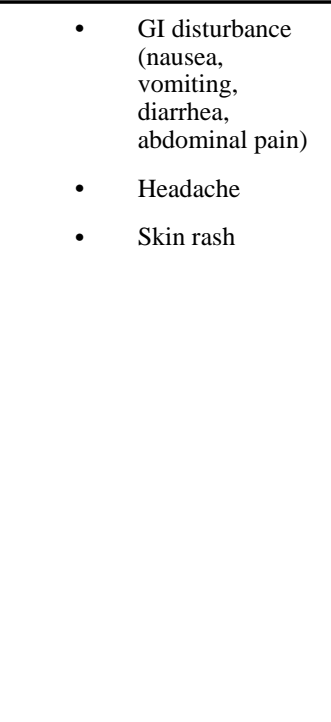 & $\begin{array}{l}\text { Monitor BUN, Cr, CBC, } \\
\text { electrolytes, and LFTs. } \\
\text { Infuse over } 2 \text { hours. } \\
\text { Consider dose reduction in } \\
\text { patients with impaired } \\
\text { renal function. }\end{array}$ \\
\hline Artesunate & $\begin{array}{ll}\text { IV: } & \\
& \\
& \text { Only available } \\
& \text { from CDC } \\
& \text { Malaria Hotline; } \\
& \text { telephone: (770) } \\
& 488-7788\end{array}$ & $\begin{aligned} \text { Rare: } & \\
\text { - } & \text { Anaphylactic reaction } \\
\text { - } & \text { Neutropenia } \\
\text { - } & \text { Bradycardia }\end{aligned}$ & $\begin{array}{ll}\text { - } & \begin{array}{l}\text { GI disturbance } \\
\text { (nausea, } \\
\text { vomiting) }\end{array} \\
\text { - } & \text { Headache } \\
\text { - } & \text { Skin rash }\end{array}$ & $\begin{array}{l}\text { Monitor CBC, LFTs, and } \\
\text { electrolytes. } \\
\sim 40 \% \text { less mortality than } \\
\text { with quinidine use in } \\
\text { severe malaria } \\
50 \% \text { lower incidence of } \\
\text { hypoglycemia than } \\
\text { quinidine }\end{array}$ \\
\hline Atovaquone (Mepron) & $\begin{array}{l}\text { Oral Suspension: } \\
\qquad \quad 150 \mathrm{mg} / \mathrm{mL}\end{array}$ & $\begin{array}{cl}\text { Frequent: } & \\
\text { • } & \text { Fever } \\
\text { • } & \text { Skin rash }\end{array}$ & $\begin{aligned} \text { Frequent: } & \\
\text { - } & \begin{array}{l}\text { GI disturbances } \\
\text { (nausea, } \\
\text { vomiting, } \\
\text { diarrhea) }\end{array} \\
\text { - } & \text { Headache } \\
\text { - } & \text { Cough } \\
\text { - } & \text { Insomnia }\end{aligned}$ & $\begin{array}{l}\text { Should be administered } \\
\text { with a meal to enhance } \\
\text { absorption; bioavailability } \\
\text { increases 3-fold when } \\
\text { administered with high-fat } \\
\text { meal. }\end{array}$ \\
\hline
\end{tabular}




\begin{tabular}{|c|c|c|c|c|}
\hline \multirow[t]{2}{*}{ Drug } & Preparations & \multicolumn{2}{|l|}{ Major Toxicities ${ }^{a}$} & \multirow[t]{2}{*}{ Special Instructions } \\
\hline & & $\begin{array}{l}\text { Indicating Need for Medical } \\
\text { Attention }\end{array}$ & $\begin{array}{l}\text { Indicating Need for } \\
\text { Medical Attention if } \\
\text { Persistent or Bothersome }\end{array}$ & \\
\hline Atovaquone/ Proguanil (Malarone) & $\begin{array}{cl}\text { Tablets: } & \\
. & \text { Pediatric tablets; } \\
& 62.5 \mathrm{mg} / 25 \mathrm{mg} \\
. & \text { Adult tablets; } \\
& 250 \mathrm{mg} / 100 \mathrm{mg}\end{array}$ & 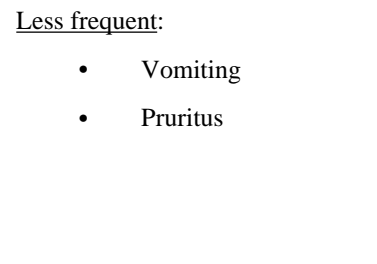 & N/A & $\begin{array}{l}\text { Pediatric tablets are } \\
\text { available to make dosing } \\
\text { easier. } \\
\text { Side effects requiring } \\
\text { discontinuation in } ~ 1 \%- \\
2 \% \text { of patients } \\
\text { Not recommended for } \\
\text { prophylaxis in patients } \\
\text { with } \mathrm{CrCl}<30 \mathrm{~mL} / \mathrm{min} \text {. }\end{array}$ \\
\hline Azithromycin (Zithromax) & \begin{tabular}{cc}
\multicolumn{2}{l}{ Oral Suspension: } \\
• & $20 \mathrm{mg} / \mathrm{mL}$ \\
• & $40 \mathrm{mg} / \mathrm{mL}$ \\
Tablets: & \\
• & $250 \mathrm{mg}$ \\
• & $500 \mathrm{mg}$ \\
• & $600 \mathrm{mg}$ \\
IV &
\end{tabular} & $\begin{array}{ll}\text { More Frequent: } \\
\text { - } & \begin{array}{l}\text { Thrombophlebitis } \\
\text { (IV form) }\end{array} \\
\text { Rare: } & \\
\text { - } & \begin{array}{l}\text { Acute interstitial } \\
\text { nephritis }\end{array} \\
\text { - } & \begin{array}{l}\text { Allergic reactions/ } \\
\text { anaphylaxis } \\
\text { (dyspnea, hives, rash) }\end{array} \\
\text { - } & \begin{array}{l}\text { Pseudomembranous } \\
\text { colitis }\end{array}\end{array}$ & $\begin{array}{ll}\text { - } & \begin{array}{l}\text { GI disturbances } \\
\text { (abdominal } \\
\text { discomfort or } \\
\text { pain, diarrhea, } \\
\text { nausea, vomiting) }\end{array} \\
\text { - } & \text { Dizziness, } \\
\text { headache }\end{array}$ & $\begin{array}{l}\text { Administer } 1 \text { hour before } \\
\text { or } 2 \text { hours after a meal; do } \\
\text { not administer with } \\
\text { aluminum-and } \\
\text { magnesium-containing } \\
\text { antacids. } \\
\text { IV should be infused at } \\
\text { concentration of } 1 \mathrm{mg} / \mathrm{mL} \\
\text { over a 3-hour period, or } 2 \\
\mathrm{mg} / \mathrm{mL} \text { over a } 1 \text {-hour } \\
\text { period; should not be } \\
\text { administered as a bolus. } \\
\text { Use with caution in } \\
\text { patients with hepatic } \\
\text { function impairment; } \\
\text { biliary excretion is the } \\
\text { main route of elimination. } \\
\text { Potential drug interactions. }\end{array}$ \\
\hline Capreomycin (Capastat) & $\mathrm{IM}$ & \begin{tabular}{ll}
\multicolumn{2}{l}{ More Frequent: } \\
$\qquad \quad$ & Nephrotoxicity \\
Less Frequent: & $\begin{array}{l}\text { Hypersensitivity } \\
\text { (rash, fever) }\end{array}$ \\
- & Hypokalemia \\
- & $\begin{array}{l}\text { Neuromuscular } \\
\text { blockade }\end{array}$ \\
- & $\begin{array}{l}\text { Ototoxicity, both } \\
\text { auditory and } \\
\text { vestibular }\end{array}$ \\
- & $\begin{array}{l}\text { Injection site pain, } \\
\text { sterile abscess }\end{array}$
\end{tabular} & N/A & $\begin{array}{l}\text { Requires dose adjustment } \\
\text { in patients with impaired } \\
\text { renal function. } \\
\text { Administer only by deep } \\
\text { IM injection into large } \\
\text { muscle mass (superficial } \\
\text { injections may result in } \\
\text { sterile abscess). } \\
\text { Should monitor renal } \\
\text { function and hearing } \\
\text { periodically (e.g., } \\
\text { monthly) in children on } \\
\text { prolonged therapy. } \\
\text { Monitor LFTs and } \\
\text { electrolytes. }\end{array}$ \\
\hline Caspofungin (Cancidas) & IV & $\begin{array}{ll}\text { More Frequent: } \\
\text { - } & \begin{array}{l}\text { Histamine-mediated } \\
\text { symptoms (fever, } \\
\text { facial swelling, } \\
\text { pruritus, } \\
\text { bronchospasm) }\end{array} \\
\underline{\text { Rare: }} & \\
\text { - } & \text { Hypokalemia } \\
\text { - } & \text { Anaphylactic reaction }\end{array}$ & 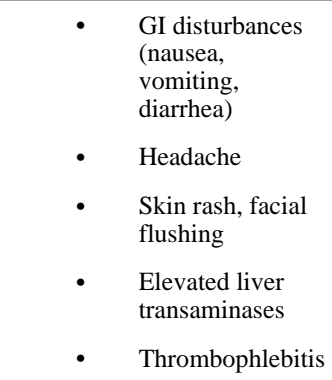 & $\begin{array}{l}\text { Requires dose adjustment } \\
\text { in moderate-to-severe } \\
\text { hepatic insufficiency. } \\
\text { IV infusion over } 1 \text { hour in } \\
\text { normal saline (do not use } \\
\text { diluents containing } \\
\text { dextrose) }\end{array}$ \\
\hline Chloroquine Phosphate (Aralen) & $\begin{array}{rr}\text { Tablets: } & \\
\text { • } & 500 \mathrm{mg} \\
\text { • } & 250 \mathrm{mg}\end{array}$ & $\begin{array}{ll}\text { More Frequent: } \\
& \begin{array}{l}\text { Pruritus: Common in } \\
\text { individuals of black } \\
\text { race }(25 \%-33 \%)\end{array}\end{array}$ & $\begin{array}{ll}- & \begin{array}{l}\text { Psoriasis } \\
\text { exacerbations }\end{array} \\
\text { - } & \begin{array}{l}\text { GI disturbances } \\
\text { (nausea, }\end{array}\end{array}$ & $\begin{array}{l}\text { Store in child-proof } \\
\text { containers and protect } \\
\text { from light. } \\
\text { Can be toxic in overdose. }\end{array}$ \\
\hline
\end{tabular}




\begin{tabular}{|c|c|c|c|c|}
\hline \multirow[t]{2}{*}{ Drug } & Preparations & \multicolumn{2}{|l|}{ Major Toxicities ${ }^{a}$} & \multirow[t]{2}{*}{ Special Instructions } \\
\hline & & $\begin{array}{l}\text { Indicating Need for Medical } \\
\text { Attention }\end{array}$ & $\begin{array}{l}\text { Indicating Need for } \\
\text { Medical Attention if } \\
\text { Persistent or Bothersome }\end{array}$ & \\
\hline & & $\begin{array}{cl}\text { Less Frequent, but More Severe: } \\
\text { - } & \text { Auditory toxicity } \\
\text { - } & \text { Ocular toxicity } \\
\text { - } & \begin{array}{l}\text { Neuropsychiatric } \\
\text { disorders }\end{array} \\
\text { - } & \text { QT prolongation } \\
\text { - } & \text { Hepatitis } \\
\text { - } & \begin{array}{l}\text { Bone marrow } \\
\text { suppression }\end{array} \\
\text { - } & \begin{array}{l}\text { Peripheral } \\
\text { neuropathy }\end{array}\end{array}$ & $\begin{array}{ll} & \text { vomiting, } \\
& \text { diarrhea) } \\
\text { - } & \text { Visual } \\
\text { disturbances } \\
\text { including } \\
\text { photosensitivity } \\
\text { - } \quad \text { Tinnitus } \\
\text { - } \quad \text { Muscle weakness }\end{array}$ & $\begin{array}{l}\text { Bitter tasting, so consider } \\
\text { administering with foods } \\
\text { that can mask the taste. } \\
\text { Solution available } \\
\text { worldwide, but not in } \\
\text { United States. } \\
\text { Caution in patients with } \\
\text { G6PD deficiency or } \\
\text { seizure disorder. } \\
\text { Monitor CBC; periodic } \\
\text { neurologic and } \\
\text { ophthalmologic exams in } \\
\text { patients on prolonged } \\
\text { therapy. }\end{array}$ \\
\hline Cidofovir (Vistide) & IV & \begin{tabular}{ll}
\multicolumn{2}{l}{ More Frequent: } \\
- & Nephrotoxicity \\
- & Neutropenia \\
Less Frequent: & $\begin{array}{l}\text { Fever and allergic } \\
\text { reactions }\end{array}$ \\
Rare: & $\begin{array}{l}\text { Vision changes due } \\
\text { to ocular hypotony }\end{array}$ \\
- & Metabolic acidosis
\end{tabular} & $\begin{array}{l}\text { - } \begin{array}{l}\text { GI disturbances } \\
\text { (anorexia, } \\
\text { diarrhea, nausea, } \\
\text { vomiting) }\end{array} \\
\text { - } \quad \text { Headache } \\
\text { - } \\
\text { - } \\
\text { Psthenia } \\
\end{array}$ & $\begin{array}{l}\text { Infuse over } 1 \text { hour. } \\
\text { Should not be used in } \\
\text { patients with severe renal } \\
\text { impairment. } \\
\text { Nephrotoxicity risk is } \\
\text { decreased with pre- } \\
\text { hydration with IV normal } \\
\text { saline and probenecid with } \\
\text { each infusion. Probenecid } \\
\text { is administered prior to } \\
\text { each dose and repeated for } \\
\text { two additional doses after } \\
\text { infusion. Additional } \\
\text { hydration after infusion is } \\
\text { recommended if tolerated. } \\
\text { Concurrent use of other } \\
\text { nephro-toxic drugs should } \\
\text { be avoided. } \\
\text { Monitor renal function, } \\
\text { urinalysis, electrolytes, } \\
\text { and CBC and perform } \\
\text { ophthalmologic exams. }\end{array}$ \\
\hline Ciprofloxacin (Cipro) & 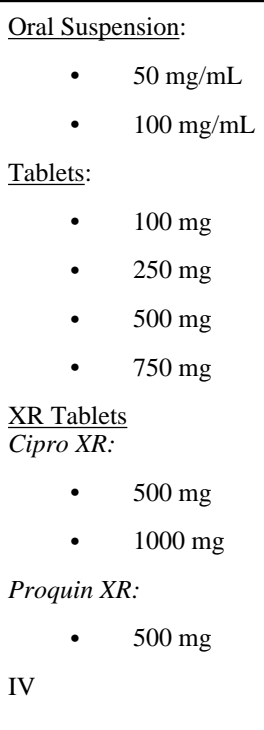 & \begin{tabular}{ll}
\multicolumn{2}{l}{ Less Frequent: } \\
\multicolumn{1}{c}{ Rare: } & Phototoxicity \\
• & CNS stimulation \\
- & Hepatotoxicity \\
- & $\begin{array}{l}\text { Hypersensitivity } \\
\text { reactions (rash, } \\
\text { pruritus, and } \\
\text { exfoliative skin } \\
\text { disorders including }\end{array}$ \\
& $\begin{array}{l}\text { SJS, dyspnea, and } \\
\text { vasculitis) }\end{array}$ \\
- & $\begin{array}{l}\text { Interstitial nephritis } \\
\text { - }\end{array}$ \\
& $\begin{array}{l}\text { Phlebitis (at injection } \\
\text { sites) }\end{array}$ \\
- & $\begin{array}{l}\text { Pseudomembranous } \\
\text { colitis }\end{array}$ \\
- & $\begin{array}{l}\text { Tendonitis or tendon } \\
\text { rupture }\end{array}$
\end{tabular} & $\begin{array}{cl}\text { More Frequent: } \\
\text { - } \\
\begin{array}{l}\text { GI disturbances } \\
\text { (abdominal } \\
\text { discomfort or } \\
\text { pain, diarrhea, } \\
\text { nausea, vomiting) }\end{array} \\
\text { - } \quad \begin{array}{l}\text { CNS toxicity } \\
\text { (dizziness, } \\
\text { headache, } \\
\text { insomnia, } \\
\text { drowsiness) }\end{array} \\
\text { Less Frequent: } \\
\text { - } \quad \text { Change in taste } \\
\text { - Photosensitivity }\end{array}$ & $\begin{array}{l}\text { Administer oral } \\
\text { formulations at least } 2 \\
\text { hours before, or } 6 \text { hours } \\
\text { after, sucralfate or } \\
\text { antacids or other products } \\
\text { containing calcium, zinc, } \\
\text { or iron (including daily } \\
\text { products or calcium- } \\
\text { fortified juices). Take with } \\
\text { full glass of water to avoid } \\
\text { crystalluria. } \\
\text { Possible phototoxicity } \\
\text { reactions with sun } \\
\text { exposure. } \\
\text { IV infusions should be } \\
\text { over } 1 \text { hour. } \\
\text { Do not split, crush, or } \\
\text { chew extended-release } \\
\text { tablets. }\end{array}$ \\
\hline
\end{tabular}




\begin{tabular}{|c|c|c|c|c|}
\hline \multirow[t]{2}{*}{ Drug } & Preparations & \multicolumn{2}{|l|}{ Major Toxicities $^{a}$} & \multirow[t]{2}{*}{ Special Instructions } \\
\hline & & $\begin{array}{l}\text { Indicating Need for Medical } \\
\text { Attention }\end{array}$ & $\begin{array}{l}\text { Indicating Need for } \\
\text { Medical Attention if } \\
\text { Persistent or Bothersome }\end{array}$ & \\
\hline & & $\begin{array}{ll}\text { - } & \text { QT interval } \\
\text { prolongation }\end{array}$ & & \\
\hline Clarithromycin (Biaxin) & $\begin{array}{cc}\text { Oral Suspension: } \\
\text { • } & 25 \mathrm{mg} / \mathrm{mL} \\
\text { • } & 50 \mathrm{mg} / \mathrm{mL} \\
\text { Tablets: } & \\
\text { • } & 250 \mathrm{mg} \\
\text { - } & 500 \mathrm{mg}\end{array}$ & $\begin{aligned} \text { Rare: } & \\
\text { - } & \text { Hepatotoxicity } \\
\text { - } & \begin{array}{l}\text { Hypersensitivity } \\
\text { reaction (rash, } \\
\text { pruritus, dyspnea) }\end{array} \\
\text { - } & \begin{array}{l}\text { Pseudomembranous } \\
\text { colitis }\end{array} \\
\text { - } & \text { Thrombocytopenia } \\
\text { - } & \text { QT interval } \\
& \text { prolongation }\end{aligned}$ & \begin{tabular}{ll}
\multicolumn{2}{l}{ More Frequent: } \\
$\begin{array}{l}\text { GI disturbances } \\
\text { (abdominal } \\
\text { discomfort or } \\
\text { pain, diarrhea, } \\
\text { nausea, vomiting) }\end{array}$ \\
Less Frequent: \\
- & $\begin{array}{l}\text { Abnormal taste } \\
\text { sensation }\end{array}$ \\
- & Headache \\
- & Rash
\end{tabular} & $\begin{array}{l}\text { Requires dose adjustment } \\
\text { in patients with impaired } \\
\text { renal function. } \\
\text { Can be administered } \\
\text { without regard to meals. } \\
\text { Reconstituted suspension } \\
\text { should not be refrigerated. } \\
\text { Potential drug interactions }\end{array}$ \\
\hline Clindamycin (Cleocin) & 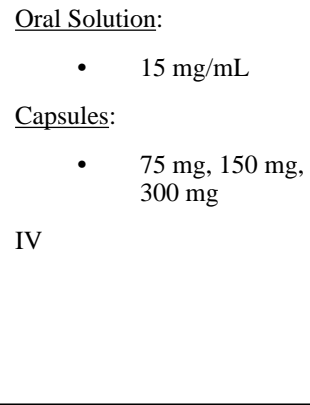 & 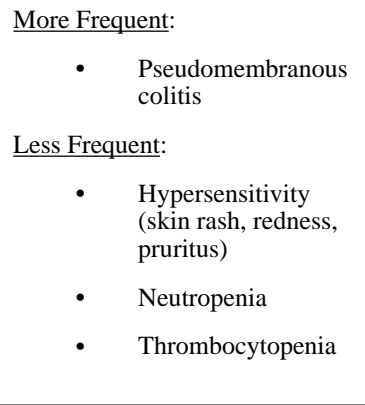 & $\begin{array}{ll}\text { More Frequent: } \\
\begin{array}{l}\text { GI disturbances } \\
\text { (abdominal pain, } \\
\text { nausea, vomiting, } \\
\text { diarrhea) }\end{array} \\
\text { Less Frequent: } \\
\begin{array}{l}\text { Fungal } \\
\text { overgrowth, } \\
\text { rectal and genital } \\
\text { areas }\end{array}\end{array}$ & $\begin{array}{l}\text { IV preparation contains } \\
\text { benzyl alcohol, not } \\
\text { recommended for use in } \\
\text { neonates. } \\
\text { IV preparation must be } \\
\text { diluted prior to } \\
\text { administration. } \\
\text { Capsule formulation } \\
\text { should be taken with food } \\
\text { or a full glass of water to } \\
\text { avoid esophageal } \\
\text { irritation. } \\
\text { Reconstituted oral solution } \\
\text { should not be refrigerated. }\end{array}$ \\
\hline Cycloserine (Seromycin) & $\begin{array}{l}\text { Capsules: } \\
\bullet \quad 250 \mathrm{mg}\end{array}$ & $\begin{array}{ll}\text { More Frequent: } \\
\qquad \begin{array}{l}\text { CNS toxicity } \\
\text { (including confusion, } \\
\text { anxiety) }\end{array} \\
\text { Less Frequent: } \\
\text { - } & \begin{array}{l}\text { Hypersensitivity } \\
\text { (skin rash) }\end{array} \\
\text { - } & \begin{array}{l}\text { Peripheral } \\
\text { neuropathy }\end{array} \\
\text { - } & \text { Seizures } \\
\text { - } & \text { Psychosis } \\
\text { Rare: } & \\
\text { - } & \text { Cardiac arrhythmias }\end{array}$ & $\begin{array}{ll}\text { - } & \begin{array}{l}\text { Headache, } \\
\text { dizziness, } \\
\text { drowsiness, } \\
\text { confusion }\end{array} \\
\underline{\text { Rare: }} & \\
\text { - } & \text { Photosensitivity }\end{array}$ & $\begin{array}{l}\text { Take with food to } \\
\text { minimize gastric irritation. } \\
\text { Neurotoxicity is related to } \\
\text { excessive serum } \\
\text { concentrations; serum } \\
\text { concentrations should be } \\
\text { maintained at } 25-30 \\
\text { mcg/mL. } \\
\text { Requires dose adjustment } \\
\text { in patients with impaired } \\
\text { renal function. } \\
\text { Do not administer to } \\
\text { patients with severe renal } \\
\text { impairment (because of } \\
\text { increased risk of } \\
\text { neurotoxicity). Should } \\
\text { monitor serum levels, if } \\
\text { possible. } \\
\text { Should administer } \\
\text { pyridoxine at the same } \\
\text { time. } \\
\text { Monitor renal function, } \\
\text { LFTs, and CBC. }\end{array}$ \\
\hline Dapsone & 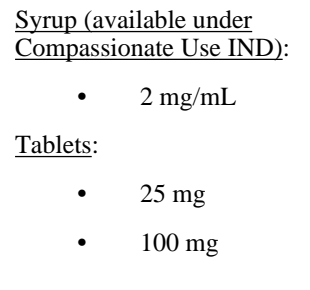 & $\begin{aligned} & \text { More Frequent: } \\
& \text { • } \begin{array}{l}\text { Hemolytic anemia } \\
\text { (especially if G6PD } \\
\text { deficiency) }\end{array} \\
& \text { • } \text { Methemoglobinemia } \\
& \text { - } \text { Skin rash } \\
& \text { Rare: }\end{aligned}$ & 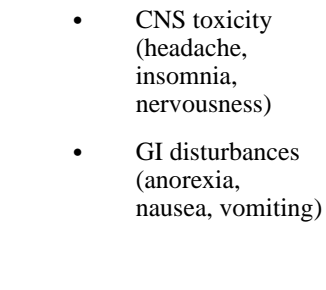 & $\begin{array}{l}\text { Protect from light; } \\
\text { dispense syrup in amber } \\
\text { glass bottles. } \\
\text { Monitor CBC and LFTs. }\end{array}$ \\
\hline
\end{tabular}




\begin{tabular}{|c|c|c|c|c|}
\hline \multirow[t]{2}{*}{ Drug } & \multirow[t]{2}{*}{ Preparations } & \multicolumn{2}{|l|}{ Major Toxicities ${ }^{a}$} & \multirow[t]{2}{*}{ Special Instructions } \\
\hline & & $\begin{array}{l}\text { Indicating Need for Medical } \\
\text { Attention }\end{array}$ & $\begin{array}{l}\text { Indicating Need for } \\
\text { Medical Attention if } \\
\text { Persistent or Bothersome }\end{array}$ & \\
\hline & & $\begin{array}{ll}\text { - } & \text { Blood dyscrasias } \\
\text { - } & \text { Exfoliative skin } \\
& \text { disorders (including } \\
& \text { SJS) } \\
\text { - } & \text { Hepatic toxicity } \\
\text { - } & \begin{array}{l}\text { Mood or other mental } \\
\text { changes }\end{array} \\
\text { - } & \text { Peripheral neuritis } \\
\text { - } & \begin{array}{l}\text { Hypersensitivity } \\
\text { reaction (fever, rash, } \\
\text { jaundice, anemia) }\end{array}\end{array}$ & $\begin{array}{ll} & \begin{array}{l}\text { Photosensitivity } \\
\text { reactions }\end{array}\end{array}$ & \\
\hline Doxycycline (Vibramycin) & 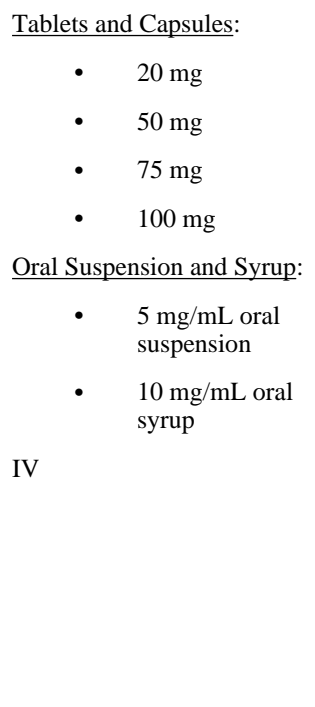 & 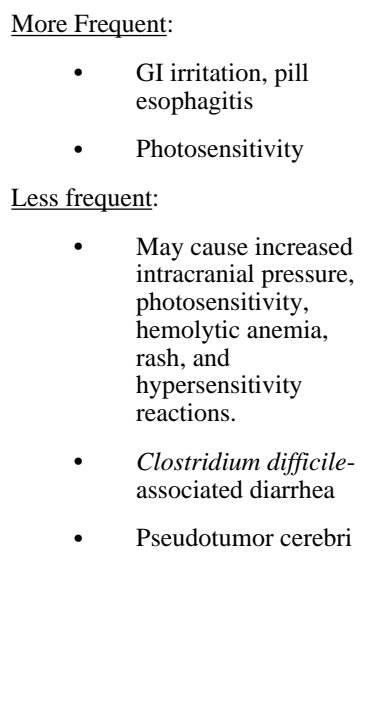 & $\begin{array}{l}\text { - } \quad \begin{array}{l}\text { Staining of teeth } \\
\text { a concern for } \\
\text { individuals aged } \\
<8 \text { years }\end{array} \\
\text { - } \begin{array}{l}\text { Photo- } \\
\text { onycholysis }\end{array} \\
\text { - } \quad \begin{array}{l}\text { GI disturbances } \\
\text { (nausea, } \\
\text { vomiting, } \\
\text { abdominal } \\
\text { cramps) }\end{array}\end{array}$ & $\begin{array}{l}\text { Swallow with adequate } \\
\text { amounts of fluids } \\
\text { Avoid antacids, milk, } \\
\text { dairy products, and iron } \\
\text { for } 1 \text { hour before or } 2 \\
\text { hours after administration } \\
\text { of doxycycline. } \\
\text { Use with caution in } \\
\text { hepatic and renal disease. } \\
\text { IV doses should be } \\
\text { infused over } 1 \text { to } 4 \text { hours. } \\
\text { Patient should avoid } \\
\text { prolonged exposure to } \\
\text { direct sunlight (skin } \\
\text { sensitivity). } \\
\text { Generally not } \\
\text { recommended for use in } \\
\text { children aged <8 years } \\
\text { because of risk of tooth } \\
\text { enamel hypoplasia and } \\
\text { discoloration, unless } \\
\text { benefit outweighs risk. } \\
\text { Monitor renal function, } \\
\text { CBC, and LFTs if } \\
\text { prolonged therapy. }\end{array}$ \\
\hline Erythromycin & $\begin{array}{l}\text { Erythromycin- Base Tablet: } \\
\text { • } \quad 250 \mathrm{mg} \\
\text { • } \quad 333 \mathrm{mg} \\
\text { • } \quad 500 \mathrm{mg} \\
\text { Delayed-Release Tablet: } \\
\text { • } \quad 250 \mathrm{mg} \\
\text { • } \quad 333 \mathrm{mg} \\
\text { • } \quad 500 \mathrm{mg} \\
\text { Delayed-Release Capsule: } \\
\text { • } \quad 250 \mathrm{mg} \\
\text { Erythromycin Ethyl } \\
\text { Succinate } \\
\text { Suspension: } \\
\text { • } \quad 200 \mathrm{mg} \\
\text { • } \quad 400 \mathrm{mg} / 5 \mathrm{~mL} \\
\text { Oral Drops: } \\
\text { • } \quad 100 \mathrm{mg} / 2.5 \mathrm{~mL}\end{array}$ & $\begin{array}{ll}\text { Less Frequent: } \\
\text { - } & \begin{array}{l}\text { Estolate may cause } \\
\text { cholestatic jaundice, } \\
\text { although } \\
\text { hepatotoxicity is } \\
\text { uncommon (2\% of } \\
\text { reported cases). }\end{array} \\
\text { Rare: } \quad \text { QT prolongation } \\
\text { - } \quad \begin{array}{l}\text { Hypersensitivity } \\
\text { reactions (rash, } \\
\text { exfoliative skin } \\
\text { disorders including } \\
\text { SJS) }\end{array}\end{array}$ & $\begin{array}{ll}\text { - } & \text { GI disturbances } \\
\text { (nausea, } \\
\text { vomiting, } \\
\text { abdominal } \\
\text { cramps) } \\
\text { - } & \text { Rash, urticaria } \\
\text { - } & \text { Increased LFTs }\end{array}$ & $\begin{array}{l}\text { Use with caution in liver } \\
\text { disease. } \\
\text { Oral therapy should } \\
\text { replace IV therapy as soon } \\
\text { as possible. } \\
\text { Give oral doses after } \\
\text { meals. } \\
\text { Parenteral administration } \\
\text { should consist of a } \\
\text { continuous drip or slow } \\
\text { infusion over 1 hour or } \\
\text { longer. } \\
\text { Adjust dose in renal } \\
\text { failure. } \\
\text { Erythromycin should be } \\
\text { used with caution in } \\
\text { neonates; hypertrophic } \\
\text { pyloric stenosis and life- } \\
\text { threatening episodes of } \\
\text { ventricular tachycardia } \\
\text { associated with prolonged } \\
\text { QTc interval have been } \\
\text { reported. } \\
\text { High potential for } \\
\text { interaction with many } \\
\text { ARVs and other drugs. }\end{array}$ \\
\hline
\end{tabular}




\begin{tabular}{|c|c|c|c|c|}
\hline \multirow[t]{2}{*}{ Drug } & \multirow[t]{2}{*}{ Preparations } & \multicolumn{2}{|l|}{ Major Toxicities $a$} & \multirow[t]{2}{*}{ Special Instructions } \\
\hline & & $\begin{array}{l}\text { Indicating Need for Medical } \\
\text { Attention }\end{array}$ & $\begin{array}{l}\text { Indicating Need for } \\
\text { Medical Attention if } \\
\text { Persistent or Bothersome }\end{array}$ & \\
\hline & 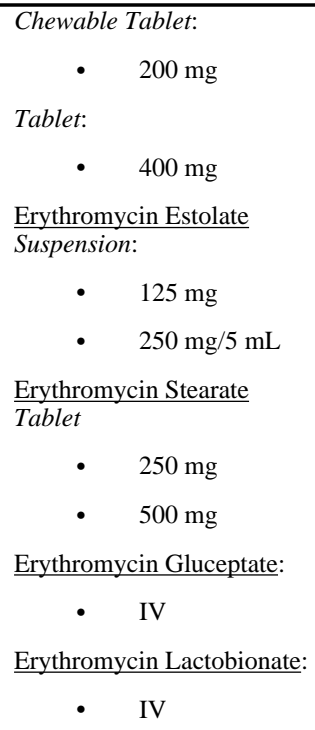 & & & \\
\hline Ethambutol (Myambutol) & $\begin{array}{rr}\text { Tablets: } & \\
\text { • } & 100 \mathrm{mg} \\
\text { • } & 400 \mathrm{mg}\end{array}$ & $\begin{array}{ll}\text { Less Frequent: } \\
\text { - } & \begin{array}{l}\text { Acute gouty arthritis } \\
\text { (secondary to } \\
\text { hyperuricemia) }\end{array} \\
\text { Rare: } & \begin{array}{l}\text { Hypersensitivity } \\
\text { (rash, fever, joint } \\
\text { pain) }\end{array} \\
\text { - } \quad \begin{array}{l}\text { Peripheral } \\
\text { neuropathy }\end{array} \\
\text { - } \quad \begin{array}{l}\text { Retrobulbar optic } \\
\text { neuritis, decreased } \\
\text { visual acuity, loss of } \\
\text { red-green color } \\
\text { discrimination }\end{array} \\
\text { Bone marrow } \\
\text { suppression } \\
\text { - } \quad \begin{array}{l}\text { Abnormal LFTs, } \\
\text { hepatotoxicity }\end{array}\end{array}$ & $\begin{array}{ll}\text { - } & \begin{array}{l}\text { GI disturbances } \\
\text { (abdominal pain, } \\
\text { anorexia, nausea, } \\
\text { vomiting) }\end{array} \\
\text { - } & \text { Confusion } \\
\text { - } & \text { Disorientation } \\
\text { - } & \text { Headache }\end{array}$ & $\begin{array}{l}\text { Requires dose adjustment } \\
\text { in patients with impaired } \\
\text { renal function. } \\
\text { Take with food to } \\
\text { minimize gastric irritation. } \\
\text { Monitor visual acuity and } \\
\text { red-green color } \\
\text { discrimination regularly. } \\
\text { Monitor renal function, } \\
\text { LFTs, and CBC. } \\
\text { Avoid concomitant use of } \\
\text { drugs with neurotoxicity. }\end{array}$ \\
\hline Ethionamide (Trecator-SC) & $\begin{array}{c}\text { Tablets: } \\
\text { • }\end{array}$ & \begin{aligned} & \multicolumn{2}{l}{ Less Frequent: } \\
& • Hepatitis, jaundice \\
& - Peripheral neuritis \\
& - $\begin{array}{l}\text { Psychiatric } \\
\text { disturbances }\end{array} \\
&$ Rare: \\
& - $\begin{array}{l}\text { Goiter or } \\
\text { hypothyroidism }\end{array} \\
&$ - Hypoglycemia \\
& - Optic neuritis \end{aligned} & $\begin{array}{ll}\text { More Frequent: } \\
& \begin{array}{l}\text { GI disturbances } \\
\text { (anorexia, } \\
\text { metallic taste, } \\
\text { nausea, vomiting, } \\
\text { stomatitis) }\end{array} \\
\text { - } & \begin{array}{l}\text { Orthostatic } \\
\text { hypotension }\end{array} \\
\text { Rare: } & \\
\text { - } & \text { Gynecomastia }\end{array}$ & $\begin{array}{l}\text { Avoid use of other } \\
\text { neurotoxic drugs that } \\
\text { could increase potential } \\
\text { for peripheral neuropathy } \\
\text { and optic neuritis. } \\
\text { Administration of } \\
\text { pyridoxine may alleviate } \\
\text { peripheral neuritis. } \\
\text { Take with food to } \\
\text { minimize gastric irritation. } \\
\text { Monitor LFTs, glucose, } \\
\text { and thyroid function. } \\
\text { Perform periodic } \\
\text { ophthalmologic exams. }\end{array}$ \\
\hline
\end{tabular}




\begin{tabular}{|c|c|c|c|c|}
\hline \multirow[t]{2}{*}{ Drug } & Preparations & \multicolumn{2}{|l|}{ Major Toxicities $a$} & \multirow[t]{2}{*}{ Special Instructions } \\
\hline & & $\begin{array}{l}\text { Indicating Need for Medical } \\
\text { Attention }\end{array}$ & $\begin{array}{l}\text { Indicating Need for } \\
\text { Medical Attention if } \\
\text { Persistent or Bothersome }\end{array}$ & \\
\hline & & - $\quad$ Skin rash & & \\
\hline Fluconazole (Diflucan) & \begin{tabular}{cc}
\multicolumn{2}{l}{ Oral Suspension: } \\
• & $10 \mathrm{mg} / \mathrm{mL}$ \\
• & $40 \mathrm{mg} / \mathrm{mL}$ \\
Tablets: & \\
• & $50 \mathrm{mg}$ \\
• & $100 \mathrm{mg}$ \\
• & $150 \mathrm{mg}$ \\
• & $200 \mathrm{mg}$ \\
IV &
\end{tabular} & \begin{tabular}{ll}
\multicolumn{2}{l}{ Less Frequent: } \\
- & $\begin{array}{l}\text { Hypersensitivity } \\
\text { (fever, chills, skin } \\
\text { rash) }\end{array}$ \\
Rare: & $\begin{array}{l}\text { Agranulocytosis, } \\
\text { eosinophilia, } \\
\text { leucopenia, } \\
\text { thrombocytopenia }\end{array}$ \\
- & $\begin{array}{l}\text { Exfoliative skin } \\
\text { disorders (including }\end{array}$ \\
& SJS) \\
- & Hepatotoxicity \\
- & QT prolongation \\
- & Thrombocytopenia
\end{tabular} & 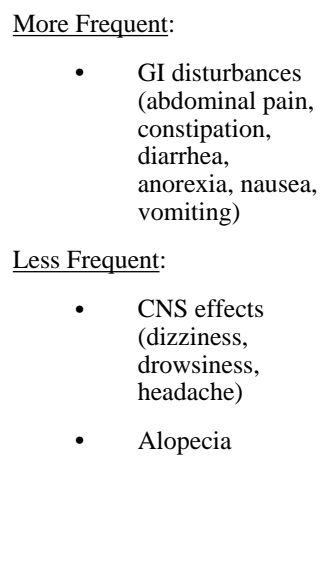 & $\begin{array}{l}\text { Can be given orally } \\
\text { without regard to meals. } \\
\text { Shake suspension well } \\
\text { before dosing. } \\
\text { Requires dose adjustment } \\
\text { in patients with impaired } \\
\text { renal function. } \\
\text { IV administration should } \\
\text { be administered over } 1-2 \\
\text { hours at a rate } \_00 \mathrm{mg} / \\
\text { hour. } \\
\text { Daily dose is the same for } \\
\text { oral and IV } \\
\text { administration. } \\
\text { Multiple potential drug } \\
\text { interactions } \\
\text { Monitor periodic LFTs, } \\
\text { renal function, and CBC. }\end{array}$ \\
\hline Flucytosine (Ancobon) & 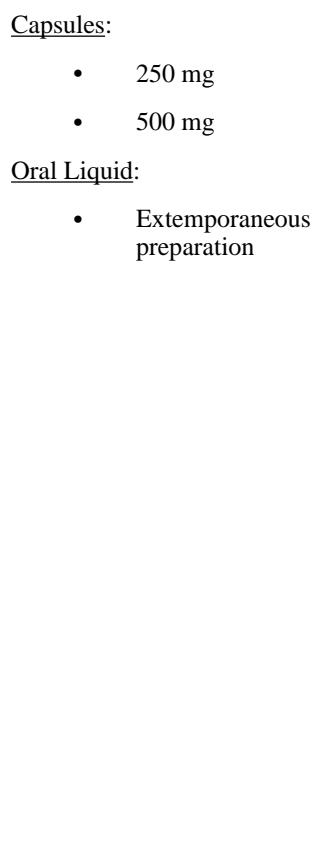 & 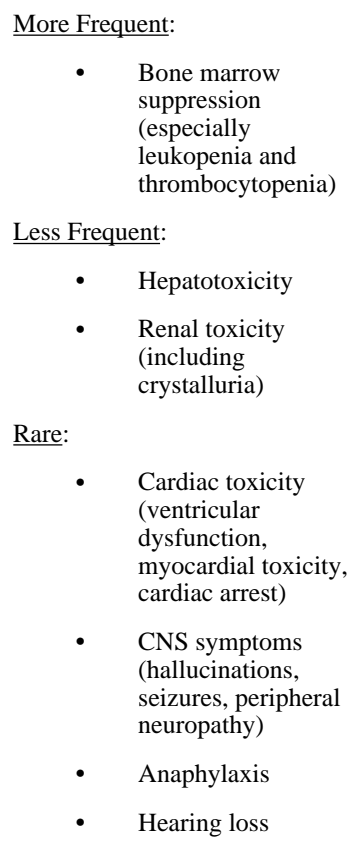 & $\begin{array}{ll}\text { - } & \begin{array}{l}\text { GI disturbances } \\
\text { (abdominal pain, } \\
\text { constipation, } \\
\text { diarrhea, } \\
\text { anorexia, nausea, } \\
\text { vomiting) }\end{array} \\
\text { - } \quad \begin{array}{l}\text { Elevated liver } \\
\text { transaminases }\end{array} \\
\text { - } \quad \text { Skin rash } \\
\text { Rare: } \quad \begin{array}{l}\text { CNS symptoms } \\
\text { (headache, } \\
\text { drowsiness, } \\
\text { confusion, } \\
\text { vertigo) } \\
\text { Crystalluria }\end{array} \\
\text { - }\end{array}$ & $\begin{array}{l}\text { Monitor serum } \\
\text { concentrations and adjust } \\
\text { dose to maintain } \\
\text { therapeutic levels and } \\
\text { minimize risk of bone } \\
\text { marrow suppression. } \\
\text { Requires dose adjustment } \\
\text { in patients with impaired } \\
\text { renal function; use with } \\
\text { extreme caution. } \\
\text { Fatal aplastic anemia and } \\
\text { agranulocytosis have been } \\
\text { rarely reported. } \\
\text { Oral preparations should } \\
\text { be administered with food } \\
\text { over a 15-minute period to } \\
\text { minimize GI side effects } \\
\text { Monitor CBC, LFTs, renal } \\
\text { function, and electrolytes. }\end{array}$ \\
\hline Foscarnet (Foscavir) & IV & $\begin{array}{ll}\text { More Frequent: } \\
& \text { Nephrotoxicity } \\
\text { - } & \begin{array}{l}\text { Serum electrolyte } \\
\text { abnormalities } \\
\text { (hypocalcaemia, } \\
\text { hypophosphatemia, } \\
\text { hypomagnesemia, } \\
\text { hypokalemia) }\end{array} \\
\text { Less Frequent: }\end{array}$ & $\begin{aligned} \text { Frequent: } & \\
\text { - } & \begin{array}{l}\text { GI disturbances } \\
\text { (abdominal pain, } \\
\text { anorexia, nausea, } \\
\text { vomiting) }\end{array} \\
\text { - } & \begin{array}{l}\text { Anxiety, } \\
\text { confusion, } \\
\text { dizziness, } \\
\text { headache }\end{array} \\
\text { - } & \text { Fever }\end{aligned}$ & $\begin{array}{l}\text { Requires dose adjustment } \\
\text { in patients with impaired } \\
\text { renal function. } \\
\text { Use adequate hydration to } \\
\text { decrease nephrotoxicity. } \\
\text { Avoid concomitant use of } \\
\text { other drugs with } \\
\text { nephrotoxicity. } \\
\text { Monitor serum } \\
\text { electrolytes, renal } \\
\text { function, and CBC. }\end{array}$ \\
\hline
\end{tabular}




\begin{tabular}{|c|c|c|c|c|}
\hline \multirow[t]{2}{*}{ Drug } & Preparations & \multicolumn{2}{|l|}{ Major Toxicities ${ }^{a}$} & \multirow[t]{2}{*}{ Special Instructions } \\
\hline & & $\begin{array}{l}\text { Indicating Need for Medical } \\
\text { Attention }\end{array}$ & $\begin{array}{l}\text { Indicating Need for } \\
\text { Medical Attention if } \\
\text { Persistent or Bothersome }\end{array}$ & \\
\hline & & $\begin{array}{ll}- & \begin{array}{l}\text { Hematologic toxicity } \\
\text { (anemia, } \\
\text { granulocytopenia) }\end{array} \\
\text { - } \quad \begin{array}{l}\text { Neurotoxicity } \\
\text { (muscle twitching, } \\
\text { tremor, seizures, } \\
\text { tingling around } \\
\text { mouth) }\end{array} \\
\text { - } \quad \begin{array}{l}\text { Cardiac } \\
\text { abnormalities } \\
\text { secondary to } \\
\text { electrolyte changes }\end{array} \\
\text { - } \quad \begin{array}{l}\text { Phlebitis (at site of } \\
\text { injection) }\end{array} \\
\text { Rare: } \quad \begin{array}{l}\text { Sores or ulcers mouth } \\
\text { or throat }\end{array} \\
\end{array}$ & & $\begin{array}{l}\text { Consider monitoring } \\
\text { serum concentrations } \\
\text { (TDM) } \\
\text { IV solution of } 24 \mathrm{mg} / \mathrm{mL} \\
\text { can be administered via } \\
\text { central line but must be } \\
\text { diluted to a final } \\
\text { concentration not to } \\
\text { exceed } 12 \mathrm{mg} / \mathrm{mL} \text { if given } \\
\text { via peripheral line. } \\
\text { Must be administered at a } \\
\text { constant rate by infusion } \\
\text { pump over } \geq 2 \text { hours (or no } \\
\text { faster than } 1 \mathrm{mg} / \mathrm{kg} / \\
\text { minute). }\end{array}$ \\
\hline Ganciclovir (Cytovene) & 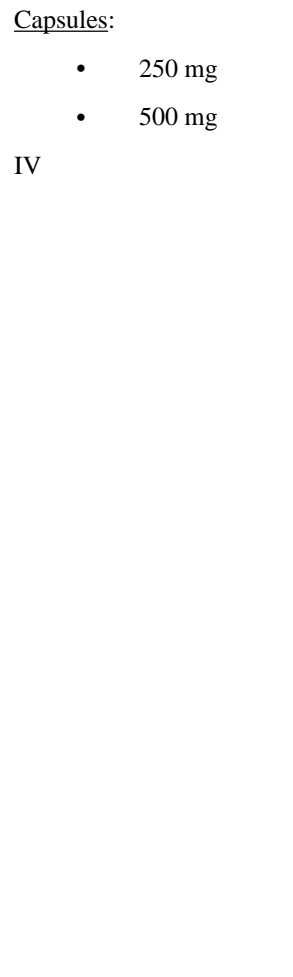 & \begin{tabular}{cl} 
More Frequent: \\
\multicolumn{1}{l}{ - } & Granulocytopenia \\
- & Thrombocytopenia \\
Less Frequent: \\
- & Anemia \\
- & $\begin{array}{l}\text { CNS effects } \\
\text { (confusion, }\end{array}$ \\
& headache) \\
- & Hypersensitivity \\
& (fever, rash) \\
- & $\begin{array}{l}\text { Elevated } \\
\text { transaminase } \\
\text { enzymes }\end{array}$ \\
- & Increase in creatinine, \\
& BUN \\
- & $\begin{array}{l}\text { Phlebitis (at injection } \\
\text { sites) }\end{array}$ \\
Rare: & \\
- & Retinal detachment \\
- & Seizures \\
- & Psychosis \\
- & $\begin{array}{l}\text { Cardiac } \\
\text { (hypertension, chest } \\
\text { pain) }\end{array}$ \\
&
\end{tabular} & 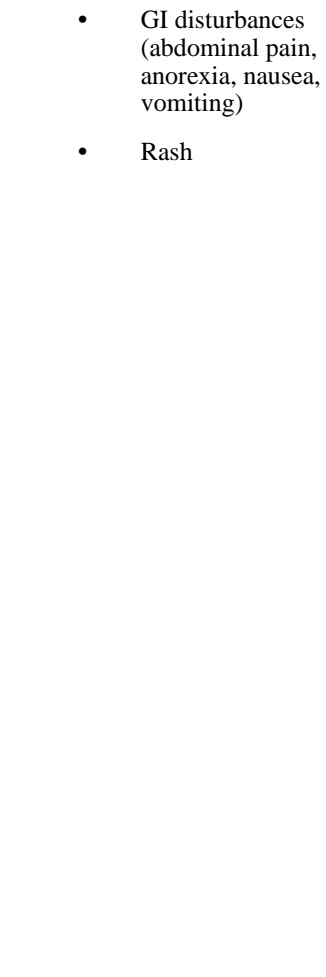 & $\begin{array}{l}\text { Requires dose adjustment } \\
\text { in patients with renal } \\
\text { impairment. } \\
\text { Avoid other nephrotoxic } \\
\text { drugs. } \\
\text { IV infusion over at least } 1 \\
\text { hour. In-line filter } \\
\text { required. } \\
\text { Maintain good hydration. } \\
\text { Undiluted IV solution is } \\
\text { alkaline (pH 11); use } \\
\text { caution in handling and } \\
\text { preparing solutions and } \\
\text { avoid contact with skin } \\
\text { and mucus membranes. } \\
\text { Administer oral doses with } \\
\text { food to increase } \\
\text { absorption. Do not open or } \\
\text { crush capsules. } \\
\text { Monitor CBC, LFTs, renal } \\
\text { function; conduct } \\
\text { ophthalmologic } \\
\text { examinations. }\end{array}$ \\
\hline Interferon-alfa-2B (IFN-a-2B; Intron) & Parenteral (SQ or IV use) & $\begin{array}{ll}\text { More Frequent: } \\
& \begin{array}{l}\text { Hematologic toxicity } \\
\text { (leukopenia, } \\
\text { thrombocytopenia) }\end{array} \\
\text { - } & \begin{array}{l}\text { Neurotoxicity } \\
\text { (confusion, } \\
\text { depression, insomnia, } \\
\text { anxiety) }\end{array}\end{array}$ & 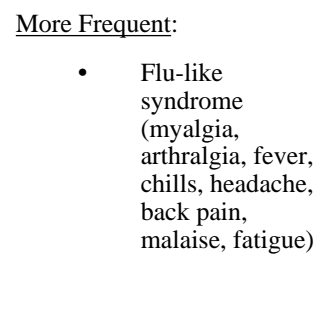 & $\begin{array}{l}\text { Severe adverse effects less } \\
\text { common in children than } \\
\text { adults. } \\
\text { Toxicity dose-related, with } \\
\text { significant reduction over } \\
\text { the first } 4 \text { months of } \\
\text { therapy. } \\
\text { For non-life-threatening } \\
\text { reactions, reduce dose or } \\
\text { temporarily discontinue } \\
\text { drug and restart at low }\end{array}$ \\
\hline
\end{tabular}




\begin{tabular}{|c|c|c|c|c|}
\hline \multirow[t]{2}{*}{ Drug } & Preparations & \multicolumn{2}{|l|}{ Major Toxicities ${ }^{a}$} & \multirow[t]{2}{*}{ Special Instructions } \\
\hline & & $\begin{array}{l}\text { Indicating Need for Medical } \\
\text { Attention }\end{array}$ & $\begin{array}{l}\text { Indicating Need for } \\
\text { Medical Attention if } \\
\text { Persistent or Bothersome }\end{array}$ & \\
\hline & & \begin{tabular}{cl} 
& Injection erythema \\
Less Frequent: & \multicolumn{1}{c}{$\begin{array}{l}\text { Cardiovascular } \\
\text { effects (chest pain, } \\
\text { hypertension, } \\
\text { arrhythmias, } \\
\text { hypotension) }\end{array}$} \\
- & $\begin{array}{l}\text { Hypoesthesia/ } \\
\text { paresthesia }\end{array}$ \\
Rare: & $\begin{array}{l}\text { Abnormality or loss } \\
\text { of vision }\end{array}$ \\
- & $\begin{array}{l}\text { Allergic reaction } \\
\text { (rash, hives) }\end{array}$ \\
- & $\begin{array}{l}\text { Hypothyroidism } \\
\text { - }\end{array}$ \\
& $\begin{array}{l}\text { Development of } \\
\text { antinuclear } \\
\text { antibodies }\end{array}$
\end{tabular} & $\begin{array}{ll} & \begin{array}{l}\text { GI disturbances } \\
\text { (abdominal pain, } \\
\text { anorexia, nausea, } \\
\text { vomiting, } \\
\text { diarrhea, } \\
\text { dyspepsia) }\end{array} \\
\text { - } \quad \begin{array}{l}\text { Pharyngitis, dry } \\
\text { mouth }\end{array} \\
\text { Less Frequent: } \\
\text { Alopecia } \\
\text { - } \quad \text { Epistaxis } \\
\text { Elevated serum } \\
\text { transaminases, } \\
\text { serum creatinine } \\
\text { and BUN, } \\
\text { glucose, } \\
\text { triglycerides }\end{array}$ & $\begin{array}{l}\text { doses with stepwise } \\
\text { increases. } \\
\text { If patients have visual } \\
\text { complaints, an } \\
\text { ophthalmologic exam } \\
\text { should be performed to } \\
\text { detect possible retinal } \\
\text { hemorrhage or retinal } \\
\text { artery or vein obstruction. } \\
\text { Should not be used in } \\
\text { children with } \\
\text { decompensated hepatic } \\
\text { disease, significant } \\
\text { cytopenia, autoimmune } \\
\text { disease, or significant pre- } \\
\text { existing renal or cardiac } \\
\text { disease. } \\
\text { If symptoms of hepatic } \\
\text { decompensation occur } \\
\text { (ascites, coagulopathy, } \\
\text { jaundice), IFN-a-2B } \\
\text { should be discontinued. } \\
\text { Reconstituted solution } \\
\text { stable for } 24 \text { hours when } \\
\text { refrigerated. } \\
\text { Monitor CBC, renal } \\
\text { function, LFTs, thyroid } \\
\text { function, and glucose. }\end{array}$ \\
\hline Isoniazid (Nydrazid) & \begin{tabular}{ll}
\multicolumn{2}{l}{ Oral Syrup: } \\
$\qquad$ & $10 \mathrm{mg} / \mathrm{mL}$ \\
Tablets: & \\
$\qquad$ & $100 \mathrm{mg}$ \\
$\bullet$ & $300 \mathrm{mg}$ \\
$\mathrm{IM}$ &
\end{tabular} & $\begin{array}{ll}\text { More Frequent: } \\
\text { - } & \begin{array}{l}\text { Hepatitis prodromal } \\
\text { syndrome (anorexia, } \\
\text { weakness, vomiting) }\end{array} \\
\text { • } & \text { Hepatitis } \\
\text { - } & \text { Peripheral neuritis } \\
\underline{\text { Rare: }} & \\
\text { - } & \text { Blood dyscrasias } \\
\text { - } & \begin{array}{l}\text { Hypersensitivity } \\
\text { (fever, rash, joint } \\
\text { pain) }\end{array} \\
\text { - } & \begin{array}{l}\text { Neurotoxicity } \\
\text { (includes seizure) }\end{array} \\
\text { - } & \text { Optic neuritis }\end{array}$ & $\begin{array}{ll}\text { - } & \begin{array}{l}\text { GI disturbances } \\
\text { (abdominal pain, } \\
\text { nausea, vomiting, } \\
\text { diarrhea) }\end{array} \\
\text { - } & \begin{array}{l}\text { Elevated liver } \\
\text { transaminases }\end{array} \\
\text { - } & \begin{array}{l}\text { Pyridoxine } \\
\text { deficiency }\end{array}\end{array}$ & $\begin{array}{l}\text { Take with food to } \\
\text { minimize gastric irritation. } \\
\text { Take } \geq \text { hour before } \\
\text { aluminum-containing } \\
\text { antacids. } \\
\text { Hepatitis less common in } \\
\text { children. } \\
\text { Use with caution in } \\
\text { patients with hepatic } \\
\text { function impairment, } \\
\text { severe renal failure, or } \\
\text { history of seizures. } \\
\text { Pyridoxine } \\
\text { supplementation should be } \\
\text { provided for all HIV- } \\
\text { infected children. } \\
\text { Monitor LFTs and } \\
\text { periodic ophthalmologic } \\
\text { examinations. }\end{array}$ \\
\hline Itraconazole (Sporanox) & $\begin{array}{l}\frac{\text { Oral Solution: }}{\bullet} \quad 10 \mathrm{mg} / \mathrm{mL} \\
\frac{\text { Capsules: }}{\bullet} \quad 100 \mathrm{mg} \\
\text { IV }\end{array}$ & \begin{tabular}{ll}
\multicolumn{2}{l}{ Less frequent: } \\
- & $\begin{array}{l}\text { Hypersensitivity } \\
\text { (fever, chills, skin } \\
\text { rash) }\end{array}$ \\
- $\quad \begin{array}{l}\text { Hypokalemia (can be } \\
\text { associated with } \\
\text { cardiac arrhythmias) }\end{array}$ \\
Rare: \\
- $\quad \begin{array}{l}\text { Hepatotoxicity } \\
\text { - }\end{array}$ \\
$\begin{array}{l}\text { Hematologic } \\
\text { abnormalities } \\
\text { (thrombocytopenia, } \\
\text { leukopenia) }\end{array}$
\end{tabular} & $\begin{array}{ll}\text { More Frequent: } \\
\text { - } & \begin{array}{l}\text { GI disturbances } \\
\text { (abdominal pain, } \\
\text { constipation, } \\
\text { diarrhea, } \\
\text { anorexia, nausea, } \\
\text { vomiting) }\end{array} \\
\text { Less Frequent: } \\
\text { - } & \begin{array}{l}\text { CNS effects } \\
\text { (dizziness, } \\
\text { drowsiness, } \\
\text { headache) }\end{array} \\
\text { - } & \text { Rash }\end{array}$ & 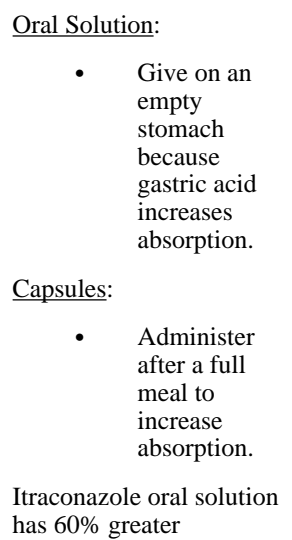 \\
\hline
\end{tabular}




\begin{tabular}{|c|c|c|c|c|}
\hline \multirow[t]{2}{*}{ Drug } & Preparations & \multicolumn{2}{|l|}{ Major Toxicities ${ }^{a}$} & \multirow[t]{2}{*}{ Special Instructions } \\
\hline & & $\begin{array}{l}\text { Indicating Need for Medical } \\
\text { Attention }\end{array}$ & $\begin{array}{l}\text { Indicating Need for } \\
\text { Medical Attention if } \\
\text { Persistent or Bothersome }\end{array}$ & \\
\hline & & & & $\begin{array}{l}\text { bioavailability compared } \\
\text { with capsules, and the oral } \\
\text { solution and capsules } \\
\text { should not be used } \\
\text { interchangeably. } \\
\text { IV infusion over } 1 \text { hour. } \\
\text { Multiple potential drug } \\
\text { interactions } \\
\text { Monitor LFTs and } \\
\text { potassium levels. } \\
\text { Monitor serum } \\
\text { concentrations (TDM) in } \\
\text { severe infections. }\end{array}$ \\
\hline Kanamycin & $\begin{array}{l}\text { IV } \\
\text { IM }\end{array}$ & 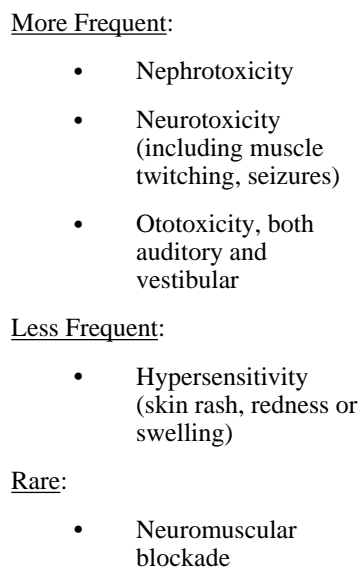 & N/A & $\begin{array}{l}\text { Must be infused over } 30 \text { to } \\
60 \text { minutes to avoid } \\
\text { neuromuscular blockade. } \\
\text { Requires dose adjustment } \\
\text { in patients with impaired } \\
\text { renal function. } \\
\text { Should monitor renal } \\
\text { function and hearing } \\
\text { periodically (e.g., } \\
\text { monthly) in children on } \\
\text { prolonged therapy. } \\
\text { Monitor serum } \\
\text { concentrations (TDM). } \\
\text { Monitor renal function; } \\
\text { conduct, hearing exams } \\
\text { for patients receiving } \\
\text { prolonged therapy. }\end{array}$ \\
\hline Ketoconazole (Nizoral) & 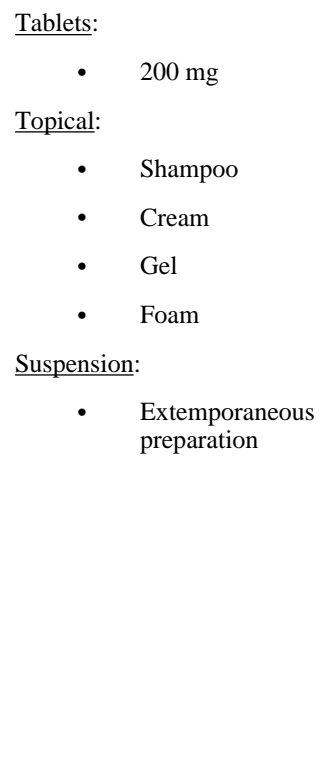 & $\begin{array}{ll}\text { Less Frequent: } \\
\text { - } & \begin{array}{l}\text { Hypersensitivity } \\
\text { (fever, chills, skin } \\
\text { rash) }\end{array} \\
\text { Rare: } & \begin{array}{l}\text { Hepatotoxicity } \\
\text { (including hepatic } \\
\text { failure) }\end{array}\end{array}$ & $\begin{array}{cl}\text { Frequent: } & \\
\text { - } & \begin{array}{l}\text { GI disturbances } \\
\text { (abdominal pain, } \\
\text { constipation, } \\
\text { diarrhea, } \\
\text { anorexia, nausea, } \\
\text { vomiting) }\end{array} \\
\text { Less Frequent: } \\
\text { - } & \begin{array}{l}\text { CNS effects } \\
\text { (dizziness, } \\
\text { drowsiness, } \\
\text { headache) }\end{array} \\
\text { Rare: } & \begin{array}{l}\text { Gynecomastia } \\
\text { - }\end{array} \\
\text { - } & \begin{array}{l}\text { Impotence } \\
\text { irregularities }\end{array} \\
\text { - } & \text { Photophobia } \\
& \end{array}$ & $\begin{array}{l}\text { Adverse GI effects occur } \\
\text { less often when } \\
\text { administered with food. } \\
\text { Drugs that decrease gastric } \\
\text { acidity or sucralfate } \\
\text { should be administered } 22 \\
\text { hours after ketoconazole. } \\
\text { Disulfiram-like reactions } \\
\text { have occurred in patients } \\
\text { ingesting alcohol. } \\
\text { Hepatotoxicity is an } \\
\text { idiosyncratic reaction, } \\
\text { usually reversible when } \\
\text { stopping the drug, but rare } \\
\text { fatalities can occur any } \\
\text { time during therapy; more } \\
\text { common in females and } \\
\text { adults }>40 \text { years, but cases } \\
\text { reported in children. } \\
\text { High-dose ketoconazole } \\
\text { suppresses corticosteroid } \\
\text { secretion, lowers serum } \\
\text { testosterone concentration } \\
\text { (reversible). } \\
\text { Multiple potential drug } \\
\text { interactions. } \\
\text { Monitor LFTs. }\end{array}$ \\
\hline Mefloquine (Lariam) & $\begin{aligned} \text { Tablets: } & \\
\bullet & 250 \mathrm{mg}\end{aligned}$ & 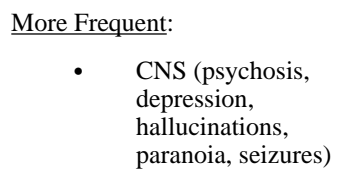 & $\begin{array}{ll}\text { - } & \text { Rash } \\
\text { - } & \text { GI disturbances } \\
\text { (abdominal pain, } \\
\text { constipation, } \\
\text { diarrhea, }\end{array}$ & $\begin{array}{l}\text { Side effects less prominent } \\
\text { in children. } \\
\text { Administer with food and } \\
\text { plenty of water. } \\
\text { Tablets can be crushed } \\
\text { and added to food; bitter }\end{array}$ \\
\hline
\end{tabular}




\begin{tabular}{|c|c|c|c|c|}
\hline \multirow[t]{2}{*}{ Drug } & \multirow[t]{2}{*}{ Preparations } & \multicolumn{2}{|l|}{ Major Toxicities ${ }^{a}$} & \multirow[t]{2}{*}{ Special Instructions } \\
\hline & & $\begin{array}{l}\text { Indicating Need for Medical } \\
\text { Attention }\end{array}$ & $\begin{array}{l}\text { Indicating Need for } \\
\text { Medical Attention if } \\
\text { Persistent or Bothersome }\end{array}$ & \\
\hline & & $\begin{aligned} \text { Rare: } & \\
\text { - } & \text { Blood dyscrasias } \\
\text { - } & \begin{array}{l}\text { Cholestasis, elevated } \\
\text { bilirubin }\end{array} \\
& \end{aligned}$ & $\begin{array}{ll} & \text { anorexia, nausea, } \\
& \text { vomiting) } \\
\text { - } & \text { CNS (dizziness, } \\
\text { vivid dreams, } \\
\text { insomnia) } \\
\text { - } \quad \begin{array}{l}\text { Tinnitus, blurred } \\
\text { vision }\end{array}\end{array}$ & $\begin{array}{l}\text { tasting so administer with } \\
\text { foods that can mask the } \\
\text { taste } \\
\text { Monitor LFTs. }\end{array}$ \\
\hline Nitazoxanide (Alinia) & $\begin{array}{l}\text { Oral Suspension: } \\
\qquad \quad 20 \mathrm{mg} / \mathrm{mL} \\
\underline{\text { Tablets: }} \\
\qquad \quad 500 \mathrm{mg}\end{array}$ & N/A & \begin{aligned} & \multicolumn{2}{l}{ More Frequent: } \\
& - $\begin{array}{l}\text { GI disturbances } \\
\text { (abdominal pain, } \\
\\
\text { nausea, vomiting) }\end{array} \\
&$ - Headache \\
& Rare: \\
& - Scleral icterus \\
& - Rash \end{aligned} & $\begin{array}{l}\text { Should be given with } \\
\text { food. } \\
\text { Shake suspension well } \\
\text { prior to dosing. }\end{array}$ \\
\hline P-Aminosalicyclic Acid (Paser) & $\frac{\text { Delayed Release Granules: }}{\text { - } 4 \text { g per packet }}$ & $\begin{array}{ll}\text { Rare: } & \\
\text { - } & \text { Hypersensitivity } \\
& \text { (fever, skin rash, } \\
& \text { exfoliative dermatitis, } \\
& \text { mono-like or } \\
& \text { lymphoma-like } \\
\text { syndrome, jaundice, } & \text { hepatitis, pericarditis, } \\
& \text { vasculitis, } \\
\text { hematologic } \\
\text { abnormalities } \\
\text { including hemolytic } \\
\text { anemia, } \\
\text { hypoglycemia, optic } \\
\text { neuritis, } \\
\text { encephalopathy, } \\
\text { reduction in } \\
\text { prothrombin) } \\
\text { Crystalluria } \\
\text { Hemolytic anemia }\end{array}$ & $\begin{array}{l}\text { GI disturbances } \\
\text { (abdominal pain, } \\
\text { nausea, vomiting, } \\
\text { diarrhea) }\end{array}$ & $\begin{array}{l}\text { Should not be } \\
\text { administered to patients } \\
\text { with severe renal disease. } \\
\text { Drug should be } \\
\text { discontinued at first sign } \\
\text { of hypersensitivity } \\
\text { reaction (rash, fever, and } \\
\text { GI symptoms typically } \\
\text { precede jaundice). } \\
\text { Vitamin B12 therapy } \\
\text { should be considered in } \\
\text { patients receiving for >1 } \\
\text { month. } \\
\text { Administer granules by } \\
\text { sprinkling on acidic foods } \\
\text { such as applesauce or } \\
\text { yogurt or a fruit drink like } \\
\text { tomato or orange juice. } \\
\text { Maintain urine at neutral } \\
\text { or alkaline pH to avoid } \\
\text { crystalluria. } \\
\text { The granule soft } \\
\text { "skeleton" may be seen in } \\
\text { the stool. } \\
\text { Monitor CBC and LFTs. }\end{array}$ \\
\hline $\begin{array}{l}\text { Pegylated Interferon Alfa-2A } \\
\text { (Pegasys) }\end{array}$ & $\begin{array}{cl}\text { Injection: } & \\
- & \begin{array}{l}\text { Vials and } \\
\text { prefilled } \\
\text { syringes }\end{array}\end{array}$ & $\begin{array}{ll}\text { More Frequent: } \\
\text { - } & \begin{array}{l}\text { Hematologic toxicity } \\
\text { (leukopenia, } \\
\text { thrombocytopenia) }\end{array} \\
\bullet \quad \begin{array}{l}\text { Neurotoxicity } \\
\text { (confusion, } \\
\text { depression, insomnia, } \\
\text { anxiety) }\end{array} \\
\text { - } \quad \text { Injection erythema } \\
\text { Less Frequent: } \\
\text { - } \\
\begin{array}{l}\text { Cardiovascular } \\
\text { effects (chest pain, } \\
\text { hypertension, } \\
\text { arrhythmias, } \\
\text { hypotension) }\end{array}\end{array}$ & 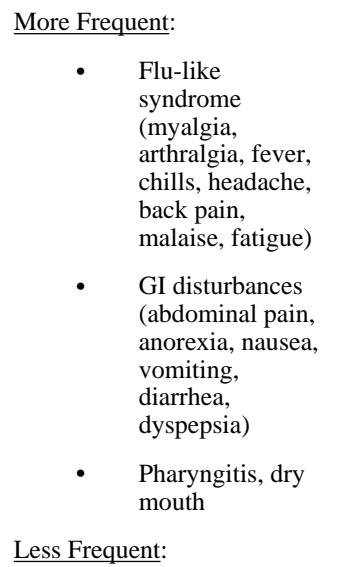 & $\begin{array}{l}\text { Toxicity dose-related. } \\
\text { Dose modifications based } \\
\text { on type and degree of } \\
\text { toxicity. } \\
\text { For non-life threatening } \\
\text { reactions, reduce dose or } \\
\text { temporarily discontinue } \\
\text { drug and restart at low } \\
\text { doses with stepwise } \\
\text { increases. } \\
\text { If patients have visual } \\
\text { complaints, an } \\
\text { ophthalmologic exam } \\
\text { should be performed to } \\
\text { detect possible retinal } \\
\text { hemorrhage or retinal } \\
\text { artery or vein obstruction. } \\
\text { Should not be used in } \\
\text { children with } \\
\text { decompensated hepatic }\end{array}$ \\
\hline
\end{tabular}




\begin{tabular}{|c|c|c|c|c|}
\hline \multirow[t]{2}{*}{ Drug } & Preparations & \multicolumn{2}{|l|}{ Major Toxicities ${ }^{a}$} & \multirow[t]{2}{*}{ Special Instructions } \\
\hline & & $\begin{array}{l}\text { Indicating Need for Medical } \\
\text { Attention }\end{array}$ & $\begin{array}{l}\text { Indicating Need for } \\
\text { Medical Attention if } \\
\text { Persistent or Bothersome }\end{array}$ & \\
\hline & & $\begin{array}{cl}\text { - } & \begin{array}{l}\text { Hypoesthesia/ } \\
\text { paresthesia }\end{array} \\
\text { Rare: } & \\
\text { - } & \begin{array}{l}\text { Vision abnormalities } \\
\text { or loss of vision }\end{array} \\
\text { - } & \begin{array}{l}\text { Allergic reaction } \\
\text { (rash, hives) }\end{array} \\
\text { - } & \text { Hypothyroidism } \\
\text { - } & \begin{array}{l}\text { Development of } \\
\text { antinuclear } \\
\text { antibodies }\end{array}\end{array}$ & $\begin{array}{ll}- & \text { Alopecia } \\
\text { - } & \text { Epistaxis } \\
\text { - } & \text { Elevated serum } \\
\text { transaminases, } \\
\text { serum creatinine } \\
\text { and BUN, } \\
\text { glucose, } \\
\text { triglycerides }\end{array}$ & $\begin{array}{l}\text { disease, significant } \\
\text { cytopenia, autoimmune } \\
\text { disease, or significant pre- } \\
\text { existing renal or cardiac } \\
\text { disease. } \\
\text { If symptoms of hepatic } \\
\text { decompensation occur } \\
\text { (ascites, coagulopathy, } \\
\text { jaundice),Peg- IFN-a-2A } \\
\text { should be discontinued. } \\
\text { Monitor CBC, renal } \\
\text { function, LFTs, thyroid } \\
\text { function, and glucose. } \\
\text { Store vials and syringes in } \\
\text { refrigerator. Protect from } \\
\text { light. } \\
\text { Administer SQ in } \\
\text { abdomen or thigh. Rotate } \\
\text { injection sites. }\end{array}$ \\
\hline $\begin{array}{l}\text { Pegylated Interferon Alfa-2B } \\
\text { (Pegintron) }\end{array}$ & $\begin{array}{cl}\text { Injection: } & \\
\bullet & \begin{array}{l}\text { Vials and } \\
\text { prefilled } \\
\text { syringes }\end{array}\end{array}$ & 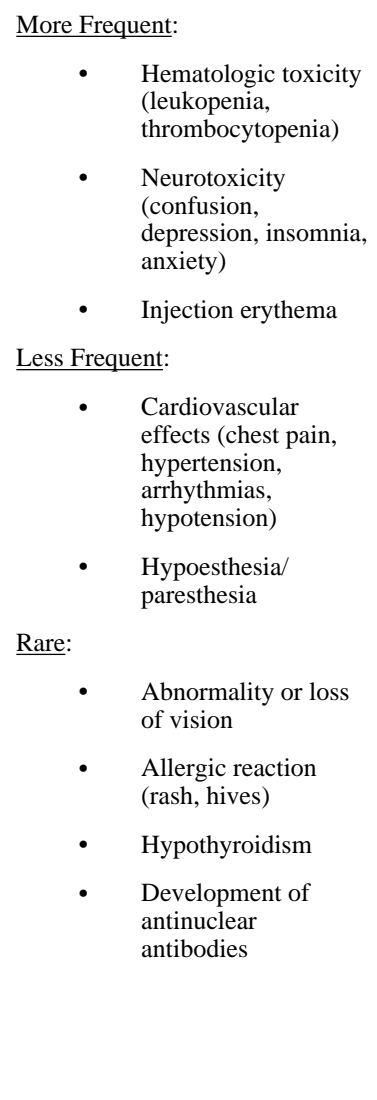 & 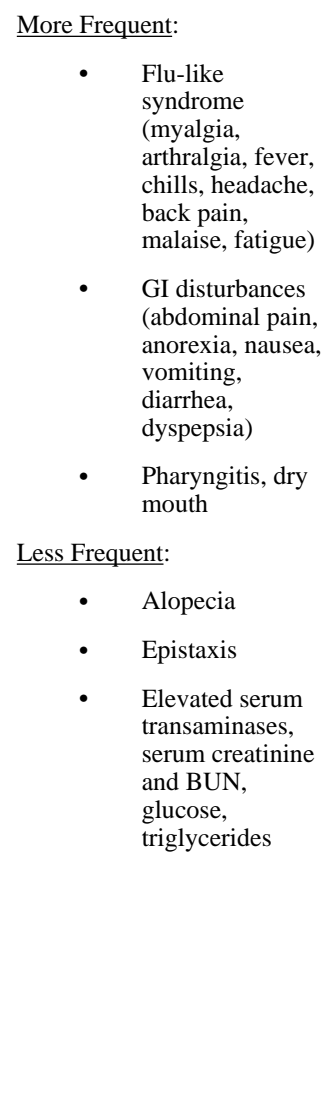 & $\begin{array}{l}\text { Toxicity dose-related. } \\
\text { Dose modifications based } \\
\text { on type and degree of } \\
\text { toxicity. } \\
\text { For non-life threatening } \\
\text { reactions, reduce dose or } \\
\text { temporarily discontinue } \\
\text { drug and restart at low } \\
\text { doses with stepwise } \\
\text { increases. } \\
\text { If patients have visual } \\
\text { complaints, an } \\
\text { ophthalmologic exam } \\
\text { should be performed to } \\
\text { detect possible retinal } \\
\text { hemorrhage or retinal } \\
\text { artery or vein obstruction. } \\
\text { Should not be used in } \\
\text { children with } \\
\text { decompensated hepatic } \\
\text { disease, significant } \\
\text { cytopenia, autoimmune } \\
\text { disease, or significant pre- } \\
\text { existing renal or cardiac } \\
\text { disease. } \\
\text { If symptoms of hepatic } \\
\text { decompensation occur } \\
\text { (ascites, coagulopathy, } \\
\text { jaundice),Peg- IFN-a-2A } \\
\text { should be discontinued. } \\
\text { Monitor CBC, renal } \\
\text { function, LFTs, thyroid } \\
\text { function, and glucose. } \\
\text { Store vials and syringes in } \\
\text { refrigerator. Protect from } \\
\text { light. } \\
\text { Administer SQ in } \\
\text { abdomen or thigh. Rotate } \\
\text { injection sites. }\end{array}$ \\
\hline Pentamidine (Pentam) & $\begin{array}{l}\text { IV } \\
\text { Aerosol }\end{array}$ & $\begin{array}{ll}\frac{\text { IV }}{\text { More }} & \text { Frequent: } \\
\text { • } & \text { Nephrotoxicity } \\
\text { - } & \text { Hypoglycemia } \\
\text { - } & \begin{array}{l}\text { Hyperglycemia or } \\
\text { diabetes mellitus }\end{array}\end{array}$ & $\begin{array}{l}\frac{\text { IV }}{\text { More Frequent: }} \\
\qquad \begin{array}{l}\text { GI disturbances } \\
\text { (anorexia, } \\
\text { nausea, vomiting, } \\
\text { diarrhea) }\end{array} \\
\text { Less Frequent: }\end{array}$ & $\begin{array}{l}\text { Rapid infusion may result } \\
\text { in precipitous } \\
\text { hypotension; IV infusion } \\
\text { should be administered } \\
\text { over } \geq 1 \text { hour (preferably } 2 \\
\text { hours). } \\
\text { Cytolytic effect on } \\
\text { pancreatic beta islet cells, } \\
\text { leading to insulin release, } \\
\text { can result in prolonged }\end{array}$ \\
\hline
\end{tabular}




\begin{tabular}{|c|c|c|c|c|}
\hline \multirow[t]{2}{*}{ Drug } & \multirow[t]{2}{*}{ Preparations } & \multicolumn{2}{|l|}{ Major Toxicities $a$} & \multirow[t]{2}{*}{ Special Instructions } \\
\hline & & $\begin{array}{l}\text { Indicating Need for Medical } \\
\text { Attention }\end{array}$ & $\begin{array}{l}\text { Indicating Need for } \\
\text { Medical Attention if } \\
\text { Persistent or Bothersome }\end{array}$ & \\
\hline & & $\begin{array}{cl} & \begin{array}{l}\text { Elevated liver } \\
\text { transaminases }\end{array} \\
\text { - } & \text { Hypotension } \\
\text { - } & \text { Leukopenia or } \\
& \text { neutropenia } \\
\text { - } & \text { Thrombocytopenia } \\
\text { Less Frequent: } \\
\text { - } & \text { Anemia } \\
\text { - } & \text { Cardiac arrhythmias } \\
\text { - } & \begin{array}{l}\text { Hypersensitivity } \\
\text { (skin rash, fever) }\end{array} \\
\text { - } & \text { Pancreatitis } \\
\text { - } & \text { Phlebitis } \\
\text { - } & \begin{array}{l}\text { Sterile abscess (at } \\
\text { site injection) }\end{array} \\
\text { Aresol } & \\
\text { More Frequent: } \\
\text { - } & \text { Sneezing } \\
\text { - } & \text { Cough } \\
& \end{array}$ & $\begin{array}{ll} & \begin{array}{l}\text { Unpleasant } \\
\text { metallic taste }\end{array} \\
\text { Aresol } & \\
\text { More Frequent: } \\
\qquad \quad \text { Bronchospasm }\end{array}$ & \begin{tabular}{l} 
severe hypoglycemia \\
(usually occurs after 5-7 \\
days of therapy, but can \\
also occur after the drug is \\
discontinued); risk \\
increased with higher \\
dose, longer duration of \\
therapy, and re-treatment \\
within 3 months of prior \\
treatment. \\
Hyperglycemia and \\
diabetes mellitus can \\
occur up to several months \\
after drug discontinued. \\
Monitor LFTs, renal \\
function, glucose, \\
electrolytes, BP. \\
Inhalation: \\
\multicolumn{1}{c}{ A special } \\
nebulizer is \\
required for \\
aerosol \\
administration. \\
Medical \\
personnel \\
should be \\
trained in the \\
proper \\
administration \\
of aerosolized \\
pentamidine.
\end{tabular} \\
\hline Posaconazole (Noxafil) & $\begin{array}{l}\text { Oral Solution: } \\
\qquad \quad 40 \mathrm{mg} / \mathrm{mL}\end{array}$ & 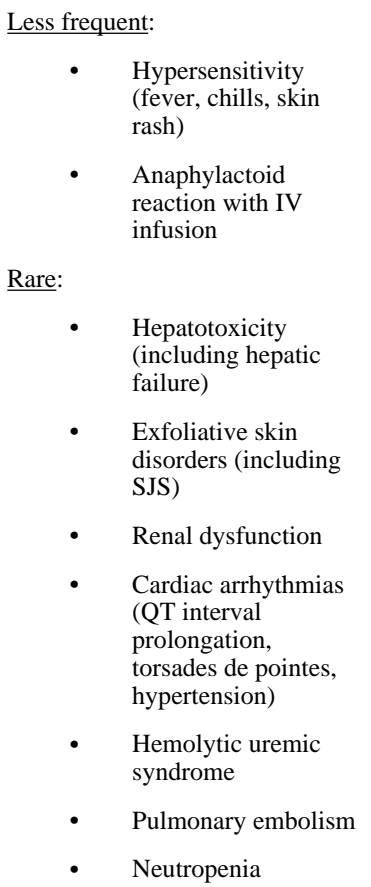 & 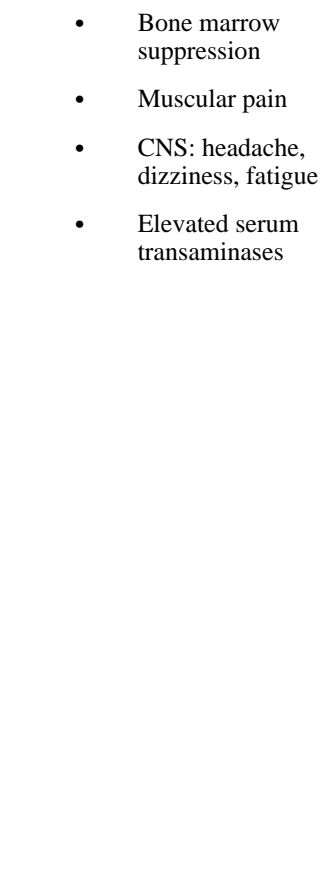 & $\begin{array}{l}\text { Must be given with meals. } \\
\text { Adequate absorption is } \\
\text { dependent on food for } \\
\text { efficacy. } \\
\text { Monitor LFTs, renal } \\
\text { function and electrolytes. } \\
\text { Monitor serum drug } \\
\text { concentrations (TDM). } \\
\text { Shake suspension prior to } \\
\text { dosing. }\end{array}$ \\
\hline
\end{tabular}




\begin{tabular}{|c|c|c|c|c|}
\hline \multirow[t]{2}{*}{ Drug } & \multirow[t]{2}{*}{ Preparations } & \multicolumn{2}{|l|}{ Major Toxicities $^{a}$} & \multirow[t]{2}{*}{ Special Instructions } \\
\hline & & $\begin{array}{l}\text { Indicating Need for Medical } \\
\text { Attention }\end{array}$ & $\begin{array}{l}\text { Indicating Need for } \\
\text { Medical Attention if } \\
\text { Persistent or Bothersome }\end{array}$ & \\
\hline Primaquine & 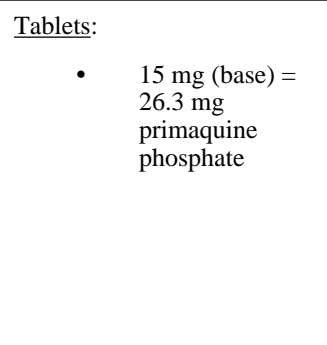 & $\begin{array}{l}\text { More Frequent: } \\
\qquad \quad \begin{array}{l}\text { Hemolytic anemia } \\
\text { (with G6PD } \\
\text { deficiency) }\end{array} \\
\text { Less Frequent: } \\
\text { • } \quad \text { Methemoglobinemia } \\
\underline{\text { Rare: }} \\
\text { • } \quad \text { Leukopenia }\end{array}$ & $\begin{array}{l}\text { GI disturbances } \\
\text { (nausea, } \\
\text { vomiting) }\end{array}$ & $\begin{array}{l}\text { Take with meals or } \\
\text { antacids to minimize } \\
\text { gastric irritation. } \\
\text { Store in a light-resistant } \\
\text { container. } \\
\text { Bitter taste. } \\
\text { Monitor CBC. }\end{array}$ \\
\hline Pyrazinamide & $\begin{array}{l}\text { Tablets: } \\
\qquad \quad 500 \mathrm{mg} \\
\text { Oral Suspension: } \\
\qquad \quad \begin{array}{l}\text { Extemporaneous } \\
\text { preparation }\end{array}\end{array}$ & $\begin{array}{l}\text { More Frequent: } \\
\qquad \quad \text { Arthralgia } \\
\text { Less Frequent: } \\
\qquad \begin{array}{l}\text { Hepatotoxicity (dose- } \\
\text { related) }\end{array} \\
\text { Rare: } \\
\text { • } \quad \begin{array}{l}\text { Acute gouty arthritis } \\
\text { secondary to } \\
\text { hyperuricemia }\end{array} \\
\text { - } \quad \begin{array}{l}\text { Thrombocytopenia, } \\
\text { anemia }\end{array} \\
\text { - } \quad \begin{array}{l}\text { Interstitial nephritis } \\
\text { Porphyria }\end{array}\end{array}$ & $\begin{array}{ll}\text { - } & \begin{array}{l}\text { Skin rash, } \\
\text { pruritus }\end{array} \\
\text { - } & \text { Photosensitivity } \\
\text { - } & \text { Malaise } \\
\text { - } & \begin{array}{l}\text { GI disturbances } \\
\text { (nausea, }\end{array} \\
\text { vomiting) } \\
\text { - } & \text { Arthralgia } \\
\text { - } & \text { Hyperuricemia }\end{array}$ & $\begin{array}{l}\text { Avoid in patients with } \\
\text { severe hepatic impairment. } \\
\text { Reduce dose in patients } \\
\text { with renal or hepatic } \\
\text { impairment. } \\
\text { Monitor LFTs and uric } \\
\text { acid. }\end{array}$ \\
\hline Pyrimethamine (Daraprim) & $\begin{array}{l}\text { Tablet: } \\
\qquad \quad 25 \mathrm{mg} \\
\text { Oral Suspension: } \\
\qquad \quad \begin{array}{l}\text { Extemporaneous } \\
\text { preparation }\end{array}\end{array}$ & \begin{tabular}{cl}
\multicolumn{2}{l}{ Less Frequent: } \\
• & Neutropenia \\
• & Thrombocytopenia \\
• & $\begin{array}{l}\text { Megaloblastic } \\
\text { anemia }\end{array}$ \\
Rare: & \\
- & SJS \\
- & Seizure
\end{tabular} & $\begin{array}{ll}\text { - } & \text { Skin rash } \\
\text { - } & \text { Photosensitivity } \\
\text { - } & \text { Dry mouth } \\
\text { - } & \begin{array}{l}\text { GI disturbances } \\
\text { (nausea, }\end{array} \\
\text { vomiting) } \\
\text { - } \quad \text { CNS (depression, } \\
\text { insomnia }\end{array}$ & $\begin{array}{l}\text { To prevent hematologic } \\
\text { toxicity, administer with } \\
\text { leucovorin. } \\
\text { Monitor CBC. }\end{array}$ \\
\hline Quinidine & IV & $\begin{aligned} \text { Serious: } & \\
\text { - } & \text { Cardiac arrhythmias } \\
\text { - } & \text { QT interval } \\
& \text { prolongation } \\
- & \text { Hypoglycemia } \\
\text { - } & \begin{array}{l}\text { Hemolytic anemia } \\
\text { (with G6PD } \\
\text { deficiency) } \\
\text { - }\end{array} \\
& \end{aligned}$ & $\begin{array}{l}\text { Very Frequent: } \\
\text { Cinchonism- } \\
\text { syndrome of } \\
\text { tinnitus, } \\
\text { reversible high- } \\
\text { frequency } \\
\text { hearing loss, } \\
\text { deafness, vertigo, } \\
\text { blurred vision, } \\
\text { diplopia, } \\
\text { photophobia, } \\
\text { headache, } \\
\text { confusion, and } \\
\text { delirium; dose } \\
\text { dependent }\end{array}$ & $\begin{array}{l}\text { EKG monitoring is } \\
\text { standard of care. } \\
\text { Do not give by bolus } \\
\text { infusion. } \\
\text { If EKG changes observed, } \\
\text { slow infusion rate. } \\
\text { Monitor CBC and LFTs. }\end{array}$ \\
\hline $\begin{array}{l}\text { Ribavirin } \\
\text { Virazole } \\
\text { Powder for solution for nebulization }\end{array}$ & $\begin{array}{l}\text { Powder for Solution for } \\
\text { Nebulization: }\end{array}$ & $\begin{array}{l}\text { Hemolytic anemia } \\
\text { (with associated } \\
\text { potential for increase }\end{array}$ & $\begin{array}{l}\text { CNS effects } \\
\text { (fatigue, } \\
\text { headache, }\end{array}$ & $\begin{array}{l}\text { Should not be used in } \\
\text { patients with severe renal } \\
\text { impairment. }\end{array}$ \\
\hline
\end{tabular}




\begin{tabular}{|c|c|c|c|c|}
\hline \multirow[t]{2}{*}{ Drug } & \multirow[t]{2}{*}{ Preparations } & \multicolumn{2}{|l|}{ Major Toxicities ${ }^{a}$} & \multirow[t]{2}{*}{ Special Instructions } \\
\hline & & $\begin{array}{l}\text { Indicating Need for Medical } \\
\text { Attention }\end{array}$ & $\begin{array}{l}\text { Indicating Need for } \\
\text { Medical Attention if } \\
\text { Persistent or Bothersome }\end{array}$ & \\
\hline $\begin{array}{l}\text { Rebetol } \\
\text { Oral capsules and oral solution } \\
\text { Copegus, Ribasphere, Ribapak } \\
\text { Oral tablets and capsules }\end{array}$ & 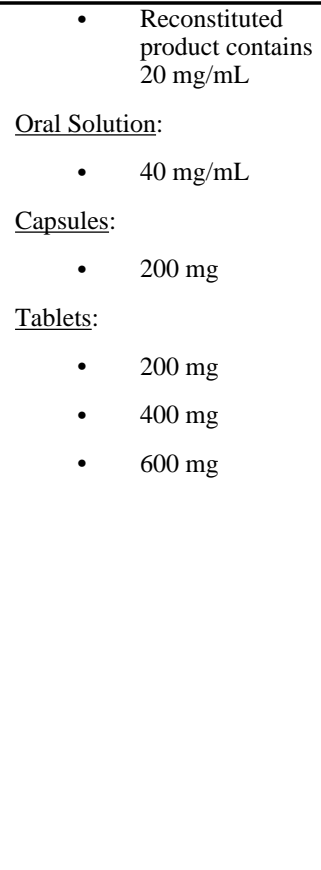 & $\begin{array}{l}\text { in unconjugated } \\
\begin{array}{l}\text { bilirubin and uric } \\
\text { acid) }\end{array} \\
\text { Less Frequent: } \\
\text { - } \quad \begin{array}{l}\text { Neutropenia, } \\
\text { thrombocytopenia, } \\
\text { anemia }\end{array} \\
\text { - } \quad \text { Pancreatitis }\end{array}$ & 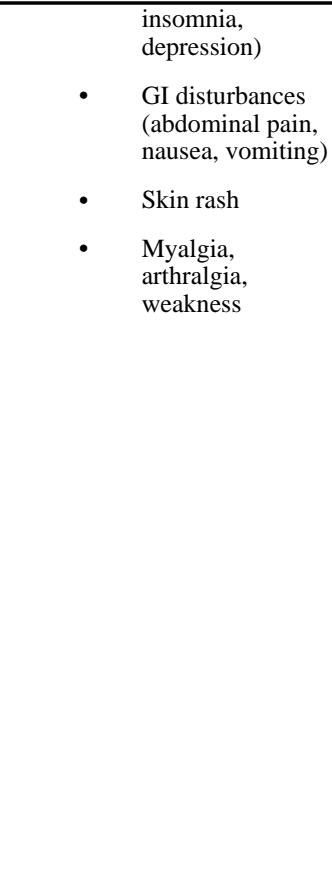 & $\begin{array}{l}\text { Should not be used as } \\
\text { monotherapy for treatment } \\
\text { of hepatitis C, but used in } \\
\text { combination with IFN-a. } \\
\text { Intracellular } \\
\text { phosphorylation of } \\
\text { pyrimidine nucleoside } \\
\text { analogues (zidovudine, } \\
\text { stavudine, zalcitabine) } \\
\text { decreased by ribavirin, } \\
\text { may have antagonism; use } \\
\text { with caution. } \\
\text { Enhances phosphorylation } \\
\text { of didanosine; use with } \\
\text { caution because of } \\
\text { increased risk of } \\
\text { pancreatitis/mitochondrial } \\
\text { toxicity. } \\
\text { Oral solution contains } \\
\text { propylene glycol. } \\
\text { Teratogenic/embryocidal. } \\
\text { Contraindicated in } \\
\text { pregnant women and their } \\
\text { male partners. Avoid } \\
\text { pregnancy for additional } 6 \\
\text { months after treatment. } \\
\text { Monitor CBC, renal } \\
\text { function, LFTs, and } \\
\text { thyroid function. Perform } \\
\text { pregnancy tests regularly } \\
\text { while on therapy. }\end{array}$ \\
\hline Rifabutin (Mycobutin) & $\begin{array}{l}\frac{\text { Capsules: }}{\bullet} \quad 150 \mathrm{mg} \\
\frac{\text { Oral Suspension: }}{\bullet \quad \begin{array}{l}\text { Extemporaneous } \\
\text { preparation }\end{array}}\end{array}$ & \begin{tabular}{cc}
\multicolumn{2}{l}{ More Frequent: } \\
• & $\begin{array}{l}\text { Allergic reaction } \\
\text { (rash, pruritus) }\end{array}$ \\
• & Neutropenia \\
Less Frequent: \\
- & Asthenia \\
Rare: & \\
- & Arthralgia, myalgia \\
- & Change in taste \\
- & Pseudojaundice \\
- & Thrombocytopenia \\
- & Uveitis
\end{tabular} & 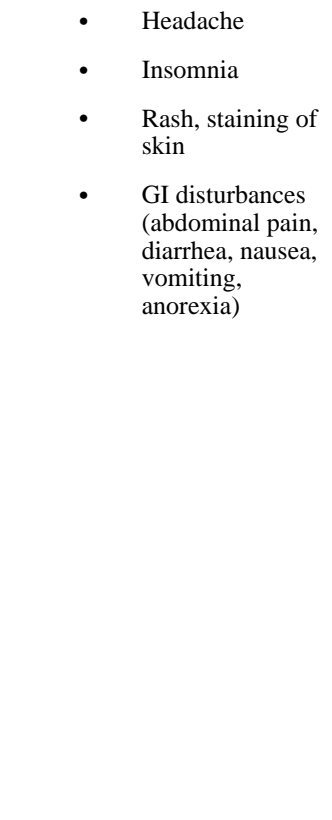 & $\begin{array}{l}\text { Preferably take on empty } \\
\text { stomach, but may be } \\
\text { administered with food in } \\
\text { patients with GI } \\
\text { intolerance. } \\
\text { The contents of capsules } \\
\text { may be mixed with } \\
\text { applesauce if patient is } \\
\text { unable to swallow capsule. } \\
\text { May cause reddish to } \\
\text { brown-orange color urine, } \\
\text { feces, saliva, sweat, skin, } \\
\text { or tears (can discolor soft } \\
\text { contact lenses). } \\
\text { Uveitis seen with high- } \\
\text { dose rifabutin (i.e., adults } \\
>300 \text { mg/ day), especially } \\
\text { when combined with } \\
\text { clarithromycin. } \\
\text { Multiple potential drug } \\
\text { interactions } \\
\text { Use with caution in } \\
\text { patients with renal or } \\
\text { hepatic impairment. } \\
\text { Monitor CBC, LFTs; } \\
\text { conduct ophthalmologic } \\
\text { examinations. } \\
\text { Reduce dose in patients } \\
\text { with renal impairment. }\end{array}$ \\
\hline Rifampin (Rifadin) & \begin{tabular}{ll}
\multicolumn{2}{l}{ Oral Suspension: } \\
$\qquad \begin{array}{l}\text { Extemporaneous } \\
\text { preparation }\end{array}$ \\
Capsules: \\
$\cdot \quad 150 \mathrm{mg}$
\end{tabular} & $\begin{array}{l}\text { Less Frequent: } \\
\text { • } \quad \text { Flu-like syndrome } \\
\underline{\text { Rare: }} \\
\text { • } \quad \text { Blood dyscrasias }\end{array}$ & $\begin{array}{l}\text { - } \begin{array}{l}\text { GI disturbances } \\
\text { (abdominal pain, } \\
\text { diarrhea) }\end{array} \\
\text { - } \quad \begin{array}{l}\text { CNS effects } \\
\text { (fatigue, } \\
\text { headache, }\end{array}\end{array}$ & $\begin{array}{l}\text { Preferably take on empty } \\
\text { stomach, but can be } \\
\text { administered with food in } \\
\text { patients with GI } \\
\text { intolerance; take with full } \\
\text { glass of water. }\end{array}$ \\
\hline
\end{tabular}




\begin{tabular}{|c|c|c|c|c|}
\hline \multirow[t]{2}{*}{ Drug } & \multirow[t]{2}{*}{ Preparations } & \multicolumn{2}{|l|}{ Major Toxicities ${ }^{a}$} & \multirow[t]{2}{*}{ Special Instructions } \\
\hline & & $\begin{array}{l}\text { Indicating Need for Medical } \\
\text { Attention }\end{array}$ & $\begin{array}{l}\text { Indicating Need for } \\
\text { Medical Attention if } \\
\text { Persistent or Bothersome }\end{array}$ & \\
\hline & $\begin{array}{ll} & \bullet 300 \mathrm{mg} \\
\mathrm{IV} & \end{array}$ & $\begin{array}{ll}\text { - } & \begin{array}{l}\text { Hepatitis prodromal } \\
\text { syndrome (anorexia, } \\
\text { nausea, vomiting, } \\
\text { weakness) }\end{array} \\
\text { - } & \text { Hepatitis } \\
\text { - } & \text { Interstitial nephritis } \\
\text { - } & \begin{array}{l}\text { Exfoliative skin } \\
\text { disorders (including } \\
\text { SJS) }\end{array}\end{array}$ & $\begin{array}{ll} & \begin{array}{l}\text { insomnia, } \\
\text { depression) }\end{array} \\
\text { - } & \text { Rash } \\
\text { - } & \begin{array}{l}\text { Discoloration of } \\
\text { body fluids }\end{array} \\
\text { - } & \begin{array}{l}\text { Elevated serum } \\
\text { transaminases }\end{array} \\
\text { - } & \text { Visual changes }\end{array}$ & $\begin{array}{l}\text { Suspension formulation } \\
\text { stable for } 30 \text { days. Shake } \\
\text { well prior to dosing. } \\
\text { May cause reddish to } \\
\text { brown-orange color urine, } \\
\text { feces, saliva, sweat, skin, } \\
\text { or tears (can discolor soft } \\
\text { contact lenses). } \\
\text { Multiple potential drug } \\
\text { interactions } \\
\text { Use with caution in } \\
\text { patients with hepatic } \\
\text { impairment. } \\
\text { Administer IV by slow } \\
\text { infusion. Extravasation } \\
\text { may cause local irritation } \\
\text { and inflammation. } \\
\text { Monitor CBC and LFTs. }\end{array}$ \\
\hline Streptomycin & $\mathrm{IM}$ & 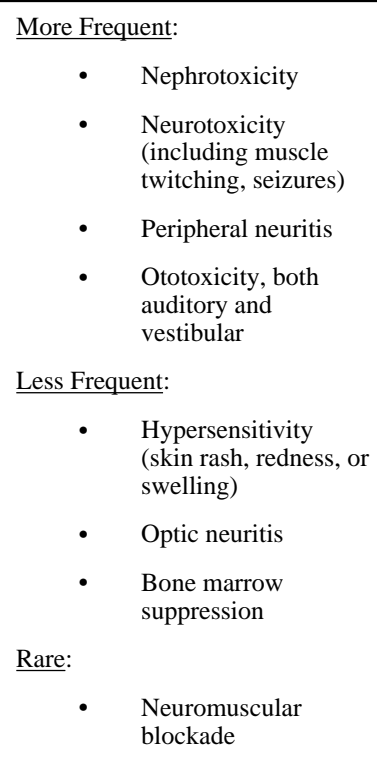 & $\begin{array}{l}\text { - CNS effects } \\
\text { (headache, } \\
\text { ataxia, dizziness ) }\end{array}$ & $\begin{array}{l}\text { Usual route of } \\
\text { administration is deep IM } \\
\text { injection into large muscle } \\
\text { mass. } \\
\text { For patients who cannot } \\
\text { tolerate IM injections, } \\
\text { dilute to } 12-15 \mathrm{mg} \text { in } 100 \\
\text { mL of } 0.9 \% \text { sodium } \\
\text { chloride; must be infused } \\
\text { over } 30 \text { to } 60 \text { minutes to } \\
\text { avoid neuromuscular } \\
\text { blockade. } \\
\text { Requires dose adjustment } \\
\text { in patients with impaired } \\
\text { renal function. } \\
\text { Monitor renal function and } \\
\text { hearing periodically (e.g., } \\
\text { monthly) in children on } \\
\text { prolonged therapy. } \\
\text { Monitor serum } \\
\text { concentrations (TDM). }\end{array}$ \\
\hline Sulfadiazine & $\begin{array}{l}\text { Tablet: } \\
\qquad \quad 500 \mathrm{mg} \\
\frac{\text { Oral Suspension: }}{\bullet \quad \begin{array}{l}\text { Extemporaneous } \\
\text { preparation }\end{array}}\end{array}$ & $\begin{array}{ll}\text { Rare: } & \\
\text { - } & \begin{array}{l}\text { Crystalluria, renal } \\
\text { failure }\end{array} \\
\text { - } & \text { Bone marrow } \\
& \begin{array}{l}\text { suppression/ blood } \\
\text { dyscrasias }\end{array} \\
\text { - } & \begin{array}{l}\text { Severe } \\
\text { hypersensitivity } \\
\text { syndrome }\end{array} \\
\text { - } & \begin{array}{l}\text { Hemolytic anemia } \\
\text { (with G6PD } \\
\text { deficiency) }\end{array}\end{array}$ & $\begin{array}{ll}\text { - } & \begin{array}{l}\text { GI disturbances } \\
\text { (abdominal pain, } \\
\text { diarrhea, nausea) }\end{array} \\
\text { - } & \begin{array}{l}\text { CNS effects } \\
\text { (headache, } \\
\text { dizziness) }\end{array} \\
\text { - } & \text { Rash } \\
\text { - } & \text { Photosensitivity }\end{array}$ & $\begin{array}{l}\text { Ensure adequate fluid } \\
\text { intake to avoid } \\
\text { crystalluria. } \\
\text { Monitor CBC, renal } \\
\text { function, and urinalysis. } \\
\text { Monitor serum } \\
\text { concentrations (TDM) if } \\
\text { serious infection. }\end{array}$ \\
\hline $\begin{array}{l}\text { Trimethoprim-Sulfameth-oxazole } \\
\text { (TMP-SMX) (Bactrim, Septra) }\end{array}$ & $\begin{array}{l}\text { Oral Suspension: } \\
\begin{array}{l}\text { TMP } 8 \mathrm{mg} / \mathrm{mL} \\
\text { and SMX 40 } \\
\mathrm{mg} / \mathrm{mL}\end{array}\end{array}$ & $\begin{array}{l}\text { More Frequent: } \\
\quad \quad \text { Skin rash } \\
\text { Less Frequent: }\end{array}$ & $\begin{array}{ll}\text { - } & \text { GI disturbances } \\
\text { (anorexia, } \\
\text { nausea, vomiting, } \\
\text { diarrhea) }\end{array}$ & $\begin{array}{l}\text { Requires dose adjustment } \\
\text { in patients with impaired } \\
\text { renal function. } \\
\text { Maintain adequate fluid } \\
\text { intake to prevent } \\
\text { crystalluria and stone }\end{array}$ \\
\hline
\end{tabular}




\begin{tabular}{|c|c|c|c|c|}
\hline \multirow[t]{2}{*}{ Drug } & \multirow[t]{2}{*}{ Preparations } & \multicolumn{2}{|l|}{ Major Toxicities $a$} & \multirow[t]{2}{*}{ Special Instructions } \\
\hline & & $\begin{array}{l}\text { Indicating Need for Medical } \\
\text { Attention }\end{array}$ & $\begin{array}{l}\text { Indicating Need for } \\
\text { Medical Attention if } \\
\text { Persistent or Bothersome }\end{array}$ & \\
\hline & 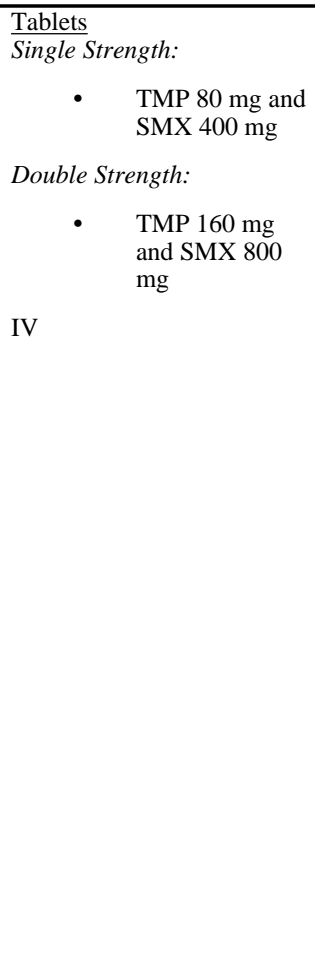 & $\begin{array}{ll} & \begin{array}{l}\text { Hypersensitivity } \\
\text { reactions (skin rash, } \\
\text { fever) }\end{array} \\
\text { - } & \begin{array}{l}\text { Hematologic toxicity } \\
\text { (leukopenia, } \\
\text { neutropenia, } \\
\text { thrombocytopenia, } \\
\text { anemia) }\end{array} \\
\text { Rare: } & \text { Exfoliative skin } \\
\text { disorders (including } \\
\text { - }\end{array}$ & $\begin{array}{ll} & \text { Rash }\end{array}$ & $\begin{array}{l}\text { formation (take with full } \\
\text { glass of water). } \\
\text { Potential for } \\
\text { photosensitivity skin } \\
\text { reaction with sun } \\
\text { exposure. } \\
\text { IV infusion over } 60 \text { to } 90 \\
\text { minutes } \\
\text { Monitor CBC, renal } \\
\text { function. }\end{array}$ \\
\hline Valacyclovir (Valtrex) & $\begin{array}{cl}\text { Tablets: } & \\
- & 500 \mathrm{mg} \\
& 1 \mathrm{~g} \\
& \text { Note: An oral } \\
& \text { suspension } \\
& \text { formulation } 50 \\
& \mathrm{mg} / \mathrm{mL} \text { can be } \\
& \text { prepared in Ora- } \\
& \text { Sweet or } \\
& \text { Syrpalta syrups) }\end{array}$ & $\begin{aligned} & \text { Rare: } \\
& \text { - } \text { Renal failure } \\
& \text { - } \text { Bone marrow } \\
& \text { suppression } \\
& \text { - } \text { Thrombotic } \\
& \text { microangiopathy/ } \text { hemolytic uremic } \\
& \text { syndrome } \\
& \text { - } \text { CNS (psychosis, } \\
& \text { seizures, delirium) }\end{aligned}$ & 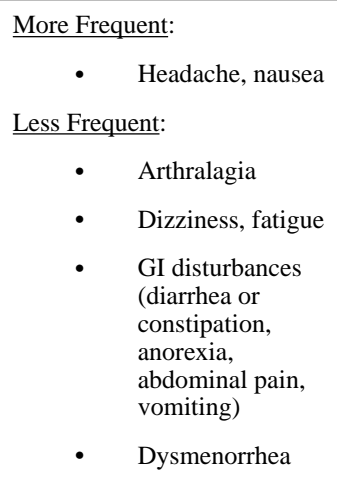 & $\begin{array}{l}\text { Thrombotic } \\
\text { thrombocytopenia } \\
\text { purpura/hemolytic uremic } \\
\text { syndrome has been } \\
\text { reported in HIV-infected } \\
\text { adults with advanced } \\
\text { disease receiving high } \\
\text { (i.e., } 8 \mathrm{~g} / \text { day) but not low } \\
\text { doses. } \\
\text { Monitor CBC and renal } \\
\text { function. }\end{array}$ \\
\hline Valganciclovir (Valcyte) & $\begin{array}{l}\frac{\text { Tablets: }}{\bullet} \quad 450 \mathrm{mg} \\
\text { Oral Solution: } \\
\qquad \quad 50 \mathrm{mg} / \mathrm{mL}\end{array}$ & \begin{tabular}{ll}
\multicolumn{2}{l}{ More Frequent: } \\
- & $\quad$ Granulocytopenia \\
- & Thrombocytopenia \\
Less Frequent: & \\
- & Anemia \\
- CNS effects \\
(seizures, psychosis, \\
hallucinations \\
- $\begin{array}{l}\text { Hypersensitivity } \\
\text { (fever, rash) }\end{array}$
\end{tabular} & $\begin{array}{l}\text { GI disturbances } \\
\text { (abdominal pain, } \\
\text { anorexia, nausea, } \\
\text { vomiting) } \\
\text { - CNS effects } \\
\text { (headache, } \\
\text { insomnia) }\end{array}$ & $\begin{array}{l}\text { Requires dose adjustment } \\
\text { in patients with renal } \\
\text { impairment. } \\
\text { Avoid other nephrotoxic } \\
\text { drugs. } \\
\text { Tablets should not be } \\
\text { broken or crushed. } \\
\text { Monitor CBC and renal } \\
\text { function. } \\
\text { Potentially teratogenic and } \\
\text { carcinogenic. }\end{array}$ \\
\hline
\end{tabular}




\begin{tabular}{|c|c|c|c|c|}
\hline \multirow[t]{2}{*}{ Drug } & Preparations & \multicolumn{2}{|l|}{ Major Toxicities $^{a}$} & \multirow[t]{2}{*}{ Special Instructions } \\
\hline & & $\begin{array}{l}\text { Indicating Need for Medical } \\
\text { Attention }\end{array}$ & $\begin{array}{l}\text { Indicating Need for } \\
\text { Medical Attention if } \\
\text { Persistent or Bothersome }\end{array}$ & \\
\hline & & $\begin{array}{ll}- & \begin{array}{l}\text { Elevated } \\
\text { transaminase } \\
\text { enzymes }\end{array} \\
\text { - } & \begin{array}{l}\text { Increase in creatinine, } \\
\text { BUN }\end{array} \\
\text { - } & \text { Retinal detachment }\end{array}$ & & \\
\hline Voriconazole (VFEND) & 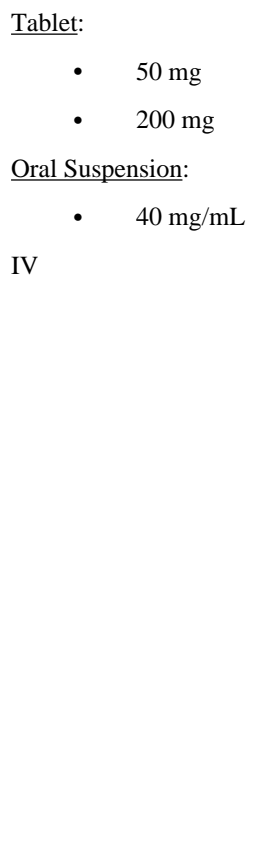 & 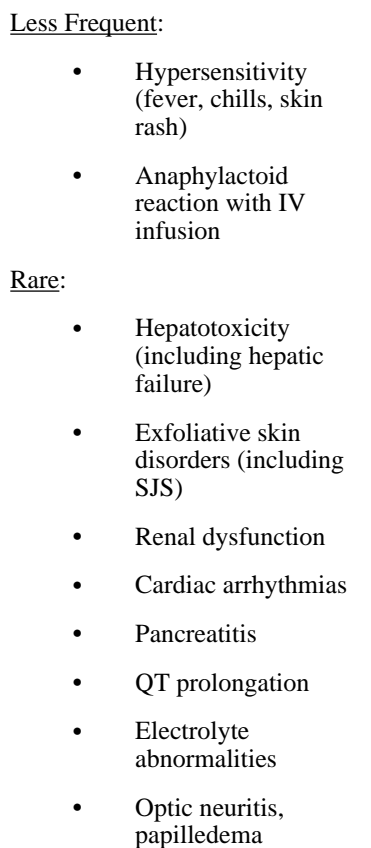 & $\begin{array}{ll}\text { More Frequent: } \\
\text { - } & \begin{array}{l}\text { Visual changes, } \\
\text { dose-related } \\
\text { (photophobia, } \\
\text { blurry vision) }\end{array} \\
\text { - } & \begin{array}{l}\text { CNS effects } \\
\text { (dizziness, } \\
\text { drowsiness, } \\
\text { headache) }\end{array} \\
\text { - } & \begin{array}{l}\text { GI disturbances } \\
\text { (abdominal pain, } \\
\text { constipation, } \\
\text { diarrhea, } \\
\text { anorexia, nausea, } \\
\text { vomiting) }\end{array} \\
\text { - } & \text { Photosensitivity } \\
\text { Rare: } & \\
\text { - } & \begin{array}{l}\text { Gynecomastia } \\
\text { - }\end{array} \\
& \begin{array}{l}\text { Elevated serum } \\
\text { transaminases }\end{array} \\
& \end{array}$ & $\begin{array}{l}\text { Oral tablets should be } \\
\text { taken } 1 \text { hour before or } \\
\text { after a meal. } \\
\text { Shake oral suspension } \\
\text { well prior to dosing. } \\
\text { Maximum IV infusion rate } \\
3 \mathrm{mg} / \mathrm{kg} / \text { hour over } 1 \text { to } 2 \\
\text { hours. } \\
\text { Oral administration to } \\
\text { patients with impaired } \\
\text { renal function if possible } \\
\text { (accumulation of IV } \\
\text { vehicle occurs in patients } \\
\text { with renal insufficiency) } \\
\text { Dose adjustment needed if } \\
\text { hepatic insufficiency. } \\
\text { Visual disturbances } \\
\text { common (>30\%) but } \\
\text { transient and reversible } \\
\text { when drug is discontinued. } \\
\text { Multiple potential drug } \\
\text { interactions } \\
\text { Monitor renal function, } \\
\text { electrolytes, and LFTs } \\
\text { Consider monitoring } \\
\text { serum concentrations } \\
\text { (TDM). }\end{array}$ \\
\hline
\end{tabular}

${ }^{a}$ The toxicities listed in the table have been selected based on their potential clinical significance and are not inclusive of all side effects reported for a particular drug.

Key to Acronyms: ARV = antiretroviral; $\mathrm{BP}=$ blood pressure; $\mathrm{BUN}=$ blood urea nitrogen; $\mathrm{CBC}=\mathrm{complete}$ blood count $\mathrm{CDC}=\mathrm{Centers}$ for Disease Control and Prevention; $\mathrm{CNS}=$ central nervous system; $\mathrm{Cr}=$ creatinine $\mathrm{CrCl}=$ creatinine clearance $\mathrm{EKG}=\mathrm{electrocardiogram} ; \mathrm{G} 6 \mathrm{PD}=$ Glucose-6-phosphate dehydrogenase; GI = gastrointestinal; IFN- = interferon alfa; IM = intramuscular; IND = investigational new drug; IV = intravenous; LFT = liver function test; SJS = Stevens-Johnson Syndrome; SMX = sulfamethoxazole; SQ = subcutaneous; TDM = therapeutic drug monitoring; $\mathrm{TMP}=$ trimethoprim 


\section{Table 5}

Significant Drug Interactions for Drugs Used to Treat or Prevent Opportunistic Infections (Last updated November 6, 2013; last reviewed November 6, 2013)

There is the potential for significant drug interactions and overlapping toxicities in patients receiving medications for treatment or prevention of opportunistic infections (OIs). These patients often are receiving other medications, including antiretrovirals that interfere with metabolism or elimination of OI medications. In particular, protease inhibitors and non-nucleoside reverse transcriptase inhibitors affect the CYP450 or other transporter systems and may be associated with clinically significant drug interactions. The integrase inhibitor raltegravir is metabolized by UGT1A1 and may be a suitable option when trying to minimize interactions with other drug classes.

Table 5 provides clinicians with information regarding known or suspected drug interactions between drugs commonly used for treatment or prevention of HIV-associated OIs and treatment of HIV infection. Drug interaction information is generally obtained from studies involving healthy adult volunteers. Some pharmacokinetic (PK) data are available from studies involving HIV-infected adults, whereas data in children are extremely limited. New information continues to become available and it is important to carefully review a patient's current medications, including prescription and over-the-counter medications. It is difficult to predict the interaction potential when three or more drugs with similar metabolic pathways are co-administered and there is substantial inter-patient variability in the magnitude of these interactions. When possible, alternative agents with less drug interaction potential or use of therapeutic drug monitoring should be considered.

Table 5 contains only a partial listing of drug interactions for drugs used to treat or prevent OIs. The links below are excellent resources for investigating the potential for drug interactions. These tools include more comprehensive information and provide up-to-date information as new PK data become available. http://www.hiv-druginteractions.org/ http://tdm.pharm.buffalo.edu/home/di_search/ http://www.aidsinfo.nih.gov/guidelines/html/1/adult-and-adolescent-arv-guidelines/32/drug-interactions/ http://www.drugs.com/drug_interactions.html http://hivinsite.ucsf.edu/InSite?page=ar-00-02 http://www.nynjaetc.org/clinical_support.html http://www.clinicaloptions.com/inPractice.aspx http://epocrates.com

\begin{tabular}{|c|c|c|}
\hline Drug Name & Overlapping Toxicities & Recommendation \\
\hline \multicolumn{3}{|c|}{$\begin{array}{l}\text { The drug interactions included in this table were selected on the basis of their potential clinical significance and are not } \\
\text { inclusive of all potential drug interactions (see drug label and the drug interaction websites listed for complete information } \\
\text { on drug interactions). }\end{array}$} \\
\hline \multirow[t]{2}{*}{ Acyclovir (Zovirax) } & $\begin{array}{l}\text { Overlapping Toxicities: } \\
\qquad \quad \text { Nephrotoxic drugs }\end{array}$ & Monitor for toxicities of these drugs. \\
\hline & $\begin{array}{l}\text { Increased Concentrations (Both Drugs) and Overlapping } \\
\text { Toxicities: } \\
\text { - Antivirals: valacyclovir, valganciclovir, ganciclovir, } \\
\text { cidofovir } \\
\text { ARVs: tenofovir }\end{array}$ & Monitor for toxicities of these drugs. \\
\hline Albendazole & Increases Albendazole Concentrations: & Caution advised. \\
\hline
\end{tabular}




\begin{tabular}{|c|c|c|}
\hline Drug Name & Overlapping Toxicities & Recommendation \\
\hline & • $\quad$ Anthelmintic drugs: praziquantel & \\
\hline Amikacin & $\begin{array}{ll}\text { Overlapping Toxicities: } \\
\text { - } & \text { Anti-tuberculosis drugs (injectable): streptomycin, } \\
& \text { kanamycin } \\
\text { - } & \text { Nephrotoxic or ototoxic drugs } \\
\text { - } & \text { Antimycobacterial drugs: capreomycin } \\
\text { - } & \text { Antivirals: cidofovir }\end{array}$ & $\begin{array}{l}\text { Caution advised. Avoid combination of } \\
\text { amikacin and cidofovir. }\end{array}$ \\
\hline $\begin{array}{l}\text { Amphotericin B } \\
\text { Amphotericin B } \\
\text { Lipid Complex } \\
\text { (Abelcet) } \\
\text { Amphotericin B } \\
\text { Liposome } \\
\text { (Ambisome) }\end{array}$ & $\begin{array}{cl}\text { Overlapping Toxicities: } \\
\text { - } & \text { Bone marrow suppressant drugs: corticosteroids } \\
\text { - } & \text { Nephrotoxic drugs } \\
\text { - } & \text { Neuromuscular blocking drugs }\end{array}$ & Caution advised. \\
\hline Atovaquone & $\begin{array}{ll}\text { Decreases Atovaquone Concentrations: } \\
\text { - } & \text { Antimycobacterial drugs: rifampin, rifabutin } \\
\text { - } & \text { ARVs: lopinavir/ritonavir, atazanavir/ritonavir } \\
\text { - } & \text { Antibiotics: doxycycline }\end{array}$ & $\begin{array}{l}\text { Co-administration of atovaquone and rifampin } \\
\text { should be avoided. }\end{array}$ \\
\hline Azithromycin & $\begin{array}{l}\text { Overlapping Toxicities: } \\
\qquad \quad \text { Artemether/lumefantrine, chloroquine, quinine }\end{array}$ & $\begin{array}{l}\text { Caution advised. Increased risk of QT } \\
\text { prolongation. }\end{array}$ \\
\hline Boceprevir & \multicolumn{2}{|c|}{$\begin{array}{l}\text { Please see Adult OI guidelines for information about drug interactions, including warnings about interactions between } \\
\text { boceprevir and HIV protease inhibitors. }\end{array}$} \\
\hline Capreomycin & $\begin{array}{ll}\text { Overlapping Toxicities: } \\
\text { • } & \text { Nephrotoxic or ototoxic drugs } \\
\text { - } & \text { Neuromuscular blocking drugs } \\
\text { - } & \text { Antibacterial drugs: aminoglycosides (parenteral) }\end{array}$ & Caution advised. \\
\hline Caspofungin & $\begin{array}{cl}\text { Decreases Caspofungin Concentrations: } \\
\text { - } & \text { Anticonvulsant drugs: phenytoin } \\
\text { - } & \text { Antimycobacterial drugs: rifampin } \\
\text { - } & \text { ARV drugs: efavirenz, nevirapine }\end{array}$ & $\begin{array}{l}\text { Increase in dose of caspofungin is } \\
\text { recommended when co-administered with } \\
\text { CYP450 inducers. }\end{array}$ \\
\hline Cidofovir & $\begin{array}{ll}\text { Overlapping Toxicities: } \\
\text { - } & \text { Antibacterial drugs: aminoglycosides } \\
\text { - } & \text { Antiviral drugs: foscarnet } \\
\text { - } & \text { Nephrotoxic drugs }\end{array}$ & Monitor for toxicities of these drugs. \\
\hline \multirow[t]{2}{*}{ Ciprofloxacin } & $\begin{array}{ll}\text { Decreases } & \text { Ciprofloxacin Absorption: } \\
\text { - } & \text { ARV drugs: didanosine } \\
\text { - } & \text { Minerals: ferrous sulfate, zinc } \\
\text { - } & \begin{array}{l}\text { Gastrointestinal drugs: antacids, sucralfate, } \\
\text { magnesium-containing laxatives }\end{array}\end{array}$ & $\begin{array}{l}\text { Give oral ciprofloxacin } 2 \text { hours before or } 6 \\
\text { hours after drugs that may interfere with } \\
\text { absorption. }\end{array}$ \\
\hline & Overlapping Toxicities: & Caution advised. \\
\hline
\end{tabular}




\begin{tabular}{|c|c|c|}
\hline Drug Name & Overlapping Toxicities & Recommendation \\
\hline & • Artemether/lumefantrine, clarithromycin, quinine & \\
\hline \multirow[t]{3}{*}{ Clarithromycin } & $\begin{array}{ll}\text { Increases Clarithromycin Concentrations: } \\
\text { - } & \text { ARV drugs: atazanavir/ritonavir, lopinavir/ritonavir } \\
\text { - } & \text { Antifungals: itraconazole (itraconazole concentrations } \\
& \text { also increased) }\end{array}$ & $\begin{array}{l}\text { Caution advised. Concern for QTc } \\
\text { prolongation. Decrease clarithromycin dose or } \\
\text { consider switching to azithromycin, which has } \\
\text { less potential for drug interactions. }\end{array}$ \\
\hline & $\begin{array}{l}\text { Increases Concentration of Other Medications: } \\
\text { - ARV drugs: etravirine }\end{array}$ & Consider alternative agent. \\
\hline & $\begin{array}{ll}\text { Decreases } & \text { Clarithromycin Concentrations: } \\
& \text { ARV drugs: efavirenz, etravirine, nevirapine } \\
\text { - } & \begin{array}{l}\text { Antimycobacterial drugs: rifampin, rifabutin } \\
\text { (rifabutin concentrations also increased) }\end{array}\end{array}$ & $\begin{array}{l}\text { Consider switching to azithromycin, which has } \\
\text { less potential for drug interaction. } \\
\text { For concomitant use of rifabutin and } \\
\text { clarithromycin, consider decreasing dose of } \\
\text { rifabutin or switching to azithromycin. }\end{array}$ \\
\hline Clindamycin & $\begin{array}{l}\text { Decreases Clindamycin Antibacterial Efficacy: } \\
\text { • Antibacterial drugs: chloramphenicol, erythromycins }\end{array}$ & Avoid concomitant use. \\
\hline Cycloserine & $\begin{array}{l}\text { Overlapping Toxicities: } \\
\qquad \quad \text { Antimycobacterial drugs: ethionamide, isoniazid }\end{array}$ & Caution advised. \\
\hline \multirow[t]{3}{*}{ Dapsone } & $\begin{array}{l}\text { Decreases Dapsone Concentrations: } \\
\text { • Antimycobacterial drugs: rifampin }\end{array}$ & $\begin{array}{l}\text { Co-administration should be avoided if } \\
\text { possible. Consider alternatives for dapsone or } \\
\text { use rifabutin. }\end{array}$ \\
\hline & $\begin{array}{l}\text { Decreases Dapsone Absorption: } \\
\text { - } \\
\text { ARV drugs: didanosine suspension } \\
\text { - Gastrointestinal drugs: antacids }\end{array}$ & $\begin{array}{l}\text { For co-administration with antacids or } \\
\text { didanosine suspension, give dapsone } 1 \text { hour } \\
\text { before or } 4 \text { hours after the other medication. }\end{array}$ \\
\hline & $\begin{array}{l}\text { Overlapping Toxicities: } \\
\qquad \quad \begin{array}{l}\text { Bone marrow suppressant drugs or drugs associated } \\
\text { with hemolysis }\end{array}\end{array}$ & Caution advised. \\
\hline Doxycycline & $\begin{array}{l}\text { Decreases Doxycycline Concentrations: } \\
\text { - } \\
\text { - Anticonvulsant drugs: phenytoin, carbamazepine } \\
\text { Antimycobacterial drugs: rifampin }\end{array}$ & $\begin{array}{l}\text { Potential for decreased doxycycline efficacy. } \\
\text { Monitor for therapeutic failure. }\end{array}$ \\
\hline Erythromycin & $\begin{array}{l}\text { Increases Concentrations of Erythromycin and Co-Administered } \\
\text { Medication: } \\
\text { - Antifungals: itraconazole }\end{array}$ & $\begin{array}{l}\text { Monitor for toxicities of both drugs, potential } \\
\text { for QT prolongation. }\end{array}$ \\
\hline Ethambutol & $\begin{array}{l}\text { Overlapping Toxicities: } \\
\qquad \quad \text { Neurotoxic drugs }\end{array}$ & Caution advised. \\
\hline Ethionamide & $\begin{array}{cl}\text { Potential for Increased Toxicity Due to Overlapping Toxicity: } \\
\text { - } & \text { Neurotoxic drugs } \\
\text { - } & \text { Antimycobacterial drugs: cycloserine, isoniazid }\end{array}$ & Caution advised. \\
\hline Fluconazole & $\begin{array}{l}\text { Decreases Fluconazole Levels: } \\
\text { • Anticonvulsant drugs: phenytoin }\end{array}$ & $\begin{array}{l}\text { Monitor for efficacy. May need to increase } \\
\text { fluconazole dose. }\end{array}$ \\
\hline
\end{tabular}




\begin{tabular}{|c|c|c|}
\hline Drug Name & Overlapping Toxicities & Recommendation \\
\hline & $\begin{array}{ll} & \text { Antimycobacterial drugs: rifampin } \\
\text { - } & \text { ARV drugs: rilpivirine }\end{array}$ & \\
\hline & $\begin{array}{l}\text { Increases Concomitant Drug Concentrations: } \\
\qquad \quad \begin{array}{l}\text { ARV drugs: saquinavir, tipranavir, nevirapine, and } \\
\text { etravirine }\end{array}\end{array}$ & $\begin{array}{l}\text { May need to decrease dose of saquinavir. Avoid } \\
\text { tipranivir with high doses of fluconazole } \\
\text { (maximum fluconazole dose in adults: } 200 \mathrm{mg} \text { ). } \\
\text { Caution advised with etravirine. }\end{array}$ \\
\hline & - Antimycobacterial drugs: rifabutin & May need to decrease dose of rifabutin. \\
\hline & - $\quad$ Statins: simvastatin, lovastatin, atorvastatin & $\begin{array}{l}\text { Do not co-administer with simvastatin or } \\
\text { lovastatin. Avoid use of atorvastatin if possible. } \\
\text { Alternative statins such as fluvastatin, } \\
\text { rosuvastatin, pravastatin are preferred or } \\
\text { discontinue statin during antifungal therapy. }\end{array}$ \\
\hline Flucytosine & $\begin{array}{l}\text { Increases Flucytosine Concentrations: } \\
\qquad \quad \text { Nephrotoxic drugs }\end{array}$ & Caution advised. \\
\hline Foscarnet & $\begin{aligned} & \text { Overlapping Toxicities: } \\
& \text { - } \text { Antiviral drugs: cidofovir } \\
& \text { - } \text { Anti-pneumocystis drugs: pentamidine } \\
& \text { - } \text { Nephrotoxic drugs }\end{aligned}$ & Monitor for toxicities of these drugs. \\
\hline \multirow[t]{3}{*}{ Ganciclovir } & $\begin{array}{l}\text { Increases Ganciclovir Concentrations : } \\
\qquad \quad \text { ARV drugs: tenofovir (concentrations also increased) }\end{array}$ & Monitor for toxicities of these drugs. \\
\hline & $\begin{array}{l}\text { Increases Concomitant Drug Concentrations: } \\
\qquad \quad \text { ARV drugs: didanosine, tenofovir }\end{array}$ & Caution advised. \\
\hline & $\begin{array}{ll}\text { Overlapping Toxicities: } \\
\text { - } & \text { Antibacterial drugs: imipenem-cilastatin } \\
\text { - } & \text { ARV drugs: zidovudine } \\
\text { - } & \text { Bone marrow suppressant drugs } \\
\text { - } & \text { Nephrotoxic drugs }\end{array}$ & $\begin{array}{l}\text { Caution advised. Increased risk of seizures with } \\
\text { imipenem-cilastatin. }\end{array}$ \\
\hline Interferon-Alfa & $\begin{array}{l}\text { Overlapping Toxicities: } \\
\qquad \quad \text { ARV drugs: zidovudine, lamivudine } \\
\text { - } \quad \text { Bone marrow suppressant drugs }\end{array}$ & $\begin{array}{l}\text { Co-administration of zidovudine and } \\
\text { lamivudine should be avoided if possible. } \\
\text { Caution advised with other bone marrow } \\
\text { suppressant drugs. }\end{array}$ \\
\hline \multirow[t]{4}{*}{ Isoniazid } & $\begin{array}{l}\text { Decreases Isoniazid Concentrations: } \\
\quad \text { - Corticosteroids: glucocorticoids (e.g., prednisolone) }\end{array}$ & Use with caution. \\
\hline & $\begin{array}{l}\text { Decreases Isoniazid Absorption: } \\
\qquad \quad \text { Gastrointestinal drugs: antacids }\end{array}$ & Caution advised. \\
\hline & $\begin{array}{l}\text { Increases Concomitant Drug Concentrations: } \\
\qquad \quad \text { Diazepam }\end{array}$ & Caution advised. \\
\hline & Decreases Concomitant Drug Concentrations: & $\begin{array}{l}\text { Co-administration should be avoided, if } \\
\text { possible. }\end{array}$ \\
\hline
\end{tabular}




\begin{tabular}{|c|c|c|}
\hline Drug Name & Overlapping Toxicities & Recommendation \\
\hline & - $\quad$ Antifungal drugs: ketoconazole, itraconazole & \\
\hline & $\begin{array}{ll}\text { Overlapping Toxicities: } \\
\text { - } & \text { Antimycobacterial drugs: rifampin, cycloserine, } \\
\text { ethionamide } \\
\text { - } & \text { Hepatotoxic drugs } \\
\text { - } & \text { Neurotoxic drugs }\end{array}$ & Caution advised. \\
\hline \multirow[t]{10}{*}{ Itraconazole } & $\begin{array}{l}\text { Increases Itraconazole Concentration: } \\
\begin{array}{l}\text { Antibacterial: clarithromycin, erythromycin, } \\
\text { ciprofloxacin }\end{array} \\
\text { - ARVs: protease inhibitors }\end{array}$ & $\begin{array}{l}\text { Monitor for toxicities. Monitor itraconazole } \\
\text { concentration. Consider azithromycin instead of } \\
\text { other macrolides. High doses of itraconazole are } \\
\text { not recommended with PIs. }\end{array}$ \\
\hline & $\begin{array}{l}\text { Increases Concomitant Drug Concentrations: } \\
\text { - ARV drugs: etravirine, maraviroc, protease inhibitors }\end{array}$ & $\begin{array}{l}\text { Caution advised. Monitor for toxicities. } \\
\text { Decrease adult maraviroc dose to } 150 \mathrm{mg} \text { twice } \\
\text { daily. }\end{array}$ \\
\hline & - Statins: lovastatin, simvastatin, atorvastatin & $\begin{array}{l}\text { Do not co-administer with simvastatin or } \\
\text { lovastatin. Avoid use of atorvastatin if possible. } \\
\text { Alternative statins such as fluvastatin, } \\
\text { rosuvastatin, pravastatin are preferred or } \\
\text { discontinue statin during antifungal therapy. }\end{array}$ \\
\hline & - Antibacterial: clarithromycin, erythromycin & $\begin{array}{l}\text { Consider switching to azithromycin, which has } \\
\text { less potential for drug interaction. }\end{array}$ \\
\hline & $\begin{array}{l}\text { Sedatives/hypnotics: midazolam, alprazolam, } \\
\text { diazepam }\end{array}$ & $\begin{array}{l}\text { Co-administration of midazolam and } \\
\text { alprazolam should be avoided. Co- } \\
\text { administration of diazepam should be avoided, } \\
\text { if possible. }\end{array}$ \\
\hline & - Cardiac: quinidine & $\begin{array}{l}\text { Co-administration of quinidine should be } \\
\text { avoided. QT prolongation. }\end{array}$ \\
\hline & $\begin{array}{l}\text { Decreases Itraconazole Concentrations: } \\
\qquad \begin{array}{l}\text { ARV drugs: efavirenz, etravirine, nevirapine, } \\
\text { rilpivirine }\end{array}\end{array}$ & $\begin{array}{l}\text { Monitor itraconazole concentration. Co- } \\
\text { administration of efavirenz should be avoided if } \\
\text { possible. }\end{array}$ \\
\hline & - Anticonvulsant drugs: carbamazepine, (fos)phenytoin & Monitor itraconazole concentration. \\
\hline & $\begin{array}{l}\text { Antimycobacterial drugs: rifampin, rifabutin, } \\
\text { rifapentine, isoniazid }\end{array}$ & $\begin{array}{l}\text { Co-administration with rifampin should be } \\
\text { avoided. Co-administration with rifabutin } \\
\text { should be avoided, if possible. Monitor for } \\
\text { toxicities. Monitor itraconazole concentration. }\end{array}$ \\
\hline & $\begin{array}{ll}\text { Decreases Itraconazole Absorption: } \\
\text { - } & \text { ARV drugs: didanosine } \\
\text { - } & \begin{array}{l}\text { Gastrointestinal drugs: antacids, anticholinergics/ } \\
\text { antispasmodics, histamine } \mathrm{H}_{2} \text {-receptor antagonists, } \\
\text { omeprazole, sucralfate }\end{array}\end{array}$ & Monitor itraconazole concentration. \\
\hline \multirow[t]{2}{*}{ Lumefantrine } & $\frac{\text { Increases Concomitant Drug Levels: }}{\text { - ARV drugs: nevirapine }}$ & Monitor for nevirapine toxicity. \\
\hline & $\begin{array}{ll}\text { Overlapping Toxicities: } \\
\text { - } & \text { ARV drugs: protease inhibitors } \\
\text { - } & \text { Antibacterial drugs: macrolides, fluoroquinolones } \\
\text { - } & \text { Antifungal drugs: fluconazole, voriconazole }\end{array}$ & $\begin{array}{l}\text { Co-administration with fluconazole or } \\
\text { voriconazole should be avoided. For all other } \\
\text { drugs, co-administration should be avoided, if } \\
\text { possible; monitor for toxicities (QT } \\
\text { prolongation). }\end{array}$ \\
\hline
\end{tabular}




\begin{tabular}{|c|c|c|}
\hline Drug Name & Overlapping Toxicities & Recommendation \\
\hline & $\begin{array}{ll}- & \text { Antimalarial drugs: quinine, quinidine } \\
\text { - } & \text { Psychotropic drugs: quetiapine, tricyclic } \\
\text { antidepressants }\end{array}$ & \\
\hline \multirow[t]{3}{*}{ Mefloquine } & $\begin{array}{cc}\text { Decreases Mefloquine Concentrations: } \\
\text { - } & \text { Antimalarial drugs: quinine } \\
\text { - } & \text { Antimycobacterial: rifampin }\end{array}$ & $\begin{array}{l}\text { Monitor for decreased mefloquine efficacy. } \\
\text { Co-administration of rifampin should be } \\
\text { avoided, if possible; use rifabutin instead. }\end{array}$ \\
\hline & $\begin{array}{l}\text { Decreases Concomitant Drug Concentrations: } \\
\qquad \begin{array}{l}\text { ARV drugs: ritonavir, possibly other protease } \\
\text { inhibitors }\end{array}\end{array}$ & $\begin{array}{l}\text { Monitor for virologic failure of protease } \\
\text { inhibitor-containing ART regimen. }\end{array}$ \\
\hline & $\begin{array}{ll}\text { Overlapping Toxicities: } \\
\qquad \quad \text { Anti-malarial drugs: quinine } \\
\text { • } & \text { Other drugs that can cause prolonged QT }\end{array}$ & $\begin{array}{l}\text { Avoid co-administration, if possible. Monitor } \\
\text { for toxicities (EKG changes, cardiac arrest; also } \\
\text { seizures with quinine). If co-administered with } \\
\text { quinine, give mefloquine at least } 12 \text { hours after } \\
\text { last dose of quinine. }\end{array}$ \\
\hline Nitazoxanide & $\begin{array}{l}\text { Increases Concomitant Drug Concentrations: } \\
\text { • Phenytoin }\end{array}$ & $\begin{array}{l}\text { Potential for interaction with other medications } \\
\text { that are highly protein bound. Use with caution } \\
\text { as interaction will increase concentrations of } \\
\text { concomitant medication. }\end{array}$ \\
\hline Paromomycin & $\begin{array}{l}\text { Overlapping Toxicities: } \\
\qquad \quad \text { Neuromuscular blocking drugs }\end{array}$ & Use with caution. \\
\hline \multirow[t]{5}{*}{ Pentamidine } & $\begin{array}{l}\text { Overlapping Toxicities: } \\
\qquad \quad \text { Antiviral drugs: foscarnet }\end{array}$ & $\begin{array}{l}\text { Co-administration should be avoided, if } \\
\text { possible. Monitor for toxicities (hypocalcaemia, } \\
\text { QT prolongation). }\end{array}$ \\
\hline & - $\quad$ ARV drugs: protease inhibitors, didanosine & $\begin{array}{l}\text { Co-administration should be avoided, if } \\
\text { possible. Monitor for toxicities (QT } \\
\text { prolongation with protease inhibitors; } \\
\text { pancreatitis for didanosine). }\end{array}$ \\
\hline & - Bone marrow suppressant drugs & Monitor for toxicities. \\
\hline & - $\quad$ Nephrotoxic drugs & Monitor for toxicities. \\
\hline & - Other drugs that can cause prolonged QT & $\begin{array}{l}\text { Monitor for toxicities. Avoid co-administration, } \\
\text { if possible. }\end{array}$ \\
\hline \multirow[t]{4}{*}{ Posaconazole } & $\begin{array}{l}\text { Decreases Posaconazole Drug Concentrations: } \\
\text { - ARV drugs: efavirenz, fosamprenavir, rilpivirine }\end{array}$ & $\begin{array}{l}\text { Co-administration of fosamprenavir should be } \\
\text { avoided. Co-administration of efavirenz should } \\
\text { be avoided, if possible. If co-administered, } \\
\text { monitor posaconazole concentrations and adjust } \\
\text { dose accordingly. }\end{array}$ \\
\hline & - Anticonvulsant drugs: phenytoin & $\begin{array}{l}\text { Co-administration should be avoided, if } \\
\text { possible. If co-administered, monitor } \\
\text { posaconazole concentrations and adjust dose } \\
\text { accordingly. }\end{array}$ \\
\hline & - $\quad$ Antimycobacterial drugs: rifabutin, rifampin & $\begin{array}{l}\text { Co-administration should be avoided, if } \\
\text { possible. If co-administered, monitor } \\
\text { posaconazole concentrations and adjust dose } \\
\text { accordingly. }\end{array}$ \\
\hline & $\begin{array}{l}\text { Increases Concomitant Drug Concentrations: } \\
\quad \begin{array}{l}\text { ARV drugs: atazanavir, saquinavir, lopinavir, } \\
\text { etravirine, and ritonavir }\end{array}\end{array}$ & $\begin{array}{l}\text { Co-administration should be avoided, if } \\
\text { possible. Monitor for toxicities. Consider } \\
\text { monitoring concentrations and adjust dose as } \\
\text { necessary. }\end{array}$ \\
\hline
\end{tabular}




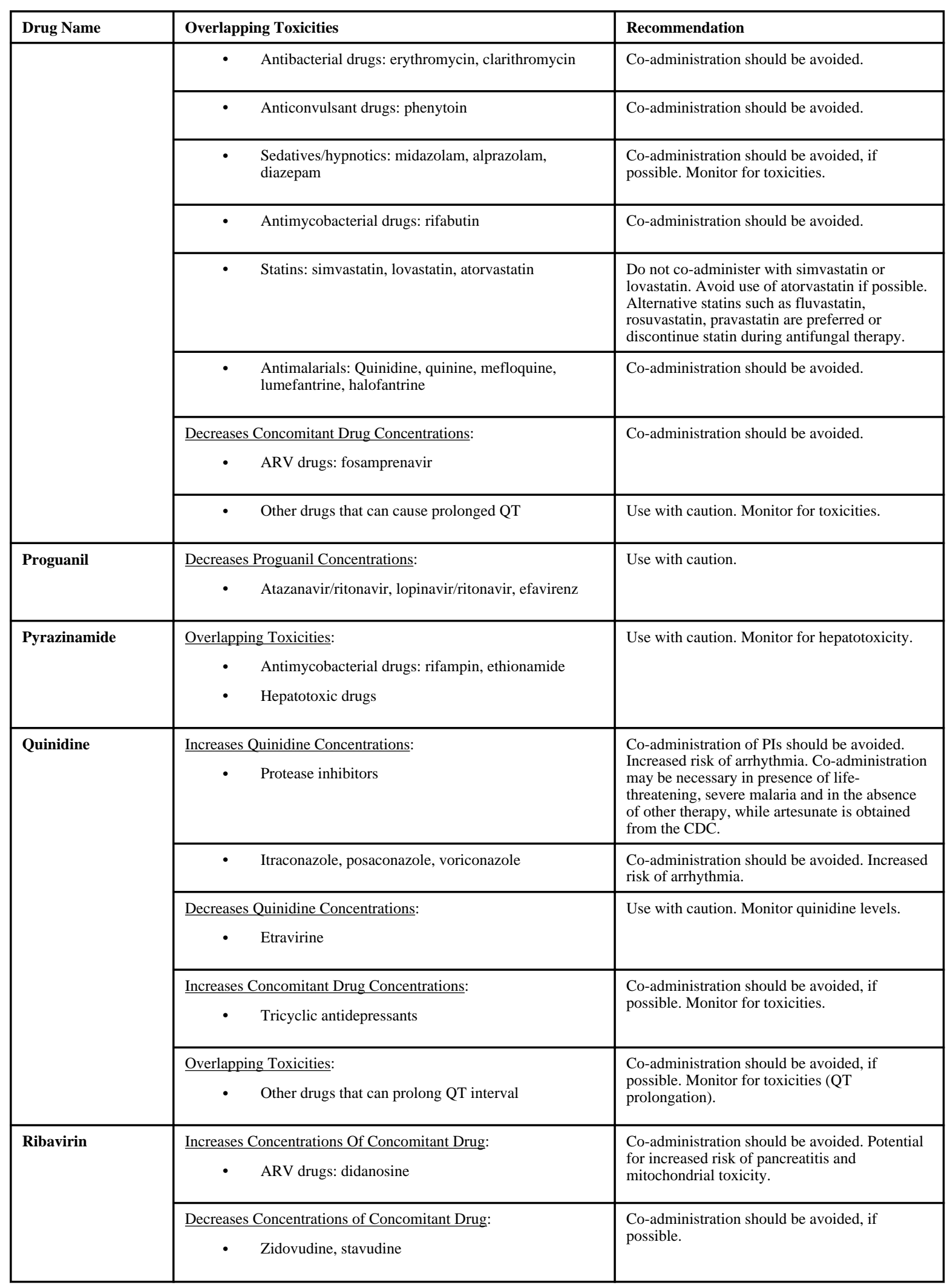




\begin{tabular}{|c|c|c|}
\hline Drug Name & Overlapping Toxicities & Recommendation \\
\hline & $\begin{array}{l}\text { Overlapping Toxicities: } \\
\qquad \quad \text { Zidovudine, all NRTIs }\end{array}$ & $\begin{array}{l}\text { Co-administration should be avoided, if } \\
\text { possible. Monitor for toxicities (anemia for } \\
\text { zidovudine; lactic acidosis for all NRTIs). }\end{array}$ \\
\hline \multirow[t]{11}{*}{ Rifabutin } & $\begin{array}{r}\text { Increases Rifabutin Concentrations: } \\
\qquad \quad \text { HIV protease inhibitors }\end{array}$ & $\begin{array}{l}\text { Use with caution. Monitor for rifabutin toxicity. } \\
\text { Reduce rifabutin dose if co-administered with } \\
\text { PIs. }\end{array}$ \\
\hline & - $\quad$ Fluconazole & $\begin{array}{l}\text { Use with caution. Monitor for rifabutin toxicity. } \\
\text { Consider rifabutin dose reduction. }\end{array}$ \\
\hline & - Voriconazole, itraconazole, posaconazole & $\begin{array}{l}\text { Co-administration should be avoided, if } \\
\text { possible. If co-administered, consider TDM and } \\
\text { monitor for rifabutin toxicities (and azole } \\
\text { clinical efficacy). }\end{array}$ \\
\hline & - $\quad$ Clarithromycin & $\begin{array}{l}\text { Co-administration should be avoided, if } \\
\text { possible. Monitor for rifabutin toxicity. } \\
\text { Consider rifabutin dose reduction or using } \\
\text { azithromycin instead. }\end{array}$ \\
\hline & $\begin{array}{l}\text { Increases Concomitant Drug Concentrations: } \\
\qquad \quad \text { Didanosine }\end{array}$ & $\begin{array}{l}\text { Use with caution. Monitor for didanosine } \\
\text { toxicity. }\end{array}$ \\
\hline & $\frac{\text { Decreases Rifabutin Concentrations: }}{\text { E } \quad \text { Efavirenz, etravirine }}$ & $\begin{array}{l}\text { Use with caution. Higher rifabutin dose } \\
\text { required when efavirenz co-administered. } \\
\text { Consider TDM. }\end{array}$ \\
\hline & $\begin{array}{l}\text { Decreases Concomitant Drug Concentrations: } \\
\text { - ARV drugs: rilpivirine }\end{array}$ & Co-administration should be avoided. \\
\hline & - $\quad$ ARV drugs: saquinavir, etravirine, maraviroc & $\begin{array}{l}\text { Co-administration should be avoided, if } \\
\text { possible. }\end{array}$ \\
\hline & - $\quad$ Antibacterial drugs: dapsone, atovaquone & $\begin{array}{l}\text { Use with caution. Monitor for dapsone } \\
\text { treatment failure. }\end{array}$ \\
\hline & - $\quad$ Antifungal drugs: azoles (except for fluconazole) & $\begin{array}{l}\text { Co-administration should be avoided, if } \\
\text { possible. If co-administered, consider TDM and } \\
\text { monitor for rifabutin toxicities (and azole } \\
\text { clinical efficacy). }\end{array}$ \\
\hline & - Contraceptives: oral & $\begin{array}{l}\text { Oral contraceptives less effective. Additional } \\
\text { non-hormonal contraceptive or alternative } \\
\text { recommended. }\end{array}$ \\
\hline \multirow[t]{4}{*}{ Rifampin } & $\begin{array}{l}\text { Decreases Concomitant Drug Concentrations: } \\
\text { - Contraceptives: oral }\end{array}$ & $\begin{array}{l}\text { Oral contraceptives less effective. Additional } \\
\text { non-hormonal contraceptive or alternative } \\
\text { recommended. }\end{array}$ \\
\hline & $\begin{array}{l}\text { ARV drugs: PIs } \pm \text { ritonavir, nevirapine, raltegravir, } \\
\text { rilpivirine }\end{array}$ & $\begin{array}{l}\text { Significantly decreases PI exposure; co- } \\
\text { administration should be avoided. Nevirapine: } \\
\text { use only if other options not available and close } \\
\text { virologic and immunologic monitoring can be } \\
\text { done; consider efavirenz instead. Raltegravir } \\
\text { dose increase may be required. Rilpivirine co- } \\
\text { administration should be avoided. }\end{array}$ \\
\hline & $\begin{array}{l}\text { Antimicrobial: atovaquone, dapsone, clarithromycin, } \\
\text { doxycycline }\end{array}$ & $\begin{array}{l}\text { Co-administration of atovaquone and rifampin } \\
\text { should be avoided. Consider switching } \\
\text { clarithromycin to azithromycin, which has less } \\
\text { potential for drug interaction. Dapsone and } \\
\text { Doxycycline efficacy may be reduced. }\end{array}$ \\
\hline & - $\quad$ Antifungal drugs: azoles, caspofungin & $\begin{array}{l}\text { Increase in dose of caspofungin is } \\
\text { recommended when co-administered with } \\
\text { CYP450 inducers. } \\
\text { Azoles: Monitor for efficacy. May need to } \\
\text { increase antifungal dose }\end{array}$ \\
\hline
\end{tabular}




\begin{tabular}{|c|c|c|}
\hline Drug Name & Overlapping Toxicities & Recommendation \\
\hline & - Other: corticosteroids, methadone & $\begin{array}{l}\text { Caution advised with corticosteroids (decreased } \\
\text { efficacy). } \\
\text { Methadone: Monitor for efficacy and/or opiate } \\
\text { withdrawal symptoms with methadone. }\end{array}$ \\
\hline & $\begin{array}{l}\text { Overlapping Toxicities: } \\
\qquad \quad \text { Bone marrow suppressant drugs } \\
\text { • } \\
\text { Hepatotoxic drugs }\end{array}$ & Monitor for toxicities of these drugs. \\
\hline Streptomycin & $\begin{array}{cl}\text { Potential for Increased Toxicity Due to Overlapping Toxicity: } \\
\text { - } & \text { Nephrotoxic drugs } \\
\text { - } & \text { Neuromuscular blocking drugs }\end{array}$ & Monitor for toxicities of these drugs. \\
\hline Telaprevir & \multicolumn{2}{|c|}{$\begin{array}{l}\text { Please see Adult OI guidelines for information about drug interactions, including warnings about interactions between } \\
\text { telaprevir and HIV protease inhibitors. Caution advised. }\end{array}$} \\
\hline Trimethoprim-Sulfame & $\begin{array}{l}\text { - } \quad \text { Folate antagonists } \\
\text { - } \quad \text { Bone marrow suppressant drugs }\end{array}$ & Monitor for toxicities of these drugs. \\
\hline Valacyclovir & $\begin{array}{l}\text { Potential For Increased Concentrations (of Both Drugs) and } \\
\text { Overlapping Toxicity: } \\
\text { - } \\
\text { Antivirals: acyclovir, valganciclovir, ganciclovir, } \\
\text { cidofovir } \\
\text { - ARVs: tenofovir }\end{array}$ & Monitor for toxicities of these drugs. \\
\hline Valganciclovir & $\begin{array}{l}\text { Potential for Increased Concentrations (of Both Drugs) and } \\
\text { Overlapping Toxicity: } \\
\text { - } \quad \begin{array}{l}\text { Antivirals: valacyclovir, acyclovir, ganciclovir, } \\
\text { cidofovir }\end{array} \\
\text { - ARVs: tenofovir }\end{array}$ & Monitor for toxicities of these drugs. \\
\hline \multirow[t]{5}{*}{ Voriconazole } & $\begin{array}{l}\text { Decreases Voriconazole Concentrations: } \\
\text { - } \quad \begin{array}{l}\text { Anticonvulsant drugs: carbamazepine, long-acting } \\
\text { barbiturates }\end{array}\end{array}$ & Caution advised. \\
\hline & - Antimycobacterial drugs: rifabutin, rifampin & $\begin{array}{l}\text { Rifabutin and Rifampin co-administration } \\
\text { should be avoided. }\end{array}$ \\
\hline & $\begin{array}{l}\text { ARV drugs: efavirenz, nevirapine, PIs boosted with } \\
\text { ritonavir }\end{array}$ & $\begin{array}{l}\text { Standard doses of efavirenz and voriconazole } \\
\text { should not be used; voriconazole dose may need } \\
\text { to be increased and efavirenz dose decreased, or } \\
\text { use alternative antifungal agent. } \\
\text { Potential for increased PI concentrations and } \\
\text { decreased voriconazole concentrations; consider } \\
\text { monitoring voriconazole concentrations and } \\
\text { adjust dose accordingly; monitor for PI- } \\
\text { associated toxicities or consider using an } \\
\text { alternative antifungal agent. }\end{array}$ \\
\hline & $\frac{\text { Increases Voriconazole Concentrations: }}{\text { - ARV drugs: etravirine }}$ & $\begin{array}{l}\text { Monitor voriconazole concentrations to reduce } \\
\text { toxicity. }\end{array}$ \\
\hline & $\begin{array}{l}\text { Increases Concomitant Drug Concentrations: } \\
\text { • Antimycobacterial drugs: rifabutin }\end{array}$ & Caution advised. \\
\hline
\end{tabular}




\begin{tabular}{|l|c|l|}
\hline Drug Name & Overlapping Toxicities & Recommendation \\
\hline \multirow{2}{*}{} & $\bullet \quad \begin{array}{l}\text { ARV drugs: protease inhibitors boosted with } \\
\text { ritonavir, efavirenz, etravirine }\end{array}$ & Caution advised. \\
\cline { 2 - 3 } & $\bullet \quad$ Statins: simvastatin, lovastatin, atorvastatin & $\begin{array}{l}\text { Statins: Do not co-administer with simvastatin } \\
\text { or lovastatin. Avoid use of atorvastatin if } \\
\text { possible. Alternative statins such as fluvastatin, } \\
\text { rosuvastatin, pravastatin are preferred or } \\
\text { discontinue statin during antifungal therapy. }\end{array}$ \\
\cline { 2 - 3 } & $\bullet \quad \begin{array}{l}\text { Sedatives/hypnotics: midazolam, alprazolam, } \\
\text { triazolam }\end{array}$ & $\begin{array}{l}\text { Co-administration should be avoided if } \\
\text { possible. Monitor for toxicities. }\end{array}$ \\
\hline
\end{tabular}

Key to Acronyms: $\mathrm{ART}=$ antiretroviral therapy; $\mathrm{ARV}=$ antiretroviral $; \mathrm{CDC}=$ Centers for Disease Control and Prevention; $\mathrm{EKG}=$ electrocardiogram; NNRTI = non-nucleoside reverse transcriptase inhibitors; $\mathrm{NRTI}=$ nucleoside reverse transcriptase inhibitors; $\mathrm{OI}=$ opportunistic infection; $\mathrm{PI}=$ protease inhibitors; $\mathrm{PK}=$ pharmacokinetic; $\mathrm{TDM}=$ therapeutic drug monitoring 